\title{
French Intensive Care Society, International congress - Réanimation 2016
}

Published: 17 June 2016

\section{PHYSICIANS ABSTRACTS}

\section{1}

Impact of tracheal cuff shape on microaspiration of gastric contents in intubated critically ill patients: a multicenter randomized controlled study (BEST CUFF)

Emmanuelle Jaillette ${ }^{1}$, Christophe Girault ${ }^{2}$, Guillaume Brunin ${ }^{3}$, Farid Zerimech ${ }^{4}$, Arnaud Chiche ${ }^{5}$, Céline Broucqsault-Dedrie ${ }^{6}$, Cyril Fayolle? Franck Minacori ${ }^{8}$, Isabelle Alves ${ }^{9}$, Stephanie Barrailler ${ }^{10}$, Laurent Robriquet ${ }^{1}$, Fabienne Tamion ${ }^{11}$, Emmanuel Delaporte ${ }^{3}$, Damien Thellier ${ }^{5}$ Claire Delcourte ${ }^{1}$, Alain Duhamel ${ }^{12}$, Saad Nseir ${ }^{1}$

${ }^{1}$ Centre de réanimation, C.h.r.u. Lille, Lille, France; ${ }^{2}$ Réanimation Médicale, Centre Hospitalier Universitaire Rouen, Rouen, France; ${ }^{3}$ Reanimation, Hospital Center De Boulogne-Sur-Mer, Boulogne-sur-Mer, France; ${ }^{4}$ Laboratoire de biochimie et biologie moléculaire, C.h.r.u. Lille, Lille, France; ${ }^{5}$ Réanimation, Centre Hospitalier de Tourcoing, Tourcoing, France; ${ }^{6}$ Réanimation, Hôpital Victor Provo, Roubaix, France; ${ }^{7}$ Reanimation, Hospital Center De Dunkerque, Dunkerque, France; ${ }^{8}$ Reanimation, Hospital Saint Philibert, Lille, France; ${ }^{9}$ Reanimation, Hospital Center De Valenciennes, Valenciennes, France; ${ }^{10}$ Reanimation, C.H. de Lens, Lens, France; ${ }^{11}$ Réanimation médicale, Hospital Center University Rouen, Rouen, France;

${ }^{12}$ Clinique de santé publique, plateforme d'aide méthodologique, C.h.r.u. Lille, Lille, France

Correspondence: Emmanuelle Jaillette - emmanuelle.jaillette@chru-lille.fr Annals of Intensive Care 2016, 6(Suppl 1):01

Introduction Ventilator-associated pneumonia (VAP) is the most common ICU-acquired infection in intubated critically ill patients. Microaspiration of gastric and oropharyngeal contaminated secretions represents the primary mechanism involved in the pathogenesis of VAP. Tracheal cuff plays an important role in stopping the progression of contaminated secretions into the lower respiratory tract. In vitro and animal studies suggested that leakage was significantly reduced with polyvinyl chloride (PVC) conical-cuffed tubes compared with barrel (standard) or cylindrical cuffs. Clinical studies found conflicting results. Therefore, the aim of this study is to determine the superiority of PVC conical- versus barrel (standard)-cuffed tracheal tube on abundant microaspiration of gastric contents in intubated critically ill patients. Materials and methods BEST CUFF is a prospective multicenter (ten French ICUs) cluster randomized controlled crossover and open-label trial performed in patients with predicted duration of mechanical ventilation $\geq 48 \mathrm{~h}$. Patients were allocated to be intubated using a PVC standard (barrel)-shaped or a PVC conical-shaped tracheal tube. Prevention measures of VAP were standardized in all ICUs. The main objective was to demonstrate the superiority of conical versus standard cuff shape in reducing abundant microaspiration of gastric contents (pepsin level $>200 \mathrm{ng} / \mathrm{ml}$ in at least $30 \%$ of tracheal aspirates). After inclusion, tracheal aspirates were collected for $48 \mathrm{~h}$ to measure pepsin and salivary amylase, and diagnose gastric and oropharyngeal microaspiration. To diagnose tracheobronchial colonization, quantitative aspirate was performed after intubation and two times a week until extubation. In patients with suspected VAP, quantitative tracheal aspirate or bronchoalveolar lavage was performed to confirm the diagnosis. We hypothesized that the use of conical-cuffed tracheal tubes would reduce the incidence of abundant microaspiration of gastric contents from 50 to $30 \%$ of study patients. With a two-sided alpha risk of $5 \%$, a power of $80 \%$, and to account for an anticipated rate of $10 \%$ of patients without any tracheal secretions, 312 patients had to be recruited.

Results A total of 326 patients were included during the study period (June 2014-September 2015). Pepsin and salivary amylase measurement is actually performed, and all results should be available in November 2015. Statistical analyses will be performed in mid-December, and study results will be presented at the 2016 Réanimation Congress.

Discussion Our study is sufficiently powered to detect a significant difference in microaspiration of gastric contents between patients intubated with conical-cuffed tracheal tubes and standard-cuffed tracheal tubes. One of the strengths of this study is the use of quantitative measurement of pepsin as a marker of microaspiration.

Conclusion BEST CUFF is the first randomized controlled study evaluating the impact of PVC tracheal cuff shape on microaspiration of gastric contents. The results will be presented at the 2016 Réanimation Congress.

\section{Competing interests None.}

\section{2}

Bicarbonate versus saline for contrast-induced acute kidney injury prevention in critically ill patients

Xavier Valette ${ }^{1}$, Isabelle Desmeulles², Benoit Savary ${ }^{3}$, Romain Masson², Amélie Seguin ${ }^{1}$, Cédric Daubin', Bertrand Sauneuf ${ }^{1}$, Jennifer Brunet ${ }^{2}$, Pierre Verrier ${ }^{1}$, Véronique Pottier ${ }^{2}$, Marie Orabona ${ }^{2}$, Désiré Samba ${ }^{2}$, Gérald Viquesnel ${ }^{2}$, Mathilde Lermuzeaux ${ }^{2}$, Pascal Hazera ${ }^{3}$, Jean-Luc Hanouz ${ }^{2}$, Jean-Jacques Parienti ${ }^{4}$, Damien Du Cheyron ${ }^{2}$

${ }^{1}$ Réanimation médicale, C.H.U de Caen, Caen, France; ${ }^{2}$ Réanimation chirurgicale, C.H.U de Caen, Caen, France; ${ }^{3}$ Réanimation polyvalente, C.H. Mémorial France - Etats-Unis (Saint-Lô), Saint-Lô, France; ${ }^{4}$ Unité de biostatistique et de recherche clinique, C.H.U de Caen, Caen, France Correspondence: Xavier Valette - valette312ax@sfr.fr Annals of Intensive Care 2016, 6(Suppl 1):O2

Introduction The administration of contrast media for imaging and interventional procedures is frequent in intensive care unit (ICU). Whether volume expansion with bicarbonate is better than isotonic saline to reduce the risk of contrast-induced acute kidney injury ( $\mathrm{Cl}$ AKI) in critically ill patients is unknown.

Materials and methods The HYDRAREA study was a randomized, double-blind, multicenter trial including adults admitted in three ICUs in France who received intravascular contrast media. Patients with unstable renal function (increase in serum creatinine level of at least $0.3 \mathrm{mg} / \mathrm{dL}$ during the previous $48 \mathrm{~h}$, anuria during the previous $12 \mathrm{~h}$, renal replacement therapy) or contraindication to volume expansion 
or bicarbonate load (uncontrolled cardiogenic pulmonary edema, metabolic alkalosis with $\mathrm{pH}>7.50$, hypokalemia $<3 \mathrm{mmol} / \mathrm{L}$, chronic hypercapnia) were not included. Patients were randomized in a 1:1 ratio to receive $0.9 \%$ sodium chloride or $1.4 \%$ sodium bicarbonate administered with the same infusion protocol: $3 \mathrm{~mL} / \mathrm{kg}$ for $1 \mathrm{~h}$ started before contrast media administration followed by $1 \mathrm{~mL} / \mathrm{kg} / \mathrm{h}$ for $6 \mathrm{~h}$. The primary outcome was the incidence of $\mathrm{Cl}-\mathrm{AKI}$ defined by the Acute Kidney Injury Network criteria within $72 \mathrm{~h}$ after contrast medium administration. Secondary outcomes included Cl-AKI using alternative definitions (AKIN without urine output criteria, RIFLE classification, creatinine increase $\geq 0.5 \mathrm{mg} / \mathrm{dL}$ or $25 \%$ ), need for renal replacement therapy, ICU length of stay and mortality.

Results Among the 307 patients randomized, the mean (SD) age was $56 \pm 15$ years and $208(68 \%)$ patients were male. Patients were admitted in ICU for medical reason in $132(43 \%)$ patients, for emergency surgery in 118 (38\%) patients, following trauma in 45 (15\%) patients and after a scheduled surgery in $12(4 \%)$ patients. The mean (SD) SAPS II and SOFA scores at ICU admission were $42 \pm 17$ and $7 \pm 4$, respectively. Contrast media were used for computed tomography in $193(63 \%)$ patients and for arteriography in $114(37 \%)$ patients. Low-osmolar contrast media were used in $276(90 \%)$ patients. At randomization, 243 (79\%) patients were under mechanical ventilation, 100 (33\%) patients needed vasopressor, 104 (34\%) patients had sepsis, and $40(13 \%)$ patients had a RIFLE stage equal or higher than the "Risk of Kidney Injury" class. Patient characteristics were well balanced between groups excepted for the use of nephrotoxic medications which was more common in saline group (40 (26\%) patients vs $59(38 \%)$ patients; $p<0.05)$.

As expected, patients receiving bicarbonate $(n=151)$ had higher urinary $\mathrm{pH}$ at the end of the infusion than patients receiving saline $(n=156)(6.7 \pm 2.1$ vs $6.2 \pm 1.8$, respectively; $p<0.001)$. The incidence of $\mathrm{Cl}-\mathrm{AKI}$ was similar in both groups: $52(33.3 \%)$ patients in saline group and 53 (35\%) patients in bicarbonate group (RR 0.96; $95 \% \mathrm{Cl}$ $[0.70-1.30] ; p=0.81$ ). There was no difference between groups whatever the definition used to define CI-AKI. The need for renal replacement therapy (five (3.2\%) patients in saline group vs six (4\%) patients in bicarbonate group; RR 0.81; $95 \% \mathrm{Cl}[0.25-2.59] ; p=0.77$ ), ICU length of stay (24.7 \pm 22.9 days in saline group vs $23 \pm 23.8$ days in bicarbonate group, $p=0.52)$ and mortality $(25(16 \%)$ patients in saline group vs $24(15.9 \%)$ patients in bicarbonate group; RR 1.00; $95 \% \mathrm{Cl}$ $[0.60-1.68] ; p=0.97)$ were also similar between groups.

Conclusion Hydration with sodium bicarbonate for $\mathrm{Cl}-\mathrm{AKI}$ prevention in critically ill patients does not provide supplementary beneficial effect in comparison with hydration by isotonic sodium chloride.

\section{Competing interests None.}

\section{3}

Neurally adjusted ventilatory assist in the early phase of weaning from mechanical ventilation: a multicenter randomized study Alexandre Demoule', Marc Clavel${ }^{2}$, Camille Rolland-Debord', Sébastien Perbet $^{3}$, Nicolas Terzi ${ }^{4}$, Achille Kouatchet ${ }^{5}$, Florent Wallet ${ }^{6}$, Hadrien Roze ${ }^{7}$, Frédéric Vargas ${ }^{8}$, Claude Guérin ${ }^{9}$, Jean Dellamonica ${ }^{10}$, Samir Jaber ${ }^{11}$,

Thomas Similowski ${ }^{12}$

${ }^{1}$ Intensive Care and Respiratory Division, Pitié-Salpêtrière Hospital, Paris, France; ${ }^{2}$ Service de réanimation polyvalente, Centre Hospitalier Universitaire de Limoges, Limoges, France; ${ }^{3}$ Service de réanimation adultes, C.H.U. Estaing, Clermont-Ferrand, France; ${ }^{4}$ Service de réanimation médicale, Clinique de Réanimation Médicale, Grenoble, France; ${ }^{5}$ Réanimation médicale, Centre Hospitalier Universitaire d'Angers, Angers, France; ${ }^{6}$ Réanimation médicale et chirurgicale, CHU Lyon Sud, Lyon, France; ${ }^{7}$ Sar 2, unité réanimation thoracique greffe, $\mathrm{CHU}$ de Bordeaux, Bordeaux, France; ${ }^{8}$ Réanimation médicale, Centre Hospitalier Universitaire de Bordeaux, Bordeaux, France; ${ }^{9}$ Réanimation médicale, Hôpital de la Croix-Rousse, Lyon, France; ${ }^{10}$ Réanimation médicale, Centre Hospitalier Universitaire Archet, Nice, France; ${ }^{11}$ DAR B, Hôpital Saint Eloi, Montpellier, France;

${ }^{12}$ Service de pneumologie et réanimation médicale, Groupe Hospitalier Pitié-Salpêtrière, Paris, France

Correspondence: Alexandre Demoule - alexandre.demoule@aphp.fr Annals of Intensive Care 2016, 6(Suppl 1):O3
Introduction Neurally adjusted ventilatory assist (NAVA) is a ventilatory mode that tailors the level of assistance delivered by the ventilator to the electromyographic activity of the diaphragm. The objective of the present study was to compare the impact of mechanical ventilation with NAVA or pressure support ventilation (PSV) on the early phase of weaning from mechanical ventilation.

Patients and methods A multicenter randomized controlled trial of 128 intubated adults recovering from an acute respiratory failure was conducted in 11 intensive care units (ICUs) from April 2010 to June 2012. Patients were randomly assigned to NAVA or PSV. The primary endpoint was the probability to remain in an assisted mode during the entire first $48 \mathrm{~h}$. Secondary endpoints included asynchrony index, duration of mechanical ventilation and hospital mortality at 28 days.

Results The proportion of patients remaining in an assisted mode during the entire first $48 \mathrm{~h}$ was $67.7 \%$ in the NAVA group versus $69.4 \%$ in the PSV group $(p=0.47)$. The time spend in an assisted mode similar in the two groups (47 [43-48] vs. 47 [40-48], $p=0.55$ ). The asynchrony index was lower in the NAVA group (19.7 vs. $32.6 \%$ ). Duration of mechanical ventilation was similar in the two groups (12.0 [7.0-17.0] in the NAVA group vs. 13.5 [10.0-21.0] in the PSV group, $p=0.121$ ) as was the ICU mortality rate (12.9 vs. $21.2 \%, p=0.21)$. More patients required post-extubation noninvasive mechanical ventilation in the PSV group (66.6 vs. $43.5 \%, p=0.008$ ).

Conclusion NAVA can be applied efficiently in a clinical setting and improves patient ventilator interaction. However, NAVA does not increase the probability to remain in an assisted mode during the first $48 \mathrm{~h}$.

Competing interests A. Demoule: reserche contract with Maquet, Drager, Philips, Covidien. Consulting for Covidien. Speaker for Covidien.

\section{4}

Very high volume hemofiltration with the Cascade system in septic shock patients

Jean-Pierre Quenot ${ }^{1}$, Christine Binquet ${ }^{2}$, Christophe Vinsonneau ${ }^{3}$, Saber-Davide Barbar ${ }^{4}$, Sandrine Vinault, ${ }^{2}$, Valérie Deckert ${ }^{5}$, Stephanie Lemaire ${ }^{5}$, Ali Ait Hssain ${ }^{6}$, Rémi Bruyère ${ }^{7}$, Bertrand Souweine ${ }^{6}$, Laurent Lagrost $^{5}$, Christophe Adrie ${ }^{8}$

${ }^{1}$ Réanimation médicale, CHU Dijon, Dijon, France; ${ }^{2}$ Centre d'investigation clinique, Chu Dijon, Dijon, France; ${ }^{3}$ Réanimation polyvalente, Centre Hospitalier Marc Jacquet, Melun, France; ${ }^{4} 30$, Chru De Nîmes, Nîmes, France; ${ }^{5} 21$, Faculté de médecine, Dijon, France; ${ }^{6}$ Réanimation médicale, CHU Gabriel-Montpied, Clermont-Ferrand, France; ${ }^{7} 01, \mathrm{CH}$ de Fleyrat, Bourg-en-Bresse, France; ${ }^{8}$ Physiologie, Hôpital Cochin, Paris, France Correspondence: Jean-Pierre Quenot - jean-pierre.quenot@chu-dijon.fr Annals of Intensive Care 2016, 6(Suppl 1):O4

Introduction We compared hemodynamic and biological effects of the Cascade system, which uses very high volume hemofiltration (HVHF) $(120 \mathrm{ml} / \mathrm{kg} / \mathrm{h})$, with those of usual care in patients with septic shock.

Patients and methods Multicenter, prospective, randomized, openlabel trial was conducted in three intensive care units (ICU). Adults with septic shock with administration of epinephrine/norepinephrine were eligible. Patients were randomized to usual care plus HVHF (Cascade group) or usual care alone (Control group). Primary endpoint was the number of catecholamine-free days up to 28 days after randomization. Secondary endpoints were number of days free of mechanical ventilation, renal replacement therapy (RRT) or ICU up to 90 days, and 7-, 28- and 90-day mortality.

Results We included 60 patients (29 Cascade and 31 usual care). Baseline characteristics were comparable. Median number of catecholamine-free days was 22 [IQR 11-23] versus 20 [0-25] for Cascade versus Control; there was no significant difference even after adjustment. There was no significant difference in number of mechanical ventilation-free days or ICU requirement. Median number of RRT-free days was 85 [46-90] versus 74 [0-90] for Cascade versus Control groups, $p=0.42$. By multivariate analysis, the number of RRT-free days was significantly higher in the Cascade group (up to 25 days higher after adjustment). There was no difference in mortality at 7,28 or 90 days. 
Conclusion Very HVHF using the Cascade system can safely be used in patients presenting septic shock, but was not associated with a reduction in the need for catecholamines during the first 28 days.

\section{Competing interests None.}

\section{5}

\section{Effect of rapid response systems on hospital mortality, a} prospective interventional study and systematic review

Boris Jung ${ }^{1}$, Aurelien Daurat ${ }^{1}$, Audrey De Jong ${ }^{1}$, Gérald Chanques ${ }^{1}$, Martin Mahul, ${ }^{1}$, Marion Monnin ${ }^{2}$, Nicolas Molinari ${ }^{3}$, Samir Jaber ${ }^{1}$

${ }^{1}$ Dar b, Hôpital Saint Eloi, Montpellier, France; ${ }^{2}$ Dar b, CHRU Hôpital Saint Eloi, Montpellier, France; ${ }^{3}$ Dim, Hôpital La Colombière, Montpellier, France Correspondence: Boris Jung - boris.jung@me.com

Annals of Intensive Care 2016, 6(Suppl 1):05

Introduction Although rapid response systems (RRSs) are known to reduce in hospital cardiac arrest rate, their effect on mortality remains in question. The present study aimed at evaluating the effect of a medical emergency team (MET) implementation on mortality in hospitalized patients.

Patients and methods A prospective study was conducted in the four hospitals of the regional healthcare center of Montpellier, France. An intensivist-led MET was implemented on a $24 / 7$ basis in only one of the four hospitals from January 2012 to June 2012. Patients $>18$ years admitted for more than $24 \mathrm{~h}$ in the medical-surgical wards from July 2010 to December 2011 (pre-MET period) and from July 2012 to December 2013 (MET period) were included. The main outcome was unexpected mortality in hospitalized patients. An updated systematic review and meta-analysis were also performed.

Results A total of 137,251 patients were admitted for $24 \mathrm{~h}$ or more in the medical-surgical wards during the pre-MET and MET periods. MET implementation was associated with a decrease in unexpected mortality rate in the hospital that implemented MET (from 21.9 to 17.4 per 1000 admissions; $P=0.002$ ). Reduction in unexpected mortality associated with MET implementation could be estimated at 1.5 lives saved per week in the MET hospital. In the three other hospitals, mortality rate was not significantly modified (from 19.5 to 19.9 per 1000 admissions; $P=0.69)$. Patients in the MET hospital were more frequently admitted to the intensive care unit (ICU) during the MET period (45.8 vs 52.9 per $1000 ; P=0.002$ ), and their sequential organ failure assessment (SOFA) score upon ICU admission significantly decreased from 7 [4-10] to 5 [2-9]; $P<0.001$. The updated meta-analysis including the present results showed that RRS was associated with a significant decrease in both unexpected (OR $0.51 ; 95 \% \mathrm{Cl} 0.35-0.76)$ and overall mortality (OR $0.89 ; 95 \% \mathrm{Cl} 0.85-0.93$ ).

Conclusion In the present prospective study, implementation of a MET was associated with a decrease in unexpected and overall mortality. Updated meta-analysis confirms the benefit of RRS on unexpected and overall mortality (Fig. 1).

\section{Competing interests None.}
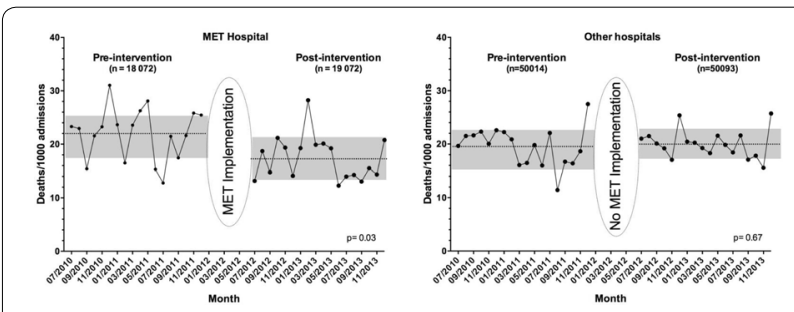

Fig. 1 Evolution of unexpected death rate per 1000 admissions by month in the MET pavilion (left side) and in the three other pavilions (right side). Dotted lines represent the mean rate per month. Grey rectangles represent the standard deviations
06

Beta-lactams serum concentrations in critically ill cirrhotic patients: a matched control study

Olivier Lheureux ${ }^{1}$, Eric Trepo ${ }^{2}$, Maya Hites ${ }^{3}$, Frederic Cotton ${ }^{4}$, Fleur Wolff ${ }^{4}$, Rudy Surin ${ }^{3}$, Jacques Créteur ${ }^{1}$, Jean-Louis Vincent ${ }^{1}$, Thierry Gustot ${ }^{2}$,

Frederique Jacobs $^{3}$, Fabio Silvio Taccone

${ }^{1}$ Service de soins intensifs, Hospital Erasme, Brussel, Belgium; ${ }^{2}$ Service de gastroentérologie, Hospital Erasme, Brussel, Belgium; ${ }^{3}$ Service de maladies infectieuses, Hospital Erasme, Brussel, Belgium; ${ }^{4}$ Service de biochimie médicale, Hospital Erasme, Brussel, Belgium

Correspondence: Olivier Lheureux - olheureu@ulb.ac.be Annals of Intensive Care 2016, 6(Suppl 1):06

Introduction Few data are available on the pharmacokinetics of $\beta$-lactams in critically ill cirrhotic patients. The objective of this study was to evaluate whether $\beta$-lactam concentrations were altered in patients with cirrhosis compared with other critically ill patients and to identify the principal risk factors for any differences.

Materials and methods We reviewed data from critically ill cirrhotic patients and matched controls in which routine therapeutic drug monitoring of broad-spectrum $\beta$-lactam antibiotics (piperacillin/ tazobactam; meropenem) was performed. Serum drug concentrations were measured twice during the elimination phase by high-performance liquid chromatography. Antibiotic pharmacokinetics was calculated using a one-compartment model. We considered therapy was adequate when serum drug concentrations were between 4 and 8 times the minimal inhibitory concentration of Pseudomonas aeruginosa during optimal periods of time for each drug $\geq 50 \%$ for piperacillin/tazobactam; $\geq 40 \%$ for meropenem).

Results We studied 38 cirrhotic patients (16 for piperacillin/tazobactam and 22 for meropenem) and 38 matched controls. Drug dosing was similar in the two groups. The pharmacokinetic analysis showed a lower volume of distribution of meropenem $(p=0.05)$ and a lower antibiotic clearance of piperacillin/tazobactam $(p=0.009)$ in patients with cirrhosis when compared to non-cirrhotic patients. Patients with cirrhosis more often had excessive (23/38) than insufficient $(5 / 38)$ or adequate (10/38) serum $\beta$-lactam concentrations; they also had excessive drug concentrations more frequently than did control patients $(23 / 38$ vs. $13 / 38-p=0.02)$, particularly for piperacillin/tazobactam ( $9 / 16$ vs. $2 / 16-p=0.01$ ). The only variable significantly associated with insufficient drug concentrations was the creatinine clearance on the day of the therapeutic drug monitoring. No specific variable was associated with excessive drug concentrations.

Conclusion This case-control study shows that standard doses of $\beta$-lactams result in excessive serum concentrations in two-thirds of critically ill patients with cirrhosis, especially in those treated with piperacillin/tazobactam. Our results support routine $\beta$-lactam therapeutic drug monitoring in this patient population.

\section{Competing interests None.}

\section{References}

1. Roberts JA, Paul SK, Akova M, Bassetti M, De Waele JJ, Dimopoulos G et al. DALI: defining antibiotic levels in intensive care unit patients: are current $\beta$-lactam antibiotic doses sufficient for critically ill patients? Clin Infect Dis. 2014;58(8):1072-1083.

2. Gustot T, Felleiter P, Pickkers P, Sakr Y, Rello J, Velissaris D et al. Impact of infection on the prognosis of critically ill cirrhotic patients: results from a large worldwide study. Liver Int. 2014;34(10):1496-1503.

07 Systematic overdosing of oxa- and cloxacillin in severe infections treated in ICU: Risk factors and side effects

Mathilde Neuville ${ }^{1}$, Jean-François Timsit ${ }^{1}$, Najoua El-Helali ${ }^{2}$, Alban Le Monnier ${ }^{2}$, Eric Magalhaes ${ }^{1}$, Aguila Radjou ${ }^{1}$, Roland Smonig ${ }^{1}$, Jean-François Soubirou ${ }^{1}$, Guillaume Voiriot ${ }^{1}$, Romain Sonneville ${ }^{1}$, Lila Bouadma $^{\uparrow}$, Bruno Mourvillier ${ }^{1}$

${ }^{1}$ Réanimation médicale et infectieuse, Hôpital Bichat-Claude Bernard, Paris, France; ${ }^{2}$ Unité de microbiologie clinique et dosages des anti-infectieux, Groupe hospitalier Paris Saint-Joseph, Paris, France 


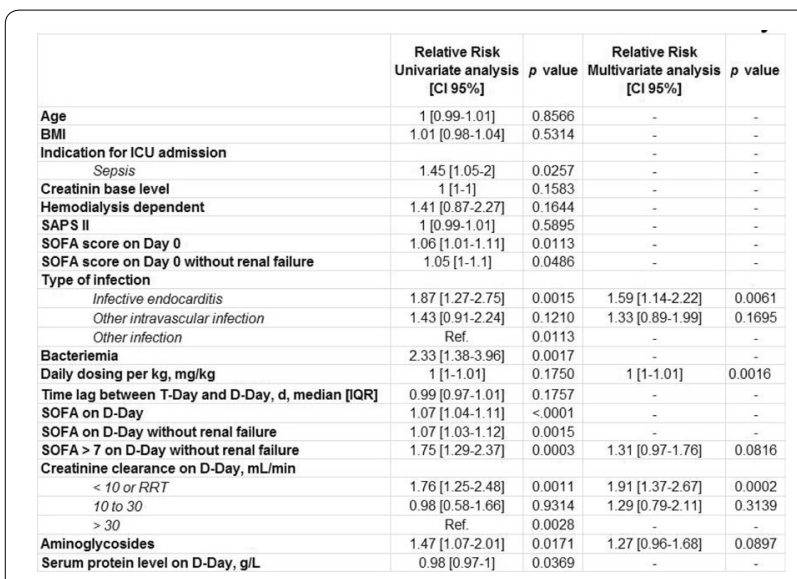

Fig. 2 Univariate and multivariate statistical analysis. Day $0=$ day of ICU admission, T-day $=$ day of treatment initiation, D-day = day of TDM

Correspondence: Mathilde Neuville - mathilde.neuville@aphp.fr Annals of Intensive Care 2016, 6(Suppl 1):07

Introduction Oxacillin and cloxacillin are the most frequently used penicillins for the treatment of severe methicillin-susceptible Staphylococcus aureus (MSSA) infections in intensive care units (ICU), especially endocarditis. No adaptation of the dosage is required in case of renal failure. We wanted to assess the risk factors for overdosing in ICU, and the related observed side effects.

Patients and methods All patients with a therapeutic drug monitoring (TDM) of oxa- or cloxa-cillin between 2008 and 2014 were included. The optimal trough concentration was considered to be $20 \mathrm{mg} / \mathrm{L}$, i.e., 10 times the minimum inhibitory concentration of cloxaxillin for MSSA. Data concerning the medical history, the characteristics of the infection, the given treatment, the renal function and the attributed side effects of overdosing were collected. A negative binomial model was used to compute the measured trough concentrations.

Results Sixty-two patients were included in this study. We found a median trough plasma concentration of $134.3 \mathrm{mg} / \mathrm{L}$ [IQR $65.3-$ $201 \mathrm{mg} / \mathrm{L}]$. Only one patient reached the target concentration; all other patients were overdosed. Eleven patients $(17.7 \%)$ experienced neurological side effects attributed to a high antibiotic concentration, i.e., persistent coma and delirium. When adjusted on the dosage used, the first trough concentration was significantly increased by a creatinine clearance below $10 \mathrm{ml} / \mathrm{min}$ (with or without hemodialysis) and by endocarditis as the source of infection (Fig. 2).

Conclusion With the recommended dose of $12 \mathrm{~g} /$ day for cloxacillin treatment in case of endocarditis and severe infections occurring in ICU, almost all patients are largely overdosed. Considering the high frequency of observed side effects, doses should be accurately monitored and reduced, particularly when renal replacement therapy is needed.

\section{Competing interests None.}

\section{8}

Amikacin peak concentrations in patients receiving extracorporeal membrane oxygenation (ECMO) support: a casecontrol study

Elodie Gélisse', Mathilde Neuville ${ }^{2}$, Etienne De Montmollin ${ }^{3}$, Guillaume Voiriot $^{2}$, Jean-François Soubirou ${ }^{2}$, Roland Smonig², Aguila Radjou², Eric
Magalhaes², Lila Bouadma², Bruno Mourvillier ${ }^{2}$, Jean-François Timsit ${ }^{2}$, Romain Sonneville ${ }^{2}$

${ }^{1}$ Unité de réanimation robert debré, CHU Reims, Reims, France; ${ }^{2}$ Service de réanimation médicale et infectieuse, Hôpital Bichat-Claude Bernard-APHP, Paris, France; ${ }^{3}$ Réanimation, C.H. Général Saint Denis hôpital Delafontaine, Saint-Denis, France

Correspondence: Elodie Gélisse - elodie.gelisse@gmail.com Annals of Intensive Care 2016, 6(Suppl 1):08

Introduction More than half of adult patients receiving extracorporeal membrane oxygenation (ECMO) require an antibiotic therapy during their stay in ICU. Amikacin is the most used aminoglycoside for the treatment of septic shock given its good bactericidal activity on Pseudomonas aeruginosa and the low resistance rate observed with the other Gram-negative bacilli. However, data on pharmacokinetics of amikacin in patients receiving ECMO support have scantly been studied in these conditions. We aimed to report on the distribution of peak serum concentration $\left(C_{\max }\right)$ of amikacin and on factors associated with insufficient $\mathrm{Cmax}$ in a population of critically ill patients receiving ECMO.

Materials and methods We conducted an observational single-center study of patients admitted to a general ICU. Inclusion criteria were: (1) age $\geq 18$ years and (2) a suspected Gram-negative infection requiring a loading dose of intravenous amikacin. Amikacin was routinely administered intravenously at a dose of $25 \mathrm{mg} / \mathrm{kg}$ of total body weight over a 30-min infusion time. $C_{\max }$ was measured $30 \mathrm{~min}$ after the end of infusion and trough serum concentration $24 \mathrm{~h}$ after the end of infusion $\left(C_{\min }\right)$. ECMO patients were matched with critically ill patients without ECMO support (controls) for characteristics that had been previously identified as independent predictors for insufficient $C_{\max }$ namely body mass index (BMI), cirrhosis and 24-h fluid balance. An amikacin $C_{\max }>60 \mathrm{mg} / \mathrm{L}$ and $<80 \mathrm{mg} / \mathrm{l}$ was considered the target concentration (adequate drug concentrations). An amikacin $C_{\max }<60 \mathrm{mg} / \mathrm{L}$ was considered as an insufficient drug concentration, and a $C_{\max }>80 \mathrm{mg} / \mathrm{l}$ was considered as an excessive one. The primary outcome was the distribution of $C_{\max }(\mathrm{mg} / \mathrm{L})$ between ECMO patients and controls.

Results A total of 50 episodes in $46 \mathrm{ECMO}$ patients were matched with 50 controls. Baseline characteristics did not differ between groups, except for age (61 vs. 63.5 years), reason for ICU admission and SOFA score (12 [10-14] vs. 9 [6-11]). At the day of $C_{\text {max }}$ in ECMO patients the SOFA score was 9 [5.3-9.8], and $100 \%$ episodes required mechanical ventilation, $96 \%$ vasopressors and $44 \%$ renal replacement therapy. There were no significant differences in serum concentrations between ECMO and non-ECMO patients: The amikacin Cmax was 71.7 [58.9-79.7] mg/l for ECMO patients versus 68.4 [53-81] mg/l for nonECMO patients $(p=0.36)$. The proportion of insufficient $[13 / 50(26 \%)$ episodes vs. $17 / 50$ (34 \%) episodes), adequate (25/50 (50\%) episodes vs. $18 / 50$ (36 \%) episodes] and excessive [12/50 (24 \%) episodes vs. $15 / 50(30 \%)$ episodes] drug concentrations was similar in ECMO and non-ECMO patients (Table 1). $C_{\min }$ was measured in $43(86 \%)$ episodes, and $28 / 43(65 \%)$ measurements were above the toxic threshold of $5 \mathrm{mg} / \mathrm{L}$ in ECMO patients versus 30/50 (60\%) in non-ECMO patients. Conclusion ECMO therapy did not significantly impact on peak and trough serum concentrations of amikacin.

Competing interests None.

\section{References}

1. Schmidt M, Brechot N, Hariri S, Guiguet M, Luyt CE, Makri R, et al. Nosocomial Infections in Adult Cardiogenic Shock Patients Supported by Venoarterial Extracorporeal Membrane Oxygenation. Clin Infect Dis. 2012 Dec 15;55(12):1633-41.

2. De Montmollin E, Bouadma L, Gault N, Mourvillier B, Mariotte E, Chemam $S$, et al. Predictors of insufficient amikacin peak concentration in critically ill patients receiving a $25 \mathrm{mg} / \mathrm{kg}$ total body weight regimen. Intensive Care Med. 2014 Jul;40(7):998-1005. 


\begin{tabular}{|c|c|c|c|}
\hline Variables & $\mathrm{ECMO}(n=50)$ & $\begin{array}{l}\text { Non-ECMO } \\
(n=50)\end{array}$ & $P$ value \\
\hline \multicolumn{4}{|c|}{ Characteristics at ICU admission } \\
\hline Age (years) & $61(43-68)$ & $63.5(54-72)$ & 0.03 \\
\hline Cirrhosis & $2(4)$ & $4(8)$ & 0.68 \\
\hline $\begin{array}{l}\text { Acute respiratory } \\
\text { failure }\end{array}$ & $7(14)$ & $13(26)$ & $<0.001$ \\
\hline $\begin{array}{l}\text { Severe sepsis/septic } \\
\text { shock }\end{array}$ & $5(10)$ & $12(24)$ & \\
\hline Cardiogenic shock & $21(42)$ & $4(8)$ & \\
\hline Cardiac surgery & $10(20)$ & $11(22)$ & \\
\hline Post-cardiac arrest & $5(10)$ & $3(6)$ & \\
\hline Others & $2(4)$ & $7(14)$ & \\
\hline BMI $\left(\mathrm{kg} / \mathrm{m}^{2}\right)$ & $26.5(24.2-29.4)$ & $26.3(24.5-30.9)$ & 0.50 \\
\hline SAPS II & $51(42.5-73.3)$ & $53.5(41-73.3)$ & 0.98 \\
\hline \multicolumn{4}{|c|}{ Characteristics at time of therapeutic monitoring } \\
\hline SOFA score & $12(10-14)$ & $9(6-11)$ & $<0.001$ \\
\hline $\begin{array}{l}\text { 24-h fluid balance } \\
(\mathrm{mL})\end{array}$ & $\begin{array}{l}100(-925 \text { to } \\
1860)\end{array}$ & $\begin{array}{l}100(-1550 \text { to } \\
1019)\end{array}$ & 0.52 \\
\hline Weight (kg) & $88.5(73-100)$ & $82(70-93)$ & 0.37 \\
\hline Amikacin dose (mg) & $2250(1825-2500)$ & $\begin{array}{l}2000(1713- \\
2300)\end{array}$ & 0.17 \\
\hline $\begin{array}{l}\text { Amikacin regimen } \\
(\mathrm{mg} / \mathrm{kg})\end{array}$ & $25(25-25.6)$ & $24.9(24.5-25.5)$ & 0.10 \\
\hline Amikacin $C_{\max }(\mathrm{mg} / \mathrm{L})$ & $71.7(58.9-79.7)$ & $68.4(53-81)$ & 0.36 \\
\hline $\begin{array}{l}\text { Patients with } C_{\max } \\
<60 \mathrm{mg} / \mathrm{L}\end{array}$ & $13(26)$ & $17(34)$ & 0.51 \\
\hline $\begin{array}{l}\text { Patients with } C_{\max } \\
>80 \mathrm{mgL}\end{array}$ & $12(24)$ & $15(30)$ & 0.65 \\
\hline Amikacin $C_{\min }(\mathrm{mg} / \mathrm{L})$ & $8.5(3-15.4)$ & $9.6(2.5-16.9)$ & 0.45 \\
\hline
\end{tabular}

\section{9}

A high aminoglycoside regimen associated with renal replacement therapy for the treatment of multi-drug-resistant pathogens

Alexandre Brasseur ${ }^{1}$, Maya Hites ${ }^{2}$, Sandrine Roisin ${ }^{3}$, Frederic Cotton ${ }^{4}$ Jean-Louis Vincent ${ }^{5}$, Daniel De Backer ${ }^{6}$, Frederique Jacobs ${ }^{2}$, Fabio Silvio Taccone $^{7}$

${ }^{1}$ Soins Intensifs, Hôpital Erasme, Bruxelles, Belgium; ${ }^{2}$ Service de maladies infectieuses, Hospital Erasme, Brussel, Belgium; ${ }^{3}$ Service de microbiologie clinique, Hospital Erasme, Brussel, Belgium; ${ }^{4}$ Service de biochimie médicale, Hospital Erasme, Brussel, Belgium; ${ }^{5}$ Service de Réanimation Médico-Chirurgicale, Hôpital Erasme, Bruxelles, Belgium; ' ${ }^{6}$ Soins intensifs, Hospital Erasme, Brussel, Belgium; ' ${ }^{5}$ Service de Soins Intensifs, Hôpital Erasme, Bruxelles, Belgium

Correspondence: Alexandre Brasseur - albrasse@ulb.ac.be Annals of Intensive Care 2016, 6(Suppl 1):09

Introduction Infections caused by multi-drug-resistant (MDR) Gramnegative (GN) organisms in critically ill patients are a therapeutic challenge. The administration of high-dose aminoglycoside (HDA) therapy coupled with high-flow continuous venovenous hemodiafiltration (CVVHDF) could allow required high drug peaks to be achieved with acceptable drug elimination.

Patients and methods All adult patients present on the intensive care unit (ICU) between October 2009 and July 2014 who had MDR-GN sepsis were considered for HDA and high-flow ( $>45 \mathrm{~mL} / \mathrm{kg} / \mathrm{h}$ ) CVVHDF when an isolated pathogen was susceptible or intermediate susceptible to aminoglycosides, and the patient's condition was not improving with conventional therapy. Optimal antibacterial activity was defined as a peak concentration of at least eight times the minimal inhibitory concentration.

Results Fifteen patients infected with MDR-GN pathogens (11 with Pseudomonas aeruginosa; ten with abdominal and five with respiratory infections) were treated with amikacin $(n=11)$, gentamicin $(n=3)$ or tobramycin $(n=1)$ and high-flow CVVHDF. A favorable clinical response was observed in eight (53\%) patients, including three in whom microbial eradication was obtained. Six patients were discharged alive from the ICU and five from the hospital. No renal toxicity was observed among survivors.

Conclusion In this cohort of septic patients with MDR-GN infections, HDA combined with high-flow CVVHDF represented a valuable therapeutic option. The effectiveness of this approach should be further evaluated in larger studies.

\section{Competing interests None.}

010

Optimization of administration of vancomycin in septic patients: a prospective randomized study

Valerie Van Ruychevelt', Eric Carlier ${ }^{1}$, Michael Piagnerelli ${ }^{1}$, Michel

Vanhaeverbeek ${ }^{2}$, Christine Danguy ${ }^{3}$, Patrick Biston

${ }^{1}$ Intensive Care Unit, Hôpital Civil Marie Curie, Charleroi, Belgium; ${ }^{2}$ Internal medicine, Hospital André Vésale, Montigny-le-Tilleul, Belgium; ${ }^{3}$ Pharmacy,

Hospital André Vésale, Montigny-le-Tilleul, Belgium

Correspondence: Valerie Van Ruychevelt - vvruyche@ulb.ac.be

Annals of Intensive Care 2016, 6(Suppl 1):010

Introduction Continuous intravenous administration of vancomycin (CIV) in intensive care unit (ICU) patients requires dose adjustment according to weight and renal function, but its assessment remains difficult. The aim of this study was to validate a new dose regimen of CIV correlated with renal function assessed by creatinine clearance (Creat $\mathrm{Cl})$.

Materials and methods In this prospective randomized study, we evaluated septic critically ill patients in whom CIV was given for more than $48 \mathrm{~h}$ in the absence of renal replacement therapy. The CIV protocol was developed based on recommendations of IDSA for the loading dose $(30 \mathrm{mg} / \mathrm{kg})$, but a new protocol for the maintenance dose was tested (depending on weight and daily Creat $\mathrm{Cl}$ ). The serum target vancomycin (vanco) concentration was $25-30 \mu \mathrm{g} / \mathrm{ml}$. Patients were randomized in three groups by daily assessment of renal function by a Creat $\mathrm{Cl}$ of $4 \mathrm{~h}$ (from 4 to 8 a.m.) or $12 \mathrm{~h}$ of Creat $\mathrm{Cl}$ (from 8 p.m. to 4 a.m.) or by the MDRD. Acute kidney injury (AKI) was defined as a daily urine output $<0.5 \mathrm{ml} / \mathrm{kg} / \mathrm{h}$ and/or an increase in the serum creatinine of $\geq 0.3 \mathrm{mg} / \mathrm{dL}$ from baseline levels. Multivariable logistic regression analysis was performed to identify predictors of CIV under-/over-dose and predictors of AKI.

Results During 23 months, in a medico-surgical 36 beds ICU (CHU Charleroi, Belgium), 67 patients were randomized: $21(31 \%)$ in the MDRD group, 21 (31\%) in the $4 \mathrm{~h} \mathrm{Creat} \mathrm{Cl}$ and $25(38 \%)$ in the $12 \mathrm{~h}$ Creat $\mathrm{Cl}$. The three groups were comparable in demographic terms, severity score, kidney function before CIV and bacteriology. For 7/67 (10\%) patients, CIV was targeted on bacteriological findings (one MRSA and six Enterococcus faecium). In the other patients, vanco was introduced empirically for clinically evolving sepsis. Vanco concentration $<25 \mu \mathrm{g} / \mathrm{ml}$ on day 1 was found in 15 patients $(22 \%)$ in the MDRD group, $11(16 \%)$ in $4 \mathrm{~h}$ Creat $\mathrm{Cl}$ and $15(22 \%)$ in $12 \mathrm{~h} \mathrm{Creat} \mathrm{Cl}$ $(p=0.5)$, and in $12(17 \%), 12(17 \%)$ and $13(19 \%)$, respectively, on day $2(p=0.8)$. In logistic regression analysis, $\mathrm{BMI}<30$ and an elevated Creat $\mathrm{Cl}>90$ were found independent predictors of under-dosing vanco $(<25 \mu \mathrm{g} / \mathrm{ml})$ at day 1 with an OR of 1.098 [1.01-1.19] and 0.935 [0.8-1], respectively. The day 1-day 2 CIV overdose was found to be an independent risk factor for AKI with an OR of 7.5 [2.2-23.7].

Discussion Our protocol cannot optimally achieve target levels on days $1-2$. However, the chosen therapeutic target $(25-30 \mu \mathrm{g} / \mathrm{ml})$ seems a posteriori not justified given the local epidemiology. With the 
target proposed in the literature $(20-30 \mu \mathrm{g} / \mathrm{ml})$, vanco concentration at day 1 was $<20 \mu \mathrm{g} / \mathrm{ml}$ in nine patients (43\%) in MDRD group, four (19\%) in $4 \mathrm{~h} \mathrm{CCU}$ and nine (36 \%) in $12 \mathrm{~h} \mathrm{CCU,} \mathrm{and} \mathrm{in} 3(14 \%), 3(14 \%)$ and $5(20 \%)$, respectively, at day 2 . It would therefore be preferable to target therapeutic levels between 25 and $30 \mu \mathrm{g} / \mathrm{ml}$ only for patients in the presence of multi-resistant germs demonstrated. For empirical antibiotic choice, therapeutic target [ $20-30 \mu \mathrm{g} / \mathrm{ml}]$ is probably enough and more careful. The assessment of renal function using three different methods failed to highlight difference in Creat $\mathrm{Cl}$. The evaluation of Creat $\mathrm{Cl}$ by the MDRD formula may be sufficient.

Conclusion Insufficient vancomycin concentration is a frequent finding at days 1-2. Predictors were $\mathrm{BMI}$ and high Creat $\mathrm{Cl}$. Vanco level $>30 \mu \mathrm{g} / \mathrm{ml}$ at days $1-2$ is an independent predictive factor for AKI with an OR of 7.5.

\section{Competing interests None.}

\section{References}

1. Rybak, M. et al. Therapeutic monitoring of vancomycin in adult patients: a consensus review of the American Society of Health-System Pharmacists, the Infectious Diseases Society of America, and the Society of Infectious Diseases Pharmacists. Am. J. Health-Syst. Pharm. AJHP Off. J. Am. Soc. Health-Syst. Pharm. 66, 82-98 (2009).

2. Ocampos-Martinez, E. et al. Determinants of early inadequate vancomycin concentrations during continuous infusion in septic patients. Int. J. Antimicrob. Agents 39, 332-337 (2012).

\section{1}

Impact of elevated intra-abdominal pressure on the ability of dynamic parameters to predict fluid responsiveness

Siu-Ming Au', Emmanuelle Begot ${ }^{2}$, François Dalmay ${ }^{3}$, Xavier Repessé ${ }^{4}$, Gwenael Prat ${ }^{5}$,Koceila Bouferrache ${ }^{6}$, Michel Slama ${ }^{7}$, Philippe Vignon², Antoine Vieillard-Baron ${ }^{4}$

${ }^{1} \mathrm{Icu}$, Hospital Ambroise Paré, Boulogne-Billancourt, France; ${ }^{2}$ Service de réanimation polyvalente, Centre Hospitalier Universitaire de Limoges, Limoges, France; ${ }^{3}$ Réanimation Polyvalente, CHU Limoges, Limoges, France; ${ }^{4}$ Réanimation médico-chirurgicale, Assistance Publique Hôpitaux de Paris, Hôpital Ambroise Paré, Boulogne-Billancourt, France; ${ }^{5}$ Réanimation médicale, CHRU de Brest, Brest, France; ${ }^{6} \mathrm{Cu}$, Hospital Foch, Suresnes, France; ${ }^{7}$ Réanimation médicale, Centre Hospitalier Universitaire, Amiens, France

Correspondence: Antoine Vieillard-Baron - antoine.vieillard-baron@apr. aphp.fr

Annals of Intensive Care 2016, 6(Suppl 1):011

Introduction Elevated intra-abdominal pressure (IAP) has been suggested to decrease the accuracy of dynamic parameters of fluid responsiveness, especially for the distensibility index of the inferior vena cava $(\Delta \mathrm{IVC})$. The goal of our study was to confirm this limitation in a large series of unselected patients.

Materials and methods This is a sub-study of an observational, prospective and multicenter study (Hemopred) that we conducted between November 2012 and November 2014. All sedated patients under mechanical ventilation who required an echocardiographic assessment for an acute circulatory failure, with an inserted central venous catheter and arterial catheter, were eligible. Acute circulatory failure was defined as a sustained hypotension (systolic blood pressure $<90 \mathrm{mmHg}$ or mean blood pressure $<65 \mathrm{mmHg}$ ) and/or the presence of clinical signs of hypoperfusion (e.g., mottled skin, oliguria), metabolic acidosis ( $\mathrm{pH}<7.35$ and base excess less than $-5 \mathrm{mmol} / \mathrm{L}$ ), elevated lactate $(>2 \mathrm{mmol} / \mathrm{L}$ ) or decreased central venous oxygen saturation $\left(\mathrm{ScvO}_{2}<70 \%\right)$. Dynamic parameters used to predict fluid responsiveness assessed in this study were pulse pressure variations $(\triangle \mathrm{PP})$ and the following echocardiographic indices: respiratory variations of aortic Doppler maximal velocity $\left(\Delta V_{\text {max }}\right)$, collapsibility index of superior vena cava $(\Delta S V C)$ and $\Delta I V C$.

At the time of echocardiography, a systematic measurement of the bladder pressure was performed as recommended and elevated IAP was defined as a pressure $\geq 12 \mathrm{mmHg}$.

Response to fluids was defined by an increase in cardiac output of more than $10 \%$ during a passive leg raising (PLR), as previously validated. Area under the ROC curve (AUC) was built for each recorded dynamic parameter.

Results Among the 540 patients included in the study, 174 (32\%) had an elevated IAP (group 1). No difference was observed compared with the remaining patients without elevated IAP (group 2), except for patients with an abdominal surgery ( 32 vs $23 \%, p=0.03$ ). Among group 1, 81 patients had an IAP between 12 and $15 \mathrm{mmHg}$ and 93 patients had an IAP $>15 \mathrm{mmHg}$. Central venous pressure (CVP) was increased in group 1 compared with group $2(11 \pm 5 \mathrm{vs} 9 \pm 5 \mathrm{mmHg}$, $p<0.001)$, as well as plateau pressure (Pplat) $\left(20 \pm 5\right.$ vs $18 \pm 5 \mathrm{cmH}_{2} \mathrm{O}$, $p<0.001)$. In group 1, $81(46 \%)$ patients were classified as responders by the PLR, compared with $124(40 \%)$ in group $2(p=0.18)$. No difference between the AUC for $\triangle \mathrm{IVC}(0.64$ vs $0.59, p=0.46), \triangle \mathrm{PP}(0.71$ vs $0.66, p=0.47)$ and $\Delta V_{\max }(0.83$ vs $0.71, p=0.06)$ was observed between groups 1 and 2, respectively, although AUC was increased in group 1 for $\triangle \mathrm{SVC}(0.72$ vs $0.82, p=0.02)$. No difference was observed regarding the feasibility between the two groups for any parameters, although feasibility for $\triangle \mathrm{IVC}$ was $74 \%$ in group 1 compared with $80 \%$ in group $2(p=0.1)$.

Conclusion Thirty-two percent of patients in our series of unselected ventilated patient with shock had an elevated IAP. They demonstrated higher CVP and Pplat and more benefited from abdominal surgery. Elevated IAP did not have significant effect on accuracy of dynamic parameters of fluid responsiveness, even though $\triangle I V C$ was less frequently recorded (nonsignificant) and $\triangle S V C$ had a better prediction.

\section{Competing interests None.}

\section{2}

Passive leg raising for predicting fluid responsiveness: a systematic review and meta-analysis

Xavier Monnet ${ }^{1}$, Paul Marik ${ }^{2}$, Jean-Louis Teboul ${ }^{1}$

${ }^{1}$ Service de réanimation médicale, inserm umr_s 999, Hôpital de Bicêtre, Université Paris-Sud, Faculté de Médecine, Université Paris-Saclay, Le Kremlin-Bicêtre, France; ${ }^{2}$ Department of medicine, division of pulmonary and critical care medicine, Eastern Virginia Medical School, Norfolk, USA Correspondence: Xavier Monnet - xavier.monnet@aphp.fr Annals of Intensive Care 2016, 6(Suppl 1):012

Introduction We performed a systematic review and meta-analysis of studies that investigated the passive leg raising (PLR)-induced changes in cardiac output (CO) or surrogates and the PLR-induced changes in arterial pulse pressure (PP) as predictors of fluid responsiveness in adults.

Patients and methods MEDLINE, EMBASE and Cochrane Database of Systematic Reviews were screened for relevant original and review articles.

Results Twenty-one studies (995 patients) were included. CO was measured by echocardiography in six studies, calibrated pulse contour analysis in six studies, bioreactance in four studies, oesophageal Doppler in three studies, either transpulmonary thermodilution or pulmonary artery catheter in one study and suprasternal Doppler in one study. The pooled correlation between the PLR-induced and the volume expansion-induced changes in $\mathrm{CO}$ was $0.76(0.73-0.80)$. The best threshold was a PLR-induced increase in $\mathrm{CO}>10 \pm 2 \%$. The pooled sensitivity was $0.85(0.81-0.88)$, and the pooled specificity was 0.91 (0.88-0.93). The area under the ROC curve was $0.95 \pm 0.01$. Eight studies (432 patients) met the selection criteria for the PLR-induced changes in PP. For the PLR-induced changes in PP, the pooled sensitivity was $0.56(0.49-0.53)$, the pooled specificity was $0.83(0.77-0.88)$, and the pooled area under the ROC curve was $0.77 \pm 0.05$.

Conclusion The changes in CO induced by a PLR test are highly reliable in predicting the response of $\mathrm{CO}$ to volume expansion in adult patients with acute circulatory failure. When its effects are assessed by changes in PP, the specificity of the PLR test remains acceptable, but its sensitivity is poor.

Competing interests XM and J-LT are members of the Medical Advisory Board of Maquet. 
013

Predicting volume responsiveness by using combined end-expiratory and end-inspiratory occlusion tests with echocardiography

Mathieu Jozwiak' , Jean-Louis Teboul ${ }^{1}$, Christian Richard ${ }^{1}$, Xavier Monnet 'Service de réanimation médicale,inserm umr_s999, Hôpital de Bicêtre, Université Paris-Sud, Faculté de Médecine, Université Paris-Saclay, Le

Kremlin-Bicêtre, France

Correspondence: Mathieu Jozwiak - mathieu.jozwiak@aphp.fr Annals of Intensive Care 2016, 6(Suppl 1):013

Introduction The changes in intrathoracic pressure induced by end-expiratory (1) and end-inspiratory occlusions induce significant changes in cardiac preload that may be used to test preload dependence. We tested whether the effects of respiratory occlusions on the velocity-time integral of the subaortic flow (VTI) could serve as a functional test for predicting volume responsiveness in patients with circulatory failure.

Patients and methods In ten mechanically ventilated patients with circulatory failure in whom volume expansion was planned, we continuously measured VTI (transthoracic echocardiography) during the 5 last seconds of 15 -s end-inspiratory and end-expiratory occlusions, separated by $1 \mathrm{~min}$, and after the subsequent infusion of $500-\mathrm{mL}$ saline. Patients in whom volume expansion induced an increase in cardiac index (transpulmonary thermodilution, $\mathrm{PICCO}_{2}$ ) $>15 \%$ were defined as "volume-responders".

Results Volume expansion increased cardiac index by more than $15 \%$ $\left(2.75 \pm 0.66-3.36 \pm 0.71 \mathrm{~L} / \mathrm{min} / \mathrm{m}^{2}, p<0.05\right)$ in four patients. In these volume-responders before volume expansion, the end-expiratory occlusion increased VTI to a significantly larger extent than in nonvolume-responders ( $9 \pm 5$ vs. $3 \pm 1 \% p=0.02$, respectively). Similarly, before volume expansion, the end-inspiratory occlusion decreased VTI to a significantly larger extent in volume-responders than in non-volume-responders ( $-11 \pm 3$ vs. $-5 \pm 2 \% p=0.01$, respectively). Volume responsiveness was predicted by an increase in VTI $>4 \%$ during the end-expiratory occlusion with a sensitivity of $75 \%(95 \% \mathrm{Cl} 19-99 \%)$ and a specificity of $100 \%$ (95 \% Cl 54-100\%). If the absolute values of the VTI changes recorded during end-expiratory and end-inspiratory occlusions were added, the predictive accuracy of volume responsiveness tended to be better than for the VTI changes recorded during the only end-expiratory occlusion [area under the ROC curve: 0.917 (95\% $\mathrm{Cl} 0.575-0.999)$ vs. 0.771 (95\% Cl 0.415-0.964), $p=0.24$, respectively]. Conclusion The sum of the absolute values of changes in VTI recorded during consecutive end-expiratory and end-inspiratory occlusions could reliably predict volume responsiveness. This prediction tends to be better than the changes in VTI during the only end-expiratory occlusion. More patients will be included in order to confirm these preliminary results.

\section{Competing interests None.}

\section{Reference}

1. Monnet et al. Crit Care Med. 2009; 37:951-956.

\section{4}

Early dynamic left intraventricular obstruction is associated with hypovolemia and hight mortality in septic shock patients Jean-Louis Chauvet ${ }^{1}$, Shari El-Dash'², Olivier Delastre ${ }^{1}$, Bernard Bouffandeau', Dominique Jusserand', Jean-Baptiste Michot ${ }^{1}$, Fabrice Bauer $^{3}$, Julien Maizel ${ }^{4}$, Michel Slama ${ }^{4}$

${ }^{1}$ Réanimation Polyvalente - USC, Centre Hospitalier Elbeuf Louviers Val de Reuil, Elbeuf, France; ${ }^{2}$ Réanimation médicale, CHU Amiens-Picardie, Salouel, France; ${ }^{3}$ Cardiology, Chu De Rouen, Rouen, France; ${ }^{4}$ Réanimation médicale, Centre Hospitalier Universitaire, Amiens, France

Correspondence: Jean-Louis Chauvet jean-louis.chauvet@ chi-elbeuf-louviers.fr

Annals of Intensive Care 2016, 6(Suppl 1):014

Introduction Based on previously published case reports demonstrating dynamic left intraventricular obstruction triggered by hypovolemia or catecholamines, this study aimed to establish: (1) IVO occurrence in septic shock patients; (2) correlation between the intraventricular gradient and volume status and fluid responsiveness; and (3) mortality rate.

Patients and methods We prospectively analyzed patients with septic shock admitted to a general ICU over a 28-month period who presented Doppler signs of left intraventricular obstruction. Clinical characteristics and hemodynamic parameters as well as echocardiographic data regarding left ventricular function, size, and calculated mass, and left ventricular outflow Doppler pattern and velocity before and after fluid infusions were recorded.

Results During the study period, 218 patients with septic shock were admitted to our ICU. Hemodynamic and echocardiographic characteristics of patients with intraventricular obstruction before and after fluid infusion are given in Table 2. Left intraventricular obstruction was observed in 47 (22\%) patients. Mortality rate at 28 days was found to be higher in patients with than in patients without left intraventricular obstruction (55 vs $33 \%, p<0.01$ ). Small, hypercontractile left ventricles (end-diastolic left ventricular surface $4.7 \pm 2.1 \mathrm{~cm}^{2} / \mathrm{m}^{2}$ and ejection fraction $82 \pm 12 \%$ ), and frequent pseudohypertrophy were found in these patients. A rise $\geq 12 \%$ in stroke index was found in $87 \%$ of patients with left intraventricular obstruction, with a drop of $47 \%$ in left intraventricular obstruction after fluid infusion.

A late velocity of diastolic mitral flow, $A^{\prime}$ late velocity of diastolic mitral annulus motion, $E$ early diastolic velocity of mitral flow, $E^{\prime}$ early velocity of diastolic mitral annulus motion, ns not significant.

Discussion The presence of left intraventricular obstruction is associated with high mortality rate. The data showed an association between the presence of this obstructive flow pattern on left ventricular Doppler flow and the presence of hypovolemia and cardiac hypercontractility and also demonstrated that these patients frequently present small and pseudo-hypertrophic left ventricular. Furthermore, this study also demonstrates the high rate of fluid responsiveness in these patients with left intraventricular obstruction despite the absence of significant pulse pressure variations.

Conclusion In our study, left intraventricular obstruction is a frequent event in septic shock patients bearing an important correlation with fluid responsiveness. Mortality rate was found to be higher in these patients in comparison with patients without obstruction.

Competing interests None.

Table 2 Hemodynamic and echocardiographic characteristics of patients with intraventricular obstruction before and after fluid infusion

\begin{tabular}{lccl}
\hline & Before & After & p value \\
\hline Heart rate (beats/min) & $109 \pm 25$ & $102 \pm 22$ & ns \\
Systolic arterial pressure $(\mathrm{mmHg})$ & $110 \pm 28$ & $141 \pm 32$ & $<0.01$ \\
Diastolic arterial pressure $(\mathrm{mmHg})$ & $54 \pm 11$ & $65 \pm 14$ & $<0.01$ \\
Mean arterial pressure $(\mathrm{mmHg})$ & $71 \pm 16$ & $89 \pm 1$ & $<0.01$ \\
Cardiac output $(\mathrm{I} / \mathrm{min})$ & $4.6 \pm 1.9$ & $5.8 \pm 2.1$ & $<0.01$ \\
Cardiac index $\left(\mathrm{I} / \mathrm{min} / \mathrm{m}^{2}\right)$ & $2.4 \pm 0.9$ & $3 \pm 1$ & $<0.01$ \\
Stroke volume $(\mathrm{ml})$ & $43 \pm 18$ & $58 \pm 22$ & $<0.01$ \\
Indexed stroke volume $\left(\mathrm{ml} / \mathrm{m}^{2}\right)$ & $23 \pm 10$ & $31 \pm 11$ & $<0.01$ \\
Intraventricular obstruction $(\mathrm{m} / \mathrm{s})$ & $1.9 \pm 0.9$ & $1 \pm 1$ & $<0.01$ \\
Intraventricular obstruction $(\mathrm{mmHg})$ & $18 \pm 18$ & $8 \pm 13$ & $<0.01$ \\
Inferior vena cava diameter $(\mathrm{mm})$ & $16 \pm 6.4$ & $18 \pm 6.6$ & $\mathrm{~ns}$ \\
E/A $^{\prime}$ & $0.8 \pm 0.46$ & $0.9 \pm 0.3$ & $\mathrm{~ns}$ \\
E $^{\prime}(\mathrm{cm} / \mathrm{s})$ & $14 \pm 5$ & $16 \pm 5$ & $\mathrm{~ns}$ \\
E'/A $^{\prime}$ & $0.77 \pm 0.26$ & $0.87 \pm 0.37$ & $\mathrm{~ns}$ \\
E/E' $^{\prime}$ & $5.8 \pm 2.1$ & $6.8 \pm 1.8$ & $\mathrm{~ns}$ \\
\hline
\end{tabular}


015

Predictive factors for poor hemodynamic tolerance to fluid removal in ICU: the DepleRea study

François Brazier', Pablo Mercado ${ }^{1}$, Loay Kontar ${ }^{1}$, Dimitri Titeca' ${ }^{1}$, Bertand De Cagny', Gaelle Bacari-Risal', Antoine Riviere', Michel Slama', Julien Maizel $^{1}$

${ }^{1}$ Réanimation médicale, Centre Hospitalier Universitaire, Amiens, France

Correspondence: Julien Maizel - maizel.julien@chu-amiens.fr Annals of Intensive Care 2016, 6(Suppl 1):015

Introduction In the intensive care unit (ICU), the presence of edema after the initial phase of fluid resuscitation is an independent poor prognostic factor. The fluid removal (using diuretics or during renal replacement therapy) is an important component of care. However, the depletion can decrease cardiac output and provoke hypotension. Therefore, it can be harmful. Our objective was to determine factors for poor hemodynamic tolerance to fluid removal.

Patients and methods We conducted a single-center prospective observational study in the medical ICU of the University Hospital of Amiens in France from March to September 2015. Inclusion criteria were critical patients initially requiring fluid therapy and then hemodynamically stabilized, in whom fluid removal was decided. Exclusion criterion was failure to perform a thoracic (or lung) or a cardiac ultrasonography. We collected the main clinical and biological characteristics. Hemodynamic status before the initiation of depletion was assessed by transthoracic echocardiography. Thoracic ultrasound was performed to estimate extravascular lung water. Our primary outcome was poor hemodynamic tolerance in the $24 \mathrm{~h}$ following the initiation of fluid, corresponding to at least one episode of hypotension (mean arterial pressure $<65 \mathrm{mmHg}$ ) or initiation/increase of catecholamines.

Results Sixteen patients were included in the study. The median age was 69 years (IQR 16.3), SAPS II was 52 (IQR 25.8), and the proportion of men was $56.3 \%$. Median time to initiation of fluid removal was 4 days (IQR 4.5). Fluid removal was performed in nine cases by loop diuretics and in eight cases with renal replacement therapy. The median depletion was $1617.5 \mathrm{ml}$ (IQR 2025.8). Fifty percent were intubated and ventilated. Seven out of eight patients had spontaneous ventilatory cycles. Pulse pressure variation could be calculated in only one patient. Nine patients (56.2 \%) did not tolerate fluid removal. The median SOFA at inclusion was significantly higher in patients who had poor hemodynamic tolerance to depletion compared with patients who well tolerated the depletion $(9 \pm 3$ vs $5 \pm 5.5, p=0.036)$. All patients with catecholamine therapy $(n=4$; median posology $0.087 \mu \mathrm{g} / \mathrm{kg} / \mathrm{min}$ ) did not hemodynamically tolerate fluid removal. Initial median cardiac index in patients who developed arterial hypotension was $2.7 \pm 1.6 \mathrm{l} / \mathrm{min} / \mathrm{m}^{2}$ and $3.6 \pm 3 \mathrm{l} / \mathrm{min} / \mathrm{m}^{2}$ among those who did not develop hypotension $(p=0.088)$. All patients having cardiac index lower than $2.7 \mathrm{l} / \mathrm{min} / \mathrm{m}^{2}$ developed hypotension. Diastolic blood pressure of patients with hemodynamic instability was significantly lower ( $56 \pm 10.5$ vs $72 \pm 18 \mathrm{mmHg}, p=0.042$ ). Four out of five ( $80 \%$ ) of the patients with positive passive leg raising test showed hemodynamic intolerance to fluid removal, while only one patient (16.7 \%) had a positive passive leg raising test in the well-tolerated depletion group $(p=0.58)$. Patients with well hemodynamic tolerance to fluid removal had approximately twice as many $B$ lines than patients having poorly tolerated fluid removal ( $14 \pm 17$ vs $7 \pm 13.3, p=0.152)$.

Discussion Our results suggest that a preload dependence (shown by a positive passive leg raising test) and a lower accumulation of extravascular lung water (shown by fewer $B$ lines) are associated with a poor hemodynamic tolerance to fluid removal. A precarious hemodynamic state (cardiac index lower than $2.7 \mathrm{I} / \mathrm{min} / \mathrm{m}^{2}$, catecholamines therapy and lower diastolic arterial pressure) also appears predictive of a poor fluid removal hemodynamic tolerance. Patients with poorly tolerated fluid depletion had a higher SOFA score, indicating a greater severity of these patients. These results must be confirmed in a larger cohort.

Conclusion Our results suggest that poor hemodynamic tolerance to fluid removal is associated with a lower diastolic pressure, the presence of catecholamine and a preload dependency as suggested by a low cardiac index and a positive passive leg raising test. The presence of many $B$ lines assessed by thoracic ultrasound seems to be protective of poor hemodynamic tolerance.

Competing interests None.

\section{6}

High-flow nasal cannula: first-line treatment of noninvasive ventilation for infants with bronchiolitis. Applicability and risk factors for failure

Camille Guillot ${ }^{1}$, Claire Le Reun ${ }^{1}$, Marie Lampin ${ }^{1}$, Ahmed Sadik', Astrid Botte $^{1}$, Alain Duhamel ${ }^{2}$, Stéphane Leteurtre ${ }^{1}$

${ }^{1}$ Réanimation et surveillance continue pédiatriques, Centre Hospitalier Régional Universitaire de Lille, Lille, France; ${ }^{2}$ Clinique de santé publique, plateforme d'aide méthodologique, C.h.r.u. Lille, Lille, France

Correspondence: Stéphane Leteurtre - stephane.leteurtre@chru-lille.fr Annals of Intensive Care 2016, 6(Suppl 1):016

Introduction Viral bronchiolitis is an acute infection disease of the lower respiratory tract. In the most severe cases, acute respiratory failure occurs. High-flow nasal cannula (HFNC) therapy is a relatively new noninvasive ventilator support that can improve respiratory distress for infants with bronchiolitis. The objectives of this study were (1) to evaluate a study design suggesting the use of HFNC as the first-line treatment for infants with bronchiolitis in a pediatric intensive and intermediate care unit (PI\&ICU) and (2) to identify risk factors for HFNC failure.

Patients and methods This study was prospective during two consecutive seasons (2013-2014 and 2014-2015) in a PI\&ICU (18 beds). Inclusion criteria were patients $<2$ years with bronchiolitis needing invasive or noninvasive ventilation. During the 2013-2014 season, no recommendation between HFNC and continuous positive airway pressure (CPAP) was proposed. During the 2014-2015 season, a study design suggested the use of the HFNC as noninvasive ventilation as first-line tratment (before C PAP) for bronchiolitis. Primary outcome was the percentage of HFNC (comparison between the two periods). For the secondary outcome, failure of HFNC was defined by the need of CPAP or invasive ventilation. Clinical and biological variables were collected during the PI\&ICU stay at different times (before ventilation, $\mathrm{H} 1-\mathrm{H} 2$, $\mathrm{H} 4-\mathrm{H} 6, \mathrm{H} 10-\mathrm{H} 12$ and $\mathrm{H} 24$ ). Comparisons of percentage and logistic regression were made with SAS software (SAS Institute, Cary, NC, USA). Results During the 2013-2014 season, 34 \% (14/41 infants) were treated by HFNC at admission compared with $90 \%$ (55/61 infants) during the 2014-2015 season $\left(p<10^{-4}\right)$ (Fig. 3). The intubation rate at admission and during the hospitalization was not different between the two seasons.

During the 2014-2015 season, HFNC therapy failed in 21 infants (38\%; 21/55). In the responders group, the use of HFNC therapy decreased the respiratory rate, the heart rate (HR) and the $\mathrm{CO}_{2}(p<0.001)$. With multivariate analysis, non-responders patients to HFNC therapy have a

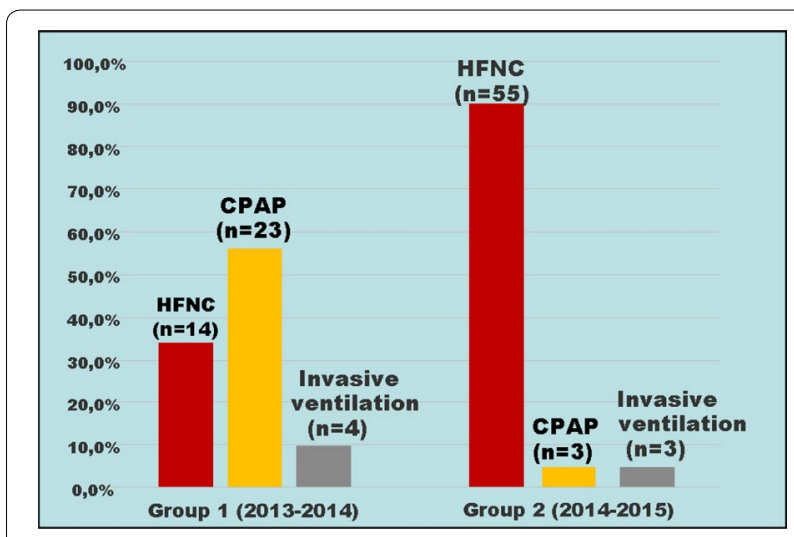

Fig. 3 Characteristics of ventilation modalities during the 2 periods 
hypercapnia higher (OR 1.136; $95 \% \mathrm{Cl} 1.04-1.24 ; p<0.01$ ) and a tachycardia lesser (OR 0.95; $95 \% \mathrm{Cl} 0.91-0.99 ; p=0.02$ ) compared with responders patients.

Discussion The use of HNCF as first-line treatment for patients with bronchiolitis has been frequently possible and accepted by all the team in our PI\&ICU. No differences for complications are observed during these two periods.

Conclusion HFNC can be used as a first-line treatment for moderate or severe bronchiolitis hospitalized in our PI\&ICU. HFNC requires a monitoring to determine the failure criteria. A multicenter study will be necessary to confirm these results.

\section{Competing interests None.}

\section{7}

Is high-flow nasal cannula better than nasal continuous positive airway pressure for bronchiolitis management in pediatric intensive care unit?

Aurélie Collins ${ }^{1}$, Céline Kempeneers ${ }^{2}$, Nathalie Cajgfinger ${ }^{3}$

${ }^{1}$ Pediatric departement, CHR de la Citadelle, Liège, Belgium; ${ }^{2}$ Pediatric respirology departement, CHR de la Citadelle, Liège, Belgium; ${ }^{3}$ Pediatric intensive care unit, CHR de la Citadelle, Liège, Belgium

Correspondence: Aurélie Collins - a.collins@student.ulg.ac.be Annals of Intensive Care 2016, 6(Suppl 1):017

Introduction Bronchiolitis is the first pathology encountered in pediatric intensive care unit during winter. In the management of respiratory failure, noninvasive ventilation may improve work of breathing and has been suggested to be effective in preventing intubation. High-flow nasal cannula had increasingly been used and tends to replace nasal continuous positive airway pressure in this indication despite the lack of evidence. Nowadays, no randomized control trial had compared nasal continuous positive airway pressure and highflow nasal cannula in this indication. Only observational studies exist. Most authors have described a superiority of high-flow nasal cannula against nasal continuous positive airway pressure but with some controversies. In this study, we compared retrospectively nasal continuous positive airway pressure and high-flow nasal cannula in patients with bronchiolitis admitted to pediatric intensive care unit during two seasons in our tertiary hospital.

Patients and methods We included 77 patients, in which 13 treated with nasal continuous positive airway pressure and 24 treated with high-flow nasal cannula. We compared vital signs at $\mathrm{HO}, \mathrm{H} 1, \mathrm{H} 2, \mathrm{H} 3$, $\mathrm{H6}, \mathrm{H} 9$ and $\mathrm{H} 12$ after device positioning, length of stay and rescue intubation.

Results There was a significantly lower respiratory rate at $\mathrm{H} 6$; fraction of inspired oxygen was significantly lower at $\mathrm{H} 2$ and $\mathrm{H} 6$, with comparable pulse oximeter oxygen saturation; length of stay tended to be shorter and intubation rate lower in the high-flow nasal cannula group.

Conclusion Our results tend to show a small advantage to use highflow nasal cannula over nasal continuous positive airway pressure in respiratory failure in bronchiolitis. Larger prospective randomized clinical trials are needed to compare these two techniques.

\section{Competing interests None.}

\section{8}

Interest and risk of high-flow cannula during acute hypoxemic pneumonia in children: a retrospective study

Camille Ohlmann', Robin Pouyau', Fabien Subtil2, Florent Baudin', Bruno Massenavette ${ }^{1}$, Etienne Javouhey ${ }^{1}$

${ }^{1}$ Réanimation pédiatrique hfme, Hospices civils de Lyon, Lyon, France; ${ }^{2}$ Service de biostatistiques, Hospices Civils de Lyon, Lyon, France

Correspondence: Robin Pouyau - robin.pouyau@chu-lyon.fr Annals of Intensive Care 2016, 6(Suppl 1):018

Introduction Pneumonia remains the world's leading cause of death among children under 5 years old. Treatment of hypoxemic pneumonia is based on oxygen therapy. High-flow nasal cannula (HFNC) is a technique whereby heated and humidified oxygen can be delivered to the nose at high flow rates. It generates a low level of positive pressure in the upper airways and decreases the work of breathing. This technique might be useful to hypoxemic pneumonia treatment and could reduce the need of invasive or noninvasive ventilation in pediatric patients.

Patients and methods This retrospective monocentric study included all the children from 1 to 18 years old, admitted in pediatric intensive care unit between January 1, 2010, and June 1, 2014, and affected by hypoxemic pneumonia. Children affected by bronchiolitis or asthma were excluded. The goals of our study were to determine predictive factors for success or failure of HFNC. The failure group was defined as the group of patients which required the switch of HFNC to another oxygen delivery system (oxygen mask, noninvasive ventilation (NIV) or invasive ventilation). We studied clinical, biological and radiological criteria at different times for the first $48 \mathrm{~h}$. Then, we compared these criteria between the failure group and the success group.

Results Ninety-two children were included. HFNC was a success for 62 patients $(67 \%)$. No side effects were reported and the tolerance was good for all the patients. There was no demographic difference between the two groups of patients. The risk of failure was an initial higher $\mathrm{FiO}_{2}$ [mean of 0.69 in failure group vs 0.46 in the other group $(p=0.001)]$, an initial lower ratio $\mathrm{SpO}_{2} / \mathrm{FiO}_{2}[164.6$ vs $260.2(p=0.001)]$ and a higher PELOD score at admission [5.27 vs $3.35(p=0.042)]$. This risk of failure of HFNC persisted for the first $48 \mathrm{~h}$. This risk was significantly higher for patients who had three or more affected quadrants in pulmonary chest X-ray $(p=0.006)$. Immunocompromised patients $(n=26)$ were at higher risk of failure of HFNC in our population $(p=0.047)$. In the failure group $(n=30), 15$ patients needed NIV, 15 patients were intubated, and four died. There was no hypercapnia in either group and no modification of the capnia at any time. We finally defined criteria and threshold for patients at higher risk of failure of HFNC: immunodepression, $\mathrm{FiO}_{2} \geq 0.6$, ratio $\mathrm{SpO}_{2} / \mathrm{FiO}_{2} \leq 194$, PELOD $\geq 6$, three or more affected quadrants in pulmonary chest $X$-ray.

Conclusion Our study is one of the first on hypoxemic pneumonia in children. HFNC could be secure, useful and effective in this indication. The successful rate is $67 \%$. Identification of criteria associated with failure could help the physician to optimize the timing of secondary support, mainly intubation. Those criteria should be validated in multicenter and prospective studies. HFNC seems to be a promising technique of ventilation for patients with hypoxemic pneumonia. The rate of failure may be higher in immunocompromised children, patients with a severe hypoxemia or with an other organ failure (Fig. 4).

\section{Competing interests None.}

\section{Reference}

1. Frat J-P, Thille AW, Mercat A, Girault C, Ragot S, Perbet S, et al. High-flow oxygen through nasal cannula in acute hypoxemic respiratory failure. N Engl J Med.2015;372(23):2185-96.

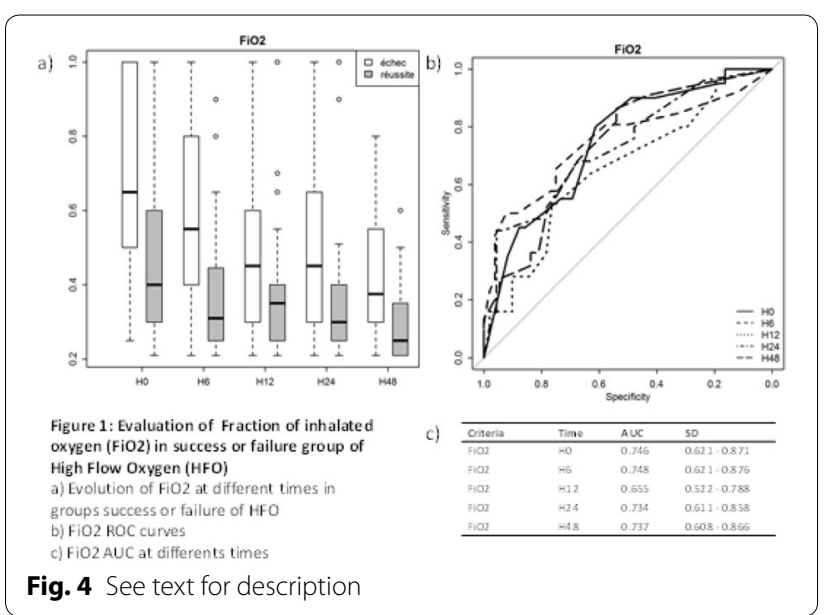


019

Interest of high-flow nasal cannula (HFNC) versus nasal continuous positive airway pressure (nCPAP) during the initial management of severe bronchiolitis in infants: a multicenter randomized controlled trial

Christophe Milesi ${ }^{1}$, Sandrine Essouri ${ }^{2}$, Robin Pouyau, ${ }^{3}$, Jean-Michel Liet ${ }^{4}$, Mickael Afanetti ${ }^{5}$, Julien Baleine ${ }^{1}$, Sabine Durand ${ }^{1}$, Philippe Durand ${ }^{2}$, Etienne Javouhey ${ }^{6}$, Jean Christophe Roze ${ }^{4}$, Didier Dupont ${ }^{5}$, Gilles Cambonie $^{1}$

${ }^{1}$ Réanimation pédiatrique, Hôpital Arnaud-de-Villeneuve, Montpellier, France; ${ }^{2}$ Service de réanimation pédiatrique, $\mathrm{CHU}$ de Bicêtre, Le Kremlin-Bicêtre, France; ${ }^{3}$ Réanimation pédiatrique, Hôpital Femme Mère Enfant, Bron, France; ${ }^{4}$ Réanimation pédiatrique, CHU de Nantes, Nantes, France; ${ }^{5}$ Réanimation pédiatrique, Hôpitaux Pédiatrique CHU-Lenval, Nice, France; ${ }^{6}$ Réanimation pédiatrique hfme, Hospices civils de Lyon, Lyon, France

Correspondence: Christophe Milesi - c-milesi@chu-montpellier.fr Annals of Intensive Care 2016, 6(Suppl 1):019

Introduction Concerning the management of severe bronchiolitis, nCPAP is the gold standard. HFNC is increasingly used in this indication even if there are no randomized studies evaluating its efficacy and safety in this population. Our objective was to evaluate the failure rate of HFNC compared with the nCPAP during the initial management of severe bronchiolitis.

Patients and methods Prospective, controlled, randomized, multicenter study was conducted in five French pediatric intensive care units (PICU). Infants from 0 to 6 months old, admitted in PICU for severe bronchiolitis (modified Wood score $>3$ ), were randomized into two groups: "HFNC" (2 l/kg/min) and "nCPAP" $\left(6 \mathrm{~cm} \mathrm{H}_{2} \mathrm{O}\right)$ during $24 \mathrm{~h}$. The primary endpoint was the percentage of failure in both arms during the first $24 \mathrm{~h}$. Failure was define as an increase in (1) clinical score for respiratory distress (modified Wood score) or (2) respiratory rate (RR) or (3) discomfort (EDIN score) or (4) apnea.

Results \{median (SD) and \%(IC)\} A total of 142 infants were included from November 2014 to March 2015. Their weight was 4100 (1200) g, and they were 40 (35) days old, the RR: 53 (13) breath/min, $\mathrm{FiO}_{2}$ : 30 (12), modified Wood score: 4.3 (1), $\mathrm{PCO}_{2}: 59$ (14) $\mathrm{mmHg}, \mathrm{pH}: 7.28$ (0.1). Seventy one were included in HFNC group and 71 in the nCPAP group. There was no difference between the two groups (weight, age, $\mathrm{HR}, \mathrm{BP}, \mathrm{RR}, \mathrm{FIO}_{2}$, modified Wood score, EDIN, $\mathrm{PCO}_{2}$ ). The failure rate was higher in the HFNC group $49(39-62)$ vs $31(20-42) \%$ [OR 0.49 $(0.2-0.9), p=0.018]$. We did not find any predictive failure criteria. The main cause of failure in the HFNC group was a raise of the respiratory distress (58 \%) and discomfort in the nCPAP group (59\%). Failure

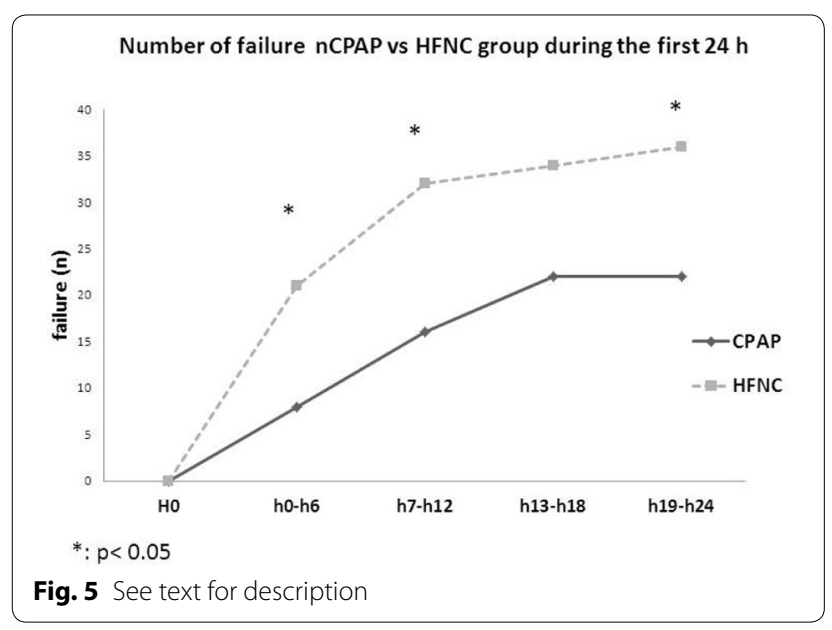

occurred rapidly during the first $18 \mathrm{~h}$ in the two groups. We did not find any difference between the two groups concerning the length of stay [6.2 (6) vs 7.5 (13) days; $p=0.46$ ] or the number of intubation (3/71 vs $5 / 71 ; p=0.51)$.

Conclusion During severe bronchiolitis management, the failure rate is higher with the high flow compared with nasal CPAP (Fig. 5).

\section{Competing interests None.}

020

Outcome of severe cerebral venous thrombosis in intensive care unit: a cohort study

Benjamin Soyer ${ }^{1}$, Marco Rusca', Anne-Claire Lukaszewicz'1 Isabelle

Crassard $^{2}$, Jean-Pierre Guichard ${ }^{3}$, Damien Bresson ${ }^{4}$, Didier Payen de la Garanderie

${ }^{1}$ Réanimation chirurgicale, CHU Lariboisière, Paris, France; ${ }^{2}$ Neurologie, CHU Lariboisière, Paris, France; ${ }^{3}$ Neuroradiologie, CHU Lariboisière, Paris, France; ${ }^{4}$ Neurochirurgie, CHU Lariboisière, Paris, France

Correspondence: Benjamin Soyer - benjaminsoyer@wanadoo.fr Annals of Intensive Care 2016, 6(Suppl 1):O20

Introduction Severity of cerebral venous thrombosis (CVT) may require the transfer to intensive care unit (ICU). This report described the context for CVT transfer to ICU, the strategy of care, and the outcome after 1 year.

Patients and methods We studied a monocentric cohort of 41 consecutive CVT in a French tertiary hospital. Data collected were as follows: demographic data, clinical course, incidence of craniectomy and/ or endovascular procedures, outcome in ICU, after 3, and 12 months.

Results

1-Characteristics $73.2 \%$ were female, 47 years old (IQ 26-53), having a SAPS II 41 (32-45), GCS 7 (5-8), and at least one episode of mydriasis in $48.8 \% .48 .8 \%$ had seizures before ICU admission and $43.9 \%$ during ICU stay.

2-Lesions Thrombosis location was $80.5 \%$ in lateral sinus and $53.7 \%$ in superior sagittal sinus; intracranial hematoma was present in $78.0 \%$, signs of intracranial hypertension in $60.9 \%$, cerebral edema in $58.5 \%$, and venous ischemia in $43.9 \%$.

3-Monitoring All patients had an imaging technique (TDM, angioTDM, and MRI). All patients were closely monitored for $\mathrm{CO}_{2}\left(\mathrm{PCO}_{2}\right.$ and end-tidal $\mathrm{CO}_{2}$ ). When feasible, transcranial Doppler was performed in $78 \%$ of patients. Despite the risk of bleeding, eight patients had a monitoring of intracranial pressure.

4-Therapies All patients received heparin therapy, and nine cases had endovascular treatment (21.9\%), osmotherapy (53.7\%), and decompressive craniectomy (16 cases, $39 \%$ ) were necessary to control intracranial hypertension. $63.4 \%$ received norepinephrine to improve brain perfusion, and $90.2 \%$ of patients were under mechanical ventilation. Thirty-one patients received antiepileptic drugs.

5 -Outcome Ten out of 41 patients (24.4\%) died in ICU, and 18/31 $(58.1 \%)$ discharged from ICU had a good outcome (mRS 0-3). After 12 months, $92 \%$ of survivors $(23 / 25)$ had a mRS between 0 and 3 . The proportion of death was $31.7 \%$ at 1 year.

Compared with ICU deaths, survivors were: predominance of female $(p=0.039)$, a worse initial GCS [4 (3-6); $p=0.0004]$, a more frequent mydriasis (100 vs. $32.3 \%, p=0.0001$ ) with more frequent signs of intracranial hypertension on imaging techniques $(p=0.003)$, with a trend for more frequent use of norepinephrine (90 and $54.8 \%$, respectively) and an anticoagulation more difficult to be adequate (50 vs. $80.6 \%$, respectively). Comparing good outcome with poor outcome after 1-year evolution, patients mRS 0-3 appeared with a higher platelets count at day $1(p=0.03)$ and day $5(p=0.06)$ during ICU stay, as a lower D-dimer level after initiation of anticoagulation $(p=0.03)$.

Conclusion The large proportion of acceptable outcome in survivors, which continue to functionally improve after 1 year, motivates the hospitalization in ICU for severe CVT. Persistence of fibrinolysis and activation of coagulation and difficulty to reach efficient anticoagulation during the initial phase might lead to a poor outcome. 


\section{1}

Brain lesion spectrum characterization in an experimental model of infective endocarditis

Marie Cantier', Candice Sabben ${ }^{1}$, Liliane Louedec', Sandrine Delbosc ${ }^{1}$ Clément Journé', Phalla Ou ${ }^{1}$, Isabelle Klein ${ }^{1}$, Françoise Chau ${ }^{2}$, Agnes Lefort $^{3}$, Jean-Philippe Desilles' ${ }^{1}$, Jean-Baptiste Michel' ${ }^{1}$, Romain Sonneville ${ }^{1}$ Mikaël Mazighi

${ }^{1}$ Inserm u1 148, laboratory for vascular translational science, Hôpital Bichat-Claude Bernard, Paris, France; ${ }^{2}$ Inserm u1 137, infection, antimicrobiens, modélisation, evolution, Hôpital Bichat-Claude Bernard, Paris, France; ${ }^{3}$ Service de médecine interne, Hospital Beaujon, Clichy, France

Correspondence: Marie Cantier - mariecantier@gmail.com

Annals of Intensive Care 2016, 6(Suppl 1):021

Introduction Infective endocarditis (IE) is a severe disease, associated with a high mortality. Neurological events are the most frequent complications of left-sided endocarditis, occurring in $20-40 \%$ of patients. Embolic events from vegetations are commonly accepted as the main mechanism responsible for brain lesions, but other hypotheses were highlighted, including a potential subacute cerebral vasculitis. The main objective of the present study was to define the cerebral lesion spectrum in an IE rat model.

Materials and methods Wistar rats underwent an experimental aortic Staphylococcus aureus or Enterococcus faecalis IE. During a surgical procedure, a catheter was inserted into the left ventricle via the right carotid artery. Twenty-four hours later, animals were inoculated with bacterial strain. A systematic brain magnetic resonance imaging (MRI) was performed 2 days after bacterial inoculation. After killing, brains and vegetations were removed. Brains were stained with Masson's trichrome, hematoxylin and eosin, diaminobenzidine-enhanced Perls, and Gram stainings. Immunohistochemistry was performed on brains, including 4',6'-diamidino-2-phenylindole, myeloperoxidase, glial fibrillary acidic protein, and ionized calcium-binding adapter molecule 1 immunostaining. Finally, DNA extraction and polymerase chain reaction (PCR) were performed on the brains and vegetations for specific bacterial DNA amplification. Three other animals groups underwent the same analyses and served as controls: rats of the sham A group were only inoculated with $S$. aureus or $E$. faecalis without any surgical procedure; rats of the sham $B$ group underwent the surgical procedure without bacterial inoculation; and rats of the control group were free of any procedure.

Results Cerebrovascular lesions were detectable on MRI in 12/23 (52\%) animals with IE. Lesions included mostly cerebral micro-bleeds $(n=8,35 \%)$ and brain infarcts $(n=9,39 \%)$. We observed no difference related to the involved microorganism. None of the sham $A$ and control rats revealed MRI lesions. In contrast, the radiological lesion type was similar in the sham B and IE rats. Neuropathological findings showed a cerebral lesion load larger than the one observed on brain MRI. In IE rats, a scattered brain injury pattern was documented made of brain infarcts $(n=12,52 \%)$, micro-hemorrhages $(n=10$, $44 \%)$, and micro-abscesses $(n=11,48 \%)$, associated with a patchy inflammatory perivascular and small vessel wall infiltrate. Reactional gliosis was present around the lesion areas. The IE rats had significantly higher pathological lesions compared with the sham A rats. In contrast, no significant difference was observed between the IE and sham B groups, except for microglial activation, higher in the IE group $(p=0.02)$. Of note, bacterial studies including Gram staining and PCR for bacterial DNA in brains were inconclusive.

Discussion We report here the first rat model of brain injury in the setting of IE. Brain lesion spectrum was relevant to clinical findings in IE. We did not find strong arguments in favor of septic embolism. Moreover, lesion spectrum found is similar to those observed in cerebral vasculitis in human. Therefore, embolism from vegetations may not be the unique mechanism involved in IE-related brain injury. We found similar lesion type in IE and sham B groups, but higher microglial activation in the IE group. Taken together, these results suggest that sepsis and valvular lesion are synergistic conditions involved in brain injury. In this hypothesis, sepsis appears to amplify observed brain injury and reactional gliosis. Finally, this model appears to be adapted for cerebral micro-bleeds detection and analysis, whose physiopathology needs to be further investigated.
Conclusion In this rat model of $\mathrm{IE}$, we characterized brain injury as scattered infarcts, micro-abscesses, micro-bleeds, and small vessel wall inflammation. This lesion spectrum probably results from a combination of thromboembolism and cerebral vasculitis, promoted by additional sepsis and systemic inflammation.

\section{Competing interests None.}

022

\section{Outcome of patients with Parkinson's disease admitted} in intensive care unit

Omar Ben Hadj Salem ${ }^{1}$, Sophie Demeret ${ }^{2}$, Alexandre Demoule ${ }^{3}$, Thomas Similowski ${ }^{3}$, Francis Bolgert ${ }^{4}$, Tarek Sharshar ${ }^{5}$, David Grabli ${ }^{6}$

${ }^{1}$ Service de réanimation médicale, Cochin, Paris, France; ${ }^{2}$ Service de réanimation neurologique, Pitié-Salpêtrière Hospital, Paris, France; ${ }^{3}$ Service de pneumologie et réanimation médicale, Groupe Hospitalier Pitié-Salpêtrière, Paris, France; ${ }^{4}$ Réanimation neurologique, Pitié-Salpêtrière Hospital, Paris, France; ${ }^{5}$ Réanimation, Hôpital Raymond-Poincaré, Garches, France; ${ }^{6}$ Féderation des maladies du système nerveux, Pitié-Salpêtrière Hospital, Paris, France

Correspondence: Omar Ben Hadj Salem - omar.bhs@live.fr Annals of Intensive Care 2016, 6(Suppl 1):022

Introduction Parkinson's disease is associated with an increased risk of severe septic complications that can lead to intensive care unit (ICU) admissions. Despite this, no prognosis data for Parkinson's disease (PD) patients in ICU are available.

Patients and methods The objective was to describe PD patients characteristics admitted to ICU and assess mortality and prognosis at the end of hospitalisation and up to 18 months after discharge. To this purpose, data on 62 PD patients admitted between 1999 and 2014 in two university hospital ICUs have been analysed.

Results The admitted patients were of variable age profiles, duration of evolution and severity of Parkinson's disease. The admission reason was sepsis for $59 \%$ of patients $(n=61)$, and $85 \%$ had at least one sepsis during their hospitalisation $(n=60)$. Mortality was $12.5 \%(n=55)$ after the ICU stay and tended to increase over the 18 months of followup. A Hoehn and Yahr score $>3$ was associated with an extra mortality at $\mathrm{M} 1$ (OR 13.22; $95 \% \mathrm{Cl}$ [1.4-124.9]). The HY average score increased at an early stage from M1 $(2.95-3.9 ; p=0.031)(n=21)$ and lasting until M18 (2.7-3.6; $p=0.049)(n=11)$. The proportion of subjects with cognitive disorders increased to reach over $30 \%$ at M18. Thirty-eight percentage of patients had returned home at M18.

Conclusion The ICU hospitalisation, mostly for a sepsis, marks a turning point in the development of Parkinson's disease. Broader prospective studies are required to assess more accurately the impact of sepsis on Parkinson's disease evolution.

\section{Competing interests None.}

\section{3}

Cerebrospinal fluid glucose and lactate concentrations after subarachnoid hemorrhage

Safa Aribํ, Ilaria Alice Crippa', Jacques Créteur ${ }^{2}$, Jean-Louis Vincent ${ }^{3}$, Fabio Silvio Taccone

${ }^{1}$ Service de soins intensifs, Hôpital Erasme, Bruxelles, Belgium; ${ }^{2}$ Service de soins intensifs, Hospital Erasme, Brussel, Belgium; ${ }^{3}$ Service de Réanimation Médico-Chirurgicale, Hôpital Erasme, Bruxelles, Belgium

Correspondence: Fabio Silvio Taccone - ftaccone@ulb.ac.be

Annals of Intensive Care 2016, 6(Suppl 1):O23

Introduction In patients with subarachnoid hemorrhage (SAH), the monitoring of brain metabolism through microdialysis catheters showed a decrease in brain glucose concentrations and an increase in lactate levels as a marker of cellular distress. However, the use of such catheters is not routinely applied. No data are available on glucose and lactate concentrations into the cerebrospinal fluid (CSF) of such patients.

Patients and methods We performed a retrospective analysis of patients admitted after a non-traumatic SAH from January 2011 to December 2014. Inclusion criteria were: (a) age $>18$ years; (b) the 
presence of an external ventricular drain (EVD) for intracranial pressure monitoring; (c) daily analysis of CSF including glucose and lactate concentrations for at least four consecutive days; and (d) concomitant analysis of glucose and lactate concentrations on the arterial blood gas analysis. Demographics and clinical characteristics were recorded on admission as the development of complications and 3-month neurological outcome (favorable outcome was defined as a Glasgow outcome scale of 4-5).

Results A total of 223 patients with SAH were admitted over the study period; among them, 131 needed a DVE placement and 54 met the inclusion criteria for the final analysis (median age: 57 [49-64] years; male gender: 28/54). A DVE was placed in most of the patients (41/54) on the day of hospital admission and was maintained for a period of 12 [9-16] days. Of the 48 identified aneurysms, 44 were treated with endovascular therapy. Median Glasgow coma scale on admission was 12 [8-14], and CT scan Fisher's scale was 4 [3-4]. A total of 16 patients developed a delayed neurological ischemic deficit (DNID); ICU mortality was $30 \%(16 / 54)$, and $20(37 \%)$ patients had a favorable neurological outcome. Despite a similar CSF red blood cells count, CSF and blood glucose levels than survivors, non-survivors patients had higher blood (1.9 [1.6-2.3] vs. $1.2[1.0-1.9] \mathrm{mEq} / \mathrm{L} ; p<0.009)$ and CSF lactate levels (4.6 [3.6-5.8] vs. $3.2[2.3-4.1] \mathrm{mEq} / \mathrm{L} ; p=0.002)$ and a lower CSF glucose/lactate ratio (21.5 [15.1-25.1] vs. $28.1[20.9-35.1] ; p=0.01)$ on the first CSF analysis. Similar results were also found when patients with poor neurological outcome were compared with others.

Conclusion Patients with poor outcome after SAH demonstrated higher CSF lactate levels and a lower CSF glucose/lactate ratio than patients with favorable outcome on the first day of DVE placement. These CSF findings may suggest a more pronounced metabolic brain distress in these patients or a need for an alternative energetic substrate to glucose.

\section{Competing interests None.}

\section{4}

Spontaneous angionegative subarachnoidal hemorrhage: neurological outcome based on a retrospective study of 68 patients

Alexis Soummer ${ }^{1}$, Nicolas Engrand ${ }^{2}$, Pierre Guedin ${ }^{3}$, Grégoire Trebbia' ${ }^{1}$ Sorin Aldea ${ }^{4}$, Charles Cerf ${ }^{1}$

${ }^{1}$ Réanimation, Hospital Foch, Suresnes, France; ${ }^{2}$ Département d'anesthésie-réanimation, Fondation Ophtalmologique Adolphe de Rothschild, Paris, France; ${ }^{3}$ Neuroradiology, Hospital Foch, Suresnes, France; ${ }^{4}$ Neurosurgery, Hospital Foch, Suresnes, France

Correspondence: Alexis Soummer - alexis_soummer@yahoo.fr Annals of Intensive Care 2016, 6(Suppl 1):O24

Introduction Non-aneurysmal angionegative subarachnoid hemorrhage (NA-SAH), which concerns about $15 \%$ of patients, is known to be associated with a good prognosis [1, 2]. Thus, systematic prolonged clinical observation in neuro-ICU might not be necessary, and to date, no recommendation exists about this point. The aim of our study was to describe the incidence of neurological complications in NA-SAH and try to identify associated risk factors.

Patients and methods We performed a retrospective analysis of NASAH patients in two university-affiliated hospital from 2008 January to 2015 September. NA-SAH was confirmed in all patients with a negative catheter cerebral angiography.

Usual clinical and demographic data and pattern of bleeding on CT scan (perimesencephalic, non-perimesencephalic, Fischer's score) on admission were recorded. During ICU stay, occurrence of neurological and medical complications was analyzed: rebleeding, hydrocephalus, requirement of external ventricular drain (EVD), symptomatic or not cerebral vasospasm, delayed ischemic deficit (DID), dysnatremia (>145 or $<135 \mathrm{mmol} / \mathrm{L}$ ), cardiac and respiratory complications. Follow-up lasted until hospital discharge, and clinical status was described with Glasgow outcome scale (GOS). Results were presented as mean \pm SD or median (first interquartile-third interquartile), and we compared patients with and without complications. Statistical analysis was performed with SPSS ${ }^{\circledR}$.
Results Sixty-eight patients were included. Mean age was $51 \pm 9$, SAPS 2 was 17 (15-20), $96 \%$ were WFNS grades $1-2$, and one patient was grade 4 . Bleeding was strictly limited to perimesencephalic spaces in $32(47 \%)$ patients, and $36(53 \%)$ had a non-perimesencephalic pattern.

22 (33\%) patients developed at least one complication. Nine patients had hydrocephalus, and five of them required EVD. Seven had nonsymptomatic vasospasm, and no patient developed DID. GOS at discharge was good in all cases.

Factors associated with occurrence of complication were non-perimesencephalic pattern compared with perimesencephalic (48 vs $18 \%, p=0.01$ ), intraventricular hemorrhage ( 54 vs $23 \%, p=0.01$ ). In perimesencephalic pattern, no patient experienced serious complication: one patient developed hydrocephalus that did not require EVD, one patient had an asymptomatic vasospasm on CT scan, and four patients had mild dysnatremia. Besides neurological complication, patients often needed multimodal analgesia including opioids to control headaches and nausea/vomiting symptoms.

Conclusion Our retrospective analysis confirms previously published results $[1,2]$ that patients presented a very low rate of complication. Serious complication occurred in $<10 \%$ of patients and consisted in requirement of EVD. Perimesencephalic was never associated with early or late serious complication.

Besides, it is admitted that repetition of brain imaging in non-perimenscephalic SAH gets an average $10 \%$ yield for detection of unrecognized vascular abnormality [1,2], but we do not confirm this in our population.

Further study on larger cohort might lead to a precision of management in patients with a perimesencephalic pattern including a proposition of a short observation in ICU before discharge to the opposite of non-perimesencephalic patients that require a close observation due to the risk of early and late neurological complication.

\section{Competing interests None.}

\section{References}

1. Dalyai R et al., Subarachnoid hemorrhage with negative initial catheter angiography: a review of 254 cases evaluating patients clinical outcome and efficacy of short- long-term repeat angiography. Neurosurgery. 2013 Apr; 72(4): 646-652.

2. Maslehaty $\mathrm{H}$ et al., Special features of subarachnoid hemorrhage of unknown origin: a review of a series of 179 cases. Neurol Res. 2012 Jan; 34(1):91-97.

025

Serious game evaluation of a one-hour training basic life support session for secondary school students: new tools for future bystanders

Victoire Desailly ${ }^{1}$, Pierre Pasquier ${ }^{2}$, Patrick Brun ${ }^{3}$, Damien Roux ${ }^{1}$, Jonathan Messika' ', Gwendoline Latournerie', Laetitia Kasprzyk' ${ }^{1}$, Vincent Grosjean', Amine Latreche', Pierre Habert ${ }^{1}$, Stephane Huot ${ }^{1}$, Timon Jobin ${ }^{1}$, Antoine Tesnière ${ }^{4}$, Didier Dreyfuss ${ }^{1}$, Jean-Damien Ricard ${ }^{1}$, Alexandre Mignon ${ }^{4}$, Stéphane Gaudry ${ }^{1}$

${ }^{1}$ Service de réanimation médico-chirurgicale, CHU Louis Mourier,

Colombes, Colombes, France; ${ }^{2}$ Service de réanimation, Hôpital

d'Instruction des Armées Percy, Clamart, France; ${ }^{3}$ Giga la vie, Institut des Hauts de Seine, Nanterre, France; ${ }^{4}$ Laboratoire ilumens, Universisté René Descartes - Paris V, Paris, France

Correspondence: Stéphane Gaudry - stephanegaudry@gmail.com Annals of Intensive Care 2016, 6(Suppl 1):O25

Introduction Cardiopulmonary resuscitation and immediate activation of emergency medical services are crucial determinants of survival after out-of-hospital cardiac arrest. All citizens should then be taught how to call for help and to perform cardiopulmonary resuscitation. Teaching basic life support to secondary school students has been proved feasible, but not much evaluated. We conducted a study to evaluate the efficacy of a 1-h basic life support training course for secondary school students.

Materials and methods From September 2014 to June 2015, six secondary schools (in two neighboring towns) were included in the study. 


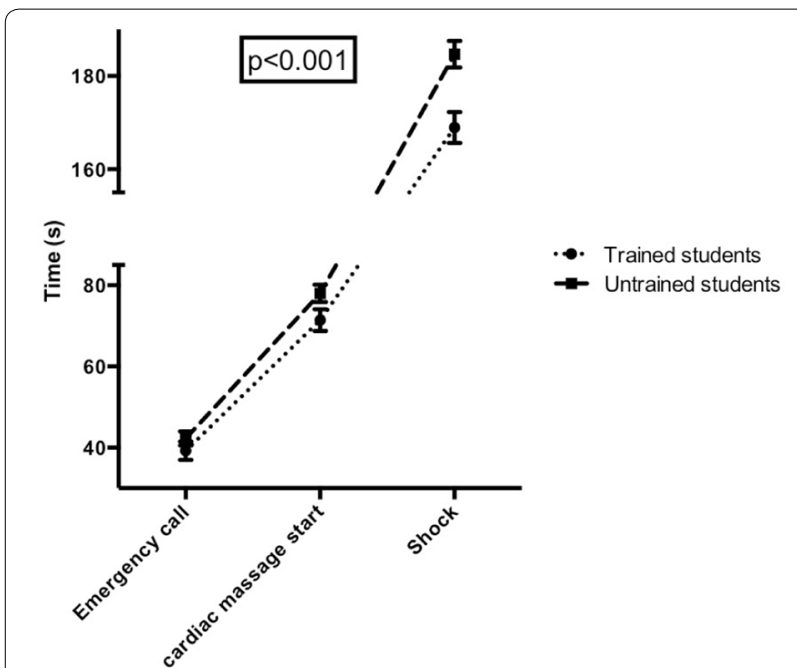

Fig. 6 See text for description

Two of them received a 1-h basic life support session conducted by an intensivist assisted by three medical students. Our work focused on youngest students (first and second year of secondary school). Sessions contained: a theoretical lecture and a practical simulation-based training (chest compression, use of automated external defibrillators) using low-fidelity manikins (MiniAnnePlus ${ }^{\circledR}$, Laerdal). Between 2 and 3 months later, both trained and untrained students were assessed using a serious game reproducing real-life cardiac arrest situation (3D real-time simulation software, Stayingalive ${ }^{\circledR}$, ILUMENS-Dassault system). Primary outcome was the time spent to complete the serious game (from onset of cardiac arrest to shock delivery and recovery). Secondary outcomes included knowledge of emergency call $(15,112$ or 18), hand placement during chest compressions and electrodes placement of automated external defibrillator.

Results Among 199 students (none of them have ever played on Stayingalive ${ }^{\circledR}$ Web site) assessed on the serious game, 72 had received the 1-h basic life support session. Children's ages did not differ between the two groups (12 [11-12] vs 12 [11-12] years old, $p=0.7)$.

The total game completion time was $169( \pm 28) \mathrm{s}$ in the trained group versus $185( \pm 32) \mathrm{s}$ in the non-trained group ( $p=0.0006$; see Fig. 6). Knowledge of emergency call did not differ between the two groups (92\% in trained group vs $86 \%$ in non-trained group, $p=0.2$ ). Hands were more often properly placed during chest compression in the trained group ( 92 vs $73 \%, p=0.002$ ). Electrodes of automated external defibrillator were more often properly placed in the trained group ( 44 vs $10 \%, p=0.0001$ ).

Conclusion A 1-h basic life support training session with low-fidelity manikin improved significantly young students' performance during a $3 \mathrm{D}$ real-life simulation session. This study forms the basis of a large educational health program in Hauts-de-Seine department in France.

Competing interests Manikins were provided by Laerdal Medical without any compensation.

\section{6}

Refractory out-of-hospital refractory cardiac arrest treated by veno-arterial extracorporeal membrane oxygenation. $A$ retrospective single-center experience from 2012 to 2015 . CARECMO program (Cardiac ARrest Extra Corporeal Membrane Oxygenation)

François-Xavier Laithier ${ }^{1}$, Antoine Kimmoun ${ }^{1}$, Tahar Chouihed ${ }^{2}$, Stéphane Albizzati ${ }^{2}$, Edoardo Camenzind ${ }^{3}$, Fabrice Vanhuyse ${ }^{4}$, Bruno Levy ${ }^{1}$

${ }^{1}$ Réanimation médicale brabois, Centre Hospitalier Universitaire de Nancy, Vandœuvre-lès-Nancy, France; ${ }^{2}$ Sau-samu-smur, C.H.U. de Nancy, Nancy,
France; ${ }^{3}$ Cardiologie, C.H.U. de Nancy, Nancy, France; ${ }^{4}$ Chirurgie cardiaque, Centre Hospitalier Universitaire de Nancy, Vandœuvre-lès-Nancy, France Correspondence: Antoine Kimmoun - akimmoun@gmail.com Annals of Intensive Care 2016, 6(Suppl 1):O26

Introduction There is a growing interest in emergency extracorporeal life support for the management of out-of-hospital refractory cardiac arrest (OHRCA). However, preliminary results are discrepancy and many questions remain unresolved. We describe here the preliminary results of our CARECMO program in Nancy, France.

Patients and methods We evaluated retrospectively our monocenter experience between September 2012 and September 2015. Patients were eligible to receive a vaECMO if they presented an out-of-hospital cardiac arrest respecting the CARECMO criteria: (1) witnessed cardiac arrest; (2) no flow <2 min; (3) patient located at $<15 \mathrm{~min}$ to the ECMO center; (3) no ROSC despite advanced cardiac life support (ACLS) provided by the medical mobile team unit; (4) first call to the ECMO center within the $15 \mathrm{~min}$ after the beginning of ACLS; and (5) transfer to the ECMO center under mechanical chest compressor.

Results Thirty-two patients presented a OHRCA respecting CARECMO criteria. Thirteen out of 32 patients had prior cardiovascular history, while $8 / 32$ had no prior medical history. Median age was 43 [IQR 34-48] and sex ratio F/M 1:4. Surgical ECMO implantation was successful in 32 patients (100\%). Immediate coronarography was performed in $28 / 32$ ( $88 \%$ ) patients, and at least one coronary artery was revascularized in 20/28 (71 \%) patients. Eight out of $28(28 \%)$ patients had no coronary artery disease, among which none survived. A total of 11/32 (34\%) patients were weaned from vaECMO, and 8/32 (25\%) were discharged from intensive care unit. In survivors, median length of stay in ICU was 30 days [10-43]. All of these patients were alive at 6 months, and seven of them had a Glasgow outcome score at 5 . Initial cardiac rhythm, prothrombin time within the $4 \mathrm{~h}$ following admission (survivors $48 \%$ [IQR 39-76] vs. non-survivors $41 \%$ [IQR 37-55], $p=0.29$ ) and duration of low flow (survivors 68 [IQR 56-76] vs. non-survivors 76 min [IQR 67-93], $p=0.16$ ) was not associated with the outcome. No difference was found between survivors and non-survivors for $\mathrm{pH}$ (7.27 [7.12-7.32] vs. 7.18 [7.12-7.30], $p=0.5)$ and lactate (7.2 [3.4-13] vs. $8.4[4.5-14], p=0.6)$ at admission in ICU.

Conclusion Following rigorous inclusion criteria, ECMO should be considered in out-of-hospital refractory cardiac arrest.

\section{Competing interests None.}

\section{7}

Decreased monocyte HLA-DR expression after out-of-hospital cardiac arrest

Martin Cour ${ }^{1}$, Fabienne Venet ${ }^{2}$, Romain Hernu ${ }^{1}$, Julie Demaret ${ }^{2}$, Guillaume Monneret ${ }^{2}$, Laurent Argaud ${ }^{1}$

${ }^{1}$ Réanimation Médicale, Hospices Civils de Lyon - Groupement Hospitalier Edouard Herriot, Lyon, France; ${ }^{2}$ Laboratoire d'immunologie, Hôpital

Édouard Herriot, Lyon, France

Correspondence: Laurent Argaud - laurent.argaud@chu-lyon.fr

Annals of Intensive Care 2016, 6(Suppl 1):027

Introduction Post-cardiac arrest syndrome leads to a systemic inflammatory response that shares many similarities with the one observed in sepsis. As a decrease in monocyte HLA-DR expression (mHLA-DR) has been repeatedly described in septic patients with an increase risk of death, we tested whether this immune alteration could also be observed after out-of-hospital cardiac arrest (OHCA).

Patients and methods Fifty-five OHCA patients sampled at day 0 (D0: within $4 \mathrm{~h}$ after OHCA), D1 (the next day), and D3 (after two additional days) were included. CD4 + lymphocyte count and mHLA-DR were evaluated by flow cytometry.

Results We observed a marked decrease in mHLA-DR as early as D0 in patients compared with normal values. This decrease persisted till D3 and was associated with a moderate decrease in the number of CD4+ lymphocytes. No correlations were identified between mHLA-DR and both usual prognostic markers after OHCA and sequential organ failure assessment (SOFA) score. However, overtime evolution in mHLADR values appeared different between survivors and non-survivors 
with a quasi-systematic decrease between D1 and D3 in non-survivors versus an increase in survivors.

Conclusion This preliminary pilot study describes the occurrence of OHCA-induced immune alterations as illustrated by a decreased $\mathrm{mHLA}$ DR and CD4+ lymphopenia. CD4+ count and mHLA-DR could be simple tools to assess the systemic inflammatory response after OHCA.

\section{Competing interests None.}

\section{8}

Is emergent $\mathrm{PCl}$ associated with a clinical benefit in post-cardiac arrest patients without ST-segment elevation pattern? Insights from the PROCAT II registry

Florence Dumas ${ }^{1}$, Wulfran Bougouin ${ }^{2}$, Guillaume Geri ${ }^{2}$, Lionel Lamhaut ${ }^{3}$, Julien Rosencher', Frédéric Pène ${ }^{2}$, Jean-Daniel Chiche2, Olivier Varenne ${ }^{5}$, Pierre Carli ${ }^{6}$, Xavier Jouven 7 , Jean-Paul Mira ${ }^{2}$, Christian Spaulding ${ }^{8}$, Alain Cariou $^{2}$

${ }^{1}$ Emergency department, Cochin hospital-APHP, Paris, France; ${ }^{2}$ Réanimation Médicale, Hôpital Cochin, Paris, France; ${ }^{3}$ Réanimation adulte, Hôpital Necker - Enfants Malades, Paris, France; ${ }^{4}$ Cardiologie, Hôpital Cochin-Port Royal, Paris, France; ${ }^{5}$ Cardiologie, Hôpital Cochin, Paris, France; ${ }^{6}$ Réanimation adulte - samu, Hôpital Necker - Enfants Malades, Paris, France; ${ }^{7}$ Cardiologie, Hôpital Européen Georges-Pompidou, Paris, France; ${ }^{8}$ Cardiologie, Hopital Europeen Georges-Pompidou, Paris, France

Correspondence: Florence Dumas - florence.dumas@aphp.fr Annals of Intensive Care 2016, 6(Suppl 1):O28

Introduction Emergent coronary angiogram and reperfusion are currently a standard of care in patients resuscitated from an out-of-hospital cardiac arrest (OHCA) with ST-segment elevation (STE). However, using a similar invasive strategy is still debated in patients without ST-segment elevation (non-STE). In a large cohort of OHCA patients without STE, we assessed the relationship between the use of an early invasive strategy and patient outcome.

Materials and methods In the absence of an obvious extra-cardiac cause, since many years our practice is to perform an emergent coronary angiogram (CAG) in all OHCA patients (STE and non-STE) at admission, followed by percutaneous coronary intervention (PCI) when required. All patients' characteristics are prospectively collected in a database (PROCAT). Focusing on non-STE patients and using logistical regression, we investigated the association between early $\mathrm{PCl}$ and favorable outcome (CPC1-2 at discharge), and we searched predictive factors for $\mathrm{PCl}$ requirement.

Results During the study period (2004-2013), we investigated 958 OHCA patients with an emergent CAG. Among them, 695/958 (73\%), mostly male (76 \%) and average age of 60 years, had no evidence of STE on the post-resuscitation ECG. A PCI was deemed necessary in 199/695 (29\%). A favorable outcome was observed in 87/200 (43 \%) in patients with $\mathrm{PCl}$ compared with $164 / 495(33 \%)$ in patients without $\mathrm{PCl}(p=0.02)$. After adjustment, $\mathrm{PCl}$ was associated with a better outcome $\left(\mathrm{OR}_{\mathrm{adj}}=1.80[1.09-2.97], p=0.02\right)$. The other predictive factors were a shorter resuscitation length $(<20 \mathrm{~min})$, an initial shockable rhythm and a lower dose of epinephrine $(p<0.001)$. An initial shockable rhythm $\left(\mathrm{OR}_{\mathrm{adj}}=2.83[1.84-4.36], p<0.001\right)$ was an independent indicator for $\mathrm{PCl}$ requirement.

Conclusion A culprit coronary lesion requiring $\mathrm{PCl}$ was found in nearly one-third of OHCA patients without STE. In these patients, emergent PCI was associated with a nearly twofold increase in the rate of favorable outcome. These findings support the use of an invasive strategy in these patients, particularly in those resuscitated from a shockable rhythm.

\section{Competing interests None.}

\section{9}

Predictors of long-term quality of life after cardiac arrest: insights from the Parisian registry

Guillaume Geri', Florence Dumas², Franck Bonnetain, ${ }^{3}$, Wulfran Bougouin', Benoit Champigneulle ${ }^{1}$, Michel Arnaout ${ }^{1}$, Pierre Carli ${ }^{4}$, Eloi Marijon ${ }^{5}$, Olivier Varenne $^{6}$, Jean-Paul Mira', Jean Philippe Empana ${ }^{7}$, Alain Cariou ${ }^{1}$

${ }^{1}$ Réanimation Médicale, Hôpital Cochin, Paris, France; ${ }^{2}$ Service d'accueil des urgences, Hôpital Cochin, Paris, France; ${ }^{3}$ Unité de méthodologie et de qualité de vie en cancérologie, CHRU de Besançon, Boulevard Alexandre Fleming, Besançon, France, Besançon, France; ${ }^{4}$ Réanimation adulte samu, Hôpital Necker - Enfants Malades, Paris, France; ${ }^{5}$ Cardiologie, Hôpital Européen Georges-Pompidou, Rue Leblanc, Paris, France, Paris, France; ${ }^{6}$ Cardiologie, Hôpital Cochin, Paris, France; Inserm u970, Paris Centre de recherche Cardio-vasculaire, Paris, France

Correspondence: Guillaume Geri - guillaume.geri@cch.aphp.fr Annals of Intensive Care 2016, 6(Suppl 1):O29

Introduction Determinants of health-related quality of life (HRQOL) are poorly known in patients resuscitated from out-of-hospital cardiac arrest (OHCA).We aimed at comparing long-term HRQOL between OHCA survivors and controls from the general population and to assess factors associated with HRQOL.

Patients and methods HRQOL was evaluated by telephone interview using SF-36 questionnaire in a large cohort of OHCA survivors. As a comparison group, each OHCA case was age- and gender-matched with four controls from the French general population. Association between pre- and in-hospital Utstein variables and early interventions with the different dimensions of the SF-36 questionnaire was investigated using MANCOVA.

Results During the study period (2000-2013), 1829 OHCA patients were admitted in our unit and 602 discharged alive. SF-36 interview took place 50 months [min, max: 22-93] after cardiac arrest in 255 patients (137 patients died and 210 were lost of follow-up) with a median age of 55 years $[45,64]$ and including $73.7 \%$ of males. Global physical and mental components did not differ between OHCA survivors and controls ( 47.0 vs. $47.1, p=0.88$ and 46.4 vs. $46.9, p=0.45$ ). Physical functioning (74.1 vs. $78.4, p=0.02$ ) and vitality (50.7 vs. 56.2 , $p<0.01$ ) were significantly altered in OHCA survivors. Younger age, male gender and initial shockable rhythm were associated with almost all SF-36 dimensions. Early coronary reperfusion was associated with better physical functioning $(+7.0, p=0.06)$, general health $(+7.3$, $p=0.02)$ and vitality $(+4.4, p=0.08)$.

Conclusion HRQOL of OHCA survivors appeared similar to that of the general population, but some physical components of SF36 scale were significantly altered. Younger age, male gender, initial shockable rhythm and early coronary reperfusion were independently associated with a better HRQOL.

\section{Competing interests None.}

\section{0}

Red blood cell transfusions in early resuscitation of severe sepsis and septic shock in patients with hematological malignancies Adrien Mirouse ${ }^{1}$, Matthieu Resche-Rigon ${ }^{2}$, Virginie Lemiale ${ }^{3}$, Djamel Mokart ${ }^{4}$, François Vincent ${ }^{5}$, Julien Mayaux ${ }^{6}$, Antoine Rabbat ${ }^{7}$, Martine Nyunga ${ }^{8}$, Anne Pascale Meert ${ }^{9}$, Dominique Benoit ${ }^{10}$, Achille Kouatchet ${ }^{11}$, Michaël Darmon ${ }^{12}$, Fabrice Bruneel ${ }^{13}$, Elie Azoulay ${ }^{14}$, Frédéric Pène ${ }^{15}$ ${ }^{1}$ Réanimation médicale, hôpital Cochin, Assistance Publique - Hôpitaux de Paris, Paris, France; '2Biostatistiques, Hôpital Saint-Louis, Paris, France; ${ }^{3}$ medical ICU, Hôpital Saint Louis, Paris, France; ${ }^{4}$ Réanimation, Institut Paoli-Calmettes, Marseille, France; ${ }^{5}$ Réanimation polyvalente, Groupe Hospitalier Intercommunal Le Raincy-Montfermeil, Montfermeil, France; ${ }^{6}$ Pneumologie et réanimation médicale, Hôpital Pitié-Salpêtrière, Paris, France; ${ }^{7}$ Réanimation pneumologique, Hôpital Cochin, Paris, France; ${ }^{8}$ Réanimation, Centre Hospitalier De Roubaix, Roubaix, France; ${ }^{9}$ Réanimation, Institut Jules Bordet, Bruxelles, Belgium; ${ }^{10}$ Réanimation polyvalente, Hopital universitaire, Gand, Belgium; ${ }^{11}$ Réanimation médicale, Centre Hospitalier Universitaire d'Angers, Angers, France; ${ }^{12}$ Réanimation Médicale, CHU Saint-Etienne - Hôpital Nord, Saint-Étienne, France; ${ }^{13}$ Réanimation médico-chirurgicale, Centre Hospitalier de Versailles, Le Chesnay, France; ${ }^{14}$ Réanimation médicale, Hôpital Saint-Louis, Paris, France; ${ }^{15}$ Réanimation Médicale, Hôpital Cochin, Paris, France

Correspondence: Adrien Mirouse - adrien.mirouse@gmail.com Annals of Intensive Care 2016, 6(Suppl 1):030

Introduction Severe sepsis and septic shock are common complications for patients with hematological malignancies. Anemia is a frequent underlying condition in such patients who are then likely to receive red blood cell transfusion as part of early hemodynamic 
resuscitation. However, the optimal threshold and the eventual benefit of red blood cell transfusion in septic acute circulatory failure remain debated. In this study, we investigated the indications of red blood cell transfusion and its impact on outcome in a large cohort of patients with hematological malignancies who were admitted to the ICU for the main diagnosis of severe sepsis and septic shock.

Patients and methods TRIAL-OH is a prospective, multicenter observational study that included 1011 patients with hematological malignancies who required ICU admission in 2010-2011 in 17 French and Belgian centers (1). We focused on patients admitted in ICU with a definite diagnosis of severe sepsis or septic shock according to the Surviving Sepsis Campaign definitions. We addressed the requirements for red blood cell transfusion during the first $48 \mathrm{~h}$ as part of initial resuscitation. Continuous variables were expressed as median [interquartile range]. Endpoints were vital status at 7 days, ICU and hospital discharge. Multivariate logistic regression was used to assess the impact of transfusion on mortality.

Results Among the 1011 patients of the whole TRIAL-OH cohort, 631 $(62.4 \%)$ were admitted to the ICU for severe sepsis $(55 \%)$ or septic shock (45\%). Patients were mostly males $(64.3 \%)$ with an age of 60 [49-70] years. The admission SOFA score was 7 [4-10]. The hemoglobin level at the time of ICU admission was 9.1 [7.9-10.4] g/dL. One hundred and forty-eight ( $23.5 \%)$ and $89(14.5 \%)$ patients received red blood cell transfusion at days 1 and 2, respectively. Overall, 210 (33.3\%) patients received 2 [1-3] packed red cells during the first 48 h. Hemoglobin levels were lower in transfused patients at day 1 (7.8 [7.1-8.8] vs. 9.7 [8.7-11] g/dL, $p<0.001)$ and day 2 (8.9 [7.9-9.9] vs. 9.3 [8.4-10.2] g/dL, $p=0.001)$ and became similar to those of non-transfused patients at day 3 (8.9 [8.2-9.8] vs. 8.9 [8-10]). Early red blood cell transfusion was more likely in patients with myeloid neoplasms and neutropenia. Transfused patients appeared with more severe presentations as assessed by higher admission SOFA scores (8 [5-11] vs. 6 [4-9], $p<0.001)$ and blood lactate levels $(2.4[1.6-5.6]$ vs. $1.9[1.1-3.6] \mathrm{mmol} / \mathrm{L}, p<0.001)$ and the requirements for organ failure supports including vasopressive drugs (78.9 vs. $49.7 \%, p<0.001)$, invasive mechanical ventilation $(75.5$ vs. $33.5 \%, p<0.001$ ) and renal replacement therapy (38.7 vs. $15.4 \%$, $p<0.001)$. In univariate analysis, red blood cell transfusion within the first $48 \mathrm{~h}$ was associated with increased mortality at day 7 (20.5 vs. $13.3 \%, p=0.02$ ), in the ICU (39 vs. $25.2 \%, p<0.001$ ) and in the hospital (51 vs. $36.6 \%, p<0.001$ ). After adjustment with other significant variables, red blood cell transfusion was no longer associated with day 7 mortality (OR 1.18 [0.78-1.98], $p=0.52$ ), but tended to be associated with in-ICU and in-hospital mortality (OR 1.48 [0.96-2.26], $p=0.07$ and OR 1.54 [1.04-2.29], $p=0.03$, respectively).

Discussion Although early red blood transfusion was provided to the most severe septic patients, it remained associated with a poor prognosis in the multivariate analysis. Whether transfusion may represent a potent marker of severity in this setting or may favor subsequent side effects in septic patients deserves further investigations.

Conclusion Red blood cell transfusion is commonly used in early resuscitation of hematological patients with severe sepsis, and particularly in those with septic shock. The poor prognostic value was associated with transfusion calls for prospective studies in this highrisk subgroup of patients.

\section{Competing interests None.}

\section{Reference}

1. Azoulay et al. J Clin Oncol 2013; 31(22): 2810-8.

\section{1}

Causal effect of transfusion on mortality and other adverse events among critically ill septic patients: an observational study with a marginal structural model

Claire Dupuis ${ }^{1}$, Michaël Darmon², Carole Schwebel ${ }^{3}$, Elie Azoulay ${ }^{4}$ Romain Sonneville ${ }^{5}$, Lila Bouadma ${ }^{6}$, Roland Smonig', Yves Cohen ${ }^{8}$, Stéphane Ruckly ${ }^{9}$, Christophe Adrie ${ }^{10}$, Dany Goldgran-Toledano ${ }^{11}$, Sébastien Bailly ${ }^{12}$, Guillaume Marcotte ${ }^{13}$, Maïté Garrouste-Orgeas ${ }^{14}$, Jean-François Timsit ${ }^{15}$

${ }^{1}$ Réanimation médicale, CHU Bichat, Paris, France; ${ }^{2}$ Réanimation Médicale, CHU Saint-Etienne - Hôpital Nord, Saint-Étienne, France; ${ }^{3}$ Réanimation médicale, C.H.U. Grenoble, Grenoble, France; ${ }^{4}$ Réanimation médicale, Hôpital Saint-Louis, Paris, France; ${ }^{5}$ Service de réanimation médicale et infectieuse, Hôpital Bichat-Claude Bernard-APHP, Paris, France; ${ }^{6}$ Réanimation médicale et des maladies infectieuses, Hôpital Bichat-Claude Bernard, Paris, France; ${ }^{7}$ Réanimation médicale, Centre Hospitalier Universitaire de Rennes, Rennes, France; ${ }^{8}$ Réanimation médico-chirugicale, Hôpital Avicenne, Bobigny, France; ${ }^{9}$ Umr 1137, Inserm iame, Paris, France; ${ }^{10}$ Réanimation, CHU De La fontaine, Saint-Denis, France; ${ }^{11}$ Réanimation, CH gonesse, Gonesse, France; ${ }^{12}$ Equipe 11, Institut Albert Bonniot - Inserm U823, La Tronche, France; ${ }^{13}$ Reanimation medicale, Assistance Publique Hôpitaux de Paris, Paris, France; ${ }^{14}$ réanimation, Fondation

Hopital Saint Joseph, Paris, France; ${ }^{15}$ Réanimation médicale et infectieuse, Hôpital Bichat-Claude Bernard, Paris, France

Correspondence: Claire Dupuis - claire.dupuis@inserm.fr

Annals of Intensive Care 2016, 6(Suppl 1):031

Introduction Septic patients tend to be anemic with limited tolerance in intensive care unit. Red blood cell transfusion (RBCT) is the main treatment but can be deleterious. In that context, one RCT(1) concluded that $7 \mathrm{~g} / \mathrm{dl}$ is a good target but excluded patients with overt bleeding or low hemoglobin level during the day of admission and showed significant increase in ischemic events. Moreover, many cohort studies estimated the impact of RBCT, but reached opposite results. One of their main limits was they did not handle time-dependent covariates and past history of treatment. In that context, the aim of our study was to estimate the causal effect on outcomes and adverse events of RBCT in septic shock patients thanks to a marginal structural model.

Patients and methods We used the prospective French multicentric Outcomerea database. Four outcomes were assessed: death, the occurrence of nosocomial infection, ischemic event and acute respiratory distress syndrome. In each analysis, we included patients with sepsis severe and septic shock since 04/01/1998 and excluded patients with hemorrhagic shock on admission. Only patients staying for more than $48 \mathrm{~h}$ were analyzed to estimate the risk of nosocomial infection. Similarly, patients having ischemic events during the first $48 \mathrm{~h}$ were not included in the estimation of the risk of ischemic event. A marginal structural cox model with ponderation on the inverse probability of treatment weight (IPTW) and inverse probability of censoring weight (IPCW) was then used to estimate the impact of transfusion with a censoring at day 30 . Subgroups with similar hematocrit evolution pattern were determined thanks to a trajectory modeling. Impact of transfusion on death was assessed among those subgroups.

Results Finally, 6016 patients were included, and 1940 (32.2 \%) of them were transfused during their ICU stay and received 3 [2-5] (median [IQR]) red cell pack per patient transfused per ICU stay. RBCT was not associated with death [HR $0.8,95 \%$ confidence interval (Cl) $0.544-1.176, p=0.255]$, with the occurrence of ischemic event (HR $0.774,95 \% \mathrm{Cl}, 0.343-1.749, p=0.538$ ) or with the occurrence of ARDS (HR 0.948, $95 \% \mathrm{Cl}, 0.811-1.107, p=0.498)$. However, RBCT was associated with the occurrence of nosocomial infections ( $\mathrm{HR} 2.149,95 \% \mathrm{Cl}$, $1.662-2.779, p<0.001)$. Four subgroups were identified thanks to our trajectory modeling. In the subgroup with the lowest hematocrit level (hematocrit 26 [24-28]), transfusion had a beneficial effect (HR 0.375, $95 \% \mathrm{Cl} 0.225-0.625, p<0.001)$, whereas in the subgroup with a hematocrit at 30 [28-31.2], transfusion had no more impact on outcome (HR $0.942,95 \% \mathrm{Cl} 0.601-1.477, p=0.795)$.

Conclusion Transfusion was not associated with death in the entire cohort. Modification of transfusion practice such as systematic deleukocytation and lower transfusion thresholds might have improve the tolerance of transfusion. The protective effect in the subgroup with the lower hematocrit level might reflect the limits of a drastic lowering of the transfusion thresholds. RBCT still remained associated with nosocomial infections. Others immunomodulating factors than leukocytes might explain this association.

\section{Competing interests None.}

Reference

1. Holst LB, Haase N, Wetterslev J, Wernerman J, Guttormsen AB, Karlsson $\mathrm{S}$, et al. Lower versus Higher Hemoglobin Threshold for Transfusion in Septic Shock. N Engl J Med. 2014 Oct 9;371(15):1381-91. 
032

Autoimmune hemolytic anemia in the intensive care unit

Antoine Lafarge ${ }^{1}$, Claire Pichereau', Sandrine Valade ${ }^{1}$, Marion Venot ${ }^{1}$, Akli Chermak', Igor Theodose', Marion Scotto', Delphine Kemlin' ${ }^{1}$, Claire Givel $^{1}$, Leïla Mourtada' ${ }^{1}$ Etienne Ghrenassia' ${ }^{1}$ Emmanuel Canet $^{1}$, Virginie Lemiale', Benoît Schlemmer', Elie Azoulay', Eric Mariotte ${ }^{1}$ ${ }^{1}$ Réanimation médicale, Hôpital Saint Louis, Paris, France

Correspondence: Antoine Lafarge - antoinelafarge@outlook.com Annals of Intensive Care 2016, 6(Suppl 1):032

Introduction Autoimmune hemolytic anemia (AlHA) is a rare, heterogeneous and potentially life-threatening disease. Data on AlHA patients requiring admission to the intensive care unit (ICU) are scarce. The aim of our study was to describe the characteristics and outcome of patients with AlHA in the ICU.

Patients and methods Patients with AlHA admitted to the ICU of Saint Louis University Hospital between years 2002 and 2015 were retrospectively identified using a computerized database. Clinical and biological data including past medical history, etiologic diagnosis, type of direct antiglobulin test (DAT), organ failures, treatments and follow-up were abstracted from the patients' charts. Primary outcome was ICU mortality. Data are presented as numbers (\%) or medians (interquartile range). Survivors (S) and decedents (D) were compared using Chi squared test for binary variables and Mann-Whitney test for continuous variables.

Results During the study period, 270 patients with hemolytic anemia required ICU admission, including $44(16.3 \%)$ patients with AIHA. Twenty patients presented with a DAT positive for immunoglobulin G (IgG) only (45.5\%), eight with a DAT positive for both IgG and complement $(36.3 \%)$ and 16 with a DAT positive for complement only (18.1\%). There were 24 men (54.5\%) with an age of 57 years (44.5-69). Twenty-five patients (56.8 \%) had a past history of malignant hemopathy, five $(11.4 \%)$ had solid cancer, and five $(11.4 \%)$ had HIV infection. Median delay from first symptoms of AlHA to hospitalization was 14 days (2-38). Patients were mostly hospitalized for hematological (23 [52.3\%]) or respiratory (11 [25\%]) failure. Median sequential organ failure assessment score (SOFA) at day 1 was 6 (3.8-8). Hemoglobin rate at admission was $6.8 \mathrm{~g} / \mathrm{dl}(4.6-9.1)$. Corticosteroids were administered to 39 patients $(88.6 \%)$ and anti-CD20 monoclonal antibody rituximab to 11 patients (25.6\%). ICU stay was 2.5 days (2-6), and hospital stay was 15 days (9-42). Follow-up was 74 days (11.5-695.2).

Thirteen patients $(29.5 \%$ ) died in the ICU, and none of the survivors died during post-ICU hospitalization. There was no difference between $S$ and $D$ regarding associated comorbidities like hemopathy (18 [58.1\%] vs $7[53.8 \%], p=0.80$ ), solid cancer (3 [9.7\%] vs 2 [15.4\%], $p=0.59)$ or HIV (4 [12.9\%] vs 1 [7.7\%], $p=0.62)$. In $\mathrm{D}$, age was higher (72 years old [57.8-76.3] vs 50 years old [34.3-64], $p<0.01$ ) and organ dysfunctions were more severe at day 1 (SOFA 8 [7-11] vs 5.5 [3-7], $p<0.01$ ). Patients with a DAT positive for lgG only (DAT-IgG) displayed poorer outcome in comparison with patients with a Coombs test positive for lgG + complement or complement only (DAT-IgG + C/C) (ICU mortality 69.2 vs $35.5 \%, p=0.04)$. In comparison with patients with DAT-IgG + C/C, DAT-IgG patients were older (65 years old [52-73.5] vs 49 years old [34.5-59.5], $p=0.01$ ), presented with higher rates of hemoglobin at admission (8.3 [6.7-9.8] vs 5.5 [3.7-7.5], $p<0.01)$ and were less likely to receive rituximab in the ICU (2 [10\%] vs 9 [39.1\%], $p=0.03$ )

Discussion Mortality rate of AlHA patients requiring ICU admission is higher than previously reported and appears to be impacted by age, organ failures and DAT-lgG. Those results are tempered by evident recruitment biases (over-representation of hemopathies, paucity of cold hemagglutinin disease), confounding factors (age, rituximab) and the small sample size. Further larger studies are needed to confirm DAT-lgG as an independent prognostic factor and explain its pathophysiology.

Conclusion The mortality rate of AIHA patients in ICU is consequential. Besides organ failures, IgG-positive DAT may be a substantial prognostic factor.

Competing interests None.
033

Pre-ICU location, lead time bias and outcomes in patients with thrombotic microangiopathies

Damien Vimpere ${ }^{1}$, Sandrine Valade ${ }^{1}$, Marion Venot $^{1}$, Claire Pichereau $^{1}$, Akli Chermak², Virginie Lemiale ${ }^{1}$, Emmanuel Canet', Lionel Galicier ${ }^{3}$, Elie Azoulay ${ }^{1}$, Eric Mariotte ${ }^{1}$

${ }^{1}$ Réanimation médicale, Hôpital Saint Louis, Paris, France; ${ }^{2}$ Réanimation polyvalente, $\mathrm{CH}$ sud Essonne, Paris, France; ${ }^{3}$ Immuno-hématologie, Assistance Publique Hôpitaux de Paris, Paris, France

Correspondence: Damien Vimpere - vimpere.damien@gmail.com Annals of Intensive Care 2016, 6(Suppl 1):O33

Introduction The thrombotic microangiopathy (TMA) syndrome encompasses a wide spectrum pathologies including thrombotic thrombocytopenic purpura (TTP) and complement-mediated TMA [formerly known as atypical hemolytic uremic syndrome (HUS)]. The aim of the present study was to describe the first clinical symptoms in critically ill TMA patients and to evaluate the impact of pre-intensive care unit (ICU) location on the patients' outcomes.

Patients and methods Patients with TMA admitted to a 12-bed medical university hospital ICU between 2009 and 2015 were identified using a computerized database. Data concerning the pre-ICU period and clinico-biological parameters were abstracted from medical charts. Data are expressed as numbers (\%) and medians (interquartile range). Comparisons were made using the Chi square test or the Mann-Whitney $U$ test as appropriate.

Results Among the 74 TMA patients admitted in our ICU (43 [58 \%] females, aged 42 [32-55] years), 51 patients (68.9\%) had TTP, 13 (17.6\%) HUS and $10(13.5 \%)$ other TMA. At ICU admission, hemoglobin rate was $8.2 \mathrm{~g} / \mathrm{dl}(6.8-9.7)$, platelet rate $17 \mathrm{G} / \mathrm{l}(9-31), \mathrm{LDH}$ level $1743 \mathrm{U} / \mathrm{l}(1064-2581)$ and creatininemia $113.5 \mu \mathrm{mol} / \mathrm{I}$ (75-184), and 43 patients $(64.2 \%)$ displayed elevated troponin levels. Day 1 SAPS2 score was $21.5(12-32)$ and SOFA score was $6(4-8)$. ICU length of stay was 7 days (4-11), and hospital length of stay was 16.5 days (12.5-28.5). Five patients (9.4\%) died during hospital stay. Earlier TMA manifestation was neurologic in 25 cases (33.8\%), digestive in 20 cases (27\%), uro-nephrologic in 14 cases (18.9\%), dermatologic in 12 cases (16.2\%), cardiologic in ten cases $(13.5 \%)$ and respiratory in nine cases $(12.2 \%)$. Sixteen patients (21.6\%) reported simultaneous occurrence of more tha one first symptom. The occurrence of diarrhea or abdominal pains as first symptoms of TMA was more frequent in HUS patients in comparison with TTP and other TMA patients (respectively, 8 [61.5\%] vs 11 [21.6\%] vs 1 [10\%], $p<0.001$ ). Time from first clinical symptom to first medical contact was 4 days (0-8.5). This time was longer when the first alleged symptom was dyspnea (14 days [10.5-18.5] vs 3.5 days [0-7], $p=0.03$ ). First medical contact consisted in hospital consultation in 58 cases $(78.4 \%$ ) including 45 emergency department (ED) consultations, six direct admissions to specialized ICUs (three neurologic and three cardiologic) and three direct admissions to medico-surgical wards. In $16(21.6 \%)$ cases, the first medical contact was community based (15 general practice and one cardiology practice). Patients were admitted in one hospital ward (range 0-5) before medical ICU referral. Only 14 patients (18.9\%) were directly admitted to a medical ICU performing PEX, after first medical contact with an ED (11 cases) or hospital-based consultations (three cases). None of these patients died. Prior to ICU admission, 21 patients were managed in the ED (27 cases [34.5\%]), another medical ICU in nine cases (15\%) or a medico-surgical ward (24 cases [40\%]). Secondary referral to ICU where PEX was available was similar in patients first admitted to ED or medico-surgical wards ( 63 vs $70.8 \%$ ), but time to ICU transfer was shorter for patients admitted first to ED than for patients directly admitted to wards ( 1 days $[0-1]$ vs 3 days [1.5-7], $p<0.01$ ). Patients admitted first to another ICU were secondarily referred to our ICU for refractory TMA within 6 days (0.5-10.5).

Discussion Our data suggest that family physicians and ED specialists must maintain a high level of suspicion for the diagnosis to appropriately refer these patients to specialized ICUs. Indeed, no patient directly admitted to ICU died, suggesting that early recognition of TMA syndrome and quick transfer to specialized centers may improve 
TMA outcomes. However, we found no association of pre-ICU characteristics and mortality, likely because of the low case fatality rate.

Conclusion TMA patients still display relatively long pre-ICU trajectories before referral to specialized ICUs, with inappropriate referral resulting in longer delays for ICU admission in centers where PEX and specific management is available. These data need to be repeated at large scale and with outcomes such as morbidity, length of stay, unresponsive TMAs and long-term outcomes.

\section{Competing interests None.}

\section{4}

Septic shocks with no early etiological diagnosis: a multicenter prospective cohort study (the shock 24 study)

Damien Contou', Damien Roux ${ }^{2}$, Sebastien Jochmans ${ }^{3}$, Remi Coudroy ${ }^{4}$ Emmanuel Guérot ${ }^{5}$, David Grimaldi ${ }^{6}$, Sylvie Ricome ${ }^{7}$, Eric Maury ${ }^{8}$, Gaëtan Plantefève ${ }^{9}$, Julien Mayaux ${ }^{10}$, Armand Mekontso Dessap ${ }^{11}$, Christian Brun-Buisson ${ }^{11}$, Nicolas de Prost ${ }^{1}$

${ }^{1}$ Medical Intensive Care Unit, Hospital Henri Mondor, Créteil, France; ${ }^{2}$ Réanimation médico-chirurgicale, Hôpital Louis-Mourier - APHP, Colombes, France; ${ }^{3}$ Service de Réanimation, Centre Hospitalier Marc Jacquet, Melun, France; ${ }^{4}$ Réanimation médicale, CHU de Poitiers, Poitiers, France; ${ }^{5}$ Réanimation médicale, Hopital Europeen Georges-Pompidou, Paris, France; ${ }^{6}$ Service de réanimation polyvalente, Centre Hospitalier de Versailles, Le Chesnay, France; ${ }^{7}$ Service de réanimation polyvalente, Centre hospitalier intercommunal Robert Ballanger, Aulnay-sous-Bois, France; ${ }^{8}$ Réanimation Médicale, Hôpital Saint-Antoine, AP-HP, Paris, France; ${ }^{9}$ Réanimation polyvalente, C.H. Victor Dupouy (Argenteuil), Argenteuil, France;

${ }^{10}$ Pneumologie et réanimation médicale, Hôpital Pitié-Salpêtrière, Paris, France; ${ }^{11}$ Réanimation Médicale, Hôpital Henri Mondor, Créteil, France Correspondence: Damien Contou - damien.contou@aphp.fr Annals of Intensive Care 2016, 6(Suppl 1):034

Introduction The lack of a patent source of infection and microbiological documentation is not uncommon during the first $24 \mathrm{~h}$ management of a septic shock, raising the possibility of non-infectious causes. Patients and methods Among patients admitted in the intensive care unit (ICU) with a "clinical phenotype" of septic shock, we aimed to determine the prevalence of shock with no early etiological diagnosis, to identify their main causes and to compare their outcome to those of patients with an "early-diagnosed" septic shock. We conducted a prospective, multicenter, observational cohort study in ten ICUs of public hospitals in France.

Results From November 2014 to June 2015, 508 patients were admitted for a suspicion of septic shock. Among them, 374 (74\%) had a proven diagnosis of septic shock made during the first $24 \mathrm{~h}$ ("early" septic shock, ESS), while the 134 (26\%) others had no source of infection nor microbiological documentation identified within the first $24 \mathrm{~h}$. These patients had either septic shock confirmed during the first $24 \mathrm{~h}$ ("late" septic shock, LSS; $n=37 / 134,28 \%$ ) or a severe systemic inflammatory response syndrome (SIRS) $(n=97 / 134,72 \%)$ encompassing patients having a non-septic severe SIRS (NS-SIRS) (i.e., a noninfectious cause was identified; $n=59 / 134,44 \%$ ) or a severe SIRS of unknown origin (SIRS-UO) $(n=38 / 134,28 \%)$. The leading causes of non-septic severe SIRS were adverse drug reactions, acute mesenteric ischemia, malignancies and inflammatory diseases. There were no differences regarding the in-ICU mortality $(n=139 / 374,37 \%$ vs. $49 / 134,37 \% ; p=0.90$ ) and the median duration of ICU stay (7 vs. 7 days, $p=0.69$ ), of tracheal intubation ( 4 vs. 4 days, $p=0.19$ ) and of vasopressor support ( 3 vs. 3 days, $p=0.55$ ) between patients with "early" septic shock (ESS) and others. A multivariable Cox model with adjustment on variables significantly associated with day- 60 mortality showed that patients with a severe SIRS of unknown origin had a higher risk of mortality than others (Fig. 7).

Conclusion One-quarter of the patients admitted in the ICU for a suspicion of septic shock had no infection identified $24 \mathrm{~h}$ after vasopressors introduction, and almost three-quarters of these had a severe SIRS that mimicked sepsis. Patient with a severe SIRS of unknown origin had a poorer outcome (Fig. 8).
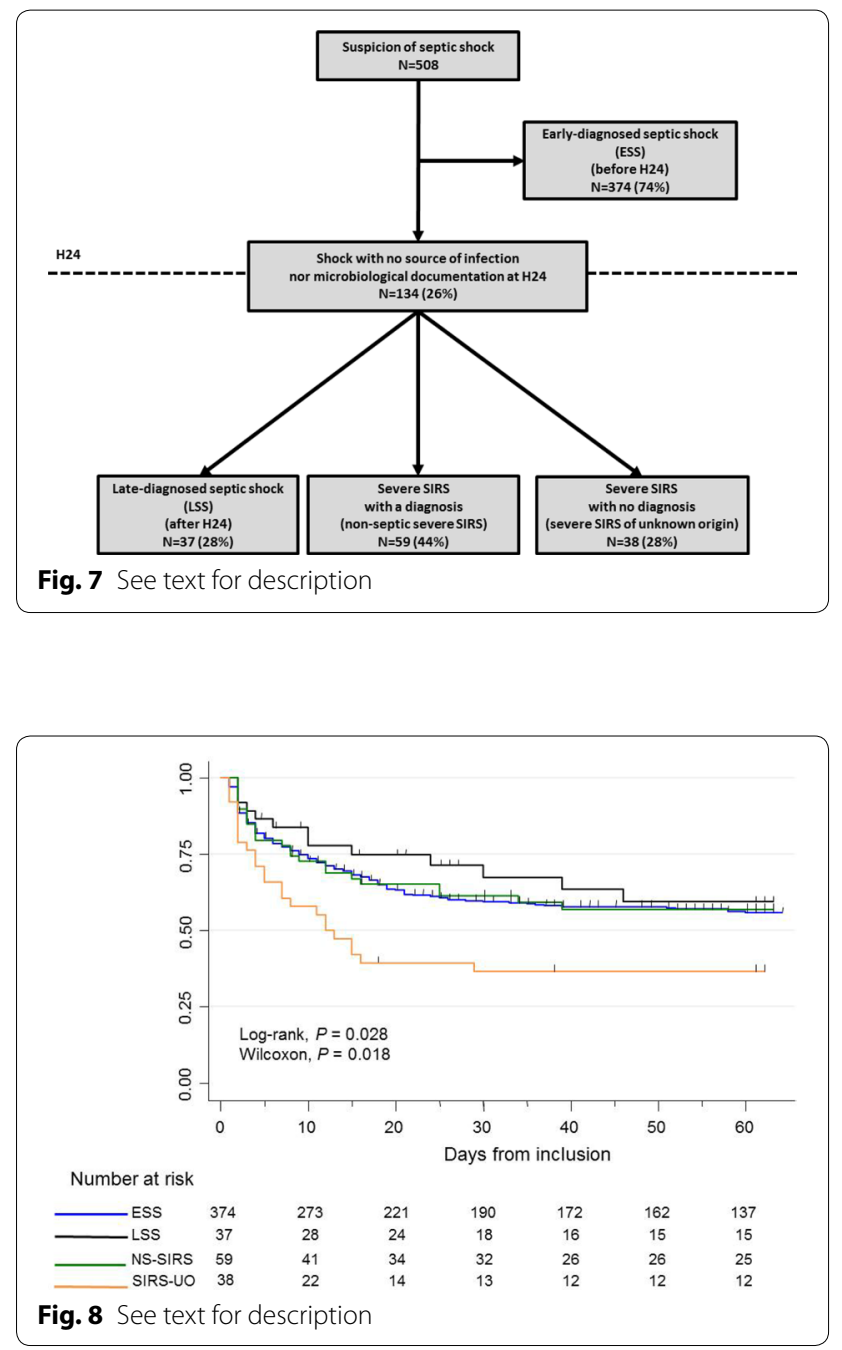

Competing interests None.

\section{References}

1. Brun-Buisson C, Meshaka P, Pinton P, Vallet B, (2004) EPISEPSIS: a reappraisal of the epidemiology and outcome of severe sepsis in French intensive care units. Intensive Care Med 30: 580-588.

2. Heffner AC, Horton JM, Marchick MR, Jones AE, (2010) Etiology of illness in patients with severe sepsis admitted to the hospital from the emergency department. Clin Infect Dis 50: 814-820.

\section{5}

Respective contribution of diaphragm and limbs muscles weakness on weaning from mechanical ventilation outcome Alexandre Demoule ${ }^{1}$, Bruno-Pierre Dubé ${ }^{1}$, Julien Mayaux', Julie Delemazure ${ }^{1}$, Thomas Similowski ${ }^{1}$, Martin Dres ${ }^{1}$

${ }^{1}$ Intensive Care and Respiratory Division, Pitié-Salpêtrière Hospital, Paris, France

Correspondence: Martin Dres - martin.dres@aphp.fr Annals of Intensive Care 2016, 6(Suppl 1):035

Introduction The role of intensive care-acquired weakness (ICU-AW) and diaphragm weakness (DW) is questioned in case of prolonged or failed weaning from mechanical ventilation. The relative contribution of DW and ICU-AW on this outcome is unclear. We aimed at assessing 
the respective prevalence and impact of DW and ICU-AW on the success (WS) or failure (WF) of the weaning process. We combined the use of twitch airway occlusion pressure and ultrasound to evaluate diaphragm stength.

Patients and methods In ICU patients undergoing a first spontaneous breathing trial, diaphragm function was evaluated by twitch tracheal pressure in response to bilateral anterior magnetic phrenic nerve stimulation (Ptr,stim) and by diaphragm ultrasonography [end-expiratory thickness (TdiEXPI), thickening fraction (TFdi), excursion (EXdi). MRC score below 48 defined ICU-AW and Ptr, stim below $11 \mathrm{cmH} 2 \mathrm{O}$ defined DD.

Results Seventy-six patients were included: age: 60 years (47-69) and duration of mechanical ventilation: 5 days (3-9). DW and ICU-AW were, respectively, present in $48(63 \%)$ and $26(34 \%)$ patients. The prevalence of DW was similar among patients with or without ICU-AW (62 vs. $64 \%, p=0.83$ ) as well was the median MRC score in patients with or without DW [51 (43-56) vs. 51 (44-59), $p=0.30]$. The proportion of patients with DW but without ICU-AW was $42 \%$, whereas the proportion of patients with both DW and ICU-AW was $21 \%(p<0.05)$. By contrast, the proportion of patients with ICU-AW but without DW was $13 \%$. The proportion of patients without ICU-AW neither DW was $24 \%$. DW was associated with WF (OR 23.7, $95 \% \mathrm{Cl}$ 5.0-112.2, $p<0.001$ ) but not ICU-AW (OR 1.9, $95 \% \mathrm{Cl} 0.73-5.0, p=0.23$ ). Patients with both DW and ICU-AW had a significantly higher ICU mortality as compared to patients with ICU-AW without DW (38 vs. $0 \%, p<0.001$ ). The areas under the receiver operating characteristic curves of Ptr, stim, TFdi and MRC score to predict weaning outcome were $0.92,0.90$ and 0.67 , respectively.

Conclusion Diaphragm weakness had higher prevalence and worst impact on weaning outcome than ICU-AW. Diaphragm ultrasound predicted weaning outcome with good performance. Determination of the respective risk factor for DW and ICU-AW deserves now further studies.

\section{Competing interests None.}

\section{6}

Impact of sleep quality on the duration of weaning

from mechanical ventilation

Ludivine Rousseau' ${ }^{1}$ Xavier Drouot ${ }^{2}$, Véronique Diaz² ${ }^{2}$ Yohann Rebollar², Jean-Pierre Frat ${ }^{1}$, Remi Coudroy ${ }^{1}$, René Robert ${ }^{1}$, Arnaud, W Thille' ${ }^{1}$, Groupe ALIVE

${ }^{1}$ Réanimation médicale, Chu De Poitiers, Poitiers, France; ${ }^{2}$ Neurophysiologie et explorations fonctionnelles, Chu De Poitiers, Poitiers, France

Correspondence: Ludivine Rousseau - ludivine.rousseau@gmail.com Annals of Intensive Care 2016, 6(Suppl 1):036

Introduction In difficult-to-wean ICU patients under mechanical ventilation, the influence of sleep quality on weaning duration has never been studied. We aimed to compare sleep quality between patients with a short weaning duration and those with a prolonged weaning duration.

Patients and methods Prospective physiological study performed in a French teaching hospital. All patients intubated at least $24 \mathrm{~h}$ and difficult-to-wean, i.e., those who experienced at least one weaning trial failure, could be included. Patients with continuous sedation, central nervous system or psychiatric disease, or peripheral neuromuscular disease were excluded. A complete polysomnography was performed as soon as possible the night following the first weaning trial failure. Peripheral muscle strength, maximal inspiratory pressure and delirium were measured at time of polysomnography. Weaning duration was defined as the time from polysomnography to extubation. Prolonged weaning was defined as a weaning duration more than or equal to 3 days (failure of at least three weaning trials) according to the international conference consensus of weaning [1].

Results Over the first 6 months of the study, 25 patients intubated for at least $24 \mathrm{~h}$ were considered as difficult-to-wean. Among the 15 patients included in the study, six patients $(40 \%)$ had short weaning period and nine patients $(60 \%)$ had prolonged weaning ( $\geq 3$ days). Patients with prolonged weaning group had less rapid eye movement
(REM) sleep episodes and shorter REM sleep duration than patients with short weaning $(0.0$ episodes [0.0-2.0] vs 6.0 [4.5-6.8] and $0 \mathrm{~min}$ [0-6] vs 43 [33-77], $p=0.02$ for both). Atypical sleep and/or pathological wake [2] was more likely to be found in the prolonged weaning group: $56 \%$ of the patients $(5 / 9)$ versus $17 \%(1 / 6)$ although this difference was not significant $(p=0.29)$. Patients with absent or altered electroencephalographic reactivity $(n=10 / 15)$ were more likely to have prolonged weaning than the others $(80 \%$ of the patients with absent/altered reactivity had prolonged weaning vs $20 \%$ of the patients with normal reactivity, $p=0.03$ ). Maximal inspiratory pressure was similar in the two groups as well proportion of delirium. The only difference was a lower peripheral muscular strength in the prolonged weaning group as indicated by a lower MRC score (32 [28-55] vs 59 [55-60], $p=0.02$ ).

Conclusion Our preliminary results show that decreased REM sleep time and altered electroencephalographic reactivity measured by polysomnography seem to be associated with prolonged weaning duration in ICU patients.

\section{Competing interests None.}

\section{References}

1. Boles JM, Bion J, Connors A, Herridge M, Marsh B, Melot C, Pearl R, Silverman $\mathrm{H}$, Stanchina M, Vieillard-Baron A, Welte T (2007) Weaning from mechanical ventilation. Eur Respir J 29:1033-1056.

2. Drouot X, Roche-Campo F, Thille AW, Cabello B, Galia F, Margarit L, d'Ortho M-P, Brochard L (2012) A new classification for sleep analysis in critically ill patients. Sleep medicine 13:7-14.

037

Integrative ultrasound assessment of lung, cardiac and diaphragm function during a successful weaning trial predicts postextubation distress

Dalinda Ait Aissa', Pierre Coquet ${ }^{2}$, Jean Ruiz ${ }^{3}$, Fabrice Ferre ${ }^{1}$, Lucille

Hoarau', Béatrice Riu-Poulenc ${ }^{1}$, Benoit Bataille ${ }^{2}$, Stein Silva ${ }^{4}$

${ }^{1}$ Réanimation, Centre Hospitalier Universitaire Toulouse, Toulouse,

France; ${ }^{2}$ Critical care unit, CHR, Narbonne, France; ${ }^{3}$ Réanimation, Institut

Universitaire du Cancer de Toulouse-Oncopole, Toulouse, France; ${ }^{4}$ Critical care unit/inserm u825, University Teaching Hospital of Purpan, Toulouse, France

Correspondence: Stein Silva - silvastein@me.com

Annals of Intensive Care 2016, 6(Suppl 1):037

Introduction Establishing the correct time of weaning from mechanical ventilation (MV) is a crucial issue in the critical care practice. We hypothesize that the use of an integrative thoracic ultrasound assessment, encompassing bedside respiratory, cardiac and diaphragm sonographic data, could significantly improve weaning decision process. The aim of the present study was to prospectively compare the predictive value of an integrated thoracic ultrasound (TUS) applied in this setting, against a standard approach (CB) elaborated from clinical and biological data.

Patients and methods We prospectively recruited consecutive patients from three ICU of an university teaching hospital. TUS assessments were determined before $(\mathrm{H} 0)$ at the end of a $60-\mathrm{min}(\mathrm{H} 1)$ spontaneous breathing trial. Clinical and biological (arterial blood gas, plasma BNP) data were recorded concomitantly to create a standard predictive mode. We applied machine-learning methods to improve the accuracy of the related predictive assessments and explore the specific predictive load of each clinical, biological and ultrasonographic parameters.

Results One hundred and thirty-four patients who succeeded a SBT test were extubated and included in the study. Among these patients, 103 were definitely weaned and 31 suffered from postextubation distress. The most relevant clinical/biological parameters (CB model) were the spontaneous respiratory rate and the $\mathrm{PaCO}_{2}$. Regarding the whole ultrasound dataset (TUS model), only lung parameters (A, B1 and B2 profiles) and the echocardiographic estimation of left ventricular filling pressures (E/e) emerged as reliable predictors. Crucially, the predictive value of TUS was significantly greater than $\mathrm{CB}$ during both $\mathrm{HO}$ and H1 assessments (Fig. 9). 


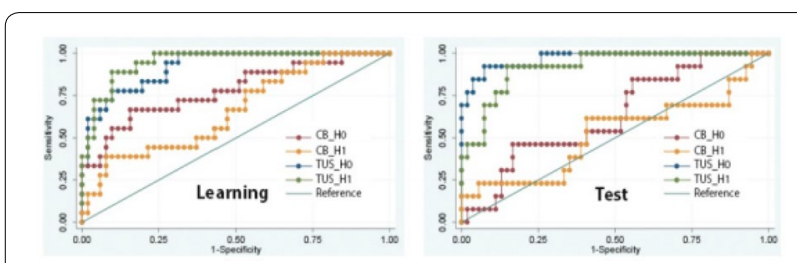

Fig. 9 See text for description

Conclusion An integrative thoracic ultrasound assessment during a successful breathing trial appears to be a most accurate predictor of weaning from MV than a standard clinical and biological approach.

\section{Competing interests None.}

\section{8}

$\mathrm{ETCO}_{2}$ improved outcome prediction of mechanical ventilation weaning

Jean-Luc Baudel ${ }^{1}$, Simon Bourcier ${ }^{1}$, Claire Pichereau², Naïke Bigé2 ${ }^{2}$ Jalel Tahiri' ${ }^{1}$ Vincent Dubée ${ }^{1}$, Bertrand Guidet ${ }^{1}$, Eric Maury ${ }^{1}$, Hafid Ait-Oufella ${ }^{1}$ Service de reanimation, Hôpital Saint-Antoine, Paris, France; ${ }^{2}$ Réanimation médicale, Hôpital Saint-Antoine, Paris, France

Correspondence: Hafid Ait-Oufella - hafid.aitoufella@sat.aphp.fr Annals of Intensive Care 2016, 6(Suppl 1):038

Introduction Weaning from mechanical ventilation (MV) is a crucial step in the management of intensive care patients. Weaning failure is associated with prolonged duration of MV leading to morbidities, increased mortality as well as increased hospital expenses. However, the prediction of weaning outcome remains challenging. We aimed to investigate the end-tidal $\mathrm{CO}_{2}\left(\mathrm{ETCO}_{2}\right)$ and hypothesized that the product "ETCO ${ }_{2} \times$ Respiratory Rate," called ETCO2 $\times$ RR, reflects alveolar ventilation and could predict MV weaning outcome.

Patients and methods We conducted aprospective observational study. All patients under MV that met the criteria for weaning as defined by the International Consensus Conference for Weaning from Mechanical Ventilation were enrolled. Patients underwent a T-piece spontaneous breath trial (SBT) for $30 \mathrm{~min}$, and the usual respiratory and hemodynamic clinical parameters were recorded every $5 \mathrm{~min}$. $\mathrm{ETCO}_{2}$ was recorded blindly by an independent physician using capnography. Patients ventilated through tracheostomy were excluded. SBT failure was defined as one or more of the clinical and biological criteria from International Consensus Conference. $\mathrm{PaO}_{2}, \mathrm{PaCO}_{2}$ and $\mathrm{pH}$ were determined at the beginning (i) and at the end of the SBT (f).

Results During a 10-month period, 310 SBTs were analyzed, 196 patients underwent $1 \mathrm{SBT}, 57$ did two trials and 48 did three or more. For the first SBT, we analyzed 196 first SBT with three groups: success $(n=139)$, hypoxemia-related failure $(n=11)$ and non-hypoxemiarelated failure $(n=46)$. Mean age was $57 \pm 17$ years, $63 \%$ were men, and SAPS II was $47 \pm 16$. Duration of mechanical ventilation was $2.0(1.0-3.4)$ days before starting $\mathrm{SBT}, \mathrm{FiO}_{2}$ was $38 \pm 18 \%$, and PEEP level was $4 \pm 1 \mathrm{~cm} \mathrm{H} \mathrm{H}_{2} \mathrm{O}$. After $30 \mathrm{~min}$ of $\mathrm{SBT}, \mathrm{ETCO}_{2}$, respiratory rate and $\mathrm{ETCO}_{2} \times \mathrm{RR}$ were predictive of MV weaning outcome as AUC was, respectively, $71 \%$ [75-94], 80 [74-89] and $88 \%$ [80-93] (all, $P<0.0001)$. A threshold of $\mathrm{ETCO}_{2} \times \mathrm{RR}$ of 860 predicted weaning failure with a sensitivity of $78 \%$ (Cl95 \% [61-88]) and a specificity of $81 \%$ (Cl95\% [72-87]). We found a significant correlation between delta $\mathrm{PaCO}_{2}(\mathrm{f}-\mathrm{i})$ and delta $\mathrm{ETCO}_{2} \times \mathrm{RR}(\mathrm{f}-\mathrm{i})(r=0.35, P<0.0001)$. During the second SBT, we analyzed 57 patients according to weaning outcome: success $(n=31)$, hypoxemia-related failure $(n=5)$ and nonhypoxemia-related failure $(n=21)$. After $30 \mathrm{~min}$ of SBT, the predictive performance of ETCO2*RR was better (AUC $84 \%$ [73-96]), $P=0.0001$ ) than ETCO2 alone (AUC $75 \%$ [59-90]), $P=0.0002$ ) or RR (68 \% [52-84], $P=0.03)$.
Conclusion In unselected mechanically ventilated patients undergoing SBT, ETCO2*RR predicts weaning outcome.

Competing interests None.

039

Characteristics of 150 cases of weaning-induced pulmonary oedema and effects of diuretics

Liu Jinglun ${ }^{1, *}$, Feng Shen ${ }^{1, *}$, Jean-Louis Teboul ${ }^{1}$, Christian Richard ${ }^{1}$, Xavier Monnet $^{1}$

'Service de réanimation médicale, inserm umr_s 999, Hôpital de Bicêtre, Université Paris-Sud, Faculté de Médecine, Université Paris-Saclay, Le Kremlin-Bicêtre, France

Correspondence: Xavier Monnet - xavier.monnet@aphp.fr

* Both first authors contributed to this work

Annals of Intensive Care 2016, 6(Suppl 1):039

Introduction Weaning-induced pulmonary oedema (WIPO) has not been investigated in large series of patients. Although diuretics are widely used to treat it, the dose that must be administered is unknown. Our aim was to describe the incidence of weaning-induced pulmonary oedema (WIPO) in a population of critically ill patients, to compare the characteristics of patients with and without WIPO and to detail the effects of diuretics on WIPO.

Patients and methods We systematically monitored all consecutive spontaneous breathing trials (SBT, 1-h trial, T-tube trial) that were performed in our unit. In the patients for whom cardiac index (Cl) was monitored (PiCCO device), a passive leg raising (PLR) test was systematically performed before SBT in order to assess preload dependence. The diagnosis of WIPO was established a posteriori by four experts who based their diagnosis on clinical, echocardiographic and biological (haemoconcentration) data and on the evolution after SBTs. Experts were unaware of the effects of PLR test in the patients in whom it had been performed.

Results From April to September 2015, 150 SBTs were performed in 36 patients. SBT failed in 67 cases (35\% of all SBTs). According to the experts, the diagnosis of WIPO was positive in 44 cases $(66 \%$ of failing cases), negative in 98 cases and inconclusive in five cases. The incidence of previous COPD was $33 \%$ in patients who experienced at least one WIPO and $0 \%$ in patients who experienced no WIPO $(p=0.03)$. The incidence of previous cardiopathy, hypertension or supraventricular arrhythmia was $78 \%$ in patients who experienced at least one WIPO and $50 \%$ in patients who experienced no WIPO $(p=0.16)$. Patients with and without WIPO were similar in terms of left ventricular ejection fraction or of mitral $E / E^{\prime}$ ratio at baseline and in terms of fluid balance of the previous day. Compared with patients without WIPO, patients with WIPO had a larger gain in weight from admission. Myocardial ischaemia was not detected during any cases WIPO. Among the 34 patients with cardiac output monitoring, the effects of PLR on $\mathrm{Cl}$ were significantly larger in patients without than with WIPO (changes in CI $13 \pm 15$ vs. $5 \pm 14 \%$, respectively, $p=0.04$ ). This confirmed our previous finding that the presence of preload dependence is associated with a better cardiac tolerance to the SBT. All patients with WIPO received diuretics. Among the six cases with WIPO in which cardiac output was monitored, the PLR was negative. The PLR test performed after diuretics administration on the following day was positive in two of these six cases. These two cases succeeded to the next SBT. The PLR performed after diuretics administration was negative in the remaining four cases. Three of these cases experienced WIPO during the next SBT.

Conclusion In this population of critically ill patients, WIPO was responsible for two-third of weaning failures. WIPO was associated with preload independence, indicating the incapacity of the heart to adapt to the changes in loading conditions during SBT. After a failing SBT, when diuretics had changed the PLR test from negative to positive, the following SBT was very likely to succeed.

Competing interests $\mathrm{XM}$ and J-LT are members of the Medical Advisory Board of Maquet. 
040

Antifungal de-escalation was not associated with adverse outcome in critically ill patients treated for invasive candidiasis Sébastien Bailly ${ }^{1}$, Olivier Leroy ${ }^{2}$, Philippe Montravers ${ }^{3}$, Jean-Michel Constantin ${ }^{4}$, Hervé Dupont ${ }^{5}$, Didier Guillemot ${ }^{6}$, Olivier Lortholary ${ }^{7}$ Jean-Paul Mira ${ }^{8}$, Pierre-François Perrigault ${ }^{9}$, Jean-Pierre Gangneux ${ }^{10}$, Elie Azoulay ${ }^{11}$, Jean-François Timsit ${ }^{12}$

${ }^{1}$ Descid - team 5, INSERM U1137, Paris, France; ${ }^{2}$ Réanimation, Centre Hospitalier de Tourcoing, Tourcoing, France; ${ }^{3}$ Anesthésie et soins intensifs, Hôpital Bichat-Claude Bernard, Paris, France; ${ }^{4}$ Réanimation adulte, C.H.U. Estaing, Clermont-Ferrand, France; ${ }^{5}$ Réanimation cardio thoracique et vasculaire, CHU Amiens-Picardie, Amiens, France; ${ }^{6}$ Umr 1181 «biostatistics, biomathematics, pharmacoepidemiology and infectious diseases», Inserm, Paris, France; ${ }^{7}$ Necker pasteur center for infectious diseases, Hospital Necker, Paris, France; ${ }^{8}$ Réanimation Médicale, Hôpital Cochin, Paris, France; ${ }^{9}$ Réanimation médicale et chirurgicale, C.h.u. De Montpellier, Prades-le-Lez, France; ${ }^{10}$ Mycologie, Hospital Center University De Rennes, Rennes, France; ${ }^{11}$ Réanimation médicale, Hôpital Saint-Louis, Paris, France; ${ }^{12}$ Réanimation médicale et infectieuse, Hôpital Bichat-Claude Bernard, Paris, France

Correspondence: Sébastien Bailly - sbailly@chu-grenoble.fr Annals of Intensive Care 2016, 6(Suppl 1):040

Introduction Systemic antifungal therapy of invasive candidiasis needs to be initiated immediately upon clinical suspicion. Unfortunately, no diagnostic tests are available to firmly confirm or discard the diagnosis of IC in the absence of positive blood cultures or non-contaminated positive sample from a sterile site. Controversies exist about adequate time and potential harms of antifungal de-escalation in documented and suspected candidiasis in intensive care unit patients. Our objective was to investigate whether de-escalation within 5 days of antifungal initiation is associated with an increase of the 28-day death in systemic antifungal therapy treated non-neutropenic adult intensive care unit patients.

Patients and methods From the 835 non-neutropenic adults recruited in a multicenter prospective observational study, we selected the patients receiving systemic antifungal therapy for a documented or suspected invasive candidiasis in the intensive care units and who were still alive 5 days after systemic antifungal therapy initiation. They were included into two groups according to the occurrence of observed systemic antifungal therapy de-escalation before day 6 . The average causal systemic antifungal therapy de-escalation effect on 28-day death was evaluated by using a double-robust inverse probability of treatment weight estimator which is a causal inference method based on observational data. The objective of this estimator is to balance the distribution of baseline confounders across de-escalation groups, in order to reach the condition of a randomized controlled trial.

Results Among the 647 included patients, early de-escalation at day 5 after antifungal initiation occurred in 142 patients ( $22 \%$ ), including $48(34 \%)$ patients whose systemic antifungal therapy was stopped before day 6 . Patients in the de-escalation group were younger and had a shorter previous intensive care unit stay, but their SAPSII or SOFA score at intensive care unit admission was similar. The rate of proven invasive candidiasis was not different between de-escalation and no de-escalation groups. After adjustment on the baseline confounders, early systemic antifungal therapy de-escalation was not associated with increased 28-day mortality (RR 1.12, $95 \% \mathrm{Cl}[0.76-1.66]$ ). Subgroup analyses did not show any effect of early de-escalation on mortality, and there was no effect of early stopping on morality either (RR 0.98 [0.71-1.35]) (Fig. 10). Finally, the number of days alive without SAT at day 28 was higher in the de-escalation group $(14$ days $[5 ; 23])$ than in the no de-escalation group (10 days $[2 ; 17] p<0.01)$ leading to a median cost difference of 1100 euros in favor of de-escalation group.

Conclusion Our causal analysis based on a large prospective observational multicenter study showed that systemic antifungal de-escalation in case of suspected or documented IC in non-neutropenic ICU patients occurred in only $22 \%$ of the cases. In non-neutropenic critically ill adult patients with documented or suspected invasive candidiasis, SAT de-escalation within 5 days was not related to increased day-28 mortality, but it was associated with a subsequent

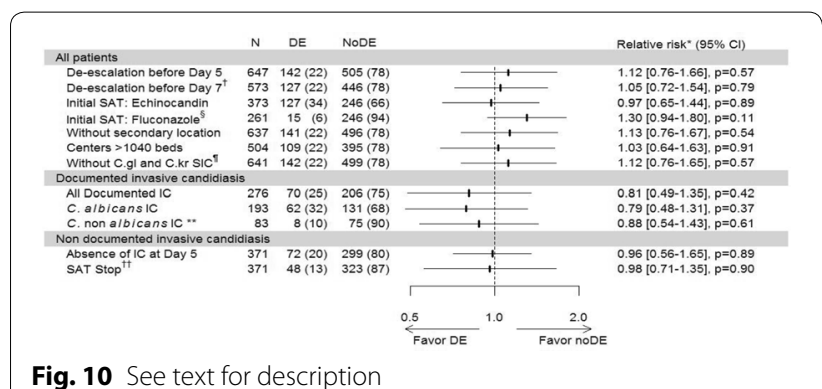

Fig. 10 See text for description

and significant decrease in the antifungal consumption. These results remained valid on different subgroups and were confirmed by sensitivity analysis. Early stop in the absence of proven invasive candidiasis or de-escalation to fluconazole at day 5 could be done safely. The latter has to be confirmed in randomized controlled trials.

Competing interests None.

\section{1}

Intensive care acquired pneumonia due to extended-spectrum $\beta$-lactamase-producing Enterobacteriaceae (ESBL-PE) among patients with prior colonization: Frequency, risk factors and prognosis

Keyvan Razazi', Armand Mekontso-Dessap², Chloe Jansen³ ${ }^{3}$ Nicolas de Prost $^{2}$, Christian Brun-Buisson ${ }^{2}$

${ }^{1}$ Réanimation médicale, Hospital Henri Mondor, Créteil, France; ${ }^{2}$ Réanimation médicale, Hôpital Henri Mondor, Créteil, France; ${ }^{3} \mathrm{Cepi}$, Hospital Henri Mondor, Créteil, France

Correspondence: Keyvan Razazi - keyvan.razazi@aphp.fr Annals of Intensive Care 2016, 6(Suppl 1):041

Introduction Pneumonia is the most frequent ICU-acquired infection. The increasing prevalence of ESBL-PE carriage on ICU admission raises important questions on empiric therapy policies in patients presenting with ICU-acquired pneumonia, which may include the use of a carbapenem as first-line therapy. The incidence of and risk factors for ICU-acquired pneumonia (ICUAP) among patients with ESBL-PE carriage are unknown.

Patients and methods This 6-year prospective study (May 2009January 2015) was conducted in the medical intensive care unit of an university-affiliated hospital. All patients with ESBL colonization or infection were prospectively recorded. A detailed clinical profile of each patient was established, and variables associated with ESBL-PE pneumonia after colonization was analyzed.

Results A total of 6168 patients were admitted to our medical ICU during the study period. Nine patients were excluded because they developed ESBL-PE ICUAP before ESBL-PE colonization. Of these patients, 826 (13.4 \%) were detected as ESBL-PE carriers on admission screening samples or acquired ESBL carriage while in the ICU. One hundred and six ( $12.8 \%$ of carriers) patients developed ICUAP after colonization, 47 (44\%) with ESBL-PE, mostly Enterobacter spp. and K. pneumoniae. By multivariable analysis, SAPS II $\geq 43$ (OR 2.6 [1.02-6.8]), more than one episode of ICUAP before the index pneumonia (OR 5.4 [1.2-24.6]), and colonization with E.cloacae or K. pneumoniae rather than with E. coli (OR 7.2 [1.8-28.9]) were independent predictive factors for ESBL-PE ICUAP with prior colonization. More than 2 days of fluoroquinolone within the past 3 months fell short of statistical significance $(P=0.058)$ Amoxicillin/ clavulanic acid during ICU was a protective factor for ESBL-PE ICUAP (OR 0.29 [0.10-0.82]), thus predictive for another microorganism than ESBLPE, mostly Pseudomonas. All patients but one (98\%) with ICU-acquired infection caused by ESBL-PE had rectal carriage of ESBL-PE with the same species. Appropriate antibiotherapy was given to most patients without difference between ESBL-PE infections and others, even if aminoglycosides were not considered. ESBL-PE ICUAP had higher SOFA score, a trend to more frequent septic shock at ICU-AP onset and worse outcome [57 \% died in ICU vs $41 \%(p=0.09)$ ]. Twenty-four patients had 
ICUAP caused by a carbapenem-resistant microorganism after colonization, most of which (87 \%) were nonfermenting gram-negative bacilli. Carbapenem exposure before or after ICU admission was associated with carbapenem-resistant microorganism.

Conclusion This study highlights the high rate (44\%) of ESBL-PE pneumonia acquired in ICU in patients with prior colonization. We identified new risk factors for ESBL-PE ICUAP after colonization. These data may be useful for identifying which patients may warrant empiric ESBL-targeted antimicrobial drug therapy, as improving carbapenem use is currently a major challenge for intensivists.

Competing interests None.

\section{2}

Epidemiology, characteristics and outcomes of septic critically ill patients after the removal of a totally implantable venous-access ports

Marie Lecronier ${ }^{1}$, Valade Sandrine ${ }^{2}$, Naïke Bigé ${ }^{3}$, Nicolas de Prost ${ }^{4}$, Damien Roux $x^{5}$, Jean-Damien Ricard ${ }^{5}$, Eric Maury ${ }^{3}$, Julien Mayaux ${ }^{1}$, Elie Azoulay ${ }^{2}$, Alexandre Demoule', Martin Dres

${ }^{1}$ Service de pneumologie et réanimation médicale, Groupe Hospitalier Pitié-Salpêtrière - APHP, Paris, France: ${ }^{2}$ Réanimation médicale, Hôpital Saint-Louis - APHP, Paris, France; ${ }^{3}$ Réanimation médicale, Hôpital Saint-Antoine - APHP, Paris, France; ${ }^{4}$ Réanimation Médicale, Hôpital Henri Mondor - APHP, Créteil, France; ${ }^{5}$ Réanimation médico-chirurgicale, Hôpital Louis-Mourier - APHP, Colombes, France

Correspondence: Marie Lecronier - lecronier.marie@gmail.com

Annals of Intensive Care 2016, 6(Suppl 1):042

Introduction International guidelines support the need to remove totally implantable venous-access ports in septic patients admitted to intensive care unit. Nevertheless, the clinical relevance of this attitude is debated. Therefore, we undertook the present study to determine the proportion of confirmed totally implantable venous-access portsrelated infection in case of removal. We also assessed the epidemiological, clinical and microbiological characteristics of these patients.

Patients and methods We conducted a multicenter, retrospective, observational study from 2012 to 2014. All patients with life-threatening sepsis in whom a decision of totally implantable venous-access ports removal was taken by the clinician in charge were included. Catheter-related infection was defined by the association of general or local signs of infection and bacteremia and/or positive culture of the catheter or differential time to positivity of simultaneous blood cultures or good evolution $48 \mathrm{~h}$ after device removal and after antibiotics initiation.

Results One hundred and fifty one patients were included ( $57 \pm 14$ years old, $61.5 \%$ of male). Totally implantable venous-access ports-related infection was confirmed in 75 patients (45\%). IGS2 was $47 \pm 15$ and $56 \pm 17(p<0.05)$ in patients with and without catheter-related infection, respectively. On admission, temperature was $38.6 \pm 1.5$ and $38.2 \pm 1.5^{\circ} \mathrm{C}(p>0.05)$ and mean arterial pressure was $69 \pm 23$ and $70 \pm 18 \mathrm{mmHg}(p>0.05)$ in patients with and without catheter-related infection, respectively. Local signs of sepsis were present in $27(18 \%)$ and $6(4 \%)$ patients with and without catheterrelated infection, respectively $(p<0.05)$. There was no difference between patients with and without catheter-related infection regarding aplasia on admission ( 30 and $37 \%$, respectively, $p>0.05$ ). The microbiological findings identified enterobacteria as the most common pathogen (37.5\%). Coagulase-negative staphylococci and Staphylococcus aureus were the others frequent pathogens (30.3 and $16 \%$, respectively).

Intensive care unit, day 28, day 90 and 6 months mortality were, respectively, for patients with and without catheter-related infection 9 and $40 \%(p<0.01), 22$ and $48 \%(p<0.01), 32$ and $53 \%(p<0.05)$ and 40 and $57 \%(p>0.05)$.

Discussion Almost half of our population had a confirmed totally implantable venous-access ports infection. It is noteworthy that patients with or without catheter-related infection had similar characteristics on admission but a clear difference regarding the short- and long-term survival. These findings support the removal of the catheter in case of life threatening.
Conclusion Almost half of the totally implantable venous-access ports removed in patients admitted for life threatening were really infected. The clinical course of these patients was better than patients with sepsis from other origin.

\section{Competing interests None.}

\section{References}

1. Mermel LA, Allon M, Bouza E, Craven DE, Flynn P, O'Grady NP, et al. Clinical practice guidelines for the diagnosis and management of intravascular catheter-related infection: 2009 Update by the Infectious Diseases Society of America. Clin Infect Dis Off Publ Infect Dis Soc Am. 2009 Jul 1:49(1):1-45.

2. Lebeaux D, Fernández-Hidalgo N, Chauhan A, Lee S, Ghigo J-M, Almirante $B$, et al. Management of infections related to totally implantable venousaccess ports: challenges and perspectives. Lancet Infect Dis. 2014 Feb;14(2):146-59.

043

Genetic risk of Pseudomonas aeruginosa ventilator-associated pneumonia: the EXTENSE study

Jean-Paul Mira', Sophie Blein², Nathalie Marin', Christophe Rousseau ${ }^{3}$ Julien Charpentier', Jean-Daniel Chiche ${ }^{1}$, Alexandre Pachot ${ }^{4}$

${ }^{1}$ Réanimation Médicale, Hôpital Cochin, Paris, France; ${ }^{2}$ Biostatistics/bioinformatics, Biomérieux, Marcy-l'Étoile, France; ${ }^{3}$ Unité 1016, 22 rue méchain, 75014, paris, nstitut National de la Santé et de la Recherche Médicale, Paris, France; ${ }^{4}$ Recherche diagnostique, Biomerieux, Rue Censier, Paris, France, Paris, France

Correspondence: Jean-Paul Mira - jean-paul.mira@cch.aphp.fr Annals of Intensive Care 2016, 6(Suppl 1):043

Introduction Genetic association studies and genome-wide association studies have demonstrated the role of human genetic variations in the epidemiology of infectious diseases in ICU. However, no study has used the power of genome or exome sequencing in this clinical condition. Hence, we design an exome study to search for genetic factors involved in the risk of ventilator-associated pneumonia (VAP) due to Pseudomonas aeruginosa $(\mathrm{Pa})$.

Patients and methods Patients had been previously participated in Pneumagene study that included 3200 ICU patients, which should be on invasive mechanical ventilation for more than 2 days and had no immunodepression, and no risk factor for Pseudomonas aeruginosa VAP (COPD, Pa colonization). For PaVAP group, inclusion criteria included the presence of two or more VAP due to $P a$ during the same ICU stay. Control group (C) included patients that had no VAP and have not been colonized by $\mathrm{Pa}$ during mechanical ventilation (MV) and that can be matched with one PaVAP patient on values of age, SAPS2, duration of MV, reason for ICU admission, potential risk factors for VAP (coma, ARDS, head trauma) and outcome. The Knome Company performed exome sequencing and analysis with the SKAT Common Rare algorithm. Multiple testing was corrected by bootstrap resampling technique with 1000 resamples and a FWER $=0.05$.

Results Fifty patients were included in each group. Median age, SAPS2, gender and Pa risk factors were not different between the groups. The control group had higher duration of MV that PaVAP group (59 vs 52.6 , respectively, $p<0.05$ ). One thousand genetic variants were finally detected and analyzed. After multiple corrections, 178 loci remain statistically associated with PaVAP risk. Interestingly, two areas appear to be of high interest on the same part of the genome chr 16 p11.2 (26 SNPs and 20 SNPs, respectively, for area 1 and area 2). These two areas are gene deserts but have already been reported in GWAs and seem to be involved in gene regulation.

Conclusion This is the first exome study in ICU patients that did not reveal clear significant genetic variant that can be associated with risk of PaVAP, despite the presence of two promising areas on the chromosome 16. This powerful approach may offer new opportunities for understanding infectious disease susceptibility, severity, treatment, control and prevention.

Competing interests None. 
044

Cytomegalovirus reactivation enhances the virulence of a Staphylococcus aureus pneumonia in a mouse model Sami Hraiech ${ }^{1}$, Julien Bordes ${ }^{2}$, Samuel Lehingue ${ }^{3}$, Lamballerie Xavier De $^{4}$, Jean-Louis Mège ${ }^{5}$, Jean-Marie Forel' ${ }^{1}$ Christophe Guervilly' ${ }^{1}$ Mélanie Adda', Didier Raoult', Laurent Papazian ${ }^{6}$

${ }^{1}$ Réanimation DRIS, Hôpital Nord APHM, Marseille, France; ${ }^{2}$ Anesthésie, Hôpital d'instruction des Armées Sainte-Anne, Toulon, France; ${ }^{3}$ Réanimation dris, APHM CHU Nord, Marseille, France; ${ }^{4}$ Unite des virus emergents umr190 "emergence des pathologies virales" faculte de medecine, 27, Aix-Marseille Université, Marseille, France: ${ }^{5}$ |hu méditerranée infection, urmite cnrs ird inserm umr 7278, marseille, france, Aix-Marseille Université, Marseille, France; ${ }^{6}$ Service de réanimation-détresses respiratoires et infections sévères, Hôpital Nord, Marseille, France

Correspondence: Sami Hraiech - sami.hraiech@ap-hm.fr

Annals of Intensive Care 2016, 6(Suppl 1):044

Introduction Cytomegalovirus (CMV) reactivation is common in immunocompetent mechanically ventilated patients. Lungs are a frequent site of reactivation. CMV reactivation may be responsible for higher mortality, length of stay and mechanical ventilation. A higher incidence of bacteremia and nosocomial pneumonia following CMV reactivation may in part explain the worse prognosis of these patients. The aim of this study was to assess the virulence of a staphylococcal pneumonia developed during CMV reactivation in a mouse model.

Materials and methods The study was approved by our local ethic committee. Female BALB/c mice were used in all experiments. CMV primoinfection was obtained by intraperitoneal inoculation of $2 \times 10^{4} \mathrm{PFU}$ of murine CMV (MCMV) Smith strain. Seropositivity was confirmed by immunofluorescence in serum. MCMV was considered to be latent 4 months later. Reactivation was triggered by cecal ligature and puncture (CLP). Mice were considered to have a CMV reactivation 2 weeks after CLP. After this, 32 MCMV-positive mice underwent an intranasal inoculation with $5 \times 10^{8}$ CFU of methicillin-susceptible Staphylococcus aureus (MSSA) to induce pneumonia. Twenty-nine MCMV-negative BALB/c mice were treated according to the same protocol, including CLP (control group). Daily weight, signs of sepsis and spontaneous mortality were noted. After 15 days, surviving mice were euthanized. Blood and lung were collected for bacterial culture. Interferon alpha and gamma were assessed in the serum of both groups. MCMV reactivation was assessed by PCR in the lungs. A second cohort of 26 mice was treated according to the same protocol (13 MCMV positive and 13 control) but were killed at day 2 and day 5 after pneumonia. The same samples were performed after killing.

Results No mortality from staphylococcal pneumonia was observed in the control group, whereas the mortality rate was of $9 \%$ in the MCMV group $(p=0.09)$. The weight loss at day 3 after pneumonia was higher in MCMV mice than in the control group (2.2 vs $0.7 \mathrm{~g}$, respectively; $p=0.005$ ). Macroscopic observation and bacteriological analysis of lungs showed staphylococcal abscesses in 5/32 mice in MCMV group as compared to $0 / 29$ in control group at day 15 . At day 5, 3/8 mice had lung abscesses in MCMV group as compared to $0 / 6$ in control group. Overall, $8 / 40$ (20\%) mice had lung staphylococcal abscesses in MCMV group as compared to $0 \%$ in control group $(p=0.011)$. Lung bacterial count (median (interquartile range) was significantly higher in MCMV-positive mice as compared to control mice at day $2\left[5 \times 10^{3}\right.$ $\left(10^{3}-3 \times 10^{5}\right) \mathrm{CFU} /$ lung vs $10^{2}\left(0-4 \times 10^{2}\right)$ CFU/lung; $\left.p=0.007\right]$ and day $5\left[2.5 \times 10^{4}\left(1.6 \times 10^{3}-6.5 \times 10^{5}\right)\right.$ vs $\left.15(10-40) ; p=0.005\right]$. Two days after staphylococcal pneumonia, MCMV-positive mice had higher interferon alpha serum levels $(p=0.023)$. No difference between the two groups was observed concerning interferon gamma levels.

Conclusion In a mouse model, CMV reactivation leads to the switch from a non-lethal to a lethal staphylococcal pneumonia, increases bacterial lung count and favors the occurrence of staphylococcal lung abscesses. The role of the immune response and especially the way of interferon alpha seems to be determining.

\section{Competing interests None.}

\section{References}

1. Jaber et al., Chest. 2005 Jan;127(1):233-41.

2. Cook et al., J Infect Dis. 2002 May 15;185(10):1395-400.
045

Families looking back: meaning-making after a decision to accept or refuse organ donation. A qualitative approach

Nancy Kentish-Barnes ${ }^{1}$, Zoé Cohen-Solal ${ }^{1}$, Elie Azoulay ${ }^{2}$

${ }^{1}$ Réanimation médicale, Assistance Publique Hôpitaux de Paris, Hôpital

Saint Louis, Paris, France; ${ }^{2}$ Réanimation médicale, Hôpital Saint-Louis, Paris, France

Correspondence: Nancy Kentish-Barnes - nancy.kentish@sls.aphp.fr Annals of Intensive Care 2016, 6(Suppl 1):045

Introduction In the context of brain death, a survey led by the French Institution, the Agence de Biomédecine, in 2007, showed that for $34 \%$ of caregivers, organ donation could increase the pain of the family. The family is at the heart of the organ donation process: if the patient is not registered as a non-donor, then discussions are led with his/her relatives. In a recent study focused on relatives' experience and grieving process after request for organ donation, relatives were followed up for 9 months to complete instruments measuring the risk of presenting anxiety, depression and post-traumatic stress symptoms as well as complicated grief. After this follow-up, in-depth interviews were led with voluntary relatives in order to permit a better understanding of their experience: understand how they construct and frame their experience 1 year after the patient's death; analyse the meaning they give to their decision; draw parallels, regarding experiences and feelings, between the experience of donor versus non-donor relatives; and qualify the impact of the decision on the grieving process.

Patients and methods In the main study, 202 relatives were followed up three times over 9 months. After this period, 24 relatives (saturation obtained at 24) participated in an in-depth interview (16 relatives whose loved-one was a donor, eight whose loved-one was not a donor). Interviews were recorded and fully transcribed. Medium length of interview was $1 \mathrm{~h} 10 \mathrm{~min}$. We privileged an inductive approach (grounded theory) and obtained four themes through thematic analysis: relationship to the patient in the context of brain death; meaning-making regarding organ donation and the decision; search for continuity (follow-up of transplantation process); and impacts on the grieving process (for oneself and for others).

\section{Results}

Relationship to the patient in the context of brain death is complex and ambiguous. Relatives question the patient's condition and wonder whether he/she can hear. Whatever their subjective answer to this question, they need to talk to the patient. Talking to the patient is a ritual that permits closure. Relatives need the clinicians' support to be able to express their feelings and say good-bye to the patient who remains a person until he/she is taken to the operating theatre.

Meaning-making regarding organ donation and the decision relatives express a strong responsibility about organ donation decision-making. Three justifications of the decision can be put forward: respect of the patient's choices; altruism; and the hope of life in spite of death.

Search for continuity Relatives stress the importance of being able to follow-up the transplantation process. If some relatives want news, they must get it; if others do not, they need to know they can. Ambiguity is central, as relatives are incapable of considering a possible failure of the organ donation process. With failure comes the risk of dehumanising the organ donation process.

Impact on the grieving process Organ donation decision impacts on relatives' experience at two levels. First, on a personal level, accepting organ donation can help give meaning to the patient's death ("He did not die for nothing") but can also be experienced as a moral duty ("I respected my son, there was no choice"). Second, on a social level, relatives who accepted become organ donation "ambassadors" by encouraging discussions about organ donation and by expressing their positive perspective on the subject.

Conclusion Relatives invest strong meaning into organ donation decision-making. The decision requires reflection prior to the experience and is made with respect to the patient and to the relative him/herself. Relatives cannot afford ambiguity as the decision impacts on their grieving process and on the meaning they give to the patient's death.

Competing interests None. 
046

The letter of condolence at the heart of family centered care. A qualitative approach of relatives' experience

Nancy Kentish-Barnes ${ }^{1}$, Zoé Cohen-Solal ${ }^{1}$, Virginie Souppart ${ }^{2}$, Elie Azoulay $^{3}$

${ }^{1}$ Réanimation médicale, Assistance Publique Hôpitaux de Paris, Hôpital Saint Louis, Paris, France; ${ }^{2}$ Réanimation médicale, Hôpital Saint Louis, Paris, France; ${ }^{3}$ Réanimation médicale, Hôpital Saint-Louis, Paris, France Correspondence: Nancy Kentish-Barnes - nancy.kentish@sls.aphp.fr Annals of Intensive Care 2016, 6(Suppl 1):046

Introduction After the death of a loved-one in the ICU, relatives are particularly vulnerable and at high risk of presenting symptoms of anxiety, depression, posttraumatic stress and complicated grief. Moreover, these relatives may experience persistent incomprehension and a feeling of abandonment regarding the ICU team. Many relatives also express the need to contact the ICU team after the death of the patient. In their official report in 2009, the French institution IGAS recommended to prevent complicated grief, emphasizing on the fact that "care of the family must not end with the death of the patient." In this perspective, a condolence letter $(\mathrm{CL})$ could help recognize both the relative's pain and his/her strong link to the ICU team. The CL could also help decrease the risk of presenting post-ICU syndrome. In a recent study by the Famiréa group aimed at measuring the impacts of the $\mathrm{CL}$ on relatives' grieving process-a randomized multicentre trial-242 relatives were followed up over 6 months, half of whom had received a CL. The objective of this presentation is to analyse spontaneous feedback by relatives having received a CL. This feedback was gathered orally during telephone follow-up and also using the letters written by relatives to the ICU teams.

Patients and methods Relatives in the intervention group received a $\mathrm{CL} 15$ days after the patient's death. They were contacted over the phone twice, 1 and 6 months after the loss. During telephone calls, the interviewers noted all spontaneous feedback regarding the CL. Additionally, all letters spontaneously written to the clinician after receiving the $\mathrm{CL}$ were retrieved and analyzed. All feedback was transcribed verbatim. This corpus was analyzed using thematic analysis. Three themes will be put forward: humanization of medical care; reflection on care received by the patient; and closure.

\section{Results}

Humanization of medical care Relatives are touched by the time spent by physicians in writing the $\mathrm{CL}$. They say that the letters are personalized and feel that they are not "just a number," but are recognized for the role they played in caring for the patient and in their pain after the loss. The $\mathrm{CL}$ helps to give a positive image of the institution: "With this letter you give me the hope that medical care can be humanized and that means a lot to me."

Reflection on care received by the patient After receiving a $\mathrm{CL}$, relatives want to thank the ICU team for the patient's care and want to renew their feeling of trust, more specifically regarding pain management. Many relatives also validate decisions made by the ICU team and show their understanding of the situation. Last, the CL helps to think the patient as a whole, with a specific trajectory and personality, thus reaffirming, from the relative's perspective, the quality of care. "Thank you for your kind letter. I am convinced that my husband received the best care during his stay. I know that he passed away without any pain and this is a great comfort to me."

Closure The $\mathrm{CL}$ is a way of remembering the person who died with the team that cared for him/her. It also confirms the relationship between the clinicians and the relatives. Remembering, sharing and expressing emotions permit closure that, in turn, helps bereaved relatives to accept the loss and to start healing. "Your letter has been a tremendous help, it really did help to ease our pain."

Conclusion The $C L$ helps relatives give meaning to the patient's trajectory. Emphasis is put of personalization, showing that these letters cannot be systematic. Writing a $\mathrm{CL}$ must also have meaning for the clinician. More importantly, offering the possibility of a last contact with the team is soothing for the relatives and has positive impacts of their grieving process.

Competing interests None.

\section{7}

Use of a children information booklet to support young relatives visiting a critically ill adult: impact on ICU practices

Lionel Kerhuel' ${ }^{1}$, Carole Haubertin ${ }^{1}$, Isabelle Exbrayat ${ }^{1}$, Elodie Rozières' Audrey Argain ${ }^{1}$, Agnès Suc ${ }^{2}$, Michel Vignes ${ }^{3}$, Pierre Cougot ${ }^{4}$, Béatrice Riu-Poulenc ${ }^{1}$, Stein Silva', Olivier Fourcade ${ }^{1}$, Elodie Brunel ${ }^{1}$

${ }^{1}$ Critical care unit - purpan hospital, CHU Toulouse, Toulouse, France; ${ }^{2}$ Pain management and palliative care unit - children hospital, CHU Toulouse, Toulouse, France; ${ }^{3} \mathrm{Child}$ and adolescent psychiatry unit - la grave hospital, CHU Toulouse, Toulouse, France; ${ }^{4}$ Critical care unit - rangueil hospital, CHU Toulouse, Toulouse, France

Correspondence: Lionel Kerhuel - lionel.kerhuel@gmail.com

Annals of Intensive Care 2016, 6(Suppl 1):047

Introduction According to the 2009 SFAR/SRLF guidelines for improving hospital conditions in ICU, the admission of children who wish to visit a critically ill relative "should be facilitated and supervised" by ICU staff members. Yet, the difficulties faced by caregivers in this challenging setting potentially induce restrictions in children visits. The objective of this work was to evaluate the impact on ICU staff practices of a specifically designed children information booklet.

Materials and methods The booklet was designed by a multidisciplinary team (nurses, intensivists, psychiatrists, cartoonist) and included both child-centred explanations (5-12 years old) and advices for accompanying adults. During the study period, booklets were provided freely to patients' relatives and ICU staff members as a practical tool to help accompanying children who were likely to visit a patient. We used a prospective before-after study design with a questionnaire at baseline and 1 year post-intervention to assess the impact of the booklet on practices in ICU staff members (nurses, auxiliary nurses and physicians). The participants were split into an intervention centre (booklet available) and a control centre (booklet not available). Both centres were general ICUs and were part of a same university hospital. These ICUs were comparable regarding the nurse-to-patient ratio, the number of visiting hours and the admission policy for family visits. Primary endpoints were the proportion of participants who supported visiting children over the 3 months prior to the questionnaire, and the Moral Distress Scale Revised (MDS-R) to evaluate the emotional experience of staff members in this setting.

Results Fifty-seven booklets were distributed in the intervention ICU (8\% of admitted patients). During the study period (07/201407/2015), 93 children were likely to visit their relative. A total of 195 staff members participated in the study ( $75 \%$ of ICU staff). They were nurses, auxiliary nurses and physicians in 56, 27 and $14 \%$ of cases, respectively. Participants were equally recruited at the intervention ICU (87 participants, $74 \%$ of ICU staff) and the control ICU (108 participants, $76 \%$ of ICU staff). In the intervention ICU, the proportion of staff members who supported at least one visiting child over the 3 months prior to the questionnaire increased from $41 \%$ before the intervention to $69 \%$ after the intervention $(p<0.001)$, whereas it was not significantly different in the control ICU (38 vs $39 \%, p=0.97$ ). A multivariate analysis confirmed that the use of the booklet was an independent factor for increasing the rate of caregivers who supported visiting children [OR 4.0, $95 \% \mathrm{Cl}[2.0 ; 7.9], p<0.001]$. No significant effect of occupation (OR 0.9, $p=0.89$ ), age (OR 1.7, $p=0.15$ ) and years of experience in critical care (OR $0.7, p=0.34$ ) emerged from this analysis. In the intervention ICU, the proportion of participants who disagreed with child visits decreased from 35 to $14 \%(p=0.002)$, whereas it remained stable in the control ICU (30 vs $34 \%, p=0.51)$. In the intervention ICU, the proportion of participants who claimed that the admission policy for child visits had to be improved decreased from 94 to $76 \%(p=0.001)$, whereas it remained stable in the control ICU ( 91 vs $91 \%, p=0.94$ ). There were no significant differences in MDS-R before and after the intervention in both the intervention ICU $(21[12 ; 30]$ vs $20[9 ; 30], p=0.44)$ and the control ICU $(18[10 ; 29]$ vs 21 $[8 ; 33], p=0.13)$.

Conclusion We reported a substantial increase in visiting children admission in ICU when a specifically designed children information booklet was available. Despite the fact that no emotional effect was 
identified, the implementation of booklets seemed useful to standardize practices regarding child visits policy among staff members. Further work would be necessary to assess the utility of the booklet regarding children experience during their visit in the ICU.

\section{Competing interests None.}

\section{8}

Withdrawal of life-support therapies reporting in ICU randomized controlled trial: Let us move to a transparent reporting-a systematic review

Jonathan Messika', Stéphane Gaudry', Florence Tubach², Emeline Dubief ${ }^{1}$, Blandine Pasquet ${ }^{2}$, Sylvie Guillo², Didier Dreyfuss ${ }^{1}$, Jean-Damien

Ricard ${ }^{1}$

'Service de réanimation médico-chirurgicale, CHU Louis Mourier, Colombes, Colombes, France; ${ }^{2}$ Département d'epidémiologie et recherche clinique, Unité de Recherche Clinique Paris Nord, Paris, France Correspondence: Jonathan Messika - jonathan.messika@lmr.aphp.fr Annals of Intensive Care 2016, 6(Suppl 1):048

Introduction Intensivists' decision to withdraw or withhold life-sustaining therapies may have considerable impact on patient outcome since data suggest that one death out of two in the intensive care unit ensures such a decision. The vast majority of decisions (including withdrawal/withholding life-sustaining therapies) in the intensive care are evidence-based, stemming from ICU trials. We therefore sought to investigate how decisions of withholding/withdrawing life-sustaining therapies are addressed in randomized controlled trials in critically ill patients.

Materials and methods This is an ancillary analysis of a systematic review of randomized clinical trials in critically ill patients. These randomized controlled trials were identified through an exhaustive search conducted in Medline (PubMed ${ }^{\circledR}$ ) over a 1-year period (2013). Data extraction was performed by two independent authors (senior intensivists). Whether or not withdrawing/withholding of life-sustaining therapies was reported in these trials was systematically reported. Data are expressed in median [IQR], or $n(\%)$ as appropriate.

Results Among the 150 randomized control trials performed on critically ill patients retrieved from the search, only 7 (4.6\%) reported the number of patients for whom decisions of withdrawal/withholding of life-sustaining therapies had been taken. In the subset of studies that had mortality as primary outcome $(n=25)$, only four reported the number of patients concerned by these end-of-life decisions.

The seven trials involved a total of 2,620 subjects (300 [80-548] patients per trial), totalizing 1055 death (150 [8-225] per trial) for which 413 (5 [4-118]-39\%) were preceded by a limitation of therapeutic effort. The median survival rate was $52 \%$.

The interventions assessed were drugs for four trials (balanced vs chloride-rich solution in brain injury patients; enoxaparin for thromboprophylaxis; vasopressin, steroids and epinephrine in cardiac arrest; and recombinant human activated protein $\mathrm{C}$ in septic shock) and therapeutic strategies for three (high-frequency oscillation ventilation; blood glucose control algorithm; and targeted temperature management after cardiac arrest). All studies reported figures regarding these patients in the results section, including one in a table, but none of them mentioned this information in the abstract. Only one trial indicated in Methods section the way these data were obtained. In the trial assessing target temperature management in cardiac arrest, pre-specified criteria for withdrawal of life-sustaining therapy were established.

Discussion Withdrawal/withholding of life support therapies is very rarely reported in randomized controlled studies in critically ill patients since $<5 \%$ numbered this information.

This is prejudicial when mortality is the primary outcome, given the huge impact on mortality when such decisions are taken in patients. We suggest that guidelines for reporting of randomized controlled trials in critically ill patients include reporting on withdrawal/withholding of life-sustaining therapies, at least in studies with mortality as primary outcome, in Methods and Results sections.

Conclusion Although accounting for a number of deaths of intensive care patients, the number of patients with withdrawal/withholding of life-sustaining therapies decisions are scarcely reported in critical care trials. Whether reporting these figures could alter conclusions of the some of these studies remains to be investigated.

Competing interests None.

049

\section{Gasp during children end-of-life: healthcare providers' feelings} and knowledge

Charlotte Pierron ${ }^{1}$, Marion Grimaud ${ }^{2}$, Caroline Farnoux ${ }^{3}$, Amélie Maillard ${ }^{4}$ ${ }^{1}$ Réanimation et surveillance continue pédiatriques, Hospital Rob-

ert-Debré, Paris, France; ${ }^{2}$ Réanimation et surveillance continue médicochirurgicales pédiatriques, Hôpital Necker Enfants-Malades, Paris, France;

${ }^{3}$ Réanimation néonatale et néonatalogie, Hôpital Robert-Debré, Paris, France; ${ }^{4}$ Réanimation et médecine néonatales, Hôpital Cochin-Port-royal, Paris, France

Correspondence: Charlotte Pierron - charlottepierron@hotmail.com Annals of Intensive Care 2016, 6(Suppl 1):049

Introduction Gasp onset is frequent during children end-of-life and may provoke distress in the witnessing parents. The knowledge and the feelings of healthcare providers confronted to the gasps have never been studied.

Materials and methods We conducted a prospective study in two neonatal and two pediatric intensive care units in Paris, France. We developed a 12-item survey, about the feelings of the healthcare providers witnessing gasps during end-of-life, their knowledge about these events and the information that should be given to the families. The survey was distributed to all healthcare providers in contact with patients.

Results Fifty-one percent of the 488 healthcare providers of the four wards responded to the survey. Among them, $77 \%$ mentioned a discomfort when confronted to gasps, when 43 and $91 \%$ of the respondents think that gasps are uncomfortable for the dying child and for the parents, respectively. Consequently, $83 \%$ of the respondents declare that gasps should be ceased, primarily for the parents or the child discomfort, but also for the healthcare providers. According to the respondents, the efficient treatments against gasps are sedation, analgesia, and neuromuscular blockers. About one-third and twothirds of the respondents reported that gasps are interfering with their accompaniment of the dying child and of their parents, respectively. Finally, one-third of the respondents declare that they are personally emotionally affected by the occurrence of gasps. Most of the respondents $(78 \%)$ state that parents should be informed before the onset of gasps. Avoiding words with negative meanings, they suggest explaining that gasps are not associated with a willingness to survive and are not respiratory movements and further that their occurrence and their length are unpredictable and above all that they are not uncomfortable or painful. Thirty-eight percent of the respondents declare not knowing the mechanism leading to gasps when $60 \%$ think that gasps are secondary to severe brainstem hypoxia or decerebration.

Discussion The prevalence of gasps is not found in the literature but seems to be high according to pediatricians and neonatologists. Gasp is a reflex of auto-resuscitation that follows a functional decerebration induced by a severe brainstem hypoxia. It is believed that gasps are not painful or uncomfortable for the dying patient although this is debated. Nonetheless, in our study, $43 \%$ of the healthcare providers declare that gasps are uncomfortable for the patient, which is indicating a treatment to stop them. Moreover, $91 \%$ think that they provoke discomfort for the parents and $77 \%$ for the healthcare providers. There exist some case reports about the negative feelings of the parents witnessing gasps, but no studies addressing how healthcare providers confronted to sign of end-of-life in dying child react. Even if gasps are not uncomfortable for the dying child, a treatment would seem to be required to avoid distress of the witnessing parents. However, neuromuscular blockers, the only active compounds against gasps, are related to active euthanasia in ventilator-free children. No official instructions exist on the timing and the content of information to be given to the parents. Some recommendations about the sign of end-of-life suggest informing the family before their onset. Only onethird of the respondents, although working in intensive care units, 
followed a specific formation for palliative cares or end-of-life. This could explain that more than one-third does not know the mechanisms leading to gasps, resuscitating in part their discomfort.

Conclusion Gasps seem to be frequent at the time of end-of-life, and they can be very impressive for the parents. In our study, we show that gasps provoke discomfort to the healthcare providers too and complicate their accompaniment of the parents and the dying child. Almost half of the healthcare providers think that gasps should be prevented, mainly for the well-being of the parents. A lack of specific formation is likely a source of the difficulties of the healthcare providers. A dedicated formation would allow the healthcare providers to better assist the parents and diminish their distress. Finally, studies on the prevalence of gasps in pediatric population and on how the parents are dealing with them, depending upon the information given by healthcare providers would help us to limit parental and professional distress.

\section{Competing interests None.}

\section{0}

\section{Prognosis of malignant primary brain tumors in intensive care} unit

Maxens Decavèle ${ }^{1}$, Nicolas Weiss² ${ }^{2}$ Hélène Prodanovic ${ }^{3}$, Julien Mayaux ${ }^{3}$, Ahmed Idbaih ${ }^{4}$, Agusti Alentorn ${ }^{4}$, Jean-Yves Delattre ${ }^{4}$, Thomas

Similowski ${ }^{3}$, Alexandre Demoule ${ }^{3}$

${ }^{1}$ Réanimation médico-chirurgicale, Hôpital Tenon, Paris, France; ${ }^{2}$ Unité de réanimation neurologique, Hôpital Pitié-Salpêtrière, Paris, France; ${ }^{3}$ Service de pneumologie et réanimation médicale, Groupe hospitalier Pitié-Salpêtrière, Paris, France; ${ }^{4}$ Service de neurologie mazarin 2, Pitié-Salpêtrière Hospital, Paris, France

Correspondence: Maxens Decavèle - maxencesar@hotmail.fr Annals of Intensive Care 2016, 6(Suppl 1):O50

Introduction In the two past decades, the admission of cancer patients to the intensive care unit (ICU) has increased steadily and accounts for $10-20 \%$ of all ICU admissions nowadays. Malignant primary brain tumors (MPBT) are rare tumors representing $<2 \%$ of all new cancer cases in Europe in 2012. Interestingly, if the prognosis improvement of hematological malignancies or solid tumors has been well documented over the past 20 years, few data exist concerning the prognosis of MPBT in ICU. Data on survival of these patients when admitted to the ICU could help accurate triage and management decision. The aim of our study was to describe the profile of MPBT patient admitted to the ICU, to assess ICU and 90-day survival and to identify factors associated with ICU and 90-day mortality in a large cohort of critical MPBT patients.

Patients and methods We performed a bi-center retrospective study of a consecutive cohort of patients with MPBT admitted to ICU over a 19-year period (March 1995-May 2014). Only non-metastatic malignant primary brain tumors were included. Were excluded brain metastases of solid cancers, diffuse lymphomas with cerebral localization and benign brain tumors. The median and the interquartile range were calculated for continuous variables and the absolute and relative frequencies for categorical variables. Statistical analysis consisted of univariate and multivariate logistic regression analysis on ICU and 90-day mortality.

Results A total of 197 patients were included. Acute respiratory failure (ARF) was the main reason for ICU admission (45\%) followed by seizures ( $25 \%$ ) non-epileptic comas (14\%) and shock (12\%). Pneumocystis pneumonia and pulmonary embolism accounted, respectively, for 18 and $9 \%$ of ARF. Admission for epilepsy was more common in patients with glial lesions ( 84.0 vs. $66.7 \%, p=0.019)$, and patients with primary brain lymphoma were more frequently admitted for shock ( 41.6 vs. $18.4 \%, p=0.015$ ). Leukopenia was more commonly found in patients with primary brain lymphoma ( 50.0 vs. $18.9 \%, p=0.005$ ). ICU and 90 -day mortality were, respectively, 23 and $51 \%$. Date of admission did not impact on mortality. The introduction of invasive mechanical ventilation (MV) in epileptic patients did not affect ICU mortality.
Table 3 See text for description

\begin{tabular}{lcrl}
\hline Variables & OR & 95 \% Cl & $\boldsymbol{p}$ value \\
\hline Multivariate analysis on 90-day mortality & & & \\
Epilepsy & 0.15 & {$[0.06-0.42]$} & 0.0002 \\
Need for MV & 4.67 & {$[1.94-11.25]$} & 0.0005 \\
Cancer progression & 6.81 & {$[2.83-16.37]$} & 0.00002 \\
Charlson comorbidity index & 1.45 & {$[1.11-1.91]$} & 0.007 \\
Systolic blood pressure & 1.02 & {$[1.00-1.03]$} & 0.019 \\
Respiratory rate & 1.06 & {$[1.01-1.10]$} & 0.016 \\
Glasgow coma scale & 0.91 & {$[0.83-0.99]$} & 0.039
\end{tabular}

The median and the interquartile range were calculated for continuous variables and the absolute and relative frequencies for categorical variables

$O R$ odds ratio, $\mathrm{Cl}$ confidence interval, $\mathrm{MV}$ mechanical ventilation

In multivariate analysis, factors associated with ICU mortality were the reason for admission for seizure, cancer progression, respiratory rate and Glasgow coma scale (GCS). Factors associated with 90-day mortality were the admission for seizure, cancer progression, the need for mechanical ventilation, systolic blood pressure, respiratory rate, GCS and the Charlson comorbidity index (Table 3). A long-term (1 year) survival was not rare and was observed in around $30 \%$ of cases.

Conclusion ICU mortality of MPBT approaches that of the other solid tumors. Among MPBT patients admitted to ICU, half are alive at 90 days. Epilepsy is associated with a particularly good short-term prognosis. Today, the existence of a MPBT does not appear to be sufficient for ICU recusal admission.

\section{Competing interests None.}

\section{1}

Impact of early immunomodulating treatment on outcome of adult patients with anti- $N$-methyl-D-aspartate receptor encephalitis requiring intensive care: a multicentre study with prospective long-term follow-up

Etienne De Montmollin ${ }^{1}$, Sophie Demeret ${ }^{2}$, Noelle Brule ${ }^{3}$, Marie

Conrad ${ }^{4}$, Frédéric Dailler ${ }^{5}$, Nicolas Lerolle ${ }^{6}$, Jean-Christophe Navellou ${ }^{7}$, Carole Schwebel ${ }^{8}$, Mikael Alves ${ }^{9}$, Martin Cour ${ }^{10}$, Nicolas Engrand ${ }^{11}$, Jean-Marie Tonnelier ${ }^{12}$, Stephane Ruckly ${ }^{13}$, Géraldine Picard ${ }^{14}$, Véronique Rogemond ${ }^{14}$, Jean-François Timsit ${ }^{15}$, Jérôme Honnorat ${ }^{14}$, Romain Sonneville ${ }^{15}$, ENCEPHALITICA study group

${ }^{1}$ Réanimation, C.H. Général Saint Denis hôpital Delafontaine, Saint-Denis, France; ${ }^{2}$ Service de réanimation neurologique, Hopital Pitié-Salpêtrière, AP-HP, Paris, France; ${ }^{3}$ Réanimation médicale, CHU, Nantes, France; ${ }^{4}$ Réanimation Médicale, Hôpital Central, C.H.U. de Nancy, Nancy, France; ${ }^{5}$ Réanimation neurologique, Groupement Hospitalier Est, Hospices Civils de Lyon, Bron, France; ${ }^{6}$ Réanimation médicale, Centre Hospitalier Universitaire d'Angers, Angers, France; ${ }^{7}$ Réanimation Médicale, $\mathrm{CHU}$ de Besançon, Besançon, France; ${ }^{8}$ Réanimation médicale, C.H.U. Grenoble, Grenoble, France; ${ }^{9}$ Réanimation médico-chirurgicale, Centre Hospitalier Intercommunal Poissy Saint Germain En Laye, Poissy, France; ${ }^{10}$ Réanimation Médicale, Hospices Civils de Lyon - Groupement Hospitalier Edouard Herriot, Lyon, France; ${ }^{11}$ Département d'anesthésie-réanimation, Fondation Ophtalmologique Adolphe de Rothschild, Paris, France; ${ }^{12}$ Réanimation médicale, CHU Brest, Brest, France; ${ }^{13}$ lame, umr1137, inserm, Université Paris Diderot, Sorbonne Paris Cité, Paris, France; ${ }^{14}$ Centre national de reference des syndromes neurologiques paranéoplasiques, Hôpital neurologique Pierre Wertheimer, Hospices Civils de Lyon, Bron, France;

${ }^{15}$ Réanimation médicale et infectieuse, Hôpital Bichat-Claude Bernard, AP-HP, Paris, France

Correspondence: Etienne De Montmollin - edemontmollin@gmail.com Annals of Intensive Care 2016, 6(Suppl 1):051 
Introduction Encephalitis due to anti- $\mathrm{N}$-methyl-D-aspartate receptor (NMDAR) antibodies is a rare neurological syndrome, representing $4 \%$ of all encephalitis. Despite the fact that intensive care need is both prognostic of a bad neurological outcome and frequent in the course of the disease ( $75 \%$ of patients), there are presently no data on this specific population. We aimed to describe patients admitted to an intensive care unit (ICU) with anti-NMDAR encephalitis and to identify prognostic factors for good neurological outcome.

Patients and methods In this multicentre study, we included consecutive patients meeting all the following criteria: (1) age $>15$ years, (2) admission to an ICU with a diagnostic of encephalitis, and (3) positive testing for anti-NMDAR antibodies by immunochemistry and cellbased assay in the cerebrospinal fluid (CSF) at the French National Reference Centre. The primary outcome was a good neurological status at 6 months, defined by a modified Rankin score $\leq 2$. Data on ICU management were retrospectively collected, and neurological outcome was prospectively assessed up to 24 months after ICU admission. Early treatment was defined as administration of the treatment $<9$ days after intensive care unit admission. Data are presented as numbers (percentages) and medians [inter-quartiles]. Clinically relevant variables with a $p$ value $\leq 0.1$ in univariate analysis were included in the multivariate analysis.

Results We identified 77 patients between 1 January 2007 and 31 December 2014 from 52 hospitals and three countries (France $n=73$, Belgium $n=2$, Switzerland $n=2$ ). One patient was excluded from analysis because of missing data on the primary outcome.

Patients (aged 24 [20-31] years, female gender 68 (88 \%)) were admitted to the ICU mainly for coma $(n=31,40 \%)$ and seizures $(n=31$, $40 \%$ ), with a Simplified Acute Physiology Score (SAPS) II of 27 [1638.5]. The delay between first neurological symptoms and ICU admission was 10 [5-26] days. A tumour was diagnosed in 31 patients (41\%) and consisted in mature teratoma $(n=23,30 \%)$, immature teratoma ( $n=6,8 \%)$ and small cell lung carcinoma $(n=2,3 \%)$.

During ICU stay, 70 patients (92\%) experienced seizures and 35 (46\%) presented a status epilepticus. ICU length of stay was 55 [24-88] days. First-line immunomodulating treatment consisted in steroids ( $n=61 / 74,82 \%$ ), intravenous immunoglobulins ( $n=71 / 74$, $96 \%)$ and plasmapheresis ( $n=17 / 74,23 \%)$. Second-line therapy was necessary for $46 / 74$ patients (62 \%) and included cyclophosphamide $(n=6 / 45,13 \%)$, rituximab $(n=24 / 45,53 \%)$ or both $(n=15 / 45,33 \%)$. At 6,12 and 24 months, good neurological outcome was achieved in, respectively, 43/76 (57\%), 59/74 (80 \%) and 49/56 (87 \%) patients. At 6 months, 3 (4\%) patients were dead.

Variables tested in the multivariate model were: age at admission, delay from ICU admission to tumour imaging, delay from ICU admission to first immunomodulating treatment, type of first treatment and white blood cell (WBC) count in the first CSF examination. By contrast, SAPS II, extra-neurological organ failures, status epilepticus, the presence of a tumour and type of second-line treatment were not

\section{Table 4 Multivariate analysis of factors for good neuro-} logic outcome at 6 months

\begin{tabular}{|c|c|c|c|}
\hline & OR & $95 \% \mathrm{Cl}$ & $p$ \\
\hline \multicolumn{4}{|l|}{ First-line immunomodulating treatment } \\
\hline Early intravenous immunoglobulins & 2.26 & $0.54-9.35$ & \\
\hline Early steroids & 3.26 & $0.62-17.19$ & \\
\hline Early combined treatment & 8.42 & $2.11-33.57$ & \\
\hline Late treatment & 1 & - & 0.026 \\
\hline \multicolumn{4}{|c|}{ White blood cell count in first CSF (cells/mm³) } \\
\hline$<10$ & 6.88 & $1.58-29.92$ & \\
\hline $10-50$ & 3.60 & $1.03-12.65$ & \\
\hline$>50$ & 1 & - & 0.026 \\
\hline
\end{tabular}

associated with outcome. Low WBC count on first CSF examination and early combined first-line treatment were independent factors for good neurological outcome at 6 months (Table 4).

Conclusion We report in this study the largest cohort to date of intensive care patients with anti-NMDAR encephalitis. We show that a low CSF inflammation and early combined immunomodulating treatment by steroids and intravenous immunoglobulins are independent prognostic factors for good neurological outcome, advocating for prompt diagnosis and aggressive treatment of this rare disease. We also show that severe neurological dysfunction and non-neurological organ failures at admission are not prognostic and that despite prolonged ICU stays, the long-term neurological outcome is good, with improvement up to 24 months after ICU admission.

\section{Competing interests None.}

\section{2}

Determinants of outcome in critically ill patients

with hematological malignancy and central neurological failure: data from the TRIAL OH study

Chiara Marzorati ${ }^{1}$, Virginie Lemiale ${ }^{2}$, Djamel Mokart ${ }^{3}$, Frédéric Pène ${ }^{4}$, Achille Kouatchet ${ }^{5}$, Julien Mayaux ${ }^{6}$, François Vincent ${ }^{7}$, Martine Nyunga ${ }^{8}$, Fabrice Bruneel ${ }^{9}$, Antoine Rabbat ${ }^{10}$, Christine Lebert ${ }^{11}$, Pierre Perez ${ }^{12}$, Dominique Benoit ${ }^{13}$, Giuseppe Citerio', Elie Azoulay ${ }^{2}$, Stéphane Legriel ${ }^{9}$ ${ }^{1}$ Anaesthesiology and critical care department, San Gerardo Hospital, Monza, Italy; ${ }^{2}$ Réanimation médicale, Hôpital Saint-Louis, Paris, France; ${ }^{3}$ Réanimation, Institut Paoli-Calmettes, Marseille, France; ${ }^{4}$ Réanimation Médicale, Hôpital Cochin, Paris, France; ${ }^{5}$ Réanimation médicale, Centre Hospitalier Universitaire d'Angers, Angers, France; ${ }^{6}$ Service de pneumologie et réanimation médicale, Groupe hospitalier Pitié-Salpêtrière, Paris, France; ${ }^{7}$ Réanimation polyvalente, Groupe Hospitalier Intercommunal Le Raincy-Montfermeil, Montfermeil, France; ${ }^{8}$ Réanimation, Centre Hospitalier De Roubaix, Roubaix, France; ${ }^{9}$ Réanimation médico-chirurgicale, Centre Hospitalier de Versailles, Le Chesnay, France; ${ }^{10}$ Réanimation pneumologique, Hôpital Cochin, Paris, France; ${ }^{11}$ Réanimation, Centre Hospitalier Départemental - site de La Roche-sur-Yon, La Roche-sur-Yon, France; ${ }^{12}$ Réanimation polyvalente, C.H.U. de Nancy, Nancy, France;

${ }^{13}$ Réanimation polyvalente, Hopital universitaire, Gand, Belgium

Correspondence: Stéphane Legriel - slegriel@ch-versailles.fr

Annals of Intensive Care 2016, 6(Suppl 1):052

Introduction Neurological failure in patients with hematological malignancy admitted to the intensive care unit has received little attention. We sought to report determinants of outcome in these patients.

Patients and methods We performed a post hoc analysis of the TRIAL $\mathrm{OH}$ database. The TRIAL OH study was prospectively carried out in 17 university or university-affiliated centers in France and Belgium (20102012). Central neurological failure was defined by any neurological disorder of central origin among impairment of consciousness, seizure with or without status epilepticus, focal neurological signs, encephalopathy, and meningeal symptoms.

Results Of the 1011 patients initially enrolled, 226 (22.4\%) demonstrated central neurological failure. There were 132 males and 94 women, aged 62 years (IQR 53-72). Median SAPS II score was 53 (4266). Median Charlson comorbidity index was $3(2-4)$ at ICU admission, and poor performance status was encountered in 54 (24\%) patients. Median time diagnosis of malignancy and median time from onset of neurological manifestations at ICU admission were 120 days (8-847) and 0 days $(0-1)$, respectively.

$42(42 \%)$ patients were managed at the diagnosis phase of hematological malignancy, and $47(21 \%)$ patients were in complete or partial remission. Underlying malignancies were as follows: non-Hodgkin's lymphoma in $85(38 \%)$, acute myeloid leukemia in $48(21 \%)$, myeloma in $22(10 \%)$, acute lymphocytic leukemia in $17(8 \%)$, chronic lymphocytic leukemia in $17(8 \%)$, myelodysplastic syndrome in $11(5 \%)$, Hodgkin's disease in $5(2 \%)$, chronic myeloid leukemia in $5(2 \%)$ and others in $16(7 \%)$. Bone marrow or hematopoietic stem cell transplantation were performed in $39(17 \%)$ patients associating 21 (9\%) autologous and $18(8 \%)$ allogenic stem cell recipients. A history of cancer chemotherapy within 30 days before ICU admission was noted 
in 95 (42\%), and neutropenia was found at ICU admission in 52 (23\%) patients.

Presenting manifestations were drowsiness (65\%), coma (32\%), tiredness $(26 \%)$, seizures (19\%), nausea/vomiting (15\%), headache $(11 \%)$, focal neurological signs (10\%), meningism (6\%) and visual disturbances (5\%).

Median SOFA score at day-1 after ICU admission was 8 (5-12) resulting to a total number of organ failures of $3(2-3)$. Median GCS score was 12 (7-15). Mechanical ventilation was required in 124 (55\%) patients, catecholamine support in 93 (41\%) and dialysis in 25 (34\%). Eleven (5\%) received chemotherapy during their ICU stay.

Neuroimaging, lumbar puncture and EEG were performed in 113 (50\%), 73(32\%) and $63(28 \%)$ patients and were deemed contributive to final diagnosis in 51 (45\%), $18(25 \%)$ and 24 (38\%) cases, respectively. Finally, a neurosurgical biopsy was performed in only one patient providing the cause of neurological impairment.

Topographic localizations of the cause of neurological failure were as follows: encephalic in 200 (88\%), meningeal in 5 (2\%), brainstem in 2, cerebellar and/or medullary in 1, and undetermined in 17 (8\%). According to the direct/indirect classification, malignancy involvement was sought being direct in 11 (5\%) (central nervous system infiltration), neurological indirect in $114(50 \%)$ (metabolic in 73, central nervous system infection in 12 , vascular damage in 14 , iatrogenic in 15), extraneurologic in 77 (34 \%) (shock in 52, sepsis in 10 and cardiac arrest in 15) and remained unknown in $24(11 \%)$.

Among 226 patients with neurological failure, hospital mortality was $50 \%$. By multivariate analysis, independent positive predictors of hospital mortality were poor performance status [odds ratio (OR) $3.99,95 \% \mathrm{Cl} 1.82-9.39, p=0.0009]$, non-Hodgkin's lymphoma [OR $2.60,95 \% \mathrm{Cl} 1.35-5.15, p=0.005]$, shock [OR $1.95,95 \% \mathrm{Cl} 1.04-3.72$, $p=0.04]$ and respiratory failure [OR $2.18,95 \% \mathrm{Cl} 1.14-4.25, p=0.02]$, whereas GCS score at day 1 [OR $0.88 /$ point, $95 \% \mathrm{Cl} 0.81-0.95$, $p=0.0009$ ] and autologous stem cell transplantation [OR $0.25,95 \% \mathrm{Cl}$ $0.07-0.75, p=0.02]$ were negative predictors of hospital mortality. The 2-year probability of survival was markedly lower in patients requiring ICU management with neurological failure than in the absence thereof (HR 1.45, $95 \% \mathrm{Cl} 1.18-1.78, p=0.0003$ ).

Conclusion In hematological patients requiring ICU management, neurological failure is frequent and associated with poor outcome. Type of underlying hematological malignancy, poor performance status, hemodynamic and respiratory failures and intensity of consciousness impairment are independently associated with poor outcome. Early goal directed management in those patients may offer opportunities for improvement.

\section{Competing interests None.}

\section{3}

Ammonemia predicts severity and outcome in cirrhotic patients with hepatic encephalopathy in ICU

Simona Tripon', Maxime Mallet ${ }^{1}$, Marika Rudler ${ }^{1}$, Francoise

Imbert-Bismut ${ }^{2}$, Dominique Thabut ${ }^{1}$, Nicolas Weiss ${ }^{3}$

'Brain-Liver Pitié-Salpêtrière Study Group (BLIPS), Soins Intensifs d'Hépatologie, Pitié-Salpêtrière Hospital, Paris, France; ${ }^{2}$ Département de biochimie, Hôpital Pitié-Salpêtrière, Paris, France; ${ }^{3}$ Brain-liver pitié-salpêtrière study group (blips), unité de réanimation neurologique, Pitié-Salpêtrière Hospital, Paris, France

Correspondence: Simona Tripon - triponsimona@yahoo.com Annals of Intensive Care 2016, 6(Suppl 1):053

Background Hepatic encephalopathy (HE), a complication of cirrhosis, presents clinically from mild neuropsychiatric symptoms to coma. It is a major public health problem, it is associated with a poor prognosis, one-half of the patients presenting HE will die in the following year. The physiopathology of HE is complex and multifactorial. Since decades, a relationship between ammonia and the occurrence $\mathrm{HE}$ has been proposed, but remains still controversial. The correlation between the severity of $\mathrm{HE}$ and the ammonia levels is thus still debated. Often in clinical practice, the serum ammonia is not measured in cirrhotic patients with altered neurological examination.
Aim The aim of this study was to determine whether ammonia was correlated with the severity of HE episode and with the outcome, in a large series of patients with cirrhosis admitted in Liver Intensive Care Unit.

Methods We included prospectively all cirrhotic patients hospitalised in Liver ICU for severe cirrhosis decompensations. Serum ammonia was measured at admission, during the hospitalisation and at the discharge from ICU. Neurological status was assessed by West-Haven (WH) and Glasgow scores. Overt HE was diagnosed as a West-Haven score ranging between 2 and 4 .

Results Ninety-eight cirrhotic patients hospitalised in ICU between May 2014 and September 2015 were included. The main clinical characteristics were as follows: mean age was $58 \pm 13$ years, 74 patients were male, and the aetiology of the liver cirrhosis was: alcoholic (48\%), virus (12\%), mixed (alcool + virus/metabolic) $(22 \%)$ and other (15\%). $91 \%$ of the patients had a decompensated cirrhosis (Child-Pugh B and C). $85 \%$ of patients had an abnormal neurological examination at admission, $27 \%$ were hospitalised for $\mathrm{HE}$, and $17 \%$ had already a treatment for $\mathrm{HE}$. The mean ammonia was $99.1 \pm 57.3 \mu \mathrm{mol} / \mathrm{L}$, and $80 \%$ of patients displayed abnormally elevated ammonia ( $>50 \mu \mathrm{mol} / \mathrm{L})$. Ammonia was higher in patients with $\mathrm{HE}$ than in those without [108 (95\% Cl 86-123) vs 57 (95\% $\mathrm{Cl} 50-81), p=0.0004]$. In patients with $\mathrm{HE}$, ammonia was significantly correlated with severity of HE assessed by GCS $(p=0.001)$. Overall, 34 $\mathrm{HE}$ patients experienced worsening of their neurological status during hospitalisation. Ammonia at admission was significantly higher in those patients [128 (95\% Cl 108-147) vs 87 (95\% Cl 64-110), $p=0.007]$. In multivariate analysis, parameters independently associated with worsening of neurological status were MELD score and ammonia levels at admission ( $p=0.01$ and 0.02 , respectively).

Conclusion Hyperammonia reflects the severity of HE and is correlated with the poor prognosis in cirrhotic patients with HE.

\section{4}

Acute varicella zoster encephalitis admitted to the ICU: a case series of 47 patients

Emmanuel Canet ${ }^{1}$, Adrien Mirouse ${ }^{2}$, Romain Sonneville ${ }^{3}$, Laurent Argaud ${ }^{4}$, Stanislas Faguer ${ }^{5}$, Keyvan Razazi ${ }^{6}$, Claude Guérin ${ }^{7}$, Pierre Perez $^{8}$, Anne-Sophie Moreau ${ }^{9}$, Claire Pichereau ${ }^{10}$, François Barbier ${ }^{11}$, Sybille Merceron ${ }^{12}$, Amélie Seguin ${ }^{13}$, Julien Mayaux ${ }^{14}$, Guillaume Geri ${ }^{15}$, Christophe Guitton ${ }^{16}$, Francois Labadie ${ }^{17}$, Elie Azoulay ${ }^{18}$

${ }^{1}$ Medical Intensive Care Unit, Hôpital Saint Louis, Paris, France; ${ }^{2}$ Réanimation médicale, hôpital Cochin, Assistance Publique - Hôpitaux de Paris, Paris, France; ${ }^{3}$ Service de réanimation médicale et infectieuse, Hôpital Bichat-Claude Bernard-APHP, Paris, France; ${ }^{4}$ Réanimation Médicale, Hospices Civils de Lyon - Groupement Hospitalier Edouard Herriot, Lyon, France; ${ }^{5}$ Département de néphrologie et transplantation d'organes, Hopital Rangueil, Toulouse, France; ${ }^{6}$ Réanimation médicale, Hospital Henri Mondor, Créteil, France; ${ }^{7}$ Réanimation médicale, Hôpital de la Croix-Rousse, Lyon, France; ${ }^{8}$ Réanimation polyvalente, C.H.U. de Nancy, Nancy, France; ${ }^{9}$ Réanimation Médicale, Hôpital Saint-Louis, Assistance Publique Hôpitaux de Paris, Paris, France; ${ }^{10} 75$, Hôpital Saint Louis, Paris, France; ${ }^{11}$ Loiret, Hôpital Régional Orléans La Source, Orléans, France;

${ }^{12}$ Réanimation médico-chirurgicale, Centre Hospitalier de Versailles, Le Chesnay, France; ${ }^{13}$ Réanimation médicale, Centre Hospitalier Universitaire de Caen, Caen, France; ${ }^{14}$ Réanimation médicale, Hôpital Pitié-Salpêtrière, Paris, France; ${ }^{15}$ Réanimation Médicale, Hôpital Cochin, Paris, France;

${ }^{16}$ Réanimation médicale, C.H.U. Hôtel Dieu, Nantes, France; ${ }^{17}$ Service de réanimation polyvalente, Hôpitaux de Saint-Nazaire, Saint-Nazaire, France; ${ }^{18}$ Réanimation médicale, Hôpital Saint-Louis, Paris, France

Correspondence: Emmanuel Canet - emmanuel.canet@aphp.fr

Annals of Intensive Care 2016, 6(Suppl 1):054

Introduction Varicella zoster virus (VZV) is responsible for human infections with various clinical presentations, followed by long-lasting viral latency in the spinal and cranial ganglia. VZV reactivation can be responsible for encephalitis. The purpose of this study is to describe the clinical features and the prognosis of VZV encephalitis in adults patients admitted to the intensive care unit (ICU).

Patients and methods $A$ retrospective multicenter cohort study was performed, including consecutive adult patients admitted to 18 
French ICUs between 01/01/1999 and 01/09/2015 with a diagnosis of VZV encephalitis. Data and follow-up were abstracted from the medical charts. Patients were evaluated for vital status at ICU discharge. A univariate analysis was performed to identify factors associated with ICU survival.

Results Forty-seven patients were included in the study. Median age was 54 (inter-quartile range 35-67) years and 22 (47 \%) were male. Thirty-nine patients $(83 \%)$ reported an underlying cause of immune deficiency. The three main causes of immune deficiency were malignancy $(12,31 \%)$ solid organ transplantation (10, $26 \%)$ and HIV infection $(8,21 \%)$. Long-term exposure to steroids was reported in 19 (49 \%) patients. Patients were admitted to the ICU 2 (1-6) days after the onset of the first neurological symptoms. Altered mental status at ICU admission was constant. The other symptoms were focal neurological signs $(17,36 \%)$, seizure $(13,34 \%)$ and status epilepticus $(9$, $19 \%)$. Thirty-three (70 \%) patients had a rash. The median coma Glasgow score (CGS) at ICU admission was 12 (8-15); 12 (27 \%) patients had a CGS $\leq 8$. Two-third of the patients had fever. The median white blood cell count in the cerebrospinal fluid (CSF) was $59(16-116) / \mathrm{mm} 3$ with $73(67-84) \%$ of lymphocytes and 21 (8-67) \% of neutrophils. Median CSF protein level was $1.4(0.7-3.8) \mathrm{g} / \mathrm{L}$, and median CSF glucose level was $3.7(2.5-5.0) \mathrm{mmol} / \mathrm{L}$. CSF PCR for VZV was tested in 37 (79\%) patients and positive in $89 \%$ of the cases.

Thirty-two (68\%) patients had a central nervous system imaging, 27 (57\%) a computed tomography (CT) and 21 a magnetic resonance imaging (MRI). Twenty (74\%) CTs were reported as normal. Main abnormal findings reported with CT were cerebral edema (2, $7.4 \%)$ and petechial hemorrhages $(2,7.4 \%)$. When performed, most MRI reported abnormal findings $(16,76 \%)$, including brainstem lesions $(5$, $29 \%)$, arteritis and ischemic infarctions (4, $20 \%)$ and demyelinating lesions (4, $20 \%)$

The median SOFA score at day 1 was 7 (4-9). Patients were treated intravenously with $10(10-15) \mathrm{mg} / \mathrm{kg} / 8 \mathrm{~h}$ of acyclovir during a median of 14 (9-20) days. In addition, three (6\%) patients received intravenous globulins. During ICU stay, 41 (87\%) patients required invasive mechanical ventilation, 20 (43\%) received vasopressors, and RRT was implemented in $14(30 \%)$ patients. The median ICU length of stay was 16 (6-39) days. Thirty-six (77 \%) patients were alive at ICU discharge. By univariate analysis, two factors were associated with an increased likelihood of ICU mortality: a SOFA score $\geq 7$ at day 1 , odds ratio (OR) $28.8,95 \%$ confidence interval (1.54-538.4), $p<0.01$, and the presence of disseminated intravascular coagulation, OR $7.0,95 \% \mathrm{Cl}(1.23-39.8)$, $p=0.03$.

Discussion We report the clinical features of 47 patients admitted to the ICU for the management of VZV encephalitis. This diagnosis may be challenging as clinical signs and symptoms may be diminished by an underlying cause of immunosuppression. Indeed, one-third of the patients had no fever and up $30 \%$ had no skin rash. Patients should be investigated for VZV-related cerebral arteritis despite the fact that its treatment remains a matter of debate.

Conclusion Severe VZV encephalitis occurs mainly in patients with a history of immune deficiency. In the ICU setting, about $20 \%$ of the patients experienced status epilepticus. MRI is useful to evaluate patients with VZV encephalitis. ICU mortality is $23 \%$ and correlates with the number of organ dysfunction at ICU admission.

Competing interests None.

\section{5}

\section{High-flow nasal cannula for acute respiratory failure} in immunocompromised patients

Virginie Lemiale ${ }^{1 *}$, Matthieu Resche-Rigon ${ }^{2}$, Djamel Mokart ${ }^{3}$, Frédéric Pène $^{4}$, Etienne Faucher ${ }^{5}$, Christophe Guitton ${ }^{6}$, Antoine Rabbat ${ }^{7}$, Christophe Girault ${ }^{8}$, Achille Kouatchet ${ }^{9}$, François Vincent ${ }^{10}$, Fabrice Brunee $^{11}{ }^{11}$, Martine Nyunga ${ }^{12}$, Amélie Seguin ${ }^{13}$, Kada Klouche ${ }^{14}$, Sylvie Chevret ${ }^{15}$, Elie Azoulay ${ }^{16}$, Groupe de Recherche en Reanimation Respiratoire du patient d'Onc-hématologie

${ }^{1}$ Medical ICU, Hôpital Saint Louis, Paris, France; ${ }^{2}$ Biostatistiques, Hôpital Saint-Louis, Paris, France; ${ }^{3}$ Réanimation, Institut Paoli-Calmettes, Marseille, France; ${ }^{4}$ Réanimation Médicale, Hôpital Cochin, Paris, France; ${ }^{5}$ Réanima- tion Médicale, Hospices Civils de Lyon - Groupement Hospitalier Edouard Herriot, Lyon, France; ${ }^{6}$ Réanimation médicale, C.H.U. Hôtel Dieu, Nantes, France; ${ }^{7}$ Réanimation pneumologique, Hôpital Cochin, Paris, France; ${ }^{8}$ Réanimation Médicale, Centre Hospitalier Universitaire Rouen, Rouen, France; ${ }^{9}$ Réanimation médicale, Centre Hospitalier Universitaire d'Angers, Angers, France; ${ }^{10}$ Réanimation polyvalente, Groupe Hospitalier Intercommunal Le Raincy-Montfermeil, Montfermeil, France; ${ }^{11}$ Réanimation médico-chirurgicale, Centre Hospitalier de Versailles, Le Chesnay, France; ${ }^{12}$ Réanimation polyvalente, Centre Hospitalier de Roubaix, Roubaix,

France; ${ }^{13}$ Réanimation médicale, Centre Hospitalier Universitaire de Caen, Caen, France; ${ }^{14}$ Service de réanimation, CHU Lapeyronie, Montpellier, France; ${ }^{15}$ Service de biostatistique et information médicale, Hôpital Saint-Louis, Paris, France; ${ }^{16}$ Réanimation médicale, Hôpital Saint-Louis,

Paris, France

Correspondence: Virginie Lemiale - virginie.lemiale@aphp.fr

Annals of Intensive Care 2016, 6(Suppl 1):055

Introduction In immunocompromised patients with acute respiratory failure (ARF), invasive mechanical ventilation remains associated with high mortality. Choosing the adequate device for oxygenation is of the utmost importance in that setting. High-flow nasal cannula (HFNC) has been associated with decreased mortality in ARF. In this study, we assess outcomes of immunocompromised patients treated with HFNC. Patients and methods We performed a post hoc analysis of iVNIctus study, a multicentre trial of NIV in critically ill immunocompromised patients admitted for ARF in 29 intensive care units in France and Belgium. Only those alive at day 2 were analyzed in this study. A propensity score-based approach was used to assess the impact of HFNC compared with oxygen only on hospital mortality. Propensity score was based on randomization group, severity at admission and etiology of acute respiratory failure. Primary endpoint was mortality at day 28. Secondary endpoints were intubation rate, duration of mechanical ventilation, ICU-acquired infection, ICU and hospital stay lengths.

Results Among 374 patients included in the study, 353 met inclusion criteria. Underlying disease included malignancy $(n=296,84 \%)$, solid organ transplantation $(n=24,6.8 \%)$ and other cause of immunosuppression $(n=33,9.3 \%)$. ARF etiologies were mostly bacterial pulmonary infections $(n=157,44.4 \%)$ and opportunistic infection $(n=76$, $21.5 \%$ ). 180 (50 \%) patients received NIV. D-28 mortality was $22.6 \%$ (80 deaths). Mechanical ventilation was ultimately needed in 142 (40.2\%) patients.

At ICU admission, 127 patients received HFNC, whereas 226 patients never received HFNC. D-28 mortality was, respectively, 33 (25.9\%) and $47(20.7 \%)(p=0.26)$. Ninety patients in each group (HFNC or oxygen only) were matched according the propensity score. Among them, 92 patients received NIV (44/90 in HFNC group vs 48/90 in oxygen group). HFNC was not associated with a decreased D-28 mortality ( 23.3 vs $25.5 \%, p=0.76)$. Intubation rate and ICU-acquired infections were, respectively ( 43.3 vs $50 \%, p=0.47$, and 23.3 vs $26.7 \%, P=0.73$ ). ICU lengths of stay and mechanical ventilation duration were not different (respectively, 8 [7-11] vs 7 [6-16 days, $p=0.89$, and 13 [4-46] vs 14 [8-33 days, $p=0.72$ ).

Conclusion In this study, HFNC during acute respiratory failure in immunocompromised patients did not improve survival compared with oxygen only. HFNC was associated with neither a decrease intubation rate nor a lower ICU-acquired infection rate.

Competing interests None.

\section{6}

High-flow oxygen therapy through a nasal cannula in immunocompromised patients with acute hypoxemic respiratory failure

Jean-Pierre Frat', Stéphanie Ragot ${ }^{2}$, Christophe Girault ${ }^{3}$, Remi Coudroy ${ }^{1}$ René Robert ${ }^{1}$, Jean-Michel Constantin ${ }^{4}$, Gwenael Prat ${ }^{5}$, Thierry Boulain ${ }^{6}$, Angéline Jamet, ${ }^{1}$, Alain Mercat ${ }^{7}$, Laurent Brochard ${ }^{8}$, Arnaud, W. Thille $^{1}$ ${ }^{1}$ Réanimation Médicale, $\mathrm{CHU}$ de Poitiers, Poitiers, France; ${ }^{2}$ Département de Biostatistiques et d'Epidémiologie, CHU de Poitiers, Poitiers, France; ${ }^{3}$ Réanimation Médicale, Centre Hospitalier Universitaire Rouen, Rouen, France; ${ }^{4}$ Réanimation adulte, C.H.U. Estaing, Clermont-Ferrand, France; ${ }^{5}$ Réanimation médicale, CHRU de Brest, Brest, France; ${ }^{6}$ Réanimation 
médicale polyvalente, Hôpital de La Source, CHR Orléans, Orléans, France; ${ }^{7}$ Service de réanimation médicale et médecine hyperbare, Centre Hospitalier Universitaire d'Angers, Angers, France; ${ }^{8}$ Interdepartmental division of critical care- university of toronto, Hospital St. Michael and Keenan research center, Toronto, Canada

Correspondence: Remi Coudroy - remi.coudroy@chu-poitiers.fr Annals of Intensive Care 2016, 6(Suppl 1):056

Introduction In the early 2000s, two randomized controlled trials showed that as compared to standard oxygen therapy, noninvasive ventilation could decrease mortality of immunocompromised patients admitted to ICU for acute respiratory failure. However, the benefits of noninvasive ventilation in immunocompetent patients with acute respiratory failure are debated. High-flow oxygen therapy through a nasal cannula may offer an alternative in hypoxemic patients. We recently found in a randomized controlled trial including 310 patients with acute respiratory failure (FLORALI study) that as compared to noninvasive ventilation, high-flow oxygen therapy decreased mortality. Immunocompromised patients could also be included in this study, except for those with profound neutropenia. Therefore, we assessed the benefits of high-flow oxygen therapy or noninvasive ventilation in this subgroup of patients. Our objective was to compare intubation and mortality rates in the subset of immunocompromised patients admitted to ICU for acute respiratory failure.

Patients and methods We performed a subgroup analysis in the framework of the FLORALI study. This study included all patients with non-hypercapnic $\left(\mathrm{PaCO}_{2} \leq 45 \mathrm{mmHg}\right)$ acute respiratory failure while excluding patients with cardiogenic pulmonary edema and those with underlying chronic lung disease. Patients were assigned to three groups according to treatment: high-flow oxygen therapy, standard oxygen therapy or noninvasive ventilation. The primary outcome was the intubation rate, and secondary outcome included 90 -day mortality. We focused on the subset of immunocompromised patients included in this study, while patients with profound neutropenia were deliberately excluded.

Results Among the 310 patients with acute respiratory failure, 82 (26\%) were immunocompromised including 26 patients in the highflow oxygen therapy group, 30 in the standard oxygen group and 26 in the noninvasive ventilation group. Intubation rates were 31,43 and $55 \%$ in the high-flow oxygen therapy, standard oxygen therapy and noninvasive ventilation groups, respectively $(p=0.04)$. The 90-day mortality rates were 15, 27 and $46 \%$ in the high-flow oxygen therapy, standard oxygen therapy and noninvasive ventilation groups, respectively $(p=0.046)$. Ventilator-free days at day 28 were $26 \pm 6,23 \pm 10$ and $14 \pm 13$ days in the high-flow oxygen therapy, standard oxygen therapy and noninvasive ventilation groups, respectively $(p<0.0001)$. Conclusion In immunocompromised patients admitted to ICU for acute hypoxemic respiratory failure, as compared to standard oxygen therapy or noninvasive ventilation, high-flow oxygen therapy was associated with lower intubation and mortality rates and reduced duration of invasive mechanical ventilation.

\section{Competing interests None.}

\section{7}

Prospective validations of the PASTEIL score to assess the clinical pretest probability of Pneumocystis jirovecii pneumonia (PjP) in patients with hematologic malignancies (HMs) and acute respiratory failure (ARF)

Elie Azoulay ${ }^{1}$, Sylvie Chevret ${ }^{2}$, Antoine Roux ${ }^{3}$, Etienne Faucher ${ }^{4}$, François Vincent ${ }^{5}$, Antoine Rabbat ${ }^{6}$, Achille Kouatchet ${ }^{7}$, Frédéric Pène ${ }^{8}$, Julien Mayaux ${ }^{9}$, Pierre Perez ${ }^{10}$, Martine Nyunga ${ }^{11}$, Christophe Guitton ${ }^{12}$, Fabrice Bruneel $^{13}$, Kada Klouche ${ }^{14}$, Djamel Mokart ${ }^{15}$, Virginie Lemiale ${ }^{16}$ ${ }^{1}$ Réanimation médicale, Hôpital Saint-Louis, Paris, France; ${ }^{2}$ Service de biostatistique et information médicale, Hôpital Saint-Louis, Paris, France; ${ }^{3}$ Réanimation pneumologique, APHP hôpital pompidou, Paris, France; ${ }^{4}$ Réanimation Médicale, Hospices Civils de Lyon - Groupement Hospitalier Edouard Herriot, Lyon, France; ${ }^{5}$ Réanimation polyvalente, Groupe Hospitalier Intercommunal Le Raincy-Montfermeil, Montfermeil, France; ${ }^{6}$ Réanimation pneumologique, Hôpital Cochin, Paris, France; ${ }^{7}$ Réanimation médicale, Centre Hospitalier Universitaire d'Angers, Angers, France; ${ }^{8}$ Réanimation Médicale, Hôpital Cochin, Paris, France; ${ }^{9}$ Réanimation pneumologiqe, Hôpital Pitié-Salpêtrière, Paris, France; ${ }^{10}$ Réanimation polyvalente, C.H.U. de Nancy, Nancy, France; ${ }^{11}$ Réanimation polyvalente, Centre Hospitalier de Roubaix, Roubaix, France; ${ }^{12}$ Réanimation médicale, C.H.U. Hôtel Dieu, Nantes, France; ${ }^{13}$ Réanimation médico-chirurgicale, Centre Hospitalier de Versailles, Le Chesnay, France; ${ }^{14}$ Service de réanimation, CHU Lapeyronie, Montpellier, France; ${ }^{15}$ Réanimation, Institut Paoli-Calmettes, Marseille, France; ${ }^{16}$ Medical ICU, Hôpital Saint Louis, Paris, France

Correspondence: Virginie Lemiale - virginie.lemiale@aphp.fr Annals of Intensive Care 2016, 6(Suppl 1):057

Introduction Pneumocystis jiroveci pneumonia (PjP) occurs increasingly in non-AIDS immunocompromised patients, chiefly those with HMs. Delayed implementation of trimethoprim-sulfamethoxazole is associated with increased mortality. A clinical score to early assess at the bedside the clinical pretest probability of PjP before proceeding with diagnostic testing would allow avoiding missed diagnosis and delayed treatment.

Patients and methods Last year, we presented at REANIMATION 2015 the PASTEIL score (see Table 5) that was developed in $1092 \mathrm{HM}$ patients with ARF (134 [12.3 \%] with proven PjP) admitted to eight ICUs between 2006 and 2012 ("learning set"). First, variables were identified through a multivariable logistic regression model after multiple imputation of missing confounders, and then, the score described below was derived from mean of beta regression coefficients and validated by bootstrap (resampling). Discrimination (i.e., ability of the score can separate PjP from non-PjP patients) was measured by the area under the receiver operating characteristic (ROC) curve (AUC, $C$ statistic). Calibration (i.e., how well predicted probabilities agree with actual observed risk) was measured by the Hosmer-Lemeshow statistic (goodness-of-fit test and calibration curves) that compares the average predicted risk within subgroups to the proportion that actually develops disease. The score was performing very well in the learning set, with a very good discrimination (AUC was 0.87 saying that predicted values for cases are all higher than for non-cases) and a very good calibration (mean goodness of fit of -0.75 and calibration curves suggesting applicability of the models to individual cases in this learning set). Here, we sought to further validate this score in a prospective and independent reliability cohort. We first imputed the missing data using mice (multiple imputation algorithm), based on 30 multiple imputed datasets. On each complete imputed dataset, the score was computed for each patient and averaged; lastly, for each patient, the predicted probability of PjP was derived from the average score and its performance assessed.

Results To validate the diagnostic score, we collected data from an independent dataset of 238 patients with HMS admitted to 26 ICUs for ARF (iVNIctus trial), including $15(6.3 \%)$ patients with PjP. When compared to patients with other ARF etiologies, PjP patients were younger (33 aged $<50$ vs. $20 \%, P=0.02$ ), had more frequently a lymphoproliferative disorder (93.3 vs. $45.7 \%, P=0.0003$ ), never received prophylaxis (vs. $36 \%$ in other ARF etiologies, $P<0.0001$ ) and had more frequently an interstitial pattern on chest imaging (67 vs. $32 \%)$. The three other components of the score, namely time since respiratory symptoms onset, shock at admission and pleural involvement, were not significantly different between PjP patients and those with other ARF etiologies. In this reliability cohort, the score has a very good discrimination [AUC was 0.83 (95\% Cl 0.73-0.93, DeLong estimates)]. However, the calibration was suboptimal with predicted probabilities of PjP above the observed proportions of actual PjP in the low deciles of the score.

\section{Table 5 See text for description}

\begin{tabular}{|c|c|c|c|c|c|c|c|c|c|c|}
\hline \multirow{2}{*}{$\begin{array}{l}\text { No } \\
\text { prophy- } \\
\text { laxis }\end{array}$} & \multicolumn{3}{|l|}{ Age } & \multirow{2}{*}{$\begin{array}{l}\text { Shock } \\
\text { at admis- } \\
\text { sion }\end{array}$} & \multicolumn{3}{|c|}{$\begin{array}{l}\text { Time since respir- } \\
\text { atory symptoms }\end{array}$} & \multirow{2}{*}{$\begin{array}{l}\text { - Pleural } \\
\text { effu- } \\
\text { - sion }\end{array}$} & \multirow{2}{*}{$\begin{array}{l}\text { Inter- } \\
\text { stitial } \\
\text { pattern }\end{array}$} & \multirow{2}{*}{$\begin{array}{l}\text { Lym- } \\
\text { phopro- } \\
\text { liferative } \\
\text { disease }\end{array}$} \\
\hline & $<50$ & $\begin{array}{l}50- \\
70\end{array}$ & $>70$ & & $<3$ & $3-5$ & $>5$ & & & \\
\hline+1 & 0 & -1.5 & -2.5 & -1.5 & 0 & +3 & +3 & -2 & +2.5 & +2 \\
\hline
\end{tabular}



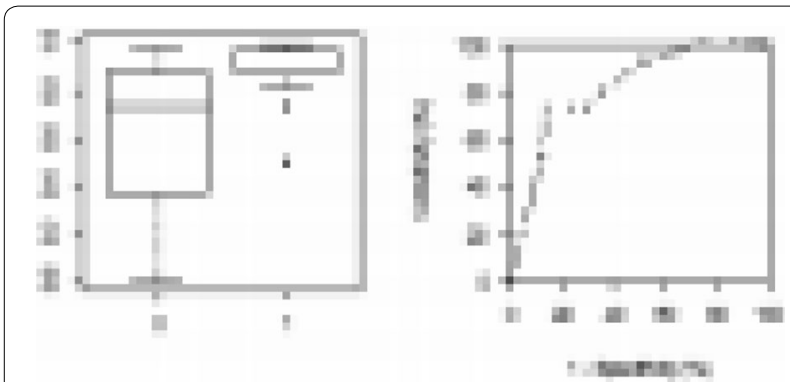

Fig. 11 See text for description

Conclusion Within hours from ICU admission, bedside clinicians are able to establish the clinical pretest probability of PjP in patients with HMs and ARF. The PASTEIL score has a very good discrimination that provides promising ways to avoid missing the diagnosis and delaying treatment. Interventional studies in high-risk patients are warranted (Fig. 11).

Competing interests None.

\section{8}

Bedside contribution of electrical impedance tomography (EIT) to set mechanical ventilation for severe acute respiratory distress syndrome (ARDS) on extracorporeal membrane oxygenation (ECMO)

Guillaume Franchineau', Nicolas Brechot ${ }^{2}$, Guillaume Lebreton ${ }^{3}$, Guillaume Hekimian ${ }^{2}$, Ania Nieszkowska ${ }^{4}$, Charles-Edouard Luyt ${ }^{5}$, Pascal Leprince ${ }^{6}$, Jean Louis Trouillet ${ }^{5}$, Alain Combes ${ }^{7}$, Matthieu Schmidt ${ }^{8}$

${ }^{1}$ Paris, Pitié-Salpêtrière Hospital, Paris, France; ${ }^{2}$ Medical intensive care unit, ican, institute of cardiometabolism and nutrition, Pitié-Salpêtrière Hospital, Paris, France; ${ }^{3}$ Cardiac and thoracic surgery, ican, institute of cardiometabolism and nutrition, Groupe Hospitalier Pitié Salpêtrière, Paris, France; ${ }^{4}$ Medical intensive care unit, ican, institute of cardiometabolism and nutrition, Hopital Pitie Salpetrière, Paris, France; ${ }^{5}$ Medical intensive care unit, ican, institute of cardiometabolism and nutrition, Groupe Hospitalier Pitié Salpêtrière, Paris, France; ${ }^{6} \mathrm{Cardiac}$ and thoracic surgery, ican, institute of cardiometabolism and nutrition, Hopital Pitie Salpetrière, Paris, France; ${ }^{7}$ Medical intensive care unit, ican, institute of cardiometabolism and nutrition, hôpital de la pi, Groupe Hospitalier Pitié Salpêtrière, Paris, France; ${ }^{8}$ Medical intensive care unit, ican, institute of cardiometabolism and nutrition, Groupe Hospitalier Pitié-Salpêtrière, Paris, France

Correspondence: Guillaume Franchineau - gfranchineau@gmail.com Annals of Intensive Care 2016, 6(Suppl 1):058

Introduction Extracorporeal membrane oxygenation (ECMO) has been proposed as a possible therapeutic option for patients with severe acute respiratory distress syndrome (ARDS) who have refractory hypoxemia or excessively high inspiratory airway pressures as well as being unable to tolerate volume and pressure limited strategy. This device also permits "ultraprotective" mechanical ventilation with further reduction in volume and pressure that might ultimately enhance lung protection and improve clinical outcomes of acute respiratory distress syndrome (ARDS) patients. In addition, high level of positive end-expiratory pressure (PEEP) during the first day on ECMO may be associated with better outcome. On the other hand, inappropriate high PEEP level may also lead to overdistension, hyperinflation and definitive lung damage. Because of these considerations, continuous monitoring of harmful effects of PEEP appears relevant. Considering the high severity of these patients, electrical impedance tomography (EIT) could allow an individual, noninvasive, real-time, bedside, radiation-free imaging of the lungs, with global and regional dynamic analysis.

The aims of this study were: (1) to evaluate the ability of EIT to monitor a PEEP trial in patients with severe ARDS on ECMO and ventilated with very low tidal volume and (2) to evaluate the benefits of EIT to provide tools to individualize and match PEEP with the regional distribution of ARDS lesions.

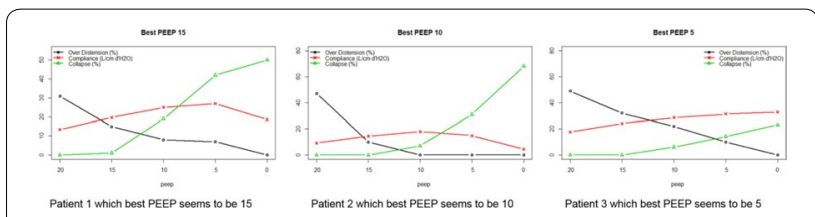

Fig. 12 See text for description

Patients and methods We conducted a monocentric observational prospective study. Inclusions criteria were: (1) sedated patients mechanically ventilated in pressure-controlled mode for severe ARDS that required veno-venous ECMO; (2) delay between ECMO initiation and inclusion $<7$ days. Exclusion criteria were manufacturer contraindications to use of a thoracic belt, cardiac pacing, pneumothorax or past history of barotrauma and hemodynamic instability. A decremental PEEP trial, using $5 \mathrm{cmH}_{2} \mathrm{O} 20$-min steps, was performed from 20 to $0 \mathrm{cmH}_{2} \mathrm{O}$. Driving pressure and respiratory frequency were maintained constant at $14 \mathrm{cmH}_{2} \mathrm{O}$ and $24 / \mathrm{min}$, respectively. At the end of each PEEP level, hemodynamic, pulmonary compliance, blood gases and EIT were recorded. Lung images were divided into four symmetrical frontal to dorsal region of interest (ROI). For each PEEP level, we recorded impedance variation $(\Delta z)$ in each $\mathrm{ROI}$, end-expiratory lung impedance (EELI) and estimation of the alveolar collapse (CL) and overdistension (OD) area.

Results We included 15 patients [age 57 (range 40-72), six males, SOFA score 15 (12-17)]. Origin of ARDS was viral pneumonia (46\%) and bacterial pneumonia for $20 \%$ of patients. Analysis was conducted after a median time of mechanical ventilation of $8(6-12)$ days and 4 (2-6) days of ECMO. Compared with PEEP 20, decremental PEEP trial led to a decrease in EELI of 14, 37, 60 and $96 \%$ at PEEP 15, 10, 5 and 0 , respectively. Regional analyses of EELI and $\Delta z$ illustrated that "ultraprotective" ventilation of the most dorsal region of the lungs did not participate in ventilation regardless the level of PEEP. However, midventral and mid-dorsal regional ventilations were more dependent on PEEP. Meanwhile, pulmonary compliance was 13.4, 18.7, 18.6, 18.7 and $12.1 \mathrm{~mL} / \mathrm{cmH}_{2} \mathrm{O}$ at the five decreasing PEEP levels, respectively, resulting to median tidal volumes ranging from 2.8 to $4 \mathrm{ml} / \mathrm{kg}$. Median overdistension decreased from 37 to $0 \%$ and collapsed area of the lung increased from 0 to $50 \%$ while decreasing PEEP level. Based on the best compromise between low overdistension and low collapse, EIT provided "best PEEP level" for each patient on ECMO. These results were variable, and depending on patients, optimal PEEP was between 5 and $15 \mathrm{cmH} 2 \mathrm{O}$ (see Fig. 12).

Conclusion Our study is the first using EIT on severe ARDS with ECMO. Our results indicate that EIT is a simple and feasible technique that could provide good assessment of regional changes during PEEP maneuvers, despite a low spatial resolution and very low tidal volumes. In our population, best PEEP level, which protects from both overdistension and collapse, did not exist. However, EIT may provide a tool to select PEEP level with the best compromise between overdistension and collapse. This level was very variable from patient to patient, illustrating the need to individualize mechanical ventilation settings based on monitoring the effects of PEEP.

Competing interests None.

059

Liberal oxygenation versus restrictive oxygenation in ICU patients: effects on proinflammatory cytokines, sRAGE and organ dysfunctions - results of an ancillary study

Loïc Barrot ${ }^{1}$, Gaël Piton ${ }^{2}$, Michael Bailey ${ }^{3}$, Rakshit Panwar ${ }^{3}$, Nicolas Belin ${ }^{1}$, François Belon, ', Cyrille Patry', Jean-Christophe Navellou', Mathilde Grandperrin', Claire Chaignat ', Guylaine Labro², Bérengère Vivet', Gilles Capellier

${ }^{1}$ Réanimation Médicale, CHU de Besançon, Besançon, France; ${ }^{2}$ Réanimation médicale, CHRU de Besançon, Besançon, France; ${ }^{3}$ Depm, monash university, ANZIC-RC, Melbourne, Australia 
Correspondence: Loïc Barrot - Ibarrot@chu-besancon.fr Annals of Intensive Care 2016, 6(Suppl 1):059

Introduction There is no clear recommendation on which level of oxygenation is better in intensive care patients. Oxygen $\left(\mathrm{O}_{2}\right)$ is known as a double-edged sword because of the risk of oxygen toxicity, especially in the lungs in balance with the risk of hypoxemia. In a previous multicenter international randomized study, we tested the safety and the feasibility of management of two levels of oxygenation in ICU patients with one group having oxygen saturation kept between 88 and $92 \%$ (conservative $=\mathrm{CO}$ ) and the other having oxygen saturation kept higher or equal to $96 \%$ (liberal = LO) (Am J Respir Crit Care Med 2015 150903080351007). Results of the pilot study confirm the feasibility and the safety of conducting a such study. Here, we report results of the French cohort with measurement of proinflammatory cytokines, soluble receptor for advanced glycation end products (sRAGE) a marker of pulmonary epithelial injury and markers of organ dysfunction.

Patients and methods This is an ancillary study of a multicenter, international, prospective, randomized study. Inclusion criteria were age higher or equal to 18 years, mechanical invasive ventilation begun not more than $24 \mathrm{~h}$, previsible length of ventilation higher than $48 \mathrm{~h}$. Exclusion criteria were pregnancy, legally incapacitated patient, lack of written consent of trusted person or next-of-kind, high risk of death during the next $72 \mathrm{~h}$ and clinician in charge of the patient refusal. The $\mathrm{FiO}_{2}$ was set to achieve targets of $88-92 \% \mathrm{SpO}_{2}$ for the $\mathrm{CO}$ group or $\geq 96 \% \mathrm{SpO}_{2}$ for $\mathrm{LO}$ group during mechanical ventilation. IL-1, IL-6, TNF-a, IL-12, IL-8 and sRAGE were dosed at days 0, 3 and 6. Troponin, catecholamines, lactates, creatininemia, platelets, fibrinogen, hemoglobin and CRP were measured at days $0,1,2,3,4,5,7$ and 10. Hemodynamic instability, new onset of ARDS and $\mathrm{PaO}_{2} / \mathrm{FiO}_{2}$ at day 7 was noticed in ICU and in the hospital, at day 90 and in ICU for mortality.

Results A total of 240 patients were screened. Twenty-two patients were recruited from June 2013 to October 2014. There were 11 patients per group. Patients characteristics were similar between the two groups except a greater number of patients with ARDS at the inclusion in $\mathrm{CO}$ group. The two groups had different mean $\mathrm{PaO}_{2}$ over time with $68 \mathrm{mmHg}$ [64-79] in CO group versus $89 \mathrm{mmHg}$ [84-106] in LO group. Variations of IL-1, IL-12 and TNF-a were not detectable.

IL-6 (pg/ml) was 129 [50-333] versus 50 [18-142] at day 0, 40 [14-109] versus 25 [8-78] at day 3, 20 [6-61] versus 21 [7-64] at day 6 in, respectively, liberal and conservative groups.

$\mathrm{IL}-8(\mathrm{pg} / \mathrm{ml})$ was $43[20-92]$ versus 36 [16-84] at day 0,18 [8-39] versus 2518 [8-44] at day 3, 14 [6-33] versus 19 [8-45] at day 6 in, respectively, liberal and conservative groups.

IL-10 (pg/ml) was 3.68 [1.08-4.46] versus $4.54[0.81-5.86]$ at day $0,2.38$ [0.68-3.19] versus $0.64[0.17-0.97]$ at day $3,1.79[0.35-4.04]$ versus 0.28 [0.14-0.77] at day 6 in, respectively, liberal and conservative group.

SRAGE $(\mathrm{pg} / \mathrm{ml})$ was $2336 \pm 305$ versus $2172 \pm 334$ at day $0,1756 \pm 316$ versus $2038 \pm 364$ at day 3, 2020 \pm 376 versus $1297 \pm 364$ at day 6 in, respectively, liberal and conservative groups.

All show significant variation over time. No significant variation overtime between the two groups was detected despite a tendency to a higher SRAGE elevation in liberal oxygenation group at day 6. There was no difference in mortality (in ICU, in hospital or at day 90). There was no difference in $\mathrm{PaO}_{2} / \mathrm{FiO}_{2}$ at day 7 , hemodynamic instability. More episodes of hypoxia were detected in the restrictive group as expected. There was no difference between the two groups over time of troponin level, catecholamine doses, creatininemia, platelet, fibrinogen, lactates and CRP. SOFA has nonsignificant tendency to be lower in the $\mathrm{CO}$ than in the LO group. There was a nonsignificant tendency for new onset of ARDS in LO group. Hemoglobin was lower in the CO group due to lower basal level.

Conclusion A liberal oxygenation strategy does not induce higher systemic inflammation neither other complication in comparison with a conservative oxygenation strategy in ICU patients. However, despite nonsignificant, SRAGE, a marker of epithelial pulmonary injury, has tendency to stay at high level at day 6 , there is tendency to have more ARDS, and SOFA score at day 6 has tendency to be higher in patients exposed to liberal oxygenation strategy in comparison with conservative oxygenation strategy. Further research has to be conducted to find the more safe level of oxygenation in ICU patients, especially for the protection of the lungs.

\section{Competing interests None.}

\section{References}

1. Panwar R, Hardie M, Bellomo R, et al.: Conservative Versus Liberal Oxygenation Targets for Mechanically Ventilated Patients - a Pilot Multicenter Randomized Controlled Trial. Am J Respir Crit Care Med 2015; 150903080351007

2. Kallet RH, Matthay MA: Hyperoxic Acute Lung Injury. Respir Care 2013; 58:123-141.

\section{0}

Immature granulocyte level at the acute phase of sepsis, a potential prognostic marker of clinical deterioration. The Septiflux 2 multicenter trial

Thomas Daix', Estelle Guérin ${ }^{2}$, Elsa Tavernier ${ }^{3}$, Emmanuelle Mercier $^{4}$, Valérie Gissot ${ }^{5}$, Christine Vallejo ${ }^{6}$, Jean-Paul Mira ${ }^{7}$, Christophe Guitton ${ }^{8}$, Bruno François ${ }^{9}$, Septiflux trial Group

${ }^{1}$ Inserm cic 1435/medical-surgical icu, Chu Limoges, Limoges, France; ${ }^{2}$ Hematology laboratory/umr cnrs 7276, Chu Limoges, Limoges, France; ${ }^{3}$ Inserm cic 1415, Chru Hôpitaux De Tours, Tours, France; ${ }^{4}$ Medical icu, Chru Hôpitaux De Tours, Tours, France; ${ }^{5}$ Emergency department/inserm cic 1415, Chru Hôpitaux De Tours, Tours, France; ${ }^{6}$ Emergency department, Chu Limoges, Limoges, France; ${ }^{7}$ Medical icu, Hôpital Cochin, Paris, France; ${ }^{8}$ Medical icu, C.H.U. Hôtel Dieu, Nantes, France; ${ }^{9}$ Inserm cic $1435 /$ medical-surgical icu/umr 1095, Chu Limoges, Limoges, France

Correspondence:Thomas Daix - thomas.daix@wanadoo.fr

Annals of Intensive Care 2016, 6(Suppl 1):060

Introduction Sepsis remains a major cause of death for intensive care unit (ICU) patients. The numerous experimental data published contrast with the few biological data (mainly neutrophil and platelet count) used by physicians at patients' bedside in such circumstances. Since there are no specific predictive clinical signs in sepsis, it would be helpful to have a biological tool to predict the progression of the disease but also to understand the pathophysiology and especially the immune status of the patients. Given the great worsening potential using a prognostic marker to early identify patients at risk of clinical deterioration could enable a more specific management. In that way, "Septiflux 1," a single-center trial (Guerin et al., Crit Care Med, 2014) on "selected septic patients," demonstrated that immature granulocyte CD10dim/CD16dim level at acute phase of sepsis can predict early clinical deterioration. We wanted to confirm these promising results in a large multicenter trial. The main objective was to evaluate the ability to predict early clinical worsening of immature granulocytes CD10dim/ CD16dim assessed by flow cytometry at the acute phase of sepsis. Secondary objectives were to appraise the capacity of other leukocyte subsets to predict clinical deterioration at D-2 and D-28 death.

Patients and methods We designed a multicenter prospective observational study. Patients hospitalized in the emergency department (ED) or in the ICU with SIRS and a clinically or microbiologically documented bacterial infection developing for $<24 \mathrm{~h}$ were eligible. Exclusion criteria were pregnancy, progressive neoplastic disease, HIV history of hematological or inflammatory disease, immunosuppressive treatment and hospitalization in ICU for more than $48 \mathrm{~h}$. Within $8 \mathrm{~h}$ after admission, flow cytometry analysis was performed to evaluate immature granulocytes CD10dim/CD16dim but also T-lymphocytes CD3pos, expression of CD24, CD64 and CD11b on granulocytes, and pro-inflammatory monocytes CD16pos. As regards their organ failure, all patients were classified as sepsis, severe sepsis and septic shock using the international sepsis classification. Sepsis deterioration was evaluated $48 \mathrm{~h}$ after inclusion and was defined as followed: sepsis developing to severe sepsis or septic shock; severe sepsis developing to septic shock. Death occurring before $48 \mathrm{~h}$ was also considered as clinical deterioration. Patients were followed until D-28. SOFA score was also calculated at inclusion and after $48 \mathrm{~h}$.

Results From November 2013 to June 2015, at 11 university sites, 1062 patients were included in this trial. Recruitment has now ended and 
780 patients have been kept for final analysis, the remaining being secondary excluded mainly because sepsis was not confirmed by the adjudication committee. At inclusion, 523 (67 \%) were hospitalized in the ICU and 257 were from the ED. All the sites have performed the related flow cytometry according to a standardized protocol. The database cleaning process and locking should be done in the coming weeks followed by the statistical analysis. Results will be available in the coming 2 months.

Conclusion Septiflux 2 is the larger multicenter trial that has evaluated the prognostic value of immature granulocytes and the overall interest of flow cytometry at the acute phase of sepsis.

\section{Competing interests None.}

\section{Reference}

1. Guérin E, Orabona M, Raquil MA, et al. Circulating immature granulocytes with T cell killing functions predict sepsis deterioration. Crit Care Med 2014:42(9):2007-18

\section{1}

\section{Analysis of microbiome and resistome using next-generation sequencing in urine samples from patients with sepsis, severe sepsis or septic shock}

Céline Ravry', Bruno François ${ }^{2}$, Emmanuelle Begot ${ }^{3}$, Nicolas Pichon ${ }^{3}$, Catherine Chapellas ${ }^{3}$, Anne-Laure Fedou ${ }^{3}$, Antoine Galy ${ }^{3}$, Marie-Cécile Ploy $^{4}$, Olivier Barraud ${ }^{4}$, Philippe Vignon

${ }^{1}$ Service de Réanimation Polyvalente/Inserm UMR-S 1092/Faculté de Médecine, Centre Hospitalier Universitaire de Limoges, Limoges, France; ${ }^{2}$ Service de Réanimation Polyvalente/Inserm CIC 1435/Inserm UMR-S 1092, Centre Hospitalier Universitaire de Limoges, Limoges, France;

${ }^{3}$ Service de Réanimation Polyvalente, Centre Hospitalier Universitaire de Limoges, Limoges, France; ${ }^{4}$ Inserm UMR-S 1092/Faculté de Médecine/ Laboratoire de Bactériologie, Centre Hospitalier Universitaire de Limoges, Limoges, France

Correspondence: Philippe Vignon - sarah.demai@chu-limoges.fr Annals of Intensive Care 2016, 6(Suppl 1):061

Introduction Early and appropriate antibiotic therapy is a prognostic factor in patients presenting with severe sepsis and septic shock. Incidence of multidrug-resistant bacteria (MRB), especially Gram-negative bacilli (GNB), continuously increases. Next-generation sequencing (NGS) is a powerful technique that enables sequencing the complete genome of bacteria, thus giving access to their precise identification and potential antibiotic resistance genes. However, this technique is currently too cumbersome and expensive to be implemented in routine practice. Accordingly, the feasibility of NGS has been scarcely assessed on biological samples obtained from patients with sepsis. This study aimed at evaluating the feasibility and the diagnostic capacity of NGS in patients with urosepsis using the detection of bacterial microbiome and resistome from urine samples. Results were compared with those of conventional microbiology.

Patients and methods In this pilot study, we analyzed the urine samples of 40 patients with urinary sepsis $(n=17)$, severe sepsis $(n=8)$ or septic choc $(n=15)$ secondary to upper tract urinary infection (17 men; mean age: 71 years). Conventional urine culture was either positive with a single isolated GNB $(n=27)$ or two GNB $(n=6)$, being MRB or not, positive with three or more bacteria $(n=3)$, or negative $(n=4)$. After total DNA extraction and partial depletion of human DNA, prokaryotic DNA from urine samples was sequenced using lonProton $^{\mathrm{TM}}$ technology. The bioinformatic analysis was conducted with the Geneious ${ }^{\circledR}$ software (microbiome) and the Web site of the Center for Genomic Epidemiology (microbiome and resistome). All NGS analyses and their final interpretation were performed by a medical fellow who had received personalized training prior to the study. NGS results were compared to those of conventional culture. Initially, microbiological results from conventional culture were known for comparison with NGS data (Group 1;n=21). Subsequently, the medical fellow who performed NGS analysis was blinded from the results of conventional cultures (Group 2; $n=19$ ).

Results For the microbiome (Table 6), NGS showed a total concordance for the detection of bacterial species (mainly Escherichia coli), in
Table 6 See text for description

\begin{tabular}{lll}
\hline & $\begin{array}{c}\text { Conventional } \\
\text { culture }\end{array}$ & NGS results \\
\hline $\begin{array}{l}\text { Urine culture with one } \\
\text { GNB }\end{array}$ & 27 & $\begin{array}{c}27 \text { (including four with one } \\
\text { supplementary bacterial } \\
\text { species) }\end{array}$ \\
$\begin{array}{l}\text { Urine culture with two } \\
\text { GNB }\end{array}$ & 6 & $\begin{array}{l}\text { (and four with only one } \\
\text { GNB) }\end{array}$ \\
$\begin{array}{l}\text { Urine culture with one } \\
\text { GNB and one Gram } \\
\text { positive }\end{array}$ & 2 & 0 (two with only GNB) \\
$\begin{array}{l}\text { Urine culture with three } \\
\text { or more bacteria }\end{array}$ & 1 & 0 (one GNB only) \\
Negative urine culture & 4 & (two with one GNB, one \\
& & $\begin{array}{l}\text { with Actinobaculum schaalii, } \\
\text { one with anaerobes) }\end{array}$
\end{tabular}

samples with single isolated bacteria. NGS enabled the identification of bacteria potentially responsible for urosepsis in three out of four urine samples with negative conventional culture. For the resistome, NGS enabled the identification of all genes explaining the acquired resistance phenotypes $(n=18)$ and especially all $c t x-m$ genes which give MRB phenotype to bacteria (ESBL, $n=11$ ). No relevant difference in NGS diagnostic capacity was observed between groups. However, the study showed that a time-consuming in-depth analysis was still required.

Conclusion NGS performed on urinary samples from patients with urosepsis appears feasible and provides concordant information with that obtained from conventional culture regarding both the identification of bacteria and associated profiles of antibiotic resistance. Even if the technical requirements, duration of computerized analysis and costs currently preclude its clinical implementation, NGS promises in the near future to provide accurate results faster than conventional bacterial culture to early guide initial empirical antibiotic therapy in patients with urosepsis.

\section{Competing interests None.}

062

Patients metabolomic profiles at intensive care admission

Aurelie Thooft ${ }^{1}$, Raphael Conotte ${ }^{2}$, Jean-Marie Colet ${ }^{2}$, Patrick Biston ${ }^{3}$

Michael Piagnerelli ${ }^{3}$

${ }^{1}$ Réanimation polyvalente, C.H.U. De Charleroi, Charleroi, Belgium; ${ }^{2}$ Laboratoire de biologie humaine et toxicologie, Université de Mons - Campus

Plaine de Nimy, Mons, Belgium; ${ }^{3}$ Réanimation polyvalente, Hôpital Civil

Charleroi, Charleroi, Belgium

Correspondence: Aurelie Thooft - athooft@ulb.ac.be

Annals of Intensive Care 2016, 6(Suppl 1):062

Introduction In stress situation, inflammation causes several metabolic modifications. To give energetic substrate to vital tissues, an activation of proteolysis, lipolysis and hepatic gluconeogenesis occurs and, at cell level, the main energy source comes from anaerobic reactions. The metabolomic analysis is an approach that enables to study many products from metabolic pathways [1]. We aimed to study metabolic profile of patients admitted in intensive care unit (ICU) to identify potential biomarkers of patients' outcome.

Patients and methods This is a prospective study in a medico-surgical ICU. We included adult patients admitted in ICU for $<24 \mathrm{~h}$ and healthy volunteers. At admission, the sera of the patients were analysed by $1 \mathrm{H}$ nuclear magnetic resonance. We first compared patients and volunteers by multivariate analysis. The patients were divided into four groups based on their diseases: sepsis, septic shock, shocks from other origins than sepsis and other pathologies. We realized a Kruskal-Wallis test followed by Dunn correction to analyse the metabolites between 
the different groups. After identification of relevant metabolites, we search correlations between these and the mortality and the length of stay.

Results A total of 117 consecutive patients were screened, of whom 111 were included in the study after informed consent signature. Mean age was $67 \pm 14$, mean SOFA score was $5 \pm 3$, and mean APACHE II score was $20 \pm 9$. The 90 -day mortality was $30 \%$. We first identified a significant difference in metabolic profile between volunteers and ICU patients (CV-ANOVA, $p<0.001)$. When septic shock patients were compared with non-septic patients, some significant differences in the metabolome were observed $(p<0.05)$ : pyruvate, lactate, carnitine, creatine, myo-inositol, creatinine, urea, phenylalanine and mannitol. Despite all these changes, there was no correlation between metabolomic profile and patients prognosis.

Discussion These metabolic differences indicate a profound change in energetic metabolism in septic shock patients. The high ratio lactate/ pyruvate indicates a trend towards anaerobic glycolysis. The lactate increasing comes from hypoxic area and can serve for hepatic gluconeogenesis via Cori cycle. The increase of carnitine reflects the free fatty acids oxidation to be used as energy source. A reflect of proteolysis is observed by the rise of phenylalanine. The amino acids are used in the Krebs cycle or are degraded in urea, which is also increased in our study. We also observed a rise of creatine-energetic substrate of muscles-and its degradation product, creatinine. The myo-inositol increase reflects an activation of intra-cellular messages. Finally, the mannitol, which does not come from human metabolism, could perhaps reflect bacterial translocation.

Conclusion Metabolomic approach is a new interesting way to characterize metabolic changes in ICU patients at their admission. In the group of septic shock, we observed an increase in anaerobic glycolysis, proteolysis, lipolysis and gluconeogenesis. Nevertheless, the differences are not correlated with prognosis.

Competing interests None.

\section{3}

Lymphocyte phenotype during severe sepsis and septic shock

Matthieu Le Dorze ${ }^{1}$, Virginie Tarazona, ${ }^{1}$, Caren Brumpt, ${ }^{2}$, Hélène

Moins-Teisserenc, ${ }^{3}$, Anne-Claire Lukaszewicz', Didier Payen de la

Garanderie

${ }^{1}$ Anesthesiology and critical care, Hospital Lariboisière, Paris, France;

${ }^{2}$ Hematology, Hospital Lariboisière, Paris, France; ${ }^{3}$ Immunology, Hôpital

Saint Louis, Paris, France

Correspondence: Matthieu Le Dorze - mledorze@yahoo.fr

Annals of Intensive Care 2016, 6(Suppl 1):063

Introduction Two major immuno-inflammatory phases are well documented during sepsis: an early active inflammatory phase and a deactivated inflammatory phase leading to immunosuppression. Early death ( $<7$ days) and late death ( $>7$ days) must be then distinguished. The immune deficiency concerned both innate and adaptive system and represents a potential indication for immunostimulation therapy (IFN- $\gamma$, IL-7, PD-1 antagonists, etc.). Finding immune parameters to be used as a monitoring of inflammatory status becomes a major goal for research. Lymphocyte as a source of mediators for innate immunity has a phenotype in severe septic patients that is not well characterized. The objective was to describe and compare lymphocyte phenotypes to healthy volunteers, measured early ( $<7$ days) in the course of severe sepsis with or without shock, and to study the relationship of this phenotype with prognosis.

Patients and methods We conducted a monocenter prospective observational study. Patients and groups: Severe Sepsis Group = severe sepsis \pm septic shock; Control group = healthy volunteers. Measurements at D2 (D1-D3): demographic, clinical severity, mortality, blood cell count, monocytic HLA-DR expression, lymphocyte phenotype (flow cytometry, CD3+, CD3+CD4+, CD3+CD8+, CD19+, CD16+CD56+).

Results Forty-eight healthy volunteers were in control group: $24 \mathrm{M} / 24 \mathrm{~F}$ and aged $42(31-54)$ years. Sixty-seven patients were in severe sepsis group: $39 \mathrm{M} / 28 \mathrm{~F}$ and aged $69(52-85)$ years. Charlson, SOFA and IGS2 scores were $5(2-7), 8(5-13)$ and $52(38-67)$, respectively; sites of
Table 7 Lymphocyte phenotype and mHLA-DR expression [values are expressed as median (first quartile-third quartile); Student's $t$ test]

\begin{tabular}{|c|c|c|c|}
\hline & $\begin{array}{l}\text { Severe sepsis } \\
\text { group }(n=67)\end{array}$ & $\begin{array}{l}\text { Control group } \\
(n=48)\end{array}$ & $p$ \\
\hline \multicolumn{4}{|c|}{ Lymphocyte phenotype and mHLA-DR expression } \\
\hline $\begin{array}{l}\text { Lymphocytes } \\
\qquad\left(/ \mathrm{mm}^{3}\right)\end{array}$ & $1000(600-1400)$ & $1586(1372-1991)$ & 0.85 \\
\hline $\mathrm{CD} 3+\left(/ \mathrm{mm}^{3}\right)$ & $535(251-832)$ & $1182(949-1523)$ & $<0.0001$ \\
\hline $\begin{array}{l}\text { CD3+CD4+ } \\
\left(/ \mathrm{mm}^{3}\right)\end{array}$ & $324(155-566)$ & $685(595-1034)$ & $<0.0001$ \\
\hline $\begin{array}{l}\text { CD3+CD8+ } \\
\left(/ \mathrm{mm}^{3}\right)\end{array}$ & $170(93-290)$ & $432(300-508)$ & 0.0003 \\
\hline $\operatorname{CD} 19\left(/ \mathrm{mm}^{3}\right)$ & $149(78-263)$ & $141(94-192)$ & 0.36 \\
\hline $\mathrm{NK}\left(/ \mathrm{mm}^{3}\right)$ & $102(54-214)$ & $161(114-268)$ & 0.39 \\
\hline $\mathrm{mHLADR}(\mathrm{AB} / \mathrm{C})$ & $\begin{array}{l}5985(3674- \\
10367)\end{array}$ & $\begin{array}{l}39,970(35,198- \\
47,478)\end{array}$ & $<0.0001$ \\
\hline
\end{tabular}

sepsis origin are abdominal (33\%), lung ( $27 \%)$ and others ( $40 \%)$; early death was at J7 (28\%) and late death was at J7-J28 (20\%) (Table 7). Lymphocyte phenotype was not related to the severity scores (IGS2 SOFA). Lymphocyte phenotype was not related to early (<day 7$)$ and late death (day 28).

Conclusion T lymphopenia (whatever the sepsis severity) is associated with a decreased monocytic HLA-DR expression suggesting a synergistic change in both innate and adaptive immunity. This phenomenon can be physiologic, but could be a marker of immunosuppression associated to secondary infections. Monitoring mHLA-DR expression and lymphocyte phenotype over the course of sepsis may argue in favor of immunostimulation therapy, especially if secondary infections occurred.

\section{Competing interests None.}

\section{References}

1. Hotchkiss RS, Monneret G, Payen D. Nature Rev Immunol, 2013.

2. Boomer JS et al. JAMA, 2012.

064

Myeloid-derived suppressor cells expressing arginase-1 and IDO play a major role in immune dysfunction during septic shock Fabrice Uhel ${ }^{1}$, Imane Azzaoui, ${ }^{2}$, Murielle Gregoire ${ }^{3}$, Céline Pangault ${ }^{4}$, Joëlle Dulong, ${ }^{4}$, Luc Cynober, ${ }^{5}$, Jean-Marc Tadié ${ }^{1}$, Mikaël Roussel, ${ }^{4}$, Yves Le Tulzo ${ }^{1}$, Karin Tarte ${ }^{3}$

${ }^{1}$ Réanimation médicale, Centre Hospitalier Universitaire de Rennes, Rennes, France; ${ }^{2}$ Pôle biologie, Centre Hospitalier Universitaire de Rennes, Rennes, France; ${ }^{3}$ Biosit and inserm u917, faculte de medecine, universite rennes i, Centre Hospitalier Universitaire de Rennes, Rennes, France; ${ }^{4}$ Inserm u917, Faculté de Médecine de Rennes - Université Rennes 1, Rennes, France; ${ }^{5}$ Service de biochimie, AP-HP, Hôpital Cochin, Paris,

France

Correspondence: Fabrice Uhel - fabrice.uhel@chu-rennes.fr Annals of Intensive Care 2016, 6(Suppl 1):064

Introduction Patients who survive the early hours of sepsis develop an acquired immune dysfunction responsible for nosocomial infections and late mortality. Myeloid-derived suppressor cells (MDSCs), a heterogeneous population characterized by their ability to suppress T-cell responses, may play a major role. They have been described in numerous tumors as well as in inflammatory and infectious diseases. However, their phenotype and mechanism of action remain unclear. The objective of this study was to broadly assess the presence and mechanisms of suppression of MDSC subsets during septic shock. 
Patients and methods In a prospective cohort of patients with septic shock ( $n=35)$ and healthy controls $(n=26)$, peripheral blood myeloid cell subsets were quantified and phenotypically analyzed by multicolor flow cytometry.

The expression of 45 myeloid genes was studied by qRT-PCT on wholeblood RNA from septic patients $(n=29)$ and healthy controls $(n=15)$. Plasma levels of MDSC mediators were quantified by ELISA and highperformance liquid chromatography (HPLC) in 73 septic patients and 19 healthy controls. In vitro, CFSE-labeled peripheral blood mononuclear cells were depleted or not of CD14pos or CD15pos cells and stimulated with anti-CD3/CD28 antibodies; T-cell proliferation was assessed by CFSE dilution. Arginase and indoleamine 2,3-dioxygenase (IDO) activities were determined by HPLC.

Results We identified a suppressive phenotype in circulating myeloid cells from septic patients, characterized by an increase in circulating monocytic M-MDSC (CD14posHLA-DRlo/neg monocytes) count $(p<0.001)$ and in CD14negCD15pos low-density granulocytes identified as granulocytic G-MDSCs $(p<0.0001)$. Further phenotypic analyses showed that G-MDSCs include subsets of both immature granulocytes and mature granulocytes expressing high levels of degranulation markers. G-MDSCs were more specifically associated with occurrence of nosocomial infections.

A panel of genes involved in MDSC biology, including S100A8, S100A9, MMP8 and ARG1, was significantly upregulated in the blood of septic patients compared with healthy controls. Plasma levels of MDSC mediators S100A8/A9, S100A12, arginase 1 as well as IDO activity were significantly increased in septic patients compared with healthy controls $(p<0.001)$.

In vitro, restoration of T-cell proliferation after CD14pos and CD15pos depletion confirmed the suppressive properties of M- and G-MDSCs, respectively. Quantitative analysis of amino acids concentrations in culture supernatants revealed an arginase activity specifically burden by G-MDSCs and an IDO activity burden by both M- and G-MDSCs.

Conclusion In patients with septic shock, M-MDSC and G-MDSC counts are increased and promote the occurrence of nosocomial infections. Those subsets bear IDO and arginase activities and exert a suppressive activity on T-cell proliferation. These new insights highlight the complex mechanisms underlying immune dysfunction in septic patients and could promote future therapeutic approaches.

This work was supported by the 2012 experimental research grant from French Intensive Care Society.

\section{Competing interests None.}

\section{5}

\section{Anti-neutrophil cytoplasmic antibody (ANCA)-associated} vasculitis admitted to the intensive care unit: a multicenter study Julien Demiselle, ${ }^{1}$, Johann Auchabie, ${ }^{2}$, Julie Boisramé-Helms ${ }^{3}$, Pierre François Dequin ${ }^{4}$, Damien Du Cheyron ${ }^{5}$, Michaël Darmon ${ }^{6}$, Nicolas Chudeau $^{7}$, Guillaume Geri ${ }^{8}$, René Robert ${ }^{9}$, François Fourrier ${ }^{10}$, Steven Grange $^{11}$, Christophe Guitton ${ }^{12}$, Lise Piquilloud ${ }^{33}$, Alexandre Lautrette ${ }^{14}$, Sonia Boyer ${ }^{15}$, Emmanuel Guérot $^{16}$, Julien Letheulle ${ }^{17}$, Nicolas Lerolle ${ }^{18}$ ${ }^{1}$ Service de néphrologie et transplantation rénale, Centre Hospitalier Universitaire d'Angers, Angers, France; ${ }^{2}$ Département de réanimation médicale et médecine hyperbare, Centre Hospitalier Universitaire d'Angers, Angers, France; ${ }^{3}$ Réanimation, CHU de Strasbourg, Strasbourg, France; ${ }^{4}$ Réanimation polyvalente, CHRU Hôpitaux de Tours, Tours, France; ${ }^{5}$ Réanimation médicale, Centre Hospitalier Universitaire de Caen, Caen, France; ${ }^{6}$ Réanimation Médicale, CHU Saint-Etienne - Hôpital Nord, Saint-Étienne, France; ${ }^{7}$ Centre hospitalier universitaire d'angers, Service de Réanimation Médicale et Médecine Hyperbare, Angers, France; ${ }^{8}$ Réanimation Médicale, Hôpital Cochin, Paris, France; ${ }^{9}$ Réanimation médicale, $\mathrm{CHU}$ de Poitiers, Poitiers, France; ${ }^{10}$ Réanimation, centre de réanimation, Lille, France; ${ }^{11}$ Réanimation médicale, Centre Hospitalier Universitaire Rouen, Rouen, France; ${ }^{12}$ Réanimation médicale, C.H.U. Hôtel Dieu, Nantes, France; ${ }^{13}$ Service de médecine intensive adulte et centre des brûlés, CHUV|Centre hospitalier universitaire vaudois, Lausanne, Switzerland; ${ }^{14}$ Réanimation médicale, CHU Gabriel-Montpied, Clermont-Ferrand, France; ${ }^{15}$ Service de réanimation médicale, CHU Nice, Nice, France; ${ }^{16}$ Réanimation médicale, Hopital Europeen Georges-Pompidou, Paris, France; ${ }^{17}$ Réanimation médicale, Centre Hospitalier Universitaire de Rennes, Rennes, France; ${ }^{18}$ Réanimation médicale, Centre Hospitalier Universitaire d'Angers, Angers, France

Correspondence: Nicolas Lerolle - nicolas.lerolle@univ-angers.fr Annals of Intensive Care 2016, 6(Suppl 1):065

Introduction Data regarding patients with the most severe manifestations of ANCA-associated vasculitis admitted to intensive care unit are scarce. The objective of the present study was to analyze the main features of ANCA-associated vasculitis patients admitted to intensive care unit for vasculitis activity and to study long-term outcome.

Patients and methods We analyzed retrospectively consecutive adult ANCA-associated vasculitis patients from 17 intensive care units admitted for active disease over a 10-year period (2002-2012). This intensive care group was compared to a group of ANCA-associated vasculitis patients admitted to a nephrology department within the same period. ANCA-associated vasculitis patients admitted to intensive care unit or nephrology department for other reasons than disease activity (i.e., complication of treatments such as sepsis) were excluded.

Results A total of 88 patients were included in the intensive care group and 55 patients in the non-intensive care group. The mean age of patients of the intensive care group was $59 \pm 16.1$ years, 54 patients had granulomatosis with polyangiitis (61\%), 32 had micropolyangiitis $(36.4 \%)$, and two had eosinophilic granulomatosis with polyangiitis (2\%). Proteinase-3 ANCAs were predominant (60\%). Seventy-four patients had newly diagnosed ANCA-associated vasculitis, and 14 were cared for a relapse. When compared to the non-intensive care group, the intensive care group had a higher Birmingham Vasculitis Activity Score $(23 \pm 8$ vs $16 \pm 4, p<0.01)$ and a higher frequency of lung (diffuse alveolar hemorrhage in 64 vs $11 \%, p<0.001$ ), heart, ear-nose-throat and central nervous system involvement. Respiratory assistance, renal replacement therapy and vasopressor amines were used in 72,54 and $27 \%$ of intensive care patients, respectively. Renal replacement therapy was required in $44 \%$ of the non-intensive group patients ( $p=0.254$ for comparison with intensive care patients). In the intensive care group, all patients were treated with steroids, $86 \%$ received cyclophosphamide, and $51 \%$ had plasma exchanges as remission induction regimen. Rituximab was used in $3 \%$ in the intensive care group. Fourteen patients (15.9\%) died during intensive care stay. The need for mechanical ventilation, the use of vasopressors and the occurrence of at least one infectious event were significantly associated with intensive care mortality in univariate analysis. The use of plasma exchange was not significantly associated with mortality change (OR 0.69 [0.24-1.98]). After adjustment, only the occurrence of an infectious event was associated with intensive care mortality in the multivariate analysis (OR 11 [1.8-64.2] $p<0.01$ ). Despite a higher in-hospital mortality rate ( 19.3 vs $3.6 \%, p=0.007$ ), the long-term mortality of the intensive care group was not different from that of the non-intensive care group (respectively, 1-year mortality, 20.4 vs $17.0 \%$, $p=0.372$, and see Fig. 13).

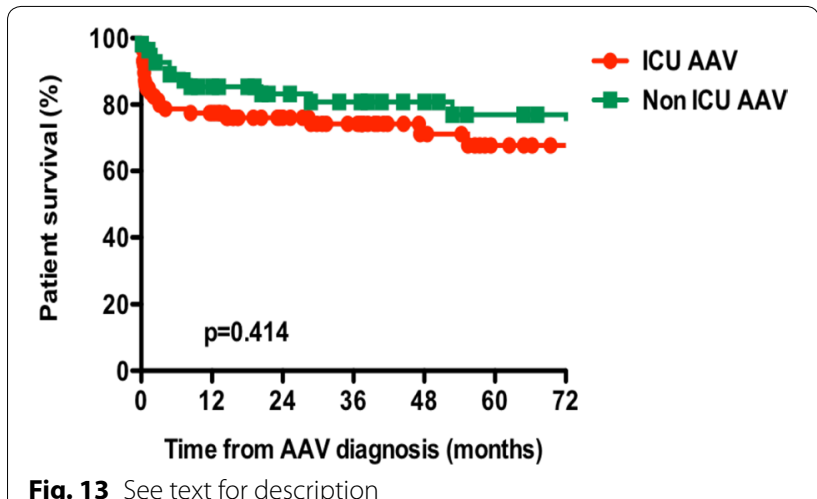


Conclusion This study represents the largest cohort of ANCA-associated vasculitis patients with active disease requiring intensive care. Even if respiratory failure appears as the main cause of intensive care admission, we identified sepsis as the main risk factor for intensive care mortality. Our results suggest that surviving patients have a longterm prognosis that is not significantly different from patients whose disease does not require intensive care admission.

\section{Competing interests None.}

\section{6}

Continuous renal replacement therapy versus intermittent hemodialysis: impact on mortality and renal recovery

Anne Sophie Truche ${ }^{1}$, Michaël Darmon ${ }^{2}$, Sébastien Bailly ${ }^{3}$, Christophe Clec' $^{\prime}{ }^{4}$, Claire Dupuis ${ }^{1}$, Benoit Misset ${ }^{5}$, Elie Azoulay ${ }^{6}$, Carole Schwebel ${ }^{7}$ Lila Bouadma ${ }^{8}$, Christophe Adrie ${ }^{9}$, Guillaume Marcotte ${ }^{10}$, Philippe Zaoui ${ }^{11}$, Virginie Laurent ${ }^{12}$, Dany Goldgran Toledano ${ }^{13}$, Romain Sonneville ${ }^{14}$, Bertrand Souweine ${ }^{15}$, Jean-François Timsit ${ }^{16}$

${ }^{1} U$ mr 1137 iame equipe 5 descid, Université Paris Diderot, Paris, France; ${ }^{2}$ Réanimation Médicale, CHU Saint-Etienne - Hôpital Nord, Saint-Étienne, France; ${ }^{3}$ Equipe 11, Institut Albert Bonniot - Inserm U823, La Tronche, France; ${ }^{4}$ Réanimation medico-chirurgicale, hopital avicenne, Bobigny, France; ${ }^{5}$ Réanimation polyvalente, Groupe Hospitalier Paris-Saint-Joseph, Paris, France; ${ }^{6}$ Réanimation médicale, Hôpital Saint-Louis, Paris, France; ${ }^{7}$ Réanimation médicale, C.H.U. Grenoble, Grenoble, France; ${ }^{8}$ Réanimation médicale et des maladies infectieuses, Hôpital Bichat-Claude Bernard, Paris, France; ${ }^{9}$ Physiology department, C.H.U Cochin, Paris, France; ${ }^{10}$ Reanimation medicale, Assistance Publique Hôpitaux de Paris, Paris, France; ${ }^{11}$ Nephrologie, C.H.U Grenoble, La Tronche, France; ${ }^{12}$ Réanimation médicale, Hospital Center De Versailles, Le Chesnay, France; ${ }^{13}$ Réanimation polyvalente, Centre Hospitalier Général, Gonesse, France; ${ }^{14}$ Service de réanimation médicale et infectieuse, Hôpital Bichat-Claude Bernard-APHP, Paris, France; ${ }^{15}$ Réanimation médicale, CHU Gabriel-Montpied, Clermont-Ferrand, France; ${ }^{16}$ Réanimation médicale et infectieuse, Hôpital Bichat-Claude Bernard, Paris, France

Correspondence: Anne Sophie Truche - astruche@chu-grenoble.fr Annals of Intensive Care 2016, 6(Suppl 1):066

Introduction The optimal initial modality of renal replacement therapy remains controversial. Available literature is limited by several biases such as lack of statistical power, the absence of standardization of renal replacement therapy initiation criterion, lack of information concerning dialysis dose and a high rate of crossover between modalities. Moreover, some area of uncertainties remains including optimal modality of renal replacement therapy during shock and their impact on long-term renal outcome. Additional studies including a sufficient number of patients were necessary.

The purpose of our study was to compare the prognostic impact on both techniques, adjusting for the probability of being treated by continuous renal replacement therapy or intermittent hemodialysis a particular day.

Patients and methods Patients of a French prospective high-quality observational multicenter cohort were included if they underwent at least one renal replacement therapy session between 2004 and 2014. The day of renal replacement therapy initiation for a patient was his study inclusion day.

Baseline and daily patients' characteristics differences between treatment groups were taken into account by using a marginal structural Cox model, allowing drawing a causal conclusion in observational longitudinal data analysis. The composite primary endpoint was 30-day mortality and dialysis dependency. Following subgroup analyses were planned in the experimental design: chronic renal or heart disease; liver cirrhosis; diabetes; hypertension; hemodynamic status at renal replacement therapy initiation defined according to SOFA hemodynamic component (strictly inferior to 3 , and equal or above 3 ); invasive mechanical ventilation at inclusion; and extent of daily weight gain between intensive care unit admission and inclusion, defined as the upper quartile of the study population.

Secondary objective was to assess 6-month prognosis in term of mortality and persistent renal dysfunction by a logistic model with inverse probability of treatment weighting, considering the treatment most received in the first 7 days.

Results Among 18,834 patients of the cohort 1360 were included, with, respectively, $544(40.0 \%)$ and $816(60.0 \%)$ patients initially treated by continuous therapies and intermittent hemodialysis. Median age was 65 [interquartile range: 54; 76], and $204(15.0 \%)$ patients suffered from chronic kidney disease, 235 (17.3\%) from chronic heart failure and $327(24.0 \%)$ from diabetes. A medical condition was present among $77.3 \%$ of patients. Median initial SOFA score was 10 [interquartile range: $7 ; 13$ ]. Invasive mechanical ventilation was required at renal replacement therapy initiation in $930(68.4 \%)$ patients. Both therapies were administered in 265 (19.5\%) patients. At day 30, 539 (39.6\%) patients died. Among survivors, 150 (23.8\%) still required renal replacement therapy. There was no difference between initial modalities for the primary endpoint (HR 1.00; $95 \% \mathrm{Cl}$ $0.77-1.29 ; p=0.97$ ).

In patients with higher weight gain at renal replacement therapy initiation, mortality and dialysis dependency were significantly lower with continuous therapies (HR $0.54 ; 95 \% \mathrm{Cl} 0.29-0.99 ; p=0.05$ ). Conversely, this technique was deleterious in patients without hemodynamic instability (HR 2.24; $95 \% \mathrm{Cl} 1.24-4.04 ; p=0.01$ ). In patients with shock at inclusion, no improvement in prognosis was noted with continuous therapies.

Six-month mortality and persistent renal dysfunction were not influenced by initial renal replacement therapy modality (OR $0.81 ; 95 \% \mathrm{Cl}$ $0.45-1.46 ; p=0.48$ ).

Discussion The marginal structural model allowed inclusion of a large number of patients while minimizing biases resulting from our observational design. Hence, variables associated with choice of treatment and outcome was taken into account by this model. Switch between treatment modalities was considered by an as-treated analysis. Lastly, the model was adjusted for timing of renal replacement therapy initiation and dialysis dose.

Main limitation of the study is that even if all known confounding factors were introduced in the model, unknown confounding factors might not have been considered.

Conclusion Continuous renal replacement therapy did not improve 30-day and 6-month patients' prognosis. It seems beneficial for patients with fluid overload, but might be deleterious in the absence of hemodynamic failure.

\section{Competing interests None.}

\section{Reference}

1. Kidney Disease: Improving Global Outcomes (KDIGO) Acute Kidney Injury Work Group. KDIGO Clinical Practice Guideline for Acute Kidney Injury. Kidney inter., Suppl. 2012; 2: 1-138.

\section{7}

Hospital survival and need of renal replacement therapy with respect to AKI duration in critically ill patients: results of a multicenter cohort study

Sophie Perinel Ragey ${ }^{1}$, Anne Sophie Truche ${ }^{2}$, Bertrand Souweine ${ }^{3}$, Sébastien Bailly ${ }^{4}$, Elie Azoulay ${ }^{5}$, Lila Bouadma ${ }^{6}$, Christophe Clec'h ${ }^{7}$, Maïté Garrouste-Orgeas ${ }^{8}$, Antoine Gros ${ }^{9}$, Carole Schwebel ${ }^{10}$, Christophe Adrie ${ }^{11}$ Anne Sylvie Dumenil ${ }^{12}$, Laurent Argaud ${ }^{13}$, Samir Jamali ${ }^{14}$, Dany Goldgran Toledano $^{15}$, Guillaume Marcotte ${ }^{16}$, Michaël Darmon ${ }^{17}$, Jean-François Timsit $^{18}$, For the OUTCOMEREA study group

${ }^{1}$ Réanimation G, CHU Hôpital Nord, Saint-Priest-en-Jarez, France; ${ }^{2}$ Medical icu, Hopital Michallon, La Tronche, France; ${ }^{3}$ Réanimation médicale, CHU Gabriel-Montpied, Clermont-Ferrand, France; ${ }^{4}$ Equipe 11, Institut Albert Bonniot - Inserm U823, La Tronche, France; ${ }^{5}$ Réanimation médicale, Hôpital Saint-Louis, Paris, France; ${ }^{6}$ Réanimation médicale et des maladies infectieuses, Hôpital Bichat-Claude Bernard, Paris, France; ${ }^{7}$ Réanimation medico-chirurgicale, hopital avicenne, Bobigny, France; ${ }^{8}$ réanimation, Fondation Hopital Saint Joseph, Paris, France; ${ }^{9}$ Réanimation médico-chirurgicale, Centre Hospitalier de Versailles, Le Chesnay, France; ${ }^{10}$ Réanimation médicale, C.H.U. Grenoble, Grenoble, France; ${ }^{11}$ Explorations fonctionnelles, Cochin Port-Royal, Paris, France; ${ }^{12}$ Réanimation chirurgicale, Hôpital Antoine Béclère, Clamart, France; ${ }^{13}$ Réanimation Médicale, Hospices Civils de Lyon - Groupement Hospitalier Edouard Herriot, 
Lyon, France; ${ }^{14}$ Réanimation médicale, Centre Hospitalier Sud Essonne, Dourdan, France; ${ }^{15}$ Réanimation polyvalente, Centre Hospitalier Général, Gonesse, France; ${ }^{16}$ Reanimation medicale, Assistance Publique Hôpitaux de Paris, Paris, France; ${ }^{17}$ Réanimation Médicale, CHU Saint-Etienne - Hôpital Nord, Saint-Étienne, France; ${ }^{18}$ Réanimation médicale et infectieuse, Hôpital Bichat-Claude Bernard, Paris, France

Correspondence: Michaël Darmon - michael.darmon@chu-st-etienne.fr Annals of Intensive Care 2016, 6(Suppl 1):067

Introduction The classical dichotomy between functional acute kidney injury (AKI) and intrinsic AKI has recently been challenged. Thus, authors suggested that transient and persistent AKI in critically ill patients might share similar pathophysiological mechanisms. A recent study confirmed these findings [1]. However, this previous study suffers a lack of statistical power, and time dependency for renal recovery was unaccounted for.

The primary objective of our study was to assess prognostic impact of AKI duration. Secondary objective was to assess relationship between renal recovery at specific time frame and need for renal replacement therapy.

Patients and methods We performed a retrospective analysis of a large prospective multicenter cohort database involving 20 French ICUs. Every adult patient with AKI at ICU admission according to the KDIGO definition was included.

Renal recovery was defined by a decrease in one stage or more according to the KDIGO classification.

Results are reported as medians (interquartile range, IQR) or numbers (\%). Variable of interest was day-28 mortality, and influence of renal recovery was assessed after adjustment in a cause-specific hazard model taking time dependency of this variable into account. Discharge alive from the ICU was used as a competing event.

Results Overall, of 18,684 patients of our database, 5229 had an AKI at ICU admission and were included in this study. 3167 patients were of male gender (60.6\%), median age was of 70.5 [57.7-78.8], and initial severity assessed by SAPS 2 was of 51 [39-70]. Main reasons for ICU admission were shock (41.0\%) and acute respiratory failure (21.2 \%). AKI severity at admission according to KDIGO classification was stage 1 in 2453 patients $(46.9 \%)$, stage 2 in $1178(22.5 \%)$ and stage 3 in 1598 (30.6\%). According to our definition, renal recovery occurred in 65.7, 62.6 and $45.7 \%$ of patients with AKI stages 1,2 and 3, respectively. Day-28 mortality was, respectively, of $22.8,27.8$ and $26.3 \%$ for patients with AKI stages 1,2 and 3 . After adjustment for confounders, renal recovery was associated with a favorable outcome (cause-specific HR $0.53 ; 95 \% \mathrm{Cl} 0.45-0.62$ ). Results were unchanged when assessing influence of a renal recovery defined by renal recovery at day 3 .

In patients alive at day 8 , rate of renal replacement therapy in patients with persistent and transient AKI defined at various time frames is reported in Fig. 14.

Conclusion These data confirm short renal dysfunction reversibility to be potent prognostic marker in AKI patients and therefore to be clinically relevant in defining severity of AKI. Additionally, our results confirm a definition of persistent AKI based upon renal recovery between day 3 and day 7 to be potent predictor for need of RRT. Distinction

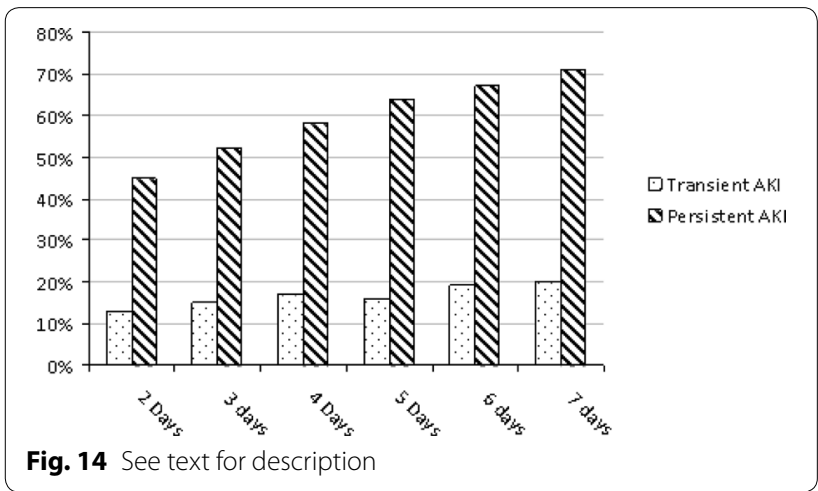

between transient and persistent AKI might therefore be clinically relevant surrogate outcome variable for diagnostic testing in critically ill patients with AKI.

\section{Competing interests None.}

\section{Reference}

1. Perinel et al. Crit Care Med 2015.

068

Early versus delayed initiation of renal replacement therapy in septic acute kidney injury: a retrospective study

Mikhael Chouraquii ', Antoine Dewitte ${ }^{1}$, Brigitte Chastel', Pauline Carles ${ }^{1}$, Catherine Fleureau', Olivier Joannes-Boyau', Alexandre Ouattara' ${ }^{1}$

${ }^{1}$ Réanimation polyvalente, service d'anesthésie-réanimation 2, CHU de Bordeaux, Maison du Haut Lévèque, Avenue de Magellan, Pessac, France Correspondence: Antoine Dewitte - antoine.dewitte@chu-bordeaux.fr Annals of Intensive Care 2016, 6(Suppl 1):068

Introduction Acute kidney injury (AKI) is a common complication in critically ill patients. Its incidence in septic shock is around $50 \%$ (1). Among patients with severe AKI, 50-70\% receives renal replacement therapy (RRT). However, the optimal timing of RRT initiation in patients with severe AKI remains controversial (2). This study compared the effect of early versus delayed RRT in patients with septic AKI.

Patients and methods This retrospective study included all patients hospitalized in our intensive care unit (ICU) between January 2008 and December 2013 for severe sepsis or septic shock complicated by AKI requiring RRT. Exclusion criteria were age $<18$ years, end-stage renal disease and RRT initiation before ICU admission. Reference creatinine was retrieved from blood samples at steady state during the year preceding admission. AKI was defined and classified according to the KDIGO criteria (both diuresis and serum creatinine). Early group was defined as an initiation of RRT within $12 \mathrm{H}$ after admission or AKI stages $0-1$ at initiation. Delayed group was defined as an initiation of RRT $>12 \mathrm{H}$ after admission and AKI stages 2-3 at initiation. The primary outcome was in-hospital mortality.

Results A total of 312 patients were treated by RRT during the study period. Two hundred and four patients suffered from severe sepsis or septic shock, and 169 patients were included in the study. In the early group $(n=79)$, SOFA score (12 vs $10, P=0.01)$ and serum creatinine level (174 $\mu \mathrm{mol} / \mathrm{L}$ [123-283] vs $154 \mu \mathrm{mol} / \mathrm{L}$ [102-229], $P=0.003)$ were higher at ICU admission. One hundred and fifty-eight (93\%) patients were receiving norepinephrine and mechanical ventilation when EER was initiated. The KDIGO score at RRT initiation was higher in the delayed group ( 3 vs $2 ; P<0.0001$ ) on both creatinine and diuresis criteria. The crude hospital mortality was $58 \%$ in the early group versus $54 \%$ in the delayed group $(P=0.6)$. A Cox regression model showed a better probability of survival adjusted to the SOFA score at ICU admission in the early group $(P=0.003$, Fig. 15$)$. Risk factors for mortality

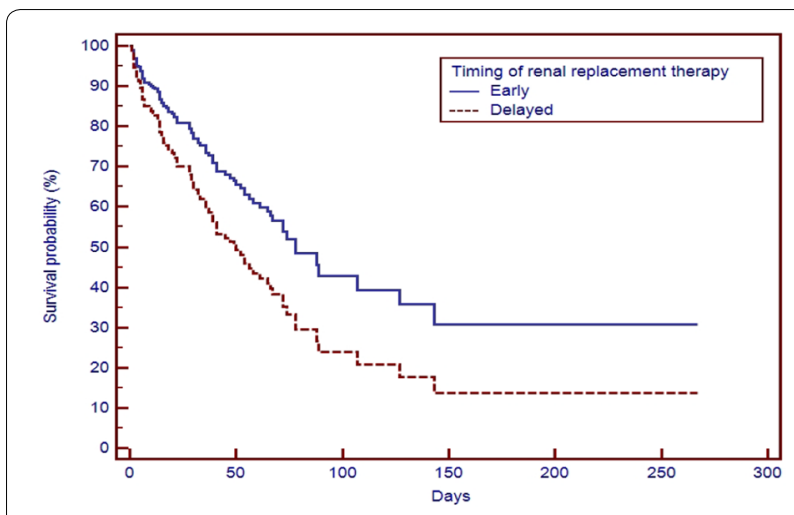

Fig. 15 See text for description 
analyzed by logistic regression were the $\mathrm{PaO}_{2} / \mathrm{FiO}_{2}$ ratio and lactate level at initiation of RRT. Survivors had a similar renal recovery in both groups, but the hospital length of stay was longer in the delayed group ( 51 vs 34 days, $P=0.05$ ).

Conclusion Our study reflecting our clinical practice reveals a better outcome when RRT is early initiated in ICU according to its timing after admission and AKI severity. The deleterious effects of fluid overload and hypoximia in case of AKI should be precise in further studies.

\section{Competing interests None.}

\section{References}

1. Quenot JP, Binquet C, Kara F, Martinet O, Ganster F, Navellou JC, Castelain V, Barraud D, Cousson J, Louis G, Perez P, Kuteifan K, Noirot A, Badie J, Mezher C, Lessire H, Pavon A: The epidemiology of septic shock in French intensive care units: the prospective multicenter cohort EPISS study. Crit Care 2013, 17:R65.

2. Karvellas CJ, Farhat MR, Sajjad I, Mogensen SS, Leung AA, Wald R, Bagshaw SM: A comparison of early versus late initiation of renal replacement therapy in critically ill patients with acute kidney injury: a systematic review and meta-analysis. Crit Care 2011, 15:R72.

\section{9}

Renal recovery after severe acute kidney injury in critically ill myeloma patients

Adrien Joseph ${ }^{1}$, Marion Venot ${ }^{1}$, Sandrine Valade ${ }^{1}$, Eric Mariotte ${ }^{1}$, Claire Pichereau', Akli Chermak², Virginie Lemiale', Elie Azoulay', Emmanuel Canet $^{1}$

${ }^{1}$ Réanimation médicale, Hôpital Saint Louis, Paris, France; ${ }^{2}$ Réanimation polyvalente, $\mathrm{CH}$ sud Essonne, Paris, France

Correspondence: Adrien Joseph - adrien.joseph@hotmail.fr

Annals of Intensive Care 2016, 6(Suppl 1):069

Introduction Despite substantial improvements in the management of multiple myeloma, renal failure remains an important burden which tremendously impairs prognosis. The purpose of this study was to describe the characteristics and prognostic factors for renal recovery in myeloma patients admitted to the intensive care unit (ICU) for acute kidney injury (AKI) stage 3 treated with renal replacement therapy (RRT).

Patients and methods A retrospective single-center cohort study was performed, including consecutive myeloma patients admitted to our ICU between 01/01/2007 and 01/09/2015 and treated with RRT. Data and follow-up were abstracted from the medical charts. Patients were evaluated 60 days after ICU discharge and divided into three groups: alive without dialysis, alive and dialysis-dependent or deceased. A univariate analysis was performed to identify factors associated with being alive without dialysis 60 days after ICU discharge.

Results Fifty-five patients were included in the study. Median age was 63 (inter-quartile range 58-70) years, and $33(60 \%)$ were male. Patients were admitted to the ICU $3(0-5)$ years after the diagnosis of myeloma, after $1(1-3)$ line of chemotherapy. Twenty-two had already been treated with autologous stem cell transplantation, at least once. The baseline renal function before ICU admission, estimated by the glomerular filtration rate (GFR), was $58(41-78) \mathrm{ml} / \mathrm{min}$. The median SOFA score at day 1 was 6 (4-8). The three main reasons for ICU admission were AKI $(33,60 \%)$, sepsis $(21,38 \%)$ and acute pulmonary edema (13, $55 \%)$. During ICU stay, 17 (31\%) patients required invasive mechanical ventilation, $13(27 \%)$ received vasopressors, and RRT was implemented in all patients (55, $100 \%$ ) during 13 (5-42) days. The median ICU and hospital length of stay were 13 (5-43) days and 24 (14-35) days, respectively. Forty (73\%) patients were alive at hospital discharge.

At day 60 after ICU discharge, $22(41 \%)$ patients were alive without dialysis, 19 (36\%) had died, and $12(23 \%)$ were still undergoing dialysis. Among the 22 patients who recovered, the median time of dialysis was $6(2-18)$ days. These patients recovered their previous renal function with a median GFR of $65(25-74) \mathrm{ml} / \mathrm{min}$ at day $60(p=0.70)$.

By univariate analysis, two factors were associated with a decreased likelihood of renal recovery at day 60: a history of autologous stem cell transplantation, odds ratio (OR) $0.27,95 \%$ confidence interval
(0.08-0.93), $p=0.04$, and a proteinuria at ICU admission $>380 \mathrm{mg} /$ mmol creatininuria, OR $0.22,95 \% \mathrm{Cl}(0.06-0.71), p=0.01$.

None of the other variables related to the hematological malignancy or to the ICU stay were associated with renal recovery at day 60 .

Discussion This study is the first to assess renal recovery in critically ill myeloma patients with severe AKI, in the era of bortezomib therapy. First of all, survival and renal recovery in our study are much higher than previously reported. About two-third of the patients were alive at day 60 , and $41 \%$ had recovered their baseline renal function. We identified two prognostic factors which can be considered as surrogates of more aggressive malignancies.

Conclusion AKI stage 3 in critically ill myeloma patients was associated with a hospital mortality $<30 \%$. The main cause of AKI is likely to influence the likelihood of renal recovery. Patients with more aggressive malignancies (history of autologous transplantation, higher proteinuria) had a poorer renal prognosis.

Competing interests None.

\section{0}

\section{Impact of proactive nurse participation in ICU family conferences:} a Mixed-Method Study

Maïté Garrouste-Orgeas ${ }^{1}$, Adeline Max², Talia Lerin ${ }^{3}$, Charles Grégoire², Stephane Ruckly ${ }^{4}$, Martin Kloeckner ${ }^{2}$, Cédric Bruel ${ }^{5}$, Sandie Brochon ${ }^{6}$, Francois Philippart ${ }^{2}$, Emmanuelle Pichot ${ }^{2}$, Clara Simons ${ }^{5}$, Jean-François Timsit ${ }^{7}$, Benoit Misset ${ }^{2}$

${ }^{1}$ réanimation, Fondation Hopital Saint Joseph, Paris, France; ${ }^{2}$ Réanimation polyvalente, Groupe Hospitalier Paris-Saint-Joseph, Paris, France; ${ }^{3} l l e$ de france, Hospital Group Paris Saint-Joseph, Paris, France; ${ }^{4}$ Statistical department, Outcomerea group, Paris, France; ${ }^{5}$ Réanimation, Hôpital Paris Saint-Joseph, Paris, France; ${ }^{6}$ Réanimation, Groupe Hospitalier Paris Saint-Joseph, Paris, France; ${ }^{7}$ Réanimation médicale et infectieuse, Hôpital Bichat-Claude Bernard, Paris, France

Correspondence: Maïté Garrouste-Orgeas - mgarrouste@hpsj.fr Annals of Intensive Care 2016, 6(Suppl 1):O70

Introduction Poor communication is associated with anxiety, distress and posttraumatic stress-related symptoms in families of both survivors and nonsurvivors. Few studies have assessed the impact on everyday medical decisions of better communication, most notably delivered by a multidisciplinary team. Our objectives are to investigate family perceptions of having a nurse participate in family conferences and to assess the psychological well-being of the same families after ICU discharge.

Patients and methods We designed a parallel-group randomized trial comparing family conferences with versus without the proactive participation of a nurse in family conferences. The study has a mixedmethod design with a qualitative study embedded in a single-center randomized study. We included one family member for each consecutive patient who received more than $48 \mathrm{~h}$ of mechanical ventilation in the ICU. We scheduled planned proactive participation of a nurse in family conferences led by a physician. In the control group, conferences were led by a physician without a nurse. We evaluated family conferences on days 1 and 3, weekly, during bad news or end-of-life. Audits by checklists were conducted for all conferences. All ICU physicians and nurses received training of communication by role players. Physicians and nurses used conferences guides created before the study. We used the NURSE acronym, the Ask-Tell-Ask concept and the VALUE strategy depending on the type of conferences. The primary outcome was the score on the Impact of Events Scale-revised (IES-R) (for posttraumatic stress-related symptoms) completed by the family member 3 months after the death or discharge of the patient. Secondary outcomes were symptoms of anxiety and depression in family members 3 months after patient death or ICU discharge. We conducted a qualitative evaluation of perceptions by family members of nurse participation in the family conferences at ICU discharge using interview guides. Each interview was transcribed verbatim and evaluated using interpretative phenomenological analysis.

Results Of the 172 eligible family members, 100 (60.2 \%) were randomized; among them, 88 underwent semi-structured interviews at ICU discharge and 86 completed the Peritraumatic Dissociative 
Experiences Questionnaire at ICU discharge and the Impact of Event Scale and Anxiety Depression Questionnaire 3 months later. The number of conferences was 188: 90 on day $1 ; 57$ on day $3 ; 24$ on day 7 and then weekly; and 17 for patients at the end-of-life. There were 96 conferences in the intervention group and 92 in the control group. The audits by checklists showed no significant between-group differences in conference contents on days 1, 3, 7 and then weekly or at the endof-life. Conference duration was about $30 \mathrm{~min}$ at all time points. Primary and secondary outcomes

The median [IQR] IES-R score was not significantly different between the control and intervention groups (24 [12.5-45] and 21 [9-23], respectively; $P=0.24$ ). IES-R scores indicated posttraumatic stressrelated symptoms in $52.2 \%$ and $50 \%$ of family members in the control and intervention groups, respectively $(P=0.83)$. Although the intervention group had significant lower anxiety (4 [1-9] vs 8 [4.5-12], $p=0.01)$ and depression (2 [0-6] vs $5.5[1-11.5], p=0.04)$ subscale scores, the prevalence of severe anxiety [14 (33.3\%) vs $23(52.3 \%)$, $p=0.08]$ and depression symptoms [10 (23.8\%) vs 17 (38.6\%), $p=0.14]$ (HADS subscale scores $>8$ ) did not differ significantly between the two groups.

Qualitative findings

The qualitative data indicated that the families valued the principle of the conference itself. Perceptions of nurse participation clustered into four main themes: trust that ICU teamwork was effective $(50 / 88$, $56.8 \%)$, trust that care was centered on the patient $(33 / 88,37.5 \%)$, trust in effective dissemination of information $(15 / 88,17 \%)$ and trust that every effort was made to relieve anxiety in family members $(12 / 88,13.6 \%)$. In conferences without nurse participation, two themes were identified: satisfaction with the information given by the physician alone $(15 / 45,33.3 \%)$ and satisfaction with the information given by the nurse in the patient's room $(7 / 45,15.5 \%)$.

Conclusion In conclusion, our study adds new insights about the role for repeated, routine, structured family conferences in the ICU. The families greatly appreciated the conferences themselves. Although the participation of a nurse in the conferences did not change significantly the post-ICU burden, it was considered positive by the family members. Our study offers some guidance on how to conduct family conferences in each specific clinical situation.

\section{Competing interests None.}

071

Duration of platelet storage and outcome of critically ill patients Andrew Flint', Cécile Aubron², Michael Bailey', Rinaldo Bellomo', David Pilcher ${ }^{3}$, Allen Cheng ${ }^{4}$, Colin Hegarty ${ }^{5}$, Anthony Martinelli ${ }^{5}$, Benjamin Howden ${ }^{6}$, Michael Reade 7 , Zoe Mcquilten ${ }^{8}$

${ }^{1}$ Depm, monash university, ANZIC-RC, Melbourne, Australia; ${ }^{2}$ Réanimation médicale, CHRU de Brest, La Cavale Blanche, Brest, France; ${ }^{3}$ Intensive care unit, Alfred Hospital, Melbourne, Australia; ${ }^{4}$ Depm, Monash University, Melbourne, Australia; ${ }^{5}$ The transfusion service, Austin Hospital, Heidelberg, Australia; Infectious diseases department, Austin Hospital, Heidelberg, Australia; ${ }^{7}$ Australian defense force and burns, trauma and critical care research center, University of Queensland, 4029, Australia, Brisbane, Australia; ${ }^{8}$ Depm, monash university, Transfusion Research Unit, Melbourne, Australia

Correspondence: Cécile Aubron - cecile.aubron@monash.edu Annals of Intensive Care 2016, 6(Suppl 1):071

Introduction Platelets (PLTs) can been stored up to 5-7 days depending on the country's transfusion policies. Changes in platelets structure and functional variables during the storage have been described in vitro. Although increase in duration of PLTs storage could improve PLTs availability in remote area and decrease waste of PLTs, information on the impact of platelets storage duration is limited and controversial. This study aims to determine whether the storage duration of PLTs transfused to critically ill patients is associated with patient outcomes.

Patients and methods We performed a retrospective analysis of patients admitted to two intensive care units (ICUs) in tertiary hospitals from 2008 to 2014. Comparison of outcomes of patients receiving only PLT with a maximum age higher than 5 days PLT versus patients receiving only PLT with a maximum age $<5$ days has been made. Outcome variables were hospital mortality and ICU-acquired infection (bacteraemia and/or bacteriuria). Associations between PLT age and outcomes were modelled using multiple logistic regression and linear regression.

Results Among 2250 ICU patients who received one or more PLTs, storage duration of all transfused PLTs was available for 1430 (64\%). These patients had a mean age of $57.5 \pm 18.1$ years, mean APACHE 3 score at admission was $65.8 \pm 29.0$, and $964(67.4 \%)$ were male. The median PLT age was 4 days (IQR 2, range 2-5). In-hospital mortality was $22.1 \%$, median ICU LOS in survivors 5.0 days (IQR 8.7) and infection rate $13.2 \%$. When comparing patients who received PLTs with a maximum age of 5 days with a maximum age $<5$ days, there were no significant differences in demographics, pre-ICU illness severity or number of transfused products. There were also no differences in mortality ( 21.6 vs. $22.8 \%, p=0.59$ ), bacteraemia (7.5 vs. $6.4 \%$, $p=0.44$ ) or LOS (median 4.9 vs. 5.1 days, $p=0.57$ ). After adjusting for confounders, age of PLTs was not independently associated with mortality (odds ratio [OR] 1.04, $95 \%$ confidence interval [CI] 0.78-1.40) or infection (OR 1.31, $95 \% \mathrm{Cl}$ 0.88-1.97). Similar results were found when considering separately bacteraemia and bacteriuria or when analysing the 639 patients who were transfused with only one PLT unit.

Conclusion In this large retrospective study, storage duration of PLTs is not associated with an increased risk of mortality or infection in critically ill patients. Our results support further research investigating safety and benefit to extend PLTs storage duration.

\section{Competing interests None.}

\section{References}

1. Inaba K, Branco BC, Rhee P, Blackbourne LH, Holcomb JB, Spinella PC, Shulman I, Nelson J, Demetriades D. Impact of the duration of platelet storage in critically ill trauma patients. J Trauma. 2011 Dec;71(6):1766-73.

2. Triulzi DJ, Assmann SF, Strauss RG, Ness PM, Hess JR, Kaufman RM, Granger S, Slichter SJ. The impact of platelet transfusion characteristics on posttransfusion platelet increments and clinical bleeding in patients with hypoproliferative thrombocytopenia.Blood. 2012 Jun 7;119(23):5553-62.

072

REA-C-SUR safety culture in intensive care in France: is there a link with morbi-mortality conferences?

Cédric Bretonnière ${ }^{1}$, Daniel Villers ${ }^{1}$, Christophe Guitton² ${ }^{2}$ Groupe RMM, Qualité et Sécurité des Soins - CBPR-CFAR

${ }^{1}$ Réanimation médicale polyvalente, C.H.U. Hôtel Dieu, Nantes, France;

${ }^{2}$ Réanimation médicale, C.H.U. Hôtel Dieu, Nantes, France

Correspondence: Cédric Bretonnière - cedric.bretonniere@reanantes.

com

Annals of Intensive Care 2016, 6(Suppl 1):072

Introduction Improving the safety of care is a challenge for the health systems. Development of a safety culture (SC) is one of the objectives. Morbidity-mortality conferences (MMC) are one tool that could help in improving SC.

The main objective of the national multicenter REA-C-SUR project is to measure the SC in ICUs in France. The secondary objective is to establish a link between SC and the characteristics of MMR.

This project was initiated by the national group 'RMM, Qualité et Sécurité des Soins en Réanimation.' It is funded by a national grant AO PREPS 2012.

Materials and methods SC was measured among professionals (doctors, managers, nurses, etc.) through the questionnaire (Hospital Survey on Patient Safety Culture, HSOPS) developed by AHRQ in the USA and validated in French. This questionnaire, through 40 items, explores ten dimensions of SC. A dimension is developed if the score is above $75 \%$ and "to be improved" if the score is $<50 \%$.

For each unit, MMCs' organizational characteristics were collected.

Results From September 2013 to September 2014, 64 units (adults or pediatric) were included from French hospitals as follows: 40 teaching, 23 non-teaching and one private sector. A total of 36,149 patients, in 2012, were admitted. 


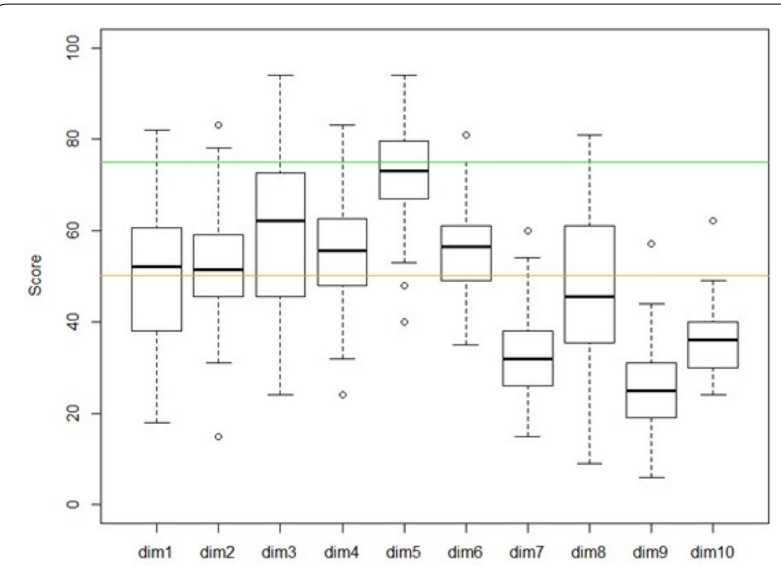

Fig. 16 See text for description

Participation rate was excellent: 3692 questionnaires filled out of 4811 (77 \%). SC overall is really undeveloped. The scores for perception by professionals in the management support for security (dim 9) or teamwork between departments ( $\mathrm{dim} 10$ ) are extremely bad: $<50 \%$ for almost all units. The dimension "non-punitive response" is also very undeveloped (Fig. 16).

There is a statistical link between MMR and SC. The more the MMR are structured, the better the scores for the overall perception of safety and support of management. There is also a link between nurses' involvement and reporting of adverse events, learning organization and human resources. Finally, the presence at MMCs, of an external participant, seems to improve communication openness.

Conclusion This prospective, multicenter study is the first in ICUs, in Europe. It might help in improving SC that is to date really low.

\section{Competing interests None.}

\section{3}

\section{Survival of critically ill solid cancer patients: results of a} retrospective multicentre study

M Soares ${ }^{1}$, Virginie Lemiale ${ }^{2}$, Djamel Mokart ${ }^{3}$, Frédéric Gonzalez ${ }^{4}$, Fabrice Bruneel $^{5}$, Yves Cohen ${ }^{4}$, Elie Azoulay ${ }^{6}$, Michaël Darmon 7 , François Vincent ${ }^{8}$, for the GrrrOH: Groupe de recherche en réanimation respiratoire en Onco-Hématologie (Group for Research in Respiratory Intensive Care Onco-Hematology)

'Post-graduation program, D'Or Institute for Research and Education, Rio de Janeiro, Brazil; ${ }^{2}$ Réanimation médicale, Hôpital Saint Louis, Paris, France; ${ }^{3}$ Réanimation médico-chirurgicale, Institut Paoli-Calmettes, Marseille, France; ${ }^{4}$ Réanimation médico-chirurgicale, Hôpital Avicenne, Bobigny, France; ${ }^{5}$ Réanimation médico-chirurgicale, Centre Hospitalier de Versailles, Le Chesnay, France; ${ }^{6}$ Réanimation médicale, Hôpital Saint-Louis, Paris, France; ${ }^{7}$ Réanimation médicale, CHU Saint-Etienne - Hôpital Nord, Saint-Étienne, France; ${ }^{8}$ Réanimation médico-chirurgicale, GHIC Le Raincy-Montfermeil, Montfermeil, France

Correspondence: François Vincent - frncsvncnt@aol.com Annals of Intensive Care 2016, 6(Suppl 1):073

Introduction We previously reported factors associated with day- 120 outcome in ICU survivors among 1053 patients with solid cancers hospitalized in intensive care unit (ICU) [1]. We performed additional analysis to assess hospital mortality and factors associated with day120 mortality in the overall population of patients.

Patients and methods We conducted an international, multicenter retrospective study. All solid cancer patients, except those admitted after scheduled surgery, admitted between 2006 and 2011 in the five participating ICUs were included. Variables of interest were in-hospital and day-120 post-ICU mortality. We performed logistic regression analyses to identify variables statistically significantly associated with

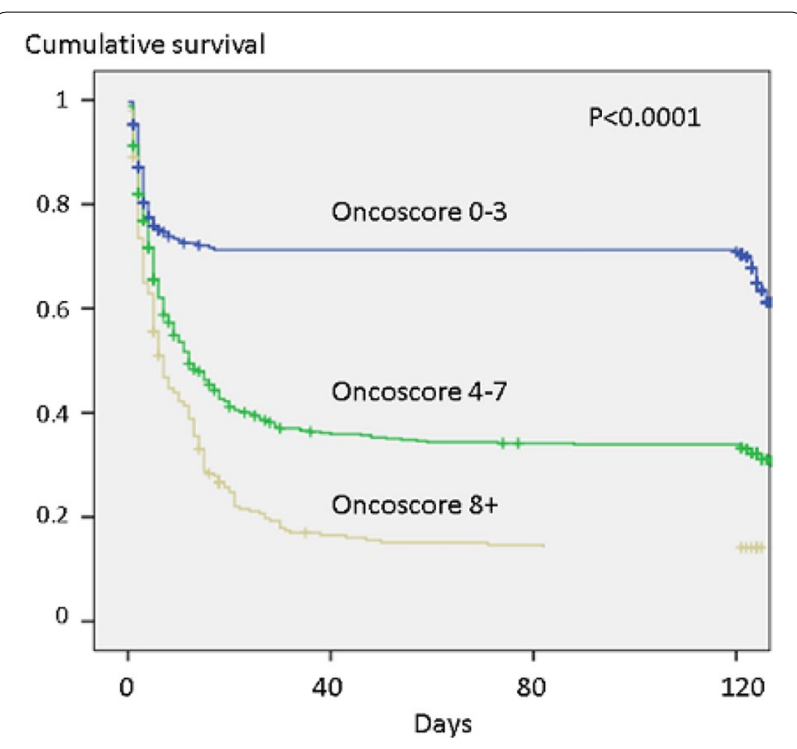

Fig. 17 See text for description

hospital mortality and day-120 post-ICU discharge mortality. A prediction score (Oncoscore) was then built and evaluated.

Results A total of 1053 patients were included. The median age was of 63 years (54-71), and $66.8 \%$ of the patients were of male gender. The principal underlying cancers were lung in $202(19.2 \%)$, colonic in $178(16.9 \%)$, breast in 129 (12.5\%), and head and neck in 125 (11.9\%). In-ICU, in-hospital, and day-120 post-ICU discharge mortalities were of, respectively, $41.3,60.7$, and $65.8 \%$. Factors independently associated with day-120 mortality were systemic extension of the cancer (OR 2.54; $95 \% \mathrm{Cl} 1.87-3.45)$ and organ support including need for mechanical ventilation (OR 2.54; $95 \% \mathrm{Cl} 1.80-3.45$ ), renal replacement therapy (OR $1.54 ; 95 \% \mathrm{Cl} 0.99-2.38$ ), or vasopressors (OR 2.35; $95 \% \mathrm{Cl} 1.66-3.29$ ). Conversely, when compared to lung cancer, other types of tumours were found to be protective from poor outcome with odds ratio ranging from 0.25 to 0.52 ( $P<0.05$ for each).

A prediction model (Oncoscore) was then built ranging from 0 to 11 . In the studied population, Oncoscore was $6(3-7)$. The overall model area under ROC curve in predicting day-120 outcome was fair $(0.74$; $95 \% \mathrm{Cl} 0.71-0.77)$. A score of 4 was found to be sensitive of poor outcome (sensitivity 0.84 ), while a score of 8 was specific (specificity 0.92). According to these cut-offs, day-120 mortality was of $40 \%(n=111)$, $70 \%(n=363)$ and $87 \%(n=216)$ with Oncoscore $<4$, of $4-7$ and $>7$, respectively. Cumulative survival is reported in Fig. 17.

Conclusion Critically ill patients with solid cancer have a meaningful day-120 survival. Conversely to critically ill patients with haematological malignancy underlying malignancy and extension of the disease seems to be independently associated with day-120 mortality. The Oncoscore developed in this study, although performing fairly in the studied population remains to be validated in an external population of solid cancer patients requiring ICU admission.

\section{Competing interests None.}

\section{Reference}

1. Abstract FC091, S175, SRLF 2014.

\section{4}

Encouraging results of the French controlled donation after circulatory death Maastricht category III Program

Clémence Fauché ${ }^{1}$, Michel Muller ${ }^{1}$, Samuel Gay ${ }^{1}$, Olivier Skowron², Albrice Levrat ${ }^{1}$, Didier Dorez ${ }^{3}$

${ }^{1}$ Haute savoie, Intensive Care Unit, Hospital Center Annecy Genevois, Metz-Tessy, France; ${ }^{2}$ Haute savoie, Department of Urology, Hospital 
Center Annecy Genevois, Metz-Tessy, France; ${ }^{3}$ Haute savoie, Organ Procurement Coordination, Hospital Center Annecy Genevois, Metz-Tessy, France

Correspondence: Samuel Gay - sgay@ch-annecygenevois.fr Annals of Intensive Care 2016, 6(Suppl 1):074

Introduction Organs from donors following circulatory death (DCD) have undoubtedly contributed to the expansion of donor organ pool. Recently, the French regulation agency, Agence de BioMédecine (ABM) and the Intensive Care Societies (SRLF/SFAR) made recommendations and authorized "controlled" donation after circulatory death (CDCD) Maastricht category III. Here, we describe the experience of one of the first authorized pilot centers in France.

Patients and methods From December 2014 to September 2015, all CDCD data were prospectively collected, in conformity with the ABM protocol [1].

The different steps of our local procedure were: (1) Withdrawal of lifesustaining therapy (WLST) decision was discussed by intensive care staff including external second opinion. This decision was independent of any discussion to donate organs for transplantation. (2) The relatives were involved in the decision of WLST. The healthcare provider ensured the understanding and acceptance of the decision. (3) Only after WLST decision, the local organ procurement coordination was contacted to indicate that the patient might be eligible for CDCD. (4) Then, a representative assessed the patient for potential donation (health security). (5) If the patient was suitable for donation, a second interview with relatives was done to research the non-opposition for donation. (6) Evaluation of kidney and liver function. (7) WLST determined by the ICU staff and relatives. (8) Declaration of death after circulatory arrest. (9) Percutaneous introduction of in situ regional normothermic recirculation (RNR) in intensive care. (10) Transfer in the operating room for organ procurement.

This prospective, single-center, descriptive study reports the first data from our cohort of CDCD.

Results From 01/12/2014 to 31/08/2015, among 742 intensive care admissions, four potentials donors were identified (three anoxic encephalopathy and one severe traumatic brain injury). All four had no opposition for donation and were eligible for the procedure. Titrated sedation and extubation were always used for WLST. Death occurred in each case in $<3 \mathrm{~h}$. There was one cannulation failure of the RNR. For the three other procedures, six kidneys were explanted and grafted. There was no delayed graft function. One liver was explanted and grafted with success. During the same period, our team accomplished 17 donation after brain death and six uncontrolled DCD (Maastricht II). Conclusion The understanding of the $C D C D$ protocol by relatives was good. The discussion of donation options was frequently initiated by family request for information. There was no procedure failure due to delayed cardiac arrest or prolonged hypoperfusion period. These results could be explicated by our procedures for WLST with titrated sedation, analgesia and comfort care. Our policies and procedures for WLST are the same for both donation and non-donation cases. Our team met technical difficulties for percutaneous RNR. This percutaneous approach needs to be evaluated in the future. CDCD represents $18 \%$ of our recovery activity for transplantation. After an intense preparation phase, essential to the proper understanding of the protocol, the establishment of a cDCD activity in our center led to six kidney and

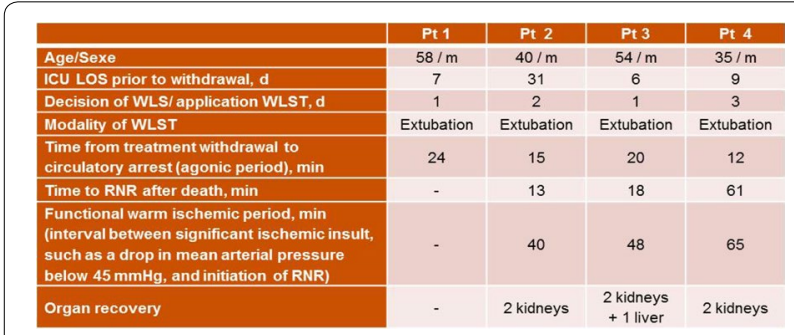

Fig. 18 See text for description one liver transplants. On this short cohort, we had no grafts dysfunction. These data showed promising results concerning $C D C D$ program but need further evaluation (Fig. 18).

Competing interests None.

Reference

1. Antoine C, Mourey F, Prada-Bordenave E: How France launched its donation after cardiac death program. Ann Fr Anesth Réanimation 2014, 33:138-143.

075

Clinical significance of cardiac troponin I release in severe trauma patients

Maxens Decavèle ${ }^{1}$, Arnaud Foucrier², Sebastian Pease², Tobias Gauss², Catherine Paugam²

${ }^{1}$ Réanimation médico-chirurgicale, Hôpital Tenon, Paris, France; ${ }^{2}$ Unité de réanimation chirurgicale polyvalente, service d'anesthésie réanimation, Hospital Beaujon, Clichy, France

Correspondence: Maxens Decavèle - maxencesar@hotmail.fr Annals of Intensive Care 2016, 6(Suppl 1):075

Introduction Elevated plasma levels of cardiac troponin I (cTn-l) have been described in about $10 \%$ of cases in the early phase of severe multiple trauma patients. However, apparently specific for myocardial cell membrane disruption, the mechanisms of its increase are still poorly understood. Moreover, if CTn-I elevation appears as an independent prognostic marker in various critical situations, its impact on severe trauma outcome in the intensive care unit (ICU) remains controversial. The main objectives of our study were first to determine the incidence and factors influencing $\mathrm{CTn}-\mathrm{I}$ release in severe multiple trauma and secondly to assess its prognosis on outcomes in intensive care unit.

Patients and methods We performed a retrospective analysis from a prospective, multicenter, regional scientific database (Traumabase $\left.{ }^{\circledR}\right)$, of all patients admitted to the Beaujon University Hospital ICU between January 2011 and December 2013. Only patients for whom a troponin assay was performed on arrival were included for analysis (immunological methods of chemiluminescence-ARCHITECT STAT Troponin- $I^{\circledR}$, positivity threshold $0.05 \mathrm{ng} / \mathrm{ml}$ ). If more than one value were available, the highest troponin value over the first $24 \mathrm{~h}$ was retained for analysis. The primary endpoint was the cTn-I elevation and the ICU mortality. Continuous variable is expressed as median and interquartile range and categorical variables as absolute value and relative frequencies. Univariable and multivariable logistic regression analysis was performed for CTn-I elevation and ICU mortality.

Results A total of 1029 patients were included (age $38 \pm 18$; Injury Severity Score $19 \pm 14$ ). Pre-hospital variables associated with cTn-I elevation in multivariable analysis are reported in Table 8 . In a multivariable model including the Injury Severity Score and the Simplified Acute Physiology Score and the presence of shock and cardiac arrest before admission, initial cTn-I elevation is not associated with ICU mortality.

Conclusion In this series of more than 1000 patients, the initial serum concentration of CTn-I does not appear as an independent predictor of mortality in the ICU. Its initial elevation seems rather to reflect the severity of the trauma. However, its association with various

\section{Table 8 See text for description}

Multivariate analysis on cTn-I elevation at admission

\begin{tabular}{llll}
\hline Variables & OR & $\mathbf{9 5} \% \mathbf{C l}$ & $\boldsymbol{P}$ value \\
\hline Minimum oxygen saturation & 0.9568 & $0.9204-0.9906$ & 0.0180 \\
Severe cranial trauma & 0.8366 & $0.3753-1.8141$ & 0.6554 \\
Hemorragic shock & 2.7104 & $1.392-5.1504$ & 0.0027 \\
Intubation in the field & 2.0988 & $1.0724-4.0273$ & 0.0275 \\
Thoracic trauma & 2.3028 & $1.2988-4.1921$ & 0.0050 \\
\hline
\end{tabular}


pre-hospital variables may highlight the complex mechanisms of its release.

\section{Competing interests None.}

\section{6}

The modified Glasgow prognostic score is helpful in screening lung cancer patient at risk of hospitalization at the emergency department

Julie Gorham 1 , Lieveke Ameye ${ }^{2}$, Marianne Paesmans², Thierry Berghmans ${ }^{1}$, Jean-Paul Sculier', Anne-Pascale Meert

${ }^{1}$ Intensive Care and Thoracic Oncology, Institute Jules Bordet, Brussel, Belgium; ${ }^{2}$ Data centre, Institute Jules Bordet, Brussel, Belgium

Correspondence: Julie Gorham - julie.gorham@ulb.ac.be

Annals of Intensive Care 2016, 6(Suppl 1):076

Introduction We previously found predictive factors for hospital admission and death during hospitalization in lung cancer patients consulting at the emergency department. Systemic inflammation is a prognostic factor in cancer patients and can be studied by inflammation-based prognostic scores [1], such as the modified Glasgow prognostic score (Table 9), a combination of C-reactive protein and albumin, biological parameters easily obtained and measured in routine. This score was initially described in 2003 in patients with inoperable lung cancer and proved to be a prognostic factor for survival [2]. Then, it was studied for its prognostic value in patients with operable cancer and receiving chemo-/radio-therapy. A predictive role for response to antineoplastic treatment was also suggested [3]. The goal of our study was to determine whether systemic inflammation measured using the modified Glasgow prognostic score could improve the predictive value of our previous models, a model for hospitalization and a model for death during hospitalization.

Patients and methods We conducted a retrospective study including all patients with lung cancer consulting at the emergency department of a cancer hospital between January 1, 2008, and December 31, 2010. In order to assess whether the Glasgow score improves the prediction of the two published models (predicting hospitalization and death during hospitalization), we have refitted the two models in the cases with the Glasgow score available, dropped the variables one per one with a nonsignificant $p$ value, and then added the Glasgow score to both models.

Results Of the 548 emergency department visits, CRP and albumin needed for calculating the modified Glasgow prognostic score were available in 291 cases. Of these, 94 (32\%) involved patients with clinically documented infection. The 291 visits resulted in 222 hospitalizations. The majority of patients had a modified Glasgow prognostic score of one and a stage IV cancer. In multivariate analysis based on the model originally developed, the modified Glasgow prognostic score is an independent predictor of hospital admissions (odds ratio 2.72 per one-unit increase; $p$ value $<0.0001$ ) as well as arrival by ambulance (odds ratio 25.93; $p$ value $<0.0001$ ) and the presence of physical signs associated with the chief complaint (odds ratio 2.83; $p$ value $=0.001$ ). One hundred and eighty-one consultations (first visit only) were considered for determining prognostic factors for death during hospitalization. The modified Glasgow prognostic score is an independent predictor of death during hospitalization (odds ratio 2.95 per one-unit increase; $p$ value $<0.001$ ) as well as arrival by ambulance (odds ratio 19.03; $p$ value $=0.0002$ ). After stratifying patients into two groups according to the presence of an infection or not, this score added significant information in the two strata ( $p$ value 0.003 and 0.001 , respectively).

\section{Table 9 The modified Glasgow prognostic score}

\begin{tabular}{ll}
\hline The modified Glasgow prognostic score & Points allocated \\
\hline C-reactive protein $\leq 10 \mathrm{mg} / \mathrm{l}$ & 0 \\
C-reactive protein $>10 \mathrm{mg} / \mathrm{l}$ and albumin $\geq 35 \mathrm{~g} / \mathrm{l}$ & 1 \\
C-reactive protein $>10 \mathrm{mg} / \mathrm{l}$ and albumin $<35 \mathrm{~g} / \mathrm{l}$ & 2
\end{tabular}

Discussion This is an original study. To our knowledge, this score has never been evaluated in emergency departments in cancer patients or even in a general population.

Conclusion The modified Glasgow prognostic score is an independent predictive factor for hospitalization and death during hospitalization in patients with lung cancer consulting at the emergency department.

\section{Competing interests None.}

\section{References}

1. Proctor MJ, Morrison DS, Talwar D, et al. A comparison of inflammationbased prognostic scores in patients with cancer. A Glasgow Inflammation Outcome Study. Eur J Cancer 2011: 47: 2633-41.

2. Forrest LM, McMillan DC, McArdle CS, Angerson WJ, Dunlop DJ. Evaluation of cumulative prognostic scores based on the systemic inflammatory response in patients with inoperable non-small-cell lung cancer. Br J Cancer 2003; 89: 1028-1030.

3. McMillan DC. The systemic inflammation-based Glasgow Prognostic Score: a decade of experience in patients with cancer. Cancer treat Rev 2013; 39: 534-40.

077

Relationship between body mass index classes and massive transfusion needs in trauma patients and predictive performance of the TASH score in obese and non-obese populations: a retrospective study on 910 trauma patients

Audrey De Jong ${ }^{1}$, Pauline Deras², Orianne Martinez², Pascal Latry ${ }^{3}$, Samir Jaber ${ }^{1}$, Xavier Capdevila ${ }^{2}$, Jonathan Charbit ${ }^{2}$

${ }^{1}$ Dar b, Hôpital Saint Eloi, Montpellier, France; ${ }^{2}$ Dar a centre régional d'accueil des polytraumatisés, Hospital Lapeyronie, Montpellier Cedex 5, France; ${ }^{3}$ Inserm u1046, Hospital Lapeyronie, Montpellier Cedex 5, France Correspondence: Audrey De Jong - audreydejong@hotmail.fr Annals of Intensive Care 2016, 6(Suppl 1):077

Introduction The early detection of patients at risk of massive transfusion (MT) is challenging in bleeding trauma patients [1]. Mortality has been shown to be higher in severely injured obese patients compared with non-obese patients. However, to our knowledge, the risk of MT has never been assessed in obese population. Moreover, although the Trauma-Associated Severe Haemorrhage (TASH) score is a robust scoring system for predicting MT requirements [2], its predictive performance has never been validated in obese patients. The main objective of the study was to compare the MT rate in trauma patients according to obesity status. The secondary objectives were to assess and revalidate the TASH score in predicting MT in population of obese and nonobese trauma patients and to use a grey zone approach to providing relevant thresholds to optimize the ability to predict MT in clinical practice.

Patients and methods All trauma obese and non-obese patients admitted in a Level I Regional Trauma Center were included. Were excluded patients who died immediately after admission or not directly admitted or with important clinical data missing in the database. MT rate and TASH score were assessed in obese [body mass index $(\mathrm{BMI}) \geq 30 \mathrm{~kg} / \mathrm{m}^{2}$ ] and non-obese $\left(\mathrm{BMl}<30 \mathrm{~kg} / \mathrm{m}^{2}\right.$ ) patients. Logistic regression was performed to assess the relationship between obesity and MT, before and after adjusting for ISS score. The odds ratio (OR) was provided for obesity status and for an increase of 5 units of BMI with the $95 \%$ confidence interval $(\mathrm{Cl})$. Received operating characteristic (ROC) curve analysis was performed for the TASH score in predicting MT in the obese and non-obese groups. Thereafter, thresholds analysis was carried out from the ROC curves using the grey zone method.

Results Between January 2008 and December 2012, 119 obese trauma patients and 791 non-obese trauma patients were included. The MT rate was $10 \%(94 / 910)$ in the whole population. The MT rate trended to be higher in obese patients versus non-obese patients: $15 \%(18 / 119)$ versus $10 \%(76 / 791)$, OR 1.68 [95 \% Cl 0.97-2.92], $p=0.07$. After adjusting for ISS score, obesity was significantly associated with MT rate (OR 1.79 [95 \% 1.00-3.21], $p=0.049$ ). BMI as a continuous variable was also significantly associated with MT before (OR 1.24 [95 \% Cl 1.00-1.54], $p=0.047$ ) and after adjusting for ISS score 


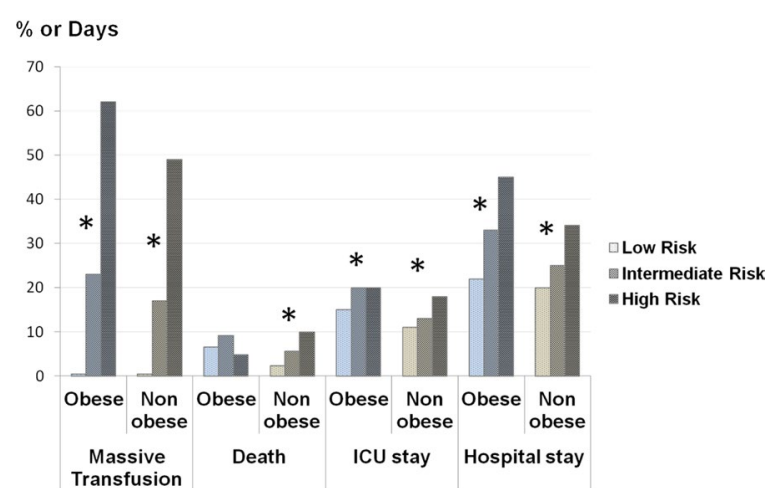

Fig. 19 Rates of massive transfusion, death, length of ICU and hospital stays according to the TASH score in the obese and non-obese groups

(OR 1.28 [95 \% Cl 1.03-1.60], $p=0.028$ ). The TASH score was higher in the obese group than in the non-obese group: $8 \pm 5$ versus $6 \pm 5$. The area under the ROC curves of the TASH score in predicting MT ( $\geq 10 \mathrm{U}$ of packed red blood cells in the first $24 \mathrm{~h}$ ) was very high and comparable between the obese and non-obese groups: $0.93(95 \% \mathrm{Cl}$ $0.89-0.98)$ and $0.94(95 \% \mathrm{Cl} 0.92-0.96)$, respectively ( $p=0.80)$. The grey zone ranged, respectively, from 10 to 13 and 9 to 12 in obese and non-obese patients and allowed to separate patients into three subgroups using the TASH score: low, intermediate and high risk, significantly associated with ICU and hospital stay (Fig. 19).

Conclusion Obesity was associated with a higher rate of MT and a higher TASH score, which was a strong and accurate predictor of MT in both obese and non-obese populations. For the first time, the TASH score was validated in obese patients and a grey zone was established for this predictive score, allowing the MT risk to be affirmed or rejected with accuracy.

\section{Competing interests None.}

\section{References}

1. Spahn DR, Bouillon B, Cerny V, et al. Management of bleeding and coagulopathy following major trauma: an updated European guideline. Crit Care 2013; 17(2):R76.

2. Yücel N, Lefering R, Maegele M, et al. Trauma Associated Severe Hemorrhage (TASH)-Score: probability of mass transfusion as surrogate for life threatening hemorrhage after multiple trauma. J Trauma 2006; 60(6):1228-1236.

\section{8}

The Eschmann stylet as first tracheal access tool during emergency difficult tracheal intubation: An initial simulation trial followed by a clinical study

Jaubert J.', Celia Etiennar², Lucie Ginoux², Jean-Luc Sebbah², Hakim Haouache', Gilles Dhonneur ${ }^{1}$

${ }^{1}$ Anesthesia and intensive care medicine, CHU Henri Mondor, Créteil, France; ${ }^{2}$ Prehospital emergency medicine unit (smur 95), Centre Hospitalier, Gonesse, France

Correspondence: Gilles Dhonneur - gilles.dhonneur@hmn.aphp.fr Annals of Intensive Care 2016, 6(Suppl 1):078

Introduction When anesthesia quality is controlled, tracheal intubation (TI) difficulty may be related to: patient's conditions and characteristics, the environment where the maneuver is performed and the skill of the physician. In case of difficult laryngoscopy, the Eschmann stylet (ES) is proposed as a second-step intubation tool to access the trachea. We have hypothesized that when difficult emergency intubation is encountered, first-step ES (ES-1) may reduce TI delay.

Materials and methods We conducted two prospective successive evaluations. The first one was a simulation trial performed over
3 months. In our airway management laboratory, 24 physicians (12 novices and 12 skilled physicians) were involved. Each of them had to intubate a manikin allowing to simulate simple or difficult TI (Cormack grade $=1$ or $3: \mathrm{C} 1$ or $\mathrm{C} 3$ ) placed in three different positions and environment: standard operating table (OT), lying on the ground (LG) and with very restricted access to the head (RA). All the sequences of TI were randomized for $\mathrm{C} 1, \mathrm{C} 3$, OT, LG, RA and to either conventional tracheal intubation technique using the tracheal tube as first tracheal assess tool (TT-1) or ES-1. With the results of this simulation trial and obtention of ethical approval, the 12 skilled physicians conducted the clinical study over 1 year in prehospital conditions. Anesthesia and difficult airway management procedures were standardized, and the patients were randomly allocated to either TT-1 or ES-1 group. Prehospital TI characteristics were recorded. The study was preemptively dimensioned and powered to demonstrate $30 \%$ reduction in TI delay for the patients with difficult laryngoscopy as attested by the low percentage of glottis inlet visible (POGO score) of below $30 \%$ (Ref). In both studies, TI difficulties (anticipated and post-maneuver) and comfort were assessed.

Results A total of 276 tracheal intubations were timed and analyzed for the simulation trial. We demonstrated that ES-1 as compared to TT-1 was a powerful mean to significantly reduce TI delay of: novices when $\mathrm{C} 3$ was imposed in OT, LG and RA; and skilled physicians when C3 was imposed for LG and RA. For the clinical trial, 250 patients were included. Details of both groups were similar. We demonstrated in the subgroup of patients with POGO score of $<30 \%(n=73)$ that ES-1 as compared to TT-1 significantly reduced TI delay.

Conclusion We have observed in a difficult TI simulation trial that ES-1, as compared to conventional TI, was a powerful strategy to shorten $\mathrm{TI}$ delay when direct laryngoscopy was difficult for novices and skilled physicians. We demonstrated that simulation results translated to clinical conditions. Our clinical data suggest that ES-1 may be useful strategy when emergency difficult TI is anticipated because of the patient's characteristics or environment. Moreover, we believe that the recommended $2 \mathrm{~d}$ TI attempt using TT is not necessary and the switch to ES could be proposed at the first difficult laryngoscopy.

Competing interests None.

079

\section{Maternal mortality risk factors for eclampsia}

Chaigar Mohammed Cheikh', I. Moussaid', O. Ghazaoui ', Kamal Belkadi', S. El Youssoufi', S. Salmi ${ }^{1}$

${ }^{1}$ Anesthesie reanimation, $\mathrm{CHU}$ Ibn Rochd Casa, Casablanca, Morocco Correspondence: Chaigar Mohammed Cheikh - chaigarmed@gmail. com

Annals of Intensive Care 2016, 6(Suppl 1):079

Introduction Eclampsia remains an important cause of maternal and perinatal mortality and morbidity worldwide [1]. The aim of this study was to determine the eclamptic patient's characteristics and to identify risk factors associated with maternal mortality in patients with eclampsia.

Patients and methods We conducted a retrospective study between January 2002 and December 2013, including all patients diagnosed as eclampsia in the obstetrical intensive care unit of our hospital.

Results During this period, a total of 1130 cases of eclampsia were collected, yielding an overall incidence rate of $1.05 \%$. Mean age was $27 \pm 6.64(15-48)$ years. The major proportion of patients was nulliparous $(63.4 \%), 819(72.4 \%)$ cases were diagnosed during the antenatal period, whereas $311(27.6 \%)$ had a postnatal onset. Mean gestational age at onset was $34.5 \pm 4.3(21-43)$ weeks of gestation. HELLP syndrome was identified as the most frequent complication in our study, associated with $34.3 \%$ of patients, while acute kidney injury, placental abruption and neurological disorders were, respectively, associated in $18.5,17.7$ and $16.5 \%$ of cases. In our study 55 patients died, yielding a case fatality rate of $4.9 \%$, cerebral hemorrhage was identified as the first cause of death associated with $25.5 \%$ of deceased patients. In univariate analysis, risk factors for maternal mortality in eclampsia were: advanced maternal age ( $\geq 30$ years), diagnosis of eclampsia during the antenatal period, low GCS $(\leq 8)$, the presence of a neurological deficit, 
oligo-anuria or jaundice, the presence of these biological disorders: low platelets count $(<100,000)$ - ASAT $>70 \mathrm{UI} / \mathrm{I}-$ ALAT $>70 \mathrm{UI} / \mathrm{I}-\mathrm{LDH}$ $>600 \mathrm{UI} / \mathrm{l}$, the occurrence of HELLP syndrome, acute kidney injury, cerebral hemorrhage, acute pulmonary edema, abruption placentae, postpartum hemorrhage, disseminated intravascular coagulation, the use of an anticonvulsant treatment other than the magnesium sulfate, subcapsular liver hematoma and long assisted ventilation (>72 h). According to logistic regression model, we have identified as risk factors for maternal mortality in eclampsia: advanced maternal age ( $\geq 30$ years), low GCS $(\leq 8)$, ALAT $>70 \mathrm{UI} / \mathrm{l}$, cerebral hemorrhage, acute pulmonary edema, disseminated intravascular coagulation, subcapsular liver hematoma and long assisted ventilation $(>72 \mathrm{~h}$ ).

Conclusion Eclampsia is still a major cause of maternal morbidity and mortality worldwide. It incidence remains high, especially for developing countries in need of health care. Several studies are needed to determine all the risk factors and improve maternal prognosis.

\section{Competing interests None.}

\section{0}

Use of aminoglycosides in cirrhotic patients admitted in intensive care for severe sepsis or septic shock: shortand intermediate-term impact on renal function and mortality Laure Pajot ${ }^{1}$, Benjamin Zuber ${ }^{1}$, Carole Schwebel ${ }^{2}$, Romain Sonneville ${ }^{3}$, Benoit Misset ${ }^{4}$, Michael Darmon ${ }^{5}$, Guillaume Marcotte ${ }^{6}$, Anne Sophie Truche ${ }^{2}$, Dany Goldgran-Toledano ${ }^{7}$, Bertrand Souweine ${ }^{8}$, Samir Jamali ${ }^{9}$, Stephane Ruckly ${ }^{3}$, Elie Azoulay ${ }^{10}$, Christophe Clec'h ${ }^{11}$, Jean Pierre Bedos ${ }^{1}$, Jean-François Timsit ${ }^{3}$, OutcomeRéa

${ }^{1}$ Réanimation médico-chirurgicale, Hospital Center De Versailles, Le Chesnay, France; ${ }^{2}$ Réanimation médicale, C.H.U. Grenoble, Grenoble, France; ${ }^{3}$ Réanimation médicale et infectieuse, Hôpital Bichat-Claude Bernard, Paris, France; ${ }^{4}$ Réanimation polyvalente, Groupe Hospitalier Paris-Saint-Joseph, Paris, France; ${ }^{5}$ Réanimation médicale, CHU Bichat Paris, France; ${ }^{6}$ Reanimation chirurgicale, CHU Lyon, Paris, France; ${ }^{7}$ Réanimation, $\mathrm{CH}$ gonesse, Gonesse, France; ${ }^{8}$ Réanimation médicale, CHU Gabriel-Montpied, Clermont-Ferrand, France; ${ }^{9}$ Réanimation médicale, Centre Hospitalier Sud Essonne, Dourdan, France; ${ }^{10}$ Réanimation médicale, Hôpital Saint-Louis, Paris, France; ${ }^{11}$ Réanimation medico-chirurgicale, hopital avicenne, Bobigny, France

Correspondence: Benjamin Zuber - bzuber@ch-versailles.fr

Annals of Intensive Care 2016, 6(Suppl 1):080

Introduction Severe sepsis and septic shock are a frequent lifethreatening cause of ICU admission of cirrhotic patients. Among the antibiotics frequently used in intensive care, the prescription of aminoglycosides to cirrhotic patients is controversial because of their nephrotoxicity. The aim of this study was to evaluate the use of aminoglycosides administration among cirrhotic patients admitted in intensive care unit for severe sepsis and septic shock and study their impact on mortality and renal function.

Patients and methods Cirrhotic patients with severe sepsis and septic shock were selected over a 18-year period (1997-2014) from a longitudinal prospective French multicenter database. We investigated the factors associated with the prescription of aminoglycosides started between day 1 and day 3 of admission, and we studied the modalities of administration of aminoglycosides. Using a competing risk analysis, we evaluated the impact of aminoglycosides administration on the risk of renal replacement therapy or death at day 28. Among survivors who required dialysis or with a renal failure when leaving ICU, we retrospectively collected renal status at day 90 after leaving intensive care.

Results Among the 332 cirrhotic patients admitted for severe sepsis or septic shock in ICU, 119 (35.8\%) received antibiotic therapy with aminoglycosides. In multivariate analysis, factors associated with aminoglycosides prescription were immunosuppression, SAPS II, and surgical cause for ICU admission. Administration of aminoglycosides was also significantly different between centers. All patients received a once-daily administration of aminoglycosides for a 2-day median time (IQR 1-5). Using a cause-specific model for competing risk adjusted on risk factors for death and renal replacement therapy, administration of aminoglycosides between days 1 and 3 was not associated with renal replacement therapy or death at day 28 (death: cause-specific hazard ratio CSHR 1.41,
$95 \% \mathrm{Cl}$ 0.98-2.02, $p=.06$; renal replacement therapy: CSHR 1.44, $95 \%$ $\mathrm{Cl}$ 0.67-3.1, $p=.35$ ). Among 195 patients discharged alive from the ICU, 49 patients had persistent kidney failure or needed renal replacement therapy during their ICU stay. Among these patients, 16 patients (33\%) died at day 90 and no patient required dialysis at day 90 .

Conclusion The use of aminoglycosides in cirrhotic patients with severe sepsis and septic shock is frequent in intensive care units. Their administration with a daily dose is not associated with renal replacement therapy or death in this study.

Competing interests None.

\section{1}

Diagnostic yield and therapeutic impact of liver biopsy in the intensive care unit

Bertrand Sauneuf ${ }^{1}$, Benoit Dupont ${ }^{2}$, Axelle Eugène ${ }^{3}$, Jean-Luc Hanouz ${ }^{4}$, Françoise Galateau-Sallé ${ }^{3}$, Nicolas Terzi ${ }^{5}$

${ }^{1}$ Réanimation Médicale Polyvalente, C.H. Public du Cotentin, Cherbourg-Octeville, France; ${ }^{2}$ Département d'hépato-gastroentérologie et nutrition, C.H.U de Caen, Caen, France; ${ }^{3}$ Laboratoire d'anatomie pathologique, C.H.U de Caen, Caen, France; ${ }^{4}$ Service d'anesthésie réanimation, Centre Hospitalier Universitaire de Caen, Caen, France; ${ }^{5}$ Service de réanimation médicale, Clinique de Réanimation Médicale, Grenoble, France

Correspondence: Bertrand Sauneuf - bertrandsauneuf@yahoo.fr Annals of Intensive Care 2016, 6(Suppl 1):081

Introduction The specific diagnostic and therapeutic yield of liver biopsy in patients hospitalized in intensive care unit (ICU) is unknown. The main goal of this study was to evaluate the contribution of liver biopsy to diagnosis and treatment in this population.

Patients and methods An 8-year (2006-2014) retrospective study was conducted in medical and surgical ICU of Caen University Hospital on patients without transplanted liver who underwent liver biopsy during their management. Medical files were retrieved for data analysis.

Results Forty-six patients underwent a liver biopsy mostly by transjugular route $(94 \%)$. The liver biopsies were performed for a suspicion of cirrhosis in $20 \%$ of cases, for acute liver failure in $26 \%$, for a suspicion of severe acute alcoholic hepatitis in $22 \%$ and for a suspicion of blood disease in $28 \%$. Biopsy-related intraperitoneal hemorrhage occurred in two (4\%) patients requiring an embolization in one case. Liver biopsy established a specific diagnosis in $38(83 \%)$ patients. Therapeutic modifications followed the results of the biopsy in 18 (39\%) patients. ICU mortality was of $57 \%$ and was not different in the group of patients with a specific diagnosis established by the biopsy and in the group of patients for which the biopsy was non-contributory ( 21 deaths/38 patients vs $5 / 7 ; p=0.68$ ).

Conclusion In this cohort highly selected by the treating physicians, liver biopsy helped to establish a specific diagnosis in the majority of the patients with a limited complications rate.

\section{Competing interests None.}

082

\section{Critically ill patients requiring liver transplantation: a 7-year} monocentric retrospective experience

Baptiste Michard', Benjamin Lebas', Alexandra Boivin', Max Guillot', Jean-Etienne Herbrecht ${ }^{1}$, Marie-Line Harlay', Ralf Janssen-Langenstein', Maleka Schenck', Bernard Ellero², Marie-Lorraine Woehl-Jaegle², Camille Besch $^{2}$, Vincent Castelain', Philippe Bachellier², Francis Schneider ${ }^{1}$

${ }^{1}$ Service de réanimation médicale, Hôpital de Hautepierre, Hôpitaux Universitaires de Strasbourg, Strasbourg, France; ${ }^{2}$ Service de chirurgie hépato-biliaire et transplantation, Hôpital de Hautepierre, Hôpitaux Universitaires de Strasbourg, Strasbourg, France

Correspondence: Benjamin Lebas - benji.lebas@neuffr Annals of Intensive Care 2016, 6(Suppl 1):082

Introduction Fulminant hepatitis or acute on chronic liver failure can result in multiple organ failure. In these settings, liver transplantation is the treatment required for recovery. However, such patients have a pejorative post-transplant prognosis when compared to patients who 
undergo transplantation with stable chronic liver disease. This results in an unresolved ethical dilemma given the context of graft shortage. Moreover, published data or guidelines to cope with this dilemma are scarce. Our objective was to assess long-term post-transplant prognosis in patients admitted to intensive care unit (ICU) in relation to multiple organ failure and acute liver failure, and to search for possible pre-transplant predictive factors for post-transplant mortality.

Patients and methods We performed a retrospective monocentric study in 84 critically ill patients requiring liver transplantation according to standard worldwide accepted criteria. These patients were admitted in our ICU from 2007 to 2014. We used $x^{2}$ or $t$ test for univariate analysis and Cox proportional hazards model for multivariate analysis to identify factors associated with mortality.

Results Pre-transplant median model for end-stage liver disease (MELD) score was 42 and mean SOFA (Sequential Organ Failure Assessment) score 15/24, reflecting, respectively, liver dysfunction and overall severity. In addition, $20 \%$ out of the patients demonstrated septicemia before transplantation (i.e., during ICU stay). Overall posttransplant mortality rate was $33 \%$ with a mean follow-up of 2.3 years. After 1 year, de novo acute liver failure patients had better survival rates than those from the French National Survey average, while the mortality rate of acute on chronic liver failure was $40 \%$. In addition, $97 \%$ of survivors were autonomous, yet $2 \%$ required chronic dialysis. Ten pre-transplant factors were significantly predictive of 90-day post-transplant mortality in univariate analysis, among which arterial lactate, low platelet count, SOFA score, SAPS (Simplified Acute Physiology) II score and respiratory failure $\left(\mathrm{PaO}_{2} / \mathrm{FiO}_{2} \leq 300 \mathrm{mmHg}\right)$ for the overall cohort $(p<0.05)$. Severe pre-transplant respiratory failure $\left(\mathrm{PaO}_{2} / \mathrm{FiO}_{2} \leq 200 \mathrm{mmHg}\right)$ and a late initiation of invasive ventilation ( $>24 \mathrm{~h}$ after admission to ICU) were strongly predictive of 90 days post-transplant mortality in multivariate analysis (hazard ratio 7.1, $p<0.04$, and $7.2, p<0.05$, respectively). In contrast, the need for norepinephrine infusion, renal replacement therapy or the occurrence of septicemia did not influence post-transplant mortality.

Discussion When excluding the seven deaths influenced by pre-transplant acute respiratory failure, the overall remote mortality is decaying to $25 \%$, which is close to post-transplant survival rates observed nationwide in liver transplantation for stable chronic liver disease. Moreover, in our cohort, long-term morbidity for survivors was low if compared to data from the literature.

Conclusion Our data suggest that liver transplantation can be safe and successful despite critical care criteria and poor underlying conditions (i.e., a context of circulatory failure requiring infusion of high doses of norepinephrine, septicemia or renal replacement therapy). They also suggest that liver transplantation should cautiously be advised in the presence of preoperative acute lung injury criteria. In the latter case, liver transplantation should (at least) temporarily be discouraged.

\section{Competing interests None.}

\section{3}

Hospital survival and access to liver transplantation of MARS-treated patients in France, 2004-2008: a retrospective multicenter study (RETROMARS)

Christophe Camus ${ }^{1}$, Fawzi Saliba ${ }^{2}$, Bernard Goubaux ${ }^{3}$, Agnès Bonadona ${ }^{4}$, Laurence Lavayssiere ${ }^{5}$, Catherine Paugam ${ }^{6}$, Alice Quinart ${ }^{7}$, Olivier Barbot ${ }^{8}$, Sebastien Dharancy ${ }^{9}$, Bertrand Delafosse ${ }^{10}$, Nicolas Pichon ${ }^{11}$, Helene Barraud $^{12}$, Arnaud Galbois ${ }^{13}$, Benoit Veber ${ }^{14}$, Sophie Cayot ${ }^{15}$, Bruno Souche $^{16}$, Clara Locher ${ }^{17}$, RETROMARS Study Group

135033, Hospital Center University De Rennes, Rennes, France; ${ }^{2}$ Villejuif, Hospital University Paul Brousse, Villejuif, France; ${ }^{3} 06000$, CHU Nice, Nice, France; ${ }^{4}$ Réanimation médicale, C.H.U. Grenoble, La Tronche, France; 531000, CHU Rangueil, Toulouse, France; ${ }^{6}$ Anesthésie-réanimation, CHU Beaujon, Clichy, France; ${ }^{7} 33000$, CHU, Bordeaux, France; ${ }^{8} 25000$, CHU, Besançon, France; ${ }^{9} 59000$, CHRU Huriez, Lille, France; ${ }^{10} 69000$, Hospices Civils de Lyon, Lyon, France; ${ }^{11}$ Service de réanimation polyvalente, Centre Hospitalier Universitaire de Limoges, Limoges, France; ${ }^{12} 54000$, Hépatogastroentérologie, CHU Nancy, Nancy, France; ${ }^{13}$ Réanimation médicale, Hôpital Saint-Antoine, Paris, France; ${ }^{14}$ Reanimation chirurgicale,
Centre Hospitalier Universitaire Rouen, Rouen, France; ${ }^{15} 63000, \mathrm{CHU}$ Clermont-Ferrand, France; ${ }^{16} 34000$, CHU Saint-Eloi, Montpellier, France; 1735000, CIC Pontchaillou, Rennes, France

Correspondence: Christophe Camus - christophe.camus@chu-rennes.fr Annals of Intensive Care 2016, 6(Suppl 1):083

Introduction There is no current recommendation for the use of albumin dialysis with the molecular adsorbent recirculating system (MARS) in patients with acute or chronic liver failure. We performed a retrospective survey in France in patients who were treated with MARS, 2004-2008. The aim of the study was to assess the indications of MARS treatments, hospital survival, with or without liver transplantation (LTX), and also long-term follow-up, especially in patients with preexisting liver disease.

Patients and methods All the hospitals in which MARS technique was regularly performed were requested to participate to the study. All patients who had received MARS therapy in adult care setting over the 5 -year study period were eligible. A questionnaire was sent to investigators and should be completed for each treated patient. The study was approved by the National Research Consultative Committee and the Institutional Ethics Committee. Variables associated with outcome were identified by multivariate analysis using logistic regression.

Results Among 25 centers, 16 participated to the study. A total of 383 patients received 393 MARS treatments (1091 sessions). Based on the examination of Mars treatment kits consumption records at all French hospitals during the study period, it could be estimated that the study accounted for $78 \%$ of total MARS sessions in adult care setting in France.

The recorded indications for MARS (\% of treatments) The indications were: major hyperbilirubinemia (37.1\%); hepatic encephalopathy ( $\mathrm{HE}$, $23.6 \%$ ); any kidney dysfunction ( $22.9 \%$; either hepatorenal syndrome [HRS, $15.3 \%$ ] or other renal dysfunction [7.6 \%]); refractory pruritus (9.9\%); acute liver failure (ALF, $32.6 \%)$; early nonfunction after LTx (6.1\%); liver failure after liver resection (3.8\%); $\geq 2$ indications (37.1\%). Hospital survival rate according to the indication (\%, $95 \% \mathrm{Cl}$ ) One hundred and thirty-one patients were listed and 86 underwent LTx. Survival was assessed according to whether or not the patients were on the transplant list. (1) Not listed (66.3\%): ALF, $46 \%$ (35-57); hyperbilirubinemia, $22 \%$ (15-31); HE, $14 \%$ (7-24); HRS, $20 \%$ (10-34); refractory pruritus, $85 \%$ (69-95); nonfunction after LTx, $0 \%$ (0-21); liver failure after liver resection. $29 \%(10-53) ; \geq 2$ indications, $17 \%(15-25)$.

(2) Listed (33.7\%): ALF, $75 \%$ (63-85); hyperbilirubinemia, $63 \%$ (48-76); $\mathrm{HE}, 41 \%$ (25-59); HRS, $44 \%$ (23-67); refractory pruritus, $100 \%$ (79100); nonfunction after LTx, $82 \%$ (55-96); $\geq 2$ indications, $59 \%$ (45-73). Hospital survival rate in patients with nonbiliary cirrhosis $(n=137)$ The overall rate was $38 \%$ (30-46) (listed for LTx: $56 \%$ [40-71]; not listed: $32 \%$ [23-41]). Variables associated with hospital death were HE (OR 3.00 [1.25-7.19], $p=0.01$ ), hyperbilirubinemia (OR 2.40 [1.08-5.38], $p=0.03$ ) and the absence of listing for LTx (OR $3.18[1.30-7.76$, $p=0.01)$.

Outcome in ALF patients Thirty-eight ALF patients (29.5\%) underwent LTx during their hospital stay. Listing with the "Superurgence" priority was the single variable associated with hospital survival (OR 3.79 [1.69-8.48], $p=0.001$ ). Hospital survival without transplantation was associated with paracetamol etiology (OR 2.41 [1.04-5.58], $p=0.04$ ) and the number of sessions performed (OR 1.49 [1.11-2.00], $p=0.009$ ).

One-year Kaplan-Meier survival estimate according to preexisting liver disease $(\%, 95 \% \mathrm{Cl})$ The patients with preexisting liver disease were classified into five main diagnostic categories and were evaluated separately whether or not they underwent LTX. Fifteen patients were lost to follow-up.

(1) Without LTx: nonfunction after LTx, $9 \%$ ( -8 to 26$)$; liver failure after liver resection. $0 \%$; late graft dysfunction, $5 \%(-5$ to 15$)$; nonbiliary cirrhosis, $15 \%$ (8-22); biliary cirrhosis/sclerosing cholangitis, $67 \%$ (29greater than 100).

(2) With LTx: nonfunction after LTx, $88 \%$ (65-greater than 100); late graft dysfunction, $80 \%$ (60-100); nonbiliary cirrhosis, $91 \%$ (80-greater than 100); biliary cirrhosis/sclerosing cholangitis, $90 \%$ (71-greater than 100). 
Discussion ALF was the recorded indication of one-third of all MARS treatments and was associated with acceptable outcome even for nontransplanted patients. In patients with preexisting liver disease who were not candidates to LTx, the prognosis of nonbiliary cirrhosis was very poor, especially when $\mathrm{HE}$ or hyperbilirubinemia was the indication for MARS. Nonfunction after LTx was fatal in all cases without reLTx. Regarding late graft dysfunction and liver failure after liver resection, MARS provided no survival benefit in the absence of LTX perspective.

Conclusion MARS therapy could be used in patients with ALF even in those for whom LTx is not considered. In patients with preexisting liver disease, MARS should be best restricted to those who are suitable for LTx. Prospective studies are required to reassess the benefit of MARS in listed patients awaiting a liver graft.

\section{Competing interests None.}

\section{References}

1. Bañares R, Nevens F, Larsen FS, Jalan R, Albillos A, Dollinger M, Saliba F, Sauerbruch T, Klammt S, Ockenga J, Pares A, Wendon J, Brünnler T, Kramer L, Mathurin P, de la Mata M, Gasbarrini A, Müllhaupt B, Wilmer A, Laleman W, Eefsen M, Sen S, Zipprich A, Tenorio T, Pavesi M, Schmidt HH, Mitzner S, Williams R, Arroyo V; RELIEF study group. Extracorporeal albumin dialysis with the molecular adsorbent recirculating system in acute-on-chronic liver failure: the RELIEF trial. Hepatology 2013;57:1153-62.

2. Saliba F, Camus C, Durand F, Mathurin P, Letierce A, Delafosse B, Barange K, Perrigault PF, Belnard M, Ichaï P, Samuel D. Albumin dialysis with a noncell artificial liver support device in patients with acute liver failure: a randomized, controlled trial. Ann Intern Med 2013;159:522-31. ${ }^{1}$

\section{P1}

\section{Severe brain injuries: epidemiology and outcome in a Tunisian} medical intensive care unit

Hassen Ben Ghezala', Salah Snouda', Rebeh Daoudi', Moez Kaddour ${ }^{1}$ ${ }^{1}$ Teaching department of emergency and intensive care, Hospital Zaghouan, Zaghouan, Tunisia

Correspondence: Hassen Ben Ghezala - hassen.ghezala@gmail.com Annals of Intensive Care 2016, 6(Suppl 1):P1

Introduction Severe brain injuries are most often due to road traffic accidents and usually occur in young persons. Despite the progress made, their management remains dependent on the availability of resources. In this study, we tried to establish the profile of severe traumatic brain injury (TBI) in the teaching department of intensive care unit of the regional hospital of Zaghouan and compare it to the literature. Then, we tried to determine outcome factors.

Materials and methods We performed a retrospective study over 04 years from 2011 to 2014. We enrolled all adult patients admitted to intensive care via emergency following a cranial trauma with GCS $\leq 8$ after normalization of hemodynamic status. We excluded from the results analysis patient records with insufficient clinical or para-clinical data.

Results During the study period, 132 patients admitted for TBI were collected representing $3 \%$ of all admissions and $12 \%$ of all head trauma hospitalized in the unit. The most common mechanism was the road traffic accident (78 \% of cases). All transport was non-medicalized. So there was no intubation-ventilation at the site of trauma. The takeover delay was $78 \mathrm{~min}$. The average age of patients was $43 \pm 16$ years with extremes ranging from 19 to 83 years. The sex ratio was 4.3. The state of consciousness was evaluated by the GCS with an average of $5 \pm 2$. Sixty-six percent of patients had a pupillary anomaly, $19 \%$ had a neurological deficit, and $81 \%$ had associated lesions. The most frequent CT lesions were subarachnoid hemorrhage and cerebral contusions. All patients required intubation and mechanical ventilation with an average duration of hospitalization of $9 \pm 2$ days. The prognosis, assessed by the Glasgow Outcome Scale (GOS), was correlated with several factors. The takeover delay $(p<0.02)$, the GCS

${ }^{1}$ O84 Noradrenaline dose levels predict futility of liver transplantation in patients with paracetamol-overdose-related acute liver failure (ALF). http:// onlinelibrary.wiley.com/doi/10.1002/hep.28236/full.
( $p<0.01)$, the presence of pupillary anomaly $(p<0.01)$, the presence of associated lesions $(p=0.01)$, the ISS score $(p<0.01)$ and the CT scan score $(p<0.01)$ were considered as outcome indicators in univariate analysis. A Kaplan-Meier survival curve showed a correlation between the age, the takeover delay and the outcome of the patients. Correlation with secondary systemic insults was not established in our study. Multivariate analysis showed a correlation between the Glasgow coma scale and mortality.

Conclusion The profile of TBI in ICU of Zaghouan is similar to data in recent literature. The prognostic factors identified in our study were: the mean delay of management, the GCS, the presence of pupillary anomalies, the presence of associated injuries, the ISS and the CT score. In order to improve the outcome of TBI, we need to use multimodal monitoring.

\section{Competing interests None.}

P2

\section{Stroke prognosis in the ICU of the regional hospital of Bizerte} and withhold and withdrawal treatment decisions

Hatem Ghadhoune', E. Rachedi ${ }^{1}$, J. Guissouma' ${ }^{1}$ A. Ben Slimene' ${ }^{1}$ W.

Azzeza ${ }^{1}, H$. Brahmi ${ }^{1}$, H. Elghord

${ }^{1}$ Service de réanimation médicale, Hôpital universitaire de Bizerte, Bizerte, Tunisia

Correspondence: Hatem Ghadhoune - ghadhoune@yahoo.fr

Annals of Intensive Care 2016, 6(Suppl 1):P2

Introduction The short-term prognosis of stroke in ICU remains very reserved. Therefore, the management of this disease remains controversial. Few Tunisian studies have examined this subject, and no withhold and withdrawal treatment decisions were established in the severe cases. The purpose of this study is to explore predictors of stroke mortality in intensive care.

Patients and methods Sixty patients with stroke confirmed by $\mathrm{CT}$ scan (either intracerebral hematoma or ischemic stroke) were included in this study from patients admitted into the ICU (six beds) of the regional hospital of Bizerte between January 2010 and August 2015. The socio-demographic, anamnestic, clinical, biological and evaluative data were evaluated by univariate and multivariate analysis.

Results The median age was 60 years (interquartile range 54-66). Twenty $(33.3 \%)$ of the patients were women with a sex ratio equal to 2 . The diagnosis of recurrent stroke was retained in $20(33.3 \%)$ patients. Sixty percent of patients had an ischemic stroke. IGS2 and APACHE 2 mean were, respectively, $41.57 \pm 15$ and $19.5 \pm 6.7$. One hundred percent of patients with GCS $<6$ died. The use of mechanical ventilation was required in $76.7 \%$ of patients $(38 / 60)$. The length of stay was $13.4 \pm$ of 18 days. Mortality was $71.7 \%$.

In univariate analysis, factors associated with death were a high IGS2 $(p=0.009)$ and APACHE $2(p=0.01)$, a low coma scale $\left(p<10^{-3}\right)$, a low $\mathrm{PaO} 2 / \mathrm{FiO} 2$ ratio $(p=0.003)$, mechanical ventilation $(p=0.002)$ and septic shock appeared during evolution $(p=0.04)$. In multivariate analysis, a low coma scale and the onset of septic shock during the evolution were the only factors associated independently with increased ICU mortality among patients admitted for stroke.

Discussion Withhold and withdrawal treatment decisions in severe stroke are related mainly to neurological status and advanced age (1). The absence of reversible complications and a deep coma (GCS $<9)$ shall qualify the usefulness of the resuscitation (2). Also, the use of mechanical ventilation is associated with high mortality (3). In this study, advanced age and mechanical ventilation were not associated with a significant mortality. For against a Glasgow score of $<6$ is associated with $100 \%$ mortality. This could be a major criterion in therapeutic decisions limiting serious stroke and help Tunisian health authorities to improve the standards established in the management of severe stroke.

Conclusion The severity of the stroke at admission is related to the depth of the coma which depends on withhold and withdrawal treatment decisions. However, prognosis mainly relies on the improvement in the quality of stroke management and the prevention of its complications. 
Competing interests None.

\section{References}

1. Crozier S, Santoli F, Outin H, Aegerter P, Ducrocq X, Bollaert PÉ. Severe stroke: Prognosis, intensive care admission and withhold and withdrawal treatment decisions. Rev Neurol (Paris). 2011 Jun-Jul;167(6-7):468-73.

2. Management of stroke in emergencies. Standards of Tunisian minister of public health. Mai 2006

3. Crozier S. Withdrawing and withholding treatments in acute severe old stroke patients. La revue du Praticien. vol. 62, n² 9, 2012, pages $1243-1245$

P3

Brain death predictive factors for traumatic brain injury

Ahmed Youssef Kada ${ }^{1}$, Radia Chikh ${ }^{1}$, Roza Slimani ${ }^{1}$, Kheireddine.a

Bouyoucef

${ }^{1}$ Neurosurgery, CHU de Blida, Blida, Algeria

Correspondence: Ahmed Youssef Kada - aykada@gmail.com

Annals of Intensive Care 2016, 6(Suppl 1):P3

Introduction Encephalic death is one of the traumatic brain injury consequences. The goal of our study case-witnesses was to determine the predictive factors for them.

Patients and methods All patients hospitalized for traumatic brain injury are prospectively included between September 2008 and September 2013, and the only criterion of exclusion was the presence of a confusing factor. If they evolved to encephalic death, we paired them with deceased ones, but not of neurological cause.

Age, sex, Glasgow score, tomodensitometric classification (Marshall classification), medical transport and kidney injury (uremia) were collected.

We carried out an univariate then multivariate analysis to identify predictive factors for encephalic death post-traumatic.

Results A total of 95 cases and 95 witnesses were included in our study. All profited from a therapeutic without limitation of care. The average score at admission was $5.40 \pm 2.29$ at the cases and $6.96 \pm 3.45$ at the witnesses $(p=0.01)$, middle age being of $25.88 \pm 15.08$ years at the cases and of $38.71 \pm 21.53$ years at the witnesses $(p=0.01)$.

Male sex $(p=0.004$; OR 3$)$, transport $>60 \mathrm{mn}(p=0.02$; OR 2$)$, age $<50$ years ( $p=0.0001$; OR 6$)$, a class IV on the scannographic classification of Marshall (Traumatic Coma Data Bank) ( $p=0.01$; OR 5.47) and high uremia ( $p=0.01$; OR 0.01) were found as predictors of encephalic death in univariate analysis.

In multivariate analysis, predictors were age lower than 50 years $(p=0.000$; OR 8.9) and a unilateral mydriasis ( $p=0.025$; OR 4.026).

Discussion The aim of the study was to provide predictive clinical elements of passage to encephalic death. Two risk factors are come out from this analysis significantly, age factor (OR 8.9) and unilateral mydriasis (OR 4). These elements will make it possible to anticipate an unfavourable evolution and to adapt the therapeutic protocol consequently.

Conclusion These elements will make it possible to anticipate an unfavourable evolution and to adapt the therapeutic protocol consequently.

\section{Competing interests None.}

\section{References}

1. Facteurs pronostics de passage en état de mort cérébrale chez les patients en coma grave admis en réanimation dans le cadre d'une réanimation d'attente pour prélèvement d'organes. J.-B. lascarrou, C. ageneau, T. Fradin, S. Valot, M. Fiancette, I. Vinatier, M. lemarié, J.-C. lacherade, C. lebert, a. Cottereau, a. Yehia, e. Clémenti, J. Reignier, I. Martin lefevre. Réanimation (2012) 21:S21-S24.

2. Facteurs de risque d'évolution vers la mort encéphalique chez les patients hospitalisés en réanimation pour hématome intracérébral.V. Jouffroy, E. Brocas, A. Rodrigues, B. Deschamps, D. Perrin-Gachadoat. Prélèvement d'organes/Annales Françaises d'Anesthésie et de Réanimation 32S (2013) A250-A254.
P4

Guillain-Barré syndrome in intensive care unit: epidemiology and prognostic factors: analysis of $\mathbf{3 0}$ patients

Kais Regaieg ${ }^{1}$, Chtara Kamilia', Najeh Baccouch², Olfa Turki ${ }^{1}$, Anis Chaari ${ }^{1}$, Hmida Chokri Ben ${ }^{1}$, Mabrouk Bahloul ${ }^{1}$, Mounir Bouaziz ${ }^{3}$

${ }^{1}$ Réanimation polyvalente, Faculté de médecine de Sfax, Sfax, Tunisia; ${ }^{2}$ Réanimation polyvalente, CHU Habib Bourguiba, Sfax, Tunisia; ${ }^{3} \mathrm{lcu}, \mathrm{CHU}$

Habib Bourguiba, Sfax, Tunisia

Correspondence: Chtara Kamilia - kamilia.chtaraelaoud@gmail.com Annals of Intensive Care 2016, 6(Suppl 1):P4

Introduction Several complications related to Guillain-Barré syndrome (GBS) can lead to admission in ICU. The objective of our study is to describe clinical and laboratory characteristics of patients with GBS in ICU and identify predictive factors for poor prognosis in this patients group.

Patients and methods Retrospective study included all patients over a period of 8 years with GBS confirmed by an electromyogram. Categorical variables were expressed as frequencies and percentage, whereas quantitative variables were expressed as media [quartiles] Two groups were compared in univariate analysis: survivors and deceased. Multivariate analysis was performed to identify the independent predictors of poor prognosis.

Results During the study period, 30 patients were admitted for GBS. The median age was 42.5 [26.3-59]. The sex ratio (M/W) was 0.6 The main indication to ICU admission was insufficient acute respiratory $(86.7 \%)$, shock (10\%) and impaired consciousness (13.3\%). The median SAPSII was 33.5 [29.5-39] points. The median SOFA score was 3 [2-5] points. The median SAPSIII score was 52 [50.3-56.5] points. The motor deficit was present in all patients. An associated sensory loss was observed in $76.7 \%$ of cases. An anomaly of the ocular mobility was observed in $6.7 \%$ of cases. Autonomic disorders were found in only one patient (3.3\%). A albumin-cytological dissociation was observed in $76.7 \%$ of cases. The electromyographic study identified axonal damage in $36.7 \%$ of cases. The mechanical ventilation was reported in 18 patients (60\%) for a median duration of 19 [8-31] days. Noninvasive ventilation has been attempted unsuccessfully in two patients. A tracheotomy was performed in nine patients (50\% of ventilated patients). Plasmapheresis was performed in 21 patients $(70 \%)$, while the immunoglobulins were administered in six patients (20\%). The ICU stay was complicated by nosocomial pneumonia in 13 patients (43.3\%) and pulmonary embolism in two patients $(6.7 \%)$. The duration median ICU stay was 11.5 [4.8-21.8] days. ICU mortality was $36.7 \%$. In multivariate analysis, only the occurrence of nosocomial pneumonia was associated with a poor prognosis (OR 44.6; $95 \% \mathrm{Cl}$ [3.5-560]; $p=0.003)$.

Conclusion GBS is a serious disease involving life threatening. Mortality of severe forms seems to be conditioned by serious complications occurring during the ICU stay, especially pneumonia nosocomial.

\section{Competing interests None.}

P5

Eclampsia: epidemiological and clinical study about 194 cases Fatma Kaaniche Medhioub ${ }^{1}$, Najeh Baccouch ${ }^{2}$, Manel Zekri ${ }^{2}$, Kais Rgieg ${ }^{2}$, Chokri Bhimada ${ }^{3}$, Bouaziz Mounir ${ }^{3}$

${ }^{1}$ Faculté de médecine de Sfax, Sfax, Tunisia; ${ }^{2}$ Réanimation polyvalente, CHU Habib Bourguiba, Sfax, Tunisia; ${ }^{3}$ Réanimation polyvalente, Faculté de médecine de Sfax, Sfax, Tunisia

Correspondence: Fatma Kaaniche Medhioub - fatma_kaaniche@yahoo. $\mathrm{fr}$

Annals of Intensive Care 2016, 6(Suppl 1):P5

Introduction Eclampsia is a serious paroxysmal gravidic-puerperal accident complicates vascular and renal conditions of pregnancy. It is characterized by convulsive seizures that can be fatal to both mother and fetus [1]. The aim of our work is to study the epidemiological and clinical characteristics in obstetric patients admitted in ICU for eclampsia 
Patients and methods Our study is retrospective and includes all cases of eclampsia collected in the medical ICU of the University Hospital of Sfax over a period of 20 years (1993-2012)

Results During our study period, 725 patients were hospitalized in the ICU. Among these patients, 194 presented eclampsia (26.7\%). Pregnancy has been followed medically in 79 women (40.6\%). The average age of patients was $28 \pm 4.3$ years. The average parity was $1.7 \pm 1.2$ with a primiparity from $49.5 \%$. The mean gestational age was $34.8 \pm 2.9$ weeks. HELLP syndrome was noted in 30 cases $(15.5 \%)$ placental retro hematoma in 15 cases $(7.87 \%)$, acute renal failure in 43 cases $(22.3 \%)$, disseminated intravascular coagulation in 27 cases $(14 \%)$, acute pulmonary edema in 17 cases $(8.8 \%)$ and state of shock in seven cases (3.6\%). The cesarean delivery was urgent in 161 cases (83\%). Mechanical ventilation more than $24 \mathrm{~h}$ was necessary in 79 cases $(40.7 \%)$, and 43 patients $(22.2 \%)$ received a transfusion (packed red blood cells and/or fresh frozen plasma and/or platelet button). Hemodialysis was carried in two cases (1\%). Antihypertensive therapy was initiated in 171 patients, magnesium sulfate in 190 patients and anticonvulsant therapy in 187 patients. The average length of ICU stay was $2.7 \pm 2.2$ days. Mortality hit nine patients $(4.6 \%)$ and 25 fetuses (12.8\%).

Conclusion Eclampsia is a serious accident complicating gestational hypertension. It particularly affects the primipara. Obstetric medical management including emergency cesarean section and adequate reanimation reduces maternal and fetal mortality.

\section{Competing interests None.}

\section{Reference}

1. Pottecher T, Luton D, Zupan V, Collet M. Réanimation des formes graves de prééclampsie. Conférence d'expert communes SFAR/CNGOF/SFMP/ SFNN; 2009.

P6

Neuroimagery in eclampsia and preclampsia

Chaigar Mohammed Cheikh ${ }^{1}$, I. Moussaid ${ }^{1}$, Kamal Belkadi ${ }^{1}$, O. Ghazaoui ${ }^{1}$ S. El Youssoufi', S. Salmi ${ }^{1}$

${ }^{1}$ Anesthesie reanimation, $\mathrm{CHU}$ Ibn Rochd Casa, Casablanca, Morocco Correspondence: Chaigar Mohammed Cheikh - chaigarmed@gmail. com

Annals of Intensive Care 2016, 6(Suppl 1):P6

Introduction Eclampsia is a severe complication of preeclampsia liable to an important maternal and fetal mortality, principally in developing countries [1]. The aim of our study was to describe neuroradiological lesions in preeclampsia/eclampsia in obstetric ICU of our hospital and to study clinical and therapeutic characteristics of this pathology as well as the outcome of our patients.

Patients and methods We conducted a retrospective descriptive study spread over 5 years, from January 1, 2010, to December 31, 2014, including all patients with posterior reversible encephalopathy syndrome (PRES), hemorrhagic stroke and cerebral ischemia occurring eclampsia or preeclampsia and hospitalized in obstetric ICU department.

Results Twenty-seven patients were included in the study. The mean age was 28.18 years. Only five patients had a well-attended pregnancy. The average systolic blood pressure was $158 \mathrm{mmHg}$, and the mean diastolic blood pressure was $96.29 \mathrm{mmHg}$. The mean Glasgow score at ICU admission was 11.18 , and the observed neurological deficits were: hemiparesis (22\%), blindness (19\%), hemiplegia (7\%) and facial paralysis (7\%). Complications were: HELLP syndrome (29.6\%), placental hematoma ( $25.9 \%)$, acute renal failure ( $3.7 \%)$ and pulmonary edema (3.7\%). All patients had conventional neuroradiological exploration showing damage in all cases. CT showed two cases of ischemia (7.4\%), seven cases of cerebral edema (six cases of PRES: $22.2 \%$; one case of diffuse edema: $3.7 \%$ ) and three cases of hemorrhagic stroke (11.11\%). MRI diffusion sequence approved in 15 patients has showed a PRES in ten cases $(37.03 \%)$, cerebral ischemia in two cases (7.4\%), one case of hemorrhagic stroke and two cases of diffuse edema. The main mode of delivery was cesarean section under general anesthesia for 22 patients $(81.48 \%)$. All patients had received antihypertensive treatment and anticonvulsant treatment. The outcome was critical in one patient how died from a septic shock. Perinatal mortality was about $25.9 \%$.

Conclusion From this study, improving maternal and fetal prognosis requires:

- Prevention, with early and regular monitoring of pregnant women;

- An appropriate treatment of pregnancy hypertension;

- And management of preeclampsia and eclampsia before complications.

\section{Competing interests None.}

\section{Reference}

1. o.collange et Al. Eclampsie. AFAR2010.

\section{P7}

Interest of brain oxygen tension measurement $\left(\mathrm{PbrO}_{2}\right)$ in the diagnosis of cerebral vasospasm after aneurismal subarachnoid hemorrhage

Elodie Lang ${ }^{1}$, Stephane Welschbillig ${ }^{1}$, Fabian Roy-Gash' ${ }^{1}$, Nicolas Engrand ${ }^{1}$ ${ }^{1}$ Département d'anesthésie - réanimation, Fondation Ophtalmologique Adolphe de Rothschild, Paris, France

Correspondence: Stephane Welschbillig - swelschbillig@fo-rothschild.fr Annals of Intensive Care 2016, 6(Suppl 1):P7

Introduction Delayed brain ischemia as a result of cerebral vasospasm is the most common cause of death and disability after aneurysmal subarachnoid hemorrhage (SAH). Because clinical signs cannot be detected for sedated patients, monitoring tools have been developed. As an example, transcranial Doppler is an easily bedside method to detect middle cerebral artery vasospasm [1], but fails in the detection of others intracranial vessels spasm. Tissue brain oxygen tension measurement $\left(\mathrm{PbrO}_{2}\right)$ has been described as a useful monitoring tool to detect tissue hypoxia before irreversible neuronal damage in trauma brain-injured patients [2]. The goal of the study was to determinate the accuracy of $\mathrm{PbrO}_{2}$ to detect cerebral vasospasm after severe aneurysmal SAH.

Patients and methods We performed a retrospective observational study on ten patients admitted in our institution from January 2014 to September 2015 for SAH and monitored with $\mathrm{PbrO}_{2}$ (Lycox). $\mathrm{PbrO}_{2}$ data were recorded retrospectively. Cerebral vasospasm was assessed with angiography and treated with in situ vasodilators. $\mathrm{PbrO}_{2}$ before and after cerebral spasm angioplasty was defined "with" and "without" cerebral spasm values of $\mathrm{PbrO}_{2}$. Continuous variable $\mathrm{PbrO}_{2}$ was presented mean (SE) and compared with paired $t$ test.

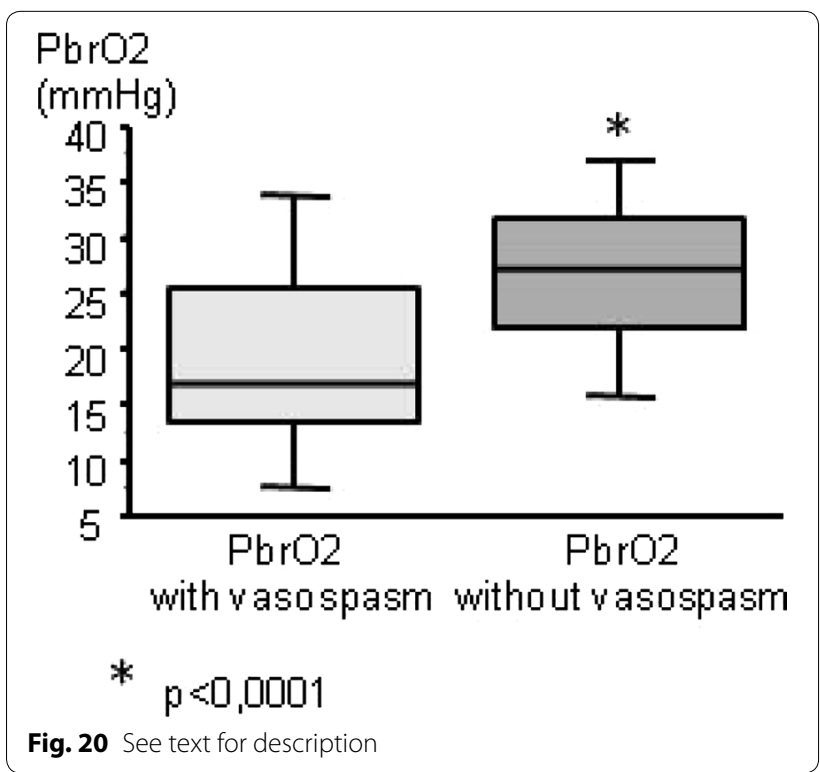


Results Ten comatose SAH patients (WNFS IV) were included, and 48 angiographies were performed for cerebral vasospasm. $\mathrm{PbrO}_{2}$ probe was inserted in all patients 7 (3-14) days after SAH without any complication. The mean duration of $\mathrm{PbrO}_{2}$ monitoring was 8 (4-16) days. Two vasospasm episodes were excluded because $\mathrm{PbrO}_{2}$ probe was located in the opposite side of the vasospasm area. In four cases of vasospasm, the angioplasty failed and it was impossible to obtain the "without spasm" value of $\mathrm{PbrO}_{2}$.

In cerebral vasospasm, mean value of $\mathrm{PbrO}_{2}$ was $19(9) \mathrm{mmHg}$ but with a large spread of values. After angiographic treatment of vasospasm, mean value of $\mathrm{PbrO}_{2}$ was significantly higher and reached $27(9) \mathrm{mmHg}(p<0.0001)$ (Fig. 20). Despite a wide overlap between the two groups of $\mathrm{PbrO}_{2}$ values, we could observe that a $\mathrm{PbrO}_{2}$ value lower than a threshold value of $22 \mathrm{mmHg}$ predicted vasospasm with both sensibility and specificity of $75 \%$, and when $\mathrm{PbrO}_{2}$ value was under $11 \mathrm{mmHg}$, all patients had a cerebral vasospasm.

Conclusion Continuous monitoring of $\mathrm{PbrO}_{2}$ could have an interest to predict the cerebral vasospasm and to avoid delayed brain ischemia in comatose patients after aneurismal subarachnoid hemorrhage.

\section{Competing interests None.}

\section{References}

1. Symptomatic vasospasm diagnosis after subarachnoid hemorrhage: evaluation of transcranial Doppler ultrasound and cerebral angiography as related to compromised vascular distribution. Suarez JI, Qureshi Al, Yahia AB, Parekh PD, Tamargo RJ, Williams MA, Ulatowski JA, Hanley DF, Razumovsky AY. Crit Care Med 2002;30(6):138-55

2. Brain oxygen monitoring: in vitro accuracy, long-term drift and responsetime of Licox and Neurotrend sensors. Hoelper BM, Alessandri B, Heimann A, Behr R, Kempski O. Acta Neurochir (Wien). 2005 Jul;147(7):767-74.

P8

Noninvasive ventilation: an alternative to the intubation during myasthenic crisis

Mathilde Perrin ${ }^{1}$, Fabian Roy-Gash², Stephane Welschbillig ${ }^{3}$, Jean-Michel Devys $^{1}$

175019, Fondation Ophtalmologique Adolphe de Rothschild, Paris, France; ${ }^{2}$ neuro intensive care, Fondation Ophtalmologique Adolphe de Rothschild, Paris, France; ${ }^{3}$ Intensive care unit, Fondation Ophtalmologique Adolphe de Rothschild, Paris, France

Correspondence: Mathilde Perrin - mathildeperrin75@gmail.com Annals of Intensive Care 2016, 6(Suppl 1):P8

Introduction Myasthenic crisis is defined by acquired myasthenic gravis severe enough to require intubation. The actual recommendations are to intubate patients who have a vital capacity $<15 \mathrm{~mL} / \mathrm{kg}$, or $30 \%$ (1) while waiting for treatment by intravenous immunoglobulins to be effective.

The use of noninvasive ventilation (NIV) to prevent intubation was described in only two retrospective studies $(2,3)$. NIV was successful for $20-25 \%$ of patients showing myasthenic crisis. We wanted to describe our experience of NIV in myasthenic crisis.

Patients and methods This is an observational prospective study from 01.11.2013 to 01.03.2015. Patients included were assisted with NIV when they showed a single myasthenic crisis, an Osserman score $<70$ and a vital capacity (VC) $<50 \%$. Durations of NIV were adapted to the patient's comfort.

The NIV failure was: inhalation, intubation, hypoxemia, major asthenia. Every day, the demographics and treatment modalities, pulmonary complications, intubation outcomes, duration of NIV and duration of stay in intensive care were recorded.

Results Eight patients were included. The median age was 53 (1681 ) years. At the admission in intensive care unit, the median Osserman's score was 50 (23-68) and VC $38 \%$ (22-43\%). Median duration of NIV was 4 days (2-10), and median ICU length of stay was 7 days (4-13). Nobody was intubated. Five patients with deglutition troubles did not show pulmonary complications.
Conclusion In this group, NIV was always successful. Systematic use of NIV seems to prevent muscular asthenia caused by gravis myasthenic crisis and avoids patient's respiratory worsening. NIV in intensive care unit seems to be a good way to not intubate patients with gravis myasthenic crisis (Fig. 21).

\section{Competing interests None.}

\section{References}

1. Reanimation 14 (2005) 118-125.

2. Neurocrit care $2009 ; 10(1): 35-42$

3. Arch Neurol 2008 Jan; 65(1):54-8.

\section{P9} Interest of the transcranial Doppler ultrasound in the management of severe traumatic brain injury

Soumia Benbernou', Houria Mokhtari-Djebli', Sofiane Ilies ${ }^{1}$, Khalida Bouyacoub ${ }^{2}$, Abdelkader Azza ${ }^{2}$

1 Urgences Medicales Chuoran, Faculté de médecine d'Oran, Oran, Algeriav; ${ }^{2}$ Urgences Medicales, Chu Oran, Oran, Algeria

Correspondence: Soumia Benbernou - gsoumia@hotmail.com Annals of Intensive Care 2016, 6(Suppl 1):P9

Introduction After a serious traumatic brain injury (TBI), the occurrence of intracranial hypertension (intracranial hypertension) is associated with an increased risk of morbidity and mortality. The appearance of intracranial hypertension at an early stage worsens the prognosis, and thus, through the clinic, brain $\mathrm{CT}$, monitoring intracranial pressure and evaluation of cerebral circulation by transcranial Doppler ultrasound treatment can be given quickly. The objective of the study was to compare the advantage brought by one or other of the means used for the diagnosis and monitoring of intracranial hypertension.

Patients and methods The work was exhibited/performed at the ICU of the UMC CHUOran.

This is a prospective study over 2 years (2012 and 2013) of 59 patients who received simultaneously a monitoring intracranial pressure (ICP) and a transcranial Doppler monitoring by the TCDU. Simultaneous recording of intracranial pressure, cerebral perfusion pressure, the pulsatility index, systolic and diastolic velocity has been achieved.

Results The number of STBI compiled was 59 . The study population was young, the median age was 29 years, and $80 \%$ were trauma patients. No pupillary changes are observed for higher numbers of ICP $>45 \mathrm{mmHg}$. There is a linear relationship between the ICP and the pulsatility index since the correlation coefficient was 0.43 and $p=0.019965$ and between the CFC and the pulsatility index since the correlation coefficient: $r^{2}=0.60$ and $p=0.000613$. The literature is contradictory on this issue.

Discussion A study was conducted in 1994 by Boishardy and collaborators in France, out of a total of ten serious head trauma whose age was between 17 and 37 years and that benefited dice admission and then twice a day, of a simultaneous measurement SV, DV, PI and ICP by means of an intraparenchymal catheter to show a significant correlation between mean ICP and PI $r=0.837$ and between the PI and CPP $(r=0.563 ; p<0.001)[1]$.

By against the US study conducted by Thomas in California on a group of 99 severe TBI patients showed that the correlation between ICP and PI was classified as moderate to zero [2].

Conclusion All severe TBI monitoring means have their place, with superior non-invasive tests on simple clinical surveillance, and indicate the need for the installation of the monitoring of the ICP in due time, since many patients can develop at any time during their stay an ICHT.

\section{Competing interests None.}

\section{References}

1. Boishardy, J.C. Granry, J.P. Jacob, N. Houi, D. Fournier, A. Delhumeau. Intérêt du doppler transcrânien dans la prise en charge du traumatisés crâniens graves. Annales Françaises d'Anesthésie et de Réanimation, 13: $172-176,1994$ 


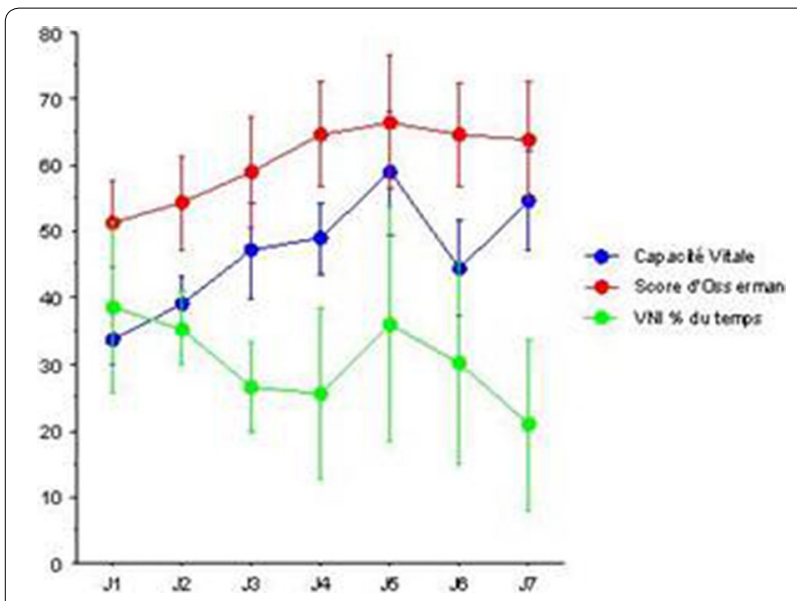

Fig. 21 See text for description

2. Thomas C. Glenn, Arun K. Sherma, David L. McArthur, Xiao Hu, Christopher R. Hanuscin, Mehjabeen S. Furreedan, David A. Hovda, Paul M. Vespa, and Neil A. Martin The Linear Relationship Between Transcranial Doppler Pulsatility Indices and Intracranial Pressure Is Influenced by Traumatic Brain Injury and Vasospasm University of California Neurotrauma Initiative and NIH/NINDS 1P01NS058489-01.

\section{P10}

VV-ECMO on ARDS: toward a lower anticoagulation ratio?

Elie Zogheib ${ }^{1}$, Joseph Nader ${ }^{2}$, Léonie Villeret ${ }^{1}$, Mathieu Guilbart ${ }^{1}$, Patricia Besserve ${ }^{1}$, Thierry Caus ${ }^{2}$, Hervé Dupont ${ }^{1}$

${ }^{1}$ Réanimation cardio thoracique et vasculaire, CHU Amiens-Picardie, Salouel, France; ${ }^{2}$ Chirurgie cardiaque, CHU Amiens-Picardie, Salouel, France

Correspondence: Elie Zogheib - eliezogheib1@yahoo.fr

Annals of Intensive Care 2016, 6(Suppl 1):P10

Introduction Veno-venous ECMO (VV-ECMO) is a rescue therapy in refractory ARDS. Despite technological progress and enhanced biocompatibility, anticoagulation with unfractionated heparin remains the most often used as recommended. Bleeding complications remain a main cause of increasing morbidity and mortality in patient treated with VV-ECMO. The aim of our study was to study the effectiveness of the dosage of unfractionated heparin (aPTT, anti-Xa activity) in patients on VV-ECMO for ARDS

Patients and methods We performed a retrospective observational study stretching from 2008 to the first quarter 2015. Patient's baseline characteristics, the dose of UFH anticoagulation and biological data of anticoagulation (aPTT, anti-Xa activity, Hemochron ACT, ATIII, fibrinogen) were collected. We studied the incidence of hemorrhagic or thromboembolic events under UFH anticoagulation.

Results Forty-eight patients with ARDS requiring VV-ECMO were included, with a majority of men [35 (63.6\%) vs $20(36.4 \%)$ $(p<0.5)$. The mean age was 47 years ( \pm 15 years). In $35.4 \%$ of cases, a double cannula Avalon common type used (mean diameter of $27 \mathrm{~GB}$ ), while the others had a femoro-jugular cannulation with an average diameter of femoral vein of $25 \mathrm{~GB}$. UFH anticoagulant was used in most patients. During installation of VV-ECMO, $33 \%$ of patients did not have unfractionated heparin (UFH). The average value of aPTT was $35 \pm 8 \mathrm{~s}$ and the anti-Xa was $0.14 \pm 0.06$. The average of the Hemochron was $171 \pm 23 \mathrm{~s}$. The values of UFH administered averaged $778 \pm 601$ IU/h IVSE. Bleeding complications were noted in $56.25 \%$ of cases. None of our patients had
ECMO thrombosis cannulas or oxygenator. Thirty-nine percent of patients had positive anti-PF4, and only 11 patients had a change in anticoagulation.

Conclusion Anticoagulation used in our study appears lower than recommendations without increasing thromboembolic complications, with persistence of a high rate of bleeding complications with or without a direct connection with ECMO. These results would reinforce the possibility of using ECMO with less or no anticoagulant?

Competing interests None.

Reference

1. Extracorporeal life support for patients with acute. Ann Intensive Care 2014 May 24;4:15. doi: 10.1186/2110-5820-4-15. eCollection 2014

P11

Inter-hospital transportation of patients implanted with veno-venous ECMO: Experience of the Pitié-Salpêtrière mobile ECMO team

Nicolas Brechot ${ }^{1}$, Ciro Mastroianni ${ }^{2}$, Matthieu Schmidt ${ }^{1}$, Francesca Santi ${ }^{2}$, Guillaume Lebreton ${ }^{2}$, Charles-Edouard Luyt ${ }^{1}$, Jean Louis Trouillet ${ }^{1}$, Ania Nieszkowska1', Guillaume Hékimian¹, Leprince Pascal², Jean Chastre1, Alain Combes $^{1}$

${ }^{1}$ Service de réanimation médicale, Groupe Hospitalier Pitié-Salpêtrière, Institut de Cardiométabolisme et Nutrition, Paris, France. ${ }^{2}$ Service de chirurgie cardio-vasculaire et thoracique, Groupe Hospitalier Pitié-Salpêtrière, Paris, France

Correspondence: Nicolas Brechot - nicolas.brechot@aphp.fr

Annals of Intensive Care 2016, 6(Suppl 1):P11

Introduction The way to organize veno-venous circulatory assistance in France is still a matter of debate. Particularly, data on the efficiency and safety of mobile ECMO teams are still scarce. The aim of this study was to evaluate the safety of the mobile ECMO team in our center.

Patients and methods In this retrospective monocenter study, the outcome of patients implanted with a veno-venous ECMO by the mobile ECMO team of la Pitié-Salpêtrière hospital from 2009 to 2012 was compared with patients who were implanted on site. Results are expressed as median (25th-75th IQR) or $n(\%)$.

Results A total of 118 patients were implanted by the mobile ECMO team and 39 on site. Both groups were quite comparable and consisted in very severe patients, reflected by a high SAPS-II score of 71 (61-81) and $\mathrm{a} \mathrm{PaO}_{2} / \mathrm{FiO}_{2}$ of 58 (50-73). Major complications at ECMO implantations were identical between both groups [3 $(2.5 \%)$ in the mobile ECMO group vs. 2 (5.1\%) in the onsite group, $p=0.6]$. All transportations were performed by the SAMU with ground facilities, in the presence of a specialized perfusionist. Median time between call and ECMO implantation was $192 \mathrm{~min}$ (154-225). Distances ranged from 0.5 to $241 \mathrm{~km}$ (median 15 (6-25)), for a median time of $35 \mathrm{~min}$ (25-35). No major incident was observed during transportation, except $1(0.8 \%)$ patient who died from hemodynamic deterioration. Eight $(6.8 \%)$ patients in the mobile group needed a subsequent switch to venoarterial ECMO due to hemodynamic deterioration, compared with two $(5.1 \%)$ in the onsite group ( $p=0.7)$. ICU mortality was comparable, $46.6 \%$ in the mobile group versus $53.8 \%$ in the onsite group, $p=0.5$. In multivariable analysis, implantation by the mobile ECMO team was not associated with ICU mortality (OR 1.1 [0.4-1.7], $p=0.85$ ). Severe ECMO-related complications were frequent but comparable between groups [63 (53.4\%) in the mobile group vs. 21 (53.8\%) in the onsite group, $p=1.0]$. Particularly, the rate of subsequent recannulation, bleedings or infection at the insertion site and bacteremia did not differ between groups.

Conclusion Implantation of a veno-venous ECMO by a mobile ECMO team and subsequent transportation of the patient to an experienced ECMO center appears to be feasible and safe.

Competing interests None. 
P12

Evolution of tidal volume and compliance

under pressure-controlled mode during the first $\mathbf{2 4}$ hours of ECMO ARDS patients transported to a referral center

Hadrien Roze ${ }^{1}$, Benjamin Repusseau', Jean-Christophe Richard ${ }^{2}$, Virginie Perrier $^{1}$, Alexandre Ouattara ${ }^{1}$

${ }^{1}$ Sar 2, unité réanimation thoracique greffe, $\mathrm{CHU}$ de Bordeaux, Bordeaux, France; ${ }^{2}$ Samu 74 et service des urgences, Centre Hospitalier Annecy Genevois, Annecy, France

Correspondence: Hadrien Roze - hadrien.roze@chu-bordeaux.fr Annals of Intensive Care 2016, 6(Suppl 1):P12

Introduction Extracorporeal membrane oxygenation (ECMO) is proposed for patients with severe acute respiratory distress syndrome (ARDS), which may be defined as not being effectively and/or safely treated by current clinical types of mechanical ventilation. Once on ECMO, it is immediately possible to limit stress and strain with a volume- and pressure-limited protective ventilation strategy. This study focuses on the mechanical ventilation during the first $24 \mathrm{~h}$ of ARDS patients transferred with an ECMO mobile team (UMAC) to the referral center of Aquitaine region.

Patients and methods All data were prospectively recorded, $1 \mathrm{~h}$ before ECMO $(\mathrm{H}-1)$, at ECMO start before transportation (Humac), at $1 \mathrm{~h}(\mathrm{H} 1)$ and $24 \mathrm{~h}(\mathrm{H} 24)$ in the referral center. Patients were transported by road with SAMU 33 using a Cardiohelp (Maquet, Sweden) and a ventilator Elisé 350 (ResMed, Australia). During the first $24 \mathrm{~h}$ under ECMO, patients were paralyzed and ventilated with a pressurecontrolled mode targeting a plateau pressure of $24 \mathrm{cmH}_{2} \mathrm{O}$, a respiratory frequency of 12 and a PEEP of $10 \mathrm{cmH} 2 \mathrm{O}$. Results are expressed as median (interquartile range $25-75 \%$ )

Results We analyzed 15 ARDS patients, between 2013 and 2014, requiring veno-venous ECMO. Median age was 35 (30-55) years; IGS2 was 50 (43-59). At $\mathrm{H}-1, \mathrm{PaO}_{2} / \mathrm{FiO}_{2}$ was 49 (42-54), and thoracopulmonary compliance (Ctp) was $16(13-25) \mathrm{ml} / \mathrm{cmH}_{2} \mathrm{O}$. Between $\mathrm{H}-1$ and Humac, protective ventilation decreased driving pressure and VT from 25 (16-27) to $12(12-15) \mathrm{cmH} 2 \mathrm{O}$ and $6.4(5.6-7.1)$ to $3.8(2.5-5.8) \mathrm{ml} \mathrm{kg}^{-1}$ PBW, respectively, $p<0.0001$, whereas PEEP did not change $8(8-12)$ versus $12(10-12) \mathrm{cmH}_{2} \mathrm{O}, p=0.12$. At Humac, $\mathrm{Ctp}$ was unchanged with 15 (10-27) $\mathrm{ml} / \mathrm{cmH}_{2} \mathrm{O}$. At $\mathrm{H} 24$, VT and Ctp were significantly lower than Humac $2.6(1.5-3.2) \mathrm{ml} \mathrm{kg}^{-1}$ PBW $p=0.01$ and 11 (8-14) $\mathrm{ml} / \mathrm{cmH}_{2} \mathrm{O}$ $p=0.03$, respectively. At $\mathrm{H} 1$, eight patients $(53 \%)$ had a decrease of VT $>15 \%$ during transportation, median reduction was $-44(19-73) \%$, and VT was $1.5(1.3-3.7) \mathrm{ml} \mathrm{kg}^{-1}$ PBW. No patient responds to alveolar recruitment procedures at H24. Duration of ECMO was 14 (7-24) days, and three patients died in the referral center (20\%) (Fig. 22).

Discussion ECMO allowed immediate reduction in driving pressure with a stable Ctp. We found a significant reduction in VT and Ctp during the first $24 \mathrm{~h}$ and for some patients during transportation. This might be explained by the worsening of ARDS, by alveolar derecruitment due to a pressure-controlled mode (1), by the use of a transport ventilator under extremely low Ctp and curare, by the disconnections of the patient from the ventilators at each steps of transportation or by the airway suctions. Conclusion Limiting driving pressure of severe ARDS under ECMO is of high clinical interest. By nature, pressure-controlled ventilation permits to achieve this goal, but it may also promote derecruitment and affect compliance, especially during patient's transportation.

Competing interests None.

\section{Reference}

1. Rittayamai et al.Chest 2015;148:340-355.

\section{P13}

Extracorporeal dioxide carbon removal $\left(\mathrm{ECCO}_{2} \mathrm{R}\right)$, a French national survey

Benjamin Deniau', Jean-Damien Ricard ${ }^{1}$, Jonathan Messika', Didier Dreyfuss ${ }^{1}$, Stéphane Gaudry ${ }^{1}$

${ }^{1}$ Service de réanimation médico-chirurgicale, CHU Louis Mourier, Colombes, Colombes, France

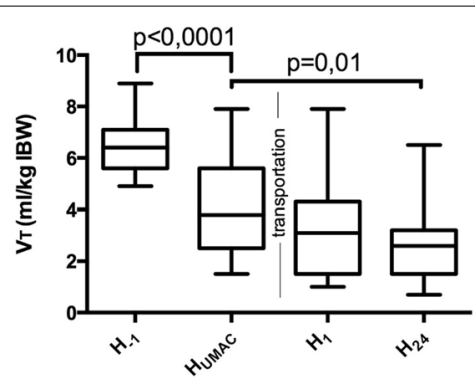

Fig. 22 See text for description

Correspondence: Stéphane Gaudry - stephanegaudry@gmail.com Annals of Intensive Care 2016, 6(Suppl 1):P13

Introduction Extracorporeal dioxide carbon removal $\left(\mathrm{ECCO}_{2} \mathrm{R}\right)$ is an extracorporeal decarboxylation technology described in 1978. Physiological studies showed that $50 \%$ of produced $\mathrm{CO}_{2}$ were eliminated. Potential indications are: ultra-protective mechanical ventilation for acute respiratory distress syndrome (ARDS) and hypercapnic patients at risk of non-invasive ventilation failure. Because of the lack of scientific evidence, $\mathrm{ECCO}_{2} \mathrm{R}$ is not available in the USA. We sought to assess the use of $\mathrm{ECCO}_{2} \mathrm{R}$ in France.

Materials and methods This retrospective, observational study was performed in French intensive care units (ICUs) from January 2010 to January 2015. A phone interview was conducted with French ICUs affiliated to national societies and public and private hospitals registries. Data recorded were the following: use and indications of $E C \mathrm{CO}_{2} \mathrm{R}$, type of $E C C_{2} R$, number of patients treated during the study period, complications associated with the technique, satisfaction rates (in terms of efficacy, tolerance and global) based on a scale [0 (total unsatisfaction)-10 (total satisfaction)], and concomitant use of extracorporeal membrane oxygenation (ECMO) in the unit.

Results A total of 239 French ICUs were contacted ( 147 mixed, 55 medical, 25 surgical, four cardio-thoracic, six paediatric and two neurosurgical ICU). Only three refused to participate. Thirty-five (15\%) ICUs had used $\mathrm{ECCO}_{2} \mathrm{R}$ at least once in the past 5 years, in 303 patients. The most frequently used devices were: iLA ${ }^{\circledR}$ (Novalung) $\left(63 \%\right.$ ) and Hemolung ${ }^{\circledR}$ (Alung) (37\%). The median number of patients treated per ICU was 3 [1-8]. The most frequent indication was ultra-protective ventilation for ADRS (54\%). Other indications were: failure of non-invasive ventilation during chronic obstructive pulmonary disease exacerbation (30\%), weaning from invasive mechanical ventilation in chronic obstructive pulmonary disease patients (12\%) and miscellaneous (4\%).

Among ICUs using $\mathrm{ECCO}_{2} \mathrm{R}$, 22 (63\%) reported at least one complication. The most frequent complications were bleeding (45\%) and membrane failure $(18 \%)$. Satisfaction rates were: in terms of decarboxylation $7.9 \pm 2.4$; tolerance $6.9 \pm 2.6$; and overall satisfaction $6.8 \pm 2.2$. Twenty-one (63\%) of the $35 \mathrm{ICUs}$ using $\mathrm{ECCO}_{2} \mathrm{R}$ also used ECMO. The main reasons for not using $\mathrm{ECCO}_{2} \mathrm{R}$ were the lack of trained staff, unavailability of the device and the lack of scientific evidence (for, respectively, 53, 39 and $17 \%$ of responders). Among 204 ICUs not using $\mathrm{ECCO}_{2} \mathrm{R}, 20(10 \%)$ had the project to start $\mathrm{ECCO}_{2} \mathrm{R}$ in the coming months.

Conclusion These results show that $\mathrm{ECCO}_{2} \mathrm{R}$ is not widely used in French ICUs. The lack of strong scientific data on outcome is probably the main reason behind the limited use of $\mathrm{ECCO}_{2} \mathrm{R}$. French studies currently in progress will help define indications of $\mathrm{ECCO}_{2} \mathrm{R}$ and impact on outcome.

Competing interests None. 


\section{P14}

Levels of microparticles in acute respiratory distress syndrome with veno-venous extra-corporeal membrane oxygenation Christophe Guervilly' ${ }^{1}$ Fanny Klasen ${ }^{2}$, Sami Hraiech'1 , Jean-Marie Forel' Laurent Papazian ${ }^{3}$

${ }^{1}$ Réanimation DRIS, Hôpital Nord APHM, Marseille, France; ${ }^{2}$ réanimation DRIS, Hospital Nord, Marseille, France; ${ }^{3}$ Service de réanimation-détresses respiratoires et infections sévères, Hôpital Nord, Marseille, France Correspondence: Fanny Klasen - fanny.klasen@gmail.com Annals of Intensive Care 2016, 6(Suppl 1):P14

Introduction Veno-venous extra-corporeal membrane oxygenation ( $v v E C M O)$ allows blood oxygenation and $\mathrm{CO}_{2}$ removal for the most severe case of acute respiratory distress syndrome (ARDS). One of the major complications associated with this technique is the occurrence of microthrombi on the ECMO membrane oxygenator which can require the change in circuit. The microparticles (MPs) are proinflammatory and procoagulant nanovesicles derived from circulating cells, and their circulating levels may be elevated in many pathologies associated with inflammatory, thrombotic and infectious disorders. The main objective of our study is to quantify the presence and to characterize the cellular origin of MPs in patients treated by vvECMO for severe ARDS. Secondary objectives are to analyze the kinetic of MPs rates between ECMO application and removal and to establish a link between the need for a circuit change and MPs evolution.

Materials and methods This is a monocentric pilot study, conducted between January 2012 and June 2014, which included patients with severe ARDS treated with vVECMO. We measured total MPs, platelet-, erythrocyte-, endothelial- and leukocyte-derived MPs, coagulation and hemolysis parameters within $6 \mathrm{~h}$ after the ECMO application. The same parameters were measured at the time of the circuit removal (either during weaning or during a circuit change for biological complication or because of death).

Results Nineteen patients were included in the study analysis. Leukocyte-derived $(p<0.0001)$ and erythrocyte-derived $(p=0.04)$ MPs were higher at the admission in patients who died under ECMO compared with those who have been weaned alive. Leukocyte-derived $(p=0.01)$ and endothelial-derived $(p=0.001)$ MPs were higher in patients who needed a circuit change.

Discussion There are subpopulations of MPs associated with the prognosis of patients with severe ARDS treated with veno-venous ECMO. Conclusion This pilot study suggests a potential role of these biomarkers as diagnostic or prognostic factor in the occurrence of complications related to the technique.

Competing interests None.

\section{P15}

In patients under extracorporeal $\mathrm{CO}_{2}$ removal therapy $\left(\mathrm{ECCO}_{2} \mathrm{R}\right)$ for ARDS can we do prone position? Efficiency, stability and safety of the maneuver

Jean Pierre Ponthus ${ }^{1}$, Polysie Ngasseu' ${ }^{1}$, Virginie Amilien', Elise Barsam', Pierre Lehericey ${ }^{1}$, Martial Tchir ${ }^{1}$, Jean Francois Georger ${ }^{1}$

${ }^{1}$ Réanimation polyvalente - surveillance continue, Ctre Hospitalier

Intercommunal de Villeneuve Saint Georges, Lucie et Raymond AUBRAC, Villeneuve-Saint-Georges, France

Correspondence: Jean Francois Georger - georger.chiv@gmail.com Annals of Intensive Care 2016, 6(Suppl 1):P15

Introduction The mechanical ventilation of some patients with ARDS could be facilitated by $\mathrm{ECCO}_{2} \mathrm{R}$ allowing the reduction in blood acidosis and the reduction in tidal volume for the application of the protective ventilation. Prone position (PP) could be used for some patients with $\mathrm{PaO}_{2} / \mathrm{FiO}_{2}<150$. We do not know whether we could associate PP and $\mathrm{ECCO}_{2} \mathrm{R}$ in $\mathrm{ARDS}$ patients

The aim of this study is to describe the feasibility of PP under $E C C_{2} R$, the stability of the parameters of the device and whether we have side effects of the PP under $\mathrm{ECCO}_{2} \mathrm{R}$.

Materials and methods In our intensive care unit of 15 beds with a large experience of $\mathrm{PP}$, we have retrospectively included all sessions of $\mathrm{PP}$ (at least $16 \mathrm{~h}$ of PP) performed on patients under $\mathrm{ECCO}_{2} \mathrm{R}$ therapy between August 2014 and March 2015. We used ILA ACTIVVE ${ }^{\circledR}$ device (NOVALUNG ${ }^{\circledR}$ ) with MINILUNG ${ }^{\circledR}$ membrane and a double-line femoral catheter (NOVAPORT TWIN ${ }^{\circledR} 24 \mathrm{~F}$ ). The gas flow was $10 \mathrm{l} / \mathrm{min}$. For each session, we compared $\mathrm{PaO}_{2} / \mathrm{FiO}_{2}$ and the $\mathrm{PaCO}_{2}$ before and after $1 \mathrm{H}$ of PP. The device records the flow and pressure parameters every $10 \mathrm{~s}$, and we recovered these parameters after each prone position session. For each session, we did the mean of blood flow and drainage pressure $(P 1)$ during a length of $1 \mathrm{~h}$ : during the last hour before $\mathrm{PP}$, the first hour after PP and the last hour before stopping PP. We compared with a Freidman's test, the mean and the coefficient of variation of each parameter to evaluate the stability of the device. We noted all the side effects of the PP (bleeding, decannulation, etc.).

Results We performed nine PP sessions on five patients, one in three patients and three in two patients. The $\mathrm{PaO}_{2} / \mathrm{FiO}_{2}$ ratio was higher during PP [136 (78-250)] than before PP [126 (58-145)] $(p<0.05)$. There is no difference of $\mathrm{PaCO}_{2}$ before PP [55 $\mathrm{mmHg}$ (34-80)] than during PP [54 (34-70)]. Between before, the beginning and the end of PP, we did not find difference in blood flow, respectively, $1472 \mathrm{ml} / \mathrm{min}$ (1201$1971), 1403 \mathrm{ml} / \mathrm{min}(1216-1850)$ and $1447 \mathrm{ml} / \mathrm{min}(1231-2012)$, and in $\mathrm{P} 1$, respectively, $-37 \mathrm{mmHg}(-46$ to -25$),-41 \mathrm{mmHg}(-50$ to -28$)$ and $-41 \mathrm{mmHg}(-47$ to -29$)$. The coefficient of variation of the blood flow was low, and we did not find variations of it between these three moments, respectively $0.9 \%(0.7-2.8), 0.7 \%(0.4-2.1)$ and $0.6 \%(0.4-$ 1.6). The coefficient of variation of P1 was low, and it was lower at PP than before PP $(p<0.05)$, respectively: $8.2 \%$ (3.7-9.9), $5.6 \%(2.8-6.8)$ and $4.2 \%(2.9-5.8)$. We did not find side effects of the PP maneuver. There were no decannulation, bleeding and skin lesion on the tubing paths.

Conclusion Prone position under $\mathrm{ECCO}_{2} \mathrm{R}$ with a femoral catheter is possible. We found no side effects of this technique. No differences in the blood flow, in the drainage pressure and in the stability of the blood flow were found. The stability of the drainage pressure is better in PP. The $\mathrm{PaO}_{2} / \mathrm{FiO}_{2}$ ratio is better in PP.

Competing interests None.

\section{P16}

Feasibility of the implementation of a technique of extra-corporeal $\mathrm{CO}_{2}$ removal $\left(\mathrm{ECCO}_{2} \mathrm{R}\right)$ in an intensive care unit which does not use ECMO and its real utilization

Virginie Amilien', Jean Pierre Ponthus ${ }^{1}$, Polysie Ngasseu' ${ }^{1}$, Martial Tchir ${ }^{1}$, Elise Barsam ${ }^{1}$, Pierre Lehericey ${ }^{1}$, Jean Francois Georger '

${ }^{1}$ Réanimation polyvalente - surveillance continue, Ctre Hospitalier Intercommunal de Villeneuve Saint Georges, Lucie et Raymond AUBRAC, Villeneuve-Saint-Georges, France

Correspondence: Jean Francois Georger - georger.chiv@gmail.com Annals of Intensive Care 2016, 6(Suppl 1):P16

Introduction The utility and feasibility of $\mathrm{ECCO}_{2} \mathrm{R}$ in an intensive care unit (ICU) which does not use ECMO has not been described. In our general ICU of 15 beds, we do not use ECMO, and we decided to introduce $\mathrm{ECCO}_{2} \mathrm{R}$ in COPD and ARDS patients.

The purpose is to describe the feasibility of this technique in our ICU and the actual uses of it.

Materials and methods We chose ILA ACTIVVE ${ }^{\circledR}$ device (NOVALUNG ${ }^{\circledR}$ ) with MINILUNG $^{\circledR}$ membrane and a double-line femoral catheter (NOVAPORT TWIN 24F). A training for each member of the team was conducted (physicians and nurses), and we wrote a detailed procedure between February 2014 and July 2014. We wrote a procedure for: the preparation of the device, laying the catheter, monitoring, removal of the catheter and the machine, any malfunctions, etc.). Between august 2014 and February 2015, data were collected for each patient receiving $\mathrm{ECCO}_{2} \mathrm{R}$. Indication, mortality at J28, duration of ECCO2R, complications and success of the technique were evaluated.

Results During this period, ten of the 348 patients admitted to our ICU have received ECCO2R therapy. We included five patients with ARDS (we laid the device because tidal volume $\left(V_{t}\right)$ was $6 \mathrm{ml} / \mathrm{kg}$ PBW with a $\mathrm{PaO}_{2} / \mathrm{FiO}_{2}$ ratio between 80 and 150 , after at least one prone position session, and with a plateau pressure between 25 and $30 \mathrm{~cm}$ of water and high level of $\mathrm{PaCO}_{2}$ ). We included three COPD patients in failure of non-invasive ventilation (NIV) in a hypercapnic coma, one asthmatic 
patient in severe respiratory acidosis under invasive ventilation (IV) and one severe COPD patient unweaning of the IV. Patients with ARDS normalized arterial $\mathrm{pH}$, and we decreased $V_{\mathrm{t}}(3-4 \mathrm{ml} / \mathrm{kg})$ and respiratory rate. Three of them are alive at J28 and had received 5, 7 and 14 days of $E_{C C O} R$, and two of them died under ECCO2R after 16 and 9 days of $\mathrm{ECCO}_{2} \mathrm{R}$. The reason of the death was nosocomial lung infection. None of the COPD patients with hypercapnic coma were intubated, and they received 5,3 and 6 days of $\mathrm{ECCO}_{2} \mathrm{R}$ and were alive at J28. The asthmatic patient received 7 days of $E C C_{2} R$, was extubated under $\mathrm{ECCO}_{2} \mathrm{R}$ and was alive at J28. The unweaning COPD patient was extubated two times and reintubated two times due to severe neuropathy and tracheal inhalation. He was tracheotomized and weaned from the $\mathrm{ECCO}_{2} \mathrm{R}$ after 19 days, and he was alive at J28, but died at J76. There was no bleeding leading by catheter but one hemothorax requiring a blood transfusion during an overdose of heparin

Conclusion The $\mathrm{ECCO}_{2} \mathrm{R}$ in a general ICU could have multiple indications in COPD and ARDS patients. These indications remain to be validated, but $\mathrm{ECCO}_{2} \mathrm{R}$ could improve the management of those patients. The implementation of $\mathrm{ECCO}_{2} \mathrm{R}$ is possible but requires a great investment, a training of the medical and the paramedical team and a written detailed procedure. Nevertheless, the installation of the device for each patient must for now be discussed in a team taking into account the risk-benefit ratio of each procedure.

\section{Competing interests None.}

\section{P17}

Six-hour daily session of high-frequency oscillatory ventilation in moderate-to-severe ARDS: impact on the end-expiratory lung volume, compliance and oxygenation

Bérénice Puech', David Vandroux', Arnaud Roussiaux', Dominique

Belcour ${ }^{1}$, Cyril Ferdynus ${ }^{2}$, Olivier Martinet ${ }^{1}$, Julien Jabot ${ }^{1}$

${ }^{1}$ Réanimation Polyvalente, CHU La Réunion, Saint-Denis, France; ${ }^{2} U$ nité de soutien méthodologique, CHU La Réunion, Saint-Denis, France Correspondence: Bérénice Puech - berenice.puech@gmail.com Annals of Intensive Care 2016, 6(Suppl 1):P17

Introduction High-frequency oscillatory ventilation (HFOV) is usually considered as an alternative continuous technique to conventional mechanical ventilation (CMV) with the aim of improving the oxygenation. Our goal was to use HFOV not as a surrogate to CMV but as a recruitment process during only $6 \mathrm{~h}$ a day alternating with the CMV and to test whether this new sequential HFOV use could increase the end-expiratory lung volume (EELV) and the respiratory system compliance (used as recruitment markers) and thus improve the oxygenation. Patients and methods Interventional prospective study, in singlecenter ICU, was performed in patients with moderate-to-severe ARDS $\left(\mathrm{PaO}_{2} / \mathrm{FiO}_{2}\right.$ ratio $<150$ after a 6 -h period of optimized $\mathrm{CMV}$ for maximal alveolar recruitment). Patients were placed under HFO sequentially ( $6 \mathrm{~h}$ a day) up to 4 days in a row, as long as $\mathrm{PaO}_{2} / \mathrm{FiO}_{2}$ ratio was $<200$. The primary endpoint was the before/after HFO session EELV difference measured by the nitrogen wash-in/wash-out technique. Secondary endpoints were the before/after $\mathrm{HFO}$ session $\mathrm{PaO}_{2} / \mathrm{FiO}_{2}$ ratio and respiratory system compliance differences.

Results Twenty-two patients (mean IGS $55 \pm 14$ ) were enrolled between November 2012 and May 2015 for a total of 29 sessions. Three of these (10\%) were stopped (two for hemodynamic failure and one for respiratory failure). After the 6-h HFOV session, the EELV increased significantly by more than $30 \%$, while the $\mathrm{PaO}_{2} / \mathrm{FiO}_{2}$ ratio and the respiratory system compliance increased significantly by more than $90 \%$ and more than $20 \%$, respectively. By considering as responder to the HFOV session a patient with an EELV increase of more than $15 \%$ after the HFOV, we obtained 19 responders (66\%) for 10 non-responders. By considering as responder to the HFOV session a patient with an EELV increase of more than $15 \%$ and a $\mathrm{PaO}_{2} / \mathrm{FiO}_{2}$ ratio increase of more than $30 \%$ after the HFOV, we obtained 16 responders (55\%) for 13 nonresponders. No significant change in hemodynamic parameters was found, except the significant $10 \pm 6 \%$ cardiac index decrease after $1 \mathrm{~h}$ of HFOV for the 15 sessions monitored by transpulmonary thermodilution. No pneumothorax occurred during the protocol.
Conclusion In ARDS patients with a $\mathrm{PaO}_{2} / \mathrm{FiO}_{2}$ ratio $<150$, an early 6-h HFOV session provides a significant improvement in oxygenation and ventilatory mechanics by alveolar recruitment. HFOV could be used sequentially in addition to CMV as a recruiting technique and not as an alternative rescue method.

\section{Competing interests None.}

P18

Can we optimize prescription of laboratory tests in surgical intensive care unit (ICU)? Study of appropriateness of care Marion Fresco ${ }^{1}$, Gaëlle Demeilliers-Pfister ${ }^{1}$, Véronique Merle ${ }^{2}$, Valéry Brunel $^{3}$, Benoit Veber ${ }^{1}$, Bertrand Dureuil ${ }^{4}$

${ }^{1}$ Reanimation chirurgicale, Centre Hospitalier Universitaire Rouen, Rouen, France; ${ }^{2}$ Département d'épidémiologie et de santé publique, Centre Hospitalier Universitaire Rouen, Rouen, France; ${ }^{3}$ Institut de biologie clinique, Centre Hospitalier Universitaire Rouen, Rouen, France; ${ }^{4}$ Anesthésie, Centre Hospitalier Universitaire Rouen, Rouen, France

Correspondence: Marion Fresco - fresco.marion@gmail.com

Annals of Intensive Care 2016, 6(Suppl 1):P18

Introduction In the lack of guidelines, prescription of laboratory tests in ICU sometimes seems to pertain to individual or team habits. We aimed to assess the impact of clinical guidelines to improve the volume of laboratory tests performed.

Materials and methods It was a monocentric, comparative study, before and after guidelines implementation at patient bedside, in a surgical ICU of a French university hospital. All consecutive patients present between May 1 and October 31, 2013, and between May 1, and October 31, 2014, were enrolled. The prescription help-guide was displayed in each patient room from May 2, 2014. Physicians were encouraged to rely on this help-guide for laboratory tests prescription, once patients stabilized. The primary outcome measure was the variation of routine laboratory tests per patient ICU day, before compared with after guidelines. Secondary outcomes were the number of ICU days, the mortality, the variation of blood transfusion, the variation of nosocomial infections potentially related to blood samplings and the economic impact after guidelines implementation. We also assessed the variation of laboratory tests per patient ICU day, during the same periods, in the medical ICU of the university hospital, without any guidelines.

Results A total of 274 patients were admitted, staying for 3167 ICU days during Period-1, and 342 patients were admitted, for 2799 ICU days during Period-2. The patients' general characteristics were similar, except for the ICU days significantly shorter for Period-2. A $27.21 \%$ relative reduction in routine laboratory tests per patient ICU day was observed after guidelines implementation. A $27.45 \%$ relative reduction in blood transfusion was also observed, without difference neither on mortality in ICU, nor on nosocomial infections potentially related to blood samplings. The mean decrease per laboratory test was significantly more important in the surgical ICU than in the medical ICU ( 27.21 vs. $15.13 \%, p=0.00837)$. There were a $124,000 €$ overall cost reduction on 6 months related to the guidelines implementation, and a transfusion economy of $53,000 €$

Conclusion This help-guide induced an important decrease in the volume of routine laboratory tests performed. We also demonstrated a major reduction in blood transfusion, without difference either on mortality, or on nosocomial infections potentially related to blood samplings. We observed a very important economic impact of our clinical guidelines.

\section{Competing interests None.}

\section{References}

1. Prat G, Lefèvre M, Nowak E, Tonnelier JM, Renault A, L'Her E, \& al. Impact of clinical guidelines to improve appropriateness of laboratory tests and chest radiographs. Intensive Care Med. 2009 Jun; 35(6):1047-1053.

2. Lehot JJ, Heuclin C, Neidecker J, Cartier R, French PF, Reverdy ME et al. How can we optimize medical orderings in intensive care unit? Ann Fr Anesth Reanim. 2010 Oct; 29(10):682-686. 


\section{P19}

Impact of the implementation of guidelines for laboratory testing in an intensive care unit

Sébastien Leydier ${ }^{1}$, Isabelle Clerc-Urmes ${ }^{2}$, Jérémie Lemarie ${ }^{1}$, Charles-Henri Maigrat ${ }^{1}$, Marie Conrad ${ }^{1}$, Aurélie Cravoisy-Popovic ${ }^{1}$, Damien Barraud ${ }^{1}$, Lionel Nace', Sébastien Gibot ${ }^{1}$, Nelly Agrinier ${ }^{2}$, Pierre-Edouard Bollaert ${ }^{1}$ Réanimation médicale, hôpital central, C.H.U. de Nancy, Nancy, France; 2Epidémiologie et evaluation clinique, C.H.U. de Nancy, Nancy, France Correspondence: Pierre-Edouard Bollaert - pe.bollaert@chu-nancy.fr Annals of Intensive Care 2016, 6(Suppl 1):P19

Introduction The aim of this study was to evaluate the long-term effects of the implementation of "good laboratory test practices" procedure on blood test prescription, cost cutting laboratory test and patient outcome and red cells transfusion needs. In addition, we looked for factors associated with transfusion.

Patients and methods This is a retrospective, single-center study. Considering that prescription of laboratory tests was excessive in our ICU, we developed in 2009 a procedure aiming at limiting assays and blood sampling. All adult patients over 18 years admitted in the ICU from January 1, 2008, to December 31, 2012, were consecutively included. For each patient, the following data were retrospectively obtained from medical records and institutional databases: demographic and severity of illness parameters, the amount and costs of laboratory tests, daily blood volume drawn, number of red cells transfusions and ICU and in-hospital outcomes.

Results A total of 3568 patients were included between January 1 2008, and December 31, 2012. Patient's characteristics were similar during the study with respect to overall severity and vital organ support, except the need for vasoactive drugs which increased across time. After implementation of guidelines, the total number of laboratory tests decreased from 78,406 in 2008 (18.1 tests/patient/day) to 27,514 in 2012 (6.4 tests/patient/day). 318,000€ were saved in 2012 on the five more expensive tests as compared to 2008. Average daily blood drawn volume decreased from $29.2 \pm 13 \mathrm{ml} /$ day in 2008 to $22.4 \pm 10.1 \mathrm{ml} /$ day $(p<0.001)$ in 2012 in the whole population. The decline was larger for transfused patients $(33.8 \pm 15.6 \mathrm{ml} /$ day in 2008 to $23.6 \pm 10.7 \mathrm{ml} /$ day in $2012 p<0.001$ ). We observed a nonsignificant reduction in PRBC administered after the institution of guidelines. Hemoglobin threshold for blood transfusion remained unchanged over time. There was a significant relationship between daily blood volume loss and blood transfusion [OR 1.012 per $\mathrm{ml}$ volume drawn $\mathrm{Cl}$ (1.009-1.014), $p<0.001]$, but mechanical ventilation, renal replacement, invasive monitoring and use of vasoactive drugs were more potent independent factors for transfusion. ICU and in-hospital mortality did not change across time $(p=0.367$ and $p=0.361)$

Conclusion Guidelines of laboratory tests prescription led to a sustained reduction in the number of laboratory tests and related costs, the average daily blood volume drawn, without changing the ICU and in-hospital mortality. Although there was a correlation between the amount of blood sampled and the transfusion of PRBC, the number of patients transfused and the amount of red blood cells transfused did not significantly decrease.

\section{Competing interests None.}

\section{P20}

\section{Identifying useless precaution to reduce costs in ICU}

Vincent Peigne ${ }^{1}$, Jean-Louis Daban ${ }^{1}$, Mathieu Boutonnet ${ }^{1}$, Guillaume Dumas ${ }^{1}$, Elisabeth Falzone ${ }^{1}$, Patrick Jault ${ }^{2}$, Bernard Lenoir ${ }^{2}$

${ }^{1}$ Réanimation, Hôpital d'Instruction des Armées Percy, Clamart, France;

${ }^{2}$ Département d'Anesthésie-Réanimation, Hôpital d'Instruction des Armées Percy, Clamart, France

Correspondence: Vincent Peigne - vincentpeigne@yahoo.fr Annals of Intensive Care 2016, 6(Suppl 1):P20

Introduction ICU caregivers can achieve useless precautions to limit stress and feeling of uncertainty about their patients.

We tried to identify and eradicate some behaviors of useless precaution inducing supplementary use of medical devices of drugs in our
ICU. We made the hypothesis that suppressing these practices could reduce the variable costs of our 12-bed unit.

Patients and methods In a first step, two senior intensivists analyzed the expenditures of drugs and medical devices during 1 year to identify excessive consumptions related to useless precautions. Strategies were set on to reduce these behaviors. In a second step, the impact of these measures was analyzed 1 year after their implementation.

Results Three behaviors of useless precaution were identified: (1) the daily systematic preparation of two sets of intubation drugs (succinylcholine, ketamine, atropine, and epinephrine), (2) routine bed-side hematocrit measurement in non-bleeding patients and (3) use of carbapenem for community-acquired infections.

Corrective measures included: (1) intubation drugs were routinely gathered but were prepared only if an intubation was decided by the attending physician, (2) bed-side hematocrit was performed only after medical prescription and physicians were encouraged to limit its use to bleeding patients, and (3) recommendations encouraging use of ceftriaxone instead of ertapenem for community-acquired pneumonia and intra-abdominal infections were implemented in the unit.

One-year consumption of the drugs and devices related to these measures decreased dramatically after the intervention: 245 doses of succinylcholine versus 860 (1896€ vs $6656 €$ ), 448 doses of ketamine versus 925 (821€ vs 2081€), 750 doses of atropine versus 3234 (133€ vs $728 €$ ) and 1660 doses of epinephrine versus 2400 (1444€ vs $2087 €$ ). Only 4800 bedside measurements of the hematocrit were performed during the year after the intervention versus 13,200 before (5052€ vs $14,841 €)$. The consumption of ertapenem decreased from 330 doses $(12,359 €)$ to 87 doses $(3198 €)$.

No adverse event was related to the new practices.

Conclusion Useless precautions were frequent reassurance behaviors in our unit. Identification of such practices and strategies to limit them allowed us to decrease the consumption of drugs and medical devices. The reduction in annual expenditures was superior to $26,000 €$.

\section{Competing interests None.}

\section{P21}

\section{The ICU of the teaching hospital of Kinshasa}

Joseph Nsiala Makunza', Nathalie Mejeni

${ }^{1}$ Anesthésie-Réanimation, Hôpital Privé d'Athis-Mons - Site Caron, Athis-Mons, France; ${ }^{2}$ Anesthésie-réanimation, Clinique universitaire de Kinshasa, Kinshasa, Democratic Republic of the Congo

Correspondence: Joseph Nsiala Makunza - mnsiala78@gmail.com Annals of Intensive Care 2016, 6(Suppl 1):P21

Introduction The objective of an ICU is to take care of critically ill patients who are presenting at least one potential or effective organ failure impairing directly with the vital prognosis and requiring the setting in emergency of replacement techniques.

According to the complexity of the care bring to these patients, a special attention is focused on the quality of the care in ICU.

Very few studies concerning the quality of the care in ICU are made in countries with low incomes.

The objective of this study was to have a global overview of our ICU and to evaluate our activities.

Patients and methods We made an evaluation of professional practices by following-up indicators of structure, of procedures of care and of results. All medical records of patients admitted in our ICU between 1 January and 30 June 2015 were analysed retrospectively. An investigation of satisfaction a day given was carried out, to collect the opinion of families of patients on the quality of the care given to their neighbours during their stay in our ICU.

Our service is a multipurpose ICU with a capacity of eight beds. It is held by a professor of university assisted by 14 specialists in resuscitation and 34 doctor-assistants (internal) in formation. Only the doctorassistants take night duties our ICU.

Our equipment is composed by a life support machine and a multiparametric monitor multiparametric for two beds. We have no equipment for measuring gases of blood, neither bronchial fibroscope, nor cardiac echography device. No technique of renal replacement is available. 
Results During 6 months, 140 patients ( $71 \%$ men) were admitted in our service. The mean age was of $46 \pm 22$ years. The main reasons for admissions were related to medical pathologies $(60 \%)$, followed by surgical pathologies (35.6\%) and traumatic pathologies (4.4\%). No gravity score was recorded at the admission

During the stay in ICU, $39 \%$ of patients were ventilated, $27 \%$ received vasoactives amines, no patient received a parenteral nutrition, and only three patients were dialysed. Especially severe septic patients who represent the majority of our admissions, the global compliance in the recommendations of the Surviving Sepsis Campaign "SSC" passed from $50 \%$ during the second half-year 2014 to $33.3 \%$ in the first half-year 2015.

The rate of observance of hand hygiene practice was low $(11 \%)$ and the rate of nosocomial infections was high (31.1\%). The mean duration of stay was $8.2 \pm 4.2$ days, and the mortality rate was $86.6 \%$.

Surprisingly, the majority of the questioned people declared very satisfied with the care given to their relatives in our ICU.

\section{Discussion}

- Multipurpose ICU of young adults;

- Numerous specialists in resuscitation (2md/bed vs $1 \mathrm{MD} / 4 \mathrm{beds})$;

- No specialists on duty;

- Lack of nurses (1 inf/4 beds);

- Lack of equipment;

- Most patients are ventilated and got nosocomial infections (NI $31.1 \%$, SAPI $5-40 \%$, France $5.4 \%$ );

- Lack of protocols;

- Not use of gravity score;

- High mortality.

Conclusion This study shows that the equipment of our intensive care unit has to be optimised. During the first half of 2015, the mortality rate was high and the compliance to recommendations, of SSC, was low. It will be advisable to develop and to promote protocols of care and to ensure the presence of a specialist in resuscitation in our ICU $24 \mathrm{~h}$ a day and $7 \mathrm{j} / 7$.

\section{Competing interests None.}

\section{References}

1. Martin W. Dünser, MD; Inipavudu Baelani, MA; Lundeg Ganbold, MD A review and analysis of intensive care medicine in the least developed countries. Crit Care Med. 2006 Apr;34(4):1234-42.

2. SRLF. Structures et organisation des unités de réanimation: 300 recommandations. Réanimation 12/2012; 21(3). doi:10.1007/s13546-012-0510-9.

P22

Security culture and risk management in intensive care units: risk cartography in a French intensive care unit

Amélie Mazaud', Sébastien Béague ${ }^{2}$, Anne-Flore Rousselle ${ }^{3}$, Alain Durocher $^{4}$

${ }^{1}$ Service d'anesthésie réanimation, Hôpital Édouard Herriot, Lyon, France; ${ }^{2}$ Réanimation polyvalente adulte, Hospital Center De Dunkerque, Dunkerque, France; ${ }^{3}$ Cellule de management de la qualité et de la gestion du risque, Hospital Center De Dunkerque, Dunkerque, France; ${ }^{4}$ Réanimation médicale, Centre Hospitalier Régional Universitaire de Lille, Lille, France

Correspondence: Amélie Mazaud - ameliemazaud@me.com

Annals of Intensive Care 2016, 6(Suppl 1):P22

Introduction During their stay in hospital, patients might be confronted to many risks due to health care. In France, health authorities command to identify those risks: either in an a posteriori way for intensive care units (ICU) like morbidity-mortality meetings, or in an a priori way, that is to say before the occurrence of undesirable events, in such process that operating rooms. A priori risk identification is not recommended for ICU, yet ICU patients are very exposed to iatrogenic events, which can eventually conduct to permanent after effects, or even death. Our aim was to identify risks of patients during their stay in ICU, in an "a priori" way, by realising a risk cartography.
Materials and methods This study took place in a French ICU of a general hospital. The methodology used is the one described in French quality reference documents. A volunteer multi-professional group was created, including doctors, nurses, auxiliary nurses, head nurses, and quality engineer. Risks identification meetings took place from April to September 2014. Patient's general process through ICU was firstly described, from the admission to the discharge of the unit. Risk quotation was made through scales about frequency, seriousness and control of the risks, concerning each risk of the patient's process. From these quotations, gross criticality was calculated by multiplying frequency and seriousness, and residual criticality by multiplying gross criticality and control. Residual criticalities were classified into three groups: criticality bearable without any action (up to 6), criticality bearable, but actions will be needed later (up to 27), and unbearable criticality, needing immediate action (over 28). Cartography representation was made, as a Kiviat diagram, using the mean, minimal and maximal values of residual criticality for each step of the patient's process.

Results A 188 risks have been identified during the different steps of the patient's process in ICU, as follows: admission/welcoming, circulatory care, ventilatory care, blood purification, hygiene/isolation, and discharge. Cartographic representation shows that none of the mean residual criticalities of those previous steps has reached the threshold of unbearable residual criticality. Hygiene/isolation has the worst mean residual criticality (18). Inside every steps but one (blood purification) of the process, some individual risks reached the threshold of unbearable residual criticality, requiring correcting measures. Many actions, including additional lecture of previous procedures, creation of new procedures, creation of healthcare pathways, and training courses for nurses and physicians, were recorded in the 2015 action plan. A new assessment of those actions shall take place in 2016-2017 (Fig. 23).

Conclusion Our study of a priori risks during the whole ICU stay of patients is completely unique to our knowledge. It has shown that many point of our practice can be improved rapidly, without waiting for the undesirable event to occur, avoiding potential iatrogenic complications for patients. Furthermore, it is a complete match with the current French trend, commanded by our healthcare authorities, of healthcare management through security culture and risk management. However, the results of this cartography cannot be applied directly to any ICU: The identified risks are specific only to the ICU where has taken place the study. Nevertheless, the methodology used in this study, and probably a good part of the patient's process through ICU, can be reused in other ICU. That way our study could be an inspiration tool for other ICU eager to improve their risk management.

\section{Competing interests None.}

\section{References}

1. Moulaire M. La cartographie des risques, un outil de management des risques en établissement de santé. Risques Qual. 2007;IV(4):221-9.

2. Garrouste-Orgeas M, Timsit JF, Vesin A, et al. Selected medical errors in the intensive care unit: results of the IATROREF study: parts I and II. Am J Respir Crit Care Med. 2010 Jan 15;181(2):134-42

\section{P23}

Acquiring messages of medical articles according to the reading style: the BIBLIOFLASH multicenter study

Romain Sonneville ${ }^{1}$, Benjamin Sztrymf ${ }^{2}$, Emmanuel Canet $^{3}$, for the BIBLIOFLASH study group

${ }^{1}$ Service de réanimation médicale et infectieuse, Hôpital Bichat-Claude Bernard-APHP, Paris, France; ${ }^{2}$ Réanimation polyvalente, Hôpital Antoine Béclère, Clamart, France; ${ }^{3}$ Réanimation médicale, Hôpital Saint-Louis, Paris, France

Correspondence: Romain Sonneville - romain.sonneville@bch.aphp.fr Annals of Intensive Care 2016, 6(Suppl 1):P23

Introduction Reading medical articles represents one of the main sources of information for medical doctors. However, the exponential availability of medical articles leads to difficulty in the selection of 


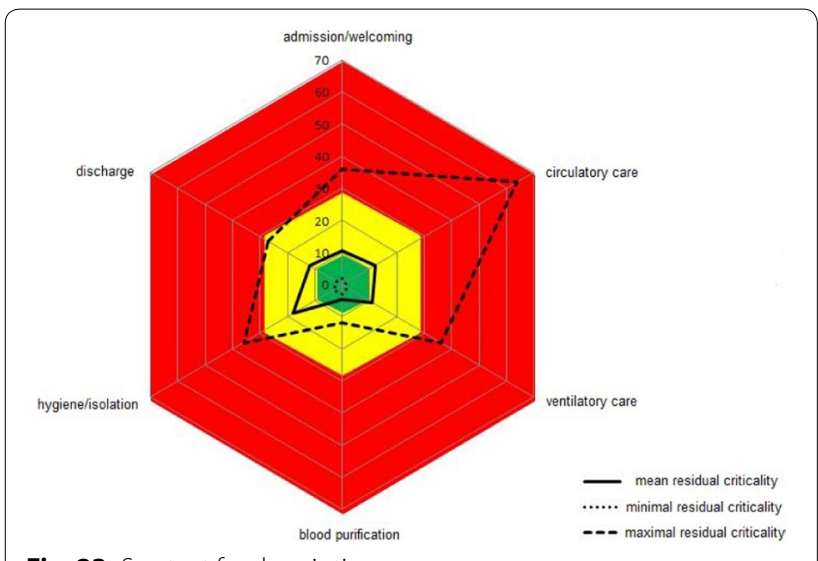

Fig. 23 See text for description articles and reading the full version of an article is time-consuming. A reading of a shorter version of the article could represent an interesting and time-saving alternative to the full version.

Materials and methods We aimed to test the impact of an abbreviated reading of a medical article on the understanding and acquisition of medical messages by residents in intensive care medicine. We performed a multicenter prospective study in three medical intensive care units of the Paris area over a 6-month period. Residents in intensive care medicine read articles selected by senior intensivists. For each article, residents were randomly assigned to one of the three following versions: (1) full version of the article; (2) incomplete version, without discussion; and (3) flash version, restricted to abstract, tables and figures. The evaluation of the understanding of the article was evaluated by a senior physician according to a standardized grid, assessing six main points: (1) objectives, (2) study population, (3) design, (4) main outcomes, (5) main bias and (6) external validity. Residents were asked to give the main message of the study. All articles were then discussed with the senior intensivists during a journal club session. One month later, residents were asked to recall the main message of each article. Data are presented as percentages or median (interquartile range).

Results Twenty residents evaluated 25 different articles (randomized controlled studies $n=16$, cohorts $n=7$ ). A total of 131 articles were read in three different versions, as follows: full version $(n=50)$, incomplete version without discussion $(n=35)$ and flash version $(n=46)$. The characteristics of the three centers, of residents and of the studied articles were not different between groups. The median time spent on reading the article was significantly lower for the flash version group [15 (10-16) $\mathrm{min}]$ than for the complete version group [40 $(30-50) \mathrm{min}]$ and for the incomplete version group [30 (21-30) $\mathrm{min}$ ] $(p<0.0005)$. Reading only the flash version of the article allowed residents to accurately identify the objective, the population, the design and the primary outcome of the study, with no significant difference compared with the other groups. However, accurate identification of potential bias and of external validity was significantly less frequently observed in the flash version group compared with the two other groups. Moreover, an accurate identification of the main message of the study was less frequently observed in the flash version group (52\%) than in the full version (78 \%) and the incomplete version (71\%) groups $(p=0.02)$. At 1 month, the recalling of the main message from the initial reading was significantly reduced in the full version group ( 52 vs. $78 \%, p=0.006$ ) and in the incomplete version group (46 vs. $71 \%, p=0.03)$, while there was no change in the flash version group (61 vs. $52 \%, p=0.4)$.

Conclusion The reading of a shorter version of a medical article by residents in training accurately identifies the objective, the population, the design and the main outcome of a study. However, it is associated with a decrease in the understanding of important methodological issues which may lead to inappropriate translation of the results into clinical practice. An accurate recalling of the main message of the article at 1 month was observed in approximately $50 \%$ of cases, irrespective of the reading style.

\section{Competing interests None.}

\section{P24}

ICU portrayal in social media channels

Pauline Perez ${ }^{1}$, Alexandra Grinea', Nicolas Weiss ${ }^{2}$

${ }^{1}$ Unité de réanimation neurologique, féderation de neurologie 1, pole des maladies du systeme nerv, Groupe Hospitalier Pitié-Salpêtrière Charles Foix, Assistance Publique - Hôpitaux de Paris, Paris, France; ${ }^{2}$ Unité de réanimation neurologique, Hôpital Pitié-Salpêtrière, Paris, France Correspondence: Pauline Perez - perez.pauline@gmail.com Annals of Intensive Care 2016, 6(Suppl 1):P24

Introduction Intensivists communicate daily with family members about the main diagnosis ('cardiac arrest' and 'acute respiratory distress syndrome') and various medical complications ('tracheal intubation', 'artificial ventilation' and 'septic shock'). Frequently family members go on home and make internet searches concerning the terms they have heard.

Concordance between information obtained on the internet, medical publications and social media channels have rarely been studied.

Materials and methods We used the Google Trend application to retrieve search volumes about the aforementioned diagnosis and complications. We compared search volumes on Google to the number of publications on PubMed from 2004 to 2015. In addition to that, a search through Ritetag (a site for trends in tweets) was conducted.

Results 'Cardiac arrest' and 'septic shock' searches on Google had a constant distribution over the years. Meanwhile, searches conducted for 'artificial ventilation', 'acute respiratory distress syndrome' and 'tracheal intubation' had a burst starting in 2008. Occurrences in PubMed followed a similar distribution with the exception of 'cardiac arrest' that showed two downfalls in 2008 and in 2010. The trends over the time period were, however, different between Google and PubMed. For Tweeter, we have found a median of four tweets per hour for the term 'cardiac arrest', whereas for the other terms it was difficult to retrieve any viable information (Fig. 24).

Conclusion This preliminary study suggests that searches for medical terms related to intensive care unit show similar results in Google and PubMed. This could suggest that intensivists and family members tend to have access to similar information. Meanwhile, the social media channels have yet to catch up in this domain.

\section{Competing interests None.}

\section{P25}

The burnout syndrome in a Tunisian intensive care unit: what is the truth?

Hassen Ben Ghezala', Naoures Bedoui', Salah Snouda', Rebeh Daoudi', Moez Kaddour

${ }^{1}$ Teaching department of emergency and intensive care, Hospital Zaghouan, Zaghouan, Tunisia

Correspondence: Hassen Ben Ghezala - hassen.ghezala@gmail.com Annals of Intensive Care 2016, 6(Suppl 1):P25

Introduction Burnout syndrome results from a strong emotional interpersonal implication in help professions. It is composed of three dimensions: emotional distress, depersonalization and professional fulfillment. It is frequent in intensive care units all over the world. The aim of our study was to identify the prevalence and to describe the epidemiological profile of burnout syndrome in a Tunisian medical intensive care unit. We tried also to compare the prevalence of the burnout syndrome between caregivers working in the intensive care unit and between the departments of the hospital.

Materials and methods We performed a prospective observational study in the teaching department of emergency and medical intensive care medicine (EMICU) in Zaghouan Hospital in Tunisia. All caregivers who gave their approval to participate were enrolled in the study. Each 


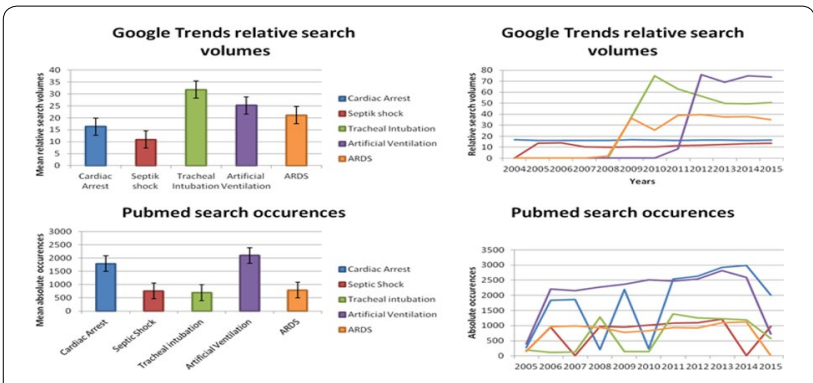

Fig. 24 See text for description

caregiver answered to a questionnaire about socio-demographic characteristics (age, sex, marital status) and the work conditions (intensive care unit conditions, number of hours of work per day, the number of on-call duty hours at the hospital). The Malasch Burn Inventory score was used to assess the burnout syndrome in our sample.

Results One hundred questionnaires were distributed in the departments of EMICU, surgery and pediatrics. Only 79 were collected, registered and then analyzed.

The participation rate was about $79 \%$. We found that $58 \%$ of all caregivers working in EMICU had a burnout syndrome and $26 \%$ of them were seriously affected. Forty-two percent of the affected caregivers had a high level of emotional distress, $43 \%$ had a high level of depersonalization, and $39 \%$ had a low level of professional fulfillment.

The mean average age of our sample was 33 years with a standard deviation of 9 . We have a female predominance with a sex ratio of 0.46 . Fifty-six percent of the caregivers enrolled in the study were married. From all the persons who answered to the questionnaire, $47 \%$ chose their department of work. More than half of our population (72\%) works in the hospital from $<10$ years. The average number of hours of work per week was $41 \mathrm{~h}$. Twenty-seven percent of all caregivers had to work at least two on-call duties per week.

Analytical study showed that among the socio-demographic characteristics, the female sex was significantly associated with the lowest score of professional fulfillment $(p=0.03)$. Seventy-two percent of caregivers affected by burnout were male with $40 \%$ who were seriously affected. Sixty-one percent of our affected population was married with a severe score in $22.7 \%$ of cases.

General surgery department had the highest level of emotional distress and depersonalization (73 and $64 \%$ ) followed by our EMICU department (56 and $60 \%$ ), while the level of professional fulfillment was the lowest in pediatric department $(14.3 \%)$ followed by our EMICU department (23.5\%).

Conclusion As expected, the prevalence of burnout syndrome is particularly high in our Tunisian emergency and intensive care unit. We found also that it was correlated with socio-demographic characteristics. Our department had one of the highest levels of burnout syndrome in the hospital. We are trying to identify the causes of this high level. Preventive and interventive measures against burnout syndrome should be started. This work is the first step of a multicenter Tunisian study.

\section{Competing interests None.}

\section{P26}

Resistance of endotracheal tubes measured after extubation in ICU patients

Alina Stoian'1, Loredana Baboi ${ }^{1}$, Florent Gobert ${ }^{1}$, Hodane Yonis' ${ }^{1}$, Romain Tapponier', Jean-Christophe Richard', Claude Guérin ${ }^{1}$

${ }^{1}$ Réanimation médicale, Hôpital de la Croix-Rousse, Lyon, France Correspondence: Claude Guérin - claude.guerin@chu-lyon.fr Annals of Intensive Care 2016, 6(Suppl 1):P26

Introduction Increase in resistance of endotracheal tube (RETT) during mechanical ventilation in ICU should reflect reduction in internal diameter due to accumulation of secretions. The aim of this study was to measure RETT after extubation in ICU patients. Our hypothesis was that RETT increased with the length of use of invasive mechanical ventilation.

Patients and methods The study was performed over patients intubated for at least 1 day in our ICU. Once the patient was extubated the tube was immediately stored in a plastic bag at room temperature and kept in a safe place until bench assessment. This was performed maximal $24 \mathrm{~h}$ after extubation as follows. The endotracheal tube was attached to a filter (Hygrobac), and both were set to ASL 5000 active servo lung (IngMar Medical). The lung model was set in passive condition in order to deliver two consecutive breaths at constant flow from 2 to $-2 \mathrm{~L} / \mathrm{s}$. The filter was tested first, and then, the filter and the endotracheal tube were run. The relationship of pressure $(P)$ to flow was fitted to the following equation $P=K 1$ flow $+K 2$ flow2, where $K 1$ and $K 2$ are constants. $P$ pertaining to endotracheal tube was obtained by subtracting $P$ from filter to $P$ from filter and endotracheal tube. Dividing $P$ by flow led to RETT $=K 1+K 2$ flow. RETT at $1 \mathrm{~L} / \mathrm{s}\left(\mathrm{cm} \mathrm{H}_{2} \mathrm{O}\right)$ was equal to $K 1+K 2$. The relationships of $K 1, K 2$ or RETT to length of intubation was analysed by linear mixed model where tube brand and size were factors with random effects.

Results We included 52 patients ( 34 male) of median (first-third quartiles) age 68 (61-78) years. The median duration of intubation was 5.5 (2-9) days (min 1-max 19 days). Endotracheal tubes were from Mallinckrodt $(n=45)$, TaperGuard $(n=4)$ or Rush $(n=3)$ brands and internal diameter $7.0(n=10), 7.5(n=39), 8.0(n=3) \mathrm{mm}$. The relationships of K1, K2 or RETT to length of intubation were not significant taking into account both brand and size of endotracheal tubes (Fig. 25).

Conclusion Increase in resistance of endotracheal tube used in the ICU is not related to the length of tracheal intubation.

\section{Competing interests None.}

\section{P27}

Length of endotracheal tube, humidification system and airway resistance: an experimental bench study

Frédéric Duprez', Arnaud Bruyneel2 ${ }^{2}$, Thierry Bonus', Grégory Cuvelier ${ }^{3}$, Sharam Machayeckhi', Sandra Olieuz', Alexandre Legrand ${ }^{4}$

${ }^{1}$ ICU, C.H. Epicura, Hornu, Belgium; ${ }^{2}$ Nursing school, Condorcet, Mons,

Belgium; ' ${ }^{3}$ Laboratoire de l'effort et du mouvement, Condorcet, Tournai, Belgium; ${ }^{4}$ Physiologie et pharmacologie, Université de Mons - Campus Plaine de Nimy, Mons, Belgium

Correspondence: Frédéric Duprez - dtamedical@hotmail.com Annals of Intensive Care 2016, 6(Suppl 1):P27

Introduction After intubation, the resistance of the inspiratory line is usually increased. This rise in airway resistance is associated with the presence of the endotracheal tube, a heat and moisture exchanger (HME) and/or any another tubing in the breathing circuit. During assisted ventilation or in spontaneously breathing patient, these high resistances will increase the work of breathing and negatively impact the chance of weaning. To limit this elevation in resistance, the endotracheal tube is sometime shortened and a heated humidifier used instead of a HME. The aim of this study was to evaluate the actual effect of these strategies on the airway resistance.

Materials and methods A two-compartment model of adult lung (DTL: TTL 1600 Dual Test Lung, Michigan Instrument) was connected to a Dragër Evita $4^{\circledR}$ Ventilator. The ventilator was set in volume-controlled mode $\left(V_{\mathrm{t}}: 0.5 \mathrm{~L}\right.$; respiratory frequency: $20 \mathrm{bpm}$; inspiratory flow: $70 \mathrm{~L} / \mathrm{min}$ ). To simulate normal and obstructive clinical conditions, two different resistances were placed at the entry of the airline connected to DTL by using Pneuflo ${ }^{\circledR}$ (parabolic resistor, Michigan Instrument; mean \pm SD: $2.5 \pm 0.06$ and $\left.17.04 \pm 0.03 \mathrm{cmH}_{2} \mathrm{O} / \mathrm{L} \mathrm{s}^{-1}\right)$. Compliance of the artificial lung was set to $70 \mathrm{ml} / \mathrm{cmH}_{2} \mathrm{O}$.

The airline was compounded of an endotracheal tube and a humidification system. Three different inside diameters (ID) (Portex ${ }^{\circledR}$ endotracheal tube of 7,8 and $9 \mathrm{~mm}$ ) and two humidification systems (HME (Gibbeck Humid Vent ${ }^{\circledR}$ Adult) or Fisher and Paykel MR850 Heated Humidifier ${ }^{\circledR}$ ) were evaluated before and after shortening of the tube at $10 \mathrm{~cm}$ length. The change in pressure was measured by an analog 


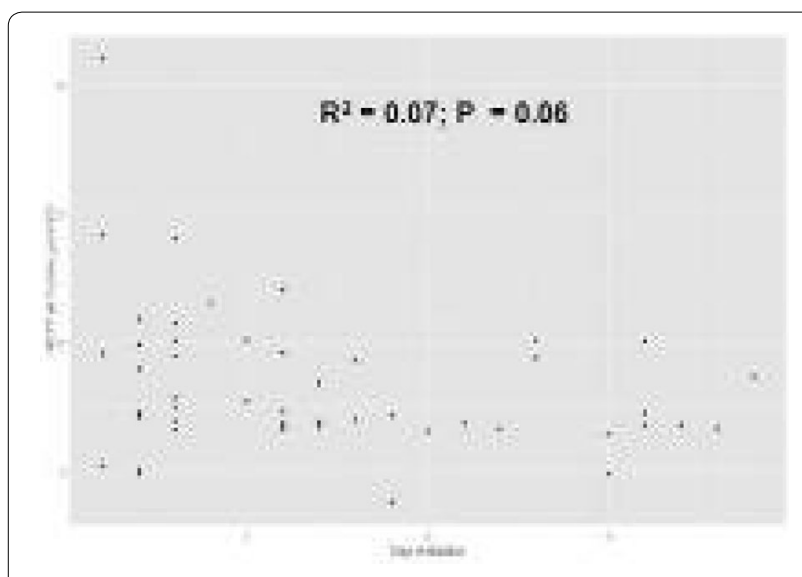

Fig. 25 See text for description

iWorx station/digital IWx/214. Resistance value was calculated from the following equation:

$$
\begin{aligned}
& \text { Inspiratory Resistance } \\
& \quad=(\text { Peak pressure }- \text { Plateau pressure/Inspiratory flow })
\end{aligned}
$$

Statistical test (Student's test) was performed. Values are presented as mean \pm standard deviation.

\section{Results}

Conclusion The use of heated humidifier instead of HME and shortening of endotracheal tube ( $10 \mathrm{~cm}$ of length) allow significant decrease in airway resistance. Both strategies may be helpful for the weaning of obstructive patients. However, the shortening of the tube (relative impact) has a decreasing impact when the tube diameter is growing and even for smaller tube the impact of humidifier is more important (Table 10).

\section{Competing interests None.}

\section{Reference}

1. Morgan NJ. Air flow resistance of three heat and moisture exchanging filter designs under wet conditions: implications for patient safety. Br J Anaesth 2001; 87: 289-91.

\section{P28}

Diagnosis contribution and safety of bronchoalveolar lavage in intensive care unit

Fatma Feki ${ }^{1}$, Amira Jamoussi ${ }^{1}$, Takoua Merhebene ${ }^{1}$, Emna Braham² Asma Ghariani ${ }^{3}$, Faouzi El Mezni², Leila Slim³ ${ }^{3}$ Jalila Ben Khelil', Mohamed Besbes $^{1}$

${ }^{1}$ Respiratory icu, Hospital Abderrahmen Mami De Pneumo-Phtisiologie, Ariana, Tunisia; ${ }^{2}$ Pathology, Hospital Abderrahmen Mami De Pneumo-Phtisiologie, Ariana, Tunisia; ${ }^{3}$ Microbiology, Hospital Abderrahmen Mami De Pneumo-Phtisiologie, Ariana, Tunisia

Correspondence: Amira Jamoussi - dr.amira.jamoussi@gmail.com Annals of Intensive Care 2016, 6(Suppl 1):P28

Introduction Bronchoalveolar lavage (BAL) is a diagnostic tool that explores the deep lung; it can provide useful histological and microbiological information. However, endobronchial injection of important volumes of saline serum may cause hypoxemia. This risk is particularly important to consider in ICU patients. The aim of this study was to determine the diagnostic value and to identify incidents attributable to BAL in intensive care unit.

Patients and methods This was a retrospective study conducted from January 2011 to December 2014 at the respiratory ICU of the Abderrahmen Mami Hospital in Ariana (Tunisia). Were included all patients who underwent BAL in intensive care unit. We recorded demographic,

\begin{tabular}{|c|c|c|c|}
\hline \multicolumn{4}{|c|}{ Resistance value ( $\mathrm{cm} \mathrm{H} 2 \mathrm{O} / \mathrm{L} . \mathrm{sec}-1)$ and variation in \% } \\
\hline \multicolumn{2}{|c|}{ Normal initial Resistance } & \multicolumn{2}{|c|}{ High initial Resistance } \\
\hline HME & Heated humidifier & HME & Heated humidifier \\
\hline $4,88(+/-0,04)$ & $2,5(+/-0,06)$ & $19,73(+/-0,03)$ & $17,04(+/-0,03)$ \\
\hline \multicolumn{2}{|c|}{$(* *)-95 \%$} & \multicolumn{2}{|c|}{$\left({ }^{* *}\right)-15,8 \%$} \\
\hline Tube 7 & Tube 7 cut down & Tube 7 & Tube 7 cut down \\
\hline $13,36(+/-0,06)$ & $11,74(+/-0,03)$ & $26,19(+/-0,04)$ & $25,13(+/-0,02)$ \\
\hline \multicolumn{2}{|c|}{$\left({ }^{* *}\right)-13,8 \%$} & \multicolumn{2}{|c|}{$(* *)-4,2 \%$} \\
\hline Tube 8 & Tube 8 cut down & Tube 8 & Tube 8 cut down \\
\hline $8,36(+/-0,05)$ & $7,27(+/-0,01)$ & $22,99(+/-0,02)$ & $22,2(+/-0,01)$ \\
\hline \multicolumn{2}{|c|}{ (***) $-15 \%$} & \multicolumn{2}{|c|}{$(* *)-3,6 \%$} \\
\hline Tube 9 & Tube 9 cut down & Tube 9 & Tube 9 cut down \\
\hline $4,39(+/-0,05)$ & $3,98(+/-0,10)$ & $18,79(+/-0,04)$ & $18,42(+/-0,02)$ \\
\hline \multicolumn{2}{|c|}{$(*)-10,3 \%$} & \multicolumn{2}{|c|}{$(*)-2 \%$} \\
\hline & $* * \mathrm{p}$ & .001 & ${ }^{*} p<0,01$ \\
\hline
\end{tabular}

Table 10 See text for description

clinical and paraclinical characteristics of patients, results and incidents of BAL.

Results During the 4 years of the study, 100 BALs were performed in 100 patients ( 55 men and 45 women) with a mean of age of 44 years [15 years -87 ans].

Respiratory history was present in 22 patients and systemic disease in 31 patients, and 42 patients were smokers.

The main cause of admission was acute respiratory failure (97 patients).

On chest $X$ ray, alveolar and/or interstitial damage was found in 93 patients; 78 were bilateral.

BAL was performed under invasive ventilation in 20 patients, under NIV in 20, on oxygen in 54 and for six patients in ambient air.

BAL was contributory to diagnosis in 77 patients: 28 cases of intra-alveolar hemorrhage, 26 cases of bacterial pneumonia (ten communityacquired pneumonia and 16 nosocomial), eight cases of pulmonary pneumocystosis, six cases of active pulmonary tuberculosis, six cases of malignant pulmonary infiltrate, two cases of eosinophilic pneumonia and one case of histiocytosis $X$.

The major occurring incident was severe hypoxemia in four patients among which three had required endotracheal intubation. In the 96 remaining patients, BAL was safe and harmless.

Conclusion In ICU, BAL is a good and safe tool; it contributes to diagnosis in $77 \%$ of cases. However, the risk of worsening breathing after BAL should be evaluated, especially in critically ill patients.

\section{Competing interests None.}

\section{P29}

Impact of fiberoptic bronchoscopy performed under noninvasive ventilation on the outcome of critically ill patients: a cohort study over 11 years

Antoine Marchalot ${ }^{1}$, Christophe Girault ${ }^{2}$, Gaetan Beduneau ${ }^{2}$, Dorothée Carpentier ${ }^{2}$, Steven Grange ${ }^{2}$, Emmanuel Besnier ${ }^{3}$, Gioia Gastaldi², Julien Abily ${ }^{2}$, Marion Beuzelin ${ }^{2}$, Fabienne Tamion ${ }^{4}$

${ }^{1}$ Seine-Maritime, CHU Charles Nicolle Rouen, Rouen, France; ${ }^{2}$ Réanimation Médicale, Centre Hospitalier Universitaire Rouen, Rouen, France;

${ }^{3}$ Pôle Réanimations Anesthésie SAMU, Hospital Center University Rouen, Rouen, France; ${ }^{4}$ Réanimation médicale, Hospital Center University Rouen, Rouen, France

Correspondence: Antoine Marchalot - amarchalot@gmail.com Annals of Intensive Care 2016, 6(Suppl 1):P29

Introduction Fiberoptic bronchoscopy (FOB) is frequently performed in intensive care unit (ICU) for diagnostic and/or therapeutic procedures. The main complication of FOB is hypoxemia, which can lead 
to intubation and mechanical ventilation in patients with hypoxemic acute respiratory failure (ARF), and heavily strike the prognosis, particularly in cases of underlying immunosuppression. Noninvasive ventilation (NIV) has been proposed to improve oxygenation during FOB. The purpose of this study was to evaluate the impact of FOB performed under NIV on the outcome of patients with hypoxemic ARF.

Materials and methods We conducted a retrospective cohort study, in the medical ICU of the Rouen University Hospital from January 2003 to December 2013 and compared two different strategies of $\mathrm{FOB}$, under either NIV or spontaneous breathing with standard oxygen therapy by mask $\left(\mathrm{O}_{2}\right)$. The main evaluation criterion was the incidence of intubation after FOB within $48 \mathrm{~h}$. Secondary criteria were ICU mortality, predictive factors for intubation and ICU mortality, as well as diagnostic and therapeutic yield of FOB.

Results Ninety-two patients were included. In the FOB-NIV, 14 of 39 patients were intubated ( $36 \%$ ) versus 8 of 53 patients in the FOB-O2 group (17\%) (HR 2.58; $p=0.03$ ). Mortality in the FOB-NIV group was 46 versus $12 \%$ in the $\mathrm{FOB}-\mathrm{O}_{2}$ group (HR 1.66; $p=0.29$ ). Patients in the FOB-NIV group were older (64 vs $60, p=0.028$ ), more severe (SAPS2, SOFA, $\mathrm{PaO}_{2} / \mathrm{FiO}_{2}$ ratio) on admission or the day of $\mathrm{FOB}$. In multivariate analysis, predictive factors for intubation were higher heart rate (HR per heart beat: 1.03, $p=0.02$ ), SpO2 $<95 \%$ (HR 5.07; $p=0.001$ ) and SAPS2 score the day of FOB (HR per unit: 1.06; $p<0.0001$ ). The type of ventilatory support during FOB was not found as an independent predictive factor for intubation $(p>0.05)$.

Conclusion Performing FOB under NIV in hypoxemic ARF patients seems to be associated with an increased risk of intubation as compared to $\mathrm{FOB}$ with $\mathrm{O}_{2}$, but without increase in their ICU mortality. As the ventilatory support used during the FOB does not appear as a predictive factor for intubation within the $48 \mathrm{~h}$ after the FOB, this risk appears closely related to the underlying severity of patients. Finally, the choice to perform FOB under NIV by the clinician appears to be guided by this severity.

\section{Competing interests None.}

\section{Reference}

1. Cracco C, Fartoukh M, Prodanovic H, Azoulay E, Chenivesse C, Lorut C, et al. Safety of performing fiberoptic bronchoscopy in critically ill hypoxemic patients with acute respiratory failure. Intensive Care Med. 2013;39(1):45-52.

P30

New single-use flexible bronchoscope dedicated for the invasive mechanical ventilation: a proof of concept

Mai-Anh Nay ${ }^{1}$, Adrien Auvet ${ }^{2}$, Julie Mankikian², Virginie Hervé ${ }^{3}$, Antoine Guillon ${ }^{2}$

${ }^{1}$ Réanimation médicale polyvalente, Hôpital de La Source, CHR Orléans, Orléans, France; ${ }^{2}$ Réanimation polyvalente, CHRU Hôpitaux de Tours, Tours, France; ${ }^{3}$ Inserm u1 100, centre d'étude des pathologies respiratoires, Université François Rabelais, Tours, France

Correspondence: Mai-Anh Nay - vannavy@free.fr

Annals of Intensive Care 2016, 6(Suppl 1):P30

Introduction Bronchoscopy can be particularly challenging in patients with acute respiratory distress (ARDS) who required lungprotective ventilation strategies. In a previous study, we showed that a bronchoscope with an external diameter of $4 \mathrm{~mm}$ (or less) would allow safer bronchoscopic interventions. Diameter reduction cannot be at the expense of the suction channel which needs to be sufficient to easily removed thick secretion. From the single-use bronchoscope technology, we proposed an innovation of flexible bronchoscope with a variable outer diameter as follows: a proximal $4 \mathrm{~mm}$ diameter for the part inserted in the endotracheal tube, distal head camera with classical diameter $(5.3 \mathrm{~mm})$, and $2 \mathrm{~mm}$ diameter for the suction channel. The aim of our study was to demonstrate that our prototype of flexible bronchoscope: (1) does not disrupt the protective ventilation, while it is in place in the endotracheal tube (ETT); and (2) enables an efficient suctioning capacity.

Materials and methods In vitro We simulated ARDS lungs mechanically ventilated according to actual guidelines. We used four models of flexible bronchoscope (FB): reusable adult, single-use adult, reusable paediatric, and prototype. All combinations between FB and ETTs (7.0-8.0 mm of internal diameters) were evaluated in pressure-controlled ventilation mode (inspiratory pressure of $22 \mathrm{cmH}_{2} \mathrm{O}$ for a target tidal volume of $380 \mathrm{~mL}$, respiratory rate at $27 / \mathrm{min}, \mathrm{PEEP}$ at $10 \mathrm{cmH}_{2} \mathrm{O}$, inspiratory flow at $60 \mathrm{~L} / \mathrm{min}$, compliance at $35 \mathrm{~mL} / \mathrm{cmH}_{2} \mathrm{O}$, inspiratory/ expiratory 1:2). We measured the percentage of minute ventilation (MV) delivered compared with baseline and the increase in total positive end-expiratory pressure ( $\Delta$ totalPEEP). To test the capacity of the suction channel, we measured the suction flow (water and viscous fluid) with two vacuum levels.

In vivo We described and compared the consequences of bronchoscopy during protective ventilation on two ventilated pigs with a single-use FB and our prototype of FB.

Results In vitro Inserting adult FB in ETTs severely altered ventilation: 19-61 \% of the baseline MV was delivered (7.0-8.0 ETTs). On the contrary, the modifications on the ventilation were reduced with the prototype $\mathrm{FB}(72-87 \%$ of the baseline MV). When the inspiratory pressure was set to guaranty the targeted tidal volume, the $\triangle$ totalPEEP was +13 and $+11 \mathrm{cmH}_{2} \mathrm{O}$, respectively, for the reusable and for the single-use adult $F B$ (ETT 7.0), whereas totalPEEP was unmodified for the prototype (as for the paediatric FB). At $-150 \mathrm{mmHg}$ vacuum level, prototype FB suction flow was identical $(247 \mathrm{ml} \pm 12 \mathrm{~mL} / \mathrm{min}$ for water and $176 \mathrm{~mL} \pm 4 \mathrm{~mL} / \mathrm{min}$ for viscous fluid) to the adult $\mathrm{FB}$ and was clearly superior to the suction flow provided by paediatric FB $(147 \pm 10 \mathrm{~mL} /$ min for water and $100 \pm 6 \mathrm{~mL} / \mathrm{min}$ for viscous fluid). Similar observations were made for the $300 \mathrm{mmHg}$ vacuum level.

In vivo

When the adult FB was inserted in the 7.0 ETT of the ventilated pigs, it was not anymore possible to ventilate them with the initial settings (inspiratory pressure of $15 \mathrm{cmH}_{2} \mathrm{O}$, respiratory rate at $27 / \mathrm{min}, \mathrm{PEEP}$ at $10 \mathrm{cmH}_{2} \mathrm{O}$ ). Indeed, the inspiratory pressure was increased up to $52 \mathrm{cmH}_{2} \mathrm{O}$ to deliver the targeted tidal volume, leading to a total PEEP of $16 \mathrm{cmH}_{2} \mathrm{O}$. On the contrary, no significant difficulties were observed with the prototype of FB.

Conclusion Our prototype of single-use FB did not disrupt the lungprotective ventilation strategies and had still efficient capacity of suction.

\section{Competing interests None.}

P31

Incidence of and risk factors for difficult intubation in ICU

Marie Soulie', Pierrick Cronier', Elie Kantor', Laura Federici', Marion

Gilbert', Ilham Mezhari', Gérald Choukroun', Sophie Marque ${ }^{1}$

${ }^{1} \mathrm{ICu}, \mathrm{C} . \mathrm{H}$. Sud Francilien, Corbeil-Essonnes, France

Correspondence: Sophie Marque - ellayon@yahoo.fr Icu

Annals of Intensive Care 2016, 6(Suppl 1):P31

Introduction Difficult intubation (DI) remains an issue of concern in critically ill patients. Though its incidence and risk factors are well described in anesthesiology, few authors studied difficult airway management in ICU patients (1). The aim of our study was to describe the incidence and risks factors for DI in our ICU.

Patients and methods We conducted a monocentric, prospective observational study between January 2014 and May 2015. All patients admitted to our ICU and requiring intubation were included in the study. Exclusion criteria comprised age under 18 and refusal from the patients or their relatives to participate.

An intubation procedure was defined as difficult whenever it required more than two laryngoscopic attempts by a senior intensivist or the use of a different technique (stylet, gum bougies, laryngeal masks, fiberoptic intubation, cricothyroidotomy or tracheostomy). Patients comorbidities, demographic data and classical risk factors for difficult intubation in anesthesiology, as well as the MACOCHA score, were collected on admission. For each intubation, modalities of the procedure were recorded. A multivariate logistic regression was used to identify risk factors for DI. Variables were selected after univariate analysis with a significant association $(p<0.20)$.

Results During the study period, 263 intubation procedures were studied. Patients were mostly men $(n=173,65.3 \%)$, with a mean IGS 
II of 54 (52-56). The cause of ICU admission was medical for the great majority of them $(n=239,90 \%)$. A total of $33(12.6 \%$ [8.8-17.2]) procedures out of 263 turned out to be difficult.

When the MACOCHA score was recorded ( $n=153,58 \%$ ), it was significantly higher in the DI group (median = 2[1-7] vs 1 [1-2]). In this subgroup of 153 patients, the MACOCHA score appeared to be the only predictive factor for $\mathrm{DI}$ (OR per point $=1.47,95 \% \mathrm{Cl}$ [1.23-1.75]).

In the global population $(n=173)$, patients with a DI had a significantly higher Mallampati score, had a more frequent history of head and neck disease $(n=7[21 \%]$ vs $n=4[1,72 \%], p<0.0001)$ and presented more often with a low thyro-mental distance $(n=13$ [39\%] vs $n=34[15 \%])$ or a reduced mobility of cervical spine ( $n=5[15 \%]$ vs $n=7[3 \%]$ ). After adjustment, a Mallampati score higher than 2 (OR 37.8 [10.6-135.1]) and a limited mouth opening (OR 5.38, $95 \% \mathrm{Cl} 1.1-$ 26.4) were both associated with DI.

Discussion In our study, the incidence of DI was comparable to previous studies. Predictive independent factors for DI (Mallampati score higher than 2 and limited mouth opening) differed from those which recently led to the validation of the MACOCHA score in a medico-surgical ICU population 1. In our medical ICU population, the MACOCHA score often showed to be difficult to record. The Mallampati classification, which represents one of its fundamental items, was not hardly ever feasible in emergency in medical patients, nor was it predetermined by the anesthesiologist (as in scheduled surgery patients).

Conclusion Two clinical items predictive of difficult intubation were identified in our study. The MACOCHA score seemed to be difficult to record in a medical ICU population, but confirmed to be a helpful score when available.

\section{Competing interests None.}

\section{Reference}

1. De Jong A et al., Early Identification of Patients at Risk for Difficult Intubation in the Intensive Care Unit. Am J Respir Crit Care Med 2013.

\section{P32}

Endotracheal self-extubation in the intensive care unit: a two-year observational study

Z. Coppere ${ }^{1}$, Maxens Decavèle $^{1}$, Jean-Pierre Fulgencio ${ }^{1}$, Clarisse Blayau', $^{1}$ Tai Pham', Muriel Fartoukh'1, Michel Djibre ${ }^{1}$

${ }^{1}$ Réanimation médico-chirurgicale, Hôpital Tenon, Paris, France

Correspondence: Michel Djibre - michel.djibre@tnn.aphp.fr

Annals of Intensive Care 2016, 6(Suppl 1):P32

Introduction Endotracheal self-extubation (ESE) is a common adverse event in the intensive care unit (ICU). It may carry significant safety risks for the patients and exceptionally cause death. We aimed to investigate the incidence and consequences of ESE in ICU patients and to assess the risk factors for complicated ESE

Materials and methods This is a single-center prospective observational study conducted in a medical and surgical ICU and affiliated intermediate care of 26 beds. From January 2013 to December 2014, all ESEs were prospectively recorded including the circumstances of the event, the characteristics of the patients and the outcome. Quantitative and qualitative variables are presented as mean (standard deviation), median [interquartile range] or number (\%), as appropriate.

Results During the study period, 42 episodes of ESE occurred in 41 patients, among 690 intubated patients (6\%). The patients (31 males) were aged of 56.5 [47-72] years and had a median Charlson comorbidity index of 5 [4-7] points. The main reasons for ICU admission were respiratory failure $(54 \%)$, postoperative monitoring $(14 \%)$, and severe sepsis or septic shock (10\%). The SAPSII score was 42 [33-58]. ICU length of stay (LOS) was 16 [9-26] days, and ICU mortality rate was $19.5 \%$ [95 \% Cl 0.07-0.32].

At the time of ESE, 35 patients (83\%) were on a respiratory weaning program (PSV), with a pressure support of 12 [8-13] $\mathrm{CmH}_{2} \mathrm{O}$ and a $\mathrm{FiO}_{2}$ of $35 \%$ [30-40]; 18 patients (43\%) were still sedated, 15 (36\%) had agitation, and $15(36 \%)$ had physical restraints. ESE occurred 4 [3-9] days after intubation and 64.5 [20-102] h after PSV onset. Nine patients ( $21 \%$ ) had a spontaneous breathing trial before ESE.
Altogether, $66 \%$ of ESE occurred around 7:00 a.m. $(n=7)$, 13:00 p.m. $(n=15)$ and 7:00 p.m. $(n=6)$ and $31 \%(n=13)$ occurred during the weekend. ESE resulted in a complicated outcome (CO) in 24 patients $(57 \%)$, including non-invasive ventilation (NIV) $(n=11 ; 26 \%)$, reintubation (RI) $(n=16 ; 38 \%)$, bacterial pneumonia $(n=7 ; 17 \%)$ and death $(n=8 ; 19 \%)$. On univariate analysis, the need for RI was associated with a prolonged ICU LOS $(p=0.003)$, an increased ICU mortality $(p=0.03)$ and a trend for an increased pulmonary infection $(p=0.08)$ Analysis of specific circumstances before ESE highlights a shorter time from the beginning of weaning $(p=0.03)$ and a higher pressure support level $(p=0.05)$ in case of CO.

Discussion Most of ESE occurred during the weaning period and around some critical hours during the day, particularly during the work shifts in the morning and the evening, and at noon. Risk factors associated with a $\mathrm{CO}$, like a shorter time from the beginning of weaning and a higher pressure support level, might reflect insufficient preparation for extubation.

Conclusion ESE is a common and serious adverse event in the ICU, and the need for RI may potentially be associated with higher morbidity and mortality. A prevention policy of ESE is warranted to minimize ESE, particularly in patients at risk of CO.

Competing interests None.

\section{P33}

Criteria to assess extubation readiness and prediction

of successful weaning

Matthieu Reffienna', Michel Arnaout', Sébastien Lefort ${ }^{1}$, Anne-Sophie

Debue ${ }^{1}$, Fabrice Daviaud', Sarah Cabon'1, Frédéric Pène', Julien

Charpentier ${ }^{1}$, Alain Cariou', Jean-Paul Mira', Jean-Daniel Chiche" Working Group on Mechanical Ventilation

${ }^{1}$ Réanimation médicale, Hôpital Cochin, Paris, France

Correspondence: Jean-Daniel Chiche - jean-daniel.chiche@cch.aphp.fr Annals of Intensive Care 2016, 6(Suppl 1):P33

Introduction Daily evaluation of patients for weaning from mechanical ventilation (MV) by nurses has been shown to decrease duration of MV. Evaluation is based on the search of weaning criteria allowing the start of a T-piece trial and the assessment of patient tolerance during spontaneous breathing. Nonetheless, reintubation occurs in $5-10 \%$ of cases [1], increases the duration of MV and ICU stay and is associated with significant morbidity and mortality. Failure to wean patients may result from respiratory failure (high respiratory workload, poor gas exchange) or extubation failure (inability to clear secretions, laryngospasm). Weaning criteria commonly used are poor predictors of extubation failure. We have tested the interest of adding to our weaning test-specific criteria to assess muscle strength, cough effectiveness, airway patency and patient's consciousness (Table 11).

Patients and methods We have retrospectively reviewed charts of all patients mechanically ventilated in the 24-bed MICU of Cochin University Hospital from $01 / 2007$ to $12 / 2013$. These patients were assessed twice a day by nurses who look for the presence of weaning criteria. Patients who met those criteria underwent a spontaneous breathing trial on T-piece during which tolerance was assessed. All patients data were continuously recorded in a clinical information management system (Clinisoft ${ }^{\circledR}$, GE Healthcare), including data related to weaning success/failure. In 08/2008, we progressively introduced a fiveitem new component to our weaning protocol to improve our ability to test muscle strength, cough effectiveness, airway patency and patient's consciousness. To evaluate the effectiveness of this strategy, we assessed the incidence of weaning failure (defined as respiratory or extubation failure requiring reintubation within $48 \mathrm{~h}$ ), the incidence of self-extubation, the duration of MV and ICU stay, and survival before (T1), during (T2) and after (T3) implementation of the new component. Informed consent was waived by our IRB for this study. Data are reported as median and interquartile ranges. A $P<0.05$ was considered significant for all statistical tests performed.

Results A total of 4488 treated with invasive MV have been included in the study from $01 / 2007$ to $08 / 2008(T 1, n=982), 08 / 2008$ to $08 / 2009$ ( $\mathrm{T} 2, n=642)$ and $08 / 2009$ to $12 / 2009$ (T3, $n=2864)$. Main results are summarized below. 
Conclusion Addition of a new component to an existing nurse-driven weaning protocol to evaluate readiness for extubation was not associated with improved ability to predict successful extubation. These results mandate a thorough analysis of causes of reintubation.

\section{Competing interests None.}

\section{Reference}

1. Thille AW et al. AJRCCM 2013,187;12: 1294-1302.

\section{P34}

Domestic accidents during school holidays in children

Nabil Tabet Aoul', Zakaria Addou' ${ }^{1}$, Ali Douah ${ }^{3}$, Mohamed Moussati ${ }^{3}$, Kamel Belhabiche ${ }^{3}$, Nabil Aouffen ${ }^{3}$

${ }^{1}$ Réanimation Pédiatrique Canastel, Faculté de médecine d'Oran, Oran, Algeria; ${ }^{2}$ Réanimation Pédiatrique de Canastel d'oran, Departement de medecine d'Oran Algerie, Oran, Algeria; ${ }^{3}$ Anesthésie Réanimation Pédiatrique, Etablissement Hospitalier Spécialisé en Pédiatrie Canastel, Oran, Algeria

Correspondence: Nabil Tabet Aoul - tabetrea@yahoo.fr Annals of Intensive Care 2016, 6(Suppl 1):P34

Introduction The accident represents the first cause of death, handicap and hospitalization in children. Most of the accidents arise further to a relaxation of the surveillance and the awareness of the parents. The risk is everywhere, in the house and in the street. Some situations favor the relaxation of awareness as it is the case during the school holidays.

Our aim is to describe the epidemiological profile of the accidents during the school holidays

Materials and methods It is a descriptive observational cohort study during the school holidays from December 2014 to January 2015, at the pediatrics hospital in Canastel in Oran. All children under age of 15 years who are victims of accident were included.

Results One hundred and fifteen children were enrolled. The mean of age was 5.2 years $( \pm 4.1)$ with sex ratio of 1.78 . The most frequent consultation in the pediatric emergency was the traumatology $(62.6 \%)$ of the cases followed by the burn (32.2\%) of the cases. The majority of the children are from Oran (63.5\%). Eight couples were divorced. The child was alone at the time of the accident in $80.3 \%$ of the cases. $61.3 \%$ of the children live with the big family. The age of the father was between 30 and 40 years in $46.2 \%$ and more than 40 years in $41.5 \%$, while the age of the mother was between 20 and 30 years in $49.2 \%$ of the cases and between 30 and 40 years in $43.1 \%$. No attempts of suicide or voluntary accident were noted. The children required a hospitalization in $24.3 \%$ and stay in pediatric intensive care in $4.3 \%$ of the cases. A 9-month-old child died following an inhaled foreign body. Conclusion The most of the accidents could be avoided. The implementation of a preventive strategy to emphasize the information and the education of the family and on the necessity of creating a safer environment for children seems to be necessary in our city.

Competing interests None.

\section{Table 11 See text for description}

\begin{tabular}{lllll}
\hline & T1 & T2 & T3 & $\boldsymbol{P}$ \\
\hline Sex (M/F) & $602 / 380$ & $375 / 267$ & $1818 / 1046$ & 0.04 \\
Age & $58[43 ; 72]$ & $60[47 ; 72]$ & $62[48 ; 74]$ & 0.001 \\
SAPS2 & $57[43 ; 70]$ & $58[45 ; 74]$ & $59[45 ; 74]$ & 0.001 \\
Reintubation (\%) & 4.7 & 4.4 & 6.7 & 0.01 \\
Self-extubation (\%) & 3.7 & 6.7 & 6.6 & 0.01 \\
Duration of MV (days) & $4[2 ; 9]$ & $3[2 ; 8]$ & $3[2 ; 8]$ & NS \\
ICU LOS (days) & $4.6[1.7 ; 10.8]$ & $5[2.1 ; 11]$ & $5.5[2.5 ; 10.7]$ & 0.05 \\
Death (\%) & 28.9 & 31.9 & 27.6 & NS \\
\hline
\end{tabular}

P35

Pediatric ARDS: interest of prone positioning and NAVA

ventilation during veno-venous ECMO

Sylvie Soulier ${ }^{1}$, Philippe Mauriat ${ }^{2}$, Nadir Tafer $^{2}$, Alexandre Ouattara $^{1}$

${ }^{1}$ Sar 2, Hôpital Haut-Lévèque, Pessac, France; ${ }^{2}$ Sar 2, unité cardiopathies

congénitales, Hôpital Haut-Lévèque, Pessac, France

Correspondence: Sylvie Soulier - sylvie.soulier@ymail.com

Annals of Intensive Care 2016, 6(Suppl 1):P35

Introduction The mortality of ARDS is related to several factors: etiologies, severity of hypoxemia $\left(\mathrm{PaO}_{2} / \mathrm{FiO}_{2}\right.$ ratio or oxygenation index value) and prolonged mechanical ventilation. Sometimes, VV ECMO has been used to rescue patients suffering from pulmonary failure unresponsive to conventional therapies (2). Due to high risk of thrombotic and bleeding complications, its duration and effectiveness may be limited. Prone position (PrP) and lung-protective and ultraprotective mechanical ventilation (tidal volume $<2 \mathrm{ml} / \mathrm{kg}$ with Peep $>10 \mathrm{cmH}_{2} \mathrm{O}, \pm \mathrm{HFOV}$ ) are recognized pulmonary rehabilitation strategies. The increase in oxygenation has been reported, associated with VILI reduction and improvement in alveolar recruitment (1). The main objective of the study was to reduce the duration of VV ECMO and ARDS morbi-mortality, by using these respiratory strategies in pediatric $A R D S$, requiring $V V$ support.

Patients and methods Following the recommendations of Pediatric Acute Lung Injury Consensus conference, we used double-lumen venous cannula VV support, in right internal jugular vein, with echographic control (3). During ECMO, the respiratory strategies were: ultra-protective controlled ventilation (UCV), PrP for $12 \mathrm{~h}$ daily, early reversal neuromuscular blockade (NMB) and spontaneous neurally adjusted ventilatory assist (NAVA) ventilation. Durations of ECMO, UCV and NMB were collected, as well as the time of introduction of NAVA during ECMO and extubation after the weaning off ECMO.

Results Six patients suffering from ARDS were admitted in our ICU and included in this study. The age ranged from 21 days to 4 years. The diverse causes of ARDS were VRS bronchiolitis, Enterobacter cloacae pneumonia, systemic inflammatory reaction syndrome (SIRS) and stage 4 drowning and macrophage activation syndrome. No increased cannulation site bleeding and flow issues, notably during the PrP procedure, were observed. The reversal NMB was at 3.7 days $( \pm 2.3)$ during the ECMO. NAVA ventilation was initiated also during the ECMO at 7 days $( \pm 2.6)$, and the duration of VV support was 7.3 days $( \pm 3)$. The patients were extubated 3.9 days $( \pm 2.6)$ after decannulation. All children survived.

Discussion The durations of ECMO and invasive ventilation observed in our study were dramatically short regarding the severity of ARDS (2). Our study showed the successful of respiratory rehabilitation strategies during VV support that included rapid improvement in oxygenation, early NMB weaning, using NAVA ventilation before decannulation and then decrease ECMO duration.

Conclusion VV ECMO, with double-lumen venous cannula, in severe ARDS in pediatric patients, can be optimized by respiratory rehabilitation strategies such as prolonged PrP, UCV and early respiratory weaning with NAVA ventilation. All these strategies seem to improve outcomes of these patients. However, further studies still required to confirm our encouraging results (Table 12).

Competing interests None.

\section{References}

1. Fineman LD et al. Pediatric Crit Care 2006;7(5):413-422.

2. Flamant C et al. Eur J Pediatrci 2005;164:93-98.

\section{P36}

Esogastric pressure measurement as a guide for noninvasive ventilation in unexplained hypercapnic respiratory failure in infants

Guillaume Mortamet ${ }^{1}$, Alessandro Amaddeo ${ }^{2}$, Sonia Khirani ${ }^{2}$, Brigitte Fauroux ${ }^{2}$

${ }^{1}$ réanimation médico-chirurgicale pédiatrique, Hôpital Necker, Rue de Sèvres, Paris, France; ${ }^{2}$ Pediatric non invasive ventilation and sleep unit, Hôpital Necker, Rue de Sèvres, Paris, France, Paris, France 
Table 12 See text for description

\begin{tabular}{lccrrrrc}
\hline Case & $\mathbf{1}$ & $\mathbf{2}$ & $\mathbf{3}$ & $\mathbf{4}$ & $\mathbf{5}$ & $\mathbf{6}$ & Mean $\mathbf{\text { SD }}$ \\
\hline ECMO duration (days) & 9 & 9 & 11 & 5 & 3 & 7 & $7.3 \pm 3$ \\
NMB duration (days) & 8 & 4 & 3 & 3 & 3 & 1 & $3.7 \pm 2.3$ \\
$\begin{array}{l}\text { Mechanical ventilation } \\
\quad 12\end{array}$ & 18 & 17 & 10 & 8 & 14 & $13.2 \pm 4$ \\
$\quad \begin{array}{l}\text { duration (days) } \\
\text { NAVA on ECMO (days) }\end{array}$ & 9 & 7 & 11 & 5 & 4 & 6 & $7 \pm 2.6$ \\
$\begin{array}{l}\text { Extubation after } \\
\quad\end{array}$ & 1 & 1.5 & 6 & 5 & 5 & 5 & $3.9 \pm 2.6$ \\
\hline
\end{tabular}

Correspondence: Guillaume Mortamet - mortam@hotmail.fr Annals of Intensive Care 2016, 6(Suppl 1):P36

Introduction In critically ill patients, noninvasive ventilation is classically set on a variable association of clinical parameters. However, this "clinical" setting provides no accurate data regarding the improvement in the respiratory effort and the synchronization of the patient with the ventilator. Esophageal pressure and gastric pressure have been shown to be useful in the ventilatory management of adult patients with acute respiratory failure. The aim of the present study is to describe the value of esophageal pressure and gastric pressure measurements for determining the usefulness and optimal setting of noninvasive ventilation in children with respiratory failure admitted to the pediatric intensive care unit (PICU).

Patients and methods We conducted a one single-center retrospective descriptive study. After the insertion of the esogastric catheter, the study started with a period of spontaneous breathing. After this period, a series of different noninvasive ventilation settings were performed with the first aim to normalize or to maximally reduce the patient's respiratory effort, reflected by the normalization of the esophageal pressure and transdiaphragmatic pressure swings. The second aim was to obtain the best synchrony between the patient and ventilator.

Results All the six patients had a severe underlying condition with an associated lung disease. Four patients were naive to noninvasive ventilation. The median age of the patients was $7.7 \pm 2.8$ months. All the patients had severe hypercapnic respiratory failure with a mean PvCO2 of $71.1 \pm 8.9 \mathrm{mmHg}$ and an increase in respiratory rate (mean $61.8 \pm 20.4$ breaths/min). Four patients were assisted by CPAP ventilation and two with BiPAP ventilation.

NPPV indication was proved by Pes measurement in four patients, as assessed on a reduction in the PTPes/min and PTPdi/ min from $303.0 \pm 112.0$ and $329.0 \pm 141.0$ to $169.0 \pm 115.0$ and $194.0 \pm 106.0 \mathrm{cmH}_{2} \mathrm{O} \mathrm{s} \mathrm{m^{-1 }}$ during NPPV, respectively $(p<0.05)$. In these four patients, two died and one was still on noninvasive pressure ventilation (NPPV) at PICU discharge. This decrease in respiratory effort was associated with a decrease in $\mathrm{RR}$ and $\mathrm{PvCO}_{2}$.

NPPV non-indication was proved by esophageal pressure measurement in two patients. In these two patients, despite the physiological findings, NPPV was continued in one patient after esophageal pressure and gastric pressure measurements because of an important clinical improvement. One of these two patients died.

Conclusion This descriptive and retrospective study shows that a physiological approach, based on the esophageal and gastric pressures measurement, may be gainful to manage noninvasive ventilation, to indicate or non-indicate NPPV and to optimize its settings, especially in a heterogeneous population of critically ill children.

Competing interests None.
P37

Experience Of Antibacterial Treatment Of Bone And Joint Sepsis Of Young Children

Gennadiy Khanes', Olga Liutko², Svitlana Bidnenko ${ }^{2}$

${ }^{1}$ Neonatal Surgery, National Ukrainian Pediatric Hospital OkhMatDyt, Kyiv,

Ukraine; ${ }^{2}$ Microbiology and chemotherapy, Research Institute of Orthopedics of Medical Sciences of Ukraine, Kyiv, Ukraine

Correspondence: Gennadiy Khanes - androshchuk@hotmail.com

Annals of Intensive Care 2016, 6(Suppl 1):P37

Introduction An adequate choice of antibiotics and antifungal drugs with the help of microbiological studies decides the fate of infants with bone and joint sepsis.

Materials and methods Over the past 10 years, microbiological studies were carried out in accordance with the standards and CLSI and EUROCAST. To establish the etiological diagnosis of the disease, we investigated punctate synovial fluid (mycological and bacteriological analyzes) and venous blood (serological and PCR investigations). Simultaneously, we examined the activity of the inflammatory process by determining blood levels of CRP and PCT.

Results Isolated from the synovial fluid of 16 cultures of microorganisms: nine staphylococcus (three S. aureus, three S. epidermidis and three S. heamolyticus); six Enterococcus (four E. faecalis and two T. faecium); and one Bacillus spp.

CRP was 12-96 mmol/l and PCT was $0.5-2.3 \mathrm{ng} / \mathrm{LB}$ depending on the timing of onset of the disease.

With active septic process (data CRP and PCT)-staphylococci isolated antibody titer of 1: 1280-2560: and PCR in different staphylococci (Figs. 26, 27).

Table sensitivity to the antibiotics and the schedule of the selected microorganisms.

Conclusion Studies have demonstrated the practical value of microbiological research in the treatment of bone and joint sepsis.

Competing interests None.

\section{P38}

\section{Comparison of toxic and septic shocks in children}

Solenn Remy ${ }^{1}$, Emilie Doye ${ }^{2}$, Nicolas Voirin ${ }^{3}$, Delphine Maucort-Boulch ${ }^{4}$, Karine Kolev-Descamp 1 , Guillaume Monneret ${ }^{5}$, Etienne Javouhey ${ }^{6}$

${ }^{1}$ Réanimation pédiatrique, Hôpital Femme Mère Enfant, Bron, France; ${ }^{2} 69$,

Hôpital Femme Mère Enfant, Bron, France; ${ }^{3}$ Cnrs umr 5558, Hospices Civils de Lyon, Lyon, France; ${ }^{4}$ Cnrs umr5558, Hospices civils de Lyon, Lyon, France; ${ }^{5}$ Laboratoire d'immunologie, Hôpital Édouard Herriot, Lyon, France; ${ }^{6}$ Réanimation pédiatrique hfme, Hospices civils de Lyon, Lyon, France

Correspondence: Solenn Remy - solenn.remy@hotmail.fr Annals of Intensive Care 2016, 6(Suppl 1):P38

Introduction Toxic shocks (TS) and septic shocks (SS) share many common clinical signs of an exacerbated inflammatory response but with different pathophysiological mechanisms. The objectives were to compare their characteristics of shock and their cellular immune response.

Patients and methods We did a monocentric retrospective study of children $<18$ years old admitted to PICU for TS or SS between January 2011 and September 2014. Immunocompromised and precociously died patients were excluded (death within the first $24 \mathrm{~h}$ ).

Comparisons of the time course of leukocytes, organ dysfunction (PELOD2 score) and nosocomial infections were made between TS and SS groups. We used time course of lymphocytes, precisely persistent lymphopenia at day 3 , like a marker of immunoparalysis.

Results Seventy-nine patients were included, 42 of SS and 37 of TS. Most of them were lymphopenic at day 1 (83.3 vs $97.3 \%$ in SS and TS groups, respectively), and, respectively, 45.2 and $35.1 \%$ were still lymphopenic at day 3.

Significantly, children with TS were more initially lymphopenic and corrected faster than septic patients $\left(p=2.52 \times 10^{-5}\right)$.

In both groups, the PELOD2 score was higher at day 1 in patients who are more lymphopenic. 


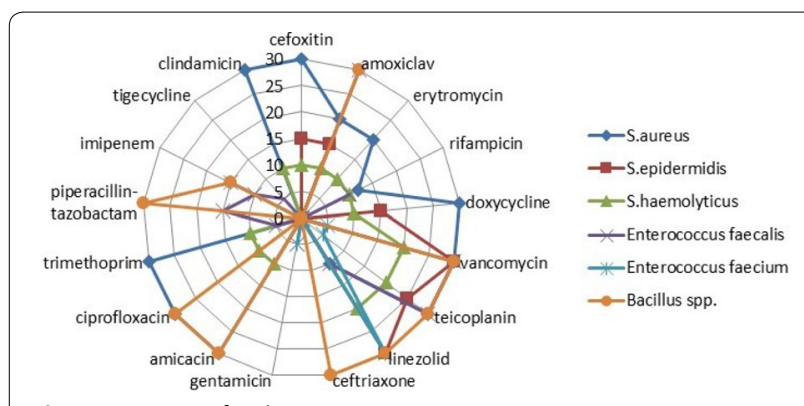

Fig. 26 See text for description

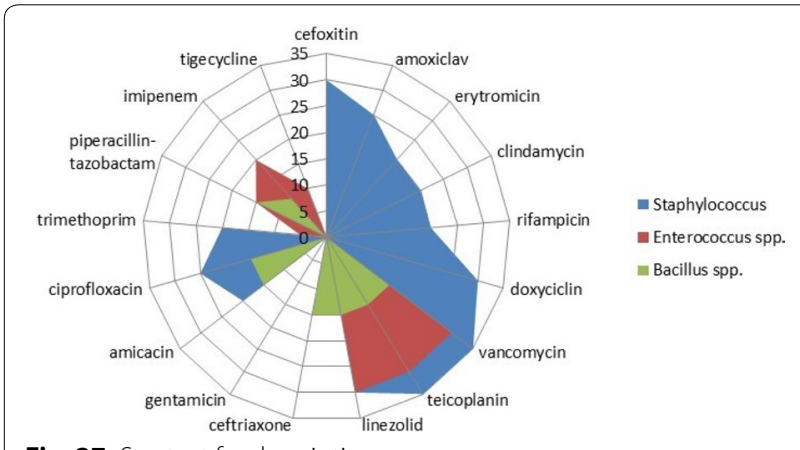

Fig. 27 See text for description

The rate of nosocomial infection was similar between groups and was higher in children with persistent lymphopenia at day 3. In SS, $26.3 \%$ patients with nosocomial infections were still lymphopenic at day 3 and $17.6 \%$ were not, while In TS, 23.0 versus $11.8 \%$. But the differences were not statistically significant: in SS OR $1.64,95 \% \mathrm{Cl}[0.3-$ 12.7], and in TS OR 2.2, $95 \% \mathrm{Cl}$ [0.2-30.7].

TS were less lymphopenic at day 3 and had less secondary nosocomial infections than SS.

Discussion Whether children who had at day 3 lymphopenia are more prone to develop nosocomial infection, as demonstrated in adults, may require a larger cohort.

Conclusion The difference in time course of lymphocytes between TS and SS groups may be explained by the superantigenic activation of lymphocytes in TS group. Our hypothesis is that superantigenic activation leads to a deeper and more precocious immunosuppression, but the recovery is faster than in patients with septic shock.

Immunomodulation therapy perspectives need better knowledge of immunosuppression induced by different severe infectious shocks.

\section{Competing interests None.}

\section{References}

1. Decreased T-Cell Repertoire Diversity in Sepsis: a preliminary study. F. Venet, G. Monneret and all. Critical Care Medicine 2013.

2. Hall MW, Knatz NL, Vetterly C, Tomarello S, Wewers MD, Volk HD, et al. Immunoparalysis and nosocomial infection in children with multiple organ dysfunction syndrome. Intensive Care Med. 2011 Mar;37(3):525-32.

\section{P39}

Pediatric carbon monoxide poisoning in the lle-de-France Region, Link between $\mathrm{HbCO}$ level and clinical presentation

Imane El Aouane', Aben Essid', Wallid Hammami², Isabelle Haegy', Jacques Bataille ${ }^{2}$, Jean Bergounioux ${ }^{3}$

192380, Hopital Raymond Poincare - Medecine Phys, Garches, France;

${ }^{2} 92380$, Hopital Raymond Poincare - Medecine Phys, Boulevard Raymond

Poincaré, Garches, France, Garches, France; ${ }^{3}$ Unité de réanimation pédiat- rique, Hopital Raymond Poincare - Medecine Phys, Garches, France Correspondence: Jean Bergounioux - jean.bergounioux@aphp.fr Annals of Intensive Care 2016, 6(Suppl 1):P39

Introduction Carbon monoxide poisoning remains a threat for children. Clinical severity and presentation of carbon monoxide (CO) poisoning are dependent of $\mathrm{COHb}$ level. Our aim was to describe the children hospitalized in our department for $\mathrm{CO}$ poisoning and to compare $\mathrm{HbCO}$ level to clinically reported symptoms.

Materials and methods We are a secondary care center hospital with hyperbaric oxygen therapy facilities and represent the reference center for lle-de-France region for $\mathrm{CO}$ poisoning. The medical records of patients aged between 0 and 16 years with a confirmed diagnosis of $\mathrm{CO}$ poisoning, defined as the presence of a $\mathrm{COHb}$ level of more than $5 \%$, were evaluated. Relevant information such as age, sex, source of $\mathrm{CO}$, coaffected family members, month of presentation, time of presentation and presenting symptoms, duration of oxygen treatment in the emergency department, need for admission to an inpatient ward or intensive care unit, and Glasgow coma scale scores was recorded for each patient on preprepared forms.

Results The records of 50 patients were included. Of them, 26 $(57.1 \%)$ were female and $24(42.9 \%)$ were male, with a median age of 7.0 years (range 1 month-16 years) and a mean $\mathrm{COHb}$ level of $7 \%$. Thirty-two patients (64\%) had a COHb between 1.5 and $10 \%$ on presentation, whereas the remaining 18 patients (36\%) had a presenting $\mathrm{COHb}$ of $>10 \%$. Neurologic symptoms such as headache, syncope, seizures, and confusion were encountered more frequently in the $\mathrm{COHb}>10 \%$ group compared with the group with $1.5-10 \% \mathrm{COHb}$ levels.

Conclusion In this study, we managed to demonstrate the presence of more frequent symptoms in patients with a $\mathrm{COHb}$ level of $10 \%$ or greater. Further analysis revealed that severe symptoms were more pronounced in adolescents and that the severity of symptoms increased with age.

\section{Competing interests None.}

\section{P40}

\section{Refractory septic shock in children: an ESPNIC definition}

Luc Morin', Samiran Ray ${ }^{2}$, Graeme Maclaren ${ }^{3}$, Etienne Javouhey ${ }^{4}$, Simon Nadel $^{5}$, Martin Kneyber ${ }^{6}$, Mark Peters ${ }^{2}$, Koos Jansen 7 , Daniele De Luca ${ }^{8}$, Clare Wilson ${ }^{5}$, Luregn Schlapbach ${ }^{9}$, Pierre Tissières ${ }^{1}$, The infection,

inflammation and sepsis section of the ESPNIC

${ }^{1}$ Réanimation et surveillance continue pédiatriques, CHU Kremlin Bicêtre, Le Kremlin Bicêtre, France; ${ }^{2}$ Pediatric intensive care unit, Great Ormond Street Hospital, London, United Kingdom; ${ }^{3}$ Pediatric intensive care unit, The Royal Children's Hospital, Parkville, Australia; ${ }^{4}$ Réanimation pédiatrique hfme, Hospices civils de Lyon, Lyon, France; ${ }^{5}$ Pediatric intensive care unit, Saint Mary's Hospital, London, United Kingdom; ${ }^{6}$ Pediatric intensive care unit, Beatrix Children's Hospital, Groningen, Netherlands; ${ }^{7}$ Pediatric intensive care unit, Wihelmina Children's Hospital, Utrecht, Netherlands;

${ }^{8}$ Neonatal intensive care unit, Hospital Antoine Béclère, Clamart, France; ${ }^{9}$ Pediatric intensive care unit, Royal Children hospital, Brisbane, Australia Correspondence: Luc Morin - lucpmorin@gmail.com Annals of Intensive Care 2016, 6(Suppl 1):P40

Introduction Septic shock is a constantly evolving challenge for pediatric intensive care units (PICU) physicians. Despite a decrease in the trend of mortality, part of these patients dies in the first hours of care from refractory septic shock (RSS). A definition for RSS is needed to help its earlier diagnostic, have a better understanding of the disease and evaluate specific treatments. We aimed to define RSS with a tworound Delphi study among the European Society of Pediatric and Neonatal Intensive Care (ESPNIC), to create septic shock prognostic scores on a development cohort and to validate these tools as diagnostic of RSS on a multicentric cohort.

Patients and methods A total of 114 PICU physicians from 27 countries answered a clinical case-based two-round Delphi study. Criteria most associated with diagnosis of RSS in clinical cases were selected, then validated in the second round of the study 
and compiled in a draft definition. During a 4-year period (20102013), all patients admitted for septic shock without limitation of active treatment were screened. Seventy patients from one PICU (21 deaths, $30 \%$ ) were included in the development population and 324 patients from four PICUs (39 deaths, $12 \%$ ) in the validation population. Two septic shock scores were created after combination of the survey results and the multivariate analysis on the development population.

Results The resulting draft definition for pediatrics constitutes myocardial dysfunction with high blood lactate levels despite adequate vasopressor treatment. Analyzed in the development population, each item of the draft definition was independently associated with need for ECMO or death. Two septic shock scores were created. A simplified septic shock score $(4 \mathrm{~S}) \geq 2 / 5$ had a mortality positive predictive value of 60.4 and $84.6 \%$ if $\geq 4 / 5$. The complete septic shock score (cSSS) had a discriminative power measured with the area under the responderoperating curve of $95 \%$ (95\% Cl 92-98\%).

Discussion There is no existing score to assess risk of death in children with septic shock. Blood lactates, VIS and septic cardiomyopathy are known factors associated with mortality in septic shock. The combination of the three criteria is a powerful and sensitive way to diagnose patients in RSS. Cutoff proposed for this diagnosis is a $4 \mathrm{~S} \geq 2 / 5$ which is associated with a hazard ratio of death of 68.69 (95 \% Cl 30.47-154.9). Conclusion Refractory septic shock in pediatrics defined with the ESPNIC selects patients with high mortality rate in PICU. Septic shock scores are useful tools to assess risk of death or need for ECMO at the bedside of the patient (4S) or to compare populations of septic shock patients (CSSS).

\section{Competing interests None.}

\section{P41}

Impact of the Leonetti law on end-of-life practices in a French NICU and PICU, from January 2011 to December 2014

Anne-Claire Girard', Elodie Savajols², Antoine Burguet ${ }^{2}$, Denis Semama²,

Stephanie Litzler-Renault ${ }^{3}$

${ }^{1}$ Côte d'or, C.H. Chalon sur Saône William Morey, Chalon-sur-Saône,

France; ${ }^{2}$ Pédiatrie 2, Chu Dijon, Dijon, France; ${ }^{3}$ Réanimation pédiatrique polyvalente, Chu Dijon, Dijon, France

Correspondence: Stephanie Litzler-Renault - stephanie.litzler@yahoo.fr

Annals of Intensive Care 2016, 6(Suppl 1):P41

Introduction End-of-life is a highly debated subject. In France, the Leonetti law was promulgated in 2005 to clarify medical practices regarding this topic. It draws attention to the aspect of unreasonable obstinacy and authorizes withholding or withdrawal of treatments such as mechanical ventilation, vital drugs or artificial nutrition when they appear "useless or disproportionate." It also emphasizes the necessity to alleviate pain, even when doing so could result in shortening life. Ten years after its promulgation, this law still seems not fully implemented in French Neonatal Intensive Care Unit (NICU) and Pediatric Intensive Care Unit (PICU).

The aim of this study is to assess the implementation of the Leonetti law in our NICU and PICU and to study the evolutions of this implementation over time.

Materials and methods We performed a retrospective observational monocentric study using data collected from medical and nursing charts. For each case, four criteria to be respected regarding the Leonetti law were sought from the charts: collegiate decision regarding the implementation of end-of-life care, parental involvement in the decision, traceability of the decision within the patient medical file, and the way dignity and quality of life that were maintained throughout end of life. This classification has been done by a physician outside our unit, unaware of the child's situation, in order to lead to a consensual classification. The interpretation of the data was based on univariate analysis, descriptive at first. We then compared those data year by year. Results Over this period, 94 cases out of 119 were included. The four criteria were met in $40 \%$ of the cases, and this value significantly improved over time [8-52\% between 2011 and 2014] $(p<0.01)$. Three criteria out of four were met in on average $76 \%$ of the cases. The legal obligation of collegiate decision was respected in $73 \%$ of the cases [58-76\%] ( $p 0.3)$, parental involvement in $85 \%$ [58-92 \%] $(p<0.01)$ and traceability in $99 \%$. Dignity and quality of life were maintained suitably in $53 \%$ of the cases [21-72\%] $(p<0.01)$. While three criteria out of four tended to improve over time, the traceability rate was stable around $99 \%$.

Discussion Despite the biases caused by its retrospective nature, our study allowed us to confirm the improvement in our practices in children end of life. However, there is still room for improvement, particularly regarding collegiate decision (e.g., systematic presence of an external consultant) and assessment of pain and discomfort in end-oflife situations.

Conclusion Laws and medical recommendations cannot bring all answers to end-of-life situations. However, many elements regarding the implementation of the Leonetti law within our units still need to be improved. Following this study, a series of reflections on ethical issues aiming at fulfilling the predefined legal criteria will be set up within our units.

\section{Competing interests None.}

\section{References}

1. Loi n²005-370 du 22 avril 2005 relative aux droits des malades en fin de vie. J Off Repub Fr Ed Lois Decrets 2005; 95: 7089. Disponible sur: http:// www.legifrance.gouv.fr.

2. Limitations et arrêts des traitements en réanimation pédiatrique de la décision à son application. Hubert P., Centre Laennec 2009; 57:22-34.

\section{P42}

\section{Mortality risk factors for anticoagulant-related bleeding}

Ines Sedghiani ${ }^{1}$, Hana Fredj ${ }^{1}$, Malek Hassouna ${ }^{1}$, Youssef Zied El Hechmi ${ }^{1}$ Mohamed Ali Cherif', Jerbi Zouheir

${ }^{1}$ Emergency and intensive care department, Hopital Habib Thameur, Tunis, Tunisia

Correspondence: Ines Sedghiani - sedghiani.ines@gmail.com Annals of Intensive Care 2016, 6(Suppl 1):P42

Introduction Vitamin K antagonists (VKA) have served as the cornerstone of long-term anticoagulant therapy. Out of the respect of the recommendations of prescription and biological monitoring, this treatment can be complicated by life-threatening bleeding events.

Patients and methods We carried out a retrospective study of 58 patients taking oral anticoagulants and who were hospitalized for hemorrhagic events from January 2013 to June 2015.

The aim of this study is to describe the characteristics of patients treated with VKA presented a bleeding event, to study the hemorrhagic event and its management and to search for prognostic factors. Results Among the 58 patients (24 men and 34 women, aged $69 \pm 11$ years) with anticoagulant-associated bleeding event, 41 patients survived and 17 patients died. All patients were already taking acenocoumarol for $65 \pm 61$ months. VKA were used for the prevention of stroke due to atrial fibrillation in $41(70 \%)$ cases, prosthetic cardiac valve replacement in 13 (22\%) cases and venous thromboembolism in four $(7 \%)$ cases.

Conclusion The results of this study showed that IGSII $\geq 37$, intracranial and gastrointestinal bleeding and elevated AST level are useful prognostic factors for predicting anticoagulant-associated bleeding event mortality (Table 13).

Competing interests None.

\section{P43}

Management of the polytrauma in the emergency department of CHUOran

Houria Mokhtari-Djebli' ${ }^{1}$ Soumia Benbernou², Ahmed Dernane ${ }^{3}$, Fouad Bouakkaz ${ }^{2}$, Khalida Bouyacoub ${ }^{4}$, Abdelkader Azza ${ }^{4}$

${ }^{1}$ Urgences médicales et réanimation chuoran, Faculté de Médecine d'Oran, Oran, Algeria; ${ }^{2}$ Urgences Medicales Chuoran, Faculté de médecine d'Oran, Oran, Algeria; ${ }^{3} U \mathrm{mc}$, Hospital Center And University D'oran, Oran, Algeria; ${ }^{4}$ Urgences medicales, chu oran, oran, Algeria 
Correspondence: Soumia Benbernou - gsoumia@hotmail.com Annals of Intensive Care 2016, 6(Suppl 1):P43

Introduction The polytrauma is a major public health problem, and assessment of severity is an important part of the initial management which determines prehospital means necessary.

The host structure must take into account the particularities of these patients.

The need for a complete and rapid lesion balance makes desirable the management of trauma patients in centers with a complete technical platform.

Some studies have shown that up to $30 \%$ of deaths in trauma patients could have been avoided by better management.

Goal

The goal of this study was to define the epidemiological profile of polytrauma at the service of medical and surgical emergencies CHUOran. Patients and methods Retrospective study of trauma patients admitted to the intensive care unit of the UMC from January 1, 2009, to December 31, 2011.

Data collection was carried out through a pre-established record where data are noted. The coding data entry and data analysis are performed using the Epilnfo Version 6 software.

The main items of the plug are age, sex, mechanism, collection, time of admission hemodynamic, respiratory and neurological constants, initial lesion balance, surgery, secondary lesion balance, ICU hospitalization and evolution of the patient.

Results A total of 217 severe trauma patients were collected during the period from January 2009 to December 2011.

\begin{tabular}{|c|c|c|c|}
\hline & $\begin{array}{l}\text { Survivors } \\
(n=41)\end{array}$ & $\begin{array}{l}\text { Nonsurvi- } \\
\text { vors }(n=17)\end{array}$ & $p$ \\
\hline Intracranial & $4(9 \%)$ & $5(29 \%)$ & \\
\hline Alveolar & $5(12 \%)$ & 0 & \\
\hline Gastrointestinal & $9(22 \%)$ & 7 (41 \%) & \\
\hline Gynecologic & $1(2 \%)$ & $1(5 \%)$ & \\
\hline Hematuria & $7(17 \%)$ & 0 & \\
\hline Intraabdominal & $3(7 \%)$ & $1(5 \%)$ & \\
\hline Pericardial & 0 & $1(5 \%)$ & \\
\hline Initial prothrombin ratio (\%) & 10 & 10 & 0.47 \\
\hline Initial INR & 10 & 15 & 0.14 \\
\hline Cephalin kaolin time (s) & $76 \pm 30$ & $74 \pm 38$ & 0.85 \\
\hline Hemoglobin (g/dl) & $9.7 \pm 3.3$ & $8.9 \pm 4.4$ & 0.54 \\
\hline $\begin{array}{l}\text { Fall in hemoglobin level } \\
(\mathrm{g} / \mathrm{dl})\end{array}$ & 1 & 1 & 0.76 \\
\hline Platelet count $\left(\times 10^{3} \mathrm{e} / \mathrm{mm}^{3}\right)$ & $308 \pm 131$ & $261 \pm 134$ & 0.25 \\
\hline $\begin{array}{l}\text { Aspartate aminotransferase } \\
\text { (AST) (UI/L) }\end{array}$ & 27 [17-38] & 68 [34-244] & 0.007 \\
\hline$A S T \geq 34 \mathrm{UI} / \mathrm{L}$ & $12(29 \%)$ & $11(64 \%)$ & 0.008 \\
\hline $\begin{array}{l}\text { Alanine aminotransferase } \\
\text { (ALT) (UI/L) }\end{array}$ & 22 [12-35] & 45 [16-136] & 0.08 \\
\hline Bilirubin $(\mu \mathrm{mol} / \mathrm{l})$ & 20 [14-39] & 35 [22-63] & 0.05 \\
\hline Blood urea $(\mathrm{mmol} / \mathrm{l})$ & $13 \pm 8$ & $22 \pm 14$ & 0.1 \\
\hline Serum creatinine $(\mu \mathrm{mol} / \mathrm{l})$ & 118 & 153 & 0.22 \\
\hline
\end{tabular}

Table 13 See text for description

\begin{tabular}{|c|c|c|c|}
\hline & $\begin{array}{l}\text { Survivors } \\
(n=41)\end{array}$ & $\begin{array}{l}\text { Nonsurvi- } \\
\text { vors }(n=17)\end{array}$ & $p$ \\
\hline Sex ratio (M/F) & 0.57 & 1.1 & 0.25 \\
\hline Age, mean (SD), years & $68 \pm 12$ & $72 \pm 8$ & 0.2 \\
\hline IGS II, mean (SD) & $27 \pm 10$ & $41 \pm 17$ & 0.006 \\
\hline$|G S| \mid \geq 37$ & $7(17 \%)$ & $9(53 \%)$ & 0.005 \\
\hline SOFA, median (IQR) & $2(2-4)$ & $3(2-4.5)$ & 0.1 \\
\hline \multicolumn{4}{|l|}{ Comorbidities, n (\%) } \\
\hline Hypertension & $25(61 \%)$ & $13(76 \%)$ & 0.25 \\
\hline Diabetes & $10(24 \%)$ & $8(47 \%)$ & 0.08 \\
\hline Congestive heart failure & $14(34 \%)$ & $6(35 \%)$ & 0.93 \\
\hline Atrial fibrillation & $31(75 \%)$ & $13(76 \%)$ & 0.94 \\
\hline Liver cirrhosis & $3(7 \%)$ & 0 & 0.54 \\
\hline Alternate-day dosing & $12(29 \%)$ & $6(35 \%)$ & 0.22 \\
\hline Indication of VKA & & & 0.3 \\
\hline Atrial fibrillation & $27(65 \%)$ & $14(82 \%)$ & \\
\hline $\begin{array}{l}\text { Prosthetic valve replace- } \\
\text { ment }\end{array}$ & $10(24 \%)$ & $3(17 \%)$ & \\
\hline Thromboembolic events & $4(9 \%)$ & 0 & \\
\hline Daily dose (mg) & $0.5 \pm 0.27$ & $0.5 \pm 0.22$ & 0.71 \\
\hline $\begin{array}{l}\text { Timing of anticoagulant } \\
\text { therapy (months) }\end{array}$ & $65 \pm 57$ & $66 \pm 92$ & 0.97 \\
\hline Antiplatelet therapy & $7(17 \%)$ & $3(17 \%)$ & 1 \\
\hline HAS-BLED >3 & $2[1.75 ; 3]$ & $3[2 ; 4]$ & 0.19 \\
\hline Bleeding site & & & 0.05 \\
\hline Cutaneous & $12(29 \%)$ & $2(11 \%)$ & \\
\hline
\end{tabular}

We note a clear predominance of the male sex with a sex ratio of 8.77. Most traumatized patients were young with an average age of 34.217 years.

The accident in the way was the origin of the trauma in $77.4 \%$ followed by the fall with a frequency of $22.6 \%$.

The collection was medicalized in $39.6 \%$ of cases. $11.5 \%$ were transferred from other cities.

$20.7 \%$ had systolic blood pressure $\leq 90 \mathrm{mmHg}$ and $23 \%$ had $\mathrm{SpO}_{2}$ $\leq 90 \%$.

$11.1 \%$ had a Glasgow score $\leq 3 / 15$. For $4.6 \%$ of them, it was of $4 / 15$, $6 \%$ had a score of $5 / 15,8.3 \%$ had a score of $6 / 15,13.8 \%$ had a score of $7 / 15,11.2 \%$ had a score of $8 / 15$, and $45 \%$ had a score $\geq 9 / 15$ on the Glasgow coma scale. $18 \%$ had anisocoria on admission and $1 \%$ was in mydriasis.

The revisited trauma score was $\leq 5$ in $19.4 \%$ of patients.

$43.78 \%$ had an orthopedic injury. $62.7 \%$ were intubated dice admission.

Chest radiography was performed in $92.6 \%$ of patients, and that of the basin and abdominal ultrasound was made in $94 \%$ of them.The computed tomography was performed in $95.4 \%$ of traumatized patients, and that of the thorax and abdomen was with a respective proportion of 76 and $39.6 \% .85 .3 \%$ of our patients were polytraumatized, and trauma of the head was the most frequent lesion with a proportion of $85.3 \%$ followed by the thorax with a frequency of $62.8 \%$ and finally the abdomen with a rate of $21 \%$. The average ICU stay was 19.19 days. The outcome was unfavorable in $30.9 \%$ of patients

Discussion In our study, we found that the polytrauma mainly touched young males under 40 years old. This constitution is similar to that reported by David J. Thurman and collaborators in the published report on the brain injury in the USA. In the Annual Report of 2013 of the Trauma Data Bank and by the state of 805 health centers in the USA, the fall was the leading cause of head injury with a frequency of $40.66 \%$ followed by accident thoroughfare of $28.1 \%$. 
At a time where the prehospital medicalization is the subject of numerous publications and a wide debate between two different management systems:

- In the USA, initial support is provided by paramedics, who after treatment of respiratory and hemodynamic distress use the shortest time possible to transfer the patient to the nearest trauma center, and this concept is called "scoop and run."

- In France and in some European countries, the management is performed by physicians who take all the time necessary for the treatment of different afflictions before transferring the patient to the hospital, and it is called "stay and play."

Our study has found that the initial management of patients was not medicalized in $60 \%$ of patients. First, the multicenter study was able to show that prehospital medicalization of patients was associated with a significant reduction in risk of death in 30th post-traumatic day.

Conclusion The care of the injured polytrauma is complex and must not be improvised in any case.

This management must be rational and constantly evaluated in order to improve the quality and results. It concerns civil society, hospital practitioners and public authorities.

\section{Competing interests None.}

\section{References}

1. David J. Thurman, Clinton Alverson, Doug Browne Traumatic Brain Injury in the United States: A Report to Congress 1999.

2. J.-M. Yeguiayan, D. Garrigue, C. Binquet, C. Jacquot, J. Duranteau, C. Martin, F. Rayeh, B. Riou, C. Bonithon-Kopp, M. Freysz*. Prise en charge actuelle du traumatisé grave en France : premier bilan de l'étude FIRST (French Intensive care Recorded in Severe Trauma) Ann. Fr. Med. Urgence (2012) 2:156-163. doi:10.1007/s13341-012-0181-1.

\section{P44}

\section{Realization of a feedback system of respiratory parameters} during CPR

Lhoucine Ben Taleb' ${ }^{1}$ Elmaati Essoukaki ${ }^{1}$, A Zeddine Mouhsen'1, Aissam Lyazidi', Jean-Christophe Marie Richard ${ }^{3}$, Ahmed Mouhsen ${ }^{4}$, Mohammed Harmouchi', Mourad Rattal ${ }^{2}$

'Laboratoire rayonnement-matière et instrumentation, université hassan 1er, Faculté des Sciences et Techniques, Settat, Morocco; Institut supérieur des sciences de la santé-laboratoire rayonnement-matière et instrumentation, université hassan 1er, Settat, Morocco; ${ }^{3}$ Emergency and intensive care department, General Hospital of Annecy, Annecy, France, France; ${ }^{4}$ Laboratoire d'ingénierie, management industriel et innovation, Faculté des Sciences et Techniques, Settat, Morocco

Correspondence: Lhoucine Ben Taleb - Ihoucine.fsts@gmail.com Annals of Intensive Care 2016, 6(Suppl 1):P44

Introduction The objective of this work is to achieve a system for monitoring parameters of respiratory tract and ensure feedback on the quality of chest compressions during cardiopulmonary resuscitation (CPR).

Materials and methods This prototype is designed by flow and pressure sensors that plug at the respiratory tract, as well as efficient data acquisition card which processes and monitors signals. Communication with the user is done by visual interfaces (graphical and numerical) via an efficient development tool LABVIEW 2014 (National Instrument).

The settings for the compression quality, frequency, flow, volume and respiratory tract pressure are recorded and displayed during CPR.

Results The figure presents a preliminary test and calibration of the system's sensors on a graphical interface using the measurement system (Fig. 28).

Conclusion The realized system will give a feedback on respiratory parameters generated by chest compressions. Additional tests are expected to validate this prototype in more realistic situations.

\section{Competing interests None.}

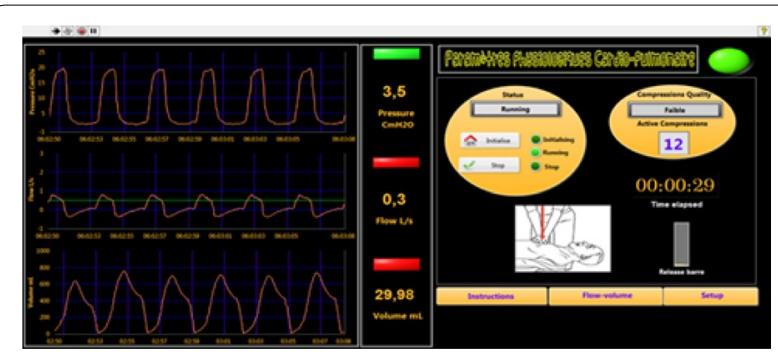

Fig. 28 See text for description

\section{P45}

Burns from French military operations: a 14-year retrospective observational analysis

Matthias Huck' ${ }^{1}$, Thomas Leclerc ${ }^{1}$, Nicolas Donat ${ }^{1}$, Audrey Cirodde',

Jean-Vivien Schaal', Yannick Masson', Clément Hoffmann'

${ }^{1}$ Centre de traitement des brûlés, Hôpital d'Instruction des Armées Percy, Clamart, France

Correspondence: Clément Hoffmann - clement.hoffmann@yahoo.fr Annals of Intensive Care 2016, 6(Suppl 1):P45

Introduction Burns in combat usually represent 5-20\% of injuries [1]. The purpose of the study was to describe epidemiological characteristics and the management of burns during French military operations.

Patients and methods $A$ retrospective observational study was performed of French casualties with burns, transported to Percy Burn Center (R4 Medical Treatment Facility) between January 1, 2001, and December 31, 2014.

Results Forty-six patients were included. Seventy-six percent of burns analyzed were non-battle injuries, mainly due to mishaps occurring with the burning of waste and the use of fuel. Combat burns $(n=11 / 46)$ were all explosions related (improvised explosive devices). The median total body surface area burned was $15 \%[9 ; 27]$. Major anatomical regions affected were upper limbs $(91 \%)$, head/ neck $(78 \%)$ and hands (70\%). Battle-related burns resulted in higher median Injury Severity Score, more patients under mechanical ventilation, more blood transfusions and surgeries, respectively, $16[6 ; 23]$ versus $4[1 ; 8], 64$ versus $17 \%, 8[0 ; 17]$ versus 0 and $3[0 ; 4]$ versus 0 $(p<0.05)$. The case fatality rate was $2.2 \%(n=1 / 46)$.

Discussion Majority of burns are non-battle injuries, small in size and accessible to prevention [2]. For battle-related burn injuries, the anatomical topography can be explained by the personal protective equipment and the higher severity score due to the associated trauma and mechanism. This study is only the visible tip of the iceberg.

Conclusion All military care providers should be familiar with the assessment and treatment of burns in military settings.

\section{Competing interests None.}

\section{References}

1. Cancio LC et al. J Burn Care Rehabil 2005;26:151-161.

2. Kauvar DS et al. J Burn Care Res 2009;30:700-704

\section{P46}

Drowning in Martinique-characteristics and infectious complications

Dabor Resiere', Laura Cerland ${ }^{1}$, Ruddy Valentino', C Chabartier ${ }^{1}$, Laurent Villain-Coquet ${ }^{2}$, Jean-Louis Ferge ${ }^{1}$, Y Brouste ${ }^{2}$, Bruno Megarbane ${ }^{3}$, Hossein Mehdaoui $^{1}$

'Service de réanimation médico-chirurgicale, $\mathrm{CHU}$ de Fort de

France, Fort-de-France, France; ${ }^{2}$ Samu 972, CHU de Fort de France,

Fort-de-France, France; ${ }^{3}$ Service de Réanimation Médicale et Toxi-

cologique, CHU Lariboisière, Paris, France

Correspondence: Dabor Resiere - dabor.resiere@chu-fortdefrance.fr Annals of Intensive Care 2016, 6(Suppl 1):P46 
Introduction Drowning is a public health problem, with a high incidence of 500,000 people worldwide, and 150,000 deaths each year in mainland France and the overseas territories. This is one of the leading causes of accidental death in children and adolescents. Immersion exposes them to multiple complications that are often life threatening. Infectious complications are, however, poorly understood and not well described. The objective of this study was to define the frequency and nature of lung infections in victims of drowning admitted to the emergency and ICU departments at the University Hospital of Martinique.

Patients and methods We conducted a retrospective study from victims of drowning cases admitted to the ER and ICU CHU Martinique over a period of 3 years (2012-2015), supplemented by data from SAMU 972 records, including presentation of descriptive data and univariate predictors of death.

Results One hundred and forty-four patients were included [102 men/42 women, mean age 36.5 years (range 1-83 years)]. Drownings occurred in seawater $(N=120,83 \%)$; swimming $(N=15,10 \%)$; freshwater $(N=5,3 \%)$ or polluted water $(N=1,1 \%)$. Seventy-eight patients (54\%) had comorbidities including hypertension $(N=14$, $10 \%)$; diabetes $(N=10,7 \%)$; epilepsy $(N=12,8 \%)$; heart disease rhythm and ischemic $(N=10,7 \%)$; chronic alcoholism $(N=8,6 \%)$; and obesity $(N=6,4 \%)$. The majority of victims (31\%) were in deep coma (score Glasgow: 3 ) and severe hypoxia; 32 victims (22\%) suffered cardiac arrest. Complications were as follows: multiple organ failure $(97 \%)$, lactic acidosis ( $75 \%$, mean arterial $\mathrm{pH} 7.20)$, acute respiratory failure (49\%) and ARDS (16\%, 2 refractory ARDS patients required veno-venous ECMO). Bacterial pneumonia $(N=22,15 \%)$ was the main cause of infectious complications, early onset (within $24 \mathrm{~h}$ ). Antibiotic therapy was started probabilistically on admission $(N=85$, $59 \%$ ), with amoxicillin/clavulanic acid (92\%), cefotaxime (6\%) or cefotaxime metronidazole $+(1 \%)$. Only $17 \%$ of victims had blood cultures and $52(36 \%)$ lung sampling. Antibiotic therapy was administered before performing lung samples $(82 \%)$. The most frequently encountered germs were Aeromonas hydrophilia, Enterobacter aerogenes, Enterobacter cloacae, Morganella and Morgani. The average length of ICU stay was 7 days. Eighteen of these patients (13\%) died in the ICU. Predictors of mortality endpoints were: the occurrence of a prehospital cardiac arrest $(p<0.0001)$, ARDS $(p<0.001)$, the immersion time $(p<0.05)$, metabolic acidosis $(p<0.05)$, the Glasgow coma scale score $<5(p<0.01)$, the resuscitation period $(p<0.01)$ and hypothermia $(p<0.05)$.

Conclusion The prognosis remains poor after immersion in the absence of a rapid response and appropriate treatment, especially in cases of occurrence of out-of-hospital cardiac arrest (OHCA).

\section{Competing interests None.}

\section{Reference}

1. Ouanes-Besbes L, F Dachraoui, Ouanes I Abroug F. Drowning pathophysiological and therapeutic. Réanimation 2009.

\section{P47}

Prevalence, characteristics, and associated factors to physician's conflicts in Emergency Department: A multicentric Moroccan study

Jihane Belayachi' ${ }^{1}$, Maha Louriz ${ }^{1}$ Naoufal Madani ${ }^{1}$, Fatiha Amlaiky ${ }^{1}$, Tarek Dendane ${ }^{2}$, Khalid Abidi², Aicha Zekraoui ${ }^{1}$, Amine Ali Zeggwagh², Redouane Abouqal

'Medical Emergency Department, Ibn Sina University Hospital, Rabat, Morocco; ${ }^{2}$ Service de réanimation médicale et de toxicologie cliniquechu ibn sina-universite mohamed v, Hopital Ibn Sina, Rabat, Morocco Correspondence: Jihane Belayachi - jihanebelayachi@gmail.com Annals of Intensive Care 2016, 6(Suppl 1):P47

Introduction Despite evidence that conflicts are common and harmful in the Emergency Department (ED), no study has been described in the literature. This study aimed to evaluate the prevalence and characteristics of conflict in ED physicians and factors associated with still ongoing conflict.

Materials and methods This was an observational cross-sectional survey conducted among physicians in 12 Moroccan ED, from June
2012 to January 2013. Socio-demographics, work characteristics, conflict-related variables, burnout level and self-estimation measures were included. Conflict-related question was formulated in the following way: In the past years, have you been drawn into any kind of conflict in ED? Response alternatives were (yes or no). To those who answered in the affirmative, the followed characteristics of conflict were collected; numbers of episodes of conflicts $(<5$ or $\geq 5)$. (a) The source of the conflict (Control, Prevision, Resources, Organisation, Coordination); (b) the form of the conflict; (Conflict of content, Conflicts of persons, Conflicts of procedure); (c) the degrees of the conflict: (Confrontation of viewpoint, Destructive personal attacks); (d) the conflict is important or unimportant; (e) the result of the conflict is good or poor; (f) The conflict is terminated or still ongoing; $(\mathrm{g})$ the response to the conflict: (Avoidance, Accommodation, Competition, Collaboration). Comparison between "terminated conflict" emergency physicians group and "still ongoing conflict" group was made. Multiple logistic regression was used to evaluate factors associated with still ongoing conflict.

Results A total of 101 emergency physicians were included with a response rate of $81 \%$. The mean age of ED physicians was $40 \pm 8$ years, and $70.3 \%$ among them are men. Conflicts were reported in $97 \%$ of emergency physicians, $49.5 \%$ reported more than five episodes of conflicts, and $41 \%$ of conflict is still ongoing. The principal sources of conflict were: organisation in $82.2 \%$ of case, resources $87.1 \%$ of case and coordination $81.2 \%$ of case. Comparison between the two groups showed that ED physicians with still ongoing conflict have a longer years of practices ( $11.6 \pm 5$ vs $9.1 \pm 6.8, p=0.02)$. Concerning burnout, ED physicians with still ongoing conflict are more emotionally exhausted (37.3 \pm 9.7 vs $32.6 \pm 12.8, p=0.04)$ and have lesser personal accomplishment $(27.5 \pm 3.6$ vs $32.6 \pm 9.4, p=0.01)$. Concerning self-rated scales, ED physicians with still ongoing conflict perceived a worst mental health $(6.6 \pm 1.3$ vs $7.3 \pm 1.3, p=0.01)$ are less joyful $(6 \pm 1.2$ vs $6.7 \pm 1.5, p=0.02)$.

Multiple logistic regression showed that longer years of practice (OR1.2, $95 \% \mathrm{Cl} 1.02-1.31 ; p=0.02)$ and emotional exhaustion (OR $1.2 ; 95 \% \mathrm{Cl} 1.004-1.11 ; p=0.03$ ) were the factors associated with still ongoing conflict.

Conclusion The prevalence of conflict is very high in ED. Longer years of practice and emotional exhaustion in physicians was associated with still ongoing conflict. This high prevalence of conflict suggests that conflict in the ED may be a universal phenomenon that should be addressed.

\section{Competing interests None.}

P48

Serious peripartum complications needing admission in obstetrical ICU: retrospective study about 127 cases

Affra Brahim ', Fehmi Ferhi', Hosni Khouadja', Mohamed Amine Bouslama' ', Khalii Tarmiz², Khaled Benjazia'

${ }^{1}$ Anesthesia and intensive care, Chu Farhat Hached, Sousse, Tunisia;

${ }^{2}$ Sousse centre, Chu Farhat Hached, Sousse, Tunisia

Correspondence: Khalil Tarmiz - khalil.tarmiz@gmail.com

Annals of Intensive Care 2016, 6(Suppl 1):P48

Introduction Peripartum morbidities present a clinical challenge due to maternal physiological adaptations related to pregnancy. Pregnant women may be afflicted with surgical condition and/or medical requiring admission to intensive care unit. The aim of our study is to identify serious complications occurred during pregnancy and childbirth in our health structure and analyze the epidemiological aspects, the management and the development of these complications.

Patients and methods We conducted a retrospective descriptive study of 127 patients admitted in obstetric ICU for severe peripartum complications, during the period from January 2011 to December 2013.

Results The average age of patients was $31 \pm 5$ years, ranging from 19 to 45 years. Eighty-six women were classified as ASA 1 (67\%), and 41 had a medical history including cardiomyopathy (29\%). The average gestity in these patients was $2 \pm 1$ [1-8], the parity was 1 [0-6], and the medium-term pregnancy at the time of hospitalization was 
34 weeks [28-42]. The main reasons for admission were the management of postpartum hemorrhage in $36 \%$ of cases followed by severe preeclampsia in $31 \%$ of cases. The admission to gravity scores were $10 \pm 7$ for APACHE 2 [0-35], $17 \pm 13$ for the IGS2 and $3 \pm 3$ for the SOFA score. Artificial ventilation was necessary in $42 \%$ of cases. The duration of ventilation was $2 \pm 2$ days [0-16]. The main therapeutic implemented during the ICU stay has been in order of frequency: the antibiotic therapy in $43 \%$ of patients, anticonvulsants (magnesium sulfate) and antihypertensives (40\%), transfusion of blood products (27\%) and use of vasopressors ( $15 \%$ of cases). Fifty-six patients had at least one complication during hospitalization. The most frequent one was acute kidney failure, infectious and respiratory complications. The average hospital stay was 5 days [1-85]. Five patients died.

Conclusion Our study confirms that direct obstetric complications, mainly hypertensive and hemorrhagic, predominate. Severity scores are lower than for the general population resuscitation, the observed mortality was lower than the predicted mortality, and longer durations of hospitalization and ventilation are shorter. A structure of obstetric ICU could promise earlier management of maternal conditions at high risk of serious complications.

\section{Competing interests None.}

\section{P49}

Epidemiology of suicide in emergency department

Neila Maaroufi', Abid Zeineb², Dhaouadi Mahassen ${ }^{3}$

${ }^{1}$ General Hospital, Tunis, Tunisia; ${ }^{2}$ Emergency, General Hospital, Tunis,

Tunisia; ${ }^{3}$ Tunisia, General Hospital, Tunis, Tunisia

Correspondence: Neila Maaroufi - maaroufineila@gmail.com

Annals of Intensive Care 2016, 6(Suppl 1):P49

Introduction This study aimed to illustrate the characteristics of suicide attempters treated in the Emergency Departments of a general hospital in Tunisia and to provide relevant data for early psychological treatment.

Patients and methods Between January 2009 and December 2014, 571 suicide attempters were treated in the Emergency Departments. Data were collected using a semi-structured questionnaire. Descriptive statistics, Chi square tests, and multivariate analyses were used to identify the factors associated with suicidal behaviors

Results Females outnumbered males at a ratio of 3.5-1. The greatest proportion of cases was in the age group of 15-30 years (62.7\%). Patients who finished middle school or high school accounted for most of the suicide attempters. The most common method used for attempted suicide was drug ingestion (85.5\%). The majority of cases attempted suicide at home. Marriage frustration, work and study problems, family fanaticism and conflict, somatic disease, and history of mental disorders were all significantly associated with suicide attempts. The ratio of patients to be discharged or to die was similar in occupation, marital status, and the place of suicide attempt; however, the results were different in gender, age, educational level, methods used for suicide, time of day, and reason.

Discussion and conclusion Suicide is an important public health problem. A previous suicide attempt is a risk factor for suicide; thus, assessing the characteristics of suicide attempts or instrumental suicide-related behaviors with/without injuries is necessary to prevent these attempts.

\section{Competing interests None.}

\section{P50}

Beneficial cardiovascular effects of O-GIcNAc stimulation in early phase of septic shock

Julien Cadiet ${ }^{1}$, Marine Ferron ${ }^{2}$, Valentine Prat ${ }^{2}$, Angelique Erraud ${ }^{2}$, Virginie Aillerie'2, Mathieu Mevel ${ }^{3}$, Amandine Grabherr², John C. Chatham4,

Chantal Gauthier ${ }^{2}$, Benjamin Lauzier ${ }^{2}$, Bertrand Rozec ${ }^{5}$

${ }^{1}$ Department of anesthesiology and intensive care unit, Centre hospitalier universitaire de Nantes, Nantes, France; ${ }^{2}$ L'institut du thorax, INSERM UMR 1087, Nantes, France; ${ }^{3}$ Atlantic gene therapies, INSERM UMR 1089, Nantes, France; ${ }^{4}$ Division of molecular and cellular pathology, University of Alabama at Birmingham, Birmingham, United States of America;
${ }^{5}$ Réanimation CTCV Transplantation thoracique, CHU de Nantes - Hôpital Nord Laennec, Saint-Herblain, France

Correspondence: Julien Cadiet - julien.cadiet@gmail.com

Annals of Intensive Care 2016, 6(Suppl 1):P50

Introduction Septic shock is a worldwide burden with over 750,000 cases per year in the US only. This pathology, characterized by a systemic inflammation, is associated with a severe cardiovascular dysfunction. Currently, there is no specific treatment for the septic shock beside antibiotherapy, fluid resuscitation and vasopressor amine. Finding new therapeutic leads in septic shock is important because this disease is a major public health problem. Recently, Not et al. showed, during hemorrhagic shock in rats, a beneficial effect of the O-GlcNAcylation stimulation on cardiovascular parameters. O-GlcNAcylation, a posttranslational modification, is the end product of the hexosamine biosynthetic pathway (HBP). We postulated that increase in total protein O-GlcNAcylation at the early phase of septic shock could improve cardiovascular function and reduce mortality.

Materials and methods To induce an endotoxemic shock, rats $(n=6-8)$ received $i v$ either lipopolysaccharide (LPS, $5 \mathrm{mg} / \mathrm{kg}$ ) or saline (CTRL). After $1 \mathrm{~h}$, fluid resuscitation (FR, $15 \mathrm{ml} / \mathrm{kg}$ of colloid, iv) was associated or not with HBP substrate: glucosamine $(\mathrm{GlcN}, 180 \mathrm{mg} / \mathrm{kg})$ or an O-GlcNAcase inhibitor (NButGT, $10 \mathrm{mg} / \mathrm{kg}$ ). Two hours later, echography and mean arterial pressure (MAP) evaluation were performed; blood samples and heart were then collected to evaluate biological parameters (lactate, troponin T, creatinine), inflammation, autophagy and total O-GlcNAcylation by western blot. Survival analysis was performed in parallel.

Results In vivo studies showed an hypotension in LPS restored by FR and treatments (MAP: CTRL $87 \pm 1$, LPS $73 \pm 5^{*}$, LPS-FR $93 \pm 6 \#$, NButGT $83 \pm 5$, GlcN $92 \pm 4 \mathrm{mmHg}^{*}: p<0.01$ vs CTRL, \#: $p<0.01$ vs LPS) and a systolic dysfunction in LPS with a trend toward improvement by NButGT and GICN (ejection fraction: CTRL $81 \pm 2$, LPS $65 \pm 3 *$, LPS-FR $69 \pm 2$, NButGT $75 \pm 2 \#$, GlcN $74 \pm 2 \%, *: p<0.01$ vs CTRL, \#: $p<0.05$ vs LPS). NButGT and GlcN efficiently increased total O-GlcNAc (200 and $300 \%$, respectively, vs LPS-FR). This effect was associated with a reduced cardiomyocyte insult, tissue dysoxia and renal dysfunction (creatinine: CTRL $25.4 \pm 1.2$, LPS $51.4 \pm 5.8^{*}$, LPS-FR $77.5 \pm 6.8^{*}$, NButGT $33.7 \pm 4.7 £$, GlcN $38.7 \pm 8.2 \mu \mathrm{mol} / \mathrm{I} £^{*}: p<0.01$ vs CTRL, $£$ : $p<0.05$ vs LPS-FR). Moreover, while all LPS $+\mathrm{R}$ rats did not survive after $10 \mathrm{~h}, 20 \%$ of treated rats survived after $18 \mathrm{~h}$. Interestingly, NButGT and GlcN effects were not associated with modification of autophagic pathway (Beclin-1, LC3, BCl-2) or inflammation (TNF-a, IL-6).

Conclusion At the early phase of septic shock, NButGT and GlcN induce an increase in cardiac O-GlcNAcylation leading to improve tissue oxygenation and renal function and to reduce cardiac injury. These treatments improved cardiovascular function. The next step is to determine the mechanisms involved in these beneficial effects.

\section{Competing interests None.}

\section{References \\ 1. PMID: 20016375. \\ 2. PMID: 17909453.}

P51

MiR-21 deficiency increases mortality during experimental septic shock

Jeremie Joffre ${ }^{1}$, Xavier Loyer ${ }^{2}$, Jean-Rémi Lavillegrand ${ }^{1}$, Lynda Zeboudj ${ }^{2}$, Ludivine Laurans ${ }^{2}$, Bruno Esposito' ${ }^{2}$, Alain Tedgui², Ziad Mallat ${ }^{2}$, Hafid Ait-Oufella

${ }^{1}$ Service de reanimation, Hôpital Saint-Antoine, Paris, France; ${ }^{2}$ U970, Inserm, Paris, France

Correspondence: Hafid Ait-Oufella - hafid.aitoufella@sat.aphp.fr Annals of Intensive Care 2016, 6(Suppl 1):P51

Introduction MicroRNAs (miRs) are noncoding RNAs found in all eukaryotic cells that regulate the human genome, primarily through translational repression. MiR-21 is expressed by inflammatory and endothelial cells in response to bacterial LPS, TNF-a or apoptotic 
debris. Mir-21 limits the production of cytokines and the expression of endothelial adhesion molecules and modulates cell survival. Most of the miR-21 targets being involved in the pathophysiology of septic shock, we hypothesized that miR-21 could play a role in tissue injury following bacteria invasion.

Patients and methods We demonstrated genetic invalidation (miR$21-/-$ ) or pharmacological inhibition of miR-21 (antagomiR-21). We reviewed mouse models of severe infections, endotoxinemia (LPS $15 \mathrm{mg} / \mathrm{kg}$ ) and non-resuscitated peritonitis.

Results In vitro, LPS-stimulated miR-21-/- splenocytes produced more IL-12p70 than miR-21+/+ splenocytes $(p<0.05)$. In addition, miR-21 - /- bone marrow-derived macrophages stimulated by LPS produced more IL-12p70, II- $1 \mathrm{~b}$, TNF-a and less II-10 than control miR$21+/+$ macrophages $(P<0.05)$. In a mouse model of endotoxinemia, genetic invalidation (miR-21-/-) of miR-21 significantly increased mortality $(P<0.01)$. Pharmacological inhibition of miR-21 using antagomiR-21 significantly increased mortality in two models of lethal infections $(P<0.01$, endotoxinemia and peritonitis). MiR-21+/+ and miR-21-/- mice were irradiated and retransplanted with MiR-21+/+ and miR-21-/- bone marrow. Four groups of chimeric mice were generated and challenged intraperitoneally with LPS. Interestingly, both miR-21 deficiency in leucocytes and in endothelial cells increased mortality in a non-redundant manner.

Conclusion MiR-21 deficiency induced a deviation of the immune response towards a pro-inflammatory profile and increased mortality during experimental shock septic.

\section{Competing interests None.}

\section{Reference}

1. Sheedy et al. Frontiers in Immunology 2015.

\section{P52}

Isolated heart rate reduction by ivabradine does not improve cardiac and vascular function in experimental septic shock Chaojie Wei ${ }^{1}$, Narimane Al Kattani ${ }^{1}$, Huguette Louis ${ }^{2}$, Sophie Orlowski ${ }^{3}$, Bruno Levy ${ }^{4}$, Antoine Kimmoun ${ }^{4}$

${ }^{1}$ Inserm u1116, Université de Lorraine, Vandœuvre-lès-Nancy, France;

${ }^{2}$ Dcac, Inserm, Vandœuvre-lès-Nancy, France; ${ }^{3}$ Service de biochimie,

Centre Hospitalier Universitaire de Nancy, Vandœuvre-lès-Nancy, France;

${ }^{4}$ Réanimation Médicale Brabois, Centre Hospitalier Universitaire de Nancy, Vandœuvre-lès-Nancy, France

Correspondence: Antoine Kimmoun - akimmoun@gmail.com

Annals of Intensive Care 2016, 6(Suppl 1):P52

Introduction In recent studies, $\beta 1$-adrenergic blocker demonstrated cardiovascular protection in sepsis. Experimental research showed that these benefits are associated with a down-regulation of inflammatory pathways. However, the specific impact of heart rate reduction on the inflammatory pathways remains unknown. Ivabradine is a pure heart rate-lowering drug that acts specifically on the sinoatrial node by selectively inhibiting the If current. The present study was designed to assess the effects of heart rate reduction by ivabradine on cardiovascular function and on the inflammatory pathways involved in peritonitis-induced septic rats.

Materials and methods Sixteen Wistar male rats were treated with cecal ligation and perforation (CLP) to evoke peritonitis. Four hours after CLP, rats were randomly allocated to $\operatorname{CLP}(n=8)$ and ivabradine ( $n=8$, administrated per os at H4) groups. Another eight Wistar male rats underwent sham operation. All rats received a continuous infusion of saline [10 mL/( $\mathrm{kg} \mathrm{h})$ ], analgesic [nalbuphin: $0.2 \mathrm{mg} /(\mathrm{kg} \mathrm{h})]$ and antibiotic (imipenem and cilastatin sodium: $10 \mathrm{mg} / \mathrm{kg}$ ) $4 \mathrm{~h}$ after the surgery. Assessment at $18 \mathrm{~h}$ included in vivo cardiac function by echocardiography and ex vivo vasoreactivity by myography. Cardiac and vascular protein expressions of NF-kB and eNOS/Akt/iNOS pathways were also assessed by western blotting.

Results Compared with Sham animals, CLP induced tachycardia (376 \pm 26 vs. $431 \pm 22 \mathrm{bpm}, p<0.05)$, hypotension $(111 \pm 7$ vs. $80 \pm 7 \mathrm{mmHg}, p<0.05)$, decrease in cardiac output $(95 \pm 13$ vs. $56 \pm 15 \mathrm{~mL} / \mathrm{min}, p<0.05)$, hyperlactatemia $(1.2 \pm 0.3$ vs. $2.5 \pm 0.5 \mathrm{mmol} / \mathrm{L}, p<0.05$ ) and vascular hypo-responsiveness to vasopressors. Adjunction of ivabradine decreased heart rate $(329 \pm 38$ vs. $431 \pm 22$ bpm, $p<0.05$ ) without any impact on blood pressure ( $73 \pm 10$ vs. $80 \pm 7 \mathrm{mmHg}, p>0.05)$, cardiac output ( $37 \pm 18$ vs. $56 \pm 15 \mathrm{~mL} / \mathrm{min}, p>0.05)$, lactatemia ( $2.6 \pm 0.6$ vs. $2.5 \pm 0.5 \mathrm{mmol} / \mathrm{L}$, $p>0.05$ ) and vascular responsiveness to vasopressors, compared with CLP group. Results of western blot will be provided later.

Conclusion Isolated reduction in heart rate by ivabradine in an experimental model of septic shock does not result in any effect on cardiovascular function.

\section{Competing interests None.}

\section{References}

1. Morelli A, Ertmer C, Westphal M, et al. Effect of heart rate control with esmolol on hemodynamic and clinical outcomes in patients with septic shock: a randomized clinical trial. JAMA. 2013 Oct 23;310(16):1683-91.

2. Katsuya Mori, Hiroshi Morisaki, Satoshi Yajima, et al. Beta-1 blocker improves survival of septic rats through preservation of gut barrier function. Intensive Care Med (2011) 37:1849-1856.

3. Gareth L. Ackland, Song T. Yao, Alain Rudiger, et al. Cardioprotection, attenuated systemic inflammation, and survival benefit of b1-adrenoceptor blockade in severe sepsis in rats. Crit Care Med 2010 Vol. 38, No. 2.

4. Jerome Aboab, Veronique Sebille, Mercé Jourdain, et al. Effects of esmolol on systemic and pulmonary hemodynamics and on oxygenation in pigs with hypodynamic endotoxin shock. Intensive Care Med (2011) 37:1344-1351.

5. Takeshi Suzuki, Hiroshi Morisaki, Ryohei Serita, et al. Infusion of the b-adrenergic blocker esmolol attenuates myocardial dysfunction in septic rats. Crit Care Med 2005 Vol. 33, No. 10.

6. Satoshi Hagiwara, Hideo Iwasaka, Hayato Maeda, et al. Landiolol, an ultrashort-acting beta1-adrenoceptor antagonist, has protective effects in an LPS-induced systemic inflammation model. SHOCK, Vol. 31, No. 5, pp. 515-520, 2009.

7. Antoine Kimmoun, Huguette Louis, Narimane AlKattani, et al. B1-Adrenergic Inhibition Improves Cardiac and Vascular Function in Experimental Septic Shock. Crit Care Med. 2015 Sep;43(9):e332-40.

8. Otilia Postea and Martin Biel. Exploring HCN channels as novel drug targets. Nat Rev Drug Discov. 2011 Nov 18;10(12):903-14.

9. Otilia Postea and Martin Biel. Exploring HCN channels as novel drug targets. Nat Rev Drug Discov. 2011 Nov 18;10(12):903-14.

\section{P53}

Exploration and modulation of the EGF-R during experimental septic shock Jean-Rémi Lavillegrand', Lynda Zeboudj ${ }^{2}$, Jeremie Joffre, ${ }^{1}$, Ludivine Laurans, ${ }^{2}$, Bruno Esposito ${ }^{2}$, Alain Tedgui ${ }^{2}$, Pierre-Louis Tharaux ${ }^{2}$, Hafid Ait-Oufella ${ }^{1}$

${ }^{1}$ Service de reanimation, Hôpital Saint-Antoine, Paris, France; ${ }^{2}$ U970, Inserm, Paris, France

Correspondence: Hafid Ait-Oufella - hafid.aitoufella@sat.aphp.fr Annals of Intensive Care 2016, 6(Suppl 1):P53

Introduction The receptor of epidermal growth factor (EGF-R) is expressed by mesenchymal and immune cells. EGF-R is involved in survival, migration and proliferation. Pharmacological EGF-R inhibiting therapy using either neutralizing $\mathrm{mAbs}$ or small-molecule tyrosine kinase inhibitors (TKI) has been successfully used in cancer treatment. Few evidences suggested that EGF-R could modulate directly the immune system, but the consequences in response to bacteria injury remain unknown. We aimed to investigate the effects of EGF-R blocking in an experimental model of septic shock.

Patients and methods We demonstrated the specific invalidation of Egf-r in myeloid cells (LysMCre Egf-rlox/lox) or pharmacological inhibition of EGF-R using TKI in vitro (AG-1478) and in vivo (Erlotinib, Tarceva $\left.{ }^{\circledR}\right)$. We reviewed the model of endotoxinemia by intraperitoneal injection of LPS (15 mg/kg). Cell culture of splenocytes, purified bone marrow neutrophils (isolation kit Miltenyi) and bone marrowderived macrophages (BMDMs) were tested.

Results In vitro, LPS-stimulated LysMCre Egf-rlox/lox splenocytes produced more IL-6 $(+300 \%, P<0.05)$ and more IL-10 $(+70 \%, P<0.05)$ than control LysMCre Egf-r+/+ but no difference regarding TNF-a. 
LPS-stimulated LysMCre Egf-rlox/lox BMDMs produced significantly more IL-6 (+70 \%) and more IL-10 (+180 \%) than control LysMCre Egf$r+/+$ cells. In addition, LysMCre Egf-rlox/lox neutrophils stimulated by LPS produced more TNF-a (+48 \%) and have a higher MPO activity $(+50 \%, P<0.05)$ than control cells. Genetic invalidation and pharmacological inhibition of EGF-R significantly decreased the recruitment of immune cells within the peritoneal cavity following LPS injection (respectively, -65 and $-42 \%, P<0.05$ ). However, genetic invalidation or pharmacological inhibition of Egf-r did not impair mouse survival in a model of endotoxinemia.

Conclusion EGF-R deficiency/inhibition modulated innate immune response but did not impair survival during experimental sepsis.

\section{Competing interests None.}

\section{P54}

An intravenous omega-3 bolus at reperfusion time improves shock and vascular dysfunction in a myocardial ischemiareperfusion rat model

Mélanie Burban ${ }^{1}$, Grégory Meyer ${ }^{2}$, Anne Olland ${ }^{3}$, Blandine Yver ${ }^{4}$, Florence Toti ${ }^{1}$, Valérie Schini-Kerth ${ }^{1}$, Alexandra Monnier ${ }^{5}$, Alexandra Boivin ${ }^{6}$, Pierrick Leborgne ${ }^{7}$, Ferhat Meziani ${ }^{5}$, Julie Boisramé-Helms ${ }^{8}$

${ }^{1}$ Umr7213, Strasbourg University, Strasbourg, France; ${ }^{2}$ Ea 4278, Université d'Avignon, Avignon, France; ${ }^{3}$ Chirurgie thoracique, Nouvel Hôpital Civil, CHU Strasbourg, Strasbourg, France; ${ }^{4}$ Ea7293, Strasbourg University, Strasbourg, France; ${ }^{5}$ Réanimation médicale, Nouvel Hôpital Civil, CHU Strasbourg, Strasbourg, France; ${ }^{6}$ Réanimation médicale, C.H.R.U. Hôpitaux Universitaires Strasbourg, Strasbourg, France; ${ }^{7}$ Service d'accueil des urgences, Nouvel Hôpital Civil, CHU Strasbourg, Strasbourg, France; ${ }^{8}$ Réanimation, CHU de Strasbourg, Strasbourg, France

Correspondence: Julie Boisramé-Helms - julie.helms@libertysurf.fr Annals of Intensive Care 2016, 6(Suppl 1):P54

Introduction Omega-3 supplementation for several weeks has been shown to improve myocardial resistance to ischemia-reperfusion in rats. In a rat model of myocardial ischemia-reperfusion, we investigated the effect of an intravenous bolus of omega-3, associated or not with iodinated contrast media (used for reperfusion process during coronarography), at reperfusion time, so as to mimic emergency clinical management of myocardial infarction and reperfusion-induced shock.

Materials and methods A myocardial ischemia-reperfusion model was performed by left anterior descending coronary artery ligation in rats. After a 30-min ischemia, but before reperfusion, an intravenous bolus of EPA and DHA (6:1), associated or not with iodinated contrast media, was administered. After a 4-h reperfusion, circulating procoagulant microparticles were quantified phenotyped. Vascular and heart inflammation, oxidative and nitrosative stresses were assessed. Ex vivo vascular reactivity was performed with a pharmacological selective inhibitor of iNOS. Infarct size was assessed by triphenyltetrazolium chloride staining.

Results Treating rats with an EPA/DHA bolus before reperfusion significantly improved the ischemia-reperfusion syndrome, increasing mean arterial pressure $(151 \pm 13$ vs $122 \pm 17 \mathrm{mmHg}, p<0.05)$, coronary and carotid blood flow, and decreasing infarct size $(39.9 \pm 2.3$ versus $26.8 \pm 5.7 \%$ of left ventricle, $p<0.05$ ). Moreover, ex vivo mesenteric resistance artery sensitivity to phenylephrine was improved. Finally, endothelial CD54+ microparticle release was decreased $(9.1 \pm 2.5$ vs. $4.8 \pm 2.0 \mathrm{nM}$ EqPhtdSer, $p<0.05)$, as well as vascular inflammation and oxidative stress, reflecting a reduced vascular dysfunction.

Discussion In our model, the omega-3 vascular beneficial effects may partly result from endothelial protection. Indeed, ischemia-reperfusion-induced endothelial dysfunction results in phenotypic and physical changes in the endothelium, with a deregulated release of potent vasodilators nitric oxide and prostacyclin, reduced vascular reactivity to vasoconstrictors, associated with leukocyte and platelet aggregation and deregulation of nitric oxide signaling. Rat hemodynamic parameters were subsequently improved. Associating omega-3 to iodinated contrast media in the reperfusion process therefore deserves further investigating and might be a promising line of development in humans.

Conclusion In this rat model, an intravenous omega-3 bolus before reperfusion significantly improved cardiovascular resistance to ischemia-reperfusion-induced cardiovascular failure and shock.

\section{Competing interests None.}

\section{P55}

Impact of urantide antagonist of urotensinergic system on myocardial function during a murine model of septic shock Emmanuel Besnier ${ }^{1}$, Thomas Clavier ${ }^{1}$, Mulder Paulus ${ }^{2}$, Hélène Castel ${ }^{3}$, Vincent Richard ${ }^{2}$, Fabienne Tamion

${ }^{1}$ Pôle Réanimations Anesthésie SAMU, Hospital Center University Rouen, Rouen, France; ${ }^{2}$ U1096, inserm, Rouen, France; ${ }^{3}$ U982, Inserm, Mont-Saint-Aignan, France

Correspondence: Emmanuel Besnier - emmanuel.besnier@chu-rouen.fr Annals of Intensive Care 2016, 6(Suppl 1):P55

Introduction New therapies to improve prognosis of patients with septic shock are a major area of research. Urotensinergic system plays a role in both modulation of inflammation and cardiovascular system and thus could be a therapeutic target. Unpublished work previously done highlighted an improvement in inflammatory parameters by administration of the antagonist urantide in an endotoxinic model. The aim of our study was to evaluate survival and myocardial function after administration of urantide during sepsis.

Materials and methods This animal study was subdivided into three experimental process involving different mice (C57BI/6): a survival analysis ( $n=10 /$ group), an echocardiographic analysis ( $n=5 /$ group) and a study of tissular inflammation markers ( $n=5$ /group). Three groups of mice were made, a sham group without sepsis, a control group (LPS-NaCl) and an urantide-treated group (LPS-urantide). Intraperitoneal injection of $30 \mathrm{mg} / \mathrm{kg}$ of lipopolysaccharide (LPS) from Escherichia coli was administered for septic groups. Echocardiographic analysis was performed every $3 \mathrm{~h}$ during $9 \mathrm{~h}$ for left ventricular ejection fraction (LEVF), cardiac output, aortic flow, heart rate and the E/A ratio obtained from mitral flow. Injection of either $\mathrm{NaCl}$ or urantide $\left(10^{-4} \mathrm{M}\right)$ at hour 3 and 6 was administered. Analysis of survival was performed according to the same experimental process, and tissular expression of hepatic, renal and myocardial NF-KB was performed at hour 9.

Results Survival was significantly improved from $30 \%$ up to $88.9 \%$ by urantide. Administration of LPS induced impairment of LVEF $9 \mathrm{~h}$ after the onset of sepsis. Urantide induced a significant difference in the LVEF and cardiac output at hour 9 with an increase up to $46 \%(p<0.05)$ and $51 \%(p<0.01)$, respectively, versus the control group. No difference was reported for heart rate or E/A ratio. Renal and hepatic expression of NF-KB was reduced down to 60 and $65 \%$, respectively, by urantide administration. Myocardial expression was not performed because of technical issues.

Discussion Our results highlighted a key role of urotensinergic system in cardiac performance during septic shock and a potential beneficial effect of its antagonizing. Nevertheless, our study was not able to determine the preload status of mice. Thus, it was not possible to discriminate a beneficial effect due to vascular or inotropic modulation.

Conclusion Urantide administration induced an improvement in survival and systolic myocardial function with increase in LVEF and cardiac output in an endotoxinic model of sepsis. Improvement in tissular inflammation may be involved. Further study is necessary in order to explore the effect on myocardial performance independently from vascular function.

\section{Competing interests None.}




\section{P56}

Association of dexamethasone and antibodies

to poly- $\beta(1,6)-n$-acetyl-glucosamine in prevention and treatment of neonatal bacterial meningitis: an experimental study

Stéphanie Pons ${ }^{1}$, Bruno Mourvillier² ${ }^{2}$ David Skurnik

${ }^{1}$ Division of Infectious Diseases, Brigham and Women's Hospital, Boston, MA, USA; ${ }^{2}$ Réanimation Médicale et Infectieuse, GH Bichat Claude Ber-

nard, Paris, France

Correspondence: Stéphanie Pons - pons.stephanie0@gmail.com

Annals of Intensive Care 2016, 6(Suppl 1):P56

Introduction Bacterial meningitis is still a severe infectious disease worldwide, with high rates of morbidity and mortality in children and adults. Dexamethasone (DXM) is recommended in association with antibiotics in some specific cases to decrease the risk of sequelae and possibly the mortality rate. The poly- $\beta-(1,6)-N$-acetyl-glucosamine (PNAG) is a surface polysaccharide expressed by numerous pathogenic bacteria, including species responsible for meningitis as Streptococcus pneumoniae, Neisseria meningitidis, Haemophilus influenzae, or Escherichia coli K1. Passive immunotherapy by monoclonal (MAb) to PNAG and polyclonal antibodies (PAb) to deacetylated PNAG (dPNAG) can prevent and treat various infections. The aim of this study was to analyze the association of DXM and antibodies to PNAG in neonatal bacterial meningitis prevention and treatment, using an E. coli $\mathrm{K} 1$ neonatal meningitis murine model.

Materials and methods The presence of PNAG on E. coli K1 surface in vitro and in vivo was assessed by confocal microscopy using fully human immunoglobulin G1 MAb with V-regions encoding specificity for either $P$. aeruginosa alginate, MAb F429 (control), or PNAG, MAb F598. In vitro experiments of bacterial adhesion on blood brain barrier (BBB) human cells were conducted using hCMEC/D3 cell line. Confluent cells were infected with $3.5 \times 10 \mathrm{e} 8$ of the well-described $E$. coli $\mathrm{K} 1 \mathrm{~S} 88$, alone or with DXM, goat PAb to dPNAG or normal goat serum (NGS). Experiment in the neonatal meningitis model was performed in 3-day-old CD1 mice by intraperitoneal (IP) administration of E. coli S88 K1 $\left(10^{-6} /\right.$ mouse). The IP administration of PBS or goat PAb to dPNAG (50 $\mathrm{\mu L} /$ mouse) was performed $24 \mathrm{~h}$ before and $2 \mathrm{~h}$ after challenge. DXM $(0.7 \mathrm{mg} / \mathrm{kg})$ was also injected $2 \mathrm{~h}$ after infection. The newborn mice were killed $24 \mathrm{~h}$ after challenge, the brain harvested and the colony-forming unit (CFU) count of $E$. coli determined.

Results In vitro, bacterial growth of $E$. coli $\mathrm{K} 1$ was not affected by the presence of different concentrations of DXM, antibodies to PNAG or both. PNAG expression was conserved in vitro on the bacterial surface of $E$. coli $\mathrm{K} 1$ in the presence of different concentrations of DXM, meaning that antibodies to PNAG could be associated with DXM. PNAG was also found on E. coli K1 surface in positive brains' homogenates of newborn mice, confirming that PNAG could effectively serve as a therapeutic target in vivo. hCMEC/D3 experiments showed a $50 \%$ decrease of bacterial adhesion in the presence of PAb to dPNAG compared to E. coli K1 alone $(p=0.001)$ or NGS $(p=0.007)$. DXM was responsible for a significant increase of $E$. coli adhesion in a dose-dependent manner (DXM $100 \mu \mathrm{M}$ per well $2 \times 10^{-7}$ vs $\left.8.2 \times 10^{-6}, p=0.001\right)$. The addition of different concentrations of DXM to PAb or to NGS did not change $E$. coli adhesion on hCMEC/D3 compared, respectively, to PAb alone $(p=0.98)$ or NGS alone $(p=0.48)$. In vivo, E. coli K1 meningitis rate was $100 \%$ after IP administration. Compared with PBS, the administration of PAb decreased significantly the CFU per brain $\left(4.1 \times 10^{-5}\right.$ vs $4 \times 10^{-2}$, $p<0.001)$. DXM administration after bacterial challenge did not change the CFU in the brain of neonates compared with bacteria alone $\left(1.7 \times 10^{-5}\right.$ vs $\left.1.3 \times 10^{-5}, p=0.43\right)$. The association of PAb to DXM showed a significant decrease in CFU in the brain compared with PBS $\left(4.1 \times 10^{-5}\right.$ vs $\left.10 \times 10^{-3}, p=0.007\right)$ but no difference with PAb alone $\left(10^{-} 3\right.$ vs $\left.4 \times 10^{-2}, p=0.33\right)$.

Conclusion PAb to dPNAG decrease E. coli $\mathrm{K} 1$ adhesion on the BBB in vitro and $E$. coli CFU per brain in vivo. DXM in association with PAb to dPNAG does not change the effect of PAb alone in vitro neither in vivo. Further experiments using the $E$. coli $\mathrm{K} 1$ neonatal model as well as adult animal models of meningitis when testing $S$. pneumoniae or $N$. meningitidis will better determine the possible use of DXM in association with PAb or MAb F 598 in bacterial meningitis treatment as well as the general and local levels of inflammation with both treatments.

\section{Competing interests None.}

References

1. Brouwer MC, et al. Corticosteroids for acute bacterial meningitis. Cochrane Database Syst Rev. 2013 Jun 4;6:CD004405

2. Wang $X$, et al. The pgaABCD locus of Escherichia coli promotes the synthesis of a polysaccharide adhesin required for biofilm formation. J Bacteriol. 2004 May;186(9):2724-34.

\section{P57}

Hyperoxemia as a risk factor for ventilator-associated pneumonia Sophie Six ${ }^{1}$, Karim Jaffal ${ }^{1}$, Saad Nseir

${ }^{1}$ Centre de réanimation, Centre Hospitalier Régional Universitaire de Lille, Lille, France

Correspondence: Saad Nseir - s-nseir@chru-lille.fr

Annals of Intensive Care 2016, 6(Suppl 1):P57

Introduction Several studies demonstrated an increased mortality rate in critically ill patients with hyperoxemia. Reactive oxygen species production, atelectasis, alveolar macrophage dysfunction, and acute lung injury are well-known consequences of hyperoxemia. In addition, these factors have been reported to increase the risk of pneumonia in intubated patients. To our knowledge, no study has previously evaluated the relationship between hyperoxemia and ventilator-associated pneumonia (VAP).

Patients and methods This retrospective observational study was performed during an 18-month period in a 30-bed medical and surgical ICU. All intubated patients requiring mechanical ventilation for more than $48 \mathrm{~h}$ were eligible. The primary objective was to determine the impact of arterial hyperoxemia (defined as $\mathrm{PaO}_{2}>120 \mathrm{mmHg}$ ) on the risk of VAP occurrence. VAP definition included clinical, radiological, and quantitative microbiological criteria. Patient characteristics and information on VAP occurrence were prospectively collected. Only data on arterial blood gases were collected retrospectively. Each day with at least one $\mathrm{PaO}_{2}>120 \mathrm{mmHg}$ accounted for $24 \mathrm{~h}$ with hyperoxemia. Risk factors for VAP were determined using univariate analysis and logistic regression multivariate analysis.

Results VAP was diagnosed in 141 (28\%) out of the 503 included patients. The incidence rate of VAP was 14.7 per 1000 ventilator-days. Pseudomonas aeruginosa (34\%) and Staphylococcus aureus (11.3\%) were the most frequently isolated bacteria. Hyperoxemia at ICU admission (67 vs. $53 \%$, OR [95 \% Cl] 1.8 [1.2-29], $p=0.004)$ and number of days spent with hyperoxemia ( 5 vs 3 days, $p<0.001$ ) were significantly higher in patients with VAP, compared with those without VAP. Univariate analysis also identified $(p<0.05)$ age, SAPS II, LOD score, shock at ICU admission, proton-pump inhibitor (PPI) use, red blood cell transfusion, sedation, and duration of mechanical ventilation as risk factors for VAP.

Multivariate analysis identified the number of days spent with hyperoxemia (OR [95 \% Cl] 1.1 [1.04-1.2] per day, $p<0.05)$, SAPS ॥ (1.01 [1.002-1.024] per point, $p<0.05)$, red blood cell transfusion (1.8 [1.12.7], $p=0.01)$ and PPI use (1.9 [1.03-1.2], $p<0.05)$ as independent risk factors for VAP. Two other logistic regression models were performed to determine the relationship between the percentage of days spent with hyperoxemia, or hyperoxemia at ICU admission and VAP. In these secondary analyses, the percentage of days spent with hyperoxemia, and hyperoxemia at ICU admission were also independently associated with VAP occurrence.

Conclusion Hyperoxemia is an independent risk factor for VAP. Further prospective large multicentre studies are required to confirm our results.

\section{Competing interests None. ${ }^{2}$}

${ }^{2}$ P58 Effects of Proanthocyanidins on Adhesion, Growth, and Virulence of Highly Virulent Extraintestinal Pathogenic Escherichia coli Argue for Its Use to Treat Oropharyngeal Colonization and Prevent Ventilator-Associated Pneumonia. http://www.ncbi.nlm.nih.gov/pubmed/25803655. 
P59

Significance of prior digestive colonisation

with extended-spectrum beta-lactamase producing Enterobacteriaceae in patients with ventilator-associated pneumonia

Rémi Bruyère ${ }^{1}$, Clara Vigneron ${ }^{1}$, Audrey Large ${ }^{1}$, Julien Bador ${ }^{2}$, Serge Aho ${ }^{3}$, Sebastien Prin ${ }^{1}$, Jean-Pierre Quenot ${ }^{1}$, Pierre-Emmanuel Charles ${ }^{1}$

${ }^{1}$ Réanimation médicale, Chu Dijon, Dijon, France; ${ }^{2}$ Laboratoire de bactériologie, Chu Dijon, Dijon, France; ${ }^{3}$ Service d'epidemiologie et d'hygiène hospitalière, Chu Dijon, Dijon, France

Correspondence: Rémi Bruyère - rbruyere@ch-bourg01.fr Annals of Intensive Care 2016, 6(Suppl 1):P59

Introduction Ventilator-associated pneumonia (VAP) is frequent in intensive care units (ICU). Extended-spectrum beta-lactamase-producing Enterobacteriaceae (ESBL-EB) are difficult-to-treat pathogens. We sought to assess the interest of screening for ESBL-Eb rectal carriage as a way to predict their involvement in VAP.

Patients and methods $A$ retrospective cohort study of patients with suspected VAP in a medical ICU was conducted. Every patient admitted between January, 2006, and August, 2013, was eligible if subjected to mechanical ventilation for more than $48 \mathrm{~h}$. Each patient with suspected VAP was included in the cohort. Active surveillance culture for ESBL-EB detection was routinely performed in all patients on admission and then weekly throughout the study period. ESBL colonisation was defined by the isolation of at least on ESBL-EB from rectal swab culture.

Results Among 587 patients with suspected VAP, 40 (6.8\%) were colonised with ESBL-EB prior to the development of pneumonia. Over the study period, 20 patients ( $3.4 \%$ ) had VAP caused by ESBL-EB, of whom 17 were previously detected as being colonised with ESBL-EB. Sensitivity and specificity of prior ESBL-EB colonisation as a predictor of ESBLEB involvement in VAP were 85.0 and $95.7 \%$, respectively. The positive and negative predictive values were 41.5 and $99.4 \%$, respectively. The positive likelihood ratio was 19.8 .

Conclusion Screening for ESBL-EB digestive colonisation by weekly active surveillance cultures could reliably exclude the risk of the involvement of such pathogens in VAP in low prevalence area.

\section{Competing interests None.}

\section{References}

1. Razazi K, Derde LP, Verachten M, et al. Clinical impact and risk factors for colonization with extended-spectrum beta-lactamase-producing bacteria in the intensive care unit. Intensive Care Med 2012.

2. Blot S, Depuydt P, Vogelaers D. Maximizing rates of empiric appropriate antibiotic therapy with minimized use of broad-spectrum agents: are surveillance cultures the key? Intensive Care Med 2008.

\section{P60}

Lower respiratory tract colonization in mechanically ventilated patients: Incidence, risk factors and impact on prognosis

Yosr Touil', Soufia Ayed ${ }^{1}$, Habiba Sik Ali', Najla Tilouch'1 , Houda Mateur', I

Talik', Rim Gharbi', Mohamed Fekih Hassen'1, Souheil Elatrous ${ }^{1}$

${ }^{1}$ Réanimation médicale, EPS Taher Sfar Mahdia, Mahdia, Tunisia

Correspondence: Souheil Elatrous - souheil.elatrous@rns.tn

Annals of Intensive Care 2016, 6(Suppl 1):P60

Introduction Ventilator-associated pneumonia (VAP) complicates the course of $8-28 \%$ of patients receiving mechanical ventilation (MV). Its mortality rate can reach $76 \%$ when lung infection is caused by highrisk pathogens as Acinetobacter baumannii. Bacterial colonization of the trachea and bronchi preceding VAP is well recognized as part of the pathogenic mechanisms. The objectives of this study were to determine the incidence, the associated risk factors and the impact on prognosis of lower respiratory tract colonization in patients under MV. Patients and methods It is a monocentric prospective study performed within 3 years. All patients admitted to our intensive care unit requiring invasive mechanical ventilation for more than $48 \mathrm{~h}$ were included. Eligible patients had a bacteriological follow-up that starts at the third day of hospitalization by realizing tracheal aspirate (TA) which is repeated every 3 days during MV length. Lower respiratory tract colonization is defined by a positive TA culture in the absence of clinical suspicion of VAP.

Results Two hundred and sixty-six patients on MV for more than $48 \mathrm{~h}$ were prospectively followed in this study. The airway colonization occurrence was $38 \%$ with a maximum between day 3 and day 6 of MV. The two most frequently isolated germs are Acinetobacter baumannii and Pseudomonas aeruginosa with a significant increase in their occurrence in time.

In multivariate analysis, the use of proton pump inhibitors, MV's duration and length of stay are identified as associated factors to the onset of airway colonization.

The incidence of VAP was significantly higher in colonized versus noncolonized patients (32 vs $18 \% ; p=0.006$ ). The onset of airway colonization was significantly associated with prolonged duration of $\mathrm{MV}$ and an increased length of stay $[19 \pm 17$ vs $9 \pm 7$ days $(p<0.0001)$ and $25 \pm 23$ vs $12 \pm 9$ days $(p<0.0001)$, respectively)] The mortality rate was comparable between colonized and non-colonized patients.

Conclusion The lower respiratory tract colonization is frequent in patients under MV. The use of proton pump inhibitors, MV's duration and length of stay are identified as associated factors to the onset of airway colonization. Colonized patients had a greater risk of developing VAP. The onset of airway colonization was significantly associated with prolonged duration of $\mathrm{MV}$ and an increased duration of stay.

Competing interests None.

\section{P61}

Epidemiology of ventilator-associated pneumonia in the ICU among patients already hospitalized: early- versus late-onset pneumonia

Mathilde Grandperrin ${ }^{1}$, Isabelle Patry ${ }^{2}$, Xavier Bertrand ${ }^{3}$, Jean-Christophe Navellou', Gaël Piton ${ }^{1}$, Gilles Capellier ${ }^{1}$

${ }^{1}$ Réanimation Médicale, CHU de Besançon, Besançon, France; ${ }^{2}$ Bactériologie, CHRU de Besançon, Besançon, France; ${ }^{3}$ Hygiène hospitalière, Centre

Hospitalier Régional Universitaire de Besançon, Besançon, France

Correspondence: Gaël Piton - piton.gael@wanadoo.fr

Annals of Intensive Care 2016, 6(Suppl 1):P61

Introduction Initiation of antibiotherapy is of major importance in the treatment of nosocomial pneumonia in the ICU. Antibiotherapy must be early, appropriated to the presumed bacteria, and must take into account epidemiology of the ICU and of the hospital. The aim of this study was to describe epidemiology of ventilator-associated pneumonia (VAP) in the ICU for patients already in hospital before ICU admission (HCAP) and to identify risk factors for multidrug-resistant pathogens.

Patients and methods This was a monocentric retrospective study of patients admitted to a single ICU in a large regional university hospital, with a diagnosis of ventilator-associated pneumonia, originating from another unit in the same hospital. Patients coming from home and from another hospital, and immunocompromised patients were excluded. Ventilator-associated pneumonias were divided into early onset ( $<5$ days) and late onset ( $\geq 5$ days). Duration of hospitalization before ICU admission, and the main risk factors for multidrug-resistant pathogens were collected.

Results Between January 2014 and May 2015, 114 patients developed ventilator-acquired pneumonia in the ICU. There were $69(61 \%)$ early-onset pneumonia and $45(39 \%)$ late-onset pneumonia. Bacterial diagnoses were made on tracheal aspirations $(99.1 \%)$ and broncho alveolar lavages (35.9). There were no significant differences in the bacteriology of early- and late-onset VAP. Gram negative bacteria were predominant ( 30.4 vs $31.1 \%, p=0.94$ ). There were $18.4 \%$ of multidrug-resistant pathogens in the overall population. Multidrug-resistant pathogens were mainly gram-negative bacteria (four BLSE, 11 cephalosporinase, four multiresistant pseudomonas aeruginosa, one stenotrophomonas maltophilia, and 1 SARM). There was a trend toward a higher prevalence of multidrug-resistant pathogen among patients with late-onset VAP (13 vs $27 \%, p=0.07)$. In univariate analysis, multidrug-resistant pathogens were associated with duration of hospitalization before ICU admission ( $5.9 \pm 8.5$ vs $24.8 \pm 74.5, p<0.05)$, 
duration stay in the ICU ( $8.8 \pm 6.8$ vs $27.2 \pm 25.9, p=0.004)$, and duration of mechanical ventilation (7.1 \pm 5.9 vs $20.6 \pm 25.3, p=0.04$ ).

Conclusion This work did not find significant differences in the epidemiology of early-onset versus late-onset VAP for patients already in hospital before ICU admission. However, there was a trend toward a higher rate of multidrug-resistant pathogens in late-onset VAP. Multidrug-resistant pathogens were mainly gram-negative bacteria, whereas prevalence of SARM was anecdotal. Duration of hospital stay before ICU admission, duration of ICU-stay, and duration of mechanical ventilation were risk factors for multidrug-resistant pathogens.

\section{Competing interests None.}

\section{P62}

Systematic review of inclusion/exclusion criteria, judgment criteria and statistical hypothesis in randomized controlled trials assessing the efficacy of antimicrobials for hospital-acquired and ventilator-associated pneumonia treatment

Emmanuel Weiss ${ }^{1}$, Jean-Ralph Zahar ${ }^{2}$, Christophe Adrie ${ }^{3}$, Wafa Essaied ${ }^{4}$, Martin Wolkewitz ${ }^{5}$, Jean-François Timsit ${ }^{6}$

'Département d'anesthésie-réanimation, Hôpital Beaujon, Boulevard du Général Leclerc, Clichy, France, Clichy, France; ${ }^{2}$ Unité de prévention et de lutte contre les infections nosocomiales, C.H.U. d'Angers, Rue Larrey, Angers, France, Angers, France; ${ }^{3}$ Explorations fonctionnelles, Cochin Port-Royal, Paris, France; ${ }^{4}$ Umr 1137 iame, Université Paris Diderot, Paris, France; ${ }^{5}$ Biostatistics, Biostatistical institute, Freiburg, Germany; ${ }^{6}$ Réanimation médicale et infectieuse, Hôpital Bichat-Claude Bernard, Paris, France Correspondence: Emmanuel Weiss - manuweiss@yahoo.fr Annals of Intensive Care 2016, 6(Suppl 1):P62

Introduction Hospital-acquired pneumonia (HAP) is the second most common nosocomial infection in hospitalized adults and is a leading cause of death among critical infections. To face the dramatically increasing prevalence of multidrug-resistant pathogen-related HAP, new therapeutic options are currently in development. The evaluation of these new treatments will require properly designed studies with appropriate inclusion criteria and endpoints. During the past 10 years, the regulatory agencies repeatedly updated guidance for the design of randomized controlled trials (RCT) aiming to evaluate the efficacy of antimicrobials for treatment of hospital-acquired and ventilator-associated pneumonia (HAP/VAP). However, our hypothesis is that, to date, HAP/VAP RCTs greatly differ in population enrolled, in HAP/VAP definition criteria used and in primary and secondary outcomes assessed. Such differences between studies may be of importance because they may impact results of studies. Thus, we performed a systematic review of characteristics of enrolled populations, inclusion/exclusion criteria and endpoints in RCT addressing the efficacy of antimicrobials for HAP/VAP treatment.

Patients and methods A double data search (PubMed-EMBASE, ICAAC, ESCMID) was performed of all RCT of the past 20 years evaluating antimicrobials for treatment of intensive care unit (ICU)-HAP/VAP.

Results The search retrieved 997 abstracts, of which 93 studies were reviewed. After exclusion of duplicates, post hoc analyses, studies without precise inclusion/exclusion criteria, studies without VAP and studies performed only outside the ICU, 25 studies were analyzed. One-third of studies included only VAP, while the other two-third included both HAP and VAP. The definition of HAP/VAP used various clinical radiological and biological criteria. Variable mandatory duration of prior hospital stay was used and length of mechanical ventilation was included in inclusion criteria in $36 \%$ of VAP studies only. Microbiological criteria were required in only $48 \%$ (with a microbiological threshold in $36 \%$ of studies). Severe patients (APACHE score $>25$ ) were excluded in $24 \%$ of studies. Most studies tested a hypothesis of non-inferiority (36\%) or equivalence ( $24 \%$ ), while $16 \%$ of studies aimed to demonstrate superiority. Surprisingly, statistical hypothesis was not clearly defined and sample size calculation not mentioned in 28 and $64 \%$ of studies, respectively. Clinical cure was considered as a primary endpoint in $92 \%$ of studies. However, the primary endpoint was dual and even triple in, respectively, 40 and $12 \%$ of studies because it was studied in different analysis population ( $28 \%$ of studies) or it was associated with microbiological cure (16\%) and/ or safety. Assumed clinical cure rate was anticipated in only one-third of manuscripts. Mortality was used as a primary endpoint only in two studies and safety in one. Definitions of clinical cure consisted in complete or partial remission of clinical signs and symptoms associated with pneumonia in $100 \%$ of studies, but improvement in chest radiography was associated in only two-third of the studies. Interestingly, the lack of additional antibiotic requirement during follow-up was included in clinical cure definition in $29 \%$ of studies. The test-of-cure visit evaluating the clinical cure ranged from the end of treatment to 28 days later.

Conclusion Our study provides a description of populations and endpoints of RCTs evaluating antimicrobials for treatment of ICU-HAP/VAP. We show a significant heterogeneity in enrollment criteria, endpoints and statistical design that may influence the ability of studies to demonstrate differences between studied drugs.

COMBACTE is supported by IMI/EU and EFPIA.

\section{Competing interests None.}

\section{P63}

Gram stain identification resulting from blindly inserted telescoping plugged catheter material. Does it contribute to diagnosis and treatment of ventilator-associated pneumonia?

Mickaël Soued ${ }^{1}$, Fabrice Cook ${ }^{1}$, David Lobo ${ }^{1}$, Roman Mounier ${ }^{1}$, Aurélie

Emirian², Jean Winoc Decousser², Gilles Dhonneur

${ }^{1}$ Anesthesia and intensive care medicine, Hospital Henri Mondor, Créteil,

France; ${ }^{2}$ Microbiology, Hospital Henri Mondor, Créteil, France

Correspondence: Fabrice Cook - fabrice.cook@aphp.fr

Annals of Intensive Care 2016, 6(Suppl 1):P63

Introduction Ventilator-associated pneumonia (VAP) is a frequent infection that complicates the course of many critical care patients. Early antimicrobial therapy, initiated before the results of pulmonary specimen cultures, seems to improve outcome (1). However, empiric treatment strategies expose the risk of inappropriate treatment, or unnecessary broad-spectrum antibiotic. Best bacteriological technique for the diagnosis of VAP is still debated. Blindly inserted telescoping plugged catheter (TPC) sampling seems reliable and is recommended methods for the bacteriological diagnosis of VAP. The threshold of $10^{3}$ colony-forming units (cfu) $\mathrm{mL}^{-1}$ provides a good sensitivity and specificity. Because 24- to 48-h delay is needed for quantitative cultures, early Gram's stain identification performed upon TCP samples could guide empirical antibiotic therapy. However, accuracy of such strategy is controversial because true sensitivity and specificity of Gram's stain for diagnosis of VAP remain questionable. In the present study, we evaluate whether the Gram stain upon initial TPC samples could predict the results of quantitative culture and identification of pathogens in patients suspected for VAP. We analyze associated factors that may influence accuracy of Gram's stain identification and the resulting empiric antibiotic treatment adequacy.

Patients and methods We retrospectively analyzed 122 TPC samples from 77 patients between January and December 2012, corresponding to all TPC samples retrieved for VAP diagnosis in a 20-bed surgical intensive care unit of a University Hospital. Positive Gram's stains and culture for any microorganisms, the presence of Gram-positive cocci (GPc) and/or Gram-negative rods (GNr) on Gram's stain and culture were collected. Cultures were considered positive if they yielded organisms on final culture with a threshold value $\geq 10^{3} \mathrm{cfu} \mathrm{mL}^{-1}$. The presence of leukocytes, moment of the day sampling being perform, prior to sampling antibiotic therapies, and duration of mechanical ventilation before sampling were analyzed as possible associated factors that may influence results of Gram's stain. Fisher's exact test was used for comparison of sensitivity and specificity between groups. Data are expressed as number ( $95 \%$ confidence interval); $p<0.05$ was considered to be statistically significant.

Results A total of 122 TPC samples were analyzed. Details of diagnosis performance (sensitivity, specificity, predictive value and likelihood 
ratio) of Gram staining for positive quantitative culture of any microorganism, GPc and GNr are summarized in Table 14.

Among associated factors, the presence of leukocytes on Gram's stain was associated with an improvement in sensitivity versus the absence of leukocytes for any microorganism $(0.87, p<0.001)$, for GPC $(0.83$, $p=0.04)$ and for $\mathrm{GNr}(0.63, p=0.036)$. Other associated factors failed to influence the results of Gram's stains. False negative Gram's stain for any microorganism was significantly associated with non-adapted empirical antibiotic treatment $(p=0.008)$.

Conclusion Gram's stain identification upon TPC samples has acceptable accuracy for predicting positive culture for the diagnosis of VAP. Prediction of GPc or GNr bacteria involved in VAP has poor accuracy excepted for GNr specificity. The presence of leukocytes on TPC results of Gram's stain improves sensitivity for VAP-positive culture. Taking Gram's stain information into account for establishing empiric antibiotic treatment may generate non adapted therapy. Results must be confirmed by prospective and multicenter studies.

\section{Competing interests None.}

\section{Reference}

1. Irequi M, Ward S, Sherman G, Fraser VJ, Kollef MH. Clinical importance of delays in the initiation of appropriate antibiotic treatment for ventilatorassociated pneumonia. Chest. 2002 Jul;122(1):262-268.

\section{P64}

\section{Colistin in nosocomial pneumonia in intensive care}

Hanane Ezzouine ${ }^{1}$, Mohamed Toufik Slaoui ${ }^{1}$, Abdellatif Benslama ${ }^{1}$

${ }^{1}$ Anesthesiology and intensive care department, University Teaching Hospital Ibn Rushd-Casablanca, Casablanca, Morocco

Correspondence: Hanane Ezzouine - ezzouinehanane@yahoo.fr Annals of Intensive Care 2016, 6(Suppl 1):P64

Introduction The increase in the incidence of ventilator-acquired pneumonia (VAP) Acinetobacter baumannii to multiresistant (ABMR) is responsible for a high morbidity and mortality in intensive care environment worldwide. The gold standard treatment of these serious infections remains carbapenems, including imipenem and meropenem. Sulbactam is a validated therapeutic alternative. Few studies have proved the effectiveness of VAP colimycin onto ABMR.

Patients and methods This is a prospective observational study in the medical ICU of CHU IBN Rushd of Casablanca for 24 months (October 2012-October 2014). Inclusion criteria were patients hospitalized in intensive care and who developed nosocomial pneumonia after $48 \mathrm{~h}$ of mechanical ventilation, with isolation of Acinetobacter Baumanii

\section{Table 14 See text for description}

\begin{tabular}{|c|c|c|c|}
\hline & $\begin{array}{l}\text { Any microor- } \\
\text { ganism }\end{array}$ & GPc & $\mathrm{GNr}$ \\
\hline Positive Gram's strain & 74 & 47 & 43 \\
\hline Positive culture & 85 & 32 & 66 \\
\hline Sensitivity & $0.81(0.71,0.89)$ & $0.78(0.60,0.91)$ & $0.58(0.45,0.70)$ \\
\hline Specificity & $0.86(0.71,0.95)$ & $0.76(0.65,0.84)$ & $0.91(0.80,0.97)$ \\
\hline $\begin{array}{l}\text { Positive predictive } \\
\text { value }\end{array}$ & $0.93(0.85,0.98)$ & $0.53(0.38,0.68)$ & $0.88(0.75,0.96)$ \\
\hline $\begin{array}{l}\text { Negative predictive } \\
\text { value }\end{array}$ & $0.67(0.52,0.80)$ & $0.91(0.82,0.96)$ & $0.65(0.53,0.75)$ \\
\hline $\begin{array}{l}\text { Positive likelihood } \\
\text { ratio }\end{array}$ & $\begin{array}{l}6.01(2.64 \\
13.66)\end{array}$ & $3.20(2.13,4.80)$ & $6.45(2.72,15.27)$ \\
\hline $\begin{array}{l}\text { Negative likelihood } \\
\text { ratio }\end{array}$ & $0.22(0.14,0.34)$ & $0.29(0.15,0.56)$ & $0.47(0.35,0.62)$ \\
\hline
\end{tabular}

only sensitive to colimycin, using the protected distal sampling. After the confirmation of the presence of a susceptibility to ABMR, antibiotic therapy was started.

Results In our study, 120 cases of ventilator-acquired pneumonia were collected. The average age of the patients was $40 \pm 15$ years, and the sex ratio was 1.5 with a male predominance. The reason for hospitalization in the ICU was dominated by diabetics ketoacidosis and serious head injuries. The simple gravity index (SAPS II) of the patients was $35 \pm 12$. The mean time to onset of VAP was $6 \pm 1$ days. Treatment with intravenous colimycine was considered effective in 114 patients ( $95 \%$ of cases). The overall mortality rate was $25 \%$ (30 cas) in our series, and only six cases (5\%) were attributable to VAP AB complicated multi-resistant refractory septic shock. There were no patients with impaired kidney function or clinical neurological impairment. Conclusion Our study suggests that colimycin could be a first-line therapy for VAP in ABMR in our Third World countries due to its efficacy, safety and low cost.

\section{Competing interests None.}

\section{P65}

Bedside tissue perfusion evaluation predicts intradialytic hemodynamic instability in critically ill patients

Naïke Bigé ${ }^{1}$, Claire Pichereau', Jean-Rémi Lavillegrand', Arnaud Galbois ${ }^{1}$

Mikael Alves ${ }^{1}$, Jean-Luc Baudel', Simon Bourcier ${ }^{1}$, Vincent Dubée ${ }^{1}$, Jalel

Tahiri ${ }^{1}$, Eric Maury', Bertrand Guidet', Hafid Ait-Oufella'

${ }^{1}$ Réanimation médicale, Hôpital Saint-Antoine, Paris, France

Correspondence: Naïke Bigé - naikebige@gmail.com

Annals of Intensive Care 2016, 6(Suppl 1):P65

Introduction Intermittent hemodialysis is a key support therapy in ICU. Despite protocol-based optimization, arterial hypotension during intermittent hemodialysis remains a frequent issue ranging from 10 to $60 \%$ in critically ill patients. Our objective was to test whether tissue perfusion parameters assessed at the bedside (mottling, index capillary refill time (CRT), and lactate) predict intradialytic hemodynamic instability ( $\mathrm{HI})$.

Patients and methods We conducted a prospective observational study in a 18-bed medical ICU in a tertiary university hospital including hemodialysis sessions performed for acute kidney injury. Exclusion criteria were the following: patients with dark skin and dialysis performed in extreme emergency. Mean arterial pressure (MAP), cardiac index, mottling, index CRT and lactate level were recorded just before starting hemodialysis. $\mathrm{HI}$ requiring fluid resuscitation or vasopressors introduction/increase was recorded 60, 120, and $240 \mathrm{~min}$ after hemodialysis starting.

Results One hundred and six hemodialysis sessions performed in 45 patients were recorded. Patients received vasopressors in $43(41 \%)$ sessions. HI occurred in $22(21 \%)$ of all the sessions and was more frequent among patients receiving vasopressors ( 42 vs $6 \%, P<0.0001$ ). Mottling was more frequent (77 vs $40 \%, P<0.0001$ ), index CRT was higher (3.3 [1.6-4.6] vs $1.1[0.8-1.9] \mathrm{s}, P<0.0001)$, and lactate level was higher (2.8 [1.4-6.9] vs $1.1[0.8-1.5] \mathrm{mmol} / \mathrm{L}, P<0.0001)$ before sessions with $\mathrm{HI}$ in comparison with sessions without. The incidence of $\mathrm{HI}$ increases with the number of tissue perfusion alterations $(2,14,31$, and $65 \%$ for $0,1,2$, and 3 alterations, respectively, $P<0.0001)$, independently of MAP $(P<0.0001)$. A tissue perfusion score, defined as "index CRT(s) + lactate level (mmol/L) + 0/1 if mottling were absent/ present", was predictive of HI independently of MAP (OR 1.18 [1.051.32], $P<0.0001$ ). According to ROC curves analysis, a threshold of 4.2 predicted $\mathrm{HI}$ with a sensitivity of $82[60-95] \%$ and a specificity of 82 [72-90] \%.

Conclusion The incidence of $\mathrm{HI}$ increases with the number of tissue perfusion alterations independently of MAP. At the bedside, a combined tissue perfusion score including mottling, index CRT, and lactate level helps to identify patients at risk.

Competing interests None. 


\section{P66}

Evaluation of electrolytes and acid-base disorders in patients undergoing citrate-based anticoagulation for continuous renal replacement therapy in intensive care unit

Benjamin Delmas', Olivier Joannes-Boyau², Antoine Dewitte'2, Julien Jabot', Dorothée Valance ${ }^{1}$, Nicolas Allou', Jérôme Allyn ${ }^{1}$, Catherine Fleureau, ${ }^{2}$, Alexandre Ouattara ${ }^{2}$

${ }^{1}$ Réanimation polyvalente, CHU La Réunion, Saint-Denis, France; ${ }^{2}$ Service d'anesthésie-réanimation 2, CHU de Bordeaux, Maison du Haut Lévèque, Pessac, France

Correspondence: Benjamin Delmas - benjamindelmas.desar@yahoo.fr Annals of Intensive Care 2016, 6(Suppl 1):P66

Introduction Specific electrolytes and acid-base disorders may occur using citrate-based anticoagulation (CBA) for continuous renal replacement therapy (CRRT). The aim of this study is to evaluate and to compare the impact of two CBA-CRRT protocols on electrolytes and acid-base status: one continuous veno-venous hemodialysis (CVVHD) protocol using $4 \%$ citrate (group A) and one continuous veno-venous hemofiltration (CVVH) protocol using 18/0 citrate (group B), both of which use phosphate-containing solutions.

Patients and methods We conducted a retrospective study from January 2013 to March 2014. All patients treated by CBA-CRRT admitted in three medico-surgical ICUs have been included. We collected demographic, clinical and biological data and performed Wilcoxon's matched-pairs signed-ranks test to compare for quantitative values. Data are expressed as median, first and third quartile.

Results Forty-seven patients were included, 30 in group $A$ and 17 in group B. No severe metabolic alkalosis occurred. One severe metabolic acidosis occurred in group $A$ as a complication of citrate accumulation syndrome $(\mathrm{pH} 6.99$, base excess $-19 \mathrm{mmol} / \mathrm{L}$ and bicarbonate $12 \mathrm{mmol} / \mathrm{L}$ ), diagnosed as a total calcium over ionized calcium ratio of 2.54. This was also the only case of severe hypocalcemia (ionized calcium $0.89 \mathrm{mmol} / \mathrm{L})$. Seven patients ( $15 \%$ ) presented mild hypophosphoremia, but no severe hypophosphoremia occurred. We did not notice any hyperphosphoremia in our patients. Median $\mathrm{pH}$ was 7.41 $(7.38-7.46)$ on day 3 and $7.34(7.28-7.39)$ on day $1(p<0001)$. Median natremia on day 3 was $141(139-141)$ and $135(134-135) \mathrm{mmol} / \mathrm{L}$ in group $\mathrm{A}$ and $\mathrm{B}$, respectively $(p=0.002)$. Median chloremia was 108 (106-109) and 95 (95-96) $\mathrm{mmol} / \mathrm{L}$ in group $A$ and $B$, respectively $(p=0.0002)$. In group $A$, one severe hypernatremia occurred on day 1 , corrected on day 2 , and two moderate hypernatremia occurred on day 1 , corrected on day 2 . No hypernatremia occurred in group $B$ $(p=0.29)$. Hypomagnesemia occurrence constantly increased from day 1 to day 3 , from 0 to $71 \%$.

Discussion This study pinpoints three interesting findings. First, it is well known that hypernatremic metabolic alkalosis may occur under CBA-CRRT in case of using trisodium citrate, which contains a high sodium load $(408 \mathrm{mmol} / \mathrm{L})$ as compared to $18 / 0$ citrate $(140 \mathrm{mmol} / \mathrm{L}$ of sodium), but the mild risk of alkalosis due to hypochloremia using dilute citrate $(86 \mathrm{mmol} / \mathrm{L}$ in $18 / 0)$ is mostly unrecognized. However, no patient presented severe metabolic alkalosis, but natremia was significantly higher in group A and chloremia lower in group B. Second, hypophosphoremia rate, which has been diagnosed in as many as $65 \%$ of the patients undergoing CRRT when using dialysis or replacement solutions without phosphate, is effectively decreased by using phosphate containing solutions. Finally, attention must be drawn on the high proportion of hypomagnesaemia, even if dialysis and replacement solutions contain magnesium, as citrate creates complex with magnesium as well as calcium.

Conclusion Both protocols are effective for correcting acid-base disorders, but in different ways, one by increasing natremia and the other by reducing chloremia. Phosphate-containing solutes are effective to reduce hypophosphoremia occurrence, but not sufficient to avoid hypomagnesaemia.

Competing interests None.
P67

Continuous venovenous hemofiltration in critically ill patients with acute kidney injury: a cost study impact of replacement fluids consumptions

Elodie Jean-Bart ${ }^{1}$, Floriane Bel.' ', Fabienne Morey ${ }^{1}$, Rémi Bruyère², Nicholas Sedillot ${ }^{2}$

${ }^{1}$ Pharmacy, Hospital Center Fleyriat, Bourg-en-Bresse, France; ${ }^{2}$ Intensive care unit, Hospital Center Fleyriat, Bourg-en-Bresse, France

Correspondence: Elodie Jean-Bart - elodiejb@yahoo.fr

Annals of Intensive Care 2016, 6(Suppl 1):P67

Introduction For patients with acute kidney injury (AKI), in our intensive care unit (ICU), continuous venovenous hemofiltration (CVVH) with citrate has been implemented since 2013. This has been conducted to change replacement fluids (RF), needing inside addition of ionic (phosphore and/or calcium), and had a cost impact.

The aim of the study was to assess the cost impact of citrate CVVH versus non-citrate CVVH and to estimate the cost impact of different RF use. Patients and methods We performed a retrospective study about all ICU patients in AKI and requiring CVVH in 2014. Data collected were about: patients characteristics (age, sex ratio, BMI, IGS2); CVVH (indications, effective duration, filters, RF, calcium and phosphore consumption). For citrate $\mathrm{CVVH}$, RF used were citrate anticoagulation solution and bicarbonate-buffered solution and for heparin or no anticoagulation, only bicarbonate-buffered solution. To estimate the economic impact of other RF, in citrate group, cost of restitution solution with integrated phosphore was used. In non-citrate group, cost of bicarbonate-buffered solution with calcium integrated was used. Results about costs were expressed for $24 \mathrm{~h}$ of effective CVVH. Differences between citrate and non-citrate group were assessed with Student's test with $p<0.05$. The cost impact simulation of different RF was assessed with appariate Student's test with $p<0.05$.

Results In 2014, 66 patients received CVVH in ICU. We included 64 patients (two excluded because of missing data). They had a mean age of $68.1 \pm 16.6$ years old, a mean IGS2 of $58.2 \pm 20.5$, a mean stay in ICU $9.0 \pm 9.6$ days and a mortality rate of $28.1 \%$. There was no difference between citrate and non-citrate group. Mean effective duration of CVVH was $52.1 \mathrm{~h}$. Citrate anticoagulation was used for $40.0 \%$ of them. About hemofiltration, there was no statistical difference between mean cost $/ 24 \mathrm{~h}$ in citrate and non-citrate group. Cost simulations with bicarbonate-buffered solution with integrated ionic were significantly less expensive with a mean economy of $48.3 € / 24 \mathrm{~h}$, so an annual total economy of $5726.3 €$ (Table 15).

Conclusion This study highlighted an interesting assessment of $\mathrm{CVVH}$ practices. There was no statistically difference between citrate and non-citrate group about the mean cost $/ 24 \mathrm{~h}$. But cost simulations with ionic integrated RF seem to be less expensive, especially as these results are not taking into account human costs. Most CVVH was shorter than $24 \mathrm{~h}$, and reflection about the intermittent hemofiltration could be needed. Simulations showed that $5726.3 €$ could be spare. With this methodology, only evaluation of cost impact of fluids and materials consumptions in ICU is able to help us to identify where some interesting economies could be made.

\section{Competing interests None.}

\section{P68}

Acute kidney injury in patients receiving liposomal amphotericin $B$ in an intensive care unit: register of 104 patients from 2008 to 2014

Marion Venot ${ }^{1}$, Lucie Biard ${ }^{2}$, Sylvie Chevret ${ }^{2}$, Sandrine Valade ${ }^{1}$, Eric Mariotte', Claire Pichereau' ${ }^{1}$, Virginie Lemiale', Elie Azoulay ${ }^{1}$, Emmanuel Canet $^{1}$

${ }^{1}$ Réanimation médicale, Hôpital Saint-Louis, Paris, France; ${ }^{2}$ Service de biostatistique et information médicale, Hôpital Saint-Louis, Paris, France

Correspondence: Marion Venot - marionvenot@free.fr

Annals of Intensive Care 2016, 6(Suppl 1):P68 
Table 15 CVVH characteristics and cost impact simulations

\begin{tabular}{|c|c|c|c|c|c|}
\hline & $\begin{array}{l}\text { All }(n=74) \\
\text { Mean (SD) }\end{array}$ & & $\begin{array}{l}\text { Citrate }(n=31) \\
\text { Mean (SD) }\end{array}$ & No citrate $(n=43)$ Mean (SD) & $p^{*}$ \\
\hline CVVH dose (ml/kg/h) & $36.4(8.3)$ & & $36.2(9.2)$ & $36.5(7.6)$ & 0.89 \\
\hline Hemofiltration rate (\%) & $14.8(3.7)$ & & $16.5(1.7)$ & $13.5(4.3)$ & $<0.0001$ \\
\hline Mean effective duration (h) & $52.1(60.7)$ & & $53.8(60.8)$ & $50.8(61.3)$ & 0.16 \\
\hline Total cost $(€)$ & $70,385.1$ & & $26,024.1$ & $44,361.0$ & - \\
\hline Simulated cost $(€)$ & $64,658.8$ & & $25,052.5$ & $39,606.3$ & - \\
\hline Mean cost/24 h $(€)$ & $590.9(433.0)$ & $p^{\prime}=0.0005$ & $535.2(383.6)$ & $631.0(465.6)$ & 0.33 \\
\hline Mean simulated cost/24 h $(€)$ & $564.5(396.2)$ & & $525.3(387.3)$ & $561.8(406.3)$ & 0.69 \\
\hline
\end{tabular}

$p^{*} p$ value statistical differences between citrate and no citrate group, $p^{\prime} p$ value statistical impact cost of different RF, SD standard deviation

Introduction Fungal infections represent an increasing and challenging issue in intensive care unit patients. Antifungal drugs have considerably evolved, with new molecules, whose choice depends on spectrum and adverse effects. Amphotericin B is an old drug, with a wide spectrum and highly efficient, but associated with renal toxicity. Liposomal amphotericin B is supposed to be less nephrotoxic, but other molecules may be preferred in case of renal dysfunction. We aimed to assess the impact of liposomal amphotericin B administration on renal function in critically ill patients.

Patients and methods $A$ retrospective single-center cohort study was performed, including consecutive patients admitted to our intensive care unit between 31/01/2008 and 12/07/2014, and receiving liposomal amphotericin B for at least 2 days. Acute kidney injury was defined using the KDIGO criteria. Patients were divided into four groups: those who did not develop any acute kidney injury, those who developed acute kidney injury 1 day or more after liposomal amphotericin B initiation (de novo), those who had acute kidney injury at initiation and worsened their renal function 1 day or more after initiation and those who had acute kidney injury at initiation and did not worsen their acute kidney injury. Patients with worsening renal function after liposomal amphotericin B (de novo or on previous acute kidney injury) were compared to patients without worsening renal function (no acute kidney injury or not worsening previous acute kidney injury). The analysis of factors associated with liposomal amphotericin B-related acute kidney injury was performed in a competing risks framework (death and discharge without acute kidney injury considered as competing events).

Results One hundred and four patients were included. Median age was 51 [interquartile ranges $31 ; 58$ ], 60 (58 \%) were male, and median SAPS 2 was 50 [38; 68]. Main comorbidities were: hematologic malignancy in $88(85 \%)$, cardiovascular diseases in $12(12 \%)$, diabetes mellitus in seven $(7 \%)$ and chronic kidney disease in eight $(8 \%)$. During the week before intensive care unit admission, $36(35 \%)$ received contrast agents, $37(36 \%)$ vancomycin and 35 (34 \%) aminoglycosides. At admission, 15 (14\%) were already treated by liposomal amphotericin B. Reasons for intensive care unit admission were hemodynamic failure in $48(46 \%)$, respiratory distress in $40(38 \%)$, neurologic disorder in seven $(7 \%)$ and close monitoring in nine (9\%). During intensive care unit stay, 71 patients $(68 \%)$ underwent invasive mechanical ventilation, $65(62 \%)$ needed vasopressors, 29 (28\%) received contrast agents, 68 (65\%) vancomycin and 77 (74\%) aminoglycosides. Intensive care unit, hospital and 3-month survivals were, respectively, 56.5, 52.5 and $35.5 \%$. Patients received liposomal amphotericin B during 14 days (from 2 to 64 days). $29(28 \%)$ patients worsened their renal function after liposomal amphotericin B initiation: $19(18 \%)$ de novo and $10(10 \%)$ on previous acute kidney injury. There was no renal degradation in the other 75 (72\%) patients: no acute kidney injury in $40(38 \%)$ and not worsening acute kidney injury in 35 (34\%). The only factor associated with the development of liposomal amphotericin B-related acute kidney injury was initiation of liposomal amphotericin $B$ before intensive care unit admission (hazard ratio $3.75,95 \%$ confidence interval [1.70-8.26], $p$ value $=0.001$ ). Among the seven patients who worsened their renal function with liposomal amphotericin B and were alive 3 months after intensive care unit discharge, six ( $86 \%$ ) recovered their previous renal function. Among the 10 patients alive at month 3 who had an acute kidney injury at liposomal amphotericin B initiation and did not worsen their renal function, nine $(90 \%)$ recovered their previous renal function.

Discussion While liposomal amphotericin $\mathrm{B}$ has been reported to be less nephrotoxic than conventional amphotericin $B$, intensivists are often reluctant to use this drug in critically ill patients because they are at high risk of acute kidney injury. In our experience, liposomal amphotericin B can be used in critically ill patients with a good safety profile. Less than $30 \%$ of the patients experienced acute kidney injury related to the drug and most of them recovered. Renal function should be carefully monitored in patients admitted to the intensive care unit and already treated with liposomal amphotericin B.

Conclusion In this retrospective single-center study, more than $70 \%$ of the patients treated with liposomal amphotericin B did not worsen their renal function. In case of worsening, about $90 \%$ recovered their previous renal function.

Competing interests None.

P69

Regional citrate-based anticoagulation for dummies in continuous venovenous hemofiltration: does it work?

Dorothée Valance ${ }^{1}$, Richard Galliot ${ }^{1}$, Romain Zunarelli', David Vandroux ${ }^{1}$ Cyril Ferdynus², Bernard-Alex Gauzere', Olivier Martinet ${ }^{1}$, Julien Jabot ${ }^{1}$ ${ }^{1}$ Réanimation polyvalente, CHU La Réunion, Saint-Denis, France; ${ }^{2}$ Unité de soutien méthodologique, CHU La Réunion, Saint-Denis, France

Correspondence: Julien Jabot - jabot974@gmail.com

Annals of Intensive Care 2016, 6(Suppl 1):P69

Introduction Regional citrate-based anticoagulation (RCA) has proven effective to increase the filter lifespan during continuous venovenous hemofiltration $(\mathrm{CVVH})$ [1] whenever performed by experienced practitioners in highly specialized teams. Moreover, from the perspective of beginners, such technique may seem to require more nursing time give to the numerous interventions to modify citrate doses and/or calcium intake. In our 23-bed ICU (University Teaching Hospital, Saint-Denis, Reunion island, France), at the decision to initiate RCA for CVVH, we had no medical expertise in dealing with the protocol, technical issues and training nurses. Therefore, we looked at reaching the same results as an experienced team with regard to the filter lifespan and the nursing workload.

Patients and methods A homemade protocol primarily tested on five patients was used in all patients meeting the criteria for CVVH with no contraindication for RCA. This protocol contained a target-oriented algorithm for post-filter ionized calcium concentration (between 0.15 and $0.4 \mathrm{mmoL} \mathrm{L}^{-1}$ ) and arterial ionized calcium concentration (between 1.00 and $1.15 \mathrm{mmoL} \mathrm{L}^{-1}$ ). Primary endpoints were filter lifespan and number of handlings for RCA therapy's adaptation (changes in citrate anticoagulation and calcium compensation). Secondary endpoints were safety, changes in biological parameters and reasons to terminate the technique. 
Results Thirty patients ( 20 men and 10 women, age $57 \pm 12$ years, SAPS2 $53 \pm 11$ ) were included from November 2013 to April 2014. The protocol was set to deliver a renal replacement therapy dose of $30 \mathrm{~mL} \mathrm{~kg}^{-1} \mathrm{~h}^{-1}$. The median filter lifespan was 51 [37-65] h, and the median number of handlings was 2 [1-4]. Only six patients had to receive low dose of heparin in order to prevent thromboembolism. The 24 other patients received no anticoagulation. Reasons for terminating the therapy were 13 filter clottings, 12 interruption for CT scan, operating room or discharge, two catheters dysfunctions, two technical problems and one elective filter change $(>72 \mathrm{~h}$ ). We did not record any complication. Electrolytes and acid-base disturbances were uncommon and transient in patients treated with RCA: median initial pH was 7.39 [7.33-7.41], 7.44 [7.42-7.47] $24 \mathrm{~h}$ later and 7.40 [7.38-7.45] $48 \mathrm{~h}$ later; median initial serum magnesium level was $0.85[0.74-0.94] \mathrm{mmol} \mathrm{L}^{-1}$ $0.69[0.62-0.77] \mathrm{mmol} \mathrm{L}^{-1}$ at $24 \mathrm{~h}$ and $0.68[0.60-0.81] \mathrm{mmol} \mathrm{L}^{-1}$ at $48 \mathrm{~h}$. Discussion Our "RCA for dummies" protocol fulfilled its objectives far beyond our expectations. The median filter lifespan $(51 \mathrm{~h})$ was surprisingly as high as that of reported by experts ( $49 \mathrm{~h}$ ) [1]. The number of RCA therapy's adaptation was low (one intervention every $20 \mathrm{~h}$ ) with no significant nursing overwork.

These first results are promising ones for a non-expert team, and we wonder whether we were not too cautious than needed with this new technique. As RCA is a routine technique on our hands, we are conducting a second survey in order to rule out a "starter effect."

Conclusion We confirm that RCA is both efficient and safe since its early use in ICUs by inexperienced practitioners, with fairly acceptable achievements in terms of filter lifespan, number of handlings and nursing overwork.

\section{Competing interests None.}

\section{Reference}

1. Efficacy and safety of citrate-based anticoagulation compared to heparin in patients with acute kidney injury requiring continuous renal replacement therapy: a randomized controlled trial. Stucker F, Ponte B, Tataw J, Martin PY, Wozniak H, Pugin J, Saudan P. Crit Care. 2015 Mar 18;19:91. doi: 10.1186/s13054-015-0822-z.

\section{P70}

Incidence of and risk factors for acute kidney injury in early postoperative liver transplantation according to AKIN classification

Jean-Charles Cartier ${ }^{1}$, Thomas Jouve ${ }^{2}$, Marie-Noëlle Hilleret ${ }^{3}$, Rebecca Hamidfar-Roy ${ }^{4}$, Claire Ara-Somohano ${ }^{1}$, Clémence Minet ${ }^{5}$, Agnès Bonadona $^{6}$, Carole Schwebel ${ }^{5}$

${ }^{1}$ Réanimation médicale, C.H.U de Grenoble C.H.U, La Tronche, France; ${ }^{2}$ Néphrologie, hémodialyse, transplantation rénale, C.H.U de Grenoble C.H.U, La Tronche, France; ${ }^{3}$ Hépato-gastro-entérologie, C.H.U de Grenoble C.H.U, La Tronche, France; ${ }^{4}$ Réanimation médicale, C.h.u., La Tronche, France; ${ }^{5}$ Réanimation médicale, C.H.U. Grenoble, Grenoble, France; ${ }^{6}$ Réanimation médicale, C.H.U. Grenoble, La Tronche, France

Correspondence: Jean-Charles Cartier - jccartier1@chu-grenoble.fr Annals of Intensive Care 2016, 6(Suppl 1):P70

Introduction Liver transplantation (LT) is the reference treatment for hepatocellular terminal chronic diseases. Early postoperative acute kidney injury (AKI), which incidence varies between 29 and $60 \%$ depending on the definitions and cohorts, is a factor of poor prognosis, especially when renal replacement therapy (RRT) is necessary. The objective of this study is to assess the incidence of early AKI (in the first $48 \mathrm{~h}$ ) of a LT considering AKIN classification with consideration of diuresis and to identify associated risk factors.

Patients and methods Patients who received a first non-emergency LT orthotopic cadaveric graft between January 2011 and December 2014 at Grenoble University Hospital were included in this retrospective study. Eleven patients were excluded because of the need for RRT before the LT or related kidney transplant. Demographic and clinical and biological parameters pre-, intra- and postoperative, including comorbidities, etiology and staging of liver disease, anesthetic management, graft function, use of renal replacement therapy, diuresis by $6 \mathrm{~h}$ and evolution, were collected. Serum creatinine at $24 \mathrm{~h}$ was considered to reflect kidney function between $\mathrm{H} 0$ and $\mathrm{H} 24$ and $1-48 \mathrm{~h}$ to reflect that between $\mathrm{H} 24$ and $\mathrm{H} 48$. Logistic regression was used for multivariate analysis (alpha risk 0.05)

Results Of the 155 patients in the study, 129 (83.2 \%) were male, median MELD and Child-Pugh scores were, respectively, 14.5 (11-23) and 9 (7-11). Seventy-three percent of patents had alcoholic cirrhosis, and median creatinine clearance (according aMDRD) was $95 \mathrm{ml} / \mathrm{min}$ with 26 patients (16.8\%) below $60 \mathrm{ml} / \mathrm{min} .128(82.6 \%)$ had an AKI according to the AKIN criteria, and 31 (20\%) required at least one RRT in ICU. The inclusion of diuresis in the definition of the AKI increases the number of patients with AKI in $32(20 \%)$ with peak incidence between $\mathrm{H} 24$ and $\mathrm{H} 30$. During first $48 \mathrm{~h}$, incidence of IRA varies from 54.4 to $78.1 \%$ depending on the time slot. The median plasma volume was 3 liters of crystalloid intraoperatively and 4 liters during the first $24 \mathrm{~h}$ without significant differences by AKI as albumin dose. Patients who develop early AKI were more transfused in labile blood products. Incidence of nephrotoxics as iodine injection and aminoglycosides during first $48 \mathrm{~h}$ were, respectively, 20.6 and $6.5 \%$ without significant difference. Only the IGS2 and monitoring by Swan-Ganz are significant independent predictive factors for AKI. Diabetics, admission norepinephrine concentration and high first residual tacrolimus dosage are significant independent predictive factors for RRT (respective OR of 3.75 (1.15 to -12.12$), 2.04$ (1.13 to -3.69$)$ and 1.07 (1.05 to -1.09$)$ per point of tacrolimus). No patient without early AKI according to these criteria has used an RRT during the ICU stay.

Conclusion The incidence of early post-LT AKI with the AKIN criteria is high. The use of AKIN classification shows dynamic nature of AKI in this context. These criteria appear prognostic for the use of renal replacement therapy in the ICU. Diabetic patients and high norepinephrine dose in immediate postoperative time seem to be related to AKI requiring RRT and should have special attention. These results need to be validated in prospective studies to clarify the impact of graft quality in the complex determinism of post-transplant AKI.

Competing interests None.

P71

Metabolic acidosis uncompensated by kidney in septic patients: a predictive factor for acute kidney injury?

Aline Pourcelet ${ }^{1}$, Jean-Michel Hougardy ${ }^{1}$, Pierre Defrance ${ }^{2}$, Patrick Biston ${ }^{2}$ Michael Piagnerelli

${ }^{1}$ Nephrology, Hospital Erasme, Brussel, Belgium; ${ }^{2}$ Intensive care, Hospital

Civil Charleroi, Charleroi, Belgium

Correspondence: Aline Pourcelet - aline.pourcelet@gmail.com

Annals of Intensive Care 2016, 6(Suppl 1):P71

Introduction Metabolic acidosis is a frequent acid-base disturbance observed in septic patients. Normally, the physiological adaptive response of the kidney is an increased urinary excretion of $\mathrm{NH} 4 \mathrm{Cl}$ inducing a negative urinary anion gap $(\mathrm{UAG}=[\mathrm{Na}+] \mathrm{u}+[\mathrm{K}+]$ $\mathrm{u}-[\mathrm{Cl}-] \mathrm{u})$.

We investigated, in septic patients, the evolution of the urinary anion gap and the correlation with acute kidney injury according to kidney disease improving global outcomes (KIDGO) criteria.

Materials and methods This prospective study included patients with severe sepsis or septic shock admitted in a 24-bed medico-surgical intensive care unit of the CHU-Charleroi Marie Curie (Belgium). Blood and urinary chemistry were performed every $12 \mathrm{~h}$ after the admission during a maximum of 96th hour of intensive care unit length of stay. Metabolic acidosis was defined according to Stewart's method (SIDa $<39 \mathrm{mmol} / \mathrm{L}$ ) over a period of $24 \mathrm{~h}$. Acute kidney injury was defined according kidney disease improving global outcomes (increased creatinine concentration $\geq 0.3 \mathrm{mg} / \mathrm{dL}$ unless $48 \mathrm{~h}$ or decreased diuresis $<0.5 \mathrm{~mL} / \mathrm{kg} / \mathrm{h}$ on $6-12 \mathrm{~h}$ ). We also defined renal tubular acidosis by a serum anion gap $<16 \mathrm{mmol} / \mathrm{L}$ and urinary anion gap $>0 \mathrm{mmol} / \mathrm{L}$.

Data were presented in median values with [25th-75th] percentiles and compared by the Mann-Whitney test. A value of $p<0.05$ was considered as significant.

Results Among the 40 patients included, 23 (58\%) have a metabolic acidosis at admission and 20 (50\%) developed an acute kidney injury. At the intensive care unit admission, no significant differences 
were observed between patients with and without metabolic acidosis according to the APACHE 2 score, creatinine (1.12 [0.78-1.85] vs $1.0[0.62-2.04] \mathrm{mg} / \mathrm{dL}$ ) and lactate concentrations (1.6 [1.0-4.2] vs 2.2 [1.5-3.2] $\mathrm{mmol} / \mathrm{L}$ ), quantity and the type of liquid received within $24 \mathrm{~h}$ (4800 [3438-6528] vs 4475 [2610-4830] mL).

On the 23 patients with metabolic acidosis, 17 (74 \%) presented a renal tubular acidosis. All, except one, did not have kidney adaptation to metabolic acidosis. This inadequate response persisted during the $96 \mathrm{~h}$ of this study despite normalization of the acid-base status. Despite inadequate kidney response to metabolic acidosis, there was no difference in the onset of acute kidney injury between the two groups (10 acute kidney injury - group 1 in majority -in each group). Conclusion Inadequate kidney response to metabolic acidosis was frequent in septic patients and persists despite normalization of the acid base status. Nevertheless, this tubular dysfunction does not correlate with the onset of acute kidney injury.

\section{Competing interests None.}

\section{References}

1. De Mendonça A, Vincent JL, Suter PM, Moreno R, Dearden NM, Antonelli $\mathrm{M}$, et al. Acute renal failure in the ICU: risk factors and outcome evaluated by the SOFA score. Intensive Care Med. 2000;26(7):915-21.

2. Kellum $\mathrm{J}$ a. Determinants of blood pH in health and disease. Crit Care. 2000;4(1):6-14.

\section{P72}

Blood volume monitoring is useful to predict intradialytic hypotension during intermittent hemodialysis

Louis De Laforcade', Alexandre Boyer², Didier Gruson², Benjamin Clouzeau ${ }^{2}$

${ }^{1}$ C.H. Lyon Sud, Pierre-Bénite, France; ${ }^{2}$ Réanimation médicale, Centre Hospitalier Universitaire de Bordeaux, Bordeaux, France

Correspondence: Louis De Laforcade - louis.de-laforcade@chu-lyon.fr Annals of Intensive Care 2016, 6(Suppl 1):P72

Introduction During intermittent hemodialysis (HD), intradialytic hypotension (IDH) is a frequent complication, associated with worse outcomes. Blood volume monitoring (BV) by dialysis generator has shown its interest in chronic dialysis to predict IDH due to hypovolemia, but its utility is unclear in intensive care units.

Patients and methods We conducted a monocentric, prospective, observational study. The primary outcome was to assess whether IDH (defined by mean arterial pressure lower than $65 \mathrm{mmHg}$ ) was associated with a decrease in the ratio between BV and ultrafiltration (UF). Secondary outcome was to assess the association between dialysis parameters and IDH. Every dialysis session in our unit has been screened for analysis. When BV assessment was not reliable or when no UF was realized, the session was excluded of the analysis. Dialysis generators, paramedical monitoring and dialysis consumables were not modified compared with usual standard of care in our unit. Dialysis settings were left to the choice of the medical team.

Results A total of 204 sessions were screened, and 112 (corresponding to 42 different patients) were included. Median dialysate sodium was 140 , and median dialysate temperature was $36^{\circ} \mathrm{C}$. Under pressor amines, $18.8 \%$ of the sessions were made. IDH occurred in $40 \%$ of the sessions.

BV/UF ratio was lower in session with IDH $(-7.7$ vs $-10.5 \% / \mathrm{L}$, $p=0.027$ ). ROC curve showed an AUC of 0.624 , but a cutoff value of $-8.9 \% / \mathrm{L}$ was associated with a sensibility of $80 \%$ to predict IDH.

Others parameters associated with IDH in univariate analysis were a higher natremia, a higher bicarbonate dialysate, a lower systolic, diastolic or mean arterial pressure at the beginning of the session, the use of pressor amines and sedation and a low difference between sodium dialysate and natremia. In multivariate analysis, the use of pressor amines and a low mean arterial pressure were associated with IDH.

\section{Discussion}

Our study is the first to show an association with BV/UF and IDH for adults admitted in intensive care units. Good sensitivity of this test may be useful to detect IDH.

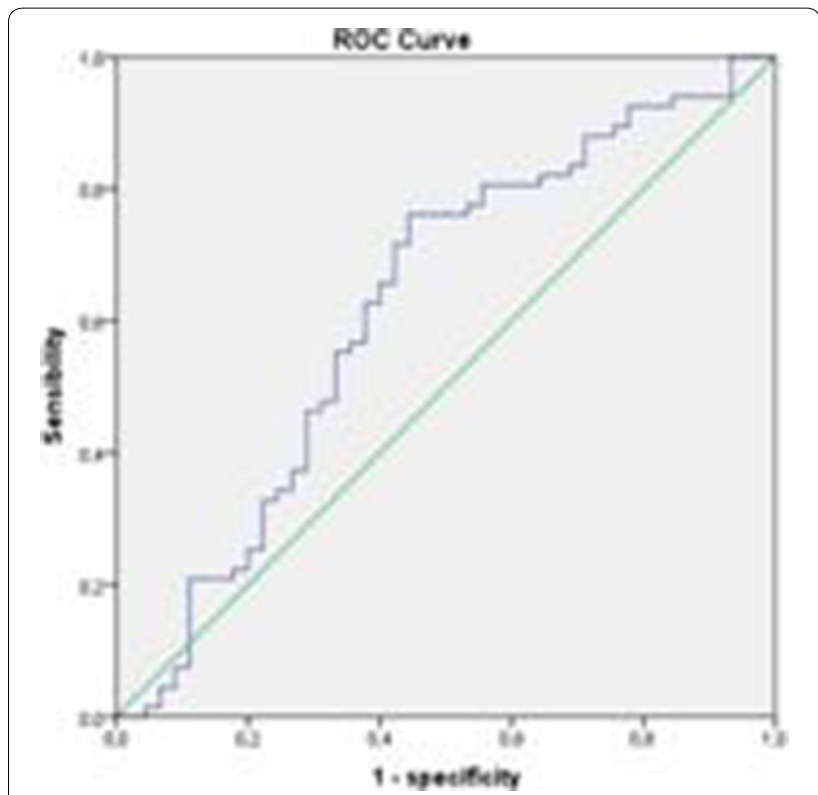

Fig. 29 See text for description

$\mathrm{BV}$ is widely used since 20 years in chronic hemodialysis and is now recommended by international guidelines. In intensive care unit, two studies have shown no interest in adults, and another did not find a decrease in IDH using a BV feedback protocol. In children, BV using was associated with a higher total UF without increasing IDH.

Other guidelines has been proposed in order to reduce IDH: high sodium dialysate concentration $(145 \mathrm{mmol} / \mathrm{l})$, or moderately cool dialysate temperature $\left(0.5^{\circ} \mathrm{C}\right.$ beyond body temperature). Our study did not find any association between these settings and IDH.

Conclusion Decrease in BV/UF ratio is associated with IDH in our study. A $8.9 \% / \mathrm{L}$ threshold may predict IDH with a sensitivity of $80 \%$ (Fig. 29).

\section{Competing interests None.}

\section{References}

1. De Vries JP. Kidney International (1993) 44, 851-854.

2. Tonelli M. Kidney International (2002) 62, 1075-1080.

P73

Risk factors for occurrence of acute kidney injury in diabetic ketoacidosis

Najah Hajjam ${ }^{1}$, Ines Sedghiani ${ }^{1}$, Houda Nasri ${ }^{1}$, Hamdi Doghri ${ }^{1}$, Youssef Zied El Hechmi ${ }^{1}$, Mohamed Ali Cherif ${ }^{1}$, Jerbi Zouheir

${ }^{1}$ Emergency and intensive care department, Hopital Habib Thameur, Tunis, Tunisia

Correspondence: Ines Sedghiani - sedghiani.ines@gmail.com

Annals of Intensive Care 2016, 6(Suppl 1):P73

Introduction Acute kidney injury (AKI) is a common complication of non-equilibrated diabetes, and it may occur in patients with ketoacidosis (DKA) either in admission or during hospitalization

Patients and methods It is a retrospective study including all cases of diabetic ketoacidosis between January 2014 and August 2015. Patients with chronic renal failure were not included.

The diagnosis of diabetic ketoacidosis was defined by the association of blood glucose $>11 \mathrm{mmol} / \mathrm{L}$, ketonuria and metabolic acidosis with a $\mathrm{pH}<7.30$ and/or plasma bicarbonate $<15 \mathrm{mmol} / \mathrm{L}$.

On admission, acute kidney injury was defined by a reversible increase in serum creatinine $>110 \mu \mathrm{mol} / \mathrm{L}$ or in blood urea $>9 \mathrm{mmol} / \mathrm{l}$ or a reversible decrease in the urinary output $<0.5 \mathrm{ml} / \mathrm{kg} /$ day. 
Table 16 See text for description

\begin{tabular}{|c|c|c|c|}
\hline & With AKI $(n=26)$ & $\begin{array}{l}\text { Without AKI } \\
(n=27)\end{array}$ & $p$ \\
\hline Sex ratio (M/F) & 1.1 & 1.45 & 0.69 \\
\hline Age (years) & $48 \pm 24$ & $34 \pm 15$ & 0.02 \\
\hline Age $\geq 49$ & $16(61 \%)$ & $10(37 \%)$ & 0.000 \\
\hline Type 2 diabetes & 15 (57\%) & $6(22 \%)$ & 0.008 \\
\hline $\begin{array}{l}\text { Time of diagnosis of } \\
\text { diabetes (years) }\end{array}$ & $5[3.25-13.75]$ & $5[5-10]$ & 0.78 \\
\hline $\begin{array}{l}\text { Blood glucose } \\
(\mathrm{mmol} / \mathrm{l})\end{array}$ & $39 \pm 13$ & $26 \pm 8$ & 0.00 \\
\hline Blood glucose $\geq 28$ & $21(80 \%)$ & 10 (37\%) & 0.001 \\
\hline Initial blood pH & $7.18 \pm 0.09$ & $7.21 \pm 0.08$ & 0.25 \\
\hline $\mathrm{HCO}_{3}-(\mathrm{mmol} / \mathrm{l})$ & $9.08 \pm 5$ & $9.4 \pm 3.6$ & 0.77 \\
\hline $\begin{array}{l}\text { Blood osmolarity } \\
\text { (mosmol/l) }\end{array}$ & $287 \pm 14$ & $278 \pm 10$ & 0.01 \\
\hline Osmolarity $\geq 282$ & 17 (65\%) & $8(29 \%)$ & 0.009 \\
\hline $\begin{array}{l}\text { Serum sodium } \\
(\mathrm{mmol} / \mathrm{L})\end{array}$ & $135 \pm 4.8$ & $133 \pm 5.5$ & 0.2 \\
\hline Etiology of DKA & & & 0.26 \\
\hline Treatment omission & $9(34 \%)$ & 12 (44 \%) & \\
\hline Infections & $8(30 \%)$ & $8(29 \%)$ & \\
\hline Diet errors & $5(19 \%)$ & $1(3 \%)$ & \\
\hline
\end{tabular}

Results In this study, 53 patients with a mean age $41 \pm 21$ years were included. They have been diagnosed with type 1 diabetes in $60 \%$ of the cases and with type 2 diabetes in $40 \%$ of the cases, on average 5 years before admission. The majority of the patients were female $(56 \%)$. Sixteen of the patients have had a previous episode of DKA. The origin of DKA was attributed to treatment omission in 21 (39\%) patients, infections in $16(30 \%)$ patients and diet errors in six (11\%) patients.

On admission, they have a mean serum glucose $32 \pm 12 \mathrm{mmol} / \mathrm{L}$, mean pH 7.2 \pm 0.08 and a bicarbonate level of $9.2 \pm 4.2 \mathrm{mmol} / \mathrm{L}$.

On admission, patients were dichotomized in with AKI and without AKI. Clinical and biological parameters were compared in these two groups. Twenty-six patients (49\%) have acute renal failure on admission, with a mean serum creatinine of $136 \pm 41 \mu \mathrm{mol} / \mathrm{L}$ and a mean blood urea of $10 \mathrm{mmol} / \mathrm{L}$ compared with $72 \pm 32 \mu \mathrm{mol} / \mathrm{L}$ and $6.6 \pm 4 \mathrm{mmol} / \mathrm{L}$ at discharge (Table 16).

Conclusion Acute kidney injury complicates frequently diabetic ketoacidosis on admission in ICU. Age, blood glucose, high osmolarity and occurrence in type 2 diabetes are their risk factors.

\section{Competing interests None.}

\section{P74}

Face-to-face tracheal intubation with the video laryngoscope Airtraq in the sitting patients: a report of 3-year experience in patients after failed conventional tracheal intubation technique

Gilles Dhonneur ${ }^{1}$, Nicolas Mongardon', Eric Levesque ${ }^{1}$, Hakim Haouache ${ }^{1}$ ${ }^{1}$ Anesthesia and Intensive Care Medicine, CHU Henri Mondor, Créteil, France

Correspondence: Gilles Dhonneur - gilles.dhonneur@hmn.aphp.fr Annals of Intensive Care 2016, 6(Suppl 1):P74

Introduction The video laryngoscope Airtraq (VAQ) is now placed in the difficult airway management algorithm as second-step device to intubate the tracheal of patients when conventional tracheal intubation (TI) technique with Macintosh laryngoscope (ML) has failed (1). VAQ was also shown in simulation conditions to promote simple face-to-face (F2F) tracheal intubation in the patients placed in sitting position (2). With significant clinical experience acquisition, we have decided to propose F2F-VAQ-TI as an alternative to awake fiberscopeassisted nasotracheal intubation (AANI) in the patients after failed ML. Patients and methods After informed consent of the patient initially scheduled for elective AANI was obtained, those with previous failed $M L$, showing inter-incisor distance of $>20 \mathrm{~mm}$, with no expansive tumoral process in the upper airway and those with easy identification of anatomical cricothyroid membrane landmarks were proposed F2FVAQ-TI in sitting position. An Eschmann stylet (ES) was systematically armed in the tracheal tube inserted in the VAQ channel. Preoxygenation and anesthesia induction techniques were standardized. Filmed TI maneuver was started $30 \mathrm{~s}$ after succinylcholine IV bolus $\left(1 \mathrm{mg} \mathrm{kg}^{-1}\right)$ was injected. Insertion of an LMA Fastrach was considered as a Plan B in case of difficulty to maintain $\mathrm{SaO}_{2}>90 \%$. The fiberscope used with VAQ or LMA Fastrach was recommended as impossible tracheal access Plan B. In the PACU, the patients were asked to evaluate the level of comfort of the proposed airway management strategy using a visual analogue scale (VAS $0-100$ ). For those patients that had previously been proposed AANI, they were asked to quote their comfort during both previous AANI and F2F-VAQ-TI. Moreover, they were asked to choose for the next surgery step the airway management technique among the two proposed options.

Results Over 3 years, 106 F2F-VAQ-TI were performed. The surgical indications requesting $\mathrm{TI}$ were as follows: maxillofacial reconstruction $(41 \%)$, orthopedic (32\%), neurological and visceral. The reported reasons for previous $\mathrm{ML}$ failure were as follows: restricted mouth aperture of below $25 \mathrm{~mm}$, major abnormality of anterior neck structure, severe cervical spine ankylosis, previous causes combined with post-surgery/ radiation sequels or unknown non-tumoral causes. Mean age of the patients was 49 years. Among them, 3 patients benefited of 3 F2FVAQ-TI and 5 of 2 . TI succeeded in all patients. TI duration was $<15 \mathrm{~s}$, 15 to $<30 \mathrm{~s}, 30 \mathrm{~s}$ to $<1 \mathrm{~min}, 1-2$ and $>2 \min : 24,25,42,6$, and $3 \%$, respectively. ES was used in $61 \%$ of cases. Plan B was used twice. One tracheal intubation was performed using the fiberscope inserted in the tracheal tube armed in the VAQ. Unrecognized laryngeal cyst prevented both tracheal tube and ES tracheal penetration. The second Plan B concerned a 39-year-old woman (1.29 m height; $35 \mathrm{~kg}$ weight) that was oxygenated and intubated through the LMA Fastrach (size 3). $\mathrm{SaO}_{2}<90 \%$ episodes occurred in 2 patients (nadir 86 and $78 \%$ ). All the patients $(n=36)$ that had received AANI for previous surgery would ask F2F-VAQ-TI for the next surgery. The mean (SD) comfort of F2F-VAQ-TI was remarkably high: 90 (6).

Conclusion We have shown that with respect to our selection process and airway management procedures F2F-VAQ-TI in the sitting patient was a safe alternative to AANI. This new airway management option seems simple for trained skilled operators and very comfortable for the patient. Comparison of the learning process of AANI and F2F-VAQ$\mathrm{Tl}$ is now made in our laboratory and department.

\section{Competing interests Consulting activity for PRODOL Company.}

\section{References}

1. Anesthesiology, 2011;114:25-33.

2. Br J Anaesth, 2012; 108: 140-50.

\section{P75}

Ultrasound-guided central venous access technique among French intensivists

Marie-Anaïs Bastide ${ }^{1}$, Jack Richecoeur ${ }^{2}$, Eric Frenoy ${ }^{3}$, Christian Lemaire ${ }^{4}$, Bertrand Sauneuf ${ }^{5}$, Fabienne Tamion ${ }^{6}$, Saad Nseir ${ }^{7}$, Damien Du Cheyron ${ }^{8}$, Hervé Dupont ${ }^{9}$, Julien Maizel', BoReal Study Group

${ }^{1}$ Réanimation médicale, Centre Hospitalier Universitaire, Amiens,

France; ${ }^{2}$ Réanimation polyvalente, C.H. de Beauvais, Beauvais, France; ${ }^{3}$ Réanimation polyvalente, Hospital Center General Du Havre, Le Havre, France; ${ }^{4}$ Réanimation polyvalente, Hospital Center De Roubaix, Roubaix, France; ${ }^{5}$ Réanimation Médicale Polyvalente, C.H. Public du Cotentin, Cherbourg-Octeville, France; ${ }^{6}$ Réanimation médicale, Hospital Center University Rouen, Rouen, France; ${ }^{7}$ Centre de Réanimation, Centre Hospi- 
talier Régional Universitaire de Lille, Lille, France; ${ }^{8}$ Réanimation médicale, Centre Hospitalier Universitaire de Caen, Caen, France; ${ }^{9}$ Réanimation cardio thoracique et vasculaire, CHU Amiens-Picardie, Amiens, France Correspondence: Julien Maizel - maizel.julien@chu-amiens.fr Annals of Intensive Care 2016, 6(Suppl 1):P75

Introduction For many years, the use of ultrasound has been recommended to secure central venous catheter placement in the intensive care units. Many studies and meta-analysis have demonstrated that ultrasound-guided technique increases the success rate and decreases the number of complications by novice and experienced operators. However, several surveys showed that only $50 \%$ of the CVC procedures are performed using the ultrasound-guided technique. The reason why physicians continue to use landmarks was the lack of formation and the absence of ultrasound device. In our area (northwest of France), ultrasounds have been taught to our residents and learning courses were implemented for senior physicians. Therefore, we designed a survey to elicit information on physician's characteristics, experience in CVC placement, training on ultrasound technique, the use of ultrasound for CVC placement, reasons for nonuse of ultrasound and their opinion on the necessity to continue to teach the landmark technique to residents.

Patients and methods This survey (14 questions) has been electronically addressed by email (using the Survey Monkey online software) to every physician belonging to the group of research on clinical practice in the northwest of France composed of 8 university's and 20 community's ICU. The survey was sent to the 289 physicians ( 166 seniors and 121 residents) working in those ICUs.

Results We received 190 responses (response rate $66 \%$ ). Among the respondents, $66 \%$ were less than 40 years old, $34 \%$ were residents, $41 \%$ presented an experience in CVC placement $\leq 5$ years and $53 \%$ declared putting more than 1 CVC per week. The preferred site of insertion was the jugular for $70 \%$, subclavian for $21 \%$ and femoral for $9 \%$. Seventy-one percentage of the residents declared having learned both landmark and ultrasound-guided techniques during their residency. Only $18 \%$ reported using always the ultrasound to put CVC. The main reasons why they do not use ultrasound were as follows: (1) they think they do not need it (36\%), (2) the ultrasound was not available (33\%) while $3 \%$ declare the absence of ultrasound device in their setting and (3) $11 \%$ reported the absence of formation on ultrasound technique. The ultrasound technique used was ultrasound-guided in $66 \%$. Coagulation abnormalities (64\%), obesity (54\%) and anatomical difficulties (52\%) were the main motivations to use ultrasound reported. Fifty-three percentage of the respondents declared having confronted at least one time during the last 12 months to an urgent situation where the ultrasound was not available quickly enough. Finally, $91 \%$ think that the landmark technique should still be taught to the residents.

Conclusion Our survey shows that a large majority of the intensivists declare still performing landmark procedures while almost every physician received a formation on the ultrasound technique. Ultrasound device is present in the large majority of the institution; however, their availability is still scarce. Despite the several guidelines, physicians still do not use ultrasound for every procedure and think that landmark technique should still be taught to residents.

Competing interests None.

\section{P76}

Ultrasound-guided venous catheter insertion. What do French intensivists really do?

Hafid Ait-Oufella', Eric Maury', Julien Maizel'², Frédérique Schortgen ${ }^{3}$ Cécile Aubron ${ }^{4}$, Christophe Clec' $h^{5}$, Christophe Guitton ${ }^{6}$, Kada Klouche', Nicolas Lerolle ${ }^{8}$, Frédéric Pène ${ }^{9}$, Sebastien Preau ${ }^{10}$, Carole Schwebel ${ }^{11}$, Nicolas Terzi ${ }^{12}$, Frédéric Vargas ${ }^{13}$, Vincent Castelain ${ }^{14}$

${ }^{1}$ Service de Reanimation, Hôpital Saint-Antoine, Paris, France; ${ }^{2}$ Réanimation médicale, Centre Hospitalier Universitaire, Amiens, France;

${ }^{3}$ Réanimation médicale, Hôpital Henri Mondor, Créteil, France; ${ }^{4}$ Réanimation Médicale, $\mathrm{CHU}$ de la Cavale Blanche, Brest, France; ${ }^{5}$ Réanimation medico-chirurgicale, hopital avicenne, Bobigny, France; ${ }^{6}$ Réanimation médicale, C.H.U. Hôtel Dieu, Nantes, France; ${ }^{7}$ Service de réanimation,
CHU Lapeyronie, Montpellier, France; ${ }^{8}$ Réanimation médicale, Centre Hospitalier Universitaire d'Angers, Angers, France; ${ }^{9}$ Réanimation Médicale, Hôpital Cochin, Paris, France; ${ }^{10}$ Réanimation médicale, Centre Hospitalier Régional Universitaire de Lille, Lille, France; ${ }^{11}$ Réanimation médicale, C.H.U. Grenoble, Grenoble, France; ${ }^{12}$ Service de réanimation médicale, Clinique de Réanimation Médicale, Grenoble, France; ${ }^{13}$ Réanimation médicale, Centre Hospitalier Universitaire de Bordeaux, Bordeaux, France;

${ }^{14}$ Hautepierre réanimation médicale, C.H.R.U. Hôpitaux Universitaires Strasbourg, Strasbourg, France

Correspondence: Hafid Ait-Oufella - hafid.aitoufella@sat.aphp.fr Annals of Intensive Care 2016, 6(Suppl 1):P76

Introduction Accumulating evidence suggests that ultrasoundguided venous catheter insertion decreases insertion failure and immediate mechanical complications rates. However, due to misleading opinion, ignorance or absence of training, this method has not been widely adopted. Moreover, the exact utilization of this method among French intensivists is unknown. The aim of this prospective survey was to assess knowledge and utilization of ultrasound guidance for venous catheter insertion.

Patients and methods A questionnaire (16 questions) assessing knowledge, training, mastery and use of ultrasound guidance for central venous catheter (CVC) insertion, constructed by the educational section of the National Intensivists French College (CNER), was electronically sent to French intensivists between May and June 2015 (CNER and SRLF Web site).

Results Answers to the questionnaire were obtained from 300 intensivists [attending physicians (50\%), residents (16\%), fellows (16\%), professor or assistant professor $(16 \%)]$ with initial training in anesthesiology (56\%), pulmonology (13\%), internal medicine $(10 \%)$ or cardiology (7\%) and who had for most of them (84 \%) inserted more than $100 \mathrm{CVCs}$. Ultrasonography use was significantly higher for internal jugular vein $(75 \%)$ than for femoral vein $(45 \%)$ or subclavian vein (29\%). Dynamic ultrasonic guidance isolated use was reported by $38 \%$ of respondents while $45 \%$ used both screening before cannulation and dynamic guidance. Use of short-axis approach, as well as the long-axis one according to the site, was reported by $56 \%$ of respondents. The majority of respondents $(85 \%)$ reported having never used a specific guiding device. Whereas $88 \%$ of respondents considered specific training as mandatory or absolutely mandatory, only $44 \%$ of them (essentially the youngest ones) reported having received a specific training (most of the them on an inanimate mankind).

Conclusion Despite insufficient training, most of French intensivists report relevant knowledge on ultrasound guidance for venous catheter insertion.

\section{Competing interests None.}

\section{P77}

Can we trust new connected devices recording heart rate and oxygen saturation? A concordance assessment in intensive care unit

Franck Ehooman', Yacine Tandjaoui-Lambiotte', Claire Montlahuc², Guillaume Van Der Meersch ${ }^{1}$, Johanna Oziel ${ }^{1}$, Jean Bardon ${ }^{3}$, Matthieu Resche-Rigon ${ }^{2}$, Nicolas Postel-Vinay ${ }^{4}$, Yves Cohen ${ }^{1}$

${ }^{1}$ Réanimation médico-chirurgicale, Hôpital Avicenne, Bobigny, France: ${ }^{2}$ Service de biostatistique et information médicale, Hôpital Saint Louis, Paris, France; ${ }^{3}$ Réanimation chirurgicale, Hôpital Henri Mondor, Créteil, France; ${ }^{4}$ www.automesure.com, Unité d'hypertension artérielle, Hôpital Européen Georges Pompidou, Paris, France

Correspondence: Yacine Tandjaoui-Lambiotte -yt1r82@gmail.com

Annals of Intensive Care 2016, 6(Suppl 1):P77

Introduction Connected devices have become increasingly common, including for healthcare purposes, since some items now allow a continuous measure of the heart rate (HR) and percutaneous oxygen saturation $\left(\mathrm{SpO}_{2}\right)$. These devices are sold for wellness, and to date, they are not approved by health authorities as medical devices. While their use for chronically or acutely ill patients would be of great interest, there are no existing data regarding the reliability of their measurements. 
Materials and methods We performed a prospective monocentric study in the intensive care unit (ICU) of an university hospital to assess the concordance of $\mathrm{HR}$ and $\mathrm{SpO}_{2}$ measured using the three connected devices available on the market to an ICU monitor as gold standard. All consecutive patients hospitalized in ICU during September 2015 were included except for those under vasopressor or mechanical ventilation.

\section{- Connected devices:}

Withings Pulse Ox

Safe Heart Smartphone iOximeter

iHealth Wireless Pulse Oximeter

- Monitor:

Philips MP60 or MP70.

Measurements were taken during the first $24 \mathrm{~h}$ after ICU admission during nurses' usual round or when an intercurrent event occurred. A maximum of 12 measures per patient per day were performed, and measures could be registered over several days. At each time point, HR and $\mathrm{SpO}_{2}$ were measured using the three connected devices and the gold standard.

A concordance analysis was performed to assess the reliability of each connected device compared to the gold standard. Intraclass correlation coefficients and $95 \%$ confidence intervals $(\mathrm{Cl})$ were estimated using a single pair of measures per patient. Bland-Altman diagrams for repeated measures were used to illustrate concordances.

Results Twenty-four patients, among which 16 (67\%) men, were included in the analysis. The median age was 60 years [interquartile range (IQR) 54-72], and the main cause admission was a medical issue [23 (96\%)]. Overall, 119 different measures were analyzed with a mean of 5 per patient ranging from 1 to 12 measures per patient. The median $\mathrm{SpO}_{2}$ measured on the monitor was 96 [IQR 94-99]. The corresponding measures from devices 1,2 and 3 were, respectively, 93 [IQR 90-95], 94 [IQR 91-96] and 98 [IQR 97-99]. The median HR measured on the monitor was 98 [90-109]. The corresponding measures from devices 1, 2 and 3 were, respectively, 95 [IQR 86-105], 95 [91-102], 98 [90-109]. For $\mathrm{SpO}_{2}$, the intraclass correlation coefficient between the monitor and the device 1 was 0.12 , between the monitor and device 2 was 0.32 and the monitor and device 3 was 0.17 . Concerning HR, the intraclass correlation coefficient between the monitor and device 1 was 0.68 , between the monitor and device 2 was 0.67 and the monitor and device 3 was 0.08 . Moreover, devices failed to provide any $\mathrm{SpO}_{2}$ measure in $0,0.8$ and $23 \%$ of cases for devices 1, 2 and 3, respectively. Device 3 also failed to measure any HR measure in $12 \%$ of the cases, while devices 1 and 2 always succeeded in measuring HR.

Conclusion We observe a poor correlation between devices and the gold-standard measures, especially for $\mathrm{SpO}_{2}$. These results need to be confirmed in larger studies. For now, these connected devices should not be recommended for $\mathrm{SpO}_{2}$ and $\mathrm{HR}$ measurement in critically ill patients admitted in ICU.

\section{Competing interests None.}

\section{P78}

Hemoglobin monitoring with HemoCue $\odot$, spectrophotometry and blood cells analyzer: comparison of agreement, completion time and costs

Mehdi Lafi', Frédéric Jacobs', Dominique Prat' ${ }^{1}$, Matthieu Le Meur', Olfa Hamzaoui ${ }^{1}$, Anne Sylvie Dumenil', Guy Moneger ${ }^{1}$, Nadège Demars', Pierre Trouiller', Benjamin Sztrymf ${ }^{1}$

${ }^{1}$ Réanimation polyvalente, Hôpital Antoine Béclère, Clamart, France Correspondence: Benjamin Sztrymf - benjamin.sztrymf@abc.aphp.fr Annals of Intensive Care 2016, 6(Suppl 1):P78
Introduction Anemia is frequent in the critical care setting. It is the result of several mechanisms such as hemodilution, hemorrhage or inflammation consequences. Blood spoliation due to repeated blood punctures is also one of the factors leading to anemia. The reference method to determine the hemoglobin concentration is the cyanohemoglobin method performed with an automatic hematology analyzer $(\mathrm{HA})$. The main pitfalls of this method are to require a certain volume of blood, further enhancing the blood spoliation, and to be completed in a laboratory, increasing the lag time to diagnosis. Alternate methods such as HemoCue@ $(\mathrm{HC})$ or spectrophotometry with the blood gas analysis (BGA) have been developed to address these issues. Nevertheless, accuracy of these methods remains debatable. Therefore, we conducted a study to compare the aforementioned techniques agreement, completion time and costs in ICU.

Patients and methods This is a single-center prospective study. All patients undergoing both a blood cell count and an arterial blood gas analysis were included. A capillary HemoCue $\odot$ was performed just after the blood puncture and the capillary hemoglucotest. The completion time was registered, as well as the hour of the blood puncture. We also registered the hours at the blood sample arrival at the laboratory and at the availability of the result on the hospital intranet. The HA was considered as the reference method. Costs were estimated according to the French health system charts. Agreement between the methods has been estimated with Bland and Altman test. Results are given in mean \pm SD. Comparisons have been made with Student's $t$ test, ANOVA or Chi square test as appropriate.

Results Fifty-one samples have been compared in 37 patients (age $70 \pm 15$ years, SAPS II $48 \pm 20$ ). We found a difference when comparing the hemoglobin concentration with $\mathrm{HA}, \mathrm{BGA}$ and $\mathrm{HC}$ (respectively, $9.9 \pm 1.8,10.2 \pm 2.1$ and $9.5 \pm 2.5 \mathrm{~g} / \mathrm{dl}, p=0.003$ ). The comparison between $\mathrm{HA}$ and BGA evidenced a systematic positive deviation $(+0.25 \mathrm{~g} / \mathrm{dl})$. Limits of agreement $( \pm 2 \mathrm{SD})$ were $\pm 1.94 \mathrm{~g} / \mathrm{dl}$. Three measurements were outside these limits. The comparison between HA and $\mathrm{HC}$ evidenced a systematic negative deviation $(-0.4 \mathrm{~g} / \mathrm{dl})$. Limits of agreement were $\pm 2.92 \mathrm{~g} / \mathrm{dl}$. Three measurements were outside these limits. A transfusion was indicated once according to recommended transfusion thresholds by HA. This transfusion was also indicated by BGA. HC would have indicated nine transfusions, only one being confirmed by HA.

HC was performed in $1.2 \pm 0.5 \mathrm{~min}(\mathrm{~min})$. The time between the blood sample and the result's availability was significantly lower for the BGA as compared to HA ( $34 \pm 19.9$ vs. $54.5 \pm 32.6 \mathrm{~min} ; p=0.01)$. It was the result of a faster laboratory technique $(23.7 \pm 15.5$ vs. $31.6 \pm 20.2 \mathrm{~min}$; $p=0.03$ ), whereas the transport time of both samples was not different. The costs of $\mathrm{HA}, \mathrm{BGA}$ and $\mathrm{HC}$ were estimated to be, respectively, $426.87,1032.75$ and 45.9 euros. Nevertheless, when summing these "direct costs" with the price of the transfusions that would have been indicated according to the tests, $\mathrm{HC}$ became the most expensive tool (5022.9 euros) as compared to HA (979.87 euros) or BGA (1585.75 euros).

Discussion Among the three methods, $\mathrm{HC}$ was the fastest to perform, but seemed to be the less accurate as well. Its precise position in the ICU setting must be discussed, since the reference test appears to be mandatory to indicate a transfusion. Its utility may be more substantial in the pre-hospital setting where no other hemoglobin measurement tool is available. The blood gas analysis, often performed in ICU, offers the advantage to be more accurate than HemoCue $\odot$ and to provide other useful information.

Conclusion Alternate methods of hemoglobin monitoring allow a faster result, but their accuracy is debatable. Their respective utility might depend on the clinical setting.

Competing interests None. 


\section{P79}

Activated partial thromboplastin time and anti-Xa measurements in heparin monitoring among critical care patients Noémie Tencé ${ }^{1}$, Inès Zaien ${ }^{1}$, Martine Wolf², Pierre Trouiller ${ }^{1}$, Frédéric Jacobs $s^{1}$

${ }^{1}$ Réanimation polyvalente, Hôpital Antoine Béclère, Clamart, France; ${ }^{2}$ Service d'hématologie biologique, Hôpital Antoine Béclère, Clamart, France Correspondence: Frédéric Jacobs - frederic.jacobs@abc.aphp.fr Annals of Intensive Care 2016, 6(Suppl 1):P79

Introduction Monitoring the anticoagulant effect of unfractionated heparin (UFH) is mandatory. This monitoring can be done by the mean of the activated partial thromboplastin time (aPTT) or by anti-Xa level measurements. Compared with anti-Xa levels testing, aPTT is more frequently impacted by preanalytic variables and biologic factors (increased levels of acute-phase reactants, consumption coagulopathy) often encountered among critically ill patients. We studied the agreement of both test results in unselected critically ill patients.

Patients and methods aPTT and anti-Xa levels were simultaneously monitored in patients treated by continuous intravenous infusion of UFH. Blood samples were drawn into sodium citrate tubes (Greiner Bio-One SAS, France). aPTT was measured with TriniCLOT Automated aPTT reagent (Tcoag, Ireland) and anti-Xa levels with Biophen Heparin (LRT) (HYPHEN Biomed, France). An aPTT of 2-3 times the control and anti-Xa levels between 0.3 and $0.7 \mathrm{IU} / \mathrm{ml}$ were defined as therapeutic.

Results Forty-four patients (mean age $71.13 \pm 15.7$ years; mean SAPSII $39.1 \pm 14.4$ ) were included. Reasons for admission were medical in 31 and surgical in 13. The indications for UFH therapy were atrial fibrillation (26), venous thromboembolism/pulmonary embolism (13), thrombophilia (2), acute coronary syndrome (1), and arterial thrombosis (2). Paired measurements of aPTT and anti-Xa were taken on 353 samples. Linear regression analysis was used to evaluate the relationship between aPTT and anti-Xa.

The correlation between aPTT and anti-Xa levels was low $(r=0.495)$.

The concordance of tests results is shown in Table 17.

Concordant aPTT and anti-Xa values were observed in $213(60.3 \%)$ data pairs. aPTT was discordantly high in $96(29.9 \%)$ data pairs and discordantly low in 44 (12.5\%) ones.

Discussion In an unselected population of critically ill patients, the concordance of aPTT and anti-Xa levels was low. Considering anti-Xa as gold standard, monitoring anticoagulation treatment by aPTT leads to a high risk of misdosing. aPTT is frequently impacted by biologic factors. Although less commonly, anti-Xa levels can also be influenced by biologic cofounders.

Poor correlation between aPTT and anti-Xa could result from alterations in FII and FVIII activity.

Conclusion Use of aPTT and anti-Xa levels to guide heparin therapy may lead to different estimates of UFH concentration in the same patient.

\section{Table 17 Cross-tabulation of clinically relevant aPTT and anti-Xa levels}

aPTT below therapeutic range/anti-Xa below therapeutic range $\quad 105$

aPTT below therapeutic range/anti-Xa in therapeutic range

aPTT below therapeutic range/anti-Xa above therapeutic range

aPTT in therapeutic range/anti-Xa below therapeutic range

aPTT in therapeutic range/anti-Xa in therapeutic range

aPTT in therapeutic range/anti-Xa above therapeutic range

aPTT above therapeutic range/anti-Xa below therapeutic range

aPTT above therapeutic range/anti-Xa in therapeutic range

aPTT above therapeutic range/anti-Xa above therapeutic range
Both aPTT and anti-Xa have limitations when used for UFH monitoring and may not accurately assess anticoagulant status. Further investigation (using thromboelastometry or thrombin generation assays) could be useful to determine the optimal anticoagulation testing protocol in critically ill patients.

\section{Competing interests None.}

P80

Agreement between the Point-Of-Care Siemens RAPIDPoint ${ }^{\circledR}$ 500 Blood gas system and central laboratory measurement of hemoglobin, hematocrit, glucose and electrolytes in ICU patients

Jérôme Allardet-Servent ${ }^{1}$, Melissa Lebsir², Christian Dubroca², Mireille Portugal ${ }^{2}$, Matthias Castanier', Thomas Signouret', Guillemette Thomas', Rettinavelou Soundaravelou', Anne Lepidi', Philippe Halfon'2, Jean-Marie Seghboyan ${ }^{1}$

${ }^{1}$ Réanimation, Hôpital Européen Marseille, Marseille, France; ${ }^{2}$ Laboratoire européen, Laboratoire d'analyses de Biologie Médicale Alphabio, Marseille, France

Correspondence: Jérôme Allardet-Servent - j.allardetservent@

hopital-europeen.fr

Annals of Intensive Care 2016, 6(Suppl 1):P80

Introduction Several blood analyses are performed in critically ill patients. Rapid detection of abnormal values, by point-of-care testing, allows clinicians to promptly deliver the appropriate therapy. We sought to investigate the agreement between the last generation of blood gas analyzer and central laboratory measurement of electrolytes (sodium, potassium, chloride, bicarbonate), hemoglobin, hematocrit and glucose.

Patients and methods Three hundred and fourteen paired samples were collected prospectively in 51 patients. All samples were drawn from an arterial line in the morning at the 06:00 am. Biological tubes were collected through a Vacutainer ${ }^{\circledR}$ and analyzed in the central laboratory (Lab) using Beckman Coulter ${ }^{\circledR}$ automates (DXH and AU 5800). BD Preset ${ }^{\circledR}$ heparinized syringe was filled with $1.6 \mathrm{ml}$ of arterial blood and analyzed immediately in the ICU using the Point-of-Care Siemens RAPIDPoint ${ }^{\circledR} 500$ Blood gas system (POC). Measurements obtained by the two methods were compared using the Bland-Altman method (bias and limit of agreements), Deming regression analysis and Pearson correlation. We use the proficiency testing criteria to define acceptable analytical performance (CLIA 1992): $\pm 4 \mathrm{mmol} / \mathrm{l}$ for sodium, $\pm 0.5 \mathrm{mmol} / \mathrm{l}$ for potassium, $\pm 5 \%$ for chloride, $\pm 0.6 \mathrm{~g} / \mathrm{l}$ for glucose, $\pm 6 \%$ for hematocrit and $\pm 7 \%$ for hemoglobin.

Results The main results are presented in the Table 18.

Conclusion The Point-of-Care Siemens RAPIDPoint ${ }^{\circledR} 500$ Blood gas system satisfied CLIA criteria of acceptable analytical performance for all tested parameters except for hemoglobin (difference $=7.6 \%$ ). We identified a systematic overestimation of hemoglobin (mean $0.8 \mathrm{~g} / \mathrm{dl}$ ) with the RAPIDPoint ${ }^{\circledR} 500$ Blood gas system.

\section{Competing interests None.}

\section{References}

1. Ehrmeyer SS, Laessig RH, Leinweber JE, Oryall JJ: 1990 Medicare/CLIA final rules for proficiency testing: minimum intralaboratory performance characteristics (CV and bias) needed to pass. Clin Chem 1990, 36:1736-1740.

2. CLSI. Measurement Procedure Comparison and Bias Estimation Using Patient Samples; Approved Guideline-Third Edition. CLSI document EP09A3. Wayne, PA: Clinical and Laboratory Standards Institute; 2013.

P81

Clinical features and prognosis of pesticide poisoning

Neila Maaroufi', Dhaouadi Mahassen², Abid Zeineb ${ }^{3}$

${ }^{1}$ General Hospital, Tunis, Tunisia; ${ }^{2}$ Tunisia, General Hospital, Tunis, Tunisia;

${ }^{3}$ Emergency, General Hospital, Tunis, Tunisia

Correspondence: Neila Maaroufi - maaroufineila@gmail.com

Annals of Intensive Care 2016, 6(Suppl 1):P81 
Table 18 See text for description

\begin{tabular}{|c|c|c|c|c|c|c|c|}
\hline & Min lab & Max lab & Bias & $\begin{array}{l}\text { Lower limit } \\
\text { of agreement }\end{array}$ & $\begin{array}{l}\text { Upper limit } \\
\text { of agreement }\end{array}$ & Regression & Correlation \\
\hline Sodium (mmol/l) & 119.4 & 150.2 & 1.3 & -2.1 & 4.7 & $\mathrm{Lab}=16.007+0.891 \mathrm{POC}$ & 0.936 \\
\hline Potassium (mmol/l) & 2.76 & 5.8 & 0.2 & -0.2 & 0.6 & $\mathrm{Lab}=0.435+0.939 \mathrm{POC}$ & 0.944 \\
\hline Chloride (mmol/l) & 86 & 116 & -0.9 & -3.7 & 1.9 & $\mathrm{Lab}=-1.329+1.004 \mathrm{POC}$ & 0.967 \\
\hline Bicarbonate (mmol/l) & 13.5 & 43.1 & 0.8 & -1.9 & 3.4 & $\mathrm{Lab}=0.272+1.019 \mathrm{POC}$ & 0.968 \\
\hline Glucose $(g / l)$ & 0.5 & 3.97 & -0.1 & -0.3 & 0.1 & $\mathrm{Lab}=-0.0964+0.992 \mathrm{POC}$ & 0.972 \\
\hline Hemoglobin (g/dl) & 6.1 & 15.5 & -0.8 & -1.3 & -0.2 & $\mathrm{Lab}=-0.0201+0.926 \mathrm{POC}$ & 0.985 \\
\hline Hematocrit (\%) & 18 & 46 & -1.1 & -2.9 & 0.7 & $\mathrm{Lab}=-1.353+1.008 \mathrm{POC}$ & 0.981 \\
\hline
\end{tabular}

Introduction Deliberate self-poisoning with pesticide continues to be a major public health concern in many developing countries. This study aimed to evaluate the data on cases of acute pesticide poisoning and to compare different variables between survivors and non-survivors.

Patients and methods In this cross-sectional study, medical records of all pesticide-intoxicated patients were reviewed from 2009 to 2013, retrospectively. Demographics, clinical features and laboratory findings were evaluated. The variables compared between survivors and non-survivors were the amount of pesticide ingested, occurrence of vomiting after ingestion, time and place of hospital admission, length of hospital stay, leukocytosis, serum creatinine level and the outcomes. Results A total of 154 patients were evaluated. The mean \pm standard deviation of patients' age was $32 \pm 18$ years. The length of hospital stay was $4.76 \pm 4.8$ days. Most poisonings occurred in spring and summer. The in-hospital fatality rate was $36 \%$. Statistically significant associations were found between the outcome of patients and the amount ingested $(P=0.002)$, vomiting $(P=0.003)$, early need to intensive cares $(P=0.005)$, leukocytosis $(P=0.001)$ and serum creatinine levels $(P=0.001)$.

Discussion and conclusion Prompt vomiting, early need to intensive cares, leukocytosis and multi-organ failures are major determinants for fatal outcome of pesticide poisoning. It may be useful to educate health professionals and the general population about the serious consequences of exposure to pesticide.

\section{Competing interests None.}

P82

Variability of neurobehavioral toxicity of naphyrone, a new synthetic cathinone, according to its acute or binge administration

Camille Gamblin ${ }^{1}$, Nadia Benturquia' ${ }^{1}$, Olivier Roussel' ${ }^{1}$, Lucie Chevillard ${ }^{1}$ Patricia Risede ${ }^{1}$, Jacques Callebert ${ }^{1}$, Bruno Megarbane $^{2}$

${ }^{1}$ Inserm u1 144, Université Paris Descartes, Paris, France; ${ }^{2}$ Service de Réanimation Médicale et Toxicologique, CHU Lariboisière, Paris, France Correspondence: Bruno Megarbane - bruno.megarbane@lrb.aphp.fr Annals of Intensive Care 2016, 6(Suppl 1):P82

Introduction Naphyrone is a new psychoactive substance and a cathinone derivate. Naphyrone was reported to be used by approximately $2 \%$ of recreational drug users and is frequently present in bath salts. Scarce data are available regarding its toxicity, pharmacokinetics and effects on brain monoamines. Our objectives were to study naphyrone-related effects on behavior and brain monomaine content according to two administration modalities (acute and binge) mimicking its use in humans.

Materials and methods We performed an experimental study on the effects of naphyrone-induced effects after acute and repeated administration (binge) on the locomotor activity, anxiety (openfield), resignation (forced swimming), memory (Y-maze), hedonic status (sucrose consumption) of Swiss mice. We also investigated the effects of naphyrone-induced on monoamines in the prefrontal cortex. Plasma naphyrone concentrations were measured using high-performance liquid chromatography (HPLC) coupled to mass spectrometry (MS) and brain monoamine concentrations using HPLC coupled to fluorometry. For each animal and each time, we calculated the difference between the parameter value at that time and baseline and the area under the curve of its time course. Comparisons were made using two-way ANOVA followed by posttests using Bonferroni correction. Pharmacokinetics (PK) was modeled and parameters calculated using WinNonlin ${ }^{\circledR}$ software.

Results Naphyrone induced dose-dependent stimulation of locomotor activity that appeared more marked and prolonged than MDPV- and cocaine-related effects (used as positive controls), additionally increasing after its binge administration $(p<0.001)$. Significant increase in the distance walked at the periphery of the openfield was observed up to $24 \mathrm{~h}$ postinjection $(p<0.001)$, corresponding to the behavior compensation of enhanced frighten. During binge administration ( 3 times per day, 3 successive days), increased locomotor effects at day 3 (after the 9th injection) in comparison with day 1 (after the 1st injection) supported naphyrone-induced hyper-sensitization process. Similarly, significant effects were observed on mice depression $(p<0.0001)$ and memory $(p<0.005)$ but not on hedonic status. Significant dose-dependent increase in aggressively social relationships was also reported among naphyrone-treated mice $(p<0.001)$. The neurochemical study revealed significant increase in dopamine and norepinephrine concentrations in the prefrontal cortex, without significant modifications in serotonin concentrations. This monoamine profile was similar after repeated naphyrone administration. Naphyrone PK profile was described after acute and binge administration and effects correlated with plasma concentrations.

Conclusion Neurobehavioral disorders induced by acute and repeated naphyrone administration mainly consist in the stimulation of locomotor activity. The increase in the cortical dopamine concentrations may suggest an addictive potential that should be further investigated.

Competing interests None.

\section{P83}

Acute acenocoumarin poisoning: an epidemiological and clinical study

A. M'rad ${ }^{1}$, Hana Fredj ${ }^{1}$, Messaouda Khelfa ${ }^{1}$, Youssef Blell ${ }^{1}$, H. Thabet ${ }^{2}$, Nozha Brahmi ${ }^{1}$, M. Amamou ${ }^{1}$

${ }^{1}$ Department of intensive care and toxicology, Centre d'Assistance Médicale Urgente, Tunis, Tunisia; ${ }^{2}$ Department of emergency, Centre d'Assistance Médicale Urgente, Tunis, Tunisia

Correspondence: A. M'rad - mrad.aymen@gmail.com

Annals of Intensive Care 2016, 6(Suppl 1):P83

Introduction Acenocoumarin is the most commonly prescribed oral anticoagulant in our country. Because of the paucity of data on acute acenocoumarin poisoning, we undertook this study, which aimed to determine the epidemiologic, clinical, therapeutic characteristics and outcome of this intoxication.

Patients and methods It was a retrospective study performed over a 9-year period (from June 2006 to June 2015). Demographic characteristics, acenocoumarin long-term treatment history, clinical signs, treatment and outcomes of this intoxication have been analyzed. 
Results Fifty-eight patients aged $28 \pm 11$ years were eligible; their sex ratio was of 1 . All cases were self-inflicted. Among them 11 males were detained. Acenocoumarin long-term treatment was noted in $40 \%$ $(n=23)$ patients, for thromboembolic disease $(n=17)$ or valvular replacement $(n=6)$. Coingestion of other drugs was reported in $43 \%$ of cases. The mean ingested dose was $56 \pm 48 \mathrm{mg}$ (extremes 8 and $240 \mathrm{mg}$ ). The average time of consultation was $9 \pm 11 \mathrm{~h}$. In the majority of cases, patients were asymptomatic. Bleeding was reported in 4 detained patients but not observed by physician. Gastric lavage was performed in $17 \%$ of cases within $1 \mathrm{~h}$ after ingestion. Vitamin $\mathrm{K}$ was administrated in $52 \%$ of cases. No patient required PPSB perfusion or transfusion. The outcome was favorable in all cases. No deaths were reported. Length of stay was $30 \pm 24 \mathrm{~h}$.

Discussion Acenocoumarin overdose was well described in the literature, and the treatment is well codified.

However, few studies were interested in acute poisonings and their management. Our study suggested that acute acenocoumarin poisoning is often not serious if patients were early managed. The administration of vitamin K could be necessary and safety, especially for patients without long-term anticoagulant treatment.

Conclusion Acenocoumarin poisoning is increasingly frequent in our country; its prognoses are generally favorable. Hospitalization must be indicated in symptomatic patients who presented active bleeding or those with long-term anticoagulant treatment. The others can be managed ambulatory.

\section{Competing interests None.}

\section{References}

1. Granditsch G, Pilgerstorfer HW. Acenocoumarin (Sintrom) poisoning in a child Wien. KlinWochenschr. 1971;83(4):62-3.

2. Isbister GK, Whyte IM. Management of anticoagulant poisoning. Vet Hum Toxicol. 2001;43(2):117.

\section{P84}

Death by deliberate self-poisoning in Sfax, Tunisia: Victims characteristics and toxic substance profile

Youssef Nouma', Wiem Ben Amar², Sami Bardaa', Karama Regaieg',

Kaouthar Jammeli ${ }^{1}$, Mabrouk Bahloul ${ }^{3}$, Zouheir Hammami ${ }^{1}$, Maatoug Samir ${ }^{1}$

${ }^{1}$ Forensics, Habib Bourguiba University Hospital, sfax, Tunisia; ${ }^{2}$ Foren-

sics, Habib Bouarguiba, Sfax, Tunisia; ${ }^{3}$ Réanimation polyvalente, Faculté de

médecine de Sfax, Sfax, Tunisia

Correspondence Maatoug Samir - wiembenamar@yahoo.fr

Annals of Intensive Care 2016, 6(Suppl 1):P84

Introduction Deliberate self-poisoning is a serious problem in Tunisia. However, our understanding of the problem of suicidal intoxication remains limited. This study aims to reveal the frequency of suicide by self-poisoning in the region of Sfax, to describe the characteristics of victims and to identify the difficulties often encountered in determining the toxic substance.

Patients and Methods This study is a retrospective study conducted during a period of 08 years (2007-2014), about all cases of self-poisoning collected in the Department of Forensic Medicine of Habib Bourguiba University Hospital of Sfax, Tunisia.

Results During the study period, 299 cases of suicide are compiled. In 42 cases $(14 \%)$, suicide is committed by self-poisoning, representing a little more than 5 cases per year. The sex ratio is 0,7 . The mean age of victims is $32.9 \pm 7,6$ years with extremes ranging from 18 to 63 years. In 25 cases $(60 \%)$ the victim is single, in 15 cases (35\%) she is married and she is divorced in only 2 cases (5\%). She is unemployed in 18 cases ( $43 \%$ ) and she is a daily-worker in 16 cases (38\%). In half of cases the victim doesn't have any known psychiatric illness history. The antecedent of suicide attempts is found in 8 cases (19\%).

In only 14 cases (33\%) death occurred at home. Most of victims were hospitalized in intensive care unit ( 28 cases, $67 \%$ ). The average time interval between hospitalization and death is 32.45 hours with extremes ranging from 8 to 96 hours.

The forensic autopsy is often performed more than one day after the intoxication date. The post-mortem toxicological samples systematically made during the autopsy were positive in only 5 cases (12\%). Toxic samples are often not made during the hospitalization. The nature of the toxic substance is determined based only on history and clinical information. In our study the nature of the toxic substance is a pesticide in 16 cases ( $38 \%$ ) and a medication in 14 cases (34\%). It is still undetermined in 6 cases (14\%).

Conclusion Toxicological samples performed after a prolonged interval of time from the intoxication moment are often negative. Thus it is fundamental to realize them systematically at the time of admission into intensive care unit in case of intoxication suspicion. The determination of toxics allows not only to orientate the therapeutic management but also to strengthen preventive strategies against suicide. Competing interests None.

\section{P85}

Adverse drug reactions (ADRs) collected by medical-staffed ambulances: pilot study

A. Lillo-Le Louët ${ }^{1}$, F. Baud ${ }^{2}$, C. Le Beller ${ }^{1}$, B. Vivien ${ }^{2}$, L. Soufir ${ }^{3}$, P. Carli ${ }^{2}$ and H. Le Louët ${ }^{4}$

${ }^{1}$ Centre Régional de Pharmacovigilance (CRPV) Hôpital Européen Georges Pompidou; ${ }^{2}$ SAMU de Paris, Hôpital Necker EM; ${ }^{3}$ Réanimation, Groupe hospitalier Saint-Joseph; ${ }^{4}$ CRPV Hôpital Henri Mondor, AP-HP, France

Introduction In Paris area, emergency medical assistance is run by the "SAMU de Paris." Medical-staffed ambulances go directly to take care of any patient with an acute medical problem after an evaluation of the clinical situation made by phone.

Aim: Collection, analysis, and evaluation of adverse drug events (ADRs), whatever it is, acute intoxication, organ failure, allergy, and whatever its clinical presentation.

Methods: Every month, forms from all medical interventions performed daily by the SAMU de Paris are analyzed retrospectively by a physician trained in pharmacovigilance. The following information is available: patient's main characteristics, description of the medical event, main diagnosis, and outcome. If an ADR is present or even suspected, a copy of the form is made. The pharmacovigilance centers contact medical staff that took care of the patient to retrieve more information about the final diagnosis and the drug involvement. If a drug adverse effect is retained, the case is anonymously registered in the national database, and a specific letter is sent to describe and sum up the case.

Results From January 1, 2015, to July 31, 2015, 274 cases of possible ADRs have been collected.

- 203 cases of ADRs (74 \% of the total) have been immediately diagnosed and registered.

- The drug causality is pending for 29 cases. For the last 37 cases, a drug implication has been definitely ruled out, with no drug intake, another causative agent, illicit substances and food.

- For all ADRs retained $(n=203)$, a fatal outcome occurred in 14 cases ( $7 \%$ of ADRs). The main organ failure was central nervous system $(n=107,53 \%)$, cardiovascular $(n=55,27 \%)$, digestive $(n=21,10 \%)$, and lung $(n=8)$.

- The type of ADRs was voluntary overdose $(n=60,30 \%)$, bleeding $(n=48,24 \%)$, hypoglycemia $(n=40,20 \%)$, allergy $(n=15$, $7 \%)$, and medication error $(n=11,6 \%)$.

- The following drugs were involved for all ADRs retained $(n=203)$ : psychotropic drugs $(n=73,36 \%)$; drugs acting on hemostasis $(n=48,24 \%)$; antidiabetics $(n=41,6 \%)$; pain killers $(n=12,6 \%)$; antiinfectious $(n=11,6 \%)$, and various $(15)$ NSAID (8) - anesthetic agents (3) - corticosteroid (3) - contrast media (2) - potassium (1), vaccine (1), GcSF (1), colchicine (1).

Discussion and conclusion To our knowledge, this is the first time that serious adverse drug reactions are collected directly from the first step of emergency care, before hospitalization. Excepted intentional drug overdose for suicide attempts, the main ADRs observed such as bleeding with anticoagulants or hypoglycemia with insulin, are expected and evitable. Further analysis on cardiovascular effects 
is pending. This study increases awareness of physicians working in SAMU. The usefulness of toxicological analysis must be discussed.

\section{P86}

Prognostic value of plasma concentration of acebutolol in acute poisoning

A. M'rad ${ }^{1}$, Youssef Blel ${ }^{1}$, Fatma Essafi ${ }^{1}$, Nasreddine Foudhaili', Abdelaziz Ben Slimen ${ }^{1}$, Nozha Brahmi ${ }^{1}$, M. Amamou'

'Department of Intensive Care and Toxicology, Centre d'Assistance Médicale Urgente, Tunis, Tunisia

Correspondence: A. M'rad - mrad.aymen@gmail.com

Annals of Intensive Care 2016, 6(Suppl 1):P86

Introduction Beta-blockers could be responsible of severe poisoning leading to death. The knowledge of prognostic factors is essential and could guide the management of these patients.

The purpose of our study is to determine whether the plasma concentrations of acebutolol can predict clinical symptoms and prognosis.

Patients and methods It was a retrospective study conducted over 2 years in a 16-bed toxicological unit including all admitted patients for acebutolol poisoning, presenting with shock or cardiac arrest. A systematic serum measure of acebutolol was done at least once hospital stay.

Results Eleven patients were eligible. The mean age was $23.0 \pm 6.6$ years with a female predominance $(9 / 11)$.

The average of the presumed ingested dose was $8050 \pm 4812 \mathrm{mg}$.

On admission, cardiovascular manifestations were predominant; the main presenting symptoms were bradycardia $(n=5)$, shock $(n=10)$ and cardiac arrest with favorable evolution in one case.

Electrocardiogram showed a heart block in 7 cases: first degree $(n=4)$ and third degree $(n=3)$, wide QRS complex with sodium channelblocking effect in 7 cases (mean QRS duration was $220 \pm 77 \mathrm{~ms}$ ) and a prolongation of the QT interval in 8 patients (the mean QT interval was $521 \pm 79 \mathrm{~ms}$ ). The mean plasma concentration of acebutolol at admission was $19.3 \pm 16.6 \mathrm{mg} / \mathrm{l}$. Six patients had required mechanical ventilation due to hemodynamic instability. Inotrope drugs were required in eight patients; semi-molar sodium bicarbonate was infused in seven patients with an average volume of $500 \pm 144 \mathrm{ml}$.

Three patients died. Two early deaths were related to intoxication, and the later was related to nosocomial complication.

The analysis of prognostic factors showed the absence of correlation between acebutolol plasma concentration either on admission or any time and clinical or electrocardiographic signs in serious poisoning. Conclusion The severity of acebutolol poisoning must be assessed by clinical and electrocardiographic manifestations. The plasma concentration of acebutolol should not be considered as prognostic factor.

Competing interests None.

\section{Table 19 See text for description}

\begin{tabular}{ll}
\hline Variables & All patients $(\boldsymbol{n}=\mathbf{4 4 0})$ \\
\hline Age [median (IQR)] & $68(61-74)$ \\
Gender (M/F) & $372 / 68$ \\
pH at ICU admission [median (IQR)] & $7.28(7.23-7.32)$ \\
Time course of COPD (years) [median (IQR)] & $7(4-15)$ \\
Baseline treatment & \\
Aminophylline $[n(\%)]$ & $107(24.3)$ \\
Short-acting $\beta 2$ mimetics $[n(\%)]$ & $197(44.7)$ \\
Long-acting $\beta 2$ mimetics $[n(\%)]$ & $46(10.4)$ \\
Inhaled anticholinergics $[n(\%)]$ & $39(8.9)$ \\
Inhaled corticosteroids $[n(\%)]$ & $116(26.4)$ \\
Systemic corticosteroids $[n(\%)]$ & $37(8.4)$ \\
\hline
\end{tabular}

P87

Baseline pharmacologic treatment for patients admitted in ICU for AECOPD: trends in use and conformity to the GOLD guidelines Islem Ouanes ${ }^{1}$, Rami Jabla', Hedia Hammed', Mahdi Marzouk', Nesrine Boujelbene ${ }^{1}$, Fahmi Dachraoui ${ }^{1}$, Asma Hachani', Saousen Ben Abdallah'1, Hend Ben Lakhal', Chaima Ghribi', Imen Ben Ali', Imen Abdellaoui', Lamia Ouanes-Besbes ${ }^{1}$, Fekri Abroug ${ }^{1}$

${ }^{1}$ Réanimation Polyvalente, CHU Fattouma Bourguiba, Monastir, Tunisia Correspondence: Islem Ouanes - ouanes.islem@gmail.com Annals of Intensive Care 2016, 6(Suppl 1):P87

Introduction The quality of baseline treatment of COPD patients is important in the prevention of acute exacerbation. The aim of our study is to describe trends in baseline pharmacologic treatment (BPT) use guidelines in a cohort of patients admitted to a Tunisian ICU for AECOPD and to assess its conformity with GOLD, which is intended to classify patient's COPD severity and to guide patient's management.

Patients and methods In a cohort study of consecutive patients admitted between 2000 and 2012 for AECOPD in our ICU, we analysed BPT and its conformity with GOLD guidelines issued in 2001 and 2007. Results During the study period, 440 patients admitted for $552 \mathrm{epi}$ sodes of severe AECOPD requiring ventilatory support (NIV in $67.8 \%$ ). Table 19 shows patients' characteristics and baseline medications used in this cohort:

Aminophylline use decreased significantly from around $50 \%$ (in 2000) to $21.4 \%$ (in 2012), short-acting 32 mimetic use remained unchanged (70.1\% in 2000 and $64.3 \%$ in 2012), whereas inhaled anticholinergics and corticosteroids increased significantly during the same period (respectively, from 0 to $18 \%$ and from 13 to $60 \%$ ). In patients in whom FEV1 was measured and GOLD classification was possible, BPT was compliant with 2001 and 2007 recommendations in 58.9 and $15.6 \%$, respectively.

Conclusion In this cohort of severe COPD admitted to ICU for acute exacerbation, compliance with GOLD 2001 and 2007 varied substantially and could be explained by changes regarding long-acting 32 mimetic use.

Competing interests None.

Table 20 See text for description

\begin{tabular}{|c|c|c|c|}
\hline & Eo > $2 \%(n=22)$ & Eo $\leq 2 \%(n=20)$ & $p$ \\
\hline $\begin{array}{l}\text { Age (years) [median } \\
\text { (IQR)] }\end{array}$ & $70.5(61-73.2)$ & $63(57.2-77.5)$ & 0.207 \\
\hline Gender (M/F) & $16 / 6$ & $19 / 1$ & 0.096 \\
\hline $\begin{array}{l}\text { NIV at ICU admission } \\
{[n(\%)]}\end{array}$ & $22(100)$ & $18(90)$ & 0.221 \\
\hline $\begin{array}{l}\mathrm{pH} \text { at ICU admission } \\
\text { median (IQR) }\end{array}$ & $7.32(7.26-7.36)$ & $7.29(7.25-7.33)$ & 0.390 \\
\hline CRP median (IQR) & $44(14-76.5)$ & $17(7-58)$ & 0.045 \\
\hline $\begin{array}{l}\text { Procalcitonin median } \\
\text { (IQR) }\end{array}$ & $0.16(0.14-0.31)$ & $0.11(0.05-0.24)$ & 0.092 \\
\hline $\begin{array}{l}\text { NT-proBNP median } \\
\text { (IQR) }\end{array}$ & $365(168-3174)$ & $400(94-1800)$ & 0.911 \\
\hline 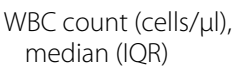 & $8010(6282-10,475)$ & $\begin{array}{l}11,240(9005- \\
13,145)\end{array}$ & 0.016 \\
\hline $\begin{array}{l}\text { NIV duration (days) } \\
{[n(\%)]}\end{array}$ & $8(5-14.5)$ & $7(3.75-11)$ & 0.530 \\
\hline $\begin{array}{l}\text { MV duration (days) } \\
{[n(\%)]}\end{array}$ & $13(10.5-17)$ & $5(3.5-7.5)$ & 0.048 \\
\hline $\begin{array}{l}\text { ICU LOS (hours) [n } \\
(\%)]\end{array}$ & $12(6.5-13)$ & $8(6-24)$ & 0.718 \\
\hline
\end{tabular}




\section{P88}

Blood eosinophils levels and acute exacerbations of COPD

Islem Ouanes ${ }^{1}$, Asma Hachani ${ }^{1}$, Lamia Ouanes-Besbes ${ }^{1}$, Saousen Ben Abdallah', Rami Jabla', Hedia Hammed', Mahdi Marzouk', Hend Ben Lakhal ${ }^{1}$, Imen Ben Ali ${ }^{1}$, Imen Abdellaoui ${ }^{1}$, Chaima Ghribi ${ }^{1}$, Fahmi Dachraoui', Fekri Abroug

${ }^{1}$ Réanimation Polyvalente, CHU Fattouma Bourguiba, Monastir, Tunisia

Correspondence: Islem Ouanes - ouanes.islem@gmail.com

Annals of Intensive Care 2016, 6(Suppl 1):P88

Introduction Increased blood eosinophil count (greater than $2 \%$ ) has been described by several studies as reflecting eosinophilic airway inflammation exacerbation and a useful marker to guide systemic corticosteroids administration in acute exacerbations of COPD (AECOPD) $[1,2]$. The aim of this study is to describe the rate and the patterns of hyper-eosinophilia in a population of patients admitted to an intensive care unit (ICU) for severe AECOPD requiring ventilatory support.

Patients and methods This is a retrospective cohort study, including patients consecutively admitted to our ICU between January and August 2015 for severe AECOPD requiring ventilatory support. COPD patients with an obvious cause of decompensation (pulmonary embolism, acute pulmonary edema, pneumothorax, pneumonia) were excluded. Continuous variables are presented as medians and interquartile ranges (IQRs) and compared by the Mann-Whitney test, whereas dichotomous variables are presented in percentage and compared by the Chi square test.

Results During the study period, 42 patients were admitted for AECOPD [median age was 66.5 years (IQR 60-74) with $83.5 \%$ male]. Hyper-eosinophilia (>2 \%) was present in $52.4 \%$ of patients with a median value of $3.25 \%$ (2.2-4.0). Table 20 compares the characteristics of patients with and without hyper-eosinophilia.

Conclusion Hyper-eosinophilia ( $>2 \%$ ) appears to be common in patients admitted for AECOPD ( $52.4 \%$ of patients in our study) and seems associated with inflammation markers and a trend toward longer ventilation and ICU length of stay durations. A policy of corticosteroids prescription guided by blood eosinophilia count seems reasonable in our population.

Competing interests None.

Table 21 See text for description

\begin{tabular}{|c|c|c|c|c|}
\hline $\begin{array}{l}\text { Acute respiratory } \\
\text { postoperative com- } \\
\text { plications (ARPC) }\end{array}$ & $\begin{array}{l}\text { Overall } \\
\text { population } \\
(n=98)\end{array}$ & $\begin{array}{l}\text { Not EF } \\
(n=77)\end{array}$ & $\mathrm{EF}(n=21)$ & $p$ \\
\hline Hospital stay (days) & 11.4 & 11.5 & 11.2 & 0.64 \\
\hline IGS & 17 & 17 & 23 & 0.9 \\
\hline $\begin{array}{l}\text { Postoperative pneu- } \\
\text { monia }[n(\%)]\end{array}$ & $24(25 \%)$ & $17(22 \%)$ & $7(33 \%)$ & 0.18 \\
\hline $\begin{array}{l}\text { Postoperative exacer- } \\
\text { bation }[n(\%)]\end{array}$ & $45(46 \%)$ & $33(43 \%)$ & $12(57 \%)$ & 0.11 \\
\hline Atelectasis [n (\%)] & $47(48 \%)$ & $36(47 \%)$ & $11(52 \%)$ & 0.38 \\
\hline $\begin{array}{l}\text { Acute respiratory } \\
\text { failure }[n(\%)]\end{array}$ & 35 (36 \%) & $25(32 \%)$ & $10(48 \%)$ & 0.1 \\
\hline $\begin{array}{l}\text { Acute kidney injury } \\
{[n(\%)]}\end{array}$ & 14 (14\%) & $12(16 \%)$ & $2(10 \%)$ & 0.63 \\
\hline $\begin{array}{l}\text { Cardiovascular com- } \\
\text { plication }[n(\%)]\end{array}$ & 19 (19\%) & $18(23 \%)$ & $1(5 \%)$ & 0.09 \\
\hline Sepsis & 15 (15\%) & $13(17 \%)$ & $2(10 \%)$ & 0.5 \\
\hline $\begin{array}{l}\text { Noninvasive ventila- } \\
\text { tion }[n(\%)]\end{array}$ & $22(22 \%)$ & $15(19 \%)$ & $7(33 \%)$ & 0.1 \\
\hline Bronchoscopy & 20 (20 \%) & $14(18 \%)$ & $6(29 \%)$ & 0.2 \\
\hline
\end{tabular}

\section{References}

1. Saha S, Brightling CE. Eosinophilic airway inflammation in COPD. Int J Chron Obstruct Pulmon Dis. 2006;1 (1):39-47.

2. Bafadhel M, McKenna S, Terry S et al. Blood eosinophils to direct corticosteroid treatment of exacerbations of chronic obstructive pulmonary disease: a randomized placebo-controlled trial. Am J Respir Crit Care Med. 2012 Jul 1;186(1):48-55.

P89

POFE: postoperative outcomes of chronic obstructive pulmonary disease (COPD) frequent exacerbators after pulmonary resection-a pilot study

Suela Demiri ${ }^{1}$, Christine Lorut ${ }^{2}$, Van Lang, Daniel Luu ${ }^{3}$, Antoine Rabbat ${ }^{2}$, Aurélie Lefebvre ${ }^{2}$, Marco Alifano ${ }^{4}$, Jean-Francois Reignard ${ }^{4}$, Marc

Samama ${ }^{3}$, Daniel Dusser ${ }^{2}$, Nicolas Roche ${ }^{2}$

${ }^{1}$ Hôpital Cochin, Paris, France; ${ }^{2}$ Réanimation pneumologique, Hôpital Cochin, Paris, France; ${ }^{3}$ Anesthésie réanimation, Hôpital Cochin, Paris,

France; ${ }^{4}$ Chirurgie thoracique, Hôpital Cochin, Paris, France

Correspondence: Suela Demiri - demiri.suela@gmail.com Annals of Intensive Care 2016, 6(Suppl 1):P89

Introduction After pulmonary resection, a postoperative pulmonary complication is present in $12-40 \%$ of cases. Risk factors such as COPD are well identified. The phenotype of frequent exacerbator (FE) has been recently described. No existing study has evaluated the rate of patients called ' $\mathrm{FE}$ ' among COPD patients requiring pulmonary resection and the relations between exacerbations history and the incidence of acute respiratory postoperative complications (ARPC).

The main objective was to determine the frequency of ARPC (atelectasis, acute respiratory failure, pneumonia) following lung resection in COPD patients. The secondary objectives aimed to determine the frequency of extra-pulmonary postoperative complications and the prevalence of the 'FE' phenotype in this population, as well as its relation with the risk of ARPC.

Patients and methods It was a prospective, observational, singlecenter study of patients with COPD hospitalized for elective thoracic surgery in the center of Hôpital Cochin, Paris, France. The inclusion criteria were as follows: male or female adults, permanent airflow obstruction (VEMS/CV $<70 \%$ ), scheduled oncologic pulmonary resection. Collected data were as follows: COPD symptoms and severity scores, preoperative severity scores, comorbidities, per operative data, postoperative complications and 30-day mortality.

Results Since July 2014 to September 2015, 98 COPD patients were included for analysis. Twenty-one were considered as FE (21.6\%). COPD classification was as follows: I-5 (\%), II-11 (\%), III-2 (\%). There was no difference in demographic or COPD severity score (CAT score, IPAQ, MRC) between FE and the rest of the population. There was no difference in the 30-day mortality.

Results are in Table 21.

Conclusion FE history does not seem to affect the ARPC rate following oncologic pulmonary resection in COPD patients.

Competing interests None.

\section{References}

1. McGarvey L, Lee AJ, Roberts J, Gruffydd-Jones K, McKnight E, Haughney J Characterisation of the frequent exacerbator phenotype in COPD patients in a large UK primary care population. Respir Med. 2015 Feb;109(2):228-37.

2. Nojiri T, Inoue M, Takeuchi Y, Maeda H, Shintani Y, Sawabata N, Hamasaki T, Okumura M. Impact of cardiopulmonary complications of lung cancer surgery on long-term outcomes. Surg Today. 2015 Jun;45(6):740-5.

P90

Optimal delivery of salbutamol with a pressurized metered-dose inhaler within a high-flow nasal therapy circuit

François Réminiac ${ }^{1}$, Cassandre Landel ${ }^{2}$, Samuel Gensburger ${ }^{2}$, Thomas Bocar $^{2}$, Lydiane Mordier ${ }^{2}$, Marion Philippe 2 , Déborah Le Pennec ${ }^{2}$, Laurent Vecellio ${ }^{2}$, Stephan Ehrmann ${ }^{3}$

${ }^{1}$ Anesthésie-réanimation, CHRU Hôpitaux De Tours, Tours, France; ${ }^{2}$ Inserm u1100, aérosolthérapie et biomédicaments à visée respiratoire, Université François Rabelais, Tours, France; ${ }^{3}$ Réanimation polyvalente, CHRU Hôpi- 
Aerosol mass of salbutamol potentially reaching the lungs (respirable mass)

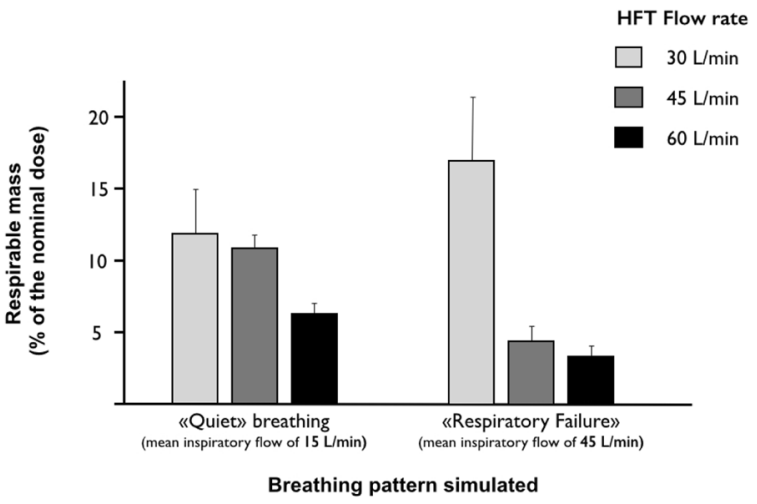

Fig. $\mathbf{3 0}$ See text for description

taux de Tours, Tours, France

Correspondence: François Réminiac - f.reminiac@yahoo.fr Annals of Intensive Care 2016, 6(Suppl 1):P90

Introduction Nasal high-flow therapy (HFT) is increasingly used in intensive care for patients suffering from respiratory failure who also often require inhaled bronchodilators. Pressurized metered-dose inhalers (pMDls) combined with spacers are convenient and costeffective to generate aerosols for both ambulatory and mechanically ventilated patients. Therefore, administration of salbutamol within a spacer, placed in the HFT circuit, using a pMDI, could be relevant. The aim of this bench study was to determine optimal settings in this regard.

Materials and methods We assessed the mass of salbutamol delivered downstream of a tridimensional anatomical model reproducing aerosol deposition and leakage at the nose and rhinopharynx level after ten pMDI actuations (delivery of $1000 \mu \mathrm{g}=$ nominal dose) of a canister connected with a spacer chamber (CombiHaler $\left.{ }^{\circledR}\right)$ placed in an HFT circuit and synchronized with inspiration. The aerosol was collected on a filter and the mass of salbutamol measured by spectrophotometry. The spacer was placed either before the humidification chamber or immediately before the nasal cannula, and we assessed the influence of different HFT flow rates $(30,45$ and $60 \mathrm{~L} / \mathrm{min}$ ) and breathing patterns ("respiratory failure" with mean patient inspiratory flow of $45 \mathrm{~L} / \mathrm{min}$ vs. "quiet" breathing with a mean patient inspiratory flow of $15 \mathrm{~L} / \mathrm{min}$ ) on the delivered mass of salbutamol (respirable mass). Six experiments were performed for each condition tested.

Results Connection of the spacer before the humidification chamber was always poorly efficient, whatever the HFT flow rate used or respiratory pattern simulated, with masses collected ranging from 1.5 to $2.5 \%$ of the nominal dose. When the spacer was placed immediately upstream from the cannula, the masses collected were 11.8, 10.7 and $6.2 \%$ of the nominal dose for HFT flow rates of 30,45 and $60 \mathrm{~L} / \mathrm{min}$, respectively, with the quiet breathing setting. During simulation of respiratory distress, the amount of salbutamol collected on the filter was higher at the HFT flow rate of $30 \mathrm{~L} / \mathrm{min}(16.8 \mathrm{vs} .11 .8 \%)$ while it was lower at 45 and $60 \mathrm{~L} / \mathrm{min}$, compared to the quiet breathing pattern (respectively 4.4 vs. $10.7 \%$ and 3.3 vs. $6.2 \%$ ): Fig. 30 .

Conclusion Placing the spacer immediately upstream from the cannula was the most efficient configuration for pMDI salbutamol administration in an HFT circuit, actuation being synchronize with inspiration. The masses observed are likely to produce a therapeutic effect in the clinical setting, even in case of respiratory failure and high patients inspiratory flows. Indeed, an inspiratory flow matching the HFT may minimize aerosol leakage during inspiration. These findings may lay the foundations for the clinical evaluation of salbutamol delivery with a pMDI during HFT.

Competing interests None.
P91

Differences in perceptions between the various healthcare professionals working at the bedside of the risks related to the rehabilitation procedures

Laurent Poiroux ${ }^{1}$, David Thevoz ${ }^{2}$, Julien Simons ${ }^{2}$, Cheryl Hickmann ${ }^{3}$, Jean Roeseler ${ }^{3}$, Anthéa Loiez ${ }^{4}$, Stéphanie Gérard ${ }^{5}$, Nicolas Dousse ${ }^{6}$, Tai Pham? Nicolas Terzi ${ }^{8}$, Lise Piquilloud ${ }^{9}$

${ }^{1}$ Département de réanimation médicale et médecine hyperbare, $\mathrm{CHU}$ d'Angers, Angers, France; ${ }^{2}$ Service de physiothérapie cardio-respiratoire,

CHUV, Lausanne, Switzerland; ${ }^{3}$ Service des soins intensifs, Cliniques Univer-

Table 22 See text for description

\begin{tabular}{|c|c|c|c|}
\hline & Nurses (\%) & $\begin{array}{l}\text { Physiotherapists } \\
\text { (\%) }\end{array}$ & Physicians (\%) \\
\hline \multicolumn{4}{|l|}{$\begin{array}{l}\text { Intubation for less } \\
\text { than } 12 \mathrm{~h}\end{array}$} \\
\hline Passive (in bed) & 0 & 5 & 5 \\
\hline Out of bed & 39 & 37 & 26 \\
\hline \multicolumn{4}{|c|}{$\begin{array}{l}\text { Fraction of inspired } \\
\text { oxygen }\left(\mathrm{FIO}_{2}\right) \\
>0.6 \text { and/or } \mathrm{O}_{2} \\
\text { partial pressure } \\
\text { over } \mathrm{FIO}_{2} \text { ratio } \\
<150 \mathrm{mmHg}\end{array}$} \\
\hline Passive (in bed) & 5 & 5 & 5 \\
\hline Out of bed & 59 & 50 & 50 \\
\hline \multicolumn{4}{|l|}{$\begin{array}{l}\text { Respiratory rate at } \\
\text { rest }>30 / \mathrm{min}\end{array}$} \\
\hline Passive (in bed) & 0 & 0 & 5 \\
\hline Out of bed & 25 & 10 & 32 \\
\hline \multicolumn{4}{|c|}{$\begin{array}{l}\mathrm{O}_{2} \text { saturation }<88 \% \\
\text { at rest }\end{array}$} \\
\hline Passive (in bed) & 5 & 10 & 11 \\
\hline Out of bed & 53 & 21 & 44 \\
\hline \multicolumn{4}{|c|}{$\begin{array}{l}\text { Severe respiratory } \\
\text { acidosis }(\mathrm{pH}<7.2)\end{array}$} \\
\hline Passive (in bed) & 11 & 20 & 16 \\
\hline Out of bed & 71 & 56 & 74 \\
\hline \multicolumn{4}{|l|}{$\begin{array}{l}\text { Positive expiratory } \\
\text { pressure (PEEP) } \\
>10 \mathrm{cmH}_{2} \mathrm{O}\end{array}$} \\
\hline Passive (in bed) & 0 & 10 & 5 \\
\hline Out of bed & 76 & 25 & 53 \\
\hline \multicolumn{4}{|l|}{ Prone position } \\
\hline Passive (in bed) & 42 & 37 & 21 \\
\hline Out of bed & 100 & 100 & 100 \\
\hline \multicolumn{4}{|c|}{ Venous femoral line } \\
\hline Passive (in bed) & 0 & 0 & 0 \\
\hline Out of bed & 17 & 11 & 11 \\
\hline \multicolumn{4}{|l|}{$\begin{array}{l}\text { Dialysis catheter in } \\
\text { femoral position }\end{array}$} \\
\hline Passive (in bed) & 0 & 5 & 0 \\
\hline Out of bed & 29 & 22 & 17 \\
\hline \multicolumn{4}{|l|}{ Arterial femoral line } \\
\hline Passive (in bed) & 0 & 5 & 0 \\
\hline Out of bed & 33 & 16 & 11 \\
\hline
\end{tabular}


sitaires Saint-Luc, Bruxelles, Belgium; ${ }^{4}$ Département de réanimation médicale et de médecine hyperbare, C.H.U. d'Angers, Angers, France; ${ }^{5}$ Service de physiothérapie, Hôpital neuchâtelois, Neuchâtel, Switzerland; ${ }^{6}$ Service de soins intensifs, Hôpitaux Universitaires de Genève, Genève, Switzerland; ${ }^{7}$ Réanimation médico-chirurgicale, Hôpital Tenon, Paris, France; ${ }^{8}$ Service de réanimation médicale, Clinique de Réanimation Médicale, Grenoble, France; ${ }^{9}$ Service de médecine intensive adulte et centre des brûlés, CHUV Centre hospitalier universitaire vaudois, Lausanne, Switzerland

Correspondence: Laurent Poiroux - lapoiroux@chu-angers.fr Annals of Intensive Care 2016, 6(Suppl 1):P91

Introduction In intubated patients, early mobilization reduces the incidence of ICU-acquired weakness but also mechanical ventilation duration and length of stay both in the ICU and in the hospital. Even if a relative consensus about the definition of early mobilization has emerged (i.e., rehabilitation interventions that are initiated immediately after stabilization of physiologic derangements), there is no definite consensus about the safety criteria required to initiate the rehabilitation process in acutely ill patients. This is probably at least partly due to very different perceptions, particularly between the various healthcare professionals working at the bedside of the risks related to the rehabilitation procedures.

Materials and methods The aim of the study was to compare what the different members of the ICU multidisciplinary team consider as contraindications to perform rehabilitation in mechanically ventilated critically ill patients. Practically, as preliminary part of a multicenter interventional study on early mobilization, a questionnaire was sent to the referent nurse, physiotherapist and physician of each participating center. Each healthcare professional was asked to define whether passive or active mobilization, inside or outside the bed, could safely be performed in different clinical situations and with various ICU equipment. Each participant was asked to answer by "No" if the mobilization was contraindicated in the described situation and by "Yes" if the mobilization was possible.

Results Fifty-one questionnaires were analyzed (17 from nurses, 17 from physiotherapists and 17 from physicians). The percentages of "No" answers meaning that mobilization is contraindicated are reported in Table 22

Conclusion In general, passive mobilization is considered as safe and possible by all the healthcare professionals in the majority of the clinical situations described. Oppositely, when active mobilization outside the bed is considered, major differences in perception of mobilization associated safety emerged within the various healthcare professionals. In particular, we noticed that the physiotherapists were less afraid by poor respiratory conditions than nurses and doctors. On the other hand, the presence of an arterial femoral line or of a dialysis catheter in femoral position was mainly considered as a contraindication of mobilization outside the bed by the nurses.

\section{Competing interests None.}

\section{References}

1. Pohlman MC, Schweickert WD, PohIman AS, et al. Feasibility of physical and occupational therapy beginning from initiation of mechanical ventilation. Crit Care Med 2010;38(11):2089-2094.

2. Bourdin G, Barbier J, Burle JF, et al. The feasibility of early physical activity in intensive care unit patients: a prospective observational one-center study. Respir Care 2010;55(4):400-407.

\section{P92}

\section{Early rehabilitation in critically ill patients: a prospective} multicenter assessment of real-life practice

Lorraine Ducroux', Sylvie L'hotellier², Elodie Baumgarten ${ }^{3}$, Stéphanie Borschneck ${ }^{1}$, Francis Schneider ${ }^{4}$, Vincent Castelain

${ }^{1}$ Hautepierre réanimation médicale, C.H.R.U. Hôpitaux Universitaires Strasbourg, Strasbourg, France; ${ }^{2}$ Service de réanimation, C.H.R.U. Hôpitaux Universitaires Strasbourg, Strasbourg, France; ${ }^{3} 67000$, Cabinet libéral de masso kinésithérapie, Strasbourg, France; ${ }^{4}$ Réanimation médicale, C.H.R.U. Hôpitaux Universitaires Strasbourg, Strasbourg, France
Table 23 See text for description

\begin{tabular}{|c|c|c|c|}
\hline Cases & Rehabilitation & $\%$ & Limits \\
\hline \multirow{5}{*}{$\begin{array}{l}\text { Resolving coma, } \\
\text { tapering dose } \\
\text { of } \mathrm{NE} \text {, ongoing } \\
\text { respiratory } \\
\text { weaning }\end{array}$} & a. & $\begin{array}{l}\text { Often 3; rarely } \\
96.9\end{array}$ & \multirow[t]{10}{*}{$\begin{array}{l}\text { Consciousness: } \\
69.7 \% \text { presence } \\
\text { of low doses } \\
\text { of } \mathrm{NE}\end{array}$} \\
\hline & b. & 84.8 & \\
\hline & c. & 15.1 & \\
\hline & $\mathrm{d}$ & 6 & \\
\hline & e. & 0 & \\
\hline \multirow{5}{*}{$\begin{array}{l}\text { 2. Chronic } \\
\text { obstructive } \\
\text { pulmonary } \\
\text { disease on } \\
\text { noninvasive } \\
\text { ventilation } \\
(1 \mathrm{~h} / 3)\end{array}$} & a. & $\begin{array}{l}\text { Daily } 65.5 \text {; often } \\
34.5\end{array}$ & \\
\hline & b. & 89 & \\
\hline & c. & 75 & \\
\hline & $\mathrm{d}$ & 86 & \\
\hline & e. & 65 & \\
\hline \multirow{5}{*}{$\begin{array}{l}\text { 3. Acute on } \\
\text { chronic liver } \\
\text { failure with } \\
\text { tapering NE }\end{array}$} & a. & $\begin{array}{l}\text { Daily } 54.5 \text {; never } \\
45.5\end{array}$ & \multirow[t]{5}{*}{ Low-dose NE } \\
\hline & b. & 85 & \\
\hline & c. & 66 & \\
\hline & d. & 60 & \\
\hline & e. & 30 & \\
\hline \multirow{5}{*}{$\begin{array}{l}\text { 4. Respiratory } \\
\text { weaning } \\
\text { failure after } \\
\text { laparotomy } \\
\text { (many drains) }\end{array}$} & a. & $\begin{array}{l}\text { Infrequently } \\
66.6\end{array}$ & \multirow[t]{5}{*}{$\begin{array}{l}\text { Drains, lapa- } \\
\text { rotomy }\end{array}$} \\
\hline & b. & 96 & \\
\hline & c. & 59 & \\
\hline & d. & 39 & \\
\hline & e. & 15 & \\
\hline \multirow{5}{*}{$\begin{array}{l}\text { 5. ARDS under } \\
\text { MV (with seda- } \\
\text { tion + curare) }\end{array}$} & a. & 0 & \multirow[t]{5}{*}{ Curare } \\
\hline & b. & 44.5 & \\
\hline & $c$. & 0 & \\
\hline & $\mathrm{d}$ & 0 & \\
\hline & e. & 0 & \\
\hline
\end{tabular}

a: transferring from a flat bed to chair; $b$ : bed in armchair position; $c$ : transferring to edge of bed; $d$ : standing position; e: marching; NE: norepinephrine

Correspondence: Lorraine Ducroux - ducroux.lorraine@gmail.com Annals of Intensive Care 2016, 6(Suppl 1):P92

Introduction Early mobilization of critically ill patients is a major issue when it comes to ICU stay shortening for multiple organ failure patients. It is well accepted that "bed-in-chair" sitting, first chair sitting exercise, passive verticalization and walking are crucial steps to better rehabilitation in the ICU and after patients have left the ICU. We aimed at assessing the real-life conditions performance of early rehabilitation practices in ICUs and to study why there are some limitations to a large use of such training for patients.

Materials and methods A prospective telephone inquiry was performed from February to March 2015 in 35 ICU (25 from university hospitals), representing 1046 beds; it recorded data on ICUs and on the real-life practice of rehabilitation in the setting of 5 clinical cases (1 coma patient undergoing mechanical ventilation weaning yet he was on low tapering NE infusion, 1 ARDS, 1 COPD under noninvasive ventilation, 1 acute/chronic liver failure patient infused tapering doses of norepinephrine, 1 postoperative patient). For each case, staff was questioned on the practice of transferring from flatbed to chair; bed 
in armchair positioning; transferring to edge of bed; standing position; and marching. In addition, we collected the reasons for not practicing early mobilization.

Results One hundred percentage of responses were made by physiotherapists, $64.5 \%$ of which had less than 5 -year working experience. The median number of patients taken in care by one physiotherapist was 14 [range (3.5-52)], whereas simultaneously each nurse had 2.5 patients in charge, and each nurse auxiliary 4.2. Shift hours were on $12 \mathrm{~h}$ a-day in $58.8 \%$ of the ICUs (Table 23 ).

The main reasons for not proposing early rehabilitation are low consciousness level, NE infusion, curare infusion and the simultaneous presence of many surgical drains in the same patient.

Conclusion Despite low sedation, many critically ill patients are readily installed in a bed in armchair position because this position does not include danger and allows easy visual control. Postoperative patients with complicated surgical devices are not often proposed for early mobilization from bed: nurses and physiotherapists bring up the problem of understaffing. Patients with low doses (or tapering low doses) of NE are infrequently proposed for early mobilization; although there are no reliable data concerning the doseeffect relationship with negative side effects in such a population, a specific study is deserved to understand whether such patients would benefit from such rehabilitation in terms of shorter ICU stay and outcome.

\section{Competing interests None.}

\section{P93}

Extracorporeal membrane oxygenation

for pheochromocytoma-induced cardiogenic shock: report of nine cases

Guillaume Hékimian ${ }^{1}$, Nicolas Brechot ${ }^{1}$, Matthieu Schmidt ${ }^{1}$, Fatima Kharcha ${ }^{2}$, Cécile Ghander ${ }^{2}$, Guillaume Lebreton ${ }^{3}$, Christophe Tresallet ${ }^{4}$,
Jean Louis Trouillet', Pascal Leprince ${ }^{3}$, Alain Combes ${ }^{1}$, Charles-Edouard Luyt $^{1}$

'Service de réanimation médicale, Groupe Hospitalier Pitié Salpêtrière, Paris, France; ${ }^{2}$ Endocrinologie, Groupe Hospitalier Pitié-Salpêtrière, Paris, France; ${ }^{3}$ Chirurgie cardiaque, Hopital Pitie Salpetrière, Paris, France; ${ }^{4} \mathrm{Chir}-$ urgie digestive, Groupe Hospitalier Pitié-Salpêtrière, Paris, France

Correspondence: Guillaume Hékimian - g.hekimian@free.fr

Annals of Intensive Care 2016, 6(Suppl 1):P93

Introduction Pheochromocytoma is a rare catecholamine-producing tumor that might occasionally provoke a stress-induced takotsubo-like cardiomyopathy and severe cardiogenic shock. Since the recourse to veno-arterial extracorporeal membrane oxygenation (VA-ECMO) has rarely been reported in this setting, we reviewed the presentation and outcomes of patients admitted to our ICU for pheochromocytomainduced refractory cardiogenic shock that was rescued by VA-ECMO.

Patients and methods All cases of pheochromocytoma-induced refractory cardiogenic shock managed with VA-ECMO in our ICU between January 2007 and March 2015 were retrospectively reviewed. Results Nine patients were included, 7 women and two men, aged 31-51 years (median: 43, IQR [36-49]). None of them were previously known to suffer pheochromocytoma. The medical history of these patients was unremarkable except for 4 of them: one had isolated hypertension, one had chronic headache, one had a type 1 multiple endocrine neoplasia syndrome and another reported clinical symptoms suggestive of the disease in the last 12 months. At hospital admission, 3 patients had hypertensive crisis and required antihypertensive therapy, 3 had chest pain mimicking acute coronary syndrome, five had dyspnea related to congestive heart failure. For two patients, a surgical triggering factor (hysterectomy and hernia surgery) was identified with hemodynamic instability and acute pulmonary edema during anesthesia. One patient had cardiac arrest (Ventricular Fibrillation) before admission. All 9 patients rapidly developed refractory cardiogenic shock with very severe left ventricular impairment (left ventricular ejection fraction

Table 24 See text for description

\begin{tabular}{|c|c|c|c|c|c|}
\hline & $\begin{array}{l}1997-2000 \\
(n=3248)\end{array}$ & $\begin{array}{l}2001-2004 \\
(n=4602)\end{array}$ & $\begin{array}{l}2005-2008 \\
(n=5179)\end{array}$ & $\begin{array}{l}2009-2012 \\
(n=6387)\end{array}$ & $\begin{array}{l}\text { Percentage change } \\
\text { from } 1997 \text { to } 2012 \\
(95 \% \mathrm{Cl})\end{array}$ \\
\hline Age, mean (SD) (years) & $66.4 \pm 16.1$ & $65.1 \pm 16.5$ & $63.6 \pm 16.9$ & $63.7 \pm 16.6$ & $-2.7(-2.0 \text { to }-3.4)^{*}$ \\
\hline Men & $1977(61)$ & $2877(62.5)$ & $3315(64)$ & $4152(65)$ & $-4.0(-6.0 \text { to }-1.9)^{*}$ \\
\hline SAPS $\|$ & $58.7 \pm 25.3$ & $59.3 \pm 24.7$ & $63.8 \pm 23.7$ & $64.5 \pm 23.3$ & $5.8(4.8 \text { to } 6.8)^{*}$ \\
\hline Acute respiratory failure & $1149(35)$ & $2175(53)$ & $2984(58)$ & $3410(53)$ & $18.0(16.0 \text { to } 20.1)^{*}$ \\
\hline Coma & $506(16)$ & $1181(26)$ & $1631(31.5)$ & $1673(26)$ & $10.6(8.9 \text { to } 12.2)^{*}$ \\
\hline Acute renal failure & $1039(32)$ & $1936(42)$ & $2807(54)$ & $3468(54)$ & $22.3(20.3 \text { to } 24.3)^{*}$ \\
\hline Sepsis & $649(20)$ & $1135(25)$ & $1552(30)$ & $1815(28)$ & $8.4(6.7 \text { to } 10.2)^{*}$ \\
\hline Cardiac arrest & $870(27)$ & $1400(30)$ & $1721(33)$ & $2142(33.5)$ & $6.8(4.8 \text { to } 8.7)^{*}$ \\
\hline Acute myocardial infarction & $771(24)$ & $1018(22)$ & $1052(20)$ & $1167(18)$ & $-5.5(-7.2 \text { to }-3.7)^{*}$ \\
\hline Drug intoxications & $160(5)$ & $230(5)$ & $265(5)$ & $269(4)$ & $-0.7(-1.6 \text { to } 0.2)^{*}$ \\
\hline Pulmonary embolism & $220(7)$ & $240(5)$ & $244(5)$ & $304(5)$ & $-2.0(-3.1 \text { to }-1.0)^{*}$ \\
\hline \multicolumn{6}{|l|}{ In-hospital management } \\
\hline Invasive ventilation & $2377(73)$ & $3446(75)$ & $3987(77)$ & $4765(75)$ & $1.4(-0.4 \text { to } 3.3)^{*}$ \\
\hline Circulatory support & $2895(89)$ & $4052(88)$ & $4172(81)$ & $5096(80)$ & $-9.3(-10.8 \text { to }-7.9)^{*}$ \\
\hline Renal support & $466(14)$ & $924(20)$ & $1342(26)$ & $1709(27)$ & $12.4(10.8 \text { to } 14.0)^{*}$ \\
\hline In-CCU Death & $1635(50)$ & $2294(50)$ & $2416(46.5)$ & $2860(45)$ & $-5.6(-7.7 \text { to }-3.5)^{*}$ \\
\hline Outcomes in survival patients & $n=1613$ & $n=2308$ & $n=2763$ & $n=3527$ & \\
\hline Discharge at home & $63(4)$ & $107(5)$ & $103(4)$ & $78(2)$ & $-0.7(-1.3 \text { to }-0.2)^{*}$ \\
\hline To another hospital & $431(27)$ & $567(24)$ & $628(23)$ & $733(21)$ & $-1.8(-3.2 \text { to }-0.4)^{*}$ \\
\hline Intrahospital transfer & $1114(69)$ & $1633(71)$ & $2009(73)$ & $2716(77)$ & $8.3(6.2 \text { to } 10.3)^{*}$ \\
\hline
\end{tabular}

* $P<0.001$ 
ranged from 5 to $20 \%$, left ventricular outflow tract velocity time integral ranged from 3 to $8 \mathrm{~cm}$ ). Pheochromocytoma was highly suspected on abdominal CT scan showing adrenal gland tumor in 7 cases (the two others did not have $\mathrm{CT}$ scan during ICU stay). Three patients died from refractory cardiogenic shock despite ECMO implantation. For the 6 others, myocardial function improved and ECMO was removed 3-7 days after initiation. Alpha and beta blockers were progressively introduced after ECMO removal. Five survivors had adrenalectomy 6 weeks after ICU discharge and had satisfactory outcome. In one patient, pheochromocytoma was diagnosed 1 year after the index event and surgical adrenalectomy was performed without complication.

Conclusion Pheochromocytoma is a rare but reversible cause of cardiogenic shock that might be rescued by VA-ECMO. Radiographic imaging with echography and CT scan of the adrenal gland should be obtained in all patients with unexplained cardiogenic shock. Lastly, adrenalectomy might be safely performed in the weeks following the initial catastrophic presentation, after complete recovery of LV systolic function.

\section{Competing interests None.}

\section{P94}

Mortality related to cardiogenic shock in critically ill patients in France, 1997-2012

Etienne Puymirat', Jean-Yves Fagon², Philippe Aegerter ${ }^{3}$, Florence

Boissier $^{4}$, Jean-Luc Diehl ${ }^{2}$, Caroline Hauw-Berlemont ${ }^{2}$, Bertrand Guidet ${ }^{5}$, Gilles Chatellier ${ }^{6}$, Nicolas Danchin ${ }^{7}$, Nadia Aissaoui ${ }^{2}$

${ }^{1}$ Cardiology, Hôpital Européen Georges-Pompidou, Paris, France; ${ }^{2}$ Réanimation médicale, Hopital Europeen Georges-Pompidou, Paris, France;

${ }^{3}$ Urc, Hospital Ambroise Paré, Boulogne-Billancourt, France; ${ }^{4}$ Réanimation Médicale, Hôpital Henri Mondor, Créteil, France; ${ }^{5}$ Réanimation Médicale, Hôpital Saint-Antoine, Paris, France; ${ }^{6}$ Unité de recherche clinique, Hopital Europeen Georges-Pompidou, Paris, France; ${ }^{7}$ Cardiologie, Hopital Europeen Georges-Pompidou, Paris, France

Correspondence: Nadia Aissaoui - nadia.aissaoui@egp.aphp.fr Annals of Intensive Care 2016, 6(Suppl 1):P94

Introduction There is a lack of data concerning the characteristics, the outcome and temporal trends in mortality of cardiogenic shock (CS) patients admitted in critical care unit (CCU). Accordingly, we provide an extended 15 years long perspective into changing trends in key features of patients, use of life-supporting therapies and in-CCU death rates.

Patients and methods We conducted a retrospective study using prospectively collected data from the 1997-2012 database of Parisian area critical care units-the CubRea (Intensive Care Database User Group). We assessed temporal trends in the use of life-supporting therapies and patient outcomes (Crude and SAPS II-Standardized Mortality, discharge location).

Results Among 316,905 patients admitted in CCUs, 19, 416 (6.1\%) exhibited CS, with an increasing incidence between 1997 and 2012 from 4.1 to $7.7 \%, P<0.001$. Baseline characteristics of patients according to the study period are reported in Table 24 . Over time, the age of CS patients decreased $66.4 \pm 16.1-63.7 \pm 16.6, P<0.001$ and mean SAPS II increased from $58.7 \pm 25.3-64.5 \pm 23.3, P<0.001$. Use of lifesupporting therapies increased with increase in renal replacement therapy by 12.4 percentage points $(95 \% \mathrm{Cl} \mathrm{10.8-14.0)}$ and of invasive ventilation by 1.4 percentage points $(95 \% \mathrm{Cl}-0.4$ to 3.3 ). However, crude in-CCU mortality declined from 50 to $45 \%, P<0.001$. SAPS IIStandardized in-CCU mortality rates also declined from 56.5 to $44.2 \%$, $P<0.001$. Among survivors, the numbers of patients discharged at home was low and decreased from 4 to $2 \%, P<0.001$.

Conclusion In France, the profile of CS patients admitting in CCUs changed over a 15-year study period. From 1997 to 2012, CS patients are younger but with a higher severity and need for respiratory and renal support. Yet, both crude and SAPS II-Standardized Mortality decreased, suggesting an improvement in the management and prognosis.

Competing interests None.
P95

Benefits of extracorporeal life support (ECLS) in elderly patients with postcardiotomy refractory cardiogenic shock

Nadia Aissat', R. Bernard', Guillaume Lebreton², Dimitri Margetis', Julien Amour $^{3}$, Pascal Leprince ${ }^{4}$, Adrien Bouglé ${ }^{1}$

${ }^{1}$ Ile de France, Pitié-Salpêtrière Hospita, ICAN, Paris, France; ${ }^{2}$ Service de chirurgie thoracique et cardiovasculaire, Groupe Hospitalier Pitié Salpêtrière, Paris, France; ${ }^{3}$ Dar, Groupe Hospitalier Pitié Salpêtrière, Paris, France; ${ }^{4}$ Chirurgie cardiaque, Hopital Pitie Salpetrière, Paris, France

Correspondence: Nadia Aissat - nadia_aissat@yahoo.fr Annals of Intensive Care 2016, 6(Suppl 1):P95

Introduction Because of the sanitary and social progress, proportion of elderly patients undergoing cardiac surgery constantly increases. Nevertheless, in case of postcardiotomy refractory cardiogenic shock, the need for extracorporeal life support (ECLS) is questionable because of the lack of survey data in this population. The aim of this study was to investigate the 28-day mortality after cardiac surgery in patient $\geq 80$ years old with or without ECLS.

Materials and methods In a monocentric retrospective study, we included all patients $\geq 80$ years old undergoing on pump cardiac surgery between January 2013 and December 2014. Primary endpoint was the 28-day mortality. Data are expressed in median (25th-75th quartile) or percentage. Fisher's test for qualitative variable or Wilcoxon's test for quantitative analysis was used.

Results We included 225 consecutive patients. The median age was $82(81-85)$ with $62.5 \%$ of men. The Euroscore 2 was $9.1 \%$ (7.1-10.8). ECLS was needed in $8.7 \%$ of cases $(n=20)$. In control group, without ECLS, median age was $82(81-85)$, the Euroscore 2 was $4 \%(3-9)$, the CPB duration was 84 (86-106) min, the initial SAPS2 was 37 (27-49), initial SOFA score was $5(3-6)$, and postoperative mechanical ventilation duration was $0(0-1)$ days. In ECLS group, the median age was 83 $(82-84)(p=0.74)$. The Euroscore2 was higher, $13 \%(4-25)(p=0.007)$. Both SAPS2 $64(52-73)(p<0.0001)$ and initial SOFA score increased [8 (6-9), $p=0.0001]$. Postoperative mechanical ventilation duration increased (5.5 (2.0-11.0) days, $p<0.0001)$. In ECMO group, in-hospital mortality ( 80 vs $7 \%$ ), 28 -day mortality ( 85 vs $11 \%$ ) and 6-month mortality ( 85 vs $15 \%)$ increased significantly in comparison with control group ( $p<0.0001$ for all).

Conclusion In elderly patients undergoing on pump cardiac surgery, refractory cardiogenic shock is associated with high morbidity and mortality. Nevertheless, in this particular severe cohort, ECLS seems to offer a valuable alternative and could be beneficial in some selected patients.

\section{Competing interests None.}

\section{P96}

Heart transplantation versus ventricular assist device: which therapy for patients with refractory cardiogenic shock? Charles Vidal', Guillaume Lebreton², Djavidi Nima' ${ }^{1}$, Varnous Shaida² Adrien Bouglé ${ }^{1}$, Pascal Leprince ${ }^{2}$, Julien Amour ${ }^{1}$

${ }^{1}$ Réanimation chirurgicale cardio-vasculaire et thoracique, Groupe Hospitalier Pitié-Salpêtrière, Paris, France; ${ }^{2}$ Service de chirurgie cardio-vasculaire et thoracique, Groupe Hospitalier Pitié-Sal pêtrière, Paris, France

Correspondence: Charles Vidal - charlesvidal@orange.fr

Annals of Intensive Care 2016, 6(Suppl 1):P96

Introduction Since 2004, patients needing heart transplantation in emergency benefit from national prioritization "super urgency 1" (SU1) for graft attribution. The efficiency of this new modality of access to transplantation in emergency is uncertain. Several transplantation teams report increased transplantation survey in patients assisted previously with ventricular device (VAD). The aim of this work was to compare the survey of patients in SU1 who were transplanted during this period in comparison with patients who failed 
to be transplanted and who benefited of VAD treatment in bridge to transplantation.

Patients and methods This monocentric retrospective study included all the patients in SU1 between January 1, 2008, and June 30, 2013. Primary endpoint was the hospital mortality after either transplantation or VAD treatment. The second endpoint was the 1-year mortality. Data are expressed to median (25th-75th quartile) and percentage. Groups were compared using Mann-Whitney and Fischer tests.

Results During the period study, 227 patients were included in SU1 program. In total, 162 patients received a cardiac graft and 65 failed to be transplanted during the SU1 period. In failed attempt group, 33 were treated with VAD therapy [22 biventricular assist device (BiVAD) and 11 left ventricular assist device (LVAD)], 15 patients died without transplantation or any other device and 15 patients were excluded of the study because they were transplanted after the "SU1" period. One patient survived without any transplantation or device at 1 year. Between transplanted and VAD groups, there was no difference in terms of age [51 (40-59) vs 49 (40-59) years, $p=0.82$ ], preoperative extracorporeal life support (38 vs $36 \%$, $p=1.0$ ) or SOFA score at the time of heart transplantation or VAD implantation [5 (4-6) vs 5 (4-6), $p=0.19$ ]. There was more male gender in the VAD group than in the transplant group ( 97 vs $82 \%$, $p=0.03$ ). Both hospital mortality and 1-year mortality were lower in the transplant group than the VAD group, $23 \%$ versus $55 \%$ $(p<0.001$, OR $0.25[0.11-0.58])$ and $25 \%$ versus $61 \%(p<0.001$, OR 0.22 [0.09-0.50]) respectively. Mortality of patients assisted by BiVAD tended to be more important than patients assisted by LVAD [72 vs $36 \%, p=0.06]$. After 1 year, all survivors pretreated with LVAD $(n=11)$ were hundred percent successful transplanted after transplantation.

Conclusion Heart transplantation is the best treatment in SU1 conditions for patients with refractory cardiogenic shock. In case of failed attempt in SU1, VAD implantation needs meticulous revaluation in particular of BiVAD because of high mortality.

\section{Competing interests None.}

\section{Reference}

1. Attisani M, Centofanti P, Ribezzo M, et al. Advanced heart failure in critical patients (INTERMACS 1 and 2 levels): Ventricular assist devices or emergency transplantation? Interact cardiovasc thorac surg; Oct 2012.

\section{P97}

Refractory cardiogenic shock in immunocompromised patients rescued by veno-arterial extracorporeal membrane oxygenation Amandine Dorget ${ }^{1}$, Nicolas Bréchot ${ }^{1}$, Guillaume Lebreton², Guillaume Hekimiann ${ }^{1}$, Ania Nieszkowska' ${ }^{1}$, Charles-Edouard Luyt ${ }^{1}$, Pascal Leprince ${ }^{2}$, Jean Louis Trouillet ${ }^{1}$, Alain Combes ${ }^{1}$, Matthieu Schmidt ${ }^{1}$

'Medical intensive care unit, ican, institute of cardiometabolism and nutrition, Groupe Hospitalier Pitié-Salpêtrière, Paris, France; ${ }^{2}$ Cardiac and thoracic surgery, ican, institute of cardiometabolism and nutrition, Groupe Hospitalier Pitié-Salpêtrière, Paris, France

Correspondence: Amandine Dorget - amandedorget@gmail.com Annals of Intensive Care 2016, 6(Suppl 1):P97

Introduction Veno-arterial extracorporeal membrane oxygenation (VA-ECMO) is used as a salvage therapy for refractory cardiogenic shock. Since major improvement in the prognosis of immunocompromised patients admitted to the intensive care unit (ICU) has been reported in recent years, our objective was to analyze short- and longterm outcomes of immunocompromised refractory cardiogenic shock patients supported by VA-ECMO.

Patients and methods We retrospectively collected demographic, admission severity scores, pre- and post-ECMO data, ECMO-related complications, and 6-month survival of immunocompromised patients who received VA-ECMO for refractory cardiogenic shock in our 26-bed intensive care unit (ICU) from June 2006 to June 2015. Immunocompromised status was defined as hematological malignancy, solid cancer $<5$ years, solid organ transplant, human immunodeficiency virus (HIV) infection, long-term corticosteroid or immunosuppressive therapies. Patients who received ECMO within 1 year after cardiac transplantation or after left ventricular assist device were excluded from this cohort. Patients who survived were contacted in September 2015 and assessed for health-related quality of life, psychological and post-traumatic stress disorder status.

Results Eighty-two immunocompromised patients were included (median age 43 years, [interquartile range 30-60]; $54 \%$ male, Simplified Acute Physiology Score II (SAPS II) 72 [55-89]). Reasons for immunodeficiency were hematological malignancies $(n=17)$, solid cancers $(n=14)$, HIV $(n=8)$, solid organ transplantation $(n=17)$ and autoimmune connective tissue disorders or vasculitis or long-term corticosteroid therapy $(n=26)$. Main causes of cardiogenic shock were myocarditis $(n=24)$, dilated cardiomyopathy $(n=12)$, acute myocardial infarction $(n=10)$, septic shock $(n=8)$, cardiac graft rejection $(n=8)$ or post-cardiotomy $(n=5)$. Twenty-nine patients $(35 \%)$ had pre-ECMO cardiac arrest, 11 of them (13\%) were cannulated during cardiopulmonary resuscitation. Median IGS II score at admission and mean SOFA score at cannulation were, respectively, 72 [IQR 55-89] and $12 \pm 5$. Pre-ECMO left ventricle ejection fraction (LVEF), $\mathrm{pH}$, blood lactate were, respectively, $10 \%$ [5-18], 7.27 [6.99-7.43] and 10 [7-15] $\mathrm{mmol} / \mathrm{L}$. Severe hemorrhage and at least one episode of nosocomial infection were reported in, respectively, 34 and $50 \%$ patients with no impact on survival. Six-month survival was $32 \%$ after, respectively, 7 days [5-14] on ECMO and a length of stay of 26 days [15-33] in ICU and 57 days [33-74] in hospital. Independent factors associated with 6-month mortality according to logistic regression analysis were SAPS II score > 65 (OR 4.26 [95\% Cl 1.03-17.62]) and LVEF < $10 \%$ at the time of cannulation (OR 8.14 [95 \% Cl 2.15-62.33]). On the other hand, autoimmune connective tissue disorders or vasculitis or long-term corticosteroid therapy seemed to be protective (OR 0.11 [95 \% Cl 0.02-0.61]). Lastly, 34 months [23-55] after ICU discharge, no dependency for the performance of daily living activities was reported. However, compared with age- and sex-matched controls, our ten responding immunocompromised survivors had significantly lower SF-36 physical domain scores and role emotional component $(p<0.001)$.

Conclusion Despite extreme disease severity and major underlying comorbidities, we observed that $32 \%$ of immunocompromised patients rescued with VA-ECMO for refractory cardiogenic shock were alive at 6 months with acceptable long-term quality of life. Higher severity score and profound cardiac impairment at ECMO initiation were independently associated with poor outcomes. Although survival was less in this group of patients than in non-immunocompromised patients, recourse to this salvage therapy should not be denied based only on this pre-morbid condition. Lastly, the type of immunosuppression might also impact on the outcomes of these patients.

Competing interests None.

\section{P98}

Which index severity to use in obstetric medium? (Comparison of 4 scores)

Rabi Toufiki ${ }^{1}$, Youssef Mouaffak', Ahmed Rhassan El Adib ${ }^{1}$, M. Amine ${ }^{2}$ ${ }^{1}$ Service Anaesthesia and Intensive Care Hospital Mother - Child, CHU Marrakech, Marrakech, Morocco; ${ }^{2}$ Clinical Laboratory of Epidemiology, $\mathrm{CHU}$ Marrakech, Marrakech, Morocco

Correspondence: Rabi Toufiki - toufiki.rabi@yahoo.fr

Annals of Intensive Care 2016, 6(Suppl 1):P98

Introduction Maternal mortality constitutes an indicator impossible to circumvent the development in any country and its monitoring remains fully of news. So evaluating the risk of mortality and reducing it to a certain number of scores of gravity were developed in the intensive care units, but their application in obstetric medium remains discussed.

We aimed in this study to check and to compare the capacity of forecast of four scores of gravity SAPS II, APACHE II, OSF and SAPSO among allowed parturient in the gynecologist-obstetric intensive care unit in order to be able to determine the most relevant score of gravity to use for the obstetric population of our context. 
Materials and methods Four hundred and forty-eight patients were studied prospectively the beginning of January until the end of December 2013. The four scores were calculated during the first $24 \mathrm{~h}$. The comparison of the variables called on Student's $t$ test and Chi square $(p<0.05)$ and with a multivariate analysis. We compared discrimination and the calibration, respectively, by the sectors under curve ROC (AUC ROC) and standardized mortality ratios (SMR) calculated for each score.

Results Four predictive factors independent of mortality were found: score of Glasgow, creatininemy, diastolic blood pressure and rate of plates.

Discrimination was excellent for the four scores AUR ROC $>0.75$.

The SAPS II had an overall good calibration $(S M R=1171)$. APACHE II and the OSF over-estimate mortality, APACHE II and the OSF over-estimate mortality, with a better calibration of the OSF (SMR, respectively, 0.454 and 0.771 ), and the SAPSO underestimates mortality in our context $(S M R=2173)$.

Conclusion Severity scores are applicable in our context. They provide important information on the severity of patients and allow people to categorize in order to predict their evolution.

Competing interests None.

\section{References}

1. T0245/2005: -Validation a severity score-OBST SAPS (simplified acute physiology score adapté to obstetric patien) in obstetric intensive care unit of 541 cases.

2. Le Gall J, Alberti C. Severity Ratings and applications in intensive care. Medico-Surgical Encyclopaedia 36-700-A-10

\section{P99}

Maternal mortality in obstetric department of UH IBN ROCHD, Casablanca, Morocco

Kamal Belkadi', I. Moussaid', Chaigar Mohammed Cheikh', S. Moumine', S. El Youssoufi', S. Salmi ${ }^{1}$

${ }^{1}$ Anesthesie reanimation, $\mathrm{CHU}$ Ibn Rochd Casa, Casablanca, Morocco Correspondence: Chaigar Mohammed Cheikh - chaigarmed@gmail. com

Annals of Intensive Care 2016, 6(Suppl 1):P99

Introduction Maternal mortality (MM) remains a real problem of public health worldwide and stills recognized as an indicator of care's quality in obstetrics; it reflects risks incurred by mothers during pregnancy and delivery. According to the WHO, the maternal mortality is defined as the death of a pregnant woman or within 42 days of ending pregnancy, from any cause related to pregnancy or aggravated by it or its management but not from accidental or incidental causes (1). The aim of this study was to specify the incidence of the MM in our hospital and to analyze its epidemiological factors and causes for suggesting ways of its prevention.

Patients and methods Retrospective study spread of 5 years, from January 2010 to December 2014, including all maternal deaths in obstetric department of our hospital, with epidemiological analysis of risk factors and mortality causes.

Results In this period, 130 cases of maternal deaths have been collected among 43,735 living births let a rate of MM about 297/100,000 living births. The main causes of those maternal deaths are direct obstetric ones in $80 \%$, including obstetrical hemorrhage $(35.38 \%)$ and hypertensive diseases (28. $46 \%$ ). The average age of our patients was 31.34 years, with a peak frequency recorded in the age range of 35 years and more $(34 \%)$. The primipars $(36.15 \%)$ and the paucipare $(36.92 \%)$ were the most affected. Most deaths (53.05\%) carried their pregnancies to term; only $19 \%$ were followed in prenatal consultation. The rate of parturient admitted directly from home to the UH was $40 \%$, while $60 \%$ of women passed by another before $\mathrm{UH}$, especially peripheral hospitals ( $37 \%)$. The rate of patients given birth by caesarian is superior to those given birth by low way, with 60.77 and $31.54 \%$, respectively. Most patients were admitted in severe status, $37.69 \%$ of the deaths occurred less than $24 \mathrm{~h}$ after admission, while $19.23 \%$ of patients died after 7 days of resuscitation's care.
Conclusion MM rate obtained in our study (297/100000LB) is overestimated comparing to actual rate of MM in our country, because $\mathrm{UH}$ is a referral center and tends to receive serious and complicated cases with higher risk of death. To reach the objective of the WHO telling that all women over the world have the right of not to die while procreating, it seemed urgent to mobilize communities for education and information of the women to get better outcome of pregnancy, as well as an engagement from government and healthcare professionals, associated with an adequate equipment of the maternities and optimize transportation covering the whole population.

Competing interests None.

Reference

1. WHO. Maternal mortality fact sheet N 348 updated May2014.

P100

Disseminated intravascular coagulation in pregnant women: evaluation of a new score

Marie Jonard', Anne Sophie Ducloy-Bouthors ${ }^{2}$, François Fourrier ${ }^{3}$

${ }^{1}$ Nord, centre de réanimation, Lille, France; ${ }^{2}$ Anesthésie obstétricale, Hos-

pital Jeanne De Flandre, Lille, France; ${ }^{3}$ Réanimation, centre de réanimation, Lille, France

Correspondence: Marie Jonard - marie.jonard@wanadoo.fr

Annals of Intensive Care 2016, 6(Suppl 1):P100

Introduction Many studies have shown a good correlation between the value of the International Society of Thrombosis and Hemostasis (ISTH) score, diagnosis of severe coagulopathy and mortality, especially during severe sepsis and polytrauma. However, physiological changes in hemostasis during pregnancy probably alter the significant thresholds of the ISTH score compromising the application and interpretation of it for the diagnosis of disseminated intravascular coagulopathy (DIC). The morbidity and mortality associated with severe hemorrhage and consumption coagulopathy leading to DIC during pregnancy emphasize the need for the adjustment of this ISTH DIC score to these patients On this basis, an Israeli team recently created from a large retrospective database study ( $n=24,693$ deliveries), a score of DIC. The components and the coagulation parameter thresholds were determined according to the physiological changes of the coagulation of pregnant women.

Primary objective of the study was to test a new scoring system for diagnosing disseminated intravascular coagulopathy (DIC), which is adapted to hemostasis changes in pregnant women and to compare this new score to the International Society of Thrombosis and Hemostasis (ISTH) score. Secondary objectives were to explain differences in diagnostic predictive value between the two scores and to assess DIC and its evolution of DIC according to the main obstetrics diseases causing ICU admission.

Materials and methods This is a population-based retrospective study of 154 patients admitted for severe postpartum complications from 2008 to 2014 at intensive care center in Lille University Hospital. The new score (based on 3 components: platelet count, prothrombin time difference and fibrinogen) and the ISTH score (based on 4 components: platelet count, fibrinogen, prothrombin time, and fibrin-related marker) were calculated from biological data on the day of delivery and repeated at ICU admission (day 0) until second day included (day 2) with reference to the consensus diagnostic analysis by two experts. The sensitivity, specificity, and area under the curve (AUC) for each score were calculated for each time and overall by generalized linear mixed model. The agreement between the two scores was evaluated with the Kappa coefficient.

Results The new score allowed us to make the diagnosis of DIC with a sensitivity of 0.78 , a specificity of $0.97(p<0.01)$, and total AUC of $96 \%$, while the ISTH score had a sensitivity of 0.31 , a specificity of 0.99 and an AUC of $94 \%(p<0.01)$. Their Kappa correlation coefficient was 0.35 . The discriminating thresholds of the components of the new score had a good correlation with the expert analysis regarding the difference in prothrombin time and fibrinogen while the platelet count threshold was less adapted. Analysis of ISTH score thresholds showed a high prevalence of DIC for high-weighted thresholds and also a 


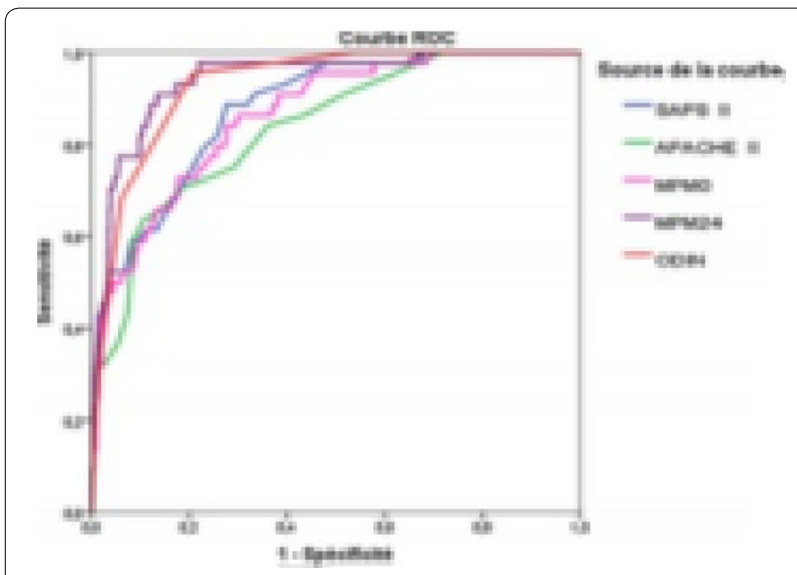

Fig. 31 ROC curves showing the correlation between SAPS II, APACHE II, MPM II 0, MPM II 24 and ODIN and mortality (area under the respective curves: $0.88,0.84,0.86,0.94$ and 0.93 )

significant prevalence for the thresholds considered as not markers of DIC. DIC complicated mostly acute fatty liver of pregnancy (AFLP) and postpartum hemorrhage and especially if it was associated with HELLP syndrome. The correction of the DIC was faster in the postpartum hemorrhage compared with preeclampsia, HELLP syndrome or AFLP. Conclusion The ISTH score should not be used in pregnant women because of its poor sensitivity. The new score established from the physiological changes of pregnancy seems highly discriminating and it assesses the evolution of DIC and the effects of its treatment.

\section{Competing interests None.}

\section{References}

1. Erez. O., Novack. L., Beer-Weisel. R., et coll. DIC score in pregnant womena population based modification of the International Society on Thrombosis and Hemostasis score. PloS One. 2014; 9:93240.

2. Rattray. DD., O'Connell. CM., Baskett. TF. Acute disseminated intravascular coagulation in obstetrics: a tertiary centre population review (19802009). J Obstétrique Gynécologie Can JOGC. 2012; 34:341-7.

\section{P101}

\section{Validity of severity scores in critically ill obstetric patients}

Fatma Kaaniche Medhioub ${ }^{1}$, Anis Chaari'2, Olfa Turki ${ }^{3}$, Kais Rgieg ${ }^{3}$, Mabrouk Bahloul2 ${ }^{2}$, Hmida Chokri Ben², Bouaziz Mounir ${ }^{2}$

${ }^{1}$ Faculté de médecine de Sfax, Sfax, Tunisia; ${ }^{2}$ Réanimation polyvalente, Faculté de médecine de Sfax, Sfax, Tunisia; ${ }^{3}$ Réanimation polyvalente, Chu habib bourguiba, Sfax, Tunisia

Correspondence: Fatma Kaaniche Medhioub - fatma_kaaniche@yahoo. $\mathrm{fr}$

Annals of Intensive Care 2016, 6(Suppl 1):P101

Introduction The severity scores have been developed in order to assess the severity of patients in the ICU and predict their subsequent evolution and prognosis [1]. Obstetric patients are a particular subgroup for which the interest of these scores is understudied. The objective of our study was to evaluate the interest of severity scores SAPS II, APACHE II, MPMII 0, MPMII 24 and ODIN in obstetric patients admitted to ICU.

Patients and methods $A$ retrospective study includes all patients admitted in ICU for 20 years (1993-2012). The discriminatory power was investigated by ROC curve method. The calibration was based on comparing the observed mortality (OM) and the predicted mortality $(M P R)(S M R=O M / P M R)$ and verified by the Hosmer-Lemeshow $(\mathrm{H})$ goodness-of-fit test.

Results Seven hundred and twenty-five patients were included. The average age was $31.2 \pm 6.4$ years. On admission, 124 patients $(17.1 \%)$ were in shock. Ninety-four patients $(13 \%)$ had the acute pulmonary edema. The acute renal failure was observed in 367 patients (50.6\%). Two hundred and one patients $(27.7 \%)$ had intravenous disseminate coagulation. Eighty-five patients (11.7\%) had disorders of consciousness. All these systemic complications were significantly associated with high mortality. The childbirth was by cesarean section in 462 patients $(63.7 \%)$. Mechanical ventilation for more than $24 \mathrm{~h}$ was performed in 233 women (32.1\%). The average stay of our patients was $2.8 \pm 4.2$ days. Mortality has affected 44 patients (6\%).

The areas under the ROC curve for SAPS II, APACHE II, MPM II 0, MPM II 24 and ODIN were, respectively, $0.8895 \% \mathrm{Cl}[0.82 ; 0.93], 0.8495 \% \mathrm{Cl}$ [0.77; 0.89], $0.8695 \% \mathrm{Cl}[0.81 ; 0.91], 0.9495 \% \mathrm{Cl}$ [0.90; 0.91$]$ and 0.93 $95 \%$ CI $0.90 ; 0.95$ ] (Fig. 31). SMR was close to 1 for SAPS II and MPM II 24 scores (respectively, 0.90 and 0.97 ), whereas it was $0.53,0.54$ and 0.6 , respectively, for APACHE II, MPM II 0 and ODIN scores. The value of $\mathrm{H}$ was $13.65(P>0.05)$ and $2.14(P>0.05)$, respectively, for scores SAPS II and MPM II 24, while it was $32.48(P<0.001), 32.47(P<0.001)$ and $37.56(P<0.001)$, respectively, for scores APACHE II, MPM II 0 and ODIN. Conclusion For each of the 5 studied scores, we found an excellent discriminatory power in terms of mortality. SAPS II and MPM II 24 had a perfect calibration to predict satisfactorily the probability of death. Other scores APACHE II, MPM II 0 and ODIN give an overestimation of the mortality.

\section{Competing interests None.}

\section{Reference}

1. Bertrand Guidet, Philippe Aegerter. Indices de gravité et applications en réanimation. Le Praticien en Anesthésie Réanimation 2009; 13: 6-18.

P102

Evaluation of a neuromuscular blockade with benzylisoquinoliniums: a comparison of a train of four with a clinical assessment

Pierre Bouju ${ }^{1}$, Nicolas Barbarot ${ }^{2}$, Arnaud Gacouin ${ }^{1}$, Fabrice Uhel ${ }^{1}$, Julien Letheulle ${ }^{1}$, Pierre Fillatre ${ }^{1}$, Guillaume Grillet ${ }^{1}$, Angélique Goepp ${ }^{2}$ Jean-Marc Tadié ${ }^{1}$, Yves Le Tulzo ${ }^{1}$

${ }^{1}$ Service de maladies infectieuses et réanimation médicale, Centre Hospitalier Universitaire, Rennes, France; ${ }^{2}$ Service de réanimation polyvalente,

Centre hospitalier, Saint Brieuc, France

Correspondence: Pierre Bouju - pierre.bouju@chu-rennes.fr

Annals of Intensive Care 2016, 6(Suppl 1):P102

Introduction Despite few studies, a monitoring of a neuromuscular blockade with a train of four (TOF) is recommended in intensive care unit (ICU) (1). The role of neuromuscular blocking agents (NMBAs), without TOF monitoring, was recently enhanced in acute respiratory distress syndrome (2). Our objective was to compare the results of a TOF with a clinical assessment of a neuromuscular blockade in ICU patients treated with recommended doses of benzylisoquinoliniums.

Patients and methods Design: An observational prospective study. Setting: Two polyvalent ICUs.

Patients: A total of 119 critically ill patients who required a neuromuscular blockade for more than $24 \mathrm{~h}$.

Interventions: None.

Measurements: Based on recommendations (1), three levels of neuromuscular blockade were defined: "over-paralyzed" (TOF =0), "wellparalyzed" (TOF = 1-2), and "under-paralyzed" (TOF = 3-4). Similarly, physicians were asked to classify patients as "over-," "well-," or "under-" paralyzed. Two measurements per day of the TOF on the facial nerve were taken and compared to the clinical assessment made by physicians who were blinded to the TOF results.

Results During the study period, 996 measurements of the facial TOF were taken, and only $8.8 \%$ of them were in the category "well-paralyzed." The agreement between the facial TOF and clinical assessment was very poor (kappa: 0.06; weighted kappa: 0.09). The proportion of "under-paralyzed" TOF measurements increased with the duration of the neuromuscular blockade $(p<0.05)$, suggesting tachyphylaxis. No significant difference was found when the plateau pressure and $\mathrm{PaO}_{2} / \mathrm{FiO}_{2}$ ratio were compared between the three levels of muscle relaxation defined by the TOF in the subgroup of patients with acute respiratory distress syndrome. The risk of ICU-acquired weakness was 
associated with a median infusion rate of NMBAs. It was also associated with a higher number of "over-paralyzed" TOF measurements $(p<0.05)$.

Conclusion Our results suggest that, according to the facial TOF, less than $10 \%$ of patients are "well-paralyzed" with recommended doses of benzylisoquinoliniums and that the clinical evaluation of neuromuscular blockades is inaccurate. However, the clinical impact of adjusting doses of NMBAs according to the TOF is still a matter of debate.

\section{Competing interests None.}

\section{References}

1. Sauder P, Andreoletti M, Cambonie G, Capellier G, Feissel M, Gall O, et al. [Sedation and analgesia in intensive care (with the exception of new-born babies). French Society of Anesthesia and Resuscitation. French-speaking Resuscitation Society]. Ann Fr Anesthésie Rèanimation. 2008;27(7-8):541-51.

2. Papazian L, Forel J-M, Gacouin A, Penot-Ragon C, Perrin G, Loundou A, et al. Neuromuscular blockers in early acute respiratory distress syndrome. N Engl J Med. 2010;363(12):1107-16.

\section{P103}

The use of enteral route for drug administration in the ICU is dimly rational

Anne-Laure Clairet ${ }^{1}$, Gilles Capellier ${ }^{1}$, Samuel Limat ${ }^{2}$, Christian Cornette ${ }^{2}$, Gaël Piton ${ }^{1}$

${ }^{1}$ Réanimation Médicale, $\mathrm{CHU}$ de Besançon, Besançon, France; ${ }^{2}$ Pôle pharmaceutique, $\mathrm{CHU}$ de Besançon, Besançon, France

Correspondence: Gaël Piton - piton.gael@wanadoo.fr

Annals of Intensive Care 2016, 6(Suppl 1):P103

Introduction Many patients are fed enterally $48 \mathrm{~h}$ after their admission to the ICU, and the administration of enteral nutrition is usually subjected to the use of a protocol. In contrast, enteral administration of drugs, and the shift from parenteral to enteral route of drugs, is out of protocol. We aim to evaluate the use of enteral route in the ICU for nine drugs of interest (linezolid, amoxicillin-clavulanic acid, metronidazole, spiramycin, fluconazole, levetiracetam, furosemide, amiodarone and esomeprazole). These drugs have a suitable form for enteral administration, an excellent oral bioavailability, and are often used in ICU.

Patients and methods This was a retrospective analysis of all the patients staying more than $72 \mathrm{~h}$ in our ICU and receiving one of the nine drugs of interest, either parenterally or enterally. At $72 \mathrm{~h}$ of admission, we considered eligible for enteral route patients receiving enteral nutrition, presenting without shock, small bowel disease, hematological disease, or severe sepsis requiring specifically the parenteral route. Results Seventy-two hours after ICU admission, $45 \%$ of patients were eligible for the use of enteral route. Ineligibility to the enteral route was most often due to either hemodynamic instability (patients with vasopressors) or a non-fed enterally patient. $18.6 \%$ of patients $(n=40)$ received no drug of interest enterally. Linezolid was the only drug that was never switched from parenteral to enteral route. Among patients eligible for enteral route, $14 \%$ of patients were using enteral route from the beginning of treatment and $25 \%$ with a switch from parenteral to enteral route. For patients ineligible for enteral route, only $22.6 \%$ have received their medication by strict parenteral route throughout the duration of treatment.

Discussion To our knowledge, this is the first description of the use of enteral route for drug administration in ICU. It included 215 patients representing 13,660 drug administrations. It was neither eligibility to enteral route ( $14 \%$ of eligible patients had no drug of interest enterally, whereas $77.4 \%$ of non-eligible patients received at least one drug of interest enterally) nor the bioavailability of the drug of interest (drugs selected for this study had excellent bioavailability) or the severity of the patient (patients receiving amiodarone had higher SAPS II score than the general population of the study, whereas the drug was one of the most enterally given) that dictated the primary or secondary use of the enteral route in critically ill patients. This suggests that there are barriers against the use of enteral route in ICU: ignorance of practices, human factors, and ethical considerations.
Conclusion This first inventory of the use of enteral route of drugs in the ICU suggests that the choice of enteral administration is poorly rational. It permits the identification of points of challenges, including human factors.

\section{Competing interests None.}

\section{P104}

Microcirculatory effects of norepinephrine in septic shock: a muscle microdialysis study

Amira Fatnassi' ${ }^{1}$, Zied Hajjej', Chiheb Romdhani' ${ }^{1}$, Walid Sammoud ${ }^{1}$, Iheb

Labbene $^{1}$, Mustapha Ferjani ${ }^{1}$

${ }^{1}$ Department of Critical Care Medicine and Anesthesiology, Military

Hospital of Tunis, Tunisia, Tunis, Tunisia

Correspondence: Zied Hajjej - hajjej_zied@hotmail.com

Annals of Intensive Care 2016, 6(Suppl 1):P104

Introduction The management of septic shock includes after optimization of patient's preload, administration of alpha-adrenergic drug such as norepinephrine, to maintain adequate mean arterial pressure. However, alterations of tissue perfusion, source of cellular hypoxia, can coexist with optimal macrocirculatory parameters. The aim of our study was to analyze the effects of norepinephrine on metabolic parameters of microcirculation in septic patients studied by muscle microdialysis.

Patients and methods It was a prospective, randomized, doubleblind study, including all patients aged over 16 years with septic shock defined by the bone criteria of the consensus conference of the American College of Chest Physicians/Society of Critical Care Medicine (ACCP/SCCM) 1991. Patients were randomized to receive either continuous infusion of levosimendan at $0.2 \mu \mathrm{g} / \mathrm{kg}$ per minute over $24 \mathrm{~h}$ (levosimendan group) or a continuous infusion of dobutamine at $5 \mu \mathrm{g} / \mathrm{kg}$ per minute during all the study period (dobutamine group) or a continuous infusion of a normal saline solution (placebo group). Before inclusion, norepinephrine should be at stable flow for more than an hour. Hemodynamic monitoring of macrocirculation was performed by echocardiography. Analysis of microcirculation parameters (lactate, pyruvate and lactate/pyruvate ratio) was made every $6 \mathrm{~h}$ during the first 3 days by muscle microdialysis (CMA600, CMA microdialysis $A B$,

Table 25 Drugs' modes of administration

\begin{tabular}{|c|c|c|}
\hline & & $\begin{array}{l}\text { Respect of } \\
\text { guidelines }\end{array}$ \\
\hline Stop feeding before drugs & Yes: $100 \%$ & Yes \\
\hline Rinsing with water & No: $42 \%$; yes: $58 \%$ & $\begin{array}{l}\text { No: } 42 \% ; \\
\text { yes: } 58 \%\end{array}$ \\
\hline Wait 30 min/stop feeding & No: $100 \%$ & No \\
\hline Direct refeeding after drug & Yes: $100 \%$ & No \\
\hline \multicolumn{3}{|l|}{ Drugs by shape } \\
\hline Liquids/orodispersible & $\begin{array}{l}\text { Dilution/dispersion in } \\
\text { water }\end{array}$ & Yes \\
\hline Pills/capsules & $\begin{array}{l}\text { Crush/open }+ \\
\text { dilution in water }\end{array}$ & No \\
\hline \multicolumn{3}{|l|}{ Drugs in time } \\
\hline One by one & Yes: $42 \%$ & Yes: $42 \%$ \\
\hline At the same time & Yes: $58 \%$ & No: $58 \%$ \\
\hline Equipment used & $\begin{array}{l}\text { Mortar/pestle/60-ml } \\
\text { syringe }\end{array}$ & Yes \\
\hline \multirow[t]{2}{*}{ Solution for blocked tube } & $\begin{array}{l}\text { Injection of water to } \\
\text { flush it }\end{array}$ & Yes \\
\hline & Changing the tube & No \\
\hline
\end{tabular}


Table 26 Drug's list administrated by stomach tube in ICU and their management

\begin{tabular}{|c|c|c|c|}
\hline Drugs R (DCl) & Presentation & Cut into 2 & Crushing \\
\hline Additiva (vit A, B, C, E) & Dispersible & & \\
\hline Amep (amlodipine) & Pills & Yes & Yes \\
\hline Aspirine (acide acetyl-salicylique) & Bags & & \\
\hline Atarax (hydroxyzine) & Pills & Yes & Yes \\
\hline Cardioaspirine & Pills & No & Yes \\
\hline Plavix (Clopidogrel) & Pills & Yes & Yes \\
\hline Cordarone (amiodarone) & Pills & Yes & Yes \\
\hline $\begin{array}{l}\text { Depakine chrono (valproate de } \\
\text { sodium) }\end{array}$ & Pills & Yes & Yes \\
\hline Deroxat (paroxétine) & Pills & Yes & Yes \\
\hline Digoxin (digoxin) & Pills & Yes & Yes \\
\hline Haldol (haloperidol) & Pills & Yes & Yes \\
\hline Mag 2+ (magnesium) & Liquid & & \\
\hline Minirin (desmopressin) & Pills & Yes & Yes \\
\hline Neurontin (gabapentin) & $\begin{array}{l}\text { Pills } \\
\text { Capsules }\end{array}$ & Yes & Yes \\
\hline Selenium (selenium) & $\begin{array}{l}\text { Pills } \\
\text { Capsules }\end{array}$ & & Yes \\
\hline Sintrom (acenocoumarol) & Pills & Yes & Yes \\
\hline Tramal (tramadol) & Pills & Yes & Yes \\
\hline $\begin{array}{l}\text { Ultralevures (saccharomyces } \\
\text { boulardii) }\end{array}$ & Bags & & \\
\hline
\end{tabular}

Stockholm, Sweden). Correlations between microcirculation parameters and norepinephrine doses were studied.

Results Thirty patients were included in the study (ten patients in each group). Demographic characteristics including age, sex, weight, medical history as well as the sepsis site, SAPS II score and mortality were comparable across the three groups. In total, 390 samples of muscle interstitial fluid were analyzed.

No correlation was found between norepinephrine doses and rate of muscle lactate and muscle lactate/pyruvate ratio $(p>0.05)$ for all groups: levosimendan, dobutamine and placebo. We found a weak inverse correlation between norepinephrine dose and muscle pyruvate levels $(p<0.05)$ for the control group and dobutamine group but not for levosimendan group.

Conclusion The use of vasopressors in the treatment of septic shock involves non-selective molecules with an action on macrocirculation easily clinically observable but with effects on microcirculation (modified heterogeneously between organs) remains not entirety assessable by current surveillance devises. Norepinephrine is currently the preferred vasopressor, although some molecules look promising (vasopressin, levosimendan, etc.).

\section{Competing interests None.}

\section{P105}

Administration of drugs per os by stomach tube for enteral feeding in surgical ICU

Hind Taibi', Ijlal Elatiqi', A Iraqui', Rachid Cherkab', Wafae Haddad', Chafik Elkettani ${ }^{1}$, Lahoucine Barrou'

${ }^{1}$ Anesthésie Réanimation, Chu Ibn Rochd, Casablanca, Morocco;

${ }^{2}$ Anesthésie réanimation, CHU Ibn Rochd Casa, Casablanca, Morocco

Correspondence: Hind Taibi - taibihind@hotmail.fr

Annals of Intensive Care 2016, 6(Suppl 1):P105
Introduction Drugs administration by stomach tube often needs the grinding of the tablets or the opening of the pills, which may seem consequent less, but can cause some transformations that can damage patient's health or cancel the product's effect [1]. The purpose of this work is to evaluate our practice concerning the drug's administration per os, in surgical ICU department comparing to international guidelines.

Materials and methods This is a prospective descriptive study for a 3-month period (From May to August 2015), including all the patients of surgical ICU department and collecting all the daily pharmaceutical analysis's data from all the medical prescriptions.

Results During the period of the study, 62 patients have been studied, and all had a stomach tube for enteral feeding (Tables 25,26 ).

Conclusion This study emphasizes the lack of respect of the recommendations and the urgent need of changing attitudes, by adapting the pharmaceutical form, the consequent risk of transformation and the means of grinding respecting the rules of good practice. Doctors have few clinical data, and studies at a bigger scale are required in order to highlight the drugs' mode of administration, more significantly on ICU's patients.

Competing interests None.

\section{Reference}

1. ADMINISTRATION DES MEDICAMENTS PAR SONDE CHEZ L'ADULTE. Pharmacie des Hopitaux Universitaires Genève (2013).

P106

Implementation of an intensive care follow-up clinic

Fabien Cave ${ }^{1}$, Alain Mercat ${ }^{2}$, Nicolas Lerolle ${ }^{1}$

${ }^{1}$ Réanimation médicale, Centre Hospitalier Universitaire d'Angers, Angers, France; ${ }^{2}$ Service de réanimation médicale et médecine hyperbare, Centre Hospitalier Universitaire d'Angers, Angers, France

Correspondence: Nicolas Lerolle - nicolas.lerolle@univ-angers.fr Annals of Intensive Care 2016, 6(Suppl 1):P106

Introduction The consequences of an ICU stay are marked by increased long-term mortality and morbidity. The post-intensive care syndrome (PICS) is increasingly well described and is characterized by psychiatric, cognitive and functional impairments, and the possible persistence of organ failures. Management strategies to date are still in their infancy, but are a major public health challenge. Since April 2015, we initiated an ICU follow-up day clinic (IFUDC) program to assess patients' health and provide care in coordination with the treating physician.

Patients and methods We describe here the implementation of the IFUDC program with a day care clinic at 1 and 6 months after ICU discharge and a first assessment after 10 sessions. The aim of this first step was to assess patients need and program feasibility.

Results From April 2015, patients expected to be discharged alive from the ICU were screened on a daily basis according to the following criteria: age between 18 and 80 years; living in the department; endotracheal intubation for more than 5 days; absence of follow-up program already initiated (i.e., chronic dialysis); absence of severely debilitating neurological or psychiatric disorders; good long-term prognosis. Eight patients were selected between April and July 2015. On the day of ICU discharge, they received information about IFUDC program and were offered to participate (no patient refused). Two weeks after, patients were convoked for the 1 month day clinic, with the accompanying person of their choice. Questionnaires such as HADS (anxiety and depression), the IES-R (post-traumatic stress disorder) and the SF-36 (quality of life) were sent to be completed and returned on the day of the clinic. On the day of the IFUDC, patients had a measurement of vital parameters, fasting blood and urine collection (dipstick test, blood and urine electrolytes and creatinine, proteinuria, blood count, hemostasis, lipid status, iron status, liver function test), electrocardiogram, pulmonary function test (spirometry) and a chest $\mathrm{X}$-ray. Breakfast was then served. They were then received in consultation by an intensivist to detail the history of their ICU stay, a therapist doctor and a psychiatrist. All data and observations were gathered in a computer form specifically designed for IFUDC program. After lunch, 
patients and accompanying persons were invited (all patients agreed) to return to visit the ICU in which they were admitted. They were able to meet the team that cared for them. This visit was each time seen as very positive and full of emotions as by caregivers as by patients and their families. If abnormal cardiac, renal and pulmonary examinations were observed, a call to a specialist was made to define the necessary actions. By afternoon, patients received an oral summary of diagnoses and actions implemented. A computerized report was generated and sent to the treating physician, the therapist doctor, the psychiatrist and other specialists as needed. The patients ( 2 patients at the moment) were reconvened at 5-6 months of discharge from ICU for a second IFUDC day clinic session. For the eight patients seen at 1 month, 19 diagnoses were made, notably: persisting renal failure (4 patients), iron deficiency anemia (3 patients), persistent physical weakness (3 patients), shoulder impingement syndrome (2 patients), posttraumatic stress disorder ( 2 patients), anxiety of the accompanying person ( 1 ) and 28 therapeutic actions were implemented ( 8 treatment modifications, 6 physiotherapy prescriptions, 6 referral to specialist, 2 specialist advices during IFUDC session, etc.). The valuation of each session according to French T2A (pay upon activity) system was 502 euros.

Conclusion ICU follow-up day clinic sessions were well appreciated both by patients and caregivers. Frequent diagnoses and therapeutic actions were made, but health impact of these measures cannot be measured. The sustainability of the IFUDC program will involve significant human and financial resources.

\section{Competing interests None.}

\section{P107}

\section{Needs assessment of adults post-intensive care rehabilitation} department in Amiens University Hospital

Gaelle Bacari-Risal', Pierre Louis Doutrellot ${ }^{2}$, Benoit Vaysse ${ }^{3}, M^{\prime}$. Inan ${ }^{4}$ K. Khelfoun ${ }^{5}$, M. Nasserallah' ${ }^{6}$, Julien Maizel', C. Legrand-Monteil ${ }^{8}$, S. Tasseel-Ponche ${ }^{2}$

${ }^{1}$ Médecine physique réadaptation plateau technique et unité mobile de mpr et réanimation médicale, Centre Hospitalier Universitaire, Amiens, France; ${ }^{2}$ Médecine physique et réadaptation neurologique et ufr de médecine upjv, pôle santé, Centre Hospitalier Universitaire, Amiens, France; ${ }^{3}$ Service d'information médicale, service d'épidémiologie, Centre Hospitalier Universitaire, Amiens, France; ${ }^{4}$ Soins service, Service d'hospitalisation à domicile, Rivery, France; ${ }^{5}$ Service des urgences, C.H. Compiègne-Noyon, Compiègne, France; ${ }^{6}$ Département de médecine générale, Centre Hospitalier Universitaire, Amiens, France; ${ }^{7}$ Réanimation médicale, Centre Hospitalier Universitaire, Amiens, France; ${ }^{8}$ Médecine physique et réadaptation plateau technique et unité mobile de $\mathrm{mpr}$, Centre Hospitalier Universitaire, Amiens, France

Correspondence: Julien Maizel - maizel.julien@chu-amiens.fr Annals of Intensive Care 2016, 6(Suppl 1):P107

Introduction A part of patients transferred from intensive care unit (ICU) to continuing care services is due to the persistence of important functional failures. The role of early rehabilitation in the management of these patients has shown interest. The creation of unit, with both expertise of intensivists and physics Doctor, would improve the healthcare network. But physical and rehabilitation medicine requires specialized human resources and equipment. This retrospective epidemiological pilot study was to determine the needs of Amiens University Hospital in post-intensive care rehabilitation department (PICRD). Patients and methods We determined the number of patients eligible to PICRD, during 2013. The «Programme Médicalisé des Systèmes d'Information» allowed to count all patients admitted to medical-surgical ICUs in Amiens University Hospital. Patients who had brain injury, spinal cord injury, or severe peripheral neurological damage regardless of origin were included in the study among the ICU patients. Even if they did not suffer from those pathologies, patients with duration of hospitalization (DH) over 7 days in the ICU were also included. The need for PICRD has been evaluated by computing the number of eligible patients in PICRD. They were eligible to PICRD: patients with persistent neurological impairment in their day 10 of hospitalization, without hemodynamic disability in absence of catecholamine, with a tracheotomy when a ventilator weaning was impossible. It was applied to the group "eligible" a new release date, which corresponded to day 2 after the realization of tracheotomy and/or stopping of catecholamine, and/or stopping continuous invasive treatments. This allowed calculating a new $\mathrm{DH}$ and therefore a new average length of stay. Over 70 and under 18 years old and those with undergoing neoplasia were excluded from the study.

Results A total of 2690 hospitalizations in ICU were counted at the Amiens University Hospital in 2013. Seven hundred and seventeen patients were included in the study; after application of the exclusion criteria, a total of 388 were analyzed. This group of patients consisted of $72 \%$ men, and the median age was 54 years old $(I Q R=20.25)$. The median SAPS II was $42(\mathrm{IQR}=28)$ with a median length of stay of 12 days (IQR $=14)$ and a mortality rate of $19.6 \%$. As regards the reasons for hospitalization: over half, $56 \%$, fell to neurological impairment, with stroke $(25 \%)$, severe traumatic brain injury (STBI $=22 \%)$ and spinal cord injury ( $\mathrm{SCl}=6 \%$ ). The other half consisted mostly shocks (19\%), acute respiratory failure (8\%), acute respiratory distress syndrome (ARDS $=4.5 \%$ ), severe sepsis $(5 \%)$, cardiopulmonary arrest $(2.5 \%)$. Nearly $22 \%$ of patients $(n=84)$ were eligible for PICRD. They were young: median of age 55 years old $(I Q R=20)$, mostly men $(81 \%)$. The median SAPS II was $38(\mathrm{IQR}=23.25)$. The main diseases were also neurological for $46 \%$ with: stroke, STBI and SCl. Other causes were: shock for $14 \%$, acute respiratory distress and ARDS for a total of $28 \%$, severe sepsis for $11 \%$. Seventy-seven of them came out alive from the ICU, $20 \%$ in intensive care in "Berck", $10 \%$ in physical and rehabilitation medicine unit, $50 \%$ in medicine or surgery services. After application of the new date of stay, a significant difference in hospital stay of 40 days $(p<0.0001)$ was demonstrated.

Conclusion There is a need of adults PICRD at Amiens University Hospital. It would allow an early medical and rehabilitative treatment, improving the fluidity of the post-intensive care discharge, and lead to a reduction in socioeconomic costs of care of these patients who need no more ICU care.

\section{Competing interests None.}

P108

Factors of degradation on independence and quality of life at 3 and 6 months after stay in intensive care unit

Benjamin Kowalski ${ }^{1}$, Anne Guaguere ${ }^{2}$, Claire Boulle-Geronimi ${ }^{3}$ ${ }^{1}$ Reanimation, Centre Hospitalier de Douai, Douai, France; ${ }^{2}$ Réanimation polyvalente, Hospital Center De Douai, Douai, France; ${ }^{3}$ Réanimation polyvalente, Hôpital de Douai, Douai, France

Correspondence: Benjamin Kowalski - kowalskibenj@orange.fr Annals of Intensive Care 2016, 6(Suppl 1):P108

Introduction Although survival of patient remains the priority in intensive care unit (ICU), quality of life and level of dependence when returning home have become important issues. This study aims to assess these two parameters after a stay in intensive care unit (ICU) and to identify the factors involve in their impairment.

Patients and methods This prospective and observational study focuses on all the patients admitted into intensive care unit. Patients who presented immediately deteriorating of mental status such as low or moderate memory disorders and psychiatric disorders were excluded. Patients or their relatives were interviewed during their stay. Then, patients were clinically reevaluated at third month (M3) and interviewed by phone at sixth month (M6) after admission date. The independence degree had been measured using IADL (Instrumental Activities of Daily living) test score and their quality of life using WHOQOL-BREF test score (World Health Organization Quality of Life). Data from stay in intensive care, onset of delirium, autonomy before hospitalization, physical capacities at home, presence of posttraumatic stress disorder assessed by the Post traumatic Scale Score 10 (PTSS10) and anxio-depressive symptoms evaluated by Hospital Anxiety Depression Score (HADS).

Results In total, thirty-five patients were included at the beginning. Four patients died at M3. Three patients cannot be evaluated for the level of dependence at M3. Seven patients left 
the follow-up at M6. All patients showed decreased quality of life at M3. At 3 months, 28 patients were evaluated for the independence. Thus, 20/28 patients who were more dependent at 3 months had also a significantly use of nurses at home $(p<0.02)$, a use of bequille $(p<0.02)$, a decrease in psychic health $(p<0.044)$ and an increase in score of post-traumatic stress disorder $(p<0.05)$ and in anxiety score $(p<0.019)$. Between M3 and M6, 15/24 (65\%) patients showed an improvement of their quality of life. Patients with impaired quality of life $9 / 24(25 \%)$ showed significant health degradation $(p<0.05)$ and a decrease in social relationships $(p=0.084)$. These alterations were associated with increase medical support at home $(p<0.02)$ and required more institutionalization $(p<0.02)$. At 6 months, $12 / 24$ showed decrease independence score. These patients experienced more delirium during stay $(p<0.05)$, needed more medical support at home $(p<0.05)$ and were delayed to come back home $(p<0.05)$.

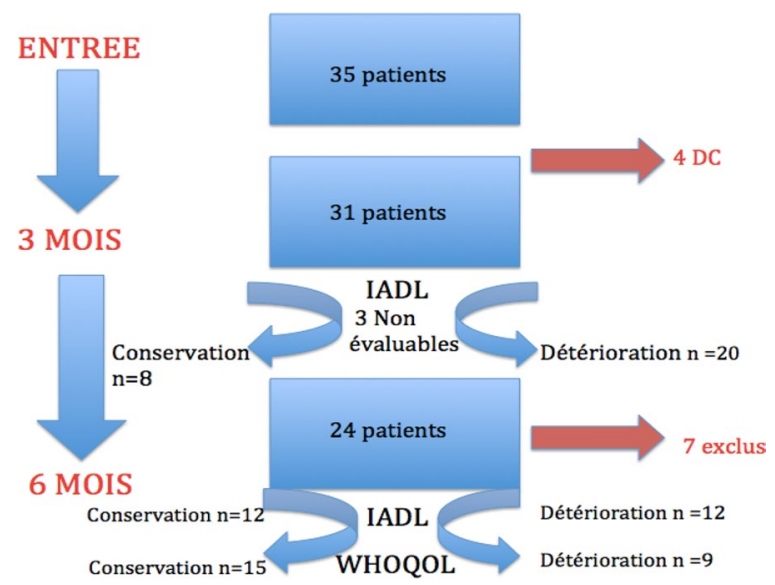

Conclusion Impaired quality of life and increase of dependence after stay in ICU were observed for all patients of our study. The early screening and adapted therapy for psychiatric disorders, such as delirium, post-traumatic stress disorder and anxio-depressive symptoms, may decrease the onset of this impairment.

\section{Competing interests None.}

\section{References}

1. García Lizana F, Peres Bota D, De Cubber M, Vincent J-L. Long-term outcome in ICU patients: what about quality of life? Intensive Care Med. 2003 Aug;29(8):1286-93.

2. Dowdy DW, Eid MP, Sedrakyan A, Mendez-Tellez PA, Pronovost PJ, Herridge MS, et al. Quality of life in adult survivors of critical illness: A systematic review of the literature. Intensive Care Med. 2005 Apr 1;31(5):611-20.

\section{P109}

\section{Octavie: Epidemiological study of mortality and autonomy 3} and 6 months after ICU stay for very elderly patients

Juliette Masse ${ }^{1}$, Clément Vanbaelinghem², Patrick Herbecq ${ }^{2}$

${ }^{1}$ Pôle de réanimation hôpital roger salengro, C.H.R.U. Lille, Lille, France;

${ }^{2}$ Réanimation polyvalente, C.H. de Roubaix, Roubaix, France

Correspondence: Juliette Masse - juliette.masse2911@gmail.com Annals of Intensive Care 2016, 6(Suppl 1):P109

Introduction Admitting very elderly patients in ICU has always been questionable as the benefit for these patients in terms of survival remains unclear. Furthermore, their potential of recovery from loss of autonomy induced by the ICU stay is still not well known. The main objective was to compare 3 and 6 months mortality between patients over and under 80 years old and to identify risk factors associated with 3 months mortality for those over 80 years old. We also compared evolution of autonomy between the two groups.

Materials and methods Prospective observational study conduced from November 2012 to January 2014 in the Hospital of Roubaix (France) comparing patients more than 80 years old to younger ones. Each very elderly patient hospitalized in the ICU was matched by drawing lots to a younger one hospitalized at the same time. Patients with an expected length of stay shorter than $48 \mathrm{~h}$ were excluded. Data were collected at admission, during the whole ICU stay and at 3 and 6 months from discharge. Patients were followed by a post-ICU clinical consultation and/or by phone interviews. ADL and IADL scores were chosen to evaluate autonomy. Loss of self-dependence was defined by loss of $\geq 1$ point and for ADL score and for IADL score by a raise of $\geq 3$ points. Severe limitation was defined by $A D L$ score $\leq 3 / 6$ or IADL score $\geq 15 / 31$.

Results Two hundred and forty-eight consecutive patients were included, 124 in each group. Median age was, respectively, 83 [8186.5] and 62 [55-69.5] years for each group. Admission was mainly medical in the 2 groups. The gravity score SAPS II minus the points for age was 32.5 [23-48.5] for $\geq 80$ years and 41 [28.5-54] for $<80$ years. Mortality rates were, respectively, 47 versus $37 \%(p=0.12)$ and 51 versus $39 \%(p=0.055)$ at 3 and 6 months. Analysis adjusted on chronic organ failure, gravity score and withholding and withdrawing life-sustaining treatment did not show any difference of mortality at 3 and 6 months. In multivariate analysis, age was not an independent risk factor for mortality. Three months risk factors for mortality for very elderly patients were withholding and/or withdrawing life-sustaining treatments (HR [95 \% Cl] 6.19 [3.10-12.35]; $p<0.001$ ) and SAPS II score minus points for age (1.02 [1.01-1.03]; $p=0.02)$. Loss of self-dependence on $A D L$ score was more frequent in very elderly compared to younger ones at 3 ( 43 vs $19 \%, p=0.005$ ) and 6 months ( 54 vs $13 \%$, $p<0.0001)$. At 6 months, $19 \%$ of the elderly and $5 \%$ of the $<80$ years had severe functional limitations $(p=0.02)$.

Conclusion No statistically significative difference of mortality was found between the 2 groups. Withholding and/or withdrawing lifesustaining treatments were the main factor for death at 3 months for more than 80-year-old patients. More than half of very elderly patients were dead at 6 months. Older survivors suffered from major autonomy deterioration and became more frequently self-dependent than younger patients after an ICU stay.

\section{Competing interests None.}

\section{P110}

Analysis of burn injuries in the elderly

Amel Mokline', Syrine Draief ${ }^{1}$, Lazhari Gharsallah¹, Imen Rahmani ${ }^{1}$, Bahija Gasri ${ }^{1}$, Sofiene Tlaili ${ }^{1}$, Rym Hammouda ${ }^{1}$, Amen Allah Messadi ${ }^{1}$

${ }^{1}$ Intensive Burn Care Department, Burn and Trauma Center, Tunis, Tunisia Correspondence: Amel Mokline - amokline@yahoo.fr Annals of Intensive Care 2016, 6(Suppl 1):P110

Introduction Burns in the elderly are still common cause of morbimortality due to impaired immunological function and preexisting cardiopulmonary diseases. They cause devastating complications and long-term socioeconomic impact. The purpose of this study was to review the characteristics of burn injuries in elderly patients and to assess their prognosis.

Patients and methods A retrospective study was conducted in a 20-bed adult burn ICU in Tunis between January 2009 and December 2012. Elderly burned patients aged over 60 years were enrolled. Characteristics of elderly patients, burn injuries and prognostic factors were collected. Statistical analysis was performed using the SPPS 20. 
Results During the period of study, 1417 patients were admitted with 144 of elderly patients ( $10 \%$ of total admissions). The mean age was $74 \pm 8$ years old. There were 63 males and 81 females. The average TBSA was $25 \pm 22 \%$ with an UBS of $56 \pm 74$. The major mechanism of injury for the patients was scalding or flame burns $(97 \%)$. Elderly burn patients suffer from premorbid conditions: diabetes $(n=38)$, hypertension $(n=30)$ and cardiovascular diseases ( $n=16)$. In our series, burns affected limbs $(75 \%)$ and the head/ neck (57\%). Inhalation injuries were noted in $17 \%$ of cases, and mechanical ventilation was required in fifty-three patients (36\%) for a duration of 5 days (1-30 day). Our patients developed hypovolemic shock at admission in $22 \%$ of cases. Clinical complications were sepsis $(46 \%)$, acute renal failure $(40 \%)$ and thromboembolic complications (11\%). The mortality rate was $43 \%$ and occurred at 12 days after burn injury. In our study, factors associated with death in elderly burned patients were: secondary transfer, inhalation injury, ARDS, sepsis, mechanical ventilation, acute renal failure and septic shock.

Conclusion Burns in elderly patients are frequent (10\%) and serious. Burn prevention education and implementation of safety measures at home would help reduce such incidences.

\section{Competing interests None.}

\section{P111}

\section{ICU admission criteria for patients 85 years and older}

Philippe Michel ${ }^{1}$, Bruno Gelée ${ }^{2}$, Fouad Fadel ${ }^{1}$, Marie Thuong ${ }^{1}$

${ }^{1}$ Réanimation médico-chirurgicale, CH René Dubos, Pontoise, France;

${ }^{2}$ Réanimation polyvalente, C.H. de Beauvais, Beauvais, France

Correspondence: Philippe Michel - philippe.michel@ch-pontoise.fr Annals of Intensive Care 2016, 6(Suppl 1):P111

Introduction To determine whether the criteria for admission in intensive care unit (ICU) were different for patients aged 85 or older compared to patients aged 70-84.

Patients and methods This analysis is part of a multicenter, prospective, observational study (six centers) on the outcome of patients aged 85 or older (very old group) after an hospitalization in ICU.

The control group consists of patients, from the same centers, aged 70-84 (mild-old group) randomly selected.

The autonomy-related variables collected were: ALD score six items, living conditions (at home, alone in couple or with family, or in retirement homes), comorbidities (cardiac failure NYHA 3 or 4 , respiratory failure Gold 4, chronic renal disease, dialysis), the pathway before resuscitation and ICU stay. Results are given as mean \pm standard deviation or percentage.

Results The very old group included 34 patients (age 86 years \pm 1.6 IGSII $50.7 \pm 27$ ); the medium-old group included 57 patients (age 77.6 years \pm 4.7 , IGS II $54.2 \pm 24.1$ ). The admission mode in ICU was the same (Emergencies Department: medium-old $=32 \%$, very old $=22 \%$, home by SMUR: medium-old $=2.8 \%$, very old $=6.5 \%$, already hospitalized: medium-old $=39 \%$, very old $=34 \%, p=0.34$ ). There was no difference about their living conditions nor about crippling heavy histories.

The ALD autonomy score did not exhibit differences, whether item by item or on total score (medium-old $=5.35 \pm 1.5$, very old $=5.11 \pm 1.81, p=0.49$ ). There was no difference for the Knaus score noted on the admission. The surgical pathologies were more frequent for older patients (medium-old $8.7 \%$; very old $13.5 \%, p$ 0.001 ) as well as admissions for cardiac pathologies, particularly for cardiogenic pulmonary edema (medium-old $=8 \%$, very old $=58 \%$, $p=0.01$ ).

The duration of invasive ventilation was shorter for older patients: medium-old: $4.82 \pm 7.55$; very old: $1.16 \pm 1.62, p=0.005$. There was no difference on the need for noninvasive ventilation, renal replacement therapy or surgery. The length of stay in ICU was also shorter for the oldest ( $\mathrm{Mb} 8.84 \pm 8.61$; vo $5.41 \pm 8.0, p=0.05$ ). Duration of invasive ventilation was Mb $4.82 \pm 7.55$; vo $1.16 \pm 1.62, p=0.005$.

Discussion In this study, older patients admitted in ICU are not in a more serious state, do not have more extensive histories and do not usually live more in retirement homes than the younger age group, which does not match the expected evolution of the overall population. The ICU stay data (length of stay, invasive techniques requirement, mortality) seem very similar to data observed for younger patients. The admission patterns, known to be rapidly favorable, prevail, raising the question of more stringent admission criteria applied by physicians.

Conclusion Despite the absence of any literature or recommendations besides precise situations, the oldest patients are admitted in ICU only after a serious sorting process. Data collected on the outcome after the stay in ICU will allow for a more comprehensive analysis of the selected strategy.

\section{Competing interests None.}

\section{P112}

Facteurs pronostiques chez les patients âgés admis en réanimation médicale. Prognostic factors in elderly patients admitted in medical intensive care

Sandra Ait-Aoudia', Salima Laddi', Mohamed-Mohcen Sahraoui ${ }^{1}$ Réanimation medicale, Hopital central de l'armee (HCA), Alger, Algeria

Correspondence: Sandra Ait-Aoudia - sandrait2004@hotmail.com Annals of Intensive Care 2016, 6(Suppl 1):P112

Introduction It is customary to say that the Algerian population is characterized by its youth, but keep in mind that the proportion of elderly is increasing. Currently, half of the doctors of activities is devoted to the elderly; this patient population is predominant in hospitals and represents almost half of the recruitment of intensive care units.

Materials and methods This is a single-center study, observational and descriptive, conducted in medical intensive care unit (Algiers, Algeria).

Inclusion criteria: Age $\geq 65$ years and a hospital stay $\geq 48 \mathrm{~h}$.

Results During the period of our study, 852 patients were admitted to our service. The average age of these patients was $66.11 \pm 18.26$ years [range 15-98 years] and sex ratio was 1.50. Patients aged 65 and over represent $65 \%$ of all admissions to intensive care during this period. For our study, 400 elderly patients are retained. The average age of these patients was $77.19 \pm 7.05$ years [range $65-98$ years]; there is a male predominance $(61 \%)$ with a sex ratio of 1.56 . Elderly patients mainly come from the emergency department $(59.50 \%)$, and the main reason for admission is the altered state of consciousness ( $56.25 \%)$. The average number of comorbidities by elderly patient is $3.17 \pm 1.40$ medical conditions with an average of $4.16 \pm 2.16$ medications per patient. The average scores at admission in ICU, respectively: $24.62 \pm 6.41$ points for the Acute Physiology and Chronic Health Evaluation II (APACHE II); $47.80 \pm 15.12$ points for the Simplified Acute Physiology Score II (SAPS II); $13.60 \pm 4.68$ points for the Short Emergency Geriatric Assessment (SEGA) and $6.74 \pm 2.22$ points for the Charlson score (comorbidities). The average length of stay in intensive care for older patients was $10.66 \pm 11.25$ days [range $2-160$ days]. The mortality rate is high, of the order of $57 \%$. Univariate analysis has allowed us to retain 11 predictors of mortality, which are: APACHE $\| \geq 22$ points (OR 8.65 with $95 \% \mathrm{Cl}[5.13-14.71] ; p<0.000001)$ ) SAPS II $\geq 40$ (OR 5.18 with $95 \% \mathrm{Cl}[3,22-8.36] ; p<0.000001)$, SEGA score $\geq 8$ points (OR 5.18 with $95 \% \mathrm{Cl}[3.22-8.36] ; p<0.000001)$, antecedent of hypertension $(O R$ 1.73 with $95 \% \mathrm{Cl}[1.09-2.75] ; p=0.01)$, antecedent of heart disease (OR 1.70 with $95 \% \mathrm{Cl}[1.14-2.48] ; p=0.02)$, antecedent of stroke (OR 2.23 with $95 \% \mathrm{Cl}[1.31-3.82] ; p=0.001)$, acute renal failure (OR 1.81 with $95 \% \mathrm{Cl}[1.13-2.91] ; p=0.008)$, nosocomial infection (OR 3.22 with $95 \%$ Cl [1.93-5.38]; $p<0.0001)$, extra-renal purification (OR 2.16 with $95 \%$ Cl $[1.22-3.83] ; p=0.004)$, mechanical ventilation (OR 42.95 with $95 \%$ $\mathrm{Cl}[22.68-82.24] ; p<0.000001)$, and finally the use of vasoactive drugs (OR 17.46 with $95 \%$ Cl [10.23-29.96]; $p<0.000001$ ). Multivariate analysis by logistic regression allows us to retain the final as 4 parameters as independent predictors of mortality among our elderly patients: an APACHE II score $\geq 22$ points (OR 2.40 with 95 IC $\%$ [1.65-3.51]; $p=0.036$ ), SAPS II score $\geq 40$ (OR 2.02 with $95 \% \mathrm{Cl}$ [1.54-3.26]; $p=0.047$ ), use of mechanical ventilation (OR 14.53 with $95 \% \mathrm{Cl}[8.1528 .9]$; $p<0.0001)$, and the use of vasoactive drugs (OR 10.17 with $95 \% \mathrm{Cl}$ [4.67-22.2]; $p<0.0001)$. 
Table 27 Global overview of the uDCD activity in Annecy and France

\begin{tabular}{|c|c|c|}
\hline & $\begin{array}{l}\text { Annecy } \\
n \text { (\% of national activity) }\end{array}$ & $\begin{array}{l}\text { France } \\
n\end{array}$ \\
\hline Identified potential donors & $45(11 \%)$ & 401 \\
\hline Effective donors & $31(18 \%)$ & 170 \\
\hline Explanted kidneys & $61(18 \%)$ & 336 \\
\hline Transplanted kidneys & $54(22 \%)$ & 242 \\
\hline \multicolumn{3}{|l|}{ Conversion rates } \\
\hline Effective donors/potential donors & 0.69 & 0.42 \\
\hline $\begin{array}{l}\text { Transplanted kidneys/potential } \\
\text { kidneys }\end{array}$ & 0.6 & 0.3 \\
\hline $\begin{array}{l}\text { Transplanted kidneys/explanted } \\
\text { kidneys }\end{array}$ & 0.89 & 0.72 \\
\hline
\end{tabular}

Conclusion The admission of elderly ICU patients raises medical, ethical, sociological, and economic factors. Although the aging trend of the population admitted to intensive care continues, no statistical relationship was found between chronological age and mortality. The physiological age conditioned by concomitant, illness, and associated organ failure is of benefit to consider the actual age of the patients. Keywords: elderly patients, intensive care, comorbidity, prognostic factors.

\section{Competing interests None.}

\section{P113}

Uncontrolled donation after circulatory determination of death: 42 months of experience in a general hospital

Clémence Fauché', Samuel Gay ', Michel Muller', Anna Faucher², Olivier Skowron ${ }^{3}$, Albrice Levrat', Didier Dorez ${ }^{2}$

${ }^{1}$ Réanimation polyvalente, Hospital Center Annecy Genevois, Metz-Tessy, France; ${ }^{2}$ Coordination des dons d'organe et de tissus, Hospital Center Annecy Genevois, Metz-Tessy, France; ${ }^{3}$ Urologie, Hospital Center Annecy Genevois, Metz-Tessy, France

Correspondence: Michel Muller - mmuller@ch-annecygenevois.fr Annals of Intensive Care 2016, 6(Suppl 1):P113

Introduction Following the encouraging results of other countries, the uncontrolled donation after circulatory determination of death (uDCD) procedure has been introduced in France in 2006 and started at the Annecy General Hospital in July 2011. This work presents the first 42 months of our experience.

Patients and methods Our hospital is a reference center for the medical care of cardiorespiratory arrest (CRA) for the department of Haute Savoie, and a part of Savoie and Ain (a population of about 1 million inhabitants). Distinct indications of UDCD and therapeutic extracorporeal life support have been beforehand clearly defined by a team of cardiologists, emergency doctors and intensivists.

For each CRA, the opportunity of a UDCD is systematically evaluated according to the national criteria edicted by the Agence de la Biomédecine. If appropriate, after hospital admission of the potential donor verification of the inclusion criteria and a 5 -min period of no-touch, death was declared and a Regional Normothermic Recirculation (RNR) was surgically implemented. The donor relatives were then informed and interviewed. If there was no opposition to the organ donation, the donor could be transferred to the operative room for explantation. We reviewed the charts of uncontrolled donors from July 2011 to December 2014. We analyzed the times, conversion rates, and outcomes of procured organs. Results are given as median [1st-3rd quartile].

Results The total number of cardiac arrests over the period was 2170; 45 potential uDCDs were referred to our hospital. The median no flow was $10 \mathrm{~min}$ [5-20]. Time from CRA to arrival at the hospital was $95 \mathrm{~min}$
[72-107]. We faced 4 technical failures, 6 family refusals, 1 family could not be contacted, and there were 3 medical contraindications. Thirtyone patients became donors, for whom the mean time from CRA to effective RNR was 137 min [125-143], and time from RNR to organs explantation was 232 min [216-240].

Sixty-one kidneys could be explanted, among which 6 had anatomical problems and 1 was of bad quality. The mean resistance index at $\mathrm{H} 4$ was 0.15 . Finally, 54 kidneys were transplanted (organ conversion rate $60 \%$ ) with a cold ischemia time of $11.1 \mathrm{~h}$ [9.1-13.2]. There was $50 \%$ of delayed graft function with 2 [0-5] dialysis sessions. The recipients mean creatininemia at 6 months was $121 \mu \mathrm{mol} / \mathrm{l}$ [100-149].

A comparison of our results with the national data is exposed in Table 27.

After authorization in January 2014, 2 livers were retrieved and transplanted. The first recipient died of sepsis at 3 months with a functional graft, the other is still living without complication.

Discussion Organization matters appear to be the main strength of the procedure: strong involvement of the prehospital teams, unique place to admit all CRA, organ preservation by RNR.

Ethical concerns were raised before and during the program, but regular discussions between teams and successes of the program maintained a stable recruitment with a good attendance of involved professionals.

Conclusion uDCD can be implemented in a general hospital and yields success in kidney and liver transplantation despite procedural and ethical challenges.

\section{Competing interests None.}

\section{P114}

Epidemiology of long stays in ICU

Bertrand Hermann', Caroline Hauw Berlemont ${ }^{1}$, Alexandra Monnier', Florence Boissier ${ }^{1}$, Nadia Aissaouii ${ }^{1}$, Jean-Yves Fagon ${ }^{1}$, Jean-Luc Diehl' ${ }^{1}$, Emmanuel Guérot ${ }^{1}$

${ }^{1}$ Réanimation médicale, Hopital Europeen Georges-Pompidou, Paris, France

Correspondence: Caroline Hauw Berlemont - alexis.berlemont@gmail. com

Annals of Intensive Care 2016, 6(Suppl 1):P114

Introduction Little is known about the epidemiologic description and prognosis of patients undergoing a prolonged stay in ICU. Our descriptive study focused on patients who were hospitalized 30 days or more, representing the 95th percentile of the duration of all stays in our medical ICU; it aimed at describing the characteristics of patients, searching for risk factors for mortality and comparing them with patients who stayed less than 30 days.

Patients and methods We conducted a retrospective observational study, in a medical ICU of a Parisian university hospital between July 2000 and December 2013, including all patients who stayed 30 days or more (long stay patients $=$ LSP). Collected data carried on McCabe comorbidity score, autonomy score, diagnosis on admission in ICU, SAPSII, using of organ support (mechanical ventilation and/or renal replacement therapy-RRT), complications during ICU stay (either evolutive, infectious or intercurrent), and in-ICU/in-hospital mortality. We compared them with patients who stayed less than 30 days (short stay patients $=$ SSP), for some data available in our database (age, sex, SAPSIl and mortality).

Results Out of 8906 patients hospitalized in the period of the study, $416(4.67 \%)$ were hospitalized 30 days or more. The median length of stay was 39 days (ranging from 462 to 30 ). LSP had a mean age of $65.7 \pm 14.7$ years versus $62.7 \pm 18.6$ years for the SSP $(p<0.0001)$. The majority of the LSP, $72 \%$, came from in-hospital transfer, rather than from direct admission $28 \%$. This was significantly different from the SSP, where in-hospital transfer and direct admission were more well balanced (respectively, 52 and $48 \%, p<0.0001$ ). Sixty-one percentage had a preserved autonomy (autonomy score A and B) and only $38 \%$ had a life-threatening comorbidity (defined as a McCabe score of 2 or 3). The reasons for admission in the ICU were: acute respiratory failure $(43 \%)$, sepsis $(29 \%)$, weaning from ventilation $(10 \%)$, neurological failure $(7.5 \%)$, cardiac arrest $(4.5 \%)$, circulatory failure (3.5\%), 
metabolic abnormality (1\%), and others (1.5\%). There was no significant difference between the severity of illness at admission assessed by the SAPSII score between LSP and SSP $(53.0 \pm 18.5$ vs. $52.0 \pm 25.6)$. Ninety-nine percentage of LSP underwent mechanical ventilation versus $68 \%$ of SSP $(p<0.0001)$, with a mean of $36.6 \pm 28.0$ days of mechanical ventilation. There was the same significant difference for the use of RRT between long stay group versus short stay group (33 vs. $19 \%, p<0.0001$ ). The rate of complications was $37 \%$ for the LSP. Overall observed in-ICU mortality was $39 \%$, which was significantly higher than mortality of SSP, $30 \%(p<0.001)$. The in-hospital mortality of the LSP rose up to $48 \%$. Among the LSP the in-hospital mortality increased with the McCabe score: $34 \%$ for a score of $0,42 \%$ for a score of $1,60 \%$ for a score of 2 , and $75 \%$ for a score of $3(p<0.0004)$. The SAPSII was higher among the deceased, $57.0 \pm 18.1$ vs. $48.9 \pm 18.1$ $(p<0.0001)$. There was a higher rate of death among patients who had a complication than among those who had not (55 vs. $35 \%$, $p<0.0004)$. Interestingly, there was no difference between the length of stay of the deceased and the survivors $(47.2 \pm 22.6$ vs. $48.7 \pm 36.9)$. Conclusion Prolonged ICU stays are associated with a slight increase of mortality rate and a more frequent use of organ support, but more than half of the concerned patients were finally discharged alive from the hospital. Mortality is associated with comorbidities, severity at admission and occurrence of complications but does not increase the length of stay. Identification of LSP at the time of admission seems difficult.

\section{Competing interests None.}

\section{P115}

\section{Are daily sedation stops safe in a medical ICU?}

Michel Arnaout ${ }^{1}$, Julien Charpentier ${ }^{1}$, Benoit Champigneulle ${ }^{1}$, Julie Busson ${ }^{1}$, Anne-Sophie Debue ${ }^{1}$, Julie Dhumeaux' Guillaume Geri' ${ }^{1}$, Wulfran Bougouin', Nicole Ericher', Sébastien Lefort', Pierre Lucas', Alain Cariou', Jean-Paul Mira', Frédéric Pène' ${ }^{1}$, Jean-Daniel Chiche' ${ }^{1}$, Groupe de travail sur la ventilation

${ }^{1}$ Réanimation médicale, hôpital Cochin, Paris, France

Correspondence: Michel Arnaout - michel.arnaout@gmail.com Annals of Intensive Care 2016, 6(Suppl 1):P115

Introduction Despite evidence that daily sedation stops (DSS) by nurses reduce duration of mechanical ventilation (MV), DSS are not part of routine practice in most ICUs. Reasons for slow adoption include scepticism over efficacy, logistical burden in initiating changes, and fear of adverse events during sedation stops. Whether sedation stops are associated with significant adverse events is unknown.

This study aims to evaluate adverse events occurring during 2-h DSS in a medical ICU.

Patients and methods We have retrospectively reviewed charts of all patients mechanically ventilated in the 24-bed MICU of Cochin University Hospital. In the absence of contraindication, all patients underwent a 2-h DSS as part of our combined sedation and weaning protocols. During and at the end of DSS, adverse events need for bolus or infusion of sedative agents, and RASS scores were prospectively recorded in a clinical information management system (Clinisoft ${ }^{\circledR}, \mathrm{GE}$ Healthcare). Data are reported as median and interquartile ranges. A $P<0.05$ was considered significant for all statistical tests performed.

Results Between 03/08 and 04/15, 9799 patients (5760 M/4039 F, 62 [46-76] y.o., SAPS2 43 [29-60], SOFA 5 [2-8]) underwent 12,474 DSS. No significant adverse event occurred in 12,217 (97.9\%) of the DSS Adverse events included patient-ventilator asynchrony ( $n=159$ DSS), $\mathrm{O}_{2}$ desaturation ( $\left.n=119 \mathrm{DSS}\right)$, accidental catheter removal $(n=22)$, fall $(n=9)$. However, mild to moderate agitation occurred during 2483 DSS (19.9\%) and led to prematurely resume continuous sedation $(n=1707,13.7 \%)$ or to administer boluses of sedative agents $(n=776,6.2 \%)$.

\begin{tabular}{lllll}
\hline & BZD & Opiates & Propofol & Others \\
\hline Infusion & $984(57.6 \%)$ & $575(33.7 \%)$ & $127(7.5 \%)$ & $21(1.2 \%)$ \\
Bolus & $182(23.4 \%)$ & $240(30.9 \%)$ & $26(3.3 \%)$ & $241(31 \%)$ \\
\hline
\end{tabular}

Sedatives administered during DSS

Conclusion Overall, daily sedation stops are safe and associated with few adverse events in a setting with nurse-driven monitoring of neurological status. Administration of sedative agents may occasionally be needed to control mild to moderate agitation that may lead to adverse events.

\section{Competing interests None.}

\section{P116}

Delay in awakening after stopping continuous sedation in critically ill patients: incidence, risk factors and impact on ventilator weaning

Magalie Dumas', Celine Chapelle², Benedicte Philippon-Jouve³, Jerome Morel $^{4}$, Pascal Beuret ${ }^{3}$

${ }^{1}$ Réanimation polyvalente urgences, Centre Hospitalier Universitaire, Saint-Priest-en-Jarez, France; ${ }^{2}$ Unité de recherche clinique innovation et pharmacologie, Centre Hospitalier Universitaire, Saint-Priest-en-Jarez, France; ${ }^{3}$ Réanimation polyvalente, Centre Hospitalier, Roanne,

France; ${ }^{4}$ Réanimation polyvalente, Centre Hospitalier Universitaire, Saint-Priest-en-Jarez, France

Correspondence: Magalie Dumas - magaliedumas@hotmail.fr Annals of Intensive Care 2016, 6(Suppl 1):P116

Introduction In critically ill patients, the use of continuous sedation increases the morbidity by lengthening the duration of mechanical ventilation. Numerous studies have shown that maintaining light levels of sedation improves clinical outcome. However, delay in awakening seems ever frequent after stopping sedation. We therefore performed a study to assess the incidence, associated factors and impact on duration of mechanical ventilation of a delay in awakening.

Patients and methods It was a retrospective study, including all consecutive adult patients under mechanical ventilation and continuous sedation for at least $48 \mathrm{~h}$, admitted between January 2012 and September 2013 in two medico-surgical ICUs. Each of these two units used a protocol to titrate the depth of sedation. The primary endpoint was the time to awakening, defined as the time (in hours) between stopping continuous sedation and the appearance of a response to a simple order.

Results Two hundred and thirty-one patients were included in the study, with a mean age of $63 \pm 15$ years, SAPS II at admission $44 \pm 15$, total duration of sedation $147 \pm 161 \mathrm{~h}$. The mean time to awakening was $26 \pm 36 \mathrm{~h}$. Forty-four out of two hundred and thirty-one patients $(19 \%)$ experienced a delay in awakening $\geq 48 \mathrm{~h}$. The patients with a delay in awakening $\geq 48 \mathrm{~h}$ were older $(70 \pm 11$ vs $62 \pm 15, p=0.003)$, had a higher SAPS II ( $54 \pm 15$ vs $42 \pm 14, p<0.0001)$, lower level of creatinine clearance using MDRD ( $54 \pm 41$ vs $84 \pm 53, p=0.0006$ ), lower level of prothrombin ratio $(60 \pm 19$ vs $71 \pm 16, p=0.0003)$, and lower left ventricular ejection fraction at admission $(43 \pm 15$ vs $53 \pm 12$, $p<0.0001)$, they received more frequently renal replacement therapy during the first 2 days ( 45 vs $19 \%, p=0.0004$ ) than patients with time to awakening $<48 \mathrm{~h}$. Concerning the sedation by itself, the patients with a delay in awakening $\geq 48 \mathrm{~h}$ had received more frequently midazolam ( 93 vs $74 \%, p=0.01$ ); conversely, duration of sedation, average dose of sedative and incidence of use of a score to titrate sedation were similar between the two groups. Multivariate analysis identified three factors independently associated with delay in awakening $\geq 48$ h: age $\geq 70$ years (OR 5.84; $95 \% \mathrm{Cl} 2.52-13.5$ ), SAPS II at admission >50 (OR 5.22; $95 \% \mathrm{Cl} 2.31-11.8$ ) and use of midazolam (OR 8.78; $95 \% \mathrm{Cl} 2.32-33.2$ ). Patients with a delay in awakening experienced a longer duration of mechanical ventilation $(404 \pm 257$ vs $206 \pm 191 \mathrm{~h}$, $p=0.0001)$, notably the duration without sedation $(240 \pm 150$ vs $61 \pm 72, p<0.0001)$ than patients with time to awakening $<48 \mathrm{~h}$.

Conclusion Despite the use of protocols to titrate sedation, delay in awakening after stopping continuous sedation remains common, increasing the duration of mechanical ventilation. This study shows that age, severity at admission and use of midazolam expose the patients to a delay in awakening. These data suggest to use non-benzodiazepine sedation in these patients.

Competing interests None. 
Table 28 Prevalence of asynchrony between measurement of neural inspiratory time and visual inspection

\begin{tabular}{lccr}
\hline & With EAdi & Visual inspection & $\boldsymbol{p}$ \\
\hline Asynchrony index (\%) & $24.5(15.0-38.1)$ & $1.5(1-4)$ & $<0.0001$ \\
Ineffective triggering (\%) & $0(0-0.2)$ & $0.1(0-0.6)$ & 0.0410 \\
Double triggering (\%) & $3.1(0.8-8.9)$ & $0.6(0.2-1.5)$ & $<0.0001$ \\
Auto-triggering (\%) & $1.7(0.8-3.8)$ & $0(0-0)$ & $<0.0001$ \\
Short cycle (\%) & $4.2(2.3-9.5)$ & $0.15(0.1-0.5)$ & $<0.0001$ \\
Prolonged cycle (\%) & $5.1(1.4-14.0)$ & $0.1(0-0.3)$ & $<0.0001$ \\
\hline
\end{tabular}

\section{P117}

Risk factors and prognosis impact of decreased patient-ventilator synchrony in mechanically ventilated patients-a prospective study

Côme Bureau ${ }^{1}$, Camille Rolland-Debord ${ }^{1}$, Tymothée Poitou ${ }^{2}$, Marc Clave ${ }^{3}$, Sébastien Perbet ${ }^{4}$, Nicolas Terzi ${ }^{5}$, Achille Kouatchet ${ }^{6}$, Thomas Similowski ${ }^{7}$, Alexandre Demoule ${ }^{1}$, Université Paris 6-Pierre et Marie Curie and Institut National pour la Santé et la Recherche Médicale, UMRS1158, Paris ${ }^{1}$ Intensive care and respiratory division, Pitié-Salpêtrière Hospital, Paris, France; ${ }^{2}$ Paris, Pierre and Marie Curie University, Paris, France; ${ }^{3}$ Service de réanimation polyvalente, Centre Hospitalier Universitaire de Limoges, Limoges, France; ${ }^{4}$ Service de réanimation adultes, C.H.U. Estaing, Clermont-Ferrand, France; ${ }^{5}$ Service de réanimation médicale, Clinique de Réanimation Médicale, Grenoble, France; ${ }^{6}$ Réanimation médicale, Centre Hospitalier Universitaire d'Angers, Angers, France; ${ }^{7}$ Intensive care and respiratory division, Groupe Hospitalier Pitié-Salpêtrière, Paris, France

Correspondence: Côme Bureau - come.bureau@gmail.com Annals of Intensive Care 2016, 6(Suppl 1):P117

Introduction Patient-ventilator asynchrony is defined as a mismatch between patient and ventilator inspiratory and expiratory times and is associated with negative outcomes such as longer duration of mechanical ventilation and higher mortality and tracheotomy rates. To date, 5 major patterns of asynchrony are described. However, there is no universal agreement on the definition of these patterns, resulting in heterogeneity of the prevalence of asynchrony and of the association between asynchrony and outcome. The aim of our study was to prospectively and noninvasively compare the prevalence of patientventilator asynchrony according to two distinct methods. In addition, we determined the factors associated with asynchrony and the prognostic impact of asynchrony.

Patients and methods This is a ancillary study of a multicentre, randomized controlled trial comparing neurally adjusted ventilator assist to pressure support ventilation during the early phase of weaning. Airway flow and pressure and (EAdi) were recorded during 4 sequences of $20 \mathrm{~min}$. Main asynchronies were further quantified according to two methods: (1) using electrical activity of the diaphragm (EAdi) as a reference and (2) based on the only visual inspection of the flow and airway pressure signal traces. The 5 main asynchronies were quantified, and the asynchrony index defined as the proportion of asynchronous cycles was calculated.

Results One hundred and six patients mechanically ventilated for 5 days (1-35) were included, 72 men (68\%), aged 66 (37-86) years, SAPS II 44 (14-96), $60 \%$ had a history of hypoxemic respiratory failure. The distribution of asynchrony and the asynchrony index varied according to the method used to quantify asynchronies (Table 28).

Older age [77 (54-86) vs $66(37-83)$ years, $p=0.02]$ and a higher Charlson score [7.5 (5-11) vs $5(2-9), p=0.004]$ were associated with a higher rate of patient ventilator asynchrony. Asynchrony was not associated with a higher intensive care unit mortality, length of stay or duration of mechanical ventilation. Subgroup analysis showed that of hospital length of stay was significantly lower in patients with a low prevalence of double triggering [28 (10-71) vs 35 (6-96), $p=0.04$ ].

Conclusion The prevalence of patient-ventilator asynchrony varies according to the methods and definitions used to quantify asynchrony, which suggests the need for a consensus statement in asynchrony's definition. In one decade, the prevalence of patient-ventilator asynchrony has decreased, suggesting an improvement in mechanical ventilation practices. Patient-ventilator asynchrony was not associated with a poorer outcome.

\section{Competing interests None.}

\section{P118}

High-flow oxygen therapy through a nasal cannula versus noninvasive ventilation versus in immunocompromised patients with acute respiratory failure

Remi Coudroy ${ }^{1}$, Angéline Jamet ${ }^{1}$, Philippe Petua ${ }^{1}$, René Robert ${ }^{1}$, Jean-Pierre Frat ${ }^{1}$, Arnaud, W. Thille ${ }^{1}$

${ }^{1}$ Réanimation médicale, CHU de Poitiers, Poitiers, France

Correspondence: Remi Coudroy - remi.coudroy@chu-poitiers.fr

Annals of Intensive Care 2016, 6(Suppl 1):P118

Introduction Acute respiratory failure is the leading cause of ICU admission of immunocompromised patients. In the early 2000s, two randomized controlled trials suggested that as compared to standard oxygen therapy, noninvasive ventilation could decrease mortality of immunocompromised patients admitted to ICU for acute respiratory failure. However, the benefits of noninvasive ventilation in immunocompetent patients with acute respiratory failure are still debated. High-flow oxygen therapy through a nasal cannula may offer an alternative in hypoxemic patients, and we recently found, in a randomized controlled trial including 310 patients with acute respiratory failure, that as compared to noninvasive ventilation, high-flow oxygen therapy decreased mortality. In our unit, high-flow oxygen therapy is increasingly used, gradually replacing noninvasive ventilation. Our objective was to compare outcomes of immunocompromised patients admitted to ICU for acute respiratory failure and treated with either high-flow oxygen therapy or noninvasive ventilation as first-line therapy.

Patients and methods All patients admitted for acute respiratory failure to our 15-bed medical ICU of a teaching hospital from January 1, 2007, to December 31, 2014, were retrospectively screened. Among them we included patients with immunosuppression caused by hematological or solid cancer, neutropenia, immunosuppressive therapy such as corticosteroids or oral chemotherapy, and who received highflow oxygen therapy or noninvasive ventilation as first-line therapy. Patients with acute-on-chronic respiratory failure, those treated with standard oxygen alone or needing immediate intubation, and those with a do-not-intubate order were excluded.

Results Over this 8-year period, 1299 patients were admitted for acute respiratory failure including $267(21 \%)$ immunocompromised patients. After excluding 142 patients, 125 patients were analyzed including 52 patients $(42 \%)$ treated with high-flow oxygen therapy and 73 patients $(58 \%)$ with noninvasive ventilation. Baseline characteristics between the 2 groups were not different. Patients were more likely to be treated with noninvasive ventilation in the first period of study (2007-2010): 77 versus $47 \%$ in the second period (2011-2014), $p=0.0015$. Intubation rate was lower in patients treated with high-flow oxygen therapy (33\%) than in those treated with noninvasive ventilation $(53 \%, p=0.03)$. In-ICU mortality and day 28 mortality were significantly lower in patients treated with high-flow oxygen therapy than in those treated with noninvasive ventilation ( 15 vs. $33 \%, p=0.04$; and 17 vs. $38 \%$, respectively, $p=0.02$ ). After adjustment on Simplified Acute Physiology Score II, the use of noninvasive ventilation remained independently associated with day 28 mortality with an adjusted odds ratio of 3.04 [1.366.78], $p=0.005$.

Conclusion In immunocompromised patients admitted to ICU for acute respiratory failure, intubation and mortality rates were lower in patients treated with high-flow oxygen therapy than in those treated with noninvasive ventilation as first-line therapy. The use of noninvasive ventilation was independently associated with day 28 mortality.

Competing interests None. 
P119

The use of dexmedetomidine in patients with noninvasive ventilation: a preliminary study

Hassen Ben Ghezala', Salah Snouda', Rebeh Daoudi', Moez Kaddour ${ }^{1}$ ${ }^{1}$ Teaching department of emergency and intensive care, Hospital Zaghouan, Zaghouan, Tunisia

Correspondence: Hassen Ben Ghezala - hassen.ghezala@gmail.com Annals of Intensive Care 2016, 6(Suppl 1):P119

Introduction Noninvasive ventilation (NIV) is indicated in the treatment of acute respiratory failure associated with acute cardiogenic pulmonary edema (CPE) and exacerbation of chronic obstructive pulmonary disease (COPD). Dexmedetomidine is an alpha 2 adrenoceptor agonist with a unique mechanism of action providing sedation and anxiolysis via receptors within the locus ceruleus. The aim of the study was to show the feasibility and the effectiveness of dexmedetomidine for patients with NIV.

Patients and methods It was a prospective clinical investigation performed in the medical teaching department of emergency and intensive care medicine in Zaghouan in Tunisia from January to June 2015. All patients had acute respiratory failure due to CPE or COPD and required NIV but could not continue NIV sessions because of discomfort and agitation. They had 1 on the Ramsay score and +1 on the Richmond Agitation-Sedation Scale (RASS). We excluded patients with contraindications to NIV. The experimental protocol was approved by our local review board.

Results Twelve patients were enrolled in the investigation, which represents 12/85 (14\%) of all patients who needed NIV in this period.

There were seven men and five women. The mean age was 65 . The indications of NIV were CPE in five cases and hypercapnic exacerbation of COPD in seven cases. All patients received an initial loading dosage of $3 \mathrm{yg} / \mathrm{kg} / \mathrm{h}$ followed by a continuous infusion of $0.5 \mathrm{yg} / \mathrm{kg} / \mathrm{h}$.

At baseline, all patients showed response levels of Ramsay score 1 and RASS score 1.5. Maintenance of Ramsay score at $2.9 \pm 1$ and RASS score at $1.2 \pm 1$ and obtainment of effective sedation were reached in all cases during dexmedetomidine infusion. All settings of NIV were in bilevel-PAP. Baseline measures were made at an average of $6 \mathrm{~h}$ from the time NIV was started. The total infusion time of dexmedetomidine was about $12 \mathrm{~h}$. Heart rate, mean arterial pressure and respiratory rate decreased significantly $(p<0.05)$ after the infusion of dexmedetomidine. Blood gas exchange $\left(\mathrm{PaO}_{2}\right.$ and $\left.\mathrm{PaCO}_{2}\right)$ improved significantly $3 \mathrm{~h}$ after the start of infusion $(p<0.05)$.

All patients were weaned successfully from NIV, and we did not need endotracheal intubation for all our patients. All patients were

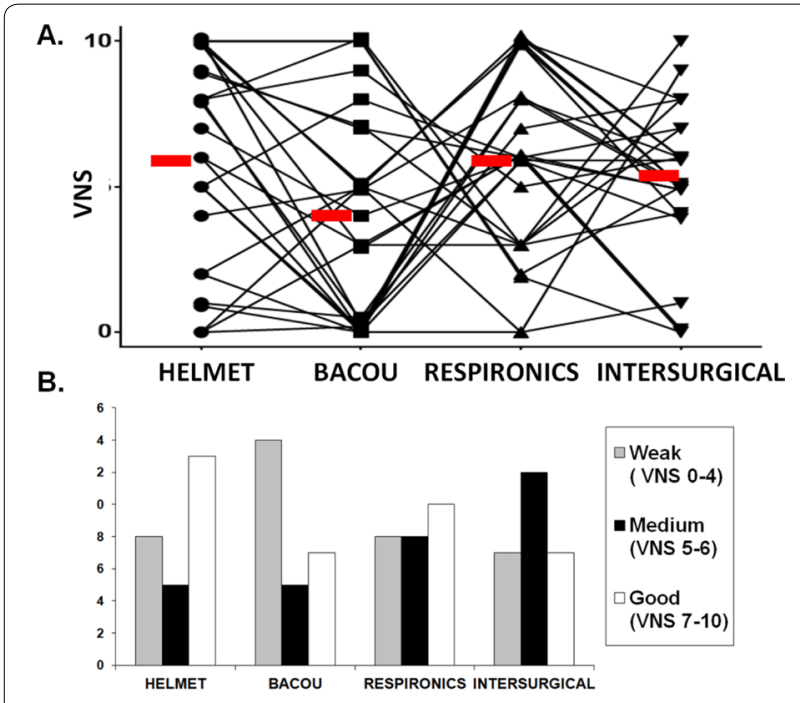

Fig. 32 Comfort assessed by patients for each interface discharged from intensive care and from hospital alive. They could cough and expectorate without assistance. No patients had respiratory infections or presented adverse effects like vomiting and no patients from those who developed bradycardia needed an intervention.

Conclusion It is at our knowledge the first Tunisian study of dexmedetomidine in patients requiring NIV in a medical intensive care department. This study shows that we can use this drug for patients presenting difficulties to perform NIV because of agitation. This drug appears to provide several advantages and safe control for NIV sedation in patients with CPE and COPD. Comparative studies are needed to assess these findings.

\section{Competing interests None.}

\section{P120}

Comparison of tolerance of 4 interfaces for preventive noninvasive ventilation after abdominal surgery in intensive care units assessed by patients and caregivers

Audrey De Jong ${ }^{1}$, Albert Prades ${ }^{1}$, Marion Monnin ${ }^{1}$, Martin Mahul ${ }^{1}$, Marion Basty ${ }^{1}$, Clément Monet ${ }^{1}$, Benjamin Mounet ${ }^{1}$, Emmanuel Futier ${ }^{2}$, Gérald Chanques' ${ }^{1}$, Samir Jaber ${ }^{1}$

'DAR B, Hôpital Saint Eloi, Montpellier, France; ²Dar, CHU Estaing,

Clermont-Ferrand, France

Correspondence: Audrey De Jong - audreydejong@hotmail.fr Annals of Intensive Care 2016, 6(Suppl 1):P120

Introduction The use of noninvasive ventilation (NIV) to prevent acute respiratory failure following abdominal surgery has been associated with a better outcome, when done immediately after extubation (1). However, NIV still fails in one-third of patients. Even if the causes of NIV failure are not totally cleared, patient and interface selection seem to be the major determinant (2). The aim of this study was to compare the tolerance of 4 interfaces used during preventive NIV in intensive care units (ICUs), assessed both by patients and by caregivers.

Patients and methods Patients admitted to the ICU after abdominal surgery for preventive NIV were included in a prospective, monocenter, randomized, crossover study. Four interfaces $\left(\right.$ Helmet $^{\circledR}$, Bacou $^{\circledR}$, Intersurgica ${ }^{\circledR}$, and Respironics ${ }^{\circledR}$ ) were studied. The general patient characteristics and physiological parameters (saturation, respiratory rate) were collected. Patients and caregivers estimated with a visual numeric scale (VNS) tolerance parameters: comfort $(0=$ maximum discomfort, $10=$ perfect comfort), leaks $(0=$ maximum leaks, $10=$ no leak $)$, and communication $(0=$ no communication, $10=$ optimal communication). Positioning $(0=$ very difficult, $10=$ very easy) was estimated by caregivers.

Results Twenty-six patients were included. The mean Simple Acute Physiologic Score II was $37 \pm 10$, the $\mathrm{PCO}_{2}$ of $39 \pm 7$, the $\mathrm{PO}_{2}$ of $88 \pm 22$. The level of comfort, self-assessed by patients, did not significantly differ between interfaces ( $p=0.07$, Fig. 32). However, each interface was not equivalent for a single patient: The correlations between interfaces were either weak, nulls or negatives. The abilities of communication and of positioning, evaluated by patients and caregivers, were significantly increased for the Helmet ${ }^{\circledR}$ interface $(p<0.001)$. Caregivers overestimated the comfort for each interface and the abilities of communication compared to patients $(p<0.008)$. The main physiological parameters did not differ between groups. Conclusion The main result of this study is that comfort of NIV after abdominal surgery did not differ across interfaces. However, comfort differed between interfaces for a given patient: The correlation of comfort between interfaces was weak or null, showing that there is no universal interface, but rather an optimal interface for each patient Moreover, comfort and communication assessment strongly differed between patients and caregivers, caregivers underestimating the inconvenient of interfaces compared with patients. Helmet ${ }^{\circledR}$ mask was the best assessed interface in both patients and caregivers for communication and positioning of the interface.

\section{Competing interests None.}

\section{References}

1. Jaber S, Delay JM, Chanques G, Sebbane M, Jacquet E, Souche B et al. Outcomes of patients with acute respiratory failure after abdominal 
surgery treated with noninvasive positive pressure ventilation. Chest. 2005;128(4):2688-95.

2. Esteban A, Ferguson ND, Meade MO, Frutos-Vivar F, Apezteguia C, Brochard $L$ et al. Evolution of mechanical ventilation in response to clinical research. Am J Respir Crit Care Med. 2008;177(2):170-7.

\section{P121}

Dexmedetomidine, new approach in sedation analgesia: a prospective and observational study of mechanically ventilated patients in adults intensive care units in a university hospital during the first year of use

Audrey Leroux ${ }^{1}$, Xavier Oudinot ${ }^{1}$, Gaëlle Demeilliers-Pfister ${ }^{2}$, Marc Laurent $^{1}$

${ }^{1}$ Pharmacy, CHU - Hôpitaux de Rouen, Rouen, France; ${ }^{2}$ Anesthésie réanimation chirurgicale, C.H.U. Rouen, Rue de Germont, Rouen, France, Rouen, France

Correspondence: Audrey Leroux - audrey.leroux@chu-rouen.fr Annals of Intensive Care 2016, 6(Suppl 1):P121

Introduction Sedation and analgesia are essential in the care of ventilated patients in intensive care. It focuses on patient comfort, calmness, pain relief and facilitates mechanical ventilation. Dexmedetomidine is an a-2 agonist sedative, selective of the $a-2$ central presynaptic receptors. It is indicated in ventilated patients requiring mild to moderate sedation and is an alternative to conventional treatments such as midazolam and propofol. Light sedation, increasingly sought in sedation analgesia, is part of a practice change and is involved in an "arousable" sedation.

Patients and methods The objective of this study was to describe the use of dexmedetomidine in mechanically ventilated patients in medical, surgical, cardiac and neurosurgical intensive care units of a university hospital during the first year of use. All patients receiving dexmedetomidine were included, and data sheets notably including assessment of sedation, indication, dosage, duration and adverse effects were completed in a prospective way.

Results A total of 87 patients were enrolled and 83 were analyzed. The assessment of sedation through RASS (Richmond Agitation-Sedation Scale) score was performed at initiation of dexmedetomidine therapy in 49 patients $(59 \%)$. The definition by the clinician of the RASS target objective appeared in $50 \%$ of the prescriptions. There was a significant relationship between define a RASS target and assess it $(p=0.006)$. Treatment indications were gradual awakening in $44 \%$ (with and without agitation history, $22 \%$ each), respiratory weaning in $39 \%$ and maintaining light sedation to better tolerate orotracheal intubation in case of laryngeal edema in $13 \%$. Initial and mean dosages varied significantly between services $(p<0.001)$. Mean treatment duration was 3.0 days, and mean daily treatment cost was $166 €$. Dexmedetomidine was prescribed as second line in $80 \%$ of patients. Over the first 5 days of treatment, $72 \%$ of RASS scores were within the therapeutic range (RASS 0 to -3 ). Extubation was possible under dexmedetomidine (21 extubations on 25 attempts), and no withdrawal symptoms have been reported. Self-extubation rate was $11 \%$. Agitation rate under dexmedetomidine was $22 \%$, and it could necessitate discontinuation of treatment but was associated with surgical profile $(p=0.03)$. It was also related to gradual awakening with agitation history $(p=0.049)$. The incidence of adverse cardiovascular effects (bradycardia and hypotension) was $23 \%$. There was a significant association between incidence of cardiovascular adverse effects and hypertension $(p=0.016)$. Medical satisfaction was good for the patient's ability to communicate his pain (7.1/10), to cooperate to care (6.9/10) and quality of waking (6.9/10).

Discussion The incidence of cardiovascular adverse events was high, according to literature descriptions. However, these effects, due to the molecule pharmacology, are predictable, dose-dependent, and no clinical consequences were reported. Sedation assessment through RASS score was not always carried out, representing the opportunity to remind good sedation practices and use of validated scale to adjust dosage. Conclusion This descriptive study reflects the complexity and heterogeneity of mechanically ventilated patients. It evaluated dexmedetomidine effectiveness in maintaining a target level of sedation and prescribers adaptation to this new a-2 agonist treatment. This work is an opportunity to formalize dexmedetomidine sedation by protocol.
Competing interests None.

\section{P122}

Pain, confusion, and agitation associated with noninvasive ventilation

Nesrine Boujelbene ${ }^{1}$, Rami Jabla', Fahmi Dachraoui', Islem Ouanes ${ }^{1}$, Saousen Ben Abdallah', Mahdi Marzouk ${ }^{1}$, Asma Hachani ${ }^{1}$, Hedia Hammed ${ }^{1}$, Hend Ben Lakhal ${ }^{1}$, Imen Ben Ali', Chaima Ghribi ${ }^{1}$, Imen Abdellaoui', Lamia Ouanes-Besbes ${ }^{1}$, Fekri Abroug ${ }^{1}$

${ }^{1}$ Réanimation polyvalente, CHU Fattouma Bourguiba, Monastir, Tunisia Correspondence: Islem Ouanes - ouanes.islem@gmail.com Annals of Intensive Care 2016, 6(Suppl 1):P122

Introduction Noninvasive ventilation (NIV) is widely used in ICUs, but could be associated with pain, discomfort, and claustrophobia leading in some patients to NIV failure. The aim of the present study is to measure the frequency and magnitude of pain, confusion, and agitation in a population of ICU patients receiving NIV for COPD exacerbation.

Patients and methods In a prospective cohort study conducted in ICU between May 1, 2015, and August 31, 2015, we systematically recorded a pain visual analogic scale (VAS), CAM-ICU and RASS scales, in consecutively admitted patients requiring NIV for COPD exacerbation. Measures were repeated at H0, H1, H24, H72, Day 5, Day 7 following ICU admission, and at ICU discharge.

Results During the study period, 32 patients were admitted for COPD exacerbation and received NIV [median age 72 years (64-76.5), median $\mathrm{pH} 7.30$ (7.26-7.34), and sex ratio of 2.5]. The following table summarizes variation of the recorded scores during ICU stay.

\begin{tabular}{|c|c|c|c|c|c|c|c|c|}
\hline & $\mathrm{HO}$ & $\mathrm{H} 1$ & $\mathrm{H} 24$ & $\mathrm{H} 72$ & D5 & D7 & $\begin{array}{l}\text { ICU dis- } \\
\text { charge }\end{array}$ & \\
\hline $\begin{array}{l}\mathrm{pH} \\
\text { [median } \\
(\mathrm{IQR})]\end{array}$ & $\begin{array}{l}7.30 \\
(7.26- \\
7.34)\end{array}$ & $\begin{array}{l}7.33 \\
(7.31- \\
7.39)\end{array}$ & $\begin{array}{l}7.36 \\
(7.34- \\
7.40)\end{array}$ & $\begin{array}{l}7.38 \\
(7.35- \\
7.42)\end{array}$ & $\begin{array}{l}7.37 \\
(7.36- \\
7.40)\end{array}$ & $\begin{array}{l}7.38 \\
(7.36- \\
7.41)\end{array}$ & - & $<0.001$ \\
\hline $\begin{array}{l}\text { VAS } \\
\qquad \text { [median } \\
\text { (IQR)] }\end{array}$ & $\begin{array}{l}8 \\
(6.25- \\
8)\end{array}$ & $\begin{array}{l}7.5 \\
(6-8)\end{array}$ & $7(6-8)$ & $6(5-7)$ & $5(5-6)$ & $5(4-5)$ & $4(3-4)$ & $<0.001$ \\
\hline $\begin{array}{l}\text { CAM-ICU } \\
\text { [median } \\
(\mathrm{IQR})]\end{array}$ & $\begin{array}{l}2 \\
(2-2)\end{array}$ & $2(1-2)$ & $1(1-2)$ & $1(0-1)$ & $0(0-1)$ & $0(0-1)$ & $0(0-0)$ & $<0.001$ \\
\hline $\begin{array}{l}\text { RASS } \\
\text { [median } \\
(\mathrm{IQR})]\end{array}$ & $\begin{array}{l}2 \\
(1-3)\end{array}$ & $2(1-2)$ & $\begin{array}{l}1.5 \\
(1-2)\end{array}$ & $1(0-1)$ & $\begin{array}{l}0.5 \\
(0-1)\end{array}$ & $0(0-1)$ & $0(0-0)$ & $<0.001$ \\
\hline
\end{tabular}

At ICU admission, patients suffered from pain (median VAS: 8/10), confused (median CAM-ICU: 2) and agitated (median RASS: 2). These complaints have gradually improved in an inverse way than $\mathrm{pH}$ Conclusion Our study shows that pain, confusion, and agitation were common in patients receiving NIV. These complaints improve in parallel with clinical recovery.

\section{Competing interests None.}

\section{P123}

Impact of metformin on prognosis of ICU patients

Sebastien Jochmans ${ }^{1}$, Jean-Emmanuel Alphonsine ${ }^{1}$, Ly Van Vong ${ }^{1}$, Nathalie Rolin ', Oumar Sy ${ }^{1}$, Jean Serbource-Goguel ${ }^{1}$, Jonathan Chelly², Olivier Ellrodt ${ }^{1}$, Mehran Monchi ${ }^{1}$, Christophe Vinsonneau ${ }^{1}$

${ }^{1}$ Service de Réanimation, Centre Hospitalier Marc Jacquet, Melun, France; ${ }^{2}$ Médecine intensive, Centre Hospitalier Marc Jacquet, Melun, France Correspondence: Sebastien Jochmans - sebastien.jochmans@ ch-melun.fr Annals of Intensive Care 2016, 6(Suppl 1):P123

Introduction The impact of preadmission metformin treatment remains controversial regarding lactate levels, intensity of supportive therapies and outcome in all ICU patients and particularly in patients with septic shock $[1,2]$. 
Patients and methods We performed a retrospective study of patients with diabetes admitted to our ICU over a period of 3 years. We focused on lactate levels, supportive therapies and outcome whether or not they had preadmission metformin. Then, we analyzed the subgroup of septic shock patients who is most at risk of tissue hypoperfusion and use of heavy supportive treatments.

Results Six hundred and thirty-five diabetics were included from 3871 admissions: age 71 [61-79], SAPS 239 [31-52], septic shock 131 (20.6) [pneumonia 71 (54.2), urinary sepsis $16(12.2)]$.

\begin{tabular}{|c|c|c|c|}
\hline & $\begin{array}{l}\text { No met- } \\
\text { formin }\end{array}$ & Metformin & $P$ \\
\hline Population ( $n, \%)$ & $395(62.2)$ & $240(37.8)$ & - \\
\hline Age & $73[63-80]$ & $68[60-78]$ & 0.03 \\
\hline SAPS 2 & $40[32-52]$ & $38[29-51]$ & 0.41 \\
\hline Renal failure $(n, \%)$ & $268(67.8)$ & $124(51.7)$ & $<0.001$ \\
\hline $\operatorname{RRT}(n, \%)$ & $72(18.2)$ & $41(17.1)$ & 0.71 \\
\hline INR & $\begin{array}{l}1.85 \pm 1.74 \\
{[1.67-2.03]}\end{array}$ & $\begin{array}{r}1.74 \pm 1.43 \\
{[1.68-1.8]}\end{array}$ & 0.42 \\
\hline Invasive ventilation $(n, \%)$ & $139(35.2)$ & $91(37.9)$ & 0.48 \\
\hline Vasopressors $(n, \%)$ & $136(34.4)$ & $93(38.8)$ & 0.27 \\
\hline $\mathrm{HCO} 3(\mathrm{mmol} / \mathrm{L})$ & $\begin{array}{l}21.9 \pm 8 \\
{[21.1-22.7]}\end{array}$ & $\begin{array}{l}19.9 \pm 7.7 \\
{[19-20.9]}\end{array}$ & $<0.005$ \\
\hline Lactate (mmol/L) & $\begin{array}{l}1.78 \pm 2.02 \\
{[1.58-1.98]}\end{array}$ & $\begin{array}{l}3.07 \pm 3.3 \\
{[2.65-3.49]}\end{array}$ & $<0.001$ \\
\hline $\begin{array}{l}\text { Lactate without renal } \\
\text { failure }(\mathrm{mmol} / \mathrm{L})\end{array}$ & $\begin{array}{l}1.39 \pm 0.81 \\
{[1.25-1.53]}\end{array}$ & $\begin{array}{l}2.18 \pm 1.82 \\
\quad[1.85-2.52]\end{array}$ & $<0.001$ \\
\hline ICU LOS (days) & $6[4-10]$ & $6[3-9]$ & 0.95 \\
\hline ICU death $(n, \%)$ & $74(19.7)$ & $41(17.1)$ & 0.60 \\
\hline Septic shock $(n, \%)$ & $79(60.3)$ & $52(39.7)$ & - \\
\hline Age & $62[64-78]$ & $66[61-78]$ & 0.13 \\
\hline SAPS 2 & $59[40-68]$ & $57[46-68]$ & 0.11 \\
\hline Renal failure $(n, \%)$ & $62(78.5)$ & $42(80.8)$ & 0.75 \\
\hline $\operatorname{RRT}(n, \%)$ & $24(30.4)$ & $27(51.9)$ & 0.02 \\
\hline INR & $\begin{array}{l}2.62 \pm 2.40 \\
{[2.09-3.15]}\end{array}$ & $\begin{array}{l}1.89 \pm 1.40 \\
{[1.49-2.28]}\end{array}$ & 0.11 \\
\hline Invasive ventilation $(n, \%)$ & $56(70.9)$ & $40(76.9)$ & 0.44 \\
\hline $\begin{array}{l}\text { Maximum dose of nor- } \\
\text { epinephrine }(\mathrm{mg} / \mathrm{h})\end{array}$ & $\begin{array}{l}3.23 \pm 4.28 \\
{[2.21-4.24]}\end{array}$ & $\begin{array}{l}4.38 \pm 4.57 \\
{[3.04-5.72]}\end{array}$ & 0.03 \\
\hline $\begin{array}{l}\text { Duration norepineph- } \\
\text { rine (h) }\end{array}$ & 48 [19-71.25] & $36[15-59.25]$ & 0.25 \\
\hline $\mathrm{HCO} 3(\mathrm{mmol} / \mathrm{L})$ & $\begin{array}{l}19.8 \pm 7.7 \\
{[18.1-21.5]}\end{array}$ & $\begin{array}{l}15.3 \pm 8.3 \\
{[13.1-17.6]}\end{array}$ & $<0.002$ \\
\hline Lactate (mmol/L) & $\begin{array}{l}2.78 \pm 3.61 \\
{[1.99-3.58]}\end{array}$ & $\begin{array}{l}5.78 \pm 5.0 \\
{[4.42-7.14]}\end{array}$ & $<0.001$ \\
\hline ICU LOS (days) & $9[6-19]$ & $7[4-13]$ & 0.16 \\
\hline ICU death $(n, \%)$ & $31(39.2)$ & $20(38.5)$ & 0.93 \\
\hline Hospital death $(n, \%)$ & $33(41.8)$ & $20(38.5)$ & 0.63 \\
\hline
\end{tabular}

Values are mean $\pm \mathrm{SD}[95 \% \mathrm{CI}]$, median [IQR], $n(\%)$

$I C U$ intensive care unit, $L O S$ length of stay, INR international normalized ratio, $R R T$ renal replacement therapy

Conclusion Metformin is associated with higher lactate level whatever the kidney function. In septic shock, metformin is associated with higher level of catecholamines without any significant impact on mortality.

Competing interests None.

\section{References}

1. Christiansen, C. F. et al. Preadmission metformin use and mortality among intensive care patients with diabetes: a cohort study. Crit. Care Lond. Engl. 17, R192 (2013)

2. Salpeter, S. R., Greyber, E., Pasternak, G. A. \& Salpeter, E. E. Risk of fatal and nonfatal lactic acidosis with metformin use in type 2 diabetes mellitus. Cochrane Database Syst. Rev. CD002967 (2010). doi:10.1002/14651858. CD002967.pub4.

P124

Serum cholinesterase activity in the diagnosis of septic shock due to bacterial infections: a new specific biomarker of sepsis

Mabrouk Bahloul ${ }^{1}$, Turki Olfa ${ }^{1}$, Najeh Baccouche ${ }^{2}$, Kais Regaieg ${ }^{2}$, Chtara Kamilia' ${ }^{1}$, Benhamida Chokri ${ }^{1}$, Hedi Chelly ${ }^{2}$, Mounir Bouaziz ${ }^{2}$

${ }^{1}$ Réanimation polyvalente, Faculté de médecine de Sfax, Sfax, Tunisia; ${ }^{2}{ }^{2} \mathrm{cu}$, CHU Habib Bourguiba, Sfax, Tunisia

Correspondence: Mabrouk Bahloul - bahloulmab@yahoo.fr

Annals of Intensive Care 2016, 6(Suppl 1):P124

Introduction We aimed to investigate whether serum cholinesterase (SchE) activity could be helpful for the diagnosis of septic shock and to evaluate its utility in comparison with procalcitonin (PCT) and C-reactive protein (CRP).

Patients and methods A prospective single-blinded study conducted in intensive care unit of university hospital. Patients were classified as having cardiogenic shock, septic shock or haemorrhagic shock. Moreover, we have included a control group without hemodynamic instability and without sepsis. For all included patients, blood samples were obtained (Serum ChE, procalcitonin and CRP) at the same time.

Results The comparison of sepsis markers between all groups showed that the mean value of PCT and CRP was significantly higher in patients with septic shock group. However, serum chE activity was significantly lower in this last group. Serum chE activity was found to be the finest marker for the diagnosis of septic shock in comparison with PCT and CRP. In fact, a SchE activity $\leq 4000 \mathrm{UI} / \mathrm{I}$ was associated with the diagnosis of septic shock with sensitivity at $78 \%$, specificity at $89 \%$, a negative predictive value at $97 \%$ and positive predictive value at $65 \%$. However, despite the fact that the low value of ChE activities was found to be well correlated with the diagnosis of septic shock, it was not associated with a poor outcome (death) in multivariate analysis.

Discussion The SchE activity level was significantly decreased in patients with septic shock and was not correlated with morality.

Conclusion The SchE activity level was significantly decreased in patients with septic shock and was not correlated with morality. Our results suggest that SchE activity might play an important role in the positive diagnosis of septic shock. Others studies are needed on this subject.

Competing interests None.

P125

Is hyperoxaemia a rare event during septic shock patients management?

Amélie Trichot ${ }^{1}$, Gwenhaël Colin ${ }^{1}$, Jean-Baptiste Lascarrou², Isabelle Vinatier ${ }^{2}$, Konstantinos Bachoumas' ${ }^{2}$, Jean-Claude Lacherade ${ }^{2}$, Maud Fiancette ${ }^{1}$, Aurélie Joret ${ }^{1}$, Aihem Yehia ${ }^{2}$, Christine Lebert ${ }^{2}$, Matthieu Henry-Laguarrigue ${ }^{2}$, Laurent Martin-Lefèvre ${ }^{1}$

${ }^{1}$ Réanimation polyvalente, Centre Hospitalier Départemental - site de La Roche-sur-Yon, La Roche-sur-Yon, France; ${ }^{2}$ Réanimation, Centre Hospitalier Départemental - site de La Roche-sur-Yon, La Roche-sur-Yon, France

Correspondence: Gwenhaël Colin - gwenhael.colin@chd-vendee.fr Annals of Intensive Care 2016, 6(Suppl 1):P125

Introduction There is increasing evidence of hyperoxaemia-associated harmful effect in different conditions, such as stroke, myocardial infarction and cardiac arrest. All these pathological situations induce ischaemia-reperfusion injuries. A major cause of ICU admission and ischaemia-reperfusion injury is septic shock. We wanted to investigate whether or not hyperoxemia was common in mechanically ventilated patients with septic shock in our ICU.

Patients and methods We performed a single-centre retrospective study. 
Inclusion criteria: We screened all patients with the diagnosis code of septic shock admitted in our ICU from January 1, 2010, to December 31, 2013.

Exclusion criteria: Patients were excluded if they were younger than 18 , if they did not actually have septic shock, if they were not mechanically ventilated, if they received less than $0.25 \mathrm{mg} / \mathrm{kg} / \mathrm{min}$ of norepinephrine, if they deceased before the 24th hour, if they were already included in this study or if less than 3 blood gases analysis was available during the first $72 \mathrm{~h}$ of their ICU care.

For each included patient, we estimated, in the first $72 \mathrm{~h}$ of ICU care, a hourly arterial partial pressure of oxygen $\left(\mathrm{PaO}_{2}\right)$ based on the one hand on $\mathrm{PaO}_{2} / \mathrm{FiO}_{2}$ ratio (assuming a linear variation of this ratio between two blood gas analysis) and on the other hand on the automatic report of $\mathrm{FiO}_{2}$ in our electronic chart. According to the British Thoracic Society guidelines (1), the hyperoxaemia threshold was $120 \mathrm{mmHg}$. We differentiated mild hyperoxaemia $(120-200 \mathrm{mmHg}$ ), moderate hyperoxaemia $(200-300 \mathrm{mmHg}$ ) and severe hyperoxaemia $(>300 \mathrm{mmHg}$ ). We also investigated whether patients were exposed to hypoxaemia $(<60 \mathrm{mmHg})$.

Results During the study period, 3866 patients were admitted in our ICU. A diagnosis code of septic shock was associated with 646 admissions. Two hundred and ten patients did not meet the exclusion criteria and were included in this study. There was a majority of medical patients $(67.1 \%)$, with a median SAPS2 of 65.5 (IQ1 57; IQ3 74) and with a median age of 68.5 years (IQ1 59.25; IQ3 75). The ICU mortality was $36.7 \%$. We were able to estimate $13,836 \mathrm{PaO}_{2}$ in the first $72 \mathrm{~h}$ of ICU care for septic shock. The proportion of hyperoxaemia was $19.9 \%$ (17.3\% of mild hyperoxaemia, $2.1 \%$ of moderate hyperoxaemia and $0.5 \%$ of severe hyperoxaemia). By contrast, hypoxemia occurred only for $4.5 \%$ of all the $\mathrm{PaO}_{2}$ estimates.

Hyperoxaemia was more frequent during the first hours of management $(26.2 \%$ in the first $24 \mathrm{~h}$ and $34.5 \%$ in the first $6 \mathrm{~h})$. Moreover, $82.9 \%$ of the included patients experienced at least one episode of hyperoxaemia.

Discussion In this single-centre retrospective study, hyperoxaemia is four times more frequent than hypoxaemia. Even if in most of the cases, the level of hyperoxaemia remains mild, a wide majority of patients are exposed. Whether or not hyperoxaemia may influence the outcome remains to be determined.

Conclusion In this single-centre retrospective study, hyperoxaemia exposure is not a rare event during the management of septic shock patients, especially in the first hours of ICU care. Since it has been established that hyperoxaemia is associated with a poor outcome in cardiac arrest, stroke and myocardial infarction, a possible association between hyperoxaemia and outcome should also be investigated in septic shock.

\section{Competing interests None.}

\section{Reference}

1. O'Driscoll BR and al BTS guideline for emergency oxygen use in adult patients. Thorax. 2008 Oct;63 Suppl 6:vi1-68.

P126

\section{Myocardial depressant factor during human septic shock:} screening of twenty-three cytokines and chemokines

Keyvan Razazi ${ }^{1}$, Florence Boissier ${ }^{2}$, Mathieu Surenaud ${ }^{3}$, Alexandre Bedet ${ }^{2}$, Nicolas de Prost ${ }^{2}$, Aurelien Seemann ${ }^{2}$, Christian Brun-Buisson ${ }^{2}$, Sophie Hüe $^{4}$, Armand Mekontso-Dessap ${ }^{2}$

${ }^{1}$ Réanimation médicale, Hospital Henri Mondor, Créteil, France; ${ }^{2}$ Réanimation Médicale, Hôpital Henri Mondor, Créteil, France; ${ }^{3}$ Inserm u955, Hôpital Henri Mondor, Créteil, France; ${ }^{4}$ Laboratoire d'immunologie, Hôpital Henri Mondor, Créteil, France

Correspondence: Keyvan Razazi - keyvan.razazi@aphp.fr Annals of Intensive Care 2016, 6(Suppl 1):P126

Introduction The mechanisms of myocardial depression during sepsis are not fully understood, but the hypothesis of a myocardial depressant factor (MDF) has been suggested. Our aim was to scrutinize the role of a large panel of cytokines/chemokines using methodological approaches derived from functional genomics.
Patients and methods Echocardiographies were performed during the first 3 days of septic shock to assess left ventricle ejection fraction (LVEF). Patients with immunosuppression and those with chronic heart failure were excluded. Blood samples were collected on the first day of septic shock, and plasma concentration of 23 cytokines/chemokines was assessed using a multi-analyte Milliplex human cytokine kit (Millipore Corporation, Billerica, MA, USA). Analyses were corrected for false discovery rate.

Results Seventy-four patients were prospectively included. The main source of infection was the lung (49\%). Median SOFA score was 10 [7-12]. Thirty patients (40 \%) died in ICU. Twenty-four (32\%) patients had left ventricular hypokinesia (LVEF < $45 \%), 32(43 \%)$ were normokinetic (LVEF between 45 and $60 \%$ ) and $18(24 \%)$ were hyperkinetic (LVEF $>60 \%$ ). Plasma concentration (as assessed by fluorescence intensity) of the majority of cytokines/chemokines was above normal values and closely correlated with each other. Hierarchical clustering identified three main clusters of cytokines/chemokines involving the following pathways: inflammatory response (Th1-Th17), innate immunity, and cell death, respectively. Matrix correlation and principal component analysis revealed that septic myocardial dysfunction was closely related to the cluster of innate immunity.

Conclusion Our comprehensive analysis did not identify a single MDF during septic shock. Cytokines/chemokines involved in innate immunity were associated with septic myocardial dysfunction.

Competing interests None.

\section{P127}

Plasma of septic shock patients modifies in vitro mitochondrial respiration of human PBMC as well as lymphocytes and monocytes cell lines

Raphaël Clere-Jehl' ${ }^{1}$, Julie Boisramé-Helms ${ }^{1}$, Anne-Laure Charles ${ }^{2}$, Pierrick Leborgne $^{3}$, Xavier Delabranche', Bernard Geny ${ }^{2}$, Ferhat Meziani ${ }^{1}$, Pascal Bilbault ${ }^{3}$

${ }^{1}$ Réanimation médicale, Nouvel Hôpital Civil, CHU Strasbourg, Strasbourg, France; ${ }^{2}$ Equipe d'accueil 3072, laboratoire de physiologie, Faculté de Médecine, Strasbourg, France; '3ervice d'accueil des urgences, Nouvel Hôpital Civil, CHU Strasbourg, Strasbourg, France

Correspondence: Raphaël Clere-Jehl - raphael.clere@chru-strasbourg.fr Annals of Intensive Care 2016, 6(Suppl 1):P127

Introduction Peripheral blood mononuclear cells (PBMCs) might play a role during compensatory anti-inflammatory response syndrome (CARS) following septic shock. This study investigated the time course of septic shock-induced mitochondrial respiration alterations of human PBMC.

Patients and methods Septic shock patients admitted in intensive care unit (ICU) of a university hospital were included following the 2012 Surviving Sepsis Campaign diagnosis criteria and compared to healthy volunteers. After isolating PBMC using Ficoll separation medium, we evaluated PBMC mitochondrial capacities by measuring oxygen consumption in several plasma media, following two steps: firstly a "cellular" step and secondly a "plasmatic" step. The cellular step of our work measured septic PBMC mitochondrial respiration at days 1, 2 and 7, compared with healthy PBMC. Two plasma treating methods were used at this cellular step: fresh homologous plasmaplasma from same donor used immediately — and frozen heterologous plasma-frozen plasma from another donor from same group and time. The plasmatic step compared effect of day 1 septic plasma versus non-septic plasma on oxygen consumption of three types of cells: septic PBMC at day 1, U937 and CEM cell lines. All oxygen consumption measures, expressed in $\mathrm{pmol} / \mathrm{s} / \mathrm{million}$ cells, were done using high-resolution oxygraphy (oxygraph-2k, Oroboros Instruments, Austria). We assessed also PBMC apoptosis by annexin $\mathrm{V}$, quantified with flow cytometry, plasma $\mathrm{pH}$, and superoxide $\left(\mathrm{O}_{2}^{--}\right)$production in whole peripheral blood using electron spin resonance.

Results We investigated 22 septic shock patients and 12 healthy volunteers. Our cellular step showed an increased mitochondrial respiration of septic PBMC compared to healthy volunteers: at days 1,2 and 7 PBMC from septic shock patients consumed, respectively, $8.18 \pm 2.77$, $9.50 \pm 1.60$ and $10.72 \pm 2.34 \mathrm{pmol} \mathrm{O}_{2} / \mathrm{s} /$ millions cells, significantly 
higher than healthy volunteers at $4.56 \pm 1.29 \mathrm{pmol} \mathrm{O}_{2} / \mathrm{s} /$ millions cells $(p<0.01)$, in fresh homologous plasma. Similar results were observed in frozen heterologous plasma, showing septic PBMC oxygen consumption of $9.65 \pm 6.34,11.32 \pm 5.27$ and $11.65 \pm 5.28$ pmol, respectively, at days 1, 2 and 7, significantly higher $(p<0.05)$ than healthy PBMC at $5.45 \pm 0.98 \mathrm{pmol} \mathrm{O}_{2} / \mathrm{s} /$ millions cells. The plasmatic step showed an inhibitory effect of day 1 septic plasma compared with non-septic plasma: On day 1 septic PBMC, we observed a nonsignificant higher oxygen consumption in healthy plasma versus septic shock plasma from day $1(14.02 \pm 10.12$ vs $9.65 \pm 6.34 ; p=0.13)$. On both cell lines, oxygraphy showed a significant increase of oxygen consumption in healthy versus septic shock plasma from day 1: $13.11 \pm 0.59$ versus $9.67 \pm 1.77(p=0.002)$ in U937 cells, and $7.54 \pm 1.39$ versus $6.87 \pm 1.11(p=0.03)$ in CEM cells. No significant differences in terms of plasma $\mathrm{pH}$, superoxide $\left(\mathrm{O}_{2}^{--}\right)$production or lympho-monocytes apoptosis were observed in healthy or septic shock patients.

Conclusion Our study showed a significant increase of lympho-monocytes mitochondrial respiration of septic patients, from day 1 to day 7 , compared with healthy volunteers, whereas the septic plasma has an inhibitory effect on mitochondrial respiration, suggesting a mitochondrial compensatory process, probably by mitochondrial biogenesis, in response to sepsis process. The inhibitory role of septic plasma points out that this initial sepsis insult on mitochondrial respiration seems to be mediated by circulating plasma factors, which are not determined yet. Further investigations will allow us to better understand the involved mechanisms in both initial mitochondrial injury and secondary mitochondrial compensation during CARS.

\section{Competing interests None.}

\section{P128}

Assessment of skin endothelial functions during severe infections

Simon Bourcier ${ }^{1}$, Claire Pichereau', Vincent Dubée', Gabriel Lejour', Jean-Luc Baudel', Arnaud Galbois' ${ }^{1}$, Jalel Tahiri', Naïke Bigé ', Eric Maury' Bertrand Guidet ${ }^{1}$, Hafid Ait-Oufella ${ }^{1}$

${ }^{1}$ Réanimation médicale, Hôpital Saint-Antoine, Paris, France

Correspondence: Simon Bourcier - simon_bourcier@hotmail.com

Annals of Intensive Care 2016, 6(Suppl 1):P128

Introduction Mottling extension and capillary refill time have been identified as strong predictive factors during septic shock. Based on experimental and sublingual capillaroscopy-based studies, we speculated that both skin disorders are due to skin hypoperfusion and local endothelial dysfunction. The goal of this study was to confirm this hypothesis and to investigate skin endothelial functions in ICU patients admitted for severe infections and to compare forearm and knee skin area.

Patients and methods In a prospective monocentric observational study including patients with severe sepsis and septic shock in an 18 -bed ICU in a teaching hospital $6 \mathrm{~h}$ after resuscitation, we recorded global hemodynamic and organ perfusion parameters, demographic data and severity of illness evaluated by the sequential organ failure assessment (SOFA) score. Skin endothelial functions were evaluated by local administration of acetylcholine by iontophoresis. Iontophoresis is a technique that uses an electric current to transport drugs in ionic form across the skin. In combination with laser Doppler, the vascular reaction was studied by measuring changes in microcirculatory blood flow. Global skin microcirculatory blood flow was measured at the baseline and monitored after acetylcholine challenge during $10 \mathrm{~min}$. The peak of skin blood flow and the area under the curve on both forearm and knee sites were computed.

Results Thirty-one patients were included, 8 with severe sepsis and 23 patients with septic shock. Six patients died of multi-organ failure (MOF). Knee endothelial functions decreased significantly with sepsis severity: peak value 56 (43-61) in severe sepsis versus 17 (11-45) in septic shock survivors and 8 (5-17) in MOF dead patients (KruskalWallis test, $P=0.01)$, area under the curve $20,494(16,853-29,586)$ in severe sepsis versus $7791(5762-15,892)$ in septic shock survivors and 3847 (2664-7807) in MOF dead patients (Kruskal-Wallis test $P=0.009$ ). After exclusion of 3 patients with dark skin, we compared skin microcirculatory parameters in septic shock patients according to the absence $(n=8)$ or the presence of mottling $(n=12)$. Endothelial dysfunction was much more pronounced in mottling skin in comparison with non-mottling skin: basal value 7 (6-8) versus 5 (4-6) (Mann-Whitney test, $P=0.01)$, peak value $17(8-52)$ versus $7(5-15)$ (Mann-Whitney test, $P=0.05$ ), area under the curve 7981 (424319,612 ) versus 3302 (2643-6531) (Mann-Whitney test, $P=0.03$ ). Noteworthy, endothelial function assessed on the forearm area was not different between mottling and non-mottling group. Finally, iontophoresis-derived endothelial parameters were highly correlated with SOFA score: Knee basal value $P=0.005, r=-0.53$, knee peak value knee $P<0.001, r=-0.62$, area under the curve $P=0.001, r=-0.61$.

Conclusion Skin endothelial functions are markedly impaired during severe infections. These heterogenous alterations are more pronounced in area with mottling.

\section{Competing interests None.}

\section{P129}

Toe-to-Room temperature gradient correlates with tissue perfusion and predicts outcome during septic shock

Simon Bourcier ${ }^{1}$, Claire Pichereau', Pierre Yves Boêlle ${ }^{2}$, Nemlaghi Safaa ${ }^{3}$, Vincent Dubée', Gabriel Lejour', Jean-Luc Baudel'1, Arnaud Galbois ${ }^{3}$, Jean-Rémi Lavillegrand ${ }^{1}$, Naïke Bigé1, Jalel Tahiri', Eric Maury ${ }^{1}$, Bertrand Guidet ${ }^{1}$, Hafid Ait-Oufella'

${ }^{1}$ Réanimation médicale, Hôpital Saint-Antoine, Paris, France; ${ }^{2}$ Service de santé publique, Hôpital Saint-Antoine, Paris, France; ${ }^{3}$ Réanimation polyvalente, Hospital Center Private Claude Galien, Quincy-sous-Sénart, France

Correspondence: Simon Bourcier - simon_bourcier@hotmail.com

Annals of Intensive Care 2016, 6(Suppl 1):P129

Introduction Microcirculatory disorders leading to tissue hypoperfusion play a central role in the physiopathology of organ failure during septic shock and have been identified as strong outcome predictive factors. It is therefore important to develop accurate parameters at the bedside to evaluate tissue perfusion. The objectives were to evaluate whether Central-to-Toe, Central-to-Knee, Toe-to-Room and Knee-to-Room temperature gradients are related to sepsis severity and could predict ICU outcome in critically ill patients with severe infection.

Patients and methods This is a prospective monocentric observational study including patients with severe infections. During a 10-month period, all patients, older than 18 years, admitted for a severe infection were included. There were no exclusion criteria. Patients were classified as sepsis, severe sepsis and septic shock according to 2001 International Sepsis Definitions conference. Patients were included at admission for patients with sepsis and severe sepsis, and when vasopressors were started (within $24 \mathrm{~h}$ of admission) for patients with septic shock. General characteristics of the patients were recorded: demographic data, diagnoses, severity of illness evaluated by the sequential organ failure assessment (SOFA) score (within the $6 \mathrm{~h}$ of inclusion) and Simplified Acute Physiologic Score II (SAPS II). We collected data that reflected macrocirculation and organ perfusion at inclusion, corresponding to admission or vasopressors infusion for septic shock, and at 6,12 and $24 \mathrm{~h}$ after inclusion. Global hemodynamic status was assessed using mean arterial blood pressure, heart rate and cardiac index. Microcirculatory dysfunction and organ perfusion were assessed through arterial lactate level, urinary output, mottling score and knee capillary refill time. Toe and knee skin temperature were measured using skin temperature sensor applied at patient inclusion and let in place during the first $24 \mathrm{~h}$. Central body temperature was measured with an electronic rectal thermometer, and room temperature was also recorded.

Results One hundred and twenty-five patients were enrolled, 22 sepsis, 40 with severe sepsis and 63 with septic shock. Temperature 
gradients were significantly different according to infection severity; Central-to-Toe and Central-to-Knee temperature gradients increased with disease severity, but Toe-to-Room and Knee-to-Room temperature gradients decreased. During septic shock, mortality due to multi-organ failure (MOF) occurred in $31 \%$ of the cases. After initial resuscitation, Toe-to-Room temperature gradient was significantly lower in the MOF death group than in the survivors $(-0.3[-1.0 ;+1.1]$ vs $\left.+2.4[+0.3 ;+5.8]^{\circ} \mathrm{C}, P<0.01\right)$ and differences increased during the first $24 \mathrm{~h}$. There was no correlation between Toe-to-Room temperature gradient and cardiac index or vasopressor doses. However, Toe-toRoom temperature gradient was related to tissue perfusion parameters like arterial lactate level $(r=-0.42, P=0.001)$, urinary output $(r=0.29, P=0.02)$, capillary refill time $(r=-0.39, P=0.004)$ and mottling score $(P=0.004)$.

Conclusion Toe-to-Room temperature gradient reflects tissue perfusion at the bedside and is a strong prognosis factor in septic shock patients.

\section{Competing interests None.}

\section{P130}

Determination of HLA-DR expression on monocytes in heart recipients and clinical outcome correlation

Sylvie Paulus ${ }^{1}$, Claire Flamens ${ }^{1}$, Guillaume Monneret ${ }^{2}$, Julie Demaret ${ }^{3}$, Jean Neidecker ${ }^{4}$

${ }^{1}$ Anesthésie-réanimation, Hopital Louis Pradel, Bron, France; ' 2 Laboratoire d'immunologie, Hôpital Édouard Herriot, Lyon, France; ${ }^{3}$ Cellular immunology laboratory, Hospices Civils de Lyon - Groupement Hospitalier Edouard Herriot, Lyon, France; ${ }^{4}$ Anesthésie-réanimation, Groupement Hospitalier Est, Hospices Civils de Lyon, Bron, France

Correspondence: Sylvie Paulus - sypaulus@gmail.com

Annals of Intensive Care 2016, 6(Suppl 1):P130

Introduction In intensive care unit, patients display immune failure associated with an inflammatory response usually after surgery or sepsis, and a low expression of HLA-DR on monocytes (mHLA-DR) is a predictive factor of nosocomial infections. After heart transplantation ( $(\mathrm{TT} x)$, recipients' immunosuppressive therapy increases this sepsis risk. The primary endpoint of the study was to observe a correlation between $M H L A-D R$ value and clinical outcome.

Patients and methods Eight recipients were prospectively included in ICU, 5 with septic risk: 3 with left ventricular assist device (LVAD), 1 with biventricular assist device and 1 with extracorporeal life support. Infection was controlled in LVAD patients. Expression of mHLA-DR was measured by flow cytometry on whole blood. A single measure was performed between day 12 and day 30 post-HTx. Immune failure was classified according to $\mathrm{mHLA}-\mathrm{DR}$ expression: severe if antibody bound per cell $(A B / C)<5000$, marked if $5000-10,000$, moderate if $10,000-$ 15,000 . Immune response was considered normal up to 20,000 with a grey zone between 15,000 and 20,000. Immunosuppressive therapy was standard. Follow-up was 2 months.

Results Five HTx recipients had marked immune failure, and they present neither sepsis nor rejection, patients with LVAD belonged to this group. Two patients had severe immune failure (2700 and $4400 \mathrm{AB} / \mathrm{C}$ ) and presented nosocomial infections. One patient without immune failure $(17,755 \mathrm{AB} / \mathrm{C})$ presented acute antibody-mediated rejection. All patients were discharged from hospital.

Conclusion The grading of immune failure according to a single measure of monocytes HLA-DR expression between day 12 and day 30 after $H T x$ is clearly correlated with clinical outcome, infection or rejection. It allows to adapt the immunosuppressive therapy to individual patient immune status. Immune monitoring with several measures $\mathrm{mHLA}$-DR is probably useful in case of sepsis, and it should be investigated in further study.

\section{Competing interests None.}

\section{Reference}

1. Low monocyte human leukocyte antigen-DR is independently associated with nosocomial infections after septic shock. Landelle C1, Lepape A, Monneret G. Intensive Care Med. 2010 Nov;36(11):1859-66.
P131

Nosocomial infections in neonates under ECMO

Jerome Rambaud ${ }^{1}$, Cecile Allioux' ${ }^{1}$, Romain Guedj ${ }^{2}$, Isabelle Guellec ${ }^{1}$, Julia Guilbert', Pierre-Louis Leger', Maryne Demoulin', Amélie Durandy', Sandrine Jean ${ }^{1}$, Ricardo Carbajal

${ }^{1}$ Réanimation néonatale et pédiatrique, Hôpital Trousseau, Paris, France; ${ }^{2}$ Urgences pédiatriques, Hôpital Trousseau, Paris, France

Correspondence: Jerome Rambaud - jerome.rambaud@aphp.fr Annals of Intensive Care 2016, 6(Suppl 1):P131

Introduction Neonatal ECMO is used as last resort in refractory hypoxemia or refractory shock. These techniques can lead to complications such as hemorrhaging, nosocomial infections, renal and neurologic injury. Incidence and impact of nosocomial infection occurring during neonatal ECMO have rarely been described.

Patients and methods We retrospectively reviewed all neonatal ECMO between January 2010 and June 2015, in our neonatal intensive care unit. We review incidence, risk factors, infectious organism and outcomes for bloodstream infection, ventilation-acquired pneumonia and urinary infection.

Results Ninety patients underwent ECMO support for more than $48 \mathrm{~h}$. Forty patients developed nosocomial infections ( $44 \%$ with an incidence of 13.8 infections for 1000 days of hospitalization). Initial C-reactive protein was lower in infected patients. Bloodstream infections, ventilation-acquired pneumonia and urinary infections occurred in $65,27.5$ and $7.5 \%$, respectively. Staphylococcus was the most frequent organism in bloodstream infection, and enterobacter was the most frequent organism for ventilation-acquired pneumonia. Mean time for the first nosocomial infection was 15.3 days. Infected patients had longer duration of ECMO support, mechanical ventilation, central venous catheter and hospital stays. Furthermore, infected patients needed more blood transfusion $(p<0.0001)$ and platelets $(p<0.0001)$ No significant difference between survival rates was noticed.

Conclusion Neonatal ECMO is at high risk of developing nosocomial infections. Strategies aimed at prevent these infections, as early ECMO weaning may prevent from improved outcome and effective cost of these patients.

\section{Competing interests None.}

P132

Point-of-care ultrasound in pediatric and neonatal intensive care units: prospective, observational transversal study

Aurélie Morand ${ }^{1}$, Laurent Zieleskiewicz ${ }^{2}$, Olivier Brissaud ${ }^{3}$, Stéphane Le Bel ${ }^{4}$, Mickael Afanetti ${ }^{5}$, Zoé Meresse ${ }^{6}$, Astrid Botte $^{7}$, Michel Panuel $^{8}$, Laurent Thomachot ${ }^{9}$, Jean-Michel Constantin ${ }^{10}$, Marc Leone ${ }^{11}$, Fabrice Michel ${ }^{9}$

${ }^{1}$ Anesthésie-réanimation pédiatrique, Hospital Nord, Marseille, France; ${ }^{2}$ Bouches du rhône, Hôpital Nord, Chemin des Bourrely, Marseille, France; ${ }^{3}$ Service de réanimation pédiatrique et néonatale, Centre Hospitalier Universitaire de Bordeaux, Bordeaux, France; ${ }^{4}$ Anesthésie-réanimation pédiatrique, Hospital Timone, Marseille, France; ${ }^{5}$ Réanimation pédrique, hopital Lenval, Nice, France; ${ }^{6}$ Anesthésie-réanimation, Hospital Nord, Marseille, France; ${ }^{7}$ Réanimation pédiatrique, Centre Hospitalier Régional Universitaire de Lille, Lille, France; ${ }^{8}$ Imagerie médicale, Hôpital Nord, Marseille, France; ${ }^{9}$ Anesthésie-Réanimation Pédiatrique, Hôpital Nord, Marseille, France; ${ }^{10}$ Réanimation adulte, C.H.U. Estaing, Clermont-Ferrand, France; ${ }^{11}$ Département d'anesthésie réanimation, Hôpital Nord, Chemin des Bourrely, Marseille, France

Correspondence: Fabrice Michel - fabrice.michel@ap-hm.fr

Annals of Intensive Care 2016, 6(Suppl 1):P132

Introduction The Echoday study has evaluated the prevalence of POCUS use in ICU patients and assessed the diagnostic performance and therapeutic impact of POCUS in a large cohort of ICU patients including pediatric and neonatal ICU (1). We present here detailed results of the Echoday study in PICU and NICU.

Patients and methods The prospective multicentric observational transversal study Echoday has included 142 ICUs. Among them, 22 were pediatric and/or neonatal ICUs, all tertiary centers ( 5 neonatal ICUs, 7 mixed ICUs and 10 pediatric ICUs). All the POCUS procedures 
performed during a 24-h period were prospectively analyzed. Data regarding patient condition and the POCUS procedures were collected.

Results Among 284 pediatric patients hospitalized during the study period, 154 (54.2\%) POCUS/day were performed in 87 (30.6\%) patients. POCUS served for diagnostic assessment in 141 (91.6\%) cases and procedural guidance in $13(8.4 \%)$ cases. Among the diagnosis POCUS: transthoracic echocardiography accounted for $72(51.1 \%)$ of procedures, brain, transcranial vessel and optic nerve ultrasound for $43(30.5 \%)$, lung ultrasound for $9(6.4 \%)$, renal artery and other vessel ultrasound for $5(3.6 \%)$, vesical ultrasound for $4(2.8 \%)$, abdominal ultrasound for $3(2.1 \%)$ and FAST.

ultrasound for 2 (1.4\%). POCUS confirmed 42 diagnosis (29.8\%), changed 17 diagnosis (12.1\%), spared imagery or monitoring prescription in 5 cases $(3.5 \%)$, induced imagery or monitoring prescription in 29 cases (20.6\%), induced modification or discontinuation of treatment in 30 cases $(21.3 \%)$, induced the continuation of treatment in 30 cases $(21.3 \%)$, induced the continuation of hospitalization in ICUs in 25 cases $(17.7 \%)$, induced the change of department of hospitalization in 9 cases $(6.4 \%)$. The operator was formed to diagnosis ultrasound in 105 cases $(74.5 \%)$. In 95 (67.4\%) cases of diagnosis ultrasound, patients were admitted for medical indications, in $41(29.1 \%)$ cases for surgical indications and in $4(2.8 \%)$ cases for traumatism. Fifteen (11.6\%) diagnosis POCUS were realized at the entrance of the patient. Nine $(7.8 \%)$ cases were life-threatening emergencies, 51 (36.2\%) cases were relative emergencies and 75 (53.2\%) cases were not emergencies. Diagnosis POCUS were performed on newborn in $93(66.0 \%)$ cases. Procedural guidance ultrasound was used in $12(92.3 \%)$ cases for insertion of central venous line and in $1(7.7 \%)$ case for insertion of pleural drain. Twelve $(92.3 \%)$ were successful, $8(61.5 \%)$ on the first time and $4(30.8 \%)$ on the second time. There were complications in $4(30.8 \%)$ cases. The operator was formed to echo guidance in 5 $(38.5 \%)$ cases. Eight $(61.5 \%)$ procedural guidance ultrasounds were realized on newborn.

Conclusion POCUS use in pediatric and neonatal ICUs for diagnosis is high and impacts the management of patients, but POCUS use for procedural guidance remains low. Impact of US in PICUs and NICUs seems to be quite similar than in adult population but some more investigations are necessary.

\section{Reference}

1. Zieleskew icz Intensive Care Medicine 2015.

\section{P133}

Use of continuous infusion of clonidine for sedation in critically ill children: indications, efficacy and side effects

Anna Deho'1, Laïly Sadozai ${ }^{2}$, Stéphane Dauger ${ }^{1}$, Sonia Prot-Labarthe ${ }^{2}$

${ }^{1}$ Service de réanimation et surveillance continue pèdiatriques, Hospital Robert-Debré, Paris, France; ${ }^{2}$ Département de pharmacie, Hospital Robert-Debré, Paris, France

Correspondence: Anna Deho' - anna.deho@aphp.fr

Annals of Intensive Care 2016, 6(Suppl 1):P133

Introduction The provision of adequate sedation and analgesia is required in critically ill children to reduce anxiety, pain, and enable synchronization during mechanical ventilation. This is commonly achieved through a combination of opioids and benzodiazepines. Prolonged use of these agents is associated with tolerance and withdrawal. Recent literature suggests that sedation regimens incorporating a2-adrenergic agonists may offer several benefits. In 2013, we introduced a new sedation protocol including clonidine as an adjunctive sedative agent. We aim to report our experience with clonidine used as a sedative in combination with morphine and midazolam and to prevent withdrawal syndrome.

Patients and methods We retrospectively reviewed medical records of patients admitted in our 20-bed tertiary paediatric intensive care unit between November 2013 and April 2015, who received clonidine. The study was approved by the local ethics committee. We recorded demographic data, indications for intensive care admission, paediatric index of mortality 2 , duration of mechanical ventilation, length of stay, morphine and midazolam doses, clonidine indications and doses, side effects, efficacy and withdrawal symptoms. To asses level of sedation in ventilated patients, we used the COMFORT behaviour scale. We hypothesized a potential opioid- and benzodiazepine-sparing effect of clonidine, and we analysed morphine and midazolam consumption during two time periods: 18 months before and 18 months after the introduction of clonidine in the unit. Data are expressed as numerical values and percentages for categorical variables, and as means and standard deviations for continuous variables.

Results During the study period, 57 patients received clonidine and 48 were enrolled. Mean age at admission was 2.3 years, $36.9 \%$ were male. Reasons for intensive care admission were mainly respiratory failure (46\%) followed by haemodynamic failure, postoperative care, and neurologic failure. Mean paediatric index of mortality 2 was $10.2 \%$. Mean duration of mechanical ventilation was 10.2 days. The main reasons for clonidine administration were: increasing morphine and midazolam (>100 $\mu \mathrm{g} / \mathrm{kg} / \mathrm{h}$ ) requirements $(64 \%)$, difficulties in achieving optimal level of sedation (38\%), difficult management of sedation and analgesia in the post-operative period (38\%), preventing withdrawal syndrome after prolonged administration of opioids and benzodiazepines $(7.7 \%)$, and treatment of withdrawal syndrome $(5.13 \%)$. The mean duration of clonidine infusion was 8 days. Mean COMFORT score value was 15.02 before clonidine was started and 13.69 after beginning of clonidine infusion. The clonidine dose range used was $0.2-2 \mu \mathrm{g} / \mathrm{kg} / \mathrm{h}$. During clonidine infusion, 4 patients (10.2\%) experienced bradycardia, $3(7.7 \%)$ hypotension and $5(12.8 \%)$ both. Fifty-eight per cent of patients who showed haemodynamic instability were less than 1 year old. These effects did not result in increased demand for intravenous fluid or catecholamines. We observed a significant lower consumption of morphine and midazolam after clonidine was introduced. Compared with the 18-month pre-study period, the consumption decreased by $9.3 \%$ for morphine and by $47.1 \%$ for midazolam (calculated as $\mathrm{mg} / \mathrm{months} /$ hospitalized patients).

Discussion Adequate sedation is important in paediatric intensive care. By contrast, oversedation promotes drugs tolerance and withdrawal leading to a delayed recovery. In the effort to reduce prolonged opioid and benzodiazepine exposure, we introduced clonidine in our sedation protocol. We found an acceptable side-effects profile and a clinical efficacy both in ventilated and in non-ventilated patients. Our data also confirm a benzodiazepine and opioid sparing effect of clonidine, with significant reduction in midazolam and morphine consumption.

Conclusion The use of clonidine in critically ill children is safe and effective. Clonidine can be beneficial to reduce the complications associated with prolonged use of opioids and benzodiazepines, and in the prevention and treatment of withdrawal syndrome. A prospective study is needed to better define the role of clonidine in paediatric intensive care.

\section{Competing interests None.}

\section{References}

1. Hünseler C, Balling G, Röhlig C, Blickheuser R, Trieschmann U, Lieser $\mathrm{U}$, et al. Continuous Infusion of Clonidine in Ventilated Newborns and Infants: A Randomized Controlled Trial*. Pediatr Crit Care Med. July 2014;15(6):511-522

2. Duffett M, Choong K, Foster J, Cheng J, Meade MO, Menon K, et al. Clonidine in the sedation of mechanically ventilated children: A pilot randomized trial. J Crit Care. Oct 2014;29(5):758-763.

\section{P134}

Pharmacokinetic analysis of continuous infusion vancomycin therapy in critically ill children

Mathieu Genuini', Mehdi Oualha², Moulin Florence ${ }^{2}$, Jean-Marc Treluyer ${ }^{3}$, Fabrice Lesage ${ }^{4}$, Sylvain Renolleau ${ }^{5}$

${ }^{1}$ Hospital Necker, Paris, France; ${ }^{2}$ Réanimation et surveillance continue médico-chirurgicales, Hôpital Necker - Enfants Malades, Paris, France; ${ }^{3}$ Centre d'investigation clinique, Institut Imagine, Paris, France; ${ }^{4}$ Réanimation et surveillance continue médico chirurgicales, Hôpital Necker - Enfants Malades, Paris, France; ${ }^{5}$ Réanimation pédiatrique polyvalente, Hôpital Necker - Enfants Malades, Paris, France 
Correspondence: Mathieu Genuini - mathieu.genuini@hotmail.fr Annals of Intensive Care 2016, 6(Suppl 1):P134

Introduction Vancomycin is commonly administered for methicillinresistant staphylococcal infections. Resulting plasmatic vancomycin concentration is associated with clinical outcome. While continuous infusion for adult severe infection was previously described, very poor data related to paediatric population are available. The aim of the present study is to (i) describe and investigate continuous infusion vancomycin therapy in critically ill children; (ii) to assess exposure and proper dosing taking into account estimated pharmacokinetic parameters.

Patients and methods This is a chart retrospective observational study conducted at Necker-Enfants Malades teaching hospital. Children (aged less than 18 years) admitted in paediatric intensive and intermediate care unit (PICU) from January to June 2015, who received a continuous infusion vancomycin therapy for suspected or confirmed $\beta$-lactams-resistant cocci gram-positive infection with plasma vancomycin level monitoring were included. General characteristic, clinical and bacteriological data [isolated microorganism and minimum inhibitory concentration (MIC)], organ function, creatinine clearance, vancomycin dosing regimens and measured plasmatic level were described. Using the population pharmacokinetic model of vancomycin published by Le et al. we derived the individual pharmacokinetics parameters of each patient (taking into account age, daily individual dose, body weight, creatinin level and vancomycin concentration). Using these parameters, we simulated the plasma time course of vancomycin in every child. We could then describe the concentration after the loading dose and calculate the area under the curve (AUC24) from the first dose.

Results We analysed 29 patients aged from 1 month to 17 years and weighted from 3 to $53 \mathrm{~kg}$ (median 2.7 years and $12 \mathrm{~kg}$ ). Fifteen patients (52\%) had one or more organ failure and 4 patients (14\%) received vasopressor or inotropic agents. Median creatinine clearance was $176 \mathrm{ml} / \mathrm{min}$ (range 75-343), and no patient required dialysis during the time course of study.

Fourteen patients (48\%) received vancomycin for catheter-related infection mostly caused by coagulase-negative Staphylococcus [WU1] $(n=11)$, and empirically for 13 patients $(45 \%)$, MIC value ( $\mathrm{mg} / \mathrm{L})$ was available for 3 documented infections $(1 ; 1.5$ and 1.5). Median vancomycin loading dose was $14.8 \mathrm{mg} / \mathrm{kg}$ (range 12-16) over $1 \mathrm{~h}$ followed by a continuous infusion of $44 \mathrm{mg} / \mathrm{kg} /$ day (range 35-61). Median delay for first plasmatic level after loading dose was $20 \mathrm{~h}$ (range 10-51) with a level of $14.5 \mathrm{mg} / \mathrm{L}$ (range 9.8-46.9). Eleven patients (38\%) reached a level $>15 \mathrm{mg} / \mathrm{L}$. Sixteen patients (55\%) have had one or more dosing adjustment. Median of the 11 increases of vancomycin daily dose was $+25 \%(\mathrm{IQR}+11$ to +57$)$, resulting in a median plasmatic drug level enhancement from 12.1 to $15.5 \mathrm{mg} / \mathrm{L}$. Five patients (46\%) kept a vancomycin level $<15 \mathrm{mg} / \mathrm{L}$.

During the first $24 \mathrm{~h}$ of treatment, assuming that MIC was equal to $1 \mathrm{mg} / \mathrm{L}$, AUC24/MIC was estimated to 349 (range [WU2] 201, 1001) and only 7 patients ( $24 \%$ ) reached an AUC24/MIC > 400. Median simulated concentrations of vancomycin at the end of the loading dose was $20.4 \mathrm{mg} / \mathrm{L}$ (17.1-23.7), showing that the loading dose was appropriate to reach the target of $15-25 \mathrm{mg} / \mathrm{L}$ [WU3].

Clinical and bacteriological failure occurred for one of the 16 patients who had a confirmed infection. One patient exhibited a renal function deterioration $24 \mathrm{~h}$ after the start of the cure.

Discussion This is the first study to focus on the use of a loading dose of vancomycin followed by continuous infusion in critical ill children. Initial vancomycin loading and continuous doses were very similar among the patients. Loading dose seems adequate to achieve a sufficient vancomycin level, but the following continuous dose was too low to maintain a level $>15 \mathrm{mg} / \mathrm{L}$ and to achieve the target of AUC24/ MIC-1 > 400 for most of patients. In addition, the various practices in dosing adjustment failed to reach these objectives for half of the patients after several days of therapy initiation.

Conclusion Continuous infusion vancomycin therapy in critically ill children dosing as well as adjustments could not to be appropriate and lead to under exposure. Using pharmacokinetics models should be useful to achieve target concentration and AUC.

\author{
Competing interests None. \\ Reference \\ 1. Improved vancomycin dosing in children using Area under the curve \\ exposure. Jennifer le et al. pediatric infectious dis J 2013.
}

P135

Incidence of central venous catheter-associated bacteraemia in a paediatric intensive care unit

Emmanuelle Chalavon 1, Claire Le Reun ${ }^{1}$, Rodrigue Dessein², Bruno Grandbastien ${ }^{3}$, Alain Duhamel ${ }^{4}$, Sarah Pesin ${ }^{4}$, François Dubos 5 , Stéphane Leteurtre

${ }^{1}$ Réanimation pédiatrique, Centre Hospitalier Régional Universitaire de Lille, Lille, France; ${ }^{2}$ Center for infection and immunity, CHRU de Lille, Lille, France; ${ }^{3}$ Department of epidemiology and public health, Centre Hospitalier Régional Universitaire de Lille, Lille, France; ${ }^{4}$ Clinique de santé publique, plateforme d'aide méthodologique, C.H.R.U. Lille, Lille, France; ${ }^{5}$ Pediatric emergency unit and infectious diseases, Centre Hospitalier Régional Universitaire de Lille, Lille, France

Correspondence: Stéphane Leteurtre - stephane.leteurtre@chru-lille.fr Annals of Intensive Care 2016, 6(Suppl 1):P135

Introduction The fight against nosocomial infections is a public health issue. Nosocomial infections increase patient's morbidity and mortality and are expensive to treat. The risk of nosocomial infection is higher with patients in intensive care unit (ICU) than those hospitalised in conventional unit (respectively, 23 vs $5 \%$ according to the national survey (adult and paediatric) executed in France in 2012 in 1938 institutions). Identified risks factors are mainly due to invasive equipments, such as central venous catheter (CVC). In French paediatric ICU, the incidence of CVC-associated bacteraemia is not described. The aim of this study was to define the incidence of CVC-associated bacteraemia per 1000 CVC-days in a French paediatric ICU (PICU).

Patients and methods This study was retrospective, descriptive, and monocentric in a PICU (14 beds) between the 01/01/2012 and the $01 / 09 / 2014$. Inclusion criteria were patients with 1) a short- or longterm CVC and 2) a bacteraemia during hospitalisation, according to the National Healthcare Safety Network definition. Exclusion criteria were separated into (1) clinical aspect: patient with a bacteraemia related to an other infection site than the CVC (non-CVC-associated bacteraemia) (a) and (2) bacteriological analyses: epidemiological duplicates (b), non-nosocomial bacteraemia (c), bacteraemia induced by a common commensal not cultured from two or more blood cultures, drawn on separate occasions (d), patient without a CVC at the time of the bacteraemia (e). Clinical and biological results were collected by using ICCA ${ }^{\circledR}$ software (Philips Society) and bacteriological results by using MOLIS ${ }^{\circledR}$ software (Vision4health Society). Statistical analyses were performed using Fisher's exact test and Mann-Whitney test. Differences were considered significant at $p<0.05$. The CVC utilisation ratio was calculated by dividing the number of CVC-days by the number of patient-days.

Results During the time period (33 months), 1898 patients were eligible and 99 patients with 221 bacteraemia were included. The following were excluded: 17 non-CVC-associated bacteraemia (a), 73 epidemiological duplicates (b), 61 non-nosocomial bacteraemia (c), 52 bacteraemia induced by a common commensal not cultured from two or more blood cultures, drawn on separate occasions (d) and 4 bacteraemia in patients without CVC at the time of the bacteraemia (e). For the 1898 patients, the median age was 33 months (IQR 9-113), the median weight was $12 \mathrm{~kg}$ (IQR 7-25), the mean PICU length of stay was 5.7 days (SD 18), the mean length of invasive ventilation was 6.4 days (SD 20), and the mortality rate was $4.6 \%(87 / 1898)$.

There was 439 CVC and 14 CVC-associated bacteraemia corresponding to 11 patients. The incidence of CVC-associated bacteraemia was 3.9 per $1000 \mathrm{CVC}$-days ( $95 \% \mathrm{Cl}$ 2.1-6.5). The CVC utilisation ratio was 0.75 . Between patients without CVC-infections $(n=366)$ and patients with CVC-associated bacteraemia $(n=11)$, the median PICU length of stay and the median length of invasive ventilation were statistically longer for patients with CVC-associated bacteraemia (respectively, $p=0.0001$ and $p=0.025$ ). There was no statistical difference concerning the age and the mortality between the two groups. 
Discussion According to the National Healthcare Safety Network report realised in 2011 in 300 medical and surgical PICU in the USA, the incidence of CVC-associated bacteraemia was 1.8 per 1000 days with short- and long-term CVC [median (IQR) 1.3 $(0.0-2.6)$ and 90th percentile 4.1]. Our results are closer to the 90th percentile of this American study. However in our study, the CVC utilisation ratio was higher ( 0.75 vs 0.47 in the National Healthcare Safety Network study), showing a longer use of CVC and therefore an increased risk of presenting a CVC-associated bacteraemia during hospitalisation.

Conclusion This study helped define the incidence of CVC-associated bacteraemia in a French paediatric intensive care unit and to find the measurement tools required for an automised extraction of the data. The prospect is to create an informatic tool allowing a continuous surveillance of the CVC-associated bacteraemia in a paediatric intensive care unit.

\section{Competing interests None.}

P136

Outcome at $\mathbf{2}$ months at the infectious level of the pediatric central venous catheters: prospective evaluation of the guidelines optimization impact

Marie-Aude Puel ${ }^{1}$, Marie Szulc ${ }^{2}$, Anais Philippe ${ }^{2}$, François Severac ${ }^{3}$,

Dominique Astruc', Pierre Kuhn', Philippe Desprez ${ }^{4}$

${ }^{1}$ Réanimation néonatale, CHU de Strasbourg, Strasbourg, France; ${ }^{2}$ Pédiatrie, CHU de Strasbourg, Strasbourg, France; ${ }^{3}$ Laboratoire de biostatistique et d'informatique médicale, CHU de Strasbourg, Strasbourg, France; ${ }^{4}$ Réanimation pédiatrique, CHU de Strasbourg, Strasbourg, France

Correspondence: Marie-Aude Puel - matophe@gmail.com

Annals of Intensive Care 2016, 6(Suppl 1):P136

Introduction Increasing proportion of hospitalized patients in the pediatric department of Strasbourg University Hospital benefits from a central venous catheter (CVC) placement. One of the main complications of this device is the central-line-associated bloodstream infection. These hospital-acquired infections are responsible for an important morbidity and mortality. We tried to estimate the outcome at 2 months at the infectious level, of central venous catheter placement in our department, comparing two periods separated by an update of the guidelines with the paramedical and medical teams.

Patients and methods This is a prospective study realized in the whole pediatric department of the Strasbourg University Hospital, comparing two periods: from September 1 to October 31, 2014 (period 1), and from March 1 to April 30, 2015 (period 2). All the CVC placement in the department during those periods was collected. We have recorded all the central-line-associated bloodstream infection (positive blood culture and clinical and/or biological signs of infection). Between those two periods (from November 2014 to February 2015), we have worked with the hygiene team to update, standardize and broadcast protocols of CVC placement and maintenance in the department.

Results Four hundred and one CVC were included: 198 during the period 1 and 203 during the period 2. The incidence rate of central-line-associated bloodstream infection has decreased by 7.7$5.0 / 1000$ days of use $(p=0.122)$. The effect was particularly important in neonatology, in which the rate has fallen from 13.4 to $5.8 / 1000$ days of use $(p=0.07)$.

Discussion This study is the first French pediatric study including the whole pediatric population. The insufficient number of included patients did not permit to highlight a statistically significant effect. However, the clinical efficiency was real and has confirmed the importance of protocols implementation and regular updates as well as the ongoing education of the healthcare teams.

Conclusion Although these results were not statistically significant, they show a positive effect regarding the teams' awareness to new guidelines.

Competing interests None.
P137

Ten years of intensive care in children hospital in Oran, Algeria

Nabil Tabet Aoul ${ }^{1}$, zakaria addou ${ }^{2}$, Ali Douah ${ }^{3}$, Mohamed Moussati ${ }^{3}$, Kamel Belhabiche ${ }^{3}$, Nabil Aouffen ${ }^{3}$

${ }^{1}$ Réanimation pédiatrique canastel, Faculté de médecine d'Oran, Oran, Algeria; ${ }^{2}$ Réanimation pédiatrique de Canastel d'oran, Departement de medecine d'Oran Algerie, Oran, Algeria; ${ }^{3}$ Anesthésie réanimation pédiatrique, Etablissement hospitalier spécialisé en pédiatrie Canastel, Oran, Algeria

Correspondence: Nabil Tabet Aoul - tabetrea@yahoo.fr

Annals of Intensive Care 2016, 6(Suppl 1):P137

Introduction The pediatric intensive care unit of the pediatric hospital of Professor BOKHROFA Abdelkader, Oran, Algeria, was inaugurated July 5, 2005, with 04 intensive care beds.

Materials and methods Evaluation of activity in 10 years (2005-2015) in an intensive care unit at a pediatric hospital in Oran, Algeria.

Results Business service of pediatric resuscitation:

A major change in 2008 when the number of beds has doubled to 08 beds and activity recent years has shown a marked increase in hospitalizations increased from 230 in 2008 to 520 in 2013. The number of children on average breakdown in our service pediatric intensive care is on the increase (40\% in 2014) against (20\% in 2005) with a occupancy rate has increased from $71 \%$ in 2011 to $94 \%$ in 2014.

The service always welcomes children from 1 month to 15 years for mainly respiratory distress, acute hemodynamic or neurological. The population welcomed comes from hospital medical pediatric services (infectious, hematology, gastroenterology, nephrology and pulmonology) and postoperative.

The calls are on average 60 per month. The average residence time is 7 days with prolonged periods, and this is up to 60 days due to the absence of a post-resuscitation service.

New techniques/units:

- Noninvasive ventilation introduced in 2008.

- Opening of two new units in 2013: A post-consultation resuscitation unit and a unit for sedation outside the block since 2012 (puncture marrow biopsy, bronchial endoscopy, CT and MRI)

Conclusion The decline in 10 years (2005 and 2015) and the service activity is increasing, requiring an increase in hospital capacity. There are one million newborns per year and thus there is the need to create new pediatric intensive care unities.

\section{Competing interests None.}

P138

High-frequency percussive ventilation during neonatal transportation: a pilot study

Benoît Colomb ${ }^{1}$, Karine Kolev-Descamp ${ }^{2}$, Anne Marie Petion ${ }^{3}$, Laurent Queudet $^{3}$, Elodie Savajols ${ }^{4}$, Stephanie Litzler-Renault ${ }^{1}$, Denis Semama ${ }^{4}$ ${ }^{1}$ Réanimation pédiatrique polyvalente, Chu Dijon, Dijon, France; ${ }^{2}$ Réanimation pédiatrique, Hôpital Femme Mère Enfant, Bron, France; ${ }^{3}$ Smur pédiatrique, Chu Dijon, Dijon, France; ${ }^{4}$ Pédiatrie 2, Chu Dijon, Dijon, France

Correspondence: Benoît Colomb - benoit.colomb@chu-dijon.fr Annals of Intensive Care 2016, 6(Suppl 1):P138

Introduction Neonatal respiratory distress syndrome (RDS) is a frequent medical condition encountered in preterm neonates. Outborn neonates with RDS may require mechanical ventilation (MV) and surfactant therapy before their transportation to the tertiary neonatal intensive care unit (NICU). High-frequency percussive ventilation (HFPV) has previously been used for management of RDS in neonates hospitalized in NICU. The aim of the present study was to assess the feasibility and safety of HFPV for the management of neonatal RDS during transportation.

Patients and methods We performed a retrospective observational monocentric study from September 2008 to August 2011. All outborn neonates requiring invasive mechanical ventilation for RDS before 
their transportation were included. Neonates with severe malformations and those transported after $48 \mathrm{~h}$ of life were not included. When required, HFPV was provided by the Sinusoidal Bronchotron ${ }^{\mathrm{TM}}$ device $\left(I M A P e^{\circledR}\right)$. For each infant, we recorded the mode of mechanical ventilation (conventional vs. $\mathrm{HFPV}$ ), the evolution of $\mathrm{SpO}_{2} / \mathrm{FiO}_{2}$ ratio, and maternal and neonatal characteristics. Our main outcome was $\mathrm{SpO}_{2} /$ $\mathrm{FiO}_{2}$ ratio, $1 \mathrm{~h}$ after initiation of either conventional mechanical ventilation or HFPV (M60). The comparison was stratified by range of gestational age: $<28,28-31,32-37,>37$ weeks GA. In order to examine $\mathrm{SpO}_{2} / \mathrm{FiO}_{2}$ ratio in relation to mode of ventilation, we defined potential confounding variables: baseline $\mathrm{SpO}_{2} / \mathrm{FiO}_{2}$ ratio observed at the initiation of $\mathrm{MV}$, obstetrical, maternal and neonatal characteristics. Categorical variables were compared using Chi square or Fisher's test as needed while continuous variables were compared using Student's t test. For main outcome, a multivariate analysis was performed using linear regression. $p$ values $<0.05$ were considered statistically significant.

Results Out of the 169 neonates included in the study, 57 received HFPV while 112 were placed on conventional mechanical ventilation (CMV). Univariate analysis for obstetrical, demographic and neonatal data showed no statistical difference between HFPV and CMV group whatever the gestational age, except for Apgar score at $1^{\prime}$ and $5^{\prime}$ which was significantly lower in the neonates aged 37 weeks or more and receiving HFPV. In 28-31 and 32-37 weeks subgroups, baseline $\mathrm{SpO}_{2} / \mathrm{FiO}_{2}$ ratio was found significantly lower in infants receiving HFPV and this ratio remained lower after $1 \mathrm{~h}$ of ventilation. In the more mature infants, HFPV was associated with a higher increase in $\mathrm{SpO}_{2} / \mathrm{FiO}_{2}$ ratio when compared to $\mathrm{CMV}$, but this difference failed to reach statistical significance. After multivariate analysis, the main determinant for $\mathrm{SpO}_{2} / \mathrm{FiO}_{2}$ ratio at $\mathrm{M} 60$ was the baseline value of this ratio. Noteworthy that complications usually associated with mechanical ventilation were not reported in this study.

Discussion This observational study emphasizes the feasibility and safety of HFPV in neonatal transportation. The use of both HFPV and $\mathrm{CMV}$ resulted in favorable respiratory outcome. The significant initial lower values of $\mathrm{SpO}_{2} / \mathrm{FiO}_{2}$ ratio in neonates between 28 and 37 weeks requiring HFPV may be explained by the retrospective design of the study. It is indeed reasonable to suspect that the more severe forms of RDS were immediately ventilated with HPFV.

Conclusion To our knowledge, this is the first study to assess the feasibility and safety of HFPV during neonatal transportation. More studies are necessary to confirm these results and define the indications for HFPV.

\section{Competing interests None.}

\section{References}

1. Bougatef A. High frequency percussive ventilation: principle and 15 years of experience in preterm infants with respiratory distress syndrome. Cuidados respiratorios y tecnologias aplicadas. 2007; Nov 2.

2. Greenough A, Dimitriou G, Prendergast M, Milner AD. Synchronized mechanical ventilation for respiratory support in newborn infants. Cochrane Database Syst Rev. 2008;(1):CD000456.

\section{P139}

Lymphopenia after severe infectious shock in children: association with severity and risk for nosocomial infection Emilie Doye ${ }^{1}$, Solenn Remy ${ }^{2}$, Nicolas Voirin ${ }^{3}$, Delphine Maucort-Boulch ${ }^{4}$, Karine Kolev-Descamp ${ }^{2}$, Guillaume Monneret ${ }^{5}$, Etienne Javouhey ${ }^{6}$ 169, Hôpital Femme Mère Enfant, Bron, France; ${ }^{2}$ Réanimation pédiatrique, Hôpital Femme Mère Enfant, Bron, France; ${ }^{3}$ Cnrs umr 5558, Hospices Civils de Lyon, Lyon, France; ${ }^{4}$ Cnrs umr5558, Hospices civils de Lyon, Lyon, France; ${ }^{5}$ Laboratoire d'immunologie, Hôpital Édouard Herriot, Lyon, France; ${ }^{6}$ Réanimation pédiatrique hfme, Hospices civils de Lyon, Lyon, France

Correspondence: Solenn Remy - solenn.remy@hotmail.fr Annals of Intensive Care 2016, 6(Suppl 1):P139

Introduction To describe the time course of lymphocytes in children with severe infectious shock and to search for an association between persistent lymphopenia, initial severity and risk for nosocomial infection.

Patients and methods This monocentric retrospective study enrolled all children aged less than 18, admitted to PICU for severe infectious shock between January 2011 and September 2014, excluding immunosuppressed and died within the first day. Results are expressed in percentages, medians and interquartile ranges. Fisher's exact test was used to compare groups.

Results One hundred and six patients were analysed: 27 (25.4\%) with severe sepsis (S), 42 (39.6\%) with septic shock (SS) and 37 (34.9\%) with toxic shock (TS). Eight cases were fatal (7.5\%) and 15 patients developed a nosocomial infection (14.2\%). Initial lymphopenia was found, respectively, in 17 (63\%), 35 (83.3\%) and 36 (97.3\%) children with S, SS and TS. Day3 lymphopenia was found in 6 (22.2\%), 19 $(45.2 \%)$ and $13(35.1 \%)$ patients, respectively. Only 6 patients $(5.6 \%)$ presented neutropenia. An association was found between initial severity scores (PIM2, PELOD2, Goldstein) and initial lymphopenia. The most severe patients were more prone to develop day 3 lymphopenia. The rate of nosocomial infections was $10 \%$ in the group without day 3 lymphopenia compared to $23 \%$ in the day 3 lymphopenia group $(p=0.14)$.

Conclusion Initial lymphopenia in children with infectious shock was linked to initial severity of shock, and the most severe patients were more at risk of day 3 lymphopenia. The higher rate of nosocomial infections found in children with day 3 lymphopenia should be confirmed in a larger study.

\section{Competing interests None.}

\section{References}

1. Hall MW, Knatz NL, Vetterly C, Tomarello S, Wewers MD, Volk HD, et al. Immunoparalysis and nosocomial infection in children with multiple organ dysfunction syndrome. Intensive Care Med. 2011 Mar;37(3):525-32.

2. Hotchkiss RS, Monneret G, Payen D. Immunosuppression in sepsis: a novel understanding of the disorder and a new therapeutic approach. Lancet Infect. Dis. 2013 Mar;13(3):260-8.

\section{P140}

Importance of troponin evaluation in scorpion envenomation in pediatric intensive care unit

Mouaffak Youssef ${ }^{1}$

${ }^{1}$ Service Anaesthesia and Intensive Care Hospital Mother - Child, C.H.U Médical, Marrakech, Morocco

Correspondence: Mouaffak Youssef - ymouaffak@yahoo.fr Annals of Intensive Care 2016, 6(Suppl 1):P140

Introduction The scorpion envenomation is the first cause of accidental poisoning. One-third of the envenomed population corresponds to children under the age of 15 years which is constitute a vulnerable population group and consequently displayed greater frequency of serious complications. The cardiogenic shock that accompanies the motion of enzymes affects the prognostic of this pathology and grafts pediatric patients with a significant morbidity and mortality. The aim of the study was to analyze the correlation between admission troponin levels and patient outcomes.

Patients and methods This was a retrospective study, spread over a period of 2 years (2012-2013) and conducted in the pediatric intensive care unit of a University Hospital Mohammed VI Marrakech. We included all patients admitted to pediatric intensive care unit for scorpion envenomation. The management of patients was codified according to a national protocol, and the troponin level was assessed in all the patients on admission. The cutoff level is $0.003 \mathrm{UI} / \mathrm{L}$. The statistical program used was SPSS version 21.

Results Among the 252 of envenomed children, the troponin assay is performed for 172 cases (68.2\%). Males were predominant, and the mean age was 6 years. On admission, $24.5 \%$ of patients had a pulmonary edema, and $21.8 \%$ of patients had signs of collapse. Neurological manifestations were observed in $36.4 \%$ of patients. Troponin levels were positive in $69.8 \%$ on admission. All patient received dobutamine, $15 \%$ of them epinephrine with or without noradrenaline, while $18 \%$ of 
Table 29 Mean intracranial pressure ( \pm SD) before, and in the $4 \mathrm{~h}$ post-hyperosmolar bolus

\begin{tabular}{lllllll}
\hline Agent & Pre-bolus & $\mathbf{1 h}$ & $\mathbf{2 h}$ & $\mathbf{3 h}$ & $\mathbf{4 h}$ & $\mathbf{\boldsymbol { H } ^ { * }}$ \\
\hline Mannitol (20\%) & $28.2 \pm 6.6$ & $21.2 \pm 7.8$ & $21.9 \pm 6.3$ & $22.9 \pm 6.0$ & $23.9 \pm 5.0$ & 0.016 \\
Hypertonic saline (3 \%) & $25.3 \pm 5.4$ & $23.0 \pm 6.0$ & $19.1 \pm 6.5$ & $19.6 \pm 5.7$ & $20.9 \pm 7.1$ & 0.047 \\
\hline
\end{tabular}

* Repeated-measures ANOVA

patients required mechanical ventilation. The average length of stay in hospitals was $50 \mathrm{~h}$. Favorable evolution was noted in $901 \%$. The correlation between the troponin levels and the severity of clinical situation, the need for mechanical ventilation, vasoactive drugs, the length of hospitalization and the unfavorable evolution is positive and statistically significant.

Conclusion As a myocardial enzyme marker, the troponin levels seem to be an interesting element in the immediate assessment of patients with severe envenomation and in the identification of people at risk.

\section{Competing interests None.}

\section{P141}

\section{Hyperosmolar therapy in pediatric traumatic brain injury: a} retrospective study

Nadia Roumeliotis' ${ }^{1}$ Christian Dong ${ }^{2}$, Geraldine Pettersen ${ }^{1}$, Louis Crevier ${ }^{3}$, Guillaume Emeriaud

${ }^{1}$ Soins intensifs pédiatriques, CHU Sainte Justine, Montréal, Canada; ${ }^{2}$ Centre de recherche, CHU Sainte-Justine, Montréal, Canada; ${ }^{3}$ Neurochirurgie, CHU Sainte-Justine, Montréal, Canada

Correspondence: Nadia Roumeliotis - nadia.roumeliotis@gmail.com Annals of Intensive Care 2016, 6(Suppl 1):P141

Introduction Hyperosmolar agents are commonly used agents for the first-tier management of intracranial hypertension in traumatic brain injury (TBI). Pediatric guidelines have highlighted the lack of pediatric literature on the effectiveness of hyperosmolar agents in TBI management and stressed the need for further studies to support their use. The specific objective of this study was to describe the use of hyperosmolar therapy in pediatric TBI and examine its effect on intracranial pressure (ICP) and cerebral perfusion pressure (CPP). In addition, the study will aid in assessing feasibility and preparation of a future controlled trial.

Patients and methods $A$ retrospective revue of all TBI patients admitted to CHU Sainte-Justine between 2007 and 2014 was conducted. Eligible patients were age 1 month to 18 years old, had severe TBI as defined by GCS $\leq 8$ on admission, and were admitted to the critical care unit. Specific inclusion criteria were invasive intracranial pressure monitoring and administration of a hyperosmolar agent within $48 \mathrm{~h}$ of admission to pediatric intensive care unit. Hyperosmolar agents included boluses of $20 \%$ mannitol or $3 \%$ hypertonic saline received after ICP placement. For the first 2 boluses received for increased ICP ( $>20 \mathrm{mmHg}$ ), the impact on ICP and CPP was assessed during the following $4 \mathrm{~h}$. Co-interventions to control ICP (additional hyperosmolar agent, propofol or barbiturate bolus), diuresis and serum sodium were also documented. Missing values at 4 th hour ( $n=3$ values) for ICP and CCP were treated by imputation. Repeated measure ANOVA was used for analysis of trending mean ICP and CPP post bolus, with significance set at $p=0.05$.

Results Sixty-four patients were eligible, of which 16 met inclusion criteria. Average age was 11 years (SD \pm 4 ), and median GCS was 6 (range 4-7). Thirteen patients were stabilized in another center prior to transfer, and median time from Trauma to ICP monitor was $8.5 \mathrm{~h}$ (range 7-12 h). Average number of hyperosmolar boluses per patient was 6.6 (SD \pm 3.4 ) over $48 \mathrm{~h} ; 70 \%$ of these were $3 \%$ hypertonic saline and $30 \%$ were $20 \%$ mannitol. There was no difference in patient characteristics between the two groups. For the first two boluses received, both mannitol and hypertonic saline were followed by a decrease in ICP in the following 4-h period (mannitol $n=8$, $p<0.05$ and hypertonic saline $n=14, p<0.05$ ), see Table 29. There was no significant change in CPP post-bolus (mannitol $p=0.8$ and hypertonic saline $p=0.4$ ). Eleven patients received a co-intervention to decrease ICP within the $4 \mathrm{~h}$ post-hyperosmolar agent. Diuresis was not significantly increased post-mannitol or hypertonic saline.

Conclusion In pediatric TBI patients with intracranial hypertension, mannitol and hypertonic saline are commonly used, and we did not identify clear determinants of the choice of the solute. The use of both agents was associated with a reduction in ICP. The sample size, retrospective design and non-randomized choice of the treatment limit further interpretation. This study provides an important basis for the development of a future controlled, prospective study, which is needed to determine the respective effects of mannitol and hypertonic saline on intracranial hypertension in pediatric TBI. The high proportion of non-eligible patients and frequent co-interventions will be potential barriers of crucial importance in such trial.

Competing interests None.

\section{P142}

Cerebral sinovenous thrombosis in children: report of seven cases Mouaffak Youssef ${ }^{1}$

${ }^{1}$ Service Anaesthesia and Intensive Care Hospital Mother - Child, C.H.U Médical, Marrakech, Morocco

Correspondence: Mouaffak Youssef - ymouaffak@yahoo.fr

Annals of Intensive Care 2016, 6(Suppl 1):P142

Introduction Cerebral sinovenous thrombosis (CSVT) in children is a rare disorder. However, it is increasingly recognized and diagnosed in children with the advent of new technical means both the imaging plane as clinically, allowing an early and adapted medical care.

Patients and methods It is a retrospective and descriptive study based on cases of children hospitalized over 7 years from January 2008 to December 2014. All children having a CSVT in the imagery were included. Results Seven cases were reported. The mean age was 5.3 years, with predominance in males (sex ratio $=1.3$ ). $71.4 \%$ of case with CSVT presented with an underlying disease: The infection was most frequently noted in all age groups (57.1\%), the dehydration is more common in infants $(28.57 \%)$, and the Behcet's disease in $14.2 \%$ of cases. The CVST were acute in $57 \%$ of cases, subacute in $42.8 \%$ of cases, with a polymorph clinical expression: intracranial hypertension syndrome $(42.8 \%)$, seizures $(42.8 \%)$, focal neurologic signs $(85.7 \%)$ and disturbances of consciousness (71.4\%).

The CVST diagnosis was made by a cerebral CT (computed tomography) (direct or indirect signs) in $71.4 \%$ of case and by a CT angiography (when CT was non conclusive) in $51.4 \%$ of cases.

The superficial venous systems was involved in $42.8 \%$ of cases and extended in $28.5 \%$ of cases.

The antithrombin therapy was prescribed in $28.5 \%$ of children, corticosteroid therapy in $14.2 \%$ of cases and an etiologic treatment (antibiotic treatment for cases involving infection + correction of rehydration...) in $85.7 \%$ of cases. The evolution was favorable in $28.5 \%$ of cases. Death occurred in $14 \%$ of cases, and $42.8 \%$ of children kept neurological sequelae damage.

Conclusion Compared to the data of the literature, the CVST is characterized by the large diversity of clinical presentations and etiologies. The small number of patients who received a treatment by antithrombotic does not provide a conclusion based on statistically solid results. However, the prognosis remains dreadful against the death risk and the sensorineural sequelae.

Competing interests None. 
P143

Optimising nurse confidence and skill acquisition in paediatric continuous renal replacement therapy (pCRRT) through a training programme: result of the first-year experience in a tertiary care centre

Jérôme Naudin ${ }^{1}$, Laetitia Abdallah¹, Marion Ganier ${ }^{1}$, Karine Frannais', Dylia Douglas ${ }^{1}$, Cecile Flusin ${ }^{1}$, Anna Deho' ${ }^{1}$, Stéphane Dauger ${ }^{1}$

${ }^{1}$ Réanimation et surveillance continue pédiatriques, CHU Robert Debré, Paris, France

Correspondence: Jérôme Naudin - jeromenaudin@gmail.com Annals of Intensive Care 2016, 6(Suppl 1):P143

Introduction pCRRT use has grown in recent years in paediatric intensive care unit (PICU) and with the demands for adequate intensive care staffing levels. Because pCRRT number per year is low, the weight range of patients is very large (from $<3$ to $>60 \mathrm{~kg}$ ), and the turnover of nurses is high, the provision of a safe and sustainable pCRRT service, is a big challenge in PICUs. A pCRRT working group was created in August 2014 to perform a training programme and to go with a switch in CRRT device. We evaluated this programme after the first operating year. Materials and methods Staff (doctors and nurses) training programme was organised twice a year ( 2 sessions each time). Each session ( $7 \mathrm{~h}$ and $30 \mathrm{~min}$ ) consisted of theoretical aspects of CRRT and practical simulation (low-fidelity) with peer feedback about assembly and troubleshooting of alarms and presentation of new visual tools. Fifty-two nurses $(80 \%)$ with more than 6-month experiences were trained during the first year.

Results A test ( $n=29$ nurses) was realised in September to evaluate theory and practical knowledge (6 months after the last session). Questions concerning theory of pCRRT were correct in $38 \%$ and incomplete in $45.7 \%$. Questions about alarms management had the lowest ratio of correct answers with only 16.5 and $61.4 \%$ of incomplete answer. However, a majority was more confident to manage children on pCRRT after theoretical teaching (72.1\%) and simulation (86.2\%).

Conclusion Post-programme assessment was positive, but anxiety about knowledge and practice persisted. Consolidation of skills and implementation of the training course with high-fidelity simulation, especially alarms management scenario, will be interesting to offer the opportunity for doctors and nurses to progress into expert CRRT users in PICU.

\section{Competing interests None.}

\section{P144}

From high-quality electronic database to the new concept of perpetual patient. Preliminary results

David Brossier ${ }^{1}$, Redha Eltaani ${ }^{2}$, Michaël Sauthier ${ }^{1}$, Guillaume Emeriaud ${ }^{1}$, Bernard Guillois ${ }^{3}$, Philippe Jouvet

${ }^{1}$ Soins intensifs pédiatriques, CHU Sainte-Justine, Montréal, Canada;

¿Unité de recherche clinique appliquée, CHU Sainte-Justine, Montréal, Canada; ${ }^{3}$ Néonatalogie, CHU, Caen, France

Correspondence: David Brossier - david_brossier@yahoo.fr

Annals of Intensive Care 2016, 6(Suppl 1):P144

Introduction Informatics improvement and electronic device expansion have led to massive data collection at bedside over the past decades. Thanks to the information systems and network architecture already available through fully electronic charting in our paediatric intensive care unit (PICU) at CHU Sainte Justine, we collected cardiorespiratory and clinical data within an electronic database (EDtb).

Patients and methods With the CHU Sainte Justine ethical committee approval, all patients under 18 years old admitted in the PICU were included. All data from admission to discharge were gathered in the EDtb. Cardiorespiratory data were collected from monitors every $5 \mathrm{~s}$, thanks to the Mirth software, and every $30 \mathrm{~s}$ from mechanical ventilators and infusion pumps. Data were then anonymized prior to organization in tables.
Results Between May 21 and September 10, 2015, 260 patients were included. The EDtb contained 3 categories of data: 24841592 entries from the cardiac monitors for a storage volume of $21 \mathrm{GO}$, and 2 tables of $56,351,811$ and 2,624,480 entries from the ventilator and infusion pumps for a storage volume of $8 \mathrm{GO}$ and $340 \mathrm{MO}$ respectively.

Discussion The EDtb will soon be linked with the electronic medical record of critical care patients. The EDtb will then be able to reproduce the patients'entire ICU course (perpetual patient) and be searchable for research purposes. The primary objective of this EDtb is to serve as virtual patient for the validation of computational models.

Conclusion Through an ongoing database, clinical and physiological data gathering in critical care and its reorganization in time can lead to the new concept of the perpetual patient.

\section{Competing interests None.}

\section{P145}

Transportation of children on extracorporeal membrane oxygenation: 1-year experience of a tertiary referral center in the Paris region

Jerome Rambaud ${ }^{1}$, Pierre-Louis Leger ${ }^{1}$, Michelle Larroquet ${ }^{2}$, Alain Amblard', Noella Lode ${ }^{3}$, Julia Guilbert', Sandrine Jean', Isabelle Guellec', Isabelle Casadevall ${ }^{3}$, Katia Kessous ${ }^{3}$, Hervé Walti ${ }^{1}$, Ricardo Carbajal

${ }^{1}$ Réanimation pédiatrique et néonatale, Hopital pour enfants Trousseau,

Paris, France; ${ }^{2}$ Chirugie pédiatrique, Hopital pour enfants Trousseau, Paris,

France; ${ }^{3}$ Smur pédiatrique, Robert Debré, Paris, France

Correspondence: Jerome Rambaud - jerome.rambaud@aphp.fr

Annals of Intensive Care 2016, 6(Suppl 1):P145

Introduction Extracorporeal membrane oxygenation (ECMO) is used as a rescue therapy in patients with severe and refractory respiratory and/or hemodynamic failure. Ideally, neonates or children candidates for ECMO support should be transferred to a referral ECMO center. However, sometimes patients are too ill to be safely moved with conventional ventilator support. In these situations, ECMO cannulation at the referring facility and transfer by a transport ECMO team is a potentially lifesaving intervention. With a 25 -year experience on neonatal and pediatric ECMO, the pediatric intensive care unit and the pediatric surgery unit of the Armand-Trousseau Hospital in Paris developed in November 2014 a transport ECMO team. This mobile team has been developed in collaboration with the Robert Debré emergency transport unit and the Civil Security of Paris. We report the first-year experience of this mobile team.

Patients and methods We retrospectively reviewed all neonatal and pediatric ECMO transports from November 2014 through September 2015. Reviewed data included referring facility, mode and duration of transport, type of ECMO, clinical severity score $\left(\mathrm{PaO}_{2} / \mathrm{FiO}_{2}\right.$, inotrope score) and laboratory tests (lactate, $\mathrm{pH}$ ) before and after transport.

Results Twenty-two requests for intervention of the mobile team were received. In 8 cases, the team intervention was not deemed necessary or exceeded status. In 14 cases, the mobile team travelled to the referring center. In one case the child was transported on conventional ventilation, and in 2 patients ECMO cannulation was not possible because of vascular problems. Eleven patients, including 4 neonates and 7 children, were transported on ECMO support. One patient was cannulated in our PICU and then transported to a pulmonary transplantation center. The median (range) run distance and round-trip duration were $117 \mathrm{~km}(4-392 \mathrm{~km})$ and $8 \mathrm{~h}(2-13 \mathrm{~h})$, respectively. Nine ( $81 \%)$ transports were on venous-arterial ECMO and two (19\%) on venous-venous ECMO. Median (range) pre-ECMO cannulation $\mathrm{pH}$, lactate, $\mathrm{PaO}_{2} / \mathrm{FiO}_{2}$ and inotrope score were, respectively, $7.03(6.67-7.27), 5.5 \mathrm{mmol} / \mathrm{L}$ (0.6-13.8 mmol/L), 36 (24-127) and 568 (50-890). Corresponding post-ECMO cannulation values were 7.33 (7.17-7.43), $2.8 \mathrm{mmol} / \mathrm{L}$ $(1-12.6 \mathrm{mmol} / \mathrm{L})$ and 60 (0 à 310). No adverse events during ECMO transport were noticed. 
Discussion This first-year experience suggests that the development of the first neonatal and pediatric mobile ECMO team in the north of France. This rapidly increased activity meets a significant need for ECMO support in children too critically ill to be moved on conventional therapy. The ECMO rapidly improved respiratory and hemodynamic parameters allowing transportation in good conditions to our ECMO center. It allows children hospitalized in non-ECMO centers to benefit from this rescue treatment. It contributes to improve the access to the continuity of health care in France.

Conclusion Implantations and transportations of children on ECMO supports can be proposed to children with refractory respiratory or circulatory diseases and now accessible in a large perimeter around Paris region.

\section{Competing interests None.}

\section{P146}

\section{Acute neurotoxicity of bath salts combining}

3,4-methylenedioxypyrovalerone and mephedrone in the rat Christophe Poiré', Nadia Benturquia', Olivier Roussel', Lucie Chevillard ${ }^{1}$ Patricia Risede', Jacques Callebert ${ }^{1}$, Huixiong Chen ${ }^{2}$, Bruno Megarbane ${ }^{3}$ ${ }^{1}$ Inserm u1 144, Université Paris Descartes, Paris, France; ${ }^{2}$ Cnrs umr8601, Université Paris Descartes, Paris, France; ${ }^{3}$ Service de Réanimation Médicale et Toxicologique, CHU Lariboisière, Paris, France

Correspondence: Bruno Megarbane - bruno.megarbane@lrb.aphp.fr Annals of Intensive Care 2016, 6(Suppl 1):P146

Introduction Synthetic cathinones, beta-keto-amphetamine derivates, are new psychoactive and stimulant substances with exponentially increasing use since 10 years. Bath salts, often sold legally, contain mixtures of several cathinones like 3,4-methylenedioxypyrovalerone (MDPV) and mephedrone (4MMC). Toxicity of combination of two cathinones, mimicking their real use in humans, has never been studied. Our objective was to investigate possible synergy of MDPV/4MMC combination on their stimulant effects in the SpragueDawley rat and analyze their mechanisms of interaction.

Materials and methods MDPV and $4 M M C$ were synthesized in our laboratory. The effects of MDPV/3MMC mixture (administered by the intragastric route) were studied on rat locomotor activity in an openfield using videotaping. Plasma concentrations of MDPV, 4MMC and their 3 major metabolites were measured using liquid chromatography coupled to high-resolution mass spectrometry. Brain monoamine concentrations were determined using high-performance liquid chromatography coupled to fluorometry. For each animal and each time, we calculated the difference between the parameter value at that time and baseline and the area under the curve of its time course. Comparisons were made using two-way ANOVA followed by posttests using Bonferroni correction. Pharmacokinetics (PK) was modeled and parameters calculated using WinNonlin ${ }^{\circledR}$ software.

Results MDPV (3 mg/kg)/4MMC (30 mg/kg) combination was responsible for a significant increase in rat locomotor activity in comparison with saline, MDPV and $4 \mathrm{MMC}$ alone $(p=0.02)$, with a synergic interaction between the two drugs. 4MMC PK was not altered in the presence of MDPV, while MDPV absorption was reduced in the presence of $3 \mathrm{MMC}$ without significant modifications in its half-life and clearance. The neurochemical study revealed significant increase in dopamine and serotonin but not in norepinephrine concentrations in the prefrontal cortex, when combining both drugs in comparison with each drug administered separately.

Conclusion MDPV/4MMC mixture as found in bath salts significantly increases locomotor activity in rats in comparison with each substance administered alone. Mechanisms of interaction include significant decrease in MDPV gastrointestinal absorption and significant increase in monoamine reuptake inhibition in the brain with reinforcement of both the dopaminergic and serotonergic profiles. However, further investigations at the level of the involved gastrointestinal transporters and monoamine transporters are required.

Competing interests None.
P147

Acute organophosphate poisoning: what about pralidoxime use?

A. M'rad ${ }^{1}$, Sahar Habacha', Bassem Chatbri', Youssef Blel ${ }^{1}$, H Thabet $^{2}$, Nozha Brahmi', M. Amamou'

'Department of Intensive Care and Toxicology, Centre d'Assistance Médicale Urgente, Tunis, Tunisia; ${ }^{2}$ Department of emergency, Centre d'Assistance Médicale Urgente, Tunis, Tunisia

Correspondence: A M'rad - mrad.aymen@gmail.com

Annals of Intensive Care 2016, 6(Suppl 1):P147

Introduction Organophosphates insecticides (OPI) are commonly used as acetylcholinesterase inhibitor all over the word, especially in developing countries. OPI poisoning (OPIP) can be accidental or suicidal. Its diagnosis is based on clinical presentation and decrease of acetylcholinesterase activity. Specific treatment is based on atropine for cholinergic crisis, and pralidoxime as acetylcholinesterase regenerator. Our study aimed to assess efficiency of pralidoxime in acute OPIP, concerning complications and outcomes.

Patients and methods We carried out a retrospective study of patients who had been admitted to our intensive care unit between January 1, 2010, and December 31, 2014, for occupationally related organophosphate intoxication. Seventy-five cases were 1.2 paired according to sex, age and Simplified Acute Physiology Score (SAPSII).

Results Eligible patients ( 39 females and 36 males) were aged of $30 \pm 17$ years; their mean SAPSII was $26.5 \pm 12$. Dichlorvos was the main OPI responsible of poisoning. Muscarinic syndrome was present in $100 \%$ of cases, followed by nicotinic syndrome $(60 \%)$ and then encephalic syndrome $(20 \%)$. The most frequent complications were acute pancreatitis (16\%), circulatory failure $(13 \%)$ and pulmonary edema (5\%). Mechanical ventilation was required for thirteen patients for neurological distress $(n=10)$ and respiratory distress $(n=3)$. The serum acetylcholinesterasic activity (AChE) rate was $571 \pm 1399 \mathrm{UI} / \mathrm{I}$ (NI 5000-12,000 UI/I), and the erythrocyte acetylcholinesterasic (AChEEr) rate was $3 \pm 1 \mathrm{UI} / \mathrm{ml}(\mathrm{NI} 6-9 \mathrm{UI} / \mathrm{ml}$ of red blood cell). Pralidoxime was given to 25 patients (treated group) over $24 \mathrm{~h}$ as bolus of $5 \mathrm{mg} / \mathrm{kg}$ and continuous infused dose of $50 \mathrm{mg} / \mathrm{kg}$.

The comparison between the treated group and the control showed no difference in complications occurrence, requiring atropine dose, mechanical ventilation necessity and prognosis.

Conclusion The regimens of pralidoxime use in the present study do not seem to be necessary in moderate dichlorvos poisoning.

\section{Competing interests None.}

\section{P148}

Acute nifedipine poisoning: clinical and prognosis aspects A. M'rad ${ }^{1}$, Fatma Essafi ${ }^{1}$, Nasreddine Foudhaili ${ }^{1}$, Youssef Blel ${ }^{1}$, H. Thabet $^{2}$, Nozha Brahmi', M. Amamou'

${ }^{1}$ Department of Intensive Care and Toxicology, Centre d'Assistance Médicale Urgente, Tunis, Tunisia; ${ }^{2}$ Department of emergency, Centre d'Assistance Médicale Urgente, Tunis, Tunisia

Correspondence: A M'rad - mrad.aymen@gmail.com

Annals of Intensive Care 2016, 6(Suppl 1):P148

Introduction Calcium channel blockers (CCB) represent a heterogeneous class of drugs, which are commonly used to treat hypertension, arrhythmia and angina. Nifedipine is the most frequently prescribed CCB in our country. The aim of our study was to describe the clinical presentation, the management and the outcome of nifedipine poisoning.

Patients and methods It was a retrospective study conducted over 20 months, including all patients admitted to intensive care unit for nifedipine overdose between January 2013 and August 2015. Demographics data, details of ingestion, the clinical signs, complications and management were analyzed.

Results Sixty-three patients $(12 \mathrm{M} / 51 \mathrm{~F})$ aged of $26 \pm 6$ years were eligible. Nifedipine was the only drug ingested in 30 cases (47.6\%). The mean ingested dose was $300 \mathrm{mg}$ [100-1200]. The delay of consultation period was $5 \pm 4.5 \mathrm{~h}$ post-ingestion. Symptoms occurred 
in $47.6 \%$ of cases ( $n=30$ ) including digestive vomiting, dizziness, headache and cardiovascular disorders such as palpitation, chest pain and syncope. Tachycardia was the most frequent electric sign found in $68.3 \%$ of patients $(n=43)$. Fifteen patients $(28.3 \%)$ had a shock which occurred on average of $6 \mathrm{~h}$ [2.75-9.5] after nifedipine ingestion and requiring vascular filling in all cases and norepinephrine in only 7 cases. By comparing shock and non-shock groups, only the ingested dose was significantly higher in shocked patients (400 vs $200 \mathrm{mg}, p=0.007$ ). Shock was associated with prolonged ICU stay: $30 \pm 10 \mathrm{~h}$ in shock group versus $20 \pm 4 \mathrm{~h}$ in non-shock group, $p=0.004$. One patient with theophylline coingestion died from refractory shock.

Conclusion Our results confirmed that nifedipine poisoning could be considered as benign compared to other CCB because of their predominant vascular effect and scarcity of cardiac damage. Outcome of pure poisoning is generally favorable. Coingestion of other drugs with cardiovascular toxicity worsens the prognosis.

\section{Competing interests None.}

\section{P149}

Acute behavioral toxicity and addictive liability of the new synthetic cathinone 3,4-methylenedioxypyrovalerone (MDPV) in the rat

Cyril Masniere', Nadia Benturquia', Olivier Roussel', Lucie Chevillard', Patricia Risede ${ }^{1}$, Jacques Callebert ${ }^{1}$, Bruno Megarbane ${ }^{2}$

${ }^{1}$ Inserm u1 144, Université Paris Descartes, Paris, France; ${ }^{2}$ Service de Réanimation Médicale et Toxicologique, CHU Lariboisière, Paris, France

Correspondence: Bruno Megarbane - bruno.megarbane@lrb.aphp.fr Annals of Intensive Care 2016, 6(Suppl 1):P149

Introduction 3,4-Methylenedioxypyrovalerone (MDPV), a synthetic cathinone with important reported toxicity and high abuse potential, has been increasingly used since the last 5 years. To date, scarce experimental studies have investigated MDPV-related behavioral effects. We aimed at studying MDPV-related neurobehavioral effects according to various modalities of administration, mimicking human uses.

Materials and methods Sprague-Dawley rat investigation of MDPVinduced effects on the locomotor activity, anxiety (openfield), resignation (forced swimming), memory (Y-maze), hedonic status (sucrose consumption) and brain neurochemistry (monoamines content in the prefrontal cortex), using three different administration patterns including acute ( $3 \mathrm{mg} / \mathrm{kg} I P$ ), binge-like (MDPV $3 \mathrm{mg} / \mathrm{kg} \mathrm{IP}$, 3 times per day, 3 days) and prolonged treatment (MDPV $1 \mathrm{mg} / \mathrm{kg} I P$, 10 days). Plasma concentrations of MDPV and its two major metabolites were measured using liquid chromatography coupled to highresolution mass spectrometry. Brain monoamine concentrations were measured using high-performance liquid chromatography (HPLC) coupled to fluorometry. For each animal and each time, we calculated the difference between the parameter value at that time and baseline and the area under the curve of its time course. Comparisons were made using two-way ANOVA followed by posttests using Bonferroni correction.

Results Acute MDPV administration was responsible for locomotor hyperactivity with onset of stereotypies, significant decrease in anxiety, anhedonic status and memory impairment $(p<0.05$ for each neurobehavioral measurement). Following binge-like MDPV administration, tolerance to immediate locomotor effects after drug withdrawal and significant decrease in anxiety, hedonic status and food consumption were observed $(p<0.05$ for each neurobehavioral measurement). Increase in the prefrontal cortex serotonin levels occurred, in accordance with the observed behavioral effects. Prolonged MDPV treatment resulted in tolerance development after 1 week of drug withdrawal.

Conclusion Our results suggest MDPV-induced marked neurobehavioral effects in the rat with the development of tolerance after bingelike and prolonged repeated administration.

Competing interests None.
P150

Clinical and therapeutic characteristics of metformin poisoning

A. M'rad ${ }^{1}$, Messaouda Khelfa ${ }^{1}$, Hana Fredj ${ }^{1}$, Youssef Blel ${ }^{1}$, H. Thabet ${ }^{2}$, Nozha Brahmi' ${ }^{1}$ M. Amamou' ${ }^{1}$

${ }^{1}$ Department of Intensive Care and Toxicology, Centre d'Assistance Médicale Urgente, Tunis, Tunisia; ${ }^{2}$ Department of Emergency, Centre d'Assistance Médicale Urgente, Tunis, Tunisia

Correspondence: A M'rad - mrad.aymen@gmail.com

Annals of Intensive Care 2016, 6(Suppl 1):P150

Introduction Deliberate metformin poisoning was rarely reported in the literature. Lactic acidosis is the common complication of metformin intoxication, either in intentional or in incidental cases. Our study aims to report the experience of metformin poisoning in a specialized center of toxicology.

Patients and methods It was a retrospective study over a period of 7 years (2008-2015). From patient's files, we reported the demographic characteristics, clinical and biological presentations and the outcomes of patients admitted to the intensive care department.

Results Seventy-eight patients were included. Sex ratio was $1 \mathrm{M} / 5 \mathrm{~F}$. The median age was $23[19 ; 30]$ years (extremes 13 and 62). It was a deliberate poisoning in all cases. Multidrug poisoning was reported in $67 \%(n=53)$ of cases. Among them, coingestion of sulfonylureas was the most frequent $(n=20)$. Median ingested dose was $10.2[8 ; 17] \mathrm{g}$. Our patients were without history of cardiovascular disease, hepatic or renal failure in $93 \%(n=73)$. The average time of consultation was 3 $[1.6 ; 5] \mathrm{h}$.

Gastrointestinal disorders including nausea, vomiting and abdominal pain were the most reported symptoms in $57.7 \%(n=45)$ of patients. Polypnea occurred in $5.1 \%$ of cases and hypotension in $3.8 \%$. Hyperlactatemia was noted in $51.3 \%$ of cases $(n=40)$, with a mean value of $5 \pm 4 \mathrm{mmol} / \mathrm{l}$ and metabolic acidosis in $20.5 \%$ of cases $(n=16)$. Clinical symptoms worsen by coingestants, especially hypoglycemia with sulfonylureas in $9.2 \%$ of cases $(n=7)$, hypotension and bradycardia with cardiotropics $(n=2)$.

Digestive decontamination was performed for $17.5 \%$ of patients $(n=13)$ : gastric lavage $(n=9)$ and activated charcoal in a single dose $(n=5)$. Conservative therapies were sufficient in $96 \%$ of cases. The hemodialysis was performed only for three cases with severe acidosis. The median of hospital stay was $24[18 ; 38] \mathrm{h}$. There was no death in our cases.

Discussion The prevalence of metformin poisoning is $6.4 \%$ of hospitalizations in our center.

Lactic acidosis, due to impairment of the mitochondrial respiratory function, is the most serious complication. But it was often spontaneously reversible with conservative therapies in our patients. These favorable outcomes may be explained by the absence of previous chronic conditions in our population.

Conclusion Metformin overdose is characterized by digestive symptoms and high plasma lactate levels. Although lactic acidosis is considered to be a serious condition, it was easily managed with conservative therapy.

\section{Competing interests None.}

P151

\section{Calcium channel blockers poisoning}

Samar Souissi ${ }^{1}$, Hatem El Ghord ${ }^{2}$, N. Kouraichi ${ }^{1}$, N. Brahmi ${ }^{3}$, H. Thabet ${ }^{1}$, M. $\mathrm{Amamou}^{3}$

${ }^{1}$ Service de réanimation, centre d'assistance médicale-urgente, Tunis, Tunisia; ${ }^{2}$ Reanimation médicale, Centre d'assistance médicale urgente, Tunis, Tunisia; ${ }^{3}$ Service de réanimation médicale, Centre d'assistance médicale-urgente, Tunis, Tunisia

Correspondence: Samar Souissi - souissi_samar@yahoo.fr Annals of Intensive Care 2016, 6(Suppl 1):P151

Introduction Calcium channel blockers are increasingly used in the treatment of hypertension and arrhythmias. In the same way, calcium channel blockers poisoning is increasing in frequency, and it remains serious despite therapeutic progress. The aim of our work was to study 
the epidemiology, clinical presentation, therapeutic management and prognosis of calcium channel blockers poisoning and to identify gravity factors.

Patients and methods We conducted a retrospective study during 6 years and 6 months, from January 2007 to June 2013 in a medical and toxicological intensive care unit, including cases of calcium channel blockers poisoning not associated with another cardiovascular drug.

Results We collected 74 cases. Sex ratio was $0.13(9 / 65)$. Mean age was $22.7 \pm 7.3$ years ( $12-52$ years). Fifty-five percent of patients were aged between 20 and 29 years. Most patients had no medical history (62\%). Hypertension was noted in $8 \%$ of cases and coronaropathy in $1 \%$. In $9 \%$ of cases, patients had history of suicide attempt. The agents responsible for poisoning were: nifedipine, nicardipine, amlodipine, diltiazem and lercanidipine. Nifedipine was the most found (67\%). Poisoning was intentional in $42 \%$ of cases and unintentional in $3 \%$ of cases. The reason of ingestion was unknown in $55 \%$ of cases. Patients have presented tachycardia in $92 \%$ of cases, bradycardia in $4 \%$, hypotension in $30 \%$, shock in $16 \%$ and pulmonary edema in $9 \%$ of cases. Tachycardia was isolated in $51 \%$ of cases. A patient has developed first-degree atrioventricular block, and two patients have manifested subepicardial ischemia. QT prolongation was noted in $4 \%$ of cases. Leucocytosis was found in $42 \%$ of cases, hypokalemia in $31 \%$, metabolic acidosis in $30 \%$, hyperlactatemia in $15 \%$, hyperglycemia in $11 \%$, hypocalcemia in $9 \%$ and acute renal failure in $4 \%$ of cases. Fluid replacement was conducted in $86 \%$ of patients, vasopressors in $16 \%$ (norepinephrine in $16 \%$, associated with dobutamine in $4 \%$, switched by epinephrine in $1 \%$ ), atropine in $3 \%$ and mechanical ventilation in $7 \%$ of cases. Gastric lavage was performed in $11 \%$ of cases. Charcoal was administrated in $34 \%$ of cases. Gastric lavage and charcoal were associated in $4 \%$ of cases. Calcium gluconate was used in $20 \%$ of cases. Insulin euglycemia therapy was performed in $4 \%$ of cases. Most of our patients had good outcome. Only one case of death was recorded after 25 days of ICU stay. Hospital stay ranges from 1 day to 25 days (median $=1$ day). Risk factors identified in the univariate analysis were the ingestion of nicardipine $(p=0.02$; OR 5.7 [1.4-23.2]), the ingestion of an amount superior to $150 \mathrm{mg}$ of amlodipine $(p=0.036$; OR undefined), SAPS II score $\geq 16(p<0.0001$; OR 61.6 [10.5-358.9]), APACHE II score $\geq 7$ ( $p=0.002$; OR 9 [2.3-34.5]), SOFA score $\geq 2(p<0.0001 ;$ OR 135 [13.5-1347.2]), SAPS II expanded score $\geq 1.9(p<0.0001$; OR 110 [12.1-999.9]), hypotension $(p<0.0001$; OR 1.6 [1.2-2.1]), faintness ( $p=0.01$; OR 13.6 [1.9-94.6]), electrocardiographic abnormalities $(p=0.044$; OR $4.1[1-15.8])$, subepicardial ischemia $(p=0.033$; OR 6 [3.5-10.4]), metabolic acidosis $(p<0.0001$; OR 61.2 [7.1-525.2]), hyperlactatemia ( $p=0.01$; OR 21.3 [1.8-251.2]), hypocalcemia $(p=0.001$; OR $18.4[3-111.3])$ and hyperglycemia $(p<0.0001$; OR 13.2 [5.6-30.6]). Nifedipine ingestion was associated with less severe intoxications ( $p<0.0001 ;$ OR 0.08 [0.02-0.3]). Multivariate analysis was inconclusive.

Conclusion Calcium channel blockers poisoning is severe and may be life-threatening. Its management is primarily symptomatic and must be early. The development of new antidotes may improve the prognosis of these intoxications.

\section{Competing interests None.}

\section{P152}

\section{Characteristics and outcomes in patients}

with community-acquired pneumonia admitted to intensive care unit

Takoua Marhbène ${ }^{1}$, Islam Mejri ${ }^{2}$, Amira Jamoussi ${ }^{3}$, Douha Lakhdar ${ }^{4}$, Asma Ghariani ${ }^{5}$, Leila Slim ${ }^{6}$, Jalila Ben Khelil', Mohamed Besbes ${ }^{1}$

${ }^{1}$ Réanimation médicale, Hôpital Abderrahmen Mami, Ariana, Tunisia; ${ }^{2}$ Réanimation respiratoire, Hôpital Abderrahmen Mami de pneumo-phtisiologie, Ariana, Tunisia; ${ }^{3}$ Respiratory icu, Hospital Abderrahmen Mami De Pneumo-Phtisiologie, Ariana, Tunisia; ${ }^{4}$ Réanimation médicale, Hospital Abderrahmen Mami De Pneumo-Phtisiologie, Ariana, Tunisia; ${ }^{5}$ Laboratoire de microbiologie, Hôpital Abderrahmen Mami, Ariana, Tunisia; ${ }^{6}$ Microbiology, Hospital Abderrahmen Mami De Pneumo-Phtisiologie, Ariana, Tunisia
Correspondence: Takoua Marhbène - takouamg@yahoo.fr Annals of Intensive Care 2016, 6(Suppl 1):P152

Introduction Community-acquired pneumonia (CAP) is a leading infectious cause of hospitalization and death among adults admitted in intensive care unit (ICU). Despite advances in critical care management and antimicrobial therapy, its morbi-mortality still remains high. Our aims were to study characteristics and outcomes of patients admitted in ICU with severe community-acquired pneumonia over 8 years.

Patients and methods It was a retrospective cohort study from January 1,2008 , to September 15, 2015. We described epidemiology, etiology, treatment and outcomes of patients admitted to ICU during this period.

Results Three hundred and twenty-five patients were enrolled; the overall cumulative incidence was 85 episodes/1000 admissions with progressive increase over the years. The median age was $59 \pm 18$ years and sex ratio $=3 / 1$. Seventy-five percentage of patients were hospitalized from emergency room. One hundred and twenty-one patients had received antimicrobial treatment before ICU admission. Comorbidities were observed in $84 \%$ of patients (253): Chronic lung disease in $59.3 \%$, chronic heart disease in $40.3 \%$, diabetes mellitus in $21 \%$ and immunosuppression in $9.85 \%$. Fine score was high (class IV et V) in $67.4 \%$ of patients. The mean IGS II and SOFA scores at admission were, respectively, 37 and 6 . Major criteria of ATS score were found in $72 \%$ (more than half have at least 2 major criteria). All patients had an acute respiratory failure with mean $P / F=200 \pm 87$. Mechanical ventilation was needed in $64 \%$ initially NIV for $75 \%$ of them $(n=156)$. The median duration of mechanical ventilation was 3 days [1-135]. Septic shock was observed in $27 \%$ (89). Radiographic finding are: alveolar infiltrate $99 \%$, pleural effusion $27 \%$, multilobular and bilateral involvement in 66 and $49 \%$, respectively. Acute respiratory distress syndrome was present in 131 cases (27.6\%); it was classified as severe or mild $\left(\mathrm{PaO}_{2} / \mathrm{FiO}_{2} \leq 150\right)$ in 75 patients. Pathogen was detected in $62 \%$ of cases. It was complicated with bacteremia within 11 patients. The most frequent microorganisms isolated were Hemophilus influenzae, Streptocoque pneumoniae, Pseudomonas aeruginosa and Legionella pneumophila; the respective averages were 11, 10. 7 , 4.6 and $3.4 \%$. More than one bacterium was identified in $14.8 \%$ cases (48). All patients had empiric antibiotherapy at admission; it was given to $90 \%$ of patients in the first $24 \mathrm{~h}$ and to $52.4 \%$ in the first 6 h. Combination antibiotic therapy (betalactam + macrolide or fluoroquinolone) was prescribed in $33.8 \%(n=110)$. The median length of ICU stay was $13 \pm 15$ days. Mortality rate was $30 \%$ (99). The common cause of death was multivisceral dysfunction $17 \%$ (57). Univariate analysis showed that age $\geq 65$ years, chronic respiratory disease, respiratory failure, septic shock at admission, bilateral involvement and bacteremia were predictive factors for mortality. Multivariate analysis showed that only multiorgan failure was an independent factor related to ICU mortality [OR 0.95; $95 \% \mathrm{Cl}$ (0.214-1.11)].

Conclusion Our study confirms that CAP requiring hospitalization in the ICU is associated with high mortality. Presence of multiorgan failure at admission is the major factor for worse prognosis.

Competing interests None.

P153

Comparison of characteristics and outcomes of pneumococcal community-acquired pneumonia versus other pathogens

Takoua T. Merhabane ${ }^{1}$, Héla Maamouri ${ }^{2}$, Amira Jamoussi ${ }^{3}$, Asma Ghariani ${ }^{4}$, Leila Slim ${ }^{5}$, Jalila Ben Khelii ${ }^{6}$, Mohamed Besbes ${ }^{6}$

${ }^{1}$ Réanimation respiratoire, Hôpital Abderrahmen Mami de pneumo-phtisiologie, Ariana, Tunisia; ${ }^{2}$ Service de réanimation médicale, Centre d'assistance médicale-urgente, Tunis, Tunisia; ${ }^{3}$ Respiratory icu, Hospital Abderrahmen Mami De Pneumo-Phtisiologie, Ariana, Tunisia; ${ }^{4}$ Laboratoire de microbiologie, Hôpital Abderrahmen Mami, Ariana, Tunisia; ${ }^{5}$ Microbiology, Hospital Abderrahmen Mami De Pneumo-Phtisiologie, Ariana, Tunisia; ${ }^{6}$ Réanimation médicale, Hôpital Abderrahmen Mami, Ariana, Tunisia 
Correspondence: Héla Maamouri - helamaamouri@yahoo.fr Annals of Intensive Care 2016, 6(Suppl 1):P153

\section{Introduction}

Background Community-acquired pneumonia (CAP) is a leading cause of mortality in intensive care units. It has not been determined whether CAP is associated with increased mortality among patients with specific pathogens as Streptococcus pneumoniae.

Purpose To compare demographic, clinical data, therapeutic and outcomes of patients admitted to our ICU for pneumococcal communityacquired pneumonia versus other microorganisms.

\section{Patients and methods}

Methods It was a retrospective study, which included 325 CAP over a period of 8 years from January 1, 2008, to September 15, 2015. We have defined 2 groups: GI (71 pneumococcal pneumonia) and GII (254 other microorganisms).

\section{Results}

Results No significant difference was found in mean age (60 vs 57 years), sex ratio (2.19 vs 3.09), IGSII (38 vs 37), and SOFA score (5 in two groups). Comorbidities such as diabetes and COPD were more frequent on group I. All patients had an acute respiratory failure. At admission, radiographic findings were similar in two groups. In group I, Streptococcus Pneumoniae was isolated in respiratory samples in all cases. Positive pneumococcal antigenuria was detected in $92.5 \%$, which allowed an early appropriate antibiotic therapy. CAP was associated with bacteremia in $50.7 \%$ in pneumococcal group versus $31 \%$ in second group. Initially, patients in group I were more severe: $18 \%$ had an acute respiratory distress versus $12 \% ; 62 \%$ needed mechanical ventilation versus $64 \% ; 31 \%$ had septic shock versus $26 \%$ and acute kidney failure (50.7 vs $41 \%)$. These differences were not significant. Delay of starting antibiotic was shorter in group I with a median of $5.22 \mathrm{~h}$ versus 15.68 ( $p=0.004)$. All strains of pneumococcal were susceptible to penicillin. Median duration of mechanical ventilation (8 vs 10 ) and length of ICU stay (13 vs 17 days) were shorter in group I. Mortality rate was similar in two groups (30.6 vs $31.4 \%$ ).

\section{Conclusion}

Conclusion In comparison with other microorganism, pneumococcal community-acquired pneumonia requiring hospitalization in ICU is associated with severe clinical presentation without increasing mortality.

\section{Competing interests None.}

\section{P154}

Outcome of $L$. pneumophila pneumonia and risk factors for ICU admission

Oestreicher Charlotte ${ }^{1}$, Helena Wegelius ${ }^{1}$, Caroline Landelle ${ }^{2}$, Jacques Schrenzel ${ }^{3}$, Laurent Kaiser ${ }^{3}$, Jérôme Pugin ${ }^{1}$

'Intensive care, University Hospitals of Geneva, Geneva, Switzerland;

${ }^{2}$ Infection control programme, University Hospitals of Geneva, Geneva,

Switzerland; Infectious diseases, University Hospitals of Geneva, Geneva, Switzerland

Correspondence: Jérôme Pugin - jerome.pugin@unige.ch

Annals of Intensive Care 2016, 6(Suppl 1):P154

Introduction L. pneumophila pneumonia is a rather frequent pathogen of community-acquired pneumonia (CAP) and can lead to severe complications such as septic shock, acute renal failure and ARDS, requiring admission to the ICU. Its rapid diagnosis has become easier using the urinary antigen detection.

Patients and methods We reviewed demographical, clinical, radiological, biological and outcome data from all patients presenting to the emergency department with CAP, and who had a positive urinary antigen for type 1 L. pneumophila and/or positive L. pneumophila cultures between 2009 and 2014. We compared data from patients admitted to the ICU and those admitted to the ward. Both univariate and multivariate analysis were performed to identify risk factors for ICU admission.

Results Except for one patient, all received an initial antibiotic therapy containing an anti-legionella antibiotic (macrolide or quinolone). Comorbidities frequently associated with $L$. pneumophila CAP were heavy smoking $(43.7 \%)$, cardiovascular disease (34.5\%), immune suppression (29.9\%), alcoholism (28.7\%), diabetes ( $23 \%)$, and neoplastic disease $(20.7 \%)$. Of these 87 patients, 31 were admitted to the ICU, whereas 56 patients were sent to the ward. The CURB65 score was not found to be statistically different between the 2 groups. Patients admitted to the ICU had a significantly higher proportion of chronic pulmonary disease (mainly COPD), 29 versus $8.9 \%$, higher plasma urea, percentage of band forms, procalcitonin levels, a lower plasma albumin, blood lymphocyte count, $\mathrm{PaO}_{2} / \mathrm{FiO}_{2}$ ratio, arterial $\mathrm{pH}$, and a multilobar pneumonia (4 quadrants, 45 vs. $3.6 \%$ ). Hospital mortality was higher in ICU-admitted patients (38.7 vs. $12.5 \%, p=0.005$ ). Patients admitted to the ICU required mechanical ventilation (87.1\%) for a median duration of 6 days, $25.8 \%$ received inhaled NO, $22.6 \%$ CVVHD, $19.4 \%$, glucocorticoids for ARDS, and $12.9 \%$ prone positioning. $67.7 \%$ developed septic shock. Only 2 patients developed a pneumothorax requiring drainage. A multivariate analysis identified plasma urea (adjusted odds ratio (aOR) per 1-unit increment $1.12[95 \% \mathrm{Cl}$ $1.03-1.23], p=0.01$ ), percentage of band forms (aOR per 1-unit increment 1.09 [1.03-1.17], $p=0.006$ ), and a multilobar pneumonia (OR 24.80 [4.10-149.88], $p<0.0001)$ as independent predictors for ICU admission.

Discussion L. pneumophila CAP remains a serious condition frequently requiring admission to the ICU, with hemodynamic, ventilatory, and renal support, particularly those with preexisting lung disease. Some of these patients also required specific therapies such as inhaled NO, prone positioning, and glucocorticoids. Patients admitted to the ICU were also severely hypoxemic.

Conclusion High plasma urea, band forms, and a multilobar pneumonia are independent risk factors for ICU admission in patients presenting with L. pneumophila CAP. In contrast, the CURB65 score did not identify high-risk patients. Mortality of patients admitted to the ICU was 3.1 times higher than that of patients admitted to the ward.

\section{Competing interests None.}

\section{P155}

The descending necrotizing mediastinitis: report of 60 cases Hind Belhadj

${ }^{1}$ Reanimation-anesthesie, CHU Mohamed VI, Marrakesh, Morocco

Correspondence: Hind Belhadj - hinda_0611@hotmail.fr

Annals of Intensive Care 2016, 6(Suppl 1):P155

Introduction The descending necrotizing mediastinitis is a very serious infectious disease life-threatening in 30-40\%. The prognosis depends on the speed and type of care, the patient's condition at the time of diagnosis. The purpose of this study was to report the experience of our intensive care's department in the management of this disease.

Materials and methods This is transversal study area between 2011 and 2015 relating 60 patients treated for complicating the descending necrotizing mediastinitis facial cellulitis. We excluded from this study mediastinitis complicating postoperative mediastinitis and cases of cellulitis limited to the cervical region.

Results The mean age was 27 years. We identified 12 diabetics, 6 cases in pregnancy and 30 cases of anti-inflammatory prior decision. Fiftyfour patients had dental entrance door so that was a cervical metallic foreign body. The gaseous accounted for $60 \%$, while $40 \%$ phlegmonous. In $50 \%$ of cases, the infection was polymicrobial. Involvement of the anterior mediastinum was observed in $70 \%$ of cases, the average posterior mediastinum and in $20 \%$, one case of pericardial and pleural effusion and 5 cases of pleuresy. The postoperative course was marked by the death in 10 cases by septic shock or following an extensive infectious pneumonitis.

Discussion The descending necrotizing mediastinitis is very serious infections whose mortality remains high. The most of the deaths are in keeping with a cardiovascular collapse, a septic shock or a visceral multi-failure. The clinical diagnosis is easy when the clinical picture is complete but is often evoked with delay. The germs most frequently isolated is cocci Gram with which the antibiotic treatment has to cover Bacilli to negative Gram, the anaerobic germs, the hulls with positive Gram and must be of long term. The surgical care must be the most earliest to evacuate the abscesses. The supervision operating 
comment is before all private hospital and is done in an middle of reanimation.

Conclusion Mediastinitis secondary to extension of head and neck cellulite remains a redoubtable complication burdened with a high mortality rate; the diagnosis is often mentioned with delay stage septic shock. The surgical management should be as early as possible.

\section{Competing interests None.}

\section{References}

1. Estrera AS, Landay MJ, Grisham JM, Sinn DP, Platt MR. Descending necrotizing mediastinitis. Surg Gynecol Obstet. 983; 157: 54552.

2. A. Benzaouia et al.: Cellulite cervicofaciale: study over 1 year about 106 cases; French Annals of Otolaryngology and Pathology Cervico-faciale, Volume 130: 4, October on 2013

\section{P156}

Characteristics, mortality risk factors and therapeutic approach of ARDS in patients with severe leptospirosis in Reunion Island Paul Chanareille ${ }^{1}$, Julien Jabot ${ }^{1}$, Benjamin Delmas ${ }^{1}$, Arnaud Roussiaux ${ }^{1}$, Cyril Ferdynus ${ }^{2}$, Bernard-Alex Gauzere', Olivier Martinet ${ }^{1}$, David Vandroux ${ }^{1}$ Réanimation polyvalente, CHU La Réunion, Saint-Denis, France; ${ }^{2}$ Unité de soutien méthodologique, CHU La Réunion, Saint-Denis, France

Correspondence: Paul Chanareille - pmchana@hotmail.fr

Annals of Intensive Care 2016, 6(Suppl 1):P156

Introduction Leptospirosis is a major public health concern in Reunion Island and in the other French overseas territories. The clinical manifestations are extremely varied (from flu-like condition to multiorgan failure), and significant mortality is described in some parts of the world. The pulmonary forms have a major impact on patients' prognosis. The objective of this study is to describe characteristics of patients with leptospirosis presenting acute respiratory distress syndrome (ARDS) criteria, therapeutic approach and mortality risk factors in this specific population.

Patients and methods A retrospective study of 28 patients with leptospirosis and ARDS at University Hospital of Reunion Island (SaintDenis) was performed from May 2004 to April 2015.

Results Demographic, clinical, biological and therapeutic data were collected. A mortality risk factors bivariate analysis was performed in 24 men and 4 women aged $42 \pm 18$, and IGS 2 to admission of $58 \pm 21$. Hemorrhagic pulmonary forms are the most frequent $(89 \%)$ and mortality is $25 \%$. In situation of massive hemorrhagic syndromes, desmopressin was used in 11 patients: hemoptysis was under control and nine of them left the unit alive. Five patients underwent circulatory/respiratory support (ECMO). 80 \% (ECMO) and $82 \%$ (desmopressin) survival rates are observed with these two therapeutics alone or associated. Last, seven biological parameters are independently associated with mortality (survivors vs non-survivors for each parameter):

Table 30 See text for description

\begin{tabular}{|c|c|c|c|c|}
\hline \multirow{2}{*}{ SOFA 3 or 4 } & Time & $\begin{array}{c}\text { Non survivors } \\
\text { N (\%) }\end{array}$ & $\begin{array}{c}\text { Survivors } \\
\text { N (\%) }\end{array}$ & P \\
\hline \multirow{3}{*}{ Renal } & Day1 & $10(71)$ & $29(61)$ & 0.02 \\
\cline { 2 - 5 } & Day 3 & $6(66)$ & $22(55)$ & 0.7 \\
\hline \multirow{2}{*}{ Cardiovascular } & Day1 & $7(50)$ & $16(34)$ & 0.02 \\
\cline { 2 - 5 } & Day 3 & $6(66)$ & $12(30)$ & 0.06 \\
\hline \multirow{3}{*}{ Liver } & Day1 & $9(15)$ & $24(41)$ & 0.02 \\
\cline { 2 - 5 } & Day 3 & $6(66)$ & $22(55)$ & 0.7 \\
\hline \multirow{2}{*}{\begin{tabular}{c} 
Coagulation \\
\cline { 2 - 5 }
\end{tabular}} & Day1 & $3(21)$ & $7(15)$ & 0.7 \\
\cline { 2 - 5 } & Day 3 & $2(22)$ & $6(15)$ & 0.6 \\
\hline \multirow{2}{*}{$\begin{array}{c}\text { Central Nervous } \\
\text { System }\end{array}$} & Day1 & $8(14)$ & $23(39)$ & 0.03 \\
\cline { 2 - 5 } & Day1 & $3(33)$ & $12(30)$ & 1 \\
\hline & Day 3 & $1(11)$ & $2(5)$ & 0.5 \\
\hline
\end{tabular}

total bilirubin $[135 \pm 147$ vs $403 \pm 359 \mu \mathrm{mol} / \mathrm{L}(p=0.01)]$, transaminases: AST [159 \pm 176 vs $256 \pm 134 \mathrm{UI} / \mathrm{L}(p=0.04)]$ and ALT $[77 \pm 60$ vs $216 \pm 189 \mathrm{UI} / \mathrm{L}(p=0.02)]$, prothrombin time $[82 \pm 12$ vs $58 \pm 29 \%$ $(p=0.05)]$, base excess $[-1 \pm 5 \mathrm{vs}-11 \pm 8 \mathrm{mmol} / \mathrm{L}(p=0.002)]$, potassium $[3.4 \pm 0.4$ vs $4.2 \pm 1.1 \mathrm{mmol} / \mathrm{L}(p=0.02)]$ and lactate $[3.4 \pm 3.6$ vs $12.8 \pm 7.4 \mathrm{mmol} / \mathrm{L}(p=0.002)]$.

Conclusion ARDS development is very important in severe leptospirosis prognosis. Quick identification of clinical and laboratory parameters on admission can contribute to early establishment of protocolised specific treatment such as desmopressin and/or circulatory/ respiratory support.

Competing interests None.

\section{P157}

Characterization of organ dysfunction in severe leptospirosis Adélie Ladureau', Alice Sanna², Amélie Rolle', Bertrand Pons', Pascale Piednoir ${ }^{1}$, Fanny Ardisson ${ }^{1}$, Frédéric Martino ${ }^{1}$, Thomas Bernos ${ }^{1}$, Khalid Elkoun', Elie Elain', Roland Lawson³, Matthieu Resh-Rigon², Sophie Belorgey ${ }^{4}$, Guillaume Thiery ${ }^{1}$

${ }^{1}$ Réanimation - grands brulés, CHU Pointe à Pitre - Abymes, Pointe à Pitre, France; ${ }^{2}$ Département de biostatistiques, Hôpital Saint Louis, Paris, France; ${ }^{3}$ Centre de réanimation, Centre Hospitalier Régional Universitaire de Lille, Lille, France; ${ }^{4}$ réanimation, Centre Hospitalier Général de Saint-Denis, Saint-Denis, France

Correspondence: Guillaume Thiery - guillaume.thiery@chu-guadeloupe.fr Annals of Intensive Care 2016, 6(Suppl 1):P157

Introduction Leptospirosis is the most widespread zoonotic disease in the world. Its incidence is particularly important in the French overseas territories, including Guadeloupe. This study aims to characterize organ failures in patients with severe forms of leptospirosis.

Patients and methods This is a retrospective study conducted between November 2009 and November 2014 in the ICU of Pointe à Pitre University Hospital in Guadeloupe. All patients admitted to intensive care during this period with a confirmed diagnosis of leptospirosis were included. We collected demographic, physiological, biological and radiographic data for each patients as well as sequential assessment of SOFA score of each organ on D1, D3, D6, D9, D12 and D15. Severe organ failure was defined by a SOFA score of 3 or 4 . Univariate analysis was performed to identify organ failures associated with mortality.

Results Sixty-one patients were included, of whom $90.2 \%$ were male, with a median age of 54 years [41-66]. A severe organ failure was met in renal, liver, hematological and hemodynamic failure in 66,58 , 53 and $39 \%$ of the patients, respectively. Mechanical ventilation was needed in $38 \%$ patients, renal replacement therapy in $48 \%$ and vasoactive drugs in $52 \%$.

ICU and hospital mortality were $20 \%$ and $23 \mathrm{M} \%$, respectively. In univariate analysis, factors associated with hospital mortality were SASP II, chronic hypertension, hyperlactatemia, mechanical ventilation and renal replacement therapy. Organ dysfunctions associated with mortality are given in Table 30.

Conclusion Organ failure predominantly met in severe forms of leptospirosis is kidney, liver and hematological failure as historically described in Weils triade. All but respiratory failure at day 1 is associated with mortality but none of them at day 3 .

Competing interests None.

P158

Report of experience: management of a pre-XDR and XDR-TB population hospitalized in a medical ICU

Caroline Hauw Berlemont ${ }^{1}$, Cyprian Pricopi ${ }^{2}$, Florence Boissier ${ }^{1}$, Alexandra Monnier ${ }^{1}$, Françoise Barthes ${ }^{2}$, Jean-Luc Diehl', Jean-Yves Fagon', Emmanuel Guérot

${ }^{1}$ Réanimation médicale, Hopital Europeen Georges-Pompidou, Paris, France; ${ }^{2}$ Chirurgie thoracique, Hopital Europeen Georges-Pompidou, Paris, France

Correspondence: Caroline Hauw Berlemont - alexis.berlemont@gmail. com

Annals of Intensive Care 2016, 6(Suppl 1):P158 
Introduction Treatment of pre-eXtensively drug-resistant tuberculosis (pre-XDR-TB, i.e., resistant to isoniazid, rifampicin and fluoroquinolone or second-line injectables) and eXtensively drugresistant tuberculosis (XDR-TB, i.e., resistant to fluoroquinolones and second-line injectables) is a challenge because it needs a networking and a specific organization of care, to avoid the spread of mycobacteria, like it is specified in several reports, like that of the Haut Conseil de Santé Publique of December 2014. Pre-XDR and XDR-TB patients are managed in our university medical ICU, in negative pressure containment rooms with reverse air. We report our experience for the management of this population of pre-XDR and XDR-TB patients.

Patients and methods We collected data carried on pre- and XDR-TB patients hospitalized in our university medical ICU, between July 2012 and July 2015: epidemiology, tuberculosis and treatment history, indication of surgery if required, events during post-surgery period, delay of sputum smear conversion and mortality.

Results Twenty-one tuberculosis patients were hospitalized in our ICU since 3 years: 11 XDR-TB (52 \%) and 10 pre-XDR-TB (48\%). Two lived in France since more than 10 years, while others came from the Caucasus. The mean age was $34.8 \pm 9$ years. The body mass index was $19.75 \pm 4.12$. Fourteen percentage did not receive any treatment before the diagnosis in France; 86 \% received at least five antibiotics molecules and on average 7.93 ; the mean period of antibiotic treatment before ICU admission was $112 \pm 74$ days. On admission, 6 patients (39\%) had converted their sputum smear. Among the 21 patients, 5 were admitted for vascular access complications and 1 for acute respiratory distress. Fifteeen patients (71\%) were admitted for surgical reasons: The using of surgery was decided because the sputum cultures remained positive for $66 \%$ of them and for irreversible pulmonary damages in the other cases. The surgery consisted of a lobectomy for 9 patients and pneumonectomy for 6 patients. One patient had a life-threatening complication during the surgery (bleeding by artery wound). Thirteen $(87 \%)$ were extubated in few hours, while 2 patients have been intubated for 6 and 20 days. During the immediate post-surgery period, 2 patients experienced an extra-pulmonary complication, while 4 had postoperative complication: 2 atelectasis, treated with medical care, 1 fistula and 1 pleural bleeding, needed a second surgery. Among the 21 patients, 5 were admitted for vascular access complication and 1 for acute respiratory distress. Ninety-five percentage of patients were alive on leaving the ICU. For now, 1 medical patient died because of a septic shock; 2 surgical patients (13\%) died, $3(20 \%)$ are cured, 1 is lost sight and $8(53 \%)$ are always under treatment and are followed. Among 10 surgical patients whose sputum smear remained positive, 9 converted their sputum smear in a median time of 45 days. Among 5 medical patients, the median sputum smear conversion was observed in 143 days after the beginning of treatment. Among every patient, there was no complication attributable to the specific organization of care, in particular the conditions of containment.

Conclusion The management of pre- and XDR-tuberculosis patients is binding, time-consuming and resources consuming, needing a very specific organization of care. Experience shows that an ICU stay does not generate more complications for these tuberculosis patients and can improve their global outcome.

\section{Competing interests None.}

\section{P159}

Benefit of magnesium sulfate for tetanus management in adults: case of the provincial general referral hospital of Kinshasa

Thansya Doddie ${ }^{1}$, Mj Nsiala $^{2}$, Nh Situakibanza ${ }^{3}$, Be Amisi², Mj Kazadi ${ }^{4}$, Ma Kilembe ${ }^{2}$

${ }^{1}$ Anesthésie-réanimation, Cliniques universitaires de Kinshasa, Kinshasa, Democratic Republic of the Congo; ${ }^{2}$ Anaesthesiology -reanimation,

Cliniques universitaires, Kinshasa, Democratic Republic of the Congo;

${ }^{3}$ Internal medicine, tropical medicine, infectious and parasitic diseases,
University of Kinshasa, Kinshasa, Democratic Republic of the Congo; ${ }^{4}$ Anaesthesiology-reanimation, Provincial General Referral Hospital of Kinshasa, Kinshasa, Democratic Republic of the Congo

Correspondence:Thansya Doddie - doddiethansya@yahoo.fr

Annals of Intensive Care 2016, 6(Suppl 1):P159

Introduction The effectiveness of magnesium sulfate for tetanus treatment has already been proven in several studies [1-4]. However, despite the existing data regarding this treatment, the adverse reactions remain the only fright, sometimes severe, suchlike respiratory depression, caused their stop being abandoned.

Objectives: To assess the relationship between the profit and the risk due to the treatment comparatively to diazepam during our daily tetanus management in adults.

Patients and methods The present study compares a series of 63 patients with tetanus, who were treated by magnesium sulfate versus a historical serioes of 67 patients with tetanus, who were conventionally treated with diazepam. It took place within the reanimation multiservice room of the provincial general referral hospital of Kinshasa in the Democratic Republic of the Congo (August 2013 through September 2015). All the consecutive patients, of over 15 years of age, admitted for tetanus management during the study period were included, without any selection. Patients with kidney failure, shock or well known as allergic to magnesium sulfate were all excluded. Apart from the treatment of muscular spasm, all the patients in both groups were identically managed in accordance with their usual treatment as provided by the unit. The collected data were performed retrospectively from the medical forms of all the control patients and prospectively for the study group. The decisional main criterion was the total end of muscular spasm for effectiveness delay and the occurring frequency of adverse reactions for tolerance. The statistical data were obtained via SPSS software 21.0. This study was recorded at the Ethical Office of the School of Public Health of the University of Kinshasa, approval date: ESP/CE/043/2014.

Results Between the two groups, all the patients globally presented the same features. The average age was $31.54 \mathrm{y}-\mathrm{o} \pm 16.56$, and $72 \%$ of those patients were males. The complete spasm stops were obtained in short course when treated by magnesium sulfate $(5.8 \mathrm{~d} \pm 1.5$ vs $9.8 \mathrm{~d} \pm 2.6 ; p=0.000$ ). The occurrence of respiratory depression was minimally observed in the category treated by magnesium sulfate (19 vs $46.3 \%, p=0.001$ ). This situation clearly showed the decrease use of mechanically based ventilation (12.7 vs $35.8 \%, p=0.002)$. Moreover, the duration of stay in resuscitation room decreased for this category $(9.3 \mathrm{~d} \pm 1.8$ vs $22.6 \mathrm{~d} \pm 10.1 ; p=0.000)$, with the reduced risk of sepsis ( 14.3 vs $31.3 \% ; p=0.023$ ). Finally, the hospital mortality of any origin was also significantly weak for this group of patients treated by magnesium sulfate ( 25.4 vs $50.7 \% ; p=0.004$ ).

Conclusion During our study, the balance profit risk was favorable to magnesium sulfate comparatively with diazepam. This molecule, even though slightly expensive, requires a good monitoring and frequent and rigorous control of tendinous reflexes, the respiratory beats, as well as the diuresis in order to primarily detect any overdosage.

\section{Competing interests None.}

\section{References}

1. Attygalle D, Rodrigo N: Magnesium as first line therapy in the management of tetanus: a prospective study of 40 patients. Anaesthesia 2002, 57:811-817.

2. Osalusi BS, Ogun SA, Ogunniyi A, Kolapo KO. Comparison of the efficacy of magnesium sulphate and diazepam in the control of tetanus spasms. Scientific Research and Essays 2008; 3: 571-6.

3. Ali G, Kamal M, Khan AN. Comparison of the efficacy of magnesium sulphate and diazepam in the control of tetanus spasm. Journal of Postgraduate Medical Institute 2011; 25: 106-10.

4. Thwaites CL, Yen LM, Loan HT, Thuy TT, Thwaites GE, Stepniewska K, Soni N, White NJ, Farrar JJ: Magnesium sulphate for treatment of severe tetanus: a randomised controlled trial. Lancet 2006, 368:1436-1443. 
P160

Ebola fever outbreak: questioning the delivery of care

Christophe Clement ${ }^{1}$, François Lamontagne ${ }^{2}$

${ }^{1}$ RMC, Polyclinique Bordeaux Nord Aquitaine, Bordeaux, France; ${ }^{2}$ Intensive care unit, Université de Sherbrooke, Sherbrooke, Canada

Correspondence: Christophe Clement - cclement7452@gmail.com

Annals of Intensive Care 2016, 6(Suppl 1):P160

Introduction Over 20,000 people were infected during the 2014 Ebola fever outbreak in West Africa, with a mortality rate of approximately $40 \%$.

The authors, who participated in two missions in various treatment units in Guinea and Liberia, systematically reviewed the literature and provide a historical perspective on the delivery of care in Ebola treatment units.

Patients and methods We systematically reviewed published articles that described the clinical management of patients infected by Ebola virus since 1976 . We compared with reports on the 2014 West African outbreak with previous publications and describe historical trends in monitoring, treatment and prognosis.

Results Since 1976, monitoring and treatment of patients treated within Ebola treatment units have not evolved. Monitoring of vital signs after the day of admission is never documented. Supportive care measures, such as fluid and electrolyte therapy, titrated to compensate losses, are also not mentioned. Survival has not significantly improved over time.

In contrast, in another filovirus infection, Marburg hemorrhagic fever which is very like Ebola fever, mortality rate was approximately $25 \%$ in Germany (1967), but approximately 80 \% in sub-Saharan Africa.

Discussion As reported, monitoring and treatment of patients isolated in Ebola treatment units have not evolved since early reports. This potentially explains why mortality rates have remained high. Failure to ensure proper monitoring of vital signs and correction of fluid losses and electrolytes anomalies appears suboptimal and misaligned with clinical description of severe shock that are consistent across time. Expert recommendations for monitoring and treatment remain unchanged since 1976. Our inability to comply with these recommendations in Africa may explain why outcomes are poor, whereas survival was much higher, even during early outbreaks, in Europe.

Conclusion Improving the delivery of care for the next outbreak or a resurgence of the current Ebola fever outbreak will require that we collectively address the issues of accountability.

\section{Competing interests None.}

\section{P161}

Marked decrease in the incidence of ventilator-associated pneumonia using a bundle of care containing selective oropharyngeal decontamination

Boyer, Valérie Nocquet ${ }^{1}$, Caroline Landelle ${ }^{2}$, Sébastien Naimo ${ }^{1}$, Ronan Raulais ${ }^{1}$, Emilie Genevois ${ }^{1}$, Lucie Bouchoud ${ }^{3}$, Filippo Boroli ${ }^{1}$, Nour Abidi ${ }^{1}$ Mohamed Abbas ${ }^{4}$, Stefan Harbarth², Jérôme Pugin ${ }^{1}$

${ }^{1}$ Intensive care, University Hospitals of Geneva, Geneva, Switzerland; ${ }^{2}$ Infection control programme, University Hospitals of Geneva, Geneva, Switzerland; ${ }^{3}$ Pharmacy, University Hospitals of Geneva, Geneva, Switzerland; ${ }^{4}$ Infectious diseases, University Hospitals of Geneva, Geneva, Switzerland

Correspondence: Jérôme Pugin - jerome.pugin@unige.ch Annals of Intensive Care 2016, 6(Suppl 1):P161

Introduction Ventilator-associated pneumonia (VAP) remains a significant complication of mechanically ventilated patients. VAP is associated with costs, increased duration of mechanical ventilation (MV), ICU stay and mortality. Our project aimed at decreasing the incidence of VAP using a multifaceted quality program, a bundle of care composed of 10 measures introduced stepwise, including selective oropharyngeal decontamination (SOD) with topical antibiotics.

Patients and methods The incidence density of VAP (number of VAP/1000 ventilation days in patients ventilated for $\geq 48 \mathrm{~h}$ ) was measured monthly before the introduction of the VAP bundle (baseline period, 8 months) and every month thereafter (implementation period, 11 months). VAP was diagnosed as "probable" if the patient had clinical, biological, and radiological signs of pneumonia (CPIS $\geq 6$ ), and a potential pathogen in tracheal aspirates after $48 \mathrm{~h}$ of MV. VAP was "certain" if a pathogen was cultured $\geq 10 \mathrm{E} 3$ in BAL fluid obtained by a protected Combicath ${ }^{\circledR}$ catheter or $\geq 10$ E4 by bronchoscopy. The bundle was introduced sequentially in blocks: first block: hand hygiene, bed head $>30^{\circ}$, oral care using chlorhexidine mouth wash, daily evaluation of separation from the ventilator, daily awakenings, early mobilization, and use of the Combicath ${ }^{\circledR}$ to diagnose VAP; second block: implementation of endotracheal tubes with subglottic aspiration and cuff pressure maintained $\geq 30 \mathrm{cmH} 2 \mathrm{O}$ using an automatic controller; third block: SOD administration TID containing colistin, tobramycine and nystatin. Small groups teaching allowed to train $>90 \%$ of the caregivers to the bundle within 3 months after the beginning of the implementation period. The compliance of the nurses to each measure of the VAP bundle was assessed 4 days per week during all study period in all intubated patients. A prevalence study of antibiotic resistance in stool bacteria was performed each month in all ICU patients.

Results Episodes of intubations $\geq 48 \mathrm{~h}$ were 318 (baseline period) and 468 (implementation period). The compliance of nurses to the elements of bundles significantly increased during the implementation period for bed head $>30^{\circ}$, oral care, cuff pressure $\geq 30 \mathrm{cmH}_{2} \mathrm{O}$, evaluation of extubability, daily awakenings, use of subglottic endotracheal tube, and use of SOD. The monthly incidence density of VAP ("probable" + "certain" VAPs) was $21.5 \pm 7.8$ before versus $10.8 \pm 5.6$ $\mathrm{VAP} / 1000$ days of ventilation after the implementation of the bundle $(p=0.003)$. During the last 4 months, after the introduction of the SOD, the incidence density of VAP was $5.3 \pm 1.4 \mathrm{VAP} / 1000$ ventilation days. The number of first episode of VAP fell from 20.1 to $9 \%$ $(p<0.0001)$. The median duration of mechanical ventilation in patients intubated $\geq 48 \mathrm{~h}$ fell from 7.14 (before) to 6.36 (after) days ( $p=0.004$ ). The use of Combicath ${ }^{\circledR}$ to diagnose VAP increased from 54 (before) to $81 \%$ (after, $p=0.003$ ), with a concomitant increase in "certain" VAP diagnoses, from 56 to $88 \%(p=0.001)$. In monthly prevalence studies, the rate of fecal multiresistant bacteria was similar before and after the beginning of the SOD.

Discussion The implementation of VAP bundles based on measures recognized to prevent the development of VAP has invariably shown to decrease the incidence of VAP. Herein, we show that it is possible to significantly decrease the incidence density of VAP using a block of "classical" preventive measures, including the use of endotracheal tubes with subglottic aspiration and cuff pressure controller. The addition of SOD to the preventive measures was associated with further decrease of the VAP incidence density, constantly below 10 VAP/1000 ventilation days since SOD introduction. This resulted in a significant decrease in the duration of mechanical ventilation in this patient population. The increase in the use of Combicath ${ }^{\circledR}$ in patients suspect of having VAP resulted in an increase of the diagnosis of "certain" VAP.

Conclusion A multifaceted program implementing sequentially measures known to prevent VAP was able to significantly decrease the incidence of VAP by more than half and to significantly decrease the duration of mechanical ventilation.

\section{Competing interests None.}

References

Bouadma et al. Clin Infect Dis 2010;51:1115.

Marra et al. Am J Infect Control 2009;37:619. 
P162

Ventilator-associated pneumonia prevention by oral care improvement: a before-after study

Pierre-Emmanuel Charles ${ }^{1}$, Caroline Dereux ${ }^{2}$, Audrey Large ${ }^{2}$, Rémi $^{2}$

Bruyère $^{3}$, Sebastien Prin ${ }^{2}$, Julien Bador ${ }^{2}$, Jean-Pierre Quenot ${ }^{1}$

${ }^{1}$ Réanimation médicale, Chu Dijon, Dijon, France; ${ }^{2} 21000$, Chu Dijon,

Dijon, France; ${ }^{3} 01$, CH de Fleyrat, Bourg-en-Bresse, France

Correspondence: Pierre-Emmanuel Charles-pierre-emmanuel.charles@ chu-dijon.fr

Annals of Intensive Care 2016, 6(Suppl 1):P162

Introduction Ventilator-associated pneumonia (VAP) is the most frequently encountered hospital acquired infection in the patients subjected to mechanical ventilation (MV). Significant mortality and prolonged length of stay (LOS) are generally related to VAP. Oral care including antiseptic agents application is part of bundles likely to prevent VAP. Tooth brushing efficacy is, however, controversial. We hypothesized that implementation of an enhanced oral care protocol including tooth brushing could reduce VAP incidence. Ventilator-associated pneumonia (VAP) is the most frequently encountered hospitalacquired infection in the patients subjected to mechanical ventilation (MV). Significant mortality and prolonged length of stay (LOS) are generally related to VAP. Oral care including antiseptic agents application is part of bundles likely to prevent VAP. Tooth brushing efficacy is, however, controversial. We hypothesized that implementation of an enhanced oral care protocol including tooth brushing could reduce VAP incidence.

Patients and methods We conducted a before-after study including all the patients admitted to our ICU and submitted to MV for at least $48 \mathrm{~h}$ during the "before" (April 1 to December 31, 2013) and the "intervention" period (April, 1 to December 31, 2014). Standard care in our ICU relied on basic oral care with chlorhexidine $0.5 \% 6$ times a day. Intervention consisted on the implementation of a new oral care kit including tooth brushing and oral cleansing solution containing chlorhexidine $0.12 \%$ (Q-Care ${ }^{\circledR}$, Sage products, Cary, IL). Other infection control interventions were not modified between the 2 study periods. VAP occurrence was recorded prospectively during both periods as well as relevant clinical data. The primary outcome was VAP density of incidence expressed as the number of episodes/1000 MV days. The secondary outcomes were ICU LOS and mortality.

Results Three hundred and three patients (2714 MV days) and 304 patients (3032 MV days) were analyzed during the "before" and the "intervention" periods, respectively. According to the study period, they were comparable regarding age, gender, main diagnosis and SAPS II value. Fifty-eight VAP episodes were recorded before the intervention as compared to 41 after. As a result, VAP incidence was significantly lower after intervention (13.5 vs. 21.4/1000 MV-days; odds ratio $=0.63[0.42-0.94], 95 \% \mathrm{Cl} ; p=0.029)$. However, neither LOS $(9.9$ [13.0] vs. 8.7 [10.4]; $p=0.21$ ) nor mortality ( 39.1 vs. $33.0 \% ; p=\mathrm{NS}$ ) was significantly reduced.

Conclusion Implementation of a new oral care kit including tooth brushing in our ICU was likely to significantly reduce VAP incidence, highlighting the need for a tight control of oral hygiene in the patients subjected to MV, as part of VAP prevention bundles.

\section{Competing interests None.}

\section{References}

1. Gu WJ, et al. (2012) Impact of oral care with versus without toothbrushing on the prevention of ventilator-associated pneumonia: a systematic review and meta-analysis of randomized controlled trials. Crit Care 16: R190

2. Heo SM, et al. (2008) Genetic relationships between respiratory pathogens isolated from dental plaque and bronchoalveolar lavage fluid from patients in the intensive care unit undergoing mechanical ventilation. Clinical infectious diseases: an official publication of the Infectious Diseases Society of America 47: 1562-1570.
P163

Impact of a multifaceted bundle ventilator-associated pneumonia prevention program in a secondary care medical-surgical intensive care unit

Stanislas De Guillebon ${ }^{1}$, Eloise Morisse ${ }^{2}$, Julie France ${ }^{2}$, Deborah Gasser², Philippe Badia ${ }^{2}$, Walter Picard ${ }^{2}$

${ }^{1} \mathrm{CHU}$ de BORDEAUX, Bordeaux, France; ${ }^{2}$ Pyrenées-atlantiques, Hospital

François Mitterrand, Pau, France

Correspondence: Stanislas De Guillebon - stanislas.deguillebon@

hotmail.fr

Annals of Intensive Care 2016, 6(Suppl 1):P163

Introduction Ventilator-associated pneumonia (VAP) is the most frequent nosocomial infection in the ICU and has a clear impact on patient outcome (duration of mechanical ventilation, length of stay and mortality). The aim of this study is to evaluate the impact of a multifaceted VAP prevention program on VAP rates in a medical-surgical ICU in a secondary care hospital.

Patients and methods We conducted a retrospective observational monocentric study for two 6-month study period (from January to June, 2013 and 2014). All patients admitted in the ICU for more than $48 \mathrm{~h}$ were included. The "Bundle" program involved all healthcare workers and included educational sessions, direct observations with performance feedback, and reminders. The bundle focused on a few number of targeted measures: Subglottic aspiration, tracheal cuff pressure monitoring, gastric overdistension avoidance, backrest elevation, chlorhexidine oral hygiene, nonessential tracheal suction elimination, sedation protocols, hand hygiene and glove and gown use. VAP was diagnosed on the basis of clinical and radiological criteria and quantitative cultures of distal specimens. The primary outcome was the VAP rate. Secondary outcomes included compliance rates with bundle measures, patient outcome and risk factors for VAP in our population. Results We included 189 patients in 2013 and 171 in 2014. Patient characteristics and comorbidities were similar between the two study periods (average age 62 years, average IGS2 $=44.8$, average SOFA $\max =6.35$ ). Our population was composed of $64 \%$ of medical patients, with $40 \%$ of them admitted for respiratory failure as main diagnosis. Our ICU also had trauma patients, with $6 \%$ of them admitted for severe head injury. Compliance with preventive measures significantly increased between the study periods (overall compliance: $2.2 \%$ versus $38.8 \% ; p<0.001$ ). Among intubated patients (105 in 2013, 108 in 2014), mechanical ventilation duration was higher during the intervention period $(9.7 \pm 6.9$ vs $7.4 \pm 5.5$ days; $p<0.05$ ). Baseline and intervention VAP rates were 18.7 and 24.4 per 1000 ventilator days, respectively $(p=0.71)$. However, duration of mechanical ventilation without VAP was longer in the intervention group ( $8.7 \pm 7.5$ vs $6.9 \pm 5.0$ days; $p<0.05)$. VAP risk factors in our population were: ARDS, severe head injury, duration of mechanical ventilation. The only protective factor was antibiotic therapy at admission.

Conclusion A multifaceted bundle program for VAP prevention did not reduce VAP rates but increased duration of mechanical ventilation without VAP and had a significant effect on VAP prevention measures adherence.

\section{Competing interests None.}

P164

Daily chlorhexidine bathing in a medical intensive care unit: effects on healthcare-associated infections and on acquisition of multidrug-resistant bacteria

P. Corne ${ }^{1}$, Nicolas Molinari², I. De Boulastel ${ }^{1}$, S. Seguin ${ }^{1}$, A. Blasco ${ }^{3}$, V. Ledur $^{4}$, Olivier Jonquet ${ }^{1}$, Kada Klouche ${ }^{1}$

${ }^{1}$ Réanimation médicale, Hôpital Lapeyronie, Montpellier, France; ${ }^{2}$ Département de l'information médicale, Hôpital La Colombière, Montpellier, France; ${ }^{3}$ Unité d'hygiène hospitalière et de prévention, Hôpital Lapeyronie, Montpellier, France; ${ }^{4}$ Direction des achats et des approvisionnements, Centre André Benech, Montpellier, France

Correspondence: P. Corne - p-corne@chu-montpellier.fr Annals of Intensive Care 2016, 6(Suppl 1):P164 
Introduction Healthcare-associated infections (HAl) are important causes of morbidity and mortality in intensive care units (ICUs) and mostly caused by multidrug-resistant (MDR) bacteria. The aim of this study was to evaluate the potential effect of chlorhexidine (CHX) bathing on HAI and MDR bacteria acquisition incidence rates.

Patients and methods This observational prospective study was conducted in an ICU over a 10-month period, divided into two 5-month periods: a control period (CP: September 9, 2013, to January 30, 2014) during which patients were bathed in the traditional manner with soap and water, an intervention period (IP: September 9, 2014, to January 30,2015 ) during which patients were bathed daily with $2 \%$ $\mathrm{CHX}$-impregnated wipes. Incidence rates of ICU-acquired bloodstream infection (BSI), ventilator-associated pneumonia (VAP), catheter colonization, and MDR bacteria acquisition were collected and compared among the 2 study periods.

Results Two hundred and fifty-three patients were included in the study with 121 during the CP and 132 during the IP. Age, sex, SAPSII score, duration of mechanical ventilation and length of ICU stay did not differ significantly across the 2 periods. ICU-acquired $B S I$ rates per 100 patients were 7.6 in IP versus 12.4 in $\mathrm{CP}(p=0.2)$. Infections rates stratified by type were as follows: catheter-associated BSI: 3 cases in CP and none in IP; VAP per 100 ventilated patients: 19.5 in IP and 16.3 in $\mathrm{CP}(p=0.6)$. During the CP, BSIs (15) were caused in $41 \%$ of cases by gram-positive, in $35 \%$ by gram-negative species, and in $24 \%$ by fungi. During the IP, BSIs (10) were caused in $50 \%$ of cases by gram-positive, in $40 \%$ by gram-negative, and in $10 \%$ by fungi. Rates of colonized central lines per 100 catheters were 1.8 in IP versus 5.5 in CP $(p=0.07)$. MDR bacteria acquisition rates were 6.1 and $9.1 \%$ in IP and CP, respectively $(p=0.33)$. Methicillin-resistant Staphylococcus aureus, extended spectrum beta lactamase bacteria, MDR Pseudomonas aeruginosa and Acinetobacter baumannii accounted, respectively, for acquisition incidence rates per 100 patients of $0.8,4.9,3.3,2.5$ and $2.3,2.3,1.5,0 \%$ in $\mathrm{CP}$ and IP; differences were not statistically significative. Overall mortality rates of patients of $\mathrm{CP}$ and IP were 22 and $17 \%$, respectively. Conclusion $\mathrm{CHX}$ daily cleansing did not induce a significant but only a slight reduction of ICU-acquired BSI, catheter colonization, and MDR bacteria acquisition incidence rates. Further and larger studies are required to confirm its effect as a component of an infection prevention bundle in ICU settings.

\section{Competing interests None.}

\section{P165}

Impact of twin bedrooms intensive care unit on the epidemiology of extended-spectrum beta-lactamase-producing enterobacteriaceae

Paktoris-Papine Sophie ${ }^{1}$, Margaux Artiguenave ${ }^{1}$, Faten El Sayed ${ }^{2}$, Florence Espinasse ${ }^{3}$, Aurélien Dinh ${ }^{4}$, Cyril Charron' 1 Xavier Repessé ${ }^{1}$, Antoine Vieillard-Baron ${ }^{1}$

${ }^{1}$ Réanimation médico-chirurgicale, Assistance Publique - Hôpitaux de Paris, Hôpital Ambroise Paré, Boulogne-Billancourt, France; ${ }^{2}$ Service de microbiologie, Assistance Publique - Hôpitaux de Paris, Hôpital Ambroise Paré, Boulogne-Billancourt, France; ${ }^{3}$ Equipe opérationnelle d'hygiène hospitalière, Assistance Publique - Hôpitaux de Paris, Hôpital Ambroise Paré, Boulogne-Billancourt, France; ${ }^{4}$ Equipe mobile de microbiologie, Assistance Publique - Hôpitaux de Paris, Hôpital Ambroise Paré, Boulogne-Billancourt, France

Correspondence: Xavier Repessé - xavier.repesse@apr.aphp.fr Annals of Intensive Care 2016, 6(Suppl 1):P165

Introduction Multi-drug-resistant pathogens, and specifically thirdgeneration cephalosporin-resistant Enterobacteriaceae, represent a major issue in hospital. In intensive care unit (ICU), Gram-negative pathogens that are resistant to antibiotics by production of extendedspectrum beta-lactamase (ESBL) are responsible for longer hospitalizations and poorer outcomes. The epidemiology of ESBL-producing enterobacteriaceae (PE) has been recently modified by the emergence of new ESBL. In the absence of new broad-spectrum molecules, it is crucial to control antibiotic consumption and prevent transmission between ICU patients. Our 12-bed ICU is made of three units of 2 twin bedrooms each so that recommendations on contact precautions cannot be thoroughly followed, since patients cannot be isolated in a single room. In our unit, contact precautions focus on hand washing with hydro-alcoholic solutions, on wearing a single-use gown before entering the room and on the use of gloves for soiled contacts. In this prospective study, we report the colonization and transmission rates of ESBL-PE in order to study whether this peculiar form of ICU with twin bedrooms could be responsible for increased transmission rates of ESBL-PE among ICU patients. Our secondary objectives were to describe the epidemiology of ESBLs in our ICU and to identify risk factors for their transmission.

Patients and methods This pure observational study based on our usual practices was prospectively conducted in the 12-bed ICU of a university hospital (Ambroise Paré, Boulogne-Billancourt, France), organized as a six twin bedrooms divided into three units. Inclusion criteria were: 1) adult patients and 2) a hospitalization for a period during which the patient was nursed by at least two paramedical teams. Characteristics of patients at admission (age, sex, SAPSII) and clinical data during hospital stay (duration of mechanical ventilation, duration of ICU stay, outcome) were collected. Microbiological data concerning colonization and/or acquisition of ESBL-PE were monitored, as usually done in our unit, by rectal swabs collected at admission and once weekly for the whole duration of the ICU stay. Each strain of ESBL-PE was then identified and the enzyme type sequenced by PCR.

Results From June 2014 to April 2015, 580 patients were admitted in the ICU, among which 448 (77\%) were included in the study. On the 132 patients excluded, 27 patients were admitted for a conventional hemodialysis, which is conducted in a special room and 105 patients (among which 29 died) were nursed only by one paramedical team. Mean age was $65 \pm 16$ years, and mean IGS II was $47 \pm 21$. Sixty-one percent were male. The mean duration of ICU stay was $7.4 \pm 8.8$ days, and the mean duration of mechanical ventilation was $4.5 \pm 8.1$ days. Origin of patients was home/emergency for $49 \%$ of them and another unit or hospital in $51 \%$.

The rate of ESBL colonization at admission was $13.2 \%$, mainly with Escherichia coli. The incidence of ESBL transmission among patients who were monitored by two or more rectal swabs was $4.3 \%(11$ patients on 251), also mainly with Escherichia coli. Mortality did not differ for patients colonized or not with ESBL at admission, nor for patients for whom a transmission occurred during the ICU stay. In this cohort, neither the colonization with ESBL-PE, nor the transmission of ESBL-PE was associated with antibiotic exposure or hospitalization during the three months before admission.

Conclusion Prevalence of ESBL colonization in our ICU was $13.2 \%$ Escherichia coli being the most frequent bacteria identified. This rate of ESBL carriage on admission was comparable to other rates in French ICUs (15\%). Despite the unfavorable twin-bed architecture of our ICU, the incidence of ESBL acquisition was $4.3 \%$, which was actually lower than transmission rates previously published in other ICUs. Our results question whether the strict isolation in single bedrooms plays a major role for the prevention of ESBL transmission in ICU. They rather put forward the crucial role of hand washing with hydroalcoholic solutions and of the gown for the prevention of ESBL-PE transmission. This study seems to corroborate many studies, suggesting that the routine contact isolation could be challenged in a non-epidemic setting and that environmental contamination may not play a substantial role in the transmission of ESBL-PE.

\section{Competing interests None.}

\section{P166}

Prophylactic confinement after liver transplant: a necessity or a useless precaution?

Benjamin Lebas ${ }^{1}$, Sophie Hatsch 1 , Quentin Maestraggi ${ }^{1}$, Anne-Florence Dureau', Vincent Castelain', Bernard Ellero², François Faitot ${ }^{2}$, Max Guillot ${ }^{1}$, Francis Schneider ${ }^{1}$

${ }^{1}$ Service de réanimation médicale, Hôpital de Hautepierre, Hôpitaux Universitaires de Strasbourg, Strasbourg, France; ${ }^{2}$ Service de chirurgie hépato-biliaire et transplantation, Hôpital de Hautepierre, Hôpitaux Universitaires de Strasbourg, Strasbourg, France 
Correspondence: Benjamin Lebas - benji.lebas@neuf.fr Annals of Intensive Care 2016, 6(Suppl 1):P166

Introduction Patients benefiting from a liver transplantation are systematically placed in a postoperative protective confinement during 5 days (unit protocol, no institutional guideline). This confinement is realized for a variable period according to the teams on the grounds that immediate postoperative period is a time of major pharmacologic immunodepression and acquired because of the surgical stress. Literature's data do not allow to standardize these measures.

Patients and methods We have retrospectively analyzed data from liver transplant recipients from January 2012 to December 2013 in our intensive care unit. The type of hepatopathy and the severity at admission were collected. We have also gathered bacterial colonization data before and after the transplantation by distinguishing the multiresistant bacteria and the sensitive germs. Finally, we have recorded the time for these bacteria to appear after surgery.

Results During this period, 32 patients benefited from a liver transplant. Among them, 9 (28\%) had fulminant hepatitis and $23(72 \%)$ an acute-on-chronic liver disease decompensation. The IGS II score upon admission was, respectively, 48 and 51. Bacterial colonization before and after surgery is shown in Table 31.

Twenty-one (66\%) patients acquired new germs during isolation time. The unit protocol of 5 days was followed in 7 (22\%) cases.

Discussion Patients having a chronical hepatopathy have a more frequent bacterial colonization due to preoperative sensitive germs. Indeed, infection is often the triggering factor of decompensation. This difference is not significative for resistive germs. The confinement period was often extended arbitrary (i.e.,: without reason justifying not to respect the protocol); nonetheless, we observe the acquisition of a lot of new bacteria during this time independently of the initial hepatopathy.

Conclusion An early postoperative protective confinement immediately after liver transplant does not prevent the acquisition of new bacteria. The reason behind this practice is therefore questionable, and randomized trials are necessary to allow a conclusion on the interest of such a practice.

Competing interests None.

Table 31 Bacterial colonization before and after surgery

\begin{tabular}{|c|c|c|c|}
\hline & $\begin{array}{l}\text { Global popula- } \\
\text { tion }(n=32)\end{array}$ & $\begin{array}{l}\text { Acute-on- } \\
\text { chronic } \\
\text { decompensa- } \\
\text { tion }(n=23)\end{array}$ & $\begin{array}{l}\text { Fulminant } \\
\text { hepatitis }(n=9)\end{array}$ \\
\hline $\begin{array}{l}\text { Preoperative } \\
\text { sensitive } \\
\text { germs coloni- } \\
\text { zation }\end{array}$ & 17 (53\%) & 15 (65 \%) & $2(22 \%)$ \\
\hline $\begin{array}{l}\text { Preoperative } \\
\text { multidrug- } \\
\text { resistant } \\
\text { organisms } \\
\text { colonization }\end{array}$ & 7 (22 \%) & 7 (30 \%) & $0(0 \%)^{*}$ \\
\hline $\begin{array}{l}\text { Postoperative } \\
\text { sensitive } \\
\text { germs coloni- } \\
\text { zation }\end{array}$ & 25 (78 \%) & 19 (83 \%) & 6 (67\%) \\
\hline $\begin{array}{l}\text { Postoperative } \\
\text { multidrug- } \\
\text { resistant } \\
\text { organisms } \\
\text { colonization }\end{array}$ & 12 (38 \%) & 10 (43\%) & $2(22 \%)$ \\
\hline
\end{tabular}

${ }^{*} p<0.05$
P167

Proteomic response and adaptation mechanisms of E. coli 536 to overcome the inhibitory effect of cranberry proanthocyanidins Dimitri Margetis ${ }^{1}$, Odile Bouvet ${ }^{2}$, Didier Chevret $^{3}$, Didier Dreyfuss ${ }^{4}$, Erick Denamur ${ }^{5}$, Jean-Damien Ricard ${ }^{6}$

${ }^{1}$ Anesthésie réanimation, Pitié-Salpêtrière Hospital, Paris, France; ${ }^{2}$ lame - inserm umr1 137, Université Paris Diderot, Paris, France; ${ }^{3}$ 78, I.N.R.A

(Institut National de Recherche Agronomique), Jouy-en-Josas, France; ${ }^{4}$ Inserm, iame, umr 1137, Université Paris Diderot, Sorbonne Paris Cité, Paris, France; ${ }^{5}$ lame inserm umr1137, Université Paris Diderot, Paris, France; ${ }^{6}$ Service de Réanimation Médico-Chirurgicale, CHU Louis Mourier, Colombes, France

Correspondence: Dimitri Margetis - dimargetis@free.fr Annals of Intensive Care 2016, 6(Suppl 1):P167

Introduction The cranberry fruit (Vaccinium macrocarpon) is particularly rich in polyphenols (tannins), and among these, the primary active compound is the A-type proanthocyanidin (CPAC), which exhibit potent antibacterial properties. In a previous study, we have made evidence of the strong inhibitory effect of CPAC on growth, adhesion and virulence of oropharyngeal and lung isolates of E. coli [1].

The present study was undertaken to establish a baseline of knowledge of the molecular responses of the highly virulent $E$. coli strain 536 during growth in the presence of CPAC. We employed proteomic analysis and made complementary experiences to characterize the global response of exponential and stationary phases E. coli 536 grown in a nutrient-rich broth medium supplemented with $1 \mathrm{mg} / \mathrm{mL}, \mathrm{CPAC}$ as compared with a reference culture grown without CPAC.

Materials and methods $E$. coli 536 exposed or not to $1 \mathrm{mg} / \mathrm{ml}$ of CPAC proteome was obtained in exponential and stationary growth phase by mass spectrometry proteomic analysis LC-MS/MS.

Evaluation of the intracellular ROS level by the dichlorofluorescein diacetate DCFH-DA method was evaluated using a spectrofluorimeter. Membrane potential, cell size and cell viability were evaluated using a flow cytometer (Guava EasyCyte Plus, Millipore). To examine the changes of membrane potential after CPAC exposure, the cells were stained with DiOC6(3) (3,3'-dihexyloxacarbocyanine iodide) and then immediately analyzed using a flow cytometer. The fluorescence-based LIVE/DEAD kit was used to examine the cell viability.

Results A total of 641 proteins were detected, and among these, 483 were in both exponential and stationary growth phases, whereas 506 and 618 proteins were detected only during the exponential and stationary growth phases, respectively. Proteins were manually grouped into 25 metabolic pathway categories. Among the 641 identified proteins, 117 had a significant effect, and among these, 11 were in both exponential and stationary growth phases, whereas 68 and 60 proteins were detected only during the exponential and stationary growth phases, respectively. Considerable differences were also observed in protein abundances between the untreated and treated cells. During the exponential and stationary growth phases, many proteins were over-abundant ( 31 and 29 , respectively) and under-abundant (37 and 31 , respectively).

The characterization of 117 proteins with a significant effect revealed that more proteins were over-abundant in different functional categories, especially those involved in glycolyse, fermentation, iron metabolism and detoxification. In contrast, the under-abundant proteins were mainly implicated in transport, TCA cycle and respiration.

CPAC exposure mainly affected the pathways of iron metabolism with strong evidence of iron privation, cellular detoxification as response to the oxidative stress exerted by CPAC and a redirection of respiratory metabolism through the fermentative pathways. CPAC treatment also alters many membrane functions by the strong inhibition of membrane proteins transport and secretion, affecting the respiratory chains, fimbrial expression, cell division and proton-motrice force. Supplementary experiments showed no effect of CPAC on bacterial viability, a size increase as result of cellular division inhibition and a drastic reduction of the proton-motrice force.

ROS dosage showed a significant decrease during stationary phase of growth in CPAC treated bacteria as response to the oxidative stress exerted by proanthocyanidins. 
Conclusion Proteomic analysis indicates the metabolic pathways affected by CPAC and E. coli adaptation. Treated bacteria are in a state of iron limitation, energy metabolism is redirected to fermentation and membrane proteins secretion and transport systems strongly inhibited. As a result, we observe a fimbriation inhibition affecting bacterial mobility, adhesion and virulence and alteration of the proton-motrice force and cellular division in E. coli. Our results highlight strategy adapted by E. coli 536 to resist the toxicity of tannins. During exposure to cranberry, E. coli is subjected to complex metabolic adaptations that aim to reduce the rate of intracellular ROS. The lack of production of fimbriae caused by cranberry contributes to the permanent activation of the oxidative stress response.

\section{Competing interests None.}

\section{Reference}

1. Margetis, D., D. Roux, et al. (2015). "Effects of Proanthocyanidins on Adhesion, Growth, and Virulence of Highly Virulent Extraintestinal Pathogenic Escherichia coli Argue for Its Use to Treat Oropharyngeal Colonization and Prevent Ventilator-Associated Pneumonia." Crit Care Med 43(6): e170-8.

\section{P168}

Is daily measurement of CRP useful to detect earlier ventilator-associated pneumonia?

Justine Simonet ${ }^{1}$, Mathieu Boutonnet ${ }^{1}$, Jean-Louis Daban ${ }^{1}$, Elisabeth Falzone ${ }^{1}$, Guillaume Dumas ${ }^{1}$, Philippe Vest ${ }^{2}$, Vincent Peigne ${ }^{1}$ ${ }^{1}$ Réanimation, Hôpital d'Instruction des Armées Percy, Clamart, France; ${ }^{2}$ Biochimie, Hôpital d'Instruction des Armées Percy, Clamart, France Correspondence: Vincent Peigne - vincentpeigne@yahoo.fr Annals of Intensive Care 2016, 6(Suppl 1):P168

Introduction C-reactive protein (CRP) is widely used as a biomarker of infection in ICU patients in spite of a lack of data supporting this practice. We evaluated whether the routine measurement of CRP could be a tool to detect ventilator-associated pneumonia (VAP) in a 12-bed unit where VAP is diagnosed using bronchoscopy and where CRP was measured on a daily routine in all patients until January 2015.

Patients and methods Before-after study led during two 6-month periods, with or without daily measurement of CRP. We compared 1) the delay between intubation and the diagnosis of VAP and 2) the number of bronchoscopy performed during the two periods.

Results In total, 152 and 153 patients were included during the periods with and without daily CRP measurement, respectively. Mortality rate was 16 and $20 \%(p$ 0.47). The number of patients who developed VAP during the two periods was 12 and 19 ( $p 0.19$ ), and the mean time between intubation and VAP was 6.3 and 8.9 days ( $p$ 0.52 ). The proportion of contributive bronchoscopy was 18 and $26 \%$ ( $p$ 0.25).

Conclusion We could not identify any significant difference in the delay of diagnosis and the use of bronchoscopy during the periods with and without daily measurement of CRP. However, there is a trend toward an earlier diagnosis with an increased use of endoscopy when CRP is routinely measured. Further studies including more patients and evaluating cost-effectiveness should be performed to assess this hypothesis.

\section{Competing interests None.}

\section{P169}

Clinical and biological features of healthcare-associated coagulase-negative Staphylococci meningitis: results of a retrospective study

Severine Couffin', David Lobo², Gilles Dhonneur ${ }^{3}$, Roman Mounier ${ }^{2}$ ${ }^{1}$ Surgical intensive care, Hospital Henri Mondor, Créteil, France; ${ }^{2}$ Anes-

thesia and surgical intensive care, Hospital Henri Mondor, Créteil, France; ${ }^{3}$ Anesthesia and intensive care medicine, CHU Henri Mondor, Créteil, France

Correspondence: Severine Couffin - scouffin@gmail.com

Annals of Intensive Care 2016, 6(Suppl 1):P169
Introduction Healthcare-associated meningitis is described as indolent, with poor symptomatology and mildly inflammatory biology [1]. Bacteria responsible belong mostly to cutaneous commensal flora, especially coagulase-negative staphylococci (CNS); these germs have the ability to adhere to indwelling medical devices and to persist in biofilms, but lack classical virulence factors. Thus, they are considered as low or accidental pathogens and could be the reason behind those clinical and biological features. The main objective of this study was to compare clinical and biological characteristics of CNS meningitis with those of real pathogens healthcare-associated meningitis. The secondary objectives were to collate their bacteriological data and their outcome.

Patients and methods This retrospective study included consecutive bacterial healthcare-associated meningitis admitted to University Medical Center Henri Mondor, in Créteil (France), between January 2007 and December 2014. Patients were divided into two groups: CNS and real pathogens (control group). The diagnosis of meningitis was based on isolation of a bacterium in one cerebrospinal fluid culture, as reiterated by Mayhall et al. [2]. We decided to add an exception for CNS: Two positive samples with the same strain (same antibiotype) were necessary for the diagnosis. Infections due to other low pathogens (Corynebacterium spp and Propionibacterium spp) were excluded, as well as those complicated by an abscess or an empyema, or without appropriate antibiotic treatment. We compared demographic characteristics, clinical, biological and bacteriological profiles between two groups with Mann-Whitney and Fisher's tests. Mortality was analyzed with Kaplan-Meier method.

Results Seventy-one meningitis were identified, 18 CNS and 53 controls. Patients in both groups were not different in terms of age, sex, comorbidities, reason for admission, initial severity and presence of neurosurgical device. CNS meningitis occurred within a median of 17 days after surgery, versus 12 days for the control group $(p=0.029)$. The Glasgow coma score at time of diagnosis was 14 among CNS infections versus 13 among controls $(p=0.038$ ). The cerebrospinal fluid analysis revealed less pleocytosis ( 18 versus 1330 white cells per mm3, $p<0.001$ ), less polymorphonuclear neutrophils ( 55 vs. $90 \%, p<0.001$ ), higher glucose level $(4.1 \mathrm{vs} 0.8 \mathrm{mmol} / \mathrm{L}, p<0.001)$ and lower protein level (482 vs $1608 \mathrm{mg} / \mathrm{L}, p<0.001$ ) in the CNS group. In terms of bacteriology, CNS meningitis was more often bacteraemic than controls. Finally, the survival probabilities at 28 days and 1 year were more elevated in the CNS infections.

Conclusion The indolent, paucisymptomatic and mildly inflammatory nature of healthcare-associated meningitis is related to one dominant low pathogen, the CNS. The lack of virulence of this bacterium could also explain a relatively low mortality.

Competing interests None.

\section{References}

1. Van de Beek D, Drake JM, Tunkel AR. Nosocomial bacterial meningitis. N Engl J Med 2010; 362: 146-54

2. Mayhall CG, Archer NH, Lamb VA, Spadora AC, Baggett JW, Ward JD et al. Ventriculostomy-related infections. A prospective epidemiologic study. N Engl J Med 1984; 310(9): 553-9.

\section{P170}

Prognostic value of electrocardiographic abnormalities in patients with acute pulmonary embolism

M. Cheikh Bouhlel', Ahmed Khedher ${ }^{1}$, Kaoula Meddeb ${ }^{1}$, A. Azouzi', Y. Hamdaoui', Jihene Ayachi', W. Brahim', Rania Bouneb', Mohamed Boussarsar

${ }^{1}$ Réanimation médicale, CHU Farhat Hached, Sousse, Tunisia Correspondence: Mohamed Boussarsar - hamadi.boussarsar@gmail. com

Annals of Intensive Care 2016, 6(Suppl 1):P170

Introduction Acute pulmonary embolism (APE) is a rare life-threatening complication. Prognosis prediction often challenges the clinician, even assisted with different scores. Electrocardiographic (EKG) abnormalities, even polymorphous and with poor specificity have not been widely investigated in this issue. 
Objective: To assess the prognostic value of EKG abnormalities in patients with acute pulmonary embolism.

Patients and methods We performed a retrospective analysis including all patients admitted to ICU between 2004 and 2014 with a confirmed diagnosis of APE. Patients' characteristics were recorded, especially regarding catecholamine's use, ventilator support and vital status at discharge. Pulmonary Embolism Severity Index (PESI), proposed by the European Society of Cardiology, was calculated. Patients with PESI class $\geq 3$ were considered with high early mortality risk. First available EKG records were used for analysis to identify signs of right ventricular strain, arrhythmias and repolarization disorders.

Results Twenty-eight patients with APE were collected. Mean age was $54.6 \pm 19.2$ years. Fifteen patients $(53.6 \%)$ had high risk of early mortality (PESI class $\geq 3$ ). Fourteen $(50 \%)$ had received mechanical ventilation and 15 (53.6\%) had received catecholamines. Mean ICU stay was $5.39 \pm 4.74$ days. Thirteen patients (46.3\%) died in the ICU. There was no significant difference between deaths and survivals regarding EKG abnormalities. However, arrhythmia was more frequent in patients with high PESI $(p=0.007)$. Multivariate analysis failed to identify any independent electric predictor of ICU mortality probably because of the small sample size.

Conclusion In a selected population of APE critically ill patients, EKG abnormalities failed to demonstrate any prognostic predictive value. Severe APE as assessed by PESI was associated with supraventricular arrhythmias.

\section{Competing interests None.}

\section{P171}

Does mean arterial pressure alarm improve the time spent within blood pressure target range in septic shock? A before and after study

Nans Florens' ${ }^{1}$ Martin Cour ${ }^{1}$, Julie Varéon ${ }^{1}$, Arnaud Malatray ${ }^{1}$, Romain Hernu', Laurent Argaud ${ }^{1}$

${ }^{1}$ Réanimation médicale, Hospices Civils de Lyon - Groupement Hospitalier Edouard Herriot, Lyon, France

Correspondence: Laurent Argaud - laurent.argaud@chu-lyon.fr Annals of Intensive Care 2016, 6(Suppl 1):P171

Introduction Norepinephrine is a widespread used catecholamine in the hemodynamic management of septic shock. As under-dosage or overdosage can be harmful for the patients, it is necessary to maintain mean arterial pressure (MAP) in preset bounds. We hypothesized that setting a MAP alarm on the cardiovascular monitor could lead to a better correlation between effective MAP and prescribed targets.

Patients and methods During two successive periods (P1 and P2), patients treated with norepinephrine for a septic shock for at least $24 \mathrm{~h}$ were included ( $n=50$ per period). The protocol of norepinephrine's administration was similar during the two periods including the prescription of MAP targets for all patients with a $10 \mathrm{mmHg}$ range (e.g., $65-75 \mathrm{mmHg}$ ). Effective MAP and doses of norepinephrine were stored every hour. During P2, a MAP alarm was set on the cardiovascular monitor with upper and lower bounds corresponding with prescribed MAP targets.

Results During the study period, 460 patients were admitted in the ICU. Of them, $134(29 \%)$ had a septic shock. Baseline characteristics of the $100(22 \%)$ patients who met the inclusion criteria were similar among both periods (sex ratio: 1.4; age: $67 \pm 15$ years; IGSII: $59 \pm 18$ ). Time spent out of MAP preset ranges was significantly lower in P2 when compared to $\mathrm{P} 1: 27 \pm 2$ and $53 \pm 2 \%$, respectively $(p<0.0001)$.

Table 32 See text for description

\begin{tabular}{llllll}
\hline & $\boldsymbol{\Delta C O}$ & $\boldsymbol{\Delta}$ APm & $\boldsymbol{\Delta P A P m}$ & $\boldsymbol{\Delta L V P W}$ & $\Delta$ RVPW \\
\hline DPP & 0.08 & 0.03 & 0.19 & 0.10 & 0.05 \\
RAP & $0.33^{*}$ & 0.16 & 0.17 & $0.42^{* *}$ & $0.38^{*}$ \\
PWP & 0.25 & 0.28 & 0.19 & 0.28 & $0.33^{*}$ \\
\hline
\end{tabular}

MAP was higher than the upper limit of the target in $31 \pm 3 \%$ of time in $\mathrm{P} 1$ and $15 \pm 1 \%$ in P2 $(p<0.0001)$. MAP was under the target limit in $22 \pm 2 \%$ of time in P1 and $11 \pm 18 \%$ in P2 $(p<0.001)$. MAP alarm leads nurses to change more frequently the dose of norepinephrine to maintain MAP in the target during P2 ( $p<0.01$ vs P1). MAP alarm did not decrease norepinephrine dose during the first day of administration as compared to $\mathrm{P} 1(0.50 \pm 0.07 \mathrm{vs} 0.47 \pm 0.06 \mu \mathrm{g} / \mathrm{kg} / \mathrm{min}$, $p=0.75)$. In the same way, MAP alarm did not accelerate withdrawal of norepinephrine and did not reduce length of stay and mortality $(p=\mathrm{NS})$.

Conclusion Our study suggests that setting MAP alarm could enhance time within a MAP target range and therefore may prevent patients from pernicious effects of hypotension and side effects of norepinephrine overdose.

\section{Competing interests None.}

\section{P172}

Respiratory change of pulse pressure is not indicative of preload reserve in cardiac surgery patients

Pierre Dupland', Thomas D'humières², Romain Vergier ${ }^{2}$, Pauline Issaurat ${ }^{2}$, Philippe Estagnasié ${ }^{1}$, Pierre Squara ${ }^{1}$, Alain Brusset ${ }^{1}$

${ }^{1}$ Reanimation, Clinical Ambroise Paré, Neuilly-sur-Seine, France; ${ }^{2} \mathrm{cu}, \mathrm{Clini-}$ cal Ambroise Paré, Neuilly-sur-Seine, France

Correspondence: Pierre Dupland - pierre.dupland@gmail.com

Annals of Intensive Care 2016, 6(Suppl 1):P172

Introduction Predicting fluid response is an important issue in ICU. Within the scope, the interest of monitoring continuously right atrial pressure (RAP) and periodically pulmonary wedge pressure (PWP) has been challenged. Dynamic change of pulse pressure during a respiratory cycle $(\triangle \mathrm{PP})$ has been proposed as an alternative in sedated, mechanically ventilated patients. However, in postoperative cardiac surgery, the consequences of the extracorporeal circulation on lung compliance and the thoracic effusion/drainage may alter the relationships between intra-alveolar and heart extra-wall pressures. We designed this study to assess the predictability of monitorable preload indices to changes in cardiac performance during a fluid challenge.

Patients and methods This is a single-centre prospective cohort study. We included all consecutive patients who required a pulmonary artery catheter according to our standard protocol. We performed a complete hemodynamic analysis immediately after surgery, while patients were still under anaesthesia (baseline, $\mathrm{H} 0$ ). Three sequential fluid boluses of $5 \mathrm{ml} / \mathrm{kg}$ were eventually performed if RAP or PWP $<20 \mathrm{mmHg}$. After each fluid bolus, another hemodynamic standpoint was collected $\left(\mathrm{H} 1, \mathrm{H}_{2}\right.$ and $\left.\mathrm{H} 3\right)$. For each fluid challenge, initial preload indices were RAP, PWP and $\triangle$ PP. The fluid-induced cardiac performance changes were: cardiac output $(\triangle C O)$, mean aortic pressure $(\mathrm{APm})$, mean pulmonary artery pressure (PAPm), left ventricle power $(\triangle \mathrm{LVPW})$ and right ventricle power ( $\triangle \mathrm{RVPW})$. LVPW was derived as CO x APm and RVPW by CO x PAPm. In order to identify the best possible predictor, a systematic analysis of all possible couples of thresholds was done using the $S$ ratio [1].

Results Forty-two fluid challenges were realized in 17 patients. Twelve were men $(66 \%)$, and age was $68.9 \pm 11.4 \mathrm{y}$. The number of grafts, valves, and combined surgery were 7,6 , and 4 , respectively. Left ventricle ejection fraction was $37 \pm 15$. Right ventricle function was considered normal in 14 patients, 14 patients received inotropic support. Table 32 summarizes the $R$ values of correlations between initial preload indices (columns) and fluid-induced change in cardiac performance (lines), ${ }^{*} p<0.05,{ }^{* *} p<0.01$.

The best correlation was observed between RAP and $\triangle \mathrm{LVPW}$. Among all possible couples of thresholds, the $\mathrm{S}$ ratio selected RAP $<10 \mathrm{mmHg}$ as predicting $\Delta \mathrm{LVSW}>42 \mathrm{~g} . \mathrm{m}$. with a selectivity of 0.63 and a specificity of 0.61 .

Discussion Although the small number of patients limits the analysis, possibly leading to a bias of selection, the number of correlated variables was suitable for showing a clinically relevant correlation, which was not observed. The systematic analysis of best couples of thresholds did not allow identifying a good predictor of fluid responsiveness. 
Table 33 Performances of each test to predict the fluid responsiveness. Results are given as percentage $195 \%$ confidence interval)

\begin{tabular}{llllll}
\hline Test used & Threshold & Sensitivity & Specificity & PPV & NPV \\
\hline PPV & $13 \%$ & $19(4-34) \%$ & $88(65-100) \%$ & $83(54-100) \%$ & $25(9-41) \%$ \\
PPV & $8 \%$ & $73(56-90) \%$ & $75(45-100) \%$ & $90(78-100) \%$ & $46(19-73) \%$ \\
SWV & $13 \%$ & $23(7-39) \%$ & $75(45-100) \%$ & $75(45-100) \%$ & $23(7-39) \%$ \\
EEO & $6 \%$ & $69(51-87) \%$ & $75(45-100) \%$ & $90(77-100) \%$ & $43(17-69) \%$ \\
PLR & $10 \%$ & $66(47-83) \%$ & $75(45-100) \%$ & $86(76-100) \%$ & $50(23-77) \%$ \\
PPVxDAP & 432 & $92(82-100) \%$ & $75(45-100) \%$ & $92(82-100) \%$ & $75(45-100) \%$ \\
Optimized $\%) \%(88-100) \%$ & $96(51-100) \%$ \\
\hline
\end{tabular}

Conclusion Although low values of RAP and PWP were correlated with fluid responsiveness, and conversely, our study confirmed that RAP and PWP were poor predictors of preload reserve. $\triangle P P$ is not a valid alternative in postoperative cardiac surgery.

\section{Competing interests None.}

\section{Reference}

1. Squara P: A new ratio for protocol categorization. Comput Math Methods Med 2014; 2014: 389845.

\section{P173}

Importance of diastolic arterial pressure to optimize pulse pressure variation performances for patients receiving norepinephrine

Vincent Génin' ${ }^{1}$ David Vandroux ${ }^{1}$, Cyril Ferdynus², Arnaud Roussiaux Jérôme Allyn', Nicolas Allou', Olivier Martinet ${ }^{1}$, Julien Jabot ${ }^{1}$ ${ }^{1}$ Réanimation polyvalente, CHU La Réunion, Saint-Denis, France; ${ }^{2}$ Unité de soutien méthodologique, CHU La Réunion, Saint-Denis, France Correspondence: Julien Jabot - jabot974@gmail.com Annals of Intensive Care 2016, 6(Suppl 1):P173

Introduction Pulse pressure variation (PPV) is the most validated test among the different dynamic predictors of fluid responsiveness [1]. Nevertheless, testing its reliability depending on the hemodynamic conditions of use remains poorly studied. Norepinephrine as a vasoactive drug interferes on both systemic arterial rigidity and pulse pressure (PP). These changes might subsequently alter PPV's ability to predict fluid responsiveness. We therefore thought to study the prediction's accuracy of this test among a very homogenous selected group of ICU patients with PPV's validity criteria and mean arterial pressure (MAP) restored between 65 and $90 \mathrm{mmHg}$ using norepinephrine. PPV was therefore modified into a new index called "optimized PPV" in which diastolic arterial pressure (DAP) was integrated, considering that it can account for the vasoconstrictor effect of norepinephrine. We then compared the respective performances of these two tests in this specific ICU population.

Patients and methods Patients monitored by transpulmonary thermodilution $\mathrm{PiCCO}_{2}^{\circledR}$ system and whose MAP was between 65 and $90 \mathrm{mmHg}$ using norepinephrine were prospectively included. Hemodynamic parameters (as well as arterial pressure, cardiac index $(\mathrm{Cl})$ and PPV) were collected serially in six different conditions: at baseline, before and after an end-expiratory occlusion (EEO) test, before and after a passive leg raising (PLR) test, and lastly after volume expansion (VE) $(500 \mathrm{~mL}$ saline). We studied the diagnostic accuracy of PPV, stroke volume variation (SVV), EEO test and PLR test for predicting the response to volume expansion (i.e., VE-induced increase in $\mathrm{Cl} \geq 15 \%$ ). From PPV, we performed two additional tests:

- the product of PPV by DAP (PPVxDAP),

- a composite index obtained from a logistic regression model adjusted on PPV and DAP called "optimized PPV."

For each test, we evaluated its diagnostic accuracy using its area under the ROC curve (AUC) and its $95 \%$ confidence interval $(95 \% \mathrm{Cl})$.
Results Thirty-four VE tests were collected, concerning 26 VE responders (76\%) and 8 VE non-responders in 25 patients (age $62 \pm 13$ years; SAPS $261 \pm 21$ ). At baseline, mean MAP, PPV and Cl were, respectively, $77 \pm 7 \mathrm{mmHg}, 11 \% \pm 5$ and $2.94 \pm 0.93 \mathrm{~L} \mathrm{~min}^{-1} \mathrm{~m}^{-2}$ for a mean norepinephrine dose of $1.09 \pm 3.06 \mu \mathrm{g} \mathrm{kg}^{-1} \mathrm{~min}^{-1}$.

During the 34 procedures, EEO test, PLR test and VE increased significantly the $\mathrm{Cl}$ by $8 \pm 8 \%, 12 \pm 23$ and $22 \pm 15 \%$, respectively.

With a threshold of $13 \%$, PPV showed the lowest sensitivity of all tests (see Table 33). The sensitivity was increased when reducing the threshold to $8 \%$. Taking into account DAP as well as PPV increased both sensitivity and specificity: "Optimized PPV" (given by the formula: $0.57 \times D A P+0.56 \times P P V)$ reached the best performance with a AUC of $94 \%$ (95\% Cl 87-100 \%), while PPV got only $75 \%$ (95\% Cl 49-100\%), $p<0.05$.

Conclusion In a selected group of ICU patients with PPV's validity criteria and whose MAP is restored between 65 and $90 \mathrm{mmHg}$ using norepinephrine, PPV is an inefficient test to predict fluid responsiveness. Optimizing the PPV through a composite index including DPA, seen as a reflection of the vasoconstrictor effect of norepinephrine, improves its performance under such conditions of use.

\section{Competing interests None.}

\section{Reference}

1. Yang X, Du B. Does pulse pressure variation predict fluid responsiveness in critically ill patients? A systematic review and meta-analysis. Crit Care. 2014;18(6):650

\section{P174}

Multi-parameter monitoring for tissue perfusion in septic shock: correlations between macrohemodynamic and metabolic parameters: Preliminary results

Ali Jendoubi ${ }^{1}$, Rifka Jlassi ${ }^{1}$, Bassem Hamrouni ${ }^{1}$, Yahia Marzouqui ${ }^{1}$, Jihen Kouka', Salma Ghedira', Mohamed Houissa'

${ }^{1}$ Anesthesia and Intensive Care, Charles Nicolle Teaching Hospital, Tunis, Tunisia

Correspondence: Ali Jendoubi - jendoubi_ali@yahoo.fr

Annals of Intensive Care 2016, 6(Suppl 1):P174

Introduction Bedside monitoring of tissue perfusion is mandatory in septic shock. Macrovascular indices [mean arterial pressure (MAP), cardiac index $(\mathrm{Cl})$ ] and microvascular parameters (base excess $\mathrm{BE}$, lactate, mixed $\left(\mathrm{SvO}_{2}\right)$ or central venous oxygen saturations $\left(\mathrm{ScvO}_{2}\right)$ have been utilized to evaluate the perfusion status and the effectiveness of resuscitation process. Recently, the central venous-to-arterial carbon dioxide Gap $\left(P(v-a) \mathrm{CO}_{2}\right)$ and the $P(v-a) \mathrm{CO}_{2} /$ arterial-venous oxygen content difference ratio $\left(P(v-a) \mathrm{CO}_{2} / \mathrm{C}(v-a) \mathrm{O}_{2}\right)$ have been proposed as additional resuscitation targets.

Aim

To analyze the correlations between hemodynamic, oxygenation and tissue perfusion values in septic shock.

Patients and methods Thirty patients with septic shock were prospectively enrolled. All patients were resuscitated following the 
Table 34 Relationship between hemodynamic variables and oxygenation parameters

\begin{tabular}{|c|c|c|c|c|c|c|c|c|c|c|c|c|c|c|}
\hline & \multicolumn{2}{|l|}{$\mathrm{PH}$} & \multicolumn{2}{|l|}{$\mathrm{BE}$} & \multicolumn{2}{|c|}{ Lactate } & \multicolumn{2}{|c|}{$\mathrm{ScvO}_{2}$} & \multicolumn{2}{|c|}{$P(v-a) \mathrm{CO}_{2}$} & \multicolumn{2}{|l|}{ Ratio } & \multicolumn{2}{|l|}{ OER } \\
\hline & $r$ & $p$ & $r$ & $p$ & $r$ & $p$ & $r$ & $p$ & $r$ & $p$ & $r$ & $p$ & $r$ & $p$ \\
\hline MAP & 0.61 & 0.11 & 0.77 & 0.026 & -0.78 & 0.023 & 0.96 & $<0.001$ & -0.89 & 0.003 & -0.28 & 0.5 & -0.88 & 0.004 \\
\hline $\mathrm{Cl}$ & 0.70 & 0.051 & 0.60 & 0.12 & -0.59 & 0.12 & 0.21 & 0.61 & 0.053 & 0.9 & 0.04 & 0.92 & -0.37 & 0.365 \\
\hline
\end{tabular}

Ratio: $P(v-a) \mathrm{CO}_{2} / C(a-v) \mathrm{O}_{2}$

Table 35 Relationship between metabolic parameters

\begin{tabular}{|c|c|c|c|c|c|c|c|c|c|c|}
\hline & \multicolumn{2}{|l|}{$\mathrm{PH}$} & \multicolumn{2}{|l|}{ BE } & \multicolumn{2}{|l|}{$\mathrm{DO}_{2}$} & \multicolumn{2}{|l|}{$\mathrm{VO}_{2}$} & \multicolumn{2}{|l|}{ OER } \\
\hline & $r$ & $p$ & $r$ & $p$ & $r$ & $p$ & $r$ & $p$ & $r$ & $p$ \\
\hline Lactate & -0.91 & 0.002 & -0.88 & 0.004 & -0.5 & 0.21 & 0.35 & 0.39 & 0.70 & 0.054 \\
\hline $\mathrm{ScvO}_{2}$ & 0.51 & 0.20 & 0.65 & 0.081 & 0.19 & 0.66 & -0.70 & 0.052 & -0.92 & 0.001 \\
\hline$P(v-a) \mathrm{CO}_{2}$ & -0.55 & 0.16 & -0.71 & 0.048 & -0.14 & 0.75 & 0.55 & 0.16 & 0.68 & 0.066 \\
\hline$P(v-a) \mathrm{CO}_{2} / C(a-v) \mathrm{O}_{2}$ & -0.29 & 0.47 & -0.27 & 0.52 & -0.35 & 0.40 & -0.30 & 0.48 & -0.1 & 0.86 \\
\hline Inotropic index & -0.95 & $<0.001$ & -0.96 & $<0.001$ & -0.53 & 0.17 & 0.31 & 0.45 & 0.71 & 0.051 \\
\hline
\end{tabular}

Surviving Sepsis Campaign guidelines. Transpulmonary thermodilution (TPTD) was used to monitor $\mathrm{Cl}$, oxygen delivery $\left(\mathrm{DO}_{2}\right)$ and consumption $\left(\mathrm{VO}_{2}\right)$. Simultaneous blood samples were obtained from a central venous line and an arterial catheter. Correlations between macrohemodynamic and metabolic parameters were explored with the Pearson test. Two-tailed $P<0.05$ was taken to indicate statistical significance.

Results Between March 2015 and August 2015, 30 septic shock patients (sex ratio M/W 1.5) were studied. The average age of the patients was $57 \pm 19$ years. The sources of infection were as follows: the lungs $(n=13)$, the urinary tract $(n=10)$, the abdomen $(n=5)$ and the central nervous system $(n=2)$. The mean SOFA score at T0 was $10 \pm 4$ points, and the mean IGS2 score was $54 \pm 23$.

We investigate the relationship between hemodynamic variables and metabolic parameters (Table 34).

There was a significant correlation between $\mathrm{ScvO}_{2}$ and $P(v-a)$ $\mathrm{CO}_{2}(r=-0.75, p=0.033)$. Both parameters showed strong correlations with oxygen extraction OER (Table 35). A significant correlation was found between $P(v-a) \mathrm{CO}_{2}$ and arterial BE values $(r=-0.71$, $p=0.048$ ). The preliminary results of our study demonstrated the significant impact of the cumulative inotrope index (CII) [1] on $\mathrm{PH}$ $(r=-0.95, p<0.001), \mathrm{BE}(r=-0.96, p<0.001)$, lactate $(r=0.95$, $p<0.001), P(v-a) \mathrm{CO}_{2}(r=0.74,0.037)$ and OER $(r=0.71, p=0.051)$. The $\mathrm{Cll}$ was not related to the $P(v-a) \mathrm{CO}_{2} / \mathrm{C}(a-v) \mathrm{O}_{2}$.

Conclusion All individual perfusion parameters have limitations. A multimodal monitoring integrating macrohemodynamic and microcirculatory perfusion parameters may overcome those limitations and rule out persistent tissue hypoxia.

\section{Competing interests None.}

\section{Reference}

1. Cruz, D. N. et al. JAMA 2009;301:2445-52.

\section{P175}

Correlation between changes in levels of $\mathrm{N}$-terminal pro-brain natriuretic peptide (NT-pro-BNP) and weight before and after dialysis

Kaouther Dhifaoui ${ }^{1}$, Zied Hajjej', Walid Sammoud ${ }^{1}$, Iheb Labbene ${ }^{1}$, Mustapha Ferjani

${ }^{1}$ Department of Critical Care Medicine and Anesthesiology, Military Hospital of Tunis, Tunisia, Tunis, Tunisia
Correspondence: Zied Hajjej - hajjej_zied@hotmail.com Annals of Intensive Care 2016, 6(Suppl 1):P175

Introduction Volume status is difficult to predict in patients with chronic renal failure before hemodialysis. This parameter is very important to know and to define the volume of fluid to be removed during dialysis.

We undertook this study to measure baseline $\mathrm{N}$-terminal pro-brain natriuretic peptide (NT-pro-BNP) plasma concentration and weight gain derived for "dry weight" and to correlate their levels with changes in levels of NT-pro-BNP and weight before and after dialysis.

Patients and methods Over 8 months (February 2014-September 2014), a prospective, observational study was performed in an 18-bed medical surgical intensive care unit at Tunis Military Hospital. Patients under hemodialysis (HD) treatment for end-stage renal disease (ESRD) were included in this study. All patients were on regular dialysis treatment for at least 6 months, had no residual kidney function and underwent a $4 \mathrm{~h}$ HD session 3 times a week. Patients with clinical evidence of congestive heart failure (CHF) (NYHA class III and higher) were excluded. Dry weight was determined by a clinical approach. Weight and levels of NT-pro-BNP were measured immediately before and after hemodialysis. The correlation between weight changes during the session and variation of levels of NT-pro-BNP before and after hemodialysis was studied.

Results A total of 22 patients were included. The mean age was 50 years. There was a significant reduction in the values of weight after $\mathrm{HD}$ compared to the values before HD $(p=0.001)$. There was a significant reduction in NT-pro-BNP levels after HD compared to the values before $\mathrm{HD}(p=0.03)$. Using regression analysis, we found a weak correlation between change in NT-pro-BNP and weight loss $\left(r^{2}=0.43\right.$, $p=0.04$ ) before and after HD. There was also a correlation between levels of NT-pro-BNP and weight gain relative to the dry weight before dialysis $\left(r^{2}=0.63, p=0.02\right)$.

Conclusion NT-pro-BNP can be used in patients with chronic renal failure undergoing dialysis for the assessment of volume status, especially if patient's dry weight is unknown. But it must be interpreted according to clinical settings, particularly in case of heart disease.

Competing interests None. 


\section{P176}

Impact of colonization with multidrug-resistant bacteria on the outcome of patients with hematological malignancies admitted to the intensive care medicine

Johanna Oziel ${ }^{1}$, Carole Schwebel ${ }^{2}$, Elie Azoulay ${ }^{3}$, Michaël Darmon $^{4}$, Bertrand Souweine ${ }^{5}$, Lila Bouadma ${ }^{6}$, Guillaume Marcotte ${ }^{7}$, Christophe Clec' $h^{8}$, Yves Cohen', Outcomerea Study Group

193, Hôpital Avicenne, Bobigny, France; ${ }^{2} 38$, C.H.U de Grenoble C.H.U, La Tronche, France; ${ }^{3}$ Réanimation médicale, Hôpital Saint-Louis, Paris, France; ${ }^{4}$ Réanimation Médicale, CHU Saint-Etienne - Hôpital Nord, Saint-Étienne, France; ${ }^{5}$ Réanimation médicale, CHU Gabriel-Montpied, Clermont-Ferrand, France; ${ }^{6}$ Réanimation médicale et des maladies infectieuses, Hôpital Bichat-Claude Bernard, Paris, France; ${ }^{7}$ Reanimation medicale, Assistance Publique Hôpitaux de Paris, Paris, France; ${ }^{8}$ Réanimation medico-chirurgicale, hopital avicenne, Bobigny, France; ${ }^{9}$ Réanimation médico-chirugicale, Hôpital Avicenne, Bobigny, France

Correspondence: Johanna Oziel - johanna.oziel@gmail.com

Annals of Intensive Care 2016, 6(Suppl 1):P176

Introduction While colonization with multidrug-resistant bacteria is a frequent issue in the ICU, its impact on patients' outcome remains unclear. Particularly, patients with hematological malignancies may be at high risk of colonization, subsequent infection and mortality, but convincing evidence is lacking.

The aim of our study was to assess whether colonization with MDR bacteria was associated with increased length of stay and mortality in this specific population of immunocompromised patients.

Patients and methods We performed a multicenter database study. Hematological patients (i.e., patients with hematological malignancies) were identified in the database using the International Classification of Diseases coding or through the specific disease-related variable of the SAPS II score.

Only extended-spectrum beta-lactamase-producing Enterobacteriaceae (ESBL-PE) or methicillin-resistant Staphylococcus aureus (MRSA) colonization was considered. Intestinal carriage of ESBL-PE and nasal carriage of MRSA were routinely screened by rectal and nasal swabbing on ICU admission and weekly thereafter in all participating units.

Exclusion criteria were as follows: multiple ICU stays, ESBL-PE or MRSA colonization on ICU admission, and length of ICU stay of $<7$ days.

Patients with and without ICU-acquired colonization were first compared by univariate analyses. Then, a multivariate logistic regression model was used to test the association of colonization with mortality, adjusting for confounding variables such as the SAPS II score.

Results A total of 343 patients were included. Mean age and SAPS II score were $55 \pm 16.3$ years and $52.2 \pm 14.4$ points, respectively. The global rate of ICU-acquired colonization was $9.6 \%$. Remarkably, the rate of MRSA colonization was lower than that of ESBL-PE colonization (1.7 vs $7.9 \%)$

ICU-acquired colonization significantly increased the lengths of ICU and hospital stays (medians in days [interquartile range]: 23 [15-39] vs 9 [7-13], and 41 [26-67] vs 29 [18-48], respectively, $p<0.001$ ).

Although having a similar SAPS II score, ICU and hospital mortality rates were higher among colonized patients ( 28.9 vs $20.8 \%$, and 43.3 vs $31.6 \%$, respectively, $p<0.001$ )

In multivariate analysis, ICU-acquired colonization remained significantly associated with ICU and hospital mortality (odds ratio, $95 \%$ confidence interval 3.58, 1.60-8.03, $p<0.001$, and 2.65, 1.18-5.92, $p=0.02$, respectively)

Conclusion Our results suggest that, although relatively infrequent, ICU-acquired colonization in hematological patients is associated with increased lengths of stays and mortality rates.

Accordingly, strengthened isolation precautions should be considered in these patients.

\section{Competing interests None.}

P177

ICU moving followed by a drop of imipenem-resistant Pseudomonas aeruginosa

Alexy Tran Dinh ${ }^{1}$, Caroline Neulier ${ }^{1}$, Marlène Amara ${ }^{1}$, Nicaise Nebot ${ }^{1}$, Gilles Troché ${ }^{1}$, Nelly Breton ${ }^{1}$, Benjamin Zuber $^{2}$, Sébastien Cavelot ${ }^{1}$, Béatrice

Pangon ${ }^{1}$, Jean Pierre Bedos ${ }^{3}$, Jacques Merrer ${ }^{1}$, David Grimaldi ${ }^{1}$

178150, Hospital Center De Versailles, Le Chesnay, France; ${ }^{2}$ Intensive care

unit, Hospital Center De Versailles, Le Chesnay, France; ${ }^{3}$ Réanimation médico-chirurgicale, Centre Hospitalier de Versailles, Le Chesnay, France

Correspondence: Alexy Tran Dinh - alexy.trandinh@gmail.com

Annals of Intensive Care 2016, 6(Suppl 1):P177

Introduction Pseudomonas aeruginosa (PA), a ubiquitous environmental Gram-negative bacillus, is highly endemic in intensive care units (ICU) and is responsible for severe nosocomial infections. Transmission pathways and reservoirs of PA in ICUs are still a matter of debate. However, several studies underpinned the importance of environment colonization to explain patient colonization with PA. The increasing antibiotic resistance of PA isolated from ICUs is of particular concern; our ICU had, for instance, a high proportion of imipenem-resistant (imi-R) PA. In October 2014, our ICU moved to a new place inside our hospital. We took advantage of this unique opportunity to study the effect of a change of ICU location on the proportion of imipenem resistance across all PA isolated from clinical samples.

Materials and methods This observational before/after study is conducted over 24 months separated in 2 periods: October 2013 to October 2014 (before the moving) and October 2014 to October 2015 (after the moving). All clinical samples growing with PA were identified. We excluded samples drawn within the first 2 days of patient admission and doublets. Imipenem resistance was determined with the disk method and confirmed with E-test. OprD phenotype was suspected when PA strains were sensitive to piperacillin and ceftazidime and resistant to carbapenem. We analyzed also routine environmental bacteriological samples in water, which were drawn every 3 months in a constant place of the ICU. We collected defined daily doses (DDD) of carbapenem (except ertapenem), monthly consumption of hydroalcoholic solution (HAS), number of admissions $\geq 48 \mathrm{~h}$, number of mechanically ventilated patients, percentage of immunosuppressed patients, number of intravascular catheters, mean age, mean IGS II score and mean mortality. Finally, individual risk factors for colonization with imi-R PA were assessed: previous treatment with carbapenem, history of recent hospitalization in ICU and immunosuppression.

Results We provide below intermediate results of the study censured at the end of June 2015. In the first period, there were 77 bacteriological clinical samples positive to PA in 65 patients, and 30 in 25 patients in the second period, distributed as follows: lower respiratory tract samples (56 and 15), catheters (9 and 4), abscesses (4 and 2), blood samples (3 and 6) and urine samples (5 and 3). The proportion of imi-R PA decreased dramatically in the second period: 36 versus $10 \%$ ( $p=0.008)$. Of note, imipenem resistance appeared to be mostly due to an OprD phenotype in both periods (75 vs $67 \%$ ). Interestingly, we found isolates of PA with an OprD phenotype in routine environmental bacteriological samples in both periods. During the study, DDD of carbapenem was similar during the 2 periods (103 vs 108 per 1000 patient-days), and HAS consumption was stable around 180-200 L per 1000 patient-days. Finally, the 2 periods were comparable in terms of number of admissions $\geq 48$ h (566 vs 578 ), number of mechanically ventilated patients (338 vs 340 ), percentage of immunosuppressed patients (26.7 vs $21.3 \%$ ), number of intravascular catheters (407 vs 397 ), mean age (64.3 vs 65.4), mean IGS II score (50.6 vs 49.2) and mortality (19.4 vs $15.4 \%$ ). Finally, the drop in imipenem resistance was not explained by a difference in the proportion of patients that had a previous treatment with carbapenem (7.7 vs $6.7 \%$ ), immunosuppression (13.8 vs $23.3 \%$ ) or a recent history of hospitalization in ICU (0 vs $2 \%$ ).

Conclusion Our results showed that a change of the ICU location was associated with a dramatic decrease in imi-R PA clinical isolates, mainly OprD phenotype. We did not observe any differences in DDD of 
carbapenem, HAS consumption, or any other changes between the 2 periods that could explain this drop in imi-R PA. In parallel, we isolated OprD mutants in routine environmental samples. Altogether these findings in this quasi-experimental design suggest that environmental source is a critical factor in PA colonization. Final results with the data from June to October 2015 and genotype analysis of clinical and environmental isolates of PA are ongoing.

\section{Competing interests None.}

\section{P178}

Risk factors of carbapenem resistance acquisition in intensive care unit

François Labaste' , Jean-Marie Conil' ${ }^{1}$ Julia Grossac' ${ }^{1}$ Stéphanie Ruiz'1, Marion Grare ${ }^{2}$, Olivier Fourcade ${ }^{3}$, Vincent Minville ${ }^{1}$, Bernard Georges ${ }^{1}$ Réanimation polyvalente, CHU Toulouse Rangueil, Avenue du Professeur Jean Poulhes, Toulouse, France; ${ }^{2}$ Service de bactériologie, Hospital Center University Toulouse - Casselardit Ancely, Toulouse, France; ${ }^{3}$ Réanimation purpan, CHU Toulouse, Toulouse, France

Correspondence: François Labaste - labaste.f@chu-toulouse.fr Annals of Intensive Care 2016, 6(Suppl 1):P178

Introduction In intensive care units, life-threatening bacillus infections may occur. It is a very common situation. Probabilist extendedspectrum antibiotic therapy has to be promptly instituted. The spectrum of this antibiotic should include extended-spectrum betalactamases enterobacteria. Because of their broad spectrum of action, carbapenems are the standard treatment, especially in cases of nosocomial infections. Lately, bacterial strains have acquired resistance mechanisms to carbapenems, including carbapenemase production. Because of few therapeutic options, the occurrence of bacterial resistance to carbapenems is a real public health problem. The use of carbapenem is limited by expert's recommendations to limit the risk of therapeutic impasse. The aim is to preserve their effectiveness. The objective of this work was to assess the incidence and risk factors for resistance acquisition in an intensive care unit, while rigorously good use of these antibiotics was applied.

Materials and methods We conducted a prospective observational cohort study in medical and surgical ICU of CHU Toulouse Rangueil between May and November 2014. The patients undergoing antibiotic therapy with carbapenem more than $48 \mathrm{~h}$ were included. Biological samples were performed according to the current practices of the service. The primary endpoint was the occurrence of bacterial resistance to carbapenems diagnosed between the beginning of the treatment and the patient discharged from the intensive care unit. Univariate and multivariate analyses were performed.

Results Of 364 patients admitted to the service, 78 were included in our study, $16(20.51 \%)$ developed a carbapenem resistance. The two main risk factors found were: duration of hospitalization in the ICU over 29 days (HR $=3.61 ; p=0.01)$ and presence of Pseudomonas aeruginosa in samples taken before the start of treatment $(\mathrm{HR}=5.31 ; p=0.002)$. No carbapenemase production was observed in our work.

Discussion Our prospective study evaluated risk factors of carbapenem resistance acquisition. It is an original study because of few dates on this topic. Other cohort included all patients, not just patients treated with carbapenems. Carbapenem antibiotic therapy is known to increase the risk of resistance. We specifically choose to study other risk factors in this population. In our service, we found Pseudomonas aeruginosa represents a higher risk of carbapenem resistance. Tough bacterial ecology is variable between two ICUs. Result should be interpreted with caution.

Conclusion The prescription of carbapenems in intensive care has to respect the recommendations of experts. Regarding our results, attention should be paid to patients whose ICU stay is extended and to patients in whom bacteriological samples were positive to Pseudomonas aeruginosa if diagnosed before the initiation of antibiotic therapy.

Competing interests None.

\section{References}

1. Carbapenem resistance: Toward a new dead end?; N.Grall; Journal des anti-infectieux, 2011.

2. Recommandations de bon usage des carbapénèmes; R. Gauzit; Antibiotique, 2010.

\section{P179}

Risk factors for subsequent infection among patients with extended-spectrum beta-lactamase-producing enterobacteriaceae colonization: a retrospective study

Guillaume Van Der Meersch', Johanna Oziel', Yacine

Tandjaoui-Lambiotte ${ }^{1}$, Frédéric Gonzalez ${ }^{2}$, Philippe Karoubi ${ }^{2}$, Christophe Huang ${ }^{2}$, Christophe Clec'h², Yves Cohen

${ }^{1}$ Réanimation médico-chirurgicale, CHU Avicenne, Université Paris XIII, Bobigny, France; ${ }^{2}$ Réanimation médico-chirurgicale, CHU Avicenne, Bobigny, France

Correspondence: Guillaume Van Der Meersch - gvdm75@gmail.com

Annals of Intensive Care 2016, 6(Suppl 1):P179

Introduction Intestinal carriage of extended-spectrum beta-lactamase-producing enterobacteriaceae (ESBL-PE) is a common issue in ICU patients and may be associated with subsequent infection. Thus, carbapenems are often administered as first-line antibiotic therapy in patients with prior known colonization who develop severe sepsis or septic shock.

However, while ESBL-PE-related infections rarely occur in patients without prior colonization, infections occurring among colonized patients may be due to other less resistant pathogens.

In this view, it would be interesting to be able to predict, which patients will actually develop ESBL-PE-related infections, so as to control the increasing use of carbapenems that may jeopardize their efficacy in a near future. The aim of this study was to identify simple risk factors for subsequent infection among ESBL-PE-colonized patients, likely to limit the use of carbapenems as first-line antibiotic therapy in case of severe sepsis or septic shock.

Patients and methods We performed a retrospective study in a 16-bed ICU of a university hospital. All patients with ESBL-PE colonization were considered, irrespective of whether colonization was present on ICU admission or acquired during the ICU stay. Rectal swabs were performed on admission and on a weekly basis thereafter. The following data were collected: age, sex, severity scores, admission category (medical or surgical), underlying diseases, antibiotic exposure within 3 months before ICU admission, invasive procedures, type of ESBL-PE, and outcome. The number of infected patients without prior colonization was also recorded.

Results A total of 239 colonized patients were screened over a 3-year period, of which 37 were excluded because of missed rectal swabs on admission. Among the remaining 202 patients, 142 (70.3\%) were colonized on ICU admission. Escherichia coli $(n=101,50 \%)$, Klebsiella pneumoniae ( $n=59,29.2 \%)$, and Enterobacter cloacae $(n=39,19.3 \%)$ accounted for $98.5 \%$ of species recovered from rectal swabs.

During the study period, only five patients developed ESBL-PE infection without prior colonization. Among colonized patients, 24 (11.9\%) developed subsequent infections. There was a trend toward a higher rate of infection for Klebsiella pneumoniae and Enterobacter cloacae colonization than for Escherichia coli colonization (15.3 and 17.9 vs $7.9 \%$, respectively). Overall, colonized patients with subsequent infection had received more carbapenems within 3 months before ICU admission, had a longer time to colonization, and were more likely to be admitted after planned or unplanned surgical procedures than colonized patients without subsequent infection.

Independent risk factors for subsequent infection were: prior exposure to carbapenems (OR 3.40; $95 \% \mathrm{Cl} 1.03-11.20$ ), median time to colonization greater than 14 days (OR 3.76; $95 \% \mathrm{Cl} 1.21-11.67)$, and surgical admission category (OR 4.05; $95 \% \mathrm{Cl} 1.38-11.88$ ).

Conclusion We were able to identify three simple risk factors that may be considered in clinical practice to reduce carbapenems use as first-line antibiotic therapy in case of severe sepsis or septic shock in patients with known ESBL-PE colonization. 
Reducing carbapenems use in those patients is all the more important, as ESBL-PE infection following colonization seems to be a relatively rare issue.

Our results have to be confirmed by further multicenter prospective evaluations.

\section{Competing interests None.}

\section{P180}

Is rectal colonisation predictive of extended-spectrum

$\beta$-lactamase-producing enterobacteriaceae presence

in respiratory samples performed in the intensive care unit?

Helene Carbonne', Matthieu Le Dorze ${ }^{2}$, Rishma Amarsy ${ }^{3}$, Joaquim

Mateo $^{2}$, Didier Payen de la Garanderie ${ }^{4}$

${ }^{1}$ Hospital Lariboisière, Paris, France; ${ }^{2}$ Anesthesiology and critical care, Hospital Lariboisière, Paris, France; ${ }^{3}$ Equipe opérationnelle d'hygiène, Hospital Lariboisière, Paris, France; ${ }^{4}$ Réanimation chirurgicale, CHU Lariboisière, Paris, France

Correspondence: Helene Carbonne - hcarbonne@gmail.com Annals of Intensive Care 2016, 6(Suppl 1):P180

Introduction Empirical antimicrobial therapy is a major issue in pneumonia management in intensive care unit (ICU) [1]. It can be challenging due to the presence of multidrug-resistant (MDR) pathogens, especially extended-spectrum $\beta$-lactamase-producing enterobacteriaceae (ESBL-E), with a rising incidence. Indeed, rectal colonisation with ESBL-E concerns $15 \%$ of patients admitted in ICU [2]. Many ICUs have organised MDR pathogen screening strategies; however, the impact of a MDR pathogen colonisation on empirical antimicrobial therapy for infection is unknown [3]. Regarding empirical antimicrobial therapy for pneumonia, the place of carbapenem in the strategy is not established.

The objective was to evaluate whether ESBL-E rectal colonisation was predictive of ESBL-E presence in the early ( $\leq 5$ days) or late ( $>5$ days) respiratory samples performed in the ICU.

Patients and methods Retrospective observational study performed between January 2012 and August 2014 in a 21-bed surgical ICU. Every early ( $\leq 5$ days of ICU hospitalisation) and late ( $>5$ days) positive respiratory sample was collected, along with rectal swab performed within $48 \mathrm{~h}$ before respiratory sample. Positive and negative predictive values (PPV, NPV) of an ESBL-E positive rectal swab to predict the presence of ESBL-E in respiratory sample were analysed. Early and late samples were analysed separately.

Results During the study period, 425 (231 early and 194 late) positive respiratory samples were included, corresponding to 356 patients. Concerning early samples, $3 / 231(1.3 \%)$ respiratory samples and $32 / 231$ (13.9\%) rectal swabs were positive for ESBL-E. Among the 32 ESBL-E positive rectal swabs, 2 respiratory samples were positive for ESBL-E. The PPV of ESBL-E positive rectal swab was $5.9 \%(2.9-8.9)$ and the NPV was $99.5 \%$ (98.0-100.0). Concerning late samples, 26/194 $(13.4 \%)$ respiratory samples and 66/194 $(34.0 \%)$ rectal swabs were positive for ESBL-E. Among the 66 ESBL-E positive rectal swabs, 20 respiratory samples were positive for ESBL-E. The PPV of ESBL-E positive rectal swab was $30.8 \%$ (24.1-37.4) and the NPV was $96.1 \%$ (93.4-98.9).

Conclusion ESBL-E rectal colonisation did not predict presence of ESBL-E in respiratory sample performed in the ICU, especially for early respiratory samples. Absence of ESBL-E rectal colonisation predicted the absence of ESBL-E in early and late respiratory samples with an excellent NPV. This strategy could be used to reduce carbapenem prescription for empirical antimicrobial therapy of pneumonias in ICU.

\section{Competing interests None.}

\section{References}

1. Iregui M et al. (2002), Clinical importance of delays in the initiation of appropriate antibiotic treatment for VAP., Chest.

2. Razazi K et al. (2012), Clinical impact and risk factors for colonization with ESBL-producing bacteria in the ICU., Intensive Care Med.

3. Luna CM et al. (2014), The role of surveillance cultures in guiding ventilator-associated pneumonia therapy., Curr Opin Infect Dis.
P181

Antibiotic therapy in intensive care unit patients: appraisal of medical attitude related to therapy appropriateness, severity of infection and outcome

Sophie Leloup Von Edelsberg ${ }^{1}$, Christelle Vercheval ${ }^{2}$, Monique Nys ${ }^{3}$, Pierre Damas $^{1}$

${ }^{1}$ Intensive care, CHU Sart-Tilman, Liège, Belgium; ${ }^{2}$ Clinical pharmacy, CHU Sart-Tilman, Liège, Belgium; ${ }^{3}$ Soins intensifs généraux, CHU Sart-Tilman, Liège, Belgium

Correspondence: Pierre Damas - pdamas@chu.ulg.ac.be Annals of Intensive Care 2016, 6(Suppl 1):P181

Introduction Antibiotic therapy (AT) has to be reviewed within 3 days and adapted with regard to confirmation of infection and appropriateness.

Aim of the study: To audit the present practice in AT in 5 ICUs totalizing 44 beds from a tertiary hospital, to compare it between severe and non-severe infections, to evaluate the level of inappropriate therapy and its impact on the outcome of patients.

Patients and methods This was a prospective study conducted from March 1 to August 31, 2015. All AT begun for infection were reappraised at day 4. Infectious episodes were defined as confirmed or absent or uncertain. Treatments were classified as appropriate, inappropriate or not evaluable. Severe infections included severe sepsis and septic shock. Results In total, 424 AT given to 327 patients were analyzed. Fifty cases were not confirmed as infectious. Among the others, there were 219 appropriate AT (51.6\%), 46 inappropriate (10.8\%) and 109 not documented $(25.8 \%)$.

Table 36 shows the medical decisions at day 4 according to the severity of infection and the 28-day ICU mortality of all the subgroups of patients.

Discussion (1) Half of the infections were severe $(169 / 374=45 \%)$ regardless of the appropriateness of AT. (2) Appropriate AT could be reduced in $36 \%$; this did not depend on the severity of infection. (3)

\section{Table 36 See text for description}

\begin{tabular}{|c|c|c|c|c|}
\hline \multirow{2}{*}{$\begin{array}{l}\text { Medical decision } \\
\text { at day } 4\end{array}$} & \multicolumn{2}{|c|}{ Not severe 28-day ICU } & \multicolumn{2}{|c|}{ Severe 28-day ICU } \\
\hline & Infections & Mortality & Infections & Mortality \\
\hline \multicolumn{5}{|c|}{219 appropriate treatments } \\
\hline Continued & $69(31.5 \%)$ & $15(21.7 \%)$ & $40(18.3 \%)$ & $9(22.5 \%)$ \\
\hline Reduced & $37(16.9 \%)$ & $2(5.4 \%)$ & $42(19.2 \%)$ & $9(21.5 \%)$ \\
\hline $\begin{array}{l}\text { Patient dis- } \\
\text { charged }\end{array}$ & $1(0.5 \%)$ & $0(0 \%)$ & $4(1.8 \%)$ & $0(0 \%)$ \\
\hline Early death & $1(0.5 \%)$ & 1 (100\%) & 25 (114\%) & $25(100 \%)$ \\
\hline Total & $108(49.3 \%)$ & $18(16.7 \%)$ & $111(50.7 \%)$ & $43(38.7 \%)$ \\
\hline \multicolumn{5}{|l|}{$\begin{array}{l}46 \text { inappropriate } \\
\text { treatments }\end{array}$} \\
\hline Continued & $1(2.2 \%)$ & $0(0 \%)$ & $0(0 \%)$ & $0(0 \%)$ \\
\hline Adapted & $18(39.1 \%)$ & $4(22.2 \%)$ & $21(45.6 \%)$ & $6(28.6 \%)$ \\
\hline $\begin{array}{l}\text { Patient dis- } \\
\text { charged }\end{array}$ & $4(8.7 \%)$ & $0(0 \%)$ & $0(0 \%)$ & $0(0 \%)$ \\
\hline Early death & $1(2.2 \%)$ & $1(100 \%)$ & $1(2.2 \%)$ & $1(100 \%)$ \\
\hline Total & $24(52.2 \%)$ & $5(20.8 \%)$ & $22(47.8 \%)$ & $7(31.8 \%)$ \\
\hline \multicolumn{5}{|c|}{109 non-evaluable or uncertain treatment } \\
\hline Continued & $20(18.2 \%)$ & $4(20 \%)$ & $19(17.3 \%)$ & $5(26.3 \%)$ \\
\hline Stopped & $3(2.7 \%)$ & $1(33.3 \%)$ & $2(1.8 \%)$ & $2(100 \%)$ \\
\hline Changed & $14(12.7 \%)$ & $4(28.6 \%)$ & $8(7.3 \%)$ & $2(25 \%)$ \\
\hline $\begin{array}{l}\text { Patient dis- } \\
\text { charged }\end{array}$ & $10(9.1 \%)$ & $0(0 \%)$ & $0(0 \%)$ & $0(0 \%)$ \\
\hline Early death & $2(1.8 \%)$ & $2(100 \%)$ & $7(6.4 \%)$ & $7(100 \%)$ \\
\hline Total & $49(44.5 \%)$ & $11(22.4 \%)$ & $36(32.7 \%)$ & $16(44.4 \%)$ \\
\hline
\end{tabular}


Continuation of therapy was also the same in non-documented or uncertain infections regardless of the severity. (4) Inappropriate AT occurred in $10.8 \%$ of the cases. (5) Inappropriate AT did not increase early $(2 / 46$ vs $26 / 219 p=0.18)$ or late ICU mortality $(10 / 46$ vs $37 / 219$ $p=0.405)$. (6) Mortality of severe infection was in the range of other European series. 7) $50 \%$ of mortality of severe infections occurred unexpectedly within 3 days (33/66).

Conclusion The severity of infection did not interfere with medical decision regarding the step-down therapy.

Competing interests None.

\section{P182}

Epidemiology and associated factors of ICU-acquired infections in critically ill patients: a prospective cohort study

Zied Hajjej', Chaker Bouguerra², Noura Naas', Walid Sammoud', Iheb Labbene', Mustapha Ferjani ${ }^{1}$

'Department of Critical Care Medicine and Anesthesiology, Military Hospital of Tunis, Tunisia, Tunis, Tunisia; ${ }^{2}$ Department of preventive medicine, Military Hospital of Tunis, Tunisia, Tunis, Tunisia

Correspondence: Zied Hajjej - hajjej_zied@hotmail.com

Annals of Intensive Care 2016, 6(Suppl 1):P182

Introduction Acquired infections are common in intensive care units. The aims of our study were to evaluate the epidemiology of intensive care unit (ICU)-acquired infections and to determine potential associated factors to such infections.

Patients and methods Patients with longer than a 48-h stay in an adult mixed medical-surgical ICU in a teaching hospital were included. The incidence (per cent) and incidence density (per 1000 patient days) of ICU-acquired infections were analysed prospectively in a 12-month study. Infections were diagnosed according to Centers for Disease Control and Prevention definitions. Risk factors for infection were determined by multivariable logistic regression.

Results One hundred and thirty-nine (28.8\%) of 482 patients, whose ICU stay was longer than $48 \mathrm{~h}$, acquired a total of 174 infections (1.25 per patient) during their ICU stay, with an infection rate of 66 per 1000 patient days.

The most common infections were ventilator-associated pneumonia $(35.6 \%)$, central catheter-related or primary bloodstream infections $(25.7 \%)$ and urinary tract infections ( $16 \%)$.

Infections were most commonly caused by K. pneumoniae (34.2\%), followed by P. aeruginosa (25.1\%), A. baumannii (11.6\%) and E. coli (2.6\%).

Multivariate analysis showed that clinical factors associated with new ICU-acquired infections were age $\geq 45$ years $(p=0.029,95 \% \mathrm{Cl}[1082-$ 4316], OR 2.161), surgical admission $(p=0.002,95 \%$ Cl [1.437-5.291], OR 2.757), ICU length of stay more than 7 days $(p=0.0001,95 \% \mathrm{Cl}$ [5.398-17.999], OR 9.857), duration of mechanical ventilation more than 5 days $(p=0.002,95 \% \mathrm{Cl}[1.485-5.941], \mathrm{OR} 2.970)$ and a use of an urinary catheter more than 6 days $(p=0.004,95 \% \mathrm{Cl}[1.487-7.804]$, OR 3.406).

Mortality among infected patients was $38.1 \%$, and the risk of death was increased as patients were infected $(p<0.001,95 \% \mathrm{Cl}[1.782-$ 4.021], OR 3.784).

Conclusion ICU-acquired infections were common in a cohort of mixed medical-surgical patients. Only age $\geq 45$ years, surgical admission, ICU length of stay more than 7 days, duration of mechanical ventilation more than 5 days and a use of an urinary catheter more than 6 days were found to be independently associated with these infections; they were found to have a significant effect on ICU mortality.

\section{Competing interests None.}

\section{P183}

The impact of nosocomial infection acquired in intensive care units on mortality and length of stay until hospital discharge: a retrospective, monocentric, 10-year study

Joris Muller ${ }^{1}$, François Severac ${ }^{2}$, Mickael Schaeffer ${ }^{2}$, Pierre Tran Ba Loc $^{2}$, Stéphanie Deboscker', Maleka Schenck ${ }^{3}$, Marie-Line Harley ${ }^{3}$, Ralf
Janssen-Langenstein ${ }^{3}$, Jean-Etienne Herbrecht ${ }^{3}$, Benjamin Lebas ${ }^{3}$, Max Guillot $^{3}$, Vincent Castelain ${ }^{3}$, Francis Schneider ${ }^{3}$, Thierry Lavigne ${ }^{1}$

${ }^{1}$ Infection control unit, Strasbourg University Hospital, Strasbourg, France; ${ }^{2}$ Public health service, Strasbourg University Hospital, Strasbourg, France; ${ }^{3}$ Medical intensive care unit, Strasbourg University Hospital, Strasbourg, France

Correspondence: Joris Muller - joris.muller@jom.link

Annals of Intensive Care 2016, 6(Suppl 1):P183

Introduction The impact of nosocomial infections (NI) in intensive care unit (ICU) on the whole hospital stay (from ICU admission to hospital discharge) is seldom studied and is still a subject of controversy. Large raw data collections are available on this subject among various prospective cohort surveys like the REA-RAISIN, OUTCOMEREA and INICC programs. Nevertheless, statistical modelling of the mortality and excess in length of stay due to these NI may be challenging and should explain contradictory results.

The aim of our study was to compare, among ICU patients, the risk of death and the hospital length of stay between those with acquired $\mathrm{NI}$ in the ICU and those without acquired NI in the ICU.

Patients and methods Data were collected continuously from 2004 to 2014 for each patient hospitalised more than 2 days in the 30 beds medical ICU of Strasbourg University Hospital (France). The surveillance focused on ventilation-associated pneumonia, central venous catheter-related infection or bacteraemia and bloodstream infection according to the French's Survey Network for ICU-acquired infection protocol (Réseau d'Alerte, d'Investigation et de Surveillance des Infections Nosocomiales-REA-RAISIN). The analysis included patient's characteristics, devices exposure and ICU-acquired infections.

We merged this database with the local diagnosis-related group database (PMSI-Programme de Médicalisation des Systèmes d'Information). In this way, we obtained the full hospital stay information, including main diagnosis, SAPSII, length of stay and mortality. We assessed the mortality risk and the length of stay with various models: linear and logistic regressions, Cox models and multistate models.

Results In the 7465 inpatient stays, 882 (12\%) presented at least one NI. Mean age was statistically the same for both groups (65 years), and the rate of antimicrobial therapy at admission in the ICU was the same (79 vs. $74 \%$ ). Patients with NI were more likely men (64 vs. $56 \%$ ), had a higher SAPSII score (54 vs. 49) and were more likely immunosuppressed (31 vs. $22 \%$ ).

Crude mortality during hospital stay was, respectively, 44 and $27 \%$ for patients with and without NI. The mean length of stay was more important for patients with $\mathrm{NI}$ (51 days vs. 21). This last difference remained significant with multistate models.

Other models did not meet the necessary validity conditions. The proportion of patients with NI seemed to increase slightly during the 10 years (11\% in 2004, $14 \%$ in 2014).

Discussion Multistate models have to be used to study this association because the competitive risk between the events "Death" and "Discharge" cannot be handled with the others statistical models. These multistate models seem to be underused in the literature on this subject. A wider use of these models should be obtained by teaching them in statistical classes and by using statistical software including them like R or SAS ${ }^{\circledR}$.

The increase of $\mathrm{NI}$ rates could be due to a change of practices in this ICU, change in data record definition along time or a better detection. Our systematic review of the literature on MEDLINE did not find another French study on this subject with such a long follow-up period.

The main limitations of our study are the lack of adjustment of our models and the monocentric data. A multicentric study and more comprehensive statistical models are planned in later studies.

Conclusion Our results are in favour of a significant effect of the ICUacquired $\mathrm{NI}$ on length of stay but remain unclear about a possible effect on mortality. This field of search is open to larger, such as multicentric studies or to alternative statistical models.

Competing interests None. 


\section{References}

1. Savey A, Machut A \& RAISIN group. Surveillance des infections nosocomiales en réanimation adulte. Réseau REA-Raisin, France, Résultats 2013. (2015). at http://opac.invs.sante.fr/index.php?lvl=notice_display\&id $=12414$

2. Nelson, R. E. et al. The Magnitude of Time-Dependent Bias in the Estimation of Excess Length of Stay Attributable to Healthcare-Associated Infections. Infection Control \& Hospital Epidemiology 36, 1089-1094 (2015).

\section{P184}

Incidence and prognosis of catheter-associated urinary tract infections in intensive care unit: the IPRIUS study Jennifer Brunet ${ }^{1}$, Bertrand Canoville', Cédric Daubin', Amélie Seguin', Nicolas Terzi ${ }^{1}$, Xavier Valette ${ }^{1}$, Pierre Verrier ${ }^{1}$, Damien Du Cheyron ${ }^{1}$ ${ }^{1}$ Réanimation médicale, Centre Hospitalier Universitaire de Caen, Caen, France

Correspondence: Jennifer Brunet - brunet-j@chu-caen.fr Annals of Intensive Care 2016, 6(Suppl 1):P184

Introduction Catheter-associated urinary tract infections (UTIs) are one the most common hospital- and intensive care unit (ICU)-acquired infection and are often used as healthcare quality indicator. Because their diagnosis is not well defined, epidemiologic data vary between studies, and their impact on the prognosis of critically ill patients remain unclear. The objectives of this study were to describe the incidence and the prognosis of catheter-associated UTI in a medical intensive care unit.

Patients and methods A prospective observational study was conducted in the medical intensive care unit of a French University Hospital, over a period of 1 year between February 1, 2014, and January 31, 2015. All patients admitted to the ward for at least $48 \mathrm{~h}$ were included. Results Four hundred and eighty-four patients were included. The incidence of catheter-associated UTI was 6.4 per 100 catheterized patients and 5.9 per 1000 days of urinary catheter. Among 133 patients who had at least one urine culture during their ICU stay, those with a diagnosis of infection $(n=31)$ were more likely to be admitted for a neurologic issue. They had an ICU length of stay significantly longer than patients without infection (24 [16-45] vs 17 days [11-27] $p=0.0076)$, and a significantly longer length of urinary catheterization (23 [16-38] vs 17 days [11-26] $p=0.013)$. In univariate and multivariate analyses, the occurrence of catheter-associated UTI was not found as an independent risk factor associated with mortality, unlike age and SAPS II score at ICU admission.

Conclusion Development of catheter-associated UTI is common in critically ill patients. Classic criteria for diagnosis have, however, a poor specificity. These infections are not associated with increased mortality, and related morbidity seems to be low. Because incorrect diagnosis of catheter-associated UTI treatment may have economic and ecologic impacts, in particular by the selection pressure induced by unnecessary prescription and improper use of antibiotics by patients, diagnostic criteria in the ICU setting need to be better defined, and catheter-associated UTI screening could be limited to a second step of evaluation of fever of unknown origin.

\section{Competing interests None.}

\section{P185}

Automatic tube compensation during mechanical ventilation in ICU patients. Meta-analysis of trials on weaning outcome and physiologic effects

Alina Stoian ${ }^{1}$, Claude Guérin

${ }^{1}$ Réanimation médicale, Hôpital de la Croix-Rousse, Lyon, France Correspondence: Claude Guérin - claude.guerin@chu-lyon.fr Annals of Intensive Care 2016, 6(Suppl 1):P185

Introduction Automatic tube compensation (ATC) is a feature available in some ICU ventilators to compensate for the resistive work of breathing (WOB) due to endotracheal tube. We performed a systematic review and meta-analysis on the effects of ATC on physiologic respiratory variables and weaning outcome.

Materials and methods We searched for automatic tube compensation in PubMed and Google scholar limiting to English language. Hand search in the retrieved studies was also performed. Studies were included if there were original, performed in ICU patients intubated, designed as randomized between an experimental group with ATC and at least one control group without ATC. Abstracted forms only and reviews were excluded. We separated the studies into two groups: group 1 on weaning outcome and group 2 on respiratory physiologic effects. For the first group, the main endpoint was weaning failure defined as reintubation or death within the $48 \mathrm{~h}$ after scheduled extubation. For the second group, we analyzed respiratory rate, tidal volume, minute ventilation, pressure-time product of the diaphragm and work of breathing. Two reviewers independently screened titles and abstracts and analyzed included studies using a dedicated form. Disagreements were solved by consensus. The quality of studies was assessed from ten items. Data were pooled using random-effect model. Statistical heterogeneity across the studies was assessed by using 12 . Sensitivity analyses were performed for specific levels of pressure support ventilation. Analysis was performed by using R software. Results Search retrieved 65 studies of which 7 were included in group 1 and 9 in group 2. For the group 2 studies, 3 of them investigated 2 specific groups of patients (postoperative and medical patients in one study, high and low ventilation in one study and with and without dead space in one study). We used them as independent studies leading to 12 studies in group 2.

Regarding the quality criteria for group 1 studies, 2 studies met 6 criteria, 4 met 5 criteria, and 1 met 4 criteria. Regarding group 2 studies, 3 studies had 5 quality criteria, 4 had 4 criteria and 2 had 3 criteria.

For group 1 studies, 250 patients were included in the experimental group (ATC + PEEP $5 \mathrm{cmH} 2 \mathrm{O}$ in 6 studies and ATC + pressure support in 1 study) and 227 patients in the control group (pressure support in 4, CPAP in 2 and T-tube in 1). The failure of extubation was not different between experimental and control groups (relative risk 1.02 [0.97$1.08])$. 12 was not statistically significant $\left[I^{2}=0 \%(P=0.43)\right]$.

For group 2 studies, the experimental group consisted of ATC alone in 9 studies, proportional assist ventilation + ATC in two and APRV + ATC in one. The corresponding control groups were pressure support, proportional assist ventilation and APRV without ATC. Furthermore, in the control groups with pressure support ventilation, different levels of pressure support were used. Therefore, we performed two sensitivity analyses. The first analysis was done for $15 \mathrm{cmH}_{2} \mathrm{O}$ pressure support level in 5 studies, $5 \mathrm{cmH}_{2} \mathrm{O}$ in 3 and $7 \mathrm{cmH}_{2} \mathrm{O}$ in 1 . The second analysis was done for $5 \mathrm{cmH}_{2} \mathrm{O}$ pressure support level in 8 studies and $7 \mathrm{cmH}_{2} \mathrm{O}$ in 1 . In both sensitivity analyses, there were no statistical differences between any respiratory variable between experimental and control groups.

Conclusion ATC was not associated with any significant improvement neither in respiratory physiologic variables nor in weaning outcome.

\section{Competing interests None.}

P186

Automated oxygen titration during high-flow oxygen therapy. Evaluation of the feasibility in healthy subjects

François Lellouche ${ }^{1}$, Erwan L'her ${ }^{2}$, Pierre Alexandre Bouchard' ${ }^{1}$, Mathieu Delorme ${ }^{3}$, Tamer Elfaramawy ${ }^{4}$, Benoit Gosselin ${ }^{4}$

${ }^{1}$ Centre de recherche de l'iucpq, Institut Universitaire de Cardiologie et de Pneumologie de Québec, Québec, Canada; ${ }^{2}$ Réanimation médicale, CHRU de Brest, Brest, France; ${ }^{3} 33$, Hôpital Haut-Lévêque, Avenue MagelIan, Pessac, France, Pessac, France; ${ }^{4}$ Département de génie électrique et informatique de l'université laval, Université Laval, Québec, Canada Correspondence: Mathieu Delorme - mathieu.delorme@chu-bordeaux. $\mathrm{fr}$ 


\section{Introduction}

Hyperoxia-induced hypercapnia has been described more than 60 years ago [1], and first recommendations to avoid hyperoxia in COPD patients were provided more than 50 years ago [2]. Despite abundant literature on this issue, it is still overlooked and alarm bell was recently raised [3] after the demonstration that high oxygen flows could increase mortality in patients with respiratory distress even within short exposure such as during pre-hospital transport [4]. We demonstrated that in this setting, high oxygen flows were also utilized in our country [5], in agreement with other reports [6, 7].

\section{Materials and methods}

Objectives We developed a device that continuously adjusts the $\mathrm{FiO}_{2}$ with high flows of air/oxygen based on the FreeO2 system that titrates oxygen flow delivered to patients with the aim to maintain a constant oxygenation [8].

Results The FreeO2 system was modified to allow a mixture of oxygen and air administration with a constant gas flow. The proportion of oxygen is based on an advanced closed loop to maintain a constant $\mathrm{SpO}_{2}$. We compared in healthy subjects this new prototype with Airvo2 set with minimal $\mathrm{FiO}_{2}$ of $30 \%$ (3L/min of oxygen). Both devices were set at $30 \mathrm{~L} / \mathrm{min}$, and we plan to recruit 10 healthy subjects. The experimental conditions were the following: healthy subjects will initially breath with high-flow therapy in normoxic conditions ( $5 \mathrm{~min})$, followed by 5 min of induced hypoxemia (nitrogen administration), and return to initial conditions for $5 \mathrm{~min}$. We record $\mathrm{SpO}_{2}$, respiratory rate, heart rate and $\mathrm{FiO}_{2}$ delivered.

We conducted an evaluation of automated oxygen titration during a longer period of $30 \mathrm{~min}$ with induced hypoxemia.

Discussion Data concerning the first 5 healthy subjects are presented here healthy subjects. Initial data demonstrate the feasibility to deliver variable oxygen flows administered with air at high flows (from 20 to $60 \mathrm{~L} / \mathrm{min}$ ). In this study, the flow was maintained constant at $30 \mathrm{~L} / \mathrm{min}$. During nitrogen administration to the healthy subjects, with the new prototype controlling oxygen/air administration, the oxygen increases (leading to a $\mathrm{FiO}_{2}$ increase) to maintain constant the $\mathrm{SpO}_{2}$ level (set at $96 \%$ in this study), and the air flow decreases to maintain constant the total flow $(30 \mathrm{~L} / \mathrm{min})$. After cessation of nitrogen, the oxygen flow (and $\mathrm{FiO}_{2}$ ) is automatically reduced. The percentage of time in the $\mathrm{SpO}_{2}$ target $(96 \pm 2 \%)$ was significantly more important with automated titration during the induced hypoxemia (50 vs. $24 \%, P<0.05$ ), and the percentage of time with hypoxemia was reduced with automated oxygen titration ( 11 vs. $55 \%, P<0.05)$.

In 4 subjects, the mean time in the target during the 30-min evaluation was $94.4 \%$.

\section{Conclusion}

Based on the results with this specific model, this new device may help to optimize oxygenation to maintain adequate oxygenation and avoid desaturation during high-flow oxygen therapy.

Competing interests Co-fondateur et actionnaire de Oxynov.

\section{References}

1. Davies CE, Mackinnon J. Neurological effects of oxygen in chronic cor pulmonale. Lancet. 1949;2(6585):883-5.

2. Campbell EJ. A method of controlled oxygen administration which reduces the risk of carbon-dioxide retention. Lancet. 1960;2(7140):12-4.

3. Beasley R et al. High-concentration oxygen therapy in COPD. Lancet. 2011;378(9795):969-70.

4. Austin MA et al. Effect of high flow oxygen on mortality in chronic obstructive pulmonary disease patients in prehospital setting: randomised controlled trial. BMJ. 2010;341:C5462.

5. Lellouche $\mathrm{F}$ et al. Pre-hospital oxygen administration in patients with respiratory distress. Why do we give too much oxygen? Am J Respir Crit Care Med $2012 \mathrm{Ab}$

6. Hale KE et al. Audit of oxygen use in emergency ambulances and in a hospital emergency department. Emerg Med J. 2008;25(11):773-6.

7. Wijesinghe $\mathrm{M}$ et al. Pre-hospital oxygen therapy in acute exacerbations of chronic obstructive pulmonary disease. Intern Med J. 2011;41 (8):618-22.

8. Lellouche F, L'her E. Automated oxygen flow titration to maintain constant oxygenation. Respir Care. 2012 Aug;57(8):1254-62.
P187

Accuracy of ventilators for intermediate care to deliver tidal volume. A bench study

Claude Guérin', Loredana Baboi', Sarah Guegan², Fabien Subtil ${ }^{3}$

${ }^{1}$ Réanimation médicale, Hôpital de la Croix-Rousse, Lyon, France; ${ }^{2}$ Stagiaire 5ème année, ProG'HEl, Lille, France; ${ }^{3}$ Biostatistiques, Hospices Civils de Lyon, Lyon, France

Correspondence: Claude Guérin - claude.guerin@chu-lyon.fr Annals of Intensive Care 2016, 6(Suppl 1):P187

Introduction There is an ongoing development of home-care turbinepowered ventilators able to support patients with mild acute respiratory failure in the intermediate care environment at the hospital. The capability for these ventilators to deliver accurate tidal volume (VT) is an important endpoint for patient's safety. In present study, we aimed at comparing on the bench the performance of respirators for intermediate care to deliver VT.

Materials and methods The following ventilators were used: Astral 150, Elisée 150, Trilogy 200, Monnal T50 equipped with double-limb circuit and Evita XL. They were set in volume-controlled mode at constant flow inflation shape and connected to ASL 5000 lung model. Four conditions were set at the ASL.

Three adult conditions pertaining to normal [compliance (C) $60 \mathrm{ml} /$ $\mathrm{cmH} 2 \mathrm{O}$, inspiratory (Ri) and expiratory (Re) resistance $\left.5 \mathrm{cmH}_{2} \mathrm{O} / \mathrm{LS}\right]$, COPD (C 75, Ri 15, Re 25), and ARDS (C 33, Ri 15, Re 25) profiles. Furthermore, a mechanical condition pertaining to children (C10, Ri 50, and $\operatorname{Re} 50$ ) was used.

For each adult lung condition, two targeted VT (300 and $500 \mathrm{ml}$ ) and two PEEP levels ( 5 and $10 \mathrm{cmH}_{2} \mathrm{O}$ ) were tested. For the paediatric lung condition, targeted VT was $50 \mathrm{ml}$

VT was measured with Citrex $\mathrm{H}^{\mathrm{TM}}{ }^{\mathrm{TM}}$ (imtmedical, Switzerland) over the last 10 breaths of the recording at the steady state defined as changes in VT less than $10 \%$ from the previous sampling window. The sampling rate was $200 \mathrm{~Hz}$. Citrex H4 ${ }^{\mathrm{TM}}$ device was calibrated before each experiment and set in ATP condition. Ventilators were set in BTPS conditions. Values of measured VT were expressed in BTPS conditions by using the following correction factor [PA/(PA-Pwater)] $\times(T B / T A)$, in which TA is $273 \mathrm{~K}+$ ambient temperature, $\mathrm{PA}$ the ambient pressure continuously measured by Citrex H4 ${ }^{\mathrm{TM}}$, Pwater $47 \mathrm{mmHg}$ and TB $310 \mathrm{~K}$. Measured VT was multiplied by this correction factor to obtain corrected VT. The values of VT error (measured corrected VT-target VT/target VT*100) were expressed as mean \pm SD. A negative VT error value means that delivered VT was lower than targeted VT while a positive value has opposite significance.

Given the nature of the investigation, i.e. a bench experiment, low within-ventilator variability for the values of VT was anticipated, and hence, we a priori decided not to perform statistical tests if this assumption was verified. Therefore, any observed differences in means for VT were considered as true.

We a priori defined $\mathrm{a} \pm 10 \% \mathrm{VT}$ error as a threshold for accuracy range limit.

Results The VT error is shown in the table below for the different lung conditions across the ventilators and PEEP levels, for set VT $300 \mathrm{ml}$ in lung adult and $50 \mathrm{ml}$ in paediatric lung. Same results were observed for $500 \mathrm{ml}$ set VT. As anticipated, the variability was very low and hence no statistical test performed.

\begin{tabular}{|c|c|c|c|c|c|c|c|c|}
\hline \multirow{2}{*}{$\begin{array}{l}\text { PEEP } \\
\left(\mathrm{cmH}_{2} \mathrm{O}\right)\end{array}$} & \multicolumn{2}{|l|}{ Normal } & \multicolumn{2}{|l|}{ COPD } & \multicolumn{2}{|l|}{ ARDS } & \multicolumn{2}{|l|}{ Children } \\
\hline & 5 & 10 & 5 & 10 & 5 & 10 & 5 & 10 \\
\hline Astral 150 & $6.7 \pm 0$ & $3.3 \pm 0$ & $0-0.5 \pm 0$ & $-3.6 \pm 0$ & $1.9 \pm 0$ & $2.8 \pm 0$ & $0.5 \pm 0$ & $0.6 \pm 0$ \\
\hline Elisée 150 & $1.6 \pm 0$ & $2.4 \pm 0$ & $6.4 \pm 0$ & $4.9 \pm 0$ & $0-2.5 \pm 0$ & $1.0 \pm 0$ & $0-38.0 \pm 0$ & $-38.0 \pm 0$ \\
\hline Trilogy 200 & $02.1 \pm 0$ & $0.5 \pm 0$ & $16.2 \pm 0$ & $13.2 \pm 0$ & $4.7 \pm 0$ & $2.2 \pm 0$ & $0-9.0 \pm 0$ & $-10 \pm 0$ \\
\hline $\begin{array}{c}\text { Monnal } \\
\text { T50 }\end{array}$ & $15.3 \pm 0$ & $13.7 \pm 0$ & $0 \quad 19.6 \pm 0$ & $16.4 \pm 0$ & $11.3 \pm 0$ & $11.8 \pm 0$ & $0-29.0 \pm 0$ & $-7.0 \pm 0$ \\
\hline Evita XL & $-2.5 \pm 0$ & $-1.9 \pm 0$ & $0-3.7 \pm 0$ & $-3.6 \pm 0$ & $0-10.9 \pm 0$ & $-10.5 \pm 0$ & $0-36.0 \pm 0$ & $-37.0 \pm 0$ \\
\hline
\end{tabular}


Discussion Every turbine ventilator was within the $10 \%$ accuracy range to deliver VT except for Monnal that over delivered the targeted VT. VT delivered by ICU ventilator was systematically lower than expected within the $10 \%$ range except for the ARDS condition. In the children lung configuration, Astral 150 was very accurate to deliver VT as low as $50 \mathrm{ml}$.

ConclusionThere were differences in VT delivery across ventilators. Some ventilators were particularly accurate in delivering low VT.

\section{Competing interests None.}

\section{P188}

Efficiency of ventilators for intermediate care to deliver adequate $\mathrm{FIO}_{2}$. A bench study

Claude Guérin ${ }^{1}$, Loredana Baboi ${ }^{1}$, Fabien Subtil ${ }^{2}$, Sarah Guegan ${ }^{3}$

${ }^{1}$ Réanimation médicale, Hôpital de la Croix-Rousse, Lyon, France; ${ }^{2}$ Bio-

statistiques, Hospices Civils de Lyon, Lyon, France; '3 Stagiaire 5ème année, ProG'HEl, Lille, France

Correspondence: Claude Guérin - claude.guerin@chu-lyon.fr Annals of Intensive Care 2016, 6(Suppl 1):P188

Introduction There is an ongoing development of home-care turbinepowered ventilators. These can be used in the intermediate care environment at the hospital. The capability for these home-care ventilators to deliver adequate $\mathrm{FIO}_{2}$ is an important endpoint for patient's safety. Therefore, our goal was to measure $\mathrm{FIO}_{2}$ delivered on the bench by such ventilators when non-vented (double-limb) circuit was used.

Materials and methods The following home-care turbine-powered ventilators, Astral 150, Elisée 150, Trilogy 200 and Monnal T50, equipped with non-vented double-limb circuit or equivalent, were set in volume-controlled mode (tidal volume $500 \mathrm{ml}$, breathing frequency 15 cycles/min, inspiratory time $0.8 \mathrm{~s}$, constant flow inflation shape, positive end expiratory pressure $5 \mathrm{cmH} 2 \mathrm{O}$ ) and connected to ASL 5000 lung model set in a condition mimicking COPD patient (compliance $75 \mathrm{ml} / \mathrm{cmH} 2 \mathrm{O}$, inspiratory and expiratory airways resistance 15 and $25 \mathrm{cmH}_{2} \mathrm{O} / \mathrm{L} / \mathrm{s}$, respectively). Oxygen was supplemented by plugging the low-pressure oxygen port at the rear of ventilator to the wall oxygen supply via a flowmeter. Three oxygen flow rates were delivered: 0,3 and $15 \mathrm{~L} / \mathrm{min}$. $\mathrm{FIO}_{2}$ was measured with Citrex $\mathrm{H} 4^{\mathrm{TM}}$ (imtmedical, Switzerland) and continuously monitored on the screen of the device. Once $\mathrm{FIO}_{2}$ reached a plateau, oxygen administration was maintained for an additional 1 min and $\mathrm{FIO}_{2}$ was measured over the last 10 breaths of the recording at the steady state defined as a change in $\mathrm{FIO}_{2}$ of less than $10 \%$ from the previous sampling window. The sampling rate was $200 \mathrm{~Hz}$. Citrex $\mathrm{H}^{\mathrm{TM}}$ device was calibrated before each experiment. The values of $\mathrm{FIO}_{2}$ were expressed as mean \pm SD. As we anticipated very low variability across the measurement at the steady state, we decided not to perform formal statistical comparisons between the values.

Results The values of $\mathrm{FIO}_{2}$ are shown in the table below. The baseline values of $\mathrm{FIO}_{2}$ were similar across the ventilators. The $\mathrm{FIO}_{2}$ was greater with Astral and Elisé 150 at $15 \mathrm{~L} / \mathrm{min}$.

Mean values of $\mathrm{FIO}_{2}$ between ventilators at different oxygen supply rates

\begin{tabular}{llll}
\hline & $\mathbf{0 ~ \mathbf { L }} \mathbf{m i n}$ & $\mathbf{3 ~ \mathbf { L }} \mathbf{m i n}$ & $\mathbf{1 5} \mathbf{~ \mathbf { m i n }}$ \\
\hline Astral 150 & $22 \pm 0.0$ & $42 \pm 0.0$ & $99 \pm 0.0$ \\
Elisée 150 & $21 \pm 0.0$ & $35 \pm 0.0$ & $94 \pm 0.0$ \\
Monnal T 50 & $21 \pm 0.0$ & $40 \pm 0.0$ & $86 \pm 0.0$ \\
Trilogy 200 & $22 \pm 0.0$ & $30 \pm 0.0$ & $62 \pm 0.0$ \\
\hline
\end{tabular}

Conclusion $\mathrm{FIO}_{2}$ generated by home-care turbine-driven ventilators fed by low-pressure oxygen port was different across ventilators. At the highest oxygen supply rate, the values of $\mathrm{FIO}_{2}$ are close to $100 \%$ with some ventilators.

Competing interests None.
P189

In vitro comparison of inspiratory-synchronized and continuous nebulization modes during noninvasive ventilation: analysis of inhaled and lost doses

Jean-Bernard Michotte ${ }^{1}$, Enrico Staderini ${ }^{2}$, Jonathan Dugernier ${ }^{3}$, Rares Rusu $^{2}$, Jean Roeseler ${ }^{3}$, Giuseppe Liistro ${ }^{4}$, Gregory Reychler ${ }^{4}$

${ }^{1}$ Haute école de santé vaud, University of Applied Sciences and Arts Western Switzerland (HES-SO), Lausanne, Switzerland; ${ }^{2}$ Haute école d'ingénierie et de gestion du canton de vaud, University of Applied Sciences and Arts Western Switzerland, Yverdon-les-Bains, Switzerland; ${ }^{3}$ Sevice des soins intensifs, Cliniques Universitaires Saint-Luc, Bruxelles, Belgium; ${ }^{4}$ Service de pneumologie, Cliniques Universitaires Saint-Luc, Bruxelles, Belgium

Correspondence: Jean-Bernard Michotte - jean-bernard.michotte@ hesav.ch

Annals of Intensive Care 2016, 6(Suppl 1):P189

Introduction A breath-synchronized nebulization option that could potentially improve drug delivery by limiting drug loss during exhalation is currently not available on bi-level ventilators. The aim of this in vitro study was to compare aerosol delivery of amikacin with an experimental vibrating mesh nebulizer coupled to a single-limb circuit bi-level ventilator, using a conventional continuous (Conti-Neb) and an experimental inspiratory-synchronized (Inspi-Neb) nebulization modes.

Materials and methods Using an adult lung bench model of noninvasive ventilation, we tested a vibrating mesh device coupled with a bi-level ventilator in both nebulization modes. Inspi-Neb delivered aerosol during only the whole inspiratory phase, whereas Conti-Neb delivered aerosol continuously. The nebulizer was charged with amikacin solution $(250 \mathrm{mg} / 3 \mathrm{~mL})$ and placed at two different positions: before and after the exhalation port (starting from the lung model). Inhaled, expiratory wasted and circuit lost doses were assessed by residual gravimetric method.

Results Irrespective of the nebulizer position, Inspi-Neb produced higher inhaled dose ( $p<0.01 ;+6.3$ to $+16.8 \%$ of the nominal dose), lower expiratory wasted dose $(p<0.05 ;-2.7$ to $-42.6 \%$ of the nominal dose) and lower total lost dose ( $p<0.05 ;-6.3$ to $20.6 \%$ of the nominal dose) than Conti-Neb.

Conclusion During simulated noninvasive ventilation with a singlelimb circuit bi-level ventilator, the nebulization mode of the vibrating mesh device impacts significantly the aerosol delivery of amikacin. The use of an inspiratory synchronized nebulization mode improves inhaled dose by reducing drug loss of amikacin compared to continuous nebulization mode.

\section{Competing interests None.}

P190

Short-term physiological effects of nasal high flow in patients with respiratory distress. Impact of flow rates on the work of breathing

Mathieu Delorme ${ }^{1}$, Pierre Alexandre Bouchard ${ }^{2}$, Mathieu Simon ${ }^{2}$, François Lellouche ${ }^{2}$

133, Hôpital Haut-Lévêque, Avenue Magellan, Pessac, France, Pessac, France; ${ }^{2}$ Centre de recherche de l'iucpq, Institut Universitaire de Cardiologie et de Pneumologie de Québec, Québec, Canada

Correspondence: Mathieu Delorme - mathieu.delorme@chu-bordeaux. $\mathrm{fr}$

Annals of Intensive Care 2016, 6(Suppl 1):P190

Introduction Nasal high flow (NHF) is increasingly used in the management of acute respiratory failure. Recent clinical data support its use as a first-line therapy in the treatment or prevention of hypoxemic respiratory failures. However, little is known about its impact on respiratory effort, which could explain some of the benefits in terms of comfort and efficiency.

Materials and methods We conducted a randomized controlled crossover study in patients with respiratory distress in order to evaluate the short-term effects of NHF. Patients were included if they met the following criteria: respiratory rate $>20$ breaths/min, associated 
with either hypercapnia $\left(\mathrm{pH}<7.38\right.$ and $\mathrm{PaCO}_{2}>45 \mathrm{mmHg}$ ) or hypoxemia $\left(\mathrm{SpO}_{2}<90 \%\right.$ with oxygen flow $\left.>3 \mathrm{~L} / \mathrm{min}\right)$. Four sessions of $15 \mathrm{~min}$ were conducted under various conditions: a baseline period with conventional oxygen therapy (nasal cannulas or face mask), followed by three periods of NHF set at 20, 40 and $60 \mathrm{~L} / \mathrm{min}$ and delivered in a randomized order. During the whole protocol, $\mathrm{FiO}_{2}$ was continuously adjusted to achieve a target $\mathrm{SpO}_{2}$ of $94 \%$ in hypoxemic and $90 \%$ in hypercapnic patients. Work of breathing (WOB) was computed from esophageal pressure and tidal volume, which was estimated by respiratory inductive plethysmography. Calibration of the flow with a pneumotachograph was conducted at the end of each condition and used to optimize the plethysmography accuracy. We also measured and calculated the esophageal pressure-time product (PTPeso), minute ventilation, capillary blood gases at the end of each tested condition, airway resistance and compliance.

Results Recruitment is still in progress, and we report here the data of a preliminary analysis performed in 8 patients. Both WOB and PTPeso were significantly reduced with the use of NHF as compared to baseline $(5.68 \pm 2.35$ vs. $3.39 \pm 1.72$ vs. $4.22 \pm 1.86$ vs. $3.61 \pm 2.77 \mathrm{~J} / \mathrm{min}$ for WOB; $p=0.04$; and $187.5 \pm 69.1$ vs. $129.9 \pm 67.4$ vs. $151.6 \pm 78.6$ vs. $115.9 \pm 87.0 \mathrm{cmH}_{2} \mathrm{O}$ s/min for PTPeso; $p=0.004$; respectively, for baseline, 20,40 and $60 \mathrm{~L} / \mathrm{min})$. The maximal reduction in WOB was encountered for $20 \mathrm{~L} / \mathrm{min}$ in hypercapnic patients $(n=4)(7.09 \pm 2.12$ at baseline vs. $3.43 \pm 1.74 \mathrm{~J} / \mathrm{min}$ at $20 \mathrm{~L} / \mathrm{min} ; p=0.04$ ) and for 60 $\mathrm{L} / \mathrm{min}$ in hypoxemic patients $(n=4)(4.27 \pm 1.76$ at baseline vs. $1.93 \pm 1.13 \mathrm{~J} / \mathrm{min}$ at $60 \mathrm{~L} / \mathrm{min} ; p=0.04$ ). Minute ventilation tended to decrease in hypercapnic patients between baseline and $20 \mathrm{~L} / \mathrm{min}$ $(5.24 \pm 0.57$ vs. $4.37 \pm 0.33 \mathrm{~L} / \mathrm{min}$ respectively; NS) and in hypoxemic patients between baseline and $60 \mathrm{~L} / \mathrm{min}(5.83 \pm 1.64$ vs. $4.69 \pm 1.0 \mathrm{~L} /$ min; NS). However, $\mathrm{PaCO}_{2}$ was not significantly modified throughout the study periods $(50.2 \pm 12.3$ vs. $49.5 \pm 12.1$ vs. $47.3 \pm 11.7$ vs. $47.4 \pm 13.9 \mathrm{mmHg}$; NS). Mean inspiratory resistance was significantly reduced with the use of NHF from $50.6 \pm 21.0 \mathrm{cmH}_{2} \mathrm{O} / \mathrm{L} / \mathrm{s}$ at baseline to $39.3 \pm 23.2 ; 43.9 \pm 24.0$ and $30.5 \pm 25.7 \mathrm{cmH}_{2} \mathrm{O} / \mathrm{L} / \mathrm{s}$ at 20,40 and 60 $\mathrm{L} / \mathrm{min}$, respectively $(p=0.02)$. Compliance was significantly increased with the use of NHF $(0.036 \pm 0.031$ vs. $0.045 \pm 0.021$ vs. $0.048 \pm 0.036$ vs. $0.084 \pm 0.074 \mathrm{~L} / \mathrm{cmH}_{2} \mathrm{O} ; p=0.01$; respectively).

Discussion To our knowledge, this is the first study to evaluate the work of breathing during NHF in adult patients. This lack of data may be attributable to the technical complexity of evaluating respiratory flow during NHF. Here, we used respiratory inductive plethysmography, which has been previously used by several authors, and has shown to provide an acceptable estimation of tidal volume.

Conclusion This preliminary analysis of the short-term physiological effects of NHF suggests that the use of NHF may significantly reduce respiratory effort in patients exhibiting respiratory distress symptoms. Optimal flow may differ according to the population. These effects may be related to an improvement in respiratory compliance and resistance, but inclusions must be carried on to confirm these results in a larger cohort.

\section{Competing interests None.}

\section{P191}

\section{Design and implementation of an active artificial lung}

Elmaati Essoukaki', Lhoucine Ben Taleb ${ }^{1}$, Mohammed Harmouchi ${ }^{1}$ Mourad Rattal ${ }^{2}$, Ahmed Mouhsen ${ }^{3}$, Jean-Christophe Marie Richard ${ }^{4}, \mathrm{~A}$ Zeddine Mouhsen ${ }^{1}$, Aissam Lyazidi ${ }^{2}$

${ }^{1}$ Laboratoire rayonnement-matière et instrumentation, université hassan 1er, Faculté des Sciences et Techniques, Settat, Morocco; ${ }^{2}$ Institut supérieur des sciences de la santé-laboratoire rayonnement-matière et instrumentation, Universite Hassan 1er, Settat, Morocco; ${ }^{3}$ Laboratoire d'ingénierie, management industriel et innovation, université hassan 1 er, Faculté des Sciences et Techniques, Settat, Morocco; ${ }^{4}$ Emergency and intensive care department, General Hospital of Annecy, Annecy, France, France

Correspondence: Elmaati Essoukaki - e.essoukaki@uhp.ac.ma Annals of Intensive Care 2016, 6(Suppl 1):P191

Introduction Available lung models to test ventilators during spontaneous ventilation present several limitations due to their intrinsic functioning, especially during expiration. This artificial lung model was specifically designed to simulate complete spontaneous inspiratory and expiratory passive activity.

Materials and methods The lung model is composed of variable resistor orifice and adjustable compliance cylindrical lung (balloon). Respiratory effort is provided by the shifting of a piston. These efforts are simulated in real time by a programmed script on graphic interface based on NI LabView 2014 software (National Instruments, Texas). The system balloon/piston is located in a cylindrical vacuum box, which represents the thoracic cage. This vacuum allows the passive return of the balloon during the expiratory phase.

The acquisition and display device were tested at a frequency of $20 \mathrm{cpm}$, an inspiratory pressure of $20 \mathrm{cmH} 2 \mathrm{O}$ and an inspiratory time of a second, using a ventilator (Vivo 40; Breas Medical) on a first version of the prototype.

Results The figure below shows a screenshot of pressure curves, flow and volume recorded and displayed by the sensors and the measurement system of our test lung.

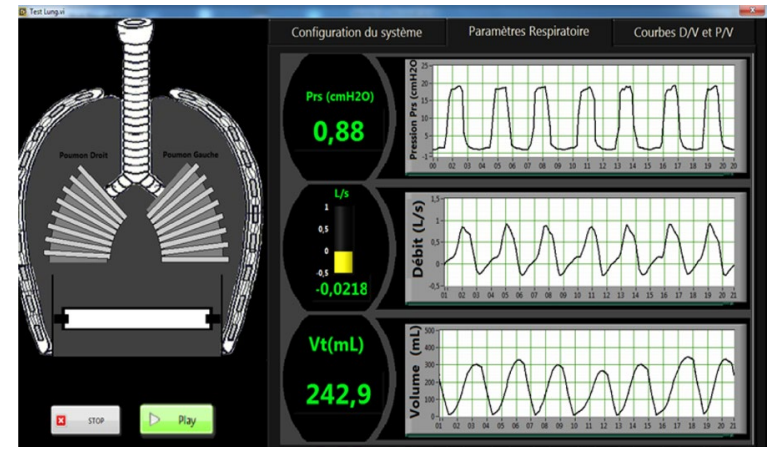

Conclusion This original lung model prototype is the first to our knowledge to combine complete active inspiratory activity and passive expiratory activity. First recordings show the feasibility of the concept and validate the acquisition system. The next step is to validate the intrapleural pressure effect, simulated by vacuum generated between the cylindrical walls of the system and lung. This validation will give the originality of this work by allowing a closer simulation of the natural functioning of the human respiratory system.

\section{Competing interests None.}

\section{P192}

\section{Assessment of the accuracy of ventilator simulation softwares}

Frédéric Duprez ${ }^{1}$, Jean Bernard Michotte ${ }^{2}$, Olivier Contal ${ }^{2}$, Thierry Bonus ${ }^{1}$, Sandra Olieuz', Grégory Cuvelier ${ }^{3}$, Sharam Machayeckhi

${ }^{1}$ ICU, C.H. Epicura, Hornu, Belgium; ${ }^{2}$ Physiotherapy, Haute École de Santé Vaud, Lausanne, Switzerland; ${ }^{3}$ Laboratoire de l'effort et du mouvement, Condorcet, Tournai, Belgium

Correspondence: Frédéric Duprez - dtamedical@hotmail.com

Annals of Intensive Care 2016, 6(Suppl 1):P192

Introduction In intensive care unit, many simulators are used to teach clinicians, nurses and respiratory therapists the principles of mechanical ventilation. Modelling the mechanical properties of the respiratory system allows them to learn settings and monitoring of mechanical ventilators. In volume-controlled mode, the airway pressure (Paw) is an important parameter to monitor for an optimal management of ventilated patients. It can be calculated from the following equation of motion of the inspired gas (EMIG):

$$
\text { Paw }=P 0+(\text { Ers } \times \mathrm{Vt})+(\operatorname{Rrs} \times Q)
$$

P0: start pressure, Ers: elastance of respiratory system, Vt: tidal volume, Rrs: resistance of respiratory system, $Q$ : inspiratory flow. 
The purpose of the study was to compare Paw calculated from the EMIG and Paw measured with 3 different teaching tools: a two-compartment adult lung model and 2 ventilator simulation softwares. Materials and methods Using the EMIG formula, we measured Paw of 50 lung mechanics (range of elastance and resistance, respectively, 11-77 $\mathrm{cmH}_{2} \mathrm{O} / \mathrm{L}$ and $5-31 \mathrm{cmH}_{2} \mathrm{O} / \mathrm{L} \mathrm{s}^{-1}$ ). These values were compared in the same conditions, with Paw measured with a Maquet Servo $i^{\circledR}$ ventilator connected to a two-compartment lung model (Dual Test Lung ${ }^{\circledR}$ Michigan Instrument-TTL 1600) and 2 ventilator simulation softwares [Dräger Evita $4{ }^{\circledR}$ simulator SW 3.99 (DS) and Hamilton simulator version 2.2. (HS)], all set in volume-controlled mode (Vt: $0.5 \mathrm{~L}$; Rf: $20 \mathrm{cpm}$; inspiratory flow: $30 \mathrm{~L} \mathrm{~min}^{-1}$; $1 / \mathrm{E}$ ratio:1/2; pressure trigger sensitivity: $-20 \mathrm{~cm} \mathrm{H} 20$; inspiratory plateau pressure: $25 \%$; inspiratory rise time: $0 \%$; peep: $0 \mathrm{cmH}_{2} \mathrm{O}$ ). Statistical method: Bland-Altman method and multiple linear regression.

\section{Results}

Pressure (Paw) measured by different techniques (EMIG, DTL, HS, DS)

\begin{tabular}{|c|c|c|c|c|c|c|c|}
\hline & $\begin{array}{l}\text { Bias (SD) } \\
(\mathrm{cmH2O})\end{array}$ & $\begin{array}{l}\text { Limits } \\
\text { of agree- } \\
\text { ment } \\
\left(\mathrm{cmH}_{2} \mathrm{O}\right)\end{array}$ & $\begin{array}{l}\text { Bias } \\
-(95 \% \mathrm{Cl}) \\
\left(\mathrm{cmH}_{2} \mathrm{O}\right)\end{array}$ & $\begin{array}{l}\text { Lower } \\
\text { limit } \\
\text { of agree- } \\
\text { ment } \\
(95 \% \mathrm{Cl})\end{array}$ & $\begin{array}{l}\text { Upper } \\
\text { limit } \\
\text { - of agree- } \\
\text { ment } \\
(95 \% \mathrm{Cl})\end{array}$ & $R^{2}$ & $P$ value \\
\hline $\begin{array}{l}\text { EMIG } \\
\text { versus } \\
\text { DTL }\end{array}$ & $1.6(1.1)$ & $\begin{array}{c}-0.7 \text { to } \\
3.8\end{array}$ & 1.2 to 1.9 & $\begin{array}{c}-1.2 \text { to } \\
-0.1\end{array}$ & $3.2-4.4$ & 0.99 & $<0.001$ \\
\hline $\begin{array}{l}\text { EMIG } \\
\text { versus } \\
\text { HS }\end{array}$ & $-0.2(1.9)$ & $\begin{array}{c}-3.8 \text { to } \\
3.5\end{array}$ & $\begin{array}{c}-0.7 \text { to } \\
0.3\end{array}$ & $\begin{array}{c}-4.8 \text { to } \\
-2.9\end{array}$ & $2.5-4.4$ & 0.98 & $<0.001$ \\
\hline $\begin{array}{l}\text { EMIG } \\
\text { versus } \\
\text { DS }\end{array}$ & $-1.0(2.1)$ & $\begin{array}{c}-5.1 \text { to } \\
3.1\end{array}$ & $\begin{array}{c}-1.6 \text { to } \\
-0.4\end{array}$ & $\begin{array}{c}-6.2 \text { to } \\
-4.1\end{array}$ & $2.1-4.2$ & 0.97 & $<0.001$ \\
\hline $\begin{array}{l}\text { HS versus } \\
\text { DS }\end{array}$ & $-0.8(1)$ & $\begin{array}{c}-2.7 \text { to } \\
1.1\end{array}$ & $\begin{array}{c}-1.1 \text { to } \\
-0.5\end{array}$ & $\begin{array}{c}-3.2 \text { to } \\
-2.2\end{array}$ & $0.6-1.6$ & 0.99 & $<0.001$ \\
\hline
\end{tabular}

$E M I G$ equation of motion of inspired gas, DTL Dual Test Lung ${ }^{\circledR}, H S$ hamilton simulator, $D S$ Dragër simulator

Conclusion Responses to several respiratory system characteristics showed that the teaching tools react appropriately. The bias between EMIG and different tools was small with very narrow limits of agreement.

We conclude that pressure values obtain from EMIG are very close of values obtain from a two-compartment adult lung model connected to a volume ventilator, HS and DS. These methods are relevant and realistic for teaching principles of mechanical ventilation.

\section{Competing interests None.}

\section{Reference}

1. Yan Shi. Pressure Dynamic Characteristics of Pressure Controlled Ventilation System of a Lung Simulator.Comput and Mathematical Methods in Medicine. Vol 2014 (2014)

\section{P193}

\section{Outcome and early prognostic factors in neutropenic patients} admitted to the intensive care unit

Becem Trabelsi', Zied Hajjej', Walid Sammoud', Hedi Garsallah', Mustapha Ferjani

${ }^{1}$ Department of Critical Care Medicine and Anesthesiology, Military Hospital of Tunis, Tunisia, Tunis, Tunisia

Correspondence: Zied Hajjej - hajjej_zied@hotmail.com

Annals of Intensive Care 2016, 6(Suppl 1):P193

Introduction Admission to the intensive care unit (ICU) carries a very high risk of mortality for neutropenic patients [1]. That is why early prognostic indicators should be identified to discriminate patients who are more or less likely to benefit from advanced ICU support. The aim of our study was to identify outcome and early prognostic factors in critically ill neutropenic patients.
Patients and methods It was a retrospective review of the medical records of 42 neutropenic patients admitted to a medical surgical intensive care unit (ICU) over an 8-year period. Neutropenia was defined as an absolute neutrophil count of less than $1.5 \times 10^{9} / \mathrm{l}$.

Results Of the 42 patients included in the study, 22 (52\%) were men and 20 (48\%) women. The mean age was $51 \pm 19$ years.

Medical histories consisted of hematologic malignancy $(n=18)$, solid tumors $(n=9)$, chronic disease under immunosuppressive therapy $(n=9)$ and toxic bone marrow aplasia $(n=6)$. Almost half of patients with hematologic malignancies were in remission.

Reasons for ICU admission were septic shock $(n=27)$, severe sepsis $(n=5)$, severe acute pancreatitis $(n=3)$, metabolic disorders $(n=2)$, hemorrhagic shock $(n=2)$, status epilepticus $(n=2)$ and high-risk pulmonary embolism $(n=1)$.

APACHE II, SAPS II and SOFA scores at admission were $21 \pm 6.4,38 \pm 16$ and $9 \pm 4.6$, respectively.

Mortality in our ICU was $67 \%$ (28 deaths). At admission, only three variables were independently associated with outcome: pancytopenia $(p=0.03)$, high SOFA score $(p=0.04)$ and lactates $\geq 3 \mathrm{mmol} \mathrm{L}^{-1}$ $(p=0.01)$. These factors were associated with an increased risk of death. Conclusion In our study, we identified three independent early predictors of outcome that may be of value in deciding in which neutropenic patients, when admitted to ICU, advanced life support must be questioned.

\section{Competing interests None.}

Reference

1. Clinical Infectious Diseases 2003; 36:149-58.

\section{P194}

Blood loss and transfusion practice in medical surgical intensive care unit: 1-year study

Walid Sammoud ${ }^{1}$, Zied Hajjej', Khaoula Harira' ${ }^{1}$, Hedi Garsallah' ${ }^{1}$, Mustapha Ferjani'

${ }^{1}$ Department of Critical Care Medicine and Anesthesiology, Military

Hospital of Tunis, Tunisia, Tunis, Tunisia

Correspondence: Zied Hajjej - hajjej_zied@hotmail.com

Annals of Intensive Care 2016, 6(Suppl 1):P194

Introduction Anemia is very common in critically ill patients. There are numerous possible causes for the anemia of critical illness, including chronic anemia, overt and occult blood loss. As a consequence of this anemia, critically ill patients receive a large number of RBC transfusions. In this work, we study the contribution of phlebotomy to anemia and need for RBC transfusion in critically ill patients.

Patients and methods A retrospective observational study was conducted in a medical surgical intensive care unit at Tunis Military Hospital during a period of 1 year extending from September 1, 2012, to December 31, 2012. Inclusion criterion was an ICU stay more than $48 \mathrm{~h}$. Exclusion criteria included: patients with obvious causes of hemorrhage on admission or during stay in the hospital and patients with history which can lead to hemoglobin rate below the transfusion threshold (chemotherapy, Sickle cell disease, hemorrhage, etc.). Patients included were subdivided, according to baseline hemoglobin, into two groups: one for transfusion study (patients with admission hemoglobin $\geq 10 \mathrm{~g} / \mathrm{dl}$ for cardiovascular history, and $\geq 7 \mathrm{~g} / \mathrm{dl}$ for the others), second group for anemia study including patients with baseline hemoglobin concentration $\geq 12 \mathrm{~g} / \mathrm{dl}$. Main data collected included the following: demographics, frequency of blood sample and associated volume of blood drawn collected over $24 \mathrm{~h}$, transfusion (rate, threshold pre-transfusion hemoglobin), hemoglobin variation and biological indicators: CRP, creatinine.

Results Over 382 consecutive patients admitted to ICU, 155 were included. The mean ICU length of stay was 8 days. Admission Acute Physiology and Chronic Health Evaluation II (APACHE II) score was $17 \pm 5$. The mean baseline hemoglobin level was $11.6 \pm 2.4 \mathrm{~g} / \mathrm{dl}$. In total, $25.2 \%$ of patients had a martial treatment with $9 \pm 1.1 \mathrm{~g} / \mathrm{dl}$ as hemoglobin indication value. The mean blood loss via phlebotomy was $160 \pm 150 \mathrm{ml}$ overall hospital stay and $21 \pm 9 \mathrm{ml}$ daily per patient. Twenty-six percentage of blood draw were for biological analysis. 
Overall, $24.5 \%$ of patients received $6 \pm 4$ units. The mean pre-transfusion hemoglobin was $7.8 \pm 1.3 \mathrm{~g} / \mathrm{dl}$. The number of RBC transfusion was independently associated with: low baseline hemoglobin, length of stay more than 9 days, blood loss more than $110 \mathrm{ml}$, septic shock at admission and the need for dialysis. Anemia was independently associated with low baseline hemoglobin, length of stay more than 9 days and blood loss more than $75 \mathrm{ml}$.

Conclusion Phlebotomy was a significant modifiable predictor of anemia and RBC transfusion and therefore constitutes a target for action to improve the quality of care for patients in intensive care unit.

\section{Competing interests None.}

\section{P195}

Atypical hemolytic and uremic syndrome in critically ill adult patients

Elie Azoulay' ${ }^{1}$, Akli Chermak' ${ }^{1}$ Cédric Rafat ${ }^{2}$, Claire Pichereau', Emmanuel Canet $^{1}$, Virginie Lemiale ${ }^{1}$, Valade Sandrine ${ }^{1}$, Marion Venot ${ }^{1}$, Benoît

Schlemmer', Eric Mariotte ${ }^{1}$

${ }^{1}$ Réanimation médicale, Hôpital Saint-Louis, Paris, France; ${ }^{2}$ Service de réanimation médico-chirurgicale, Hôpital Louis-Mourier - APHP, Colombes, France

Correspondence: Eric Mariotte - eric.mariotte@aphp.fr

Annals of Intensive Care 2016, 6(Suppl 1):P195

Introduction The intensive care unit (ICU) is the place of choice for initial management of patients with thrombotic microangiopathies (TMAs). Thrombotic thrombocytopenic purpura, which is characterized by undetectable ADAMTS13 activity, is now well recognized by critical care specialists. However, atypical hemolytic and uremic syndrome (aHUS), another rare life-threatening TMA caused by uncontrolled activation of the complement system, remains a diagnostic challenge. The disease is mostly due to mutations in the complement regulatory proteins (factor $\mathrm{H}$, factor $\mathrm{I}$, or membrane cofactor protein $[\mathrm{MCP}])$, or occasionally due to acquired neutralizing autoantibodies inhibitors of these complement system components (i.e., anti-factor $\mathrm{H}$ antibodies). As recent advances in aHUS therapy have been achieved, additional descriptive data are more than ever required to overcome diagnostic issues. As ICU data are scarce, we sought to describe a cohort of critically ill aHUS adult patients.

Patients and methods Single-center, retrospective study of aHUS patients managed between 2005 and 2015. Patients' clinical and biological data, management and outcomes are reported.

Results Among the 34 patients with HUS managed in our ICU during the study period, 5 presented with typical HUS (Shiga toxin related) and 29 with aHUS. There were 14 men and 15 women, aged 47 (21-66) years. All met diagnostic criteria for TMA, including hemolytic anemia (median hemoglobin rate $9 \mathrm{~g} / \mathrm{dl}, \mathrm{IQR}$ [5.5-9.9]), presence of schistocytes and negative Coombs test, increased LDH levels (median 1627U/l, IQR [750-4738]), undetectable haptoglobin (80\%), thrombocytopenia (median 44G/L, IQR [20-145]), and elevated free bilirubin (60\%). Arterial blood pressure was $153 \mathrm{mmHg}$ [125-205]/90 mmHg [80-122]. Five patients had fever. At ICU admission, 13 (45\%) patients presented with neurologic symptoms (9 confusion, 8 headaches, 6 seizures, 5 focal defects, 4 coma and 2 blurred vision). One patient had posterior reversible encephalopathy syndrome, one had cerebellar syndrome and one had ischemic stroke. Ten patients (35\%) had digestive symptoms, including diarrhea in 8 , and abdominal pain in 5 . Five patients had aHUS-related cardiac involvement, all with negative T waves on ECG, 3 with elevated troponin levels and one with chest pain.

All patients presented with acute kidney injury, with median urea at $19 \mathrm{mmol} / \mathrm{I}$ (IQR [9-45]) and median creatinine at $273 \mu \mathrm{mol} / \mathrm{I}$ (IQR [143859]). Eleven (38\%) patients had significant proteinuria and 13 (45\%) hematuria. All patients had detectable ADAMTS13 activity. Complement data were available in 19 patients, including 7 patients with low C3 activity, and 4 with decreased CD46 rates. Four patients had documented complement factor $\mathrm{H}$ mutation (heterozygous) and $1 \mathrm{MCP}$ mutation. No case of detectable E. coli-related Shiga toxin was found. Treatment included dialysis for 13 patients ( 6 at admission), mechanical ventilation for 9, and vasopressors for 2. Only 15 patients underwent plasma exchange therapy. All patients but two needed 2 (1-4) antihypertensive drugs. Last, 6 recent patients received humanized monoclonal anti-C5 antibody (eculizumab). Ten (34\%) patients died before hospital discharge, and ten other patients were dialysis free. Half the patients who did not receive plasma exchange died.

Conclusion Patients with aHUS are far from presenting an isolated kidney disease. Multiple organ involvement, chiefly cardiac and neurologic, occurs in up to half the patients. Plasma exchanges were not implemented in half of the cases. Large studies are needed to correlate severity at ICU admission and mortality. Also, as complement protein C5 blockade seems to greatly improve the prognosis of aHUS patients, the impact of ICU management on outcomes (mortality and chronic kidney disease) needs to be elucidated.

Competing interests None.

\section{P196}

Outcome of critically ill patients after allogeneic hematopoietic stem cell transplantation: comparison of cord blood cells with others cells as hematopoietic stem cells source

Caroline Le Jeune ${ }^{1}$, Florent Wallet ${ }^{2}$, Pascal Roy ${ }^{3}$, Laurent Argaud ${ }^{4}$, Mauricette Michallet

${ }^{1}$ Hematology, CHU Lyon Sud, Lyon, France; ${ }^{2}$ Critical care unit, CHU Lyon Sud, Lyon, France; ${ }^{3}$ Biostatistics, Hospices Civils de Lyon, Lyon, France;

${ }^{4}$ Réanimation Médicale, Hospices Civils de Lyon - Groupement Hospitalier Edouard Herriot, Lyon, France

Correspondence: Caroline Le Jeune - caroline-lejeune@wanadoo.fr

Annals of Intensive Care 2016, 6(Suppl 1):P196

Introduction The lack of donors for allogeneic hematopoietic stem cell transplantation (HSCT) has led to use unrelated umbilical cord blood (UCB) units as an alternative graft. Despite improvement in management of critically ill allogeneic HSCT recipients, survival rate remains low. Extended aplasia, poor and delayed immune reconstitution and higher rates of severe infection after UCB transplantation compared to the evolution after allogeneic bone marrow (BM) or peripheral blood stem cells (PBSC) HSCT may lead to consider UCB transplantation recipients as poor candidates for intensive care unit admission. We compared the short- and long-term outcome of allogeneic recipients requiring admission to the intensive care unit according to the source of hematopoietic stem cells.

Patients and methods Retrospective chart review of allogeneic HSCT patients admitted to intensive care unit from 2008 to 2013. The data were retrieved from the databases of the adult HSCT unit and the intensive care unit. The short-term outcome was assessed through the intensive care unit and in-hospital mortality rates. Overall survival was the retained long-term outcome criteria and was calculated from intensive care unit admission to death from any cause. Observations of patients still alive on January 1,2015, were censored at this date.

Results Of the 103 patients, 23 received UCB transplantation. Intensive care unit admission rate was higher for UCB recipients $(33 \%)$ than for BM and PBSC recipients $(22 \%)(p=0.048)$. The most common reasons for intensive care unit admission were acute respiratory failure regardless of the hematopoietic stem cells source. Intensive care unit mortality was $65 \%(15 / 23)$ for UCB transplantation and $62.5 \%(50 / 80)$ for BM and PBSC transplantation $(p=0.812)$. In-hospital mortality was $74 \%$ in UCB recipients and $82.5 \%$ in BM and PBSC recipients (odds ratio: $0.601,95 \%$ confidence interval $0.201-1.796, p=0.371$ ). In multivariate analysis, performance status $>2(p<0.001)$ and mechanical ventilation $(p=0.039)$ were associated with worse in-hospital mortality. The 100day survival was $26.1 \%$ for UCB transplantation and $25 \%$ for BM and PBSC transplantation. The 1-year survival was $26.1 \%$ in UCB recipients and $11.2 \%$ in BM and PBSC recipients. While overall survival median was 21 days in UCB recipients and 19 days in BM and PBSC recipients, survival distribution was not significantly different according to the source of hematopoietic stem cells (log-rank test $p$ value $=0.232$ ). Poor performance status remained correlated with lower overall survival $(p=0.003)$ in multivariate analysis. After adjustment on variables independently associated with outcome, the in-hospital mortality remained similar between UCB and BM and PBSC recipients (odds ratio: 0.647, $95 \%$ 
confidence interval $0.175-2.394, p=0.517$ ) as well as the overall survival (hazard ratio: $0.828,95 \%$ confidence interval $0.491-1.396, p=0.470$ ).

Discussion The source of hematopoietic stem cell was not a predictor of short- or long-term outcome. Survival of allogeneic HSCT recipients after intensive care unit admission remained low, and we have not pointed out any significant transplant-related factors. Collaboration between intensive care specialists and hematologists is undoubtedly the key to select patients who may benefit from intensive management, considering the underlying disease, its therapeutical strategy and prognosis.

Conclusion The identification of the important role of the performance status on the evolution of critically ill allogeneic HSCT recipients may help to clarify the decision-making process of intensive care unit transfer after allogeneic HSCT and to implement intensive care unit admission policies. Therefore, substantial intensive care unit survival may be achieved in these selected patients regardless the source of graft.

\section{Competing interests None.}

\section{References}

1. Azoulay E, Mokart D, Pène F, et al. (2013) Outcomes of critically ill patients with hematologic malignancies: prospective multicenter data from France and Belgium - a groupe de recherche respiratoire en réanimation onco-hématologique study. J Clin Oncol Off J Am Soc Clin Oncol 31:2810-2818. doi: 10.1200/JCO.2012.47.2365.

2. Pène F, Aubron C, Azoulay E, et al. (2006) Outcome of critically ill allogeneic hematopoietic stem-cell transplantation recipients: a reappraisal of indications for organ failure supports. J Clin Oncol Off J Am Soc Clin Oncol 24:643-649. doi:10.1200/JCO.2005.03.9073.

\section{P197}

Outcome of patients with organ failures related to solid malignancies and receiving chemotherapy in the intensive care unit

Yoann Zerbib ${ }^{1}$, Julien Maizel ${ }^{2}$, Naïke Bigé ${ }^{3}$, Benoit Misset ${ }^{4}$, Nicolas de Prost ${ }^{5}$, Virginie Lemiale ${ }^{6}$, Sylvie Ricome ${ }^{7}$, François Baudin ${ }^{8}$, Muriel Fartoukh ${ }^{9}$, Frédéric Jacobs ${ }^{10}$, François Blot ${ }^{11}$, Matthieu Schmidt ${ }^{12}$, Antoine Rabbat $^{13}$, Julien Mayaux ${ }^{12}$, Frédéric Gonzalez ${ }^{14}$, Caroline Bornstain ${ }^{15}$, Elie Azoulay $^{16}$, Frédéric Pène ${ }^{1}$

${ }^{1}$ Réanimation Médicale, Hôpital Cochin, Paris, France; ${ }^{2}$ Réanimation médicale, Centre Hospitalier Universitaire, Amiens, France; ${ }^{3}$ Réanimation médicale, Hôpital Saint-Antoine, Paris, France; ${ }^{4}$ Réanimation polyvalente, Groupe Hospitalier Paris-Saint-Joseph, Paris, France; ${ }^{5}$ Réanimation Médicale, Hôpital Henri Mondor, Créteil, France; ${ }^{6}$ Medical ICU, Hôpital Saint Louis, Paris, France; ${ }^{7}$ Service de réanimation polyvalente, Centre hospitalier intercommunal Robert Ballanger, Aulnay-sous-Bois, France; ${ }^{8}$ Réanimation chirurgicale, Cochin Port-Royal, Paris, France; ${ }^{9}$ Réanimation médico-chirurgicale, Hôpital Tenon, Paris, France; ${ }^{10}$ Réanimation polyvalente, Hôpital Antoine Béclère, Clamart, France; ${ }^{11}$ Réanimation médicale, Institut Gustave Roussy, Villejuif, France; ${ }^{12}$ Service de pneumologie et réanimation médicale, Groupe Hospitalier Pitié-Salpêtrière, Paris, France; ${ }^{13}$ Réanimation pneumologique, Hôpital Cochin, Paris, France; ${ }^{14}$ Réanimation medico-chirurgicale, hopital avicenne, Bobigny, France; ${ }^{15}$ Réanimation polyvalente, Groupe Hospitalier Intercommunal Le Raincy-Montfermeil, Montfermeil, France; ${ }^{16}$ Réanimation médicale, Hôpital Saint-Louis, Paris, France

Correspondence: Yoann Zerbib - yoanz@hotmail.com Annals of Intensive Care 2016, 6(Suppl 1):P197

Introduction Both hematological and solid malignancies may be directly responsible for life-threatening organ failures including obstruction of anatomical structures, tissue infiltration, tumor lysis syndrome, or coagulation disorders. Besides advanced life support and eventual instrumental interventions, the treatment of cancer-related organ failures relies on timely administration of chemotherapy. Published data about requirements of chemotherapy in the ICU are mostly related to patients with hematological malignancies, while reports of patients with solid tumors are scarce. In this study, we addressed the features and outcomes of patients with organ failures directly related to solid tumors.

Patients and methods We performed a retrospective multicenter study within the GrrrOH research network. All adult patients who were admitted to the ICU with organ failures related to solid malignancies and treated with chemotherapy between 2000 and 2015 were enrolled into the study. Patients were retrieved through the information systems from hospital pharmacy units involved in the delivery of cytostatic drugs. Data were collected from individual files and included the overall severity through the SOFA score computed at the time of ICU admission, the type and mechanism of organ failures, and the modalities of chemotherapy administration. Endpoints were the in-ICU and in-hospital vital status.

Results We identified 48 patients [ 26 males/22 females; median age 59 years (range 21-79)] from 5 centers. The median SOFA score at the time of admission was 4 (range $0-10$ ). The causes of admission were acute respiratory failure $(n=37,77 \%)$, thrombotic microangiopathy $(n=3,6 \%)$, septic shock $(n=2,4 \%)$, coma $(n=2,4 \%)$, hypercalcemia $(n=1,2 \%)$, cardiac arrest $(n=1,2 \%)$, acute kidney injury $(n=1,2 \%)$, and acute liver failure $(n=1,2 \%)$. Primary tumors were lung cancer in 28 patients (58\%), including 21 (44\%) with small cell lung cancer, breast cancer in $3(6 \%)$, digestive tract cancer $5(10 \%)$, and others in $7(14 \%)$. The underlying disease was metastatic in 30 patients (62\%), and the primary site remained unknown in $5(10 \%)$ patients. The mechanisms of cancer-related organ failures were tissue infiltration in 25 patients (52\%), compression of anatomical structures in 21 patients (44\%) and paraneoplastic syndrome in $2(4 \%)$. Invasive mechanical ventilation was required in 32 patients $(66 \%)$, vasopressor therapy in $14(29 \%)$, renal replacement therapy in $4(8 \%)$ and venovenous extracorporeal membrane oxygenation in $2(4 \%)$. Chemotherapy was complicated by tumor lysis syndrome in 7 patients (14\%). ICU-acquired infections occurred in 18 patients (37\%), of whom $12(25 \%)$ developed septic shock. End-oflife decisions were taken in 26 patients (54\%). The in-ICU and in-hospital survival rates were 50 and $13 \%$, respectively.

Discussion This abstract reports on a limited but preliminary cohort of patients. The collection of cases is ongoing in a number of additional centers. The results from the whole cohort will be presented at the congress.

Conclusion Acute respiratory failure related to lung cancer represents the main indication for prompt administration of chemotherapy in the ICU. Despite most patients presented with single organ failures and low severity scores, this condition is associated with a dismal prognosis.

\section{Competing interests None.}

\section{References}

Azoulay E, Thiéry G, Chevret S, Moreau D, Darmon M, Bergeron A, et al. The Prognosis of Acute Respiratory Failure in Critically III Cancer Patients: Medicine. 2004 Nov;83(6):360-70.

Taccone FS, Artigas AA, Sprung CL, Moreno R, Sakr Y, Vincent J-L. Characteristics and outcomes of cancer patients in European ICUs. Crit Care. 2009;13(1):R15.

\section{P198}

Characteristics and outcomes of patients with hematological malignancies (HMs) admitted to the intensive care unit Stéphanie Gelinotte', Dorothée Carpentier², Steven Grange ${ }^{2}$, Julien Abily², Marion Beuzelin², Emmanuel Besnier², Gioia Gastaldi', Gaetan Beduneau', Jean-Philippe Rigaud', Christophe Girault2 ${ }^{2}$,Fabienne Tamion ${ }^{2}$ ${ }^{1}$ Reanimation polyvalente, Centre Hospitalier de Dieppe, Dieppe, France; ${ }^{2}$ Réanimation médicale, Centre Hospitalier Universitaire Rouen, Rouen, France

Correspondence: Fabienne Tamion - fabienne.tamion@chu-rouen.fr Annals of Intensive Care 2016, 6(Suppl 1):P198

Introduction Despite an improvement in prognosis of patients with hematologic malignancy in recent years, the prognosis for patients 
with hematological malignancies (HMs) admitted to the intensive care unit (ICU) remains poor. However, it is difficult to predict the outcome of these patients at their admission. The objective of this study was to evaluate the clinical characteristics and hospital outcomes of critically ill patients with HMs admitted to ICU to identify risk factors for mortality.

Patients and methods We conducted a retrospective, observational cohort study, over 2010-2014, in a medical ICU of the University Hospital of Rouen. We analyzed 134 patients with HMs admitted to ICU. Data included: demographic characteristics, hematologic diagnosis, reasons for ICU admission, transplant status, the presence of neutropenia, number of organ failures and level of organ support.

Results The mean age was $57 \pm 14.5$ years, and the sex ratio was 1.48 . The hematologic disease represented was the LAM (37\%), non-Hodgkin lymphoma (31\%) and LAL (10 \%). Ten percentage had undergone an autologous stem cell and an allograft $6 \%$. At the time of ICU admission, sixty-one patients ( $46 \%$ ) were at the stage of diagnosis and/or initial treatment of their disease, $32(24 \%)$ in complete remission, $11(8 \%)$ in partial remission and $30(22 \%)$ had relapsed. The main indication for admission was: acute respiratory failure in 65 cases (49\%), septic shock in 30 patients $(22 \%)$, severe sepsis in 15 patients $(11 \%)$ and acute renal failure for 13 patients $(10 \%)$. Sixty-seven patients $(50 \%)$ were neutropenic at admission, 87 (65\%) during the ICU stay. The average SAPS II score on admission was $60 \pm 21$, the SOFA score was $10 \pm 4$ at day 1 and $9.5 \pm 5$ at day 3 . Sixty-seven patients were intubated, and the average duration of ventilation was $9.5 \pm 11.7$ days, 51 (38\%) underwent non-invasive ventilation, as exclusive treatment of respiratory failure for 19 patients ( $14 \%), 44$ patients $(33 \%)$ required dialysis, 84 patients (63\%) received vasopressors. The average length of ICU stay was $10.3 \pm 13$ days, the average length of stay in hospital $20.6 \pm 17.2$ days. Mortality in the ICU was $37 \%$, in-hospital mortality was $52 \%$, mortalities at day 28, day 90, 6 months and 1 year were, respectively, 43, 56, 64 and $71 \%$. Use of invasive mechanical ventilation, renal replacement therapy and vasopressors were associated with higher ICU mortality $(69,64$ and $55 \%$ respectively, $p<0.001)$. Patients with SOFA score increased at least 2 points over the third day had a mortality rate significantly higher $(68 \%, p<0.001)$ while those with SOFA score decreased at least 2 points had lower mortality $(10 \%, p<0.001)$.

Conclusion The prognostic factors of in-hospital death were the need for invasive mechanical ventilation, the need for vasopressors and the need for renal replacement therapy in patients with hematologic malignancy admitted to ICU. The trend of organ failures in the first days of ownership also determines the outcome of these patients in ICU.

\section{Competing interests None.}

\section{P199}

Influence of neutropenia on mortality of critically ill cancer patients: Results of a systematic review and meta-analysis of available evidences

Marie Bouteloup 1 , Sophie Perinel Ragey ${ }^{2}$, Elie Azoulay ${ }^{3}$, Djamel Mokart ${ }^{4}$, Michaël Darmon ${ }^{5}$, Groupe de Recherche en Réanimation Respiratoire et Onco-Hematologique (GRRROH)

${ }^{1}$ Réanimation médicale, Hospital Center Valence, Valence, France;

${ }^{2}$ Réanimation G, CHU Hôpital Nord, Saint-Priest-en-Jarez, France;

${ }^{3}$ Réanimation médicale, Hôpital Saint-Louis, Paris, France; ${ }^{4}$ Réanimation, Institut Paoli-Calmettes, Marseille, France; ${ }^{5}$ Réanimation Médicale, CHU Saint-Etienne - Hôpital Nord, Saint-Étienne, France

Correspondence: Michaël Darmon - michael.darmon@chu-st-etienne.fr Annals of Intensive Care 2016, 6(Suppl 1):P199

Introduction Prognostic impact of neutropenia in critically ill cancer patients (CICP) remains controversial. Hence, although neutropenia is an independent risk factor for poor outcome in the general ICU population with severe sepsis or septic shock, several studies in critically ill cancer patients failed to demonstrate impact of neutropenia on outcome [1, 2]. This lack of statistical association might, however, reflect a lack of statistical power.

The aim of this study was to assess influence of neutropenia on mortality of critically ill cancer patients. Secondary objectives were to assess influence of neutropenia on mortality of critically ill patients while taking into account underlying malignancy or changes related to period of admission.

Materials and methods This systematic review and meta-analysis was performed according to the PRISMA statements. Public domain databases including PubMed and the Cochrane database were searched by using predefined keywords. The research was restricted to articles published in English and studies focusing on critically ill adult patients from May 2005 to May 2015.

Results were analyzed using Review Manager 5.1 software. Overall, mortality is reported as median (IQR). Publication bias was assessed by visually inspecting the funnel plot. Then, summary estimates of risk difference were then calculated using the random-effect model.

Results Our initial search yielded 1528 citations, of which 744 were excluded as irrelevant for the scope of this review or being duplicate. Among the 784 remaining records, abstracts and full-text articles were scrutinized for further evaluation. Finally, 38 studies with a total of 6054 patients (including 2097 neutropenic patients) fulfilled our eligibility criteria and were included.

Overall, mortality was of $54.1 \%$ (3275/6054; $95 \%$ Cl 52.8-55.4). Mortality of neutropenic and non-neutropenic patients was of, respectively, $62.8 \%$ (1316/2097; $95 \%$ Cl 60.7-64.8) and $49.5 \%$ (1959/3957; $95 \% \mathrm{Cl} 47.9-51.0)$. Funnel plot failed to identify publication bias.

Neutropenia was associated with an increased risk of mortality of $11 \%$ $\left(9-14 \% ; P 0.0002 ; I^{2}=50 \%\right)$.

When analyzed separately, underlying malignancy did not modify the influence of neutropenia on outcome (respective risk difference of mortality in neutropenic patients of $11 \%$ [95 \% Cl -4-27], $8 \%$ [95 \% Cl 0-15] and $12 \%$ [95 \% Cl 6-17] in patients with solid tumours $(n=428)$, hematological malignancy $(n=1354)$ or both $(n=4272)$, respectively).

Mortality in studies with inclusion period before 2005 was higher in the overall population and in non-neutropenic patients (Fig. 33).

The overall impact of neutropenia on outcome was, however, unchanged by the inclusion period (respective risk difference of mortality in neutropenic patients of $10 \%$ [95 \% Cl 4-15] before 2005 vs. $10 \%$ [95 \% Cl 3-17]).

Conclusion This systematic review suggests a meaningful survival in neutropenic critically ill cancer patients despite an $11 \%(95 \% \mathrm{Cl}$ 9-14) raw increase in mortality compared to non-neutropenic critically ill cancer patients.

Neither underlying malignancy nor period of admission was associated with the prognostic impact of neutropenia. The meaningful survival in neutropenic critically ill patients strongly suggests that ICU admission denial based upon neutropenia should be discouraged.

\section{Competing interests None.}

\section{References}

1. Azoulay E et al. Outcomes of critically ill patients with hematologic malignancies: prospective multicenter data from France and Belgium. J Clin Oncol 2013.

2. Mokart $D$ et al. Prognosis of neutropenic patients admitted to the intensive care unit. Intensive Care Med 2015.

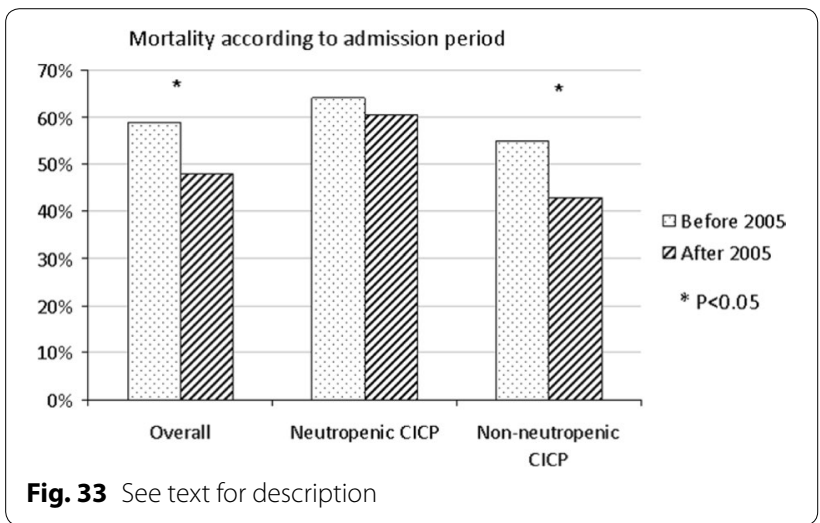


Table 37 Thrombotic microangiopathy characteristics

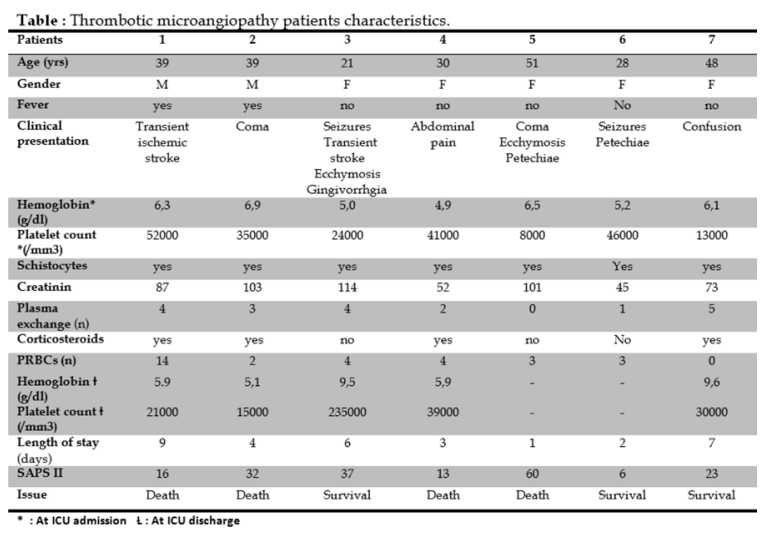

P200

Thrombotic Microangiopathy (TMA) Syndromes in the ICU: experience of a Tunisian ICU

Jihene Ayachi ${ }^{1}$, Ahmed Khedher ${ }^{2}$, Kaoula Meddeb ${ }^{1}$, A. Azouzi ${ }^{1}, Y$

Hamdaoui ${ }^{1}$, H. Nouira ${ }^{1}$, Rania Bouneb ${ }^{3}$, Mohamed Boussarsar ${ }^{1}$

${ }^{1}$ Réanimation médicale, CHU Farhat Hached, Sousse, Tunisia; ${ }^{2}$ Pneumologie, CHU Farhat Hached, Sousse, Tunisia; ${ }^{3}$ Réanimation médicale sousse, chu, Sousse, Tunisia

Correspondence: Mohamed Boussarsar - hamadi.boussarsar@gmail. com

Annals of Intensive Care 2016, 6(Suppl 1):P200

Introduction Thrombotic microangiopathy syndromes are an uncommon cause of ICU admission. They include several conditions characterized by clinical and biological manifestations such as neurological disorders, renal failure, hemolytic anemia and peripheral thrombocytopenia. Thrombotic thrombocytopenic purpura (TTP) and hemolytic uremic syndrome (HUS) are the two classic forms of TMA.

Objective: To analyze the clinical and biological features and evaluate the prognosis of TMA in the ICU.

Patients and methods It is a retrospective study performed in an 8-bed medical ICU during a period of 15 years from 2000 to 2014 . Clinical, biological, therapeutic and outcome characteristics of patients were analyzed.

Results Seven cases of TTP were collected during this period. Clinical, biological, therapeutic and outcome characteristics are displayed in Table 37.

In the present series, patients were rather young. Neurological symptoms were present in six patients and hemorrhagic signs only in three. Plasma exchange could be achieved in six patients with a favorable outcome only in three.

Conclusion Despite therapeutic progress and prognosis improvement by plasma exchange, this series of TMA patients reveals relatively high mortality.

\section{Competing interests None.}

\section{P201}

"Immunonutrition" failed to improve septic shock-induced vascular dysfunction in a rat model

Julie Boisramé-Helms ${ }^{1}$, Grégory Meyer ${ }^{2}$, Su Degirmenci ${ }^{3}$, Mélanie Burban $^{3}$, Valérie Schini-Kerth' ${ }^{3}$, Alexandra Boivin ${ }^{4}$, Raphaël Clere-Jehl ${ }^{5}$, Alexandra Monnier ${ }^{5}$, Christine Kummerlen ${ }^{5}$, Ferhat Meziani ${ }^{5}$

${ }^{1}$ Réanimation, CHU de Strasbourg, Strasbourg, France; ${ }^{2}$ Ea 4278, Université d'Avignon, Avignon, France; ${ }^{3}$ Umr7213, Strasbourg University, Strasbourg, France; ${ }^{4}$ Réanimation médicale, C.H.R.U. Hôpitaux Universitaires Strasbourg, Strasbourg, France; ${ }^{5}$ Réanimation médicale, Nouvel Hôpital Civil, CHU Strasbourg, Strasbourg, France

Correspondence: Julie Boisramé-Helms - julie.helms@libertysurf.fr Annals of Intensive Care 2016, 6(Suppl 1):P201
Introduction While n-3 poly-unsaturated fatty acids interfere with immune and inflammatory processes, the L-arginine deficit during sepsis impairs the arginine/NO pathway, setting off a multiple organ dysfunction syndrome leading to poor clinical outcomes in septic shock. However, there is no clear clinical benefit in supplementing septic patients with either $n-3$ PUFAs and/or L-arginine. The aim of our work was to assess the effects of different enteral nutrition formulations containing $n-3$ poly-unsaturated fatty acids and/or L-arginine on septic shock-induced vascular dysfunction in rats.

Materials and methods Healthy rats were fed with different enteral products: Peptamen ${ }^{\circledR}$ HN (HN group), Peptamen ${ }^{\circledR}$ AF containing $n-3$ poly-unsaturated fatty acids (AF group) or Peptamen ${ }^{\circledR}$ AF enriched with L-arginine (AFA group). After $72 \mathrm{~h}$ of nutritional pretreatment, peritonitis by cecal ligation and puncture was performed (CLP group) and compared to a SHAM group. Once septic shock was set, rats were actively resuscitated and monitored during $4 \mathrm{~h}$. At the end of the experiment, vessels and organs were harvested to assess inflammation, oxidative and nitrosative stress, and ex vivo vascular reactivity was studied.

Results Septic rats pretreated with an enteral nutrition enriched with L-arginine (AFA) had a significantly increased mortality compared to SHAM rats and to septic rats treated with a nutrition containing $n-3$ poly-unsaturated fatty acids AF or a standard enteral nutrition HN. Norepinephrine infusion rates to reach the mean arterial pressure objective were significantly increased in septic rats treated with n-3 poly-unsaturated fatty acid (AF and AFA groups), compared to SHAM and septic $H N$ rats. Both AF and AFA significantly reduced mesenteric resistance arterial contractility, decreased vascular oxidative stress, but increased NF-KB and plкB expression, nitric oxide and prostacyclin production in septic rats.

Discussion In this experimental septic shock model, an immunonutrition diet enriched with L-arginine or n-3 poly-unsaturated fatty acids failed to improve septic shock-induced hemodynamic dysfunction. Moreover, L-arginine supplementation seems to increase septic rat mortality. The need to assess the relevance of these results in septic patients, calls for further studies, and the place for so-called immunonutrition shall be questioned.

Conclusion Although immunonutrition with $n-3$ poly-unsaturated fatty acid or L-arginine supplementation exhibited an antioxidant effect, it failed to improve the septic shock-induced vascular dysfunction.

\section{Competing interests None.}

\section{P202}

PTP1B gene deletion improves glucose metabolism and limits cardiovascular dysfunction in experimental septic shock Eugénie Delile ${ }^{1}$, Pierre-Alain Thiébaut ${ }^{1}$, Jean-Claude Do Rego ${ }^{2}$, David Coquerel $^{1}$, Rémi Nevière ${ }^{3}$, Vincent Richard ${ }^{1}$, Fabienne Tamion ${ }^{4}$

${ }^{1}$ Inserm u1096, Université de rouen, Rouen, France; ${ }^{2}$ Scac-irib, Université de rouen, Rouen, France; ${ }^{3}$ Inserm u995 equipe 4, Université Lille 2, Lille, France; ${ }^{4}$ Réanimation médicale, Hospital Center University Rouen, Rouen, France

Correspondence: Eugénie Delile - eugenie.delile@etu.univ-rouen.fr Annals of Intensive Care 2016, 6(Suppl 1):P202

Introduction Hyperglycemia is a feature of septic patient and has been associated with poor outcome and higher mortality. In contrast, insulin has been shown to decrease mortality and to prevent the incidence of multi-organ failure but is often associated with deleterious hypoglycemia. Protein tyrosine phosphatase 1B (PTP1B) is a negative regulator of insulin signaling and NO production. We found that PTP1B inhibition or gene deletion improved mesenteric endothelial function and reduced cardiac and vascular inflammatory markers, leading to reduced cardiac dysfunction. The purpose of the present study was to assess the potential therapeutic effect of total PTP1B invalidation on glucose metabolism and cardiovascular insulin resistance using experimental model of sepsis.

Materials and methods Thus, in order to address this question, we developed a cecal ligation and puncture (CLP) model of sepsis. CLP is followed by subcutaneous fluid resuscitation at 1, 5 and $9 \mathrm{~h}$ after CLP. 
To evaluate the potential therapeutic effect of PTP1B invalidation, we used PTP1B-/- mice.

Metabolic phenotype study by indirect calorimetry was realized by tracking and recording food intake, physical effort and energy consumption. To assess metabolism, glucose (GTT) and insulin tolerance tests (ITT) were performed $16 \mathrm{~h}$ after CLP. Organs and plasma are removed to evaluate inflammation and oxidative stress by q-RT-PCR, insulinemia by ELISA test and insulin pathway by Western blot. Vascular function was assessed on Halpern's arteriograph.

Results Indirect calorimetry study showed that CLP induced a significative diminution of $\mathrm{VO}_{2}$ inspired, $\mathrm{VCO}_{2}$ expired, physical activity with food and water consumption. This study characterizes a severity of our CLP model without difference between WT versus PTP1B-/-.

Although this is severe model, PTP1B-/- mice showed improved GTT and ITT. Moreover, the limited increase of insulinemia in CLP PTP1B-/- mice emphasizes the improvement of glucose metabolism. Moreover, the heart expression of TNF-a, IL1-b, Gp91phox, VCAM-1, ICAM-1, and CD45 showed a significative increase of inflammation and oxidative stress in WT CLP mice compared with PTP1B-/- mice. Plasmatic dosage of insulin before and $15 \mathrm{~min}$ after glucose injection showed an elevation of insulin concentration in WT CLP mice showing a functional pancreas.

Insulin- and flow-mediated dilatation assessed in isolated perfused mesenteric arteries was abolished in WT CLP mice and was improved in PTP1B-/- mice.

Isolated perfused heart study showed that CLP induced a significative reduction of left ventricular developed pressure and cardiac efficiency in WT and PTP1B-/- mice. However, efficiency cardiac study showed a different utilization of substrate between WT CLP and PTP1B-/CLP, WT sham and PTP1B-/- sham.

Moreover, we showed that $\mathrm{PI} 3 \mathrm{k} / \mathrm{Akt}$ molecular pathway is functional in CLP mice suggesting a GLUT4 expression or translocation alteration. In fact, GLUT4 expression study showed that in plasma membrane fraction and endosome fraction, GLUT4 expression is abolished in WT CLP mice.

We also found that PTP1B-/- mice subjected to CLP had a higher survival rate compared to WT.

Conclusion CLP-induced sepsis changes the carbohydrate mechanistic by abolishing GLUT4 expression, and PTP1B inhibition restores GLUT4 expression and improves metabolic function, cardiovascular function and survival. This suggests that PTP1B inhibition may be an attractive target for the treatment of sepsis.

Competing interests None.

\section{P203}

\section{Ascitic fluid TREM-1 for the diagnosis of spontaneous bacterial} peritonitis

Laure Ichou' ${ }^{1}$ Nicolas Carbonell ${ }^{2}$, Pierre-Emmanuel Rautou ${ }^{3}$, Ludivine Laurans ${ }^{4}$, Simon Bourcier ${ }^{1}$, Claire Pichereau ${ }^{5}$, Jean-Luc Baudel ${ }^{5}$

Jean-Baptiste Nousbaum ${ }^{6}$, Christophe Renou' ${ }^{7}$, Rodolphe Anty ${ }^{8}$, Jacques Tankovic ${ }^{9}$, Eric Maury ${ }^{1}$, Bertrand Guidet ${ }^{1}$, Luce Landraud ${ }^{10}$, Hafid $^{6}$ Ait-Oufella

${ }^{1}$ Service de reanimation, Hôpital Saint-Antoine, Paris, France; ${ }^{2}$ Service d'hépatologie, Hôpital Saint-Antoine, Paris, France; ${ }^{3}$ Service d'hépatologie, Hospital Beaujon, Clichy, France; ${ }^{4}$ U970, Inserm, Paris, France; ${ }^{5}$ Réanimation médicale, Hôpital Saint-Antoine, Paris, France; ${ }^{6}$ Service d'hépato-gastro-entérologie, CHRU de Brest Hôpital Morvan, Brest, France; ${ }^{7}$ Service d'hépato-gastro-entérologie, CH, Hyères, France; ${ }^{8}$ Service d'hepato-gastro-enterologie, CHU Nice, Nice, France; ${ }^{9}$ Service de microbiologie, Hôpital Saint-Antoine, Paris, France; ${ }^{10}$ Service de microbiologie, CHU Nice, Nice, France

Correspondence: Hafid Ait-Oufella - hafid.aitoufella@sat.aphp.fr Annals of Intensive Care 2016, 6(Suppl 1):P203

Introduction Spontaneous bacterial peritonitis (SBP) is a life-threatening complication of cirrhosis. Its diagnosis is based on ascitic fluid polymorphonuclear (PMN) count. However, manual quantification of PMN is operator-dependent and can delay the diagnosis and in fine antibiotic administration. We aimed to assess the interest of ascitic fluid TREM-1, a receptor expressed by activated PMN, for the diagnosis of SBP.

Patients and methods One hundred and thirty-two consecutive ascites samples from 112 patients with cirrhosis from 3 tertiary care medical centers were examined for PMN count. TREM- 1 concentration in ascitic fluid was determined using ELISA. SBP was defined as a PMN count $>250$ cells $/ \mathrm{ml}$ with or without a bacteria positive culture. Results were expressed as median [25th-75th percentiles].

Results Twenty-six (20\%) samples fulfilled diagnostic criteria for SBP. TREM-1 concentration in ascitic fluid was significantly higher in SBP samples (4456 [3351-5498] pg/ml) than in the non-SBP samples $(642$ [417-899] pg/ml; $P<0.0001)$. By using a threshold of $1595 \mathrm{pg} / \mathrm{ml}$, the sensitivity and specificity of the assay for SBP diagnosis were $96 \%$ (95\% Cl 80-100) and $99 \%(95 \% \mathrm{Cl} 95-100)$, respectively. The area under the receiver operating characteristic curve (AUROC) was 0.98 (95\% Cl 0.95-1.00). In an independent multicenter cohort of $205 \mathrm{sam}$ ples including 28 SBP (14\%), the powerful performance of TREM- 1 for SBP diagnosis was confirmed. The AUROC was 0.98 (95\% Cl 0.96-1.00). Conclusion Ascites TREM-1 level is a highly sensitive and specific test for the diagnosis of SBP. The development of assays for the measurement of TREM-1 at bedside can be helpful for rapid and reliable screening in patients with cirrhosis.

Competing interests None.

Reference

1. Ichou et al. Gut. 2015 Jul 3.

P204

Modulation of inflammatory response related to severe peritonitis by polymyxin-B hemoperfusion

Remi Coudroy ${ }^{1}$, Jean-Claude Lecron², Didier Payen de la Garanderie², René Robert', ABDOMIX Group

${ }^{1}$ Réanimation médicale, CHU de Poitiers, Poitiers, France; ${ }^{2}$ Litec ea4331, Chu De Poitiers, Poitiers, France; ${ }^{3}$ Réanimation chirurgicale, CHU Lariboisière, Paris, France

Correspondence: Remi Coudroy - remi.coudroy@chu-poitiers.fr

Annals of Intensive Care 2016, 6(Suppl 1):P204

Introduction Although polymyxin-B hemoperfusion is supposed to improve outcomes in patients with sepsis by adsorbing endotoxin and decreasing systemic inflammation, the recent $A B D O M I X$ study did not demonstrate any beneficial effect of polymyxin- $B$ hemoperfusion in patients with septic shock due to peritonitis. In this context, cytokine clearance induced by polymyxin- $B$ hemoperfusion is debated. The objectives of the study were to assess the influence of polymyxin-B hemoperfusion on plasma cytokine concentrations and to identify the cytokines associated with day 28 mortality.

Patients and methods This ancillary study of the ABDOMIX study investigated the impact of two polymyxin- $B$ hemoperfusion sessions on day 28 mortality in peritonitis-induced septic shock. Blood samples were taken within $10 \mathrm{~h}$ post-surgery (P1), at the end of the first hemoperfusion session in the experimental group or $2 \mathrm{~h}$ after $\mathrm{P} 1 \mathrm{in}$ the control group (P2), $24 \mathrm{~h}$ after P1 (P3), and at the end of the second hemoperfusion session in the experimental group or $2 \mathrm{~h}$ after $\mathrm{P} 3$ in the control group (P4). Cytokines such as tumor necrosis factor $a$, interferon $\gamma$, interleukin-1 $\beta$, interleukin-1 $\alpha$, interleukin-1RA, interleukin-6, interleukin-4, interleukin-10, interleukin-17A and interleukin-22 were assessed using magnetic bead-based immunology multiplex assay or enzyme-linked immunosorbent assay.

Results Among the 232 patients included in the ABDOMIX study, 19 patients were excluded due to missing sampling and 213 patients (109 in the hemoperfusion group and 104 in the control group) were consequently included in the analysis. Postoperative P1 cytokine concentrations were not different between the 2 groups. Cytokine variation between P1 and P2 was not different between the 2 groups except for interleukin-1RA and interleukin-10, which decreased more pronouncedly in the control group than in the hemoperfusion group $(p=0.016$ 
and 0.047, respectively). Cytokine variations between P3 and P4 were not different between the 2 groups except for IL-10 and IL-22, both of which decreased in the control group, whereas both of them increased in the hemoperfusion group ( $p=0.002$ and 0.04 , respectively). Using a logistic stepwise regression model, the absence of decrease in interleukin-10 and interleukin-22 between P1 and P3 was associated with day 28 mortality independently from Simplified Acute Physiology Score II ( $p=0.002$ and 0.04 , respectively).

Conclusion Polymyxin-B hemoperfusion was not associated with a decrease in cytokine concentrations as compared to the control group. Higher levels of interleukin-10 and interleukin-22 in polymyxin-B hemoperfusion group may suggest unexpected deleterious interference between these cytokines and PMX membrane. The absence of decrease in interleukin-10 or interleukin-22 blood concentrations within $24 \mathrm{~h}$ after peritonitis-related septic shock was independently associated with day 28 mortality.

\section{Competing interests None.}

\section{P205}

\section{Descriptive study of ultrasonographic data inside a surgical} intensive care unit for cholestasis diagnosis: dechorea study

Michael Helary', Jonathan Paillot' ${ }^{2}$, Sebastien Pili-Floury ${ }^{3}$

${ }^{1}$ Anesthésie-Réanimation, Hospital Jean Minjoz, Besançon, France; ${ }^{2}$ Reanimation chirurgicale, Hospital Jean Minjoz, Besançon, France; ${ }^{3}$ Réanimation chirurgicale, CHU de Besançon, Besançon, France

Correspondence: Michael Helary - michael.helary@gmail.com

Annals of Intensive Care 2016, 6(Suppl 1):P205

Introduction Acute hepatic dysfunction increases length of intensive care stay and mortality rate, but cholestasis cases during intensive care have not really been studied in medical literature. Hepatobiliary ultrasonography frequently repeated for the same patient in part of the cholestasis diagnosis process. This aim of the study is to describe the epidemiological data and the predictive factors for the patients undergoing abdominal echography aiming at a cholestasis diagnosis during surgical intensive care according to the positive or negative informative result of the examination.

Patients and methods This retrospective study being monocentric and observational includes all the patients having undergone diagnostic abdominal ultrasonography in the case of acquired cholestasis in a surgical intensive care unit in the city of Besancon, France. Cholestasis is defined by a bilirubin plasmatic rate over 30 micromol per liter, alkaline phosphatase over 195 international units per liter and gamma glutamyl transferase over 82.5 international units per liter. The demographic data, the patient's ground and medical specificities have been notified as well as the various organ failures, biological results, drugs etiology through the Roussel Uclaf Causality Assessment Method score. Two groups have thus been defined: one group with "normal ultrasonography" or only sludge and the second with "pathological ultrasonography" including the patients presenting the following ultrasonographic signs: enlarged gallbladder, thickened gallbladder wall, fluid pericholecystic area and acute cholecystitis.

Results One hundred and four patients have been included from July 2011 to November 2014. Within the pathological ultrasonographic group, the median age is of $66[52 ; 73]$ years old versus $53[37 ; 64]$ years old in the other group $p=0.001$. The Simplified Gravity Index is of $48[38 ; 59]$ in the pathological ultrasonography group versus 44 [36; $56]$ in the other group. In the pathological ultrasonography group, the patients present cardiovascular medical specificities and more acute kidney injuries. This group includes icteric patients suffering an average bilirubin rate of $34 \pm 46$ millimol per liter versus $19 \pm 23$ millimol per liter.

Three cholecystitis cases have been diagnosed, and the three of them being acalculous cholecystitis and all of them are dead.

Discussion The lack of balance between the two groups and the comparatively high death rate among the pathological group patients are the limits to the study. Even though ultrasonography is recommended in first intention, it is relevant and of high interest to use a drugs imputability score in an etiological approach.
Conclusion The patients with pathological ultrasonography within the framework of cholestasis diagnosis seem to be icteric patients with more pathological comorbidities, and they seem to have less drug etiology on univariate analysis.

\section{Competing interests None.}

\section{References}

Kramer L, Jordan B, Druml W, Bauer P, Metnitz PGH. Incidence and prognosis of early hepatic dysfunction in critically ill patients A prospective multicenter study: Crit Care Med. avr 2007;35(4):1099-e7.

Brienza N, Dalfino L, Cinnella G, Diele C, Bruno F, Fiore T. Jaundice in critical illness: promoting factors of a concealed reality. Intensive Care Med. 1 févr 2006;32(2):267-74

\section{P206}

In-hospital mortality and predictive risk factors for patients with cirrhosis

Sana Khedher ${ }^{1}$, Amira Maoui ${ }^{1}$, Asma Ezzamouri ${ }^{2}$, Mohamed Salem²

${ }^{1}$ Digestif USI, EPS Charles Nicolle, bardo, Tunisia; ${ }^{2}$ Digestif USI, EPS Charles Nicoles, Tunis, Tunisia

Correspondence: Sana Khedher - sanakhedher@hotmail.fr Annals of Intensive Care 2016, 6(Suppl 1):P206

Introduction In-hospital mortality of cirrhotic is variable in the literature. It depends on several factors. The aim of our work was to determine the in-hospital mortality rate of cirrhosis and to identify predictive factors among our cirrhotic patient.

Patients and methods A retrospective study was performed on clinical records of cirrhotic patients admitted between 2013 and 2014. We identified demographic, clinical and laboratory parameters and studied their relationship with mortality.

Results Of the 78 patients admitted during the study period, there was a male dominance with a sex ratio equal to 0.63 and an average age of 62.44 . In total, $44.9 \%$ of patients had cirrhosis secondary to hepatitis C and 15.5 had cirrhosis secondary to hepatitis B. Fiftysix percentage of our patients were Child $\mathrm{C}$ and had a MELD score more than 30 in $92.3 \%$ of cases. In-hospital mortality was $19.2 \%$ (15 deaths). Death was due to septic shock in $53.13 \%$, hepatic carcinoma in 13.3 and in the $30.7 \%$ remaining cases to diverse reasons. The inhospital mortality among cirrhotic patients in our study increased with hyponatremia $(p=0.02)$, presence of renal failure $(p=0.01)$ and level of C-reactive protein exceeding $15 \mathrm{mg} / \mathrm{l}(p=0.05)$.

Discussion In worldwide, cirrhosis is a major cause of mortality. The presence of hyponatremia, renal failure and an elevated C-reactive protein level are predictive factors of mortality. The prognosis of cirrhotic patient is affected by infection. Dysfunction of immune system could be among the potential reasons of fragility.

Conclusion Cirrhosis is a severe disease. An intensive resuscitation should be promptly undertaken, especially when infection, hyponatremia, renal failure and high levels of C-reactive protein are diagnosed.

\section{Competing interests None.}

\section{P207}

CONSTIPREA: evaluation of the management of constipation in intensive care-National Survey of French intensivits Lamia Kerdjana ${ }^{1}$, Mihaela Dumitrescu ${ }^{2}$, Olivier Mimoz ${ }^{3}$, Bertrand Debaene $^{4}$, Virginie Migeot ${ }^{2}$, Claire Dahyot-Fizelier ${ }^{5}$

${ }^{1}$ Réanimation médicale, CHU Lariboisière, Paris, France; ${ }^{2}$ Service d'épidémiologie clinique, CHU de Poitiers, Poitiers, France; ${ }^{3}$ Réanimation chirurgicale, CHU de Poitiers, Poitiers, France; ${ }^{4}$ Département d'anesthésie-réanimation, CHU de Poitiers, Poitiers, France; ${ }^{5}$ Réanimation neuro-chirurgicale, CHU de Poitiers, Poitiers, France

Correspondence: Lamia Kerdjana - I.kerdjana@gmail.com Annals of Intensive Care 2016, 6(Suppl 1):P207

Introduction Constipation (or paralysis of lower gastrointestinal tract) in intensive care is multifactorial and is defined as the absence of stool for more than 3 consecutive days without mechanical obstruction. The 
incidence of constipation in intensive care patients is more than $60 \%$. Constipation is associated with longer duration of mechanical ventilation, longer stay in intensive care unit and increased mortality (1). The Working Group on Abdominal Problems (WGAP) of the European Society of Intensive Care Medicine (ESICM) developed recommendations on the constipation management in intensive care, but their implementation varies among intensivists (2). The aim of this study was to assess knowledge, attitudes, and practices when it comes to constipation management in intensive care.

Materials and methods The questionnaire KAP (knowledge, attitude, practices) for the national survey was sent by email to more than 1600 French intensivists. It was designed to investigate the characteristics of intensivists (10 issues), to assess knowledge with 13 issues in accordance with the recommendations, each one scored 0 or 1 allowing to calculate a knowledge score, to investigate behaviors (aka attitudes) regarding constipation management in their unit (9 issues) and practices with a case scored 4 . The responses were collected anonymously. Descriptive statistics are expressed as a percentage.

Results In 2 months, 579 replies were recorded on Google Drive ${ }^{\circledR}$ for a response rate of $36 \%$. Forty-two percent of respondents are aged between 30 and 39 years, and the sex ratio is 2 males for 1 female, $77 \%$ are senior intensivists, $23 \%$ are residents, $77 \%$ work in teaching hospital. The average knowledge score among all respondents is $7.5 / 13$ (minimum 2/13, maximum 12/13). The average the case among all respondents is $2.3 / 5$ (minimum $0 / 4$, maximum 4/4). These two scores are statistically associated with the number of beds in the respondent's unit, meaning that a better knowledge score is associated with a respondent working in an important intensive care unit (regarding the number of beds in the unit), but is not related to age, sex, teaching status, or seniority of medical practice. The intensivists do not know neither drugs, nor clinical and biological disorders which promote constipation in intensive care patients (1.4 and $2.8 \%$ respectively); $77.1 \%(n=444)$ underestimate the incidence of constipation in intensive care unit and only $62.7 \%$ believe that constipation affects mortality. Only $7 \%(n=41)$ of intensivists declare having a written protocol for constipation management in their unit, while $93 \%(n=536)$ have a systematic monitoring of transit in their service and $72 \%(n=415)$ initiate a treatment of constipation in the 6 days following the admission in intensive care unit. Although $60.6 \%$ of intensivists $(n=349)$ consider that constipation is a daily concern in their unit, only $5.2 \%(n=30)$ of them plan to attend a session on gastrointestinal motility impairment during a national convention.

Conclusion This is the first national survey assessing the knowledge, attitudes and practices of French intensivists in the field of constipation management. Their knowledge is insufficient and is related to the lack of interest of intensivists in the subject. This is a matter of concern, and corrective actions are needed to improve constipation management in intensive care units, such as implementation of written protocols and evaluation of implementation programs. Finally, educational programs are necessary during initial training and more data are needed to enhance the scope of the WGAP recommendations.

\section{Competing interests None.}

\section{References}

1. Gacouin A, Crit Care Med 2010;38:1933-8.

2. Reintam BA, Intensive Care Med 2012;38:384-94.

\section{P208}

Corrosive ingestion in adults: endoscopic findings and agents involved

Sana Khedher ${ }^{1}$, Asma Ezzamouri², Amira Maoui', Mohamed Salem², Amira A. Maoui ${ }^{2}$

${ }^{1}$ Digestif USI, EPS Charles Nicolle, bardo, Tunisia; ${ }^{2}$ Digestif usi, EPS Charles Nicolles, Tunis, Tunisia

Correspondence: Sana Khedher - sanakhedher@hotmail.fr

Annals of Intensive Care 2016, 6(Suppl 1):P208
Table 38 Univariate analysis between OHCA patients admitted in ICU after ROSC who developed and non-developed a HH

\begin{tabular}{|c|c|c|c|}
\hline Variable & $\begin{array}{l}\text { Non-HH patients } \\
(n=560,88.6 \%)\end{array}$ & $\begin{array}{l}\text { HH patients } \\
(n=72,11.4 \%)\end{array}$ & $\begin{array}{l}p \\
\text { value }\end{array}$ \\
\hline Age (years) & $60.7[50.0,72.6]$ & $63.9[48.1,71.3]$ & 0.74 \\
\hline Male gender & 395 (70.5 \%) & $40(55.6 \%)$ & 0.01 \\
\hline $\begin{array}{l}\text { Preexisting hepatic } \\
\text { disease }\end{array}$ & $14(2.5 \%)$ & $4(5.6 \%)$ & 0.14 \\
\hline Public setting CA & $212(38.0 \%)$ & 16 (22.2 \%) & 0.01 \\
\hline Witnessed CA & $486(89.0 \%)$ & 59 (83.1\%) & 0.14 \\
\hline Bystander CPR & $322(59.5 \%)$ & 39 (54.9\%) & 0.46 \\
\hline $\begin{array}{l}\text { Initial shockable } \\
\text { rhythm }\end{array}$ & $326(58.4 \%)$ & $24(33.3 \%)$ & $<0.01$ \\
\hline $\begin{array}{l}\text { Time from collapse } \\
\text { to ROSC (min) }\end{array}$ & $20.0[12.0,30.0]$ & $25.0[17.0,40.0]$ & 0.01 \\
\hline $\begin{array}{l}\text { Therapeutic hypo- } \\
\text { thermia }\end{array}$ & $527(94.1 \%)$ & 68 (94.4\%) & 0.91 \\
\hline Post-CA shock & $259(46.2 \%)$ & 64 (88.9\%) & $<0.01$ \\
\hline In-ICU mortality & $305(54.5 \%)$ & 62 (86.1\%) & $<0.01$ \\
\hline
\end{tabular}

Introduction During the ingestion of caustic products, the realization of the upper gastrointestinal endoscopy is inevitable. In fact, the gravity of gastrointestinal lesions does not correlate with neither the severity of oropharyngeal lesions nor clinical symptoms.

Patients and methods It is a retrospective study based on a case study. Sixty-three caustic ingestion observations were collected from 2013 to 2015. We propose: (1) to analyze the epidemiological and clinical characteristics of patient's victims of caustic ingestion, (2) to describe the different aspects of endoscopic lesions and (3) to seek predictive factors of severe lesions.

Results The average age of our patients is 29 years (range 2-73 years). Thirty-eight were female and 25 male or a sex ratio $F / M=1.52$. The ingestion was voluntary in $50.8 \%$ of cases and accidental in $49.2 \%$. Psychiatric history was found in $17 \%$ of which six $(9 \%)$ suicide attempt. A strong alkali product was ingested by $25 \%$ victims, an acidic agent by $8 \%$ victims and $67 \%$ of accidents were secondary to ingestion of oxidants. The most commonly involved agents are bleach $(63.5 \%)$ and caustic soda $(23.8 \%)$. The functional symptoms reported were mostly gastrointestinal. Physical examination was unremarkable and that interested the oropharyngeal sphere in $23.8 \%$ of cases. It showed that the oxidants were associated with higher risk of occurrence of functional symptoms in $76 \%$ of cases versus $23 \%$ of cases with strong bases and to $1 \%$ of the strong acids ingestion. Strong alkali were more responsible for hematemesis in $67 \%$ cases and ulceration of the tongue in 57 versus 25 and $38 \%$ within strong acids and 8 and $5 \%$ with oxidants $(p=0.002)$. In our series, the stomach was the most affected organ. The gastric lesions were isolated in 21 cases (33.3\%) or associated with esophageal lesions in 9 cases $(14.3 \%)$. The only caustic esophageal burns are identified in 12 patients (19\%) and in 11 cases $(17 \%)$; it was associated with other lesional levels. Otherwise, caustic injury affected the duodenum in $6.4 \%$. The triple localization (involving stomach, esophagus and duodenum) was objectified in 2 patients (3.2\%). Endoscopic lesions are as follows: stage 0 (28.6\%), stage I $(25.4 \%)$, stage lla lesions (20.6\%). More other serious were present: stage Illb (6.3\%), stage IIla (7.9\%) and stage IIIb (3.2\%); the lesion was stenosing in $7.9 \%$ of cases. Endoscopy was normal in $100 \%$ of weak acids ingestion and in $35.7 \%$ with oxidants. A significant statistically correlation between the caustic and the stage of endoscopic lesion ( $P=0.006$ ) was found. Caustic potash was at the origin of immediate stenosing lesion in $100 \%$ of cases. Endoscopy was pathological in $93 \%$ of cases of soda-based and $35.7 \%$ of endoscopies were normal 
in the case of bleach ingestion. The stomach was the most affected after caustic ingestion. The lesion in the stomach is often classified stage I (62\%). In the esophagus, stage lla was the most present (41\%). The stage Illb is objectified in the case where the lesion coexisted at the esophagus, stomach and duodenum. Stenosis was objectified in $80 \%$ of cases in the esophagus. Endoscopy was normal in $55 \%$ of patients who had an normal initial clinical examination. Clinical signs were more remarkable in cases of the stenosis and in stage Illb lesions. Conclusion Esophagoscopy is the key to diagnosis. The oropharynx injuries cannot predict the severity.

Competing interests None.

\section{P209}

Hypoxic hepatitis after out-of-hospital cardiac arrest: incidence, determinants and prognosis

Benoit Champigneulle', Guillaume Geri ${ }^{1}$, Wulfran Bougouin ${ }^{1}$, Florence Dumas ${ }^{2}$, Michel Arnaout ${ }^{1}$, Lara Zafrani ${ }^{1}$, Frédéric Pène ${ }^{1}$, Julien

Charpentier', Jean-Paul Mira', Alain Cariou'

${ }^{1}$ Réanimation médicale, Hôpital Cochin, Paris, France; ${ }^{2}$ Service d'accueil des urgences, Hôpital Cochin, Paris, France

Correspondence: Benoit Champigneulle - benoit.champigneulle@aphp. $\mathrm{fr}$

Annals of Intensive Care 2016, 6(Suppl 1):P209

Introduction Despite significant advances in post-cardiac arrest (CA) care, mortality of resuscitated out-of-hospital cardiac arrest (OHCA) remains high in intensive care unit (ICU) and is partially triggered by post-CA shock and associated organ damages. Few studies evaluated the incidence and the prognosis impact of the organ failure after OHCA. Particularly, occurrence of hypoxic hepatitis $(\mathrm{HH})$ in this setting is understudied. Aims of this study were to assess the prevalence of $\mathrm{HH}$, to investigate the factors associated with $\mathrm{HH}$ and to investigate the association between $\mathrm{HH}$ and ICU mortality in a large population of OHCA.

Patients and methods We performed a retrospective study over a 6-year period (2009-2014) in a French CA center. All non-traumatic OHCA patients admitted in ICU after return of spontaneous circulation (ROSC) who survived more than $24 \mathrm{~h}$ were included. In the specific setting of $\mathrm{CA}, \mathrm{HH}$ was defined as an elevation of alanine aminotransferase (ALT) over 20 times the upper limit of normal during the first $72 \mathrm{~h}$ after

Table 39 See text for description

\begin{tabular}{|c|c|c|c|c|}
\hline & $\begin{array}{l}\text { No AP } \\
(n=154) \\
(58.2 \%)\end{array}$ & $\begin{array}{l}\text { Chemical } \\
\text { AP }(n=51) \\
(23.2 \%)\end{array}$ & $\begin{array}{l}\text { Bacterial } \\
\operatorname{AP}(n=45) \\
(18.6 \%)\end{array}$ & $P$ \\
\hline $\begin{array}{l}\text { Age [mean } \\
\text { (SD)] }\end{array}$ & $56.9(15.9)$ & 57.5 (16.6) & $51.2(16)$ & 0.09 \\
\hline $\begin{array}{l}\text { Sex male }[n \\
(\%)]\end{array}$ & $72(46.7 \%)$ & 35 (68.3 \%) & $33(73.3 \%)$ & 0.001 \\
\hline $\begin{array}{l}\text { BMI [mean } \\
\text { (SD)] }\end{array}$ & $26.9(5.1)$ & $26.4(5.9)$ & $25.5(4.5)$ & 0.23 \\
\hline $\begin{array}{l}\text { SAPS2 [mean } \\
\text { (SD)] }\end{array}$ & $54.3(18.5)$ & $57.4(19.5)$ & 58.8 (16.6) & 0.27 \\
\hline $\begin{array}{l}\text { Duration of } \\
\text { MV, days, } \\
\text { median } \\
\text { [Q1-Q3] }\end{array}$ & $1.1[0.5-3]$ & 2.8 [1.4-5] & 3.4 [1.6-7] & $<0.001$ \\
\hline $\begin{array}{l}\text { ICU LOS, days, } \\
\text { median } \\
\text { [Q1-Q3] }\end{array}$ & $2[1-4]$ & $4.2[2-7]$ & $4.6[2-9]$ & $<0.001$ \\
\hline $\begin{array}{l}\text { Hospital LOS, } \\
\text { days, median } \\
\text { [Q1-Q3] }\end{array}$ & $2.5[1-6]$ & 6 [2-19] & $8[3-24]$ & $<0.001$ \\
\hline $\begin{array}{c}\text { Mortality } \\
{[n(\%)]}\end{array}$ & 47 (30.5 \%) & $16(31.4 \%)$ & $12(26.7 \%)$ & 0.89 \\
\hline
\end{tabular}

occurrence of CA (i.e. $900 \mathrm{IU} / \mathrm{L}$ ). Liver function test (LFT) measurements were extracted from our local database and classified in 6 time points, every $24 \mathrm{~h}$, from T0 (ICU admission) to T5 (H120). Utstein style categories concerning CA were considered. Univariate analysis and multivariate regression analysis were performed.

Results Among the 632 OHCA patients included in the study, 72 patients developed a $\mathrm{HH}$ (Table 38). Thus, the prevalence of $\mathrm{HH}$ was $11.4 \%(95 \% \mathrm{Cl} 9.0,14.1)$. In multivariate analysis, the only prehospital variable associated with the occurrence of $\mathrm{HH}$ was the time from collapse-to-ROSC [OR 1.02 per additional minute; $95 \% \mathrm{Cl}(1.00$, 1.04); $p=0.01$ ]. After adjustment for confounding factors, $\mathrm{HH}$ was associated with a higher ICU mortality [OR 4.39; $95 \% \mathrm{Cl}(1.71,11.26)$; $p<0.01]$, and this association persisted even if occurrence of a post-CA shock was considered in the statistical model [OR 3.63; $95 \% \mathrm{Cl}$ (1.39, 9.48); $p=0.01]$.

Discussion Occurrence of $\mathrm{HH}$ may complicate the ICU course of patients admitted after OHCA and is associated with a higher ICU mortality, independently of the occurrence of post-CA shock. However, a causal relationship between $\mathrm{HH}$ and mortality cannot be affirmed by this observational study, as $\mathrm{HH}$ may also reflect the severity of the post-CA syndrome and shock.

Conclusion $\mathrm{HH}$ is a common complication after $\mathrm{CA}$, appears mainly triggered by the duration of resuscitation (no flow + low flow) and is associated with early mortality in ICU.

Competing interests None.

\section{P210}

Aspiration pneumonitis in comatose patients treated with invasive mechanical ventilation. The SPIRE prospective survey

Jean-Baptiste Lascarrou', Floriane Lissonde ${ }^{2}$, Aurélie Le

Thuaut ${ }^{3}$, Konstantinos Bachoumas ${ }^{1}$, Gwenhaël Colin ${ }^{1}$, Matthieu

Henry-Laguarrigue', Maud Fiancette', Jean-Claude Lacherade',

Christine Lebert ${ }^{1}$, Isabelle Vinatier ${ }^{1}$, Aihem Yehia ${ }^{1}$, Aurélie Joret ${ }^{1}$, Laurent

Martin-Lefèvre', Jean Reignier ${ }^{4}$

${ }^{1}$ Réanimation polyvalente, Centre Hospitalier Départemental - site de La

Roche-sur-Yon, La Roche-sur-Yon, France; ${ }^{2}$ Département d'anesthésie,

CHU de Nantes, Nantes Cedex 1, France; ${ }^{3}$ Biostatistiques, C.H.U. Hôtel

Dieu, Nantes, France; ${ }^{4}$ Réanimation médicale, C.H.U de Nantes, Nantes,

France

Correspondence: Jean-Baptiste Lascarrou - jean-baptiste.lascarrou@

chd-vendee.fr

Annals of Intensive Care 2016, 6(Suppl 1):P210

Introduction Patients in comatose state are at risk of aspiration pneumonia (AP) with increased burden in morbidity and mortality. Aspiration may result in pulmonary injury due to chemical and/or bacterial aggression. No reliable clinical or biological criteria are available to ensure diagnosis of bacterial infection on patient admission. Antibiotics in AP are thus widely prescribed, although theoretically effective only in bacterial AP. We defined a new protocol for management of comatose patients with the aim to reduce antibiotics use by treating only patients with suspected AP and by stopping antibiotics in case of negative bacteriological samples. The aim of our study was to assess effectiveness and safety of this protocol.

Patients and methods This prospective survey started in November 2012 and ended in December 2014. All the patients admitted in ICU for coma (Glasgow coma score $\leq 8$ ) requiring intubation and mechanical ventilation were included. Respiratory specimen was obtained, and antibiotic treatment was began when an AP was suspected (two of the following criteria: temperature $\geq 38.5$ ou $\leq 35.5^{\circ} \mathrm{C}$, leukocytosis or leukopenia, purulent tracheal aspirates) and new infiltrates on the chest X-ray. Patients were divided into 3 groups: no AP, chemical AP (infiltrates on chest $\mathrm{X}$-ray but negative respiratory specimen) and bacterial AP (positive respiratory specimen).

Results Two hundred and fifty patients ( 56 years; SAPSII 55 ; duration of mechanical ventilation: 1.8 days; ICU length of stay: 3 days; ICU mortality $30 \%)$ were included. An AP was suspected in 96 (38.4\%) patients. Of these patients, 45 (47 \%) had a bacterial AP and $30(53 \%)$ a chemical AP. Bacterial or chemical AP were associated with increased duration 
of mechanical ventilation and length of stay. Only 2 patients of the 34 with a chemical AP had a confirmed relapse of pneumonia after antibiotic treatment had been interrupted after initial negative respiratory specimen. They do not need invasive mechanical ventilation (Table 39). Conclusion AP chemical or bacterial is associated with increase morbidity without increase mortality. Systematic bacterial sampling and antibiotic withdrawing in case of negative culture lead to antibiotic interruption in $1 / 3$ of AP cases without increased risk in AP relapse.

\section{Competing interests None.}

\section{P211}

Ventilator-associated pneumonia (VAP) in cancer patients: impact of 1-year survival

Laura Platon', Jerôme Lambert ${ }^{2}$, David Lagier ${ }^{3}$, Jeanpaul Brun ${ }^{4}$, Antoine Sannini ${ }^{4}$, Laurent Chow-Chine ${ }^{5}$, Magali Bisbal ${ }^{4}$, Marion Faucher ${ }^{4}$, Djamel Mokart $^{6}$

${ }^{1}$ Réanimation médicale, chu lapeyronie, Montpellier, France; ${ }^{2}$ Sbim, Hôpital Saint-Louis, Paris, France; ${ }^{3}$ Hôpital la timone (marseille), CHU La Timone (Marseille), Marseille, France; ${ }^{4}$ Reanimation, Institute Paoli-Calmettes, Marseille, France; ${ }^{5}$ Réanimation, Institute Paoli-Calmettes, Marseille, France; ${ }^{6}$ Réanimation, Institut Paoli-Calmettes, Marseille, France

Correspondence: Djamel Mokart - mokartd@ipc.unicancer.fr Annals of Intensive Care 2016, 6(Suppl 1):P211

Introduction Ventilator-associated pneumonia (VAP) is the most frequent intensive care unit (ICU)-acquired infection. Data regarding onco-hematology patients are scarce and VAP might impact on prognosis.

Patients and methods Monocentric prospective and observational study, including cancer patients treated with mechanical ventilation during more than $48 \mathrm{~h}$ over a 5 -year period (from December 2008 to February 2014). We evaluated risk factors for VAP among baseline characteristics of patients and ICU therapies. Using VAP as a time-dependent variable, Cox model was fitted to assess its impact on 1-year mortality, and multistate models were used to calculate its impact on morbidity.

Results Of the 208 patients included in the study, 66 patients $(31.7 \%)$ presented a VAP. ICU mortality rate was $55.8 \%$ (118 patients). Oneyear survival was $23 \%$. Most of VAP ( $87.8 \%$ ) had a microbiological documentation (Gram-negative bacilli predominantly). The use of hydrocortisone was the only independent risk factor for VAP, with a protective effect. Patients having a VAP had a longer ICU stay (mean extra length of stay: 4.2 days, for patients survivors from ICU) and a longer mechanical ventilation (mean extra duration: 1.7 days, for patients extubated). Regarding prognosis, when adjusting on SOFA score the day of intubation (HR 1.11; $95 \% \mathrm{Cl} 1.07-1.16 ; p<0.0001$ ) and disease stage (HR 1.83; $95 \% \mathrm{Cl} 1.16-2.89 ; p: 0.01)$, the occurrence of VAP was independently associated with 1-year mortality (HR 1.50; $95 \% \mathrm{Cl} 1.05-2.15 ; p$ : 0.03).

Conclusion VAP is associated with 1-year mortality in this specific population. In association with standard preventive recommendations, the use of hydrocortisone might prevent occurrence of VAP. Randomized clinical trials are warranted.

\section{Competing interests None.}

\section{P212}

High-resolution computed tomography (HRCT) patterns in patients with pneumocystis pneumonia

Leïla Mourtada', Lemiale Virgnie', Claire Givel', Valade Sandrine', Claire Pichereau ${ }^{2}$, Emmanuel Canet ${ }^{1}$, Eric Mariotte ${ }^{1}$, Elie Azoulay ${ }^{1}$

${ }^{1}$ Réanimation médicale, Hôpital Saint Louis, Paris, France; ${ }^{2}$ Réanimation médicale, Hôpital Saint-Antoine, Paris, France

Correspondence: Leïla Mourtada - leilamourtada@hotmail.com Annals of Intensive Care 2016, 6(Suppl 1):P212

Introduction Pneumocystis jiroveci pneumonia (PCP) is a major cause of acute respiratory failure in immunocompromised patients. Although the pathogen is similar, clinical features carry significant differences across different patient subgroups. The aim of this study was to describe and compare HRCT patterns in PCP patients with either AIDS, malignancies or steroid-induced-related immune suppression.

Patients and methods We performed a retrospective study among critically ill PCP patients between January 1, 2006, and July 31, 2015. All patients with a diagnostic of PCP and available HRCT within 2 days of diagnosis were included. Only confirmed cases of PCP were included. Clinical and biological data were collected. CT scans were independently reviewed by two respiratory clinicians with a consultant radiologist available to reach consensus. We compared HRCT patterns across three groups of PCP patients: AIDS, hematological malignancies and steroid-related immunosuppression.

Results Sixty-six patients (70\% men, age 56 [47-64]) were included in this study (23 AIDS, 21 Hemato, 22 Steroids). None of the AIDS patients had lymphoma or other malignancies. Underlying malignancies included acute hematological malignancies in 9 patients, chronic hematological malignancy in 11 patients and other in $1(1 \%)$. Underlying disease in steroid group was mostly kidney transplant patients [16/22 (73\%)].

At ICU admission, respiratory rate was 29 [23-35]/min, oxygen flow was 6 [4-12] $\mathrm{l} / \mathrm{min}$, and 3 patients needed mechanical ventilation at admission ( $\left.\mathrm{FiO}_{2} 100 \%\right)$. During ICU stay, 36 (54\%) patients needed invasive mechanical ventilation during 9 [6-14] days, 24 (36\%) had shock and $13(20 \%)$ needed renal replacement therapy. All patients received trimethoprim-sulfamethoxazole for 21 days and 31 (47\%) received steroid. Twenty-five (38\%) had coinfection at ICU admission. ICU length of stay was 10 [5-16] days. Hospital mortality was $7.6 \%$ $(n=5)$.

HRCT findings included the following: ground-glass opacities in $83 \%$ $(n=55)$, ill-defined consolidations in $57 \%(n=38)$, interalveolar thickening (septal lines) in $42 \%(n=28)$, bronchiectasis in $42 \%(n=28)$, crazy paving in $32 \%(n=21)$, nodular opacities in $21 \%(n=14)$, and cysts in $15 \%(n=10)$. Diffuse ground-glass opacities and crazy paving were present in $68 \%$ of cases, and nodular opacities in $16(24 \%)$

Table 40 See text for description

\begin{tabular}{lllll}
\hline $\begin{array}{l}\text { Evaluation } \\
\text { criterion }\end{array}$ & Mean & Median & $p$ & No. of patients \\
\hline $\begin{array}{c}\text { ARDS present/ } \\
\text { absent (D0-D3) }\end{array}$ & $7.02 / 13.95$ & $5.45 / 9.33$ & $<0.002$ & $27 / 58$ \\
$\begin{array}{c}\text { ARDS appeared/ } \\
\text { absent (D1-D3) }\end{array}$ & $3.05 / 13.95$ & $2.95 / 9.33$ & $<0.0001$ & $8 / 58$ \\
$\begin{array}{c}\text { ARDS worsening/ } \\
\text { non worsening }\end{array}$ & $3.11 / 13.01$ & $3.15 / 9.23$ & $<0.00001$ & $10 / 72$ \\
(D0-D3) & & & & \\
\hline
\end{tabular}

ROC analysis revealed AUC $>0.9$, positive predictive values between 0.67 and 0.83 , and negative predictive values between 0.94 and 0.95

Table 41 See text for description

\begin{tabular}{llllllll}
\hline $\begin{array}{l}\text { Evaluation } \\
\text { criterion }\end{array}$ & $\begin{array}{l}\text { Cutoff } \\
(\mathbf{n g} / \mathbf{m L})\end{array}$ & AUC & Se & Sp & NPV & PPV & No. of patients \\
\hline $\begin{array}{l}\text { ARDS } \\
\text { presence } \\
\text { (D0-D3) }\end{array}$ & 3.58 & 0.711 & 0.30 & 0.98 & 0.75 & 0.89 & $27 / 58$ \\
$\begin{array}{l}\text { ARDS } \\
\text { appear- } \\
\text { ance } \\
\text { (D1-D3) }\end{array}$ & 3.58 & 0.945 & 0.62 & 0.98 & 0.95 & 0.83 & $8 / 58$ \\
$\begin{array}{l}\text { ARDS wors- } \\
\text { ening } \\
\text { (D0-D3) }\end{array}$ & 3.58 & 0.938 & 0.60 & 0.96 & 0.94 & 0.67 & $10 / 72$ \\
\hline
\end{tabular}


patients. Lung opacities were bilateral (97\%), located in the superior parts of the lungs, and $45 \%(n=30)$ had no pleural effusion. Pneumothoraces were rare $[n=5(7.5 \%)]$. The lung opacities on HRCT were distributed similarly among the three immunocompromised groups as none of these patterns (topographic involvement and localization) were significantly different across the three groups. Consolidations and nodular opacities were significantly associated with the existence of coinfection ( $p$ 0.03).

Conclusion During PCP disease, the most frequent HRCT pattern is bilateral diffuse ground-glass opacities or crazy paving with no pleural space involvement. Alveolar or nodular opacities are less frequent but may be encountered. Therefore, despite striking differences in pathophysiology and clinical presentation between AIDS and non AIDS patients, HRCT patterns are similar. Other HRCT findings such as consolidations and nodules might be ascribable to lung super infection.

\section{Competing interests None.}

\section{P213}

Plasma endocan predicts the occurrence of acute respiratory distress syndrome (ARDS) in severe sepsis and septic shock patients

Alexandre Gaudet ${ }^{1}$, Erika Parmentier-Decrucq ${ }^{1}$, Nathalie De Freitas Caires $^{2}$, Sylvain Dubucquoi ${ }^{3}$, Philippe Lassalle ${ }^{4}$, Daniel Mathieu

${ }^{1}$ Pôle de réanimation, hôpital salengro, Centre Hospitalier Régional Universitaire de Lille, Lille, France: ${ }^{2}$ Lunginnov, Institut Pasteur de Lille, Lille, France; ${ }^{3}$ Institut d'immunologie, centre de biologie-pathologie génétique, Centre Hospitalier Régional Universitaire de Lille, Lille, France: ${ }^{4}$ Inserm u1019 e13, Institut Pasteur de Lille, Lille, France

Correspondence: Alexandre Gaudet - alexandre.gaudet@yahoo.fr Annals of Intensive Care 2016, 6(Suppl 1):P213

Introduction In severe sepsis and septic shock patients, respiratory failure usually occurs within $72 \mathrm{~h}$ following admission into intensive care unit. Endothelial dysfunction is thought to play a major role in its occurrence and prognosis.

Endocan is an anti-inflammatory molecule produced by the lung endothelium and released in the circulation in response to an infectious or inflammatory insult. The main action of endocan relates to the inhibition of leukocyte diapedesis. By preventing leukocytes accumulation in the lung, endocan reduces excessive pulmonary inflammation. Thus, a high circulating level endocan in septic patients would protect the lung from excessive inflammation and would be of better prognosis.

This hypothesis has been comforted by 2 pilot studies. In the context of severe polytrauma, high blood levels of endocan at ICU admission $(>7 \mathrm{ng} / \mathrm{mL}$ ) are observed in patients who do not develop respiratory failure (1). We found a similar profile in severe septic patients with a cutoff of $3.55 \mathrm{ng} / \mathrm{mL}$ best distinguishing patients with ARDS in the 3 first day of ICU from patients who do not develop ARDS (2).

To extend these pilot studies, a prospective observational study in a population of severe sepsis and septic shock patients was designed in order to confirm the predictive value of blood endocan at admission for the occurrence of ARDS.

Patients and methods The objective was to compare endocan level at ICU admission in 3 different sets of 2 groups: presence or absence of ARDS between D0 and D3; appearance of ARDS or not between D1 and D3; respiratory worsening or not between D0 and D3. The inclusion criteria were severe sepsis or septic shock, as defined by the SSC 2008. The exclusion criteria were age $<18$ years, dialysis, pregnancy, immunosuppression, systemic corticosteroid therapy. Patients were classified by 2 blinded independent intensivists. Endocan was measured in plasma EDTA in blind by 2 different laboratories: CHRU Lille, Inserm U1019E13. Nonparametric test was used for statistical analysis. Results Eighty-five patients were definitively included. Fifty-eight did not develop ARDS. Among the 27 who developed ARDS, 19 had ARDS at admission. Among them, 2 worsened their ARDS at D3. Eight patients developed ARDS after admission. The mean/median levels of blood endocan in the ARDS groups (present, appeared, worsening) are significantly lower than in the control groups. The best significance is obtained when ARDS is appearing or worsening $\left(p<10^{-4}, p<10^{-5}\right)$ (Tables 40, 41).

Discussion In patients with severe sepsis or septic shock, the level of blood endocan at ICU admission predicts the occurrence of ARDS: an endocan level $<3.5 \mathrm{ng} / \mathrm{mL}$ indicates a patient at risk; an endocan level $>5.5 \mathrm{ng} / \mathrm{mL}$ indicates no risk of ARDS.

Conclusion Blood endocan may help to predict the occurrence or the worsening of ARDS in severe sepsis or septic shock patients and to early apply protective ventilation in those patients.

Competing interests None.

\section{References}

1. Mikkelsen ME, Shah CV, Scherpereel A, Lanken PN, Lassalle P, Bellamy SL, Localio AR, Albelda SM, Meyer NJ, Christie JD. Lower serum endocan levels are associated with the development of acute lung injury after major trauma. J Crit Care. 2012;27(5):522.e11-7.

2. Palud A, Parmentier-Decrucq E, Pastre J, De Freitas Caires N, Lassalle P, Mathieu D. Evaluation of endothelial biomarkers as predictors of organ failures in septic shock patients. Cytokine. 2015;73(2):213-8.

P214

Comparison of $\mathbf{3}$ diagnostic methods to identify fluid responsiveness during prone position in ARDS

Jean-Christophe Richard ${ }^{1}$, Hodane Yonis ${ }^{1}$, Florent Gobert ${ }^{1}$, Romain Tapponnier ${ }^{1}$, Claude Guérin ${ }^{1}$

${ }^{1}$ Réanimation médicale, Hôpital de la Croix-Rousse, Lyon, France

Correspondence: Jean-Christophe Richard-j-christophe.richard@ chu-lyon.fr

Annals of Intensive Care 2016, 6(Suppl 1):P214

Introduction Predicting fluid responsiveness is of paramount importance to avoid unnecessary fluid administration in ARDS patients, since a positive fluid balance is associated with ARDS mortality (1). Several tests with high reliability to predict fluid responsiveness are now available, but none of them have been validated in the prone position (PP) in ARDS patients, while this treatment is now a cornerstone of the therapeutic armamentarium of severe ARDS (2). The aim of this study was to evaluate the diagnostic performance of three methods to predict fluid responsiveness in PP: cardiac index variation during the Trendelenburg position, cardiac index variation during end-expiratory occlusion, and pulse pressure variation after increasing tidal volume (VT) to $8 \mathrm{ml} . \mathrm{kg}$ predicted body weight (PBW).

Patients and methods This prospective single-center study enrolled ARDS patients with $\mathrm{PaO}_{2} / \mathrm{FiO}_{2}<150 \mathrm{~mm} \mathrm{Hg}$ in the supine position, under invasive mechanical ventilation in PP, monitored with the PiCCO device, and with acute circulatory failure. Patients were studied at baseline with bed angulation $13^{\circ}$, during a 1 -min postural change in the Trendelenburg position with bed angulation $-13^{\circ}$, during a 1 -min increase of VT to $8 \mathrm{ml} \mathrm{kg}^{-1}$ PBW, during a 15-s end-expiratory occlusion and after IV infusion of $500 \mathrm{ml}$ crystalloids. Baseline settings were resumed for 1 min after each intervention. Cardiac index was measured with the thermodilution technique at baseline and after fluid administration. Pulse contour-derived cardiac index was continuously monitored. Fluid responsiveness was deemed present if cardiac index assessed by thermodilution increased by at least $15 \%$ after fluid administration. Numeric results are presented as median [1st-3rd quartile].

Results Twenty-three patients were included at the time of abstract submission. Patients characteristics at study inclusion were the following: SAPS II 56 [48-62], VT 6.0 [5.9-6.1] $\mathrm{ml} \mathrm{kg}^{-1} \mathrm{PBW}, \mathrm{PaO}_{2} / \mathrm{FiO}_{2}$ ratio in PP $154 \mathrm{~mm} \mathrm{Hg}$ [98-192], PEEP 8 [8-10] $\mathrm{CmH}_{2} \mathrm{O}$, and plateau pressure 23 [20-28] $\mathrm{cmH}_{2} \mathrm{O}$. Nine patients (41\%) were deemed fluid responsive after fluid administration. The area under ROC curve of the 
pulse contour-derived cardiac index change during the Trendelenburg maneuver and the end-expiratory occlusion test was 0.86 [95\% $\mathrm{Cl} 0.70-1]$ and 0.71 [95 \% Cl 0.46-0.96], respectively. An increase in cardiac index $\geq 7.5 \%$ during the Trendelenburg maneuver enabled to diagnose fluid responsiveness with a sensitivity of $0.78[95 \% \mathrm{Cl}$ $0.44-1]$, and a specificity of 0.86 [95\% Cl 0.64-1]. Cardiac arrhythmia was present at baseline in 13 patients (57\%), which were therefore excluded from pulse pressure variation analysis. The area under ROC curve of pulse pressure variation during VT increase to $8 \mathrm{ml} \mathrm{kg}^{-1}$ PBW was 0.45 [95 \% Cl 0.05-0.85].

Conclusion Cardiac index variation during a Trendelenburg maneuver is a reliable method to assess fluid responsiveness in ARDS patients in the prone position, while pulse pressure variation may be of limited interest in this context.

\section{Competing interests None.}

\section{References}

1. Sakr Y et al. High tidal volume and positive fluid balance are associated with worse outcome in acute lung injury. Chest 2005;128(5):3098-108.

2. Guerin $\mathrm{C}$ et al. Prone positioning in severe acute respiratory distress syndrome. N Engl J Med 2013;368(23):2159-68.

\section{P215}

Low pressure support-high PEEP for early severe acute respiratory distress syndrome: a retrospective analysis

\section{Luc Quintin ${ }^{1}$, C. Pichot ${ }^{2}$, F. Petitjeans ${ }^{3}$}

${ }^{1}$ Physiology, CNRS, Lyon-Villeurbanne, France; ${ }^{2}$ Physiology, U Lyon1, Lyon, France; ${ }^{3}$ Critical care, Hospital Instruction Armed Desgenettes, Lyon, France

Correspondence: Luc Quintin - lucquintin@yahoo.com

Annals of Intensive Care 2016, 6(Suppl 1):P215

Introduction Management of severe acute respiratory distress syndrome (ARDS, $\mathrm{PaO}_{2} / \mathrm{FiO}_{2}=P / F<100$ ) is: controlled mechanical ventilation (CMV), 24 (1)-48 (2) $\mathrm{h}$ of muscle relaxation, early prolonged proning (3), low driving pressure (4). This improves VA/Q ratio (Guerin 2014), ventilator-patient synchrony (5), lowers $\mathrm{VO}_{2}$ (6) and controls $\mathrm{H}+$. Mortality is halved (3). As ARDS is caused by a reduction of the surface offered for $\mathrm{O}_{2}$ diffusion, failure of the respiratory generator or the ventilatory muscles spontaneous ventilation (SV), with conditionalities, may offer to the lung better circulatory and ventilatory conditions to allow for healing.

Patients and methods A series of 6 patients in which the Berlin criteria (7) were fulfilled, and sufficient details gathered were retrospectively analyzed. P/F on PEEP $\geq 5 \mathrm{cmH}_{2} \mathrm{O}$ was $<100$ with one exception (117). The patients were already present in the critical care unit (CCU) or admitted to the CCU following tracheal intubation for pulmonary/extrapulmonary ARDS. Management was: imaging, fiberoptic bronchoscopy if needed, repeated transthoracic echocardiography (absence of right ventricular dilatation or reduction of tricuspid annular systolic excursion), venous and arterial lines insertion, circulatory optimization (8) to a $\mathrm{SscvO}_{2}>70-75 \%$, arterio-venous $\mathrm{CO}_{2}$ gradient $<6$, lactates $<2$, volume loading (little or no ventilatory changes of the inferior vena cava), normothermia $\left(35-7^{\circ} \mathrm{C}\right)$, upright (9) $\left(60^{\circ}\right.$ reverse Trendelenburg legs down) position, administration of an alpha-2 agonist (clonidine $1-2 \mu \mathrm{g} \mathrm{kg}^{-1} \mathrm{~h}^{-1}$ ) combined if needed $(-3<$ RASS $<-2)$ with loxapine $100 \mathrm{mg}$ through nasogastric tube as required. PEEP (10-20 cm H20) aimed $\mathrm{SaO}_{2} \geq 98-100 \%$; then, $\mathrm{FiO}_{2}$ lowered as early as possible to 0.4 over $24-72 \mathrm{~h}$. As soon as metabolic acidosis was controlled (with extra-renal replacement therapy if needed), CMV was switched to pressure support ( $P S=8 \mathrm{~cm} \mathrm{H} 20$ lowered to $5: \mathrm{Vt} \leq 5 \mathrm{ml}$. $\mathrm{kg}, \mathrm{PaCO} \leq 60 \mathrm{~mm} \mathrm{Hg}$, Pplat $\leq 30)$. Extubation $(P / F>150-200$, PEEP $=10$ ) was followed by continuous noninvasive ventilation (NIV, $\mathrm{FiO}_{2}=0.4, \mathrm{PEEP}=10$ to $5, \mathrm{PS}=8$ to 5$)$ for 4 days (24/24 h, 18/24 etc.). Results $P / F$ increased $>200$ over $12-72 \mathrm{~h}$ followed by improvement and then disappearance of bilateral diffuse opacities. Overall reduction in vasopressor/inotropic requirements, markers of inflammation, etc. allowed one to extubate the trachea after $72-96 \mathrm{~h}$ following the beginning of said management. In some instances, the improvement of $P / F$ was from $\approx 50$ to $>300$ over $\leq 24 \mathrm{~h}$. One patient was re-intubated because of re-infection in the presence of interrupted NIV and normothermia.

Discussion The limitations are: non-prospective non-randomized design; $n=6$ although $<50$ patients were treated without full details (CCU mortality $\approx 5 \%$ ); $\mathrm{H}+$ should be controlled before SV. The rationale behind CMV + proning (introduction) can be achieved with lowered temperature with alpha-2 agonists $(10,11)$, adequate SV (12) without ventilatory depression (13). SV improves circulatory stability and uses much higher PEEP + lower driving pressure. Fast-track extubations were observed.

Conclusion Provided stringent physiological principles are met severe ARDS may be handled with CMV + muscle relaxation + proning (state-of-the-art) or SV. Evidence-based demonstration is required.

Competing interests The speaker holds a US Patent office 8703 697, April 22 2014: Method for treating early severe diffuse ARDS.

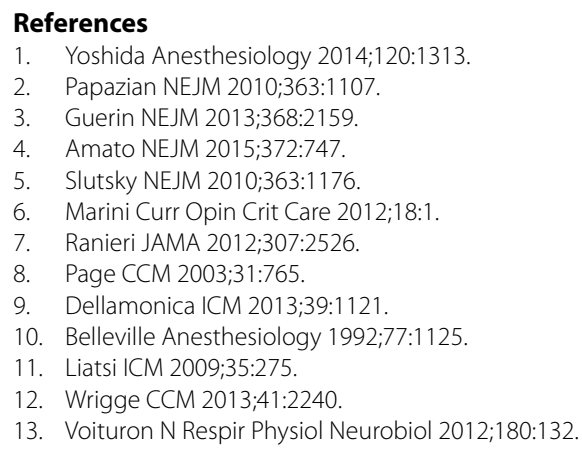

P216

Neutrophil proteases alter the interleukin-22-receptor-dependent lung antimicrobial defence

Antoine Guillon', Youenn Jouan', Daborah Breah², Fabien Gueugnon², Emilie Dalloneau², Thomas Baranek², Clemence Henry², Eric Morello², Jc Renauld ${ }^{3}$, M Pichavant ${ }^{4}$, Philippe Gosset ${ }^{4}$, Y Courty ${ }^{2}$, Patrice Diot ${ }^{5}$, Mustapha Si-Tahar ${ }^{2}$

${ }^{1}$ Réanimation polyvalente - u1100 centre d'etude des pathologies respiratoires, CHRU Hôpitaux de Tours, Tours, France; Inserm u1 100, Centre d'étude des pathologies respiratoires, Tours, France; ${ }^{3}$ Université catholique de louvain, De Duve institute, Brussels, Belgium; ${ }^{4}$ Institut pasteur de lille, Centre for infection and immunity, Lille, France; ${ }^{5}$ Pneumologie, CHRU Hôpitaux de Tours, Tours, France

Correspondence: Antoine Guillon - antoine.guillon@univ-tours.fr Annals of Intensive Care 2016, 6(Suppl 1):P216

Introduction Acute episodes of exacerbations triggered by respiratory pathogens mark the progression of chronic obstructive pulmonary disease (COPD), leading to substantial morbidity and mortality. Despite the life-threatening nature of these exacerbations, the underlying mechanisms remain unclear although a high number of neutrophils in the lungs of COPD patients are known to correlate with poor prognosis. We and others previously demonstrated that interleukin (IL)-22 protects and regenerates respiratory epithelial cells upon virus infections and also limits secondary bacterial infections. Indeed, IL-22, through its receptor (IL-22R), is a cytokine that plays a pivotal role in lung antimicrobial defence and tissue protection. Regarding the IL-22/IL22R antimicrobial pathway, we hypothesized that any alteration of IL-22R in the lungs of COPD patients may compromise innate defence mechanisms and enhance susceptibility to infections. More specifically, we examined whether IL-22R expressed on lung epithelial cells is targeted by the neutrophil proteases present at the surface of lung mucosa.

Materials and methods We exposed human bronchial epithelial cells and BALB/c mice to viral, bacterial agents or cigarette smoke and assessed IL-22R expression by RT-qPCR and flow cytometry. IL-22R expression was also examined in the lung tissue of COPD and control 
individuals. We also analysed the effect of neutrophil serine proteases on the expression, structure and function of IL-22R by flow cytometry and western blotting in mouse lungs, human epithelial cells and sputa from COPD patients with or without exacerbations.

Results Using in vitro and in vivo approaches as well as RT-qPCR, flow cytometry and/or western blotting techniques, we first showed that pathogens such as influenza virus promote IL-22R expression in human bronchial epithelial cells, whereas Pseudomonas aeruginosa, bacterial LPS, do not. We also investigated whether IL-22R lung expression was impaired by cigarette smoke in epithelial cells or in mice chronically exposed to cigarette smoke. Finally, we examined lung tissue from 129 patients (11\% nonsmokers, 41 \% "healthy" smokers, $48 \%$ COPD patients). IL-22R1 RNA expression was neither associated with the cigarette smoke exposure nor the COPD status.

In view of the foregoing data and the evidence that neutrophilia is a pathological hallmark of COPD, we hypothesized that neutrophils induce posttranslational modifications of IL-22R expression and function. We first exposed human epithelial cells to supernatant from activated neutrophils and observed that IL-22R1 protein expression was strongly decreased under these conditions, whereas $a-1$ proteinase inhibitor prevented the disappearance of the receptor. Most importantly, neutrophil proteases impaired IL-22-dependent immune signalling and expression of antimicrobial effectors such as $\beta$-defensin-2. This proteolysis resulted in the release of a soluble fragment of IL-22R, which was detectable both in cellular and animal models as well as in sputa from COPD patients with acute exacerbations.

Conclusion Hence, our study reveals an unsuspected regulation by the proteolytic action of neutrophil enzymes of IL-22-dependent lung host response. This process likely enhances pathogen replication and ultimately COPD exacerbations.

\section{Competing interests None.}

\section{P217}

Impaired phagocytosis of apoptotic neutrophils in human ARDS: beneficial effects of metformin

Fabrice Uhel', Murielle Gregoire², Arnaud Gacouin ${ }^{1}$, Yves Le Tulzo ${ }^{1}$, Karin Tarte', Jean-Marc Tadié

${ }^{1}$ Réanimation médicale, Centre Hospitalier Universitaire de Rennes, Rennes, France; ${ }^{2}$ Biosit and inserm u917, faculte de medecine, universite rennes i, Centre Hospitalier Universitaire de Rennes, Rennes, France

Correspondence: Jean-Marc Tadié - jeanmarc.tadie@chu-rennes.fr Annals of Intensive Care 2016, 6(Suppl 1):P217

Introduction ARDS is characterized by the accumulation of neutrophils (PMN) in the lung interstitium and alveolar space where they die in large numbers. The removal of apoptotic cells, a process known as efferocytosis, plays a crucial role in the maintenance of tissue homeostasis and resolution of inflammatory and immune responses. However, little is known about capacity of macrophages and PMN to phagocytes apoptotic PMN in ARDS patients.

Materials and methods In vitro experiments were carried out to investigate the efferocytosis ability of PMN and macrophages from ARDS and control patients. In a second set of experiments, we investigated the effects of bronchoalveolar lavage (BAL) fluids from ARDS and control patients on healthy donor PMN and macrophages efferocytosis capacity. Lastly, we studied the effect of metformin, an AMP kinase activator, on the phagocytic activity of neutrophils and macrophages in ARDS patients.

Results We demonstrated that PMN apoptosis was diminished in ARDS patients (Fig. 34. Fig 1). We also found that efferocytosis capacity was diminished (Fig. 34. Fig 2). Lastly, we found that metformin enhanced efferocytosis (Fig. 34. Fig 3).

Conclusion Efferocytosis is markedly decreased in ARDS. Metformin could be an interesting therapeutic approach to increase efferocytosis and reduce persistent pulmonary inflammation, thus decreasing fibrosis.

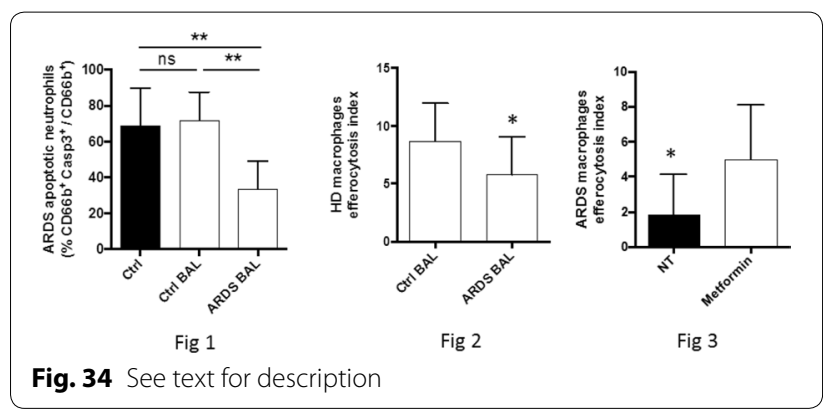

Competing interests None.

P218

What are the determinants of needle aspiration success in primary spontaneous pneumothorax?

Constance Vuillard ${ }^{1}$, Jean-Damien Ricard ${ }^{1}$, Béatrice La Combe ${ }^{1}$, Stéphane Gaudry', Didier Dreyfuss', Jonathan Messika

${ }^{1}$ Service de réanimation médico-chirurgicale, CHU Louis Mourier, Colombes, Colombes, France

Correspondence: Jonathan Messika - jonathan.messika@Imr.aphp.fr Annals of Intensive Care 2016, 6(Suppl 1):P218

Introduction Primary spontaneous pneumothorax is a common disease. Needle aspiration is recommended as first-line treatment by international guidelines and used as such in our intensive care unit. Nevertheless, little is known of factors associated with its success. Our main objective is to characterize these factors.

Patients and methods We conducted a prospective, observational, single-center study, including all patients admitted to our intensive care unit and step-down unit with a primary spontaneous pneumothorax from $01 / 2012$ to $08 / 2015$. Our primary endpoints were the number of patients with early success of needle aspiration (defined as the absence of chest-tube insertion within $24 \mathrm{~h}$ following needle aspiration), and the factors associated with early success. Secondary endpoints were number of patients with consolidated success (defined as absence of chest-tube drainage within the first week following aspiration), length of intensive care unit and hospital stay.

Results Seventy patients were admitted for primary spontaneous pneumothorax; needle aspiration was performed in 62 (89\%), 50 men (81\%), aged 31 [24-38]).

Immediate success rate was $36 \%$, and consolidated success rate was $29 \%$. Duration of evolution of pneumothorax (time from first symptoms to needle aspiration) was significantly longer when aspiration was successful (18 h [6.5-63] vs $10 \mathrm{~h}$ [5-16]; $p=0.012$ ). Factors associated with needle aspiration success were: performing needle aspiration after $24 \mathrm{~h}$ of onset of pneumothorax (success 65 vs $24 \%$; $p=0.006$ ); a smaller pneumothorax size, estimated by the Light score (29 [15-45] vs. 80 [42-94]; $p<0.0001$ ); and a lower body mass index (20 [19-22] vs 21 [20-23]; $p=0.045)$.

Patients with needle aspiration success had a significantly lower length of intensive care unit (1 [1-1.3] vs 3 days [3-5]; $p<0.0001)$ and hospital stay (1 [1-1.3] vs 6 days [3-10]; $p<0.0001)$. No adverse event of needle aspiration was reported.

Conclusion Needle aspiration was effective in a third of primary spontaneous pneumothorax treated in our institution. The immediate success rate was significantly increased when aspiration was performed after $24 \mathrm{~h}$ of onset of the pneumothorax. Needle aspiration success enabled a significant reduction in length of intensive care unit and hospital stay and hence costs.

Competing interests None. 
P219

Emergency thoracotomy: development and results in a military trauma center

Pauline Ponsin ${ }^{1}$, Jean-Louis Daban², Guillaume Boddaert ${ }^{3}$, Bertrand Grand $^{3}$, Vincent Peigne ${ }^{2}$, Yohan Baudoin ${ }^{4}$, Mathieu Boutonnet ${ }^{2}$, Patrick Jault ${ }^{5}$, Stéphane Bonnet ${ }^{4}$

${ }^{1}$ Hôpital d'Instruction des Armées Percy, Clamart, France; ${ }^{2}$ Réanimation, Hôpital d'Instruction des Armées Percy, Clamart, France; ${ }^{3}$ Chirurgie thoracique et vasculaire, Hôpital d'Instruction des Armées Percy, Avenue Henri Barbusse, Clamart, France, Clamart, France; ${ }^{4}$ Chirurgie viscérale, Hôpital d'Instruction des Armées Percy, Avenue Henri Barbusse, Clamart France, Clamart, France; ${ }^{5}$ Département d'Anesthésie-Réanimation, Hôpital d'Instruction des Armées Percy, Clamart, France

Correspondence: Pauline Ponsin - pauline_2014@hotmail.com

Annals of Intensive Care 2016, 6(Suppl 1):P219

Introduction Emergency thoracotomy is a salvage procedure for trauma patients. Successes have been reported, including in some cases of war casualties [1]. A process was implemented in our military trauma center to improve the practice of emergency thoracotomy. The aim of this study is to describe the feasibility and the results of our program of emergency thoracotomy.

Patients and methods This retrospective study included all the patients for whom emergency thoracotomy was considered between September 2013 and July 2015 at Percy Military Teaching Hospital. During this period, the equipment required to perform a resuscitative thoracotomy was gathered in a box at the emergency room, and a procedure was set on to admit trauma patients directly at the operating room.

Results During the study period, 399 trauma patients who fulfilled at least one of the Vittel's criteria of severity were admitted in our center. Resuscitative thoracotomy was considered for eight of them who were pulseless during the pre-hospital management or at admission. Two patients had cardiac arrest related to cervical spinal injury, and thoracotomy was not performed in these cases. The remaining six patients had emergency thoracotomy. The delay between admission and incision was inferior to $5 \mathrm{~min}$ for all patients. Results are reported in Table 42. Return of spontaneous circulation could not be obtained in any of the four patients with asystole. A sustained spontaneous circulation was achieved in one patient: multiple organ failure occurred and the patient died in ICU $15 \mathrm{~h}$ after the procedure.

Discussion Although resuscitative thoracotomy has been performed very quickly after admission, all our patients died. This result can be explained, at least partially, by the prolonged time of cardiac arrest before admission and the frequency of blunt trauma among our patients. Some of the procedures we performed may even be considered as futile according to recommendations suggesting not to realize thoracotomy in case of blunt chest trauma or cardiopulmonary resuscitation lasting more than $10 \mathrm{~min}$ without returning to circulatory activity [2]. However, other centers have reported such a large proportion of inadequate procedure. Reducing the delays between trauma and admission and between cardiac arrest and admission could help to improve the outcome of the patients.

Conclusion We achieved the development of a process to perform resuscitative thoracotomy less than 5 min after admission of pulseless trauma patients. Unfortunately, such a management did not improve the outcome of the patients. The duration of the pre-hospital phase and the high proportion of blunt trauma can explain at least partially these results.

\section{Competing interests None.}

\section{References}

1. Mitchell TA, Waldrep KB, Sams VG, et al. An 8-year review of operation enduring freedom and operation Iraqi freedom resuscitative thoracotomies. Mil. Med. 2015; 180: 33-36.

2. Burlew CC, Moore EE, Moore FA, et al. Western Trauma Association critical decisions in trauma: resuscitative thoracotomy. J. Trauma Acute Care Surg. 2012; 73: 1359-1363.
Table 42 See text for description

\begin{tabular}{|c|c|c|c|c|c|c|}
\hline & Patient 1 & Patient 2 & Patient 3 & $\begin{array}{l}\text { Patient } \\
4\end{array}$ & Patient 5 & Patient 6 \\
\hline $\begin{array}{l}\text { Age/gen- } \\
\text { der }\end{array}$ & 19 & 22 & 46 & 26 & 89 & 16 \\
\hline $\begin{array}{l}\text { Mecha- } \\
\text { nism of } \\
\text { injury }\end{array}$ & $\begin{array}{l}\text { Blunt } \\
\text { Crush }\end{array}$ & $\begin{array}{l}\text { Blunt } \\
\text { Fall }\end{array}$ & $\begin{array}{l}\text { Blunt } \\
\text { Car acci- } \\
\quad \text { dent }\end{array}$ & $\begin{array}{l}\text { Pen- } \\
\text { etrant } \\
\text { Ballistic }\end{array}$ & $\begin{array}{l}\text { Penetrant } \\
\text { Ballistic }\end{array}$ & $\begin{array}{l}\text { Blunt } \\
\text { Fall }\end{array}$ \\
\hline $\begin{array}{l}\text { Site of } \\
\text { admis- } \\
\text { sion } \\
\text { and } \\
\text { thora- } \\
\text { cotomy }\end{array}$ & $\begin{array}{l}\text { Operat- } \\
\text { ing } \\
\text { room }\end{array}$ & $\begin{array}{l}\text { Emer- } \\
\text { gency } \\
\text { depart- } \\
\text { ment }\end{array}$ & $\begin{array}{l}\text { Emer- } \\
\text { gency } \\
\text { depart- } \\
\text { ment }\end{array}$ & $\begin{array}{l}\text { Operat- } \\
\text { ing } \\
\text { room }\end{array}$ & $\begin{array}{c}\text { Operating } \\
\text { room }\end{array}$ & $\begin{array}{l}\text { Operating } \\
\text { room }\end{array}$ \\
\hline $\begin{array}{l}\text { Heart } \\
\text { activ- } \\
\text { ity at } \\
\text { admis- } \\
\text { sion }\end{array}$ & Asystole & $\begin{array}{l}\text { Pulseless } \\
\text { electri- } \\
\text { cal } \\
\text { activity }\end{array}$ & Asystole & Asystole & $\begin{array}{l}\text { Pulseless } \\
\text { electri- } \\
\text { cal } \\
\text { activity }\end{array}$ & Asystole \\
\hline $\begin{array}{l}\text { Duration } \\
\text { of the } \\
\text { pre- } \\
\text { hospital } \\
\text { man- } \\
\text { age- } \\
\text { ment } \\
\text { (min) }\end{array}$ & 180 & 117 & 168 & 57 & 47 & 132 \\
\hline $\begin{array}{l}\text { Duration } \\
\text { of the } \\
\text { cardiac } \\
\text { arrest } \\
\text { before } \\
\text { admis- } \\
\text { sion } \\
\text { (min) }\end{array}$ & 58 & 0 & $?$ & 60 & 0 & 40 \\
\hline $\begin{array}{c}\text { Use of an } \\
\text { auto- } \\
\text { pulse } \\
\text { shelf }\end{array}$ & Yes & No & No & Yes & No & No \\
\hline $\begin{array}{l}\text { Informa- } \\
\text { tion of } \\
\text { cardiac } \\
\text { arrest } \\
\text { given } \\
\text { by pre- } \\
\text { hospital } \\
\text { team }\end{array}$ & Yes & Yes & No & Yes & No & Yes \\
\hline \multicolumn{7}{|l|}{$\begin{array}{l}\text { Transfu- } \\
\text { sion }\end{array}$} \\
\hline $\begin{array}{l}\text { Red blood } \\
\text { cells }\end{array}$ & 4 & 5 & 4 & 8 & 10 & 6 \\
\hline Plasma & 2 & 2 & 4 & 6 & 4 & 3 \\
\hline Platelets & 0 & 0 & 0 & 0 & 1 & 0 \\
\hline Death & Yes & Yes & Yes & Yes & Yes & Yes \\
\hline
\end{tabular}

P220

Management of isthmic aortic rupture in polytraumatized patients in ICU: a report of 12 cases

Chtara Kamilia', Kais Regaieg ${ }^{1}$, Olfa Turki ${ }^{1}$, Najeh Baccouch ${ }^{2}$, Anis Chaari ${ }^{1}$, Hmida Chokri Ben ${ }^{1}$, Mabrouk Bahloul ${ }^{1}$, Mounir Bouaziz ${ }^{3}$

${ }^{1}$ Réanimation polyvalente, Faculté de médecine de Sfax, Sfax, Tunisia;

${ }^{2}$ Réanimation polyvalente, CHU Habib Bourguiba, Sfax, Tunisia; ${ }^{3} \mathrm{lcu}, \mathrm{CHU}$ Habib Bourguiba, Sfax, Tunisia

Annals of Intensive Care 2016, 6(Suppl 1):P220 
Introduction Traumatic rupture of aortic isthmus is a major problem in the management of polytraumatized patient. Except the complete rupture of the aorta, treatment should be initiated after hemodynamic and respiratory stabilization and after treating a lesion involving life-threatening.

Patients and methods This is a prospective analysis over a period of 18 months between January 2014 and July 2015. Twelve polytraumatized patients were admitted to our ICU with rupture of aortic isthmus. All patients underwent a whole body $\mathrm{CT}$ with angio-CT of mediastinal vessels.

Results During this period, among 300 polytraumatized patients were admitted to our ICU, 180 of them had chest trauma (60\%) of which 12 patients had a traumatic rupture of aortic isthmus $(4 \%)$. The median age was 43 [27-64] years. All patients were male. The mechanism of injury was road traffic accident (motorcycle in 2 cases, motor vehicle in 5 cases). All patients required mechanical ventilation due to respiratory or neurological distress. Four patients $(25 \%)$ had a Glasgow Coma Scale Score less than 12/15. Eight patients (75 \%) had a hypoxemic report $\mathrm{PaO}_{2} / \mathrm{FiO}_{2}$ less than 200 . One patient was initially in shock. The median SAPSII was 28 [22-48]. The median SOFA score was 3 [2-5]. The median Shock Index was 0.70 [0.61-0.97]. After hemodynamic and respiratory stabilization, all patients underwent a whole body $\mathrm{CT}$ with angio-CT of mediastinal vessels. We noted head trauma in 4 patients $(25 \%)$, abdominal trauma in 9 patients $(75 \%)$, fractures of pelvis in 6 patients (50\%) and fractures of the long bones in 10 patients ( $83.3 \%$ ). The rupture of aortic isthmus was associated with odds fractures in 10 patients $(83.3 \%)$, pneumothorax in 6 patients $(50 \%)$, hemothorax in 5 patients $(41 \%)$ and pulmonary contusions in all patients. A tracheotomy was performed in 8 patients (66.6 \%). The ICU stay was complicated by nosocomial pneumonia in all patients and pulmonary embolism in 6 patients $(50 \%)$, septicemia in 5 patients $(41 \%)$. Eleven of 12 patients underwent endovascular treatment. The median interval between admission in ICU and endovascular treatment was 18 [7-30] days. All patients received a single stent, mean length $25 \pm 1 \mathrm{~mm}$. There was no conversion to an open approach. The median ICU stay was 30 [14-48] days. Two patients died: the first before endovascular treatment because of cerebral herniation, the second after endovascular treatment because of a septic shock. All surviving patients had a clinical and radiological follow-up every 3 months. No morbidity was noted until this day.

Conclusion Diagnosis of traumatic rupture of aortic isthmus should be considered in any serious polytrauma. Except the complete rupture of the aorta, treatment of these lesions can be done after stabilization of a lesion involving the immediate prognosis. Endovascular treatment became the treatment of choice, especially for patients with severe associated injuries and bleeding risk. Additional data on the long-term stents are necessary in these young patients.

\section{Competing interests None.}

\section{P221}

NT-proBNP levels and echocardiography findings in the etiologic diagnosis of acute dyspnea

Salah Snouda', Hassen Ben Ghezala', Mohamed Fehmi Abbes², Rebeh Daoudi ${ }^{1}$, Moez Kaddour', Imen Benchiekh'

${ }^{1}$ Teaching department of emergency and intensive care, Regional hospital of Zaghouan, Zaghouan, Tunisia; ${ }^{2}$ Cardiology, Regional hospital of Zaghouan, Zaghouan, Tunisia

Correspondence: Salah Snouda - dr.snouda.rea@gmail.com

Annals of Intensive Care 2016, 6(Suppl 1):P221

Introduction Both NT-proBNP and Doppler echocardiography have been approved in the diagnosis of heart failure. In our study, we compared the contribution of the NT-proBNP levels with the Doppler echocardiography findings in the diagnosis of decompensated congestive left heart failure (CHF) in patients with acute dyspnea.
Patients and methods It was a prospective, observational study at the teaching department of emergency and intensive care in the regional Hospital of Zaghouan, including patients with severe dyspnea over 6 months. All patients underwent physical examination, 12-lead ECG, RX Thorax, NT-proBNP essay and echocardiography by an attending cardiologist on admission. The accuracy of the two methods for etiologic diagnosis was compared on the basis of the final diagnoses established by the medical staff.

Results Sixty-five patients were enrolled, including 45 (69\%) with CHF. Diagnosis of CHF was due to coronary artery disease, hypertension, valve disease, arrhythmia and dilated cardiomyopathy. Non-CHF was due to decompensated chronic obstructive pulmonary disease, pneumonia and severe asthma. Fifteen patients $(23 \%)$ were misdiagnosed at admission. The mean NT-proBNP concentration was 8989 [769 to 18945 ] pg/ml in the CHF subgroup and 462 [22 to 1589] pg/ $\mathrm{ml}$ in the other patients $(p<0.01)$. Systolic LV dysfunction (LVEF $<0.45$ ) was found in 31 patients with CHF $(60 \%)$ and in 7 patients with other causes of dyspnea $(15 \%)(p<0.01)$. The E/A ratio and the deceleration time of E-wave (DT) were, respectively, $1.85 \pm 0.77$ and $120 \pm 23$ in the group CHF; $0.81 \pm 0.44$ and $208 \pm 47$ in the group Non-CHF $(p<0.01)$. Impaired relaxation and restrictive mitral pattern were observed, respectively, in 28 and $30 \%$ of the patients with $\mathrm{CHF}$ and in only two patients and three patients in the other group.

Conclusion Both NT-proBNP assay and echocardiography can be used for the diagnosis of CHF in acutely dyspneic patients. However, the echocardiography is more accurate in patients with intermediate BNP levels.

\section{Competing interests None.}

\section{P222}

\section{Cardiac decompensation in peripartum}

Kamal Belkadi', I. Moussaid', Chaigar Mohammed Cheikh'1, S. El

Youssoufi', S. Salmi

${ }^{1}$ Anesthesie reanimation, $\mathrm{CHU}$ Ibn Rochd Casa, Casablanca, Morocco Correspondence: Chaigar Mohammed Cheikh - chaigarmed@gmail. com

Annals of Intensive Care 2016, 6(Suppl 1):P222

Introduction Heart diseases are not uncommon in pregnancy $(0.5-2 \%)$ (1). Physiological changes, including cardiovascular and respiratory ones observed during pregnancy, may exacerbate preexisting heart disease. The aim of our study was to describe the epidemiological, clinical profile, paraclinical and therapeutic cardiac decompensation in peripartum and to analyze the factors influencing prognosis.

Patients and methods Transversal retrospective study spread over 13 years, from January 1, 2000, to December 31, 2013, including all women in labor admitted in obstetrical ICU for heart failure, with or without known heart disease.

Results During the study period, 223 patients have been collected, with an incidence of $2.5 \%$. The average age was $32.77 \pm 6.74$, the medical story of rheumatic fever was frequently found (75\%), and $79 \%$ of women in labor were followed for rheumatic valve diseases. About $38.1 \%$ were followed by a cardiologist, while $23.8 \%$ of heart diseases have been discovered only during pregnancy. More than half of the pregnancies were not followed by an obstetrician (65.5\%). Cardiac decompensation the most common was acute heart failure in $43.7 \%$, followed by arrhythmia in $32.2 \%$ and cardiac shock in $21.9 \%$. Symptoms was dominated by dyspnea in $97 \%$ and palpitations in $84.3 \%$; majority of patients were stage III or IV of NYHA classification (92.3\%). About $62.8 \%$ of patients were delivered by cesarean and under general anesthesia in $31 \%$ of cases. The maternal and fetal mortality were, respectively, 10.8 and $26 \%$. Univariate analysis had identified the rural environment, discovering the cardiac disease during pregnancy, the lack of following in pregnancy and in heart disease, 
impaired ejection fraction, pulmonary hypertension and left obstructive heart disease as severe prognostic factors.

Conclusion Pregnancy and delivery are complicated situations with a high maternal and fetal risk, requiring early treatment and multidisciplinary care, which had to start before planning pregnancy and longlasting during the postpartum period improving maternal and fetal prognosis.

\section{Competing interests None.}

\section{Reference}

1. J.Grewal et al. pregnancy in women with heart disease: risk assessment and management of heart failure. Heart Failure Clinics 2014.

\section{P223}

\section{Peripheral intravenous catheter-related complications in an} emergency ward

Ines Sedghiani ${ }^{1}$, Hana Fredj ${ }^{1}$, Hamdi Doghri ${ }^{1}$, Youssef Zied El Hechmi ${ }^{1}$, Mohamed Ali Cherif', Jerbi Zouheir ${ }^{1}$

${ }^{1}$ Emergency and Intensive Care Department, Hopital Habib Thameur, Tunis, Tunisia

Correspondence: Ines Sedghiani - sedghiani.ines@gmail.com Annals of Intensive Care 2016, 6(Suppl 1):P223

Introduction Peripheral intravenous catheter (PIVC) is the most commonly used vascular access on hospitalized patients. It remains susceptible to infectious and non-infectious complications, which are the cause of considerable morbidity. The aim of this study was to assess the pattern and complications of PIVC among patients admitted into emergency ward.

Patients and methods We conducted a prospective observational study in an emergency ward between February 1, 2015, and April 30, 2015. All hospitalized patients who underwent PIVC insertions during the time of the study were included. PIVC-related characteristics and clinical outcomes were assessed every $8 \mathrm{~h}$. When an individual patient had more than one PIVC inserted during the study period; each was regarded as a separate event.

Results Two hundred and thirty-nine PIVC in 153 patients were followed up during a total of 2.7 catheter days. All patients were complaining of medical problems. Mean age was 68 years (52-78).

The majority ( $99 \%)$ of intravenous lines was inserted by the nursing staff in wrists (26\%), upper arms (38\%), forearms ( $22 \%)$ and ante cubital fossa (14\%). Catheter sizes were 18 Gauge in $29 \%$ of the cases, 20 Gauge in $48 \%$ of the cases and 22 Gauge in $23 \%$ of the cases. Gauze dressings were applied for the majority of patients $(97 \%)$. One hundred and eight (45\%) PIVCs were inserted in the resuscitation room, while 131 (55 \%) PIVCs were inserted in the acute care unit. PIVCrelated complications occurred in $40 \%$ of the cases after $36 \mathrm{~h}$ dwell time. Clinical outcomes were pain (5\%), erythema (5\%), palpable venous cord (4\%), infiltration (9\%), occlusion (9\%) and accidental removal $(7 \%)$.

Hydration was the most frequent infusate $(55 \%)$ followed by antibiotics $(28 \%)$ and blood products (5\%). PIVC-related complication was predicted with antibiotics administration ( $p=0.001$, OR 2.5$)$ and was associated with prolonged hospital stay ( 6 vs 2 days for non-complicated PIVC).

Conclusion Incidence of peripheral intravenous catheter-induced complications in the emergency ward was high. Antibiotic administration was a risk factor for these complications. Better insertion techniques may reduce hospital stay.

\section{Competing interests None.}

\section{P224}

\section{Burnout in the emergency room}

Neila Maaroufi', Abid Zeineb

${ }^{1}$ General Hospital, Tunis, Tunisia; ${ }^{2}$ Emergency, General Hospital, Tunis, Tunisia
Correspondence: Neila Maaroufi - maaroufineila@gmail.com Annals of Intensive Care 2016, 6(Suppl 1):P224

Introduction Burnout has been defined as a syndrome of emotional exhaustion, depersonalization and reduced personal accomplishment. Emergency services are the busiest and the most stressful units of hospitals.

Aim: To assess the prevalence of burnout among Tunisian public hospital doctors and nurses and to determine its causes and perceived repercussions.

Methods: in a first step, we assessed the work conditions, the perceived burnout and its possible consequences in 100 healthcare providers from two departments of emergency of two hospitals from the North of Tunisia. In a second step, we used the Maslach Burn Inventory to assess the burnout symptoms in ours ample.

Results More than half ( $56 \%$ ) of our sample had a burnout. Fortyseven percentage of the affected professionals had a high level of emotional distress, $36.6 \%$ had a high level of depersonalization and $33 \%$ had a low level of professional fulfillment. Burnout had a high level in nurse which affects three dimensions relative to physicians. A high level of burnout had found in healthcare provider, which had sick leave with emotional distress $(32 \%)(p=0.02)$, depersonalization (21\%) and a low level of professional fulfillment (11\%).

Discussion and conclusion Our results corroborate partially with those of the literature and illustrate some parameters that can be the cause of burnout, such as working conditions, role ambiguity, and lack of experience among the young nurse. Therefore, it is important to take into consideration this various factors and their impact on quality of life of caregivers.

\section{Competing interests None.}

P225

Prevalence, and characteristics of elderly patient admitted in a Moroccan Medical Unit for the Acute Care

Jihane Belayachi', Leila Debono ${ }^{1}$, Naoufal Madani ${ }^{1}$, Fatiha Amlaiky', Tarek Dendane², Khalid Abidi², Aicha Zekraoui', Amine Ali Zeggwagh², Redouane Abouqal ${ }^{1}$

${ }^{1}$ Medical Emergency Department, Ibn Sina University Hospital, Rabat, Morocco; ${ }^{2}$ Service de réanimation médicale et de toxicologie cliniquechu ibn sina-universite mohamed v, Hopital Ibn Sina, Rabat, Morocco

Correspondence: Jihane Belayachi - jihanebelayachi@gmail.com Annals of Intensive Care 2016, 6(Suppl 1):P225

Introduction Geriatric patients remain largely unstudied in lowmiddle income healthcare settings. The purpose of this study was to compare the characteristics and outcomes of older (age $\geq 65$ years) versus younger adults hospitalized in a Medical Unit for the Acute Care (MUAC).

Patients and methods It was a cross-sectional survey, conducted between January and June 2015 in MUAC of Ibn Sina University Hospital in Rabat, Morocco. Patient demographics, comorbidities, clinical, paraclinical characteristics, and outcomes of patient were included. Activity of daily living (ADL) 1 month before admission, on admission, and 1 month, and 3 month after discharge was assessed. Simplified Acute Physiology Score (SAPS II) and Charlson scale are also included. Outcomes measures included length of stay (in ED and hospital), and mortality (in ED and hospital, and 1 month, and three after discharge). Patients categorized as older ( $\geq 65$ years) versus younger ( $<65$ years) adults were compared for differences using descriptive statistics.

Results We included 534 patients; older patients constituted $43.1 \%$ $(n=230)$ of the cohort. Among older adults compared with younger; older patients were mainly women [136 (61\%) vs $87(39 \%)$ ] and affected by a high level of comorbidity [152 (74.1\%) vs $147(52 \%) ; p<0.001]$, with a better therapy compliance [65 (36.3\%) vs $108(51 \%) ; p=0.006]$. Concerning clinical and paraclinical characteristics, older adults had more altered mental state [39 (19.1\%) vs $33(11.8 \%) ; p=0.02]$, more extracellular dehydration signs [73 (36\%) vs $35(12.6 \%) p<0.0001]$, 
more bedsores [11 (5.4\%) vs $0 ; p<0.0001]$, more radiological abnormalities in chest X-ray [141 (68.8\%) vs $124(44.8 \%) ; p<0.0001$, more elevated CRP [113.7 \pm 105 vs $98 \pm 90 ; p=0.18]$, lower clearance of creatinine [42.6 \pm 26.8 vs $66.7 \pm 42.6 ; p<0.001]$, and higher kalemia $[4.5 \pm 0.8$ vs $4.2 \pm 0.9 ; p=0.01]$.

Relative to younger patients, older patients have higher Saps II and Charlson scale, respectively ( $18 \pm 9$ vs $30 \pm 8),(1.4 \pm 1.6$ vs $4 \pm 1.8)$, and lower ADL one month before admission ( $5.7 \pm 0.8$ vs $4.9 \pm 1.7)$, on admission ( $3.4 \pm 2.1$ vs $2.1 \pm 2$ ), and one month ( $5.1 \pm 1.7$ vs $3.8 \pm 2.3)$, and three month after discharge $[(5.2 \pm 1.6$ vs $4.6 \pm 1.9) p=0.003]$. Median [IQR] of length of stay in ED and in hospital was, respectively, 6 [3-10] and 8 [4-14] with no difference in the 2 groups. Cumulative mortality three month after discharge was $105(29.6 \%)$ with higher mortality in older patients relative to younger: (a) in ED [30 (14.3\%) vs $12(4.1 \%)]$, (b) in hospital [9 (4.2\%) vs $10(3.4 \%)]$, (c) 1 month after discharge; [21 (16.8\%) vs $15(7.5 \%)$ ], and d) three month after discharge [5 $(9.8 \%)$ vs $3(3.6 \%)$ ].

Conclusion Nearly half of Inpatients are aged 65 years and over. Older patients are more seriously ill, less independent, and with a higher mortality rate. Understanding unique needs of geriatric patients is critical to enhancing the management and prioritization of appropriate care in developing settings.

\section{Competing interests None.}

\section{P226}

Metabolic phenotyping in bronchoalveolar lavage fluids distinguishes influenza and pneumococcal pneumonia Adrien Auvet ${ }^{1}$, Antoine Guillon' ${ }^{1}$ Lydie Nadal-Desbarats ${ }^{2}$, Thomas Baranek ${ }^{3}$, Eric Morello ${ }^{3}$, François Réminiac ${ }^{3}$, Mustapha Si-Tahar ${ }^{3}$ ${ }^{1}$ Réanimation polyvalente, CHRU Hôpitaux de Tours, Tours, France;

2Université françois rabelais, INSERM U930 équipe 2, Tours, France, France; ${ }^{3}$ Inserm u1 100, Centre d'étude des pathologies respiratoires, Tours, France Correspondence: Adrien Auvet - aauvet@hotmail.fr Annals of Intensive Care 2016, 6(Suppl 1):P226

Introduction Metabolomic analysis by untargeted nuclear magnetic resonance spectroscopy (1H-RMN) allows a global assessment of endogenous metabolites within a biological system. A metabolic profile can be modified during various pathological states and can be considered as a "signature" of the disease. Here, we hypothesized that the metabolic profile of a respiratory fluid can be modified upon a lung infection and can be specific of an antimicrobial host response and/or a pathogen subtype. Consequently, metabolic analysis may help orient the diagnosis and management of severe pneumonia. As Influenza A virus (IAV) and Streptococcus pneumonia (SP) are the most commonly pathogens involved in the community-acquired pneumonia (CAP), the aim of our study was to characterize the metabolic profiles of respiratory fluids during acute lung infection by IAV or SP.

Materials and methods We developed C57BL/6 mouse models of CAP by intranasal instillation of a lethal dose of IAV or SP. Disease severity was evaluated based on clinical (i.e., lung plethysmography, animal weight), inflammatory (i.e. IL-6 release, neutrophil recruitment) and histopathological measurements. Metabolic profiles of bronchoalveolar lavage (BAL) fluids were further obtained by $1 \mathrm{H}-\mathrm{RMN}$. Data were classified according to the metabolic profile using a multivariate analysis in principal component analysis. Then, discriminatory metabolites were identified and quantified according to their spectral region.

Results We first defined the time point at which severity of lung damages was similar in both IAV- and SP-infected animals. Metabolomic analysis of BAL fluids further identified 29 metabolites, which were modified during pulmonary infection. In non-supervised principal component analysis, spectral differences could explain $94 \%$ of the variance. Moreover, metabolic profiles could differentiate IAV versus SP as the etiological agent. Metabolomic analysis correctly predicted the pathogen diagnosis in $86 \%$ of cases.
Conclusion Development of innovative microbial technologies increases the detection sensitivity of microorganisms in the airways. Nevertheless, it is still challenging to distinguish between infectious microorganisms that contribute to the disease per se and those who are merely bystanders. Here, we demonstrated that CAP due to IAV or SP results in distinct metabolic host responses (similar to a specific "metabolic fingerprint"). We believe that this innovative approach may help to match the host metabolic signature with a microorganism at the origin of lung diseases onset and/or progression.

\section{Competing interests None.}

\section{P227}

Microbiological documentation of pneumonia in the ICU

by per-bronchoscopic bronchial aspiration (PBA) is very similar to that obtained by bronchoalveolar lavage (BAL)

Jean-Luc Baudel', Jacques Tankovic², Redouane Dahoumane ${ }^{2}$, Arnaud Galbois ${ }^{1}$, Hafid Ait Oufella', Georges Offenstadt ', Eric Maury', Bertrand Guidet

${ }^{1}$ Réanimation médicale, Hôpital Saint-Antoine, Paris, France; ${ }^{2}$ Bactériologie, Hôpital Saint-Antoine, Paris, France

Correspondence: Jacques Tankovic - jtankovic@yahoo.fr Annals of Intensive Care 2016, 6(Suppl 1):P227

Introduction In patients hospitalized in the ICU for pneumonia, the realization of a bronchoalveolar lavage (BAL) for microbiological documentation can be detrimental to the respiratory function. Per-bronchoscopic bronchial aspiration (PBA) is less aggressive. We wanted to determine whether the documentation obtained by PBA is equivalent to that of BAL.

Patients and methods This is a prospective, observational study. All patients hospitalized in the ICU for community- or hospital-acquired pneumonia, who underwent fiberoptic bronchoscopy with realization of the two types of respiratory samples, were enrolled. A PBA in the bronchial segment corresponding to the pulmonary infiltrate was performed, the operative channel of the endoscope was washed with $20 \mathrm{ml}$ of $0.9 \%$ saline, and a BAL was realized with five aliquots of $20 \mathrm{ml}$ of $0.9 \%$ saline. The first was discarded as recommended. One was sent to the bacteriology laboratory together with the PBA. The samples were diluted with $0.9 \%$; homogenized and quantitative cultures were realized. Cultures were considered significant if they attained at least $105 \mathrm{CFU} / \mathrm{ml}$ for PBA and at least $104 \mathrm{CFU} / \mathrm{ml}$ for BAL.

Results During a 15-month period, 104 cases were analyzed. Ten patients were included two times. Mean age was $66 \pm 15$ years, $70 \%$ of patients were men. Fifty-eight percentage of the cases were community-acquired. In 45 cases (43\%), both samples provided identical significant (above threshold) microbiological documentation. In 3 cases $(3 \%)$, both samples provided identical nonsignificant (below threshold) microbiological documentation. In 30 cases (29\%), both samples could not document the infection. Thus, microbiological data were fully concordant in 78 cases $(75 \%)$. In 13 cases $(12.5 \%)$, the microorganisms found were identical but significant (above threshold) in one of the 2 samples only (BAL: 6, PBA: 7). In the 13 remaining cases (12.5\%), a microbiological documentation was obtained in only one sample (BAL: 5, PBA: 8), above threshold in 6 cases (BAL: 3, PBA: 3), below threshold in 7 cases (BAL: 2, PBA: 5). In total, 96 bacteria were retrieved: Enterobacteria (25 including 11 strains of Escherichia coli), Streptococcus pneumonia (20), Pseudomonas aeruginosa (19), Staphylococcus aureus (16), Haemophilus influenzae (6), various microorganisms (10). The microbial documentation was monomicrobial in 51 cases (69\%).

Conclusion Microbial documentation of pneumonia in ICU by PBA is very similar to that by BAL, suggesting that PBA could replace BAL in routine.

Competing interests None. 
P228

Compliance with the $\mathrm{H} 3$ bundle of the 2012 surviving sepsis campaign according to the chain of care of septic patients: the sepsis CHOC study

Amel Filali ${ }^{1}$, Emmanuel Faure ${ }^{2}$, Nicolas Van Grunderbeeck ${ }^{3}$, Olivier Nigeon ${ }^{4}$, Juliette Masse ${ }^{4}, \mathrm{H}_{\text {Bazus }}{ }^{5}$, Jihad Mallat ${ }^{3}$, Didier Thevenin ${ }^{3}$ ${ }^{1}$ Maladies infectieuses, C.H.R.U. Lille, Lille, France; ${ }^{2}$ Groupe de recherche translationnelle relation hôte-pathogène, Universite Lille 2 - Droit et Santé, Lille, France; ${ }^{3}$ Réanimation Polyvalente \& USC, Centre Hospitalier de Lens, Lens, France; ${ }^{4}$ Service d'accueil des urgences \& usc, Centre Hospitalier de Lens, Lens, France; ${ }^{5}$ Maladies infectieuses, Centre Hospitalier de Lens, Lens, France

Correspondence: Nicolas Van Grunderbeeck - nicovgdb9@orange.fr Annals of Intensive Care 2016, 6(Suppl 1):P228

Introduction Compliance with the Surviving Sepsis campaign (SSC 2012) bundle improves outcome of severe sepsis and septic shock. Thus, prognosis of patients partly depends on their initial management, including early diagnosis, symptomatic and etiological treatment. In this study, we compared compliance with the SSC 3-h bundle according to the initial chain of care of patients admitted in the intensive care unit for severe sepsis and septic shock.

Materials and methods We conducted a monocentric, prospective and retrospective study in a 500-bed general hospital.

For each patient, we noted initial patient severity (RISCC Score, IGS2, Sepsis score), compliance and timing of lactate dosing, blood culture sampling, empiric antibiotic therapy, fluid resuscitation and compliance to the global $\mathrm{H} 3$ bundle according to the initial chain of care: patient admitted in the intensive care unit after admission in the Emergency Department (ED), from the ward (W) or from another hospital or clinic (EXT). We also compared days 14 and 28 mortality according to the chain of care

Statistical analysis was performed with Chi square and Kruskal-Wallis test and logistic regression.

Results We included 79 patients: 43 hospitalized after emergency care, 22 from the ward and 15 from another hospital or clinic.

Significantly, compliance to the bundle is significantly lower in patients previously hospitalized in a conventional hospital care (medical or surgical) compared to patients hospitalized after emergency care.

This difference is significant for different components of the $\mathrm{H} 3$ bundle, with percentage of compliance reported as follows: $\mathrm{H} 3$ blood cultures ED $75 \%$, W 30 \%, EXT $25 \%$ ( $p=0.001)$, H3 lactate ED $83 \%$, W $30 \%$, EXT $63 \%(p=0.00)$, H3 antibiotics ED $52 \%$, W $9 \%$, EXT $20 \%$ $(p=0.001)$, with the exception of the fluid therapy timing (NS). The ED patients' management fulfilled more frequently the global $\mathrm{H} 3$ bundle $(48 \%)$ than for those from the ward $(10 \%)$ or from exterior care $(15 \%)$ $(p=0.006)$.

In multivariate analysis, survival is associated with $\mathrm{H} 1$ antibiotics (OR 7.7; $p=0.03$ ) in the emergency group and blood cultures sampling in the conventional hospital group (OR 3.3; $p=0.01$ ).

Conclusion It seems crucial to develop ways to improve the chain of care for patients at risk for poor management and poor outcome. Ward patients appear at high risk for delayed diagnosis and delayed empiric antibiotic treatment.

Improvement of sepsis chain of care involves better screening and early treatment, better "post-emergency" orientation and increase in sepsis awareness of medical and paramedical teams, especially in the ward.

\section{Competing interests None.}

\section{References}

1. Rhodes A, Phillips G, Beale R, et al. The Surviving Sepsis Campaign bundles and outcome: results from the International Multicentre

Prevalence Study on Sepsis (the IMPreSS study). Intensive Care Med. 2015 Sep;41(9):1620-8.

2. Dellinger RP, Levy MM, Rhodes A, et al. Surviving Sepsis Campaign: international guidelines for management of severe sepsis and septic shock, 2012. Intensive Care Med. 2013 Feb;39(2):165-228.
P229

CRP ratio as a predictive tool for mortality in ICU septic patients

Sophie Lempereur-Legros ${ }^{1}$, Didier Ledoux ${ }^{2}$, Monique Nys ${ }^{2}$, Pierre Damas ${ }^{3}$

${ }^{1}$ ICU, CHR Verviers, Verviers, Belgium; ${ }^{2}$ Soins intensifs généraux, CHU Sart-Tilman, Liège, Belgium; ${ }^{3}$ Intensive Care, CHU Sart-Tilman, Liège, Belgium

Correspondence: Didier Ledoux - dledoux@chu.ulg.ac.be

Annals of Intensive Care 2016, 6(Suppl 1):P229

Introduction It is well accepted that outcome of infected ICU patients depends-among other factors-on age, severity of illness and therapy appropriateness. However, the response to anti-infectious therapy could be another indicator of outcome.

Patients and methods The study was conducted from September 1,2014 , to June 30,2015 , in 5 ICU units from a tertiary hospital. ICU patients ventilated for more than 2 days and treated for an infection were prospectively followed. For patients who developed an infection, only the first episode was taken into account. Age, sex, SAPS II on admission, severity of sepsis, SOFA and CRP levels were collected, as well as, for those who were actually infected, therapy appropriateness. The response to treatment was estimated by the CRP ratio between day 5 and day 1. Delta SOFA was also calculated between day 5 and day 1. Patients were classified into three groups according to their CRP ratio: fast responders if CRP ratio was $\leq 0.4$; slow responders if CRP ratio was $>0.4$ and $\leq 0.8$; and non-responders if CRP ratio was $>0.8$.

Results Two hundred and sixty-nine ventilated patients (95 women and 174 men) were treated for an infection, 170 on admission and 99 during their ICU stay. Their mean age was $65 \pm 15$ years, and mean SAPS II was $48 \pm 15$. At day 1 of infection, mean CRP was $211 \pm 117 \mathrm{mg} / \mathrm{l}$ and the mean SOFA score $7.3 \pm 3.7$. Infection could not be confirmed in 11 (4\%) patients. According to bacteriological results, 157 (61\%) treatments were appropriate and 45 (17\%) were inappropriate. In 56 (22\%) treatment efficacy was not evaluable. At day 5 , mean CRP level was $125 \pm 93 \mathrm{mg} / \mathrm{l}$ and SOFA score $6.0 \pm 4.2$. Eighty-nine (35\%) patients were fast responders, 98 (38\%) were slow responders, and there were $71(27 \%)$ non-responders. ICU mortality was significantly higher among non-responders $(15,23.4$ and $52 \%$, respectively, for fast, slow and non-responders; $p<0.001)$. There was no statistically significant difference in mortality rate according to infection therapy appropriateness $(36 / 157,23 \%$ vs $15 / 45,33 \%$, respectively, for appropriate and inappropriate therapy $-p=0.166$ ). However, the study lacked of statistical power to assess the observed mortality difference. After adjustment for SAPS II and deltaSOFA, CRP ratio remained significantly associated with ICU $(1.08,95 \% \mathrm{Cl} 1.03-$ 1.14 for $10 \%$ CRP ratio variation, $p=0.002)$ and hospital $(1.07,95 \%$ Cl 1.02-1.13 for $10 \%$ CRP ratio variation, $p=0.009)$ mortality. In our cohort, CRP ratio had a good discrimination capacity (area under ROC curve $=0.72$ ) and a good calibration (Hosmer-Lemeshow goodnessof-fit test $=0.055$ ) for ICU mortality.

Conclusion CRP ratio is an easy tool that may help to assess prognosis of infected ICU patients.

\section{Competing interests None.}

P230

Candidemia in ICU: Epidemiology and prognostic factors

Zied Hajjej ${ }^{1}$, Walid Sammoud ${ }^{1}$, Becem Trabelsi ${ }^{1}$, Iheb Labbene ${ }^{1}$, Mustapha Ferjani $^{1}$

${ }^{1}$ Department of Critical Care Medicine and Anesthesiology, Military

Hospital of Tunis, Tunis, Tunisia

Correspondence: Zied Hajjej - hajjej_zied@hotmail.com

Annals of Intensive Care 2016, 6(Suppl 1):P230

Introduction Candidemia in patients admitted to the intensive care unit (ICU) is a serious problem and, of particular concern, associated with high mortality (1). The aim of our study was to determinate epidemiological characteristics and prognostic factors of candidemia in non-neutropenic critically ill patients. 
Patients and methods This retrospective study was carried out at the military hospital of Tunisia. From January 1, 2012, to December 31, 2013, all patients with positive blood culture for Candida species were identified.

Results Over the 2-year study period, 36 episodes of candidemia in 760 patients were reported. The incidence was 1.57 per 1.000 patient-days.

The mean age was $63 \pm 13$ years. SOFA score at admission and at day of diagnosis was $10 \pm 4$ and $15 \pm 8$, respectively.

The mean time from admission to the first positive blood specimen was $14 \pm 10.5$ days.

C. albicans was the most common pathogen $(69.5 \%, 25 / 36)$, followed by C. glabrata $(11.1 \%, 4 / 36)$ C. tropicalis $(16.6 \%, 6 / 36)$ and C. krusei $(2.7 \%, 1 / 36)$.

Candidemia was classified as catheter-related infection in $44.5 \%$ of cases and as primary in $22.2 \%$. In other cases, it was associated either with peritonitis $(22.2 \%)$ or with pneumonia $(11.1 \%)$.

At the onset of candidemia, 10 patients $(27.7 \%)$ were not colonized by any Candida specimen and $26(72.3 \%)$ patients were positive for oral carriage of yeast.

Caspofungin was the most frequently used drug as primary treatment (66.6 \%), followed by fluconazole ( $27.7 \%$ ) and amphotericin B (5.5\%). For $27.7 \%$ of cases, the initial antifungal drug was changed after a median of 5 days of treatment. The most frequent change was the substitution of fluconazole for caspofungin (6 cases) and caspofungin for fluconazole (4 cases). The median duration of treatment was 14 days (range 5-24).

Regarding bacterial strains associated with candidemia, a predominance of infection with Escherichia coli (33.3 \%) followed by Acinetobacter baumannii (27.7 \%) before the diagnosis of candidemia, was noted. While after the diagnosis of candidemia, Pseudomonas aeruginosa $(55.5 \%)$ was the most frequently organism found.

Mortality in the ICU was $45 \%$. Tow factors were associated with an increased risk of death: high SOFA score at the onset of candidemia $(p=0.01)$ and the use of steroids $(p=0.03)$.

Conclusion This report shows that candidemia is a significant source of mortality in ICU. Determining factors associated with these high rates may lead to identifying measures that can help prevent disease.

\section{Competing interests None.}

\section{Reference}

1. Curr Opin Crit Care 2008, 14:600-604.

\section{P231}

Extracorporeal membrane oxygenation cannula-associated infections: implication of virulent Escherichia coli phylogroups

Jonathan Messika ${ }^{1}$, Olivier Clermont ${ }^{2}$, Matthieu Schmidt ${ }^{3}$, Alexandra Aubry ${ }^{4}$, Romain Fernandes ${ }^{2}$, Erick Denamur ${ }^{2}$, Alain Combes ${ }^{3}$,

Jean-Damien Ricard

${ }^{1}$ Service de réanimation médico-chirurgicale, CHU Louis Mourier, Colombes, Colombes, France; ${ }^{2}$ lame, umr 1137, INSERM, Univ Paris Diderot, Sorbonne Paris Cité, Paris, France; ${ }^{3}$ Service de réanimation médicale, Groupe Hospitalier Pitié Salpêtrière, Paris, France; ${ }^{4}$ Service de microbiologie, inserm u1135, immunity and infectious diseases center, Hôpital de la Pitié-salpétrière, Sorbonne Universités, UPMC Univ Paris 06 , Paris, France

Correspondence: Jonathan Messika - jonathan.messika@lmr.aphp.fr Annals of Intensive Care 2016, 6(Suppl 1):P231

Introduction Infection is the most frequent extra-corporeal membrane oxygenation adverse event. Among all sites, cannula-related infections are mainly due to Escherichia coli. E. coli population divides into commensals, poorly virulent (phylogroups A, B1, C, E) and extraintestinal virulent (phylogroups B2, D, F) strains. Owing to the frequent femoral site of catheterization, one may wonder whether strains responsible for infection have particular traits or whether their presence simply reflects the proximity with the lower digestive tract. We therefore decided to investigate phylogenetic group belonging and antimicrobial susceptibility spectrum of $E$. coli strains from extracorporeal membrane oxygenation cannula-associated infections.
Patients and methods Retrospective study (November 2012-January 2015) of phylogenetic group belonging (by polymerase chain reaction determination) and antimicrobial susceptibility (calculation of a susceptibility score, according to the sensitivity to 17 antimicrobial agents) of $E$. coli strains of cannula-related infection or colonization, in Pitié-Salpétrière Medical Intensive Care Unit. Collection of clinical data and characteristics of infection or colonization episodes.

Results Thirty-five strains were collected ( 32 infections and 3 colonisations), in 30 patients (18 men, aged 50.7 years, median Simplified Acute Physiology Score II 45). All but 2 extracorporeal membrane oxygenation was venoarterial, implanted for cardiogenic shock (75\%), cardiac arrest $(20 \%)$ or septic shock (5\%). Four patients had more than one strain collected: two patients had two separate infectious episodes each caused by different strains; 1 patient was colonized with one strain and subsequently infected with this strain and in the last patient, the same strain was retrieved at a 1 week interval despite antibiotics.

Median time to collection of strain was 14 days after extracorporeal membrane oxygenation implantation. E. coli was associated with another pathogen in 14 episodes. E. coli cellulitis accounted for 27 episodes, of which two had concomitant bacteremia. Two other episodes of $E$. coli bacteremia were reported. Surgery was mandatory for 7 episodes, and extracorporeal membrane oxygenation was explanted in 4. Of the 35 E. coli strains collected, 12 (34\%) had a wild-type phenotype towards beta-lactams, 15 (43\%) had penicillinase secretion phenotype, $6(17 \%)$ an extended spectrum beta-lactamase secretion phenotype, and 1 cephalosporinase phenotype.

Highly virulent extra-intestinal pathogenic strains were predominant, accounting for 23 isolates (66\%, 3 colonization; phylogroups: B2 $n=13 ; \mathrm{D} n=9 ; \mathrm{F} n=1)$, whereas 11 (31\%) were grouped as commensal isolates ( $\mathrm{A} n=3 ; \mathrm{B} 1 n=3 ; \mathrm{C} n=4 ; \mathrm{E}=1)$, and 1 non-grouped. Interestingly, more virulent isolates evidenced more resistant phenotype, with a lower, albeit non-significant, susceptibility score (12 for B2, D or $\mathrm{F}$ vs 15.5 for $\mathrm{A}, \mathrm{B} 1, \mathrm{C}$, or $\mathrm{E} p=0.06$ ).

Discussion As the cannulas are placed in femoral vessels, contiguity with faecal flora may explain E. coli predominance in extracorporeal membrane oxygenation cannula-related infections, its polymicrobial feature, and the diversity of $E$. coli population. Nevertheless, among all isolates, highly virulent extra-intestinal pathogenic strains were predominantly collected. Their advantageous genetic background may explain their predominance in this context.

Conclusion As for other infectious sites, but to a lesser extent, our results confirm the predominance of highly virulent extra-intestinal $E$. coli pathogenic strains.

Competing interests None.

P232

Is diabetes mellitus a risk factor for intensive care unit-acquired infections in intensive care unit patients with hematologic malignancies?

Marion Venot ${ }^{1}$, Sylvie Chevret ${ }^{2}$, Djamel Mokart ${ }^{3}$, Frédéric Pène ${ }^{4}$, Virginie Lemiale ${ }^{1}$, Achille Kouatchet ${ }^{5}$, Julien Mayaux ${ }^{6}$, François Vincent ${ }^{7}$, Martine Nyunga ${ }^{8}$, Fabrice Bruneel ${ }^{9}$, Elie Azoulay ${ }^{1}$

${ }^{1}$ Réanimation médicale, Hôpital Saint-Louis, Paris, France: ${ }^{2}$ Service de biostatistique et information médicale, Hôpital Saint-Louis, Paris, France; ${ }^{3}$ Réanimation, Institut Paoli-Calmettes, Marseille, France; ${ }^{4}$ Réanimation Médicale, Hôpital Cochin, Paris, France; ${ }^{5}$ Réanimation médicale, Centre Hospitalier Universitaire d'Angers, Angers, France; ${ }^{6}$ Réanimation pneumologique, Hôpital Pitié-Salpêtrière, Paris, France; ${ }^{7}$ Réanimation polyvalente, Groupe Hospitalier Intercommunal Le Raincy-Montfermeil, Montfermeil, France; ${ }^{8}$ Réanimation polyvalente, Centre Hospitalier de Roubaix, Roubaix, France; ${ }^{9}$ Réanimation médico-chirurgicale, Centre Hospitalier de Versailles, Le Chesnay, France

Correspondence: Marion Venot - marionvenot@free.fr Annals of Intensive Care 2016, 6(Suppl 1):P232

Introduction Diabetes mellitus affects $10 \%$ of the general population worldwide, $10-25 \%$ of critically ill patients, and is associated with high morbidity and mortality. Sepsis is among the main complications of diabetes. Patients with hematologic malignancies also are 
immunocompromised because of underlying disease and treatments. Intensive care unit-acquired infections, especially bloodstream infections and ventilator-associated pneumonia, are challenging complications in critically ill patient. To our knowledge, no study reported the influence of diabetes mellitus on acquired sepsis in this setting. This study is a post hoc analysis of the prospective cohort TRIAL-OH to determine whether diabetes mellitus is associated with increased risk for intensive care unit-acquired infections in patients with hematologic malignancies.

Patients and methods TRIAL-OH is a prospective multicenter cohort of patients admitted to 17 intensive care units in France and Belgium. We studied the subgroup of patients with intensive care length of stay superior to 3 days and who needed invasive mechanical ventilation within the first 3 days of their admission. Patients with diabetes mellitus under treatment were compared to patients without diabetes or with only diabetic diet. A propensity score for diabetes was constructed using multivariable logistic regression and used to match patients with and without diabetes mellitus and to determine the influence of diabetes on hospital mortality and intensive care unitacquired infections (as defined by bacterial or fungal infections occurring later than the second day of intensive care unit admission).

Results Among the 1011 patients of TRIAL-OH, 378 fulfilled the inclusion criteria for this study. Forty-eight (12.7 \%) had diabetes mellitus, 38 with chronic complications and 10 uncomplicated. Diabetics were significantly older than controls (median 61.5 [interquartile ranges: $58.7 ; 73]$ vs $60[49 ; 71], p$ value $=0.019)$. Intensive care units and gender were not different between patients with and without diabetes (73 and $62 \%$ male respectively, $p$ value $=0.20)$. SOFA was close $(8[5.7$; $11.2]$ in diabetics vs $8[5.2 ; 11.7]$ in others, $p$ value $=0.94)$. There were significantly more frequently cardiovascular comorbidities in diabetics (71 vs $36 \%, p$ value $<0.0001$ ). Other comorbidities were not different, as well as hematological features: The main hematologic malignancies were acute myeloid leukemia in $27 \%$, lymphoma in $32 \%$, myeloma in $19.5 \%$, chronic lymphocytic leukemia in $19 \%$. Allogeneic and autologous bone marrow transplantation concerned 23 and $11 \%$ of patients, respectively, with no difference between groups. Throughout their stay, $79 \%$ of diabetic patients and $81 \%$ of controls required vasopressors, 25 and $21 \%$ underwent sequential renal replacement therapy and 15 and $22 \%$ continuous renal replacement therapy. Intensive care unit-acquired infections occurred in $10(21 \%)$ patients with diabetes mellitus and 91 (28\%) patients without diabetes mellitus. Intensive care unit mortality was 46 and $54 \%$ in diabetic patients and controls, respectively. Hospital mortality was 54 and $62 \%$ in diabetic patients and controls, respectively. After propensity score matching, there was no effect of diabetes mellitus on the occurrence of intensive care unitacquired infections (hazard ratio $0.57,95 \%$ confidence interval [0.26$1.24], p$ value $=0.16$ ) and on hospital mortality (hazard ratio $0.96,95 \%$ confidence interval $[0.54-1.71], p$ value $=0.88$ ).

Discussion Diabetes and hematological malignancies both are immunosuppressive conditions. However, this study suggests that diabetes does not represent an additional risk factor in patients with hematologic malignancies.

Conclusion Diabetes mellitus is not associated with increased rate of intensive care unit-acquired infections or mortality in critically ill patients with hematologic malignancies.

\section{Competing interests None.}

\section{P233}

Should we need aerobic-anaerobic or specific blood culture bottles for diagnosing candidemia in ICU patients?

Sébastien Bailly ${ }^{1}$, Jean-François Timsit ${ }^{2}$, Cécile Garnaud ${ }^{3}$, Muriel Cornet ${ }^{4}$, Patricia Pavese ${ }^{5}$, Rebecca Hamidfar-Roy ${ }^{6}$, Luc Foroni ${ }^{7}$, Sandrine Boisset ${ }^{8}$, Carole Schwebel ${ }^{9}$, Danièle Maubon ${ }^{10}$

'Equipe 11, Institut Albert Bonniot - Inserm U823, La Tronche, France; ${ }^{2}$ Réanimation médicale et infectieuse, Hôpital Bichat-Claude Bernard, Paris, France; ' ${ }^{2}$ Laboratoire de parasitologie-mycologie, C.H.U., La Tronche, France; ${ }^{4}$ Laboratoire de parasitologie-mycologie médicale, C.H.U. Grenoble, La Tronche, France; ${ }^{5}$ Maladies infectieuses, C.H.U., La Tronche, France; ${ }^{6}$ Réanimation médicale, C.H.U., La Tronche, France; ${ }^{7}$ Pharmacie, C.H.U.
Grenoble, La Tronche, France; ${ }^{8}$ Laboiratoire bactériologie-hygiène, C.H.U., La Tronche, France; ${ }^{9}$ Réanimation médicale, C.H.U. Grenoble, Grenoble, France; ${ }^{10}$ Parasitologie-mycologie médicale, C.H.U. Grenoble, La Tronche, France

Correspondence: Sébastien Bailly - sbailly@chu-grenoble.fr

Annals of Intensive Care 2016, 6(Suppl 1):P233

Introduction Candidemia has a poor prognosis and is associated with a high mortality in part because of delayed diagnosis. There is no optimal tool and, despite a rather low sensitivity, gold standard for candidemia diagnostic and follow-up still relies on blood cultures. The use of blood culture-specific media is not clearly addressed in guidelines. In vitro studies showed that the presence of an antifungal agent can significantly modify the rate and the time to positivity of blood culture not containing adsorbing agents. This can have a direct impact on diagnosis and on candidemia monitoring. Our objective was to study in clinical practice the impact of systemic antifungal therapy on the diagnostic performance of each medium and to highlight their added value in the management of candidemia in intensive care unit patients.

Patients and methods All intensive care unit patients experiencing a candidemia in a university hospital, with at least one blood sample collected, in a 4-year period (2010-2013) were included in a retrospective study. The Bactec Plus TM Aerobic/F (Aerobic) and Bactec Plus TM Anaerobic/F (Anaerobic) bottles, which both contain adsorbing resins, and the non-resin Bactec Mycosis TM IC/F (Mycosis) bottles were compared. The clinical characteristics and the presence of systemic antifungal therapy were recorded for each patient. We analyzed: (1) the positivity rate during candidemia; (2) the time to positivity; and (3) the agreement between bottles. Univariate analyses and multivariate hierarchical model for repeated measures were performed.

Results A total of 64 patients with candidemia, median age of 61 years (interquartile range: 47-74) and a median IGSII score of 47.5 [42-57] were considered. Overall, positivity rate was greater in resin bottles (Aerobic and Anaerobic: $56.6 \%$ ) than in selective Mycosis bottles (43.4\%). In the presence of systemic antifungal therapy, the positivity rate decreased significantly (OR 0.19 (95\% confidence interval [0.07; $0.52], p<0.01$ ). This decline was observed with both resin and nonresin Mycosis bottles. Overall, time to positivity was longer in resin bottles (Aerobic and Anaerobic) (30 h; IQR 19; 60) than in Mycosis bottle (27 h, IQR 16-78). Systemic antifungal therapy prolonged time to positivity by a factor $1.92([1.29 ; 2.85] ; p=0.05)$. The impact of systemic antifungal therapy on the time to positivity was more pronounced in the Mycosis bottles than in those with resins $(p<0.01)$; however, TTP remained longer in resin bottles.

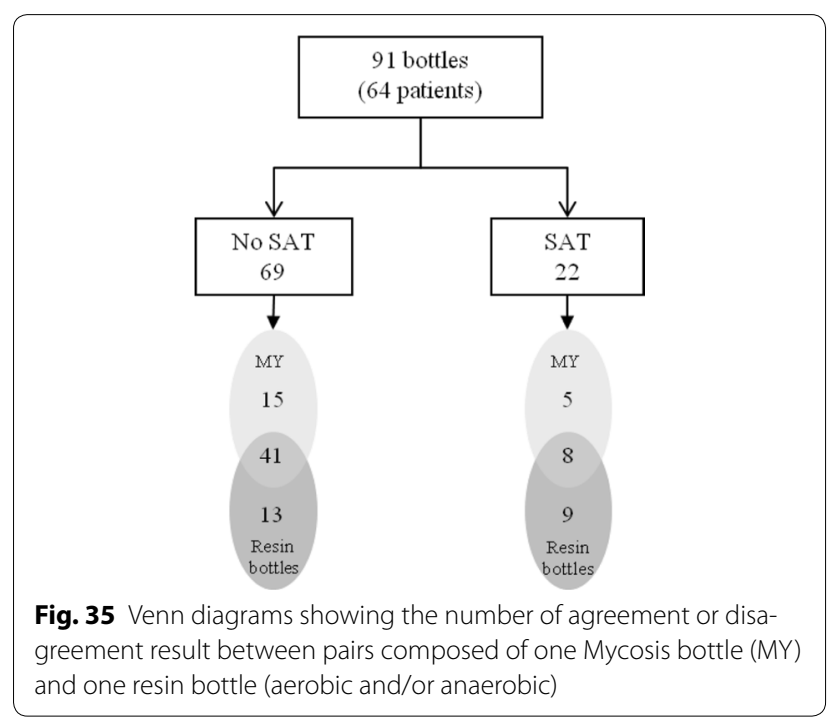


The agreement study showed that when the sampling was done the same day, on both resins and Mycosis blood cultures, in the absence of systemic antifungal therapy, $13(19 \%)$ and $15(22 \%)$ bottles were positive only on resin or only on Mycosis bottles, respectively, and in the presence of systemic antifungal therapy, $9(41 \%)$ and $5(23 \%)$ bottles were positive only on resins or only on Mycosis bottle, respectively (Fig. 35). Thus, systemic antifungal therapy decreased the agreement rate between the bottles.

Discussion Systemic antifungal therapy modifies significantly the blood culture results independently of the presence of resin in the bottles in intensive care units patients with candidemia. We also demonstrated that there is a clear gain in sensitivity to collect concomitantly resin and Mycosis bottles, especially when systemic antifungal treatment is present. However, a comparative multi-centric study would be necessary to confirm these results.

Conclusion These results encourage clinicians to use only pertinent empirical SAT prescription and to sample BC before any SAT initiation. In the presence of SAT, a systematic sampling of both Mycosis ${ }^{\circledR}$ and resins bottles may improve not only the primary diagnostic but also the time needed for the step-down or end of therapy.

Competing interests None.

\section{P234}

Invasive Candida infection in a Tunisian ICU: an observational cohort study

Islem Ouanes', Maha Abid', Hend Ben Lakhal', Saousen Ben Abdallah', Fahmi Dachraoui', Hedia Hammed', Rami Jabla', Asma Hachani', Mahdi Marzouk', Imen Abdellaoui' ', Chaima Ghribi', Imen Ben Ali', Lamia Ouanes-Besbes' ${ }^{1}$, Fekri Abroug ${ }^{1}$

${ }^{1}$ Réanimation Polyvalente, CHU Fattouma Bourguiba, Monastir, Tunisia

Correspondence: Islem Ouanes - ouanes.islem@gmail.com

Annals of Intensive Care 2016, 6(Suppl 1):P234

Introduction Invasive Candida infection is common in intensive care unit (ICU) patients and is associated with an increased mortality (up to $80 \%)(1-2)$. The aim of the current study is to describe the clinical, microbiological and management patterns of invasive Candida infections in a Tunisian ICU.

Patients and methods A retrospective cohort study including consecutive patients admitted to our ICU between January 2012 and March 2015 with invasive Candida infection, defined by a positive blood culture for Candida species and/or the presence of $50 \%$ or more colonized sites with Candida (Pittet index).

Results During the study period, invasive Candida infection was identified in 40 patients (median age 65 years, IQR 53.5-72, $55 \%$ male, median SAPS III: 63, IQR 58-70.5). The most common underlying disease was diabetes present in $65 \%$. The main reason for ICU admission was acute respiratory failure in 30 patients (75\%); $80 \%$ of patients were invasively ventilated at a moment of their ICU stay. Septic shock and ARDS were observed in 62.5 and $42.5 \%$, respectively. Candida species were isolated mainly in oropharyngeal (52\%), urinary (45\%) and rectal (32\%) sites, and blood cultures for Candida species were positive in 3 patients ( $7.5 \%)$. Candida albicans was the most prevalent species, isolated in $23 / 40$ patients $(57.5 \%$ ) [a mixed infection with other Candida species was present in $5 / 40(12.5 \%)]$; the other isolates were C. tropicalis in 12/40 (30\%) patients; C. glabrata in 7/40 (17.5\%) and other Candida species were identified in $10 \%$. Antifungal activity was tested in $21 / 40$ patients $(52.5 \%)$, showing that $17 / 21$ of species were susceptible to fluconazole $(81 \%)$ and to voriconazole in $19 / 21$ $(90.5 \%)$. Fluconazole was the first administered antifungal therapy in $97.5 \%$ of cases. Overall mortality was $55 \%$ for a median predicted mortality by SAPSIII at $41.9 \%$ (IQR: 31.5 to $58 \%$ ).

Conclusion Invasive Candida infection is mainly due to C. albicans and is associated with an increased mortality despite apparent susceptibility to fluconazole, the most empirical antifungal therapy used in this cohort.

Competing interests None.

\section{References}

1. Kett D, Azoulay E, Echeverria P et al. Candida bloodstream infections in intensive care units: analysis of the extended prevalence of infection in intensive care unit study. Crit Care Med 2011; 39: 665-70.

2. Vincent $J \mathrm{~L}$, Rello J, Marshall J et al. International study of the prevalence and outcomes of infection in intensive care units. JAMA 2009; 302: 2323-9.

P235

Causes of admission in medical intensive care of patients suffering from severe psychiatric disorders

Hanane Ezzouine ${ }^{1}$, Constant Aniolo Edith', Abdellatif Benslama

${ }^{1}$ Anesthesiology and Intensive Care Department, University Teaching

Hospital Ibn Rushd-Casablanca, Casablanca, Morocco

Correspondence: Hanane Ezzouine - ezzouinehanane@yahoo.fr

Annals of Intensive Care 2016, 6(Suppl 1):P235

Introduction The involvement of ICUs in support of vital functions of failures among patients suffering from mental disorders has been reinforced by recent years. Few studies address the issue of the reconciliation between somatic medical disciplines and psychiatry.

Patients and methods This is a prospective study between January 2012 and October 2014 including all patients admitted in medical intensive care and have had severe psychiatric history. The various data were collected from the farm returns. Only the initial diagnosis was retained.

Results During the study period of 782 hospitalizations, 26 patients (3.32\%) were admitted with psychiatric history. The sex ratio was equal to 1 and age between 18 and 60 years. Psychiatric history was essentially: schizophrenia (34.6\%), bipolar disorder (19.2\%), depression, psychosis, etc. The successful initial diagnosis: drug poisoning (34.6\%), impaired consciousness (30.7\%), shock, respiratory distress, metabolic disorders; suicide attempts; status epilepticus in $7.7 \%$ of cases. Age advanced cardiovascular history and represents poor prognostic factors. Infection remains the most serious complication. Mortality was $19.2 \%$ mainly by septic shock, with no correlation with psychiatric profile.

Conclusion The mentally ill are more exposed to various somatic pathologies that may require management in intensive care. On admission, there are more drug poisoning. Infectious complications are the cause of high mortality, not correlated with the severity score at admission or patient psychiatric history. The study was limited by its implementation on a single intensive care unit of the institution and having retained only the initial diagnosis.

Competing interests None.

\section{P236}

Unplanned extubation in critical care: still to learn from local survey

Frédéric Jacobs ${ }^{1}$, Dominique Prat ${ }^{1}$, Matthieu Le Meur ${ }^{1}$, Olfa Hamzaoui ${ }^{1}$, Anne Sylvie Dumenil', Guy Moneger ${ }^{1}$, Nadège Demars' ${ }^{1}$, Pierre Trouiller', Benjamin Sztrymf ${ }^{1}$

${ }^{1}$ Réanimation polyvalente, Hôpital Antoine Béclère, Clamart, France Correspondence: Benjamin Sztrymf - benjamin.sztrymf@abc.aphp.fr Annals of Intensive Care 2016, 6(Suppl 1):P236

Introduction The precise impact of unplanned extubation (UE) on patients' course in intensive care unit (ICU) remains elusive. While many studies have found an association between UE and increased time spent under mechanical ventilation (MV) or delayed ICU discharge, UE has also been described to carry a beneficial effect on length of MV and ICU outcome in a subgroup of patients not requiring re-intubation. We aimed to describe the incidence, characteristics and outcomes of patients experiencing UE (UE patients) and to compare these issues with patients undergoing a planned extubation (non-UE patients).

Patients and methods This is a prospective single-center study in a 15 -bed university-affiliated mixed ICU, over an 18-month period. All adult patients undergoing tracheal intubation were included. A nurse driven sedation protocol based on RASS score was used in most mechanically 
ventilated patients. Tracheal tubes were fixed with tape. The tape is fixed on the tracheal tube and wrapped around the neck. One strand of the tape sometimes passes on the bridge of the nose before reaching the neck, according to the patient's morphology. The use of physical restraints is prescribed by the attending physician but is widely used in our unit. Characteristics of UE were registered. Demographic characteristics and ICU outcomes were compared between UE and non-UE patients. Results During the study period, 882 patients were admitted and 580 patients required tracheal intubation $(65.7 \%)$. All the patients were orally intubated. Forty-six UE occurred in 42 patients $(55.1 \pm 20.6$ years, SAPS II $46 \pm 17)$. Mechanical restraints were present in $89 \%$ of the episodes. The incidence density was 10.3 events every 1000 days of MV. UE occurred equally during night and day and four times during nurse shift. Caregivers were aware of the UE risk in onethird of cases. UE was not associated with MV settings. Among the UE episodes, $21 \%$ occurred in agitated patients and 15 lead to re-intubation, within the following hour in most of the cases. Not re-intubated UE patients were younger ( $49.4 \pm 20.1$ vs. $65.3 \pm 18.6$ years, $p<0.05)$, had a shorter ICU LOS $(2.8 \pm 4.1$ vs. $9.4 \pm 11.1$ days, $p<0.05)$ and a better survival (100 vs. $79.2 \%, p<0.5)$ as compared to non-UE patients. Re-intubated UE patients were more likely to have a longer time under MV $(21.9 \pm 12.8$ vs. $9.4 \pm 11.1$ days, $p<0.05)$ and ICU LOS $(21.9 \pm 12.8$ vs. $9.4 \pm 11.1$ days, $p<0.05$ ) than non UE patients (Table 43 ).

Age, underlying systemic hypertension, time between admission and UE and between sedation withdrawal and UE were risk factors for reintubation. Voluntary intoxication as admission diagnosis was associated with UE good outcome.

Discussion Patients may self-extubate because they were not extubated in an appropriate timing. Rapid modifications of consciousness can lead them to waken in the unfriendly environment of ICU in between the nurse rounds, when deliberate UE might be unnoticed. Only one-fifth of UE patients was agitated. Nevertheless, RASS score was evaluated every $4 \mathrm{~h}$, at nurse round. Agitation may occur within these rounds. The impact of physical restraints on UE remains unclear. Conclusion UE might lead to a better outcome in the subset of not re-intubated patients. Altogether, UE underscores the need for a better involvement in sedation withdrawal, MV weaning and agitation screening.

Competing interests None.

\section{Table 43 See text for description}

\begin{tabular}{|c|c|c|c|c|c|}
\hline & \multirow{2}{*}{$\begin{array}{l}\text { Non-UE } \\
\text { patients } \\
(n=580)\end{array}$} & \multicolumn{3}{|l|}{ UE patients } & \multirow[t]{2}{*}{$p^{*}$} \\
\hline & & $\begin{array}{l}\text { Whole } \\
\text { cohort } \\
(n=42)\end{array}$ & $\begin{array}{l}\text { Not re- } \\
\text { intubated } \\
(n=27)\end{array}$ & $\begin{array}{l}\text { Re- } \\
\text { intubated } \\
(n=15)\end{array}$ & \\
\hline Age (years) & $65.3 \pm 18.6$ & $55.1 \pm 20.6$ & $49.4 \pm 20.1 \ldots$ & $67.4 \pm 16.3$ & 0.002 \\
\hline Gender (f/m) & $280 / 300$ & $16 / 26$ & $13 / 24$ & $3 / 12$ & 0.9 \\
\hline SAPS $\|$ & $49 \pm 22$ & $46 \pm 17$ & $45 \pm 18$ & $45 \pm 18$ & 0.13 \\
\hline $\begin{array}{l}\text { Died in ICU } \\
{[n(\%)]}\end{array}$ & $121(20.8)$ & $5(11.9)$ & $0(0) \ldots$ & $5(33)$ & 0.31 \\
\hline $\begin{array}{r}\text { ICU LOS } \\
\text { (days) }\end{array}$ & $9.8 \pm 11.8$ & $11.3 \pm 10.9$ & $5.4 \pm 5.9 \ldots$ & $19.8 \pm 11.2^{* *}$ & 0.1 \\
\hline $\begin{array}{l}\text { Length of MV } \\
\text { (days) }\end{array}$ & $9.4 \pm 11.1$ & $7.5 \pm 10.9$ & $2.8 \pm 4.1 \ldots$ & $21.9 \pm 12.8^{* *}$ & 0.27 \\
\hline
\end{tabular}

UE unplanned extubation, SAPS Simplified Acute Physiology Score, ICU intensive care unit, LOS length of stay, MV mechanical ventilation

* The comparison between non-UE patients and the whole cohort of UE patients

** $p<0.05$ when comparing re-intubated UE patients and non-UE patients

$\ldots p<0.05$ when comparing not re-intubated UE patients and non-UE patients
P237

Mortality associated with nights and weekends admission to intensive care unit (ICU): results of a 9-year cohort study (2006-2014)

Vincent Brunot ${ }^{1}$, Jean-Emmanuel Serre ${ }^{2}$, P. Corne ${ }^{1}$, Nicolas Molinari ${ }^{3}$, L. Landreau', Olivier Jonquet', Kada Klouche

${ }^{1}$ Réanimation médicale, chu lapeyronie, Montpellier, France; ${ }^{2}$ Néphrologie, chu lapeyronie, Montpellier, France; ${ }^{3}$ Dim, Hôpital La Colombière, Montpellier, France

Correspondence: Vincent Brunot - v-brunot@chu-montpellier.fr Annals of Intensive Care 2016, 6(Suppl 1):P237

Introduction The association between mortality and time of admission to ICUs has been extensively studied but remains a matter of debate. We aimed therefore to reassess the impact of time of admission on ICU mortality by retrospectively investigating a recent (20062014) and large ICU cohort.

Patients and methods All adults ( $\geq 18$ years) admitted between January 2006 and December 2014 to a French tertiary care medical ICU (staffed by onsite intensivist $24 \mathrm{~h}$ a day and 7 days a week) were included in the study. Patients' characteristics, medical management, and mortality were extracted from our prospective ICU database. Patients were grouped according to their admission time: weekday, weeknight, and weekend. ICU mortality was the primary outcome, and adjusted hazard ratios (HR) of death were analyzed by multivariate Cox model.

Results A total of 2.428 patients were included: age $62 \pm 18$ years, male: 1515 (62\%), and median SAPSII score: 38 (IQ, 27-52). A total of $1.449(59.7 \%)$ patients received invasive mechanical ventilation, 790 (32.5\%) patients received vasopressors (epinephrine or norepinephrine) and $162(6.7 \%)$ patients required renal replacement therapy. The overall ICU mortality rate was $13.7 \%$ (332/2428).

Admissions to ICU occurred during weekdays in 680 cases (28\%), during nighttime in 1.461 cases (60\%), and during weekends (Saturday and Sunday) in 611 cases (25\%). Baseline characteristics patients were similar between groups except that patients admitted during the second part of weeknight have a significantly higher SAPS II score than those admitted during weekdays $(41,28-56$ vs $38,26-51$; $p=0.034$, respectively). Patients admitted during weekdays have a significantly longer duration of mechanical ventilation and length of ICU stay as compared to others (7, 3-15 days vs 5, 3-13 days, $p<0.001$ and $8,3-20$ days vs $7,3-15$ days, $p<0.01$ respectively). ICU mortality was comparable for patients admitted during weekdays $(95 / 680$, $14 \%)$, weeknights $(142 / 1099,12.9 \%)$ and weekends $(94 / 649,14.5 \%)$. Univariate analysis showed that admission during the second part of the night was associated with a higher ICU mortality (HR, 1.39; $95 \%$ Cl 1.06-1.81) as compared to other time admissions. However, multivariate Cox model controlling factors associated with mortality such as SAPS II score, ICU admission source, and intensity of treatment demonstrated that admission during weeknights and weekends was not associated with an increased risk for death. During the first part of the night, the adjusted $\mathrm{HR}$ was $0.95(95 \% \mathrm{Cl} 0.66-1.37) p=0.80$ and during the second part of the night the adjusted HR was $1.24(95 \% \mathrm{Cl}$ $0.85-1.81) p=0.25$ in comparison with the weekdays admissions. During the weekend, the HR was $1.05(95 \% \mathrm{Cl} 0.82-1.33)$ in comparison with the rest of the week ( $p=0.71)$.

Conclusion Time of admission, especially weeknight and weekend (off-hours admissions), did not influence the prognosis of ICU patients. The higher illness severity of patients admitted during the second part of the weeknight may explain the observed increased mortality in this part of population.

Competing interests None. 
P238

Determinants and outcomes associated with decisions to deny admission in a Tunisian ICU

Rania Bouneb ${ }^{1}$, Kaoula Meddeb ${ }^{1}$, Y. Hamdaoui ${ }^{1}$, H Nouira ${ }^{1}$, A. Azouzi ${ }^{1}$ Jihene Ayachi ${ }^{1}$, Ahmed Khedher ${ }^{1}$, Mohamed Boussarsar'

${ }^{1}$ Réanimation médicale, CHU Farhat Hached, Sousse, Tunisia

Correspondence: Mohamed Boussarsar - hamadi.boussarsar@gmail.com Annals of Intensive Care 2016, 6(Suppl 1):P238

Introduction Decisions on whether to admit a critically ill patient to ICU are complex, since they need to balance the potential risks and benefits for the individual patient with the limited bed availability and thus the implication for future patients. Although recommendations for ICU triage are available, compliance with them has been shown to be poor. Decisions regarding ICU admission are currently not based on scientific evidence, yet refusal is associated with increased mortality.

Aim To analyze determinants and outcomes associated with decisions to deny or to delay ICU admission in critically ill patients.

Patients and methods This is an observational prospective study over a 6-month period, between January 1, 2015, and June 30, 2015, in a 7-bed medical intensive care unit. Patients' characteristics, reasons for requesting ICU admission, severity of underlying disease, severity of acute illness and mortality were recorded. Multinomial logistic regression analysis was performed to identify factors associated with ICU admission refusal.

Results ICU admission was requested for 364 patients. One hundred and four (28.6\%) were admitted, 173 (47.5\%) were never admitted and 87 (23.9\%) were admitted to the ICU at a later time. Reasons for refusal were: too sick to benefit, 62 (23.8\%); too well to benefit, 36 (13.8\%); unit full, 130 (50\%); therapeutic limitation, 28 (11\%); family order of non ICU admission, 5 (1.92 \%) and other causes, 14 (5.38\%). Multivariate analysis revealed that the non ICU admission was associated with neurological disease (OR 3.08; $95 \% \mathrm{Cl}[1.3-19.08] ; p=0.01$ ), lack of available ICU beds (OR 6.26; $95 \% \mathrm{Cl}$ [4.14-9.46]; $p=0.03$ ), cardiac disease (OR 8; $95 \%$ $\mathrm{Cl}[2.91-591.06] ; p<0.001)$, and metabolic disease (OR 2; $95 \% \mathrm{CI}[1.02-$ 10.02]; $p=0.02)$. Hospital mortality was $36.5 \%(38 / 104)$ for immediately admitted patients, $17.2 \%(15 / 87)$ for patients admitted later and $14.4 \%$ (25/173) for never admitted patients.

Conclusion Refusal of ICU admission was correlated with the severity of acute illness and lack of ICU. Further efforts are needed to define which patients are most likely to benefit from ICU admission and to improve the accuracy of data on ICU refusal rates.

\section{Competing interests None.}

\section{P239}

Characteristics and outcomes of patients not admitted to ICU

Nesrine Boujelbene ${ }^{1}$, Rami Jabla1, Fahmi Dachraoui ${ }^{1}$, Islem Ouanes', Mahdi Marzouk', Hedia Hammed ${ }^{1}$, Asma Hachani ${ }^{1}$, Hend Ben Lakhal ${ }^{1}$ Chaima Ghribi ${ }^{1}$, Imen Abdellaoui ${ }^{1}$, Imen Ben Ali ${ }^{1}$, Saousen Ben Abdallah', Lamia Ouanes-Besbes ${ }^{1}$, Fekri Abroug

${ }^{1}$ Réanimation polyvalente, CHU Fattouma Bourguiba, Monastir, Tunisia

Correspondence: Islem Ouanes - ouanes.islem@gmail.com

Annals of Intensive Care 2016, 6(Suppl 1):P239

Introduction ICU bed shortage and extremes in clinical status are among other reasons of refusals of patients proposed for ICU admission. The outcome of such patients relies heavily on local organization of healthcare facilities. The aim of this study is to describe characteristics and outcomes of non-admitted patients in a Tunisian ICU.

Patients and methods Observational prospective cohort study conducted from January 1, 2015, to August 30, 2015. All ICU admission proposals were analysed, and patients' outcome was collected after $48 \mathrm{~h}$ regardless of the type of decision: ICU admission or refusal.

Results During the study period, 320 patients were proposed for admission in our ICU (72.9\% of proposals occurred between 6:00 $\mathrm{AM}$ and 06:00 PM); $54.1 \%$ were referred from the emergency department of our hospital. One hundred and five patients $(32.5 \%)$ were admitted to the ICU. The median age of non-admitted patients was 62 years (49-75). The unavailability of free ICU beds was the most frequent reason for admission refusal (76.7 \%), followed by patient's
Table 44 See text for description

\begin{tabular}{|c|c|c|c|}
\hline & $\begin{array}{l}\text { Admitted } \\
\text { patients }(n=105)\end{array}$ & $\begin{array}{l}\text { Non-admitted } \\
\text { patients }(n=215)\end{array}$ & $P$ \\
\hline Age [median (IQR)] & $62(42.5-72.5)$ & $62(49-75)$ & 0.551 \\
\hline Gender (M/F) & $62 / 43$ & $116 / 99$ & 0.389 \\
\hline \multicolumn{4}{|l|}{ Baseline status } \\
\hline $\begin{array}{l}\text { Charlson score } \\
\text { [median (IQR)] }\end{array}$ & $5(4-8)$ & $5(3-7)$ & 0.051 \\
\hline Diabetes [n (\%)] & $77(73.3)$ & $92(42.8)$ & $<0.0001$ \\
\hline $\begin{array}{l}\text { Hypertension [n } \\
\text { (\%)] }\end{array}$ & $60(57.1)$ & $107(49.8)$ & 0.215 \\
\hline Malignancy [n (\%)] & $5(4.8)$ & $34(15.8)$ & 0.005 \\
\hline Stroke $[n(\%)]$ & $15(14.3)$ & $20(9.3)$ & 0.180 \\
\hline $\begin{array}{l}\text { Dependent } \\
\text { functional status } \\
{[n(\%)]}\end{array}$ & $18(17.1)$ & $35(16.3)$ & 0.841 \\
\hline Dementia [n (\%)] & $5(4.8)$ & $7(3.3)$ & 0.538 \\
\hline $\begin{array}{l}\text { Myocardial infarc- } \\
\text { tion }[n(\%)]\end{array}$ & $14(13.3)$ & $33(15.3)$ & 0.632 \\
\hline $\operatorname{COPD}[n(\%)]$ & $43(41)$ & $86(40)$ & 0.870 \\
\hline \multicolumn{4}{|l|}{$\begin{array}{l}\text { Organ/system } \\
\text { failure }\end{array}$} \\
\hline $\begin{array}{l}\text { Acute respiratory } \\
\text { failure }[n(\%)]\end{array}$ & 93 (88.6) & $164(76.3)$ & 0.009 \\
\hline Shock $[n(\%)]$ & 30 (28.6) & $51(23.7)$ & 0.349 \\
\hline Intubation [n (\%)] & $52(49.5)$ & $95(44.2)$ & 0.368 \\
\hline Mortality [n (\%)] & 24 (22.9) & $107(49.8)$ & $<0.0001$ \\
\hline
\end{tabular}

severity considered as extreme (hopeless cases) in $12.1 \%$, or not enough severe in $10 \%$. Among the refused patients, $44 \%$ were intubated. The Table 44 compares characteristics of admitted and nonadmitted patients.

At day 2, $15.8 \%$ of non-admitted patients died, $50.7 \%$ were still in the ward, $25.1 \%$ were transferred to another structure, and $7 \%$ were secondarily transferred to our ICU.

Conclusion Two-third of referred patients is not admitted to our ICU, and the main cause for refusal was the unavailability of free ICU beds. Mortality in non-admitted patients is high.

Competing interests None.

P240

Long-term outcomes of critically ill patients with severe acute kidney injury requiring renal replacement therapy

Vanessa Jean-Michel ${ }^{1}$, Francis Couturaud ${ }^{2}$, Elizabeth Moore ${ }^{3}$, Pierre-Yves Egreteau ${ }^{4}$, Jean-Michel Boles ${ }^{1}$, Cécile Aubron ${ }^{1}$

${ }^{1}$ Réanimation médicale, CHRU de Brest site La Cavale Blanche - Université de Bretagne Occidentale, Brest, France; ${ }^{2}$ Département de médecine interne et pneumologie, ea 3878, cic inserm 1412, ifr 148, CHRU de Brest site La Cavale Blanche - Université de Bretagne Occidentale, Brest, France; ${ }^{3}$ Department of epidemiology preventive medicine, Monash University, Melbourne, Australia; ${ }^{4}$ Réanimation, Centre Hospitalier des Pays de Morlaix, Morlaix, France

Correspondence: Vanessa Jean-Michel - vanessa.jeanmichel@gmail. com

Annals of Intensive Care 2016, 6(Suppl 1):P240

Introduction Up to $50 \%$ of critically ill patients develop acute kidney injury $(\mathrm{AKI})$ and up to 5 to $10 \%$ require renal replacement therapy (RRT) in the intensive care unit (ICU). Studies that have investigated long-term outcome in this population suggest that these patients 
have a poor prognosis and highlight the need for further investigation of factors associated with long-term outcome.

Patients and methods This is a retrospective single-centre study of patients admitted to the medical ICU of a tertiary hospital and who received RRT for AKI, between January 2008 and December 2011. AKI was defined and classified at ICU admission based on the Kidney Disease Improving Global Outcomes (KDIGO) classification using both creatinine and urine output. In order to identify predictors for death at 1 year of follow-up, a univariable analysis and a subsequent multivariable analysis were performed using a Cox proportional hazards regression model. Renal function recovery at 1 year was calculated using the following formula: [(estimated glomerular filtration Rate (e GFR) at 1 year minus baseline e GFR)/baseline e GFR], where e GFR was determined using the simplified modification of diet in renal disease equation using creatinine values within the 3 months prior to ICU admission to define baseline.

Results Of the 245 eligible patients, 214 were followed up for at least 1 year after hospital discharge. Of the 147 (68.7\%) patients who had died at 1 year, 135 (92 \%) died before hospital discharge. Using survival analysis, factors independently associated with mortality were illness' severity at admission (using the Simplified Acute Physiology Score II, hazard ratio (HR) $=1.02,95 \%$ confidence interval [1.01-1.03], $p=0.004$ ) and at RRT initiation (using the Sequential Organ Failure Assessment score, $\mathrm{HR}=1.07$ [1.01-1.12], $p=0.02$ ), use of CVVH as compared to $\mathrm{HDI}(\mathrm{HR}=3.09,[1.74-5.50], p<0.001)$, age $(\mathrm{HR}=1.02$, $[1.01-1.04], p=0.001)$ and chronic liver diseases ( $\mathrm{HR}=1.78[1.06-$ $2.96], p=0.03)$. At 1 year, renal function was known in $42 \%(28 / 67)$ of the survivors; $21 \%$ of them $(6 / 28)$ had an e GFR stage 1 while $7 \%$ $(2 / 28)$ were dialysed. Renal recovery was analysed in 19 patients: 4 patients (21\%) had a renal recovery between 50 and $89 \%, 3(16 \%)$ had a renal recovery less than $50 \%$, and $12(63 \%)$ had a complete renal recovery (more than $90 \%$ of recovery as compared to baseline e GFR).

Conclusion One-year mortality in ICU patients with AKI requiring RRT remains high with around two-thirds of patients dead at 1 year, mostly before hospital discharge. Although renal impairment persists in most survivors, only a few require long-term RRT. Prospective research is warranted to better investigate interventions associated with longterm prognosis.

\section{Competing interests None.}

\section{References}

1. Schiff H, Fischer R. Five-year outcomes of severe acute kidney injury requiring renal replacement therapy. Nephrol Dial Transplant. $2008 \mathrm{Jul}$ 1;23(7):2235-41.

2. Lins RL, Elseviers MM, Daelemans R. Severity scoring and mortality 1 year after acute renal failure. Nephrol Dial Transplant Off Publ Eur Dial Transpl Assoc - Eur Ren Assoc. 2006 Apr;21(4):1066-8.

\section{P241}

Nowadays, SAPSII better predicts 1-year mortality than hospital mortality

Jean-Etienne Herbrecht ${ }^{1}$, Max Guillot ${ }^{1}$, Quentin Maestraggi ${ }^{1}$, Charlotte Kaeuffer', Ralf Janssen-Langenstein', Sylvie L'hotellier', Sophie Hatsch', Benjamin Lebas', Alexandra Boivin'1, Maleka Schenck', Marie-Line Harlay', Anne Meyer ${ }^{1}$, Vincent Castelain ${ }^{1}$, Francis Schneider ${ }^{1}$

${ }^{1}$ Réanimation médicale, Hôpitaux Universitaires de Strasbourg (Hôpital de Hautepierre), Strasbourg, France

Correspondence: Jean-Etienne Herbrecht - jean-etienne.herbrecht@

chru-strasbourg.fr

Annals of Intensive Care 2016, 6(Suppl 1):P241

Introduction The prognosis of patients admitted in an intensive care unit (ICU) has improved thanks to new therapies and technical progresses. Concomitantly, the average age of patients admitted in an intensive care unit has increased. We studied the functional and vital prognosis of current patient population admitted at our ICU.

Patients and methods We realized this retrospective, single-center study over a 5-month period in a 30-bed medical ICU. We collected the outcome up to 1 year after hospitalization of all consecutive patients.
Table 45 See text for description

\begin{tabular}{|c|c|c|c|c|c|}
\hline \multirow[t]{2}{*}{ SAPSII } & \multirow{2}{*}{$\begin{array}{l}\text { Number } \\
\text { of patients }\end{array}$} & \multirow{2}{*}{$\begin{array}{l}\text { Prob- } \\
\text { ability } \\
\text { of mor- } \\
\text { tality } \\
(\%)\end{array}$} & \multicolumn{3}{|c|}{ Observed mortality rate } \\
\hline & & & $\begin{array}{l}\text { During } \\
\text { ICU }\end{array}$ & $\begin{array}{l}\text { During } \\
\text { whole } \\
\text { hospital } \\
\text { stay }\end{array}$ & $\begin{array}{l}\text { At 1-year } \\
\text { follow-up }\end{array}$ \\
\hline $6-40$ & 132 & $\begin{array}{r}0.5 \text { to } \\
<25\end{array}$ & $\begin{array}{c}8 \% \\
(n=11)\end{array}$ & $\begin{array}{l}12 \% \\
(n=16)\end{array}$ & $\begin{array}{l}24 \% \\
(n=32)\end{array}$ \\
\hline $41-51$ & 74 & 25 to $<50$ & $\begin{array}{l}12 \% \\
(n=9)\end{array}$ & $\begin{array}{l}22 \% \\
(n=16)\end{array}$ & $\begin{array}{l}42 \% \\
(n=31)\end{array}$ \\
\hline $52-64$ & 84 & 50 to $<75$ & $\begin{array}{l}28 \% \\
(n=24)\end{array}$ & $\begin{array}{l}39 \% \\
(n=33)\end{array}$ & $\begin{array}{l}45 \% \\
(n=38)\end{array}$ \\
\hline $65-119$ & 98 & $\geq 75$ & $\begin{array}{l}47 \% \\
(n=46)\end{array}$ & $\begin{array}{l}58 \% \\
(n=57)\end{array}$ & $\begin{array}{l}71 \% \\
(n=70)\end{array}$ \\
\hline
\end{tabular}

Results Three hundred and eighty-eight patients were admitted with an average age of 62 years ( \pm 19 years). The population over 60 years represented $61 \%$ of the admissions. The origin of the patients was equally distributed between medical hospital services, emergency services and pre-hospital origin. The median SAPSII score was 48 (range 6-119).

The main reasons for admission were respiratory distress (32\%), neurological disorders $(20 \%)$, and infections $(13 \%)$. The observed mortality in the ICU (23\%) and during the whole hospital stay (30\%) differs from mortality predicted by the SAPSII score (42\%). The 1-year mortality in our cohort was $44 \%$.

Whatever the severity groups, the mortality rates during ICU and hospital stay were lower than predicted. The mortality rate increased close to the rate predicted by the SAPSI only at 1-year follow-up.

Mortality is significantly influenced by an age over 60 years $(p<0.0001)$. There is no difference in mortality between patients over 80 years and patients of $60-79$ years $(p=0.47)$.

Twenty-one percent of patients alive at 1-year follow-up had a higher level of dependency as assessed by the Glasgow Outcome Scale. There is no link with age (Table 45).

Conclusion The SAPSII score enabled in 1993 to predict mortality during hospital stay. Thanks to the improvement in care, the observed mortality rate at end of hospital stay is now substantially lower than the predicted rate. After discharge, the mortality rate increases significantly during the first year but remains lower than predicted.

\section{Competing interests None.}

Reference

1. Le Gall JR, Lemeshow S, Saulnier F. A new Simplified Acute Physiology Score (SAPS II) based on a European/North American multicenter study. JAMA. 1993;270(24):2957-63.

P242

Impact of diabetes on burn injury: preliminary results

from retrospective study

Amel Mokline', Imen Rahmani ${ }^{1}$, Khaouther Ben Arfi', Lazhari Gharsallah', Sofiene Tlaili', Bahija Gasri', Rym Hammouda', Amen Allah Messadi ${ }^{1}$

${ }^{1}$ Intensive Burn Care Department, Burn and Trauma Center, Tunis, Tunisia Correspondence: Amel Mokline - amokline@yahoo.fr Annals of Intensive Care 2016, 6(Suppl 1):P242

Introduction Burn injuries are often associated with multisystemic complications essentially infectious, even in healthy patients. It is therefore recognized that for the diabetic patient, the underlying pathophysiologic alterations in vascular supply, peripheral neuropathy, and immune function could have a poor prognosis. The aim of this study was to determine the incidence and the characteristics of burn injuries in diabetic burn patients and to assess their prognosis. 
Patients and methods A retrospective study was conducted in a 20-bed adult burn ICU in Tunis between 2009 and 2012. Diabetic burned patients were enrolled. Characteristics of diabetes and burn injuries and prognostic factors were collected. Statistical analysis was performed using the MedCalc software.

Results Of 1205 adult burn patients, 98 (8\%) diabetic burns were admitted and only 74 medical records were available and analysed. The mean age was $58 \pm 12$ years old. There were 44 males and 30 females. The average TBSA was $21 \%$ with an UBS of 53 . The major mechanism of injury for the diabetic patients was scalding or flame burns (97\%). Diabetic patients had foot burns in $24,3 \%$. The average healing time was 103.1 days. The healing failure rate was $30 \%$, and the death rate was $30 \%$.

A poorly balanced diabetes before hospitalization doubles healing time, lengthens five times risk of sepsis and was associated with mortality in $50 \%$ of cases.

Poor glycemic control during hospitalization is multiplied by 2.5 healing period, risk of sepsis and risk of death. Increasing amounts of insulin in response to the continued high blood glucose levels was associated with lengthening healing time, a greater risk of sepsis and mortality. Severe hyperglycaemia at admission was difficult to control during hospitalization and was a marker of poor prognosis.

Conclusion Diabetics patients have a higher propensity for infection which worsens prognosis. The glycemic control during hospitalization is a first-order goal. Diabetic patient education must include caution about potential burn mishaps and the complications that may ensue from burns.

\section{Competing interests None.}

\section{P243}

\section{Withdrawal or withholding of life-sustaining treatments} decisions: Is there a risk of excessive influence of medical imaging?

Fabrice Lesage ${ }^{1}$, Sylvie Séguret ${ }^{1}$, Laurent Dupic ${ }^{1}$, Laure De Saint

Blanquat', Sylvain Renolleau'

${ }^{1}$ Réanimation et surveillance continue pédiatriques, $\mathrm{CHU}$ Necker-Enfants Malades, Paris, France

Correspondence: Fabrice Lesage - fabrice.lesage@nck.aphp.fr Annals of Intensive Care 2016, 6(Suppl 1):P243

Introduction Decisions of withdrawal or withholding of life-sustaining treatments (WWLST) almost always involve medical imaging. We worried about the risk of an excessive influence of the image on our representation of the patient, leading to a misevaluation of his prognosis, and possible inadequate WWLST decisions.

Materials and methods To investigate this point, we submitted a quiz to all the doctors of the Pediatric Intensive Care Unit of NeckerEnfants Malades Hospital (Paris). A first part consisted in questions about 6 images extracted from 6 clinical cases. For every image, they had to choose appropriate words characterizing it among a list of 18 words. Then, having only the image as information on each patient, they had to answer 2 questions: "What is the prognosis of this patient?" and "Personally would you propose a WWLST?" The second part of the study was composed with general questions about the weight of imaging in WWLST decisions.

Results Fifteen of the 20 doctors of the team completed the quiz: 6 senior doctors, 4 fellows and 5 residents. The words used to characterize the image were distributed in 2 groups: one with 8 neutral meaning words and the other one with 10 emotional meaning words. A constant number of neutral words were used to characterize the images (14-20), while the number of emotional words varied from 6 to 28 from an image to the other one. For 3 images, the prognostic evaluation agreed with the real clinical evolution. For one image, which was characterized by 6 emotional words, the suspected prognosis was "good" or "doesn't know" for 14/15 doctors. Only one doctor suspected a bad prognosis and proposed a WWLST. This patient drowned and died after a WWLST decision. For the last two images, who were characterized by 24 and 28 emotional words, respectively, the suspected prognosis was "dead" for 8 and 9/15 doctors, respectively. Respectively 5 and 10/15 proposed a WWLST. These two patients are alive after several years, respectively, without or with minor sequela. To the question "Do you think that a visually impressive image but without pejorative prognosis value for the radiologist risks to weigh wrongly in a decision of WWLST?", the mean response from 1 (not at all) to 10 (completely) was 5.7. To the question "Do you think that a not very impressive image which has a pejorative prognosis value for the radiologist has less impact on a WWLST decision than a visually impressive image with the same prognosis?", the mean response was 5.6. Moreover, the doctors express the fact that for every situation clinical assessment is much more important than imaging and express the need to have a radiologist's and an expert's point of view for every situation.

Discussion On the basis of our quiz, we could suspect that a rather unimpressive image, characterized by few emotional words, risks to lead to an excess of optimism, while a very impressive image, characterized by numerous emotional words, risks to lead to an excess of pessimism, which could lead to inadequate WWLST decisions. Our quiz overlines that the doctors are conscious of this risk and that to protect them against this risk, their WWLST decisions are based mostly on clinical examination, are helped by radiologists and experts and are always collective. In fact, real decisions for these patients weren't influenced by the impressive character of the image, as the patients with the most impressive image are alive, and the patient with the less impressive one is dead after a WWLST decision.

Conclusion Our study underlines a real risk of an excessive influence of the image on the evaluation of the patient's prognosis, which could lead to inadequate WWLST decisions. The consciousness of this risk can help the physicians to develop strategies to protect themselves against this risk, first of which is the collegiality of the process decision making during WWLST.

\section{Competing interests None.}

\section{P244}

Withholding and withdrawal of life-sustaining treatments in intensive care unit patients: predisposing factors and implementation

Anne-Sophie Baptiste ${ }^{1}$, Hugues Georges², Pierre-Yves Delannoy², Patrick Devos $^{3}$, Olivier Leroy ${ }^{2}$

${ }^{1}$ Hospital Center De Tourcoing, Tourcoing, France; ${ }^{2}$ Réanimation, Centre Hospitalier de Tourcoing, Tourcoing, France; ${ }^{3}$ Laboratoire de biostatistiques, Maison régionale de la recherche clinique, Lille, France

Correspondence: Anne-Sophie Baptiste - as.baptiste@gmail.com Annals of Intensive Care 2016, 6(Suppl 1):P244

Introduction Recommendations to withhold or withdraw life-sustaining therapies (LST) in intensive care units (ICU) patients have been published in 2009 by the French Intensive Care Society. Since then, few ICUs have reported the use of these procedures in their unit. The aim of our study was to report modalities of decisions to forgo LST (DFLST) in dying patients and characteristics of these patients in our unit.

Patients and methods We performed a retrospective study assessing all dying patients between November 2011 and December 2013 in the Tourcoing Hospital ICU. Characteristics of these patients on ICU admission and during ICU stay and modalities for withholding or withdrawing LST were recorded. Two analyses were performed: the first concerned all patients who died; the second, patients dying in the $72 \mathrm{~h}$ following ICU admission.

Results During the study period, 177 (79\%) of 224 patients dying in our unit had treatment withheld or withdrawn after a mean ICU stay of $12 \pm 14.1$ days, whereas 54 (24.1\%) patients had treatment(s) withheld (i.e., not started or not increased if already engaged), 62 (27.7 \%) had one or more treatment(s) withdrawn (i.e., invasive procedures engaged were stopped) and $61(27.2 \%)$ had a decision to withdraw LST preceded by a withholding procedure. Mean ICU stay was, respectively, $10.3 \pm 13.7$ days and $11.1 \pm 11.7$ days for withheld and withdrawn patients. Withdrawal procedures occurred after a mean of $4 \pm 3.2$ days following the decision to withhold LST in concerned patients. Cardiopulmonary resuscitation (CPR) was the most frequent withheld procedure (91.3\%) followed, respectively, by "not started or not increased" vasopressor treatment $(80 \%)$ and renal replacement therapy $(68.7 \%)$. Terminal weaning from mechanical ventilation was 
the most frequently withdrawn procedure $(73.9 \%)$ followed by discontinuation of vasopressor treatment (65.8\%). Therapeutic failure explained DFLST in 121 (68.3\%) patients, followed by an expected poor functional autonomy $(47.4 \%)$ and by the severity of underlying diseases (40.6\%). Factors associated with a decision of withholding or withdrawing LST were: cirrhosis [OR 11.7, $95 \% \mathrm{Cl}(1.1-106.4)$; $p=0.02$ ], a Knaus score $\mathrm{B}, \mathrm{C}, \mathrm{D}$ [OR 5.4; $95 \% \mathrm{Cl}(2.2-12.7) ; p=0.0001$ ], occurrence of medical complications during ICU stay [OR $3.4 ; 95 \% \mathrm{CI}$ $(1.3-8.1) ; p=0.0007]$.

Ninety-four patients (41.9\%) died in the $72 \mathrm{~h}$ following ICU admission. Among these, a procedure of withholding or withdrawing LST was recorded for 60 patients (63.8\%). Renal replacement therapy was the second most common withheld treatment (75 \%) after CPR (94.4\%). A renal SOFA score 3.4 [OR 4.1; $95 \% \mathrm{Cl}(1.2-13.2) ; p=0.0002$ ] and a Knaus score B, C, D [OR 6.9; $95 \% \mathrm{Cl}(2.4-19) ; p=0.01$ ] were associated with a decision of withholding/withdrawal of LST.

Discussion Withholding and/or withdrawal of LST in European ICUs are common and variable. They are associated with age, diagnoses, ICU stay, geographic and religious factors. In our unit, we have not identified age, presence of cancer or severe co-morbidities as conditions associated with LST procedures. This is probably due to the decision making of admission or non-admission in ICU appropriate to each hospital. Cirrhotic patients admitted to ICU have high mortality rates, but recent literature recommends to actively manage these patients in ICU during few days. This approach is applied in our unit and explains our result concerning patients with cirrhosis. Withholding RRT within 3 days following ICU admission suggests that RRT is a LST seeming excessive in some patients whose characteristics must be specified.

Conclusion Withholding/withdrawal of LST is common in ICU dying patients. Cirrhosis, poor previous functional status and occurrence of medical complications during ICU stay are associated with DFLST.

\section{Competing interests None.}

\section{Reference}

1. Ferrand E, Regnier B, Boles JM, Melot C. Limitations et arrêt des traitements en réanimation adulte :actualisation 2009 des recommandations de la SRLF. Reanimation 2010;19:679-698.

\section{P245}

\section{Advanced directives in an old patient with suicide attempt:} analysis of ICU physicians perception

Dolores Albarracin ${ }^{1}$, A. Ducousso-Lacaze ${ }^{1}$, Angéline Jamet, ${ }^{2}$, Julien Dufour ${ }^{3}$, Séverin Cabasson ${ }^{4}$, Anne Veinstein ${ }^{3}$, Delphine Chatellier ${ }^{3}$, Jean-Pierre Frat ${ }^{2}$, René Robert ${ }^{3}$

'Departement de psychologie ea 4050 caps, Université de Poitiers,

Poitiers, France; ${ }^{2}$ Réanimation médicale, CHU de Poitiers, Poitiers, France; ${ }^{3}$ Service de réanimation médicale, Chu De Poitiers, Poitiers, France; ${ }^{4}$ Service de réanimation, Centre Hospitalier la Rochelle, La Rochelle, France Correspondence: René Robert - r.robert@chu-poitiers.fr Annals of Intensive Care 2016, 6(Suppl 1):P245

Introduction The decision to admit a patient in the ICU may be complex involving both objective criterions and subjective perceptions. The aim of the study was to analyse the subjective and emotional factors justifying the decision to admit or not an old patient with suicide attempt having written advanced directives.

Patients and methods In this qualitative exploratory study, a vignette describing the case of a 84-year-old patient admitted to the ER for a poisoning with several medications. He had written a letter explaining clearly that he did not wanted any resuscitation. His clinical status was a coma (Glasgow scale 7), and he would require ICU admission and mechanical ventilation. Semi-directive interviews were conducted in five ICU-practitioner volunteers who were not prepared with the clinical case. Physicians were asked to comment and justify their decision to admit or not such a patient. Interviews were conducted by a psychologist (DA), recorded and analysed using semantic analysis content (Tropes VF8)

Results 'Difficult' was the mostly used word to qualify the current case and similar situations for which the interviewed physicians used to be faced in their clinical practice. Even though they actively set out their arguments about the case, some hesitations and contradictions were pointed out to justify the 'good' choice and the criteria proposed to evaluate this choice. Thus, pro and con factors used to present a rationalized final decision were associated with subjective factors.

The very old age of the patient was discussed a little. In contrast, the advanced directives were intensively debated. In the context of suicide attempt, they were perceived as a 'trap' or a 'alarm for help' signal. Since the acute psychiatric disease could be potentially reversible, it could not justify a non-admission decision. The final proposal for the interviewed physicians would be to admit the patient in the ICU to see the evolution and to discuss the case in collegiality.

Discussion These interviews showed the subjective dimension surrounding the decision of ICU admission or non-admission of some patients. The expressed doubts reflect the ethical dilemma related to the presented case. The suicide attempt context clearly overcomes the written advanced directives that were considered as a subjective element complicating instead of simplifying the decision. The possibility of further collegial discussion after the admission was considered as an efficient safeguard to neutralize the doubts and the risks of an individual wrong decision.

Conclusion This pilot study pointed out the ethical dilemmas associated with the complexity of medical decision in some specific context. Further studies may be of interest to analyse more deeply the emotional and subjective parts in medical decision.

\section{Competing interests None.}

P246

Having the talk about advance directives in the daily practice: Is a stay in an intensive care unit a good opportunity?

Vivien Hong Tuan Ha', Nicolas Lau', Lucie Mimounn ${ }^{2}$, Xavier Forceville ${ }^{2}$

${ }^{1}$ Intensive Care Unit, Hospital Center De Meaux, Meaux, France; ${ }^{2}$ Réanimation, Hôpital de Meaux, Meaux, France

Correspondence: Vivien Hong Tuan Ha - vdhthfirst@gmail.com

Annals of Intensive Care 2016, 6(Suppl 1):P246

Introduction The laws on advance directives arise from a transition of a paternalistic model to the deliberative model. However, the complexity of the subject and easy answer by the avoidance made that this kind of law was rarely a priority at both the legislative level and medical level. The effect of patient's rights and end-of-life laws has not had the desired result. Indeed, only $2.5 \%$ of the patient at the end of life have written advance directives (1). The objective of this study was to explore whether an ICU stay is a good opportunity to discuss advance directives.

Materials and methods Since we expected subjective answers (hard to quantify), consequently we decided to conceive a qualitative study. To answer this question, over a period of 3 months: we selected all patients over 75 years old that got out alive of an intensive care unit stay. Then, we interviewed treating physicians of those patients over the phone. We used 5 open and 1 close questions to explore the knowledge about advance directives and to know whether those physicians have ever talked about advance directives with their patients.

Results We have collected 11 testimonies from physicians. In spite of media coverage of exceptional cases, advance directives remain unknown from patients and doctors. It's hard for patients to write advance directives because it's not easy to formalize thoughts in writings and also to predict all possible clinical situations. Another issue is that treating physicians aren't used to talk about end of life and wait for their patients to ask about it.

Discussion A stay in an intensive care unit is a good opportunity to talk about advance directives because of the lack of opportunities 
and all the more since those patients have a high risk to be hospitalized again in the next few months. In interviews, treating physicians admit that the talk about advance directives is often too late in the evolution of diseases. One of the reasons for the unpopularity about advance directives: physicians aren't bind by them in the French law, but they are asked to take them into account for medical decisions.

Conclusion An ICU stay is a major event. Ideally, advance directives should have been made before that the ICU stay. That event is then an opportunity to revise them. The problem is complex and requires a solution at various levels: political, societal, economic, ethical, educational and relational. Various media coverage should allow to change mentalities. A bill is under discussion (2), and it should allow a partial response to some reluctance in the writing of advance directives.

\section{Competing interests None.}

\section{References}

1. Pennec S, Monnier A, Pontone S, Aubry R. Les décisions médicales en fin de vie en France. bulletin mensuel d'information de l'Institut national d'études démographiques. 2012 Nov.

2. Claeys A, Leonetti J. Nouveaux droits en faveur des malades et des personnes en fin de vie. Dec, 2014.

\section{P247}

Long-term outcome of patients who have withheld life support in intensive care

Adel Maamar', Stéphanie Chevalier², Vlad Botoc², Yves Le Tulzo' ${ }^{1}$ Réanimation médicale, Centre hospitalier universitaire de Rennes, Rennes, France; ${ }^{2}$ Réanimation polyvalente, C.H. de Saint Malo, Saint-Malo, France

Correspondence: Adel Maamar - adel.maamar@chu-rennes.fr Annals of Intensive Care 2016, 6(Suppl 1):P247

Introduction The incidence of withholding life support had increased during last years. While short-term outcome is frequently reported, there are no data about long-term outcome. Our objective was to report 6-month outcome of patients discharged from a general hospital ICU with treatment limitations.

Patients and methods We retrospectively collected data of patients discharged from a general hospital ICU with withheld life support from 2009 to 2011. Age, sex, McCabe's score, SAPS II score on admission and within $24 \mathrm{~h}$, SOFA score, main indication for ICU, main comorbidities, modalities of treatments limitation, length of ICU and in-hospital length of stay were recorded.

Results One hundred and fourteen patients were included. Six-month survival rate was $58.8 \%$. Survival was associated with longer length of ICU and hospital stay, a respiratory indication for ICU (47.8 vs $19.1 \%$, $p=0.006)$, a medical history of chronic respiratory disease (52.3 vs $29.8 \%, p=0.017$ ) and a lower SAPS II score on admission (44 vs 49 , $p=0.006$ ). Only a respiratory indication for ICU was identified as a prognostic factor of survival in the multivariate analysis (OR 0.31, $95 \%$ $\mathrm{Cl} 0.1-0.7, p=0.006)$.

Conclusion $58.8 \%$ of patients discharged from ICU with withheld lifesustaining therapies survived at least six months. Admission for a respiratory reason may be a prognostic factor of survival.

\section{Competing interests None.}

\section{References}

1. Ferand $E$, Robert R, Ingrand P, Lemaire F, French LATAREA Group. Withholding and withdrawal of life support in intensive-care units in France: a prospective survey. French LATAREA Group. Lancet. 2001 Jan 6;357(9249):9-14

2. Gacouin A, Jouneau S, Letheulle J, Kerjouan M, Bouju P, Fillatre P, et al. Trends in Prevalence and Prognosis in Subjects With Acute Chronic Respiratory Failure Treated With Noninvasive and/or Invasive Ventilation. Respir Care. 2015 Feb;60(2):210-8.
P248

Epidemiology of refusals in a polyvalent intensive care unit ad outcome of refused patients

Stephane Rouleau', David Schnell', Arnaud Desachy', Sylvie Calvat ${ }^{1}$, Philippe Petua', Charles Lafon', Christophe Cracco'

${ }^{1}$ Service de réanimation polyvalente, CH D'Angoulême, Angoulême, France

Correspondence: Stephane Rouleau - stephane_rouleau@yahoo.com Annals of Intensive Care 2016, 6(Suppl 1):P248

Introduction Few process of refusals in intensive care unit (ICU) has currently been evaluated. This study aimed at investigating the epidemiology of refusals, the factors influencing the decision and the outcome of refused patients.

Patients and methods All referrals not followed by admission were prospectively collected over a 6-month period in a 12-bed ICU in a general hospital of 500 beds. In our ICU, the refusals are usually recorded with a specific form collecting the identities of the patient and the calling doctor, the reason for referral (ethical advisory or medical referral), a short medical history and the reason(s) of refusal. Outcome of the refused patients is retrospectively collected from the hospital charts.

Results One hundred twelve refusals were included in the present study. Over the same period, 269 patients were admitted to the ICU. The referrals were internal in $91(81.3 \%)$ cases and from other institutions in $21(18.7 \%)$ cases. The reasons for the refusal were: supportive care deemed "futile" in 58 (51.5\%) cases, supportive care deemed unnecessary in 39 (35\%) cases, inadequate technical facilities in 10 (9\%) cases and no ICU box available in 5 (4.5\%) cases. Among patients refused for "futility," one or more criteria leaded to the decision: age ( $21.4 \%$ of cases), acute pathology not curable ( $18.8 \%$ of cases), multiple comorbidities (17.9\%), patient bedridden or suffering from severe dementia (17.9\%) and "terminal" comorbidity with poor short-term prognosis ( $11.6 \%$ of cases).

The outcome of the refused patients was evaluable in 79 of them hospitalized in the hospital. Among them, 5 patients were secondarily admitted to the ICU: 3 for worsening clinical status and 2 after revision of the initial assessment of "futility." Thirty-two patients were refused for unnecessary supportive care and $4(12.5 \%)$ died in the hospital. Forty-seven patients were not admitted to the ICU because supportive care was deemed "futile," and 31 (66\%) of them died in hospital.

Among the 47 patients refused because supportive care was deemed "futile," 25 (53.2 \%) referrals were motivated by the need of a second doctor for ethical advisory. The intensivist called felt "alone to decide" in 9 (19\%) cases, and these 9 patients died (Fig. 36).

Discussion Refusals are common in our practice. Supportive care deemed "futile" represents the leading reason for these refusals. In these settings, the outcome of refused patients is dark, similar to that

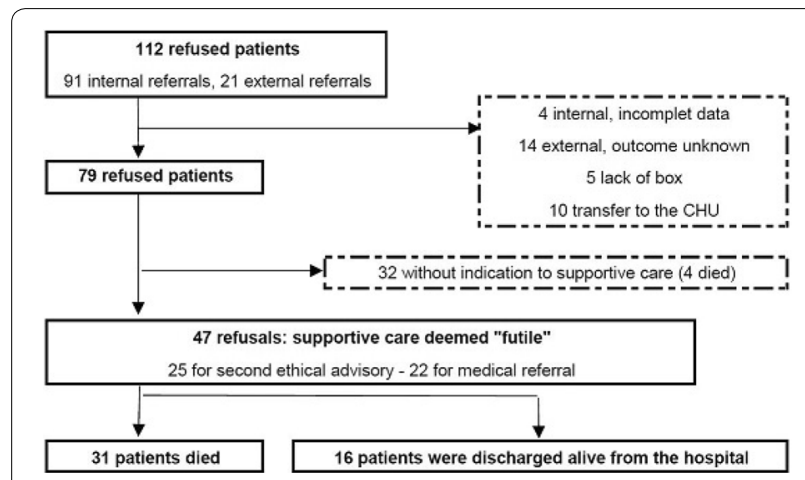

Fig. 36 See text for description 
of critically ill patients with decisions of therapeutic limitations. The ICU physician was called for ethical advisory in only half of these cases and was therefore the only decider of the therapeutic limitation in a large proportion of these patients.

Conclusion Our experience highlights the need of rethinking the process leading to ICU refusal in order to promote collegiality in these decisions of therapeutic limitation.

\section{Competing interests None.}

\section{P249}

Ten critical sentences you have already heard in the Intensive Care Unit and the Emergency Department, their real meanings and consequences

Nicolas Van Grunderbeeck', J. Temime ${ }^{1}$, Perrine Molmy ${ }^{1}$, Stephanie Barrailler', G. Gasan', Olivier Nigeon², Didier Thevenin

${ }^{1}$ Réanimation polyvalente and USC, C.H. de Lens, Lens, France; ${ }^{2}$ Service d'accueil des urgences, Centre Hospitalier de Lens, Lens, France

Correspondence: Nicolas Van Grunderbeeck - nicovgdb9@orange.fr Annals of Intensive Care 2016, 6(Suppl 1):P249

Introduction Several sentences are often used in the Intensive Care Unit (ICU) and the Emergency Department (ED) and do not exactly mean what they sound. We asked intensivists to select the most often heard sentences, what their real meaning is and which consequences their misuses reveal.

Materials and methods All the intensivists (7 physicians), fellows (5), and ED physicians (5) working in an ICU and intermediate care unit were asked which sentences with confounding meaning they have already often heard in the ICU and the ED. Their real sense was then "traducted", and their potential impact in daily practice was established. The most cited sentences are presented with their "real-life" traduction (Trad) and consequences (Csq).

Results 1st (14 votes): "it's not for full code ICU but just to overcome a hurdle". Trad: some patients with severe comorbid condition and acute organ dysfunctions are often referred to the ICU for limited care with scarce hope of recovery. Csq: inadequate intensity of care regarding severity, overuse of ICU beds, lack of palliative care, harm for other potential patients.

2nd (11 votes): "we can still give another course of chemotherapy if he/she survives after ICU admission". Trad: we have not decided about what is reasonable for this patient, so go on. Csq: patients with neoplasic disease and prolonged ICU stay are often unable to cope with subsequent toxic treatments, when they survive. There may be a lack of communication between ward physicians and intensivists, associated with lack of knowledge of each other's condition of patients' management.

3rd (9 votes): "first give antibiotics a chance". Trad: surgery is too risky right now, or for this patient. Csq: surgery is even more dangerous after surgery delay when the indication is clear.

4th (7 votes): "the patient does not sleep". Trad: I am bored of surveying and speaking to this patient to reassure him. Csq: overuse of sedation, prolonged mechanical ventilation and ICU stay, sedation-related complications (pneumonia, delirium).

5th (7 votes): "I'm saying this for the well-being of the patient". Trad: I don't want to do this. Csq: lack of beneficial care for the patient through bad (or no) communication between physicians and nurses' teams, and overall, lack of motivation.

6th (6 votes): "this patient cannot go to the ward because of his/her severity" (even when no escalation of treatment is considered regarding to age, comorbidities, or general condition). Trad: this patient cannot die in a (my) speciality ward. Csq: overuse of ICU and intermediate care unit beds, that move towards "acute palliative units".

7th (5 votes): "the patient goes alive from the ICU so for us it's a success". Trad: this patient will die anyway, but later and somewhere else. Csq: inadequate communication with family and post-ICU units. We fail to look further than the ICU doors and to plan downstream management.

8th (5 votes): "we will see -the problem/the family- tomorrow". Trad: we sometimes postpone difficulties and evade our responsibilities, waiting for someone else to do it later. Csq: in the ICU and general acute care, the sooner is often better, and we fail to remind this. "The best way to take care of the patient is to care for the patient" - right now. 9th (4 votes): "we can't allow him/her to die from sepsis/acute myocardial infarction/pneumothorax/so on". Trad: We just don't not know how to stop when the engines are on, and sometimes we do not valuate patients evolution with hindsight. Csq: everyone dies anyway at the end of his life, but we sometimes fail to remember it in the ICU and to learn it as well!

10th (3 votes): "the patient is fine". Trad: this can mean almost everything, from the patient is well educated or not dependent, or he's able to seat or eat alone, to a complete lie when it's more suited (ex: the patient is suffering dementia and needs total daily care, but looks well, and has to be transferred preferably in the ICU or in an intermediate care unit). Csq: you cannot trust anyone since you have not seen the patient by yourself first. Could telemedicine improve Critical Care as it was made by stroke teams?

Discussion What is said is not always what it means. These key sentences could be recorded in daily practice to point out some ways of care improvement in the ICU, before the ICU, or after.

Conclusion Everyone should be careful of what is said around the ICU, and be aware of lies and falsehoods.

Competing interests None.

\section{P250}

Communication with the parents: a challenge in the pediatric intensive care unit

Agathe Béranger', Charlotte Pierron², Laure De Saint Blanquat ${ }^{3}$, Sandrine Jean ${ }^{4}$, Hélène Chappuy ${ }^{5}$

${ }^{1}$ Laboratoire d'éthique médicale, Université Paris Descartes, Paris, France; ${ }^{2}$ Réanimation pédiatrique, Hospital Robert-Debré, Paris, France; ${ }^{3}$ Réanimation et surveillance continue médico-chirurgicales, Hôpital Necker - Enfants Malades, Paris, France; ${ }^{4}$ Réanimation pédiatrique et néonatale, Hopital pour enfants Trousseau, Paris, France; ${ }^{5}$ Urgences pédiatriques, Hôpital Trousseau, Paris, France

Correspondence: Agathe Béranger - agathelise@wanadoo.fr

Annals of Intensive Care 2016, 6(Suppl 1):P250

Introduction The admission of a child in the pediatric intensive care unit (PICU) is a particular situation, with a high emotional stress for the parents, bound to a threat to life for the child. A lot of complex information are given by the caregivers to the parents at the admission, during a family conference. The purpose of this study was to evaluate the comprehension of the parents in this context.

Patients and methods This prospective study took place in three university-affiliated PICU in Paris, France. Forty-two parents of 30 children who were hospitalized in the PICU more than $48 \mathrm{~h}$ were included for an audio-recorded semi-structured interview, in the first 15 days following the admission. The physician and nurse who took care of the children at the admission filled a questionnaire. The information was assessed from parents and caregivers, based on 7 prospectively defined items: diagnosis, affected organ, purpose of admission, prognosis, treatments, further investigations and the organization of the unit. The parents comprehension was graded excellent, fair or poor, depending on the comparison between the answers of the parents during the interview and the answers of the physicians and nurses in their questionnaires.

Results The comprehension was excellent for the prognosis (96.2\%), the affected organ (84.6\%) and the organization of the unit (88.9\%), fair for the purpose of admission $(68.4 \%)$ and the diagnosis $(61.9 \%)$ and poor for the treatments $(38.1 \%)$ and the further investigations $(34.5 \%)$. Only 4 parents had a complete comprehension for all the items. The prognosis was not communicated to 16 parents (12 family conferences), and 9 parents ( 6 family conferences) did not recall receiving any prognostic information. Twenty-five parents (59.5\%) thought that the given information by the physicians and the nurses were clear and sufficient. Sixty percentage of the parents expressed the need to have a good communication with the caregivers. But this parents' need was not as important as the expectation for the caregivers' professional skills (74 \%).

Discussion The comprehension of the prognosis was excellent, despite a lack of communication about prognosis by the physicians in 
the ICU and a high emotional stress for the parents. The anticipated information could be delivered before the ICU admission, by the emergency department's physician or the transport's caregivers.

Conclusion At the admission of a child in the PICU, after a family conference, the prognosis was the best understood information by the parents, although it was not always communicated. Giving information to the parents is a challenge for the caregivers and must be improve.

\section{Competing interests None.}

\section{References}

1. "Mieux vivre la réanimation" 6ème conférence de consensus SRLF - SFAR, Paris, 19 novembre 2009. Disponible sur internet: URL : http://www.sfar.org/ article/180/mieux-vivre-la-reanimation-cc-2009. Consulté le 01/07/2015.

2. Azoulay E, Chevret S, Leleu G, et al. Half the families of intensive care unit patients experience inadequate communication with physicians. Crit Care Med 2000;28:3044-9

\section{P251}

\section{End of life in the intensive care unit: between practice} and knowledge

Pierre-Marie Bertrand ${ }^{1}$, Alexandra Beurton ${ }^{2}$, Laetitia Bodet-Contentin ${ }^{3}$, Julie Boisramé-Helms ${ }^{4}$, Pierre-éRic Danin ${ }^{5}$, Elsa Dubois ${ }^{6}$, Elodie Gélisse ${ }^{7}$, Yasemin Karaca ${ }^{8}$, Pierre Mora ${ }^{9}$, Nicolas de Prost ${ }^{10}$, Damien Roux ${ }^{11}$, Alexis Soummer ${ }^{12}$, Dominique Vodovar ${ }^{13}$, Committee of young intensivists of the French Society for Intensive Care Medicine (FICS)

'Service de réanimation médico-chirurgicale, Centre hospitalier, Cannes, France; ${ }^{2}$ Réanimation médicale, Centre Hospitalier Universitaire de Nancy, Vandœuvre-lès-Nancy, France; ${ }^{3}$ Réanimation polyvalente, CHRU Hôpitaux de Tours, Tours, France; ${ }^{4}$ Réanimation médicale, Nouvel Hôpital Civil, CHU Strasbourg, Strasbourg, France; ${ }^{5}$ Réanimation chirurgicale, Hôpital l'Archet 2, Nice, France; ${ }^{6}$ Sau-samu-smur, C.H.U. de Nancy, Nancy, France; ${ }^{7}$ Unité de réanimation Robert Debré, CHU REIMS, Reims, France; ${ }^{8}$ Service de réanimation pédiatrique, CHRU de Lille - Hôpital Jeanne de Flandre, Lille, France; ${ }^{9}$ Service de réanimation médicale, CHU Cochin - Saint-Vincent de Paul - Site Cochin, Paris, France; ${ }^{10}$ Réanimation Médicale, Hôpital Henri Mondor, Créteil, France; ${ }^{11}$ Réanimation médico-chirurgicale, Hôpital Louis-Mourier - APHP, Colombes, France; ${ }^{12}$ Réanimation, Hospital Foch, Suresnes, France; ${ }^{13}$ Réanimation Médicale et Toxicologique, Hôpital Lariboisière, Paris, France

Correspondence: Julie Boisramé-Helms - julie.helms@libertysurffr Annals of Intensive Care 2016, 6(Suppl 1):P251

Introduction End of life has emerged as a major concern this year in France, with the adoption of the "Claeys-Leonetti" law, which is currently being examined by the Senate, together with the application of the "Maastricht III" (non-heart-beating donors) protocol in a few hospitals. In this context, the Committee of young intensivists of the French Society for Intensive Care Medicine (FICS) has evaluated by questionnaire both the feeling of young intensivists on everyday practices and the knowledge of the current legislation regarding the end of life ("Leonetti" and "Maastricht III").

Materials and methods A questionnaire was sent by email during the summer of 2015 to 1191 young members of the FICS ( $<35$ years of age, representing approximately one third of the SRLF members).

Results Characteristics of the members who answered One hundred and twenty-three $(10.3 \%)$ replied to the questionnaire. The sex ratio W/M was 0.78 , the mean age was 31 years old, and the geographical origin of the members who responded was not different from those who did not respond. Sixty-two percent of the members who answered declared working in a University hospital, $91 \%$ in an adult intensive care unit, and $13 \%$ in a pediatric intensive care unit. Thirty-three percent were residents, and $67 \%$ were senior intensivists.

Practice and feeling of answering members Excluding emergency situations, $90 \%$ of decisions to forgo life-sustaining treatments were declared to be decided by $\geq 2$ intensivists (including juniors and seniors). In only $13 \%$ of the decisions an external doctor was involved, whereas in $63 \%$ of the cases nurses were involved. The decision was formalized on a specific form in $69 \%$ of the cases. Young intensivists reported having been in disagreement with decisions to forgo life-sustaining treatments, because decisions were too early (65\%) or too late
(94\%) according to them. Overall, $84 \%$ of the young intensivists questioned felt qualified to announce and explain the decision to forgo life-sustaining treatments to the family members. Seventy-nine percent stated they did not have a good knowledge of the different legal texts about end of life, and $93 \%$ acknowledged that a formal update on the subject would be required.

Knowledge evaluation Overall, there were $81 \%$ of correct answers to the multiple-choice questions evaluating the knowledge on current legislation concerning the end of life ("Leonetti" and "Maastricht III"), with a better knowledge of Leonetti vs. Maastricht III texts ( 83 vs. $71 \%$, respectively). Eleven percent of the answering members did not know what the Maastricht III text was about.

Discussion Only a small number of young intensivists, members of the FICS, have answered the questionnaire, which represents one of the main limitations of this survey. This low response rate may illustrate the discomfort of young intensivists with these issues. Positive points were, however, that the questionnaire was not only declarative, but that it also evaluated the actual knowledge of young intensivists. This survey therefore provides a picture of young intensivists' knowledge, daily practices, and feelings regarding the end of life management.

Conclusion Our questionnaire sheds light on three major points: a discomfort about key decisions of active therapeutic limitations, a fair knowledge of legal documents, together with a strong demand for an update and teaching of these texts.

\section{Competing interests None.}

\section{S1} The HICU Score (Hematologic Intensive Care Unit Score): a new prognostic score for predicting 30-day and 1-year mortality of patients with hematologic malignancies admitted in ICU Yohan Desbrosses ', Guylaine Labro², Gaël Piton², Adrien Chauchet ${ }^{1}$, Etienne Daguindau', François Blot ${ }^{3}$, Eric Deconinck', Gilles Capellier ${ }^{2}$ ${ }^{1}$ Hématologie, CHRU de Besançon, Besançon, France; ${ }^{2}$ Réanimation médicale, CHRU de Besançon, Besançon, France; ${ }^{3}$ Réanimation médicale, Institut Gustave Roussy, Villejuif, France

Correspondence: Yohan Desbrosses - desbrossesyohan@gmail.com Annals of Intensive Care 2016, 6(Suppl 1):S1

Introduction Patients with hematologic malignancies $(\mathrm{HM})$ requiring intensive care unit (ICU) admission are assumed to have a poor prognosis. The aims of this study were to analyze long-term outcome and to develop a prognostic score evaluating mortality risk of patients with $\mathrm{HM}$ admitted to the ICU.

Patients and methods This was a retrospective single-center study of patients with $\mathrm{HM}$ admitted at least once to a university medical ICU, from 2003 to 2013. Hematologic history, circumstances and reason of ICU admission, life-supporting intervention were recorded. The study end points were ICU, 30 days, hospital, and 1-year survival.

Results Of 345 admissions, median age was 57.4 years $( \pm 0.9), 28.7 \%$ had received allogeneic hematopoietic stem-cell transplantation, $9.6 \%$ had newly diagnosed malignancies, and $36.2 \%$ were in remission. $39.4 \%$ had a Performans Status (PS) between 3 and 4 . The major causes of ICU admission were acute respiratory failure (38.6\%) and septic shock (27.2\%). Median SOFA score at admission was $8.8( \pm 0.2)$. Sixty-six percentage required invasive mechanical ventilation, $64 \%$ vasopressor drugs, $35 \%$ renal replacement therapy. Median time between hospital and ICU admission was 14.6 days ( \pm 1.6$)$, and $15.4 \%$ were emergency admissions. Two hundred and ninety-nine patients were admitted at least once. ICU, 30 days, hospital and 1-year survival rates were $58,51,31$ and $26 \%$, respectively.

By logistic multiple regression analysis, PS $\geq 3$, age $\geq 60$ year, SOFA score $\geq 8$, invasive mechanical ventilation and renal replacement therapy were independently associated with 30-day mortality (Fig. 37; Table 1). These factors were included in a prognostic score: the HICU Score (Hematologic ICU score) going from 0 to 5 points, for predicting 30-day and 1-year mortality of patients with HM admitted in ICU (Fig. 37; Table 2). This score appears to be able to identify patients with a poor prognosis, independently of the type of HM. An HICU Score $\geq 4$ was associated with 30-day and 1-year mortality rates of 81 and $94 \%$, respectively (Fig. 37; Table 3). 


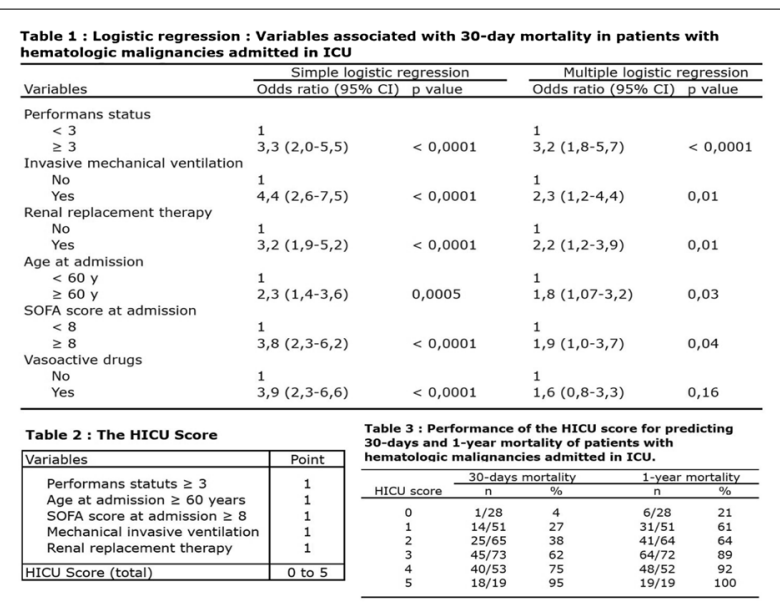

Fig. 37 See text for description

Conclusion The HICU Score is a new prognostic score, including Performans Status, age, global severity, and two modalities of therapy, for predicting 30-day and 1-year mortality of patients with hematologic malignancies admitted to the ICU.

\section{Competing interests None.}

\section{S2}

\section{Patients with multiple myeloma in the ICU: trends of use} and outcomes over time

Akli Chermak', Adrien Joseph², Virginie Lemiale ${ }^{3}$, Claire Pichereau 4 , Marinne Baron ${ }^{5}$, Emmanuel Canet ${ }^{6}$, Eric Mariotte ${ }^{2}$, Michaël Darmon ${ }^{7}$, Elie

Azoulay ${ }^{6}$

${ }^{1}$ Réanimation polyvalente, $\mathrm{CH}$ sud Essonne, Paris, France: ${ }^{2}$ Reanimation medicale, Hôpital Saint Louis, Paris, France; ${ }^{3}$ Medical ICU, Hôpital Saint Louis, Paris, France; ${ }^{4}$ Réanimation médicale, Hopital saint Louis, Paris, France; ${ }^{5}$ mmuno hematologie, Hôpital Saint-Louis, Paris, France; ${ }^{6}$ Réanimation médicale, Hôpital Saint-Louis, Paris, France; ${ }^{7}$ Réanimation Médicale, CHU Saint-Etienne - Hôpital Nord, Saint-Étienne, France

Correspondence: Akli Chermak - akli@chermak.fr

Annals of Intensive Care 2016, 6(Suppl 1):S2

Introduction Patients with multiple myeloma (MM) have experienced significant survival gains with the use of high-dose therapy regimen and newly released targeted therapies. Studies have shown continuous survival improvement over time in critically ill myeloma patients. We sought to perform a follow-up study with patients with $\mathrm{MM}$ recently managed in the ICU.

Patients and methods This is a retrospective study including all myeloma patients admitted to a 12-bed medical ICU between 2007 and 2014. Outcomes were compared with historical cohort of patients managed in the same ICU between 1999 and 2006 (control period). Determinants of hospital mortality were identified using a multivariable logistic regression model.

Results Two hundred and ninety-one patients were admitted to the ICU from 1999 to 2014: 182 in the study period and 109 in the control period. One hundred and thirty-seven (45\%) were male, aged 62 [57-70] years old. Half the patients had at least one comorbidity, and SAPSII score at admission was 45 [37-58]. Underlying myeloma required 2 [1-3] lines of therapy (chemotherapy or targeted therapy were administered in $60 \%$ of the patients), whereas 88 (30\%) patients had a newly diagnosed myeloma. Time from hospital admission to ICU admission was 1 [0-8] day. Reasons for ICU admission were acute respiratory failure (49\%), acute kidney injury (41.6\%) and shock (29.7\%). Throughout the ICU stay, patients needed mechanical ventilation (30\%), noninvasive ventilation (15\%), vasopressors $(25 \%)$ and renal replacement therapy (31\%). Hospital mortality was $32 \%$ (93). Length of follow-up was 134 [10-462] days.
By univariable analysis, patients managed during the study period were older (63 [58-71] vs. 60 [54-65], $p<0.01)$ and had more comorbidities [93 (51\%) vs. 50 (41\%), $p=0.09$ ]. In the recent period, MM was more frequently newly diagnosed [61 (34\%) vs. $27(24 \%), p<0.001]$ and targeted therapies administered more frequently [142 (78\%) vs 40 $(33 \%), p<0.01]$. In the study period, time from hospital to ICU admission was shorter ( $10-6]$ vs $3[0.25-9], p=0.02)$. Interestingly, SAPS II was lower in the second period (43 [37-55] vs 49 [38-64], $p=0.006$ ), but organ dysfunctions such as acute respiratory failure and acute kidney failure were more frequent $[99(54 \%)$ vs $49(40 \%), p=0.001$ and $107(58 \%)$ vs. $19(16 \%), p=<0.001$, respectively]. Proportions of patients receiving supportive care were not different between the 2 periods. Hospital mortality was lower in the recent period in comparison with the control period [47 (39\%) vs. $46(25 \%), p=0.01$ ]. Patients in whom life-sustaining therapies were withdrawn or withheld increased over time (5\% in the control period vs $18 \%$ in the study period, $p=0.008$ ), suggesting the increased use of ICU trials with time. By multivariate analysis, factors associated with hospital mortality were the need for mechanical ventilation [OR 2.94 (1.58-5.49), $p=0.001]$, noninvasive ventilation [OR $2.55(1.26-5.19), p=0.01]$ and vasopressors [OR 2.65 (1.38-5.12), $p=0.003$ ].

Strikingly, long-term survival was also higher in the second period. Long-term mortality was independently associated with age [OR $1.0(1.0-1.0), p=0.04]$, mechanical ventilation [OR $3.26(1.78-5.96)$, $p<0.001]$, Salmon-Durie stage III [2.0 (1.0-3.9) for class $3, p=0.03$ ] and end of life decisions [OR $3.4(1.4-8.5), p=0.01]$. Period of ICU admission was not associated with lower long-term mortality [OR 0.68 $(0.39-1.17), p=0.17]$ by multivariate analysis.

Conclusion This study reports continuous survival gains over time in critically myeloma patients resulting from a changing use of ICU in this setting. For instance, older patients were managed, with earlier ICU admission, and increased use of ICU trial. Hospital and long-term mortality seem to plateau at rates that are among the lowest mortality rates reported in critically ill hematology patients.

Competing interests None.

\section{Reference}

1. Peigne V, Rusinova K, Karlin L: Continued survival gains in recent years among critically ill myeloma patients. ICM, 2009; 35:512-518.

\section{S3}

Impact of bone marrow aspiration on the management of adult critically ill patients

Laure Calvet ${ }^{1}$, Anne Françoise Sapin² ${ }^{2}$, Bruno Pereira ${ }^{3}$, Alexandre

Lautrette', Bertrand Souweine ${ }^{1}$

${ }^{1}$ Réanimation médicale, CHU Gabriel-Montpied, Clermont-Ferrand,

France; ${ }^{2}$ Laboratoire d'hématologie, CHU Estaing, Clermont-Ferrand, France; ${ }^{3}$ Department of Clinical Research and Innovation (DRCI), CHU Clermont-Ferrand, Clermont-Ferrand, France

Correspondence: Laure Calvet - Icalvet@chu-clermontferrand.fr

Annals of Intensive Care 2016, 6(Suppl 1):S3

Introduction Bone marrow aspiration (BMA) is sometimes performed in intensive care unit (ICU) patients; however, its contribution in the management of ICU patients has not been extensively investigated yet.

Patients and methods This was a 5-year (2010-2014) retrospective cohort study in a 10-bed medical ICU on consecutive patients who underwent BMA during their ICU stay. BMA contributed in the management of patients when it yielded one of the following abnormalities nonpreviously diagnosed (increased myeloid precursors with little maturation, hemophagocytic lymphohistiocytosis (HLH), infiltration of cancer cells (solid tumor or hematologic malignancy)) and/or when it resulted in the initiation or the interruption of a specific treatment, including the decision to forgo life-sustaining therapy (DFLST).

Results Adequate BMA specimens were obtained in 193 patients (sex ratio $=1.5$ and median Simplified Acute Physiology Score $\|=60$ ). Samples were obtained by sternal aspirate in 155 patients and from the iliac crest in $38(20 \%)$. The median [interquartile range] time between ICU admission and BMA was 5 days [3-8]. The indications 
for BMA were thrombocytopenia $(N=82)$, agranulocytosis $(N=6)$, and suspicion of hematologic malignancy $(N=71)$, of solid cancer $(N=6)$, of HLH $(N=18)$, of disseminated tuberculosis $(N=5)$ and of other causes $(N=5)$. BMA findings contributed in the management of 41 patients $(21 \%)$ including increased myeloid precursors with little maturation $(N=10), \operatorname{HLH}(N=17)$, hematologic malignancy $(N=15)$, initiation of a specific treatment $(N=6)$, DFLST $(N=8)$. In multivariate analysis, the factors associated with a contributive BMA were persistent sepsis from admission to BMA (OR 2.2, $p=0.049$ ), ongoing hematologic malignancy or solid cancer (OR $3.2, p=0.03$ ), and a higher SOFA score (excluding platelet points) on the day of BMA (OR 1.1, $p=0.011$ ). Similar results were observed in the 149 patients without ongoing hematologic malignancy and solid cancer on ICU admission. Conclusion BMA has a significant impact on the management of severely ill patients with and without ongoing hematologic malignancy and solid cancer on ICU admission.

\section{Competing interests None.}

\section{S4}

\section{Red blood cells transfusion in ICU: descriptive analysis of French} cohort from "Age BLood Evaluation" clinical trial

Lucie Vettoretti', Sebastien Pili-Floury², Gaël Piton', Jacques Lacroix ${ }^{3}$, Lucy Clayton ${ }^{4}$, Elham Sabri ${ }^{5}$, Laurent Bardiaux ${ }^{6}$, Pierre Tiberghien 7 , Paul Hebert ${ }^{8}$, Gilles Capellier ${ }^{1}$, ABLE French Investigators

${ }^{1}$ Réanimation médicale, CHRU de Besançon, Besançon, France; ${ }^{2}$ Réanimation chirurgicale, $\mathrm{CHU}$ de Besançon, Besançon, France; ${ }^{3}$ Urgences et soins intensifs pédiatriques, CHU Sainte-Justine, Montréal, Canada; ${ }^{4}$ Clinical research, CHU Sainte-Justine, Montréal, Canada; ${ }^{5}$ Centre for practice-changing research, Hospital Research Institute, Ottawa, Canada; ${ }^{6}$ Distribution, Etablissement Français du Sang, Besançon, France; ${ }^{7}$ Direction de la recherche, Etablissement Français du Sang, Saint-Denis, France, ${ }^{8}$ Hospital research institute, Hospital Research Institute, Ottawa, Canada

Correspondence: Lucie Vettoretti - Ivettoretti@chu-besancon.fr Annals of Intensive Care 2016, 6(Suppl 1):S4

Introduction Red blood cells transfusions are common use in ICU to treat anemia. By enhancing oxygen delivery, fresh red cells may improve patient outcome.

Patients and methods ABLE study (multicenter, randomized, doubleblind trial) was designated to show a 90-day mortality reduction in patients who received fresh blood. Patients were eligible if a first red cells transfusion was prescribed within 7 days after admission in ICU. Enrolled patients were assigned to receive either red cells (stored less than 8 days) or standard issue red cells (oldest compatible units available in the blood bank). ABLE was an international study, and 19 French ICU spread over 10 cities were associated with this project. We report the subset group of patients included in the French cohort compared to the global one.

Results Between April 2011 and May 2014, 287 patients (169 males, 118 females) were enrolled in France while overall 2430 patients were included. The main reasons for ICU admission were respiratory or cardiovascular failure, trauma and sepsis. On the day of randomization, mean age was $59.3( \pm 16.9), 96.5 \%$ of patients were under invasive mechanical ventilation, $67.6 \%$ received vasoactive support, and $33.8 \%$ were receiving renal replacement therapy. The mean Apache II score was $24 \pm 8.4$. A total number of 1568 red cell units were transfused in 285 patients (5.5 units per patient). The first transfusion occurred between 2 and 5 days after ICU admission. Pre-transfusion hemoglobin level was $7.6 \pm 1.1 \mathrm{~g} / \mathrm{dL}$. Regarding 285 patients transfused and treatment allocation, 139 patients were assigned to receive fresh red cells and 146 were assigned to receive standard red cells. Red cells were stored a mean $( \pm S D)$ of $4.55 \pm 1.7$ days in the fresh-blood group as compared to $18.57 \pm 6.9$ days in the standard-blood group $(P<0.0001)$. Our data are different from the all cohort with lengths of storage of $6.1 \pm 4.9$ and $22.0 \pm 8.4$ days, respectively. At 90 days, 51 patients (37.2\%) in the fresh-blood group and 48 patients (33.1\%) in the standard-blood group had died (absolute risk difference, 0.041 percentage points; $95 \%$ confidence interval [CI], -0.07 to 0.15 ) compared, respectively, to 37.0 and $35.3 \%$ (absolute risk difference, 1.7 percentage points; $95 \%$ confidence interval [CI], -2.1 to 5.5 ) as reported in the international cohort. Consistent with ABLE results, no significant different 90-day mortality has been demonstrated in the French cohort but the observed larger difference needs further analysis. Data were also analyzed regarding major illnesses; duration of respiratory, hemodynamic or renal support; length of stay in ICU, hospital; adverse events. There were no differences in these secondary outcomes.

Conclusion The French cohort did not demonstrate difference in primary and secondary outcome between fresh and standard red blood cells transfusion. The larger difference between mortality in the French cohort compared to the global one still needs further analysis.

\section{Competing interests None.}

\section{S5}

Graft-versus-host disease trajectories and mortality in critically ill allogeneic stem cell recipients

Claire Pichereau', Etienne Lengliné2, Anne-Sophie Moreau', Eric Mariotte ${ }^{1}$, Marion Venot ${ }^{1}$, Sandrine Valade ${ }^{1}$, Akli Chermak', Virginie Lemiale ${ }^{1}$, Emmanuel Canet ${ }^{1}$, Gérard Socié ${ }^{3}$, Benoît Schlemmer ${ }^{1}$, Elie

Azoulay $^{\prime}$

${ }^{1}$ Réanimation médicale, Hôpital Saint-Louis, Paris, France; ${ }^{2}$ Hématologie, Hôpital Saint Louis, Paris, France; ${ }^{3}$ Hématologie-greffe de moelle, Hôpital Saint Louis, Paris, France

Correspondence: Marion Venot - marionvenot@free.fr

Annals of Intensive Care 2016, 6(Suppl 1):S5

Introduction Studies on allogeneic stem cell recipients admitted to the intensive care unit constantly report poor outcomes, even though slight improvements have been recently reported. Factors such as mechanical ventilation or acute graft-versus-host disease are associated with increased hospital mortality. In studies on unselected hematology patients, allogeneic stem cell recipients always exert highest mortality rates. However, these studies had difficulties defining graftversus-host disease, as they hardly describe graft-versus-host disease trajectory (as opposed to the binary absent/present graft-versus-host disease status).

Patients and methods This was a retrospective single-center study of allogeneic stem cell recipients patients admitted to the intensive care unit between 2005 and 2014.

Results Hundred and ninety-one patients were included. Median age was 42 [27-56], and $68.1 \%$ of them were male. Hematologic malignancy was in complete remission at intensive care unit admission in $80.6 \%$, and acute graft-versus-host disease had occurred in $61.4 \%$ before intensive care unit admission. Patients were mostly admitted for acute respiratory failure $(42.9 \%)$ or shock $(38.8 \%)$. Median SAPSII was 40 [29.5-52.5], and median SOFA at intensive care unit admission was 6 [3.3-9]. Mechanical ventilation was required in $34.6 \%$, vasopressors in $41 \%$, and renal replacement therapy in $7 \%$. Hospital mortality was $42 \%$. By univariate analysis, remission of malignancy ( 70.3 vs $51.2 \%, p=0.01$ ), time from hospital to intensive care unit admission ( 1 [0-18.5] vs 12.5 [0-27.3] days, $p=0.01)$, SAPSII score (36 [28-43] vs 48.5 [35.8-61.8], $p<0.001)$, SOFA at admission (5 [3-8] vs 8 [5.5-11], $p<0.001$ ), the use of life sustaining therapies (mechanical ventilation 24.3 vs $48.8 \%(p=0.001)$, vasopressors 32.4 vs $52.5 \%(p=0.01)$ and renal replacement therapy 2.7 vs $12.5 \%(p=0.02)$ ) and acute graftversus-host disease prior to intensive care unit admission (53.7 vs $71.6 \%, p=0.01$ ) were significantly associated with hospital mortality. Graft-versus-host disease trajectory was the main focus of interest in this study. Six groups of patients were identified: group $1=$ no graftversus-host disease $(29.8 \%)$, group $2=$ controlled acute graft-versushost disease at intensive care unit admission (38.2 \%), group $3=$ active and stable acute graft-versus-host disease at intensive care unit admission $(11 \%)$, group $4=$ newly diagnosed acute graft-versus-host disease during intensive care unit stay $(10.5 \%)$, group $5=$ improving acute graft-versus-host disease at intensive care unit admission (4.2\%) and group $6=$ worsening acute graft-versus-host disease at intensive care unit admission (4.2\%). As expected, steroid doses and the number of immunosuppressive drugs required to control acute graft-versus-host disease differed significantly between the groups. Hospital mortality also differed significantly between the groups, ranging from 
30 to $40 \%$ (group 1: $28.1 \%$, group 2: $41.1 \%$, group $4: 30 \%$. group 5: $37.5 \%$ ) to $62 \%$ (group 3) and $100 \%$ (group 6). By multivariate analysis, graft-versus-host disease groups 3 and 6 (i.e., active or worsening graft-versus-host disease at admission) remained independently associated with hospital mortality [OR $5.42(1.87-15.70), p=0.002]$. Acute respiratory failure [OR $2.90(1.26-6.68), p=0.01]$, acute kidney injury [OR 3.23 (1.33-7.84), $p=0.01$, hepatic failure [OR 9.74 (1.07-88.49), $p=0.04]$ and time from hospital to intensive care unit admission [HR 1.01 (1.00-1.03), $p=0.03$ ] were also independent risks factors for hospital mortality.

Discussion This is the first study that includes a qualitative analysis of graft-versus-host disease status at intensive care unit admission. As reported, reluctance to admit patients with graft-versus-host disease is appropriate for patients with active or worsening acute graft-versus-host disease at intensive care unit admission. Obviously, graft-versus-host disease cannot anymore be handled as a binary variable. However, the study design exposes to admission bias as the most active graft-versus-host disease cases may have not been proposed for intensive care unit admission, or not admitted to the intensive care unit after a triage procedure. Hence, a control group of patients not admitted to the intensive care unit is required. Last, these data must be validated on an independent dataset for more reliability.

Conclusion In allogeneic stem cell recipients proposed for intensive care unit admission, a careful analysis of graft-versus-host disease trajectory is mandatory as to make appropriate decisions to admit the patients. Patients with acute graft-versus-host disease may actually benefit from intensive care unit management provided the graftversus-host disease is neither unresponsive nor worsening at the time where the decision of intensive care unit admission is made.

\section{Competing interests None.}

\section{S6 \\ Outcome of 544 patients with solid cancer in the intensive care unit: the Oncoréa study \\ Edith Borcoman', Virginie Lemiale', Eric Mariotte ${ }^{1}$, Claire Pichereau', Emmanuel Canet ${ }^{1}$, Adrien Joseph ${ }^{1}$, Akli Chermak', Sandrine Valade ${ }^{1}$, Marion Venot ${ }^{1}$, Benoit Schlemmer ${ }^{1}$, Elie Azoulay ${ }^{1}$ \\ ${ }^{1}$ Réanimation médicale, Hôpital Saint Louis, Paris, France \\ Correspondence: Edith Borcoman - eborcoman@gmail.com \\ Annals of Intensive Care 2016, 6(Suppl 1):S6}

Introduction Overall mortality of patients with solid cancer has improved over the last decade with lower cancer-related mortality as the result of diagnostic and therapeutic advances. Targeted therapies have been one of the major changes in cancer treatment between 2000 and 2009. Use of these new molecules is associated with increased survival, especially in metastatic patients. However, whether these therapeutic novelties translate into better outcomes when patients become critically ill is still uncertain. We sought to determine trends in outcomes over time in critically ill patients with solid cancer.

Patients and methods Retrospective cohort of patients with solid tumors admitted to a single ICU over a 7-year period. Survival was compared between two time periods (before and after 2010). Factors independently associated with ICU mortality were identified through a multivariable logistic regression model.

Results Throughout the study period, 544 patients (age 62 years [4577], 308 [57\%] men) with solid cancer were admitted. Primary site of cancer was lung in 115 patients $(22.3 \%)$, digestive in $103(20 \%)$, urologic in $98(19 \%)$, breast in $97(18.8 \%)$, and other sites in 103 patients (19\%). At ICU admission, 290 patients (54\%) were at metastatic stage, 245 patients $(45.4 \%)$ were receiving chemotherapy, and $90(16.7 \%)$ were treated using targeted therapies (no difference between the 2 periods). Time from hospital to ICU admission was 1 day (0-17). Patients were mainly admitted in the ICU for respiratory failure (48 \%) or severe infection (27\%). Vasopressors were administered to 172 patients $(32.2 \%)$, mechanical ventilation to 247 [58 (10.7\%) received noninvasive ventilation and $175(31.6 \%)$ were intubated], and renal replacement therapy to $72(12.5 \%)$. Goals of care at ICU admission varied substantially across the 2 study periods. For instance, full code concerned $69.1 \%$ of the patients before 2010 and $50 \%$ after 2010, whereas ICU trial was $30.1 \%$ before 2010 and $47.8 \%$ after 2010. Last, exceptional admission concerned 1 patient before 2010 and 9 patients after 2010 ( $P<0.01$ for all comparisons). A decision to withhold/ withdraw life-sustaining therapies was implemented in 142 patients $(26.1 \%)$. ICU mortality decreased over time $(28.5 \%$ before 2010 vs $16.1 \%$ after $2010, P<0.01)$. By univariable analysis, mortality was significantly increased in patients with a metastatic stage (23.1 vs $14.2 \%$, $P<0.01$ ), those who were receiving chemotherapy at ICU admission ( 23.7 vs $15.3 \%, P<0.01$ ) or those with greater time from hospital to ICU admission ( 2 days [0-10.5] vs 0 day [0-4], $P=0.01$ ). Mortality was also associated with the need for vasopressors ( 44.7 vs $7.3 \%, P<0.01$ ), intubation and mechanical ventilation $(54.3$ vs $2.4 \%, P<0.01)$, or renal replacement therapy (33.3 vs $17 \%, P<0.01)$. Mortality was lower in patients with a full code status ( 13.4 vs $26 \%, P<0.01)$ or when surgery was part of the initial cancer treatment ( 14.8 vs $22.5 \%, P=0.02$ ). By multivariable analysis, factors independently associated with ICU mortality were the need for vasopressors [OR $3.17,(95 \% \mathrm{Cl} 1.61-6.14)$, $P<0.01]$ or mechanical ventilation [OR 32.9, (14.6-74.1), $P<0.01]$; the period of ICU admission after 2010 [OR $0.30,(0.15-0.63), P<0.01]$, and a full code management [OR $0.25,(0.12-0.54), P<0.01]$.

Conclusion This study reports improved survival in oncology patients admitted to the ICU. Strikingly, this cannot be ascribable to the use of targeted therapies that did not differ across the two study periods. Beside patient's severity and the need for life-sustaining therapies, the goals of care have an independent impact on mortality suggesting that oncologists and intensivists need to update their knowledge of outcomes to avoid making inappropriate end-of-life decisions.

\section{Competing interests None.}

\section{S7} Assessing the process of ICU admission refusal in critically ill patients with hematological malignancies

Katerina Rusinova ${ }^{1}$, Virginie Lemiale ${ }^{2}$, Matthieu Resche-Rigon ${ }^{3}$, Djamel

Mokart ${ }^{4}$, Antoine Rabbat ${ }^{5}$, Frédéric Pène ${ }^{6}$, Achille Kouatchet ${ }^{7}$, Fabrice Brunee $^{8}$, Julien Mayaux ${ }^{9}$, François Vincent ${ }^{10}$, Pierre Perez ${ }^{11}$, Martine Nyunga ${ }^{12}$, Elie Azoulay ${ }^{2}$, Groupe de Recherche en Réanimation Respiratoire en Onco-Hématologie

${ }^{1}$ Department of Anesthesiology and Intensive Care, First Faculty of Medicine Charles University in Prague and General University Hospital in Prague, Prague, Czech Republic; ${ }^{2}$ Réanimation médicale, Hôpital

Saint-Louis, Paris, France; ${ }^{3}$ Biostatistiques, Hôpital Saint-Louis, Paris, France; ${ }^{4}$ Réanimation, Institut Paoli-Calmettes, Marseille, France; ${ }^{5}$ Réanimation pneumologique, Hôpital Cochin, Paris, France; ${ }^{6}$ Réanimation Médicale, Hôpital Cochin, Paris, France; ${ }^{7}$ Réanimation médicale, Centre Hospitalier Universitaire d'Angers, Angers, France; ${ }^{8}$ Réanimation médico-chirurgicale, Centre Hospitalier de Versailles, Le Chesnay, France; ${ }^{9}$ Réanimation médicale, Hôpital Pitié-Salpêtrière, Paris, France; ${ }^{10}$ Réanimation polyvalente, Groupe Hospitalier Intercommunal Le Raincy-Montfermeil, Montfermeil, France; ${ }^{11}$ Réanimation polyvalente, C.H.U. de Nancy, Nancy, France;

${ }^{12}$ Réanimation polyvalente, Centre Hospitalier de Roubaix, Roubaix, France

Correspondence: Katerina Rusinova - katerina.rusinova@vfn.cz Annals of Intensive Care 2016, 6(Suppl 1):S7

Introduction Despite robust prospective outcome data in patients with hematological malignancies admitted to the ICU, a little is known about the patients who were not admitted to the ICU. The aim of the study is to describe the circumstances of refusal, the decision-making process and the outcome of these patients.

Patients and methods Circumstances of ICU admissions/refusals were analyzed prospectively in 13 university centers in France and Belgium between January 1, 2010, and May 1, 2011. Of 1041 consecutive patients considered for ICU admission, and 202 were refused. Data including reason of refusal, reason of proposal, subjective evaluation of clinicians and outcome at hospital discharge and three months later were collected in the standardized case-report form. Results were compared with cancer patients who were admitted to the ICU. 
Statistical analysis: All tests were two-sided, and $\mathrm{P}$ values $<0.05$ were considered as indicating significant association. Analyses were performed using the R statistical software version 3.0.2.

Results The refusal rate in the study group was 202 (14\%), of which 96 patients $(47.5 \%)$ were refused because they were considered too well to benefit, and 68 patients ( $33.7 \%$ ) too sick to benefit. Two patients (1\%) refused the admission themselves, and 34 patients (16.8\%) were not admitted due to lack of available ICU beds and were transferred to another hospital subsequently.

Reasons for proposition were acute respiratory failure $(47.98 \%)$, chock (33.84 \%), acute renal injury (14.1\%), need for life-saving chemotherapy $(10.6 \%)$, coma ( $8.6 \%)$ and acute liver failure $(2.5 \%)$.

Reasons for refusal included poor performance status (44\%), poor prior quality of life $(20 \%)$, no specific anticancer treatment option (72\%), allogeneic stem cell transplantation with uncontrolled graft versus host disease (13.4\%) and expected burden of ICU (15\%).

Overall, clinicians were comfortable about the decision (at 9 on the scale from 0 to 10), a consensus was reached with the addressing clinician in $86 \%$ of cases.

The hospital survival in patients considered too well to benefit was $48(75 \%)$ and $1(2 \%)$ in the group of patients too sick to benefit. The 3-month survival was $39(69.64 \%)$ and $0(0 \%)$ in both groups, respectively.

Conclusion Fourteen percent of patients considered for ICU admission are refused, and the ratio too well/too sick to benefit of 1.4 suggests an early referral practice in these patients. The reasons for refusal, e.g., performance status and quality of life, remain unchanged during the last decade. The decision of non-admission of a patient with hematological malignancy in the ICU is made consensually by participating physicians and is taken comfortably in the majority of cases.

\section{Competing interests None.}

\section{References}

1. Azoulay E, Mokart D, Pène F, et al. (2013) Outcomes of critically ill patients with hematologic malignancies: prospective multicenter data from France and Belgium-a groupe de recherche respiratoire en réanimation onco-hématologique study. J Clin Oncol Off J Am Soc Clin Oncol 31:2810-2818. doi: 10.1200/JCO.2012.47.2365.

2. Garrouste-Orgeas M, Montuclard L, Timsit J-F, et al. (2005) Predictors of intensive care unit refusal in French intensive care units: a multiple-center study. Crit Care Med 33:750-755

\section{S8}

Neurologic and hepatic failures at ICU admission are associated with worst prognosis for patients with lymphomas

Aurélien Sutra Del Galy ${ }^{1}$, Achille Kouatchet ${ }^{2}$, Mélanie Mercier ${ }^{1}$, Jean-François Hamel ${ }^{3}$, Tommy Raveau ${ }^{2}$, Marie-Pierre Moles ${ }^{1}$, Aline Clavert ${ }^{1}$, Mathilde Hunault-Berger ${ }^{1}$, Alain Mercat ${ }^{2}$, Norbert Ifrah ${ }^{1}$, Aline Schmidt-Tanguy ${ }^{1}$

${ }^{1}$ Maladies du sang, Centre Hospitalier Universitaire d'Angers, Angers,

France; ${ }^{2}$ Service de réanimation médicale et médecine hyperbare, Centre Hospitalier Universitaire d'Angers, Angers, France; ${ }^{3}$ Département de statistiques, Centre Hospitalier Universitaire d'Angers, Angers, France

Correspondence: Aurélien Sutra Del Galy - aurelien.sutradelgaly@gmail. com

Annals of Intensive Care 2016, 6(Suppl 1):S8

Introduction The prognosis of malignant lymphomas has improved over the past years. However, some patients experience life-threatening complications of treatments and need intensive care unit (ICU) admission. We conducted a retrospective study to assess factors associated with survival for these patients.

Patients and methods The files of all patients with malignant lymphoma admitted to ICU in our University Hospital between January 2008 and June 2014 were recorded. Demographic, biologic and disease characteristics as well as details of ICU admission were collected. Cox regression was performed to identify formal admission factors associated with hospital mortality. Respiratory failure was defined as previously reported (1). Hemodynamic, renal, hepatic and neurologic failures were defined as a MAP $<70 \mathrm{mmHg}$, as a creatinine 2 times baseline (KDIGO score $\geq 2$ ), as bilirubin $>50 \mu \mathrm{mol} / \mathrm{l}$ and as a Glasgow coma score of less than 13 , respectively.

Results One hundred and thirty-three patients with lymphomas were admitted to ICU during the studied period. Median age was 65 (range 18-88). Hematologic malignancies were DLBCL (41\%), Hodgkin lymphoma (11\%), follicular lymphoma (8\%), mantle cell lymphoma (8\%), Burkitt lymphoma (5\%), other low-grade B cell NHL (14\%) and T cell lymphoma (13\%). Visceral involvement was present in 103 cases ( $77 \%$ ) with bone marrow involvement in 51 cases ( $49 \%$ of them). At the time of admission, 22 patients were at the beginning of hematologic treatment $(17 \%), 30$ patients presented relapse or progressive disease $(22 \%), 28(21 \%)$ and $18(14 \%)$ patients were in complete or partial remission, respectively. Thirty-five patients $(26 \%)$ were admitted before any treatment mostly for complications of lymphoma locations ( $54 \%$ ), infections ( $29 \%$ ) and spontaneous tumour lysis syndrome (14\%). Median time from diagnosis was 144 days (interquartile range, IQR: 26-874).

ICU admissions occurred after a median of 2 days of hospitalization (IQR 0-15). In the overall population, 84 patients presented signs of sepsis (63\%) complicated by shock for 27 patients (32\% of them). The main cause of admission was respiratory distress for 48 patients ( $36 \%)$, shock for 31 patients ( $23 \%)$, acute kidney injury for 19 patients (14\%), coma for 13 patients (10\%), cardiac arrest for 5 patients $(4 \%)$ and other cause for 17 patients (13\%). Median organ failures number was 2 (IQR 1-3). $69.2 \%$ patients could be discharged from the ICU and $61.7 \%$ from hospital, whereas one-year overall survival was $37 \%$.

During the first $24 \mathrm{~h}$ after ICU admission, 50 patients (38\%) required vasopressive drugs, 57 patients $(43 \%)$ required mechanical ventilation and 18 patients $(14 \%)$ required renal replacement therapy. Number of organ replacement therapy during the first $24 \mathrm{~h}$ was greatly associated with overall survival. One-year OS rates were $51,39.8$ and $15.6 \%$ for 0 (51 patients, $38 \%$ ), 1 (45 patients, $34 \%$ ) and 2 to 3 (37 patients, $28 \%$ ) organ replacement therapy, respectively (log-rank $p<0.001)$.

In multivariate analysis, relapsing and progressive disease [HR $2.395 \%$ confidence interval (CI) 1.2-4.3; $p=0.016$ ] and neurologic or hepatic failures at admission (HR $2.295 \% \mathrm{Cl} 1.2-4.2 ; p=0.013$ and HR $3.795 \%$ Cl 1.8-7.6; $p<0.001$, respectively) were independently associated with higher risk of hospital mortality. Age $>65$ years, sepsis, neutropenia, respiratory, renal or hemodynamic failures were not independently associated with an increased risk of death. Median SOFA score (2) (Sequential Organ Failure Assessment) at ICU admission was 6 (range $0-19$ ) and appeared efficient to determine risk of mortality at hospital discharge (HR $1.395 \% \mathrm{Cl} 1.2-1.4, p<0.001$ ). Since the SOFA score is calculated after $24 \mathrm{~h}$ in the ICU and thus cannot be assessed on admission, it was not included in the Cox model.

Conclusion To our knowledge, this is the first study describing the outcome of all malignant lymphomas in the ICU. Hepatic and neurologic failures are dramatic complications for these patients. Simply and rapidly assessing organ failures in the hematology ward is required to predict prognosis before ICU admission for patients with lymphomas.

\section{Competing interests None.}

\section{References}

1. Azoulay E, Mokart D, Lambert J, et al: Diagnostic strategy for hematology and oncology patients with acute respiratory failure: Randomized controlled trial. Am J Respir Crit Care Med 182:1038-1046, 2010.

2. Vincent JL, Moreno R, Takala J, et al. The SOFA (Sepsis-related Organ Failure Assessment) score to describe organ dysfunction/failure. Intensive Care Med 22:707-710, 1996

\section{S9}

Impact of earplugs and eye mask on sleep in critically ill patients: a prospective randomized polysomnographic study

Alexandre Demoule ${ }^{1}$, Serge Carreira ${ }^{2}$, Sophie Lavault ${ }^{3}$, Olivier Pallanca ${ }^{3}$, Elise Morawiec ${ }^{1}$, Julien Mayaux ${ }^{1}$, Isabelle Arnulf ${ }^{3}$, Thomas Similowski ${ }^{1}$ ${ }^{1}$ Intensive Care and Respiratory Division, Pitié-Salpêtrière Hospital, Paris, France; ${ }^{2}$ Anesthesia and Intensice Care Unit, Hospital Saint-Camille, Bry-sur-Marne, France; ${ }^{3}$ Sleep Disorder Unit, Pitié-Sal pêtrière Hospital, Paris, France 
Correspondence: Alexandre Demoule - alexandre.demoule@aphp.fr Annals of Intensive Care 2016, 6(Suppl 1):S9

Introduction Poor sleep is common in intensive care unit (ICU) patients, and environmental factors, such as noise and light, contribute to sleep deprivation and alterations of sleep architecture. The objective of the present study was to evaluate the impact of earplugs and eye masks on sleep architecture in ICU patients.

Patients and methods A single-center randomized controlled trial of 64 ICU patients was conducted from April 2011 to June 2013. Patients were randomly assigned to sleep with or without earplugs and a facemask. A polysomnography was performed on the first day and night following inclusion. The primary end point was the proportion of sleep stage $3+4$. Secondary end points were other descriptors of sleep and major outcome variables.

Results The proportion of sleep stage $3+4$ was 11 [3-23] \% in the control group and $13[6-23] \%$ in the protective group $(p=0.72)$. Other descriptors of sleep were not different between the two groups with the exception of long awaking that were less frequent in the protective group than in the control group [21 (19-26) vs. 31 (21-47), $p=0.02]$. In the protective group, 20 patients wore the earplugs and 18 of wore their eye mask. There was no difference among the three groups in terms of sleep quality, occurrence of a delirium, ICU length of stay and mortality, anxiety and depression on ICU discharge and day 90 and the incidence of post-traumatic stress disorder.

Conclusion In ICU patients, earplugs and eye mask do not increase the proportion of sleep stage $3+4$ but decreased the number of prolonged awakenings. They had no impact on the outcome.

\section{Competing interests None.}

S10

\section{Diaphragmatic paralysis after adult cardiac surgery: incidence} and predicting factors

Thomas D'humières' ${ }^{1}$ Pierre Dupland ${ }^{2}$, Pauline Issaurat ${ }^{1}$, Romain Vergier ${ }^{1}$, Philippe Estagnasié ${ }^{2}$, Alain Brusset ${ }^{2}$, Pierre Squara ${ }^{2}$

${ }^{1}$ Icu, Clinical Ambroise Paré, Neuilly-sur-Seine, France; ${ }^{2}$ Reanimation, Clinical Ambroise Paré, Neuilly-sur-Seine, France

Correspondence: Thomas D'humières - thomasdhumieres@hotmail.fr

Annals of Intensive Care 2016, 6(Suppl 1):S10

Introduction Phrenic nerve injury is a frequent complication of cardiac surgery, leading to prolonged hospitalization, especially in patients with chronic obstructive airway disease. Several mechanisms have been recognised including hypothermia, mechanical trauma and, possibly, ischaemia. A better understanding of these mechanisms is important in order to improve both surgical techniques and postoperative care. After stopping the pericardial ice slush and generalizing the warm blood cardioplegia, we designed this study to reassess the magnitude of early changes in the diaphragmatic function after cardiac surgery and their predictive factors.

Patients and methods This was a single-centre prospective cohort study. We included all consecutive patients who were able to undergo a preoperative investigation. The diaphragmatic function was analysed by ultrasonography, performed at day 1 (reference), then days 1 and 7 , postoperatively. The collected information included bilaterally: (1) time motion in spontaneous ventilation, (2) time motion during a sniff test, (3) Doppler tissue imaging during a sniff test. The changes between pre- and postoperative values were derived and correlated with 20 pre- and peroperative data, seen as potential predicting factors, including demographic data, presence of chronic diseases, type of surgery, number of mammary arteries grafted, intraoperative durations, postoperative biomarkers.

Results One hundred and fourteen patients were included, 87 men $(75 \%)$, age $68.9 \pm 11.4$ years. The number of grafts, valves, and combined surgery were 67,39 , and 8 , respectively. One mammary artery was grafted in 8 patients and 2 in 66 . The changes in the diaphragmatic function are summarized in Table 46.

We observed a global average decrease in all diaphragmatic function indices at D1, which mostly persisted at D7. The Doppler tissue imaging correlated with time motion indices at D1 and D7 with $p<0.01$ on both sides. According to the chosen criteria, 28 to $52 \%$ of the patients did not exhibit more than $10 \%$ decrease in their diaphragmatic function. However, this change was $>50 \%$ at day 1 in 14 to $20 \%$ of the patients persisting at day 7 (7-19\%). Among the 20 data checked as potential predictive factors of diaphragmatic dysfunction, only 3 were slightly significant (height, weight, and BMI) for some of the information tested. None was linked to all criteria.

Discussion This study has limitations. First of all, diaphragmatic ultrasonography is a highly operator-dependant technique leading to inter-observer variability. Second, the lack of power at day 7 may be partly due to missing values (patients discharged). Despite these limitations, a strong pathophysiologic link between diaphragmatic dysfunction and the 20 studied factors is unlikely. In addition, due to the absence of ice slush and cold cardioplegia, the role of hypothermia is challenged.

Conclusion Cardiac surgery decreased the diaphragmatic function by $30 \%$ as assessed using ultrasonographic indices. In the least quartile, these changes reached $46-61 \%$ according to the chosen index. All time motion indices were correlated, and Doppler tissue imaging, a new index, seems to bring comparable measurement sensitivity. We were unable to identify any strong predicting factor.

Competing interests None.

\section{S11}

Ultrasound evaluation of diaphragmatic dysfunction: prognostic marker of ICU weaning failure

Cédric Carrié1, Chloé Gisbert-Mora² ${ }^{2}$ Eline Bonnardel', Matthieu Biais' Frédéric Vargas ${ }^{3}$, Gilles Hilbert ${ }^{3}$

${ }^{1}$ Réanimation des urgences, Centre Hospitalier Universitaire de Bordeaux, Bordeaux, France; ${ }^{2} 33$, Hospital Pellegrin, Bordeaux, France; ${ }^{3}$ Réanimation médicale, Centre Hospitalier Universitaire de Bordeaux, Bordeaux, France Correspondence: Chloé Gisbert-Mora - chloegisbert@gmail.com Annals of Intensive Care 2016, 6(Suppl 1):S11

Introduction Weaning from mechanical ventilation is a major issue in intensive care due to the excess mortality implies prolonged ventilation time and reintubation. Diaphragmatic dysfunction in patients during the ICU stay is a factor involved in weaning failure. It is difficult to diagnose because of a poor nonspecific and noninvasive complementary examinations carried out routinely. It therefore seems appropriate to focus on the diaphragmatic ultrasound as additional diagnostic tool, achievable by any practitioner.

Table 46 See text for description

\begin{tabular}{|c|c|c|c|c|c|c|}
\hline & $\begin{array}{l}\text { Left time } \\
\text { motion }(\mathrm{cm})\end{array}$ & $\begin{array}{l}\text { Right time } \\
\text { motion }(\mathrm{cm})\end{array}$ & Left sniff (cm) & Right sniff (cm) & $\begin{array}{l}\text { Left tissue } \\
\text { dop. }(\mathrm{cm} / \mathrm{s})\end{array}$ & $\begin{array}{l}\text { Right tissue } \\
\text { dop. }(\mathrm{cm} / \mathrm{s})\end{array}$ \\
\hline D-1 reference & $1.62 \pm 0.68$ & $1.80 \pm 0.72$ & $2.72 \pm 0.99$ & $2.72 \pm 0.97$ & $10.4 \pm 4.25$ & $10.2 \pm 4.39$ \\
\hline D1 postop. & $1.34 \pm 0.57^{* *}$ & $1.34 \pm 0.48^{* * *}$ & $1.97 \pm 0.96^{* * *}$ & $1.90 \pm 0.71^{* * *}$ & $7.40 \pm 4.34^{* * *}$ & $7.20 \pm 3.51^{* * *}$ \\
\hline Least quartile & $-0.74(-46 \%)$ & $-0.96(-53 \%)$ & $-1.60(-59 \%)$ & $-1.50(-55 \%)$ & $-6.33(-61 \%)$ & $-5.81(-57 \%)$ \\
\hline D7 postop. & $1.40 \pm 0.85^{*}$ & $1.61 \pm 0.68$ & $2.16 \pm 1.71^{*}$ & $2.41 \pm 1.21$ & $8.24 \pm 5.82$ & $8.69 \pm 4.70^{*}$ \\
\hline Least quartile & $-0.85(-52 \%)$ & $-0.91(-51 \%)$ & $-1.60(-59 \%)$ & $-0.96(-35 \%)$ & $-3.66(-35 \%)$ & $-5.38(-53 \%)$ \\
\hline
\end{tabular}


Patients and methods The main objective was to assess the capacity of the diaphragmatic excursion maximum expiration (EDEmax) to predict weaning failure (failure of extubation failure and withdrawal test). This was a prospective, observational, bi-centric study conducted in medical and surgical ICUs (CHU Bordeaux and CHR Libourne) after agreement of the CPP. It included all adult patients who received more than $48 \mathrm{~h}$ of mechanical ventilation and possessing the prerequisites for a withdrawal test. The diaphragmatic ultrasound was performed at the beginning of the weaning trial, disconnected from the ventilator patient. Three measures of EDEmax were made under diaphragmatic law, inspiration and forced expiration in TM mode. Collections of CV, PIM and MRC score were made just before the withdrawal test.

Results Sixty-seven patients were included. After the ICU stay, $33 \%$ of patients had a weaning failure. A threshold of $\leq 2.7 \mathrm{~cm}$ EDEmax in women (If $63 \%$, Sp $63 \%$ ) and $\leq 3.5 \mathrm{~cm}$ in men (in $82 \%$, Sp $58 \%$ ) was selected to predict the test fails weaning. There was a correlation between EDEmax and MRC score [rho $=0.47(0.25-0.68) ; p<0.0001]$, $\mathrm{CV}[\mathrm{rho}=0.63(0.37-0.89), p<0.0001]$ and PIM [rho $=0.54(0.22-0.87)$, $p=0.002]$. A EDEmax $\leq 2.7 \mathrm{~cm}$, a CVTh $\leq 30 \%$ and PIM $\leq 25 \mathrm{cmH}_{2} \mathrm{O}$ were significantly associated with weaning failure, respectively $\mathrm{OR}$ 3.56 (95\% Cl 1.25-10.10; $p=0.002)$, OR 6.42 (95\% Cl 1.34-30.60, $p=0.002)$ and $\mathrm{OR} 13.80(95 \% \mathrm{Cl}, 2.00$ to $94.40 ; p=0.009)$. However, an MRC score $<48 / 60$ was not associated with weaning failure.

Discussion The ability of the EDEmax predict weaning failure was not lower than the CV, MRC score, PIM, with a threshold $\leq 2.7 \mathrm{~cm}$ in women and $\leq 3.5 \mathrm{~cm}$ in man, similar thresholds being found as predictors of CVTh $\leq 30 \%$. These thresholds were sensitized in the analysis excluding patients with cardiogenic cause of failure. In addition, the MRC score in our study appeared to underestimate isolated diaphragmatic dysfunction without associated peripheral muscle deficit. The diagnosis of diaphragmatic dysfunction, associated with weaning failure, should result in a therapeutic approach to prevent this failure, such as the prophylactic administration NAV post-extubation.

Conclusion Our results confirm that the EDEmax is a useful measure to predict weaning failure, as well as the CV or PIM. In centers without access to the measurement of CV or PIM in patients intubated for resuscitation, EDEmax is probably preferable, MRC score may prove defective in patients with isolated diaphragmatic dysfunction and so does not predictive of weaning failure.

\section{Competing interests None.}

\section{References}

1. Ely EW, Baker AM, Dunagan DP, Burke HL, Smith AC, Kelly PT, Johnson MM, Browder RW, Bowton DL, Haponik EF, Effect on the duration of mechanical ventilation of identifying patients capable of breathing spontaneously. N Engl J med 1996, Dec 19;335(25):1864-9.

2. Kim WY, Suh HJ, Hong SB, Koh Y, Lim CM. Diaphragm dysfunction assessed by ultrasonography: influence on weaning from mechanical ventilation. Crit Care Med. 2011, 39:2627-30.

\section{S12}

\section{A prospective multicentric study to evaluate the impact of breathing variability on clinical outcomes in mechanically ventilated patients}

Côme Bureau', Camille Rolland-Debord', Tymothée Poitou ${ }^{2}$, Marc Clavel ${ }^{3}$ Sébastien Perbet ${ }^{4}$, Nicolas Terzi ${ }^{5}$, Achille Kouatchet ${ }^{6}$, Thomas Similowski $^{7}$, Alexandre Demoule ${ }^{1}$, Université Paris 6 _-Pierre et Marie Curie and Institut National pour la Santé et la Recherche Médicale, UMRS1158, Paris

'Intensive Care and Respiratory Division, Pitié-Salpêtrière Hospital, Paris, France; ${ }^{2}$ Paris, Pierre and Marie Curie University, Paris, France; ${ }^{3}$ Service de réanimation polyvalente, Centre Hospitalier Universitaire de Limoges, Limoges, France; ${ }^{4}$ Service de réanimation adultes, C.H.U. Estaing, Clermont-Ferrand, France; ${ }^{5}$ Service de réanimation médicale, Clinique de Réanimation Médicale, Grenoble, France; ${ }^{6}$ Réanimation médicale,

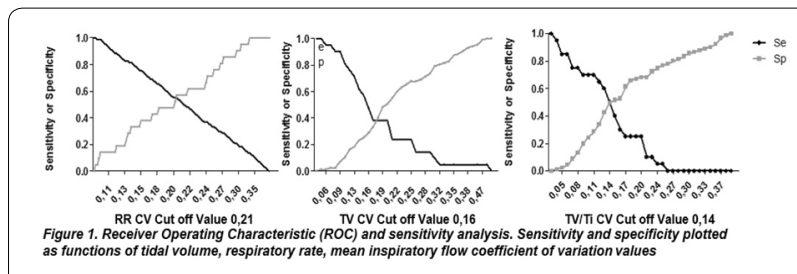

Fig. 38 See text for description

Centre Hospitalier Universitaire d'Angers, Angers, France; ${ }^{7}$ Intensive Care and Respiratory Division, Groupe Hospitalier Pitié-Salpêtrière, Paris, France Correspondence: Côme Bureau - come.bureau@gmail.com Annals of Intensive Care 2016, 6(Suppl 1):S12

Introduction Breathing is a cyclic activity that is not monotonous and is therefore variable. The variability of tidal volume, inspiratory time and mean inspiratory flow is reduced during mechanical ventilation (MV). In MV patients, breathing variability has been mostly quantified with two indices: the coefficient of variation (CV) that is defined as the standard deviation to the mean ratio and the amplitude ratio of the first harmonic peak to that of zero frequency (H1/DC).

Previous reports have suggested that a low variability was associated with higher rate of weaning failure and mortality. However, these studies have quantified variability either on very specific time points or on the contrary on the whole intensive care unit stay. The aim of our study was to quantify breathing variability early in the intensive care unit stay according to two different indices: the CV and the H1/DC. We further evaluated the factors associated with a low variability and its prognosis impact.

Patients and methods Ancillary study of a multicentre, randomized controlled trial comparing neurally ventilator adjusted assist to pressure support ventilation during the early phase of weaning. Airway flow and pressure were recorded during 4 sequences of $20 \mathrm{~min}$ Respiratory rate, tidal volume and inspiratory were measured. Variability was assessed according to H1/DC and the CV. Impact of variability on clinical outcome was determined with a receiver operating characteristic.

Results The study included 108 subjects, 72 men (66 \%), aged 68 (37$86)$ years, SAPS II 44 (14-96). Sixty-two (58 \%) subjects had hypoxemic respiratory failure. Length of mechanical ventilation prior to inclusion was 5 days long (1-35). Sensitivity and specificity were plotted as functions of the ability of the coefficient of variation of the tidal volume, respiratory rate and mean inspiratory flow to predict day- 28 mortality. The curves intersected at a coefficient of variation of 0.16 for tidal volume, 0.21 for respiratory rate and 0.14 for mean inspiratory flow (Fig. 38).

Mortality was significantly reduced in patients with a high coefficient of variation of tidal volume ( 12 vs $31 \%, p=0.024)$ and in patients with a low $\mathrm{H} 1 / \mathrm{DC}$ ratio (16 vs $60 \%, p=0.04$ ). No significant difference was observed in terms of hospital and intensive care unit length of stay, ventilator free days and duration of mechanical ventilation. Pulmonary gas exchanges were better in patients with a high coefficient of variation of tidal volume $\left(\mathrm{PaO}_{2} / \mathrm{FiO}_{2}=193(88-390)\right.$ vs $230(87-395)$, $p=0.04$ ).

Conclusion In MV patients reaching the early phase of weaning, low variability is associated with higher mortality. High variability is associated with better oxygenation. Whether a low variability is a causative factor of mortality or a consequence of severity remains to be determined.

Competing interests None. 
S13

Nurse-driven sedation protocol with sedation vacation and incidence of unplanned extubations

Pierre Lucas ${ }^{1}$, Julien Charpentier ${ }^{1}$, Michel Arnaout ${ }^{1}$, Thomas Joannon, ${ }^{1}$ Sébastien Lefort', Aude Marincamp ${ }^{1}$, Anne-Sophie Debue', Matthieu Reffienna', Frédéric Pène', Jean-Paul Mira', Alain Cariou', Jean-Daniel Chiche ${ }^{1}$, Working Group on Mechanical Ventilation

${ }^{1}$ Réanimation médicale, Hôpital Cochin, Paris, France

Correspondence: Jean-Daniel Chiche - jean-daniel.chiche@cch.aphp.fr Annals of Intensive Care 2016, 6(Suppl 1):S13

Introduction Unplanned extubation (UE) is a common complication of mechanical ventilation (MV). High incidence of UE is increasingly used as a marker of poor quality of care. Risk factors for UE depend upon the care setting (case mix, nurse to patient ratio, presence of respiratory therapists, open/closed ICUs, ...) 1 or the existence of defined care protocols aiming to reduce the duration of MV. Among those, the use of sedation and agitation scales to adjust infusion of sedatives, sedation vacation and weaning protocols may influence the incidence of UE1. We conducted this retrospective study to evaluate the incidence and risk factors for UE before/after the introduction of RASS-targeted sedation and weaning protocols, including daily sedation stops. Patients and methods Monocentre, retrospective study was conducted in the 24-bed medical ICU of Cochin University hospital. Informed consent was waived by our IRB for this observational study. Since 11/2006, all patients' data are continuously recorded in a clinical information management system (Clinisoft ${ }^{\circledR}$, GE Healthcare). Characteristics of each episode of UE (circumstances, staffing pattern, use of physical restraints, ...) are prospectively documented and reviewed during morbidity and mortality rounds. In 2009, we introduced a sedation vacation and nursedriven, RASS-targeted sedation protocol. We retrospectively reviewed all cases of UE over a 6-year period to assess the incidence, characteristics and risk factors for UE before and after implementation of the protocol. Data are presented as medians and interquartile ranges. A $P$ value $<0.05$ was considered significant for all statistical tests performed.

Results Between 11/2006 and 11/2012, 4029 pts were mechanically ventilated for 3 [2;8] days. Among them, we identified 199 patients (age $51[36,64], 132 \mathrm{M} / 67 \mathrm{~F}$, SAPS2 52 [39,62], SOFA 6 [4,9]) with 199 episodes of UE (incidence 7.5/1000 MV days). Reasons for intubation in the UE group were coma (97 pts), acute respiratory failure (61 pts), cardiac arrest (27 pts), shock (14 pts). There was a significant difference in the incidence of UE and characteristics of UE episodes before and after implementation of the RASS-targeted sedation protocols, weaning protocols and sedation vacation $(5.65 / 1000$ vs $8.38 / 1000 \mathrm{MV}$ days, $P<0.05)$. Of note, no UE occured during or within the $2 \mathrm{~h}$ following a sedation stop.

Conclusion Following implementation of a nurse-driven sedation protocol with sedation vacation, we observed an increase in the incidence of unplanned extubations although there was no temporal relationship between sedation stops and UE. These data warrant a specific analysis of risk factors for UE during both periods.

\section{Competing interests None.}

\section{References}

1. Paulo Sergio Lucas da Silva, MD, MSc, and Marcelo Cunio Machado Fonseca, MD, MsC. Unplanned Endotracheal Extubations in the Intensive Care Unit: Systematic Review, Critical Appraisal, and Evidence-Based Recommendations. Anesthesia \& Analgesia 2012 May;114(5):1003-14.

\section{S14}

Lung ultrasound enables to detect weaning-induced cardiogenic pulmonary oedema

Alexis Ferre', Max Guillot', Jean-Louis Teboul', Daniel Lichtenstein², Gilbert Meziere ${ }^{3}$, Christian Richard', Xavier Monnet ${ }^{1}$

'Service de réanimation médicale, inserm umr_s 999, Hôpital de Bicêtre, Université Paris-Sud, Faculté de Médecine, Université Paris-Saclay, Le
Kremlin-Bicêtre, France; ${ }^{2}$ Service de réanimation médicale, AP-HP, Hôpital Ambroise-Paré, Boulogne-Billancourt, France; ${ }^{3}$ Service de réanimation polyvalente, Centre hospitalier de Sens, Sens, France

Correspondence: Alexis Ferre - ferrealexis@gmail.com

Annals of Intensive Care 2016, 6(Suppl 1):S14

Introduction We tested whether performing lung ultrasound during a spontaneous breathing trial (SBT) could detect cardiogenic weaninginduced pulmonary oedema (WIPO).

Patients and methods We included 35 patients who underwent 52 consecutive SBT (1-hour T tube tests). Lung ultrasound was performed before the SBT and at the end of SBT. The reference diagnosis of WIPO was established on the evaluation by 4 experts who were blind for the lung ultrasound results. It was based on clinical, biological, chest X-ray and echocardiography data and on clinical evolution. Lung ultrasound was performed by two investigators (AF and MG) and analysed by four authors ( $A F, M G, D L$, and $G A M$ ). Each lung was divided into 3 areas. WIPO was diagnosed if a $B$ profile appeared during SBT. The $B$ profile usually indicates hemodynamic interstitial syndrome. It was defined by 1 ) the presence of three or more $B$ lines associated with lung-sliding between two ribs and 2) this pattern being disseminated at the anterior chest wall.

Results The SBT failed in 34 cases. The WIPO diagnosis could not be established in one of these cases, which was excluded from analysis. WIPO occurred in 10 of the 33 remaining cases. Among these cases, lung ultrasound detected the occurrence of interstitial syndrome during the SBT in 8 cases. Among the 41 cases without WIPO, the lung ultrasound did not find any occurrence of interstitial syndrome during the SBT, except in two cases. For detecting WIPO, the sensitivity of lung ultrasound was $80 \%(95 \% \mathrm{Cl} 44-97 \%)$, the specificity was $95 \%$ (95\% Cl 84-99\%), the positive predictive value was $80 \%$ (95\% $\mathrm{Cl} 44-97 \%)$ and the negative predictive value was $95 \%(95 \% \mathrm{Cl}$ 84-99\%).

Conclusion Lung ultrasound performed during a SBT is reliable to establish the diagnosis of WIPO. In particular, it has a very good specificity and negative predictive value.

\section{Competing interests None.}

\section{S15}

Pleural effusion during the weaning from mechanical ventilation: prevalence and impact

Damien Roux', Tai Pham², Alexandra Beurton ${ }^{3}$, Muriel Fartoukh²,

Jean-Damien Ricard ${ }^{4}$, Alexandre Demoule ${ }^{3}$, Martin Dres ${ }^{3}$

${ }^{1}$ Réanimation médico-chirurgicale, Hôpital Louis-Mourier - APHP,

Colombes, France; ${ }^{2}$ Réanimation médico-chirurgicale, Hôpital Tenon,

Paris, France; Intensive care and respiratory division, Pitié-Salpêtrière

Hospital, Paris, France; ${ }^{4}$ Service de Réanimation Médico-Chirurgicale, $\mathrm{CHU}$ Louis Mourier, Colombes, France

Correspondence: Martin Dres - martin.dres@aphp.fr

Annals of Intensive Care 2016, 6(Suppl 1):S15

Introduction Pleural effusions (PE) are common in intensive care unit (ICU) patients, especially in patients under mechanical ventilation. Since PE can alter gas exchanges, one could hypothesize that PE could play a role in the outcome of weaning from mechanical ventilation. However, no study has yet reported the incidence and characteristics of PE in the specific context of weaning failure. Therefore, the main goal of the present study was to describe the incidence of PE in patients who failed a first spontaneous breathing trial (SBT). We also compared the incidence of $\mathrm{PE}$ in patients who failed and who succeeded their first SBT.

Patients and methods We conducted a two-step prospective observational study in three medical ICU. During the first phase, all patients with a duration of mechanical ventilation $>24 \mathrm{~h}$ who failed their first SBT had a pleural ultrasonography to determinate the absence or presence of small, moderate or large PE (visual evaluation). During the 
second phase of the study, all patients ventilated more than $24 \mathrm{~h}$ performing a SBT had a pleural ultrasonography and the presence of PE was determined so far.

Results During the first phase, 336 patients were screened and 56 failed their first SBT. Among them, 28 patients $(50 \%)$ had no PE, 18 patients ( $32 \%)$ had small PE (left or right), 8 (14\%) patients had moderate PE, and 2 (4\%) patients had large PE. During the second phase, 295 patients were screened, 104 patients were included. PE was present in $27(75 \%)$ patients who failed the SBT and in $39(57 \%)$ patients who succeed the SBT $(p>0.05)$. There were no significant differences between patients without or with small PE as compared to patients with moderate or large PE regarding the rate of failed SBT (34 vs. $37 \%$, respectively). No significant difference was found regarding the length of stay in ICU, length of mechanical ventilation either the length of weaning between patients without or with small PE as compared to patients with moderate or large PE. Two patients had a pleural drainage during the study period but without evidence of clinical effect.

Conclusion Pleural effusion is often found during the weaning from mechanical ventilation. Nevertheless, no significant impact was observed. The need of drainage of PE in this context is not supported by our data and deserves further studies.

\section{Competing interests None.}

\section{S16}

Prognostic impact of left ventricular diastolic dysfunction in patients with septic shock

Céline Gonzalez ${ }^{1}$, François Dalmay ${ }^{2}$, Emmanuelle Begot ${ }^{3}$, Nicolas Pichon ${ }^{3}$, Bruno François ${ }^{3}$, Anne-Laure Fedou ${ }^{4}$, Catherine Chapellas ${ }^{5}$, Antoine Galy ${ }^{5}$, Philippe Vignon ${ }^{6}$

${ }^{1}$ Réanimation polyvalente, CHU Limoges, Limoges, France; ${ }^{2}$ Département de biostatistiques, CHU Limoges, Limoges, France; ${ }^{3}$ Service de réanimation polyvalente/inserm cic 1435, Centre Hospitalier Universitaire de Limoges, Limoges, France; ${ }^{4}$ Service de réanimation polyvalente/université de limoges, CHU Limoges, Limoges, France; ${ }^{5}$ Service de réanimation polyvalente/université de limoges, Centre Hospitalier Universitaire de Limoges, Limoges, France; ${ }^{6}$ Service de réanimation polyvalente, Centre Hospitalier Universitaire de Limoges, Limoges, France

Correspondence: Philippe Vignon - sarah.demai@chu-limoges.fr

Annals of Intensive Care 2016, 6(Suppl 1):S16

Introduction Sepsis induces a systolic and diastolic cardiac dysfunction. Echocardiography is widely used to assess left ventricular (LV) diastolic function at patients' beside. The incidence of LV diastolic dysfunction in patients with sepsis ranges between 37 and $62 \%$. The prognostic impact of LV diastolic dysfunction in patients with septic shock remains controversial. This study aimed at evaluating a potential association between LV diastolic dysfunction identified during the initial phase of septic shock and intensive care unit (ICU) mortality.

Patients and methods This descriptive, retrospective, single-center study was conducted between 2009 and 2014. Patients were eligible if: (1) they were admitted in the ICU with a septic shock defined by: (i) persistent low blood pressure despite adequate fluid resuscitation and/or (ii) clinical signs of tissue hypoperfusion confirmed by biology (lactate $>2 \mathrm{mmol} / \mathrm{L} ; \mathrm{ScvO}_{2}<70 \%$ ) and (iii) a systemic inflammatory response syndrome due to a clinically suspected or documented infection; and if (2) they underwent a transthoracic (TTE) and/or transesophageal echocardiography (TEE) during the first $24 \mathrm{~h}$ of hospitalization with digitally recorded images. LV ejection fraction (EF) was calculated from the LV end-diastolic and end-systolic volumes measured in the four-chamber view using the monoplane modified Simpson's rule. The maximal velocity of mitral Doppler $E$ and $A$ waves, $E / A$ ratio, maximal velocity $\left(V_{\max }\right)$ of $E^{\prime}$ wave measured on the lateral mitral annulus using tissue Doppler imaging, $E / E^{\prime}$ ratio, right ventricu$\operatorname{lar}(\mathrm{RV})$ and LV end-diastolic areas ratio, and the collapsibility index of the superior vena cava $(\Delta S V C)$ were obtained. LV diastolic dysfunction
Table 47 See text for description

\begin{tabular}{|c|c|c|c|c|}
\hline Parameters & $\begin{array}{l}\text { Total popula- } \\
\text { tion }(n=223)\end{array}$ & $\begin{array}{l}\text { Survivors } \\
(n=145)\end{array}$ & $\begin{array}{l}\text { Nonsurvivors } \\
(n=78)\end{array}$ & $P$ \\
\hline LVEF ( \%) & $48 \pm 17$ & $49 \pm 17$ & $46 \pm 18$ & 0.39 \\
\hline LVEDV (mL) & $93 \pm 36$ & $95 \pm 37$ & $90 \pm 34$ & 0.37 \\
\hline$E / A$ & $1.03 \pm 0.55$ & $1.05 \pm 0.58$ & $0.99 \pm 0.46$ & 0.85 \\
\hline $\begin{array}{l}V_{\max } E^{\prime} \text { lateral } \\
(\mathrm{cm} / \mathrm{s})\end{array}$ & $11.4 \pm 4.3$ & $11.9 \pm 4.5$ & $10.5 \pm 3.7$ & 0.04 \\
\hline E/E' lateral & $8.0 \pm 3.8$ & $7.7 \pm 3.7$ & $8.5 \pm 4.0$ & 0.26 \\
\hline RVEDA/LVEDA & $0.66 \pm 0.51$ & $0.59 \pm 0.17$ & $0.77 \pm 0.83$ & 0.01 \\
\hline $\begin{array}{l}\text { Paradoxical } \\
\text { septum (n) }\end{array}$ & $14(6)$ & $7(5)$ & $7(9)$ & 0.23 \\
\hline$\triangle S V C(\%)$ & $14 \pm 13$ & $15 \pm 13$ & $14 \pm 12$ & 0.97 \\
\hline
\end{tabular}

was defined by a $V_{\max } E^{\prime}$ at the lateral mitral annulus $<10 \mathrm{~cm} / \mathrm{s}$. LV dilatation was defined by an indexed LV end-diastolic volume $>75 \mathrm{~mL}$ / $\mathrm{m}^{2}$. Patients' characteristics were compared according to ICU outcome using bivariate analyses. The variables associated with ICU mortality with a $P$ value $<0.20$ were incorporated into the multivariate analysis using forward and stepwise selection procedures. Odds ratios and their $95 \%$ confidence intervals $(\mathrm{Cl})$ were estimated.

Results Among the 540 patients hospitalized in the ICU with septic shock during the study period, 223 were studied (140 men [63\%]; age: $64 \pm 13$ years; IGS2: $55 \pm 18$; SOFA: $10 \pm 3$; Charlson: $3.5 \pm 2.5$ ) and 204 of them (91\%) were mechanically ventilated. ICU mortality was $35 \%$. The prevalence of LV diastolic dysfunction was $31 \%$ and not significantly greater in nonsurvivors (28/78 [36 \%] vs. 41/145 [28\%]: $p=0.15)$. Intergroup bivariate comparisons of echocardiographic parameters are listed in the Table 47. Inappropriate initial antibiotic therapy [OR 4.17 (95 \% Cl 1.33-12.5); $p=0.03$ ], maximal dose of vasopressors [OR $1.38(95 \% \mathrm{Cl} 1.16-1.63)$ per $\mathrm{mg} / \mathrm{h} ; p=0.01]$, SOFA score [OR 1.16 (95\% Cl 1.02-1.32) per point; $p=0.02$ ] and decrease in $V_{\max }$ $E^{\prime}$ [OR $1.12(95 \% \mathrm{Cl} 1.01-1.24)$ per $\mathrm{cm} / \mathrm{s} ; p=0.02$ ] were significantly associated with ICU mortality. After adjustment on IGS2, inappropriate initial antibiotic therapy and maximal dose of vasopressors remained independent prognostic factors, whereas $V_{\max } E^{\prime}$ became quite not significantly associated with ICU death $(p=0.07)$.

Conclusion These results suggest that LV diastolic dysfunction is marginally associated with ICU mortality of septic shock patients. A larger prospective multicenter study is required to confirm these results due to the selection bias of this single-center study.

\section{Competing interests None.}

\section{S17}

Norepinephrine exerts an inotropic effect at the early phase of human septic shock

Olfa Hamzaoui ${ }^{1}$, Mathieu Jozwiak ${ }^{2}$, Thomas Geffriaud ${ }^{2}$, Benjamin Sztrymf ${ }^{1}$, Dominique Prat ${ }^{1}$, Frédéric Jacobs ${ }^{1}$, Xavier Monnet ${ }^{2}$, Pierre Trouiller ${ }^{1}$,

Christian Richard'2, Jean-Louis Teboul ${ }^{2}$

${ }^{1}$ Réanimation polyvalente, Hôpital Antoine Béclère, Clamart, France; ${ }^{2}$ Service de réanimation médicale, Hôpital de Bicêtre, Faculté de médecine Paris-Sud, université Paris-Sud, Le Kremlin-Bicêtre, France

Correspondence: Olfa Hamzaoui - olfa_hamzaoui@yahoo.fr Annals of Intensive Care 2016, 6(Suppl 1):S17

Introduction Norepinephrine (NE) is a potent vasopressor used in septic shock to reverse hypotension. An increase in cardiac output (CO) with NE has been reported in patients with septic shock, when administered early (1). This increase in CO was suggested to be mainly 
related to an increased cardiac preload via the a-agonist-mediated decrease in systemic venous capacitance. Whether NE exerts a positive effect on cardiac contractility through beta1-adrenergic stimulation is unclear. On the one hand, the sensitivity of beta1-adrenergic receptors can be abnormally reduced in septic conditions. On the other hand, the down-regulation of beta1-adrenergic receptors may occur relatively late (2) and thus could be not seen when NE is administered early. Our study was designed to examine the inotropic effects of NE when administered in the early phase of septic shock.

Patients and methods We prospectively included patients suffering from septic shock and presenting with a mean arterial pressure (MAP) lower than $65 \mathrm{mmHg}$ despite initial hemodynamic resuscitation and for whom the physician planned either to initiate Ne or to increase its dosage. Echocardiographic measurements were performed before (T0) and after either initiating NE or increasing its dosage in order to achieve a target MAP above $65 \mathrm{mmHg}$ (T1). The following variables were collected: left ventricular ejection fraction (LVEF), velocity-time integral of the subaortic flow (VTI), tissue Doppler imaging of tricuspid annular motion (Sa), tricuspid annular plane systolic excursion (TAPSE), peak early transmitral flow velocity (E), and mean systolic velocity of the lateral mitral annulus (Sm).

Results Thirty-eight patients ( $68 \pm 15$ years) were included, their mean SAPS II was $55 \pm 20$, and the main sites of infection were the lung in 15 patients, the abdomen in 8 patients, and the urinary tract in 6 patients. The mean interval time between the start of resuscitation and T0 was of $127 \pm 58 \mathrm{~min}$. At T0, patients have already received a mean volume of fluids of $1545 \pm 688 \mathrm{~mL}$ and 16 patients had not yet received NE. In the other 22 patients, the mean dose of NE was $0.17 \pm 0.45 \mu \mathrm{g} /$ $\mathrm{kg} / \mathrm{min}$ at T0. At T1, the mean dose of NE was $0.58 \pm 0.54 \mu \mathrm{g} / \mathrm{kg} / \mathrm{min}$ for the whole population. From T0 to T1, MAP increased significantly from $56 \pm 7$ to $80 \pm 9 \mathrm{mmHg}$, systolic and diastolic arterial pressures increased from $85 \pm 12$ to $124 \pm 15 \mathrm{mmHg}$ and from $45 \pm 6$ to $60 \pm 10 \mathrm{mmHg}$, respectively, and lactate decreased from $3.2 \pm 2.0$ to $2.5 \pm 1.2 \mathrm{mmol} / \mathrm{L}(p<0.05)$.

We observed significant increases $(p<0.05)$ from T0 to T1 of: LVEF (from $49 \pm 13$ to $56 \pm 13 \%$ ), Sm (from $10.8 \pm 5.1$ to $12.1 \pm 5.0 \mathrm{~cm} / \mathrm{s}$ ), VTI (from $18 \pm 5$ to $20 \pm 6 \mathrm{~cm}$ ), E (from $81 \pm 27$ to $92 \pm 28 \mathrm{~cm} / \mathrm{s}$ ), TAPSE (from $18.3 \pm 5.1$ to $20.4 \pm 4.9 \mathrm{~cm}$ ), and Sa (from $13.0 \pm 5.6$ to $15.1 \pm 6.4 \mathrm{~cm} / \mathrm{s})$. Even for the 15 patients with a LVEF $\leq 45 \%$ at T0, LVEF and VTI increased significantly from $36 \pm 7$ to $44 \pm 9 \%$ and from $16 \pm 6$ to $18 \pm 7 \mathrm{~cm}$, respectively $(p<0.05)$.

Conclusion In spite of the increase in arterial blood pressure and thus in left ventricular afterload, NE administration at the early phase of septic shock increased the indices of left ventricular systolic function, even when the left ventricular systolic function is impaired. This suggests that NE actually exerted a significant inotropic effect at the early phase of septic shock in addition to the effect on preload.

\section{Competing interests None.}

\section{References}

1. Hamzaoui et al. Crit Care. 2010;14:R142.

2. Abi-Gerges et al. Am J Respir Crit Care Med. 1999;160:1196-204.

\section{S18}

Effect of high PEEP after recruitment maneuver on right ventricular function in ARDS: Is it good for the lung and for the heart?

Shari El-Dash' ${ }^{1}$ Loay Kontar ${ }^{1}$, François Leleu' ${ }^{1}$ Bertand De Cagny ${ }^{1}$, François Brazier $^{1}$, Dimitri Titeca', Gaelle Bacari-Risal', Julien Maizel' ${ }^{1}$, Michel Slama ${ }^{1}$ Réanimation médicale, Centre Hospitalier Universitaire, Amiens, France Correspondence: Julien Maizel - maizel.julien@chu-amiens.fr Annals of Intensive Care 2016, 6(Suppl 1):S18

Introduction There is a growing body of evidence that protective ventilatory strategies for ARDS with lower tidal volumes and higher
PEEPs may benefit the lung. But high PEEP may induce right ventricular (RV) dysfunction which may severely impair hemodynamic. We hypothesized that opening the lung with recruitment maneuver followed by maintaining high PEEP may be good for the lung and for the heart.

Patients and methods We decided to include ARDS patients under mechanical ventilation sedated and paralyzed with $\mathrm{P} / \mathrm{F}<200$. An open-lung protocol consisting of a 4-step recruitment maneuver (from 25 to $40 \mathrm{~cm}$ of PEEP) and a decremental PEEP titration (to find the best PEEP named open-lung PEEP) was performed after ruling out hypovolemia and severe acidosis. We recorded clinical parameters (mean arterial pressure (MAP), heart rate (HR)) as well as rightto-left ventricle diameter ratio (RV/LV) using 2D echocardiography, tricuspid annular plane systolic excursion (TAPSE) with M-Mode echocardiography, aortic velocity time integral (using pulsed Doppler) (VTI), systolic lateral RV annulus velocity using spectral tissue Doppler imaging (S) during the recruitment maneuver at $40 \mathrm{~cm}$ $\mathrm{H}_{2} \mathrm{O}$ and 1 and $24 \mathrm{~h}$ after this maneuver at the chosen best PEEP (open-lung PEEP). Cardiac output (CO) was measured using cardiac Doppler.

Results Ten patients with ARDS (age $55 \pm 13 \mathrm{y} / 0$ ) with SAPS $70 \pm 15$ were included. Median open-lung PEEP of $21.0 \mathrm{~cm} \mathrm{H} 2 \mathrm{O}(16.0-23.0)$ was significantly higher than the baseline PEEP of 9 (6.0-13.5) $\mathrm{cm} \mathrm{H}_{2} \mathrm{O}(p<0.01)$. $\mathrm{PO}_{2} / \mathrm{FiO}_{2}$ increased from 70.6 (40.6-89.6) to $209 \mathrm{mmHg}(141-229), p<0.01$. This benefit was maintained $24 \mathrm{~h}$ later: 165.2 (107.3-199.8), $p=0.01$ with no change of lung compliance. RV/LV increased from 0.70 [0.58-0.75] to 0.80 [0.73-0.83], $p=0.02$, during PEEP of $40 \mathrm{~cm} \mathrm{H} 2 \mathrm{O}$ and returned toward baseline values (0.75 [0.63-0.89], $p=0.10)$ at open-lung PEEP and remained at the same level at $24 \mathrm{~h}$. The TAPSE showed a statistically significant drop from baseline median value of 18 [17-21] $\mathrm{mm}$ to 16 [1517] $\mathrm{mm}(p=0.02)$ at a peep of $40 \mathrm{cmH} 2 \mathrm{O}$. At open-lung PEEP, TAPSE was the same as baseline $18[17-19] \mathrm{mm}(p=1.00)$ and remained the same $24 \mathrm{~h}$ later. $\mathrm{S}$ was 16 [13-17] $\mathrm{cm} \mathrm{s}^{-1}$ at baseline and significantly decreased during at PEEP 40 and returned to the baseline value at the end of the maneuver and remained stable at $24 \mathrm{~h}$. MAP dropped from 89 [73-95] $\mathrm{mmHg}$ at baseline to a trough of $70[60-81] \mathrm{mmHg}$ ( $p=0.01$ vs baseline) during the last step of recruitment, was 70 [6977] $\mathrm{mmHg}$ at open-lung PEEP, and was 79 [67-84] $\mathrm{mmHg} 24 \mathrm{~h}$ later.

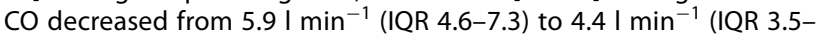
6.4) during recruitment but returned to baseline value at open-lung PEEP (5.6 I $\mathrm{min}^{-1}$ (IQR 4.4-7.1)) and remained stable at $24 \mathrm{~h}$. Ejection fraction was unchanged at open-lung PEEP and at $24 \mathrm{~h}$, compared with baseline value.

Conclusion In our ARDS patients, recruitment maneuver permitted to open the lung with a dramatic increase in $\mathrm{P} / \mathrm{F}$ and apply high PEEP of $21 \mathrm{cmH} 2 \mathrm{O}$ to maintain the lung open. Despite this high PEEP, right ventricular function was not impaired, confirming that what is good for the lung maybe not deleterious for the heart.

Competing interests None.

S19

Myocardial dysfunction during septic shock: prevalence, prognosis and role of loading conditions

Florence Boissier ${ }^{1}$, Keyvan Razazi ', Aurelien Seemann' ${ }^{1}$ Arnaud, W Thille², Nicolas de Prost ${ }^{1}$, Elise Cuquemelle, ${ }^{1}$, Filippo Boroli, ${ }^{1}$, Alexandre Bedet ${ }^{1}$,

Pascal Lim³ ', Christian Brun-Buisson 1, Armand Mekontso Dessap'

${ }^{1}$ Réanimation Médicale, Hôpital Henri Mondor, Créteil, France; ${ }^{2}$ Réanimation Médicale, CHU de Poitiers, Poitiers, France; ${ }^{3}$ Cardiologie, Hôpital Henri Mondor, Créteil, France

Correspondence: Florence Boissier - floboissier@yahoo.fr

Annals of Intensive Care 2016, 6(Suppl 1):S19

Introduction The clinical significance and prognosis of septic myocardial dysfunction is controversial. The paradoxical association between 
septic myocardial dysfunction and improved survival in some studies may be explained by the influence of loading conditions. Our aim was to assess, in a large population of patients with septic shock, the prevalence and prognosis of left ventricle systolic dysfunction, while examining the potential role of loading conditions.

Patients and methods Patients were prospectively included at the medical intensive care unit of a tertiary hospital. Hemodynamic and echocardiographic data were recorded on the first 3 days of septic shock. Septic myocardial dysfunction (hypokinesia) was defined as a left ventricle ejection fraction $<45 \%$ or by the need of inotropes to achieve a left ventricle ejection fraction $\geq 45 \%$; hyperkinesia was defined by a left ventricle ejection fraction $>60 \%$. Several indices of preload (estimates of left ventricle filling pressures and respiratory variations of vena cavae), contractility (ventricular-arterial coupling, left ventricle end-systolic maximal elastance, tissue Doppler imaging, speckle tracking imaging) and afterload (diastolic arterial pressure, systemic vascular resistance, end-systolic arterial elastance and left ventricle end-systolic wall stress) were assessed.

Results A total of 280 echocardiographies were performed in 133 patients during the first three days of septic shock. Left ventricle ejection fraction was depressed in $42(31.6 \%)$ patients, normal in 57 $(42.9 \%)$ patients and increased in $34(25.6 \%)$ patients. Patients with hypokinesia had an alteration in left ventricle contractility indices, whereas those with hyperkinesia exhibited the most severe alteration in afterload indices. Most contractility indices were inversely correlated with afterload indices. Left ventricle longitudinal peak systolic strain was altered early in patients who secondarily deteriorated their left ventricle ejection fraction. Hyperkinetic patients exhibited the most severe alteration in afterload indices and a higher in-ICU and in-hospital mortality as compared to others. Altered afterload (as assessed by lower values of left ventricle end-systolic wall stress) was significantly associated with in-ICU mortality by multivariable analysis, along with fluid balance and SOFA score.

Conclusion The alteration of left ventricle ejection fraction during septic shock was influenced by afterload, with an inverse correlation between most contractility and afterload indices. Speckle tracking-derived strain was altered early in patients who secondarily deteriorated their left ventricle ejection fraction. Hyperkinetic patients exhibited the most severe alteration in afterload indices, and a worse outcome.

Competing interests None.

\section{S20}

Echocardiography for the critically ill: 1-year experience of a noninvasive intensive care unit

W Brahim ${ }^{1}$, R Amara ${ }^{1}$, A Azouzi', Ahmed Khedher ${ }^{1}$, Jihene Ayachi', Rania Bouneb ${ }^{1}$, Y Hamdaoui ${ }^{1}$, Kaoula Meddeb ${ }^{1}$, Mohamed Boussarsar'

${ }^{1}$ Réanimation médicale, CHU Farhat Hached, Sousse, Tunisia

Correspondence: Mohamed Boussarsar - hamadi.boussarsar@gmail.

com

Annals of Intensive Care 2016, 6(Suppl 1):S20

Introduction Bedside transthoracic echocardiography (TTE) has become an important tool in the management of critically ill patients. In fact, TTE enables the intensivist to categorize the shock state and to develop an effective management strategy.

In our noninvasive ICU, we still not have bedside TTE, and this examination is made by cardiologist, in the cardiology department.

Aim To evaluate, in our context, the use of TTE and its impact on the diagnosis and/or the management of the critically ill.

Patients and methods This was a retrospective study involving all patients admitted to ICU from January to December 2014, for respiratory or circulatory failure and who may have warranted a TTE. Patients' characteristics and any change in diagnosis and/or management after TTE were recorded.

Results One hundred sixty-nine patients out of a total of 350 admissions in 2014 were included. Forty-seven (28\%) have had a TTE. Compared patient characteristics between those who underwent TTE and not are displayed in Table 48. When performed, TTE revealed left ventricle dysfunction (27.5\%) and signs of chronic pulmonary
Table 48 Compared patients' characteristics regarding TTE practice

\begin{tabular}{|c|c|c|c|}
\hline & $\begin{array}{l}\text { TTE practiced } \\
(n=47)\end{array}$ & $\begin{array}{l}\text { TTE not prac- } \\
\text { ticed }(n=121)\end{array}$ & $p$ \\
\hline Gender (M) $[n(\%)]$ & $31(66.0)$ & $82(67.8)$ & 0.48 \\
\hline $\begin{array}{l}\text { Underlying disease } \\
{[n(\%)]}\end{array}$ & $43(91.5)$ & $93(76.9)$ & 0.02 \\
\hline $\begin{array}{l}\text { Hemodynamic failure } \\
{[n(\%)]}\end{array}$ & $13(27.7)$ & $58(47.9)$ & 0.013 \\
\hline $\begin{array}{l}\text { Acute respiratory } \\
\text { failure }[n(\%)]\end{array}$ & $47(100)$ & $110(90.9)$ & 0.024 \\
\hline EKG changes [n (\%)] & $27(58.7)$ & $30(24.8)$ & 0.38 \\
\hline $\begin{array}{l}\text { Elevated cardiac } \\
\text { troponin }[n(\%)]\end{array}$ & $13(27.7)$ & $11(9.1)$ & 0.40 \\
\hline $\begin{array}{l}\text { Noninvasive ventilation } \\
{[n(\%)]}\end{array}$ & $36(76.6)$ & $50(41.3)$ & $<0.001$ \\
\hline $\begin{array}{l}\text { Invasive ventilation } \\
{[n(\%)]}\end{array}$ & $22(46.8)$ & $86(71.1)$ & 0.003 \\
\hline Vasopressors [n (\%)] & $26(55.3)$ & $32(26.4)$ & 0.11 \\
\hline
\end{tabular}

Table 49 Compared patients' characteristics according to the presence or absence of change in diagnosis and/or management of the critically ill

\begin{tabular}{llcc}
\hline & $\begin{array}{l}\text { Change+ } \\
(\boldsymbol{n = 8})\end{array}$ & $\begin{array}{l}\text { Change- } \\
(\boldsymbol{n}=\mathbf{3 9 )}\end{array}$ & $\boldsymbol{p}$ \\
\hline Gender (M) $[n(\%)]$ & $4(50.0)$ & $27(69.2)$ & 0.25 \\
Underlying disease $[n(\%)]$ & $7(87.5)$ & $36(92.3)$ & 0.53 \\
Hemodynamic failure [n (\%)] & $4(50.0)$ & $9(23.1)$ & 0.13 \\
Acute respiratory failure [n (\%)] & $8(100)$ & $39(100)$ & 0.56 \\
Invasive ventilation $[n(\%)]$ & $3(37.5)$ & $19(48.7)$ & 0.42 \\
Noninvasive ventilation $[n(\%)]$ & $6(75.0)$ & $30(76.9)$ & 0.61 \\
\hline
\end{tabular}

hypertension (6\%). However, the TTE was normal in $20 \%$ of examinations. These findings led to a change of the diagnosis in 4 patients $(10 \%)$ and in the management of $8(20.5 \%)$ patients in which $15 \%$ was considered important. Univariate analysis didn't find any factors that predict a change in the diagnosis and/or the management of the patients (Table 49).

Conclusion In our noninvasive ICU, TTE is under-prescribed. When performed, its impact on the diagnosis or the management of the patients is uncertain. Bedside TTE would be a more valuable tool giving a rapid and repeated evaluation of the critically ill patients and avoiding transport-related risk.

Competing interests None.

S21

Echocardiographic indices to predict fluid responsiveness in ventilated patients with circulatory failure: which threshold for which aim?

Emmanuelle Begot ${ }^{1}$, Xavier Repessé ${ }^{2}$, Julie Léger ${ }^{3}$, Gwenael Pratt ${ }^{4}$ Cyril Charron ${ }^{2}$, Pierre-Yves Egreteau ${ }^{5}$, Christophe Jacob ${ }^{4}$, Koceila Bouferache ${ }^{6}$, Loay Kontar? , Julien Maizel ${ }^{7}$, Michel Slama ${ }^{7}$, Antoine Vieillard-Baron², Philippe Vignon ${ }^{1}$

${ }^{1}$ Service de réanimation polyvalente/inserm cic1435, Centre Hospitalier Universitaire de Limoges, Limoges, France; ${ }^{2}$ Réanimation médico-chirurgicale, Assistance Publique - Hôpitaux de Paris, Hôpital Ambroise Paré, Boulogne-Billancourt, France; ${ }^{3}$ Inserm cic 1415, C.H. Régional Universitaire 
de TOURS, Tours, France; ${ }^{4}$ Réanimation médicale, CHRU de Brest, Brest, France; ${ }^{5}$ Réanimation, Centre Hospitalier des Pays de Morlaix, Morlaix, France; ${ }^{6}$ Réanimation polyvalente, Hôpital Foch, Suresnes, France; ${ }^{7}$ Réanimation médicale, Centre Hospitalier Universitaire, Amiens, France Correspondence: Philippe Vignon - sarah.demai@chu-limoges.fr Annals of Intensive Care 2016, 6(Suppl 1):S21

Introduction Echocardiography is increasingly used to assess patients with cardiopulmonary compromise. The identification of fluid responsiveness (FR) is key in the assessment of patients with acute circulatory failure. Several dynamic indices to predict FR obtained with transthoracic (TTE) and transesophageal echocardiography (TEE) have been previously validated in small populations of septic shock patients. Fluid resuscitation is the mainstay of acute management in most shocked patients, but a positive fluid balance is known to be detrimental on outcome. The present study aimed at determining the threshold value of echocardiographic indices to predict FR according to the clinical setting, to reduce false-negative or false-positive results. Patients and methods This descriptive, prospective, multicenter study was conducted during a 24-month period in 5 French intensive care units (ICU). All ventilated patients $>18$ year old and requiring a hemodynamic assessment using echocardiography for an acute circulatory failure (regardless of its etiology) were eligible to participate in the study. Acute circulatory failure was defined as the presence of: (1) hypotension (systolic blood pressure $<90 \mathrm{mmHg}$ or mean blood pressure $<65 \mathrm{mmHg}$ ) and/or (2) signs of tissue hypoperfusion (e.g., mottled skin, oliguria, $\mathrm{pH}<7.35$ and base deficit $>-5 \mathrm{mmol} / \mathrm{L}$, lactate $>2 \mathrm{mmol} / \mathrm{L}, \mathrm{ScvO}_{2}<70 \%$ ). Exclusion criteria were a contraindication for performing passive leg raising (PLR) or a TEE examination, or a shock secondary to a tamponade or an aortic dissection. Echocardiography was performed by an experienced operator in a patient in the semi-recumbent position $\left(45^{\circ}\right)$. The collapsibility index of superior vena cava $(\Delta S V C)$, the distensibility index of inferior vena cava $(\Delta \mathrm{IVC})$, the respiratory variations of aortic maximal doppler velocity $\left(\Delta V_{\text {max }} \mathrm{Ao}\right)$, the left ventricular ejection fraction (LVEF) and aortic Doppler velocity time integral (VTIAo) were measured. A $90^{\circ}$-PLR was then performed in all patients. VTIAo was measured after one minute of PLR. An increase in VTIAo $>10 \%$ defined fluid responsiveness.

Results During the study period, 540 patients were studied (379 men; age: $65 \pm 13$ years; SAPSII: $59 \pm 18$; SOFA: $10 \pm 3$ ), and FR was identified in 229 of them (42\%). Overall, 355 patients (66\%) had a tidal volume $<8 \mathrm{~mL} / \mathrm{kg}$ of predicted body weight, 174 patients $(32 \%)$ had an IAP $\geq 12 \mathrm{mmHg}$ and 78 patients $(14 \%)$ had a non-sinus rhythm. The origin of the acute circulatory failure was predominantly septic in 295 patients (55\%), cardiogenic in 126 patients (23\%) and hypovolemic in 112 patients (21\%). ICU mortality was $39 \%$. Mean $\triangle$ SVC, mean $\triangle I V C$ and mean $\Delta V_{\max }$ Ao were significantly greater in patients with $\mathrm{FR}(31 \pm 24 \mathrm{vs}$. $12 \pm 15 \% ; 13 \pm 14$ vs. $8 \pm 15 \% ; 15 \pm 7$ vs. $10 \pm 7 \%$, respectively; all $p<0.001)$. Patients with FR exhibited significantly lower AoVTI at baseline $(14.8 \pm 5.6$ vs. $16.9 \pm 7.2: p=0.0002)$. During $90^{\circ}-\mathrm{PLR}$, AoVTI variation reached $27 \pm 18 \%$ in patients with $\mathrm{FR}$, whereas it remained as low as $0.5 \pm 6 \%$ in their counterparts. Threshold values to optimize the sensitivity or specificity of dynamic parameters are listed in Table 50.

Conclusion Threshold values of echocardiographic indices may greatly vary according to the clinical need of optimized sensitivity or specificity to predict FR in ventilated patients with acute circulatory failure.

\section{Competing interests None.}

\section{S22}

Effects of norepinephrine on the right ventricle assessed by transthoracic echocardiography in the ICU

Claire Ragot ${ }^{1}$, Laure Corradi ${ }^{2}$, Frédéric Vargas ${ }^{3}$

133, Hopital Robert Boulin, Libourne, France; 2974 , Hopital Felix Guyon, Saint-Denis, France; ${ }^{3}$ Réanimation médicale, Centre Hospitalier Universitaire de Bordeaux, Bordeaux, France

Correspondence: Claire Ragot - cragot1@gmail.com

Annals of Intensive Care 2016, 6(Suppl 1):S22

\begin{tabular}{|c|c|c|c|c|}
\hline $\begin{array}{l}\text { Dynamic } \\
\text { parameters }\end{array}$ & $\begin{array}{l}\text { Optimized } \\
\text { sensitivity } \\
\text { (associated } \\
\text { specificity) }\end{array}$ & $\begin{array}{l}\text { Thresh- } \\
\text { old } \\
\text { value } \\
(\%)\end{array}$ & $\begin{array}{l}\text { Optimized } \\
\text { specificity } \\
\text { (associated } \\
\text { sensitivity) }\end{array}$ & $\begin{array}{l}\text { Thresh- } \\
\text { old value } \\
\text { (\%) }\end{array}$ \\
\hline $\begin{array}{l}\Delta V_{\max } A o \\
\quad(n=421)\end{array}$ & $90 \%$ (39 \%) & 7 & $90 \%$ (29\%) & 18 \\
\hline $\begin{array}{l}\Delta S V C \\
\quad(n=460)\end{array}$ & $90 \%$ (29\%) & 5 & $90 \%$ (42 \%) & 33 \\
\hline $\begin{array}{l}\Delta I V C \\
\quad(n=361)\end{array}$ & $76 \%$ (37 \%) & 3 & $90 \%$ (28 \%) & 19 \\
\hline
\end{tabular}

Introduction Right ventricular dysfunction is common in critically ill patients. Because of its disponibility, safety, and the valuable amount of information generated, transthoracic echocardiography has become the cornerstone of right ventricle exploration in the ICU. Norepinephrine could be useful in right ventricular dysfunction, by preventing right ventricular ischaemia. However, its role remains controversial, especially because of its effects on right ventricular afterload. The objective of this study was to evaluate the effects of norepinephrine on the right ventricle with use of echocardiography in critically ill patients.

Patients and methods We conducted a prospective, bicenter, physiological study. Patients included were patients remaining hypotensive after fluid resuscitation. An echocardiography was performed before and after introduction of norepinephrine. Various parameters surrogates of right ventricular systolic function were collected: right ventricular fractional area change (FAC), tricuspid annular plane systolic excursion (TAPSE), tricuspid annular motion $\left(\mathrm{s}^{\prime}(t)\right)$ by Doppler tissue imaging, right ventricular dimensions, cardiac output, pulmonary pressures and right ventricular outflow tract velocity time integral (RV VTI).

Results Fifteen patients were included. Three had an altered fractional area change and 8 a longitudinal right ventricular dysfunction defined by a TAPSE lower or equal to $16 \mathrm{~mm}$ and/or a $\mathrm{s}(\mathrm{t})$ lower or equal to $10 \mathrm{~cm} / \mathrm{s}$. In the whole population, only $\mathrm{S}(\mathrm{t})$ significantly increased from $11(8.3-14)$ to $14(12-15.8) \mathrm{cm} / \mathrm{s}, p=0.04$ under norepinephrine. FAC TAPSE, pulmonary pressures and cardiac output remained unchanged. In patients with a longitudinal right ventricular dysfunction, there was a significant increase in TAPSE from 14 (9.5-14.5) to $15(11.5-17.0) \mathrm{mm}$, $p=0.016$ and $s^{\prime}(t)$ from $8.5(8.0-10.5)$ to $12.0(8.0-13.0) \mathrm{cm} / \mathrm{s}, p=0.027$ under norepinephrine. Cardiac output and pulmonary pressures remained unchanged.

Conclusion Norepinephrine appears to improve the right ventricular systolic function in critically ill patients with compromised right ventricular function.

\section{Competing interests None.}

\section{S23}

Effect of recruitment maneuver on left ventricular systolic strain

Pablo Mercado ${ }^{1}$, Julien Maizel', Loay Kontar ${ }^{1}$, Bertand De Cagny ${ }^{1}$, François Brazier', Dimitri Titeca', Gaelle Bacari-Risal', Antoine Riviere', Shari

El-Dash', Michel Slama'

${ }^{1}$ Réanimation médicale, Centre Hospitalier Universitaire, Amiens, France

Correspondence: Julien Maizel - maizel.julien@chu-amiens.fr

Annals of Intensive Care 2016, 6(Suppl 1):S23

Introduction In ARDS patients under mechanical ventilation, recruitment maneuver may open the lung and improve blood oxygenation. During this maneuver, cardiac dysfunction may occur. We decide to analyze using speckle tracking, the effect of recruitment maneuver on the left ventricular longitudinal strain (LVLS).

Patients and methods We decided to include ARDS patients under mechanical ventilation sedated and paralyzed with $P / F<200$. An 
open-lung protocol consisting of a 4-step recruitment maneuver (from 25 to $40 \mathrm{~cm}$ of PEEP) and a decremental PEEP titration (to find the best PEEP) was performed after ruling out hypovolemia and severe acidosis. We recorded clinical parameters (mean arterial pressure (MAP), heart rate (HR)) as well as ejection fraction (EF) using 2D echocardiography, mitral annular plane systolic excursion (MAPSE) with M-Mode echocardiography, aortic velocity time integral (using pulsed Doppler) (VTI), systolic lateral LV annulus velocity using spectral tissue Doppler imaging (S) and left ventricular longitudinal stress (LVLS) using speckle tracking at baseline, during the recruitment maneuver at $40 \mathrm{~cm} \mathrm{H} 2 \mathrm{O}$ and $2 \mathrm{~min}$ after this maneuver at the chosen best PEEP.

Results Nine patients with ARDS (age $55 \pm 13 \mathrm{y} / \mathrm{o}$ ) with SAPS $70 \pm 15$, $\mathrm{P} / \mathrm{F} 130 \pm 19$, lung compliance $26 \pm 8 \mathrm{ml} / \mathrm{mmHg}$, PEEP $9 \pm 3 \mathrm{~cm} \mathrm{H} 2 \mathrm{O}$, respiratory rate $25 \pm 5 / \mathrm{mn}, \mathrm{MAP} 78 \pm 12 \mathrm{mmHg}, \mathrm{HR} 85 \pm 12 / \mathrm{mn}$, EF $60 \pm 18 \%$, VTI $22 \pm 6 \mathrm{~cm}$, MAPSE $1.3 \pm 0.5 \mathrm{~cm}, \mathrm{~S} 8.4 \pm 4.5 \mathrm{~cm} / \mathrm{s}$ were included. During recruitment at a PEEP of $40 \mathrm{cmH} 2 \mathrm{O}, \mathrm{HR} 90 \pm 14 / \mathrm{mn}$ and P/F176 \pm 85 increased; MAP $62 \pm 22 \mathrm{mmHg}$, MAPSE $1.3 \pm 0.5 \mathrm{~cm}$; $\mathrm{S} 8.4 \pm 4.6 \mathrm{~cm} / \mathrm{s} ; \mathrm{EF} 49 \pm 22 \%$, VTI17 $\pm 8 \mathrm{~cm}$ all decreased. At the best PEEP of $12 \pm 5 \mathrm{cmH} 2 \mathrm{O}, \mathrm{P} / \mathrm{F}$ dramatically increase to $242 \pm 80$ and all hemodynamical and echocardiographic and Doppler parameters returned to the baseline values. LVLS was at $-15.6 \pm 5.6 \%$ at baseline and dramatically increased to $-8.1 \pm 5.6$ at a PEEP of $40 \mathrm{cmH} 2 \mathrm{O}$ and returned to the baseline value of $-13.8 \pm 5 \%$ immediately after the recruitment maneuver. This left longitudinal strain impairment during the maneuver was more severe at the apex ( $-19 \pm 8$ vs $-7 \pm 8 \%)$.

Conclusion Recruitment maneuver induces transient LV systolic dysfunction particularly at the apex of the LV. This dysfunction may be due to a transient myocardial ischemia and disappears immediately after this maneuver.

\section{Competing interests None.}

\section{S24}

\section{Management of diabetic ketoacidosis in two pediatric intensive} care units: comparison of two care protocols

Laure Maurice ${ }^{1}$, Stéphane Dauger ${ }^{2}$, S Julliand ${ }^{3}$, Anna Deho², Fleur Le Bourgeois ${ }^{4}$

${ }^{1}$ Hospital Robert-Debré, Paris, France; ${ }^{2}$ Réanimation et surveillance continue pédiatriques, CHU Robert Debré, Paris, France; ${ }^{3}$ Reanimation pediatrique, Hospital Robert-Debré, Paris, France; ${ }^{4}$ Réanimation et surveillance continue pédiatriques, Hôpital Robert Debré, Paris, France Correspondence: Laure Maurice - maurice.laure@gmail.com Annals of Intensive Care 2016, 6(Suppl 1):S24

Introduction In France, children diagnosed with type 1 diabetes present often with diabetic ketoacidosis. In these circumstances multiple complications can occur as hydro-electrolytic disorders, hypoglycaemia and cerebral oedema, which is the most serious complication. Several potential risk factors for cerebral oedema have been identified; in particular, an early decrease in serum effective osmolality has been proposed as a possible mechanism. Thus, fast blood glucose drop, which is linked to osmolality, is supposed to be cerebral oedema main risk factor, although no evidence has yet been proven. The International Society for Pediatric and Adolescent Diabetes (ISPAD) recommends capillary blood glucose not to fall more than $5 \mathrm{mmol} / \mathrm{l} / \mathrm{h}$, and this threshold is found in several care protocols. Even though diabetic ketoacidosis complications are well known, treatment remains controversial and there is no national protocol to guide for the initial care of these patients. Thus, in two Parisians Paediatric Intensive Care Units (PICU), different care protocols are used. The main difference concerns rehydration fluids. At Necker hospital, normal saline is used during at least $4 \mathrm{~h}$ and maintained until blood glucose falls of $5 \mathrm{mmol} / \mathrm{l} / \mathrm{h}$ or under $\leq 15 \mathrm{mmol} / \mathrm{l}$. Afterward, a $0.45 \%$ sodium chloride infusion solution and dextrose $5 \%$ is used. At Robert Debré hospital, normal saline is used during $2 \mathrm{~h}$, regardless of blood glucose level, and then a $0.45 \%$ sodium chloride solution with dextrose either $5 \%$ or $10 \%$ is used. The aim of this study is to compare safety and efficacy of those two protocols. The first objective was to compare number of patients with blood glucose levels dropping more than $5.5 \mathrm{mmol} / \mathrm{l} / \mathrm{h}$ according to the protocol used. Secondary we looked for a difference between the two populations in osmotic variations, occurrence of hypoglycaemia, cerebral oedema, and delay for metabolic disorders normalization.

Patients and methods This 5-year retrospective cohort study (20102015) included 93 children with inaugural and severe diabetic ketoacidosis hospitalized in the two PICU. Severity was defined as venous $\mathrm{pH}<7.10$ or serum bicarbonate concentration $<5 \mathrm{mmol} / \mathrm{l}$ or alteration in mental status or hemodynamic disorders or age $<5$ years. First, we recorded number of patients with at least one capillary blood glucose drop more than $5.5 \mathrm{mmol} / \mathrm{l} / \mathrm{h}$ in the first $24 \mathrm{~h}$ of treatment. Second, we recorded patients characteristics, complications, maximal sodium level corrected for hyperglycaemia, osmolality decrease and maximal sodium concentration rise during the first $24 \mathrm{~h}$ of treatment.

Results No significant difference was found for demographic and clinical characteristics between the populations studied. No significant difference was noted in the number of patients with a capillary blood glucose fall more than $5.5 \mathrm{mmol} / \mathrm{l} / \mathrm{h}$, in the maximal glucose-corrected sodium and maximal effective osmolality decrease, and in sodium concentration rise during the first $24 \mathrm{~h}$ of care. Cerebral oedema and hypoglycaemia incidences were comparable in both populations. No cardiac arrhythmia was detected. Although this results are nonsignificant, delay for $\mathrm{pH}$ normalization was longer in Necker (21.9 vs $14.9 \mathrm{~h}$, $p=0.072$ ) and delay for blood glucose normalization was shorter in Necker ( 8.7 vs $10.8 h, p=0.171)$. Length of PICU stay was significantly longer in Necker (57.3 vs $37.7 \mathrm{~h}, p=0.03$ ).

Conclusion Although the care protocols for inaugural ketoacidosis in children are different, there were no significant differences in complications occurrence and in symptoms duration between both populations. Those two protocols are safe and should be combined for an easier and more efficient use in both hospitals.

\section{Competing interests None.}

S25

NUTRI-REAPED: Nutritional status on admission to the pediatric intensive care unit-a French multicentric study

Aurelien Jacquot ${ }^{1}$, Frederic Valla ${ }^{2}$, Bénédicte Gaillard-Le Roux ${ }^{3}$, Flavie Letois $^{4}$, Gilles Cambonie ${ }^{1}$, NutriSIP

${ }^{1}$ Réanimation pédiatrique, CHU de Montpellier - Hôpital Arnaud de Villeneuve, Montpellier, France; ${ }^{2}$ Réanimation pédiatrique hfme, Hospices civils de Lyon, Lyon, France; ${ }^{3}$ Pediatric Intensive Care Unit, C.H.U de Nantes, Nantes, France; ${ }^{4}$ Inserm cic 1411 , CHU de Montpellier, Montpellier, France Correspondence: Aurelien Jacquot - aurelien.jacquot@netcourrier.com Annals of Intensive Care 2016, 6(Suppl 1):S25

Introduction Children requiring intensive care are often chronically ill, and the intensive care stay may upset the balance between nutritional intakes and needs. As malnutrition is a key factor in morbidity/mortality in pediatric intensive care units (PICUs), screening for malnutrition and individualized adaptation of nutritional support are important elements of patient care. Yet, no study has previously evaluated the nutritional status of children admitted in French PICUs.

Patients and methods Principal objective: To determine the prevalence of malnutrition in the population of children admitted to intensive care.

Secondary objectives: To describe nutritional status on the basis of simple anthropometric parameters and to characterize the profile of patients with and without malnutrition.

Type of study: Observational and multicentric.

Primary assessment parameter: Malnutrition, as defined by a body mass index (BMI) value $<-2$ SD, according to the WHO Child Growth Standards.

Inclusion criteria: All children (1 month-18 years) admitted in a unit participating to the study during three one-week periods in the course of the year 2014.

Results Four hundred and forty-four patients were enrolled by the 28 participating PICUs.

The global prevalence of undernutrition was $18.5 \%$. It was particularly high among children under 5 years old: $19.6 \%$.

Young age, planned admission and chronic disease appeared as risk factors for undernutrition. 
The clinical impression of the physicians underestimated the patients' malnutrition.

Conclusion This is the first study that describes the nutritional status of children admitted to intensive care in France.

Almost 1 in 5 children is malnourished on admission in a PICU.

These results should lead to the development of training initiatives and research protocols to optimize the screening of malnutrition and the early nutritional management for children in PICUs.

\section{Competing interests None.}

\section{S26}

Apnea test in children for brain death diagnosis: efficacy and complications

Alexandre Salvadori ${ }^{1}$, Gaia Ottonello², Stéphane Blanot ${ }^{3}$, Juliette Montmayeur ${ }^{3}$, Gilles Orliaguet ${ }^{3}$

${ }^{1}$ HIA Bégin, Paris, France; ${ }^{2}$ Intensive care, Fondazione IRCCS - Policlinico San Matteo, Pavia, Italy; ${ }^{3}$ Réanimation neurochirurgicale pédiatrique, Hospital Necker, Paris, France

Correspondence: Alexandre Salvadori - alexsantard57@hotmail.fr Annals of Intensive Care 2016, 6(Suppl 1):S26

Introduction Primary outcome was to evaluate the efficacy of apnea test provided by ventilator disconnection protocol and $\mathrm{O}_{2}$ administration for the clinical confirmation of brain death in children. Secondary objectives were to analyze cardiorespiratory complications occurring during the apnea test and the evolution of the number of eligible lung transplant.

Materials and methods Retrospective single-center study performed in all pediatric intensive care unit at Necker-Enfants Malades University Hospital. From 2005 to 2014, all pediatric patients with a probable diagnosis of brain death and who required apnea test were included in the study.

Results One hundred and three patients were included from 2005 to 2014 . Apnea test was performed in $43 / 103$ patients (42 \%). Causes of brain death were trauma 14/43 (33\%), cerebral tumor $9 / 43(21 \%)$, neurovascular 8/43 (19\%), cerebral anoxia 7/43 (19\%), other 3/43 (7\%) and meningitis $2 / 43$ (5\%). Apnea test was successful in $40 / 43$ patients ( $93 \%$ ) and prematurely stopped in $3 / 43$ patients $(7 \%)$. Causes of apnea test interruption were hemodynamic failure in 2 patients and hypoxemia in 1 patient. There were no significant differences between the number of successful apnea test in children $\leq 2$ years old compared with children $>2$ years old $(13 / 15(87 \%)$ vs. $27 / 28(96 \%)$, $p=0.3) . \mathrm{PaO}_{2} / \mathrm{FiO}_{2}$ ratio $(276 \pm 126$ before apnea test vs. $179 \pm 120$ after apnea test, $p=0.0002$ ) and the number of eligible lung transplant (18/41 (44\%) before apnea test vs. 6/38 (16\%) after apnea test, $p=0.007)$ significantly decrease during the apnea test course. No serious complications occurred during apnea test courses.

Discussion The efficacy of apnea test for brain death diagnosis in children and infants is comparable with studies published in adult's population. However, gas exchange is impaired during apnea test course as showed by the drop in $\mathrm{PaO}_{2} / \mathrm{FiO}_{2}$ ratio. The decrease in $\mathrm{PaO}_{2} / \mathrm{FiO}_{2}$ ratio could be explained by atelectasis formation. Recruitment maneuvers and protective ventilation strategies could prevent $\mathrm{PaO}_{2} / \mathrm{FiO}_{2}$ ratio drop and limit the decrease in the number of available lung transplant after apnea test. Systemic hypotension occurred in 9/41 patients ( $21 \%$ ) but without metabolic impairment as shown by the stability of base excess $(-3.5 \pm 4.6$ vs. $-4.8 \pm 4.6 \mathrm{mEq} / \mathrm{L}, p=0.3)$

Conclusion Apnea test performed with a ventilator disconnection protocol is efficient for brain death diagnosis in children whatever age group without serious complication. However, prospective studies are necessary to evaluate methods such as alveolar recruitment maneuvers limiting the drop in $\mathrm{PaO}_{2} / \mathrm{FiO}_{2}$ ratio during apnea test course.

\section{Competing interests None.}

\section{References}

1. Wijdicks EFM, Rabinstein AA, Manno EM, Atkinson JD. Pronouncing brain death: Contemporary practice and safety of the apnea test. Neurology. 2008 Oct 14;71(16):1240-4.

2. Datar S, Fugate J, Rabinstein A, Couillard P, Wijdicks EFM. Completing the apnea test: decline in complications. Neurocrit Care. 2014 Dec;21(3):392-6.
S27

Interest of the ultrasound in the central venous catheterization in children poses in intensive care unit

Fehmi Ferhi' ${ }^{1}$, Hosni Khouadja', Mohamed Amine Bouslama ${ }^{1}$, Affra Brahim', Khalil Tarmiz², Khaled Benjazia ${ }^{1}$

${ }^{1}$ Anesthesia and Intensive Care, Chu Farhat Hached, Sousse, Tunisia;

${ }^{2}$ Sousse centre, Chu Farhat Hached, Sousse, Tunisia

Correspondence: Khalil Tarmiz - khalil.tarmiz@gmail.com

Annals of Intensive Care 2016, 6(Suppl 1):S27

Introduction The installation of central venous catheterization through (CVC) is a percutaneous procedure difficult in infants and children. Venipuncture is usually done blindly; however, this procedure is associated with a high morbidity and a significant risk of failure. The purpose of this study is to evaluate the usefulness of the technique of ultrasound-guided installation in reducing the failure rate and morbidity in pediatric intensive care unit.

Patients and methods Our study was conducted in the pediatric intensive care unit of University Hospital Farhat Hached of Sousse. We compared two groups. Group S: standard technique $(N=15)$ and Group E: technical guided echo $(N=15)$. We collected data related to the difficulty of installation and subsequent complications.

Results The average number of attempts and the duration of the procedure were significantly lower in group $E$, respectively, 4 and 2 versus $7 \mathrm{~min}$ versus $17 \mathrm{~min}$. The success rate at the first puncture was far more important in group $\mathrm{E}$. The arterial punctures and bruises were avoided in group $E 0 / 15$ versus $5 / 15$. The number of infections on catheters and thrombosis were significantly higher in group $\mathrm{S}$.

Conclusion Our work has helped to highlight the superiority of the installation of central venous catheters guided by real-time ultrasound in children in intensive care unit.

\section{Competing interests None.}

\section{S28}

Supraclavicular approach to the innominate vein in pediatric intensive care unit: interest in the prevention of thrombosis and catheter-related blood stream infection

Flora Habas ${ }^{1}$, Julien Baleine', Julia Lebouhellec ${ }^{1}$, Christophe Milesi ${ }^{1}$, Clementine Combes' ${ }^{1}$, Gilles Cambonie'

${ }^{1}$ Réanimation pédiatrique, Hôpital Arnaud-de-Villeneuve, Montpellier, France

Correspondence: Julien Baleine - jf-baleine@chu-montpellier.fr Annals of Intensive Care 2016, 6(Suppl 1):S28

Introduction The feasibility and the reduction of complications related to the insertion of supra clavicular central venous catheters were already demonstrated in pediatric intensive care unit. The data concerning maintenance-related complications (thrombosis and catheter-related bloodstream infections) with this insertion site are lacking We proposed to analyze the complications related to the maintenance of supraclavicular catheter versus the others sites of insertion.

Patients and methods A retrospective analysis was performed over a 4-year period in a pediatric intensive care unit. All non-tunneled central venous catheters inserted in the unit were enrolled. Dialysis catheters, peripherally inserted catheters or umbilical cord catheter were excluded. The primary outcome was the comparison of rates of infection and/or thrombosis per 100 catheters and the incidence density of the catheter related blood stream infection per 1000 catheter-days. Secondarily were considered immediate complications and characteristics of maintenance-related complications.

Results Two hundred and fifty-seven central venous catheters were included accounting for 2902 catheter-days. One hundred and fortyseven catheters were inserted in supraclavicular position $(57.2 \%)$ with an increasing proportion during the study period (from 21 to $84 \%$; $p<0.05)$. Patients were younger in the supraclavicular group $(4.6$ $(0.7-38.7)$ months vs $12.5(2.4-41.6) ; p=0.014)$ and for the proportion of neurologic or respiratory admissions. Ultrasound guidance was systematic for the supraclavicular approach compared to $56 \%$ for the others sites $(p<0.001)$. 


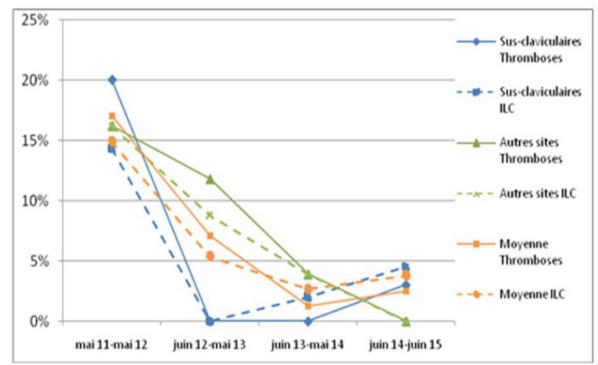

Figure1: Evolution des taux bruts des différentes complications en fonction des périodes

Fig. 39 See text for description

Complications related to the maintenance were less frequent in the supraclavicular site (incidence rate 5.4 vs $16 \% p=0.006$ ) especially regarding thrombosis ( 2.7 vs $10 \% p=0.016$ ) and approaching significance for catheter-related blood stream infections (3.4 vs $9.1 \%$, $p=0.063$ ). The incidence density of catheter-related blood stream infections was also lower (2.8 episodes per 1000 catheter-days vs 8.9, $p<0.001$ ). Time of onset of maintenance-related complications was longer with the supraclavicular approach: Concerning thrombosis, it was $15.5(9-25.5)$ vs $7(6-21)$ days for the others sites $(p=0.157)$ and 14 (6-16) vs 11 (9-15) days for the catheter-related blood stream infection $(p=0.771)$. The femoral site was found to be a significant risk factor for thrombosis in a logistic regression analysis (odds ratio $6.1195 \%$ Cl 1.77-25.6; $p=0.0064)$. Coagulase negative staphylococci was the most frequent bacteria whatever the site.

Immediate complications were less frequent with the supraclavicular approach but only arterial puncture reached significance (Fig. 39). Conclusion Supraclavicular approach of the innominate vein with ultrasound guidance is gaining interest since few years. This technique is feasible in pediatric intensive care unit even in the younger infant. We confirm that immediate complications are reduced. Furthermore, maintenance-related complications seem to be limited with this approach. Larger and eventually randomized trials are needed to confirm these results.

\section{Competing interests None.}

\section{References}

1. Supraclavicular ultrasound-guided catheterization of the subclavian vein in pediatric and neonatal ICUs: a feasibility study. Guilbert AS, Xavier L, Ammouche C, Desprez P, Astruc D, Diemunsch P, Bientz J. Pediatr Crit Care Med. 2013 May;14(4):351-5.

2. Comparison between ultrasound-guided supraclavicular and infraclavicular approaches for subclavian venous catheterization in children-a randomized trial. Byon HJ, Lee GW, Lee JH, Park YH, Kim HS, Kim CS, Kim JT. Br J Anaesth. 2013 Nov;111(5):788-92.

\section{S29}

EPURE Study: Preliminary results on the organization of pediatric acute dialysis in 2 French-speaking countries

Maryline Chomton ${ }^{1}$, Philippe Jouvet ${ }^{1}$, Bruno Ranchin², Marie Alice Macher ${ }^{3}$, Théophile Gaillot ${ }^{4}$, EPURE Study Group

${ }^{1}$ Soins intensifs pédiatriques, CHU Sainte-Justine, Montréal, Canada: ${ }^{2}$ Pédiatrie, CHU Lyon, Lyon, France; ${ }^{3}$ Pédiatrie, CHU Robert Debré, Paris, France; ${ }^{4}$ Pédiatrie, Hospital Center University De Rennes, Rennes, France

Correspondence: Philippe Jouvet - philippe.jouvet@umontreal.ca

Annals of Intensive Care 2016, 6(Suppl 1):S29

Introduction The management of children with acute kidney injury (AKI) may require a renal replacement therapy (RRT). There are three RRT methods available for children including peritoneal dialysis (PD), intermittent hemodialysis (iHD) and continuous RRT (CRRT), but it is a challenge for pediatric units to maintain staff competency and expertise for all 3 methods due to the low RRT incidence (0.7-2.4\% of pediatric intensive care units (PICU) admissions (1)). The aim of our study was to assess the RRT methods currently used in French-speaking pediatric centers.

Patients and methods We conduct a multicentric, prospective observational study in French-speaking pediatric centers $(n=24)$, both PICUs $(n=25)$ and pediatric nephrology units (PNUs, $n=6)$ from France and Canada. EPURE study started in 2012 and is financially supported by a PHRC. PICUs and PNUs characteristics are collected, and children ( 0 to 17 years old) with AKI requiring RRT are included, after parent consent, and followed up for 1 year. The study is approved by French and Canadian Institutional Review Boards. The overall distribution of RRTs modalities among children already included in the study was analyzed.

Results Two hundred forty-three children with AKI and RRT are currently included in the EPURE study, (500 are planned) and data from 181 are analyzed and reported in this abstract. The use of CCVH, PD and $\mathrm{iHD}$ among children with AKI was 43,41 and $15 \%$, respectively. The RRT methods distribution differed according to the AKI diagnosis. In hemolytic uremic syndrome (HUS), the use of CCVH, PD and iHD among children was 29,55 and $16 \%$, respectively. For the same age and AKI diagnosis, the RRT method used differed from a center to another probably because only $27 \%$ of the analyzed centers reported that they were able to provide the three RRT methods to children.

Discussion The choice of technique does not seem to only depend on age and diagnosis but also on other factors such as center expertise.

Conclusion $\mathrm{CCVH}$ and peritoneal dialysis are more frequently used than hemodialysis in children with AKI. Further analysis of EPURE study that will include patients follow-up at 1 year will inform us on the impact of these RRT choices on AKI recovery.

\section{Competing interests None.}

\section{Reference}

1. A Merouani, P Jouvet. Epuration extra-rénale en Pédiatrie. In : M Legrand, D Journois. Epuration extra-rénale en Réanimation. Ed : Paris : Arnette, 2015, 333-349.

\section{S30}

Hyperglycemia in severe scorpion envenomed children: incidence and impact outcome

Mabrouk Bahloul', Najeh Baccouche ${ }^{2}$, Kais Regaieg ${ }^{2}$, Turki Olfa' ${ }^{1}$, Chtara Kamilia', Benhamida Chokri', Hedi Chelly ${ }^{2}$, Mounir Bouaziz ${ }^{2}$

${ }^{1}$ Réanimation polyvalente, Faculté de médecine de Sfax, Sfax, Tunisia;

${ }^{2} \mathrm{CU}, \mathrm{CHU}$ Habib Bourguiba, Sfax, Tunisia

Correspondence: Mabrouk Bahloul - bahloulmab@yahoo.fr

Annals of Intensive Care 2016, 6(Suppl 1):S30

Introduction To determine the incidence of hyperglycemia and its impact prognosis in severe scorpion envenomed children requiring intensive care admission.

Patients and methods We retrospectively included 626 patients aged less than 16 years admitted for scorpion envenomation over a period of 15 years in the 22-bed intensive care unit (ICU) of our university hospital. Were excluded all patients without biological glycemia on ICU admission. All included patients were stratified in two groups according to their biological glycemia on ICU admission: hyperglycemia group (hyperglycemia $>10 \mathrm{mmol} / \mathrm{L}$ ) and hyperglycemia free group. Results There were 361 males and 265 females. The mean age $( \pm$ SD) was $5.9 \pm 3.9$ years. The hyperglycemia group included 316 patients (50.4\%). The mean delay between scorpion envenomation and ICU admission was not significantly different between two groups. The comparison between two groups with and without hyperglycemia showed that respiratory failure, the use of mechanical ventilation, the presence of pulmonary edema and the use of inotropes were significantly associated with the presence of hyperglycemia. PRISM score was significantly increased in hyperglycemia group. Finally, the mortality rate and the length of hospital stay were significantly higher in hyperglycemic patients group in comparison with normoglycemic patient group $(p=0.01)$.

Discussion Hyperglycemia is often observed in severe scorpion envenomed children. The presence of hyperglycemia represents an indicator of severity in this specific condition. 
Conclusion Our study confirms that hyperglycemia is often observed in severe scorpion envenomed children. The presence of hyperglycemia represents an indicator of severity in this specific condition. More studies are needed on this subject.

\section{Competing interests None.}

\section{References}

1. Scorpion envenomation among children: clinical manifestations and outcome (analysis of 685 cases). Am J Trop Med Hyg. 2010 Nov:83(5):1084-92.

2. Epidemiological, clinical characteristics and outcome of severe scorpion envenomation in South Tunisia: multivariate analysis of 951 cases. Toxicon. 2008 Dec 15;52(8):918-26.

\section{S31}

Prehospital care and initial hospital care of pediatric patients with severe trauma hospitalized in the ICU at Grenoble University Hospital, data from the "TRENAU" trauma-system

Murielle Moine ${ }^{1}$, François Xavier Ageron ${ }^{2}$, Claire Jannel ${ }^{1}$, Thierry Debillon', Isabelle Wroblewski ${ }^{1}$

${ }^{1}$ Reanimation pediatrique, C.H.U., La Tronche, France; ${ }^{2}$ pôle SAU - SAMU

74 - Réanimation, CHANGE, Annecy, France

Correspondence: Murielle Moine - mmoine@chu-grenoble.fr

Annals of Intensive Care 2016, 6(Suppl 1):S31

Introduction Prehospital care is crucial in the treatment of trauma patients. Nevertheless, there is a lack of data concerning the prehospital care of severely injured pediatric patients with trauma in France. The main aim of our study was to describe the prehospital and initial hospital care in children with severe trauma, hospitalized in the intensive care unit (ICU) at the Grenoble University Hospital, and were taken in charge by the trauma system "TRENAU" (trauma-system du réseau nord-alpin des urgences). The second objective was to describe the factors of the prehospital and initial care associated with the length of hospital stay and the outcome of the patients.

Patients and methods The data were retrospectively analyzed and collected from the register of the "TRENAU." Data concerning the prehospital and initial hospital care of severely injured traumatized patients (according to the Vittel Criteria) were collected. All patients with those criteria, aged 0 to 15 years and hospitalized in the ICU at the Grenoble University Hospital between January 1, 2009, and December 31, 2011, were included in the study.

Results One hundred and one severely injured traumatized pediatric patients were hospitalized in the ICU. The three main causes of trauma were road accident (39\%), mountain accident (37\%) and fall from a great height $(19 \%)$. The most frequent injury was traumatic brain injury (74\%), associated or not with other injuries. Seven percent of the patients died, all with severe head trauma. The mean duration of the prehospital care was $100( \pm 40) \mathrm{min}$.

Among the patients with hypotension, only $50 \%$ received fluid resuscitation (mean volume $16 \mathrm{ml} / \mathrm{kg}$ ). Concerning the neurological failure, $16 \%$ of the patients $(n=16)$ had a GCS less than 8 and $81 \%$ of this patients $(n=13)$ were intubated during the prehospital or initial hospital care. Concerning the intra-cranial pressure monitoring, 6 patients were monitored by an intra-cranial captor during the initial care, whereas 16 patients had an indication for it (GCS less than 8 and AIS less than 3 for the head).

In univariate analysis, many factors were associated with the outcome of the patients according to the Glasgow Outcome Scale (GOS). These factors were the age of the patient, osmotherapy, blood transfusion and the following scores: GCS, PTS, ISS, TRISS, RTS and PRISM.

In multivariate analysis, the age of the patient and blood transfusion were independently associated with the length of stay in the ICU.

Conclusion The prehospital care of pediatric patients with severe trauma, taken in charge by the TRENAU, enables guidelines compliance in several points (fast orientation to adapted trauma center, duration of prehospital care). Nevertheless, it appears that some points could be improved (intubation, fluid resuscitation volume, monitoring of intra-cranial pressure). The results of our study provides some hints as to how to improve the prehospital management of pediatric trauma patients, especially at the level of our regional trauma system, by adaptation and diffusion of pediatric protocols and formation of the medical and paramedical teams to the specificity of pediatric trauma care.

\section{Competing interests None.}

\section{S32}

Ventilator-associated pneumonia due to multi- or extensively drug-resistant Pseudomonas aeruginosa: comparison with susceptible strains

Matthieu Duprey ${ }^{1}$, Nicolas Bréchot ${ }^{1}$, Alexandra Aubry², Guillaume Hekimian ${ }^{1}$, Matthieu Schmidt ${ }^{1}$, Ania Nieszkowska' ${ }^{1}$, Jean Louis Trouillet ${ }^{1}$, Vincent Jarlier ${ }^{2}$, Alain Combes ${ }^{1}$, Jean Chastre' ${ }^{1}$, Charles-Edouard Luyt ${ }^{1}$ ${ }^{1}$ Service de Réanimation Médicale, Groupe Hospitalier Pitié Salpêtrière, Paris, France; ${ }^{2}$ Service de microbiologie, inserm u1135, Immunity and Infectious Diseases Center, Hôpital de la Pitié-salpétrière, Sorbonne Universités, UPMC Univ Paris 06, Paris, France

Correspondence: Charles-Edouard Luyt - charles-edouard.luyt@aphp.fr Annals of Intensive Care 2016, 6(Suppl 1):S32

Introduction Pseudomonas aeruginosa $(\mathrm{Pa})$ is the leading cause of ventilator-associated pneumonia (VAP) and carries a high mortality. The exact impact of resistance of Pa strains on outcome is still on debate; some studies report association between Pa resistance and morbidity/mortality, other not. We therefore performed a study to analyze the risk factors, characteristics and outcomes of Pa-VAP according to the characteristic of the strains, i.e., susceptible or resistant.

Patients and methods This was a retrospective study on data prospectively collected (1). Patients having developed bacteriologically confirmed Pa-VAP (diagnosed using bronchoalveolar lavage, tracheal aspirate or mini-bronchoalveolar lavage) were prospectively followed until ICU discharge or death. Pa strains were considered multidrug resistant (MDR) if resistant to $\geq 1$ agent in $\geq 3$ categories of antibiotics; extensively drug resistant (XDR) if resistant to $\geq 1$ or more agent in all but $\leq 2$ categories of antibiotics and pan-drug resistant (PDR) if resistant to all antibiotics tested (2). Patients infected with susceptible (S) strains were compared to patients infected with MDR and XDR strains. In a second analysis, patients infected with a MDR or XDR strains (grouped together and therefore named R) were compared to patients infected with S strains. Results are expressed as mean \pm SD or median (IQR).

Results One hundred and sixty-nine patients with at least one episode of Pa-VAP were included: 111 were males, and 58 females; they were $58 \pm 19$ year old, with a SAPS II score on admission of $51 \pm 21$ and duration of MV before VAP episode of 11 [6-20] days. Of 169, 143 ( $85 \%)$ had received antibiotics in the preceding 15 days. One hundred and fifteen patients $(68 \%)$ were infected with $S$ strain, 36 patients with MDR strain $(21 \%)$ and 18 patients $(11 \%)$ with XDR strain. No patient was infected with PDR strain. Patients infected with XDR and MDR strains had trend toward higher previous use of fluoroquinolones, ceftazidime, third-generation cephalosporins and piperacillin/ tazobactam than patients infected with S strains $(33,28$ and $16 \%$ for previous fluoroquinolones use, respectively; 22,8 and $6 \%$ for previous ceftazidime use respectively; 44,39 and $26 \%$ for previous third-generation cephalosporins use, respectively; 39,40 and $25 \%$ for previous piperacillin/tazobactam use, respectively. $P=\mathrm{NS}$ for all). No other differences were observed between patients infected with $S, M D R$ or XDR strains regarding initial characteristics of patients, VAP characteristics or outcomes (infection recurrence, ICU length of stay and mortality). Similar results were observed when comparing patients infected with $S$ strains and those infected with $R$ (MDR plus XDR grouped together): No difference at ICU admission or the day VAP was diagnosed except that patients infected with $\mathrm{R}$ strains received more frequently fluoroquinolone (30 vs. $16 \%$, respectively $p=0.03$ ) and trend toward more 
piperacillin/tazobactam (39 vs. $25 \%$, respectively, $p=0.07$ ) and thirdgeneration cephalosporins ( 41 vs. $26 \%$, respectively, $p=0.054$ ) than patients infected with $S$ strains, but exhibited similar VAP recurrence (30\% and $39 \%$, respectively, $p=N S$ ), ICU length of stay (29 [20-50] and 38 [21-53] days, respectively, $p=$ NS) and mortality rate ( $33 \%$ and $33 \%$, respectively).

Conclusion In this large cohort of Pa-VAP, resistance profile was not associated with infection recurrence, ICU length of stay or death. Except previous use of fluoroquinolones, we were not able to find any risk factor associated with resistance profile of $\mathrm{Pa}$.

\section{Competing interests None.}

\section{References}

1. Luyt CE, Aubry A, Lu Q, et al. Imipenem, meropenem, or doripenem to treat patients with Pseudomonas aeruginosa ventilator-associated pneumonia. Antimicrob Agents Chemother. 2014;58(3):1372-80.

2. Magiorakos AP, Srinivasan A, Carey RB, et al. Multidrug-resistant, extensively drug-resistant and pandrug-resistant bacteria: an international expert proposal for interim standard definitions for acquired resistance. Clin Microbiol Infect. 2012 Mar; 18(3):268-81.

\section{S33}

Can we predict a secondary documented infection in patients empirically treated for an invasive candidiasis?

Sébastien Bailly ${ }^{1}$, Olivier Leroy ${ }^{2}$, Jean-Paul Mira ${ }^{3}$, Hervé Dupont ${ }^{4}$, Jean-Pierre Gangneux ${ }^{5}$, Philippe Montravers ${ }^{6}$, Jean-Michel Constantin ${ }^{7}$, Didier Guillemot ${ }^{8}$, Olivier Lortholary ${ }^{9}$, Pierre-François Perrigault ${ }^{10}$, Elie Azoulay ${ }^{11}$, Jean-François Timsit

${ }^{1}$ Descid - team 5, INSERM U1 137 - IAME, Paris, France; ${ }^{2}$ Réanimation, Centre Hospitalier de Tourcoing, Tourcoing, France; ${ }^{3}$ Réanimation Médicale, Hôpital Cochin, Paris, France; ${ }^{4}$ Réanimation cardio thoracique et vasculaire, CHU Amiens-Picardie, Amiens, France; ${ }^{5}$ Mycologie, Hospital Center University De Rennes, Rennes, France; ${ }^{6}$ Anesthésie et soins intensifs, Hôpital Bichat-Claude Bernard, Paris, France; ${ }^{7}$ Réanimation adulte, C.H.U. Estaing, Clermont-Ferrand, France; ${ }^{8}$ Umr 1181 "Biostatistics, Biomathematics, Pharmacoepidemiology and Infectious Diseases", Inserm, Paris, France; ${ }^{9}$ Necker Pasteur Center for Infectious Diseases, Hospital Necker, Paris, France; ${ }^{10}$ Réanimation médicale et chirurgicale, C.H.U. De Montpellier, Prades-le-Lez, France; ${ }^{11}$ Réanimation médicale, Hôpital Saint-Louis, Paris, France

Correspondence: Sébastien Bailly - sbailly@chu-grenoble.fr Annals of Intensive Care 2016, 6(Suppl 1):S33

Introduction Related to the absence of effective diagnostic tools for invasive candidiasis, empirical systemic antifungal therapies are recommended and largely prescribed to the intensive care unit patients with risk factors. However, secondary documented invasive candidiasis is not observed for all the patients who receive an empirical systemic antifungal therapy. The objective of this study is to define the risk factors able to predict the probability for having a secondary documented invasive candidiasis after empirical systemic antifungal therapy by accounting for center and patient variables.

Patients and methods The patients with an empirical systemic antifungal therapy were prospectively included from a multicenter prospective observational study. Only patients without primary documented invasive candidiasis were included and were divided into two groups: patients without documented invasive candidiasis and patients with a secondary documented invasive candidiasis. A multivariate hierarchical model was performed to identify factors independently associated with a secondary documented invasive candidiasis. Two sub-group analyses were performed by considering the type of intensive care unit admission (intra-abdominal surgery vs. other).

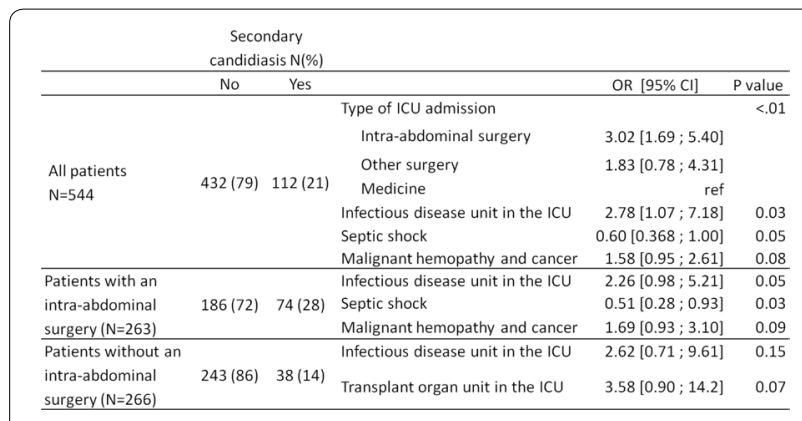

Fig. 40 Risk factors for secondary documented invasive candidiasis-multivariate analysis (OR > 1: in favor of a secondary documented infection)

Results Five hundred and forty-four patients were included in the analysis, from whom $112(21 \%)$ had a secondary documented IC (candidemia 19 (17\%), intra-abdominal IC 64 (57\%) and deep-seated IC $29(26 \%))$. The proportion of secondary documented invasive candidiasis was higher for patients admitted for an intra-abdominal surgery (28\%) compared to the patients admitted for another reason (other surgery or medicine $14 \%$ ). The multivariate analysis confirmed that the patients admitted in intensive care unit for an intra-abdominal surgery had more than three times as likely to experience a secondary documented invasive candidiasis than the patients who were admitted for a medicine reason (OR $3.02[1.69 ; 5.40]: p<0.01)$. The other factors significantly associated with a secondary documented invasive candidiasis were the presence of an infectious disease in the intensive care unit (OR $2.78[1.07 ; 7.18], p=0.03)$ and septic shock at the treatment initiation $(0.60[0.368 ; 1.00] ; p=0.05)$. When we consider only patients who were admitted in intensive care unit for an intra-abdominal surgery, the presence of an infectious disease in the intensive care unit and septic shock remained associated with secondary invasive candidiasis. Conversely, when we consider the sub-group of patients who were not admitted for an intra-abdominal surgery, there was no factor significantly associated with a secondary documented invasive candidiasis (Fig. 40).

Conclusion The patients admitted in intensive care unit for an intraabdominal surgery were more subjects to experience a secondary invasive candidiasis than other patients. Conventional risk factors for invasive candidiasis were unable to differentiate the two groups of patients, with and without secondary documented invasive candidiasis.

\section{Competing interests None.}

\section{S34}

Should aminoglycosides be administered to hematological patients with septic shock? Data from the Groupe de Recherche respiratoire en Réanimation Onco-Hématologique (Grrr-OH) Thomas Longval', Sylvie Chevret ${ }^{2}$, Fabrice Bruneel ${ }^{3}$, Djamel Mokart ${ }^{4}$, Frédéric Pène ${ }^{5}$, Julien Mayaux ${ }^{6}$, Achille Kouatchet ${ }^{7}$, Martine Nyunga ${ }^{8}$, Antoine Rabbat ${ }^{9}$, Pierre Perez ${ }^{10}$, Christine Lebert ${ }^{11}$, Dominique Benoit $^{12}$, François Vincent ${ }^{13}$, Virginie Lemiale ${ }^{14}$, Elie Azoulay ${ }^{14}$

${ }^{1}$ Réanimation médicale, Hôpital Saint Louis, Paris, France; ${ }^{2}$ Service de biostatistique et information médicale, Hôpital Saint-Louis, Paris, France; ${ }^{3}$ Réanimation médico-chirurgicale, Centre Hospitalier de Versailles, Le 
Chesnay, France; ${ }^{4}$ Réanimation, Institut Paoli-Calmettes, Marseille, France; ${ }^{5}$ Réanimation Médicale, Hôpital Cochin, Paris, France; ${ }^{6}$ Réanimation pneumologique, Hôpital Pitié-Salpêtrière, Paris, France; ${ }^{7}$ Réanimation médicale, Centre Hospitalier Universitaire d'Angers, Angers, France; ${ }^{8}$ Réan imation polyvalente, Centre Hospitalier de Roubaix, Roubaix, France; ${ }^{9}$ Réanimation pneumologique, Hôpital Cochin, Paris, France; ${ }^{10}$ Réanimation polyvalente, C.H.U. de Nancy, Nancy, France; ${ }^{11}$ Réanimation, Centre Hospitalier Départemental - site de La Roche-sur-Yon, La Roche-sur-Yon, France; ${ }^{12}$ Réanimation polyvalente, Hopital universitaire, Gand, Belgium; ${ }^{13}$ Réanimation polyvalente, Groupe Hospitalier Intercommunal Le Raincy-Montfermeil, Montfermeil, France; ${ }^{14}$ Réanimation médicale, Hôpital Saint-Louis, Paris, France

Correspondence: Thomas Longval - thomas.longval@free.fr Annals of Intensive Care 2016, 6(Suppl 1):S34

Introduction Patients with hematological malignancies (HMs) are at high risk of severe bacterial infections. Septic shock still carries high mortality for these patients. The place of aminoglycosides (AMG) in this setting is still uncertain.

Patients and methods In a post hoc analysis of the Trial-OH study, we studied 444 patients with HMs admitted to 17 ICUs for septic shock as defined by the need for vasopressors and clinically or microbiologically documented infection, or neutropenia and fever. All patients were treated using broad-spectrum beta-lactams. AMG were prescribed according to clinician's decision. The analysis was based on propensity matching (1:1 matching algorithm) and following variables such as neutropenia, acute kidney injury, myeloma, dialysis and ICU which might affect the administration of AMG in addition to age, Sepsis-related Organ Failure Assessment (SOFA), secondary acute leukemia and remission status of the HMs. The primary endpoint was hospital mortality.

Results A total of 313 patients (70.5\%) received AMG, whereas 131 did not. Hospital mortality was, respectively, 174 (55.6\%) and 73 (56.1 \%); $[\mathrm{OR}]=1.04[0.68 ; 1.54] P=0.91$. Age, SOFA, secondary acute leukemia and remission status were significant variables associated with mortality. Among the 131 patients who did not receive AMG, 123 could be matched to AMG-treated patients, resulting in a total of 246 paired observations in the propensity analysis. The proportion of hospital death was not significantly different between the two groups $56.1 \%$ (no $A M G$ ) vs $55.3 \%, P=0.89[\mathrm{OR}]=1.04[95 \% \mathrm{Cl}, 0.61-1.76]$. No center effect was reported. ICU length of stay was also not significantly different between AMG-treated patients and other patients.

Conclusion In HM patients with septic shock, up to $70 \%$ receive AMG with no center effect. These data, in line with the literature in immunocompromised patients without septic shock, do not support the use of AMG in this setting. Randomized clinical trials are warranted.

\section{Competing interests None.}

\section{S35}

Is de-escalation of antimicrobial therapy safe in hematology patients?

David Schnell', Claire Montlahuc², Matthieu Resche-Rigon ${ }^{3}$, Achille Kouatchet ${ }^{4}$, Jean-Ralph Zahar ${ }^{5}$, Michaël Darmon ${ }^{6}$, Frédéric Pène ${ }^{7}$, Virginie Lemiale $^{8}$, Antoine Rabbat ${ }^{9}$, François Vincent ${ }^{10}$, Fabrice Bruneel ${ }^{11}$, Djamel Mokart $^{12}$, Elie Azoulay ${ }^{8}$

${ }^{1}$ Réanimation médicale, Hospital Center D'angoulême, Angoulême, France; ${ }^{2}$ Service de biostatistique et information médicale, Hôpital Saint Louis, Paris, France; ${ }^{3}$ Biostatistiques, Hôpital Saint-Louis, Paris, France; ${ }^{4}$ Réanimation médicale, Centre Hospitalier Universitaire d'Angers, Angers, France; ${ }^{5}$ Unité de prévention et de lutte contre les infections nosocomiales, C.H.U. d'Angers, Rue Larrey, Angers, France, Angers, France; ${ }^{6}$ Réanimation Médicale, CHU Saint-Etienne - Hôpital Nord, Saint-Étienne, France; ${ }^{7}$ Réanimation Médicale, Hôpital Cochin, Paris, France; ${ }^{8}$ Réanimation médicale, Hôpital Saint-Louis, Paris, France; ${ }^{9}$ Réanimation pneumologique, Hôpital Cochin, Paris, France; ${ }^{10}$ Réanimation polyvalente, Groupe Hospitalier Intercommunal Le Raincy-Montfermeil, Montfermeil, France; ${ }^{11}$ Réanimation médico-chirurgicale, Centre Hospitalier de Versailles, Le Chesnay, France; ${ }^{12}$ Réanimation, Institut Paoli-Calmettes, Marseille, France Correspondence: David Schnell - david.schnell1@gmail.com Annals of Intensive Care 2016, 6(Suppl 1):S35
Introduction De-escalation of the antimicrobial therapy is recommended in severe sepsis guidelines. This study investigated the prevalence of de-escalation and the factors associated with in hematology patients admitted to the intensive care unit (ICU).

Patients and methods This study is a retrospective evaluation of a prospective observational cohort of hematology patients admitted to the ICU. Patients without antibiotics prior to ICU admission and in whom antibiotics were initiated in the first $48 \mathrm{~h}$ were included in this ancillary study. De-escalation of antimicrobial therapy was defined either by the cessation of a $\beta$-lactam or a glycopeptide or by the switch from a $\beta$-lactam to another one with a narrower spectrum (carbapenems $>$ piperacillin/tazobactam $>$ ceftazidime or cefepime $>$ piperacillin or ticarcillin > cefotaxime or ceftriaxone $>$ other penicillins) before the day 5. Characteristics and outcomes of patients with (group D+) and without de-escalation (group D-) were compared.

Results Among the 1011 patients from the prospective cohort, 124 patients were included in the present study, 82 men and 42 women with a median age of 60 (IQR 47-70) years. The underlying malignancy was acute leukemia in 46 (37\%) patients, lymphoma in 37 (30\%), myeloma in $14(11 \%)$, chronic lymphocytic leukemia in $12(10 \%)$ and miscellaneous hematologic disorders in 15 (12\%). It was newly diagnosed in $59(50 \%)$ patients and in complete remission in 22 (19\%). Twentyeight (24\%) patients were neutropenic at ICU admission. Twenty-four (19\%) had received bone marrow transplantation, including allogeneic stem cell transplantation in 11 (9\%).

The median Charlson comorbidity index was 4 (IQR 3-6), and 18 (15\%) patients had poor performance status (WHO score 3-4). The cardiovascular component of this score was present in $53(43 \%)$ patients, the pulmonary component in $33(27 \%)$, the liver component in 15 (12\%), the neurologic component in 11 (9\%), the renal component in 10 $(8 \%)$, the HIV infection component in 7 (6\%) and the diabetes component in $3(2 \%)$ patients.

The main reasons for ICU admission were acute respiratory failure in 85 $(69 \%)$ patients, shock in $46(37 \%)$, acute kidney injury in $34(27 \%)$ and coma in $26(21 \%)$. Overall, 64 (52\%) patients had multiple organ failure. The median ICU and hospital lengths of stay were 12 and 32 days, respectively. In the whole cohort, 28 (23\%) patients died in the ICU and $43(35 \%)$ in hospital.

Empirical antimicrobial therapy consisted in a $\beta$-lactam in $122(98 \%)$ patients: 66 (53\%) received piperacillin/tazobactam, 30 (24\%) cefotaxime or ceftriaxone, $20(16 \%)$ a carbapenem, $13(11 \%)$ amoxicillin/clavulanic acid, 6 (5\%) ceftazidime and $3(2 \%)$ piperacillin. Twenty-one $(17 \%)$ received a glycopeptide, combined to a $\beta$-lactam in $19(90 \%)$ of them. Thirty-five ( $27 \%)$ patients received macrolides, 29 (23\%) an aminoglycoside and 28 (23\%) a fluoroquinolone.

The antimicrobial therapy was de-escalated in $46(37 \%)$ patients (group D+). Extensive comparison of patients with and without de-escalation showed no difference in patients' characteristics, comorbidities or in the underlying malignancy status. The main reasons for ICU admission were also similar in both groups. No difference was observed as regard to ICU admission severity according to SOFA score (6 [IQR: 4-8] in group D- vs. 7 [IQR: 3-10] in group $\mathrm{D}+, P=0.55)$ or white blood cells count (9280 [IQR: 2000-24,625] vs. 7900 [IQR: $2822-17,990], P=0.48$ ). From the first to the third day spent in the ICU, the evolution of SOFA score (0 [IQR: -2 to 1$]$ vs. 0 [IQR: -4 to 3 ], $P=0.78$ ) and the rate of neutropenia recovery (6 [35\%] vs $6[55 \%], P=0.44)$ were also similar. Finally, ICU (8 [17\%] in group $D+$ vs. $20[26 \%]$ in group $D-, P=0.38$ ) and hospital mortality (12 [26\%] vs. 31 [40\%], $P=0.17$ ) were not statistically different. No difference across group in terms of mortality was noted after adjustment for confounders.

Discussion The rate of de-escalation was high in this cohort of critically ill hematological patients. De-escalation was not associated with a specific characteristic or the outcome of these high-risk patients. However, the lack of power and the design of the present study preclude any firm conclusion.

Conclusion Further studies specifically designed to explore this topic are warranted to investigate the feasibility and safety of de-escalation of the antimicrobial therapy in critically ill hematological patients.

Competing interests None. 


\section{S36}

Pharmacokinetics and absolute bioavailability of voriconazole administered through a nasogastric tube with continuous enteral feeding to critically ill ventilated patients

Philippe Karoubi ${ }^{1}$, Christophe Huang ${ }^{1}$, Antoine Rabbat ${ }^{2}$, Johanna Oziel ${ }^{3}$, Guillaume Van Der Meersch ${ }^{3}$, Yacine Tandjaoui-Lambiotte ${ }^{3}$, Frédéric Gonzalez ${ }^{1}$, Christophe Clec'h' ${ }^{1}$, Christophe Padoin ${ }^{4}$, Yves Cohen ${ }^{5}$

${ }^{1}$ Réanimation médico-chirurgicale, Hôpital Avicenne, Bobigny, France; ${ }^{2}$ Réanimation pneumologique et soins intensifs respiratoires, Hôpital Cochin, Paris, France; ${ }^{3}$ Réanimation médico-chirurgicale, Hôpital Avicenne, Université Paris 13, Bobigny, France; ${ }^{4}$ Service pharmacie, laboratoire de contrôle, hopital Avicenne, Bobigny, France; ${ }^{5}$ Réanimation médico-chirugicale, Hôpital Avicenne, Université Paris 13, Bobigny, France Correspondence: Yves Cohen - yves.cohen@avc.aphp.fr

Annals of Intensive Care 2016, 6(Suppl 1):S36

Introduction The use of nasogastric tube (NG) is one means of administering antifungal therapy to critically ill patients unable to receive medication via the oral route. The aim of the study was to determine pharmacokinetics and absolute bioavailability of voriconazole in critically ill patients receiving continuous enteral feeding.

Patients and methods A prospective multicentric, crossover study was conducted. Stable critically ill patients on mechanical ventilation and receiving continuous enteral feeding with suspected or proven fungal invasive infection (IFI) treated with voriconazole were included. Voriconazole loading dose $(6 \mathrm{mg} / \mathrm{kg}$ ) every $12 \mathrm{~h}$ for 2 doses intravenously was followed by maintenance dose of $4 \mathrm{mg} / \mathrm{kg}$ every $12 \mathrm{~h}$ during 3 days. From day 4, administration of $5 \mathrm{mg} / \mathrm{kg}$ every $12 \mathrm{~h}$ was performed through the NT. NG dose was $1 \mathrm{mg} / \mathrm{kg}$ greater than the recommended dose in order to avoid food reduction bioavailability. Blood samples were collected after the fifth intravenous (IV) infusion and after the fifth NG administration. Demographic, biologic data and concomitant drugs were collected. AUC/dose NG was compared to AUC/dose IV using a paired $T$ test.

Results Eight patients ( 5 males, 3 females) were evaluable. Median (range) age and bodyweight were 67.5 (49-86) years and 76.5 (57.3$117) \mathrm{kg}$. Only one patient did not receive esomeprazole. None had hepatic disease. Voriconazole concentrations were measured by using a validated high-performance liquid chromatographic assay (Table 51). AUC/dose were not different between route $(p=0.6)$. Mean voriconazole absolute bioavailability was $106 \%$ and range from 77 to $135 \%$. All subjects completed the study without any adverse effects.

Conclusion In tube-fed ventilated critically patients, a switch to NG voriconazole after initial IV therapy to simplify the treatment of IFI could be used. Furthermore, it suggests that concurrent enteral feeding does not interfere with the absorption of voriconazole and, thus, does not have to be interrupted during voriconazole administration. All trough concentration patients were superior to the efficacy target ( $2 \mathrm{mg} / \mathrm{L})$. However, after 6 days of treatment, 4 patients had trough concentration superior to upper therapeutic range target $5 \mathrm{mg} / \mathrm{L}$. Therapeutic drug monitoring should be performed to avoid supratherapeutic concentration and potential neurotoxicity.

Competing interests Consulting Activities for MSD and Pfizer laboratories.

\section{Table 51 See text for description}

\begin{tabular}{lllr}
\hline $\begin{array}{l}\text { Treatments } \\
(\text { mean } \pm \text { SD) }\end{array}$ & $\begin{array}{l}\text { AUC } \mathbf{0 - 1 2} \mathbf{h} / \\
\text { dose }(\mathbf{h} / \mathbf{m L})\end{array}$ & $\boldsymbol{T}_{\max }(\mathbf{h})$ & $\boldsymbol{C}_{\max }(\mathbf{m g} / \mathbf{L})$ \\
\hline IV route & $0.16 \pm 0.07$ & $0.5-1.5$ & $5.8 \pm 2.0$ \\
NG route & $0.17 \pm 0.07$ & $1-4$ & $10.2 \pm 4.2$ \\
\hline
\end{tabular}

S37

Which optimal daily dose for linezolid in burn patients?

Amel Mokline ${ }^{1}$, Lazhari Gharsallah¹, Imen Rahmani', Emna Gaies², Sofiene Tlaili', Bahija Gasri', Rym Hammouda', Sameh Triki², Anis Klouz ${ }^{2}$, Amen Allah Messadi ${ }^{1}$

${ }^{1}$ Intensive Burn Care Department, Burn and Trauma Center, Tunis, Tunisia; ${ }^{2}$ Laboratory of Pharmacology, Charles Nicolle Hospital, Tunis, Tunisia

Correspondence: Amel Mokline - amokline@yahoo.fr

Annals of Intensive Care 2016, 6(Suppl 1):S37

Introduction Linezolid was an antibiotic with time-dependent activity. It was assumed that adequate serum linezolid concentration will be achieved most of the time when using the recommended dose of $600 \mathrm{mg}$ every $12 \mathrm{~h}$. Burns induce complex physiological changes as modification of distribution volume, increased clearance of drugs and decrease in protein binding. So, the pharmacokinetic of linezolid may then be modified. This requires dose adjustment particularly in burns. The aim of our study was to assess the pharmacokinetic of linezolid in burn patients at a dose of $600 \mathrm{mg}$ three times per day. Thresholds for therapeutic efficacy of linezolid were: plasma $C_{\min }>2 \mathrm{mg} / \mathrm{l}$ and/ or AUC/MIC ratios of 80 (or AUC24 $>200 \mathrm{mg} / \mathrm{h} / \mathrm{l}$ ) or $\%$ TMIC of $>85$ (percentage of dosing interval during which linezolid concentration remains above the MIC of targeted bacteria) [1]

Patients and methods This study was conducted in burn center in Tunis. Patients with documented and/or suspected MDR Grampositive bacterium-related infections and whose burned area greater than $20 \%$ (TBSA $\geq 20 \%$ ) were included. They were excluded pregnant women and patients $<16$ years old. Enrolled patients received linezolid at a dose of $600 \mathrm{mg}$ in $1 \mathrm{~h}$ infusion three times per day. Blood samples for pharmacokinetic analysis were taken 5 min after the end of the first infusion, $5 \mathrm{~min}$ before the second and the third dose and $5 \mathrm{~min}$ before the dose of the day after. To assess linezolid serum level, $4 \mathrm{~mL}$ of blood was drawn from an arterial line, centrifuged and then stored at $-80^{\circ} \mathrm{C}$. For all patients, linezolid minimum inhibitory concentration (MIC), the AUC 0-24 h (daily area under curve) and linezolid plasma concentrations (trough $\left(C_{\min }\right)$ and peak $\left(C_{\max }\right)$ levels) were calculated.

Results During the period of study, 10 burned patients were included. The mean age was $39 \pm 22$ years old with a burned area of $30 \pm 11 \%$. No case of renal failure (creatinine $73 \pm 38 \mu \mathrm{mol} / \mathrm{l}$ ) or of thrombocytopenia was noted in our patients $(276 \pm 176 / \mu \mathrm{L})$. For all patients, linezolid administration at the dose of $600 \mathrm{mg}$ three times a day allows to reach: an adequate daily linezolid exposure with a mean AUC 0-24 h/MIC (mg/h/l)* of 126 (recommended: $80-120)$, a $C_{\min }$ of $4.84 \pm 2.33 \mathrm{mg} / \mathrm{L}$ and a \%TMIC of 100 . In our study, no case of potential overexposure was noted with this dosage of linezolid.

Conclusion Our study suggests that linezolid at the daily dosage of $600 \mathrm{mg}$ every $8 \mathrm{~h}$ in burned patients allows to reach optimal PK/PD targets for linezolid clinical efficacy: AUC/MIC of 126 and a \%TMIC of 100 , and may reduce the emergence of linezolid resistance.

\section{Competing interests None.}

\section{References}

1. Antimicrobial agents and chemotherapy, Nov. 2010, p. 4605-4610.

\section{S38}

Predictive factors of sub-therapeutic peak concentration after a single dose of amikacin in postoperative cardiovascular critically ill patients

Mouhamed Moussa ${ }^{1}$, Flavie Desrumaux ${ }^{1}$

${ }^{1}$ Nord, C.H.R.U. Lille, Lille, France

Correspondence: Flavie Desrumaux - flaviedesrumaux@yahoo.fr

Annals of Intensive Care 2016, 6(Suppl 1):S38

Introduction Therapeutic success of amikacin associated with a B-lactamin is proved to be related to an effective serum peak concentration $\left(C_{\max }\right)$. In critically ill septic patients with increased volume of distribution, failure to obtain an effective $C_{\max }\left(C_{\max }<64 \mathrm{mg} / \mathrm{l}\right)$ was observed in thirty percent of patients. Similar changes in volume of distribution are observed after cardiopulmonary bypass for cardiovascular surgery; however, there are no published data concerning the prevalence and the predictive factor of insufficient $C_{\max }$. 
Patients and methods The aim of this monocentric retrospective study was to identify predictive factors for insufficient $C_{\max }$ in a population of postoperative critically ill cardiovascular surgery patients between January 2012 and June 2015.

Results One hundred and thirty-six patients of 60 years were included. Administration of an average dose of $22 \pm 5.1 \mathrm{mg} / \mathrm{kg}$ resulted in failure to achieve a $C_{\max }>64 \mathrm{mg} / \mathrm{l}$ in $39 \%$ of patients and a $C_{\max }>60 \mathrm{mg} / \mathrm{lin}$ $30.9 \%$ of cases. More than $58 \%$ had a $C_{\max }>80 \mathrm{mg} / \mathrm{l}$.

In the univariate analysis, male gender, hypertension, diabetic and daily dose per body weight were significantly more important in the $C_{\text {max }}>64 \mathrm{mg} / \mathrm{l}$ group compared to the $C_{\max }<64 \mathrm{mg} / \mathrm{l}$ group [respectively (77.1 vs $75.5 \% ; p<0.01)$, (63.4 vs $44.2 \% ; p=0.033$ ), (38.3 vs $22 \% ; p=0.045),(23.7 \pm 4.7$ vs $19.3 \pm 4.6 ; p<0.01)]$. In multivariate analysis, the amikacin dose per body weight (OR 0.79; $p<0.001)$ and the diabetes (OR $0.35 ; p=0.03$ ) are associated with a $C_{\max }>64 \mathrm{mg} / \mathrm{l}$. There was no relation between insufficient $C_{\max }$ and outcomes. Of interest, $C_{\max }$ was significantly more important than 8 and 10 times the value of observed minimal inhibition concentration.

Conclusion In the population of postoperative cardiovascular surgery the dose weight is the most important factor to have efficiency optimal with the amikacin treatment. The diabetes is associated with a correct $C_{\max }$.

\section{Competing interests None.}

\section{Reference}

1. De Montmollin E, Bouadma L, Gault N, Mourvillier B, Mariotte E, Chemam $S$, et al. Predictors of insufficient amikacin peak concentration in critically ill patients receiving a $25 \mathrm{mg} / \mathrm{kg}$ total body weight regimen. Intensive Care Med. 2014 Jul;40(7):998-1005.

\section{S39}

Supra-therapeutic serum levels and toxicity of beta-lactam antibiotics in septic ICU patients requiring replacement therapy for acute renal failure: a single-center cohort study Faten May ${ }^{1}$, Marion Perrin ${ }^{2}$, Najoua El-Helali ${ }^{3}$, Jean-Claude Nguyen ${ }^{4}$, Kelly Tiercelet ${ }^{5}$, Stephane Ruckly ${ }^{2}$, Julien Fournier ${ }^{5}$, Mélanie Cherin ${ }^{6}$, Cédric Bruel $^{7}$, Francois Philippart ${ }^{8}$, Maïté Garrouste-Orgeas ${ }^{9}$, Marie Dominique Kitzis $^{10}$, Alban Le Monnier ${ }^{3}$, Jean-François Timsit ${ }^{11}$, Benoit Misset ${ }^{8}$ ${ }^{1}$ Réanimation polyvalente, CHU Fattouma Bourguiba, Monastir, Tunisia; ${ }^{2}$ Biostatistics, CHU, Grenoble, France; ${ }^{3}$ Unité de microbiologie clinique et dosages des anti-infectieux, Groupe hospitalier Paris Saint-Joseph, Paris, France; ${ }^{4}$ Microbiologie, Groupe Hospitalier Paris Saint-Joseph, Paris, France; ${ }^{5}$ Réanimation polyvalente, Hospital Group Paris Saint-Joseph, Paris, France; ${ }^{6}$ Réanimation polyvalente adulte, Centre Hospitalier Intercommunal André Grégoire, Montreuil, France; ${ }^{7}$ Réanimation, Hôpital Paris Saint-Joseph, Paris, France; ${ }^{8}$ Réanimation polyvalente, Groupe Hospitalier Paris-Saint-Joseph, Paris, France; ${ }^{9}$ Réanimation, Fondation Hopital Saint Joseph, Paris, France: ${ }^{10}$ Microbiologie clinique, Groupe Hospitalier Paris-Saint-Joseph, Paris, France; ${ }^{11}$ Réanimation médicale et infectieuse, Hôpital Bichat-Claude Bernard, Paris, France

Correspondence: Benoit Misset - bmisset@hpsj.fr

Annals of Intensive Care 2016, 6(Suppl 1):S39

Introduction Early and adequate antibiotic therapy increases the likelihood of survival in critically ill patients with sepsis. Optimal dosing of antibiotics requires adjustment to large variability in distribution volume and renal elimination, which are characteristic of ICU septic patients. Beta-lactams (BL) are the most frequently prescribed antibiotics because of their broad-spectrum activity and low toxicity. They are time-dependent antibiotics, meaning that their trough level should be at least 5 times over the minimal inhibitory concentration of the targeted bacteria. Supra-therapeutic serum levels have been suggested to be associated with clinical-mainly neurological-toxicity. Serum levels of $B L$ are unpredictable during acute renal failure (ARF) and renal replacement therapy (RRT).

The objective of this study was to describe the prevalence of supratherapeutic BL serum levels in septic ICU patients requiring replacement therapy for acute renal failure and their link with potential clinical toxicity.
Table 52 Antibiotics assessed, threshold used and trough serum levels (mg/l)

\begin{tabular}{lcl}
\hline & $\begin{array}{l}\text { Upper therapeutic } \\
\text { trough level }\end{array}$ & $\begin{array}{l}\text { Observed trough } \\
\text { level med [IQ] }\end{array}$ \\
\hline Piperacillin & 20 & $77[44-109]$ \\
Tazobactam & 5 & $13[6-20]$ \\
Cloxacillin & 20 & $60[35-103]$ \\
Amoxicillin & 20 & $31[19-42]$ \\
Imipenem & 3 & $2.9[1.1-4.2]$ \\
Clavulanate & 0.5 & $2.0[1.3-3.6]$ \\
Ceftazidime & 20 & $11[49-87]$ \\
Cefepime & 10 & $27[16-47]$ \\
\hline
\end{tabular}

Patients and methods Observational cohort study of patients admitted to the medical surgical ICU of the Saint Joseph Hospital from 1999 to 2013 . This was a retrospective analysis of data prospectively collected into the French multicenter Outcomerea database and into the databases of the microbiological and pharmacological laboratory of the hospital. We included consecutive patients with sepsis and ARF, who had been sampled for a BL trough serum level assessment within 7 days of sepsis and 3 days after intermittent dialysis. Sera were sampled before the next administration in case of intermittent infusion and at $24 \mathrm{~h}$ in case of continuous infusion. Assays were performed with a microbiological method (measuring the active part of the antibiotic). Table 52 shows the BL assessed, the thresholds used and the distribution of the observed trough levels. We searched an association between overdose and clinical toxicity (coma, seizures, length of mechanical ventilation, mortality) with univariate analyses.

Results One hundred and eight patients, developed 180 sepsis. 460 serum $B L$ assessments (BL inhibitor in combination with BL antibiotic is considered as 2 assays) were performed. Most assayed antibiotics were piperacillin ( $25 \%)$, tazobactam $(20 \%)$ and cloxacillin (18\%). They were delivered as follows: the mode of infusion and the interval time being decided by the attending physician according to patients characteristics: piperacillin/tazobactam: $4 \mathrm{~g} / 0.5 \mathrm{~g}$ in $30 \mathrm{~min}$, every 8 , 12 or $24 \mathrm{~h}$; cloxacillin: $2-8 \mathrm{~g}$ per $24 \mathrm{~h}$ by continuous infusion, or $2 \mathrm{~g}$ in $30 \mathrm{~min}$, every 6,8 or $12 \mathrm{~h}$. The distribution of the serum levels was very scattered (see Table 52, 3rd column). A supra-therapeutic serum level for at least one BL was observed in 96/108 (89\%) patients and 156/180 (86\%) septic events. A level in the highest quartile was observed for at least one BL in 54/108 (50 \%) patients and 80/180 (45 \%) septic events. Supra-therapeutic serum levels of piperacillin, tazobactam and cloxacillin were observed in 66, 55 and 31 and were in the highest quartile in 33,23 and 14 septic events, respectively. We did not observe a statistical link between serum overdose and clinical toxicity.

Conclusion Supra-therapeutic serum levels of beta-lactam antibiotics are commonly observed in our ICU patients with ARF and RRT. We could not find an association between supra-therapeutic levels and clinical toxicity.

\section{Competing interests None.}

\section{S40}

Post-cardiac arrest syndrome: lessons from a monocentric retrospective study

Cyrille Mathien', Antoine Poidevin' ', Luis Donatti' ${ }^{1}$ Joy Mootien', Luis Pinto ${ }^{2}$, Mathieu Egard ${ }^{2}$, Gokhan Bodur ${ }^{2}$, Guillaume Barberet ${ }^{2}$, Carmen Ionescu², Frederique Ganster², Philippe Guiot', Khaldoun Kuteifan² ${ }^{1} 68$, Hospital Center De Mulhouse, Mulhouse, France; ${ }^{2}$ Réanimation médicale, Centre Hospitalier de Mulhouse, Mulhouse, France

Correspondence: Cyrille Mathien - cyrille.mathien@gmail.com Annals of Intensive Care 2016, 6(Suppl 1):S40 
Introduction Cardiac arrest (CA) management has improved survival and functional recovery during last decades. Recently, many studies focused on the post-cardiac arrest syndrome (PCAS) so as to ameliorate patients' prognosis. PCAS is composed of four entities: persistent precipitating pathology, post-CA circulatory failure systemic, ischaemia-perfusion and post-anoxic brain injury. (1) We look forward to analyse our daily management of resuscitated patients admitted in our medical intensive care unit.

Patients and methods All patients admitted in our unit between January 2011 and June 2013 with the diagnosis of cardiac arrest were included retrospectively. We collected data about cardiac arrest itself but also those concerning PCAS. Exclusion criteria were: suspected or known acute intracranial haemorrhage or stroke, a body temperature on admission of less than $30^{\circ} \mathrm{C}$, unwitnessed cardiac arrest with initial rhythm asystole, known disease making 180 days survival unlikely, known pre-cardiac arrest Cerebral Performance Category score of 3 or 4 (2), systolic blood pressure $<80 \mathrm{mmHg}$ in spite of fluid loading, vasopressor and/or inotropic medication.

Results Eighty patients (pts) were included with average age of 65 years $( \pm 14)$ ( 57 males and 23 females). Mean SAPS II was 72, and mean SOFA score was 11. Aetiology of cardiac arrest (CA) was cardiac in 35 pts (43.8 \%), non-cardiac in 32 pts $(40 \%)$ and unknown in 13 pts ( $16 \%$ ). Coronarography has been realized for 31 pts (39\%), 21 pts of whom had a percutaneous coronary intervention (PCI). Fiftyseven pts $(71 \%)$ required catecholamine infusion during the first $48 \mathrm{~h}$. Therapeutic hypothermia $\left(32-34{ }^{\circ} \mathrm{C}\right)$ is prescribed for 46 pts $(58 \%)$. Twenty-four (52\%) had an initial shockable rhythm. ICU mortality was $55 \%$ (44 pts). Among survivors, Cerebral Performance Scale was good, moderate and severe for $33(41.25 \%), 1(1.25 \%)$ and $2(2.5 \%)$ patients, respectively.

Discussion PCAS is frequent after CA. Its management requires a rigorous control of many variables. Our retrospective study results were not far from the literature data. Only $39 \%$ of our patients underwent coronarography. Indications and timing of coronarography are still not clear except for transmural myocardial infarction. Only half of our patients had therapeutic hypothermia. This point needs clearly to be improved.

Conclusion Functional and vital prognosis of CA could probably be improved by an aggressive management of PCAS. Close control of patients' parameters is necessary to achieve such a goal. Application of guidelines must be emphasized in this setting.

\section{Competing interests None.}

\section{References}

1. Neumar et al. Circulation 2008; 118 (23):2452-83.

2. Safar et al. Resuscitation after Brain Ischemia, in Grenvik A and Safar P Eds: Brain Failure and Resuscitation, Churchill Livingstone, New York, 1981; 155-184.

\section{S41}

Out-of-hospital cardiac arrest (OHCA) score for prediction of outcome of in-hospital cardiac arrest: results of the OSPICA pilot study

Alain Mpela ${ }^{1}$, Jonathan Chelly ${ }^{1}$, Jennifer Brunet ${ }^{2}$, Stéphane Legriel ${ }^{3}$, Laurent Guérin ${ }^{4}$, Romain Persichini ${ }^{5}$, Alexis Soummer ${ }^{6}$, Bertrand Sauneuf ${ }^{7}$, Tai Pham ${ }^{8}$, Thomas Hullin ${ }^{9}$, Nicolas Deye ${ }^{10}$, Pierre Aubertein ${ }^{1}$, Mehran Monchi

${ }^{1}$ Médecine intensive, Centre Hospitalier Marc Jacquet, Melun, France; ${ }^{2}$ Réanimation chirurgicale, Centre Hospitalier Universitaire de Caen, Caen, France; ${ }^{3}$ Réanimation, Centre Hospitalier de Versailles, Le Chesnay, France; ${ }^{4}$ Réanimation, Hospital Ambroise Paré, Boulogne-Billancourt, France; ${ }^{5}$ Réanimation médicale, Hospital Bicêtre, Le Kremlin-Bicêtre, France; ${ }^{6}$ Réanimation, Hospital Foch, Suresnes, France; ${ }^{7}$ Réanimation Médicale Polyvalente, C.H. Public du Cotentin, Cherbourg-Octeville, France; ${ }^{8}$ Réanimation médico-chirurgicale, Hôpital Tenon, Paris, France; ${ }^{9}$ Réanimation, Centre Hospitalier d'Etampes, Étampes, France; ${ }^{10}$ Réanimation Médicale et Toxicologique, Hôpital Lariboisière, Paris, France

Correspondence: Jonathan Chelly jonathan.chelly@ch-melun.fr Annals of Intensive Care 2016, 6(Suppl 1):S41

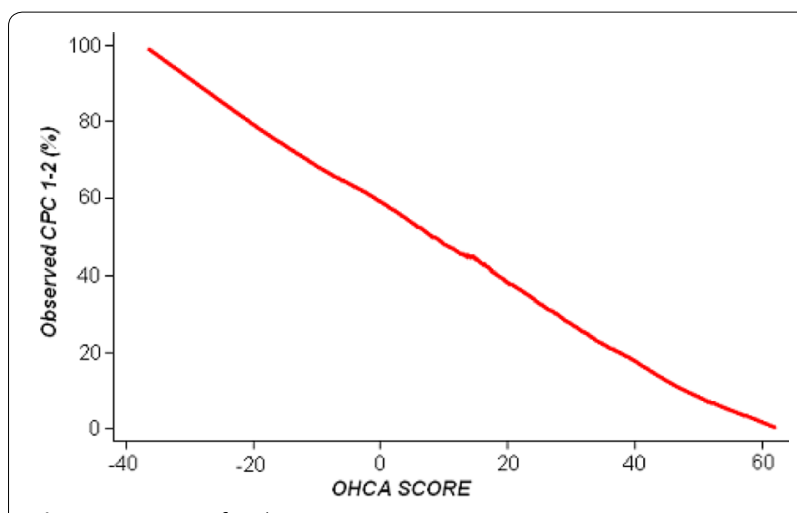

Fig. 41 See text for description

Introduction In-hospital cardiac arrest (IHCA) is still associated with poor outcomes. Early prediction of unfavourable outcomes is difficult but crucial to improve resource allocation. There is actually no predictive score for IHCA outcome. Our objective was to determine whether the out-of-hospital cardiac arrest (OHCA) score based on clinical and easily accessible variables could correctly predict outcome in patients admitted in intensive care unit (ICU) after an IHCA.

Patients and methods This was a retrospective observational multicentric cohort study including all patients admitted in 7 French medical or mixed ICUs after an IHCA between January 2009 and December 2014. Cardiac arrest (CA) occurrence in operating room or ICU was excluded. Outcome was assessed using the Cerebral Performance Categories score (CPC 1-2 for good outcome; CPC 3-4-5 for poor outcome) at hospital discharge.

Results Of the 349 patients assessed for eligibility, 297 patients were finally included. Before CA, 267 patients (90\%) were hospitalized for a non-surgical disease, 18 (6\%) for programmed surgery and 12 (4\%) for emergency surgery. IHCA occurred in a ward in 193 patients $(65 \%)$ and in the emergency room in 103 patients (35\%). IHCA was from cardiac origin in 75 patients $(25 \%)$. Median no flow duration was $0.5 \mathrm{~min}$ (interquartile range $0.5-1.0$ ), and low flow duration was $10 \mathrm{~min}$ (interquartile range 5-20). An initial shockable rhythm was found in 62 patients $(21 \%)$, and hospital survival with favourable neurological outcome occurs in $26 \%$ of cases. OHCA score was significantly lower in patients with a favourable outcome compared to unfavourable outcome (14 [interquartile range 1-25] versus 29 [interquartile range $17-40]$, respectively; $p<0.001$ ). In multivariate analysis, OHCA score was independently associated with unfavourable outcome (odds ratio per OHCA score point: $0.97,95 \%$ confidence interval [0.95-0.99], $p=0.002$ ) with a sensibility of $61 \%$ and a specificity of $85 \%$ (area under the receiver operating curve $=0.77$ ). For an OHCA score $>36$, predictive mortality was $97 \%$ (95\% confidence interval 96-97) (Fig. 41).

Conclusion In this first retrospective multicentric study, OHCA score is independently associated with IHCA outcome at hospital discharge. Early prediction of IHCA outcome by OHCA score needs further studies to confirm this hypothesis.

Competing interests None.

\section{S42}

Delayed awakening after cardiac arrest: causes and outcome in the Parisian registry

Marine Paul', Wulfran Bougouin ${ }^{2}$, Guillaume Geri ${ }^{3}$, Florence Dumas ${ }^{4}$, Benoit Champigneulle ${ }^{3}$, Stéphane Legriel ${ }^{5}$, Julien Charpentier ${ }^{3}$, Jean-Paul $\mathrm{Mira}^{3}$, Claudio Sandroni ${ }^{6}$, Alain $\mathrm{Cariou}^{3}$

${ }^{1}$ Réanimation médicale, Cochin Port-Royal, Paris, France; ${ }^{2}$ Cochin Hospital, Paris, France; ${ }^{3}$ Réanimation Médicale, Hôpital Cochin, Paris, France; ${ }^{4}$ Emergency Department, Cochin Hospital-APHP, Paris, France; ${ }^{5}$ Réanimation, Centre Hospitalier de Versailles, Le Chesnay, France; ${ }^{6}$ Depart- 
ment of Anaesthesiology and Intensive Care, Catholic University School of Medicine, Rome, Italy

Correspondence:Wulfran Bougouin - wulfran.bougouin@gmail.com Annals of Intensive Care 2016, 6(Suppl 1):S42

Introduction After cardiac arrest (CA) and therapeutic hypothermia (TH), a persisting coma despite discontinuation of sedatives is commonly considered as a trigger for initiation of neuro-prognostication. However, a persisting coma can also result from transient impairment of brain function. This study reports factors associated with delayed awakening in resuscitated patients treated with $\mathrm{TH}$.

Patients and methods All consecutive patients admitted after CA and still alive after TH were studied. Using the Richmond Agitation Sedation Scale (RASS) assessed every $3 \mathrm{~h}$, awakening was defined as 3 consecutive measurements higher than -2 and was considered delayed when occurring later than $48 \mathrm{~h}$ after discontinuation of sedation. Factors associated with duration of coma and delayed awakening were identified using multivariate analysis.

Results Three hundred and twenty-six patients were included between 2008 and 2013. Patients were mostly male (71\%), with mean age of 59 years. Post-resuscitation shock occurred in $45 \%$ of patients and $42 \%$ had renal insufficiency (GFR $<60 \mathrm{ml} / \mathrm{min}$ ) at admission. During ICU stay, 194 patients awoke in a median time of $17 \mathrm{~h}$ (IQR 7-60) after discontinuation of sedation (midazolam and fentany in all patients). Among awakeners, $29 \%$ of patients had delayed awakening. In multivariate analysis, age over 59 (OR $2.2,95 \% \mathrm{Cl} 1.1-4.6 ; p=0.03)$, initial shock (OR 2.8, $95 \% \mathrm{Cl} 1.4-5.7 ; p=0.005)$, renal insufficiency at admission (OR 3.2, $95 \%$ IC 1.4-7.0; $p=0.004$ ) and time before rewarming (OR 3.2, $95 \%$ IC 1.4-7.0; $p=0.004)$ were associated with delayed awakening.

Conclusion Among patients admitted alive after CA, nearly one-third of them awoke more than $48 \mathrm{~h}$ after discontinuation of sedation. Renal impairment, older age and initial shock can delay awakening and should lead to delayed prognostication.

Competing interests None.

\section{S43}

Incidence and characteristics of sudden unexplained cardiac death: insights from the Parisian registry

Olivier Passouant ', Guillaume Geri ${ }^{1}$, Florence Dumas ${ }^{2}$, Wulfran Bougouin ${ }^{1}$, Benoit Champigneulle', Michel Arnaout', Jonathan Chelly ${ }^{3}$, Jean-Daniel Chiche', Olivier Varenne ${ }^{4}$, Jean-Paul Mira', Alain Cariou

${ }^{1}$ Réanimation médicale, Hôpital Cochin, Paris, France; ${ }^{2}$ Service d'accueil des urgences, Hôpital Cochin, Paris, France; ${ }^{3}$ Médecine Intensive, Centre Hospitalier Marc Jacquet, Melun, France; ${ }^{4}$ Cardiologie, Hôpital Cochin, Paris, France

Correspondence: Guillaume Geri - guillaume.geri@cch.aphp.fr Annals of Intensive Care 2016, 6(Suppl 1):S43

Introduction Respective proportions of final etiologies are disparate in cohorts of cardiac arrest patients, depending on examined population and diagnostic algorithms. In particular, incidence and characteristics of sudden unexplained death (SUD) are debated. We aimed at describing etiologies in a large cohort of out-of-hospital cardiac arrest (OHCA) patients, in order to characterize victims without identified diagnosis.

Patients and methods We analyzed data from our prospective registry of OHCA patients admitted between January 2000 and December 2014. Initial diagnostic strategy included coronary angiography, brain and chest CT scan. This was completed by an extensive diagnostic strategy, encompassing biological and toxicological tests, repeated electrocardiograms and echocardiography, MRI, Holter monitoring and endocavitary explorations. Two independent investigators reviewed each final diagnosis. Baseline characteristics were compared between subgroups of patients with Chi square test and Mann-Whitney test, as appropriate. One-year mortality was compared between subgroups using univariate Kaplan-Meier curves.

Results Over the study period, 1857 patients were admitted in our unit after a resuscitated OHCA. The event was related to a non-cardiac and a cardiac cause in 526 (30.7\%) and 1057 (61.8\%) patients, respectively. The main cause of cardiac-related OHCA was ischemic heart disease $(78.4 \%)$, while non-structural cardiomyopathies accounted for only $2.2 \%$. No diagnosis was evidenced in 127 (7.4\%) patients. In these SUD patients, baseline characteristics and 1-year survival of patients under 35 years were similar to those with identified nonstructural cardiomyopathy.

Conclusion We observed that ischemic heart disease was by far the most common cause of cardiac arrest, while non-structural cardiomyopathies accounted for a very low part of diagnosis. Young patient victims of a sudden unexplained death shared similar baseline characteristics and outcome with patients with non-structural cardiomyopathies. Focusing on this subgroup of patients for further investigations and follow-up may help in managing themselves and their relatives.

\section{Competing interests None.}

\section{S44}

Supranormal oxygen tension is not associated with an impaired redox balance in patients resuscitated from cardiac arrest

Jean-François Llitjos ${ }^{1}$, Camille Chenevier-Gobeaux ${ }^{2}$, Julien Charpentier ${ }^{1}$, Florence Dumas ${ }^{3}$, Guillaume Geri ${ }^{1}$, Wulfran Bougouin' ${ }^{1}$, Benoit

Champigneulle', Michel Arnaout ${ }^{1}$, Jean-Paul Mira', Frédéric Pène' Frederic Batteux', Alain Cariou'

${ }^{1}$ Réanimation Médicale, Hôpital Cochin, Paris, France; ${ }^{2}$ Biology, Cochin Port-Royal, Paris, France; ${ }^{3}$ Service d'accueil des urgences, Hôpital Cochin,

Paris, France

Correspondence: Jean-François Llitjos - jlitjos@gmail.com

Annals of Intensive Care 2016, 6(Suppl 1):S44

Introduction Retrospective observational data suggested potential harmful effects of hyperoxia in patients resuscitated from an outof-hospital cardiac arrest (OHCA). Experimental studies have shown that this deleterious effect of $\mathrm{O}_{2}$ could be due to the worsening of ischemia-reperfusion injuries and to an oxidative burst following resuscitation. Here we analyzed the relationship between arterial oxygen tension and the pro- and antioxidant balance in a cohort of OHCA patients.

Patients and methods We analyzed a cohort of consecutive patients successfully resuscitated from an OHCA and admitted in the medical ICU of a tertiary universitary French hospital. We defined the exposure using the $\mathrm{PaO}_{2}$ value on the first arterial blood gas (ABG) at admittance. Hyperoxia was defined as $\mathrm{PaO}_{2}$ of $300 \mathrm{~mm} \mathrm{Hg}$ or greater, hypoxia as $\mathrm{PaO}_{2}$ of less than $60 \mathrm{~mm} \mathrm{Hg}$ and normoxia as a $\mathrm{PaO}_{2}$ not classified as hyperoxia or hypoxia. The primary outcome measure was the relation between $\mathrm{PaO}_{2}$ and main blood markers of oxidative stress measured at admittance in ICU: thiols, advanced oxidation protein products (AOPP), protein carbonyls, superoxide dismutase (SOD), glutathione reductase (GRx) and glutathione peroxidase activities (GPx).

Results Among 134 patients studied, 13 had hypoxia (10\%), 102 had normoxia (76 \%) and 19 had hyperoxia (14\%). The overall intra-hospital mortality was $59.7 \%(n=80)$. We found no differences between groups, with a $76.9 \%(n=10)$ mortality rate in the hypoxia group, $56.9 \%(n=58)$ in the normoxia group and $63.2 \%(n=12)$ in the hyperoxia group. Median time from cardiac arrest to $A B G$ analysis was similar between the hypoxia, the normoxia and the hyperoxia groups (respectively $278 \mathrm{~min}$, interquartile range (IQR): 153-684; $180 \mathrm{~min}$, IQR: $131-320$ and $170 \mathrm{~min}$, IQR: 103-626). There was no statistically significant correlation between any of the biological parameters and $\mathrm{PaO}_{2}$ values in the whole population, and we found no difference regarding blood markers of oxidative stress across the 3 groups (Table 53).

Conclusion In our study, $\mathrm{PaO}_{2}$ at hospital admission was not associated with differences in the ratio between oxidant and antioxidant parameters in patients resuscitated from cardiac arrest. We observed no evidence supporting a role of an impaired redox balance due to supranormal oxygen tension in this population.

Competing interests None. 
Table 53 See text for description

\begin{tabular}{|c|c|c|c|}
\hline & $\begin{array}{l}\text { Hypoxia } \\
(n=13)\end{array}$ & $\begin{array}{l}\text { Normoxia } \\
(n=102)\end{array}$ & $\begin{array}{l}\text { Hyperoxia } \\
(n=19)\end{array}$ \\
\hline Thiols ( $\mu \mathrm{M})$ & $9.7(6.3-13.8)$ & $11.3(9.2-12.9)$ & $11.3(8.2-13.1)$ \\
\hline AOPP $(\mu \mathrm{M})$ & $276(162-599)$ & $391(248-615)$ & $423(210-510)$ \\
\hline $\begin{array}{l}\text { Carbonyls } \\
\text { (nmol/mg) }\end{array}$ & $70.8(64.2-80.8)$ & $69.6(62.1-88.8)$ & $70.7(59.7-101.1)$ \\
\hline $\mathrm{SOD}(\mathrm{U} / \mathrm{mL})$ & $495(398-548)$ & $440(315-533)$ & $463(373-498)$ \\
\hline $\begin{array}{l}\text { GPx (nmol/min/ } \\
\text { mL) }\end{array}$ & $300(169-455)$ & $305(170-429)$ & $251(180-398)$ \\
\hline $\begin{array}{l}\text { GRx (nmol/min/ } \\
\text { mL) }\end{array}$ & $45(19-65)$ & $48(11-79)$ & $62(34-85)$ \\
\hline
\end{tabular}

\section{S45}

Impaired biological response to aspirin in comatose patients resuscitated from out-of-hospital cardiac arrest

Jean-François Llitjos', Ludovic Drouet ${ }^{2}$, Sebastian Voicu ${ }^{3}$, Nicolas Deye", Bruno Megarbane ${ }^{5}$, Patrick Henry ${ }^{6}$, Jean-Guillaume Dillinger ${ }^{6}$

${ }^{1}$ Réanimation Médicale, Hôpital Cochin, Paris, France; ' ${ }^{2}$ Department of Angio-Hematology, Hospital Lariboisière, Paris, France; ${ }^{3}$ Intensive Care Unit, Hospital Lariboisière, Paris, France; ${ }^{4}$ Réanimation Médicale et Toxicologique, Hôpital Lariboisière, Paris, France; ${ }^{5}$ Service de Réanimation Médicale et Toxicologique, CHU Lariboisière, Paris, France; ${ }^{6}$ Cardiology, Hospital Lariboisière, Paris, France

Correspondence: Jean-François Llitjos - jllitjos@gmail.com Annals of Intensive Care 2016, 6(Suppl 1):S45

Introduction Acute coronary syndromes are one of the main causes of out-of-hospital cardiac arrest. These patients are at high risk of persistent platelet reactivity under aspirin treatment. We herein evaluate the biological response to aspirin and ADP inhibitors in comatose patients resuscitated from out-of-hospital cardiac arrest treated by either oral or intravenous aspirin, with or without an ADP inhibitor.

Patients and methods Patients were randomized to receive longterm antiplatelet treatment with either $100 \mathrm{mg}$ oral or $100 \mathrm{mg}$ intravenous aspirin and when necessary dual antiplatelet therapy after emergency coronary angiography. Patient's blood samples were obtained at day 3 and day 7 after cardiac arrest. Biological response to aspirin was assessed $24 \mathrm{~h}$ after last aspirin intake using light transmission aggregometry with arachidonic acid and the whole blood point-of-care assay PFA- $100^{\circledR}$ system with collagen-epinephrin. Biological response to ADP inhibitors was assessed using flow cytometry (vasodilator-stimulated phosphoprotein platelet reactivity index), light transmission aggregometry with adenosine diphosphate and PFA$100^{\circledR}$ system with collagen-adenosine diphosphate.

Results Twenty-two consecutive patients were prospectively included. Thirteen patients received aspirin in the intravenous route and 9 patients in the oral route. Clinical, demographical, biological and angiographical characteristics were similar in both groups. Using light transmission aggregometry with arachidonic acid, 10 patients had persistent platelet reactivity, corresponding to $45 \%$ of the total population. At day 3 after cardiac arrest, we found a significantly higher maximum intensity in the oral group when compared to the IV group ( 29 vs. $15 \%, p=0.04$ ). The same way, closure time was significantly faster in the oral group when compared to the IV group (155 vs. $277 \mathrm{~s}, p=0.04)$. We found no difference at day 7 after cardiac arrest.
Irrespective of the biological test, clopidogrel was associated with a biological inefficacy at day 3 . Under prasugrel, persistent platelet reactivity was found in $20 \%$ of samples with inadequate effect using flow cytometry, in $10 \%$ using light transmission aggregometry with adenosine diphosphate and in $40 \%$ using the PFA- $100^{\circledR}$ system with collagen-adenosine diphosphate. Stent thrombosis occurred in 1 patient treated with oral aspirin and efficiently by prasugrel. We found no difference in mortality in both groups.

Conclusion In this study, impaired response to aspirin both oral and intravenous is frequent in comatose patients resuscitated from out-ofhospital cardiac arrest, with a higher rate using the oral route. The use of prasugrel as an ADP inhibitor seems to be associated with a highest efficiency in inhibiting platelet reactivity than clopidogrel in this population of patients.

\section{Competing interests None.}

S46

Gender differences in coronary reperfusion after cardiac arrest

Wulfran Bougouin ${ }^{1}$, Florence Dumas ${ }^{2}$, Eloi Marijon ${ }^{3}$, Guillaume Geri ${ }^{4}$, Benoit Champigneulle ${ }^{4}$, Jean-Daniel Chiche ${ }^{4}$, Olivier Varenne ${ }^{5}$, Christian Spaulding ${ }^{6}$, Jean-Paul Mira ${ }^{4}$, Xavier Jouven ${ }^{6}$, Alain Cariou ${ }^{4}$

${ }^{1}$ Cochin Hospital, Paris, France; ${ }^{2}$ Emergency Department, Cochin Hospital-APHP, Paris, France; ${ }^{3}$ Cardiologie, Hôpital Européen Georges-Pompidou, Rue Leblanc, Paris, France, Paris, France; ${ }^{4}$ Réanimation Médicale, Hôpital Cochin, Paris, France; ${ }^{5}$ Cardiologie, Hôpital Cochin, Paris, France; ${ }^{6}$ Cardiologie, Hopital Europeen Georges-Pompidou, Paris, France

Correspondence:Wulfran Bougouin - wulfran.bougouin@gmail.com Annals of Intensive Care 2016, 6(Suppl 1):S46

Introduction Early percutaneous coronary intervention (PCI) may improve survival in OHCA of ischemic cause, but selection of suitable patients remains challenging. Differences and results across gender remain unknown. We assessed the relationship between gender and coronary reperfusion after OHCA and the relationship between $\mathrm{PCI}$ and outcome according to gender.

Materials and methods All patients admitted in our center after OHCA were prospectively included in an electronic registry database, from 2000 to 2013. Characteristics were collected according to the Utstein style. We assessed the association between gender and coronary reperfusion (using multivariate logistic regression and propensity score analysis) and between $\mathrm{PCl}$ and outcome (according to gender). Results One thousand eight hundred and seventeen patients were included (520 women, $29 \%$ ). Women were older (62.8 vs 59.1 years, $P<0.0001$ ), with less cardiomyopathy. They had less bystander cardiopulmonary resuscitation ( $84 \%$ vs $88 \%, P<0.05)$ and less shockable rhythm ( $42 \%$ vs $61 \%, P<0.001)$. After multivariate logistic regression, male sex was associated with the performance of coronary angiogram (OR 1.73, $95 \% \mathrm{Cl} 1.28-2.34, P<0.001$ ). Results after propensity score matching were consistent $(P=0.02)$. Among 1157 patients who underwent coronary angiogram, rate of $\mathrm{PCl}$ did not differ between men and women (OR 1.30, $95 \% \mathrm{Cl} 0.90-1.88, P=0.17)$. Results after matching (211 men, 211 women) were consistent $(P=0.13)$. PCI was associated with favorable outcome by multivariate logistic regression (OR 1.45, $95 \% \mathrm{Cl} 1.07-1.96, P=0.02$ ) with no interaction between gender and $\mathrm{PCl}(P$ for interaction $=0.40$ ). Association between $\mathrm{PCl}$ and outcome was consistent across genders.

Conclusion After OHCA, women are less likely to undergo coronary angiogram. Rates of $\mathrm{PCl}$ after coronary angiogram do not differ across gender, and the association between $\mathrm{PCl}$ and favorable outcome is similar across gender.

Competing interests None. 
547

Prognostic factors of out-of-hospital cardiac arrests in Martinique: prospective study over a period of 29 months, about 385 cases

Dabor Resiere1, A. L. Dessoy ${ }^{1}$, Ruddy Valentino' ', C. Chabartier', Laurent Villain-Coquet ${ }^{2}$, Jean-Louis Ferge' ${ }^{1}$, Y. Brouste ${ }^{2}$, Bruno Megarbane ${ }^{3}$, Hossein Mehdaoui

${ }^{1}$ Service de réanimation médico-chirurgicale, $\mathrm{CHU}$ de Fort de France, Fort-de-France, France; ${ }^{2}$ Samu 972, CHU de Fort de France, Fort-de-France, France; ${ }^{3}$ Service de Réanimation Médicale et Toxicologique, CHU Lariboisière, Paris, France

Correspondence: Dabor Resiere - dabor.resiere@chu-fortdefrance.fr Annals of Intensive Care 2016, 6(Suppl 1):S47

Introduction Out-of-hospital cardiac arrest (OHCA) represents in 2015 a persistent public health problem, with always a bad prognosis and poor survival. Outcomes are improved by healthcare provider practices as recommended by the 2010 European Resuscitation Council Guidelines for Cardiopulmonary Resuscitation. In France, the National registry RéAC was set up in 2010 because of the lack of national and international outof-hospital cardiac arrest registry. Our study followed upon observation of the lack of data concerning the OHCA in Martinique. The aim of this study was to identify the prognostic factors of the OHCA in Martinique. Patients and methods A prospective, observational, and monocentric study was performed in Martinique over a 29-month period (from December 2012 to April 2015), including all out-of hospital cardiac arrests requiring the intervention of our emergency pre-hospital medical service (SAMU 972). We described the circumstances, demographic data, clinical and management of each link in the « chain of survival » and the different delays of care. Univariate analysis was performed to identify factors associated with mortality on hospital admission. Data from SAMU 972 were compared to other French emergency medical centers.

Results Three hundred eighty-five patients with cardiac arrest were included. Fifty-nine percent were men with a median age of 63 years. Sixty-eight percentage of OHCA occurred at home and were witnessed in $74 \%$ of cases, as compared to other French national centers. Cardiopulmonary resuscitation (CPR) was initiated by bystanders in $43 \%$. The absence of CPR and early defibrillation were associated with asystole ( $89 \%$ of the cases), when advanced cardiac life support was initiated by firefighters and/or by emergency medical service. When the emergency medical team arrived in the site, more than $80 \%$ of the patients were found in asystole and only $6 \%$ in VF/VT. Delay of departure of the emergency medical service vehicle and arrival at the scene were much more longer in comparison with other centers. The absence of CPR and delay for firefighters to reach the patients were associated with mortality on hospital admission $(p=0.03$ and $p=0.04$, respectively). The rate of return of spontaneous circulation was $18 \%$, with a $13 \%$ survival rate on hospital admission and $3.4 \%$ rate on day 30 . The overall survival rate was lower than the other French centers (RéAC). Prognostic factors significantly associated with the survival rate were $(p<0.05)$ : young age, immediate resuscitation by training bystanders, presence of emergency medical service personnel or firefighters at the time of OHCA, used of automated external defibrillator before the EMS providers arrived at the scene, delay and type of early rhythm, use of epinephrine and early therapeutic hypothermia in ICU.

Conclusion Out-of-hospital cardiac arrests in Martinique are similar to those from metropolis France with horrifying outcome. However, in Martinique, survival rate appears to be lower. Several proposals could be suggested to improve outcome, including (1) increasing population training to basic cardiac life support, (2) enhancing public access to early defibrillation, (3) reducing delays of out-of-hospital medical services interventions, (4) standardizing advanced cardiac life support, and (5) strengthening ways to identify easily house's number. However, the definitive benefit of such interventions should be further investigated.

\section{Competing interests None.}

\section{References}

1. Gueugniaud PY, Hubert H, Tazarourte K, Wiel E, Zitouni D, Vilhelm C, Escutnaire J, Cassan P, Gueugniaud PY; GR- RéAC.. RéAC, Registre électronique des Arrêts Cardiaques. Rationale, methodology, implementation, and first results of the French out-of-hospital cardiac arrest registry. Prehosp Emerg Care 2014;18(4):511-9.

S48

Incubation of immune cells in septic plasma: effect on glucose utilization and on phenotypic expression on monocytes, polymorphonuclears and lymphocytes

Benjamin Soyer ${ }^{1}$, Valérie Faivre ${ }^{1}$, Charles Damoisel ${ }^{1}$, Anne-Claire Lukaszewicz', Didier Payen de la Garanderie ${ }^{1}$

${ }^{1}$ Réanimation chirurgicale, CHU Lariboisière, Paris, France

Correspondence: Benjamin Soyer - benjaminsoyer@wanadoo.fr Annals of Intensive Care 2016, 6(Suppl 1):S48

Introduction Glucose is the key energy substrate of monocytes (Monos), polymorphonuclears (PMNs) and lymphocytes (Ly), necessary for their activation during pro-inflammatory phase. The impact of hyperglycemia $(\mathrm{HG})$ of septic plasma $(\mathrm{Spl})$ on phenotypic markers of immune cells is unknown. Objective: to assess the effect of incubation in septic plasma on glucose utilization and on phenotypic expression of immune cells.

Materials and methods Ten whole blood samples from healthy volunteers (CCPSL UNT-No13/SL/015) were incubated $90 \mathrm{~min}$ in normoglycemia conditions (NG), hyperglycemia ( $\mathrm{HG}, 12.5 \mathrm{mM}$ glucose), in the presence of deoxyglucose (DOG $12.5 \mathrm{mM}$; inhibitor of glycolysis). For 6 samples, a volume-to-volume septic plasmas pool testing the same conditions replaced healthy plasma $(\mathrm{Hpl})$. We studied the expression of surface markers on immune cells as follows:

- Monos (CD14+): HLA-DR, CD11b, CD62L (markers of cellular activation)

- PMNs (CD16+): CD11b, CD62L

- T Ly (CD3+): CTLA4 (marker of cellular inhibition)

- B Ly (CD19+): HLA-DR

Results 1. In Hpl, HG did not increase the expression of markers of interest compared to NG. DOG decreased the expression of markers of interest for Monos (HLA-DR, CD11b and CD62L) and CD62L in PMNs. 2. In Spl, there was no difference in the expression of surface markers between the 3-glycemic conditions. 3. The expression of surface markers in NG + Spl collapsed compared to Hpl. The DOG + Spl vs. $\mathrm{Hpl}$ induced increased expression of CD11b for both Monos and PMNs $(p<0.05)$; expression of CD62L was collapsed (10-15 times) for all conditions in Spl vs. Hpl ( $p<0.05$ for each one). Expression of CTLA4 for T4 and T8 Ly collapsed (about 10 times) in all conditions in Hpl vs. Spl $(p<0.05)$. There was no difference for HLA-DR on Monos and B Ly.

Discussion Our results confirm that aerobic glycolysis is a central pathway of phenotypic changes of immune cells in healthy plasma. The incubation in plasma seemed to favor septic "activated" phenotypes for both granular and T Ly independently of the use of glucose (no impact of HG on phenotypes of immune cells). No detectable effect of HG in septic plasma on studied surface makers, due to the huge changes caused by septic plasma.

Conclusion In acute phase, the impact of septic environment dominates the effects of HG. These results ask about the interest of tight glycemic control in acute septic patient in ICU.

\section{Competing interests None.}

\section{S49}

Ex vivo effects of hyperglycemia on phenotype and production of reactive oxygen species by the NADPH oxidase of human immune cells in acute inflammatory response

Benjamin Soyer ${ }^{1}$, Valérie Faivre ${ }^{1}$, Charles Damoisel ${ }^{1}$, Anne-Claire

Lukaszewicz ${ }^{1}$, Didier Payen de la Garanderie ${ }^{1}$

${ }^{1}$ Réanimation chirurgicale, CHU Lariboisière, Paris, France

Correspondence: Benjamin Soyer - benjaminsoyer@wanadoo.fr Annals of Intensive Care 2016, 6(Suppl 1):S49

Introduction Glucose is the main energy substrate (1) for immune cells (monocytes (monos) and polymorphonuclears (PMNs)). Glucose 
has also a pro-inflammatory action through the production of reactive oxygen species (ROS).

Objectives: (1) to demonstrate an increase in ROS production during moderate hyperglycemia through the NADPH oxidase (NADPHox) pathway (NADPH comes from the pentose shunt pathway (PSP)); (2) to evaluate the phenotypic consequences on immune cells: monos, PMNs, and lymphocytes (lymphos).

Materials and methods Whole blood from healthy volunteers (CCPSL UNT-No13/SL/015) was incubated $90 \mathrm{~min}$ in: 1-normoglycemia (NG) or 2-hyperglycemia ( $\mathrm{HG}$, glucose $12.5 \mathrm{mM}$ ), or with deoxyglucose, a glycolysis blocker (DOG $12.5 \mathrm{mM}$ ), or with specific inhibitors of PSP: Epi Androsterone and 6 Aminonicotinamide (EPI 6 AN).

Measurements: 1 -overall ROS production with luminometry (2) technic (area under the curve (AUC) and slope of activation) at baseline and after stimulation with PMA-lonomycin (Phorbol-12-Myristate13-Acetate, lonomycin). 2-Phenotypic changes in immune cells:

- Monos (CD14+): HLA-DR, CD11b, CD62L (markers of cellular activation)

- PMNs (CD16+): CD11b, CD62L

- T lymphos (CD3+): CTLA4 (marker of cellular inhibition)

- B lymphos (CD19+): HLA-DR

Statistics: nonparametric tests.

Results ROS production: at baseline, AUC $(n=19)$ in HG was higher than that in NG $(p<0.05)$ (increased production). After stimulation with PMA-ionomycin, this difference disappeared. Inhibitors reduced the AUC compared to the NG $(p<0.05)$. The slope of luminometry (reactivity) was higher in HG than in NG $(p<0.05)$. The slopes in NG and HG were higher than those observed with inhibitors in NG and HG $(p<0.05)$.

1. Phenotypic changes: no difference between monos, PMNs and lymphos in the 3-glycemic conditions (there were only few decreases for some markers in DOG conditions).

Discussion Hyperglycemia increased ROS production of immune cells in basal condition. However, in stimulated conditions, hyperglycemia did not increase ROS production of the NADPH oxidase system but accelerated the reactivity of the system (slope of the luminometry curve). Nonspecific inhibitor (DOG) and specific inhibitors of PSP (EPI $6 A N)$ decreased ROS production and reactivity of NADPHox system. Conclusion Our experiments confirm the implication of the PSP in ROS production. Functional changes induced by hyperglycemia were not associated with phenotypic alterations in immune cells.

\section{Competing interests None.}

\section{References}

1. Pearce, E. L. \& Pearce, E. J. Metabolic pathways in immune cell activation and quiescence. Immunity 38, 633-643 (2013).

2. Lukaszewicz, A.-C., Gontier, G., Faivre, V., Ouanounou, I. \& Payen, D. Elevated production of radical oxygen species by polymorphonuclear neutrophils in cerebrospinal fluid infection. Ann. Intensive Care 2, 10 (2012).

\section{S52}

HMGB1 induces neutrophils dysfunction after septic shock

Fabrice Uhel ${ }^{1}$, Murielle Gregoire ${ }^{2}$, Arnaud Gacouin ${ }^{1}$, Caroline Piau ${ }^{3}$, Yves Le Tulzo', Karin Tarte', Jean-Marc Tadié

${ }^{1}$ Réanimation médicale, Centre Hospitalier Universitaire de Rennes, Rennes, France; ${ }^{2}$ Biosit and inserm u917, faculte de medecine, universite rennes i, Centre Hospitalier Universitaire de Rennes, Rennes, France; ${ }^{3}$ Service de bactériologie et hygiène hospitalière, Centre Hospitalier Universitaire de Rennes, Rennes, France

Correspondence: Jean-Marc Tadié - jeanmarc.tadie@chu-rennes.fr Annals of Intensive Care 2016, 6(Suppl 1):S52

Introduction Polymorphonuclear neutrophils (PMNs) play a central role in acute inflammatory and innate immune responses through production of antibacterial peptides, cytokines, pro-inflammatory mediators such as reactive oxygen species (ROS), and extracellular traps. PMN activation and involvement in immune defense against pathogens during septic shock have been well described. However, despite evidences showing that PMN dysfunction could be found in

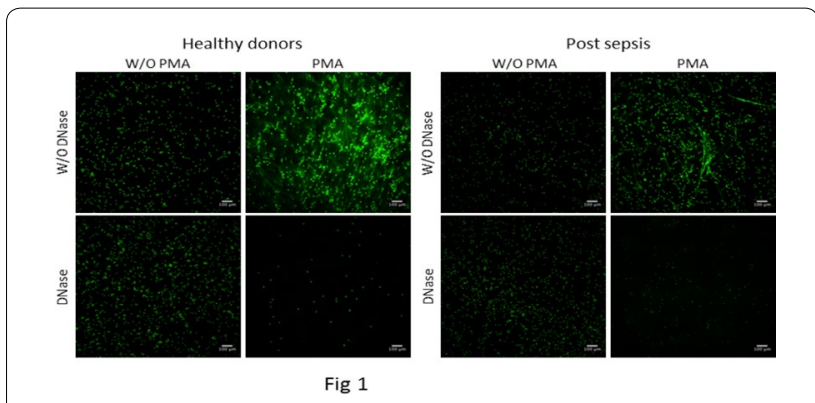

Fig. 42 See text for description

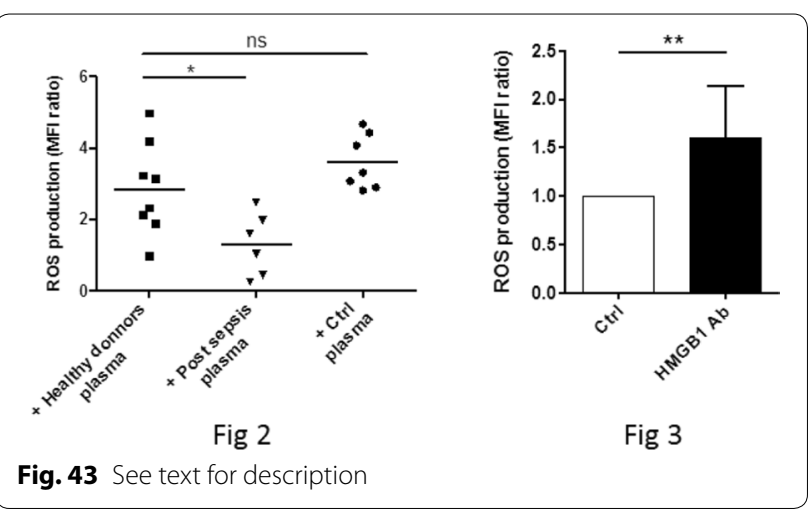

patients with nosocomial infections, very few studies have focused on PMN function after septic shock. Herein, we examined the hypothesis that HMGB1 could induce a PMN dysfunction after septic shock which could be reversed using an anti-HMGB1 antibody.

Materials and methods In a first set of experiments, we explored PMN functions (ROS production, NETosis and bacteria killing ability) during late phase of sepsis in a murine model of CLP-induced sepsis. In a second set of experiments, we compared PMN function in patients admitted in ICU for sepsis to patients admitted for non-septic reason (control) and healthy donors.

Results We found a marked PMN dysfunction after sepsis, characterized by a decreased ability to produce ROS and NETs and to kill bacteria (Fig. 42). We also found that HMGB1 induced PMN dysfunction since plasma of septic patients induced PMN dysfunction in PMN isolated from healthy donors (Fig. 43). Lastly, we found that anti-HMGB1 specific antibody could restore ROS production (Fig. 43).

Conclusion PMN dysfunction is evident after septic shock, induced by HMGB1. Specific inhibition could restore ROS production of PMN after septic shock and could be an interesting therapeutic approach.

\section{Competing interests None.}

\section{S53}

Rapid mobilization of myeloid-derived suppressor cells during sepsis and relation with clinical course

Thomas Daix ${ }^{1}$, Robin Jeannet ${ }^{2}$, Emmanuelle Begot ${ }^{3}$, Nicolas Pichon ${ }^{3}$, Anne-Laure Fedou ${ }^{3}$, Estelle Guérin ${ }^{4}$, Philippe Vignon ${ }^{5}$, Jean Feuillard ${ }^{4}$, Bruno François ${ }^{5}$

${ }^{1}$ Inserm cic 1435/medical-surgical icu, Chu Limoges, Limoges, France; ${ }^{2} U m r$ cnrs 7276, medecine university, Chu Limoges, Limoges, France; ${ }^{3}$ Medical-surgical icu, Chu Limoges, Limoges, France; ${ }^{4}$ Hematology laboratory/umr cnrs 7276, medecine university, Chu Limoges, Limoges, France; ${ }^{5}$ nserm cic 1435/medical-surgical icu/umr 1092, medecine university, Chu Limoges, Limoges, France

Correspondence:Thomas Daix - thomas.daix@wanadoo.fr

Annals of Intensive Care 2016, 6(Suppl 1):S53 
Introduction Myeloid-derived suppressor cells (MDSCs), an immature leukocyte subset composed of granulocytic (GrMDSC) and monocytic (MoMDSC), are well described in neoplastic diseases as an immunosuppressive actor. This population is also present in various clinical situations marked by activation of inflammation as trauma, burns or sepsis. During sepsis this leukocyte subset could play a key role in immunosuppressive states by killing $T$ lymphocyte. To date, only few studies specifically focused on the potential role of this leukocyte subset in sepsis. Mechanisms linked to MDSC mobilization in peripheral bloods and their origins need also to be explored to better understand their role in sepsis. The main objective of our study was to approach the kinetic of expression of MDSC in septic patients hospitalized in the intensive care unit (ICU). Secondary objectives were to assess the origin of production of MDSCs and their potential association with clinical course.

Materials and methods Patients hospitalized in our medical-surgical ICU for less than $24 \mathrm{~h}$ at inclusion with a confirmed sepsis associated with a thrombocytopenia have been prospectively studied from October 2014 to August 2015. We followed the level of MoMDSC (CD45+, CD11b+, CD14+, CD33+, HLA-DR-) and GrMDSC (CD45+, Lin-, HLA$\mathrm{DR}-, \mathrm{CD} 33+, \mathrm{CD} 14-, \mathrm{CD} 1 \mathrm{~b}+)$ by flow cytometry in the peripheral blood (leftover from complete blood count performed as part of routine care) at D0, D3, D7, D10 and D14. We analyzed the same populations in the leftover of bone marrow (BM) samples performed in thrombocytopenic septic patients at Day 0 . We recorded the site of infection, the onset date and the microorganisms involved. Each infection was adjudicated and validated according to the diagnostic criteria of the international sepsis forum consensus conference. Clinical progression during ICU stay, occurrence of secondary infection and hospital discharge were also noted. Outpatients without any hematological or infectious disorders who were recruited the time of anesthesia evaluation (peripheral blood analysis) or after a BM aspirate analysis considered as normal by hematologist were used as controls for comparison. MDSC results were expressed as percentages of CD45+ or as gram per liter $(\mathrm{G} / \mathrm{L})$.

Results A total of 16 patients ( 15 septic shocks, 1 severe sepsis) with sepsis-induced thrombocytopenia were studied, and for 7 of them kinetic of MDSCs in the peripheral blood was available. Of them, 9 patients suffered from community infections. Main sites of infection were intra-abdominal $(n=8)$, urinary tract $(n=3)$ and lung infections $(n=2)$. All infections were documented (10 gram-negative bacilli, 6 gram-positive cocci). Median ICU stay was 8 days. ICU and hospital mortality were 31 and $44 \%$, respectively. At early phase of sepsis, levels of MoMDSC subset in the BM and in peripheral blood of septic patients were increased compared to controls (BM 1.31 vs $0.39 \%$, peripheral blood 0.36 vs $0.05 \mathrm{G} / \mathrm{L}$ ). This increase was not correlated with the severity of initial sepsis. When compared to controls, septic patients had higher BM expression of GrMDCS (3.78 vs $0.18 \%$ ) but no significant increase in peripheral blood $(0.06$ vs $0.01 \mathrm{G} / \mathrm{L}, p=0.09$ ). During sepsis course, most of patients exhibited an increase in MDSC mobilization in peripheral blood until D7 (mean level D0: $0.23 \mathrm{G} / \mathrm{L}$, D3: $0.72 \mathrm{G} / \mathrm{L}, \mathrm{D} 7: 1.1 \mathrm{G} / \mathrm{L}, \mathrm{D} 14: 0.34 \mathrm{G} / \mathrm{L})$. MDSC level seems to be related to clinical course, patients with major organ failure exhibiting a higher overall MDSC level (Fig. 44). These leukocyte subset levels did not seem to be related to the site of infection, microbiological species or specific organ failure.

Conclusion During the early phase of sepsis associated with an excessive systemic inflammation, MDSC level increased secondary to a BM excretion. This result suggests that pro-inflammatory and immunosuppressive mechanisms overlap during the early phase of sepsis. MDSC level which appears to be correlated with clinical severity could be a regulation factor of immune reaction during sepsis. These hypotheses need to be confirmed in a larger prospective study.

\section{Competing interests None.}

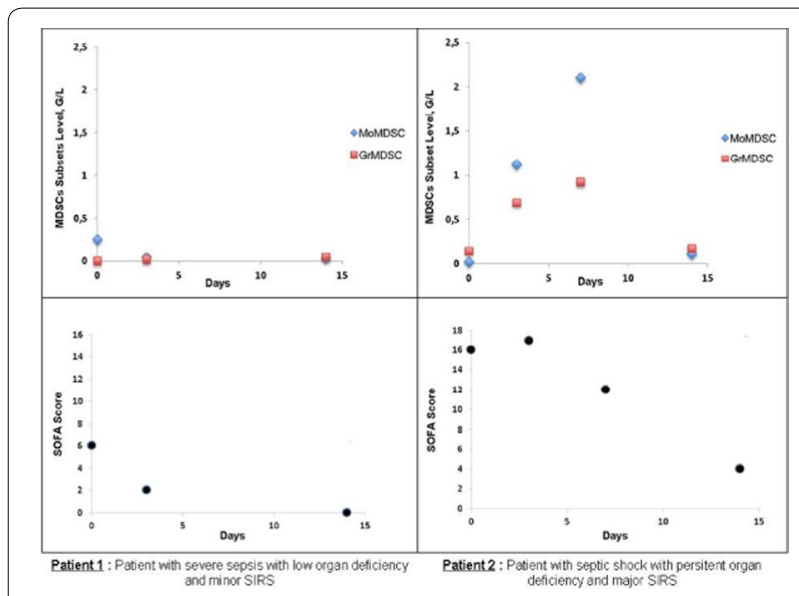

Fig. 44 See text for description

S54

Targeted endothelial TREM-1 deletion protects mice during septic shock

Lucie Jolly' ${ }^{1}$ Amir Boufenzer ${ }^{2}$, Jérémie Lemarie ${ }^{3}$, Kevin Carrasco ${ }^{1}$, Marc Derive $^{2}$, Sebastien Gibot $^{3}$

${ }^{1}$ Inserm u1116, Faculté de Médecine, Vandœuvre-lès-Nancy, France; ${ }^{2}$ Inotrem, inotrem, Nancy, France; ${ }^{3}$ Réanimation médicale, hôpital central, C.H.U. de Nancy, Nancy, France

Correspondence: Sebastien Gibot - sgibot@yahoo.fr

Annals of Intensive Care 2016, 6(Suppl 1):S54

Introduction The triggering receptor expressed on myeloid cells-1 (TREM-1) amplifies the inflammatory response driven by TLR/NLR engagement. In various models of acute inflammation, septic or not, others and we have shown that the genetic deletion or pharmacologic inhibition of TREM-1 protects animals from hyperresponsiveness and death. In this study, we demonstrate that a targeted deletion of TREM-1 on endothelial cells confers protection during septic shock in mice.

Materials and methods We generated constitutive Trem-1-/- as well as endothelium-conditional Trem-1 KO mice and submitted them to polymicrobial sepsis through CLP. Organs (BM, spleen, lungs, aorta and mesenteric artery) and blood were harvested at different time points and analysed for cellular content, gene expression, cytokine/ chemokine concentrations and vasoreactivity. Survival was monitored for $1 \mathrm{~W}$.

Results A targeting vector was designed for conditional deletion of exon 2 that encodes the extracellular domain of TREM-1. Breeding of Trem $1+/$ flox chimeric offspring mice with ubiquitous or Cadherin 5 Cre deleted mice yielded viable Trem $1+/-\times($ Cadh5) Cre+/- offspring. Interbreeding of Trem $1+/-$ mice gave rise to Trem $1-/-$ or endothelium-specific Trem1-/- (EndoTrem1-/-) mice at the expected Mendelian frequencies, and Trem1-/- mice were equal in size, weight and fertility to littermate controls. Moreover, the composition and abundance of immune cells in blood, BM, spleen and lungs did not differ between groups. Trem-1 deletion altered inflammatory cells mobilization and recruitment in lungs and spleen and favours the accumulation of reparative cells (Ly6Clow monocytes and M2 macrophages) in the lungs. This effect was even more pronounced in EndoTrem1-/-. Consequently, the activation of many inflammatory genes was reduced in the lungs as well as the concentrations of various cyto/chemokines (MCP-1, VCAM-1, IL6, ...). 
Sepsis induced a profound vascular hyporeactivity in WT mice. This phenomenon was absent in the absence of Trem1. Interestingly, in EndoTrem1-/- while vasoconstriction was still slightly impaired, endothelium-dependent vasodilation remained intact. Finally, survival was improved in the Trem $1 \mathrm{KO}$ animals and even more in the EndoTrem 1-/-group.

Conclusion The targeted deletion of endothelial Trem 1 confers protection during septic shock in modulating inflammatory cells mobilization and activation and restoring vasoreactivity. The mechanism by which cellular recruitment is reduced is probably linked to a reduction of chemokines production by endothelial cells. The effect of Trem1 on vascular tone, while impressive, deserves further investigations. The next step should be to design endothelium-specific TREM-1 inhibitors.

\section{Competing interests None.}

\section{S55}

\section{Polymicrobial sepsis inhibits tumor growth in cancer mice}

Hamid Merdji', Jean-François Llitjos ${ }^{2}$, Christophe Rousseau ${ }^{3}$, Nadia Belaidouni ${ }^{4}$, Julie Toubiana ${ }^{4}$, Jean-Paul Mira ${ }^{2}$, Jean-Daniel Chiche ${ }^{2}$, Frédéric Pène ${ }^{2}$

'Departement d'anesthésie-réanimation, CHU de Reims, Reims, France; ${ }^{2}$ Réanimation Médicale, Hôpital Cochin, Paris, France; ${ }^{3}$ Unité 1016, 22 rue méchain, 75014, paris, nstitut National de la Santé et de la Recherche Médicale, Paris, France; ${ }^{4}$ U1016, 22 rue méchain, 75014, Paris, Institut National de la Santé et de la Recherche Médicale, Paris, France

Correspondence: Hamid Merdji - hamidmerdji@hotmail.fr Annals of Intensive Care 2016, 6(Suppl 1):S55

Introduction Severe sepsis remains a frequent and dreaded complication in cancer patients. Beyond the often fatal short-term outcome, the sepsis-induced immune dysfunctions may also directly impact on the prognosis of the underlying malignancy in survivors. We are currently developing a research project in order to investigate the reciprocal relationships between bacterial sepsis and malignant tumor growth. We already reported that sepsis promoted malignant tumor growth when tumor cells were inoculated in post-septic mice. However, whether a previously established tumor might alter the septic response or whether sepsis may impact the growth of previously established tumor remains unclear. To this aim, we set up a relevant double-hit animal model through tumor inoculation followed by polymicrobial sepsis.

Materials and methods We used 8- to 12-week old female C57BL/6J mice. Mice were first subjected to malignant tumor inoculation by either subcutaneous (model of local tumor) or intravenous (metastatic model) injections of the MCA205 fibrosarcoma cell line. Seven and 14 days after tumor inoculation, respectively, corresponding to early and advanced stages of malignancy, mice were subjected to polymicrobial sepsis induced by cecal ligation and puncture (CLP), without any subsequent antibiotic treatment. Controls comprised both noncancer mice subjected to CLP and sham-operated counterparts cancer mice subjected to sham surgery. The main endpoints were survival to polymicrobial sepsis as well as local and metastatic tumor growth. The features of anti-tumoral immune response were compared between CLP- and sham-operated cancer mice and included immune cell infiltration within tumor tissues and draining lymph nodes through flow cytometry.

Results The mortality rate of polymicrobial sepsis was about $50 \%$ in non-cancer mice and was similar in cancer mice, regardless of the inoculation route and the stage of the tumoral model. However, the 24-h bacteremia was dramatically increased in cancer mice while blood cultures remained negative in non-cancer ones. As compared to their sham-operated counterparts, the local tumor growth was strongly inhibited in CLP-operated septic mice. In the same way, polymicrobial sepsis inhibited the pulmonary metastatic spread as assessed by macroscopic lung pathology and lung infiltration onto histological slides. With respect to the distribution of immune cells within tumor tissues, we observed no differences in the proportion of tumor-infiltrating CD45+ hematopoietic cells between sham- and CLP-operated mice. However, tumors from septic mice comprised an increased proportion of monocytic-myeloid-derived suppressor cells (Mo-MDSC). Sepsis also altered the cell distribution in draining lymph nodes, with a decrease in the proportion of $B$ cell lymphocytes while the proportion of monocytes and Mo-MDSC were increased.

Discussion The cellular and molecular mechanisms that account for sepsis-induced tumor inhibition are under investigation. We are currently addressing the respective contributions of host's immune response and pathogens in the control of tumor growth.

Conclusion Sepsis applied to cancer mice inhibits both local and metastatic tumor development. The current results along with our previous experiments suggest a dual impact of sepsis on tumor growth depending on the sequence of the double-hit model.

Supported by a grant from the SRLF (Bourse de Recherche Expérimentale 2013).

\section{Competing interests None.}

\section{References}

1. Fox AC, Robertson CM, Belt B, Clark AT, Chang KC, et al. (2010) Cancer causes increased mortality and is associated with altered apoptosis in murine sepsis. Crit Care Med 38: 886-893.

2. Hotchkiss, R.S., Moldawer, L.L. Parallels between cancer and infectious disease. N Engl J Med. 2014 and 371:380-383.

S56

Telestroke implementation in the biggest French region (12 millions inhabitants): the project ORTIF

Yann L'Hermitte ${ }^{1}$, France Woimant ${ }^{2}$, Catherine Oppenheim ${ }^{3}$, Christophe Couvreur ${ }^{4}$, François Dolveck ${ }^{1}$

${ }^{1}$ Fédération des urgences, hôpital marc jacquet melun, GCSdSISIF, Paris, Melun, France; ${ }^{2}$ Hôpital lariboisière paris, Agence régionnale de Santé, Paris, France; ${ }^{3}$ Neuroradiologie, C.H. Sainte Anne, Paris, France; ${ }^{4}$ GCSdSISIF, Paris, France

Correspondence: Yann L'Hermitte - yannlh@orange.fr

Annals of Intensive Care 2016, 6(Suppl 1):S56

Introduction One of the major issues of the access to urgent health for patients with stroke in acute phase is the implementation of telehealth networks. Looking back on the numerous telestroke deployments already at work, there are grounds for identifying 4 critical issues as being the 4 key success factors for achieving such project as follows: training and skills maintenance of the actors, feedback about the care of stroke, ergonomic perception of telehealth tools, actual practice analysis and any updating and adjustments of protocols in different places. Telehealth platform ORTIF has been implemented since January 2015 in one of the biggest French regions and concerns $12 \mathrm{M}$ inhabitants. It involves 43 hospitals, with a final objective of 100 expert and demanding hospitals, and covers the whole field of emergency neurology (neurosurgery, stroke, exacerbation of chronicle neurological disease). It enables through a digital interface on the web net, to share health and care contextual data, to transfer native radiology image data and to organize medical consultations on vidéo-link between multiple actors from multiple remote locations. Considering the multiplicity of actors involved, the GCSDSISF*in charge of the management of the project had to design a whole industrialization process of the project, including definition of guidelines, implementation, assistance and mentoring.

Materials and methods A scientific committee has defined the elements of the telemedical record and the organizational and medicoeconomic methods. A hotline user was put at disposal as of actors and of the protocols of formalized degraded procedures. A person receiving benefits specialized in ergonomics met the teams implied 
on the pilot phase of the project to define specifications of the evolutions desired on the product and the organizations. Meetings involving the doctors, the persons in charge, the actors of the information systems and the directions of establishment made possible to define the format of the initial and the following trainings. These formations were systematically evaluated by the listeners. A site of formation e-learning was deployed. It centralizes the whole of the documents of formation, the reference frames of operation and an access to videos of demonstration of use of the platform. An analysis of impact of the implementation of these actions is carried out over the first 7 months of operation between the months of January and July 2015 on the 43 establishments deployed in completion date of analysis.

Results Five hundred and fifteen actors could profit from an individual initial training to the tool and the procedures for use. The note of average satisfaction was of 3.43/4.

After 7 months of use, 1271 sessions of telemedicine proceeded: 1043 for neurosurgical expertise, 66 for neurovascular expertise and 162 for expertise of neuroradiology which they are diagnostic or interventional. Increasing number of procedure was on average of $177 \%$ between the months of April and May to the profit especially the neurosurgical expertise which profited from a preliminary experiment of telemedicine. The uses fewer in the field of emergency neurology are explained by the low number of neurovascular expert centers deployed during the first 5 months of the project but show also the importance of an accompaniment of the professionals in these new methods of medical evaluation. The proportion of recourse to the hotline (many incidents/number functional establishments) was of 2.4 at the end of the first month of the deployment and 0.78 in the last month of the analysis objectifying the functional quality of the platform.

Conclusion Telemedicine must be a tool support with the beforehand definite medical organizations to allow an improvement of accessibility the care and thus of their quality. The professionals must perceive these tools like a help and not a constraint. The accompaniment of the structures and their actors is a strong challenge of the success of the deployment of such projects particularly when they imply very many establishments. The industrialization of these processes is the key.

\section{Competing interests None.}

\section{S57}

Free haemoglobin concentration in patients with sickle cell disease referred to the emergency room: Is it useful?

Pierre Mora', Magali Devriese ${ }^{2}$, Sophie Dupeyrat ${ }^{1}$, Patrick Ray ${ }^{3}$, Guillaume Lefevre ${ }^{2}$, Muriel Fartoukh ${ }^{1}$

${ }^{1}$ Réanimation médico-chirurgicale, Hôpital Tenon, Sorbonne Universités, UPMC Université Paris 06, Paris, France: ${ }^{2}$ Service de biochimie et hormonologie, Hôpital Tenon, Sorbonne Universités, UPMC Université Paris 06, Paris, France; ${ }^{3}$ Service d'acceuil des urgences, Hôpital Tenon, Sorbonne Universités, UPMC Université Paris 06, Paris, France

Correspondence: Muriel Fartoukh - muriel.fartoukh@tnn.aphp.fr Annals of Intensive Care 2016, 6(Suppl 1):S57

Introduction Sickle cell disease $(\mathrm{SCD})$ is one of the most frequent inherited haemoglobin disorders caused by a structurally abnormal variant of haemoglobin ( $\mathrm{HbS}$ ). Haemolysis and vaso-occlusive phenomena account for acute, like vaso-occlusive crisis (VOC) and acute chest syndrome (ACS), and chronic complications associated with SCD. Oxidative stress has been shown to play a key role on red blood cell rigidity. Free haemoglobin ( $\mathrm{FHb}$ ) resulting from haemolysis is a powerful oxidizing agent. We aimed to analyse the usefulness of $\mathrm{FHb}$ concentration for stratifying SCD patients initially referred to the Emergency Room (ER), according to their definite site of care.

Patients and methods We conducted a retrospective monocentric observational study in all consecutive SCD patients with VOC or ACS presenting at the ER of a French university teaching hospital in Paris, between January 2013 and December 2014. The considered genotypes included $\mathrm{HbSS}$ and the compound heterozygous SC disease. Patients with no blood collection, or with unavailable or uncompleted medical records, were not included in the study. Plasma FHb concentration was determined by a photometric method. The healthcare pathways from the ED were separated into home discharge, hospitalization in acute wards or in intensive care unit (ICU). Demographics and clinical data are described with median [interquartile range 25-75] and number (percentage). Between groups comparisons used analysis of variance. A $p$ value less than 0.05 was considered statistically significant.

Results Of 506 visits to the ED during the study period, 105 were excluded due to a lack of medical records. Altogether 277 patients presenting at the ED (401 visits) were analysed, and 215 patients (78\%) were hospitalized either in the wards $(n=184)$ or in the ICU $(n=31)$. Most of them $(n=125 ; 58 \%)$ were treated with hydroxyurea. The main diagnosis at the ER guiding the decision to hospitalization was VOC $(n=204 ; 95 \%)$ evolving for 1 [0-1] day. Differences in clinics and laboratory results are detailed in Table 54, according to the site of care of the patients. Increased $\mathrm{FHb}(\geq 30 \mathrm{mg} / \mathrm{dL}$ ) was found in $40 \%$ of the blood samples. Correlation between FHb result and baseline total haemoglobin value or changes in total haemoglobin value were nonsignificant. Median values of $\mathrm{FHb}$ did not differ between discharged home patients $(16 \mathrm{mg} / \mathrm{dL}[4-57])$, patients hospitalized in the wards

Table 54 See text for description

\begin{tabular}{|c|c|c|c|c|}
\hline Variables & $\begin{array}{l}\text { Home dis- } \\
\text { charge, } n=62\end{array}$ & $\begin{array}{l}\text { Wards, } \\
n=184\end{array}$ & $\mathrm{ICU}, n=31$ & $p$ \\
\hline $\begin{array}{l}\text { Age, median } \\
\text { [IQR 25-75] }\end{array}$ & $29[27-36]$ & $28[25-30]$ & $28[25-31]$ & 0.03 \\
\hline \multicolumn{5}{|l|}{$\begin{array}{l}\text { Genotype [n } \\
(\%)]\end{array}$} \\
\hline SS & $55(89)$ & $162(88)$ & $31(100)$ & 0.13 \\
\hline SC & $7(11)$ & $22(12)$ & $0(0)$ & \\
\hline $\begin{array}{l}\text { Past medical } \\
\text { history of } \\
\text { ACS, n (\%) }\end{array}$ & $43(69)$ & 139(76) & $27(87)$ & 0.17 \\
\hline \multicolumn{5}{|c|}{ Baseline biology, median [IQR 25-75] } \\
\hline $\mathrm{Hb}(\mathrm{g} / \mathrm{dL})$ & $8[7.5-9]$ & 8 [7.5-9] & $8[7.5-8.8]$ & 0.5 \\
\hline $\begin{array}{l}\text { Free Hb (mg/ } \\
\text { dL) }\end{array}$ & $5[4-14]$ & $4[4-13]$ & $6[4-17]$ & 0.2 \\
\hline $\begin{array}{l}\text { BilirubinTot } \\
(\mu \mathrm{mol} / \mathrm{L})\end{array}$ & $44[29-71]$ & $42[29-64]$ & $40[32-64]$ & 0.9 \\
\hline ASAT (IU/L) & $41[31-62]$ & $41[31-55]$ & $46[37-54]$ & 0.6 \\
\hline ALAT (IU/L) & 28 [17-39] & 27 [18-41] & 29 [19-40] & 0.7 \\
\hline LDH (IU/L) & $372[287-511]$ & $372[277-463]$ & 443 [333-515] & 0.09 \\
\hline \multicolumn{5}{|c|}{ Biology at the ER, median [IQR 25-75] } \\
\hline $\mathrm{Hb}(\mathrm{g} / \mathrm{dL})$ & $8.7[8-9.5]$ & $8.9[8.1-9.7]$ & $8.5[7.7-9.1]$ & 0.15 \\
\hline $\begin{array}{l}\text { Free Hb (mg/ } \\
\mathrm{dL} \text { ) }\end{array}$ & $16[4-57]$ & $19[8-61]$ & $20[5-77]$ & 0.09 \\
\hline $\begin{array}{c}\text { BilirubinTot } \\
(\mu \mathrm{mol} / \mathrm{L})\end{array}$ & 49 [19-69] & $44[29-66]$ & $44[34-73]$ & 0.5 \\
\hline ASAT (IU/L) & $30[24-42]$ & 29 [19-44] & $36[22-46]$ & 0.9 \\
\hline ALAT (IU/L) & $45[36-63]$ & $50[38-68]$ & $58[46-69]$ & 0.2 \\
\hline LDH (IU/L) & $444[340-562]$ & $474[359-610]$ & $571[463-688]$ & 0.06 \\
\hline
\end{tabular}


(19 mg/dL [8-61]) or patients admitted to the ICU (20 mg/dL [5-77]). Splitting $\mathrm{FHb}$ results in three categories $(<5 ; 5-150$ and $>150 \mathrm{mg} /$ $\mathrm{dL}$ ) was significantly associated with hospitalization (OR 3.5; $95 \% \mathrm{Cl}$ 1.05-11.55).

Discussion A wide overlap in ER FHb results was observed. It might be due to the higher rate of in vitro haemolysis related to the sampling procedure and/or to the efficiency of haemoglobin clearance system. High levels of FHb should, however, be indicative for considering hospitalization.

Conclusion Although surrogate markers of haemolysis rise during VOC and ACS, FHb assay should be interpreted with caution at the ED to evaluate the severity of the acute event in patients with SCD.

\section{Competing interests None.}

\section{S58}

Evaluation of the prescription and use of antibiotics in an emergency department of a general hospital by Gyssens Method Nicolas Roothaer ${ }^{1}$, Amélie Carpentier ${ }^{1}$, Fabien Vaniet ${ }^{1}$, Antoine Maisonneuve ${ }^{1}$ Eric Wiel ${ }^{2}$

${ }^{1} 59$, Hospital Center De Valenciennes, Valenciennes, France; ${ }^{2}$ Nord, Pôle de I'Urgence-SAMU du Nord-CHRU de Lille, Lille, France

Correspondence: Nicolas Roothaer - roothaer.nicolas@gmail.com Annals of Intensive Care 2016, 6(Suppl 1):S58

Introduction Since the 2000s, the development of bacterial resistance has become a major public health problem due to unreasonable use of antibiotics despite the introduction national and international action plans. The establishment of appropriate antibiotic therapy has positive economic impact and on the patient outcome. The aim of this study was to assess antibiotic prescription in an emergency department of a general hospital by Gyssens Method, the median time between prescription and administration, and the factors that influence it.

Materials and methods This was a single-center, descriptive study. The one hundred adult consecutive patients for whom antibiotic treatment was introduced as part of hospitalization were included between the September 22, 2014, and the October 5, 2014.

Results On 100 patients (56 \% of men) with a median age of 62 years, $34 \%$ suffered from respiratory tract infection, $17 \%$ of infections were digestive, $16 \%$ of urinary origin, $14 \%$ from skin, and more rarely stomatological or neurological origin. Penicillin were the most prescribed (approximately $40 \%$ ) with cephalosporin (only in $13 \%$ of cases and in combination in $27 \%$ of cases) and quinolones. $70.7 \%$ of prescriptions were classified Gyssens I (i.e., in accordance with current recommendations). Non-compliance was essentially explained by the existence of a more effective alternative agent ( $16.1 \%$ class IV), inappropriate dosage ( $3 \%$ class Ila), improper route ( $3 \%$ class IIc), and use of any antimicrobial not indicated ( $3 \%$ class V). Most errors in prescription dealt with penicillins. Twenty-nine percentage of the patients had clinical signs of severe sepsis and $4 \%$ of septic shock (based on the surviving sepsis campaign), but nearly half of the patients with clinical signs of severity had an error antibiotic prescription due to underestimation of the severity. Median time of prescription and administration was 3.7 and $3.9 \mathrm{~h}$, respectively (significantly lengthened if the prescriber was a junior). They were not modified on period of guard, when specialized advice was requested or when the patient had clinical signs of severity (severe sepsis or septic shock). Before prescription, only $53 \%$ of patients had bacteriological sample, and $35 \%$ of patients had lactate assay.

Discussion The results of our study reported a relatively satisfactory compliance rate compared to the literature data. But in order to reduce prescription errors and to preserve the existing molecules, it is important to improve practice by developing a local guide or protocols, through working groups in consultation with the emergency's team and the infectiology specialist team of our general hospital. However, a specialized advice did not increase prescription delays of antibiotics.

Conclusion In order to reduce prescription errors and to preserve the existing molecules, efforts are to be undertaken to improve the prescription of antibiotics. This requires better training of medical staff and developing a local guide or protocols.

\section{Competing interests None.}

\section{References}

1. Gyssens IC, Kullberg BJ. Improving the quality of antimicrobial drug use can result in cost containment. Pharm World Sci 1995;17(5):163-7.

2. Goulet H, Daneluzzi V, Dupont C, Heym B, Page B, Almeida K, et al. Évaluation de la qualité des prescriptions d'antibiotiques dans le service d'accueil des urgences d'un CHU en région parisienne. Médecine et Maladies Infectieuses. Jan 2009;39(1):48-54.

S59

High dosages of norepinephrine in trauma patients

Sébastien Canu', Emmanuelle Hammad', Francois Antonini', Marion Poirier ${ }^{1}$, Laurent Zieleskiewicz'ㄹ, Coralie Vigne ${ }^{1}$, Malik Haddam', Julie Alingrin $^{3}$, Marc Leone ${ }^{4}$

${ }^{1}$ 13015, Hospital Nord, Marseille, France; ${ }^{2}$ Bouches du rhône, Hôpital Nord, Chemin des Bourrely, Marseille, France, Marseille, France; ${ }^{3}$ Service de réanimation polyvalente, Hôpital Nord, Chemin des Bourrely, Marseille, France, Marseille, France; ${ }^{4}$ Département d'anesthésie réanimation, Hôpital Nord, Chemin des Bourrely, Marseille, France

Correspondence: Sébastien Canu - seb.canu9@gmail.com

Annals of Intensive Care 2016, 6(Suppl 1):S59

Introduction In trauma patients norepinephrine is the vasopressor recommended after fluid resuscitation to preserve hemodynamic stability. The aim of this study was to determine the dosage of norepinephrine associated with an intensive care unit (ICU) death rate above $90 \%$ and whether high dosage of norepinephrine is associated with increased death rate.

Materials and methods We conducted a retrospective, noninterventional, observational study in a single ICU in an academic hospital in Marseille. From July 2011 to December 2013, severe trauma patients requiring norepinephrine were included. Data were collected at the time of ICU admission, after $24 \mathrm{~h}$, and when the maximal posology of norepinephrine was reached. Mortality was assessed at day 28.

Results Among the 179 trauma patients requiring norepinephrine, the death rate was $46 \%$. The death rate reached $50 \%$ after $24 \mathrm{~h}$ and $80 \%$ after 28 days for the quantile of patients receiving a dosage of norepinephrine above $0.75 \mu \mathrm{g} / \mathrm{kg} / \mathrm{min}$. In our cohort, five independent factors of mortality were identified: age above 40 years (odds ratio 2.32 [95\% confidence interval, 1.20-7.19]; $p=0.02$ ), Glasgow Coma Score below 9 (odds ratio 4.48 [95 \% confidence interval 3.56-24.17]; $p<0.001$ ), hemoglobin level at ICU admission below $9 \mathrm{~g} / \mathrm{dL}$ (odds ratio $1.16[95 \%$ confidence interval 1.01-1.39]; $p=0.04$ ), lactatemia at ICU admission above $3 \mathrm{mmol} / \mathrm{L}$ (odds ratio 1.95 [95 \% confidence interval 1.10-5.70]; $p<0.001$ ), and dosage of norepinephrine above $0.75 \mu \mathrm{g} / \mathrm{kg} / \mathrm{min}$ (odds ratio 2.81 [95\% confidence interval 1.44-7.54]; $p=0.005$ ) (Fig. 45).

Conclusion No dosage of norepinephrine was associated with an ICU mortality rate above $90 \%$ in trauma patients requiring norepinephrine, but a dosage of norepinephrine higher than $0.75 \mu \mathrm{g} / \mathrm{kg} / \mathrm{min}$ was an independent factor of mortality.

Competing interests None. 


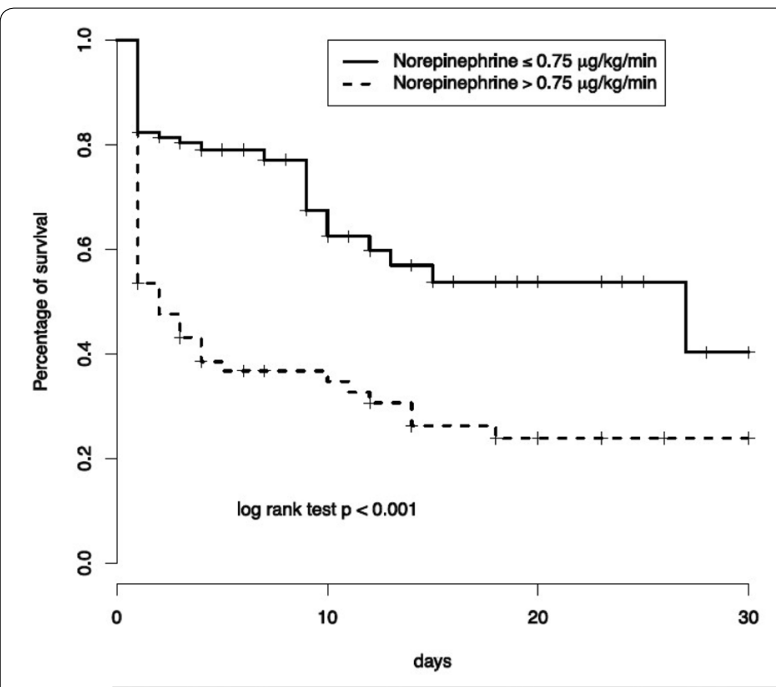

Survival of trauma patients requiring norepinephrine

Fig. 45 See text for description
Results One thousand one hundred and thirty-three pleural procedures, half in respiratory care departments and half in intensive care units $(p=0.30)$, were recorded: $130(12 \%)$ blind pleural biopsies, 625 $(55 \%)$ chest tube insertions, 378 (33\%) thoracocentesis in 185 subjects with antiplatelet therapy and 948 controls. Subjects receiving antiplatelet therapy were more frequently males ( 89 vs $64 \% ; p=0.02$ ) and were older ( $73 \pm 13$ vs $58 \pm 19$ years; $p=0.02$ ).

In the group with antiplatelet therapy, there was a higher prevalence of renal failure (42 vs $23 \% ; p<0.001$ ) and ultrasound guidance (78 vs $68 \% ; p=0.007)$ and a lesser prevalence of thrombopenia with a platelet count below $100 \mathrm{G} / \mathrm{L}$ (7.5 vs $2 \% ; p=0.006)$ in comparison with controls. There was no difference between groups concerning vitamin $\mathrm{K}$ antagonist and heparin therapies and operator's experience and qualification.

Thirteeen bleeding events occurred: 9 hemothorax, 2 hemoptysis and 2 hematomas. The incidence of bleeding events was higher in the group of patients receiving antiplatelet therapy compared to the control subjects [2.7 vs $0.8 \% ; p=0.03$; OR $3.395 \% \mathrm{Cl}(1.05-10.09)$ ].

Conclusion In this study, antiplatelet therapy provoked a 3 times increase in pleural procedures related bleeding risk.

Thus, their suspension should be considered when the arterial thrombotic risk is minor such as in primary prevention.

However, the bleeding risk related to antiplatelet therapy remains low supporting their continuation during pleural procedures when the arterial thrombosis risk is high and associated with severe outcomes such as in coronary patients.

\section{Competing interests None.}

\section{S61}

Interest of the association fibrinogen/tranexamic acid in the management of severe postpartum hemorrhage

Khalil Tarmiz' , Fehmi Ferhi², Hosni Khouadja², Affra Brahim², Mohamed

Amine Bouslama ${ }^{2}$, Khaled Benjazia ${ }^{2}$

${ }^{1}$ Sousse centre, Chu Farhat Hached, Sousse, Tunisia: ${ }^{2}$ Anesthesia

and Intensive Care, Chu Farhat Hached, Sousse, Tunisia

Correspondence: Khalii Tarmiz - khalil.tarmiz@gmail.com

Annals of Intensive Care 2016, 6(Suppl 1):S61

Introduction The association of fibrinogen $3 \mathrm{~g}$ and tranexamic acid $2 \mathrm{~g}$ was introduced into the management protocol for our maternity postpartum hemorrhage from 2012. Before that date, the use of one or both of these products was anecdotal. The objective of this study is to evaluate the effectiveness of the association (fibrinogen/exacyl) in the curative treatment of postpartum hemorrhage.

Patients and methods Our study is retrospective, descriptive and monocentric, on parturients presented with severe bleeding in the postpartum period from 2009 to 2014.

Inclusion criteria were as follows: any woman in labor who presented in the aftermath of a vaginal delivery or cesarean section, deemed severe genital bleeding (bleeding clinically abundant, significant hemodynamic changes, need for medical or surgical hemostasis). Exclusion criteria were parturients under anticoagulant treatment, parturients transferred from other health structures.

We have collected the records of all women in labor who experienced severe PPH, and we divided them into two groups: group including records of years 2009-2010-2011 and a second group including records of years 2012-2013-2014. The collection has been using a pre-established grid. The main elements of judgment were: the incidence of hemostasis hysterectomy, the average number of blood units transfused, the rate of non-transfused patients, mortality and postoperative complications (particularly acute renal failure). We also establish the transfusion ratio PFC/CG.

Results One hundred and sixty-six cases were studied (58 of 20092011: group 1 and 108 from 2012 to 2014: group 2). The two groups were comparable in terms of general and obstetric data. The association was used in $18.9 \%$ of patients in group 1 and in $71.3 \%$ group 2 . The rate of hemostasis hysterectomy decreased from $51.7 \%$ (group 1) to $19.4 \%$ (group 2). The rate of non-transfused patients increased from $6.9 \%$ (group 1) to $27.8 \%$ (group 2). The average number of transfused packed red blood cells decreased from 5.3 (group 1) to 4.2 (group 2).
Materials and methods A prospective multicentric study was conducted in 18 respiratory care departments and 11 medical intensive care units. The occurrence of bleeding events was considered within the $24 \mathrm{~h}$ following pleural procedure performance. 
There was no significant change with respect to transfusion ratio (fresh frozen plasma/red cell: PFC/CG), 1/0.79 (group 1) and 1/0.73 (group 2).

Conclusion Within the limits of this study, the association fibrinogen/ (AT) appears effective in reducing the incidence of hemostasis hysterectomy and in the reduction of transfusion requirements in the management of severe PPH.

\section{Competing interests None.}

\section{S62}

HELLP syndrome in ICU: epidemiological, clinical and prognostic study about 112 cases

Fatma Kaaniche Medhioub ${ }^{1}$, Kais Rgieg ${ }^{2}$, Olfa Turki ${ }^{2}$, Mariem Smaoui ${ }^{2}$ Anis Chaari ${ }^{3}$, Benhamida Chokri ${ }^{3}$, Mabrouk Bahloul ${ }^{3}$, Bouaziz Mounir ${ }^{3}$ ${ }^{1}$ Faculté de médecine de Sfax, Sfax, Tunisia; ${ }^{2}$ Réanimation polyvalente, CHU Habib Bourguiba, Sfax, Tunisia; ${ }^{3}$ Réanimation polyvalente, Faculté de médecine de Sfax, Sfax, Tunisia

Correspondence: Fatma Kaaniche Medhioub - fatma_kaaniche@yahoo. fr

Annals of Intensive Care 2016, 6(Suppl 1):S62

Introduction HELLP syndrome (hemolysis, elevated liver enzyme, low platelets) is a gravidarum microangiopathy at the end of second and third trimester of pregnancy [1]. The aim of our work is to study the epidemiological, clinical and prognostic characteristics in obstetric patients admitted in ICU for HELLP syndrome.

Patients and methods Our study is retrospective and includes all cases of HELLP syndrome collected in the ICU of the university hospital of Sfax over a period of 15 years (1998-2012).

Results Among the 131,631 births occurred during the study period, 506 patients had complications requiring ICU transfer of which 112 presented HELLP syndrome (22.1\%). Pregnancy has been followed in 77 women (68.7\%). The average age of patients was $31 \pm 6.2$ years. The average parity was $2.1 \pm 1.5$ with a primiparity rates at $51.4 \%$. Gestational age of discovery HELLP syndrome was $33 \pm 3.9$ weeks. Gravidarum hypertension was present in 107 patients $(95.5 \%)$. The average rate of hemoglobin was $10 \pm 2.3 \mathrm{~g} / \mathrm{dl}$, that of SGOT $203 \pm 259 \mathrm{IU} / \mathrm{l}$, of SGPT $127 \pm 137 \mathrm{IU} / \mathrm{l}$, of platelets $72,942 \pm 42,398 \mathrm{E} / \mathrm{mm} 3$, of bilirubin $54.2 \pm 59.2 \mu \mathrm{mol} / \mathrm{l}$ and of LDH $746 \pm 210 \mathrm{IU} / \mathrm{l}$. The research of schisocytes was positive in 66 patients $(58.9 \%)$. The HELLP syndrome has been associated with acute renal failure in 54 cases $(48.2 \%)$, to eclampsia in 24 cases (21.4\%), to disseminated intravascular coagulation in 23 cases (20.5\%), to retro placental hematoma in 16 cases $(18.1 \%)$, to acute pulmonary edema in 11 cases $(9.8 \%)$ and to state of shock in 11 cases $(9.8 \%)$. Childbirth was via a vaginal approach in 19 cases (16.9\%) and cesarean section in 93 cases (83.1\%). Mechanical ventilation was required in 90 cases (80.3\%). Antihypertensive therapy was initiated in 101 patients $(90 \%)$. Forty-six patients (41\%) received a transfusion. Hemodialysis was carried in 2 cases (1.8\%). The average length of stay in the ICU was $3.1 \pm 4.4$ days. The final outcome of our patients was marked by the death of eight of them (7.1\%). Mortality was significantly higher $(p<0.05)$ in patients who developed systemic (acute renal failure, disseminated intravascular coagulation, state of shock, acute pulmonary edema) and/or obstetric complications (eclampsia, retro placental hematoma).

Conclusion HELLP syndrome is a rare complication of late pregnancy, but it is a cause of severe maternal morbidity. Correctly supported in $\mathrm{ICU}$, the prognosis is better.

\section{Competing interests None.}

\section{Reference}

1. Pottecher T, Luton D, Zupan V, Collet M. Réanimation des formes graves de prééclampsie. Conférence d'expert communes SFAR/CNGOF/SFMP/ SFNN; 2009.

\section{S63}

\section{Neurological complications in eclampsia}

Chaigar Mohammed Cheikh', I. Moussaid ', Kamal Belkadi', E. El Alami' , S. El Youssoufi', S. Salmi ${ }^{1}$

${ }^{1}$ Anesthesie Reanimation, CHU Ibn Rochd Casa, Casablanca, Morocco

Correspondence: Chaigar Mohammed Cheikh - chaigarmed@gmail. com

Annals of Intensive Care 2016, 6(Suppl 1):S63

Introduction Eclampsia is a major cause of maternal and fetal morbidity and mortality, particularly in the developing world (1). These maternal deaths are essentially caused by cerebral lesions, but their precipitating factors are unknown. The aim of the study was to determine the prevalence and incidence of neurological complications of eclampsia and determine their independent risk factors.

Patients and methods A retrospective study was conducted in the obstetrical and gynecological anesthesiology and reanimation unit of the Ibn Rochd University Hospital from January 2000 to December 2013 and included all women with eclampsia. All patients with focal neurological deficit, persisting coma or convulsions after treatment underwent neuroimagery. Their epidemiological, clinical, biological, radiological, evolutive, therapeutic and obstetrical data were compared with patients without cerebral lesions through a descriptive, univariate and multivariate analysis.

Results One thousand thirty patients were included, with a global prevalence of $0.81 \%$. Sixty-five pourcents were less than 30 years of age, and gestational age was above 32 weeks in $76.8 \%$. Systolic blood pressure (SBP) was above $160 \mathrm{mmHg}$ in $74.5 \%$, diastolic blood pressure (DBP) above $110 \mathrm{mmHg}$ in $40.1 \%$ and mean arterial pressure was above $150 \mathrm{mmHg}$ in $7.9 \%$. One hundred ninety-one (14.9\%) had a neurological complication, a posterior reversible encephalopathy syndrome (PRES) in $88 \%$, and hemorrhagic stroke in $12 \%$ of patients. They were no prepartum or postpartum predominance in the occurrence of cerebral lesions, except for hemorrhagic lesions which occurred significantly in prepartum $(p=0.041)$. Neurological complications were significantly associated with SBP $\geq 180 \mathrm{mmHg}$ (unadjusted OR 1.37; $95 \% \mathrm{Cl} 1.00-1.88$ ) or DBP $\geq 110 \mathrm{mmHg}$ (unadjusted OR 1.49; $95 \% \mathrm{Cl} 1.09-2.03$ ), but these variables were not significant in the logistic regression model. HELLP syndrome in the complete (adjusted OR 3.37; $95 \% \mathrm{Cl} 1.74-6.56$ ) and partial form (AOR 2.26; $95 \% \mathrm{Cl} 1.21-4.23)$ was independently associated with neurological complications ( $p<0.001$ and $p=0.010$, respectively). Intracerebral hemorrhage was significantly associated with pulmonary edema only (unadjusted OR 9.59; $95 \% \mathrm{Cl} 2.60-35.36$ ). Unused magnesium sulfate (AOR 2.46; $95 \% \mathrm{Cl} 1.22-4.99$ ) and mechanical ventilation (AOR $5.16 ; 95 \% \mathrm{Cl} 2.90-9.15)$ were independently associated with cerebral lesions ( $p=0.012$ and $p<0.001$ ). Finally, these lesions were independently associated with poor maternal (AOR 5.18; $95 \% \mathrm{Cl} 1.77-15.10$ ) and fetal (AOR 3.15; $95 \% \mathrm{Cl} 1.16-8.57$ ) outcome ( $p=0.003$ and $p=0.025$ )

Conclusion Our study suggest that HELLP syndrome was the main risk factor for neurological complication of eclampsia, which are to link to endothelial dysfunction. These lesions were not independently associated with the elevation of BP, which contradicts recent works. Further studies are necessary to introduce new therapeutics to control the endothelial dysfunction process and improve the prognosis.

\section{Competing interests None.}

\section{Reference}

1. Abalos E. et al. Global and regional estimates of preeclampsia and eclampsia: a systematic review. European Journal of Obstetrics, gynecology and reproductive biology 2013.

S64

Brain water contain is dependent on plasmatic ammonemia levels in hyperammoniemic encephalopathy, a quantitative CT scan study

Nicolas Weiss ${ }^{1}$, Fanny Mochel ${ }^{2}$, Damien Galanaud ${ }^{3}$, Louis Puybasset ${ }^{4}$, Dominique Thabut ${ }^{5}$, Brain Liver Pitié-Salpêtrière Study Group (BLIPS), Paris, France

${ }^{1}$ Unité de réanimation neurologique, Hôpital Pitié-Salpêtrière, Paris, France; ${ }^{2}$ Unité neurométabolique, Pitié-Salpêtrière Hospital, Paris, France; ${ }^{3}$ Neuroradiologie, Pitié-Salpêtrière Hospital, Paris, France; ${ }^{4}$ Réanimation neurochirurgicale, Pitié-Salpêtrière Hospital, Paris, France; ${ }^{5}$ Unité de soins intensifs d'hépatogastroentérologie, Hôpital Pitié-Salpêtrière, Paris, France 
Correspondence: Nicolas Weiss - nicolas.weiss@aphp.fr Annals of Intensive Care 2016, 6(Suppl 1):S64

Introduction Hepatic encephalopathy (HE) has been mainly attributed to elevated levels of ammonemia associated with inflammatory response due to modification of intestinal microbiota and increased bacterial translocation. Brain edema is frequently encountered in acute liver failure and has been shown, at a lower extent, in cirrhosis. Astrocytes display cytotoxic edema due to the presence of glutamine, an osmotic agent produced by the transformation of ammonia by glutamine synthetase in the cytoplasm. This condition is not associated with increased brain volume since blood-brain barrier permeability is not modified. At the opposite, vasogenic edema is associated with an increased brain volume and a decreased specific gravity (SG) secondary to an increase in blood-brain barrier permeability. Brain volume, weight and SG, that can be measured by CT scan, can be used to estimate brain water contain and determine the type of edema. The physiopathological implication of hyperammonemia in the apparition of vasogenic brain edema is nevertheless still debated in hyperammoniemic encephalopathy. Objective: To determine the influence of ammonemia levels and their modification in the variation of brain water contain and the apparition of vasogenic edema.

Patients and methods We retrospectively included, from January 2013 to June 2015, all the patients admitted for HE in ICU that displayed ammonemia levels higher than $150 \mu \mathrm{mol} / \mathrm{L}$ and that underwent a brain CT scan. Quantitative analysis of CT was performed using the Brainview software (Institut National des Télécommunications). This software enables analyses of DICOM images and the determination of the weight, the volume and the SG of the different brain regions (hemispheres, brainstem, cerebellum, intraventricular cerebrospinal fluid).

Results Five patients were included, and 10 CT scan evaluated. Median ammonemia was $184 \mu \mathrm{mol} / \mathrm{L}$ [118-317]. Brain weights, volumes and SG were $1356 \mathrm{~g}$ [1163-1397], $1313 \mathrm{~mL}$ [1131-1355] and 1,0296 g/mL $[1,0280-1,0327]$, respectively. The volume and the SG of both the brain and the hemispheres were correlated to ammonemia levels $(p<0.05)$. The increase in volume and the decrease in SG suggested the presence of vasogenic edema. Variation in ammonemia levels was associated with a modification in volumes and SG $(p<0.05)$. The different brain regions were, however, not equally affected. Thus, brainstem and cerebellum volumes were not correlated with ammonemia levels, and it variation was not associated with modification in volumes in these regions.

Conclusion Hyperammonemia is associated with vasogenic brain edema in hyperammoniemic encephalopathy. The vasogenic edema is mainly located in cerebral hemispheres.

\section{Competing interests None.}

\section{S65}

Sodium phenylbutyrate administration to avoid neurological worsening in hepatic encephalopathy patients in ICU

Simona Tripon ${ }^{1}$, Marion Lodey ${ }^{2}$, Elsa Guiller ${ }^{2}$, Maxime Mallet ${ }^{3}$, Marika Rudler ${ }^{1}$, Dominique Thabut ${ }^{1}$, Nicolas Weiss ${ }^{4}$, Brain Liver Pitié-Salpêtrière Study Group (BLIPS), Paris, France

'Unité de soins intensifs d'hépatogastroentérologie, Hôpital Pitié-Salpêtrière, Paris, France; ${ }^{2}$ Pharmacology, Pitié-Salpêtrière Hospital, Paris, France; ${ }^{3}$ Unité de Soins Intensifs. Service d'Hépato-gastro-entérologie du Pr Poynard, CHU Pitié Salpétrière, Paris, France; ${ }^{4}$ Unité de réanimation neurologique, Hôpital Pitié-Salpêtrière, Paris, France

Correspondence: Nicolas Weiss - nicolas.weiss@aphp.fr

Annals of Intensive Care 2016, 6(Suppl 1):S65

Introduction Hepatic encephalopathy (HE) is a major clinical problem in patients with cirrhosis, which influences short- and long-term prognosis, impairs quality of life and increases costs. Clinical manifestations range from mild neurocognitive disturbances with normal clinical examination to coma. About one-third of patient with overt HE will display altered consciousness needing ICU admission and sometimes mechanical ventilation. Rapid correction of HE could avoid the need for mechanical ventilation in the most severe patients. Even if flumazenil has been proposed to reverse $\mathrm{HE}$, conflicting results exist and its use is not recommended. Recently, glycerol phenylbutyrate or sodium phenylbutyrate, that lowers ammonia by providing an alternate pathway to urea for waste nitrogen excretion, has shown to be effective in preventing the occurrence of HE in RCT.

To determine whether sodium phenylbutyrate was effective in severe HE patients to decrease ammonia and avoid neurological worsening.

Patients and methods We prospectively included all the cirrhotic patients that presented with an ammonia $>100 \mu \mathrm{mol} / \mathrm{L}$, presented $\mathrm{HE}$ and that presented no conter-indication to the treatment. Glasgow coma scale, FOUR score, West-Haven, HESA, CHESS scales and critical flicker frequency were noted in each patient in order to assess HE before and after treatment.

Neurological improvement was defined as an improvement in either Glasgow coma scale, FOUR score, West-Haven score or critical flicker frequency. Side effects were noted. Data are given as median and interquartile range.

Results Between August 2014 and September 2015, 18 patients (median age: 59 [45-68] years, male $83 \%$; aetiology of cirrhosis: alcoholic $56 \%$, viral $6 \%$, other $38 \%$; Child-Pugh 11 [8-12], MELD score 16 [14-24]) were included. Nine patients $(50 \%)$ were admitted for $\mathrm{HE}$, 4 (22\%) for gastrointestinal bleeding, 3 (17\%) for acute liver failure and 3 for miscellaneous causes. At admission, ammonia level was 151 [114-200] $\mu \mathrm{mol} / \mathrm{L}$, Glasgow coma scale 14 [13-15], FOUR score 16 [1316], West-Haven score 2 [2-3], CHESS 2 [0-6], HESA 2 [1-3] and critical flicker frequency at $33 \mathrm{~Hz}$ [30-38]. At day 1, ammonia decrease was 61 [27-83], at day 251 [10-79] and at day 343 [26-83]. Eighty-three percentage patients presented a decrease in ammonia under treatment at day $1,89 \%$ at day 2 and $94 \%$ at day 3 . Neurological improvement occured in 10 patients ( $56 \%$ ) at day 1, in $77 \%$ at day 2 and in $83 \%$ at day 3. At 6 months, 6 patients ( $33 \%)$ had died, 2 (12\%) were lost of follow-up and 10 (55\%) were still alive. No side effects were observed under treatment.

Conclusion Sodium phenylbutyrate seems effective in both reducing rapidly ammonia levels but also improve neurological status in $\mathrm{HE}$ patients with ammonia $>100 \mu \mathrm{mol} / \mathrm{L}$.

\section{Competing interests None.}

\section{S66}

Disruption of posteromedial large-scale neural communication predicts recovery from coma

Stein Silva', Béatrice Riu-Poulenc ${ }^{2}$, Jean Ruiz ${ }^{3}$, Corine Vuillaume²,

Francesco De Pasquale ${ }^{4}$, Jean Francois Demonet ${ }^{5}$

${ }^{1}$ Critical Care Unit/inserm u825, University Teaching Hospital of Purpan,

Toulouse, France; ${ }^{2}$ Réanimation, Centre Hospitalier Universitaire Toulouse,

Toulouse, France; ${ }^{3}$ Réanimation, Institut Universitaire du Cancer de

Toulouse-Oncopole, Toulouse, France; ${ }^{4}$ Neuroscience and Neuroimag-

ing, Santa Lucia, Roma, Italy; ${ }^{5}$ Applied Neurosciences, Leeanards Memory

Centera, Lausanne, Switzerland

Correspondence: Stein Silva - silvastein@me.com

Annals of Intensive Care 2016, 6(Suppl 1):S66

Introduction Recent neuroimaging studies point to the critical involvement of hubs in neural networks localized in precuneus ( $\mathrm{PrCu})$ and posterior cingulate cortex (PCC) in self-conscious processing (1). We hypothesize that the major consciousness deficit observed in coma is due to the breakdown of long-range neuronal communication supported by $\mathrm{PrCu}$ and PCC and that prognosis depends on specific connectivity pattern in these networks.

Patients and methods We compared 31 prospectively recruited comatose patients suffering from severe brain injury (Glasgow Coma Scale $<8 ; 16$ traumatic and 15 anoxic cases) to 14 age-matched healthy participants. Standardized clinical assessment and fMRI were performed on average $4 \pm 2$ days after withdrawal of sedation. Analysis of resting-state fMRI connectivity involved hypothesis-driven, ROI-based strategy. We assessed patient's outcome after 3 months using the Coma Recovery Scale-Revised (CRS-R). 

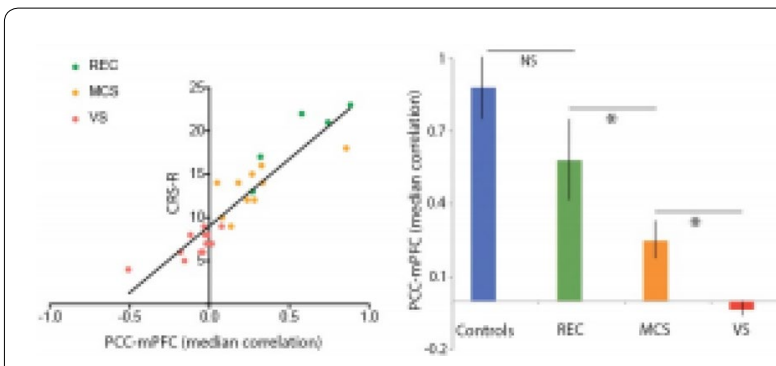

Fig. 46 The predictive role of PCC-mPFC coupling measured during coma state and neurological outcome. Functional connectivity strength between PCC-mPFC assessed at the scan time (right panel) was significantly different between comatose patients who recovered consciousness (REC) and those who evolved toward a minimally conscious state (MCS) or an vegetative state (VS) 3 month after the brain injury. PCC posterior cingulate cortex, MPFC medial prefrontal cortex

Results Comatose patients showed a significant disruption of functional connectivity of brain areas spontaneously synchronized with PCC, globally notwithstanding etiology. The functional connectivity strength between PCC and MPFC was significantly different between comatose patients who went on to recover and those who eventually scored an unfavorable outcome 3 month after brain injury (Kruskal-Wallis test, $p<0.001$; linear regression between CRS-R and PCC-mPFC activity coupling at rest, Spearman's $\rho=0.93$, $p<0.003$; see Fig. 46).

\section{Discussion}

Conclusion To summarize, the reorganization of PCC-centered, spontaneously synchronized large-scale networks, seems implicated in the loss of external and internal self-centered awareness observed during coma, largely independent of its etiology. The level of functional connectivity between PCC and MPFC appears to be related to patient neurological outcome. Future work should further explore brain intrinsic network dysfunctions in larger patient's cohort, aiming to improve patient's diagnosis, early prognostication, and enabling the development of innovative network-based personalized treatments (2).

\section{Competing interests None.}

\section{References}

1. Silva S, Alacoque X, Fourcade O, et al. Wakefulness and loss of awareness: brain and brainstem interaction in the vegetative state. Neurology 2010;74:313-320.

2. Giacino JT, Fins JJ, Laureys S, Schiff ND. Disorders of consciousness after acquired brain injury: the state of the science. Nature reviews Neurology 2014;10:99-114.

\section{7}

Loss of sleep elements on continuous EEG monitoring is associated with mortality in patients with cardiogenic shock requiring veno-arterial extra-corporeal membrane oxygenation

\section{(ECMO)}

Fabrice Sinnah ${ }^{1}$, Marie Amelie Dalloz², Eric Magalhaes ${ }^{1}$, Mathilde Neuville', Roland Smonig' ${ }^{1}$, Aguila Radjou', Bruno Mourvillier', Lila Bouadma' ', Jean-François Timsit' ${ }^{1}$, Marie Pia D'ortho ${ }^{2}$, Anny Rouvel-Tallec², Romain Sonneville

'Service de réanimation médicale et infectieuse, Hôpital Bichat-Claude Bernard, Paris, France; '² Département de Physiologie, Hôpital Bichat Claude Bernard, Paris, France

Correspondence: Fabrice Sinnah - fabrice.sinnah@gmail.com Annals of Intensive Care 2016, 6(Suppl 1):S67

Introduction Veno-arterial extra-corporeal membrane oxygenation (ECMO) is increasingly used to treat refractory shock or cardiac arrest. Use of ECMO support is associated with significant neurological complications, which may significantly impact on prognosis. Neurological status of adult patients on ECMO is hard to assess, as most of these patients are deeply sedated at the initial phase of resuscitation. Moreover, transportation for brain imaging is not easily feasible and brain MRI is contraindicated in those patients. The usefulness of electroencephalography (EEG) to predict neurological complications or outcome is poorly studied in these patients. We aimed to assess the prognostic contribution of continuous EEG monitoring (cEEG) recordings over intermittent EEG (iEEG) on outcome.

Materials and methods We performed a monocentric, prospective, observational study on adult patients with cardiogenic shock requiring veno-arterial ECMO. To be included in the study, patients had to meet the following criteria: (1) age $>18$ years; (2) initiation of venoarterial ECMO support for $<24 \mathrm{~h}$; (3) EEG recordings performed on the first day following ECMO cannulation. For each included patient, a systematic neurological assessment was conducted on the day of EEG recording, including measurements of RASS score, FOUR score, brainstem reflexes and motor responses assessments. EEG recordings consisted of iEEG, followed by CEEG monitoring overnight. An independent neurophysiologist interpreted results of iEEG and CEEG, including background abnormalities (presence of a discontinuous

\section{Table 55 See text for description}

\begin{tabular}{|c|c|c|c|c|}
\hline Variables & All $(n=22)$ & $\begin{array}{l}\text { Survivors } \\
(n=11)\end{array}$ & $\begin{array}{l}\text { Non-survivors } \\
(n=11)\end{array}$ & $p$ \\
\hline \multicolumn{5}{|l|}{$\begin{array}{l}\text { Clinical charac- } \\
\text { teristics }\end{array}$} \\
\hline SAPS2 & $55(41-78)$ & $51(37-75)$ & $60(43-82)$ & 0.36 \\
\hline SOFA & $10(8-13)$ & $9(5-12)$ & $11(10-13)$ & 0.18 \\
\hline Cardiac arrest & $4(18)$ & $2(18)$ & $2(18)$ & $>0.99$ \\
\hline RASS score & $-5(-5 ;-3)$ & $-3(-5 ;-0.5)$ & $-5(-5 ;-4)$ & 0.08 \\
\hline FOUR score & $4(0-8)$ & $6(1-9)$ & $1(0-6)$ & 0.51 \\
\hline \multicolumn{5}{|l|}{ iEEG recordings } \\
\hline Background: & $14(64)$ & $4(45)$ & $10(90)$ & 0.03 \\
\hline $\begin{array}{l}\text { Discontinuous } \\
\text { pattern }\end{array}$ & $5(23)$ & $1(9)$ & $4(36)$ & 0.12 \\
\hline $\begin{array}{l}\text { Non-reactive } \\
\text { pattern }\end{array}$ & $9(41)$ & $3(27)$ & $6(55)$ & 0.19 \\
\hline $\begin{array}{l}\text { Focal abnor- } \\
\text { malities }\end{array}$ & $5(23)$ & $2(18)$ & $3(27)$ & 0.61 \\
\hline $\begin{array}{l}\text { Paroxysmal } \\
\text { alterations }\end{array}$ & $2(9)$ & $1(9)$ & $1(9)$ & $>0.99$ \\
\hline $\begin{array}{l}\text { Non convulsive } \\
\text { seizures }\end{array}$ & $0(0)$ & $0(0)$ & $0(0)$ & $>0.99$ \\
\hline \multicolumn{5}{|l|}{$\begin{array}{l}\text { Additional find- } \\
\text { ings on CEEG }\end{array}$} \\
\hline $\begin{array}{l}\text { Background } \\
\text { pattern } \\
\text { changes }\end{array}$ & $1(5)$ & $1(9)$ & $0(0)$ & 0.23 \\
\hline $\begin{array}{l}\text { New focal } \\
\text { abnormalities }\end{array}$ & $2(9)$ & $1(9)$ & $1(9)$ & $>0.99$ \\
\hline $\begin{array}{l}\text { New paroxys- } \\
\text { mal altera- } \\
\text { tions }\end{array}$ & $3(14)$ & $1(9)$ & $2(18)$ & 0.53 \\
\hline $\begin{array}{l}\text { New periodic } \\
\text { discharges }\end{array}$ & $1(5)$ & $0(0)$ & $1(9)$ & 0.23 \\
\hline $\begin{array}{l}\text { Absence of } \\
\text { sleep ele- } \\
\text { ments }\end{array}$ & $14(64)$ & $4(45)$ & $10(90)$ & 0.03 \\
\hline
\end{tabular}


pattern and/or a non-reactive pattern), focal abnormalities, paroxysmal alterations, presence or absence of non-convulsive seizures, presence or absence of sleep elements (K-complexes, vertex sharp-waves and sleep spindles). The primary outcome was mortality 14 days following ECMO cannulation. Results are presented as number (percentages) or median (interquartile range).

Results Twenty-two patients (age: 60 (54-67) years, SAPS2: 55 (41-78), SOFA score: $10(8-13)$ ) were included. Eleven (50\%) patients were post-cardiac surgery, and $11(50 \%)$ were medical patients (including $4(18 \%)$ with refractory cardiac arrest). Five $(23 \%)$ patients had a history of stroke. Charlson score prior hospitalization was $1(0-3)$. All the patients underwent mechanical ventilation, catecholamines levels were $0.4(0-6.4) \mathrm{mg} / \mathrm{h}$, and lactate levels was $2.3(1.5-3) \mathrm{mmol} / \mathrm{l}$. Day 14 mortality was $50 \%(n=11)$. Characteristics of patients on the day of EEG recordings are presented in Table 55. Twenty (91\%) patients had ongoing sedation during EEG recordings. None of the clinical features were associated with outcome. On iEEG, only background abnormalities (discontinuous or unreactive background) were associated with outcome. Additional findings on continuous EEG recordings revealed that loss of EEG sleep elements was associated with outcome. Conclusion In adult patients with cardiogenic shock requiring venoarterial ECMO, discontinuous or unreactive iEEG background is associated with early mortality. Moreover, loss of EEG sleep elements on continuous EEG provides additional information on prognosis. These findings from this preliminary study need to be confirmed.

\section{Competing interests None.}

\section{S68}

Protective effects of high-density lipoprotein therapy in a mouse model of ischemic stroke hemorrhagic transformation due to acute hyperglycemia

David Couret ${ }^{1}$, Aurélie Catan², Brice Nativel ${ }^{2}$, Cynthia Planesse ${ }^{2}$, Nicolas Diotel $^{2}$, Olivier Meilhac ${ }^{2}$

${ }^{1}$ Neurocritical care unit, CHU de la Réunion, Groupe Hospitalier Sud Réunion, Saint-Pierre, Réunion, France; ${ }^{2}$ Inserm, UMR 1188 Diabète athérothrombose Thérapies Réunion Océan Indien (DéTROI), Sainte Clotilde, Réunion, France

Correspondence: David Couret - david.couret@chu-reunion.fr Annals of Intensive Care 2016, 6(Suppl 1):S68

Introduction Hyperglycemia during acute ischemic stroke is associated with a high risk of hemorrhagic transformation and a poor clinical outcome. High-density lipoprotein (HDL) levels are inversely associated with stroke incidence, suggesting a protective effect. Using a mouse model of ischemic stroke in hyperglycemic conditions, we tested the hypothesis that HDLs may limit hemorrhagic transformation and mortality when administered intra-arterially at the reperfusion phase.

Materials and methods After a 3-h middle cerebral artery occlusion using a 7-0 silicon-coated monofilament, C57BL6 mice were randomly treated intra-arterially with human plasma purified $\mathrm{HDLs}$ versus saline immediately after reperfusion. Four groups were used with ten animals per group: Hyperglycemic mice with HDL treatment (HG-HDL), normoglycemic mice with HDL treatment (NG-HDL), hyperglycemic mice with saline treatment (HG-S) and normoglycemic mice with saline treatment (NG-S). We used D-glucose intraperitoneal injection $(2.2 \mathrm{~g} / \mathrm{kg})$ to induce acute hyperglycemia. Blood glucose levels were followed up six times during experimental period. The effects of HDLs were assessed blindly $24 \mathrm{~h}$ later by evaluating the neurological deficit and mortality and by measuring both infarct volume and hemorrhagic transformation. Then brain blood content was estimated by hemoglobin ELISA.

Results In both hyperglycemic groups, the blood glucose levels were significantly increased from 0.5 to $3 \mathrm{~h}$ after injection of D-glucose. Hyperglycemic mice with saline treatment had severe hemorrhagic transformation (parenchymal hematoma) relative to the hyperglycemic mice with $\mathrm{HDL}$ treatment (100 vs $70 \% p=0.01)$. Hemorrhagic transformation score were: 16.6 vs $11.5 p<0.001(0=$ no hemorrhagic transformation to $20=$ parenchymal hematoma $>30 \%$ in all five brain slices). These results were confirmed by ELISA quantification of hemoglobin (HG-S: $1.911 \mathrm{ng} / \mathrm{ml}$ vs HG-HDL: $0.945 \mathrm{ng} / \mathrm{ml} p<0.001$ ). Ischemia volume was not statistically different between hyperglycemic mice with or without HDLs: $62.3 \pm 11$ vs $72.0 \pm 11.0 \mathrm{~mm} 3$. HDL treatment failed to limit the mortality (HG-S: $52 \%$ vs HG-HDL: $35 \% p=0.3$ ).

Conclusion High-density lipoprotein injection limited hyperglycemiainduced hemorrhagic transformation in an original hyperglycemic mouse model of middle cerebral artery occlusion. These results support the vasculoprotective action of HDLs and their potential use at the acute phase of ischemic stroke in hyperglycemic patients.

\section{Competing interests None.}

\section{S69}

Glucocorticoids as a "rescue therapy" for delayed intracranial hypertension after acute brain injury: a retrospective study Fabian Roy-Gash ${ }^{1}$, Stephane Welschbillig ${ }^{2}$, Elodie Lang ${ }^{2}$, Christophe Lebard $^{2}$, Nicolas Engrand ${ }^{3}$

${ }^{1}$ Neuro Intensive Care, Fondation Ophtalmologique Adolphe de Rothschild, Paris, France; ${ }^{2}$ Intensive Care Unit, Fondation Ophtalmologique Adolphe de Rothschild, Paris, France; ${ }^{3}$ Département d'anesthésie-réanimation, Fondation Ophtalmologique Adolphe de Rothschild, Paris, France Correspondence: Fabian Roy-Gash - fabian.roygash@yahoo.fr Annals of Intensive Care 2016, 6(Suppl 1):S69

Introduction Acute brain injury in neurological critical care is commonly associated with an early rise of intracranial pressure (ICP). In some patients, ICP rises sharply during a secondary stage of hypertension between 7 and 15 days. This delayed intracranial hypertension (ICHP) often evolves into a dramatic refractory edema, unresponsive to heavy therapeutic measures (deep sedation, hypertonic saline solutions, hypothermia, and decompression surgery) eventually leading to death. Many studies have shown that glucocorticoids (GC) therapy at the early stage of acute brain injury is associated with a significant rise in death rate or disability (1). However, GC therapy has not yet been investigated for treating this delayed, refractory ICHP after acute brain injury. We hypothesized that GC therapy is associated with a reduction of ICP, better cerebral perfusion pressure and survival improvement.

Materials and methods Objective: To characterize effects of GC therapy on ICP in patients with delayed refractory intracranial hypertension after acute brain injury.

Methods We retrospectively included in the Medical Surgical ICU of a French hospital from December 2011 to July 2015, all patients showing refractory intracranial hypertension defined by a rebound of ICP $(>20 \mathrm{mmHg}$ ) more than 8 days after acute brain injury and who had received GC. Patients were excluded if GC therapy was started prior to the admission in ICU for any other reason. ICP measurements were recorded every $4 \mathrm{~h}$ for each patient beginning 3 days before the GC therapy and ending 5 days after the GC therapy. Then, we studied the evolution of the median and the maximal value of ICP daily during those 8 days using pairwise comparisons (Friedman test).

Results Eighteen patients met inclusion criteria. Seven were excluded, due to GC being administered prior to the admission in order to treat preexistent neoplasic or parasitic diseases. Five were admitted in the ICU for intracerebral hemorrhage, four for severe traumatic brain injury, one for acute ischemic stroke, and one for subarachnoid hemorrhage. The median and maximal ICP at the worst stage of ICHT which prompted the rescue GC therapy were $26[\mathrm{IQR}=28] \mathrm{mmHg}$ and 37 $[\mathrm{IQR}=26] \mathrm{mmHg}$, respectively. The GC therapy was associated with significant reduction in ICP median values $(p<0.05)$ and daily maximal values $(p<0.0001)$ (Fig. 47). Two days after GC therapy, ICHT was controlled (ICP under $20 \mathrm{mmHg}$ ) in most patients, median ICP $=15$ $[\mathrm{IQR}=7]$. One week later, the median ICP was under $5[\mathrm{IQR}=10]$, and all but one patients were discharged from ICU. The rescue GC therapy consisted in methylprednisolone for all patients but with a significant variability of the initial posology (median $=2 \mathrm{mg} / \mathrm{kg} /$ day $[\mathrm{IQR}=1]$ ), the length of administration (median $=7$ days [IQR $=4]$ ). Bacterial pulmonary infections occurred in five patients after starting GC therapy. Bacterial urinary tract infections occurred in four patients. Elevated serum glucose levels requiring intravenous insulin therapy were observed in four patients.

Conclusion GC therapy could be a suitable rescue option for delayed, refractory ICHT. However, there are currently insufficient data 


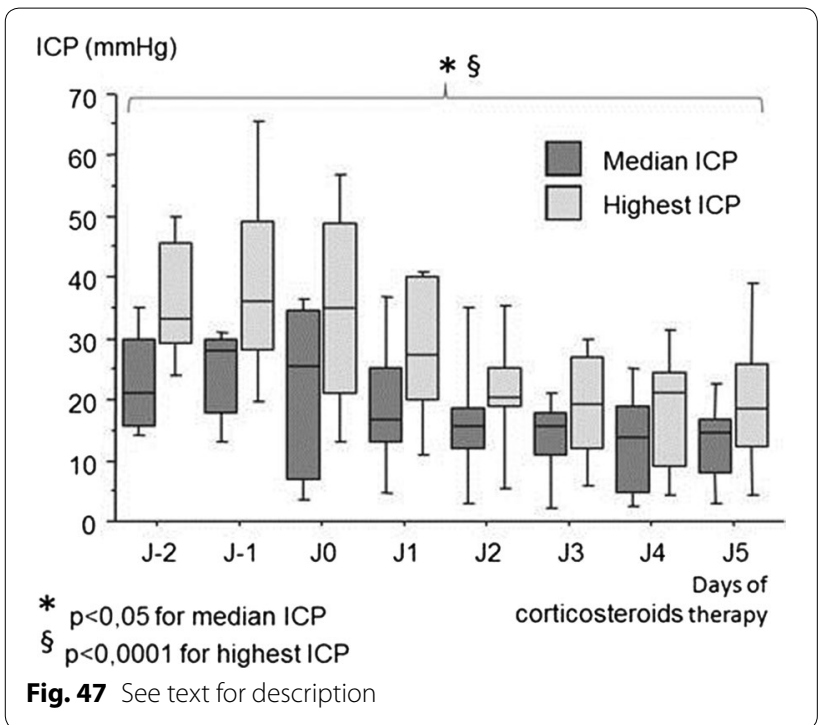

concerning the functional outcome after the discharge of ICU. Because of their side effects, they should be used with caution and further studies should be done to evaluate the benefits of GC therapy for the delayed intracranial hypertension.

\section{Competing interests None.}

\section{Reference}

1. Final results of MRC CRASH, a randomised placebo-controlled trial of intravenous corticosteroid in adults with head injury-outcomes at 6 months. Edwards P, Arango M, Balica L, Cottingham R, El-Sayed H, Farrell B, Fernandes J, Gogichaisvili T, Golden N, Hartzenberg B, Husain M, Ulloa Ml, Jerbi Z, Khamis H, Komolafe E, Laloë V, Lomas G, Ludwig S, Mazairac G, Muñoz Sanchéz Mde L, Nasi L, Olldashi F, Plunkett P, Roberts I, Sandercock P, Shakur H, Soler C, Stocker R, Svoboda P, Trenkler S, Venkataramana NK, Wasserberg J, Yates D, Yutthakasemsunt S; CRASH trial collaborators. Lancet. 2005 Jun 4-10;365(9475):1957-9.

\section{S70}

\section{Organ donation: early talks (ET) to relatives before occurence} of brain death

Anne-Gaelle Si Larbi', Virginie Gaudin 2, France Bonnin², Oceane Sultan,, Alexis Soummer ${ }^{1}$, Grégoire Trebbia', Cecile Hoc ${ }^{3}$, Emmanuel Mathieu ${ }^{3}$, Bertrand Lapergue ${ }^{4}$, Frederic Bourdain ${ }^{4}$, Charles Cerf ${ }^{1}$

${ }^{1}$ Réanimation, Hospital Foch, Suresnes, France; ${ }^{2}$ Coordination hospitalière, Hospital Foch, Suresnes, France; ${ }^{3}$ Sau, Hospital Foch, Suresnes, France; ${ }^{4}$ Neurologie, Hospital Foch, Suresnes, France

Correspondence: Anne-Gaelle Si Larbi - ag.si-larbi@hopital-foch.org Annals of Intensive Care 2016, 6(Suppl 1):S70

Introduction The aim of the early talks (ET) to the relatives is to introduce organ donation (OD) before the occurence of brain death (BD) and to search intent of the potential donor not to be a donor. This paper aims at describing the results of ET method compared to the standard practice that introduces OD once BD has occured.

Patients and methods In 2012, ET procedures have been employed among intensive care unit (ICU), emergency room (ER) and stroke unit. The ET method is used when patients are diagnosed with unrecoverable brain damage, possibly leading to $\mathrm{BD}$, with close relatives made aware of the situation and have agreed to withdraw invasive treatments. The interview highlights several points among which are patients' current state, the believed evolution of that state, OD in case of $\mathrm{BD}$, its approval or disapproval from close relatives as well as a time frame of $72 \mathrm{~h}$ after which withdrawal of treatments will be done if $\mathrm{BD}$ doesn't occur. All the collected information from potential donors is registered and helps to provide a comparison between the ET and the standard BD practice.
Table 56 Comparisons between the two groups

\begin{tabular}{llll}
\hline & $\begin{array}{l}\text { Early talks } \\
\boldsymbol{n = 9 4}\end{array}$ & $\begin{array}{l}\text { Interview after BD } \\
\boldsymbol{n = 7 1}\end{array}$ & $\boldsymbol{p}$ \\
\hline Occurence of BD $[n(\%)]$ & $42(45 \%)$ & $71(100 \%)$ & \\
Retrieval of organs $[n(\%)]$ & $31(33 \%)$ & $29(41 \%)$ & 0.3 \\
Disapproval $[n(\%)]$ & $36(38 \%)$ & $36(51 \%)$ & 0.1 \\
Sex = male $[n(\%)]$ & $46(49 \%)$ & $40(56 \%)$ & 0.3 \\
Age (years) & $69[54-82]$ & $54[44-64]$ & $<0.001$ \\
Organ retrieved/patient $(n)$ & $3[2.5-4]$ & $3[3-4]$ & 0.4 \\
$\begin{array}{l}\text { Length of stay in ICU réa, } \\
\quad \text { days }\end{array}$ & $2.7[1.3-4.2]$ & $1.6[1-2,4]$ & 0.01 \\
$\begin{array}{c}\text { Time between admission } \\
\text { and interview, days }\end{array}$ & $0.9[0.2-2.2]$ & $0.7[0.4-2]$ & 0.7 \\
\hline
\end{tabular}

Time frames are expressed in median [interquartile range].

Results Between January 1, 2012, and September 15, 2015, 165 interviews regarding organ donation and the intent of the potential donor to be or not to be a donor have been conducted. Ninety-four (57\%) of them were ET, and the remaining 71 (43\%) were done after occurence of $\mathrm{BD}$. At the time of the $\mathrm{ET}, 69$ (73\%) patients were using invasive ventilation, one being in the ER and 68 in the ICU. Among the 25 non-ventilated patients, 17 were in the stroke unit, 5 in the ER and 3 in the ICU. With the ET method, disapproval was met for 26 (38\%) invasive-ventilated patients and $10(40 \%)$ non-ventilated patients $(p=0.3)$.

Among the disapproval, 7 patients became brain dead before withdrawal treatments, 3 of which occurred quite rapidly (less than $1 \mathrm{~h}$ after the interview) and 4 between a 16 to $24 \mathrm{~h}$ time frame in the decision period requested by the relatives.

In case of organ donation rejection, a median time of $2 \mathrm{~h}$ [1.2-9.3] was found between the ET and the actual withdrawal of treatments. When organ donation has not been disapproved and patients became brain dead, the time span between ET and BD was $25 \mathrm{~h} \mathrm{[16-40].} \mathrm{In} \mathrm{only}$ one case, the patients have reached over the $72 \mathrm{~h}$ time frame ( $85 \mathrm{~h}$ ).

With organ donation not rejected and brain death not happened, the time span between the ET and the actual withdrawal of treatments was $55 \mathrm{~h}$ [48-72]. In this case, the patients could become a potential donor after circulatory death (Maastricht III). Twenty-three patients were in this case, and 9 were less than 60 years old (between the ages of 40-56), 5 of which cardiac arrest happened within $3 \mathrm{~h}$ after withdrawal of treatments (Table 56).

Discussion The ET method has become a common practice when the decision has been made to withdraw intensive treatments for patients who can potentially become brain dead. It helps providing clearer information to relatives and the disapproval rate has not risen with the ET methods. It allows an earlier withdrawal of treatments if OD was disapproved while pursuing care for a potential donor, thus increasing the chances to provide viable organ. The outcome of using ET seems overall positive for both the close relatives and medical staff. However, further study is needed to evaluate the relatives' satisfaction.

Conclusion ET has become a common practice in our unit, and it is not a associated with increasing of OD denial and has helped increasing the number of donors.

Competing interests None.

\section{S71}

Remifentanil target-controlled infusion for procedures generating pain or discomfort in the ICU: feasibility, safety and tolerance evaluation

Margot Caron ${ }^{1}$, Antoine Parrot ${ }^{1}$, Maxens Decavèle ${ }^{1}$, Clarisse Blayau', Jean-Pierre Fulgencio ${ }^{1}$, Vincent Labbe ${ }^{1}$, Michel Djibre' ${ }^{1}$ El Mahdi Hafiani ${ }^{1}$, Christophe Quesnel ${ }^{1}$, Muriel Fartoukh' ${ }^{1}$, Tai Pham ${ }^{1}$

${ }^{1}$ Réanimation médico-chirurgicale, Hôpital Tenon, Paris, France

Correspondence: Margot Caron - margotcaron@hotmail.com Annals of Intensive Care 2016, 6(Suppl 1):S71 
Introduction Remifentanil target-controlled infusion (TCl), widely used in the operating room owing to its reliability, is poorly assessed in the ICU, while several procedures may induce pain and discomfort. Only one series of 14 patients focused on Remifentanil-TCl use for performing fiberoptic bronchoscopy (FOB) in the ICU (1).

We aimed to assess Remifentanil-TCl feasibility, safety and tolerance during several procedures performed at bedside in consecutive awake ICU patients and generating pain or discomfort.

Patients and methods This was an observational prospective study of Remifentanil-TCI use during ICU procedures generating pain or discomfort, including fiberoptic bronchoscopy (FOB), digestive endoscopy (DE), transoesophageal echocardiography (TOEC) and chest tube insertion (CT).

Primary end point was the complete achievement of the procedure, without any need for general anesthesia or additional analgesia. Secondary aims were objective (respiratory and hemodynamics parameters) and subjective (pain, dyspnea and comfort assessment) tolerance and safety measurements during and after the procedure. Quantitative and qualitative variables are presented as mean (standard deviation), median [interquartile range] or number (percentage) as appropriate.

Results From November 2014 to September 2015, 79 patients (63\% men; age $61 \pm 18$ years; SAPS II score $34 \pm 16$ ) were included. The studied procedures were FOB (77 \%), CT (9\%), DE (9\%) and TOEC $(6 \%)$. The procedures were performed in patients receiving nasal oxygen $(80 \%)$, high-flow humidified oxygen (11\%), noninvasive ventilation (2\%) or invasive ventilation (5\%). Total infusion duration was 22 min [15-29], maximal TCl concentration goal was $4 \mathrm{ng} / \mathrm{mL}$ [3-5], and total drug dose infused was $252 \mu \mathrm{g}$ [184-362].

The procedures were performed completely without any additional drugs in 73 patients $(92 \%)$, whereas propofol was added in $6(8 \%)$. Six patients presented side effects including bradypnea $(n=5)$ and hypotension (systolic arterial pressure of $70 \mathrm{mmHg}, n=1$ ) that occured during the procedure and were completely reversible with $\mathrm{TCl}$ goal decrease. Thus, the procedures were performed entirely without additional anesthesia or complication in 67 patients $(87 \%)$.

Confort was assessed in 52 patients (66\%): 43 patients (8\%) stated that they felt comfortable and experienced a bearable pain or no pain at all, 9 patients $(17 \%)$ had a moderate pain, and none felt severe pain. Among the 49 patients who were asked, 43 ( $88 \%$ ) answered that they would agree to have the same procedure in the same conditions.

Conclusion Our findings suggest that TCl of Remifentanil may be useful and safe for performing procedures generating pain or discomfort in the ICU, with acceptable patient's comfort and tolerance, even in those with respiratory failure. The study completion should strengthen these preliminary results.

\section{Competing interests None.}

\section{Reference}

1. Chalumeau-Lemoine et al. Intensive Care Med (2013) 39:53-58.

\section{S72}

Minimizing dead-volume infusion devices leads to a reduction in hypoglycaemia in surgery patients admitted to a perioperative intensive care unit

Stephanie Genay ${ }^{1}$, Bertrand Décaudin', Sabine Ethgen², Pascal Odou', Gilles Lebuffe ${ }^{2}$

${ }^{1}$ Pharmacy Institute, C.H.R.U. Lille, Lille, France; ${ }^{2}$ Anaesthesia and resuscitation department, C.H.R.U. Lille, Lille, France

Correspondence: Stephanie Genay - stephanie.genay@univ-lille2.fr Annals of Intensive Care 2016, 6(Suppl 1):S72

Introduction Hyperglycaemia and insulin resistance are commonly encountered in major surgery patients. Several multicenter randomized studies have failed to provide guidelines on intensive insulin therapy because of the high risk of provoking hypoglycaemia. Moreover, no study has so far assessed the impact of the insulin infusion device on hypoglycaemia occurrence. The objective here was to compare insulin infusion modalities so as to evaluate their impact on hypoglycaemia.

Patients and methods This was an open prospective randomized clinical trial conducted in a perioperative intensive care unit composed of 15 beds. It received the ethics committee approval. All patients hospitalized between September 2012 and December 2013 were included. They received $1 \mathrm{IU} / \mathrm{mL}$ human insulin over $48 \mathrm{~h}$ infused via one of the two studied assembly lines. The glycaemic objective was between 140 and $180 \mathrm{mg} / \mathrm{dL}$. The standard infusion line consisted in insulin administration through a six-stopcock manifold connected to the distal line of a multilumen central venous catheter by $150 \mathrm{~cm}$ tubing. The optimized infusion line administered insulin infusion through a multilumen device consisting of eight accesses connected to nine separate lumens united in a 150$\mathrm{cm}$ tube. Capillary glycaemia was measured by a glucometer every $3 \mathrm{~h}$ and by an interstitial glucose sensor every $5 \mathrm{~min}$. Moderate hypoglycaemia was defined as a capillary glucose level between 54 and $70 \mathrm{mg} / \mathrm{dL}$ and severe hypoglycaemia by a level below $54 \mathrm{mg} /$ $\mathrm{dL}$. Time spent in hypoglycaemia for $1000 \mathrm{~h}$ of insulin therapy was calculated from glucose sensor data. Results are presented as mean \pm standard deviation and were compared by a Mann-Whitney $U$ test $(p<0.05)$.

Results One hundred and seventy-two patients were included. One hundred and fifty-seven patient records were workable. Twentyeight patients presented hypoglycaemia events: $0.18 \pm 0.52$ versus $0.05 \pm 0.22$ severe events $(p=0.087)$ and $0.02 \pm 0.16$ versus $0.04 \pm 0.19$ moderate events $(p=0.655)$. Time spent in hypoglycaemia was significantly different from one insulin infusion system to the other $(9.21 \pm 24.44 \mathrm{~min}$ with the standard line versus $3.91 \pm 13.95 \mathrm{~min}$ with the optimized system).

Discussion This study shows that the dead-volume measured from the point where drug and carrier streams meet to the patient's blood affects hypoglycaemia occurrence in major surgery patients. Given the severity of undiagnosed hypoglycaemia, close monitoring of blood glucose and the use of low volume infusion sets need to be considered for patients most at risk of glycaemic dysregulation.

Conclusion The use of low dead-volume infusion devices means patients undergoing intensive insulin therapy are less susceptible to hypoglycaemia.

\section{Competing interests None.}

\section{S73}

Patient-important outcomes in randomized controlled trials in critically ill patients

Stéphane Gaudry' ${ }^{1}$ Jonathan Messika', Jean-Damien Ricard ${ }^{1}$, Blandine Pasquet $^{2}$, Sylvie Guillo ${ }^{2}$, Emeline Dubief ${ }^{1}$, Didier Dreyfuss ${ }^{1}$, Florence Tubach $^{2}$

${ }^{1}$ Service de réanimation médico-chirurgicale, CHU Louis Mourier, Colombes, Colombes, France; ${ }^{2}$ Département d'epidémiologie et recherche clinique, Unité de Recherche Clinique Paris Nord, Paris, France Correspondence: Stéphane Gaudry - stephanegaudry@gmail.com Annals of Intensive Care 2016, 6(Suppl 1):S73

Introduction Intensivists' clinical decision-making should pursue two main goals: to decrease patient mortality and improve mean and longterm outcomes in survivors (termed patient-important outcomes). We sought to investigate how published randomized controlled trials (RCTs) explore these patient-important outcomes in critically ill patients.

Materials and methods We performed a systematic review of published RCTs involving critically ill patients. Literature searches were conducted in MEDLINE (Pubmed ${ }^{\circledR}$ ) over a one-year period (2013). Data extraction was conducted by 2 independent senior intensivists on standardized, pretested extract forms. We assessed patient and study characteristics. All outcomes (primary and secondary) were reviewed and classified as follows: 1/Patient-important outcomes (mortality and patient-centered outcomes after discharge from intensive care (ICU), such as quality of life and functional status); $2 /$ clinical outcomes in ICU and hospital (such as organ failure, healthcare-associated outcomes or adverse outcomes); 3/biological/physiological/radiological outcomes; 4/care provider decision-related outcomes (such as mechanical ventilation duration, length of stay or antibiotics exposure); 5/careperformance outcomes (such as care procedure quality or noise/light exposure); $6 /$ other outcomes. 


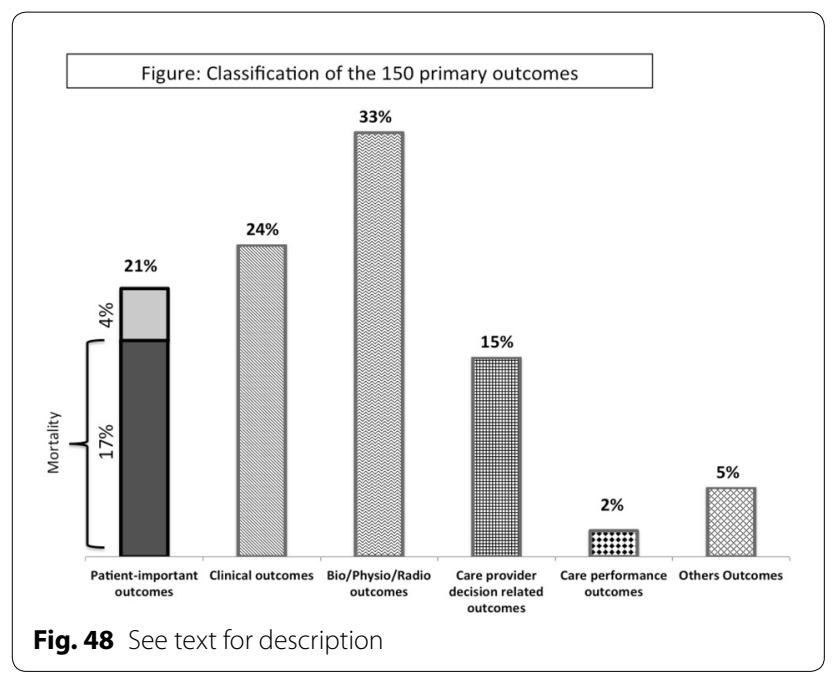

Results Of the 716 articles retrieved, we identified and assessed 150 RCTs performed on ICU patients. These totaled 141,170 patients (age: 61 [54-66] years, male sex: $62 \%)$. Most common topics of these trials were mechanical ventilation ( $31 \%)$, sepsis $(20 \%)$ and nutrition (14\%). Most frequent types of intervention were therapeutic strategy (40\%), drug (31\%) and device (15\%) evaluation.

One hundred and two (68\%) RCTs assessed the primary outcome after a fixed time-point of 7 [2-28] days (from randomization) and only 15 (10\%) assessed the primary outcome beyond 30 days. The other RCTs assessed the primary outcome at ICU discharge $(n=33,22 \%)$ or hospital discharge $(n=10,7 \%)$. Five did not specify the time from randomization to primary outcome assessment.

Among the 150 primary outcomes (Fig. 48), 31 (21\%) were patientimportant outcomes (mortality $=25(17 \%)$; functional disability $=4$ (3\%); quality of life = $2(1 \%)) ; 36(24 \%)$ were clinical outcomes in ICU and hospital; 49 (33\%) were biological/physiological/radiological outcomes; 23 (15\%) were care provider decision-related outcomes; 3 (2\%) were care-performance outcomes and $8(5 \%)$ others.

Among 794 secondary outcomes, 169 (21\%) were patient-important outcomes (mortality $=117(15 \%)$, quality of life $=22(3 \%)$, functional disability $=19(2 \%)$; neurological/cognitive performance $=7(1 \%)$; handicap $=2(0.2 \%)$; post-traumatic stress $=1(0.1 \%)$; patient satisfaction $=1(0.1 \%)) ; 218(27 \%)$ were clinical outcomes in ICU and hospital; 168 (21\%) were biological/physiological/radiological outcomes; 196 (25\%) were care provider decision-related outcomes; 12 (2\%) were care-performance outcomes and 31 (4\%) others.

Conclusion Patient-important outcomes represented only $21 \%$ of primary and secondary outcomes of RCTs performed in critically ill patients and published in 2013. The majority of them were mortality. Moreover, trial follow-up was short (only $10 \%$ of trials assessed primary outcomes beyond 30 days from randomization), precluding mean or long-term assessment of patient-centered outcomes.

Future RCTs should include patient-important outcomes as major outcome criteria since they guide, as gold standard of quantitative research, intensivists' clinical practice and patient care.

\section{Competing interests None.}

\section{S74}

Epidemiology of platelets transfusion in critically ill patients

Cécile Aubron ${ }^{1}$, Andrew Flint ${ }^{2}$, Michael Bailey ${ }^{2}$, Rinaldo Bellomo ${ }^{2}$, David Pilcher $^{3}$, Allen Cheng ${ }^{4}$, Colin Hegarty ${ }^{5}$, Anthony Martinelli ${ }^{5}$, Benjamin Howden ${ }^{6}$, Michael Reade ${ }^{7}$, Zoe Mcquilten ${ }^{8}$

${ }^{1}$ Réanimation médicale, Chru De Brest, Brest, France; ${ }^{2}$ Depm, monash university, ANZIC-RC, Melbourne, Australia; ${ }^{3}$ Intensive Care Unit, Alfred Hospital, Melbourne, Australia; ${ }^{4}$ Depm, Monash University, Melbourne, Australia; ${ }^{5}$ The Transfusion Service, Austin Hospital, Heidelberg, Australia; IInfectious Diseases Department, Austin Hospital, Heidelberg, Australia; ${ }^{7}$ Australian Defense Force and Burns, Trauma and Critical Care Research
Center, University of Queensland, 4029, Australia, Brisbane, Australia ${ }^{8}$ Depm, Monash University, Transfusion Research Unit, Melbourne, Australia

Correspondence: Cécile Aubron - cecile.aubron@monash.edu Annals of Intensive Care 2016, 6(Suppl 1):S74

Introduction Platelets (PLTs) transfusion is given to reduce the risk of bleeding or to be part of haemostatic treatment in bleeding patients. Nonetheless, some studies have reported an independent association between PLTs transfusion and adverse events including mortality and infections. The aim of this study is to describe PLTs transfusion in a large cohort of critically ill patients and to determine whether PLTs administration is associated with patient outcomes.

Patients and methods This retrospective study using prospectively maintained databases was conducted in the intensive care unit (ICU) of two tertiary hospitals. Adults admitted to ICU between 2008 and 2014 were included. Characteristics of patients who received PLTs were compared to those of patients who did not receive PLTs. The association between PLTs administration and hospital mortality or infection including bacteraemia and/or bacteriuria was modelled using multiple logistic regression. A Cox regression on outcomes using transfusion variables as time-varying variables was also performed.

Results Of the 18965 patients included, 2250 (14.5\%) received PLTs in ICU. Patients who received PLTs were younger, more often male, were more severe at ICU admission (mean APACHE III score $65 \pm 29$ versus $52 \pm 25, p<0.01$ ) and had more often comorbidities (31 versus $19 \%, p<0.01$ ) than patients without PLTs transfusion. They were more often hospitalised for cardiovascular (31 versus $27 \%, p<0.001$ ), gastrointestinal ( 26 versus $18 \%, p<0.001$ ), haematological diseases (4.3 versus $0.3 \%, p<0.001$ ) and trauma ( 17 versus $14 \%, p<0.001$ ). Invasive mechanical ventilation (87 versus $57 \%, p<0.001$ ) and renal replacement therapy ( 20 versus $4 \%, p<0.001$ ) were more frequently administered in patients receiving PLTs than in patients without PLTs. The median number of PLTs administered per patient was 1 unit (Inter quartile range $[I Q R] 1-3$ ), and this figure rose up to 4 units (IQR 2-8) in haematological patients and to 2 (IQR 1-5) in trauma patients. Patients receiving PLTs had a higher rate of infection (12.7 versus $4.7 \%, p<0.01)$ and a higher mortality $(20.1$ versus $10.2 \%, p<0.01)$ than patients who did not receive PLTs is ICU. After adjusting for confounders that included illness severity, administration of other blood components, admission diagnosis, centre and year, PLTs transfusion was independently associated with in-hospital mortality (adjusted odds ratio [OR] 1.35, $95 \%$ confidence interval [Cl] 1.15-1. 57) and infection (adjusted OR 1.62, $95 \% \mathrm{Cl} 1.30-2.01$ ).

Conclusion This large observational study shows that $12 \%$ of patients receive PLTs while in ICU. After adjustment for confounders including illness severity and administration of blood components, PLT transfusion was independently associated with mortality and infection occurrence in ICU. Prospective research is warranted to define ICU patient groups who benefit from PLT transfusion.

\section{Competing interests None.}

\section{Reference}

1. Kumar A, Mhaskar R, Grossman BJ, Kaufman RM, Tobian AA, Kleinman S, Gernsheimer T, Tinmouth AT, Djulbegovic B; AABB Platelet Transfusion Guidelines Panel. Platelets transfusion a systemic review of the clinical evidence. Transfusion 2015 May;55(5):1116-27.

\section{S75}

Outcome of selected nonagenarians admitted in ICUs: a multicenter study from the Outcomerea research group Maîté Garrouste-Orgeas', Stephane Ruckly ${ }^{2}$, Francois Philippart ${ }^{3}$, Anne Sylvie Dumenil ${ }^{4}$, Dany Goldgran Toledano ${ }^{5}$, Samir Jamali ${ }^{6}$, Carole Schwebel $^{7}$, Lilia Soufir ${ }^{3}$, Christophe Clec' $h^{8}{ }^{\prime}$, Michaël Darmon ${ }^{9}$, Bernard Allaouchiche ${ }^{10}$, Laurent Argaud ${ }^{11}$, Bruno Verdière ${ }^{12}$, Muriel Fartoukh ${ }^{13}$, Mélanie Cherin ${ }^{3}$, Elie Azoulay ${ }^{14}$, Jean-François Timsit ${ }^{15}$

${ }^{1}$ Réanimation, Fondation Hopital Saint Joseph, Paris, France; ${ }^{2}$ Statistical Department, Outcomerea Group, Paris, France; ${ }^{3}$ Réanimation polyvalente, Groupe Hospitalier Paris-Saint-Joseph, Paris, France; ${ }^{4}$ Réanimation chirurgicale, Hôpital Antoine Béclère, Clamart, France; ${ }^{5}$ Réanimation poly- 
valente, Centre Hospitalier Général, Montfermeil, France; ${ }^{6}$ Réanimation médicale, Centre Hospitalier Sud Essonne, Dourdan, France; ${ }^{7}$ Réanimation médicale, C.H.U. Grenoble, Grenoble, France; ${ }^{8}$ Réanimation medico-chirurgicale, Hopital Avicenne, Bobigny, France; ${ }^{9}$ Réanimation Médicale, CHU Saint-Etienne - Hôpital Nord, Saint-Étienne, France; ${ }^{10}$ Réanimation, Hôpital civil de Lyon, Lyon, France; ${ }^{11}$ Réanimation Médicale, Hospices Civils de Lyon - Groupement Hospitalier Edouard Herriot, Lyon, France ${ }^{12}$ Réanimation, Centre Hospitalier Général de Saint-Denis, Saint-Denis, France; ${ }^{13}$ Réanimation médico-chirurgicale, Hôpital Tenon, Paris, France; ${ }^{14}$ Réanimation médicale, Hôpital Saint-Louis, Paris, France; ${ }^{15}$ Réanimation médicale et infectieuse, Hôpital Bichat-Claude Bernard, Paris, France Correspondence: Maïté Garrouste-Orgeas - mgarrouste@hpsj.fr Annals of Intensive Care 2016, 6(Suppl 1):S75

Introduction The demands for intensive care unit admission for elderly patients are increasing throughout the world due to the increase in life expectancy and the progress of medicine. Outcome of elderly patients was most often reported for octogenarians. The mean age in studies was roughly 84 years old. The ICU and hospital mortality were 12 and $25 \%$, respectively, for scheduled surgery (1). For the same endpoints, it was 38-64 and $45-55 \%$ for medical and unscheduled surgery (2). The primary aim of this study was to precise outcome of youngest patients (80-89 years old) vs oldest ( $>90$ years old). The secondary aim was to evaluate outcome according to supporting care in wards units compared to geriatric units after ICU discharge for the nonagenarians.

Patients and methods A multicenter (14 ICUs) observational study (1997-2013) was performed in 14 ICUs belonging to the Outcomerea study group. We extracted from the prospective database Rhea

\section{Table 57 Characteristics of patients $80-89$ and $>90$ years} old

\begin{tabular}{|c|c|c|c|}
\hline Variables & 80-89 years old & 90 years old & $p$ value \\
\hline \multicolumn{4}{|l|}{ SAPS, age } \\
\hline Median (IQR) & $27(19-39)$ & $28(19-41)$ & 0.92 \\
\hline \multicolumn{4}{|l|}{ Age, years } \\
\hline Median (IQR) & $83.4(81.4-85.7)$ & $92(90.8-93.7)$ & $<0.0001$ \\
\hline \multicolumn{4}{|l|}{ Type of patients } \\
\hline Scheduled & $12(6.9)$ & $10(5.7)$ & 0.04 \\
\hline Unscheduled & $42(24)$ & $54(31)$ & \\
\hline Medical & $121(69.1)$ & $110(63.2)$ & \\
\hline \multicolumn{4}{|l|}{ NEMS day 1 and day 2} \\
\hline Median (IQR) & $26(17-37)$ & $24(14-32)$ & 0.02 \\
\hline \multicolumn{4}{|l|}{ IMV day 1 or 2} \\
\hline$N(\%)$ & $87(49.4)$ & $76(43.2)$ & 0.23 \\
\hline \multicolumn{4}{|l|}{ NIV day 1 or 2} \\
\hline$N(\%)$ & $37(21)$ & $24(13.6)$ & 0.06 \\
\hline \multicolumn{4}{|l|}{$\begin{array}{l}\text { Vasopressive treatment } \\
\text { day } 1-2\end{array}$} \\
\hline$N(\%)$ & $56(31.8)$ & $49(27.8)$ & 0.29 \\
\hline \multicolumn{4}{|l|}{ RRT day 1 or 2} \\
\hline$N(\%)$ & $14(8)$ & $5(2.8)$ & 0.03 \\
\hline \multicolumn{4}{|l|}{ ICU mortality } \\
\hline$N(\%)$ & $36(20.5)$ & $44(25)$ & 0.23 \\
\hline \multicolumn{4}{|l|}{ Hospital mortality } \\
\hline$N(\%)$ & $64(36.4)$ & $70(39.8)$ & 0.46 \\
\hline \multicolumn{4}{|l|}{ Limitations of treatment } \\
\hline Withdrawing & $4(2.3)$ & $3(1.7)$ & 0.70 \\
\hline Withholding & $3(1.7)$ & $6(3.4)$ & 0.31 \\
\hline Anticipated withholding & $18(10.2)$ & $16(9.1)$ & 0.70 \\
\hline
\end{tabular}

patient's characteristics, intensity of treatment, decisions of limitations of treatment (withholding, withdrawing and anticipated withholding), nurse workload (NEMS: Nine Equivalents of nursing Manpower Score), patient status at ICU and hospital discharge. Matching criteria were severity of illness (SAPS without age), center and year in the study.

Results The database included 2416 patients aged more than 80 years old hospitalized in 14 centers during the study period. Among them, $176(7.2 \%)$ were more than 90 years old and represented the cases patients. We matched 176 patients aged 90 years old with patients between 80 and 89 years old hospitalized in the same center and the same severity of illness at admission (Table 57 ).

10/14 (71 \%) of centers had a geriatric unit. Factors associated with transfer in a geriatric unit for nonagenarians vs other wards were severity of illness (SAPS-age, median [IQR] 35 [20.5-41] vs 23 [17-32], $p=0.03)$, use of dobutamine $(6 / 16,37.5 \%$ vs $13 / 114,11.4 \%)$, length of stay before ICU admission (0 [0-0.5] vs $1[1-0.5], p<0.01)$. The hospital mortality rate was not different $(4,25$ vs $20,17.5 \%, p=0.47)$. The main limitation of the study was the impossibility to research longterm outcome due to higher rate of missing data.

Conclusion In a cohort of mainly selected medical patients, ICU and mortality rates were not different between nonagenarians and octogenarians in spite of a lower intensity of treatment. The discharge to a geriatric unit did not change the outcome.

\section{Competing interests None.}

\section{References}

1. Bagshaw SM, Webb SA, Delaney A, George C, Pilcher D, Hart GK Bellomo $R$, (2009) Very old patients admitted to intensive care in Australia and New Zealand: a multi-centre cohort analysis Crit Care 13:R45.

2. Lerolle N, Trinquart L, Bornstain C, Tadie JM, Imbert A, Diehl JL, Fagon JY

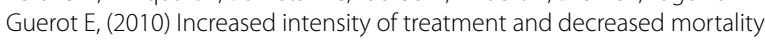
in elderly patients in an intensive care unit over a decade Crit Care Med 38:59-64.

S76

A systematic follow-up consultation guarantee quality of care in intensive care unit

Martine Nyunga', Noémie Banaias' ${ }^{1}$ Clément Vanbaelinghem¹', Céline Broucqsault-Dedrie', Delphine Colling', Marie Kauv', Erik Blondeau', Patrick Herbecq ${ }^{1}$

${ }^{1}$ Réanimation polyvalente, Centre Hospitalier de Roubaix, Roubaix, France Correspondence: Martine Nyunga - martine.nyunga@ch-roubaix.fr Annals of Intensive Care 2016, 6(Suppl 1):S76

Introduction As the number of patients surviving critical illness is increasing, since these last years, many intensive care units (ICU) developed an ICU aftercare in order to improve patient well-being and recovery. Although there is no gold standard for ICU follow-up, some medical teams showed that a single consultation is beneficial to patient. So the aim of our monocentric retrospective study was to establish that doing a systematic follow-up consultation can help to maintain quality of care for an ICU.

Patients and methods Were included all adult patients, discharged alive from ICU after at least $72 \mathrm{~h}$, coming in a follow-up consultation (FC), 3 months after their ICU stay, between February 2012 to June 2014, in general hospital of Roubaix, France. Were excluded unstable psychiatric or socially isolated patients. Were collected baseline demographics and clinical characteristics. Were evaluated during this consultation in order to define qualitative aspects of care: ICU care results (e.g., length of stay, duration of mechanical ventilation or sedation, muscle wasting and weakness, iatrogenic effects, SF 36, Barthel index); ICU healthcare conditions (e.g., questionnaire of sources of discomfort in ICU, using IPREA 2). We also performed during this consultation a multidisciplinary coordinating action to promote patient well-being and recovery (e.g., patient clinical assessment, referrals to specialists or family physician if necessary and living wills).

Results A total of 160 patients were included, mostly males (54\%), with a median age of 64 years [14-95], a median Simplified Acute Physiology Score II at 45 [6-99]. Comorbidities were mainly chronic cardiac failure $(64 \%)$, chronic respiratory failure (38\%) and chronic renal failure (17\%). 
The indications of admission were varied: $70 \%(112 / 160)$ of medical motifs, $39 \%$ of sepsis and shock, $19 \%$ of acute respiratory failure, $6 \%$ of acute cardiac failure, $6 \%$ of surgery aftermath, $6 \%$ of drug overdose, $22 \%$ of multivisceral organ failure. Management consisted of $62 \%$ of mechanical ventilation with a median duration of 8 days [0-158], $57 \%$ of noninvasive ventilation, $77 \%$ of technical gestures, $49 \%$ of amines infusion. The median length of stay in ICU was 14 days [1-162], in hospital 29 days [3-191]. Events during ICU were mainly delirium ( $28 \%$ ), ventilatory acquired pneumonia $(18 \%)$, dysphonia $(14 \%)$, bed sores $(11 \%)$ and unplanned extubation ( $8 \%)$. Hospital readmission rate in the first year ICU stay was $12.50 \%(20 / 160)$, whose 13 were admitted before their follow-up consultation for especially sepsis and shock ( $40 \%)$, and 9 readmitted post-FC. Median delay between ICU discharge and readmission was 81 days [2 - 298] and with consultation 3.5 months [0-5]. Death rate of this population was $9.38 \%(15 / 160)$.

During consultation, some data were collected: presence of close relatives $(44 \%)$, complete memory of ICU stay $(20 \%)$, partial memory (43\%), negatives memories ( $27 \%)$, feeling of non-information (41\%), nightmares $(14 \%)$, depression (11\%), posttraumatic stress disorder $(11 \%))$, fear (9\%), dyspnea (40\%), chronic pain (30\%), muscle wasting (40\%), Barthel index (91 $\pm 14 \%)$, SF36 physical (47 \pm 5.71$)$, SF 36 mental $(44 \pm 5.67)$. Evaluation of sources of discomfort during patient ICU stay using IPREA 2 questionnaire brought to light pain, fear, isolation and intimacy with high average scores.

Referrals to specialists were $57 \%$ (91/160), mainly cardiovascular $(13 \%)$, respiratory $(11 \%)$, psychiatric $(11 \%)$, pain $(5 \%)$. Twelve patients expressed their living wills $(7.50 \%)$. Patients appreciate follow-up in $74 \%$ of case because they have their issues.

In the subgroup of readmitted patients, $45 \%(9 / 20)$ were for a surgical reason, representing $1 / 5$ of surgical patients. When patients readmitted were compared to non-readmitted, it appeared that there was no causal link between readmission and surgical type of stay but a trend [18.75 (9/48) surgical readmitted vs $27.86(39 / 140)]$ surgical non-readmitted, $p=0.117$ ). There was a link between readmission and renal comorbidity [35\% (7/20) vs $14.29(20 / 140), p=0.048]$, readmission and cirrhosis [ $25 \%(5 / 20)$ vs $7.14 \%(10 / 140), p=0.024]$, and readmission and SAPS II [39.10 \pm 12.46 vs $46.43 \pm 18.59, p=0.046]$. Risk of death was higher in patients readmitted post-FC (3/9 vs 15/151, $p=0.040$ ). Readmission post-FC raised more than Barthel index was low $(82.50 \pm 16.26$ vs $91.22 \pm 13.92, p=0.071)$, and rehospitalization occurred before $\mathrm{FC}(0.89 \pm 1.27$ vs $0.11 \pm 0.34, p<0.001)$.

Discussion Our study showed that we should strengthen our active preventive measures against delirium, pneumonia, unplanned extubation and its effects, ICU sources of discomfort, muscle wasting and pain management. In view of this significant readmission rate, it seems important to identify factors of readmission; these should help us to establish an easy score useable in ICU FC. Referrals to specialists concern especially surgical patients.

Conclusion Doing a systematic ICU FC allows constantly evaluating and improving daily patient care. This clearly impacts and changes ICU behavior to focus on various aspects of long-term outcomes.

\section{Competing interests None.}

\section{Reference}

1. Jensen and al. Intensive care med (2015) 41: 763-775.

\section{S77}

Change in functional autonomy following ICU stay

Islem Ouanes ${ }^{1}$, Mahdi Marzouk', Lamia Ouanes-Besbes ${ }^{1}$, Rami Jabla$^{1}$,

Chaima Ghribi', Nesrine Boujelbene', Asma Hachani', Saousen Ben

Abdallah', Hedia Hammed', Hend Ben Lakhal', Imen Ben Ali', Imen Abdellaoui', Fahmi Dachraoui ${ }^{1}$, Fekri Abroug

${ }^{1}$ Réanimation polyvalente, CHU Fattouma Bourguiba, Monastir, Tunisia Correspondence: Islem Ouanes - ouanes.islem@gmail.com

Annals of Intensive Care 2016, 6(Suppl 1):S77

Introduction Previous studies reported the impact of ICU admission on outcome and functional status in intensive care unit (ICU) survivors in various populations. The aim of our study is to describe functional autonomy change in patients admitted to a Tunisian ICU.

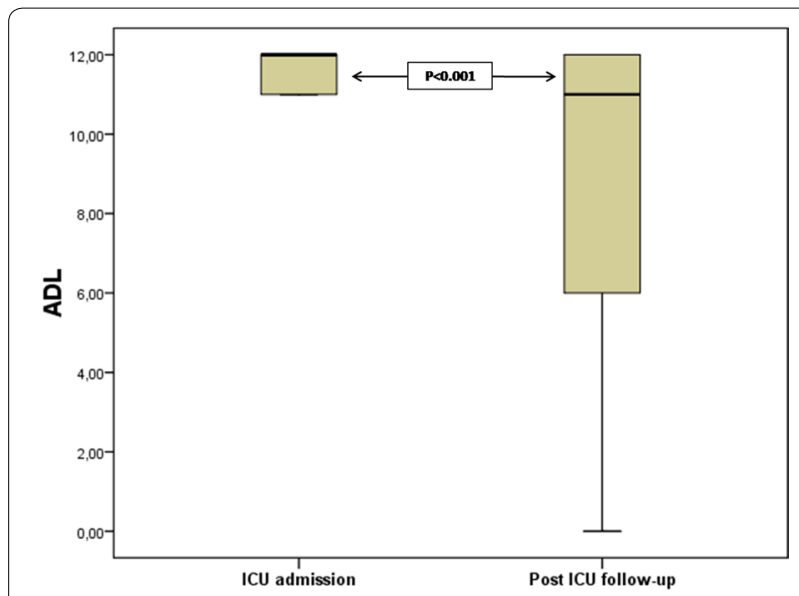

Fig. 49 See text for description

Patients and methods We analyzed a prospective collected database including patients consecutively admitted in our ICU between January 1, 2014 and June 30, 2015. Patients' autonomy was assessed by Katz's Activity of Daily Living (ADL) questionnaire recorded at admission, while current autonomy status was evaluated by phone call on August 2015. ADL scale was compared before and after ICU stay using Wilcoxon test.

Results Two hundred and ninety-five patients were admitted to our ICU, during the study period [median age: 62 years (42.5-72), median SAPS III: 50.5 (42-61), sex ratio (H/F):1.42, NIV as initial ventilatory mode in $53.3 \%$ ], median ADL scale at admission for the whole population: 12 (11-12). Fifty-seven patients died in the ICU (21\%), while 167 were discharged alive and were actually eligible for the second evaluation. The phone evaluation contact was actually performed in 131 patients $(78.4 \%)$, of whom only 111 were alive and 20 died; ADL questionnaire administration was hence made at a median followup of 12 months (IQR 6-16). Figure 49 shows a statistically significant decrease in ADL scale for the 111 patients who were still alive [12 (1112 ) versus 11 (6-12), $p<0.001]$. Autonomy impairment was significant for all items of ADL scale (bathing, dressing, toileting, transferring, continence, and feeding).

Conclusion ICU stay was associated with a significant impairment of functional autonomy in this cohort of patients admitted to a Tunisian ICU.

Competing interests None.

S78

Outcome of Intensive Care Unit (ICU) elderly patients admitted with solid tumor

Edouard Auclin', Emmanuel Guérot ${ }^{2}$, Caroline Haw-Berlemont ${ }^{2}$, Julien Taieb $^{3}$, Stéphane Oudard', Jean-Luc Diehl2 ${ }^{2}$, Jean-Yves Fagon², Nadia Aissaoui ${ }^{2}$

${ }^{1}$ Oncologie médicale, Hopital Europeen Georges-Pompidou, Paris, France; ${ }^{2}$ Réanimation médicale, Hopital Europeen Georges-Pompidou, Paris, France; ${ }^{3}$ Oncologie digestive, Hopital Europeen Georges-Pompidou, Paris, France

Correspondence: Edouard Auclin - ed.auclin@gmail.com

Annals of Intensive Care 2016, 6(Suppl 1):S78

Introduction The recent improvement of the management of patients (pts) with cancer has led to a better quality of life and also a longer survival. With the aging of the population, admission in intensive care unit (ICU) of elderly cancer pts is more often asked.

The aim of our study was to assess the outcome of pts older than 65 years with solid tumor admitted in ICU. 
Patients and methods This was a retrospective study. All pts, older than 65 years, suffering from evolutive cancer and admitted in the medical ICU of the European Georges Pompidou Hospital between August 2009 and December 2014 were included. Non-inclusion criteria were: hemopathy, cancer diagnosed during ICU stay, cancer remission superior than 5 years, limitation of active therapeutics and ICU stay inferior to $24 \mathrm{~h}$.

The primary endpoint was in-ICU mortality. The secondary endpoints were in-hospital and 90-day mortality, and resumption of anti-tumoral treatment.

Data concerning cancer (type of cancer, number of metastasis, number of chemotherapy lines), reasons for admission (classified as related to cancer progression, related to complications of chemotherapy and other), patients (age, severity at admission, need of life-supporting therapies, decision of limitation of active therapeutics after $24 \mathrm{~h}$ ) and outcome were collected.

Multivariate analysis was also performed to identify predictors of inICU mortality.

Results Baseline characteristics 398 pts with cancer were screened, and 262 pts were included. The 3 most represented cancer localizations were: gastrointestinal ( $n=71,27 \%)$, lung $(n=68,26 \%)$ and genitourinary $(n=60,23 \%)$. One hundred and fifty-eight pts (60\%) exhibited metastases.

The average IGS2 score was 62 (19-125). One hundred and thirty-five pts received mechanical ventilation (52\%), Thirty-five pts noninvasive ventilation (13\%), 126 pts inotrope drug (48\%) and 33 pts dialysis (13\%). The mean duration of ICU stay was 6.3 days (0-39), and the mean length of stay in hospital was 17 days (0-100).

Reasons for admission were distributed as follows: related to cancer progression $n=39(15 \%)$, related to anti-tumor drugs $n=49(19 \%)$ and other $n=174(66 \%)$.

Outcome In-ICU mortality was $34 \%(n=88)$. Among in-ICU died patients, 46 pts had a limitation of active therapeutics after $24 \mathrm{~h}$ (17.5\%). In-hospital mortality was $44 \%$ and 90 -day mortality $49 \%$. Among the 174 in-ICU survivors, 77 (44\%) were able to resume their anti-tumoral chemotherapy, and 32 (18\%) had no treatment because they had localized tumor and no indication of a new chemotherapy. In multivariate analysis, predictive factors of in-ICU death were IGS II, mechanical ventilation and administration of inotropes. Cancer type, presence of metastasis and number of chemotherapy lines were not associated with higher risk of death.

Conclusion The elderly patients admitted in ICU with evolutive solid cancer were severely ill. Though, $76 \%$ of them survived to ICU discharge and $44 \%$ of in-ICU survivors were able to resume anti-tumoral chemotherapy.

\section{Competing interests None.}

\section{References}

1. Unseld S, Schuepbach RA, Maggiorini M. ICU, hospital and one year mortality of patients suffering from solid or haematological malignancies. Swiss Med Wkly. 2013 Mar 26;143:0. doi: 10.4414/smw.2013.13741. PubMed PMID: 2357-2400

2. Bonomi MR, Smith CB, Mhango G, Wisnivesky JP. Outcomes of elderly patients with stage IIIB-IV non-small cell lung cancer admitted to the intensive care unit. Lung Cancer. 2012 Sep;77(3):600-4. doi: 10.1016/j. lungcan.2012.05.103. Epub 2012 Jun 17. PubMed PMID: 22709929.

\section{S79}

Risks factors for mortality in critically ill children with acute kidney injury requiring renal replacement therapy Genevieve Morissette', Wassim Kechaou' ${ }^{1}$, Catherine Litalien ${ }^{1}$, Aïcha Merouani ${ }^{2}$, Véronique Phan ${ }^{2}$, Josée Bouchard ${ }^{3}$, Philippe Jouvet ${ }^{1}$

${ }^{1}$ Pediatric Critical Care, CHU Sainte-Justine, Montréal, Canada; ${ }^{2}$ Pediatric nephrology, CHU Sainte-Justine, Montréal, Canada; ${ }^{3}$ Nephrology, Sacré-Coeur Hospital, Montréal, Canada

Correspondence: Genevieve Morissette - genevieve.morissette@ chudequebec.ca

Annals of Intensive Care 2016, 6(Suppl 1):S79
Introduction Despite major advances in blood purification technology over the last decades, the mortality rate associated with acute kidney injury (AKI) remains high and continues to exceed $50 \%$. Previous pediatric studies on renal replacement therapy (RRT) have suggested that the mortality rate is primarily related to the underlying diagnosis or the presence of a multiple organ dysfunction syndrome (MODS) rather than to a specific RRT modality or other risk factors. Several recent clinical studies have suggested that fluid overload before RRT could also influence mortality. The aim of this study was to identify the most important risk factors for 28-day mortality in patients with AKI at RRT initiation.

Patients and methods We conducted a retrospective cohort study in a tertiary care pediatric center. All critically ill children who underwent acute continuous RRT or intermittent hemodialysis for AKI between January 1998 and December 2008 were included, except those with chronic kidney disease. A case report form was developed, and specific risk factors were identified using an item generation-item selection modality with a panel of 10 pediatric intensivists and pediatric nephrologists. Risk factors analysis was made using logistic regression in SPSS version 21

Results We enrolled 70 consecutive patients. The main PICU admission reasons were respiratory failure (34 \%), septic shock (29\%), AKI (24\%) and postoperative ( $21 \%)$. The most frequent underlying diseases were bone marrow transplant in $24 \%$ and solid organ transplant in $17 \%$ of patients. The majority of patients received continuous RRT only. The median pediatric intensive care unit (PICU) length of stay was 16 days [IQR 8-27]. Thirty-three patients (47 \%) died within 28 days after PICU admission. The median age (8.9 years [IQR 1.9-14.2]), weight (28 kg [IQR 7.9-45]), PEdiatric Logistic Organ Dysfunction score (PELOD) (21 [IQR 20.3-31.8]), Pediatric Index of Mortality 2 (PIM 2) (7.7 [IQR 7.2-28.8]), creatinine (102 [IQR 70.2-154.5]), vasoactive score (3 [IQR 0-47.3]) and oxygenation index (7.3 [IQR 3.5-19.1]) at PICU admission were similar between survivors and non-survivors. We identified the following risk factors for mortality in univariate analyses: hematological underlying disease and factors at RRT initiation including: fluid overload $\geq 20 \%$, PELOD score $\geq 20$ and higher creatinine. Of those risk factors, only PELOD score $\geq 20$ was significant in the multivariate logistic regression analysis (OR 4.5; $95 \% \mathrm{Cl} 1.4-14.1 ; p=0.01$ ). After PICU discharge, 12 out of 35 survivors needed RRT. Among 31 survivors at hospital discharge, 3 patients required RRT.

Discussion Despite improvement in patient care, almost half of our patients with AKI requiring AKI died within 28 days. Our data suggest that a higher severity of illness is the most important risk factor for mortality in critical care patients with AKI requiring RRT.

Conclusion In our study, only the severity of illness, as measured by the PELOD score before initiation of RRT, was independently associated with an increased risk of mortality in critically ill children.

\section{Competing interests None.}

\section{S80}

Epinephrine use in pediatric septic shock: a retrospective, single-center study on 117 patients

Julie Starck ${ }^{1}$, Mehdi Oualha ${ }^{2}$, Nelly Briand ${ }^{3}$, Jean-Marc Treluyer ${ }^{3}$, Sylvain Renolleau4, Laurent Dupic ${ }^{5}$

${ }^{1}$ Réanimation et Surveillance Continue Médico Chirurgicale, Hôpital Necker - Enfants Malades, Paris, France; ${ }^{2}$ Réanimation et surveillance continue médico-chirurgicales, Hôpital Necker - Enfants Malades, Paris, France; ${ }^{3}$ Centre d'investigation clinique, Institut Imagine, Paris, France; ${ }^{4}$ Réanimation pédiatrique polyvalente, Hôpital Necker - Enfants Malades, Paris, France; ${ }^{5}$ Réanimation et surveillance continue pédiatriques, $\mathrm{CHU}$ Necker-Enfants Malades, Paris, France

Correspondence: Julie Starck - starck.ju@gmail.com

Annals of Intensive Care 2016, 6(Suppl 1):S80

Introduction Septic shock remains a major health issue even in our industrialized countries, with described mortality rates between 11.4 and $24.3 \%$ in the most recent European studies. Several international pediatric therapeutic guidelines have been published and widely 
diffused. As for epinephrine, they recommend its use as a first-line drug in cold shocks resistant to a well-conducted fluid resuscitation or as a second-line drug in "cold" shocks resistant to dopamine or "warm" shocks resistant to norepinephrine. However, those recommendations are only based on adult or neonatal references that can hardly be applied to children as the physiopathology of children's septic shock is different on several aspects. Thus, our study constitutes the first specifically pediatric work on epinephrine use in septic shock.

Patients and methods We conducted a retrospective, single-center, observational study of all children (newborn excluded) treated for septic shock, upon admission or during their hospitalization in our ward, between January 1, 2009, and December 31, 2013. The patients were divided in two groups: "with" and "without" epinephrine use. Medical and paramedical files were reviewed for a complete description of each group: sociodemographic, clinical and biological data, vasoactive drugs (especially epinephrine) modality of prescription, patients outcome (mortality at 28 days) were collected. We then performed a comparison between the two groups in univariate and multivariate analysis.

Results One hundred and seventeen patients corresponded to the pediatric definition of septic shock and were included: 68 (51.8\%) in the group without epinephrine use, 49 (41.9\%) in the group with epinephrine use. $78.6 \%$ of the patients had an underlying comorbidity, immunodeficiency concerned almost half of them (46.2 \%). More than half of the septic shocks were nosocomial (59.8\%), acquired in our PICU or in another ward of the hospital.

Epinephrine was prescribed for myocardic dysfunction (36.7 \%), cardiac arrest $(30.6 \%)$ and persistent hypotension (24.5\%). Compared to patients without epinephrine use, patients treated with epinephrine were more severe at the onset of the septic shock, both clinically (with $4-6$ organ dysfunction in 68.7 vs $45.2 \%$ cases; $p=0.02$ ) and biologically (lactate level of 4.15 vs $2.20 \mathrm{mmol} / \mathrm{l} ; p=0.0008$ ). Our delay before initiating epinephrine was four times longer than the one prescribed in the international pediatric guidelines, and patients were then in a severe clinical and biological state. Above all, patients treated with epinephrine had a worse outcome than patients treated without. Not only did they need continuous renal replacement therapy more frequently ( 32.7 vs $6 \% ; p=0.0002)$, but they also had a higher mortality rate at 28 days ( 63.3 vs $16.2 \% ; p<0.0001$ ) in univariate and multivariate analysis after adjustment on the initial gravity. Finally, we highlighted a potential association between the maximum epinephrine dose used and death with a $100 \%$ death rate for an epinephrine maximum dose over $1.90 \mathrm{mg} / \mathrm{kg} / \mathrm{min}$.

Conclusion Acknowledging the fact that patients treated with epinephrine have a higher mortality rate than patients treated without three questions can be raised in order to improve the patients' outcome.

Isn't a better initial monitoring of our patients necessary to determine their hemodynamic profile at best and prescribe the most appropriate drug?

As patients were clinically and biologically severe when epinephrine was prescribed, would an earlier initiation of epinephrine have a beneficial effect on the mortality rate of these patients?

As a potential relation exists between the maximum dose of epinephrine used and the mortality rate, would alternative therapeutics such as the ECMO be beneficial beyond a given maximum dose of epinephrine?

\section{Competing interests None.}

\section{References}

1. Brierley J, Carcillo JA, Choong K, Cornell T, Decaen A, Deymann A, et al. Clinical practice parameters for hemodynamic support of pediatric and neonatal septic shock: 2007 update from the American College of Critical Care Medicine. Crit Care Med. 2009 Feb;37(2):666-88.

2. Aneja R, Carcillo J. Differences between adult and pediatric septic shock. Minerva Anestesiol. 2011 Oct;77(10):986-92.
S81

Sickle cell disease in pediatric intensive care unit: a five-year retrospective monocentric study of $\mathbf{7 4}$ patients

Philippe Sachs ${ }^{1}$, S Julliand ${ }^{2}$, François Angoulvant ${ }^{3}$, Julie Sommet ${ }^{4}, \mathrm{~L}$ Holvoet $^{5}, \mathrm{M} \mathrm{Benkerrou}^{5}$, Stéphane Dauger

${ }^{1}$ Réanimation et surveillance continue pédiatriques, CHU Robert Debré, Paris, France; ${ }^{2}$ Reanimation pediatrique, Hospital Robert-Debré, Paris, France; ${ }^{3}$ Urgences pédiatriques, CHU Necker-Enfants Malades, Paris, France, France; ${ }^{4}$ Réanimation et surveillance continue pédiatriques, CHU Robert Debré, Paris, France, France; ${ }^{5}$ Centre drepanocytose, Hospital Robert-Debré, Paris, France

Correspondence: Philippe Sachs - philippe.sachs@rdb.aphp.fr Annals of Intensive Care 2016, 6(Suppl 1):S81

Introduction Acute complications of sickle cell disease can be lifethreatening and require intermediate care and/or pediatric intensive care. In this retrospective study, we describe the experience over 5 years in a tertiary pediatric intensive care unit. Our secondary goal was to specifically study the population with respiratory failure.

Patients and methods This was a retrospective monocentric descriptive study of all pediatric admissions for sickle cell disease from 2009 to 2013 in our 16 bed pediatric intensive care unit. We collected demographic data, description of admission (reason, C-reactive protein and hemoglobin level), intensive care stay (organ failures, need for artificial support, blood transfusion or exchange, complications, mortality, length of stay, Pediatric Index of Mortality 2 and Pediatric Logistic Organ Dysfunction scores from 2010 on). Organ failure definitions used were that of the 2005 International Conference on Pediatric Sepsis and of the 2015 Pediatric Acute Lung Injury Sepsis Investigator network.

Results (All patients not included yet): Primary goal: There were 74 patients accounting for 88 admissions in our pediatric intensive care unit; $60.8 \%$ were male; mean age was $9.6 \pm 5.1$ years; genotype was SS (93.2\%), SC (4.1\%), S $\beta$ (2.7\%); reason for admission was respiratory in $64.7 \%$ (acute chest syndrome $57.9 \%$ ), shock $1.1 \%$, osteomyelitis $2.2 \%$, cerebral stroke $11.4 \%$, acute anemia with hemolysis $2.2 \%$, surgery $6.8 \%$, pain management $11.3 \%$. Mean hemoglobin level at admission was $8.96 \pm 1.59$. Blood transfusion was administered in $35 \%$ before admission. Organs failure during stay were: respiratory $71.5 \%$ (52 \% on mechanical ventilation), hemodynamic $2.2 \%$ (septic shock with inotropes), neurological $11.4 \%$, hematological $9 \%$, renal $2.2 \%$, hepatic $5.7 \%$ and multi-organ failure $4.5 \%$. Blood was administered in $72.7 \%$ (transfusion $29.5 \%$, exchange in $43.2 \%$ ). Median length of stay was 3 days (25-75 \%: 2-4). Median Pediatric Index of Mortality 2 was 0.85 (25-75 \%: 0.2-3), and median Pediatric Logistic Organ Dysfunction was 1 (25-75 \%: 0-1). Two patients (2.2 \%) died: one cerebral stroke with massive oedema and one septic shock and multi-organ failure.

Secondary goal: Fifty-two admissions for acute chest syndrome $(59.1 \%)$ occurred; 43 patients $(82.7 \%)$ received mechanical ventilation (76.9\% exclusively noninvasive); 9 (17.3\%) none; $53 \%$ received blood transfusion (21.7\% before, $31.3 \%$ in our unit) and $47 \%$ blood exchange; mean admission C-reactive protein level was $144 \pm 76 \mathrm{mg} / \mathrm{l}$. Mean initial hemoglobin level was $8.8 \pm 1.5 \mathrm{~g} / \mathrm{dl}$. All received broadspectrum antibiotics without bacteriological proof of pneumonia. Almost all recovered quickly, with a median length of mechanical ventilation of 3 days (25-75\%: 2-4) and an intensive care stay of 3 days (25-75 \%: 2-4). Three of them (5.8\%) developed a more severe respiratory failure and needed invasive mechanical ventilation, and one required high-frequency ventilation and nitric oxide. Their mean initial hemoglobin level was $10.1 \mathrm{~g} / \mathrm{dl}$; all had received blood transfusion before admission. All three survived with a median length of stay of 8 days (25-75\%: 6-8.5). Overall, we found a higher rate of initial C-reactive protein ( $144.32 \mathrm{vs} .102 .6 \mathrm{mg} / \mathrm{l}, p=0.028)$ and a more elevated white blood cell count ( 23.415 vs. 17,494 cell per millimeter, $p=0.004)$ in acute chest population compared to non-acute chest syndrome patients. 
Discussion It is unclear why some patients develop life-threatening complications. In our series, acute chest syndrome is the main reason for admission. It is a hypoxic pneumonia whose criteria strongly overlap with those of acute respiratory distress syndrome but which appears to be rapidly reversible most of the time with noninvasive ventilation and blood transfusion. Antibiotics are given systematically because of a high inflammatory state but without bacteriological evidence.

Conclusion In our hospital hosting a clinic devoted to sickle cell patients, usual reasons for pediatric intensive care unit admission are acute chest syndrome and cerebral stroke. A prospective study will give more insights into the management of this specific population.

\section{Competing interests None.}

\section{S82}

Implementation and evaluation of a paediatric nurse-driven sedation protocol in a PICU

Lélia Dreyfus ${ }^{1}$, Fabienne Bordet ${ }^{1}$, Sandrine Touzet ${ }^{2}$, Angélique Denis ${ }^{2}$, Etienne Javouhey ${ }^{1}$

${ }^{1}$ Réanimation pédiatrique hfme, Hospices civils de Lyon, Lyon, France;

${ }^{2}$ Pôle information médicale évaluation recherche, Hospices Civils de Lyon, Lyon, France

Correspondence: Lélia Dreyfus - lelia.dreyfus@gmail.com

Annals of Intensive Care 2016, 6(Suppl 1):S82

Introduction Management of analgesia and sedation is an integral component of the medical care of a critically ill child. Its role is to assure comfort and safety to a patient undergoing painful cares and technical procedures; it can also be a full-processing treatment, in particular situations like acute respiratory distress syndrome or acute brain injury.

Sedation involves, most of the time, the association of an opioid and a sedative. The use of these drugs is difficult in children, because of a specific metabolism, inducing tolerance and withdrawal in case of prolonged administration.

The COMFORT-BEHAVIOR (COMFORT-B) scale is a validated, simple, reliable and reproducible score evaluating sedation and analgesia. Sedation scoring systems must be used regularly to avoid inadequate sedation.

Excessive sedation is associated with poor outcomes like prolonged mechanical ventilation, longer hospitalisation and more frequent withdrawal symptoms. Adult and paediatric data suggest that goaldirected sedation algorithms allow a more appropriate adaptation of the treatment to the patient's need and permit a reduction in the duration of mechanical ventilation.

Our objective was to evaluate the impact of a nurse-driven sedation protocol in our paediatric intensive care unit (PICU) on duration of mechanical ventilation, total doses and duration of medications, PICU length of stay, incidence of ventilator-associated-pneumonia and occurrence of withdrawal.

Patients and methods After development of the sedation protocol in January 2014, we realised a before and after protocol implementation study in a 23-bed medical-surgical PICU at a university children's hospital. We included all children aged from 0 to 18 years, requiring mechanical ventilation for at least $24 \mathrm{~h}$. Prior to implementation of the protocol, analgesia and sedation were managed by the attending physician's order. Afterwards, post-implementation, nurses managed analgesia and sedation following an algorithm, including COMFORTB scale. Data were collected from computerised medical chart, and descriptive and comparative analyses were done.

Results We included 200 patients, 107 who were consecutively admitted before the implementation of the protocol between January and December 2013 and 93 who were admitted after the implementation, between May 2014 and March 2015. The characteristics of the two populations were similar, except that there was significantly more patients admitted for chirurgical pathology in the period "after" (38 vs $24 \%, p=0.04$ ).

Median duration of mechanical ventilation was significantly reduced in the group subjected to the nurse-driven protocol: 4.8 [1.3-25.3] days versus 6 [1.1-80.5] days $(p=0.05)$. Mean total dose of midazolam was also reduced (17 vs $28 \mathrm{mg} / \mathrm{kg}, p=0.05$ ), and duration of sufentanil administration (112 vs $157 \mathrm{~h}, p=0.05$ ). We observed fewer withdrawal's symptoms ( 14 vs $24 \%, p=0.09$ ) in the period "after," but the difference did not reach statistical significance.

The use of the protocol improved sedation evaluation by increasing the number of total scores and of scores indicating adequate sedation (COMFORT-B between 11 and 17); but was, on the contrary, not associated with more agitation episodes or unplanned endotracheal extubation.

Discussion These preliminary results are promising and allow us to think that implementation of a nurse-driven sedation protocol in a PICU is feasible and present a lot of advantages. To our knowledge, this is the first study, showing a reduction in duration of mechanical ventilation. It would have to be confirmed on a larger cohort and after a longer use of the protocol. Adjusted analysis would have to be done on confounding factor like age or diagnosis at admission. In the future, it could be relevant to make a staff survey about the use of the algorithm, corresponding to a deep change in practices.

Conclusion One of the actual challenges is to enhance the sedation management of the critically ill patients. Implementation of a nursedriven sedation protocol in a PICU seems to be associated with shorter duration of mechanical ventilation, lower total doses of benzodiazepine, shorter administration of opioid and stricter evaluation of the sedation scores.

\section{Competing interests None.}

\section{S83}

Diagnostic evaluation and management of new-onset seizures and seizures with known epilepsy in children: a retrospective and comparative analysis

Julien Le Coz', Gérard Chéron ${ }^{2}$, Rima Nabbout ${ }^{3}$, Geraldine Patteau², Claire Heilbronner ${ }^{4}$, Philippe Hubert ${ }^{4}$, Sylvain Renolleau ${ }^{4}$, Mehdi Oualha ${ }^{4}$

${ }^{1}$ Urgences pédiatriques, Hospital Robert-Debré, Paris, France; ${ }^{2}$ Urgences pédiatriques, Hospital Necker - Enfants Malades, Paris, France; ${ }^{3}$ Neurologie pédiatrique, Hospital Necker - Enfants Malades, Paris, France; ${ }^{4}$ Réanimation et surveillance continue médico-chirurgicales, Hôpital Necker -

Enfants Malades, Paris, France

Correspondence: Mehdi Oualha - mehdi.oualha@nck.aphp.fr

Annals of Intensive Care 2016, 6(Suppl 1):S83

Introduction A previous history of epilepsy can influence the management of children's seizures. Assuming that there are significant differences, we described and compared the initial management of seizures between children with new-onset seizures and those with known epilepsy.

Patients and methods We conducted a single-center retrospective observational study. We included all children ( 1 month-18 years) who were admitted for seizures or status epilepticus from January to December 2014 in the emergency department and the intensive care unit at Necker-Enfants Malades Hospital.

Results One hundred and ninety patients with median age of 38 months (1-214) and sex ratio male/female of 1.08 were included: 72 with known epilepsy (38\%) and 118 with new-onset seizures (62\%) with a median age of 60 months (1-214) and 26.5 months (1-191), respectively $(p<0.001)$. Life-threatening status epilepticus was reported in 83 children (38 with known epilepsy, $52 \%$ and 45 with new-onset seizures, $38 \% ; p=0.05)$. Laboratory work and imaging were frequent in both groups $(n=104,88 \%$ for those with newonset seizures and $n=56,78 \%$ for children with known epilepsy). Children with new-onset seizures were more likely to have at least one diagnostic test performed $(p=0.005)$. For children with known epilepsy, conducting diagnostic evaluation was more likely performed in those with life-threatening status epilepticus $(p<0.02)$. Antiepileptic drugs concentration measurement were performed in 20 children, i.e., $53 \%$ of cases whenever it was possible and shown that 7 children ( $35 \%)$ of them were under target concentration. The remaining investigations had no immediate therapeutic impact except for one child. In most cases $(n=52,72 \%)$, we did not find any provoking factor for the occurring seizures in children with known epilepsy. The first-line treatment in both populations was usually diazepam, but was used more often in children with new-onset seizures ( 80 vs $46 \%, p<0.001$ ), while 
midazolam were more likely administered in children with known epilepsy ( 25 vs $1 \%, p<0.001)$. In second line, diazepam was continued for children with new-onset seizures, whereas clonazepam was used for those with known epilepsy (56 vs $34 \%, p=0.02$ and 46 vs $24 \%$, $p=0.001$, respectively). In a third line, phenytoin was administrated more frequently in children with new-onset seizures (54 vs $22 \%$, $p<0.001$ ).

Discussion The management of seizures was different in children with new-onset seizures and those with known epilepsy: (1) laboratory works and imaging except antiepileptic drug concentration measurement seem to be futile for children with known epilepsy; (2) midazolam and clonazepam are usually used for those children, whereas diazepam and phenytoin are more often administrated in children with new-onset seizures.

Conclusion Initial diagnostic evaluation as well as treatment should be considered with caution and individualized for children with known epilepsy who exhibited seizures and status epilepticus.

\section{Competing interests None.}

\section{References}

1. Abend NS, Loddenkemper T (2014) Pediatric status epilepticus management. Curr Opin Pediatr. 26(6):668-74.

2. Emily R. Freilich, John M. Schreiber, Tesfaye Zelleke, William D. Gaillard (2014) Pediatric status epilepticus: Identification and evaluation. Curr Opin Pediatr. 26(6):655-61.

\section{S84}

MRI sedation in children in pediatric hospital in Oran, Algeria

Nabil Tabet Aoul ${ }^{1}$, zakaria addou' ${ }^{2}$, Ali Douah ${ }^{3}$, Mohamed Moussati ${ }^{3}$, Kamel Belhabiche ${ }^{3}$, Nabil Aouffen ${ }^{3}$

${ }^{1}$ Réanimation pédiatrique canastel, Faculté de médecine d'Oran, Oran Algeria; ${ }^{2}$ Réanimation pédiatrique de Canastel d'oran, Departement de medecine d'Oran Algerie, Oran, Algeria; ${ }^{3}$ Anesthésie réanimation pédiatrique, Etablissement hospitalier spécialisé en pédiatrie Canastel, Oran, Algeria

Correspondence: Nabil Tabet Aoul- tabetrea@yahoo.fr

Annals of Intensive Care 2016, 6(Suppl 1):S84

Introduction Available for more than 20 years, the imaging technique by magnetic resonance (MRI) revolutionized the diagnosis of numerous diseases. However, the constraints are important and require the teams of anesthesia and intensive care for several reasons; the strict immobility is essential for the quality of the images. The environment is hostile because of noise and cold, the patient is alone in the tunnel of MRI, and an injection of contrast agent is often necessary. Children less than 6 years and with delay of development are concerned.

Our aim is to describe the characteristics of the sedation and the evaluation of its safety in the MRI suite.

Materials and methods Descriptive retrospective study over a period ranging from October 2013 to February 2015, in the radiology department of pediatric EHS PrBoukhrofa AEK, Oran, Algeria, including children aged 1 month to 15 years. All patients received a premedication with midazolam $0.3 \mathrm{mg} / \mathrm{kg} 30 \mathrm{~min}$ before and underwent induction with sevoflurane inhalation to $6 \%$ with a mixed gas rates $\mathrm{O}_{2} / \mathrm{N}_{2} \mathrm{O}$ $(60 / 40)$ and then maintains the anesthesia with sevoflurane $2 \%$.

Results We have anesthetized 212 patients, the mean age is 3 years (1 month-14 years), the sex ratio is 1.47 , and the mean weight was $14.5 \mathrm{~kg}$ (3-56 kg). $99.5 \%$ of the children was classified as ASA I and ASA II and $0.5 \%$ was ASA III. The MRI is realized in the case of emergency in $27 \%$. A cerebral MRI in $83.4 \%$ of the cases, medullary in $10.4 \%$, and in $6.2 \%$ it concerns the shoulder, the hand and the abdomino-pelvic region. The mean time of the sedation is $56 \mathrm{~min}$. Eight children complicated with bronchospasm. The variations of the FC and the systolic pressure were transient and did not require intervention.

Conclusion The immobility of the patient and the control of movement are the major problems of the MRI in pediatric practice as well as the risk of respiratory complication which requires a rigorous care and the most safety environment.

Competing interests None.
S85

Neurally adjusted ventilatory assist in pediatric patients following cardiac surgery: feasibility in clinical practice and impact on ventilation pressures

Benjamin Crulli ${ }^{1}$, Baruch Toledano ${ }^{1}$, Nancy Poirier ${ }^{2}$, Suzanne Vobecky ${ }^{2}$, Guillaume Emeriaud'

${ }^{1}$ Soins intensifs pédiatriques, CHU Sainte-Justine, Montréal, Canada; ${ }^{2}$ Chirurgie cardiaque pédiatrique, CHU Sainte-Justine, Montréal, Canada Correspondence: Benjamin Crulli - benjamin.crulli@umontreal.ca Annals of Intensive Care 2016, 6(Suppl 1):S85

Introduction Ventilator support is frequent after pediatric cardiac surgery, but high airway pressures can be detrimental on right ventricular function and pulmonary blood flow. Neurally adjusted ventilatory assist (NAVA) is a mode that improves patient-ventilator interactions, thus helping to maintain spontaneous ventilation. The use of NAVA after pediatric cardiac surgery has been seldom studied.

Our hypothesis was that using NAVA in this population is feasible and allows for lower ventilation pressures.

Patients and methods We retrospectively studied all children that were ventilated with NAVA (invasively or noninvasively) after undergoing cardiac surgery between January 2013 and May 2015 in the pediatric intensive care unit of CHU Sainte-Justine, Montreal. Baseline patient characteristics and duration of the different ventilation periods in each mode were described. For the first period of invasive NAVA in each patient, ventilatory parameters, vital signs, and available blood gas data in the $4 \mathrm{~h}$ before and after the start of NAVA were extracted from electronic patient charts.

Results Thirty-three postoperative courses were included in 28 patients with a median [25th-75th percentile] age of 3 [1-12] months. During invasive ventilation, NAVA was used in 27 postoperative courses over 49 episodes, for a total duration of 87 [15-334] hours per course. Seven (14\%) individual episodes lasted less than $2 \mathrm{~h}$, and a single patient was treated with NAVA during less than $2 \mathrm{~h}$ in total. As illustrated in Fig. 50, peak inspiratory pressures and mean airway pressures decreased significantly after the start of NAVA $(p<0.0001$ for the mode; two-factor repeated-measures ANOVA). There was no significant difference in the vital signs or blood gas values. During noninvasive ventilation, NAVA was used in 14 patients, during 79 [25-137] h.

Conclusion NAVA could be used in pediatric patients after cardiac surgery. The significant decrease in airway pressures observed after transition to NAVA could have a beneficial impact in this population. Prospective interventional trials are needed to assess the impact of NAVA on cardiac function and pulmonary vascular resistance in these patients.

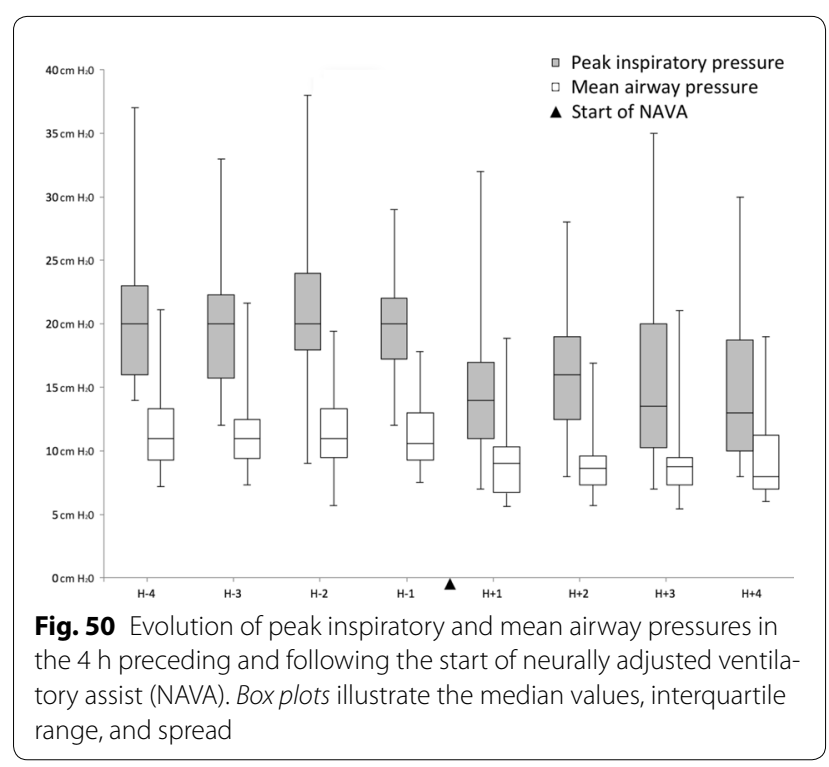




\section{Competing interests None.}

\section{S86}

Percutaneous central venous cannulation: a Seldinger technique versus an ultrasound guidance technique in a pediatric intensive care unit

Fehmi Ferhi ${ }^{1}$, Hosni Khouadja ${ }^{1}$, Mohamed Amine Bouslama ${ }^{1}$, Affra Brahim ${ }^{1}$, Khalil Tarmiz', Khaled Benjazia

${ }^{1}$ Anesthesia and Intensive Care, Chu Farhat Hached, Sousse, Tunisia

Correspondence: Hosni Khouadja - hosny_kh@yahoo.fr

Annals of Intensive Care 2016, 6(Suppl 1):S86

Introduction Percutaneous central venous cannulation (CVC) in infants and children is a challenging procedure. It is usually achieved with a blinded, external landmark technique or by vein denudation. However, these procedures are associated with a high morbidity and a significant risk of failure.

The purpose of this study was to compare landmark-to-ultrasoundguided central venous access in a pediatric intensive care unit.

Materials and methods A prospective observational study was conducted in a pediatric intensive care unit. Forty-five critically ill patients requiring central venous access were divided into two groups. In 30 patients, the cannulation of the vein was performed by ultrasound guidance (group S), and in 15 patients, the cannulation was performed with external landmark guided technique (group LM). We assessed the venous access site, the number of attempts, the access time (skin to vein), and the incidence of complications.

Results A cannulation of the central vein was achieved in 41/45 patients. In group $S$, an access site to the internal jugular vein was preferred in 29/30 patients. In group LM, an attempt to access to the right femoral vein was performed in all cases. The procedure was successful in $96.6 \%$ in the group S and $80 \%$ in the group LM. The vein was entered at the first attempt in $96.6 \%$ in group $S$ versus $40 \%$ in group LM. The median number of attempts was 1.1 in group $\mathrm{S}$ and 3 in group LM. The median access time (skin to vein) was 3 and 7 min in group S and group LM, respectively. No arterial punctures or hematomas occurred with the ultrasound technique. Three arterial punctures occurred with the seldinger technique.

Conclusion In a pediatric intensive care unit, the ultrasound-guided CVC is a more interesting technique compared to the Seldinger technique (1). In our study, ultrasound guidance seemed to reduce the number of attempts and the time of the procedure and improved the success rate and the occurrence of the complications.

\section{Competing interests None.}

\section{Reference}

1. Sidney L. et al. Central Venous Access Techniques. Atlas Oral Maxillofacial Surg. Clin. N. Am.2015;23(2):137-145.

\section{7}

Effect of remote ischemic preconditioning on renal resistive index in healthy volunteers

René Robert ${ }^{1}$, M. Vinet ${ }^{1}$, Angéline Jamet ${ }^{2}$, Remi Coudroy ${ }^{2}$, Arnaud W.

Thille

${ }^{1}$ Service de Réanimation médicale, CHU de Poitiers, Poitiers, France;

${ }^{2}$ Réanimation médicale, CHU de Poitiers, Poitiers, France

Correspondence: René Robert - r.robert@chu-poitiers.fr

Annals of Intensive Care 2016, 6(Suppl 1):S87

Introduction Recent studies showed that the remote ischemic preconditioning may provide protection of renal function after cardiac surgery and contrast-medium-induced renal failure. The mechanism is linked to the production of vasodilatory substances leading to improve organ perfusion.

The aim of our study was to determine whether a noninvasive remote preconditioning could induce a vasodilation in intraparenchymal renal circulation measured by the renal resistive index.

Patients and methods Remote preconditioning procedure was applied in six volunteers as follows: cycles of 4-min inflation of a blood pressure cuff to a pressure $50 \mathrm{mmHg}$ above the systolic arterial pressure to one upper arm, followed by 4-min reperfusion with the deflated cuff.

RRI was determined using renal Doppler-echography method performed on the interlobar arteries using a $2.5 \mathrm{MHz}$ transducer at 1.8 MHz for Doppler analysis. The RRI was calculated as follows: (peak systolic velocity - end diastolic velocity)/peak systolic velocity. Three measurements were performed on right kidney and averaged to obtain the mean RI values.

Mean values were compared at baseline (T0), during each period with the deflated cuff $(T 1, T 2, T 3)$ and at the end of the protocol (T4) using paired Wilcoxon test.

Results The mean value of RRI significantly decreased from $0.57 \pm 0.05$ before the remote conditioning to $0.49 \pm 0.04$ at the end of the experiment $p<0.01$. The progressive decrease in RRI was observed in the all six subjects. During the experiment, heart rate and arterial pressure were unchanged.

Conclusion In this pilot study, we demonstrated that the remote ischemic preconditioning induced a vasodilation of renal microcirculation assessed by the decrease in RRI in each volunteer. The precise mechanism of such a vasodilation remains to be investigated.

\section{Competing interests None.}

\section{S88}

Peak concentration of high-dose AMIKACIN does not affect renal recovery at day 90: a retrospective study of 93 patients in a single medical Intensive Care Unit

Camille Vinclair', Romain Sonneville², Bruno Mourvillier², Mathilde Neuville ${ }^{2}$, Roland Smonig ${ }^{2}$, Eric Magalhaes ${ }^{2}$, Guillaume Voiriot ${ }^{2}$, Aguila Radjou², Jean-François Soubirou², Stephane Ruckly ${ }^{3}$, Lila Bouadma², Jean-François Timsit ${ }^{2}$

${ }^{1}$ Pyrénées-atlantiques, Hospital Center De La Côte Basque, Bayonne, France: ${ }^{2}$ Service de réanimation médicale et infectieuse, Hôpital Bichat-Claude Bernard-APHP, Paris, France; ${ }^{3}$ Reanimation, hôpital, Grenoble, France

Correspondence: Camille Vinclair - cvinclair@live.fr

Annals of Intensive Care 2016, 6(Suppl 1):S88

Introduction Acute kidney injury (AKI) frequently occurs in intensive care units (ICU) and is associated with poor outcomes, including persistent kidney injury evolving to chronic kidney disease. If sepsis is the main cause for AKI, diagnostic procedures and drugs, may also contribute to renal failure. Aminoglycosides, such as Amikacin (AMK), are potent, highly bactericidal antibiotics, increasingly used in ICU to broaden the spectrum against gram-negative bacilli during septic shock. They may have an acute tubular toxicity leading to AKI. Recently, French practice guidelines recommended using AMK with once-daily dosing regimen of $25 \mathrm{mg} / \mathrm{kg}$ over $30 \mathrm{~min}$ in order to reach a peak plasma concentration ranging from 60 to $80 \mathrm{mg} / \mathrm{l} 30 \mathrm{~min}$ after the end of injection. No AMK dose adjustment is recommended for the first dose in patients with acute or chronic kidney failure. The oncedaily regimen is known to be protective for kidneys. Whether this high dose implies a greater risk of nephrotoxicity or not remains unknown. Thus, we performed a retrospective study to assess the association between the peak concentration of AMK and the class of AKI at day 90.

Patients and methods We included all patients hospitalized in a medical ICU, who had received AMK according to French guidelines, no matter their kidney function at the time of initiation of AMK. AKI was defined using Kidney Disease Improving Global Outcomes (KDIGO) 2012 classification. AKI at day 90 was assessed comparing creatinine plasma level at day 90 to steady-state pretreatment one. Clinical and biological features, including severity scores and nephrotoxic use, were recorded. Creatinine plasma levels were followed until 90 days after the first injection of AMK. We assessed the association between peak concentration of AMK and kidney function at day 90. A negative binomial model was used to compute the KDIGO class at day 90 . 
Results From March 2013 to December 2014, 93 patients were included. Median SAPS II at time of admission was 48 IQR [37.5; 62]. The median peak concentration of AMK was $74.7 \mathrm{mg} / \mathrm{I}$ IQR [62.4; 93.9], within the recommended range level according to national guidelines. Seventy-four percent of patients received only one injection of AMK. Thirty-one percent died before day 90 . Seventy percent of patients had an AKI when starting treatment. Among survivors at day $90,67 \%$ of patients had recovered their kidney function. Four patients had end-stage renal disease, all undergoing hemodialysis. No association between peak concentration of AMK and kidney function at day 90 was found (RR $=1$ (IC95 (0.99-1.01) $p=0.74$ ). In multivariate analysis, KDIGO class at day 90 was associated with the renal and non-renal components of SOFA score at admission, respectively, $\mathrm{RR}=1.44$ (IC95 (1.19-1.74); $p=0.0002)$ and $\mathrm{RR}=1.08$ (IC95 (1-1.17); $p=0.0469)$. Additional use of nephrotoxic drugs was not associated with renal outcome (for iodide contrast media in the week preceeding treatment $\mathrm{RR}=0.92$ (IC95 $(0.42-2.02) ; p=0.84)$, for iodide contrast media at day of treatment $\mathrm{RR}=1.04(\mathrm{IC} 95(0.40-2.73) ; p=0.93)$, for vancomycin $\mathrm{RR}=1.31$ (IC95 $(0.41-4.29) ; p=0.65)$, for calcineurin inhibitors $\mathrm{RR}=0.90$ (IC $95(0.32-2.53) ; p=0.84)$.

Discussion In our study, following guidelines, $75 \%$ of patients had a peak concentration of AMK above minimal concentration targets. AMK was used, regardless of kidney function at the time of the first dose. AKI class at day 90 was associated with initial intensity of extra-renal failures and kidney injury but not with the peak concentration of amikacin. The small number of patients enrolled and the high number of early death limit the power of our study.

Conclusion High-dose short-course once-daily regimen for treatment with AMK reaches the peak concentration target and may not worsen renal recovery at day 90 .

\section{Competing interests None.}

\section{S89}

Resuscitating chronic hypertensive patient with a high mean arterial pressure target during septic shock protects against acute renal failure

Julie Boisramé-Helms ${ }^{1}$, Ferhat Meziani ${ }^{1}$, Jean-François Hamel${ }^{2}$, François Beloncle ${ }^{2}$, Jean-Louis Teboul ${ }^{3}$, Peter Radermacher ${ }^{4}$, Pierre Asfar ${ }^{5}$

${ }^{1}$ Réanimation médicale, Nouvel Hôpital Civil, CHU Strasbourg, Strasbourg, France; ${ }^{2}$ Département de réanimation médicale et de médecine hyperbare, C.H.U. d'Angers, Angers, France; ${ }^{3}$ Réanimation médicale EA4533, Hôpital de Bicêtre, Université Paris-Sud, Le Kremlin-Bicêtre, France; ${ }^{4}$ Institut für anästhesiologische pathophysiologie und verfahrensentwicklung, Universitätsklinikum Ulm, Ulm, Germany; ${ }^{5}$ Réanimation, C.H.U. d'Angers, Angers, France

Correspondence: Julie Boisramé-Helms - julie.helms@libertysurf.fr Annals of Intensive Care 2016, 6(Suppl 1):S89

Introduction In SEPSISPAM study, targeting a mean arterial pressure (MAP) of $80-85 \mathrm{mmHg}$ in septic shock patients did not improve mortality compared to a lower target of $65-70 \mathrm{mmHg}$. However, the MAP that should be targeted to prevent organ dysfunction during septic shock may depend on patient medical history. The aim of the present analysis was to determine the MAP target for a specific patient profile, during septic shock resuscitation.

Patients and methods Six hundred and seventy-four of the 776 patients from SEPSISPAM prospective cohort were analyzed in per protocol, excluding patients with major protocol deviation (target pressure not reached because the vasopressor treatment was not adjusted; no septic shock) and patients for whom a decision not to resuscitate was taken after the inclusion.

Results The per-protocol analysis confirmed the intention-to-treat analysis, with no significant difference in mortality between the two groups at day 28 and day 90 . Chronic hypertensive patients resuscitated with a lowMAP target had a significantly increased risk of doubling of plasma creatinine (HR 2.14, $p=0.01$ ). Hypertensive patients without acute kidney injury at admission had a lower risk of doubling of creatinine $(p=0.01)$ when they were resuscitated with a high-target MAP compared to those in low-target group. Conversely, in patients with already installed acute kidney injury at inclusion, a high-target MAP was not associated with a better renal outcome as compared to the low-MAP target.

Discussion The MAP that should be targeted in patients with septic shock is still matter of debate. The main issue is probably that the optimal MAP target differs among patients, according to his/her medical history, and this is the point we specifically examined in the present analysis. When acute kidney injury is already installed, increasing MAP target might be detrimental due to increased vasopressor load, which in turn may increase adverse events and especially cardiac side effects. Conclusion Chronic hypertensive patients without acute kidney injury at admission benefit from resuscitation with high-MAP target during septic shock. Instead, when acute kidney injury is diagnosed, increasing MAP target might be detrimental.

\section{Competing interests None.}

\section{References}

1. Asfar P, Meziani F, Hamel JF, Grelon F, Megarbane B, Anguel N, Mira JP, Dequin PF, Gergaud S, Weiss N, Legay F, Le Tulzo Y, Conrad M, Robert R, Gonzalez F, Guitton C, Tamion F, Tonnelier JM, Guezennec P, Van Der Linden T, Vieillard-Baron A, Mariotte E, Pradel G, Lesieur O, Ricard JD, Herve E, du Cheyron D, Guerin C, Mercat A, Teboul JL, Radermacher P, Investigators S, (2014) High versus low blood-pressure target in patients with septic shock. N Engl J Med 370: 1583-1593.

S90

Guidewire exchange vs new-site placement for temporary dialysis catheters insertion in ICU patients: Is there a greater risk of colonization or dysfunction?

Elisabeth Coupez ${ }^{1}$, Jean-François Timsit ${ }^{2}$, Stephane Ruckly ${ }^{3}$, Carole Schwebel ${ }^{4}$, Didier Gruson ${ }^{5}$, Emmanuel Canet ${ }^{6}$, Kada Klouche $^{7}$, Laurent Argaud $^{8}$, Julien Bohé ${ }^{9}$, Maïté Garrouste-Orgeas ${ }^{10}$, François Vincent ${ }^{11}$ Sophie Cayot ${ }^{12}$, Michaël Darmon ${ }^{13}$, Alexandre Boyer ${ }^{14}$, Elie Azoulay ${ }^{6}$, Lila Bouadma ${ }^{15}$, Alexandre Lautrette ${ }^{16}$, Bertrand Souweine ${ }^{16}$

${ }^{1} \mathrm{CHU}$ Gabriel-Montpied, Clermont-Ferrand, France; ${ }^{2}$ Réanimation médicale et infectieuse, Hôpital Bichat-Claude Bernard, Paris, France; ${ }^{3}$ Reanimation, hôpital, Grenoble, France; ${ }^{4}$ Réanimation médicale, C.H.U. Grenoble, Grenoble, France; ${ }^{5}$ Réanimation médicale, Centre Hospitalier Universitaire de Bordeaux, Bordeaux, France; ${ }^{6}$ Réanimation médicale, Hôpital Saint-Louis, Paris, France; ${ }^{7}$ Service de réanimation, CHU Lapeyronie, Montpellier, France; ${ }^{8}$ Réanimation Médicale, Hospices Civils de Lyon - Groupement Hospitalier Edouard Herriot, Lyon, France; ${ }^{9}$ Service de réanimation, CHU Lyon Sud, Lyon, France; ${ }^{10}$ Réanimation, Fondation Hopital Saint Joseph, Paris, France; ${ }^{11}$ Réanimation polyvalente, Groupe Hospitalier Intercommunal Le Raincy-Montfermeil, Montfermeil, France;

${ }^{12} 63000$, CHU, Clermont-Ferrand, France; ${ }^{13}$ Réanimation Médicale, CHU Saint-Etienne - Hôpital Nord, Saint-Étienne, France; ${ }^{14}$ Réanimation médicale, Centre Hospitalier Universitaire de Bordeaux, Bordeaux, France; ${ }^{15}$ Réanimation médicale et des maladies infectieuses, Hôpital Bichat-Claude Bernard, Paris, France; ${ }^{16}$ Réanimation médicale, CHU Gabriel-Montpied, Clermont-Ferrand, France

Correspondence: Elisabeth Coupez - ecoupez@chu-clermontferrand.fr Annals of Intensive Care 2016, 6(Suppl 1):S90

Introduction Critically ill patients routinely require temporary dialysis catheters (DCs) for renal replacement therapy (RRT). They carry a high risk of developing end-stage renal disease. Though, their vascular accesses must be preserved. Guidewire exchange (GWE) is often used to avoid venipuncture at new site. However, the impact of GWE on infection and dysfunction of DC in ICU has never been studied. The aim of this study was to compare the effect GWE and venipuncture insertion (VPI) strategies on DC colonization and dysfunction in patients requiring $D C$ placement.

Patients and methods Using data from the ELVIS RCT (1496 critically ill adults requiring DC for RRT or plasma exchange), we performed a matched-cohort analysis with replacement. Cases were DCs inserted by GWE $(N=178)$ (only the first DC inserted by GWE was taken into account in patients with multiple GWE-DC placements), were matched with DCs inserted by VPI. Matching criteria were participating center, SAPS $\| \pm 10$, insertion site (jugular or femoral), side for jugular site, and duration between ICU admission and DC placement. DC colonization 
was defined by a quantitative DC-tip culture yielding $\geq 1000 \mathrm{CFU} / \mathrm{mL}$ with vortexing and $\geq 100 \mathrm{CFU} / \mathrm{mL}$ with sonication. DC dysfunction was defined as DC removal as a result of inadequate catheter flow despite attempts to restore DC patency.

A sensitivity analysis was performed on the specific subgroup of patients with a DC changed because of dysfunction of the previous one. We used a marginal Cox model to estimate the effect of DC insertion (GWE vs VPI) on DC colonization and dysfunction.

Results GWE-DC were not different from their matched NSP-DC for SAPS II [66 (53-84)] and rate of internal jugular catheters (25\%). However, GWE patients had longer median ICU length of stay (19 vs. 17 days; $P=0.004$ ) and median hospital length of stay (37.5 vs. 31 days; $P<0.01)$. DC colonization rate was not different between DC-GWX and DC NSP (10 (5.6 \%) vs 10 (6.7\%); hazard ratio (HR) 4.11 [0.14-122.32]; $P=0.41)$, but $D C$ dysfunction was more frequent with DC-GWX (67 (37.6 \%) vs 21 (14\%); HR, 3.56 [1.66-7.63]; $P<0.001)$. Result was similar if analysis was restricted to DC changed for dysfunction. DC-associated bloodstream infection was observed in $2 \mathrm{GWE}-\mathrm{DC}$ and in 1 VPI-DC patients.

Conclusion In ICU patients, as compared to VPI, GWE of DCs did not contribute to DC colonization infection but was associated with a more than twofold increase in DC dysfunction.

\section{Competing interests None. ${ }^{3}$}

\section{S92}

\section{Assessment of chloride levels on renal function after cardiac} arrest

Cláudia Righy Shinotsuka' ${ }^{1}$ Pietro Caironi ${ }^{2}$, Paola Villois ${ }^{1}$, Vito Fontana' Jean-Louis Vincent', Jacques Creteur', Fabio Silvio Taccone

${ }^{1}$ Service de soins intensifs, Hôpital Erasme, Bruxelles, Belgium; ${ }^{2}$ Dipartimento di fisiopatologia medico-chirurgica e dei trapianti, University of Milan, Milano, Italy

Correspondence: Cláudia Righy Shinotsuka - claudiarighy@hotmail.com Annals of Intensive Care 2016, 6(Suppl 1):S92

Introduction Restricting intravenous chloride administration in ICU patients decreased the incidence of acute kidney injury (AKI). Chloride may alter renal function by inducing vasoconstriction of renal afferent arterioles and cortical hypoperfusion. Also, tubular dysfunction may occur because of increased chloride delivery to the distal tube. There were no data on the effects of chloride balance on renal function on survivors from cardiac arrest (CA).

Aim: To investigate whether chloride serum and urinary levels as well as chloride load, balance and the anion urinary gap in the first day since admission are associated with the development of AKI among CA survivors.

Materials and methods Retrospective analysis of an institutional database including all adult comatose patients admitted to the Intensive Care Unit (ICU) after CA from January 2007 to December 2014. Inclusion criteria were as follows: age $\geq 18$; non-traumatic arrest; complete data available on serum and urinary samples for at least 3 days; survival $\geq 72 \mathrm{~h}$ after admission. Patients with previous $\mathrm{AKI}$ and with anuria on ICU admission and on chronic haemodialysis were excluded. Demographics, CA-related findings and outcome information were included in the database. We collected chloride levels on daily blood (BCI) and urinary ( $\mathrm{UCI}$ ) analyses; chloride load $(\mathrm{CL})$ was calculated by considering the amount of chloride present in the IV fluids administered daily to the patient, while chloride balance (CB) was calculated as: $\mathrm{CL}-\mathrm{CO}$, where $\mathrm{CO}$ is chloride output $(\mathrm{UCl} \times$ daily urine output). Creatinine clearance $(\mathrm{CrCl})$ was calculated on 24-h urinary collection. AKI was defined according to standard criteria.

Results Of the 404 eligible patients, 102 met the inclusion criteria and were eventually analysed. Median age was 58 [49-74] years, and

\footnotetext{
${ }^{3}$ S91 Postpartum acute renal failure: a multicenter study of risk factors in patients admitted to ICU. http://www.annalsofintensivecare.com/content/4/1/36
}

75 (70\%) were male. Median time to ROSC was 15 [9-25] min, and 46 (45\%) had a non-shockable initial rhythm. ICU mortality was $29 \%$ $(n=30)$. Forty-three patients ( $42 \%)$ developed AKI, after a median of 2 [2-4] days since admission; 13 of them (13\%) also needed continuous renal replacement therapy. Patients with AKI had similar serum creatinine on admission and 24-h fluid balance than others; however, 24-h urine output (1111 [448-1870] vs. 2130 [1125-2775] $\mathrm{mL}, p=0.004)$ and $\mathrm{CrCl}(21$ [11-44] vs. 104 [71-143] $\mathrm{mL} / \mathrm{min} ; p<0.001)$ were lower in AKI patients than those without AKI. Although the initial $\mathrm{BCl}(102$ [100-104] vs. 104 [101-107] $\mathrm{mEq} / \mathrm{L})$ and CL (341 [160-504] vs. 315 [201-436]) were similar between groups, patients with AKI had a lower $\mathrm{UCl}(66$ [33-95] vs. $106[61-138] \mathrm{mEq} / \mathrm{L} ; p=0.001)$ and CO (62 [11$125]$ vs. 177 [96-244] mEq; $p<0.001)$ than patients without AKI. This resulted in a higher CB (221 [68-462] vs. $125[6-296] \mathrm{mEq} ; p=0.02)$ and a trend towards higher AUG (45 [32-66] vs. 37 [24-54]; $p=0.16$ ) in AKI patients when compared to others.

Conclusion After CA, AKI occurred in $40 \%$ of patients and was associated with a significant alteration in renal chloride elimination, already during the first day. The pathological role of chloride and the impact of such findings on the management of fluid therapy in this setting remain to be further evaluated.

Competing interests None.

\section{References}

1. Nor'azim Mohd Yunos, Rinaldo Bellomo, David Story and John Kellum. Bench-to-bedside review: Chloride in critical illness. Critical Care 2010, $14: 226$.

2. Alexandre Toledo Maciel, Marcelo Park and Etienne Macedo. Physicochemical analysis of blood and urine in the course of acute kidney injury in critically ill patients: a prospective, observational study. BMC Anesthesiology 2013, 13:31

S93

Sustained low-efficiency dialysis with regional citrate anticoagulation is safe for patients with acute liver failure or cirrhosis

Franck Pourcine ${ }^{1}$, Ly Vong ${ }^{1}$, Jonathan Chelly' ${ }^{1}$ Sebastien Jochmans ${ }^{1}$, Oumar Sy ${ }^{1}$, Olivier Ellrodt ${ }^{1}$, Nathalie Rolin ${ }^{1}$, Jean-Emmanuel Alphonsine, ${ }^{1}$, Claire Marie Weyer', Jean Serbource-Goguel', Benoit Akando', Razach Abdallah ${ }^{1}$, Mehran Monchi ${ }^{1}$, Christophe Vinsonneau

${ }^{1}$ Service de réanimation, Centre Hospitalier Marc Jacquet, Melun, France Correspondence: Ly Vong - vong.lvp@free.fr

Annals of Intensive Care 2016, 6(Suppl 1):S93

Introduction Regional citrate anticoagulation (RCA) is one of the recommended procedures for extracorporeal circuit anticoagulation during renal replacement therapy (RRT) in intensive care unit (ICU), especially for high-bleeding risk patients. In liver failure, citrate metabolism is impaired with the risk of citrate accumulation. Total calcium/ ionized calcium ((Catot)/(Caion)) ratio is considered as a reliable sign of citrate overdose when greater than a 2.5 threshold. The aim of this study is to evaluate the security of RCA with a sustained low-efficiency dialysis (SLED) method on ICU patients with liver failure.

Materials and methods In this retrospective study, we collected data from all consecutive patients with acute liver failure or cirrhosis who required RRT on the hospitalization report from January 2014 to June 2015. All patients characteristics, severity score, and model of end-stage liver disease score (MELD score) were recorded. In addition to blood gas analysis, we measured total calcium and ionized calcium in serum at baseline and after each SLED run. The need of catecholamine and their doses are also recorded. For all SLED treatments, a blood flow (QB) of $150 \mathrm{~mL}$ / $\mathrm{min}$ and a dialysate flow (QD) of $300 \mathrm{~mL} / \mathrm{min}$ were applied. Sodium conductance was set to $150 \mathrm{mmol} / \mathrm{L}$ and bicarbonate to $35 \mathrm{mmol} / \mathrm{L}$. Each run lasted 6 to $12 \mathrm{~h}$. Sodium citrate and calcium chloride infusion was set according to a homemade protocol. The SLED run efficacy was evaluated by the online KT/N data delivered by the dialysis system.

Results A total of 41 patients who benefited from 104 RRT sessions are extracted from the database. Sixty-seven runs are exploitable due to lack of information for other runs. Nine patients (22\%) had cirrhosis, and 32 patients (78\%) had an acute liver failure. The simplified acute 
physiology score (SAPS II) is 71 [58-87]. Patients' MELD score is 27 [18.8-42]. None of the runs have thrombotic issue. There is no need of blood transfusion during RRT or $24 \mathrm{~h}$ after RRT. Noradrenaline infusion is not statistically different before and after the runs $(0.8 \mathrm{mg} / \mathrm{h}[0-3]$ vs $0.7 \mathrm{mg} / \mathrm{h}[0-4.3], p=0.80)$. None of the (Catot)/(Caion) ratio is greater than the 2.5 threshold. $\mathrm{pH}$ level is significantly higher at the end of the runs 7.22 [7.10-7.32] vs $7.34[7.24-7.40], p<0.001$. The mortality rate is $77.8 \%$ and $81 \%$, respectively, for patients with cirrhosis and acute liver failure.

Discussion Despite retrospective limitations of our study and low number of patients, RRT using SLED method with RCA appears to be safe in patients with severely impaired liver function. The efficacy, evaluated KT/V value is 0.88 [0.7-1] instead of 1.2-1.4 according to consensus, but it remains a secondary endpoint in these severely ill patients. Although there are no clinical complications and no indirect citrate overdose sign when using citrate on liver failure patients, there is no evaluation of the total amount of citrate infused to these patients with our SLED protocol.

Conclusion This study suggests that using RCA with SLED is safe for patients with acute liver failure or cirrhosis in ICU, who need RRT.

\section{Competing interests None.}

\section{S94}

The new biomarker Nephrocheck ${ }^{\mathrm{TM}}$ can discriminate the population of septic shock patients with an AKIN 1 or 2 acute renal failure who will not progress toward the AKIN 3 level Morgane Wetzstein', Loay Kontar', Dimitri Titeca', François Brazier?', Bertand De Cagny ${ }^{1}$, Gaelle Bacari-Risal ${ }^{1}$, Antoine Riviere', Michel Slama', Julien Maizel'

${ }^{1}$ Réanimation médicale, Centre Hospitalier Universitaire, Amiens, France Correspondence: Julien Maizel - maizel.julien@chu-amiens.fr Annals of Intensive Care 2016, 6(Suppl 1):S94

Introduction Patients hospitalized in intensive care unit (ICU) for septic shock have an increased risk to develop acute renal failure (ARF). A quarter of the patients developing ARF during septic shock will require a renal replacement therapy (RRT). The best delay to start RRT is a matter of debate. Starting too early can expose the patient unnecessarily to adverse event, while a proportion of those patients will restore spontaneously their renal function. Conversely starting too late the RRT exposes the patient to potential metabolic life-threatening complications. The French guidelines consider that starting RRT $48 \mathrm{~h}$ after the patient reached the KDIGO 3 level is late and starting RRT at the KDIGO 2 level is early. Therefore, if we could predict that a patient will not progress from a KDIGO level $\leq 2$ toward the KDIGO 3 level we would be able to discriminate the population that should not benefit of an early RRT. A new urine test, the Nephrocheck ${ }^{\mathrm{TM}}$ has been validated. It corresponds to the urine concentration product of 2 tubular suffering markers (TIMP2 and IGFBP7) associated with a risk of developing an ARF within $12 \mathrm{~h}$. The aim of our study was to analyze the capacity of the Nephrocheck ${ }^{\mathrm{TM}}$ to predict the absence of evolution toward the AKIN level 3 in a population of patients hospitalized for a septic shock who present an ARF AKIN 1 or 2

Patients and methods This was a prospective study. Every patient admitted for a septic shock in our 16 beds medical ICU who presented an ARF AKIN 1 or 2 within the first $12 \mathrm{~h}$ following the start of catecholamines was included. Exclusions criteria were therapeutic limitations, preterminal chronic renal failure (GFR $<15 \mathrm{ml} /$ min) or chronic dialysis, anuria, patients without ARF (AKIN 0) or with too severe ARF (AKIN 3). Nephrocheck ${ }^{\mathrm{TM}}$ urine assay was performed at baseline $(\mathrm{H} 0)$ and $\mathrm{H} 12$. Clinical and biological parameters (including AKIN staging) were collected at $\mathrm{HO}, \mathrm{H} 6, \mathrm{H} 12$ and $\mathrm{H} 24$. The performance of the Nephrocheck ${ }^{\mathrm{TM}}$ to predict the absence of evolution toward the AKIN 3 level within the first $24 \mathrm{~h}$ was tested for the baseline measurement and for the evolution of the Nephrocheck ${ }^{\mathrm{TM}}$ between baseline and $\mathrm{H} 12$ (delta $\mathrm{H} 0-\mathrm{H} 12$ ).

Results Fifty patients were included between May 2014 and September 2015. The median $(95 \% \mathrm{Cl})$ age was $63(60-66)$ years old, 31 $(62 \%)$ males and SAPSII 55 (50-61). The lung was the origin of sepsis in $70 \%$ of cases. At baseline ( $\mathrm{H} 0)$, the median arterial pressure was $73(67-78) \mathrm{mmHg}$, the median posology of noradrenaline 0.31 $(0.26-0.44) \mu \mathrm{g} / \mathrm{kg} / \mathrm{min}$, the median of fluid volume administered 15 $(9-20) \mathrm{ml} / \mathrm{kg}$, median urine output $0.57(0.4-0.8) \mathrm{ml} / \mathrm{kg} / \mathrm{h}$, median creatininemia $108(89-144) \mu \mathrm{mol} / \mathrm{l}$ and lactate $2.6(2.1-3.3) \mathrm{mmol} / \mathrm{l}$. At baseline 36 (72\%) were classified AKIN 1 and 14 (28\%) AKIN 2. Between $\mathrm{HO}$ and $\mathrm{H} 12,1$ patient died and 7 patients developed anuria. At the end of the following (24 h after baseline), 14 (28\%) patients had developed an AKIN 3 level ARF (group AKIN = 3) and 36 patients did not reached this level (AKIN $<3$ ). At $\mathrm{HO}$, the urine output was significantly different between the two groups of patients AKIN $<3$ versus AKIN $=3$ (respectively $0.71(0.4-1.5)$ versus 0.35 $(0.1-0.6) \mathrm{ml} / \mathrm{kg} / \mathrm{h} ; p=0.016)$. There was no difference between the 2 groups for the other variables at baseline. The Nephrocheck ${ }^{\mathrm{TM}}$ was significantly different between the two groups at HO (AKIN $<3$ versus $\mathrm{AKIN}=3$, respectively, $0.94(0.3-2.1)$ versus $5.96(2.5-11.6)$; $p=0.001)$ and at $\mathrm{H} 12(0.45(0.2-1.5)$ versus $3.6(1.1-9.7) ; p=0.01)$. The median delta $\mathrm{H} 0-\mathrm{H} 12$ was not significantly different between the groups $(-21 \%$ ( -66 to 150$)$ versus $-9 \%$ ( -61 to 57$) ; p=0.9)$. A Nephrocheck ${ }^{\mathrm{TM}}$ at $\mathrm{HO} \leq 2.1$ predict the absence of progression toward AKIN = 3 with a sensibility of $86 \%$ and a specificity of $78 \%$. A urine output at baseline $>0.37 \mathrm{ml} / \mathrm{kg} / \mathrm{h}$ predict the absence of progression toward AKIN $=3$ with a sensitivity of $57 \%$ and a specificity of $75 \%$. The area under the ROC curve of Nephrocheck at HO $(0.833$ (0.701-0.924)) was not significantly higher than the baseline urine output $(0.684(0.537-0.808) ; p=0.09)$.

Conclusion The Nephrocheck ${ }^{\mathrm{TM}}$ and the urine output at admission appear reliable to predict the absence of progression from AKIN 1 or 2 toward the AKIN 3 level within $24 \mathrm{~h}$ of patients hospitalized in ICU for a septic shock. In contrast baseline creatinine and delta Nephrocheck ${ }^{\mathrm{TM}}$ between baseline and $\mathrm{H} 12$ are not discriminating. Whether or not the Nephrocheck ${ }^{\mathrm{TM}}$ can help to avoid futile RRT in this population remains to be determined.

Competing interests Honoraria received from Astute.

\section{S95}

Is thrombocytopenia an early prognostic marker in septic shock?

Nadiejda Antier ${ }^{1}$, Christine Binquet ${ }^{2}$, Sandrine Vinault ${ }^{2}$, Ferhat Meziani ${ }^{3}$, Julie Boisramé-Helms ${ }^{4}$, Jean-Pierre Quenot ${ }^{5}$

${ }^{1}$ Réanimation, Ch Alès cévennes, Alès, France; ${ }^{2}$ Centre d'investigation clinique, Chu Dijon, Dijon, France; ${ }^{3}$ Réanimation médicale, Nouvel Hôpital Civil, CHU Strasbourg, Strasbourg, France; ${ }^{4}$ Réanimation, CHU de Strasbourg, Strasbourg, France; ${ }^{5}$ Réanimation médicale, CHU Dijon, Dijon, France

Correspondence: Nadiejda Antier - laitantier@gmail.com Annals of Intensive Care 2016, 6(Suppl 1):S95

Introduction Thrombocytopenia is the most common hemostatic disorder in the intensive care unit (ICU). It has been shown to be globally associated with a poor prognosis in ICU patients. However, no study has specifically investigated the impact of early thrombocytopenia in patients with septic shock.

We aimed to investigate the impact of nadir platelet count within the first $24 \mathrm{~h}$ after initiation of noradrenaline on the risk of death within the 28 days following the onset of septic shock.

Patients and methods We conducted a prospective, multicenter, observational cohort study. Consecutive adult patients with septic shock admitted in fourteen ICUs from 10 French university teaching and non-academic hospitals between November 2009 and September 2011 were eligible.

Thrombocytopenia was defined as a platelet count $\leq 150,000 / \mathrm{mm}^{3}$. We considered thrombocytopenic patients in 4 groups: platelet count $<50,000 / \mathrm{mm}^{3}$ or $[50,000-100,000] / \mathrm{mm}^{3}$ or $[100,000-150,000] / \mathrm{mm}^{3}$ or $>150,000 / \mathrm{mm}^{3}$. Patients were compared according to their platelet count using appropriate bivariate tests. Variables associated with the platelet count with a $p$ value $<0.25$ by bivariate analyses were included in a multivariate polytomic logistic regression to estimate their independent effect on platelet count. The probability of death at 28 days was estimated using the Kaplan-Meier method and compared using the log-rank test. Multivariate analyses were performed using Cox's proportional hazards model. 
Results Among 1495 eligible patients, 1486 (99.4 \%) were included. SAPSII score $\geq 56$, immunosuppression, age $>65$ years, cirrhosis, bacteraemia ( $p \leq 0.001$ for each) and urinary sepsis $(p=0.005)$ were globally associated with an increased risk of thrombocytopenia within the first $24 \mathrm{~h}$ following the onset of septic shock.

Survival at 28 days estimated by the Kaplan-Meier method was lower in patients with thrombocytopenia and decreased with thrombocytopenia severity. By multivariate Cox regression, a platelet count $\leq 100,000 / \mathrm{mm}^{3}$ was independently associated with a significant increase in the risk of death within the 28 days following septic shock onset. The risk of death increased with the severity of thrombocytopenia [hazard ratio $1.6595 \% \mathrm{Cl} 1.31-2.08$ for a platelet count below $50,000 / \mathrm{mm}^{3}$ vs $\left.>150,000 / \mathrm{mm}^{3}\left(p^{<} 0.0001\right)\right]$.

Discussion This is the first study to investigate thrombocytopenia within the first $24 \mathrm{~h}$ of septic shock onset as a prognostic marker of survival at day 28 in a large cohort of ICU patients. The increased mortality in septic thrombocytopenic patients deserves closer investigation. Current data suggest that it could be explained by the role of platelets in endothelial dysfunction and microthrombotic events leading to multiple organ dysfunction, and by a more direct pathway, since platelets can be considered as immune cells. Measuring platelet count is inexpensive and easily feasible for the physician in routine practice and thus could represent an easy "alert system" among patients in septic shock.

Conclusion This study shows that a platelet count $<100,000 / \mathrm{mm}^{3}$ during the first day after onset of septic shock is associated with a significantly increased risk of death at day 28.

\section{Competing interests None.}

\section{References}

1. Morrell CN, Aggrey AA, Chapman LM, et al. Emerging roles for platelets as immune and inflammatory cells. Blood 2014;123:2759-2767.

2. Vincent $\mathrm{L}$, Yagushi A, Pradier O. Platelet function in sepsis. Crit Care Med 2002;30:S313-S317.

\section{S96}

\section{Assessment of fibrinolysis in critically ill patients}

Yasmina Serroukh', Karim Zouaoui Boudjeltia ${ }^{2}$, Anne Joosten ${ }^{1}$, Alexandre Rousseau' ${ }^{2}$, Patrick Biston ${ }^{1}$, Michael Piagnerelli ${ }^{1}$

${ }^{1}$ Intensive Care Unit, C.H.U. De Charleroi, Charleroi, Belgium; ${ }^{2}$ Laboratoire de médecine expérimentale - université libre de bruxelles, C.H.U. De Charleroi, Charleroi, Belgium

Correspondence: Yasmina Serroukh - yasmina.serroukh@gmail.com Annals of Intensive Care 2016, 6(Suppl 1):S96

Introduction Critically ill patients present alterations of fibrinolysis, especially in the context of sepsis, and these alterations are associated with poor outcome. Euglobulin clot lysis time (ECLT) is the most commonly used test to estimate plasma fibrinolytic capacity and was correlated with organ dysfunction and C-reactive protein (CRP) levels in pilot studies. Our primary outcome was to study the correlation of ECLT with mortality in a large heterogeneous cohort of intensive care unit (ICU) patients. Our secondary objectives were to study the relationship of ECLT with organ dysfunction and inflammation.

Patients and methods After approval by the local ethical committee, we included patients with a predicted ICU length of stay of $48 \mathrm{~h}$. Exclusion criteria were transfusion of any blood product in the $48 \mathrm{~h}$ before inclusion, any known hemato-oncologic disorder, extracorporeal circulation, hemodia-filtration, burns, pregnancy and admission for thrombolysis. We assessed ECLT using a completely computerized, semi-automatic, 8-channel device (Lysis Timer, EREM, Belgium-reference values: $208 \mathrm{~min}$ for men and $117 \mathrm{~min}$ for women).

Fibrinolysis assessed by ECLT was correlated with ICU length of stay, ICU- and day-28 mortality. Organ dysfunction was assessed by the sequential organ failure assessment (SOFA) score, and inflammation was evaluated by measuring CRP concentrations. We used the MannWhitney nonparametric test to compare groups and the Spearman test for correlations. $P$ values above 0.05 were considered statistically significant. Results are expressed as median \pm interquartile ranges.
Results We included 121 patients with $(n=54)$ or without sepsis ( $n=67$ ) during the first $24 \mathrm{~h}$ of ICU stay. The admission SOFA score for all patients was 4 [2-7], and ICU- and day-28 mortality were 16 and $20 \%$, respectively. CRP level was 6.7 [2.2-18.6] mg/dl. Admission ECLT was 796 [488-1276] minutes in the whole cohort. Admission fibrinolysis was altered in septic as compared to non-septic patients: 1107 [588-1504] versus 628 [474-1130] minutes $(p=0.007)$. ECLT not correlated with mortality or organ dysfunction but positively correlated with the ICU length of stay $(R=0.55, p<0.0001)$. ECLT positively correlated with CRP in all patients $(R=0.39, p<0.0001)$ and in the septic group $(R=0.34, p=0.02)$ but not in non-septic patients $(R=0.17$, $p=0.2$ ).

Discussion We confirm that fibrinolysis assessed by ECLT is altered in critically ill patients, and this alteration is more pronounced in septic patients. ECLT does not correlate with mortality or organ dysfunction in our study, which could be due to low mortality rate and severity score of included patients. ECLT does, however, correlate with ICU length of stay. Interestingly, fibrinolysis impairment correlates with CRP levels. This relationship could be part of the link between inflammation and coagulation in critically ill patients, as suggested by murine and in vitro data. Indeed, specific effects of CRP on the fibrinolytic system were reported, including increased expression of the plasminogen activator inhibitor type 1 gene and decreased activity of tissue plasminogen activator.

Conclusion ECLT correlates with ICU length of stay but not with mortality or organ dysfunction. Fibrinolysis impairment correlates with CRP, which is a basis for further pathophysiological investigations on the link between inflammation and coagulation.

\section{Competing interests None.}

\section{S97}

Early detection of disseminated intravascular coagulation during septic shock: a multicentre prospective study Xavier Delabranche ${ }^{1}$, Jean-Pierre Quenot ${ }^{2}$, Thierry Lavigne ${ }^{3}$, Emmanuelle Mercier $^{4}$, Bruno François ${ }^{5}$, François Severac ${ }^{6}$, Lélia Grunebaum 7 , Laure Stiel $^{1}$, Florence Toti ${ }^{8}$, Ferhat Meziani ${ }^{9}$, Julie Boisramé-Helms ${ }^{10}$, CRICS Network

${ }^{1}$ Réanimation Médicale NHC, CHU de Strasbourg, Strasbourg, France; ${ }^{2}$ Réanimation médicale, CHU Dijon, Dijon, France; ${ }^{3}$ Infection Control Unit, CHU de Strasbourg, Strasbourg, France; ${ }^{4}$ Réanimation polyvalente, Centre Hospitalier Régional Universitaire de Tours, Tours, France; ${ }^{5}$ Service de réanimation polyvalente, Centre Hospitalier Universitaire de Limoges, Limoges, France; ${ }^{6}$ Laboratoire de biostatistique et d'informatique médicale, CHU de Strasbourg, Strasbourg, France; ' Laboratoire d'hématologie et hémostase, CHU de Strasbourg, Strasbourg, France; ${ }^{8}$ Umr7213,

Strasbourg University, Strasbourg, France; ${ }^{9}$ Réanimation médicale, Nouvel Hôpital Civil, CHU Strasbourg, Strasbourg, France; ${ }^{10}$ Réanimation, $\mathrm{CHU}$ de Strasbourg, Strasbourg, France

Correspondence: Xavier Delabranche - xavier.delabranche@chru-strasbourg.fr

Annals of Intensive Care 2016, 6(Suppl 1):S97

Introduction Inadequate stratification of septic shock patients may result in inappropriate treatment allocation in randomised clinical trials (RCTs), especially regarding anticoagulants. We previously reported that endothelial-derived microparticles (MPs) are relevant biomarkers of sepsis-induced disseminated intravascular coagulation (DIC). In this validation cohort, we assessed MPs as surrogates of cell activation to improve early DIC diagnosis and patient stratification.

Patients and methods This is a prospective observational study in septic shock patients in four French medical intensive care units. Two hundred and eighty-five patients with septic shock were consecutively enrolled. DIC was diagnosed according to JAAM 2006 score. Endothelial (CD105-MPs and CD31-MPs) and leucocyte-derived (CD11a-MPs) circulating procoagulant MPs were isolated and quantified by prothrombinase assay on admission (D1), D3 and D7. Clinicaltrial.gov identifier NCT \#02391792.

Results Two hundred and fifty-nine patients were analysed. Sixty-one had DIC on admission (DIC-D1), and 32 developed DIC during the first 
$24 \mathrm{~h}$ after admission (DIC-D2). These later patients were seriously ill with mortality as high as DIC-D1 patients. Multiple logistic regression model confirmed that endothelial cell-derived MPs were associated with DIC: CD105+-MPs (OR 2.13) and CD31+-MPs (OR 0.65) $(p<0.05)$. Furthermore, CD11a+-MPs to leucocyte ratio evidenced leucocyte activation (OR 1.59, $p<0.05)$. Prediction of DIC was also analysed after exclusion of DIC-D1 patients. A new multiple logistic regression analysis demonstrated the association of CD105 + -MPs ( $>0.60 \mathrm{nM}$ eq. PhtdSer, OR 1.67, $p<0.01)$, platelets count $(\leq 127 \mathrm{G} / \mathrm{L}, \mathrm{OR} 0.99, p<0.01)$ and prothrombin time $(\leq 58 \%$, OR $0.98, p<0.05)$ with DIC. A combining score on admission is predictive of the absence of DIC. The predictive equation was:

$$
\begin{aligned}
\text { preDIC }= & 1.342+(0.515 \times[\text { CD105+-MPs }]) \\
& -(0.012 \times[\text { Plt }])-(0.018 \times[\mathrm{PT}])
\end{aligned}
$$

with [CD105+-MPs] in nM eq. PhtdSer, [Plt] in G/L and [PT] in percentage (\%). The area under curve was $72.9 \%$ with $95 \%$ CI [66.2-78.9] $(p<0.001)$. Cut-off was -1.231 with a specificity of $71.2 \%$ [63.7-77.9], a sensitivity of $71.0 \%$ [52.0-85.8], a negative predictive value of $93.1 \%$ [72.5-99.6] and a positive predictive value of $31.0 \%$ [7.9-64.5].

Discussion DIC remains a challenge for many clinicians and biologists. Since coagulation constitutes a first line of defence in sepsis, accurate DIC diagnosis is essential to distinguish an appropriate patient to treat with an appropriate treatment at the right moment. DIC can be efficiently diagnosed on admission with JAAM 2006 score. Among patients who had not developed patent DIC on admission (JAAM < 4), some would present a so-called preDIC state retrospectively diagnosed during the following hours (JAAM $\geq 4)$. Early recognition of patients who will not develop DIC with a new score would be a valuable tool to avoid inappropriate antithrombin or thrombomodulin therapy. In our study, they represented about one-third of all DIC patients. ISTH "non-overt" score is not useful for DIC prediction as assessed by our false-positive and false-negative results. Non-overt DIC (according to ISTH) and non-patent DIC on admission (DIC-D2) may represent two different states of coagulopathy.

Conclusion Procoagulant MPs from endothelial cells and leucocytes reflect a vascular injury during sepsis-induced DIC that precedes obvious activation of coagulation. A combination of prothrombin time, endothelium-derived CD105+-MPs and platelet count on admission could predict the absence of DIC and allow a better stratification in future RCTs.

\section{Competing interests None.}

\section{S98}

Activation or dysregulation of coagulation during septic shock: How to stratify patients in 2016?

Xavier Delabranche ${ }^{1}$, Thierry Lavigne ${ }^{2}$, Laure Stiel', Julie Boisramé-Helms ${ }^{3}$ Achille Kouatchet ${ }^{4}$, Maleka Schenck ${ }^{5}$, Lélia Grunebaum ${ }^{6}$, Yoganaden Mootien ${ }^{7}$, Nicolas Lerolle ${ }^{4}$, Ferhat Meziani ${ }^{8}$

${ }^{1}$ Réanimation Médicale NHC, CHU de Strasbourg, Strasbourg, France; ${ }^{2}$ Infection Control Unit, CHU de Strasbourg, Strasbourg, France; ${ }^{3}$ Réanimation, CHU de Strasbourg, Strasbourg, France; ${ }^{4}$ Réanimation médicale, Centre Hospitalier Universitaire d'Angers, Angers, France; ${ }^{5}$ Réanimation médicale, C.H.R.U. Hôpitaux Universitaires Strasbourg, Strasbourg, France; ${ }^{6}$ Laboratoire d'hématologie et hémostase, CHU de Strasbourg, Strasbourg, France; ${ }^{7}$ Réanimation médicale, Hospital Center De Mulhouse, Mulhouse, France; ${ }^{8}$ Réanimation médicale, Nouvel Hôpital Civil, CHU Strasbourg, Strasbourg, France

Correspondence: Xavier Delabranche - xavier.delabranche@chru-strasbourg.fr

Annals of Intensive Care 2016, 6(Suppl 1):S98

Introduction Septic shock is associated with activation/dysregulation of blood coagulation, but assessment of this (unbalanced) adaptive response is still matter of debate and a clinical challenge. Despite experimental proof-of-concept, anticoagulant treatments failed to improve survival in large, randomised, placebo-control trials. Regarding coagulation, the inadequate stratification of septic shock patients could, at least in part, explain these negative results. This study was designed to evaluate haemostasis activation/dysregulation using routine and non-routine tests eventually combined according to DIC scoring systems.

Patients and methods Two hundred and sixty-five patients with septic shock were included in 3 medical intensive care units. Platelet count, prothrombin time, activated partial prothrombin time, fibrinogen, D-dimers, fibrin monomers, antithrombin, protein $C$ and prothrombin fragments $1+2$ were measured at admission.

Results DIC was present in 102/265 patients according to JAAM score. ISTH 'overt' and 'non-overt' scores allowed to diagnose 90 DIC and 88 pre-DIC, respectively, with a relative accordance between scores ( $k$ values were 0.78 (JAAM/'overt') and 0.70 (JAAM/'non-overt'). DIC diagnosis was associated with mortality when JAAM score was $\geq 5$ points (HR 0.53 [0.30-0.75], $p=0.006$ ). Patients were thereafter allocated to 3 groups according to their JAAM score as follows: 1-2 points (minimal, $n=128$ ), 3-4 (intermediate, $n=54$ ) and 5-8 (patent, $n=83$ ). Patients in the third group were more severely ill (SAPS2 and SOFA) with higher 28-day mortality, impaired fibrin formation and decreased inhibitors. Fibrinogen, FV, AT, PC and FM (not included in JAAM score) were not associated with DIC diagnosis in multiple logistic regression analysis. Nevertheless, abnormal values were frequently observed in septic shock patients regardless of DIC diagnosis: $56.2 \%$ for AT $(47.2 \%$ and $70.6 \%$ for non-DIC and DIC patients, respectively), $73.6 \%$ for PC (63.8 and $92.2 \%$ ) and $65.3 \%$ for FM (63.8 and $67.6 \%$ ). Fibrinogen was elevated (above $4 \mathrm{~g} / \mathrm{L}$ ) in $73.6 \%$ of patients ( 84.0 and $56.9 \%$, respectively). A low fibrinogen ( $<1 \mathrm{~g} / \mathrm{L}-\mathrm{ISTH}$ 'overt' cut-off) was present in seven patients (2.6\%), all of whom were diagnosed with DIC according to JAAM, ISTH 'overt' and 'non-overt' scores. Generated thrombin (evaluated by prothrombin fragments $1+2$ ) was elevated in two-thirds of the patients regardless the occurrence of DIC.

Discussion Physiology could explain why clinical trials, aiming at down-regulating thrombin generation, have been unsuccessful: thrombin is fundamental for survival during septic shock; it has multiple cellular and extracellular effects and may not be considered as a 'murderer' during septic shock. Excessive thrombin generation appears to play a different role in patients with or without DIC, and the lack of soluble fibrin monomers polymerization could be one. Fibrin polymerisation requires an activated transglutamase (FXIIla) to bridge soluble FM. Delayed platelet activation may explain the impairment in fibrin formation reported in DIC.

Interestingly, moderate thrombophilia, characterised by recurrent thrombosis, may favour survival during septic shock. Indeed, patients with aPC resistance (FVR506Q) had a better outcome. On the other hand, genetic (FVIII or FIX deficiencies) or drug-induced (vitamin K-antagonists) haemorrhagic diathesis failed to protect against experimental septic shock and death. Until now, anticoagulant treatment favoured survival in septic shock only when DIC was established. A better assessment of DIC may improve clinical trial design as well as patient survival.

Conclusion Low-grade activation of haemostasis assessed by JAAM score less than 5 could favour survival during septic shock. Only a rigorous scoring system may help clinicians to target the 'good treatments for the good patients'.

Competing interests None.

\section{S99}

Associated factors to early high blood pressure after weaning vasopressors during septic shock

Rostane Gaci', Jean-Romain Garric', Damien Barraud' ', Marie Conrad', Aurélie Cravoisy-Popovic ${ }^{1}$, Jérémie Lemarie ${ }^{1}$, Charles-Henri Maigrat', Pierre-Edouard Bollaert ${ }^{1}$, Sébastien Gibot ${ }^{1}$

${ }^{1}$ Réanimation médicale, Hôpital Central, C.H.U. de Nancy, Nancy, France

Correspondence: Rostane Gaci - rostane@msn.com

Annals of Intensive Care 2016, 6(Suppl 1):S99

Introduction During septic shock a life-threatening circulatory failure develops with inadequate tissue perfusion and vasoplegia. In this context, early development of arterial high blood pressure after weaning 
adrenergic vasopressors seems paradoxical. Regularly observed in our ICU, this phenomenon has not been described yet in the literature.

Patients and methods We conducted a descriptive, retrospective, single institution study to authenticate the existence of this empirical observation, specify its incidence, identify associated factors and possibly document a survival marker.

Results Two hundred and sixty-two septic shock patients were included. The median age was 66 years $(56-76)$, and median SOFA and IGS II scores were 10 (8-12) and 53 (39-77), respectively. The mortality calculated after exclusion of patients that underwent early life support therapy limitation was $28.7 \%$ at day $30(n=58)$ and $33.1 \%$ at day 90 $(n=62)$. Comparative analysis concerned 195 patients who survived to vasopressors weaning. Among them, $22 \%(n=43)$ received an early antihypertensive therapy within the $24 \mathrm{~h}$ following vasopressors weaning. Patients who received an early antihypertensive treatment after vasopressors weaning did not differ significantly in terms of age, severity scores, past history of high blood pressure or antihypertensive medication, chronic renal insufficiency, endocrinopathy, source of infection, volume resuscitation, weight gain during ICU stay, biological findings, or of norepinephrine treatment duration and maximum dose. In univariate analysis, patients with early antihypertensive therapy after vasopressors weaning presented more frequently gram-negative infection ( $33.6 \%, n=14$ vs $23.2 \%, n=35 ; p<0.001)$ and received more frequently dobutamine $(13.9 \%, n=6$ vs $5.8 \%, n=9 ; p=0.006)$, hydrocortisone opotherapy ( $55.8 \%, n=24$ vs $45.9 \%, n=70 ; p=0.02)$ and renal replacement therapy $(25.6 \%, n=11$ vs $15.3 \%, n=23$; $p=0.06$ ). In multivariate analysis, predictive factors for developing early high blood pressure were past history of ischemic heart disorder $(p=0.001)$ and support by mechanical ventilation $(p=0.01)$. Necessity of starting an early antihypertensive treatment after vasopressors weaning was associated with a better survival at day 30: $94.5 \%$ $(n=35 / 37)$ vs $82.5 \%(n=113 / 137), p<0.001$. In multivariate analysis, early high blood pressure development was predictive of survival at day 30 (OR $0.03, p=0.03$ ) whereas underlying rhythmic heart disorder seemed predictive of increased mortality (OR 24, $p=0.03$ ).

Discussion Our results suggest that the development of an arterial high blood pressure within $24 \mathrm{~h}$ following norepinephrine weaning is a frequent phenomenon affecting nearly a quarter of patients who survived the phase of initial resuscitation. The studied population was comparable to that of the recent studies about septic shock. General characteristics did not appear to be related with the arising of this event. Initial management (doses and modalities of norepinephrine and fluid resuscitation) did not seem to be involved either. The role of an underlying rhythmic or ischemic heart disorders could influence different load adaptation. Association with a favorable survival outcome at day 30 is limited but could reflect more advanced correction of tissue perfusion abnormalities at norepinephrine weaning time in patients requiring an early antihypertensive therapy.

Conclusion Development of an arterial high blood pressure within $24 \mathrm{~h}$ following vasopressors weaning in septic shock is a frequent event. It could be a protective marker regarding mortality at day 30 for patients who survived to vasopressors weaning. Physiopathological substratum of this phenomenon remains uncertain and needs further studies.

\section{Competing interests None.}

\section{S100}

Impact of endotracheal intubation on septic shock outcome: a post hoc analysis of the SEPSISPAM trial

Agathe Delbove ${ }^{1}$, Cédric Darreau ${ }^{2}$, Nicolas Lerolle ${ }^{3}$, Pierre Asfar ${ }^{4}$, CNER DESC réanimation grand ouest

'Département de soins intensifs de pneumologie, Centre hospitalier universitaire de Nantes, Nantes, France; ${ }^{2}$ Département de réanimation médicale et de médecine hyperbare, C.H.U. d'Angers, Angers, France; ${ }^{3}$ Réanimation médicale, Centre Hospitalier Universitaire d'Angers, Angers, France; ${ }^{4}$ Réanimation, C.H.U. d'Angers, Angers, France

Correspondence: Cédric Darreau - cedric.darreau@gmail.com

Annals of Intensive Care 2016, 6(Suppl 1):S100

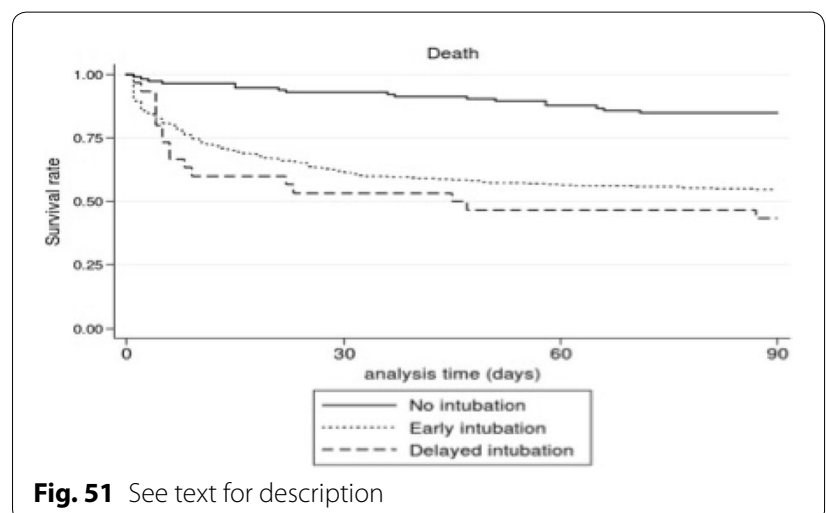

Introduction In large-scale trials, endotracheal intubation (EI) associated with mechanical ventilation is performed in $40-85 \%$ of patients with septic shock possibly indicating an important variation in practice across physicians. No study has been dedicated until now to define the place of this procedure in shock patients, and recent guidelines does not put forward any recommendation. The objective of the study was to determine the characteristics associated with endotracheal intubation in septic shock patients.

Patients and methods This is a post hoc analysis of the database of the SEPSISPAM study, including patients with septic shock.

Results Among the 776 SEPSISPAM patients, 633 (82\%) were intubated within $12 \mathrm{~h}$ of study inclusion (early intubation), $113(15 \%)$ were never intubated, and 30 (4\%) had delayed intubation. ICUs with at least 10 patients included were classified according to frequency of early intubation: early intubation $<80 \%$ of patients (lowest frequency: 7 ICUs, 254 patients), 80-90 \% (middle frequency: 5 ICUs, 170 patients), >90 \% (highest frequency: 6 ICUs, 297 patients). Type of ICU, pulmonary infection, lactate $>2 \mathrm{mmol} / \mathrm{l}$, lower $\mathrm{PaO}_{2} / \mathrm{FiO}_{2}$ ratio and absence of immunosuppression were independently associated with early intubation. Patients never intubated had a lower initial severity, less frequent pulmonary source of infection, were more likely to have immunosuppression, lower ICU and hospital length of stay and a low mortality rate. In comparison with patients intubated early, patients with delayed intubation had fewer days alive without organ support by day 28. Early El varied from 56 to $100 \%$ across ICUs. ICUs with the highest frequency of early intubation had a higher mortality rate in comparison with ICUs with middle frequency of early intubation. A nonsignificant increase in mortality was observed in ICU with lowest frequency of early intubation. No statistical difference was observed between the 3 types of ICU for university status, medical/surgical admission, number of beds per ICU and senior physician presence (Fig. 51).

Conclusion We observed a wide variation of early El practice across centers independent from acute severity parameters and presence of immunosuppression. Our result suggests that practices regarding endotracheal intubation in septic shock may impact outcome, which opens the way for future studies.

\section{Competing interests None.}

\section{References}

1. Impact of endotracheal intubation on septic shock outcome: A post hoc analysis of the SEPSISPAM trial. Delbove A, Darreau C, Hamel JF, Asfar P, Lerolle N. J Crit Care. 2015 Sep 1. doi: 10.1016/j.jcrc.2015.08.018.

2. High versus low blood-pressure target in patients with septic shock. Asfar P, Meziani F, Hamel JF, Grelon F, Megarbane B, Anguel N, Mira JP, Dequin PF, Gergaud S, Weiss N, Legay F, Le Tulzo Y, Conrad M, Robert R, Gonzalez F, Guitton C, Tamion F, Tonnelier JM, Guezennec P, Van Der Linden T, Vieillard-Baron A, Mariotte E, Pradel G, Lesieur O, Ricard JD, Hervé F, du Cheyron D, Guerin C, Mercat A, Teboul JL, Radermacher P; SEPSISPAM Investigators. N Engl J Med. 2014 Apr 24;370(17):1583-93. doi:10.1056/ NEJMoa1312173. 


\section{S101}

Corporal Contention at the Onset of Septic shock (COCOONs): study protocol and preliminary results of a phase II trial

Auguste Dargent ${ }^{1}$, Audrey Large ${ }^{2}$, Agnès Soudry-Faure ${ }^{3}$, Sebastien Prin ${ }^{2}$, Pierre-Emmanuel Charles ${ }^{4}$, Jean-Pierre Quenot ${ }^{5}$, COCOONs Study Group ${ }^{1} \mathrm{CHU}$ Dijon, Dijon, France, Dijon, France; 221000, Chu Dijon, Dijon, France; ${ }^{3}$ Direction de la recherche clinique et de l'innovation, CHU Dijon, Dijon, France, Dijon, France; ${ }^{4}$ Reanimation médicale, CHU Dijon, Dijon, France, Dijon, France; ${ }^{5}$ Réanimation médicale, CHU Dijon, Dijon, France

Correspondence: Auguste Dargent - olegg.d@gmail.com Annals of Intensive Care 2016, 6(Suppl 1):S101

Introduction Fluid overload in septic intensive care unit patients is common, and recent studies have demonstrated that it is strongly associated with a poor outcome. In fact, we think that fluid overload is mainly the reflection of the intensity of capillary leak. Capillary leak is a determining factor of shock in sepsis, leading to hypovolemia and microcirculation alteration. Thus, hypovolemia and edema are the two faces of one pathophysiologic change in wall of microvessels, i.e., capillary permeability. Unfortunately, no treatment is capable of decreasing vascular permeability in sepsis at present. Fluid replacement appears to be a poor symptomatic treatment, compensating only the leak flow and fueling formation of edema in the more elastic body tissues. Here, we propose an original therapeutic approach aiming to limit capillary leak in septic shock by means of total body contention. The principle of this method is to antagonize the distension of soft tissues of the trunk and limbs, which allows capillary fluid leak to continue. Subcutaneous tissue being by definition extensible (such as the cutaneous envelope), the volume they can "accept" can grow without a dramatic increase in pressure in the interstitial space. In this way, the pressure gradient stays favorable for the leak from vascular to interstitial compartments, despite the presence of edema. We hypothesize that capillary leak in septic shock can be quantitatively contained by limiting soft tissue extension at the onset of disease, using contention bandages on a large proportion of the body surface. The objective of this study is to evaluate the efficiency, tolerance and feasibility of a new mechanistic treatment based on this hypothesis.

Patients and methods This is a phase II multicenter single-arm trial in critically ill, non-surgical septic patients receiving mechanical ventilation. The study protocol was approved by an ethics committee (CPP). The therapeutic intervention is the application of contention bandages on more than $80 \%$ of the body surface, initiated at the onset of septic shock. Contention is maintained until fluid balance is negative on two consecutive days. We chose inelastic, short-stretch bandages to apply limited interface pressure on an already poorly perfused skin. Primary outcome is neutral or negative net fluid balance on day 7 (defined by a fluid balance $\leq+500 \mathrm{cc}$ ). Secondary outcomes are cumulative fluid balance, daily SOFA scores, death, duration of mechanical ventilation, occurrence of skin ulcers under bandages, reproducibility of interface pressure, monitoring of intra-abdominal pressure and monitoring of respiratory parameters correlated with chest wall compliance such as plateau pressure and dynamic compliance. The design is a two-staged Simon's plan, a methodology inspired by phase II cancer drug trials. Briefly, our primary outcome will be expressed as a response rate ( $\%$ of patients achieving a negative fluid balance at day 7) compared to existing data issued from a previous septic shock cohort recruited in our center. A sample size of 45 patients with available data at day 7 was calculated to demonstrate an increase in success rate of $20 \%$, from $50 \%$ (success rate of septic shock patients from our cohort) to $70 \%$.

Results The recruitment started on February 2015. We conducted a preliminary, intention-to-treat analysis with data issued from the first 22 included patients. Median Simplified Acute Severity Score (SAPS II) and Sequential Organ Failure Assessment (SOFA) are 65 and 12, respectively. Eleven patients among the $16(69 \%)$ with available data at day 7 have a neutral or negative fluid balance. Cumulative fluid balance at day 4 is $+5.9 \mathrm{~L}$. Adverse events are limited for the time being to minor skin ulcers (stage I) in 4 patients $(18 \%)$, which did not require contention retrieval. Monitoring of abdominal pressure revealed a slight increase with contention $(+2.5 \mathrm{mmHg})$, but no case of persistent abdominal hypertension is reported. Monitoring of airway pressure showed no significant increase in plateau pressure and dynamic compliance difficulties with mechanical ventilation, and in our clinical experience there were no respiratory issues attributable to contention. Conclusion The preliminary results of the COCOONs trial are encouraging, showing an increase in the percentage of patients with a neutral or negative fluid balance at day 7 compared to a local cohort, despite higher severity scores in our patients. The cumulative fluid balance at day 4 is almost half of what was reported in a reference study (Boyd et al, 2011). The tolerance is excellent. If those results are confirmed, a randomized controlled study will be conducted.

Competing interests None.

\section{S102}

\section{Veno-arterial ECMO for refractory cardiogenic shock}

\section{following cardiac arrest}

Marc Pineton De Chambrun', Nicolas Bréchot ${ }^{1}$, Guillaume Hekimian', Guillaume Lebreton², Matthieu Schmidt', Pierre Demondion'2, Ania Nieszkowska' ${ }^{1}$ Jean Louis Trouillet ${ }^{1}$, Pascal Leprince ${ }^{2}$, Jean Chastre', Alain Combes ${ }^{1}$, Charles-Edouard Luyt ${ }^{1}$

${ }^{1}$ Service de réanimation médicale, Groupe Hospitalier La Pitié-Salpêtrière, Institut de Cardiométabolisme et Nutrition, APHP, Paris, France; ${ }^{2}$ Service de chirurgie thoracique et cardiovasculaire, Groupe Hospitalier La Pitié Salpêtrière, Institut de Cardiométabolisme et Nutrition, APHP, Paris, France Correspondence: Marc Pineton De Chambrun - marc.dechambrun@ gmail.com

Annals of Intensive Care 2016, 6(Suppl 1):S102

Introduction Mortality of patients resuscitated from cardiac arrest (CA) remains particularly high, mainly due to post-cardiac arrest syndrome and anoxic cerebral lesions. Veno-arterial extracorporeal membrane oxygenation (VA-ECMO) is an effective technique to rescue patients with refractory cardiogenic shock (CS), which might benefit to some patients with refractory CS after CA. However, usefulness of VA-ECMO in refractory post-cardiac arrest syndrome is debated because most patients have profound vasoplegia, a condition in which VA-ECMO is ineffective. Furthermore, the unknown neurological prognosis at the time of implantation strongly questions the use of such an invasive and expensive technique. We therefore conducted a retrospective study on patients who received $\mathrm{VA}-\mathrm{ECMO}$ for refractory CS after CA to better describe the outcomes of such patients and to determine factors associated with poor outcome.

Patients and methods All patients who received VA-ECMO between March 2007 and January 2015 in our unit because of refractory CS after successfully resuscitated CA were included. Patients who received VAECMO for refractory $C A$ (E-CPR, ECMO under cardiopulmonary resuscitation) were not included. Continuous variables were expressed as mean (SD) or median [IQR25-IQR75] and compared using the Student's t test or Wilcoxon's rank test. Categorical variables were compared with Chi square tests. Multivariable analysis using logistic regression analysis was used to determine independent predictors of mortality.

Results We included the 94 patients who received VA-ECMO for refractory CS after CA. Sex ratio (male/female) was 3/1, mean age was 50.8 (1.5) years, and median admission SAPS II score was 82 [78$88]$. CA was due to acute myocardial infarction in 66 patients $(70 \%)$, acute decompensation of chronic cardiomyopathy in $8(8.5 \%)$, pulmonary embolism in 4 (4.3\%), drug intoxication in $3(3.2 \%)$, anaphylactic shock in $3(3.2 \%)$, and miscellaneous causes in 10 (10.6\%). CA occurred out of hospital for $78(83 \%)$ patients, and ventricular fibrillation was the first rhythm in $56(59.6 \%)$. Median no-flow and lowflow times were 0 [0-5] and 30 [15-42.75] min, respectively. Before VA-ECMO initiation, median left ventricle ejection fraction was 15 $[10-20] \%$ and aortic velocity time integral (VTI) 7 [5-9] $\mathrm{cm}$. Median time between cardiac arrest and VA-ECMO implantation was 7.4 [3.3-14] hours, and median duration of ECMO support was 4 [2-6] days. Fifty-five (59\%) patients died, while on VA-ECMO, 33 (35.1\%) were successfully weaned, $4(4.3 \%)$ were bridged to a left ventricle assist device and 2 (2.1\%) to heart transplantation. 28-day, 6-month and 12 -month survival rates were $29.8,27.7$ and $26.6 \%$, respectively. Causes of death were multiple organ failure $(65.2 \%)$, brain death 
(16.7\%), postanoxic encephalopathy (13.6\%) and CA recurrence $(4.5 \%)$. The 25 survivors at 1 -year had good neurological outcome (CPC 1). Univariable analysis retained serum lactate $>11.5 \mathrm{mmol} / \mathrm{L}$ (OR 5.6, $95 \% \mathrm{Cl}$ 2.1-15.2), prothrombin ratio <31\% (OR 7.1, $95 \% \mathrm{C}$ 2.4-20.9), admission SOFA score $>14$ (OR 9.1, $95 \%$ Cl 3.2-25.9), renal failure on admission (OR 8.6, $95 \% \mathrm{Cl} 2.9-25.6)$, SAPS 2 score $>82$ (OR $4.1,95 \% \mathrm{Cl} 1.5-10.9)$, ventricular fibrillation as first rhythm (OR 0.3, $95 \% \mathrm{Cl} 0.1-0.8$ ) as factors associated with 28-day mortality. According to multivariable analysis, independent predictors of 28-day mortality were serum lactate $>11.5(\mathrm{OR} 3.3,5 \% \mathrm{Cl} 1.1-10.3)$, prothrombin ratio $<31 \%$ (OR 4.4, $95 \% \mathrm{Cl} 1.3-14.5$ ) and admission SOFA score $>14$ (OR 4.2, $95 \% \mathrm{Cl}$ 1.3-13.4).

Conclusion In this retrospective cohort of extremely severe patients who received VA-ECMO for refractory CS after resuscitated CA, survival with good neurological outcome was similar to that of $C A$ patients who did not require ECMO support. Patients with multiple organ failures at the time of implantation had poorer outcomes, raising the question of futility in such patients. VA-ECMO should be considered as a rescue therapy in patients with refractory shock following resuscitated cardiac arrest. Further studies are, however, needed to better select patients who might benefit the most of this technique.

\section{Competing interests None.}

\section{S103}

\section{Outcome of patients with pulmonary embolism treated} by extra-corporeal life support (ECLS)

Jean-Christophe Blanchard', François Belon', Sydney Chocron², Nicolas Meneveau ${ }^{3}$, Loïc Barrot', Nicolas Belin 1 , Bérengère Vivet ${ }^{1}$, Guylaine Labro ${ }^{4}$, Jean-Christophe Navellou', Cyrille Patry', Gaël Piton', Gilles Capellier ${ }^{1}$ ${ }^{1}$ Réanimation médicale, CHU de Besançon, Besançon, France; ${ }^{2}$ Chirurgie cardiaque, CHU de Besançon, Besançon, France; ${ }^{3}$ Cardiologie, C.H. Régional Universitaire de Besançon, Besançon, France; ${ }^{4}$ Réanimation médicale, CHRU de Besançon, Besançon, France

Correspondence: Gaël Piton - piton.gael@wanadoo.fr

Annals of Intensive Care 2016, 6(Suppl 1):S103

Introduction High-risk pulmonary embolism (PE), requiring ICU admission, is associated with a high mortality rate. The potentiality for sudden and fatal hemodynamic deterioration needs early diagnostic and appropriate treatment. Veno-arterial extra-corporeal life support (ECLS) is useful for the management of obstructive shock. The aim of this study was to describe the outcome and management of patients treated with ECLS implantation for massive PE associated with obstructive shock or cardiac arrest in our university hospital.

Patients and methods This is a retrospective analysis of patients admitted to a medical ICU with massive PE and ECMO implantation.

Results From February 2009 to December 2014, 13 patients had implantation of ECLS for PE. The mean age was 41 year old, and there were mainly women $(77 \%)$. Six patients presented an extra hospital cardiac arrest, and 7 patients presented an obstructive shock. Three patients were still in cardiac arrest at ECLS implantation, and 2 patients presented a cardiac arrest during ECLS implantation. Six patients had surgical thrombectomy only; 3 patients had thrombolysis only; 2 patients had thrombolysis followed by surgical thrombectomy; 2 patients had anticoagulation only. Mean duration of ECLS was 4 days. Global mortality was $54 \%$ (7 patients) by cerebral anoxia (4 patients), hemorrhagic shock (2 patients) and stroke (1 patient). Only patients who had both ECLS and surgical thrombectomy survived.

Conclusion ECLS can be useful for the management of high gravity $\mathrm{PE}$ but the mortality of these patients is still high. The appropriate timing of ECLS implantation for high gravity PE needs to be further evaluated. In clinical practice, the decision of ECLS implantation must be early discussed by a team including intensivist, surgeon and cardiologist.

Competing interests None.
$\mathrm{S} 104$

Acute massive pulmonary embolism rescued by veno-arterial extracorporeal membrane oxygenation

Filippo Corsi', Guillaume Lebreton ${ }^{2}$, Nicolas Bréchot ${ }^{3}$, Guillaume Hékimian ${ }^{3}$, Ania Nieszkowska ${ }^{3}$, Charles-Edouard Luyt ${ }^{3}$, Pascal Leprince ${ }^{2}$, Jean Louis Trouillet ${ }^{3}$, Alain Combes ${ }^{3}$, Matthieu Schmidt ${ }^{3}$

${ }^{1}$ Dipartimento di anestesia e rianimazione, Policlinico Universitario A. Gemelli, Università Cattolica Del Sacro Cuore, Rome, Italy; ${ }^{2}$ Service de chirurgie thoracique et cardiovasculaire, Institut de Cardiométabolisme et Nutrition, Groupe Hospitalier Pitié-Salpêtrière, Paris, France; ${ }^{3}$ Service de réanimation médicale, Institut de Cardiométabolisme et Nutrition,

Groupe Hospitalier Pitié-Salpêtrière, Paris, France

Correspondence: Filippo Corsi - filippocorsi1@gmail.com

Annals of Intensive Care 2016, 6(Suppl 1):S104

Introduction High-risk acute massive pulmonary embolism (PE), defined as pulmonary embolism with the presence of persistent hypotension or shock, is a life-threatening condition, with very high mortality. Reperfusion therapy with either systemic thrombolytic agents, surgical pulmonary embolectomy or percutaneous catheter-directed treatment is mandatory for these very severe patients. Because of contraindications or important clinical instability, a small group of these patients cannot have access to reperfusion therapies. In these cases, veno-arterial extracorporeal membrane oxygenation (VA-ECMO) is one of the most reliable and quickest ways to decrease right ventricular overload, to improve hemodynamic status and to restore tissue oxygenation. We will describe herein our tertiary-care center experience in treating patients with acute massive high-risk PE who received VA-ECMO, and we will report their short- and long-term outcome.

Materials and methods We retrospectively collected demographic, admission severity scores, pre- and post-ECMO data, ECMO-related complications, and 90-day mortality of patients who received VAECMO for high-risk acute PE in our 26-bed intensive care unit (ICU) from June 2006 to June 2015. Patients who survived were contacted in September 2015 and assessed for health-related quality of life, psychological and post-traumatic stress disorder status.

Results Seventeen patients [11 females, median age 51 (range 18-70), simplified acute physiology score II (SAPS II) 78 (45-95)] were placed on VA-ECMO for high-risk pulmonary embolism, with a median duration of ECMO support of 4 days (1-12). Fifteen patients (82 \%) had preECMO cardiac arrest, with a low-flow time of $30 \mathrm{~min}(10-85), 7$ of them (41\%) were cannulated during cardio-pulmonary resuscitation. Two other patients had been placed on ECMO for refractory cardiogenic shock. Eight patients ( $47 \%$ ) received thrombolysis before ECMO, and 4 (24\%) were cannulated due to hemodynamic failure after percutaneous thromboaspiration or surgical pulmonary embolectomy. Pre-ECMO median values of mean blood pressure, $\mathrm{pH}$, blood lactate were, respectively, $42 \mathrm{mmHg}(0-106), 6.99$ (6.54-7.37) and $13 \mathrm{mmol} / \mathrm{L}$ (4-19). Major dilation of the right ventricle was noted in all patients; right ventricular/ left ventricular diameter ratio was 1.3 (0.7-1.6), and PE was diagnosed with chest CT scan in $12(70 \%)$ patients. 90-day mortality was $53 \%$. Fifteen patients $(88 \%)$ had severe hemorrhage during their ICU stay with no impact on survival. However, 90 -day survivors exhibited a lower inotrope score $24 \mathrm{~h}$ after cannulation $(p=0.001)$, and a lower SOFA score at days 1 and 3. ICU and hospital length of stay of the survivors' group were 17 (7-91) and 45 (22-135) days, respectively. Lastly, compared with ageand sex-matched controls, our seven-responding high-risk PE survivors had significantly lower SF-36 physical domain scores and role emotional component $(p<0.001) 19$ months (4-69) after ICU discharge.

Conclusion In this cohort of 17 high-risk PE patients, too unstable to tolerate at-risk thrombolytic interventions or who had failed first-line therapies, we observed that VA-ECMO can rapidly provide lifesaving hemodynamic support. Furthermore, we observed clot dissolution under conventional heparin therapy within a few days, questioning the benefit of additional therapies such as surgical thrombectomy or catheter-directed thromboaspiration in this setting.

Competing interests None. 
S105

Outcome of patient recused for extracorporeal membrane oxygenation for ARDS

Pierre-Emmanuel Meynieu', Benjamin Repusseau', Virginie Perrier' ${ }^{1}$ Arnaud Germain ${ }^{1}$, Antoine Dewitte ${ }^{1}$, Hadrien Roze ${ }^{1}$, Alexandre Ouattara ${ }^{1}$ Réanimation polyvalente, service d'anesthésie réanimation 2, CHU de Bordeaux, Bordeaux, France

Correspondence: Benjamin Repusseau - benjamin.repusseau@gmail. com

Annals of Intensive Care 2016, 6(Suppl 1):S105

Introduction Extracorporeal membrane oxygenation (ECMO) is proposed for patients suffering from severe acute respiratory distress syndrome (ARDS). ECMO allows maintaining oxygenation under protective ventilation until patient's recovery, but complications are not rare and can lead to fatal outcome. Despite few studies, the decision to initiate ECMO is controversial and an European consensus conference recommends referral centres associated with a circulatory support mobile unit available 24/7 and ready to intervene in all healthcare centres in the region concerned. 1 The aim of this study is to describe and compare outcomes of patient accepted for ECMO to those refused.

Materials and methods This is a retrospective study between 2012 and 2015 at the Universitary Hospital of Bordeaux. Indication of ECMO (veinoveinous) was decided using CESAR study criteria. 2 Recused patients stayed at the calling hospital and were categorized in "'contraindication" group $(\mathrm{Cl})$ and "to optimize" group (AO).

Results Seventy-two patients were analysed in the study, 19 (26\%) received $\mathrm{ECMO}$, and $53(74 \%)$ were refused $(34 \mathrm{Cl}$ and $19 \mathrm{AO}) . \mathrm{PaO}_{2} /$ $\mathrm{FiO}_{2}$ ratio on demand was 52 (IQR 45-63) in ECMO, 75 (IQR 62-83) in $\mathrm{AO}$ and 69 (IQR 54-81) in $\mathrm{Cl}(p=0.001)$. 90-day mortality was equivalent between ECMO and non-ECMO patients (53\%). Patients in AO group had the lower mortality $(21 \%)$, and their $\mathrm{PaO}_{2} / \mathrm{FiO}_{2}$ ratio increased significatively during the first $24 \mathrm{~h}(+71 \%, p<0.0001)$. There was no difference concerning the PRESERVE score between ECMO and non-ECMO patients (4 (IQR 3-5) vs. 5 (IQR 2-7), $p=0.144$ ), but this score was higher in the $\mathrm{Cl}$ group compared to the ECMO group (5.5 (IQR 4-8) vs. 4 (IQR 3-5), $p=0.004$ ). In the entire cohort, PRESERVE score was significantly higher in dead patients compared to those who survived (5 (IQR 4-7) vs. 3 (IQR $2-5$ ), $p=0.04$ ).

Discussion Mortality was high in both groups ECMO and non-ECMO, confirming severity of ARDS. Our study identifies a group of patients (AO) proposed to ECMO but, in our opinion, not enough severe to receive it immediately. These patients, with optimization of ventilator settings or implementation of adjuvant therapy, rapidly increase their oxygenation within $24 \mathrm{~h}$ and have a low mortality. Interestingly in non-ECMO patients, the PRESERVE score was higher in the $\mathrm{Cl}$ group. Conclusion This suggests that the decision to initiate ECMO is difficult. A referral centre with a high number of calls and a follow-up of recused patients might help to improve this decision and probably avoid ECMO with its associated complications in some cases.

\section{Competing interests None.}

\section{References}

1. Richard C, Argaud L, Blet A, et al. Extracorporeal life support for patients with acute respiratory distress syndrome: report of a Consensus Conference. Ann Intensive Care 2014;4(1):15.

2. Peek GJ, Mugford M, Tiruvoipati R, et al. Efficacy and economic assessment of conventional ventilatory support versus extracorporeal membrane oxygenation for severe adult respiratory failure (CESAR): a multicentre randomised controlled trial. Lancet 2009;374(9698):1351-63.

\section{S106}

Refractory electrical storm rescued by venoarterial extracorporeal membrane oxygenation

Guillaume Baudry', Guillaume Lebreton², Guillaume Hekimian ${ }^{1}$, Nicolas Brechot ${ }^{1}$, Ania Nieszkowska', Charles-Edouard Luyt ${ }^{1}$, Jean Louis Trouillet', Pascal Leprince ${ }^{2}$, Alain Combes ${ }^{1}$, Matthieu Schmidt ${ }^{1}$

'Service de réanimation médicale, Groupe Hospitalier Pitié Salpêtrière, Paris, France; ${ }^{2}$ Service de chirurgie thoracique et cardiovasculaire, Groupe Hospitalier Pitié Salpêtrière, Paris, France
Correspondence: Guillaume Baudry - guillaume.baudry@aphp.fr Annals of Intensive Care 2016, 6(Suppl 1):S106

Introduction Electrical storm refers to a state of cardiac electrical instability characterized by multiple episodes of ventricular tachycardia or ventricular fibrillation within $24 \mathrm{~h}$ despite antiarrhythmic drugs or device-related therapies (defibrillation or anti-tachycardia pacing) and for which overall mortality was $22-82 \%$ in previous series. Triggering factors for this condition include worsening heart failure, early postoperative period, electrolyte abnormalities, and myocardial ischemia. Since venoarterial extracorporeal membrane oxygenation (VA-ECMO) can be used as a rescue therapy in this setting, we reviewed our experience and assessed the outcomes of patients who received VA-ECMO for refractory electrical storm in our center.

Materials and methods We retrospectively collected demographic, pre-ECMO, post-ECMO data and in-ICU mortality of patients who received VA-ECMO for refractory electrical storm in our 26-bed intensive care unit from March 2007 to March 2015.

Results Of the 1286 VA-ECMO patients treated in our ICU during the study period, 38 had ECMO for refractory electrical storm after a median time of 24 (8-168) hours. Median age, SAPS II and SOFA scores at admission were 58 (49-62) years, $80(55-87)$ and $13(11-15)$, respectively. Electrical storm was secondary to myocardial infarction in 26 (68\%) patients. Cannulation was performed during and after cardiac arrest in $5(13 \%)$ and $17(45 \%)$ patients, respectively. Overall ICU survival was $47 \%$ (one patient got a heart transplant and one a left ventricular assist device). Survivors spent 5 (4-10) days on ECMO, 14 (9-25) days in ICU and 52 (34-59) days in hospital. Patients discharged alive from the ICU had lower body mass index, lower SOFA score before ECMO and at day 1 and received less inotropes in the $24 \mathrm{~h}$ following cannulation $(p=0.02)$. No difference was identified between alive and deceased patients regarding pre-ECMO anti-arrhythmic treatments and number of electrical shocks.

Conclusion Refractory electrical storm is a very severe condition that might be rescued by VA-ECMO support. The $47 \%$ ICU survival rate we observed in this series parallels that of other cardiogenic shock populations rescued by VA-ECMO. Earlier ECMO support in the course of the disease might allow a rapid decrease in inotrope doses and less organ failures, which were both associated with more favorable outcomes.

\section{Competing interests None.}

\section{S107}

Incidence and determinants of severe bleeding among patients with extra-corporeal membrane oxygenation (ECMO)

Lucie Hierle', François Belon', Guillaume Besch², Sebastien Pili-Floury²,

Loïc Barrot', Bérengère Vivet ${ }^{1}$, Guylaine Labro ${ }^{3}$, Jean-Christophe

Navellou', Cyrille Patry', Nicolas Belin', Gaël Piton', Gilles Capellier'

${ }^{1}$ Réanimation médicale, CHU de Besançon, Besançon, France; ${ }^{2}$ Réanimation chirurgicale, CHU de Besançon, Besançon, France; ${ }^{3}$ Réanimation médicale, CHRU de Besançon, Besançon, France

Correspondence: Gaël Piton - piton.gael@wanadoo.fr

Annals of Intensive Care 2016, 6(Suppl 1):S107

Introduction Bleeding and need for blood product transfusion are common among patients requiring extra-corporeal membrane oxygenation (ECMO). Transfusion is associated with a poor outcome in critically ill patients. Risk factors of bleeding among patients under ECMO are unknown. The aims of this study were to describe bleeding and to determinate risk factors of bleeding under ECMO.

Patients and methods From 2013 to 2014, we retrospectively analyzed all the patients admitted to two ICU in a university hospital that required the use of ECMO. Bleeding was defined with ELSO criteria (ELSO anticoagulation guidelines). Patients were studied according to the presence or absence of severe bleeding while they were under ECMO.

Results One hundred thirteen patients were included. One hundred two patients received venoarterial (VA) ECMO and 11 venovenous (VV) ECMO. ECMO runs for a median of 5 days. One hundred six bleeding were identified in 83 patients. Severe bleedings occured in $56(55 \%)$ 
patients with VA ECMO. Among them, 33 patients (59\%) had bleeding located on ECMO cannula. Other bleeding complications were intracranial bleeding in 4 patients, gastrointestinal bleeding, ear-nose bleeding and hemoptysis.

Totally, 849 red blood cells, 534 fresh frozen plasma and 209 platelets units were transfused. Predictive factors of severe bleeding were female sex (OR 2.8[1.4-10]), platelets <50G/L (OR 3.3 [1.3-8.7]) and antiagregant treatment (OR 3 [1.1-7.8]).

Conclusion Severe bleedings are highly more frequent during VA ECMO than VV ECMO: It occurs in more than an half of the patients with VA ECMO. Clinical management of these patients is difficult, and improving the management of severe bleeding under ECMO, in particular by defining the acceptable level of platelet count and hemostasis tests, is required.

\section{Competing interests None.}

\section{S108}

\section{Venovenous extracorporeal membranous oxygenation} device-related infections and colonizations

Guillemette Thomas ${ }^{1}$, Jean-Marie Forel ${ }^{1}$, Nadim Cassir ${ }^{2}$, Sami Hraiech', Christophe Guervilly', Fanny Klasen', Mélanie Adda', Stéphanie Dizier', Antoine Roch' ', Laurent Papazian'

${ }^{1}$ Réanimation détresses respiratoires et infections sévères, Hôpital Nord APHM, Marseille, France; ${ }^{2}$ Unité de recherche sur les maladies infectieuses tropicales et émergentes, Hôpital Nord APHM, Marseille, France

Correspondence: Guillemette Thomas - guillemette.thomas@hotmail.fr Annals of Intensive Care 2016, 6(Suppl 1):S108

Introduction Venovenous extracorporeal membrane oxygenation has become a widely accepted treatment option for life-threatening acute respiratory failure. To date, most of the studies described nosocomial infections or bloodstream infections occurring during extracorporeal membrane oxygenation support but very few studied infections directly related to extracorporeal membrane oxygenation devices. The main objective of this study was to evaluate the incidence of infections and colonizations related to extracorporeal membrane oxygenation device in venovenous extracorporeal membrane oxygenation adult patients.

Patients and methods We prospectively included all consecutive adult patients treated with venovenous extracorporeal membrane oxygenation for at least $48 \mathrm{~h}$ in our intensive care unit, from February 2013 to June 2015. At the time of extracorporeal membrane oxygenation removal, we systematically collected blood cultures, swabs on insertion cannula site (femoral and jugular) and intravascular cannula extremities. Then, we classified each extracorporeal membrane oxygenation support according to the infectious status in three groups: (1) uninfected/uncolonized extracorporeal membrane oxygenation device, (2) extracorporeal membrane oxygenation device colonization, and (3) extracorporeal membrane oxygenation device infection. Impact on outcome was analyzed.

Results Eighty-one patients underwent 85 venovenous extracorporeal membrane oxygenation support for a total of 1197 extracorporeal membrane oxygenation-days. The incidence of extracorporeal membrane oxygenation device infection was 6.7 per 1000 extracorporeal membrane oxygenation-days (8 events, $9.4 \%$ ) including 5 extracorporeal membrane oxygenation device-related bloodstream infections (4.2 per 1000 extracorporeal membrane oxygenation-days). The incidence of extracorporeal membrane oxygenation device colonization was 24.2 per 1000 extracorporeal membrane oxygenation-days (29 events, $34.1 \%)$. No difference was observed between the three groups, regarding days of mechanical ventilation, intensive care unit length of stay and mortality, and in-hospital mortality. We observed a longer extracorporeal membrane oxygenation duration (12 (9-20) days versus $7(5-15)$ days respectively, $p<0.05)$ and a higher proportion of male patients $(72.4 \%$ versus $54.2 \%, p<0.05)$ in extracorporeal membrane oxygenation device colonization group compared to uninfected/uncolonized extracorporeal membrane oxygenation device group, respectively.
Conclusion We performed a systematic analysis of extracorporeal membrane oxygenation device at the time of removal leading to the first description of incidence of extracorporeal membrane oxygenation device-related infections (6.7 per 1000 extracorporeal membrane oxygenation-days) and colonizations (24.2 per 1000 extracorporeal membrane oxygenation-days).

\section{Competing interests None.}

\section{References}

1. Roch, A. et al. Outcome of acute respiratory distress syndrome patients treated with extracorporeal membrane oxygenation and brought to a referral center. Intensive Care Med. 40, 74-83 (2014).

2. Schmidt, M. et al. Nosocomial infections in adult cardiogenic shock patients supported by venoarterial extracorporeal membrane oxygenation. Clin. Infect. Dis. Off. Publ. Infect. Dis. Soc. Am. 55, 1633-1641 (2012)

\section{S109}

High emergency lung transplant for end-stage cystic fibrosis: ECMO as a bridge to transplant for patients with temporary contraindications

Grégoire Trebbia', Kiruba Govindaradjou', Alexis Soummer², Jérôme Devaquet ${ }^{1}$, Clément Picard ${ }^{1}$, Antoine Roux ${ }^{1}$, François Parquin ${ }^{1}$, Elise Cuquemelle ${ }^{1}$, Morgan Leguen', Marie-Louise Felten ${ }^{1}$, Edouard Sage ${ }^{1}$, Alain Chapelier ${ }^{1}$, Charles Cerf ${ }^{1}$

192151, Hospital Foch, Suresnes, France; ${ }^{2}$ Réanimation, Hospital Foch, Suresnes, France

Correspondence: Grégoire Trebbia - gtrebbia@hotmail.com Annals of Intensive Care 2016, 6(Suppl 1):S109

Introduction Since 2007 in France, High Emergency Lung Transplant (HELT) combined with ECMO as a bridge to transplant represents an encouraging method to decrease mortality of patients on waiting list [1]. Acceptance for HELT is based on acute lung function worsening according to the contraindications. In our institution, we developed a new strategy for patients presenting temporary contraindications for HELT. These patients received ECMO as a bridge to evaluate before being considered eligible or not for HELT. We decided to evaluate this new strategy.

Patients and methods We reviewed the retrospective data from all patients with end-stage cystic fibrosis (CF) considered as potentially eligible for HELT and admitted in our institution since July 2007 to August 2015. The patients were classified into three groups: transplanted in HELT without ECMO (Group 1); transplanted in HELT with ECMO as a bridge to transplant (Group 2); potentially eligible for HELT but presenting one or more temporary contraindications for HELT and receiving ECMO as a bridge to wait for the recovery (Group 3). After this evaluation period, the patients were considered either eligible for HELT if the contraindication was corrected or definitively not if the contraindications persisted. Contraindications for HELT were based on the exclusion criteria defined by the French Organ Allocation Agency (l'Agence de la Biomédecine) associated with one local criteria: systemic infection, extrathoracic organ failure or multiorgan failure, denutrition (defined by an BMI $<14 \mathrm{~kg} \mathrm{~m}^{-2}$ ). For the patients not directly eligible for HELT, contraindications were listed at the admission in ICU and transplant was decided after a collegiate meeting with intensivists, lung specialists and thoracic surgeons. This study compares the patients waiting for HELT with temporary contraindications (Group 3) with the patient without contraindication (Group 2). The main outcome was the 3-month mortality.

Results Forty-nine patients with end-stage CF were admitted before HELT. Twenty-one (43\%) patients were transplanted without ECMO with a 3-month mortality of $14 \%$ (Group 1). Twenty-eight (57 \%) patients needed ECMO as a bridge to transplant or to evaluate before transplant. Among them, 16 patients presented no contraindications for HELT and were transplanted with a 3-month mortality of $0 \%$ (Group 2). Twelve (43\%) patients presented one or more temporary contraindications at the admission in ICU and received ECMO (Group 3). In this group, 3 patients were transplanted after correction of the contraindication. All of them were still alive within 3 months. Four patients were transplanted after incomplete correction of the 
contraindications. One of them presented a subocclusive syndrome, one presented an unknown Cepacia Syndrome, and two presented a persistent renal failure. All of them died within 3 months. Four patients were definitively considered unfit for transplantation, and one did not survive during the evaluation period. The 3-month mortality in the Group 3 was higher than that in the Group 2 (75 vs $0 \%, p<0.0001$ ). Conclusion ECMO could be used as a bridge to evaluate temporary contraindicated patients for HELT and allowed to transplant them after complete correction of the contraindications. However, this strategy should be used carefully to limit the risk of transplant with persistent contraindications.

\section{Competing interests None.}

\section{Reference}

1. M Lafarge MD, Pierre Mordant MD, Gabrien Tabut MD et al Experience of extracorporeal membrane oxygenation as a bridge to lung transplantation in France J Heart Lung Transplant. 2013 Sep;32(9):905-13.

\section{S110}

Malignant acute respiratory distress syndrome in diffuse lepidic adenocarcinoma (DLA): diagnosis and current issues

Maxens Decavèle ${ }^{1}$, Antoine Parrot ${ }^{1}$, Michael Duruisseaux ${ }^{2}$, Jocelyne

Fleury ${ }^{3}$, Martine Antoine ${ }^{3}$, Marie-France Carette ${ }^{4}$, Marie Wislez ${ }^{5}$, Muriel Fartoukh ${ }^{1}$

${ }^{1}$ Réanimation médico-chirurgicale, Hôpital Tenon, Paris, France; ${ }^{2}$ Service de pneumologie, C.H.U de Grenoble C.H.U, La Tronche, France; ${ }^{3}$ Département d'anatomie et cytologie pathologiques, Hôpital Tenon, Paris, France; ${ }^{4}$ Service de radiologie, Hôpital Tenon, Paris, France; ${ }^{5}$ Service de pneumologie, Hôpital Tenon, paris, France

Correspondence: Maxens Decavèle - maxencesar@hotmail.fr Annals of Intensive Care 2016, 6(Suppl 1):S110

Introduction Acute respiratory failure (ARF) is the leading cause for ICU admission of lung cancer patients. Despite its poor prognosis, recent series suggest a significant reduction in both ICU- and hospitalmortality rates. The diffuse lepidic adenocarcinoma (DLA), formerly diffuse bronchioloalveolar carcinoma, represents a rare specific pattern of worse prognostic lung neoplastic injury, with severity ranging from symptoms of limited expression to life-threatening ARDS. Mimicking non-resolving pneumonia or non-infectious inflammatory process, its diagnosis remains challenging and often delayed. Our purpose was (1) to provide a rigorous and reliable diagnosis reasoning process for guiding an early and targeted management susceptible to modify the prognosis and (2) to identify factors associated with ICU- and 90-day mortality.

Patients and methods This is a retrospective study including all consecutive patients with diffuse lepidic adenocarcinoma (DLA)-related ARDS, admitted to the intensive care unit (ICU) of Tenon hospital, Paris, France, from January 1994 to December 2014. Patients were classified according to the Berlin definition. DLA diagnosis was made on either histological or cytological samples. Specific clinical, biological, radiological and cytological patterns conducting to the diagnosis were recorded. Performance of lung sampling procedures was compared to other center when performed in other structures before ICU admission. Continuous variables were expressed as median and interquartile range and categorical variables as absolute value and relative frequencies. Univariable analysis was performed on ICU- and 90-day mortality.

Results A total of 23 patients were included ( 17 men, age 71 $[61 ; 76])$. Diagnosis was made during the ICU stay in $83 \%$ of cases. All patients presented with non-resolving pneumonia (median antibiotic lines $2[2 ; 3])$, combining, smoking history (78 \%), bronchorrhea $(83 \%)$, salty expectoration (64\%), significant weight loss $(43 \%)$, without fever ( $25 \%)$. Radiological presentation was a bilateral consolidation (95\%) with air bronchogram (90\%), base predominance $(75 \%)$, fissural bulging (55\%) and cavitations (40\%) associated with bronchiolar nodules (60\%). Bronchoalveolar lavage showed intense neutrophil alveolitis (total count 520,000 [240,000; $900,000]$, with $64 \%[48 ; 84]$ of neutrophils) and confirmed the diagnosis in $50 \%$ of cases with a better yield than in the previous
Table 58 Univariable analysis on ICU mortality

\begin{tabular}{|c|c|c|c|}
\hline Variable & ICU death $n: 5$ & ICU survival $n: 21$ & $P$ value \\
\hline $\begin{array}{l}\text { Mediastinal lymphad- } \\
\text { enopathy }\end{array}$ & $2(40 \%)$ & $1(5 \%)$ & 0.017 \\
\hline Heart rate & $115[103 ; 128]$ & $102[92,113]$ & 0.040 \\
\hline Respiratory rate & $38[32 ; 43]$ & $26[22 ; 31]$ & 0.018 \\
\hline $\begin{array}{l}\text { Delay first imaging/ } \\
\text { diagnosis }\end{array}$ & $144[126 ; 157]$ & $131[122 ; 145]$ & 0.027 \\
\hline
\end{tabular}

structures (50 vs $9 \%, p=0.027$ ). Sputum examination brought diagnosis in 5 patients. ICU- and 90-day mortality was 22 and $35 \%$, respectively. In univariable analysis, variables associated with ICUmortality were mediastinal lymphadenopathy, delay between first imaging and diagnosis, heart rate and respiratory rate on admission (Table 58).

Conclusion We reported the largest series of DLA-related ARDS. Simple rigorous physical examination and rapid on-site sputum or bronchoalveolar lavage examination may provide reliable diagnosis. Clinician should be aware of this atypic ARDS since an early recognition may impact prognosis. Prognosis is similar to the prognosis of other solid tumors in the ICU.

\section{Competing interests None.}

S111

Acute respiratory failure from pulmonary leukemic infiltrates: diagnosis contribution of high-resolution computed tomography (HRCT)

Claire Givel ${ }^{1}$, Virginie Lemiale ${ }^{2}$, Constance De Margerie-Mellon ${ }^{3}$, Etienne Lengliné ${ }^{4}$, Leïla Mourtada ${ }^{5}$, François Vincent ${ }^{6}$, Akli Chermak ${ }^{7}$, Eric De Kerviler $^{8}$, Elie Azoulay ${ }^{1}$

${ }^{1}$ Réanimation médicale, Hôpital Saint-Louis, Paris, France; ${ }^{2}$ Medical ICU, Hôpital Saint Louis, Paris, France; ${ }^{3}$ Radiologie, Saint Louis, Paris, France; ${ }^{4}$ Hématologie, Hôpital Saint Louis, Paris, France; ${ }^{5}$ Réanimation médicale, Hôpital Saint Louis, Paris, France; ${ }^{6}$ Réanimation polyvalente, Groupe Hospitalier Intercommunal Le Raincy-Montfermeil, Montfermeil, France; ${ }^{7}$ Réanimation polyvalente, $\mathrm{CH}$ sud Essonne, Paris, France; ${ }^{8}$ Radiologie, Hopital Saint Louis, Paris, France

Correspondence: Claire Givel - claire.givel@hotmail.fr Annals of Intensive Care 2016, 6(Suppl 1):S111

Introduction Acute respiratory failure (ARF) in patient at the earliest phase of acute myeloid leukemia (AML) is related to either usual (infection, overload) or specific etiologies. The latter include three different groups, namely pulmonary leukemic infiltration (PLI), leukostasis and acute lysis pneumopathy (ALP). Clinical and biological data are useful to precisely separate the three groups; however, they lack specificity. Yet, making the appropriate diagnosis is crucial as therapeutic strategy varies significantly across the three groups. In that setting, high-resolution computed tomography (HRCT) could be an interesting tool to better characterize patterns of pulmonary involvement. We report HRCT findings in 29 patients with leukemic pulmonary infiltrates.

Patients and methods Critically ill patients at the earliest phase of untreated AML were included. ARF was defined by presence of tachypnea, labor breathing, respiratory distress and/or need for $>5 \mathrm{l} / \mathrm{min}$ oxygen or mechanical ventilation. Patients who underwent HRCT within 2 days of respiratory symptoms onset were included, and their clinical and biological data were collected. ARF etiology was confirmed by 2 senior intensivists with pulmonary background and extensive experience in managing AML. HRCT were independently analyzed by two clinicians and a radiologist. Disagreements were discussed until a consensus was reached.

Results Twenty-nine patients, from 18 to 74 years old, were included. AML was mostly monocytic leukemia (Fab 4 or 5 ) with median leukocyte count of 108 (IQR $1-3(39.1 ; 224.9)$ G/L at ICU admission. 
Table 59 HRTC signs according to ARF etiology

\begin{tabular}{|c|c|c|c|c|}
\hline \multicolumn{2}{|c|}{ HACT patterns } & Leukostersis ( $n=7$ ) & PU (n=13) & APL $\langle n=B]$ \\
\hline \multicolumn{2}{|c|}{ Ground glass apacties } & 3 (43\%) & $3(23 \% 6)$ & 2 (33\%6) \\
\hline \multirow[t]{2}{*}{ Nodules } & peritrenchovisculat & $2(29 \%)$ & $3(23 \%)$ & $3(50 \%)$ \\
\hline & centrolobular & $1(14 \%)$ & $2(15 \%)$ & $3(50 \%)$ \\
\hline \multicolumn{2}{|c|}{ Iil defined Consolidation } & $3(43 \%)$ & $4(31 \%)$ & $1(17 \%)$ \\
\hline \multicolumn{2}{|c|}{ Peribronchovascular thicksaning } & $1(14 \%)$ & 0 & $1(17 \%)$ \\
\hline
\end{tabular}

Hematological diagnosis was performed 1 day before admission [IQR 1-3 $(0 ; 1)]$. At admission, mean respiratory rate was $24 \pm 7 /$ min (SD) and mean oxygen flow was $4 \pm 4 \mathrm{l} / \mathrm{min}$. Patients had extra-pulmonary signs, including fever (70 \%), gingival hypertrophy (34\%) or neurological symptom (24\%). During ICU, 9 (31\%) patients needed vasopressors and $5(17 \%)$ were intubated for $13(5-34)$ days. Twenty-six patients $(90 \%)$ received chemotherapy during ICU. All patients received antibiotics and $17(59 \%)$ patients received rescue steroids (dexamethasone $10 \mathrm{mg} \times 4 / \mathrm{j})$ until neutropenia. ICU mortality was $21 \%$, and median ICU length of stay was 6 [IQR 1-3 $(4 ; 9)]$ days. ARF etiology included leukostasis for 7 patients, PLI for 13 patients and APL for 6 patients. For three patients, association of leukemic infiltrates with infection (one patient), atelectasis (one patient) or edema (one patient) precluded HRCT evaluation. HRCT findings included ill-defined consolidation $(17,59 \%)$, mainly diffuse $(10,71 \%)$, ground-glass opacities $(14,48 \%)$, nodules $(10,34 \%)$, peribronchovascular thickening $(8,28 \%)$ or interlobular septal thickening (8.28\%). Table 59 shows HRCT signs in the 3 groups.

Kappa coefficient for interrater agreement was 0.863 [IC95 $(0.681 ; 1)$ ] for consolidation, 0.862 [IC95 $(0.677 ; 1)$ ] for ground-glass opacities, 0.473 [IC95 $(0.209 ; 0.737)$ ] for peribronchovascular thickening and 0.627 [IC95 $(0.333 ; 0.922)]$ for nodules.

Conclusion HRCT may be helpful to assess precise ARF etiology at the earliest phase of AML in patients with leukemic pulmonary infiltrates. These preliminary data can be used as a framework for validation at a larger scale. Studies to confirm benefits from HRCT in these particular patients are warranted.

\section{Competing interests None.}

\section{S112}

Characteristics and outcome of patients with interstitial lung disease admitted to the intensive care unit over a 15-year period

Yacine Tandjaoui-Lambiotte1 , Frédéric Gonzalez ${ }^{1}$, Olivia Freynet², Guillaume Van Der Meersch', Johanna Oziel' ', Christophe Huang ${ }^{1}$, Philippe Karoubi ${ }^{1}$, Christophe Clec'h ${ }^{1}$, Yves Cohen $^{1}$

${ }^{1}$ Réanimation médico-chirurgicale, Hôpital Avicenne, Bobigny, France;

${ }^{2}$ Pneumologie, Hôpital Avicenne, Bobigny, France

Correspondence: Yacine Tandjaoui-Lambiotte - yt|r82@gmail.com Annals of Intensive Care 2016, 6(Suppl 1):S112

Introduction Interstitial lung diseases (ILD) are a heterogeneous group of diseases, which can be life-threatening in case of acute respiratory failure. Until the last decade, few patients with ILD were admitted to the intensive care unit (ICU), due to the presumably poor prognosis and futility of mechanical ventilation. However, protective ventilation, spread of noninvasive ventilation, and increased consideration for urgent lung transplantation have undoubtedly changed the admission criteria and management of these patients.

The aim of this study was to describe the epidemiology of ILD patients admitted to the ICU over the past 15 years and to assess whether characteristics and outcome of these patients have changed over time.

Patients and methods We retrospectively analyzed all ILD patients admitted to the ICU of our university hospital from January 2000 to December 2014.

The following data were recorded: age, severity scores, etiology of the ILD, live-sustaining therapies, and ICU mortality.

We compared three periods:
- 2000-2006: when protective ventilation was not systematic in acute lung injury,

- 2007-2011: when protective ventilation was systematic but neither urgent lung transplantation nor ECMO were available, and

- 2011-2014: when all conditions were available.

Results During the last 15 years, 196 ILD patients were admitted to our ICU. Mean age was $64.3 \pm 12.6$ years, and mean SAPS II was $39.5 \pm 19.3$ points. Overall mortality was $47.9 \%$. Etiologies of ILD were as follows: idiopathic pulmonary fibrosis $(n=51,26 \%)$, connective tissue disease $(n=42,21.4 \%)$, miscellaneous $(n=61,31.2 \%)$ and unclassified $(n=42,21.4 \%)$.

Regarding ventilatory support, 105 (53.7\%) patients required mechanical ventilation (MV), among which 78 (74 \%) died. MV was started in $42 / 67$ patients $(62.6 \%)$ after failure of noninvasive ventilation, with a subsequent mortality rate of $76.2 \%$. The remaining 17 patients were discharged alive. Of the 9 patients who initially received high-flow nasal oxygen, 6 further required $\mathrm{MV}$, of whom 4 died.

Twelve patients were considered for urgent lung transplantation and transferred to referral centers for ECMO in the meantime (data on outcome pending).

Among nonventilatory supports, catecholamines and renal replacement therapy were provided to $80(41 \%)$ and $17(8.7 \%)$ patients, respectively, and were associated with mortality rates above $90 \%$. Regarding changes over time, the number of ILD patients admitted to the ICU kept increasing but no significant modification in their management was observed.

Finally, mortality remained constant throughout the three periods. Yet, observed mortality was much superior to mortality as predicted by the SAPS II score.

Conclusion ICU mortality of ILD patients is high and remains stable despite global improvements in ventilatory support. Nevertheless, a significant proportion of ILD patients may survive.

Our data suggest that ILD per se should not be considered as a ground for ICU admission denial.

Further analyses are warranted to identify specific prognostic factors, and subgroups of ILD patients likely to benefit most from ICU care.

Competing interests None.

S113

Outcome of patients admitted in intensive care unit for acute manifestation of small vessel vasculitis

Antoine Kimmoun ${ }^{1}$, Elisabeth Baux ${ }^{1}$, Vincent Das ${ }^{2}$, Nicolas Terzi ${ }^{3}$, Patrice Talec ${ }^{4}$, Stephan Ehrmann ${ }^{5}$, Guillaume Geri ${ }^{6}$, Steven Grange ${ }^{7}$, Nadia Anguel ${ }^{8}$, Alexandre Demoule ${ }^{9}$, Anne-Sophie Moreau ${ }^{10}$, Elie Azoulay ${ }^{11}$, Jean-Pierre Quenot ${ }^{12}$, Julie Boisramé-Helms ${ }^{13}$, Guillaume Louis ${ }^{14}$, Romain Sonneville ${ }^{15}$, Nicolas Girerd ${ }^{16}$, Bruno Levy ${ }^{1}$

${ }^{1}$ Réanimation Médicale Brabois, Centre Hospitalier Universitaire de Nancy, Vandœuvre-lès-Nancy, France; ${ }^{2}$ Réanimation polyvalente adulte, Centre Hospitalier Intercommunal André Grégoire, Montreuil, France; ${ }^{3}$ Service de réanimation médicale, Clinique de Réanimation Médicale, Grenoble, France; ${ }^{4}$ Réanimation médicale, C.H.U. d'Angers, Angers, France; ${ }^{5}$ Réanimation polyvalente, CHRU Hôpitaux de Tours, Tours, France; ${ }^{6}$ Réanimation Médicale, Hôpital Cochin, Paris, France; ${ }^{7}$ Réanimation médicale, Centre Hospitalier Universitaire Rouen, Rouen, France; ${ }^{8}$ Réanimation médicale, CHU de Bicêtre, Le Kremlin Bicêtre, France; ${ }^{9}$ Service de pneumologie et réanimation médicale, Groupe Hospitalier Pitié-Salpêtrière, Paris, France; ${ }^{10}$ Réanimation Médicale, Hôpital Saint-Louis, Assistance Publique Hôpitaux de Paris, Paris, France;

${ }^{11}$ Réanimation médicale, Hôpital Saint-Louis, Paris, France; ${ }^{12}$ Réanimation médicale, CHU Dijon, Dijon, France; ${ }^{13}$ Réanimation, CHU de Strasbourg, Strasbourg, France; ${ }^{14}$ Réanimation polyvalente, Hôpital de Mercy, Ars Laquenexy, France; ${ }^{15}$ Service de réanimation médicale et infectieuse, Hôpital Bichat-Claude Bernard-APHP, Paris, France; ${ }^{16} \mathrm{Cardi}-$ ologie - centre d'investigation clinique pierre drouin, Centre Hospitalier Universitaire de Nancy, Vandœuvre-lès-Nancy, France 
Correspondence: Antoine Kimmoun - akimmoun@gmail.com Annals of Intensive Care 2016, 6(Suppl 1):S113

Introduction The outcome of patients admitted in intensive care unit for acute manifestation of small vessel vasculitis has been poorly reported. The main goal was to determine the mortality rate and prognostic factors of patients admitted in intensive care unit for acute manifestation of small vessel vasculitis.

Patients and methods We performed a retrospective, multicenter study of patients admitted from January 2001 to December 2014 in 20 intensive care units in France specifically with acute manifestation of small vessels vasculitis. Primary end-point was assessment of mortality rate 90 days after admission.

Results Eighty-two patients were included in 20 centers, $93 \%$ of whom with a recent diagnosis ( $<6$ months) of small vessel vasculitis. Main reasons for admission were respiratory failure (33\%) and pneumo-renal syndrome (32\%). Mechanical ventilation was required in $51 \%$, catecholamines in $31 \%$ and renal replacement therapy in $71 \%$. Overall mortality at 90 days was $19 \%$. Mortality was related to infection in $69 \%$ of cases. No difference was found at admission between survivors and non-survivors at 90 days in terms of reason for admission, comorbidities, type of vasculitis diagnosis and Birmingham Vasculitis Activity Score. Risk factors associated with 90-day mortality were higher SOFA score on the day of cyclophosphamide administration and delayed administration of cyclophosphamide.

Conclusion Even in cases of multiple organ failure, patients admitted in intensive care unit for the management of acute small vessel vasculitis benefit from full intensive care support and early aggressive treatment with immunosuppressors.

\section{Competing interests None.}

\section{S114}

A monocenter retrospective study of predictive factors of prolonged intensive care unit hospitalization after a whole lung lavage procedure for patients with pulmonary alveolar proteinosis

Pauline Tallon ${ }^{1}$, Alice Hadchouel-Duvergé ${ }^{2}$, Nadège Salvi ${ }^{3}$, Pascale Cairet ${ }^{3}$, Jean-Marc Treluyer ${ }^{4}$, Mehdi Oualha ${ }^{5}$, Fabrice Lesage ${ }^{6}$, Laure De Saint Blanquat $^{5}$, Sylvain Renolleau' ${ }^{7}$, Christophe Delacourt ${ }^{2}$, Laurent Dupic ${ }^{8}$ ${ }^{1}$ Hospital Necker, Paris, France; ${ }^{2}$ Pneumologie, Hospital Necker, Paris, France; ${ }^{3}$ Anesthésie, Hospital Necker, Paris, France; ${ }^{4}$ Centre d'investigation clinique, Institut Imagine, Paris, France; ${ }^{5}$ Réanimation et surveillance continue médico-chirurgicales, Hôpital Necker - Enfants Malades, Paris, France; ${ }^{6}$ Réanimation et surveillance continue médico chirurgicales, Hôpital Necker - Enfants Malades, Paris, France; ${ }^{7}$ Réanimation pédiatrique polyvalente, Hôpital Necker - Enfants Malades, Paris, France; ${ }^{8}$ Réanimation et surveillance continue pédiatriques, CHU Necker-Enfants Malades, Paris, France

Correspondence: Pauline Tallon - benierpauli@hotmail.com Annals of Intensive Care 2016, 6(Suppl 1):S114

Introduction Pulmonary alveolar proteinosis (PAP) is a rare disease. The main symptomatic treatment is repetitive whole lung lavage (WLL). This technique is an invasive procedure that can worsen the clinical condition of the patients and lead to the intensive care unit (ICU). We hypothesized that factors related to the patient's clinical condition at the time of WLL or the procedure in itself could explain the occurrence of worsening and a prolonged ICU stay. Thus, the aim of our study was to characterize the modalities of the WLL and to highlight predictive factors of complicated WLL defined by a prolonged hospitalization in ICU after the procedure.

Materials and methods We conducted a single-center retrospective cohort study examining WLL made between 2009 and 2015 at Necker-Enfants Malades. The primary endpoint was the hospitalization in ICU greater than three days that characterized a complicated WLL. For each procedure, we identified criteria related to the patient's condition (nutritional status, respiratory status, the presence of fever) and related to the procedure (such as the experience of the endoscopist and the anesthetist, the amount of physiological saline instilled, the duration of the anesthesia). We performed univariate and multivariate analysis to identify risk factors for complicated WLL.

Results Two hundred and twenty-five procedures corresponding to 14 patients were analyzed. $27.1 \%$ of procedures were the result of hospitalization in ICU. Univariate analysis showed that the risk of WLL complicated by a prolonged hospitalization in ICU is multiplied by 54.6 for severe respiratory illness $\left(p<10^{-4}\right)$, by 6.9 if oxygeno-dependence $(p=0.03)$ and 27.1 in cases of malnutrition $\left(p<10^{-4}\right)$. The amount of saline instilled reduced the risk of hospitalization in the ICU up to $2 \%$ per $\mathrm{ml} / \mathrm{kg}(p=0.009)$. In multivariate analysis, the risk of complicated WLL prolonged hospitalization in intensive care units is multiplied by 59.7 for severe respiratory illness $\left(p<10^{-4}\right)$ by 15 if oxygeno-dependence $(p=0.02)$ and 7.3 in cases of malnutrition $(p=0.01)$. The experience of the endoscopist and anesthesiologist did not emerge as complicated WLL risk factors.

Conclusion Our study shows that the conditions attached to the WLL procedure are not predictive of prolonged ICU hospitalization. And that the clinical presentation of the patient (malnutrition and respiratory failure) seems to have an impact on complicated WLL. This result could allow us to better identify patients likely to be hospitalized in ICU and to emphasize the importance of nutritional rehabilitation of these patients.

\section{Competing interests None.}

\section{S115}

Free fatty acids plasma profile during acute chest syndrome in adult patients with sickle cell disease: a pilot study Blandine Lefebvre Blandine 1 , Guillaume Lefevre ${ }^{2}$, Laurence Beraud ${ }^{2}$, Pierre Mora ${ }^{1}$, Zoé Coppere ${ }^{1}$, Antonin Lamaziere ${ }^{3}$, François Lionnet ${ }^{4}$, Muriel Fartoukh

${ }^{1}$ Réanimation médico-chirurgicale, Hôpital Tenon, Sorbonne Universités, UPMC Université Paris 06, Paris, France; ${ }^{2}$ Service de biochimie et hormonologie, Hôpital Tenon, Sorbonne Universités, UPMC Université Paris 06, Paris, France; ${ }^{3}$ Service de spectrométrie de masse, Faculté de Médecine Saint Antoine, Sorbonne Universités, UPMC Université Paris 06, Paris, France; ${ }^{4}$ Service de médecine interne, Hôpital Tenon, Sorbonne Universités, UPMC Université Paris 06, Paris, France

Correspondence: Muriel Fartoukh - muriel.fartoukh@tnn.aphp.fr Annals of Intensive Care 2016, 6(Suppl 1):S115

Introduction Acute chest syndrome (ACS) is the main complication leading adult patients with sickle cell disease (SCD) to the intensive care unit (ICU). Fat embolism etiology is supposed to account for up to $40 \%$ of ACS. In parallel with traumatic fat embolism syndrome, a medullary fat hydrolysis by phospholipase A2 could take place in ACS, increasing free fatty acids (FFA) responsible for acute lung injury. Arachidonic acid may be one of the main FFA released, because its enzymatic conversion leads to pro-inflammatory mediators. The objective of this study was to determine whether the synthesis of arachidonic acid via secreted phospholipase A2 (sPLA2) was increased in SCD patients admitted to the ICU and leads to plasma FFA changes, as compared to steady state.

Patients and methods All SCD patients with ACS admitted to the ICU of a French University Teaching Hospital in Paris between April and June 2015 were included. Samples were collected serially at D2, D3 and at hospital discharge. FFA were determined by enzymatic (total concentration) and gas chromatography/mass spectrometry (quantitative profile). Plasma activity of SPLA2 was measured by enzymatic method. Parameters changes between times are analyzed using nonparametric Wilcoxon test. A $p$ value less than 0.05 was considered statistically significant.

Results Twelve patients (homozygous hemoglobin SS, $n=11$; compound heterozygous SC disease, $n=1$ ) were admitted to the ICU for the management of ACS. Five patients had a highly severe course during ICU stay. Twelve samples were collected at D2, 10 at D3 and 3 at hospital discharge. Increased sPLA2 activity $(202 \mu \mathrm{mol} / \mathrm{min} / \mathrm{mL}$ [188-258]) and FFA concentration (0.44 mmol/L [0.39-0.46 mmol/L]) were found. FFA qualitative analysis demonstrated an inflammatory profile, with increased levels of oleic, palmitic and linoleic acids, 
when compared to healthy subjects. Conversely, the arachidonic acid concentrations were decreased. Within-group changes suggested a decrease in the repeated concentrations of oleic and linoleic acids $(p=0.1)$.

Discussion To our knowledge, this is the first study reporting the FFA sequential assays and the profiles in SCD patients with ACS. Our findings are in accordance with Hassel et al. who reported high levels of palmitic and oleic acids in SCD patients developing ACS, as compared to vaso-occlusive crisis.

Conclusion Our findings may argue for the participation of SPLA2 and free fatty acids, especially oleic, palmitic and linoleic acids, to the pathophysiological mechanisms of ACS. The steady-state profiles remain to be collected at this stage of the research.

\section{Competing interests None.}

\section{Reference}

1. Hassell KL, Deutsch JC, Kolhouse JF, O'Connell JL, Weil JV, Lane PA. 1994. Elevated serum levels of free fatty acids in sickle cell patients with acute chest syndrome and acute multi-organ failure syndrome. Blood, 84.

\section{S116}

Extravascular lung water and lung ultrasound to predict severe primary graft dysfunction following lung transplantation Samuel Lehingue ${ }^{1}$, Christophe Guervilly'ㄹ, Stéphanie Dizier ${ }^{3}$, Laurent Zieleskiewicz', Xavier D'journo ', Guillemette Thomas ${ }^{4}$, Fanny Klasen ${ }^{5}$, Mélanie Adda ${ }^{2}$, Antoine Roch ${ }^{2}$, Forel Jean-Marie ${ }^{6}$, Marc Leone ${ }^{7}$, Laurent Papazian ${ }^{5}$

'Bouches du Rhône, Hôpital Nord, Chemin des Bourrely, Marseille, France, Marseille, France; ${ }^{2}$ Réanimation DRIS, Hôpital Nord APHM, Marseille, France; ${ }^{3}$ Reanimation DRIS Pr Papazian, Hôpital Nord, Marseille, France; ${ }^{4}$ Réanimation, Hôpital Européen Marseille, Marseille, France; ${ }^{5}$ Service de réanimation-détresses respiratoires et infections sévères, Hôpital Nord, Marseille, France; ${ }^{6}$ Réanimation des détresses respiratoires et des infections sévères, Hospital Nord, Marseille, France; ${ }^{7}$ Département d'anesthésie réanimation, Hôpital Nord, Chemin des Bourrely, Marseille, France Correspondence: Samuel Lehingue - samlehingue@gmail.com Annals of Intensive Care 2016, 6(Suppl 1):S116

Introduction Primary graft dysfunction (PGD) is the result of pulmonary edema following lung transplantation (LT). The definition of PGD is based on the ratio of the partial pressure of arterial oxygen to the fraction of inspired oxygen $\left(\mathrm{PaO}_{2} / \mathrm{FiO}_{2}\right)$ and the presence of lung infiltrates on chest X-ray. Lung ultrasonography (LU) and extravascular lung water indexed to predicted body weight (EVLWi) are reliable methods for quantifying lung edema. We assessed whether LU and EVLWi were associated with grade 3 PGD.

Patients and methods Design and patients All patients scheduled to receive single or bilateral lung transplantation were screened for inclusion between July 2013 and September 2014.

Data collection PGD was graded according to the ISHLT criteria. Chest $\mathrm{X}$-rays were interpreted independently by two physicians blinded to the clinical variables, with adjudication of disagreements. The classification $\mathrm{k}$ for agreement on grade 3 PGD was 0.93. Grading ISHLT was performed at 4 time points: within the $6 \mathrm{~h}$ after ICU admission and over the following 3 days at $10 \mathrm{am}$. The worst grading of PGD was used to classify patients. The use of rescue therapies for severe hypoxemia was considered when the following criteria were fulfilled: prone positioning (PP) if $\mathrm{PaO}_{2} / \mathrm{FiO}_{2}$ was lower than $150 \mathrm{mmHg}$, continuous neuromuscular blocking agents infusion (NMBA) if $\mathrm{PaO}_{2} / \mathrm{FiO}_{2}$ was lower than $120 \mathrm{mmHg}$, iNO if $\mathrm{PaO}_{2} / \mathrm{FiO}_{2}$ was lower than $100 \mathrm{mmHg}$ despite PP and NMBA infusion and veno-venous ECMO according to the French recommendations. Transpulmonary thermodilution and lung ultrasound During the transplantation, two thermodilution measurements were realized during chest-closed periods, one after induction of anesthesia and the second after the end of the surgical procedure. Thermodilution and LU scores were then performed during the three-day period following lung transplantation at the PGD assessment time points. Lung ultrasound examination was conducted using two specific methods: the echo comet score (ECS) and the 12-region technique (LUS). Due to barriers to US resulting from pneumothorax, subcutaneous emphysema, chest tubes or thoracic dressings, we weighted every score obtained by the number of interspaces (for the ECS) or areas (for the LUS) available.

Results Between August 2013 and September 2014, 36 patients were included prospectively. All except one underwent bilateral sequential single-lung transplantation. Thirteen patients (36\%) presented PGD grade 3 during the first 3 days following ICU admission. In the grade 3 PGD group, crystalloids infusion was larger during the transplantation procedure than the one without grade 3 PGD. Overall ICU mortality was only $3 \%$ (one death in the grade 3 PGD group). In the grade 3 PGD group, ICU length of stay and duration of mechanical ventilation were longer and the probability of rescue therapies need was higher.

No difference was observed in EVLWi during the intra-operative period. The EVLWi was higher in the grade 3 PGD group at ICU admission $(p=0.001)$, day one $(p=0.01)$ and day $2(p=0.002)$. The ROC curve analysis showed that a value of $14 \mathrm{~mL} / \mathrm{kg}$ at ICU admission was the best cutoff for predicting PGD grade 3 after lung transplantation, with a sensitivity of $82 \%$ and a specificity of $77 \%$ at ICU admission. The area under the ROC curve was 0.85 . Patients with delayed grade 3 PGD had significantly higher EVLWi at ICU admission compared with patients without grade 3 PGD at any time point. Rescue therapies were needed in $64 \%$ of patients with an EVLWi at ICU admission $\geq 14 \mathrm{~mL} / \mathrm{kg}$ and in $10 \%$ when EVLWi was $<14 \mathrm{~mL} / \mathrm{kg}(p<0.001)$.

Less than half of the patients had all LU windows available. LU scores did not discriminate patients with grade 3 PGD.

Logistic regression analysis performed with crystalloids volume infusion during surgery, mean arterial pressure, global end-diastolic volume indexed and EVLWi at ICU admission indicated that both EVLWi at ICU admission (odds ratio per $1 \mathrm{~mL} / \mathrm{kg}$ increase, $2.78,95 \% \mathrm{Cl}[1.24-$ $6.21], p=0.01$ ) and crystalloids volume infusion during surgery (odds ratio per $1 \mathrm{~L}$ increase, $1.61,95 \% \mathrm{Cl}[1.04-2.50] p=0.03$ ) were associated with grade 3 PGD.

Conclusion Unlike lung ultrasound scores, a high EVLWi measured immediately after LT is associated with grade 3 PGD. Initial EVLWi offers a great added value for the early diagnosis of grade 3 PGD when it precedes the onset of grade 3 PGD.

\section{Competing interests None.}

\section{References}

1. Christie JD, Carby M, Bag R, Corris P, Hertz M, Weill D. Report of the ISHLT Working Group on Primary Lung Graft Dysfunction part II: definition. A consensus statement of the International Society for Heart and Lung Transplantation. J Heart Lung Transplant Off Publ Int Soc Heart Transplant 2005:24(10):1454-1459.

2. LeTourneau JL, Pinney J, Phillips CR. Extravascular lung water predicts progression to acute lung injury in patients with increased risk. Crit Care Med 2012;40(3):847-854.

\section{S117}

Results of the international multicentric prospective observational study Phase Angle Project: phase angle measured by bioimpedance analysis at intensive care unit (ICU) admission predicts 28-day survival

Ronan Thibault ${ }^{1}$, Anne-Marie Makhlouf², Aurélien Mulliez ${ }^{3}$, Cristina Gonzalez ${ }^{4}$, Sylvain Dadet ${ }^{5}$, Gintautas Kekstas ${ }^{6}$, Jean-Charles Preiser ${ }^{7}$, Kozjek Nada Rotovnik ${ }^{8}$, Isabel Ceniceros Rozalen ${ }^{9}$, Kinga Kupczyk ${ }^{10}$, Zeljko Krznaric $^{11}$, Fabienne Tamion ${ }^{12}$, Noël Cano ${ }^{5}$, Claude Pichard ${ }^{2}$, Phase angle project Investigators

${ }^{1}$ Service d'Endocrinologie, Diabétologie, Nutrition, Hospital Center University De Rennes, Rennes, France; ${ }^{2}$ Nutrition Unit, Geneva University Hospital, Geneva, Switzerland; ${ }^{3}$ Drci, CHU Gabriel-Montpied, Clermont-Ferrand, France; ${ }^{4}$ Nutrition, Université de Pelotas, Pelotas, Brazil; ${ }^{5}$ Service de nutrition clinique, CHU Gabriel-Montpied, Clermont-Ferrand, France: ${ }^{6}$ Department of icu, Vilnius University Hospital, Vilnius, Lithuania; ${ }^{7}$ Service de Réanimation Médico-Chirurgicale, Hôpital Erasme, Bruxelles, Belgium; ${ }^{8}$ Department of Clinical Nutrition, Institute of Oncology, Ljubljana, Slovenia; ${ }^{9}$ Department of ICU, Clinic USP Palmaplanas, Palma, Spain; ${ }^{10}$ Department of ICU, Bydgoszcz Hospital, Bydgoszcz, Poland; ${ }^{11}$ Departement of Internal Medicine, Univ. Hospital Center Zagreb, Zagreb, Croatia;

${ }^{12}$ Réanimation médicale, Hospital Center University Rouen, Rouen, France 
Correspondence: Ronan Thibault - ronan.thibault@orange.fr Annals of Intensive Care 2016, 6(Suppl 1):S117

Introduction ICU patients prognosis is evaluated at admission by severity scores (APACHEII, SAPSII) that are time-consuming. $50 \mathrm{kHz}$ phase angle $(\mathrm{PhA})$ is measured easily and quickly by bioimpedance analysis (BIA). Lowered PhA is associated with worse prognosis of chronic diseases. The main aim was to determine whether PhA measured at ICU admission predicts 28-day survival.

Patients and methods Patients were recruited in 10 centers from 9 countries. The inclusion criteria were: age $>18$ years, absence of implantable cardioverter defibrillator or pacemaker, expected ICU length of stay $>48 \mathrm{~h}$. PhA was measured at admission (day (d) 1) by the Nutriguard (Data-Input, Germany) BIA analyzer. APACHEII and SAPSII were calculated at $\mathrm{d} 1$. The following variables were collected: 28-day mortality, duration of mechanical ventilation, ICU length of stay, infections, and expressed as mean \pm standard deviations or median \pm interquartiles accordingly. Relations between PhA and 28-day mortality, duration of mechanical ventilation or ICU length of stay were analyzed by KaplanMeier actuarial curves and Spearman test. Sensitivity, specificity and $\mathrm{PhA}$ cutoffs associated with 28-day mortality were analyzed by ROC curves and the Youden method. Variables associated with 28-day mortality were determined by multivariate logistic regression.

Results Thirty-three percentage of screened patients were analyzed: $n=918$ (/1000 planned patients according to the power analysis sample calculation): Geneva, Switzerland $(n=178)$, Pelotas, Brazil $(n=103)$, Brussels, Belgium $(n=100)$, Vilnius, Lithuania $(n=100)$, Clermont-Ferrand, France $(n=99)$, Ljubljana, Slovenia $(n=96)$, Palma de Majorque, Spain $(n=94)$, Bydgoszcz, Poland $(n=52)$, Zagreb, Croatia $(n=49)$, Rouen, France $(n=47)$. The characteristics of analyzed patients were: 549 males, $61 \pm 16$ years, body mass index $26.4 \pm 5.6$, medical $63 \%$, shock all cause $29 \%$, APACHEll $19 \pm 9$, SAPSII $43 \pm 19$, PhA d1: $4.4 \pm 1.7$ degrees $\left({ }^{\circ}\right), 28$-day mortality $21 \%$. Day $1 \mathrm{PhA}$ was lower in non-survivors than in survivors $(4.0 \pm 2.0$ vs $4.5 \pm 1.7, P=0.005)$. Sensitivity, specificity, positive predictive value (PPV) and negative predictive value (NPV) of $\mathrm{d} 1 \mathrm{PhA}<3.49$ for 28-day mortality were, respectively, 49, 74, 31 and $86 \%$ (area under the curve $(A \cup C)=0.63[0.58-0.68]$ ). The Kaplan-Meier actuarial curve indicated a better 28-day survival in patients with $d 1$ $\mathrm{PhA} \geq 3.49^{\circ}(P<0.001)$. Survival was worse in the quartile of patients with d1 $\mathrm{PhA}<3.23^{\circ}$ vs. quartiles (3.23-4.24, 4.25-5.32, > 5.32) $(P<0.001)$. Variables associated with $\mathrm{d} 28$ mortality were: SAPSII $\geq 46$ (hazard ratio (HR) 4.2 [2.1-8.3], $P<0.001$ ), $\mathrm{PhA}<3.49^{\circ}$ (HR 2.2 [1.3$3.8], P=0.005$ ) and age $>64$ years (HR 1.9 [1.0-3.5], $P=0.05$ ). Sensitivity, specificity, PPV and NPV of a multivariate model associating d1 $\mathrm{PhA}<3.49^{\circ}$ and SAPSII $\geq 46$ for 28-day mortality were $77,66,32$ and $93 \%$, respectively $(A \cup C=0.78[0.73-0.83])$. There was a reverse correlation between d1 PhA and SAPSII (rho $=-0.16)$, or APACHE II (rho $=-0.19)(P<0.001)$. The proportion of patients with mechanical ventilation was higher in patients with $\mathrm{PhA} \leq 4.2^{\circ} \mathrm{vs}$. $\mathrm{PhA}>4.2^{\circ}$ : 50 vs $31 \%, P<0.001$. There was no relation between PhA and ICU length of stay or infections.

Conclusion At ICU admission PhA is an indicator of 28-day mortality, even if SAPSII is the best predictor. A combined multivariate model associating PhA and SAPSII improves 28-day mortality predictability.

Competing interests Ronan Thibault and Claude Pichard received research grants from Bioparohm.

Funding European Society for Clinical Nutrition and Metabolism (ESPEN).

\section{S118}

Nutrireaped: a survey of physician nutrition practices and knowledge in French-speaking pediatric intensive care units Bénédicte Gaillard-Le Roux ${ }^{1}$, Aurelien Jacquot ${ }^{2}$, Corinne Jotterand ${ }^{3}$, Shancy Rooze ${ }^{4}$, Clemence Moullet ${ }^{3}$, Flavie Letois ${ }^{5}$, Frederic Valla ${ }^{6}$ ${ }^{1}$ Pediatric Intensive Care Unit, C.H.U de Nantes, Nantes, France; ${ }^{2}$ Pediatric Intensive Care Unit, CHU Arnaud de Villeneuve, Montpellier, France; ${ }^{3}$ Pedi-

\section{Table 60 See text for description}

\begin{tabular}{|c|c|c|}
\hline $\begin{array}{l}\text { Nutrition considered } \\
\text { priority }\end{array}$ & Yes (81.08 \%) & No (18.92 \%) \\
\hline $\begin{array}{l}\text { Nutrition written } \\
\text { protocols }\end{array}$ & Yes (54.05 \%) & No (45.95\%) \\
\hline $\begin{array}{l}\text { Level of knowledge of } \\
\text { the medical team on } \\
\text { nutrition }\end{array}$ & $\begin{array}{l}\text { Rather insufficient } \\
\text { (45.95\%) }\end{array}$ & $\begin{array}{l}\text { Rather sufficient } \\
\quad(37.84 \%)\end{array}$ \\
\hline $\begin{array}{l}\text { Nutrition support } \\
\text { team in PICUs }\end{array}$ & Yes (40.54 \%) & No $(59.46 \%)$ \\
\hline Indirect calorimeter & Yes (16.22 \%) & No (81.08 \%) \\
\hline $\begin{array}{l}\text { Assessment of energy } \\
\text { requirements }\end{array}$ & $\begin{array}{l}\text { Schofield equation } \\
\quad(10.81 \%)\end{array}$ & $\begin{array}{l}\text { Recommended dietary } \\
\text { allowances (70.27\%) }\end{array}$ \\
\hline $\begin{array}{l}\text { Beginning of enteral } \\
\text { nutrition (EN) }\end{array}$ & Within 24 h (54.05 \%) & $\begin{array}{l}\text { Between } 24 \text { and } 48 \text { h } \\
\quad(37.84 \%)\end{array}$ \\
\hline $\begin{array}{l}\text { Beginning of paren- } \\
\text { teral nutrition if EN is } \\
\text { insufficient }\end{array}$ & Day 1 (40.54 \%) & $\begin{array}{l}\text { Between Day } 1 \text { and Day } \\
4(48.65 \%)\end{array}$ \\
\hline $\begin{array}{l}\text { Duration of fasting } \\
\text { before extubation }\end{array}$ & Many hours (67.57\%) & $\begin{array}{l}\text { Just before extubation } \\
\qquad(32.43 \%)\end{array}$ \\
\hline
\end{tabular}

atric Intensive Care Unit, University of Lausanne, Lausanne, Switzerland; ${ }^{4}$ Pediatric Intensive Care Unit, Brussels University Hospital, Brussels, Belgium; ${ }^{5}$ Inserm CIC 1411, C.h.u. De Montpellier, Prades-le-Lez, France; ${ }^{6}$ Réanimation pédiatrique hfme, Hospices civils de Lyon, Lyon, France Correspondence: Bénédicte Gaillard-Le Roux - benedicte.gaillardleroux@chu-nantes.fr

Annals of Intensive Care 2016, 6(Suppl 1):S118

Introduction Optimal nutrition in pediatric intensive care units (PICUs) is important to improve patient outcome. The aim of our study, conducted by NUTRISIP (Nutrition French-Speaking PICU Group), was to describe the present knowledge of healthcare professionals and the practices surrounding enteral and parenteral feeding in the Frenchspeaking PICUs, in order to adapt future interventions.

Materials and methods A cross-sectional survey with 69 questions concerning nutritional practices was sent to 42 French-speaking PICUs in June 2014. A member of the medical staff answered questions.

Results Overall response rate was $88 \%$. The main answers are summarized in Table 60.

Conclusion Despite early enteral feeding in most PICUs, recommendations for nutrition in PICUs are not sufficiently known, particularly for assessment of energy requirements, or for duration of fasting before procedures. This survey highlights importance of future interventions to improve nutritional care.

Competing interests Financial support: Baxter, Nestlé, Fresenius Kabi, Nutricia.

\section{References}

1. Hamilton S, McAleer DM, Ariagno K, Barrett M, Stenquist N, Duggan CP, Mehta NM. A stepwise enteral nutrition algorithm for critically ill children helps achieve nutrient delivery goals. Pediatr Crit Care Med. 2014 Sep;15(7):583-9.

2. Mehta NM, Bechard LJ, Cahill N, Wang M, Day A, Duggan CP, Heyland DK. Nutritional practices and their relationship to clinical outcomes in critically ill children-an international multicenter cohort study* Crit Care Med. 2012 Jul;40(7):2204-11. 
S119

A computerized tool increases compliance to caloric and protein targets and may lower mortality in neutropenic hematologic patients in the ICU: the NUTCHOCO study: NUTritionnal Care in Hematologic Oncologic patients and Critical Outcome Aurélia Henin ${ }^{1}$, Florence Ettori' ${ }^{2}$, Christophe Zemmour ${ }^{3}$, Laurent Chow-Chine ${ }^{4}$, Jeanpaul Brun ${ }^{5}$, Antoine Sannini ${ }^{5}$, Magali Bisbal ${ }^{5}$, Marion Faucher $^{5}$, Jean Marie Boher ${ }^{3}$, Djamel Mokart ${ }^{6}$

${ }^{1}$ Anesthesie réanimation, Hospital Timone, Marseille, France; ${ }^{2}$ Bouches du rhône, Institute Paoli-Calmettes, Marseille, France; ${ }^{3}$ Statistics, Institute Paoli-Calmettes, Marseille, France; ${ }^{4}$ Réanimation, Institute Paoli-Calmettes, Marseille, France; ${ }^{5}$ Reanimation, Institute Paoli-Calmettes, Marseille, France; ${ }^{6}$ Réanimation, Institut Paoli-Calmettes, Marseille, France Correspondence: Florence Ettori - florenceettori@gmail.com Annals of Intensive Care 2016, 6(Suppl 1):S119

Introduction Malnutrition is frequent and deleterious in the intensive care patient. Recent attention has been brought to the challenges raised by inadequate feeding choice of the adequate route, time of onset, and nutrients. Patients with Hematologic malignancies are increasingly admitted to the intensive care and present specific hallmarks of their diseases, such as neutropenia, side effects from chemotherapy, specific gastrointestinal issues, and malnutrition preceding intensive care admission. We investigated the effects of the implementation of a computerized nutritional tool (the $\mathrm{CHOCOBOX)} \mathrm{on} \mathrm{hematologic} \mathrm{patients} \mathrm{admitted} \mathrm{to} \mathrm{the} \mathrm{intensive}$ care.

Patients and methods Our study was a before-after study comparing two prospectively collected cohorts of hematologic cancer patients chronologically admitted to a single intensive care, before and after the implementation of an optimized nutritional protocol (the $\mathrm{CHOC}$ OBOX). This protocol included a computerized prescription tool; simulation of the best combination of products to reach target; team training before the implementation of the CHOCOBOX; dietician counseling; and daily comprehensive computation of all caloric and protein intakes and of daily and total caloric and protein balances.

Our main objective was to show a $50 \%$ increase in the compliance of caloric and protein delivery, using a Poisson regression model. Compliances were defined as the numbers of days, respectively, within caloric $((25 \mathrm{kcal} / \mathrm{kg} / \mathrm{d} \pm 15 \%)$ and protein $(1.5 \mathrm{G} / \mathrm{KG} / \mathrm{D} \pm 15 \%)$ targets, adjusted on the length of ICU. Secondary objectives were to show a difference in morbidity (days of ventilation, CRRT, antibiotics, positive blood cultures, noradrenalin) and hospital mortality, in four subgroups of patients: neutropenic, bone marrow allografted, malnourished, and severe (SAPS II > 56), tested by a survival curve with a Gray test.

Results We included 275 patients: 147 patients from 2009 to 2011 and 128 patients from 2012 to 2014, using the CHOCOBOX. On admission, the $\mathrm{CHOCOBOX}$ cohort showed significantly higher rates of neutropenic and malnourished patients, number of chemotherapy lines and bone marrow transplants (BMT). Caloric compliant days increased by $50 \%$ with 4.13 vs 2.1 days (adjusted RR on neutropenia, malnutrition status, chemotherapy lines and BMT, RR $=1.42, p=0.03$ ) in the after cohort, as well as protein compliant days with 4.1 vs 0.8 days (adjusted $\mathrm{RR}=3.51 p<0.001$ )

There was no statistically significant difference between the cohorts in the number of mechanical ventilation days, nor CRRT days, amine days, antibiotic days, number of days on more than 3 antibiotics, number of positive blood cultures per patient.

There was no statistically significant difference between the cohorts for the overall survival during the hospital stay, HR 0.70 [0.39-1.28] Fine \& Gray test $p=0.26$. Overall survival was higher in the after cohort in the neutropenic subgroup $(n=100$, HR 0.53 [0.31-0.89] Fine \& Gray test $p=0.018$ ) and in the most severe patients (SAPS II $>56, n=81$, HR $0.52[0.30-0.91]$ Fine \& Gray test $p=0.023$, but was not different for allograft patients $(n=70)$ nor for malnourished patients $(n=122)$.

Discussion Our study is the first to address nutritional issues in the intensive care in hematologic patients. A nutritional protocol was able to raise caloric and protein delivery to fairer levels and decreased global caloric and protein negative balance. Early-onset enteral feeding has shown benefit in the ICU but was difficult for our patients who had specific enteral problems (esophagitis, mucitis, severe thrombopenia, intolerance, diarrhea, or neutropenic-related colitis). The standard of first choice exclusive enteral feeding may not be adequate for all these patients, but trophic feeding was possible.

Survival was better with the CHOCOBOX in patients with failures (saps II > 56) and with neutropenia, perhaps because of higher quantities of protein, or because of a "package" of integrated nutritional management, more than because of greater calories. Raised levels of compliance remained mediocre, urging us to find more effective ways to feed this specific population.

Conclusion The implementation of a computerized prescription and follow-up nutritional tool is effective in increasing the number of compliant days for protein and calorie delivery in the ICU setting. Such a protocol may lower mortality in some subgroups of patients.

Competing interests Some nutritional products were granted to the ICU by a laboratory for free.

S120

Systemic evaluation of intakes in patients receiving noninvasive ventilation: the STARVE study

Michel Arnaout ${ }^{1}$, Aude Marincamp ${ }^{1}$, Matthieu Reffienna ${ }^{1}$, Anne-Sophie Debue ${ }^{1}$, Pierre Lucas' ${ }^{1}$, Audrey Feltry ${ }^{2}$, Julien Charpentier', Benoit

Champigneulle', Wulfran Bougouin', Guillaume Geri', Frédéric Pène',

Jean-Paul Mira', Alain Cariou', Jean-Daniel Chiche', Groupe de travail sur la ventilation

${ }^{1}$ Réanimation médicale, hôpital Cochin, Paris, France; ${ }^{2}$ Equipe mobile de nutrition, Hôpital Cochin, Paris, France

Correspondence: Michel Arnaout - michel.arnaout@gmail.com

Annals of Intensive Care 2016, 6(Suppl 1):S120

Introduction Noninvasive ventilation (NIV) is increasingly used in ICUs. Whereas there are specific guidelines related to feeding of critically ill patients receiving invasive ventilatory support, there are no guidelines related to feeding pts treated with NIV. We hypothesize that pts may be significantly underfed during the first 5 days of ventilatory support with NIV. We conducted this study to evaluate caloric intakes of patients receiving NIV irrespective of the indication for NIV.

Patients and methods Prospective, multidisciplinary, observational study in a 24-bed MICU. Inclusion criteria: all patients treated with NIV for more than $4 \mathrm{H} /$ day. Exclusion criteria: patients receiving parenteral or enteral nutrition in the management of underlying diseases or comorbidities. Data collected include demographics, patients' medical characteristics, NIV indication and duration of NIV sessions, complications developed in the ICU, and nutritional data. We prospectively quantified and recorded all calories intakes through oral, enteral and parenteral routes during the whole duration of the ICU. We also measured BMI and albumin concentrations at the admission in ICU. Data are presented as median [IQR].

Results One hundred and thirteen pts $(56 \mathrm{M} / 57 \mathrm{~F}), 74$ years old [65; 82], SAPS2 40 [33; 52], SOFA 4 [2; 7], BMI 27 [23; 34] have been enrolled in the study. ICU length of stay was $6[4 ; 9]$ days. Patients were placed on NIV for $4[2 ; 5]$ days. Mortality rates was $12.4 \%$, and $24 \%$ of patients

Table 61 See text for description

\begin{tabular}{cccccc}
\hline & $\begin{array}{l}\text { Day 1 } \\
\boldsymbol{n = 1 1 3}\end{array}$ & $\begin{array}{l}\text { Day 2 } \\
\boldsymbol{n = 1 0 2}\end{array}$ & $\begin{array}{l}\text { Day 3 } \\
\boldsymbol{n = 8 1}\end{array}$ & $\begin{array}{l}\text { Day 4 } \\
\boldsymbol{n = 5 4}\end{array}$ & $\begin{array}{l}\text { Day 5 } \\
\boldsymbol{n}=\mathbf{3 4}\end{array}$ \\
\hline $\begin{array}{c}\text { Duration of } \\
\text { NIV (h) }\end{array}$ & $9[5,7 ; 12]$ & $10,2[6 ; 14.9]$ & $10[5 ; 13]$ & $6,9[4 ; 10,5]$ & $7[3,8 ; 10,7]$ \\
$\begin{array}{c}\text { Oral intakes } \\
\text { (Kcal) }\end{array}$ & $0[0 ; 305]$ & $500[0 ; 872]$ & $\begin{array}{c}660 \\
{[235 ; 1020]}\end{array}$ & $\begin{array}{c}455[0 ; \\
825]\end{array}$ & $\begin{array}{c}430[120 ; \\
950]\end{array}$ \\
$\begin{array}{c}\text { Parenteral } \\
\text { nutrition } \\
\text { (Kcal) }\end{array}$ & 58 & $89[40 ; 170]$ & $70[41 ; 193]$ & 84 & $100[40,208]$ \\
$\begin{array}{c}\text { NIV pts with } \\
<1000 \text { kcal/ } \\
\text { day (\%) }\end{array}$ & $90 \%$ & $73 \%$ & $64 \%$ & $58 \%$ & $53 \%$ \\
\hline
\end{tabular}


were intubated after NIV failure. Indication (classified according to the SRLF/SFAR consensus conference 1) were Group $1(n=85)$, Group $2+(n=11)$ or Group 2- $(n=18)$. Main results are summarized below. Median energy an proteins intakes per day under NIV was 635 [406; 925] KCal and 23 [10; 37] $\mathrm{g}$ of protein. Albumin blood concentration was $32[28 ; 36] \mathrm{g} / \mathrm{l}$ on admission (Table 61).

Conclusion Patients treated with NIV for more than $4 \mathrm{H} /$ days have limited oral intakes and are significantly underfed. Long sessions of NIV, fear of aspiration and underestimation of the patient severity may explain the failure to provide adequate nutritional support in these patients. A multicenter study is underway to assess feeding practices in NIV patients.

\section{Competing interests None.}

\section{Reference}

1. SFAR et SRLF Ventilation non invasive au cours de l'insuffisance respiratoire aigue 2006.

\section{S121}

\section{Computerized glucose control in the ICU}

Salah Adel Ben ${ }^{1}$, Juliette Audibert ${ }^{1}$, Alexandre Conia', Olivier Gontier', Mouldi Hamrouni ${ }^{1}$, Thierry Lherm ${ }^{2}$, Abdelkader Ouchenir ${ }^{1}$, Jérôme Rétif ${ }^{1}$, Alain Proudhom ${ }^{3}$, Chantal Lagardere ${ }^{3}$, Stéphane Tissot ${ }^{3}$, Pierre Kalfon ${ }^{1}$ ${ }^{1}$ Réanimation polyvalente, C.H. de Chartres, Le Coudray, France; ${ }^{2}$ Réanimation polyvalente, Hôpitaux de Chartres, Chartres, France; ${ }^{3}$ Information Technology, GE Healthcare IT France \& BeNeLux, Lyon, France

Correspondence: Pierre Kalfon - pierrekalfon@sfr.fr

Annals of Intensive Care 2016, 6(Suppl 1):S121

Introduction Stress-induced hyperglycemia is common in critically ill patients due to insulin resistance and increased hepatic output of glucose, and the relationship between stress hyperglycemia and poor outcome for patients hospitalized in the intensive care unit (ICU) was demonstrated. Although several randomized controlled studies, based on the use of whether paper protocols or computerized protocols (1), have failed to replicate any mortality benefit, the interest for computerized decision-support system (CDSS) remains because meanwhile new therapeutic goals for blood glucose (BG) control in the ICU have emerged. Furthermore, harmful effects of severe hypoglycemia and multiple hypoglycemic episodes during tight glucose control in critically ill patients have been recently demonstrated (2). The question arises whether a CDSS allowing the setting of a higher BG target and based on an enhanced algorithm for the calculation of the insulin rate to be applied after each BG measure may reduce the incidence of severe and moderate hypoglycemia.

The objective of our study pilot is to assess the impact of the use in routine care of an improved CDSS on the incidence of hypoglycemia, whether severe or moderate, in comparison with a first-generation CDSS.

Patients and methods We used in routine care a CDDS for blood glucose control in the ICU.

This CDSS is a second-generation software (CDSS-2) derived from that used during the CGAO-REA trial (NCT01002482), a nonblinded parallel-group randomized controlled trial involving adult patients admitted to ICUs and comparing tight glucose control (TGC) with a first-generation CDSS (CDSS-1, BG range between 4.4 and $6.1 \mathrm{mmol} / \mathrm{l}$ ) and conventional glucose control (CGC) protocols $(B G<10 \mathrm{mmol} / \mathrm{l})$.

The BG target has been set by default to $5-7.5 \mathrm{mmol} / \mathrm{l}$ in the CDSS-2. The embedded algorithm used for the calculation of the next insulin rate (to be applied after each BG measure) was of the type proportional integral with variable coefficients to take into account variations of insulinosensitivity throughout the ICU stay.

The CDSS- 2 was used on the admission whenever the patient required intravenous insulinotherapy and was our standard care protocol to be used (on medical prescription) by our nursing staff who had already gained experience during the CGAO-REA study.

We extracted the clinical data and that concerning BG control for all the patients treated with CDSS- 2 .
A Chi square test was used to compare the incidence of severe hypoglycemia and moderate hypoglycemia observed during the TGC arm of the CGAO-REA trial and the pilot study with CDSS-2.

Results The CGAO-REA study, carried out between October 2009 and June 2011, involved 2684 randomized patients, 1351 assigned to computerized (CDSS-1) and 1333 to conventional blood glucose control, recruited in 34 ICUs (19 in academic tertiary care hospitals and 15 in community hospitals). Thirty-six patients were discarded from the analysis: 35 because they withdrew consent and one included twice. In the end, 1336 and 1312 patients were included in the TGC (with CDSS-1) and CGC arms, respectively. Among the 1336 patients treated with CDSS-1, 19 were discarded because important data concerning BG measurement were lacking. Thus, 1317 patients were analyzed for comparison.

The pilot study, carried out between September 2012 and May 2015, involved 685 stays (and 641 patients) in the ICU among a total of 973 stays during the study period.

Severe hypoglycemia occured less frequently during the pilot study (CDSS-2) than during the TGC arm of the CGAO-REA trial (CDSS1), respectively, 58 stays of $685(8.5 \%, 95 \% \mathrm{Cl} 6.4-10.6 \%)$ and 174 stays of $1317(13.2 \%, 95 \% \mathrm{Cl} 11.4-15 \%)$. Moderate hypoglycemia $(<3.3 \mathrm{mmol} / \mathrm{l})$ occured also less frequently during the pilot study (CDSS-2) than during the TGC arm of the CGAO-REA trial (CDSS-1), respectively, 236 stays of $685(34.5 \%, 95 \% \mathrm{Cl} 30.9-38.1 \%)$ and 743 stays of 1317 ( $56.4 \%, 95 \% \mathrm{Cl} 53.7-59.1 \%)$.

Discussion Further studies are needed to improve glycemic control in the ICU and reduce the incidence of hypoglycemia. The respective roles of the increase in the BG target, the refinement of the algorithm or the use of the software in routine care could not assessed by our historical comparison. Anyway our study paves the way toward the development of the most efficient CDSS for managing BG control in the ICU.

Conclusion In comparison with the use of CDDS-1, the use of CDSS-2 was associated with a lower incidence of both severe and moderate hypoglycemia during blood glucose control in critically ill patients.

\section{Competing interests None.}

\section{References}

1. Kalfon P, Giraudeau B, Ichai C, Guerrini A, Brechot N, Cinotti R, Dequin PF, Riu-Poulenc B, Montravers P, Annane D et al: Tight computerized versus conventional glucose control in the ICU: a randomized controlled trial. Intensive Care Med 2014, 40(2):171-181.

2. Kalfon P, Le Manach Y, Ichai C, Brechot N, Cinotti R, Dequin PF, Riu-

Poulenc B, Montravers P, Annane D, Dupont $\mathrm{H}$ et al: Severe and multiple hypoglycemic episodes are associated with increased risk of death in ICU patients. Crit Care 2015, 19:153.

\section{S122}

Toxicodynetics in mono-intoxications with oxazepam and nordiazepam: an approach to a better understanding of drug-drug interaction

Lynn Sacre ${ }^{1}$, Antoine Villa1 ${ }^{1}$, Alaywa Khadija ${ }^{2}$, Robert Garnier ${ }^{1}$, Frederic Baud $^{3}$

${ }^{1}$ Hôpital fernand widal, Centre Antipoison et de Toxicovigilance de Paris, Paris, France; ${ }^{2}$ Samu de Paris, Centre antipoison de Paris, umr-8536, Hospital Necker, Paris, France; ${ }^{3} 75014$, SAMU de Paris, Réanimation polyvalente, Paris, France

Correspondence: Frederic Baud - baud.frederic@wanadoo.fr Annals of Intensive Care 2016, 6(Suppl 1):S122

Introduction Among the drugs reported in self-poisonings, benzodiazepines remain the leading class of psychotropic drugs. However, the pharmacology and the toxicology as well of these drugs remain complex due to a chain of active metabolites with a final pathway frequently involving nordiazepam and oxazepam. In spite of the progressive withdrawal of potent hypnotics, drug-induced coma remains a leading and even increasing cause of admission in intensive care units. This finding suggests potent drug-drug interactions. However, in the setting of human poisoning, basis of pharmacology failed to quantify interactions. To address this issue, we are currently developing a new 
discipline entitled toxicodynetics aiming at defining the time course of events of drugs either alone or in combination. The major aim of the present study was to report data from the Paris Poison Control Centre (PPCC) aiming at defining toxicodynetic parameters dealing with pure oxazepam and nordiazepam.

Patients and methods We used data collected at the PPCC from 1999 to 2015. Indeed, the method of collection of data was the same along this period of time. Cases of pure oxazepam and nordiazepam were selected on the basis of self-report by the patient or relatives with a particular attention paid to eliminate the concomitant alcohol ingestion. The classical toxicodynetics parameters were looked for using the individual medical record available at the PPCC. These parameters included (1) the supposed ingested dose which was expressed as a therapeutic index (TI) as defined by a percentage of the maximum recommended daily dose, (2) the time of ingestion (T0), the delay in onset (hours), (3) the rate of worsening (hours or on-off process), (4) the maximal observed effect (Emax), (5) the time of onset of the maximal effect (Tmax), (6) the shape of the duration of maximal effect as a peak or a plateau, (7) the rate of recovery, (8) the duration of hospitalization in uncomplicated poisonings.

Results During the study period the number of exposures to oxazepam and nordiazepam as a mono- or combined intoxications reported to the PPCC centre was 840 and 157, respectively. The number of mono-intoxications with oxazepam and nordazepam included in the toxicodynetic study was 257 and 74 cases, respectively. The T0 was known in all cases (100 \%). The Emax in both oxazepam and nordiazepam was sleepiness or obtundation occurring in 108 and 36 cases of oxazepam and nordiazepam, respectively. Noteworthy, coma was never used to qualify alteration in consciousness of the poisoned patients. The median delay in onset was $1 \mathrm{~h}$ in both poisonings. Interestingly in both poisonings, there was no reported delay in onset of the Emax which was reported on a "on-off" mode. The median TI resulting in the Emax effect was 3.3- and 19.6-fold, the maximum recommended daily dose in oxazepam and nordiazepam poisonings, respectively. Follow-up of the poisoned patients was performed in only 19 and $13 \%$ of the oxazepam and nordiazepam poisonings, respectively, precluding any definitive conclusion about the duration of symptoms.

Discussion Toxicodynetics is a new discipline in clinical toxicology focusing on relevant clinical toxic effects induced by a substance. Toxicodynetics may be described for all toxic substances using clinical and biological data routinely collected. Toxicodynetics does not require any toxicological analysis and may be performed in both mono- and poly-intoxication. In spite of major limitations of the study which was a monocentric, retrospective study, some information is provided regarding the early toxicodynetic phases of oxazepam and nordiazepam involving exposures to large doses of each toxicant. Both poisonings were quite similar in terms of delay in onset of about $1 \mathrm{~h}$, an "on-off" mode of occurrence of the Emax. In spite of large supposed ingested doses, limited effects on consciousness were reported, never using the term of coma. Oxazepam is the terminal common metabolite of a number of benzodiazepines, including nordiazepam. Oxazepam is both metabolized into inactive metabolites and eliminated unchanged in the urine. During the course of nordiazepam, a part of nordiazepam is metabolized into oxazepam which actually always results in a mixed nordiazepam and oxazepam poisonings. In spite of the supposed ingestion of large doses, the Emax in nordiazepam poisoning was not greater than that induced by oxazepam.

Conclusion Toxicodynetics provides information allowing going further to understand which substances may dramatically alter consciousness. These findings support the hypothesis of the role of a drug-drug intoxication. The toxicodynetic approach unveils limitation of data collected routinely in PCC, not allowing to describe the late phase of poisoning. Toxicodynetics provides quantitative information on the quality of report of data in acute poisonings.

Competing interests None.
S123

Rapid triage of victims suspected of a chemical attack: a one-page sheet allowing identifying the toxidrome: the doctors without borders (MSF) experience

Frederic Baud ${ }^{1}$, Robert Garnier ${ }^{2}$, Brigitte Vasset ${ }^{3}$, Annette Heinzelman ${ }^{4}$ ${ }^{17} 75014$, SAMU de Paris, Réanimation polyvalente, Paris, France; ${ }^{2}$ Hôpital fernand widal, Centre Antipoison et de Toxicovigilance de Paris, Paris,

France; ${ }^{3} 2$, rue saint sabin 75012 Paris, Médecins Sans Frontières, Paris, France; ${ }^{4}$, rue saint-sabin 75012, Médecins Sans Frontières, Paris, France Correspondence: Frederic Baud - baud.frederic@wanadoo.fr Annals of Intensive Care 2016, 6(Suppl 1):S123

Introduction Over the past 5 years, events in the Middle East have increased the awareness about the possible use of chemical weapons and their surrogates, the so-called Toxic Industrialized Compounds. The major issue is to identify on a clinical basis the likelihood of exposure to chemicals ranging from riot agents to lethal agents in order (1) to advice protection of rescuers and caregivers at the scene, (2) to provide adapted treatment to casualties, and (3) to address the need for antidote supply.

Patients and methods This study is a systematic review of the literature on acute chemical accidents resulting in casualties to identify easily collected clinical signs and symptoms resulting. This review provided a limited list of signs to be collected in a small subset of frankly symptomatic patients. The signs and symptoms were classified according to the corresponding organ(s) involved by chemical injury. All signs should be clinically collected using current physical examination. We hypothesized that (1) such a chemical disaster cannot injure a single patient, (2) the collection of data can be repeated due to either an immediate onset or a progressive onset of signs and symptoms on an hourly or longer basis at the request of the attending physician. We advised to examine at least three symptomatic casualties and no more than 10. There are presently three versions of the sheet: French, English, and Arabic. Owing to the political context and analytical difficulties, we never attempted at precisely determining the agent having caused signs and symptoms.

Results The one-page questionnaire was used in 5 settings, including (1) a chemical incident related to the opening of a chemical plan in Lumumbashi in 2011, (2) the repeated use of anticholinesterasic agents towards civilians in Syria from April 2013 up to that the attack launched the 21 August 2013, (3) repeated chlorine attack towards civilians in Syria since March 2014, (4) an attack using a vesicant agent towards civilians in August 2015 in Syria, and (5) the acute onset of paraplegia in Mali on Summer 2015. In each setting, the analysis of the quoted items on the sheet allowed to suggest the class of the toxicant within a few hours after exposure. In the case of exposure to chlorine and to the vesicant agent, two collections of signs over a period of a few hours were needed to draw definitive conclusion. The lowest number of casualties in an exposure was three after exposure to vesicant agents. The definitive diagnosis was in accord with the initial presumption regarding the class of toxicant. Early identification allowed to advise adapted protection of caregivers at the site as well as define the need for antidote supply and the nature of the antidote. Conversely, in one setting (Mali 2015) a toxic origin was ruled out as not fitting any known toxicant while an infectious disease was identified as the likely cause of the outbreak.

Discussion In case of suspected chemical incident or supposed terrorist attacks, a one-page sheet of the list of signs and symptoms dedicated to identity relevant signs and symptoms allowed to define a toxidrome specific of a class of toxicant, helped in protecting rescuers and caregivers at the site and refined the needs for supportive and antidotal treatment. Repeated collection of signs may be required in agents acting progressively. A close collaboration with a skilled toxicologist is needed when facing incident involving hazardous materials. At the scene only chemical incident was identified. However, repetition of bombing in the same area resulted in a more accurate and rapid response of pre-hospital and hospital at the scene.

Conclusion A one-page sheet easily filled out at the scene in the context of chemical incident allows defining the toxidrome and consequently protection of rescuers, needs for supportive and antidotal supply. Owing to the rapidity of action a number of toxicant, a single 
or repeated examination over a few hours is needed to define the toxidrome and therefore the class of toxicant.

\section{Competing interests None.}

\section{S124}

Low sensitivity of toxicological analysis in daily practice of acute poisonings: an endless rupture in spite of modern technology

Frederic Baud ${ }^{1}$, Alaywa Khadija ${ }^{2}$, Romain Jouffroy ${ }^{3}$, Lionel Lamhaut ${ }^{3}$

${ }^{1}$ 75014, SAMU de Paris; Réanimation polyvalente, Paris, France; ${ }^{2}$ Samu de paris, centre antipoison de Paris, UMR-8536, Hospital Necker, Paris, France; ${ }^{3}$ Réanimation adulte, Hôpital Necker - Enfants Malades, Paris, France Correspondence: Frederic Baud - baud.frederic@wanadoo.fr Annals of Intensive Care 2016, 6(Suppl 1):S124

Introduction The diagnosis in clinical toxicology is based on the collection of information on (1) the substance, the dose, and the route of administration, (2) signs and symptoms that have to fit the supposed ingested drug (SID), and (3) toxicological analysis (TA) which is expected to provide the definitive diagnosis. However, in the past, the development of methods based on immunoenzymology provided information on the class rather the substance by itself with questionable ability to quantify exposure. Consequently, routinely used TA was progressively discarded. TA was recommended in only a few number of toxicants and in conditions where SID does not explain major signs and symptoms. However, over the past decade, modern TA using various modes of mass spectrometry was developed; meanwhile, analysts claimed they was able to address all toxicological concerns of attending physicians. From a theoretical viewpoint, this assumption was sounded. However, the translational to practice has not been assessed to our knowledge. The aim of this retrospective study performed in a medical polyvalent intensive care unit (MPICU) was to test the hypothesis more especially as the MPICU has unlimited access to facilities provided by three University Toxicology Laboratory (Tox Lab).

Patients and methods Patients Included patients were adult patients admitted for suspicion of poisoning ranging from moderate to severe in our MPICU from the January 1, 2014, until April 2015. This period of time corresponded to the period of time at which the MPICU has unlimited access to the three Tox Lab. Toxicology analyses: According to our current practice, blood and urine specimens were collected at the time of admission and sent to the different Tox Lab owing to the facilities provided by each, including the local hospital for a limited of toxiciants, the Tox Lab on duty in our institution allowing screening and dosing, and the Tox Lab of the Forensic Department of our institution. The latter two have facilities of modern apparatus including mass spectrometry in various modes as well as separative process (gas and liquid). The Tox Lab was blinded regarding the aim of the study. Expression of results: In each patient results from the different laboratory were gathered, one positive detection/quantification of a SID was considered SID+, TA+; lack of SID with positive TA: SID-, TA+, a SID+ with negative TA was considered SID+, TA-. Unfortunately, there was no SID6, TA - as there was no need to ask for the presence of a drug to Tox Lab knowing it is was absent in a study done in current practice. Assessment of severity of exposure Severity of exposure is assessed only for drugs and alcohol using the maximum daily dose recommended for drugs (a therapeutic index $>1$ denotes an intoxication) and a blood alcohol level (BAL) of $0.4 \mathrm{~g} / \mathrm{l}$ (a BAL > $0.4 \mathrm{~g} / \mathrm{l}$ was considered toxic).

Results Over the study period, there were 224 occurrences concerning 90 SID (a number of multiple occurrences of the same drug) in 70 patients (a number of patients reported multiple drug ingestions). A SID+, TA + was recorded in only $33 \%$ of the 224 occurrences; however, the $\mathrm{TI}$ was $>1$ in $45 \%$ of the dosed drugs. In addition, in the group of SSI-, TA+, the TA added $15 \%$ of patients with SID-. However, in $79 \%$ of the SID-, TA+, the TI was less than 1. This finding suggests the detection was that of drugs prescribed or used by the patient therapeutically or recreatively in this class of patients SID6, TA+.

Discussion In the sixties, toxicological analysis was considered the gold standard to definitively assume the role of a substance in a poisoning. Surprisingly, while the methods used in analytical toxicology resulted in a manifold increase in sensitivity and specificity allowing to screen hundreds of substances in 10 microliter of whole blood, the added value of analytical toxicology is fairly low as shown by evidencing the SID in only $33 \%$ of the occurrences. We can question about the accuracy of the SID. However, until the toxicological analysis does not currently assume the substance is not present, the analytical result is subject to caution. The question is open to know whether high-resolution mass spectrometer can actually increase the added value of TA. Furthermore, closer collaboration regarding the actual needs of the clinician may help analysts in defining the lists of toxicant of interest, providing regular update of this list of toxicant.

Conclusion Gathering facilities provided by three University Tox Lab using modern technology resulted in a low added value of toxicological analysis in acute poisonings daily presenting in ICU. Improving the gap might be helped by high-resolution mass spectrometry. However, a closer collaboration between toxicologists and analysts should result in filling the gap.

\section{Competing interests None.}

\section{S125}

Context of the request and interest of toxicological screening in a medical intensive care unit

Elsa Démarest Durand ${ }^{1}$, Fabienne Tamion ${ }^{2}$, Christophe Girault ${ }^{3}$, Gaetan Beduneau ${ }^{3}$, Dorothée Carpentier ${ }^{3}$, Patricia Compagnon ${ }^{4}$, Steven Grange ${ }^{3}$ ${ }^{1}$ 76, Chu Rouen, Rouen, France; ${ }^{2}$ Réanimation médicale, Hospital Center University Rouen, Rouen, France; ${ }^{3}$ Réanimation Médicale, Centre Hospitalier Universitaire Rouen, Rouen, France; ${ }^{4} 76$, Centre Hospitalier Universitaire Rouen, Rouen, France

Correspondence: Elsa Démarest Durand - demarestelsa@hotmail.com Annals of Intensive Care 2016, 6(Suppl 1):S125

Introduction Drug poisoning is a frequent cause of hospitalization in intensive care units. The history, physical examination, electrocardiogram and standard biology are usually sufficient to make the diagnosis. In the most difficult cases, recent advances in analytical toxicology (toxicological screening) can identify possible accidental or intentional overdose or poisoning with the most frequently used drugs. Recent French guidelines indicate when this drug screening should be used, but its clinical interest is not clearly demonstrated. The purpose of this study is to evaluate the diagnostic and therapeutic usefulness of toxicological screening. We also checked whether toxicological screening requirements were consistent with indications from the 2012 French guidelines (clinical and complementary examinations incompatible with the expected toxidrome, unexplained hemodynamic failure or coma).

Patients and methods This is a retrospective single-center observational study conducted in a University Hospital. All patients hospitalized in the Medical Intensive Care Unit in 2013 and 2014 who received a toxicological screening were included. The result of toxicological screening was considered useful when it found a toxic etiology that was not initially suspected or if it concluded to a non-toxic cause in a patient suspected of drug overdose.

Results During the study period, 118 patients underwent a toxicological screening in the medical intensive care unit. The most common reasons for admission were toxic coma (39\%) and coma of other cause $(25 \%)$. The main drugs responsible of overdose were toxic substances in the central nervous system (72\%), especially benzodiazepines ( $28 \%$ ) and neuroleptics (11\%). Cardiotropic drugs were found in $8 \%$ of cases, mainly beta-blockers (5\%). The toxicology screening was asked because of a suspicion of poisoning in $58 \%$ of patients and because of clinical manifestations without obvious etiology in $42 \%$ of cases. The toxicological screening was performed according to the 2012 French guidelines in $48 \%$ of cases. The main reason for prescribing a toxicological screening without following the guidelines was a toxic coma consistent with the suspected toxidrome (54\%). The result was considered useful in the diagnosis in $27 \%$ of cases with the finding of a toxic etiology or of a non-suspected toxic in $20 \%$ of patients. However, the therapeutic management was never modified following the results.

Discussion The results of our study considering the diagnostic utility are consistent with existing data of the literature. The lack of change 
in the therapeutic approach of patients is correlated with previously published studies but is probably underestimated in the context of our retrospective study. Our toxicology screening requirements were not in agreement with the guidelines in half of the cases. A change in the way we prescribe toxicological screening is ongoing (information sheet accompanying the prescription of a toxicology screening, better collaboration between clinicians and biologists) to improve the costbenefit ratio of this technique.

Conclusion Our study highlights the diagnostic utility of toxicological screenings. A prospective study would highlight their real impact in the therapeutic management of patients.

\section{Competing interests None.}

\section{S126}

Acute poisoning in emergency department

Neila Maaroufi', Dhaouadi Mahassen²

${ }^{1}$ GENERAL HOSPITAL, Tunis, Tunisia; ${ }^{2}$ Tunisia, GENERAL HOSPITAL, Tunis, Tunisia

Correspondence: Neila Maaroufi - maaroufineila@gmail.com

Annals of Intensive Care 2016, 6(Suppl 1):S126

Introduction Acute poisoning represents a frequent reason of admission in emergency rooms and intensive care units (ICU). Evaluation of intoxication severity should rely on a rigorous approach based on analysis of prognostic factors. Existing prognostic scores are not efficient enough to predict ICU admission in daily practice. Our purpose is to identify ICU transfer risk factors for acute poisoning.

Patients and methods This was retrospective study from 2012 to 2014. All patients admitted to emergency department (ED) with a diagnosis of accidental or voluntary intoxication were included. Clinical and toxicological data were first analyzed with univariate tests.

Results During study period, 571 patients were included and $77 \%$ were women. Mean age was $26 \pm 13$ years. Toxic ingestion was voluntary in $87 \%$ of cases. The median delay of consultation was $2 \mathrm{~h}$ after ingestion. Thirty-five had psychiatric history ( $23 \%)$. The offending toxic was a drug in $83 \%$ of cases (dominated by psychotropic drug and cardiotropic drug) and a pesticide in $25 \%$ of cases. The initial examination had showed: consciousness disorders with coma, digestive disorders, hypotension $(<90 \mathrm{mmHg})$ and fasciculation. The toxidrome were identified in $55 \%$ of cases: anticholinergic, muscle relaxant, cholinergic, membrane stabilizer, opioid and adrenergic. Fifty-nine percentage of patients were admitted in ICU. The initial management in ED: digestive decontamination in $81 \%$ of cases, antidotes, intubation and vasoactive drugs. In multivariate analysis, history of previous ICU admission for poisoning (OR 3.56; IC $95 \%$ 1.08-13.22; $p=0.03$ ) and Glasgow Coma Scale (GCS) less than 13 (OR 4.47; IC $95 \%$ 2.6-7.25; $p<0.002$ ) were identified as risk factor for intensive care admission.

Discussion and conclusion Anamnestic content (history of previous ICU admission for poisoning, type of toxic $=$ chloralose) and clinical criteria at admission (GCS an anticholinergic toxidrome) are easily identified prognostic factors for early and appropriate orientation patients admitted to the ED for acute poisoning.

\section{Competing interests None.}

\section{S127}

\section{Management of poising: Are there differences}

between specialised and non-specialised emergencies?

A M'rad ${ }^{1}$, Alia Jebri ${ }^{1}$, Youssef Blel ${ }^{1}$, Hend Ben Lakhal' ${ }^{1}$, Messaouda Khelfa ${ }^{1}$ Nozha Brahmi ${ }^{1}$, H Thabet $^{2}$

${ }^{1}$ Department of intensive care and toxicology, Centre d'Assistance Médicale Urgente, Tunis, Tunisia; ${ }^{2}$ Department of emergency, Centre d'Assistance Médicale Urgente, Tunis, Tunisia

Correspondence: A. M'rad - mrad.aymen@gmail.com

Annals of Intensive Care 2016, 6(Suppl 1):S127

Introduction Acute poisoning (AP) outcome depends on an early and effective management. The aim of our study was to compare the initial management of AP in a specialised toxicology emergency to the other healthcare facilities referring to international recommendations.

Patients and methods We undertook a comparative prospective observational study over 3 months. We included ail patients aged over 14 years. The study population was divided into two groups: G1, patients directly consulting the specialised toxicology emergency; and $G 2$, patients transferred from non-specialised emergency. The primary endpoint was the initiation of symptomatic treatment, appropriate gut decontamination and antidote use. The secondary endpoints interested intensive care unit hospitalised patients and concerned the occurrence of aspiration pneumonia, nosocomial infection, length of stay, mechanical ventilation and mortality.

Results Our study included 420 patients, 123 men and 297 women, and the mean age was $26[20,35]$ years. Our two groups gathered 273 patients for the $\mathrm{G} 1$ and 147 patients for $\mathrm{G} 2$, and they were comparable for demographic and anamnestic data.

The initiation of treatment of neurologic failure was significantly more frequent in $\mathrm{G} 1(p=0.01)$. The treatment of circulatory shock and respiratory failure was comparable in both groups.

There was no significant difference between the two groups regarding the appropriate use of gastrointestinal decontamination. The antidote treatment was more appropriate for $\mathrm{G} 1(p=0.0001)$.

For hospitalised patients, the occurrence of aspiration pneumonia, nosocomial infection, length of stay, mechanical ventilation and mortality was similar in both groups.

Conclusion The initial management of AP was not always consistent with the recommendations especially in healthcare facilities other than CAMU. To overcome these shortcomings, training of primary care physicians and better collaboration with poison control centers is needed.

\section{Competing interests None.}

5128

Jusquiame poisoning: report of a family intoxication

Hassen Ben Ghezala', Salah Snouda', Rebeh Daoudi ${ }^{1}$, Moez Kaddour ${ }^{1}$

${ }^{1}$ Teaching Department of Emergency and Intensive Care, Hospital Zaghouan, Zaghouan, Tunisia

Correspondence: Hassen Ben Ghezala - hassen.ghezala@gmail.com Annals of Intensive Care 2016, 6(Suppl 1):S128

Introduction Plant intoxication is more and more frequent in Tunisia, especially in rural regions. It concerns nearly $2-3 \%$ of calls to the national Tunisian poison center in the last 10 years. They occur mainly in Spring season. Plant poisoning still be rare and unrecognized by health caregivers working in emergency and intensive care departments. The aim of our work was to report an extremely rare accidental collective plant poisoning by "hyoscyanus Niger" which happened in Zaghouan in Tunisia in a traditional celebration.

Patients and methods It was a descriptive transversal and retrospective study reporting a family collective (twenty-one members (21)) poisoning. This accidental poisoning occurred in the region of Zaghouan in the spring 2015 (May). All members of the family consumed in a traditional village marriage "couscous" prepared with an alkaloid plant identified as "jusquiame." This plant was confounded with Swiss chard.

Results On May 2015, a family composed of twenty-one members (ten men and eleven women) was admitted to the teaching emergency and intensive care department of the regional hospital of Zaghouan (Tunisia). All of them attended emergency department with a non-medical transfer.

All members of the family presented nausea, vomiting, dizziness and hallucinations.

Ten patients presented generalized seizures. All patients presented a great agitation. They reported the ingestion $12 \mathrm{~h}$ before a traditional couscous prepared specially for a traditional marriage.

They reported that it was prepared by "Swiss chard" specific to the region.

The average of blood pressure was $160 / 100 \mathrm{mmHg}$. All members of the family experienced tachycardia with extremes ranging from 100 
to 160 beats per minute. On physical examination, six members of the family had myalgia and abdominal pain without other clinical signs. We performed for all patients standard chest X-ray which was normal and laboratory tests.

Five patients (5) had hyperglycemia. Six patients (6) presented hypokalemia and hyperleukocytosis. All brain CT scans performed for the ten (10) patients presenting with seizures were normal. They received diazepam as anti-convulsive agent and were admitted in the intensive care unit for a mean hospital stay of 3 days. All patients recovered totally and were discharged from hospital after a stay ranging from 3 to 5 days.

Samples of the herbal decoction were obtained from the couscous and were sent to be analyzed in the laboratory of toxicology in the Center for Emergency Medical Assistance of Tunis in Tunisia.

Screening by GC-MS revealed the presence of scopolamine, hyoscyamine and hydroxyhyoscyamine.

Conclusion Plant poisoning still be rare in Tunisia. The plant consumed in this case series report "jusquiame" belongs to the genus "hyoscyanus" and to the variety of "solanacés." We conclude from our work that plant poisoning can be extremely severe with neurological complications like seizures. The management is mainly symptomatic. Education and prevention still be the best treatment against plant poisoning.

\section{Competing interests None.}

\section{S129}

Morbi-mortality of poisoning episodes in Paris region: a test for a multisource surveillance system

Céline Legout ${ }^{1}$, Robert Garnier ${ }^{2}$, Antoine Villa ${ }^{2}$, Frederic Baud ${ }^{3}$, Philippe Aegerter ${ }^{4}$, Anne Castot-Villepelet ${ }^{5}$

${ }^{1}$ Agence régionale de santé ile-de-france, Cellule de I'InVS en régions (Cire) Île-de-France et Champagne-Ardenne, Paris, France; ${ }^{2}$ Hôpital fernand widal, Centre Antipoison et de Toxicovigilance de Paris, Paris, France; ${ }^{3} 75014$, SAMU de Paris, Réanimation polyvalente, Paris, France; ${ }^{4}$ Urc, Hospital Ambroise Paré, Boulogne-Billancourt, France; ${ }^{5}$ Département des vigilances, Agence régionale de santé (ARS) Ile-de-France, Paris, France Correspondence: Frederic Baud - baud.frederic@wanadoo.fr

Annals of Intensive Care 2016, 6(Suppl 1):S129

Introduction The number of poisonings occurring during each year in Paris area is not known. The Paris Poison Control Centre (CAPTV) reports annually a number of exposures (asymptomatic) and poisonings (symptomatic exposure). However, a number of other medical activities deal with intoxications, including emergency department in hospitals, intensive care units, and forensic laboratories. This study aimed at addressing this issue by means of collaboration between different departments involved in the management of or dealing with acute poisonings in lle-de-France.

Patients and methods The study, led by the local team of the French Institute for Public Health Surveillance (InVS) and the Regional Health Agency of lle-de-France (ARS-IDF), is based on the descriptive analysis of patient data collected by the Paris Poison Control Center (CAPTV), an emergency department (SU) participating in the Oscour ${ }^{\circledR}$ network, a medical and toxicological intensive care unit (RMT) whose results were extended using the CUBRéa database, the regional coordination of pharmacovigilance (CRPV) in lle-de-France, and two toxicological laboratories conducting judicial forensic expertise after extra-hospital deaths. Acute poisoning having occured in the Ile-de-France (IDF) region, in 2010 and 2011, whatever the circumstances and severity were included. Deaths were collected prospectively in 2014. Druginduced adverse effects were not included. A particular attention was paid to eliminate duplicate.

Results Besides 27145 cases (annual average) of poisoning or toxic exposure/poisonings reported to CAPTV corresponding mainly to accidental poisonings of young children, it should be added 2980 annual cases for the SU, 270 in ICU, 279 for the CRPV corresponding to acute poisonings not reported to the CAPTV, and 207 toxic deaths. Based on these figures, about 63,000 cases of acute intoxications occur in IDF every year with a global death rate estimated at $0.8 \%$. The profile of the intoxicated subjects differ significantly, other partners dealing mainly voluntary poisoning in adults among them, drug poisoning predominate, associated or not with alcohol.

Conclusion This multisources cooperation confirms the hypothesis of a significant underestimation of poisonings identified by the CAPTV (one in two cases beyond him) and the possibility to improve the knowledge of the epidemiology of acute poisoning, thanks to the multiplicity of ways of observation of each partner.

\section{Competing interests None.}

\section{S130}

Atypical pneumonia in the ICU: a retrospective multicentric study Sandrine Valade ${ }^{1}$, Virginie Lemiale ${ }^{2}$, Laurent Argaud ${ }^{3}$, Frédéric Pène ${ }^{4}$ Laurent Papazian ${ }^{5}$, Fabrice Bruneel ${ }^{6}$, Amélie Seguin ${ }^{7}$, Achille Kouatchet ${ }^{8}$ Johanna Oziel ${ }^{9}$, Olivier Lesieur ${ }^{10}$, Florence Boissier ${ }^{11}$, Bruno Megarbane $^{12}$, Naïke Bigé ${ }^{13}$, Noelle Brule ${ }^{14}$, Anne-Sophie Moreau ${ }^{15}$, Alexandre Lautrette $^{16}$, Elie Azoulay ${ }^{2}$

${ }^{1}$ Réanimation médicale, Hôpital Saint-Louis, Paris, France, Paris, France; ${ }^{2}$ Réanimation médicale, Hôpital Saint-Louis, Paris, France; ${ }^{3}$ Réanimation Médicale, Hospices Civils de Lyon - Groupement Hospitalier Edouard Herriot, Lyon, France; ${ }^{4}$ Réanimation Médicale, Hôpital Cochin, Paris, France; ${ }^{5}$ Service de réanimation-détresses respiratoires et infections sévères, Hôpital Nord, Marseille, France; ${ }^{6}$ Réanimation médico-chirurgicale, Centre Hospitalier de Versailles, Le Chesnay, France; ${ }^{7}$ Réanimation médicale, Centre Hospitalier Universitaire de Caen, Caen, France; ${ }^{8}$ Réanimation médicale, Centre Hospitalier Universitaire d'Angers, Angers, France;

${ }^{9}$ Réanimation medico-chirurgicale, hopital avicenne, Bobigny, France;

${ }^{10}$ Réanimation, Centre Hospitalier la Rochelle, La Rochelle, France; ${ }^{11}$ Réanimation Médicale, Hôpital Georges Pompidou, Paris, France; ${ }^{12}$ Service de Réanimation Médicale et Toxicologique, CHU Lariboisière, Paris, France; ${ }^{13}$ réanimation médicale, Hôpital Saint-Antoine, Paris, France; ${ }^{14}$ Réanimation médicale, CHU, Nantes, France; ${ }^{15}$ Réanimation Médicale, Hôpital Saint-Louis, Assistance Publique Hôpitaux de Paris, Paris, France; ${ }^{16}$ Réanimation médicale, CHU Gabriel-Montpied, Clermont-Ferrand, France Correspondence: Sandrine Valade - valadesandrine@gmail.com Annals of Intensive Care 2016, 6(Suppl 1):S130

Introduction Chlamydia pneumoniae and Mycoplasma pneumoniae are rare pathogens that may lead to life-threatening acute hypoxemic respiratory failure. We sought to describe critically ill patients with atypical pneumonia managed in 16 French ICUs.

Patients and methods This is a retrospective cohort study of adult patients admitted to the ICU with proven atypical pneumonia (positive serology or PCR) over a 15-year study period (2000-2015).

Results Eighty-nine patients were included (62 men, 27 women) with a median age of 56 [44-66] years. They were mainly admitted for acute respiratory distress $(n=82 ; 92 \%)$. Main comorbidity was chronic respiratory failure $(n=28 ; 31 \%)$. Time from respiratory symptoms to hospital admission was 5 [3-8] days. At ICU admission all patients had tachypnea (respiratory rate was $31[27.5-36.5] / \mathrm{min}$ ) and half presented with respiratory distress. Physical examination reported fever in $71 \%$ of the patients, crackles in $50 \%$ (45 patients), ronchi in 14 (16\%), wheezing in $10(11 \%)$ and consolidation in $6(7 \%)$ patients. Extra-pulmonary symptoms were present in 32 (36\%) patients (arthritis, $n=2$; myocarditis, $n=4$; hemolytic anemia, $n=4$ and skin rash, $n=6$ ). Three patients ( $3 \%$ ) had confusion, and meningoencephalitis was diagnosed in one patient. Cold agglutinins were identified in $8(9 \%)$ patients, cytolysis was present in $9(10 \%)$ cases, and rhabdomyolysis in one patient. Ten ( $11 \%$ ) patients had shock and 27 had various neurological disorders. SOFA score was 5 [2-7], and SAPSII was 33 [25-41]. Chest X-ray disclosed alveolar opacities $(n=49,55 \%)$ or interstitial opacities ( $n=28,28 \%)$. Two [1-4] quadrants were involved on chest radiography. Pleural effusion $(n=5,6 \%)$ was a rare finding.

Pneumonia was related to Mycoplasma pneumoniae $(n=66 ; 74 \%)$ or Chlamydia pneumoniae ( $n=23 ; 26 \%)$. Microbiological diagnoses were confirmed by serology (positive IgM in favor of recent infection or elevated IgG, $n=57$ ) or positive PCR in respiratory samples $(n=34)$. Coinfection was found in 18 cases $(20 \%)$, with viruses $(n=8)$, bacteria $(n=7)$ or fungi $(n=3)$. Throughout the ICU stay, mechanical ventilation was required in $67(75 \%)$ patients and 31 (46\%) had criteria for ARDS. Four patients needed ECMO. Length of mechanical ventilation 
was 13 [8-18] days. More than one-third of the patients $(n=33)$ needed vasopressors. Ten patients required renal replacement therapy (11\%).

First-line antibiotics included active treatment on atypical bacteria in 54 cases (61\%), started 1 day [0-4] after ICU admission. Combination therapy was used in 52 patients (58\%): third-generation cephalosporin (C3G) and macrolide in $19(36 \%)$ patients, C3G and quinolone in $12(23 \%)$ patients, other betalactam and macrolide in $15(29 \%)$ patients or other betalactam and quinolone in $6(12 \%)$ patients. Antibiotics were further adjusted with macrolides mostly $(n=60)$, quinolones $(n=22)$ or cyclines $(n=2)$. Nine $(10 \%)$ patients died in the ICU. ICU length of stay was 15 [8.5-27] days. Almost half of the patients had persistent hypoxemia at ICU discharge. By univariate analysis, factors associated with mortality were duration of mechanical ventilation (18 [15.5-24.5] days vs 12 [8-17], $p=0.02$ ) and renal replacement therapy ( 3 vs 7 patients, $p=0.02$ ). Lack of respiratory distress was associated with a better outcome $(p=0.014)$.

Conclusion Atypical pneumonia is associated with $10 \%$ case fatality. At admission, extra-pulmonary symptoms were frequent and could guide intensivists to avoid missing the diagnosis. Strikingly, time to effective antibiotic against intracellular pathogens was not associated with mortality. Studies are needed to validate an algorithm for establishing clinical probability of atypical pneumonia in the critically ill.

\section{Competing interests None.}

\section{S131}

\section{Severe leptospirosis: analysis of mortality and organ failures} in 134 patients hospitalized in intensive care unit

Benjamin Delmas ${ }^{1}$, Julien Jabott ${ }^{1}$, Paul Chanareille', Jérôme Allyn ${ }^{1}$, Nicolas Allou', Cyril Ferdynus ${ }^{2}$, Bernard-Alex Gauzere' , Olivier Martinet ${ }^{1}$, David Vandroux

${ }^{1}$ Réanimation polyvalente, CHU La Réunion, Saint-Denis, France; ${ }^{2}$ Unité de soutien méthodologique, CHU La Réunion, Saint-Denis, France Correspondence: Benjamin Delmas - benjamindelmas.desar@yahoo.fr Annals of Intensive Care 2016, 6(Suppl 1):S131

Introduction Leptospirosis is a worldwide distributed zoonosis with a high mortality rate. It has been declared a major public health issue by the World Health Organization. Consensual definition of severe leptospirosis is somehow lacking. Reunion Island is one of the few regions with a high endemicity rate where every kind of organ support is available, including extra-corporeal membrane oxygenation (ECMO). The aim of this study is to determine the mortality rate of leptospirosis in an intensive care unit (ICU) where all kinds of organ supports are available and to asses the organ failures. This would help to define what severe leptospirosis is, in order to decide what patients must to be admitted in ICU.

Patients and methods This is a retrospective study from January 2004 to January 2015. All patients admitted with leptospirosis in two critical care units (Reunion Island, France) were included. We collected demographic, clinical and biological data and performed bivariate analysis to examine risk factors associated with mortality.

Results One hundred and thirty-four patients were included with biological diagnostic confirmed in $100 \%$ of the cases. Mortality rate in ICU was $6 \%$. Mean Simplified Acute Physiology Score (SAPS)-2 was $40 \pm 19$, and mean Sequential Organ Failure Assessment (SOFA) score was $10 \pm 4$. Mean age was $41 \pm 15$ years. Acute renal failure was identified in $95 \%$ of cases, thrombopenia in $93 \%$ of the cases and hyperbilirubinemia in $90 \%$ of the cases. Pulmonary involvement was frequent, with $40 \%$ alveolar hemorrhage. Twenty-one percent of the patients presented moderate-to-severe acute respiratory distress syndrome (ARDS), according to the Berlin definition. All patients have been treated by antibiotics. Fifty-six percent of the patients underwent CRRT, $31 \%$ underwent invasive mechanical ventilation and $4 \%$ underwent ECMO. Mean time for CRRT setup was 1 [1-2] day. Mean time for tracheal intubation was 12 [4-22] h. Risk factors associated with mortality were SAPS-2, SOFA score, neurological and/or respiratory failure on admission, alveolar haemorrhage, time between onset of symptoms and antibiotherapy, need for invasive mechanical ventilation, need for vasoactive support, kaliemia, $\mathrm{pH}$, lactate, base excess, bilirubinemia and blood products transfusion. Main cause for mortality was refractory ARDS (62\% of cases). All deceased patients had required oxygen support or were already under invasive mechanical ventilation upon admission.

Discussion To our knowledge, this is the largest case series of patients admitted for leptospirosis in ICU. This study pinpoints two important findings in this young population, such as the association of a very low mortality rate despite high severity scores and a strong association between respiratory failure and death. Prompt organ support, including CRRT and mechanical ventilation, is most probably the main explanation for such low mortality rate. All patients requiring oxygen support, even if as little as $1 \mathrm{~L} / \mathrm{min}$, should promptly be admitted in ICU. CRRT should in no way be delayed in patients presenting with kaliemia $>4 \mathrm{mmol} / \mathrm{L}$ and oligo-anuria. Hyperbilirubinemia is for the first time to be identified as a risk factor for mortality, but it has already been described as a risk factor for mortality in ARDS. Cause or effect, it may participate to the particular pattern of pulmonary failure in leptospirosis.

Conclusion Severe leptospirosis should be defined as leptospirosis with oxygen requirement, especially if acute renal failure is coexisting.

\section{Competing interests None.}

\section{S132}

In severe pneumonia, respiratory viruses are not passengers but pathogens

Guillaume Voiriot', Benoit Visseaux², Johana Cohen'1 Liem Binh Luong Nguyen ${ }^{3}$, Mathilde Neuville ${ }^{4}$, Caroline Morbieu ${ }^{3}$, Charles Burdet ${ }^{3}$, Aguila Radjou ${ }^{5}$, François-Xavier Lescure ${ }^{3}$, Roland Smonig ${ }^{1}$, Laurence Armand-Lefevre ${ }^{6}$, Bruno Mourvillier ${ }^{7}$, Yazdan Yazdanpanah ${ }^{3}$, Jean-François Soubirou', Stephane Ruckly ${ }^{8}$, Nadira Houhou-Fidouh², Jean-François Timsit $^{5}$

${ }^{1}$ Service de réanimation médicale et infectieuse, Hôpital Bichat-Claude Bernard-APHP, Paris, France; ${ }^{2}$ Service de virologie, Hôpital Bichat-Claude Bernard, Paris, France; ${ }^{3}$ Service de maladies infectieuses et tropicales, Hôpital Bichat-Claude Bernard, Paris, France; ${ }^{4}$ PARIS, Hôpital Bichat-Claude Bernard, Paris, France; ${ }^{5}$ Réanimation médicale et infectieuse, Hôpital Bichat-Claude Bernard, Paris, France; ${ }^{6}$ Service de microbiologie, Hôpital Bichat-Claude Bernard, Paris, France; ${ }^{7}$ Réanimation Médicale et Infectieuse, GH Bichat Claude Bernard, Paris, France; ${ }^{8}$ Reanimation, hôpital, Grenoble, France

Correspondence: Guillaume Voiriot - guillaumevoiriot@hotmail.com Annals of Intensive Care 2016, 6(Suppl 1):S132

Introduction Bacteria are usually recognized as the main causative group of pathogens in community acquired pneumonia (CAP), but the important role of respiratory viruses has recently emerged. In studies having used multiplex polymerase chain reaction (mPCR) in CAP, viruses were identified in $15 \%$ to $49 \%$ of the patients. The impact of such viral documentation in airways remains largely uncertain. We studied a cohort of adult patients with CAP requiring intensive care unit (ICU) admission and explored the impact of the viral presence in the respiratory tract.

Patients and methods This is a monocenter retrospective study. All consecutive adult patients admitted to the ICU (Hôpital Bichat Claude Bernard, AP-HP) from October 2011 to June 2015 with a final diagnosis of pneumonia were screened. Patients having undergone mPCR in the respiratory tract within the $72 \mathrm{~h}$ following the ICU admission were included. Demographics, past medical history, clinical, biologi$\mathrm{cal}$, microbiological and radiological data, organ failures and supports and outcome were collected. Four groups of patients were constituted according to the microbiological diagnosis: bacterial, viral, mixed (viral-bacterial) and no etiology groups. The primary objective was to determine the impact of the viral-bacterial coinfection on the presentation and prognosis. A composite criterion of "altered prognosis" included the hospital death or mechanical ventilation for more than 7 days. The second objective was to describe the epidemiology of respiratory viruses in adult patients admitted to ICU for a CAP in Western Europe.

Results One hundred and seventy-four patients (132 men (76\%), age 63 [53-75] years, SAPS II 38 [27;55]) were studied. Fifty-eight 
(33\%) patients were referred from medical wards or other ICU. The median PSI score at hospital admission was $106[78 ; 130]$. Pneumonia was considered healthcare-associated pneumonia in near half patients $(86,49 \%)$. At least one factor of immunosuppression was presented in 57 (33\%) of patients. A microbiological documentation was obtained in 144 (83\%) patients. At least one virus was identified in $98(56 \%)$ patients. The numbers of patients in the bacterial, viral, mixed and no etiology groups were 46 (26\%), 53 (31\%), 45 (26\%) and $30(17 \%)$ patients, respectively. S. pneumoniae was the most commonly identified microorganism, found in $40(23 \%)$ patients. Influenza viruses and rhinovirus were the most commonly identified viruses, found in $38(22 \%)$ and $22(13 \%)$ patients, respectively. All the patients infected with bacteria, except one in the bacterial group $(45 / 46,98 \%)$ and one in the mixed group (44/45, $98 \%)$, received an appropriate antimicrobial regimen within the first $24 \mathrm{~h}$ of ICU admission. The incidence of shock was higher in patients with a documented bacterial infection $30 \%$ and $24 \%$ in bacterial and mixed group, respectively) in comparison with other patients (6\% and $13 \%$ in, respectively, viral and no etiology groups, $p<0.01$ ). In terms of biological findings on ICU admission, virus-infected patients displayed a higher serum creatine kinase (viral: 231 [96;460], mixed: 381 [143;829], bacterial: 127 [74;338] and no etiology: 155 [45;259] U/L, $p<0.01$ ) and a lower platelet count (viral: 171 [116;223], mixed: 202 [129;273], bacterial: 212.5 [180;266] and no etiology: 261 [203;315] $\mathrm{ng} / \mathrm{mL}, p<0.01)$ in comparison with other patients. In terms of radiological patterns on ICU admission, a trend toward a higher frequency of alveolar-interstitial infiltrates was observed in virus-infected patients in comparison with other patients (viral: $32(62 \%)$, mixed: 23 (55\%), bacterial: 15 (33\%) and no etiology: 14 (48\%) patients, $p=0.06)$. Finally, an altered prognosis was highly frequent in the mixed group (31/45, $69 \%)$, as compared to bacterial (18/46, $39 \%)$, viral $(15 / 53,28 \%)$ and no etiology $(12 / 30,40 \%)$ groups $(p<0.01)$. In multivariate analysis, the microbiological diagnosis was identified as an independent factor of altered prognosis (reference: bacterial pneumonia; OR, 3.58; $95 \% \mathrm{Cl}, 1.16-11 ; p=0.03$ ). An additional multivariate analysis, limited to bacterial and mixed groups, identified the viral-bacterial coinfection as independently associated with the altered prognosis (reference: bacterial pneumonia; $\mathrm{OR}, 4.53 ; 95 \% \mathrm{Cl}$, 1.29-15.9; $p=0.02$ ).

Conclusion The viral-bacterial coinfection during severe CAP in adults is associated with an impaired presentation and an altered outcome. These findings highlight the key role of respiratory viruses as pathogens during CAP and provide a strong basis for considering antiviral therapies.

\section{Competing interests None.}

\section{S133}

Critical care management of patients with HHV8-associated disorders

Marine Cazaux ${ }^{1}$, Claire Pichereau ${ }^{2}$, Marion Venot $^{3}$, Sandrine Valade ${ }^{4}$, Virginie Lemiale ${ }^{5}$, Akli Chermak ${ }^{6}$, Emmanuel Canet ${ }^{3}$, Le Goff Jérôme ${ }^{7}$ Veronique Meignin ${ }^{8}$, David Boutboull ${ }^{9}$, Lionel Galicier ${ }^{9}$, Elie Azoulay ${ }^{3}$, Eric Mariotte $^{10}$

${ }^{1}$ Réanimation médicale, Hôpital Saint Louis, Paris, France; ${ }^{275}$, Hôpital Saint Louis, Paris, France; ${ }^{3}$ Réanimation médicale, Hôpital Saint-Louis, Paris, France; ${ }^{4}$ Réanimation médicale, Hôpital Saint-Louis, Paris, France, Paris, France; ${ }^{5}$ Medical ICU, Hôpital Saint Louis, Paris, France; ${ }^{6}$ Réanimation polyvalente, $\mathrm{CH}$ sud Essonne, Paris, France; ${ }^{7}$ Virology, Hôpital Saint Louis, Paris, France; ${ }^{8}$ Anatomopathology, Hôpital Saint Louis, Paris, France; ${ }^{9}$ Immuno-hématologie, Assistance Publique Hôpitaux de Paris, Paris, France; ${ }^{10}$ Service de réanimation médicale et infectieuse, Hôpital Bichat-Claude Bernard-APHP, Paris, France

Correspondence: Marine Cazaux - marine.cazaux@live.fr Annals of Intensive Care 2016, 6(Suppl 1):S133

Introduction HHV8-associated disorders (HAD), namely Kaposi sarcoma (KS), multicentric Castleman's disease (MCD) and HHV8-associated lymphomas (HAL, including primary effusion lymphoma and plasmablastic lymphoma), are a group of rare pathologies associated with poor prognosis. We sought to describe intensive care unit (ICU) management and outcomes of patients with HAP.

Patients and methods This retrospective study included patients presenting with HAD between 2002 and 2014 at our center. Factors associated with ICU mortality were identified through a multivariable logistic regression model.

Results Throughout the study period, 77 patients presented with HAD at our ICU. Twenty-nine patients (37.7\%) had only KS, 21 (27.3\%) MCD, $11(14.3 \%)$ HAL, 8 (10.4\%) had the 3 conditions and 8 other patients (10.4\%) had lymphoma not related to HHV8 but associated with KS. Sixty-one patients (79 \%) were HIV-infected with a HIV viral load of 3.3 log (1.6-5) and a CD4 count of 90/mm3 (39-192). Seven patients had received solid organ transplantation (5 kidney transplants, 1 liver and 1 pulmonary transplant), and 9 other patients had Mediterranean KS or HIV-negative MCD. Median HHV8 viral load was $2.94 \log (0-4.87)$. Reasons for ICU admission were acute respiratory failure in 33 patients $(42.9 \%)$, shock in $25(32.5 \%)$, acute kidney injury in 7 (9.1\%), hematologic failure in 7 (9.1\%) and coma in 6 patients (7.8\%). Simplified Acute Physiology Score 2 (SAPS2) at ICU admission was 47 (37.7-57). Mechanical ventilation was required in 38 patients (49\%), vasoactive agents in $32(42 \%)$, renal replacement therapy in $21(27 \%)$ and blood transfusion in 40 patients (52\%). HAD were associated with hemophagocytic lymphohistiocytosis in 34 patients $(40 \%)$. Infection occurred in 64 patients (83\%), $59 \%$ being microbiologically documented. Chemotherapy was administered in 29 patients (38\%). ICU mortality was $15 / 77$ patients (19.5\%). Lengths of ICU and hospital stays were 4 days (2-8) and 21 days (9.3-37.8), respectively. Hospital mortality was $27 / 77$ (35.1\%), and mortality at a median follow-up of 245 days was $44 / 77$ (57.1\%). By univariate analysis, factors associated with increased hospital mortality were the presence of all-cause lymphoma HHV8-associated or not (59.2 vs $22 \%$, $p=<0.01$ ), the need for mechanical ventilation ( 51.3 vs $17.9 \%, p<0.01$ ) or vasoactive agents ( 58.1 vs $19.6 \% p<0.01)$, higher SAPS2 $(53(43.2-62)$ vs $43.5(33-54), p=0.02)$, hypoprotidemia (58 (46.2-64.2) vs $63(55-75)$, $p=0.02)$, high bilirubinemia (51 (15.3-97.6) vs $15.8(12.5-34.5), p=0.04)$ high alkaline phosphatase (136 (107.2-212.2) vs $98(62-143.75), p=0.01)$ and number of involved serosae $(72.7 \%$ for 2 serosae or more vs $30 \%$ for 1 vs $28.6 \%$ for none, $p=0.02$ ). By multivariate analysis, independent risk factors were all-cause lymphoma (OR $8.31[2.31-29.95] p=0.04)$ and mechanical ventilation (OR $7.58[2.17-26.46] p=0.02)$. When entered in the multivariable model, chemotherapy administration in the ICU (including etoposide monotherapy) was independently associated with decreased mortality (OR 0.24 [0.06-0.92] $p=0.04$ ).

Conclusion HHV8 infection is responsible for a wide range of clinical diseases that display highly variable prognosis. The presence of lymphoma, whether HHV8-related or not, is the major determinant of short-term mortality for patients with HAD requiring ICU admission.

\section{Competing interests None.}

\section{S134}

Emerging moulds in critically ill patients: epidemiological trends, clinical features and ICU outcome

Laura Platon ${ }^{1}$, Philippe Rispail ${ }^{2}$, Vincent Brunot ${ }^{1}$, N. Besnard ${ }^{1}$, Patrice

Ceballos ${ }^{3}$, Kada Klouche ${ }^{1}$

${ }^{1}$ Réanimation médicale, Hôpital Lapeyronie, Montpellier, France; ${ }^{2}$ Mycologie, Hospital Arnaud-De-Villeneuve, Montpellier, France; ${ }^{3}$ Hématologie, CHRU Hôpital Saint Eloi, Montpellier, France

Correspondence: Vincent Brunot - v-brunot@chu-montpellier.fr Annals of Intensive Care 2016, 6(Suppl 1):S134

Introduction Invasive fungal infections (IFIs) are associated with high morbidity and mortality in critically ill patients, especially those immunocompromised. Aspergillus spp. remain an important cause of IFIs, but other moulds have emerged over recent years as significant opportunistic pathogens and are associated with even higher mortality rates. In this retrospective study, we aimed to describe epidemiological trends 
and clinical features of IFls caused by emerging moulds and to assess their outcome in intensive care unit (ICU) settings.

Patients and methods From our ICU database, we extracted all records of patients admitted to the ICU from 2006 to 2014, in which IFI due to emerging moulds was diagnosed. The following epidemiological and clinical data were collected: underlying disease, immunosuppressive status, severity scores, reasons for ICU admission, organ failures and therapeutic management including mechanical ventilation, vasopressives agents and renal replacement therapy. We also collected risk factors for fungal infections, mould species, span between ICU admission and IFI diagnosis, anti-fungal treatment. ICU mortality was assessed.

Results Among 3600 patients admitted in medical ICU, 17 (4.7/1000 patients) had a positive diagnosis of IFI due to emerging moulds: 3 females and 14 males, with median age 58 years (53-68), underlying disease: haematologic malignancy: 12 (70\%), diabetes: 2 (12\%), chronicle obstructive pneumonia disease: $2(12 \%)$ and solid organ transplantation: $1(6 \%)$. Most of the patients were immunocompromised (16, $94 \%)$, and 12 have been treated by corticotherapy (70\%). IFI was diagnosed early in the course of the disease, at ICU admission for 7 patients ( $41 \%$ ) and later after ICU admission for 10 patients (59\%). At IFI diagnosis, severity scores were: SOFA: $13(10-16)$ and SAPS-II: 71 (54-88). Diagnosis was made by blood cultures in 5 cases (29\%) and by bronchoalveolar lavage in 11 cases (65\%). Six patients had a retrospective diagnosis. Mould species were: Rhizopus: 7 (41\%), Trichosporon: $6(35 \%)$, Fusarium: 2 (12\%) and Scedosporium: 2 (12\%). All patients were mechanically ventilated, 15 needed catecholamines, and 11 had renal replacement therapy. An antifungal treatment was administrated to 15 patients ( $88 \%$ ) but was appropriate only for 12 of them (70\%). Two patients benefited from surgery. All patients died in ICU.

Conclusion Incidence of IFIs due to emerging moulds is far from negligible. It occurs mostly in immunocompromised patients with high severity score and still carries high ICU mortality. Early diagnosis and appropriate treatment remain a challenge. Further studies are necessary to improve our understanding of IFIs caused by emerging moulds and improve their outcome.

\section{Competing interests None.}

\section{S135}

Intensive care unit-associated bloodstream infection and ventilator-associated pneumonia in critically ill cancer patients: a 12-year retrospective study

Annabelle Stoclin ${ }^{1}$, Federico Rotolo ${ }^{2}$, Muriel Wartelle ${ }^{3}$, Yosr Hicheri ${ }^{4}$, Sylvia Maillet ${ }^{5}$, Elisabeth Chachaty ${ }^{6}$, Jean-Pierre Pignon ${ }^{7}$, François Blot ${ }^{4}$

${ }^{1}$ Réanimation-USCM, Gustave Roussy, Villejuif, France; ${ }^{2}$ Service de biostatistique et d'épidémiologie, inserm u1018, cesp, université paris-sud, Gustave Roussy, Villejuif, France; ${ }^{3}$ Direction du système d'information, Gustave Roussy, Villejuif, France; ${ }^{4}$ Service de réanimation médico-chirurgicale, Gustave Roussy, Villejuif, France; ${ }^{5}$ Service d'information médicale, Gustave Roussy, Villejuif, France; ${ }^{6}$ Service de microbiologie médicale, Gustave Roussy, Villejuif, France; ${ }^{7}$ Service de biostatistique et d'epidemiologie, inserm u1018, cesp, université paris-sud, Gustave Roussy, Villejuif, France Correspondence: Annabelle Stoclin - annabelle.stoclin@gustaveroussy. fr

Annals of Intensive Care 2016, 6(Suppl 1):S135

Introduction The prognosis of cancer patients admitted to intensive care units improves since two decades. The rare publications available suggest high rates of healthcare-associated infections (HAls) and poor prognosis of nosocomial pneumonia in such patients. There is a need for a better understanding of the HAls epidemiology in critically ill hematology and oncology patients.

Our objectives are: (1) to describe the incidence, the pathogen distribution and the risk factors for ventilator-associated pneumonia (VAP) and bloodstream infections (BSI) in critically ill cancer patients; (2) to assess the influence of ICU-acquired infections, characteristics of malignancy and patient's severity on outcome.

Patients and methods This is a retrospective review of prospectively recorded data (2000-2011) of patients treated for more than
$48 \mathrm{~h}$ in a 12-bed oncology medical-surgical ICU of a referral French cancer center. We described the development of VAP and BSI (primary, secondary and catheter-related) and evaluated the influence of clinical characteristics and device utilization on infection rates; and of infections on the ICU mortality. Logistic and Cox models were used for the study of risk factors for infection and mortality, respectively.

Results Three thousand three hundred and eighty-eight patients (first stay) acquired 198 VAP and 393 BSI (213 secondary, 103 primary and 77 catheter-related bloodstream infections [CR-BSI]). The device utilization rate was $26 \%$ for mechanical ventilation and $106 \%$ for central venous catheter (most of the patients had an indwelling intravascular device). The VAP rate was $25 / 1000$ ventilator-days; the CR-BSI rate was $2.3 / 1000$ catheter-days. The estimated cumulative incidences in the first 25 days of exposure was $59 \%$ for VAPs, $8.9 \%$ for primary, $15 \%$ for secondary and $5 \%$ for catheter-related bloodstream infection. The distribution of pathogens differed according to the type of infection: more Gram-negative bacilli in VAP and secondary BSI, Gram-positive cocci in CR-BSI; more anaerobes and polymicrobial infections in secondary BSI. Mechanical ventilation duration, older age, scheduled surgery and solid tumor were VAP-specific risk factors. ICU stay duration, high SAPS II, scheduled surgery, ECOG-PS $>2$, absence of metastases and recent leukopenia were significant primary BSI risk factors. ICU stay duration, surgery and leukopenia were significant secondary BSI risk factors. No risk factors were found significantly associated with the risk of CR-BSI due to the rarity of these events. The occurrence of neither a ventilator-associated pneumonia nor a bloodstream infection was associated with a higher risk of ICU mortality. All risk factors identified were markers of the characteristics of malignancy and of patient's severity.

Conclusion In our study, the occurrence of nosocomial infections was largely due to the characteristics of malignancy and patient's severity and did not further influence the outcome of hematology and oncology patients admitted in ICU. Our findings in a large population of critically ill cancer patients can help to identify subgroups with a particularly increased risk. These data might serve for comparative studies with other oncology ICUs and to develop quality improvement activities.

\section{Competing interests None.}

\section{References}

1. Velasco E, Thuler LC, Martins CA, Dias LM, Gonçalves VM. Nosocomial infections in an oncology intensive care unit. Am J Infect Control. 1997;25(6):458-62.

2. Azoulay E, Soares M, Darmon M, Benoit D, Pastores S, Afessa B. Intensive care of the cancer patient: recent achievements and remaining challenges. Ann Intensive Care. 2011;1(1):5

S136

Is diabetes mellitus a risk factor for intensive care unit-acquired infections?

Marion Venot ${ }^{1}$, Stephane Ruckly², Christophe Clec'h ${ }^{3}$, Michaël Darmon ${ }^{4}$, Bernard Allaouchiche ${ }^{5}$, Dany Goldgran Toledano ${ }^{6}$, Maïté

Garrouste-Orgeas ${ }^{7}$, Christophe Adrie ${ }^{8}$, Jean-François Timsit ${ }^{2}$, Elie Azoulay ${ }^{1}$ ${ }^{1}$ Réanimation médicale, Hôpital Saint-Louis, Paris, France; ${ }^{2}$ Réanimation médicale et infectieuse, Hôpital Bichat-Claude Bernard, Paris, France; ${ }^{3}$ Réanimation medico-chirurgicale, hopital avicenne, Bobigny, France; ${ }^{4}$ Réanimation Médicale, CHU Saint-Etienne - Hôpital Nord, Saint-Étienne, France; ${ }^{5}$ Réanimation, Hôpital civil de Lyon, Lyon, France; ${ }^{6}$ Réanimation polyvalente, Centre Hospitalier Général, Gonesse, France; ${ }^{7}$ Réanimation, Fondation Hopital Saint Joseph, Paris, France; ${ }^{8}$ Physiologie, Hôpital Cochin, Paris, France

Correspondence: Marion Venot - marionvenot@free.fr Annals of Intensive Care 2016, 6(Suppl 1):S136

Introduction Diabetes mellitus (DM) affects $10 \%$ of the general population worldwide, $10 \%$ to $25 \%$ of critically ill patients and is associated with high short- and long-term morbidity and mortality. Sepsis is 
among the main acute complications of diabetes. Intensive care unit (ICU)-acquired infections, especially bloodstream infections (BSI) and ventilator-associated pneumonia (VAP), are challenging complications. To our knowledge, only few studies reported the influence of diabetes mellitus on ICU-acquired sepsis in this setting. This study is an analysis of the Outcomerea database. We sought to determine whether diabetes mellitus is associated with increased risk of BSI and VAP.

Patients and methods Outcomerea is a prospective observational multicenter cohort of patients admitted to 12 French ICUs. We studied the subgroup of patients with ICU length of stay $>3$ days and who needed invasive mechanical ventilation within the first 3 days of their admission. Patients with complicated diabetes mellitus were compared to patients with uncomplicated diabetes mellitus and with patients without DM. Endpoint was occurrence of ICU-acquired sepsis (first bacterial or fungal BSI and VAP occurring later than the second day of ICU admission, and up to 2 days after extubation, exit or death, data censored at day 28). To estimate the influence of baseline covariates on sepsis occurrence, we used the proportional hazards regression Fine and Gray model for competing risk.

Results Among the 15,876 patients of Outcomerea, 5865 patients were included with 4819 controls and $1046(18 \%)$ patients with diabetes mellitus (282 with and $764(13 \%)$ without chronic complications). Age was significantly higher in DM patients (69.8 [61-77.2] in complicated DM, $69.7[60-77.6]$ in uncomplicated DM, and 63.2 [50.6-75.3] in controls, p value < 0.01). SAPS 2 score was greater in DM patients (54 [43-72] in complicated DM, 55 [43-67] in uncomplicated DM, and $51[39 ; 63]$ in controls, $p$ value $<0.01)$. Comorbidities were more frequent in patients with DM $(155(55 \%)$ in complicated DM, $386(50.5 \%)$ in uncomplicated DM, and $1968(40.8 \%)$ in controls). Main comorbidities were: respiratory $(15.1 \%)$, immunosuppression (14.8\%), cardiologic (13.3\%), hepatic $(7.2 \%)$, and renal $(5 \%)$. Main reasons for admission were: circulatory $(21.6 \%)$, neurologic (22.8\%), digestive (18.2\%), infectious (16.5\%), and metabolic (16\%). In patients with DM, reasons for admission were more often circulatory, metabolic, and infectious $(p<0.01)$. Throughout their ICU stay, 183 (64.9\%) patients with complicated DM, 450 (58.9\%) patients with uncomplicated DM, and 2680 (55.6\%) patients without DM required vasopressors $(p<0.01)$. Two hundred and forty-five $(86.9 \%)$ patients with complicated DM, 595 (77.9\%) patients with uncomplicated DM and $3610(74.9 \%)$ patients without DM underwent central venous catheterism $(p<0.01)$.

ICU-acquired sepsis occurred in 63 (22.3\%) patients with complicated DM (23 (8 \%) BSI and 44 (16\%) VAP), 140 (18.3\%) patients with uncomplicated DM (48 (6.3\%) BSI and 109 (14.3\%) VAP), and $936(19.4 \%)$ patients without DM (317 (6.6\%) BSI and 749 (15.5\%) VAP). Main pathogens in bloodstream infections were: Staphylococcus aureus (14.25\%), Pseudomonas aeruginosa (9.66 \%), Escherichia Coli (9.43\%), Enterococcus faecalis and faecium (8.74 \%), Staphylococcus epidermidis, (8.05\%) and Candida albicans (7.36\%). Main pathogens in ventilator-associated pneumonia were: Pseudomonas aeruginosa (24.40\%), Staphylococcus aureus (12.96\%), Escherichia Coli (6.88 \%), and Haemophilus influenzae $(5 \%)$. ICU mortality rates were $27.3,31.4$, and $25.8 \%$ in complicated $\mathrm{DM}$, uncomplicated DM, and controls $(p<0.01)$. Corresponding hospital mortality rates were $39.7,40.4$, and $34.6 \%(p<0.01)$.

By multivariable analysis, independent risk factors for acquired sepsis (BSI/VAP) were: the ICU, male gender (hazard ratio 1.268, $95 \%$ confidence interval [1.120-1.436], $p$ value $=0.0002)$, admission for a medical reason (1.230, [1.062-1.425], $p$ value $=0.0058)$, higher SOFA score at day 1 and day $2(1.048,[1.031-1.066], p$ value $<0.0001)$, respiratory comorbidity (1.259, [1.080-1.468], $p$ value $=0.0033)$, need for central line (1.442, [1.220-1.706], $p$ value $<0.0001)$, existence of a bloodstream infection at admission (1.269, [1.055-1.527], $p$ value $=0.0115)$, and existence of a pneumonia at admission (1.443, [1.245-1.673], $p$ value $<0.0001)$. Diabetes mellitus with and without complications were not independent risk factors for acquired infections. There was a trend toward increased Staphylococcus aureus ventilator-associated pneumonia in patients with complicated diabetes mellitus (1.748, [0.9903.088], $p$ value $=0.0544$ )

Conclusion Diabetes mellitus, whether complicated or not, does not seem to be a risk factor for intensive care unit-acquired bloodstream infection or ventilator-associated pneumonia. However, there is a trend

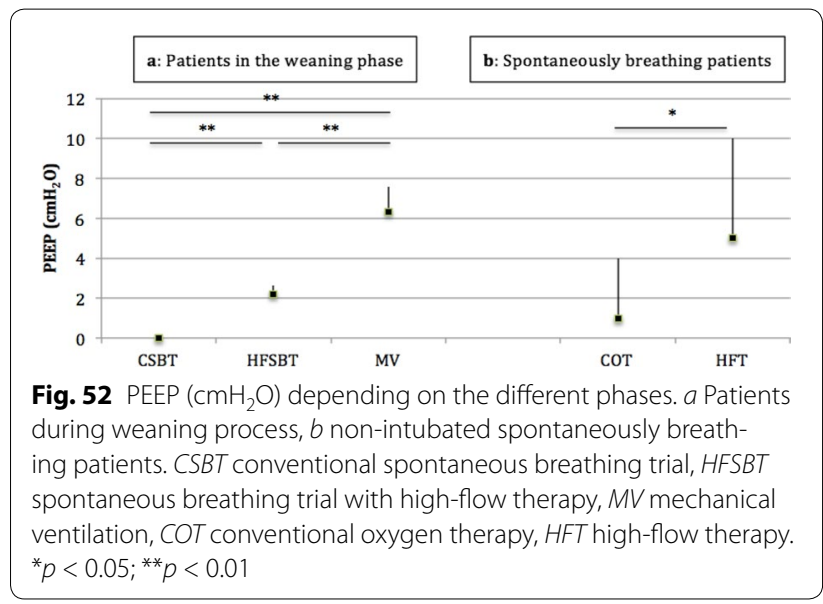

toward an association between complicated diabetes mellitus and occurrence of Staphylococcus aureus ventilator-associated pneumonia.

Competing interests None.

\section{PHYSIOTHERAPISTS ABSTRACTS}

\section{5}

Short-term physiological effects of nasal high-flow in healthy subjects. Impact of flow rates on the work of breathing Mathieu Delorme 1 , Pierre Alexandre Bouchard², Mathieu Simon², François Lellouche ${ }^{2}$

133, Hôpital Haut-Lévêque, Avenue Magellan, Pessac, France, Pessac, France; ${ }^{2}$ Centre de recherche de l'iucpq, Institut Universitaire de Cardiologie et de Pneumologie de Québec, Québec, Canada

Correspondence: Mathieu Delorme - mathieu.delorme@chu-bordeaux.fr Annals of Intensive Care 2016, 6(Suppl 1):085

Introduction Nasal high-flow (NHF) is increasingly used in the management of acute respiratory failure. Previous data suggest that the high-flow rates may result in an anatomical dead space washout. This mechanism is likely to reduce respiratory effort, but little is known about the physiological effects of NHF on the work of breathing in adults and the dose-response relationship.

Materials and methods We designed a prospective study in order to investigate the short-term effects of NHF on physiological respiratory parameters in healthy subjects. Five sessions of $10 \mathrm{~min}$ were evaluated: a baseline period with spontaneous breathing (SB), followed by a randomised sequence of four conditions: $5 \mathrm{~L} / \mathrm{min}$ with conventional nasal canulas, and 20, 40 and $60 \mathrm{~L} / \mathrm{min}$ with high-flow nasal cannulas. During the whole protocol, $\mathrm{FiO}_{2}$ was set at 0.21 . The primary outcome was the work of breathing (WOB), computed from esophageal pressure and tidal volume, which was estimated by respiratory inductive plethysmography. We also measured and calculated the esophageal pressure-time product (PTPeso), minute ventilation, capillary blood gases at the end of each tested condition, airway resistance and compliance. Results Recruitment is still under progress. We report here the data of a preliminary analysis performed in 8 subjects. No significant difference in WOB was evidenced between the study periods $(1.82 \pm 0.92$ vs. $1.71 \pm 0.80$ vs. $2.56 \pm 1.30$ vs. $2.01 \pm 0.17$ vs. $2.38 \pm 0.50 \mathrm{~J} / \mathrm{min}$; respectively, for SB, 5, 20, 40 and $60 \mathrm{~L} / \mathrm{min} ; p=0.36$ ). PTPeso was not significantly modified either $(70.2 \pm 27.3$ vs. $70.6 \pm 25.6$ vs. $87.9 \pm 34.4$ vs. $78.8 \pm 25.6$ vs. $93.4 \pm 28.0 \mathrm{cmH} 2 \mathrm{O} \mathrm{s} / \mathrm{min}$ respectively; $p=0.59$ ). However, respiratory pattern was found to be different throughout the study periods, with an increase in tidal volumes $(345 \pm 91$ vs. $377 \pm 101$ vs. $493 \pm 138$ vs. $445 \pm 157$ vs. $560 \pm 102 \mathrm{~mL}$, respectively, for SB, 5, 20, 40 and $60 \mathrm{~L} / \mathrm{min} ; p=0.002)$ and a flow-dependant decrease in respiratory rate $(16.0 \pm 1.9$ vs. $13.2 \pm 2.0$ vs. $10.6 \pm 2.6$ 
vs. $9.5 \pm 3.0$ vs. $7.6 \pm 2.4$ breaths $/ \mathrm{min}$, respectively; $p<0.0001$ ). This resulted in similar minute volumes between baseline, 5 and $20 \mathrm{~L} /$ $\min (5.42 \pm 1.02$ vs. $5.01 \pm 1.59$ vs. $5.33 \pm 2.09 \mathrm{~L} / \mathrm{min}$, respectively; $p=0.41$ ), whereas a significant decrease was found with 40 and $60 \mathrm{~L} /$ $\min (4.04 \pm 1.36$ and $4.18 \pm 1.22 \mathrm{~L} / \mathrm{min}$ respectively; $p=0.009$ vs. baseline). $\mathrm{pH}, \mathrm{PaCO}_{2}$ and $\mathrm{SpO}_{2}$ were not significantly modified during the study periods. Respiratory compliance was not significantly different between the study periods either $(p=0.74)$, but mean airway inspiratory resistances did increase with increasing flows (14.6 \pm 4.4 vs. $15.6 \pm 4.5$ vs. $18.9 \pm 6.2$ vs. $22.5 \pm 7.1$ vs. $25.7 \pm 9.6 \mathrm{cmH} 2 \mathrm{O} / \mathrm{L} / \mathrm{s}$, respectively, for $\mathrm{SB}, 5,20,40$ and $60 \mathrm{~L} / \mathrm{min} ; p=0.01$ ).

Discussion To our knowledge, this study is the first to evaluate the work of breathing during NHF in healthy adult subjects. This lack of data may be attributable to the technical complexity of measuring inspiratory flow during NHF. Here, we used respiratory inductive plethysmography, which has been previously used by several authors, and has shown to provide an acceptable estimation of tidal volume. Conclusion We found that the use of NHF above $40 \mathrm{~L} / \mathrm{min}$ led to a significant reduction of minute volume in healthy subjects, mainly driven by a dramatic decrease in respiratory rate, $\mathrm{PaCO}_{2}$ remaining constant. This finding strongly suggests a reduction in the physiological dead space and supports the necessity of such an evaluation in patients. No modification of WOB was evidenced, and this may be related to an unexpected and flow-dependant increase in inspiratory resistances in this specific population.

\section{Competing interests None.}

\section{6}

\section{High-flow therapy allows a decreased workload of accessory} respiratory muscles

Emilie Bialais ${ }^{1}$, Dupuis Julie ${ }^{1}$, Laura Paoloni ${ }^{1}$, Gregory Reychler ${ }^{2}$, Xavier Wittebolle

${ }^{1}$ Sevice des soins intensifs, Cliniques Universitaires Saint-Luc, Bruxelles, Belgium; ${ }^{2}$ Service de pneumologie, Cliniques Universitaires Saint Luc, Bruxelles, Belgium

Correspondence: Emilie Bialais - emilie.bialais@uclouvain.be Annals of Intensive Care 2016, 6(Suppl 1):086

Introduction High-flow therapy (HFT) is commonly used in ICU. We hypothesize that in patient undergoing respiratory failure, beyond the positive end-expiratory pressure (PEEP) generated, HFT provides a moderate ventilatory support. This support could be able to reduce the electrical activity of accessories inspiratory muscles. The objective of this study was to evaluate the sternocleidomastoid's (SCM) electromyographic activity during HFT versus conventional oxygen therapy (COT) in non-intubated patient and versus COT and mechanical ventilation (MV) in intubated or tracheotomized patient during difficult weaning.

Materials and methods SCM electromyographic activity (EMG) was recorded as follows: Phase I included non-intubated hypoxemic patients who needed at least $8 \mathrm{~L} / \mathrm{min}$ of COT. They received three times 20 min steps evaluation (COT-HFOT-COT). Phase II included intubated or tracheotomized patients in weaning failure. They have been randomly tested for conventional T-tube spontaneous breathing trial (CSBT) and high-flow SBT (HFSBT). These two 20-min steps were separated by a 20-min step of $\mathrm{MV}$. $\mathrm{FiO}_{2}, \mathrm{PEEP}$, arterial blood gazes and hemodynamic data were also collected.

Results Phase I included 11 patients. PEEP (Fig. 52b) was significantly higher with HFOT than with COT $\left(5 \pm 4.95\right.$ vs $1.44 \pm 3.13 \mathrm{cmH}_{2} \mathrm{O}$, respectively, $p=0.029$ ), while EMG activity (Fig. 53b) was significantly reduced with high flow $(27.35 \pm 15.77 \mathrm{mV}$ with HFOT vs $31.31 \pm 17.21 \mathrm{mV}$ with COT, $p=0.008$ ). Phase II included 10 patients. With HFSBT PEEP present an intermediary level compared with MV and with CSBT $\left(2.2 \mathrm{cmH}_{2} \mathrm{O}\right.$ in HFSBT vs $6.2 \mathrm{cmH}_{2} \mathrm{O}$ in MV vs $0 \mathrm{CmH}_{2} \mathrm{O}$ in CSBT, $p<0.001$ ) (Fig. 52a). SCM EMG differs in CSBT and MV $(32.92 \pm 8.53 \mathrm{mV}$ during CSBT vs $22.99 \pm 4.39 \mathrm{mV}$ during MV,

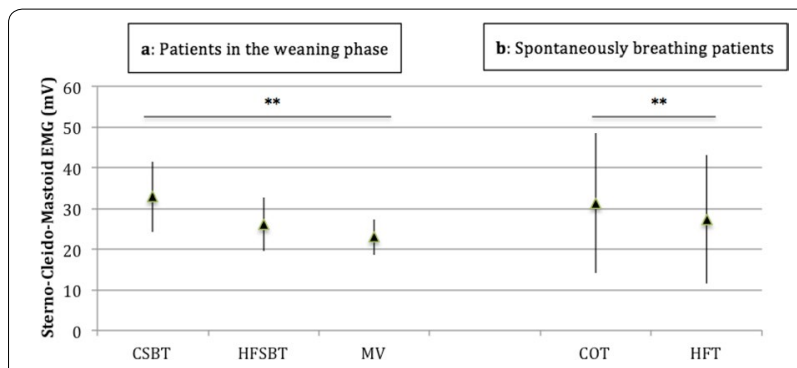

Fig. 53 Sternocleidomastoid EMG $(m V$ depending on the diferent phases. a Patients during weaning process, $b$ non-intubated spontaneously breathing patients. CSBT conventional spontaneous breathing trial, HFSBT spontaneous breathing trial with high-low therapy MV mechanical ventilation, COT conventional oxygen therapy, HFT high-low therapy. ${ }^{*} p<0.05 ;{ }^{* *} p<0.01$

$p=0.007)$, while during HFSBT, EMG presents an intermediary level (26.17 $\pm 6.56 \mathrm{mV}$ ) (Fig. 53a).

Conclusion We observed a decreased inspiratory muscle's electromyographic activity that reflects an improvement of the ventilation with HFT. This can be interesting for patient with respiratory failure, particularly for patients in weaning phase.

Competing interests None.

\section{References}

1. Ward JJ. High-Flow Oxygen Administration by Nasal Cannula for Adult and Perinatal Patients. Respiratory Care. 2013;58(1):98-122.

2. Lee JH, Rehder KJ, Williford L, Cheifetz IM, Turner DA. Use of high flow nasal cannula in critically ill infants, children, and adults: a critical review of the literature. Intensive care medicine. 2013;39(2):247-57.

087

Patient perception and feasibility of early mobilization in the critically ill

Cheryl Hickmann', Diego Castanares-Zapatero' ${ }^{1}$ Emilie Bialais ${ }^{1}$, Jonathan Dugernier ${ }^{1}$, Antoine Tordeur ${ }^{1}$, Lise Colmant ${ }^{1}$, Xavier Wittebole ${ }^{1}$, Giuseppe Tirone ${ }^{1}$, Jean Roeseler ${ }^{1}$, Pierre-François Laterre

${ }^{1}$ Intensive Care Unit, Cliniques Universitaires Saint-Luc, Bruxelles, Belgium Correspondence: Cheryl Hickmann - cheryl.hickmann@uclouvain.be Annals of Intensive Care 2016, 6(Suppl 1):087

Introduction By preventing deleterious effects of bed rest, early mobilization (EM) of critically ill patient improves various outcomes including quality of life $[1,2]$. However, no data exist on patient's perception of this EM. The aim of this study was to report on the feasibility and patient's tolerance of EM.

Patients and methods Our local standardized EM protocol was prospectively evaluated over a 2-month period in the 171 consecutive patients admitted in our mixed 14-bed ICU. Data on feasibility, adverse events, hemodynamic parameters and patient's perception were collected.

Results Three percent $(n=22)$ of the 731 patient-days reached our standard exclusion criteria for EM (prior cardiac arrest, unstable spine fracture, active bleeding and uncontrolled intracranial pressure). From the remaining 709 patient-days, $86 \%$ had at least one early mobilization intervention (physiotherapy session or transfer out of bed); $74 \%$ of patients were transferred out of bed to chair, and $59 \%$ received at least one physiotherapy session. Fifty-one percent of patients were under mechanical ventilation ( $46 \%$ patient-days), and $35 \%$ had vasopressor and $11 \%$ continuous renal replacement therapy. In patients on mechanical ventilation, transfer out of bed was achieved in $68 \%$ of patient-days and $80 \%$ received at least one EM activity. Limitations to early mobilization included staff number, diagnostic or surgical 
procedures, patients' refusal and hemodynamic instability. Very rare $(0.8 \%)$ and reversible adverse events were observed as reversible hypotension or arrhythmia. Hemodynamic parameters were not affected after active mobilization. Patient's perception was reported close to good feeling after a physiotherapy session.

Conclusion EM shows to be a common and safe procedure and is achievable in the vast majority of critical ill patients even in the presence of multiple vital organ supports. Communicant patients report a positive feeling after application of this intervention.

\section{Competing interests None.}

\section{References}

1. Schweickert WD, Pohlman MC, Pohlman AS, et al. Early physical and occupational therapy in mechanically ventilated, critically ill patients: a randomised controlled trial. Lancet. 2009;373:1874-1882.

2. Morris PE, Goad A, Thompson C, et al. Early intensive care unit mobility therapy in the treatment of acute respiratory failure. Critical care medicine. 2008;36:2238-2243.

P252

Early rehabilitation in critically ill patients: a prospective multicenter assessment of real-life practice

Lorraine Ducroux', Sylvie L'hotellier², Elodie Baumgarten ${ }^{3}$, Stéphanie Borschneck', Francis Schneider ${ }^{4}$, Vincent Castelain ${ }^{1}$

${ }^{1}$ Hautepierre réanimation médicale, C.H.R.U. Hôpitaux Universitaires Strasbourg, Strasbourg, France; ${ }^{2}$ Service de réanimation, C.H.R.U. Hôpitaux Universitaires Strasbourg, Strasbourg, France; ${ }^{3} 67000$, Cabinet libéral de masso kinésithérapie, Strasbourg, France; ${ }^{4}$ Réanimation médicale, C.H.R.U. Hôpitaux Universitaires Strasbourg, Strasbourg, France

Correspondence: Lorraine Ducroux - ducroux.lorraine@gmail.com Annals of Intensive Care 2016, 6(Suppl 1):P252

Introduction Early mobilization of critically ill patients is a major issue when it comes to ICU-stay shortening for multiple organ failure patients. It is well accepted that "bed-in-chair" sitting, first chair sitting exercise, passive verticalization and walking are crucial steps to better rehabilitation in the ICU and after patients have left the ICU. We aimed at assessing the real-life conditions performance of early rehabilitation practices in ICUs and to study why there are sometimes limitations to a large use of such training for patients.

Materials and methods A prospective telephone inquiry was performed from February to March 2015 in 35 ICU (25 from university hospitals), representing 1046 beds; it recorded data on ICUs and on the real-life practice of rehabilitation in the setting of 5 clinical cases (1 coma patient undergoing mechanical ventilation weaning yet he was on low tapering NE infusion, 1 ARDS, 1 COPD under noninvasive ventilation, 1 acute/chronic liver failure patient infused tapering doses of norepinephrine, 1 postoperative patient) For each case, staff were questioned on the practice of transferring from flat bed to chair; bed in armchair positioning; transferring to edge of bed; standing position; and marching. In addition, we collected the reasons for not practicing early mobilization.

Results One hundred percentage of responses were made by physiotherapists, $64.5 \%$ of which had less than 5 -year working experience. The median number of patients taken in care by one physiotherapist was 14 (range 3.5-52), whereas simultaneously each nurse had 2.5 patients in charge, and each nurse auxiliary 4.2. Shift-hours were on $12 \mathrm{~h}$ a day in $58.8 \%$ of the ICUs (Table 62 ).

The main reasons for not proposing early rehabilitation are low consciousness level, NE infusion, curare infusion and the simultaneous presence of many surgical drains in the same patient.

Conclusion Despite low sedation, many critically ill patients are readily installed in a bed in armchair position because this position does not include danger and allows easy visual control. Postoperative patients with complicated surgical devices are not often proposed for early
Table 62 See text for description

\begin{tabular}{|c|c|c|c|}
\hline Cases & Rehabilitation & $\%$ & Limits \\
\hline $\begin{array}{l}\text { 1. Resolving } \\
\text { coma, taper- } \\
\text { ing dose of } \\
\mathrm{NE} \text {, ongoing } \\
\text { respiratory } \\
\text { weaning }\end{array}$ & $\begin{array}{l}a \\
b \\
c \\
d \\
e\end{array}$ & $\begin{array}{l}\text { Often 3; Rarely } \\
\quad 96.9 \\
84.8 \\
15.1 \\
6 \\
0\end{array}$ & $\begin{array}{l}\text { Consciousness: } \\
69.7 \% \\
\text { Presence of low } \\
\text { doses of NE }\end{array}$ \\
\hline $\begin{array}{l}\text { 2. Chronic } \\
\text { obstructive } \\
\text { pulmonary } \\
\text { disease on } \\
\text { noninvasive } \\
\text { ventilation } \\
(1 \mathrm{~h} / 3)\end{array}$ & $\begin{array}{l}a \\
b \\
c \\
d \\
e\end{array}$ & $\begin{array}{l}\text { Daily } 65.5 \text {; often } \\
34.5 \% \\
89 \\
75 \\
86 \\
65\end{array}$ & \\
\hline $\begin{array}{l}\text { 3. Acute on } \\
\text { chronic liver } \\
\text { failure with } \\
\text { tapering NE }\end{array}$ & $\begin{array}{l}a \\
b \\
c \\
d \\
e\end{array}$ & $\begin{array}{l}\text { Daily } 54.5 \text {, never } \\
45.5 \\
85 \\
66 \\
60 \\
30\end{array}$ & Low dose NE \\
\hline $\begin{array}{l}\text { 4. Respiratory } \\
\text { weaning } \\
\text { failure after } \\
\text { laparotomy } \\
\text { (many drains) }\end{array}$ & $\begin{array}{l}a \\
b \\
c \\
d \\
e\end{array}$ & $\begin{array}{l}\text { Infrequently } \\
\quad 66.6 \\
96 \% \\
59 \\
39 \\
15\end{array}$ & $\begin{array}{l}\text { Drains, lapa- } \\
\text { rotomy }\end{array}$ \\
\hline $\begin{array}{l}\text { 5. ARDS under } \\
\text { MV (with seda- } \\
\text { tion + curare) }\end{array}$ & $\begin{array}{l}a \\
b \\
c \\
d \\
e\end{array}$ & $\begin{array}{l}0 \\
45.5 \\
0 \\
0 \\
0\end{array}$ & Curare \\
\hline
\end{tabular}

$a$ transferring from a flat bed to chair; $b$ bed in armchair position; $c$ transferring to edge of bed; $d$ standing position; e marching; NE norepinephrine

mobilization from bed: Nurses and physiotherapists bring up the problem of understaffing. Patients with low doses (or tapering low doses) of NE are infrequently proposed for early mobilization; although no reliable data concern the dose-effect relationship with negative side effects in such a population, a specific study is deserved to understand whether such patients would benefit from such rehabilitation in terms of shorter ICU stay and outcome.

Competing interests None.

\section{P253}

\section{Motor imagery and functional magnetic resonance imaging}

Claire Kemlin ${ }^{1}$, Eric Moulton², Charlotte Rosso ${ }^{3}$

${ }^{1}$ Service de médecine physique et réadaptation, Hôpital Pitié-Salpêtrière, Paris, France; ${ }^{2}$ nnstitut du cerveau et de la moelle, Hôpital Pitié-Salpêtrière, Paris, France; ${ }^{3}$ Service des urgences cérébro-vasculaires, Hôpital Pitié-Salpêtrière, Paris, France

Correspondence: Claire Kemlin - clairekemlin@gmail.com

Annals of Intensive Care 2016, 6(Suppl 1):P253

Introduction Motor imagery can be used in conjunction with fMRI (functional magnetic resonance imaging) to study brain plasticity during motor recovery for non-highly selected stroke patients. The presumed advantage of using motor imagery over overt movements is that they both share a number of behavioral (Jeannerod 1995) and 
physiological parameters (Kranczioch et al. 2009), as well as certain functional neuroanatomical correlates (recruitment of motor networks) (Confalonieri et al. 2012). Motor imagery is in fact a broad concept that includes both implicit and explicit mental imagery (Di Rienzo et al. 2014)

Clinical scores and questionnaires have been used to assess motor imagery abilities. The most frequent is the Kinesthetic and Visual Imagery Questionnaire (KVIQ), which can be somewhat subjective. In contrast, subject performance can additionally be objectively evaluated using fMRI.

Patients and methods To determine the areas and brain networks engaged during motor imagery in healthy subjects and stroke patients, a literature review was conducted. The keywords used were "motor imagery," "fMRI," and "stroke."

Results Functional imaging techniques have helped highlight the activation of a large network during motor imagery tasks, including fronto-parietal, subcortical, and cerebellar regions, as well as cortical areas involved in motor control. The activation of the primary motor cortex, however, has been controversial for many years (Lotze and Halsband 2006). Indeed, in a recent neuroimaging meta-analysis, Hétu et al. (2013) did not find the primary motor cortex to be consistently activated among subjects.

Finally, it is important to note that these activations depend on several parameters: the part of the body imagined, the mode (kinesthetic or visual), and the difficulty of the imagined motor task.

Discussion One of the limitations in assessing brain plasticity in stroke patients with motor imagery is their "chaotic" motor imagery performance, revealed by a disruption of accuracy of imagined movements and temporal coupling between executed and imagined movements (Sharma, 2006). Moreover, certain stroke locations distinctly modify patient performance in motor imagery, such as parietal lobe damage or right-sided lesions.

Malouin et al. (2012) found a lower correlation between executed and imagined movements in right-sided lesions. They hypothesized that imagining movements was more demanding in right-sided strokes due to damage to the fronto-parietal network, known to be involved in the visuospatial domain. Patients with lesions involving the parietal cortex were also selectively impaired at predicting the time required to perform an explicit motor imagery task. This is consistent with previous findings of a decorrelation between executed and imagined movements in patients with lesions restricted to the parietal lobe (Sirigu et al., 1996). The parietal lobe is indeed a complex structure responsible for several aspects involved in explicit motor imagery, such as correspondence between observed and executed movements, representation of internal models, maintaining the postural representation of the upper limb, and movement intention and motor preparation.

Conclusion These findings suggest that $\mathrm{fMRI}$ is helpful in assessing patient motor imagery ability and could be used to determine which patients would benefit from motor imagery training.

\section{Competing interests None.}

\section{Reference}

1. Hétu, S., M. Grégoire, et al. (2013). The neural network of motor imagery: An ALE meta-analysis. Neuroscience \& Biobehavioral Reviews 37(5): 930-949.

\section{P254}

Thoracic ultrasound: potential new tool for physiotherapists in respiratory management-a narrative review Aymeric Le Neindre ${ }^{1}$, Silvia Mongodi ${ }^{2}$, Francois Philippart ${ }^{3}$, Béaïd Bouhemad

${ }^{1}$ Intensive Care Unit and Department of Critical Care Medicine, Groupe Hospitalier Paris Saint-Joseph, Paris, France; ${ }^{2}$ Department of Anesthesia and Intensive care, Fondazione IRCCS Policlinico S Matteo, Pavie, Italy; ${ }^{3}$ Intensive Care Unit and Department of Critical Care Medicine, Groupe Hospitalier Paris-Saint-Joseph, Paris, France; ${ }^{4}$ Department of Anesthesia and Surgical Intensive Care Unit, CHU Dijon, Dijon, France
Correspondence: Aymeric Le Neindre - aymeric.leneindre@gmail.com Annals of Intensive Care 2016, 6(Suppl 1):P254

Introduction The use of diagnostic ultrasound by physiotherapists is not a new concept; it is frequently performed in musculoskeletal physiotherapy. Physiotherapists currently lack accurate, reliable, sensitive and valid measurements for the assessment of the indications and effectiveness of chest physiotherapy. Thoracic ultrasound may be a promising tool for the physiotherapist and could be routinely performed at patients' bedsides to provide real-time and accurate information on the status of pleura, lungs and diaphragm.

Materials and methods This is a narrative review. This review refers to lung and diaphragm ultrasound semiology and how thoracic ultrasound should be used in the physiotherapist's clinical decision-making process.

Results Lung ultrasound provides real-time and accurate information about the pleura, lung and diaphragm statuses of the patient: This allows the assessment of lung aeration, including interstitial syndrome to lung consolidation. Each ultrasound pattern is related to a level of aeration as follows: normal with A lines indicate a wellaerated lung; B lines indicate alveolar-interstitial syndrome and increased extravascular lung water; and lung consolidation indicates total loss of aeration.

A physiotherapist must to accurately evaluate a patient in order to select the most suitable intervention. Thus, to reliably assess the lung status, lung ultrasound examinations should be comprehensive (i.e., both lungs and all of the chest areas should be investigated).

In cases of ICU bedridden patients, B lines are found primarily in the most dependent parts of the lungs and are frequently associated with basal lung consolidations; thus, body positioning and mobilization might be a suitable strategy to improve lung aeration.

In case of confluent B lines, alveoli are partially filled by fluid. Techniques aimed at improving ventilation and preventing lung consolidation should be considered and include positive expiratory pressure, body positioning and mobilization.

At the patient's bedside, LUS performs much better than chest X-rays at diagnosing lung consolidation. LUS characteristics thus allow physiotherapists to quickly distinguish between atelectasis and pneumonia and to implement a suitable therapeutic strategy. For example, the dynamic air bronchogram rules out atelectasis.

In cases of lobar or hemilobar lung consolidation with fluid bronchograms in mechanically ventilated patients, the treatment should imply techniques that increase the expiratory flow rate (e.g., huffing, exsuflator) with adjustments to the ventilator settings. With lung ultrasound, the physiotherapist monitors the consolidation and evaluates the effectiveness of treatments.

If the physiotherapist identifies a pleural effusion, it seems reasonable to address the patient and wait for medical or surgical treatment to be completed. Then, the physiotherapist may help to implement supportive respiratory management if a chest drain is not required.

Discussion In addition to clinical examinations, lung ultrasound may guide the choice of chest physiotherapy strategies (e.g., alveolar syndrome, lung consolidation) or help to determine whether the patient requires medical intervention and no chest physiotherapy (e.g., pneumothorax, severe pleural effusion). An appropriate level of training is necessary to implement lung ultrasound in clinical practice. Thoracic ultrasound may be used in clinical research for chest physiotherapy because it is accurate, reliable and reproducible.

Conclusion Diagnostic ultrasound performs significantly better than chest X-rays or auscultation at diagnosing lung deficiencies that are relevant for the physiotherapist. Physiotherapists should use lung ultrasound semiology to guide, monitor and evaluate their chest physiotherapy treatments. This potential new tool for physiotherapists still requires significant development before being used commonly.

Competing interests None. 


\section{P255}

In vitro comparison of a vibrating-mesh nebulizer operating in expiratory synchronized and inspiratory nebulization modes during noninvasive ventilation

Joana Rodrigues ${ }^{1}$, Caroline Botteau ${ }^{1}$, Jonathan Dugernier ${ }^{2}$, Giuseppe Liistro $^{3}$, Gregory Reychler ${ }^{3}$, Jean-Bernard Michotte

${ }^{1}$ Haute école de santé vaud, University of Applied Sciences and Arts Western Switzerland (HES-SO), Lausanne, Switzerland; ${ }^{2}$ Sevice des soins intensifs, Cliniques Universitaires Saint-Luc, Bruxelles, Belgium; ${ }^{3}$ Service de pneumologie, Cliniques Universitaires Saint-Luc, Bruxelles, Belgium Correspondence: Joana Rodrigues - joana.rodrigues@hesav.ch Annals of Intensive Care 2016, 6(Suppl 1):P255

Introduction During nebulization associated with noninvasive ventilation using single-limb circuit bilevel ventilator, it has been shown that a part of drug nebulized during expiratory phase is held in the circuit and delivered during the next inspiratory phase. This "reservoir effect" could potentially improve drug delivery. Also, the use of an inspiratory synchronized nebulization has been shown to be effective in ventilated patient. The aim of this in vitro study was to compare aerosol delivery of amikacin using two different vibrating-mesh nebulization modes coupled to a single-limb circuit bilevel ventilator: (1) an experimental expiratory (Expi-Neb) and (2) inspiratory (Inspi-Neb) synchronized nebulization modes.

Materials and methods Using an adult lung bench model of noninvasive ventilation, we coupled a vibrating-mesh nebulizer with a bilevel ventilator. The nebulizer was charged with amikacin solution $(250 \mathrm{mg} / 3 \mathrm{~mL}$ ) and positioned before and after the exhalation port (starting from the lung model). An experimental control system was used to perform an expiratory (aerosol generated during only the whole expiratory phase) and inspiratory (aerosol generated during only the whole inspiratory phase) synchronized nebulization modes. Inhaled doses were assessed by residual gravimetric method.

Results Irrespective of the nebulizer position, Inspi-Neb produced higher inhaled dose than Expi-Neb (position before exhalation port: $75.8 \pm 0.9$ vs $55.9 \pm 0.8 \%$ of nominal dose; position after exhalation port: $34.0 \pm 0.4$ vs $15.6 \pm 0.1 \%$ of the nominal dose; $p<0.05$ for both). At position after exhalation port, adding $289-\mathrm{mL}$ corrugated piece of tubing between exhalation port and nebulizer was associated with an increase in aerosol delivery of Expi-Neb $(+5.7 \pm 0.2 \%$ of the nominal dose; $p<0.05$ )

Conclusion During simulated noninvasive ventilation with a singlelimb circuit bilevel ventilator, aerosol delivery with a vibrating-mesh nebulizer was dependent on nebulization mode and position. Expiratory synchronized nebulization mode was less efficient than the inspiratory synchronized mode.

\section{Competing interests None.}

\section{P256}

Evaluation of a new system of oxygen administration: the Wenoll system

Frédéric Duprez ${ }^{1}$, François Waroquier², Karel Christiaens², Grégory Cuvelier $^{3}$, Frank Vantrimpont ${ }^{2}$, Sharam Machayeckhi ${ }^{1}$

${ }^{1}$ ICU, C.H. Epicura, Hornu, Belgium; '2Emergency unit, C.H. Epicura, Hornu, Belgium; ${ }^{3}$ Laboratoire de l'effort et du mouvement, Condorcet, Tournai, Belgium

Correspondence: Frédéric Duprez - dtamedical@hotmail.com Annals of Intensive Care 2016, 6(Suppl 1):P256

Introduction $\mathrm{O}_{2}$ administration is essential for patient survival in clinical situation as: reanimation or anesthesia of a polytrauma, CO poisoning, decompression sickness, etc.

When the oxygen stock is limited (critical situations as war zones, disasters, etc.), the delivery of high $\mathrm{FiO}_{2}$ for an extended period may become insufficient.

To limit the $\mathrm{O}_{2}$ volume required in spontaneously breathing patients, we can use the Wenoll system (rebreather with closed circuit to recapture the exhaled $\mathrm{O}_{2}$ ).
This closed circuit provides a $100 \% \mathrm{FiO}_{2}$ for a low flow rate for patients at rest: $\mathrm{O}_{2}$ flow rate covers $\mathrm{O}_{2}$ consumption $\left(\mathrm{VO}_{2}\right)$ and a soda lime cartridge absorbs the $\mathrm{CO}_{2}$ produced by the patient's metabolism.

Since we know that an $\mathrm{O}_{2}$ flow rate of 1 to $2 \mathrm{l} / \mathrm{min}$ with an usual $\mathrm{O}_{2}$ cylinder $\left(\mathrm{O}_{2} \mathrm{C}\right)$ is generally sufficient to ensure the $\mathrm{VO}_{2}$ of a patient ventilating at rest, our goal was to determine whether the use of a portable oxyconcentrator (POC) could effectively replace the use of a $\mathrm{O}_{2} \mathrm{C}$ and thus ensure proper $\mathrm{FiO}_{2}$.

Materials and methods The study was conducted on bench with the Wenoll system connected to an a two-compartment adult lung model (Dual Test Lung ${ }^{\circledR}$ ) controlled by a Maquet Servo $I^{\circledR}$ ventilator (Vt: 0.5 L; I/E: $1 / 2$; Peep: $0 \mathrm{~cm} \mathrm{H2O}$ : Rf 10 to $40 \mathrm{cpm}$ ). Different minute ventilation (MV: 5 to $20 \mathrm{l} / \mathrm{min}$ ) were investigated. An oxygen flow rate of $2 \mathrm{l} /$ min $\mathrm{O}_{2}$ was ensured using either a $\mathrm{O}_{2} \mathrm{C}$ (B2: Air Liquide ${ }^{\mathrm{TM}}$ ) or an POC (Sequal Saros ${ }^{\circledR}$ ). Two mode of $\mathrm{O}_{2}$ delivery systems were tested: Continuous $\mathrm{O}_{2}\left(\mathrm{O}_{2} \mathrm{C}\right.$ and $\left.\mathrm{POC}\right)$ and bolus $\mathrm{O}_{2}: 74 \mathrm{ml}$ at each breath (with $\mathrm{POC}$ ). The $\mathrm{FiO}_{2}$ and $\mathrm{MV}$ measurements were made using a iWor ${ }^{\circledR}$ acquisition system (GA207 gas analyzer and analog/digital IX/228 s) and LabScribe II ${ }^{\circledR}$ software. Statistical test used: ANOVA followed by a post hoc test (Tukey).

Results After a period of $5 \mathrm{~min}$ necessary for the denitrogenation of the Wenoll system dead volume $\left(\mathrm{O}_{2}\right.$ flow rate: $\left.2 \mathrm{l} / \mathrm{min}\right)$, the $\mathrm{FiO}_{2}$ reached a value of $100 \%$ with $\mathrm{O}_{2} \mathrm{C}$ and $92 \%$ with $\mathrm{POC}$ at continuous flow $(p<0.05)$. With POC, there was no significant difference between continuous and bolus mode (pulsed volume of $74 \mathrm{ml}$ at each respiration).

Conclusion We hypothesized that the oxygen extraction ratio $(E)$ of air in the lung $\left(E=\mathrm{FiO}_{2}-\mathrm{FEO}_{2}\right)$ was stable and equal to $5 \%$. In this case, if $\mathrm{VI}$ is equal to $\mathrm{VE}, \mathrm{VO}_{2}$ is:

$$
\mathrm{VO}_{2}\left(\mathrm{Lmin}^{-1}\right)=\left(\mathrm{VI} * \mathrm{FIO}_{2}\right)-\left(\mathrm{VE} * \mathrm{FeO}_{2}\right)
$$

VI volume of air inspired per minute (I min-1)/VE volume of air expired per minute (I min-1), FiO2: Fraction inspired of $02 / F e O 2$ : fraction expired of $\mathrm{O} 2$.

Consequently, for MV from 5 to $20 \mathrm{l} / \mathrm{min}$, with the use of an POC coupled to Wenoll system, we can administer for a long period $\mathrm{FiO}_{2}$ near $92 \%$ with a very low $\mathrm{O}_{2}$ flow rate $(2 \mathrm{l} / \mathrm{min})$ to ensure the patient $\mathrm{VO}_{2}$. Note that the duration of effectiveness of the decarboxylation by soda lime cartridge of Wenoll system is estimated (by the manufactory) to be $5 \mathrm{~h}$ for a patient ventilating at rest.

\section{Competing interests None.}

\section{Reference}

1. Jurgen Graf. In-Flight Medical Emergencies. Dtsch Arztebl Int. 2012 Sep; 109(37): 591-602.

\section{P257}

Evaluation of three portable oxyconcentrators in high altitude

Frédéric Duprez ${ }^{1}$, François Waroquier ${ }^{2}$, Karel Christiaens ${ }^{2}$, Grégory

Cuvelier ${ }^{3}$, Charbel Elkhawand ${ }^{4}$, Frank Vantrimpont ${ }^{2}$, Sharam

Machayeckhi ${ }^{1}$, Frank Vermeesh ${ }^{2}$

${ }^{1}$ ICU, C.H. Epicura, Hornu, Belgium; ${ }^{2}$ Emergency Unit, C.H. Epicura, Hornu, Belgium; ${ }^{3}$ Laboratoire de l'effort et du mouvement, Condorcet, Tournai, Belgium; ${ }^{4}$ Pneumology, C.H. Epicura, Hornu, Belgium

Correspondence: Frédéric Duprez - dtamedical@hotmail.com Annals of Intensive Care 2016, 6(Suppl 1):P257

Introduction The $\mathrm{O}_{2}$ delivery in high altitude areas (HAA - > 8000 feet) is a major logistic problem. The oxyconcentrators (OCC) can be a solution to overcome this problem because they are an inexhaustible source of oxygen so far as their power supply can be ensured. Very few studies have examined the OCC in HAA. The aim of our study was to evaluate the $\mathrm{FiO}_{2}$ and $\mathrm{O}_{2}$ flow rate issued by three OCC in high altitudes.

Materials and methods Three OCC (Simply go ${ }^{\circledR}$, Devilbiss ${ }^{\circledR}$, EverFlow $\left.{ }^{\circledR}\right)$ were analyzed in continuous mode $\left(\mathrm{O}_{2}\right.$ flow rate: $\left.2 \mathrm{l} / \mathrm{min}\right)$ in an 
hypobaric chamber $(\mathrm{HC}$ ) (Aerospace Medicine Centre, Queen Astrid Military Hospital, Brussels). During the experimentation, the average temperature of the $\mathrm{HC}$ was maintained at $24{ }^{\circ} \mathrm{C}$ and relative humidity at $58 \%$. Seven altitude levels (from 0 to 18,000 Feet) were successively achieved. $\mathrm{FiO}_{2}$ was measured in continuous by an analog iWorx station/digital IWx/214 coupled to an analyzer of $\mathrm{CO}_{2} / \mathrm{O}_{2}$ iWorx GA200. $\mathrm{O}_{2}$ flow rate was analyzed by a thermal mass flow meter Vogtlyn ${ }^{\mathrm{TM}}$ Red $\mathrm{Y}$. A oxygen cylinder was used as a reference gas to recalibrate $\mathrm{O}_{2}$ analyzer at each level of altitude.

Statistical test: ANOVA.

Results For the three OCC: Average $\mathrm{FiO}_{2}$ was $93 \%( \pm 1 \%)$ at main sea level and $91 \%( \pm 2 \%)$ by 18,000 feet $(p>0.05)$.

Average $\mathrm{O}_{2}$ flow rate was $1.8 \mathrm{I} / \mathrm{min}$ at main sea level and $1.2 \mathrm{l} / \mathrm{min}$ by 18,000 feet $(p>0.05)$.
Conclusion At main sea level and 18,000 feet, the tested OCC were able to ensure a $\mathrm{FiO}_{2}$ which meets the manufacturer's standards. However, we observed a decrease oxygen flow rate during the climbing, but was not statistically significant for an $\mathrm{O}_{2}$ flow rate of $2 \mathrm{l} / \mathrm{min}$.

In our study, with an oxygen flow rate of $2 \mathrm{~L} / \mathrm{min}$, OCC are able to administer the correct oxygen concentration in high altitude.

\section{Competing interests None.}

\section{Reference}

1. Rehan Masroor. Use of a portable oxygen concentrator and its effect on the overall functionality of a remote field medical unit at 3650 meters elevation. Anaesth, Pain \& Intensive Care; Vol 17(1) JAN-APR 2013. 